



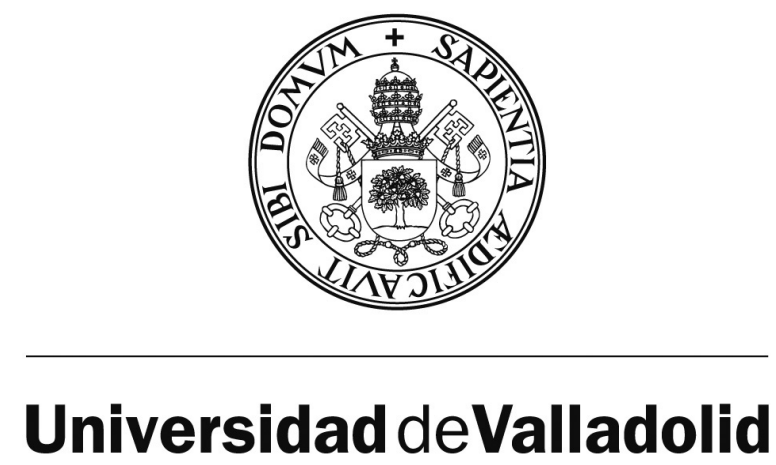

\author{
FACULTAD DE CIENCIAS \\ DEPARTAMENTO DE FÍSICA APLICADA
}

TESIS DOCTORAL:

\title{
ESTUDIO EXPERIMENTAL Y TEÓRICO DE MEZCLAS BINARIAS DE AMINAS $Y$ CETONAS
}
Presentada por D. IVÁN ALONSO MIGUEL para optar al grado de doctor por la Universidad de Valladolid

Dirigida por:

DR. JOSÉ CARLOS COBOS HERNÁNDEZ DR. JUAN ANTONIO GONZÁLEZ LÓPEZ 



\title{
Grupo Especializado en Termodinámica de los Equilibrios entre Fases
}

\author{
Departamento de Física Aplicada \\ Facultad de Ciencias. Universidad de Valladolid \\ E-47071 Valladolid (España) \\ http://www5.uva.es/getef/
}

D. José Carlos Cobos Hernández y D. Juan Antonio González López, Catedráticos de Universidad del área de Física Aplicada de la Universidad de Valladolid.

\section{CERTIFICAN:}

Que la presente memoria "ESTUDIO EXPERIMENTAL Y TEÓRICO DE MEZCLAS BINARIAS DE AMINAS Y CETONAS" ha sido realizada bajo su dirección por D. Iván Alonso Miguel en el Departamento de Física Aplicada de la Facultad de Ciencias de la Universidad de Valladolid, y constituye su Tesis para optar al grado de Doctor en Física.

Y para que conste, y en cumplimiento de la legislación vigente, firma el presente certificado en Valladolid, el 18 de agosto de 2014:

Fdo.: José Carlos COBOS HERNÁNDEZ

Fdo.: Juan Antonio GONZÁLEZ LÓPEZ 



\section{AGRADECIMIENTOS}

Me es imposible imaginar la realización de este trabajo sin la ayuda de aquellas personas que, de uno u otro modo tanto me han ayudado en esta etapa de mi vida. Entre ellos se encuentran los integrantes fundadores del grupo de investigación G.E.T.E.F. (Grupo Especializado en la Termodinámica de los Equilibrios entre Fases), con los que he trabajado codo con codo durante todo este tiempo y a quienes considero mis padres científicos. Entre las innumerables aportaciones recibidas por cada uno de ellos, a modo de ejemplo puedo citar las siguientes: de Isaías, no sólo todas las indicaciones en torno a cuestiones experimentales, las que me han servido para mejorar como físico experimental, sino también todas las gestiones realizadas para la adquisición de elementos de naturaleza variada; de Juan Antonio, la fidedigna aplicación de los distintos modelos teóricos y la concienzuda revisión de todos los Capítulos de esta Tesis; de José Carlos, las largas conversaciones telefónicas, los seminarios y sobre todo, sus excepcionales capacidades como profesor, que me indicaron inequívocamente allá por el año 2009 que debía de realizar la Tesis Doctoral bajo su tutela. Por todo ello les doy las gracias.

No merecen menor reconocimiento mis compañeros Víctor e Ismael, sin los que el trabajo diario en el laboratorio no hubiese sido tan gratificante. Al resto de compañeros que son o han sido miembros del G.E.T.E.F. también les agradezco su ayuda, pues sus trabajos han sido claves en la elaboración de la presente Memoria.

También son dignos de mención todos los integrantes del Departamento de Física Aplicada, quienes siempre se han interesado por mi trabajo. Recibid todos un fuerte abrazo. 
Especial mención merece el agradecimiento a mi hermano Ángel, cuyos conocimientos como diseñador gráfico se han plasmado en el trazado de varias de las llustraciones presentadas en los Capítulos 3 y 4 de este trabajo.

Por último, y no por ello menos importante, agradezco a mis padres, Adelina y Miguel, mis abuelos, y al resto de mi familia y mis amigos, todo el apoyo moral recibido durante el tiempo que me ha llevado la elaboración de esta Tesis Doctoral. Particularmente, a mi novia Rebeca, quien ha soportado mi presencia etérea durante todo este tiempo con total entereza, y a mi abuelo Miguel Ángel, sin quien no hubiese llegado a ser la persona que hoy en día soy y de quien tantos buenos recuerdos guardaré para siempre. 
TABLA DE CONTENIDOS

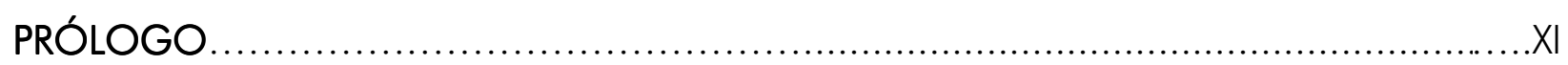

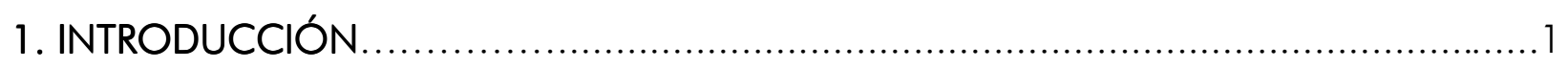

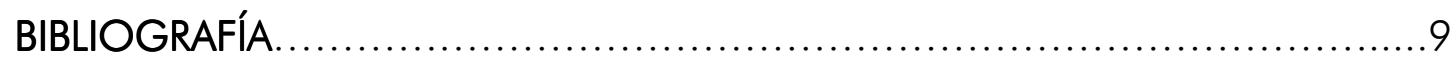

2. TERMODINÁMICA APLICADA A LOS SISTEMAS MONO Y MULTICOMPONENTES. .....13

2.1. CONCEPTOS INICIALES EN TERMODINÁMICA .................................13

2.1.1. Variables termodinámicas. Ecuaciones de estado..............................13

2.1.2. Primer principio de la Termodinámica........................................16

2.1.3. Segundo principio de la Termodinámica.................................17

2.1.4. Cambios físico-químicos. Potencial químico. Afinidad......................17

2.1.5. Ecuación fundamental de la Termodinámica. Potenciales

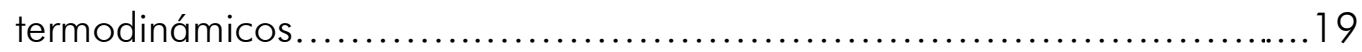

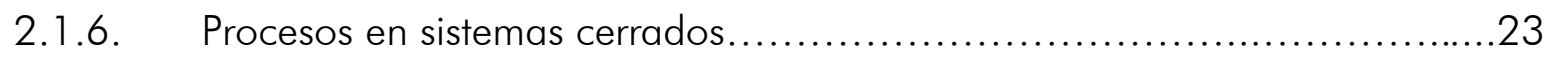

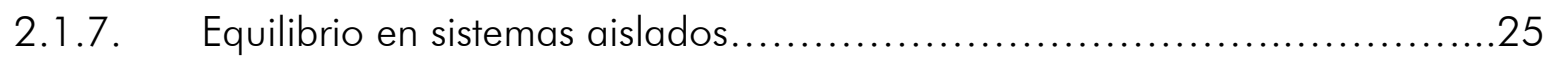

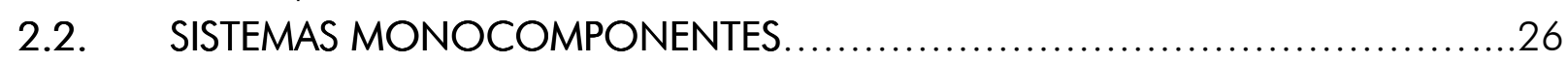

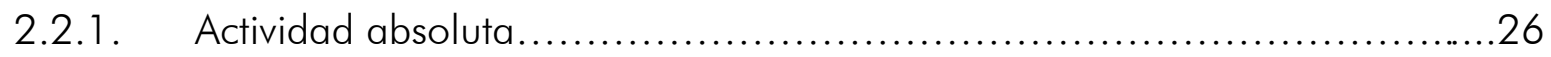

2.2.2. Ecuaciones de estado de gases puros........................................27

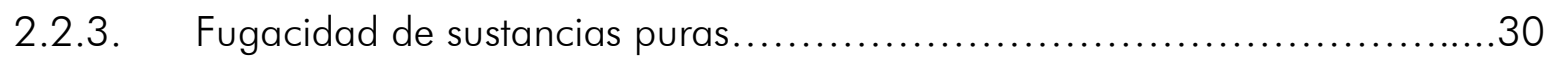

2.3. SISTEMAS DE VARIOS COMPONENTES: MEZCLAS ...............................

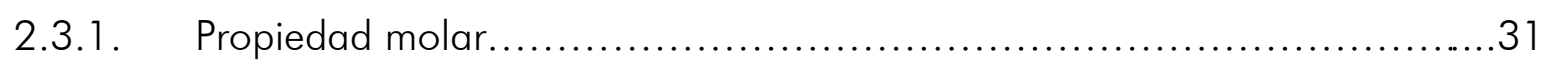

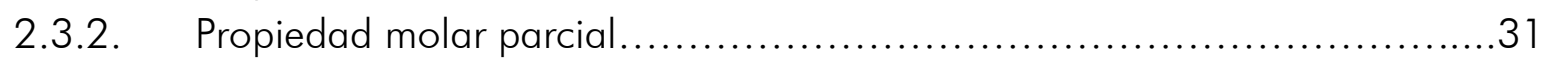


2.3.3. Actividad relativa. Fugacidad en mezclas..................................37

2.3.4. Coeficiente de fugacidad..............................................39

2.3.5. Mezcla ideal. Leyes de Lewis - Randall, Raoult y Henry........................41

2.3.6. Mezcla real. Funciones de exceso. Coeficiente de actividad...................46

2.3.7. Coeficiente de actividad a partir de funciones de exceso. Ecuaciones de ajuste.....

BIBLIOGRAFÍA

3. MEDIDA DE VOLÚMENES Y COMPRESIBILIDADES ISOENTRÓPICAS DE EXCESO

3.1. MEDIDA DEL VOLUMEN DE EXCESO .........................................62

3.1.1. Métodos indirectos................................................. 63

3.1.1.1. Agua como patrón de densidad....................................64

3.1.1.2. Mercurio como patrón de densidad......................................65

3.1.1.3. Otros líquidos como patrones de densidad............................66

3.1.1.4. Medida de la densidad de líquidos y sus mezclas......................66

3.1.2. Métodos directos..............................................................

3.1.2.1. Dilatómetros de tipo batch....................................... 76

3.1.2.2. Dilatómetros de dilución............................................. 77

3.2. MEDIDA DE LA COMPRESIBILIDAD ISOENTRÓPICA DE EXCESO .................79

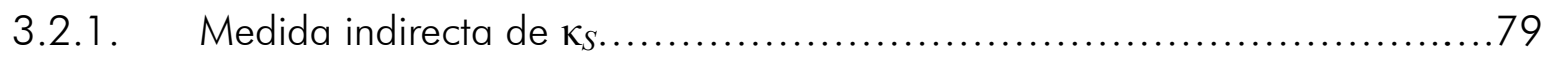

3.2.1.1. Método interferométrico............................................. 80

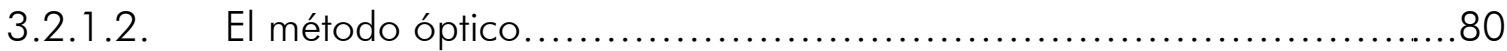

3.2.1.3. El método del pulso............................................... 80

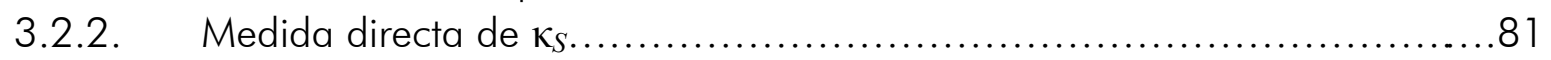

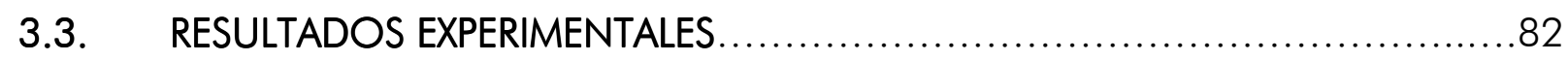

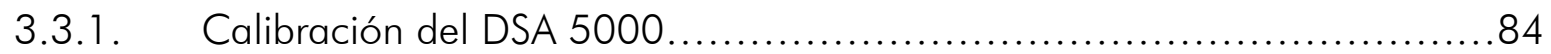

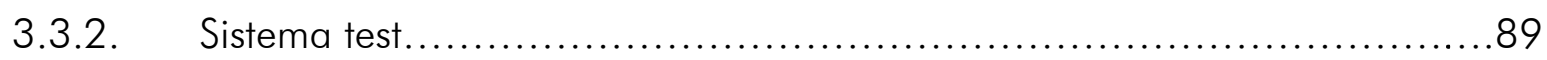

3.3.3. Mezclas cetona +amina y $\mathrm{N}$-metilanilina + alcano.........................92

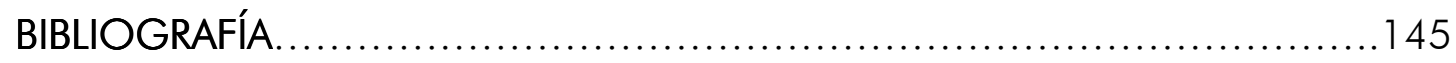

4: MEDIDA DE LAS ENTALPÍAS DE EXCESO ......................................... 153

4.1. TÉCNICAS EXPERIMENTALES PARA LA MEDIDA DE ENTALPÍA DE EXCESO ....156

4.1.1 Métodos indirectos................................................... 156

4.1.2 Métodos directos.......................................................... 157

4.1.2.1 Clasificación de las diferentes técnicas calorimétricas.................... 157

4.1.2.2 Calorímetros más usuales para la determinación....................... 165

4.1.2.3 Calorímetro Tian-Calvet........................................... 169

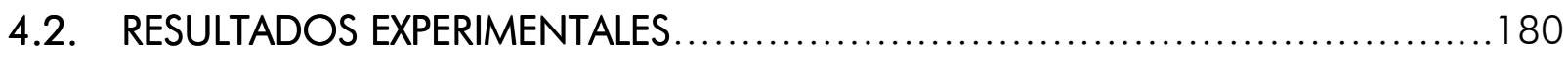

4.2.1 Calibración del microcalorímetro Tian-Calvet............................ 180

4.2.2 Sistema test....................................................... 184

4.2.3 Mezclas binarias amina + cetona y $\mathrm{N}$-metilanilina + alcano.............. 189

4.2.4 Mezcla 1-propanol (1) + trietilamina (2) + 2-butanona (3)................242

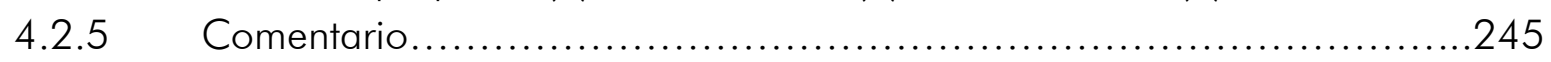

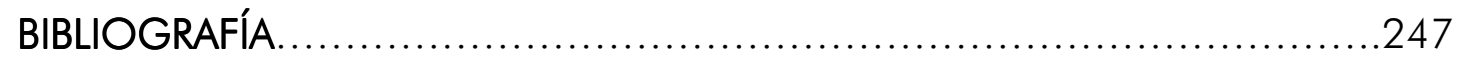


5: INTERPRETACIÓN DE LOS RESULTADOS EXPERIMENTALES .........................257

5.1. DESVIACIONES RESPECTO DEL COMPORTAMIENTO IDEAL...................257

5.2. MARCO DE REFERENCIA DE ESTE TRABAJO ....................................263

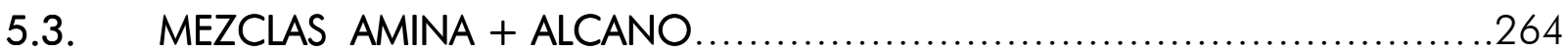

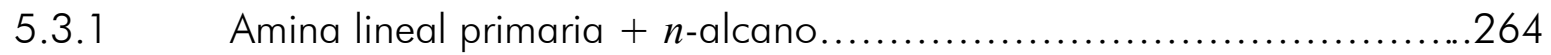

5.3.2 Amina lineal secundaria $+n$-alcano................................266

5.3.3 Amina lineal terciaria $+n$-alcano....................................268

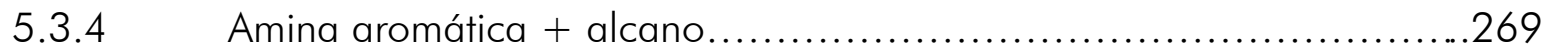

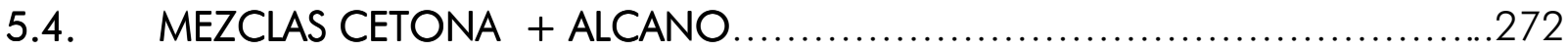

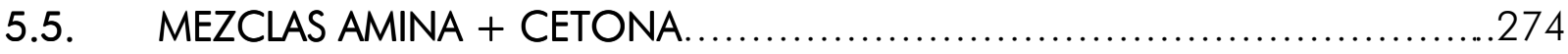

5.5.1 Amina aromática + cetona............................................275

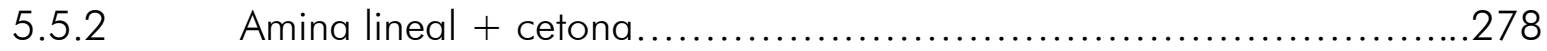

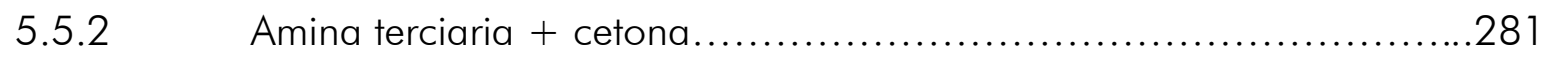

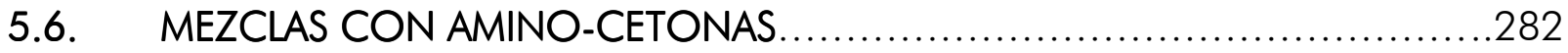

5.7. MEZCLAS 1-PROPANOL+ TRIETILAMINA+2-BUTANONA ...................283

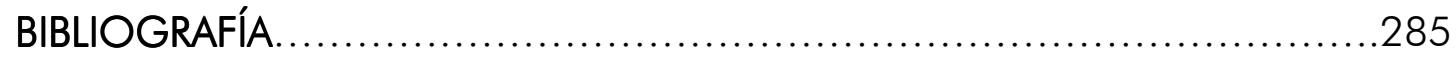

6: TEORÍAS DE LÍQUIDOS Y DISOLUCIONES DE NO ELECTROLITOS..................291

6.1 EL PROBLEMA DE LOS SISTEMAS CON MUCHAS INDIVIDUALIDADES.........291

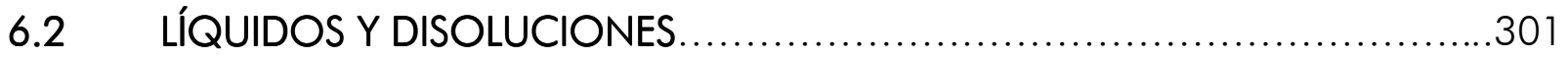

6.3 FUNCIONES DE DISTRIBUCIÓN MOLECULAR...............................314

6.3.1 Propiedades termodinámicas en función de la función de distribución

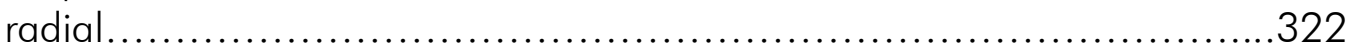

6.3.2 Determinación de la función de correlación................................323

6.3.3 Teoría de Kirkwood-Buff e inversión de Ben-Naim............................325

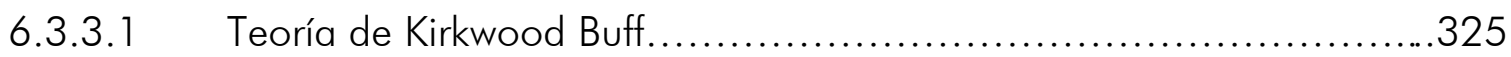

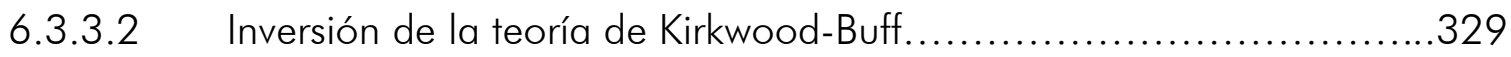

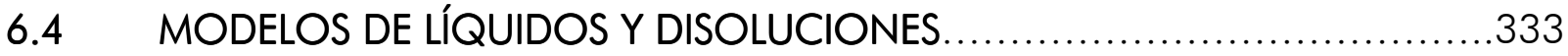

6.4.1 Principios básicos de los modelos de celdas................................335

6.4.1.1 Modelo de Guggenheim-Barker-Flory-Huggins........................343

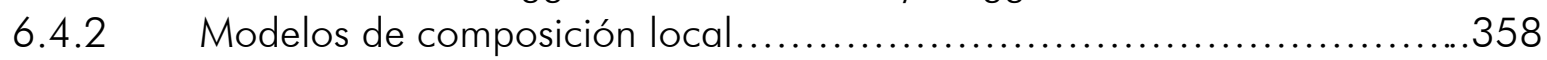

6.4.3 Teoría de disoluciones conformacionales..................................360

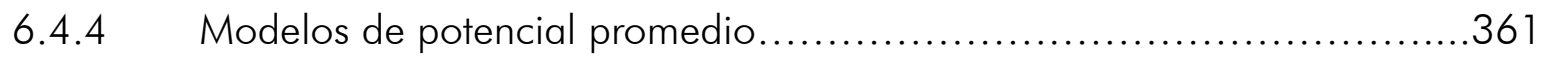

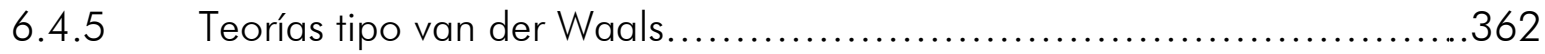

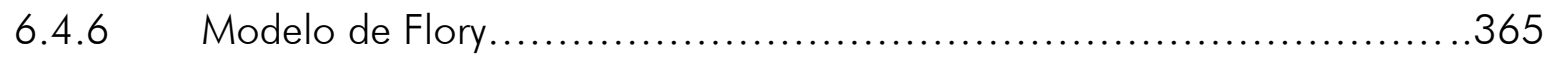

6.4.6.1 Hipótesis. Modelo de Flory para líquidos puros..........................365

6.4.6.2 Hipótesis. Modelo de Flory para mezclas.................................369

6.4.6.3 Estudio de la hipótesis de mezcla al azar. Determinación de $\chi_{A B} \ldots \ldots . .376$

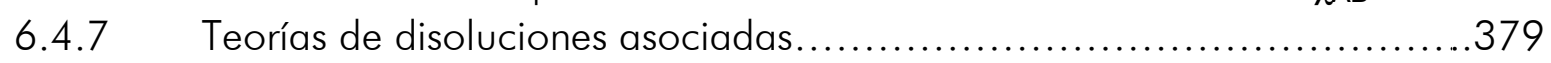

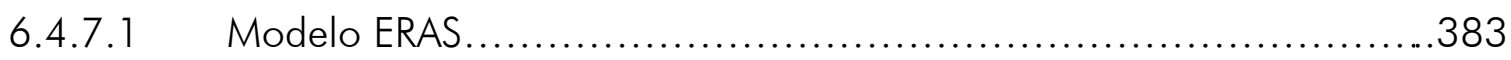

6.4.8 Métodos de contribución de grupos funcionales...........................389

6.4.9 Modelo de interacción de grupos funcionales de K-G-B. ..................392 
6.4.9.1 Definiciones e hipótesis de partida. Modelo de interacción molecula..392

6.4.9.2 Modelo estadístico. Función de partición configuracional...............395

6.4.9.3 DISQUAC (DISpersive-QUAsi Chemical).............................401

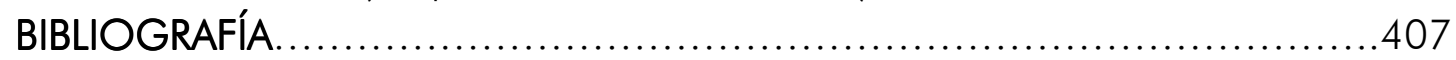

7 : APLICACIÓN DE LOS MODELOS TEÓRICOS A LOS DATOS EXPERIMENTALES ........425

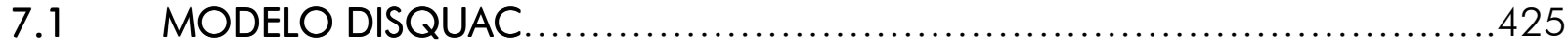

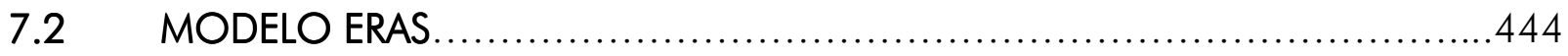

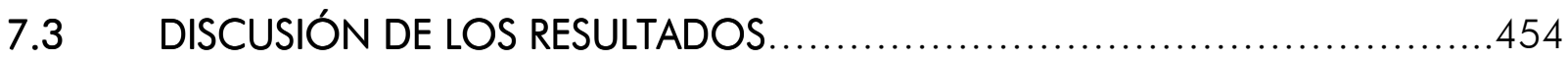

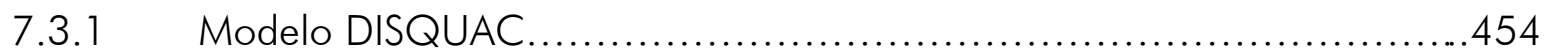

7.3.1.1 Mezclas amina + alcano......................................... 454

7.3.1.2 Mezclas amina aromática o amina lineal secundaria + cetona...........454

7.3.1.3 Mezclas amina lineal terciaria + cetona o amino-cetona + alcano....455

7.3.1.4 Mezclas ternarias..................................................... 455

7.3.2 Modelo ERAS............................................................456

7.3.2.1 Mezclas amina + alcano y alcohol + amina.........................456

7.3.2.2 Mezclas amina + cetona...........................................457

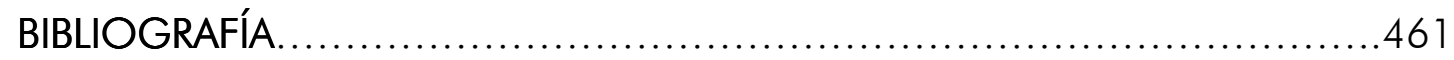

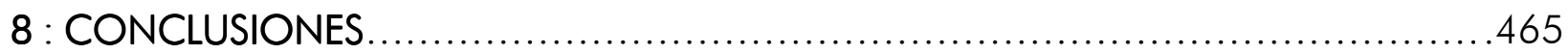

A: ELECCIÓN DEL NÚMERO DE COEFICIENTES DEL AJUSTE. CALIDAD DEL AJUSTE

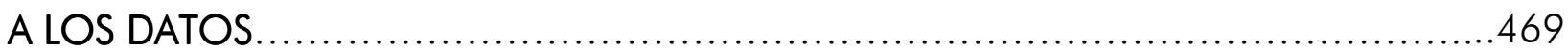

A. 1 CONSIDERACIONES GENERALES SOBRE EL AJUSTE A UNA FUNCIÓN.......469

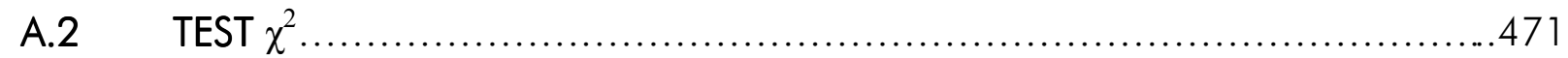

A.3 TEST F. TEST DEL TÉRMINO ADICIONAL....................................472

B: PROPIEDADES FÍSICO-QUÍMICAS Y CARACTERÍSTICAS DE INTERÉS BIOLÓGICO, FISIOLÓGICO E INDUSTRIAL DE LAS MOLÉCULAS ESTUDIADAS .....................475

B. 1 FORMACIÓN BIOLÓGICA Y CICLO DEL NITRÓGENO .......................475

B.2 EL GRUPO CARBONILO EN BIOLOGÍA Y FISIOLOGÍA .......................477

B.3 AMINAS Y CETONAS DE USO COTIDIANO ................................479

B.4 PROPIEDADES FÍSICOQUÍMICAS DE LAS AMINAS ..........................484

B.5 PROPIEDADES FISICOQUÍMICAS DE LAS CETONAS .........................492

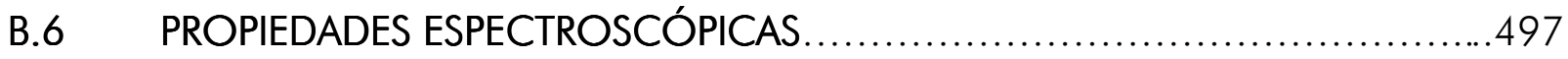

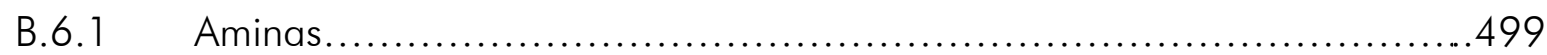

B.6.1.1 Par de electrones libres............................................499

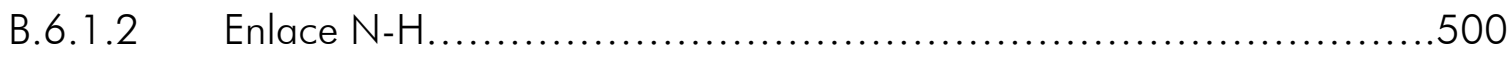

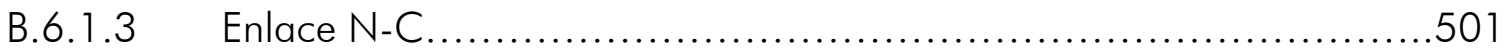

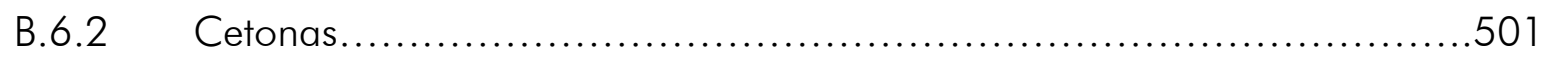

C: ANÁLISIS ESPECTROSCÓPICO DE MEZCLAS AMINA+CETONA. IMINAS..............505

BIBLIOGRAFÍA.................................................... 517 


\section{PROLOGO}

$\mathrm{D}$ entro del Illamado' Departamento de Física Aplicada de la Facultad de Ciencias de la Universidad de Valladolid existe una auténtica tradición en lo que al estudio de las propiedades termodinámicas de mezclas de líquidos no electrolitos se refiere. Estos estudios comenzaron en la década de 1970 con los trabajos de M. A. Villamañán [1], J.C. Cobos [2], [3] y C. Casanova (en el por aquel entonces llamado Departamento de Física Fundamental) y las colaboraciones con las Universidades de Santiago de Compostela, de Marsella y de Clermont-Ferrand [4]. A mediados de 1980 comenzaron a trabajar los otrora estudiantes de doctorado I. García de la Fuente [5] y J.A. González [6] que desde entonces ya nunca se separaron en lo que a tareas de investigación se refiere de J.C. Cobos, fundando los tres en 1994 el grupo de investigación G.E.T.E.F. (Grupo Especializado en Termodinámica de los Equilibrios entre Fases), dentro del cual se ha llevado a cabo la investigación que en este documento se expone.

\section{P.1 Miembros del G.E.T.E.F.}

Desde su fundación, se han ido añadiendo nuevos miembros al grupo. Así, son o han sido integrantes del mismo:

Los catedráticos:

Don José Carlos Cobos Hernández.

\footnotetext{
${ }^{1}$ A fecha de 22/10/2013.
} 
Don Isaías García de la Fuente.

Don Juan Antonio González López.

Los doctores:

Don Francisco Javier Carmona del Río.

Don Juan María Fernández Martínez.

Don Ismael Mozo Ruiz.

Don Nicolás Riesco Fernández.

Doña Susana Villa Vallejo.

Los estudiantes de doctorado:

Don Víctor Alonso Gómez.

Don Iván Alonso Miguel.

Don Francisco Javier Arroyo Maestu.

Doña Ana Cobos Huerga.

Doña Marta Fernández Regúlez.

Don Juan Lobos Martín.

Don Rubén Martínez Díez.

Don Luis Felipe Sanz del Soto.

Los licenciados:

Doña María Aboy Cebrián.

Doña Angela Mediavilla Trabada.

Don Tomás Romero Albillos.

Don Miguel Ángel Rubio Hernández.

Don Andrés Serna Gutiérrez.

\section{P.2 Líneas de investigación}

La actividad investigadora del G.E.T.E.F. viene definida por una línea general que da nombre al grupo y seis líneas específicas que desarrollan esta línea general.

\section{P.2.1 Línea General:}

Estudio termodinámico de los equilibrios entre fases que aparecen en las mezclas de gases, líquidos y sólidos.

Entre los proyectos experimentales llevados a cabo dentro de esta línea general de investigación destacan los siguientes:

a) Montaje y puesta a punto de un microcalorímetro Tian-Calvet.

b) Montaje y puesta a punto de un densímetro Anton Paar DMA-602. 
c) Montaje y puesta a punto de un densímetro y analizador de la velocidad de propagación del sonido Anton Paar DSA-5000.

d) Montaje y puesta a punto de un dispositivo experimental para la determinación de las curvas de equilibrio líquido-líquido y sólido-líquido mediante la observación del fenómeno de opalescencia crítica.

e) Montaje y puesta a punto de un refractómetro Bellingham + Stanley RFM 970.

f) Montaje y puesta a punto de un analizador de impedancias Agilent 4294A.

g) Montaje y puesta a punto de un calorímetro diferencial de barrido TA Instruments DSC 2000.

Entre los proyectos teóricos llevados a cabo dentro de esta línea general de investigación destacan los siguientes:

a) Aplicación del modelo DISCUAQ a mezclas de fluidos y aleaciones.

b) Aplicación del modelo UNIFAC en sus distintas versiones a mezclas de fluidos y aleaciones.

c) Aplicación del la teoría de Flory a mezclas de fluidos y aleaciones.

d) Aplicación del modelo ERAS a mezclas de fluidos y aleaciones.

\section{P.2.2 Líneas Específicas:}

a) Estudio experimental de mezclas asociadas. En concreto:

- Estudio termodinámico de las mezclas alcohol + hidrocarburos alifáticos o aromáticos, lineales o cíclicos.

- Estudio termodinámico de las mezclas alcohol + éter.

- Estudio termodinámico de las mezclas hidroxiéter + hidrocarburos.

- Estudio termodinámico de las mezclas hidroxiéter + alcoholes.

- Estudio termodinámico de las mezclas hidroxiéter + éter.

- Estudio termodinámico de las mezclas amina primaria, secundaria o terciaria + hidrocarburos.

- Estudio termodinámico de las mezclas amina primaria, secundaria o terciaria + alcoholes.

- Estudio termodinámico de las mezclas amina primaria, secundaria o terciaria+ cetonas.

- Calibración de un micro-DSC. Determinación de la $C_{p}^{E}$ de las mezclas alcoholes + hidrocarburos.

b) Estudio experimental de mezclas con interacciones dipolares puras. Específicamente:

- Estudio termodinámico de las mezclas cetona + éter.

- Estudio termodinámico de las mezclas cetona + alcano.

- Estudio termodinámico de las mezclas amida lineal o cíclica + alcano. 
- Estudio termodinámico de las mezclas carbonatos de alquilo + disolventes orgánicos.

- Estudio termodinámico de las mezclas anhidridos de alquilo + disolventes orgánicos.

- Determinación de las $C_{p}^{E}$ de mezclas éteres + n-alcano.

c) Estudio experimental y teórico de mezclas cuya capacidad calorífica de exceso a presión constante presenta un doble mínimo en su dependencia con la concentración. Específicamente:

- Obtención de la ecuación exacta de la capacidad calorífica de exceso para diversas teorías fundamentales de mezclas.

d) Estudio teórico de modelos de contribución de grupos funcionales. Específicamente:

- Aplicación sistemática del modelo DISQUAC para justificar todo tipo de mezclas y equilibrios entre fases.

- Aplicación del modelo DISQUAC (teoría física que no utiliza constantes de asociación ni solvatación) para justificar las propiedades termodinámicas de mezclas asociadas (alcoholes + hidrocarburos, tetraclorometano, etc.).

- Aplicación de modelos de contribución de grupos para predecir el equilibrio líquido-vapor y las funciones de exceso de mezclas líquidas multicomponentes.

e) Estudio teórico de modelos basados en la hipótesis de mezcla al azar. Aplicación del modelo de Flory para justificar las propiedades termodinámicas de las mezclas de tipo molécula polar + hidrocarburo y molécula polar + molécula polar.

f) Estudio teórico de los modelos de asociación. Específicamente:

- Aplicación del modelo ERAS para justificar las propiedades termodinámicas de las mezclas asociadas.

g) Estudio teórico de mezclas líquidas mediante el modelo de Kirkwood-Buff. En concreto:

- Análisis de las mezclas líquidas mediante la determinación del factor de correlación de Kirkwood.

La capacidad investigadora y docente del G.E.T.E.F. no se ha limitado a las mencionadas líneas específicas; ejemplos de estos proyectos no incluidos en el esquema anterior son:

- Análisis matemático a priori de la ecuación de Wilson.

- Puesta a punto y calibración de un DSC-111 SETARAM. Determinación del equilibrio sólido-líquido de mezclas que pueden formar complejos en fase condensada.

- Calibración y medida de la densidad a alta presión y temperatura de líquidos puros y mezclas, mediante un densímetro de tubo vibrante tipo R. H. Word. 
- Desarrollo de una cubierta de calor latente para invernaderos en la comunidad autónoma de Castilla y León.

- Estudio termodinámico de las unidades estructurales básicas de polímeros (oligómeros) en disolución.

- Determinación de exponentes críticos a partir de curvas de coexistencia de equilibrio líquido-líquido.

- Estudio de la influencia del término combinatorio en la predicción de propiedades termodinámicas de mezclas con componentes de cadena muy larga.

- Estudio experimental y modelado de las transiciones de fase de primer orden sólido-sólido en mezclas de alcoholes con diferentes disolventes orgánicos.

- Estudio de mezclas líquidas de interés biológico (ácidos carboxílicos).

\section{P.3 Aparatos de Investigación}

El G.E.T.E.F. dispone de los siguientes equipos para desarrollar sus tareas de investigación:

- Microcalorímetro Tian-Calvet estándar.

Con una ganancia estática del orden de $65 \mu \mathrm{V} / \mathrm{mW}$ y un umbral de detección que en las actuales circunstancias es del orden de $2 \mu \mathrm{W}$, es un calorímetro de muy altas prestaciones que habitualmente se utiliza en la medida de entalpías de exceso. Ha sido dotado de un sistema de captación automatizada de datos mediante una tarjeta GPIB y el entorno de programación VEE de Agilent.

- Equipo para la determinación del equilibrio líquido-líquido.

Basado en el método de la opalescencia crítica, consiste en una célula de vidrio donde se insertan las mezclas, que se termostatan mediante un baño GRANT LCT 640, lo que permite variar su temperatura de forma controlada. Se utiliza como termómetro una sonda estándar de platino Pt-1000, calibrada por medio de un termómetro de cuarzo Hewlett-Packard 2804A. El proceso de medida está automatizado incorporando un fotodiodo cuya señal es recogida por un ordenador.

- Densímetro Anton Paar DMA-602.

Utilizado junto con un frecuencímetro Philips PM 6669 y un baño termostático GRANT W38 para la medida de densidades y volúmenes de exceso, alcanza una resolución relativa en densidad $\Delta \rho / \rho=6 \cdot 10^{-6}$ y una exactitud en los volúmenes de exceso de $\pm\left(0,01 \cdot\left|V_{m}^{E}\right|_{\max }+0,005 \mathrm{~cm}^{3} \cdot \mathrm{mol}^{-1}\right)$. Ha sido dotado de un sistema de captación automatizada de datos mediante una tarjeta QP Computer, compatible con el estándar IEEE-488 y programa en GWBASIC.

- Densímetro y analizador de la velocidad del sonido Anton Paar DSA 5000.

Para la medida de densidades y volúmenes de exceso y velocidades de propagación del sonido y diferencias de compresibilidad isoentrópica. Se ha conectado mediante un puerto serie a un ordenador y con el entorno de programación VEE de Agilent se controla de forma automática. El intervalo de medición de densidad del equipo es de 0 a $3 \mathrm{~g} \cdot \mathrm{cm}^{-3}$, de la velocidad del sonido de 1000 a $2000 \mathrm{~m} \cdot \mathrm{s}^{-1}$ y el de temperatura de 0 
a $70{ }^{\circ} \mathrm{C}$. La exactitud de las medidas es $\pm 5 \cdot 10^{-6} \mathrm{~g} \cdot \mathrm{cm}^{-3}$ para la densidad, $\pm 0.1 \mathrm{~m} \cdot \mathrm{s}^{-1}$ para la velocidad del sonido y $\pm 0.001^{\circ} \mathrm{C}$ para la temperatura.

- Calorímetro TA Instruments DSC Q2000.

Para la medida de capacidades caloríficas. Conectado a un refrigerador de nitrógeno líquido cubre el rango de temperatura de 180 a $725^{\circ} \mathrm{C}$. El montaje por defecto proporciona una sensibilidad igual a $0.2 \mu \mathrm{W}$.

- Analizador de impedancias Agilent 4294A y celda 16452A.

Para la determinación de permitividades dieléctricas de líquidos y sus mezclas y diferencias de permitividad dieléctrica de dichas mezclas en el intervalo de frecuencias $40 \mathrm{~Hz}-30 \mathrm{MHz}$.

- Refractómetro Bellingham + Stanley RFM970.

Para la medida de índices de refracción en el intervalo de medición de los mismos comprendido entre $1.30-1.70$ con resolución $1 \cdot 10^{-5}$ y precisión $2 \cdot 10^{-5}$, en el rango de temperaturas entre 10 y $80^{\circ} \mathrm{C}$.

- Viscosímetro capilar Ubbelohde Schott Geräte.

Diseñado para operar en intervalos donde el número de Reynolds es inferior a 300. Conectado a la unidad de control automático Schott Geräte AVS-350 dotado de un sistema optoelectrónico de detección del menisco del líquido consistente en dos haces de luz que atraviesan transversalmente el capilar del viscosímetro. La unidad de control automático AVS-350 tiene un reloj de cuarzo que determina los tiempos de flujo del líquido con una precisión de $\pm 0.01 \mathrm{~s}$. El control de la temperatura se lleva a cabo sumergiendo una resistencia Pt-100 calibrada de acuerdo con la escala de temperaturas ITS-90. La estabilidad de la temperatura del conjunto es mejor que $\pm 0.01 \mathrm{~K}$.

- Otros aparatos.

Columna de rectificación de 100 platos teóricos dedicada a mejorar la pureza de los compuestos utilizados; refractometro ATAGO RX-5000 para medir índices de refracción; balanza analítica de carga A\&D Instruments HR-202; balanza analítica de precisión Sartorius MSU 125P; turbidímetro HACH $2100 N$; Espectrómetro ultravioletavisible UNICAM UV500; pHímetro CRISON GLP22; Titrator Karl-Fischer METLER DL35; Millipore Elix 3 para el tratamiento del agua por ósmosis inversa; estabilizadores de corriente; campanas para la extracción de vapores; armarios de seguridad DÜPERTHAL Typ90; baños de ultrasonidos; fuentes de corriente; fuentes de voltaje; multímetros digitales, etc.

\section{P.4 Colaboración con otros Grupos de Investigación.}

El G.E.T.E.F. mantiene o ha mantenido colaboraciones estables con varias Universidades y Centros de Investigación españoles y extranjeros, entre los que destacan:

\section{P.4.1 Centros Españoles}

- Dr. M. A. Villamañán y colaboradores. Laboratorio de Termodinámica. Departamento de Ingeniería Energética y Fluidomécanica. E.T.S. de Ingenieros Industriales. Universidad de Valladolid. 
- Dr. F. Mato, Dra. M. J. Cocero y colaboradores. Dr. A. Cartón y colaboradores. Departamento de Ingeniería Química. Universidad de Valladolid.

- Dr. R. Bravo y colaboradores. Departamento de Física Aplicada. Universidad de Santiago de Compostela.

- Dr. A. Lainez. Dr. J. A. R. Renuncio y colaboradores. Dr. R. G. Rubio y colaboradores. Departamento de Química-Física I. Universidad Complutense de Madrid.

- Dr. S. Otín y colaboradores. Departamento de Química-Física y Química Orgánica. Universidad de Zaragoza.

- Dr. C. Alonso Tristán. Departamento de Ingeniería Electromecánica. Escuela Politécnica Superior. Universidad de Burgos.

- Dr. Ma Purificación Cuadrado Curto. Departamento de Química Orgánica. Universidad de Valladolid.

\section{P.4.2 Centros Extranjeros}

- Prof. J. -P. E. Grolier, A. H. Roux, G. Roux-Desgranges, Prof. J. R. Quint y J. Y. Coxam. Laboratoire de Thermodynamique et Génie Chimique (URA/CNRS 434 ). Université Blaise-Pascal. Clermont-Ferrand (Francia).

- Dr. H. V. Kehiaian. Institut de Topologie et de Dynamique des Systèmes. Université Paris VII (LA 34, CNRS). París (Francia).

- Prof. E. Wilhelm. Institut für Physikalische Chemie. Universität Wien. Viena (Austria).

- Prof. U. Domanska. Department of Chemistry. Physical Chemistry Division. Faculty of Chemistry. Warsaw University of Technology. Varsovia (Polonia).

- Prof. S. W. Campbell. Department of Chemical Engineering. University of South Florida. Tampa, Florida (Estados Unidos de América).

- Prof. J. -P. M. Trusler y Dr. A. Fenghour. Chemical Engineering and Chemical Technology Department. Imperial College. Londres (Reino Unido).

- Dr. N. Riesco. Department of Earth Science and Engineering. Imperial College. Londres (Reino Unido).

- Prof. J. Gmehling. Technische Chemie Department. Carl von Ossietzky Universität. Oldenburg (Alemania).

Han existido otras colaboraciones más esporádicas con personas, como la Dra. Magda Sampaio de la Universidad de Lisboa (Portugal), o el Dr. A. Ait-Kaci de la Universidad Houari Boumediene, Dar el Beida (Argelia).

\section{P.5 La presente Tesis Doctoral}

La presente tesis doctoral contribuye a la extensa labor científica del G.E.T.E.F. tanto en su línea general de investigación como en varias de sus líneas específicas. 
Esta contribución ha sido dividida en dos partes: una parte experimental y una parte teórica.

\section{P.5.1 Contribución Experimental}

Dentro de la parte experimental destacan las siguientes contribuciones:

- Calibrado y puesta a punto del densímetro y analizador de la velocidad del sonido Anton Paar DSA 5000 (Capítulo 3).

- Calibrado y puesta a punto del microcalorímetro Tian-Calvet (Capítulo 4).

- Estudio sistemático de las mezclas amina + cetona o $+n$-alcanos.

Se han determinado los volúmenes de exceso, las compresibilidades isoentrópicas (Capítulo 3) y las entalpías de exceso de las mezclas binarias de aminas + cetonas o $+n$-alcanos (Capítulo 4). Asimismo el estudio se ha completado con el análisis espectroscópico de algunas mezclas de 2-butanona con amina (Anexo C).

\section{P.5.2 Contribución Teórica}

Dentro de la parte teórica destacan las siguientes contribuciones:

- Formulación de las ecuaciones de las propiedades termodinámicas de las mezclas.

Con ello se ha buscado comprender las características de las funciones termodinámicas determinadas (volumen y entalpía de exceso) para las mezclas estudiadas (Capítulo 2).

- Estudio de las teorías mecánico-estadísticas de los líquidos y de las mezclas de no electrolitos.

Se han estudiado las hipótesis fundamentales de los diferentes modelos y las ecuaciones que de ellos se obtienen (Capítulo 6). Los modelos ERAS y DISQUAC se han aplicado a las mezclas estudiadas en este trabajo, lo que ha permitido obtener algunas conclusiones importantes acerca de las propiedades de estas mezclas (Capítulo 7). Esto encuadra perfectamente en las líneas específicas de investigación del grupo G.E.T.E.F.

- Análisis de las mezclas amina + cetona o $+n$-alcano.

Haciendo uso de los resultados experimentales que se presentan en este trabajo, se presenta la aplicación de los modelos anteriores para su estudio (Capítulo 7). Además, se han discutido las propiedades generales de las mezclas de interés en base a consideraciones puramente experimentales, sin atenerse a ninguna teoría o modelo (Capítulo 5).

\section{P.6 Soporte económico}

La realización de esta tesis doctoral no hubiese sido posible sin la concesión por parte de la Junta de Castilla y León de una ayuda destinada a financiar la contratación de personal investigador de reciente titulación universitaria en el marco de la Estrategia Regional de Investigación Científica, Desarrollo Tecnológico e Innovación 2007-2013, 
cofinanciadas por el Fondo Social Europeo, según la ORDEN EDU/1933/2008, de 11 de noviembre.

Además, parte de la tesis doctoral fue desarrollada dentro del proyecto de investigación del G.E.T.E.F. "Investigación sobre la validez de la hipótesis de mezcla al azar en los sistemas alcohol + poliéter, + cetona, o + amida", referencia FIS2010-16957, financiado por el Ministerio de Ciencia e Innovación.

\section{P.7 Bibliografía}

[1]: Villamañán, M.A.; "Estudio termodinámico de mezclas líquidas alcohol+éter". Tesis Doctoral. Departamento de Física Fundamental. Facultad de Ciencias. Universidad de Valladolid, 1979.

[2]: Cobos Hernández, J.C.; "Montaje y puesta a punto de un microcalorímetro TianCalvet". Trabajo de Licenciatura. Departamento de Física Fundamental. Facultad de Ciencias. Universidad de Valladolid, 1979.

[3]: Cobos Hernández, J.C.; "Estudio termodinámico de mezclas líquidas de alcoxietanoles con disolventes orgánicos". Tesis Doctoral. Departamento de Física Aplicada II. Facultad de Ciencias. Universidad de Valladolid, 1987.

[4]: Riesco Fernández, N.; "Contribución experimental y teórica al estudio de las propiedades termodinámicas de las mezclas binarias entre compuestos orgánicos". Tesis Doctoral. Departamento de Termodinámica y Física Aplicada. Facultad de Ciencias. Universidad de Valladolid, 2003.

[5]: García de la Fuente, I.; "Estudio termodinámico de mezclas líquidas de carbonatos con disolventes orgánicos". Tesis Doctoral. Departamento de Física Aplicada II. Facultad de Ciencias. Universidad de Valladolid, 1987.

[6]: Gonzalez, J. A.; "Estudio termodinámico de las mezclas líquidas de cetonas con alcanos mediante el modelo DISQUAC. Comparación con las predicciones del modelo UNIFAC". Tesis Doctoral. Departamento de Física Aplicada II. Facultad de Ciencias. Universidad de Valladolid, 1987. 


\title{
CAPITULO 1
}

\author{
Introducción
}

$\mathrm{E}$

s suficiente con echar un vistazo a nuestro alrededor para ser conscientes de que en todo momento nos encontramos con materiales formados por la mezcla de diferentes sustancias químicos, por ejemplo el hormigón con el que se construyen nuestras casas es una mezcla de cemento, agua y áridos en las proporciones adecuadas; el aire que respiramos está formado por oxígeno, nitrógeno, dióxido de carbono, vapor de agua, y otros gases en menor proporción como el argón, el monóxido de carbono, óxidos de azufre, ozono,...; el combustible que necesitan nuestros vehículos es una mezcla de hidrocarburos, etc. Por otra parte, salta a la vista también que en cada una de las actividades diarias, ya sean de tipo fisiológico, industrial, doméstico, etc. están presentes procesos en los que ocurre una transferencia de sustancias de una fase a otra, por ejemplo, en nuestros pulmones tomamos oxígeno del aire que luego se diluye en la sangre; en nuestra lavadora, el jabón se disuelve en agua para quitar las manchas de la ropa; en las plantas químicas, se llevan a cabo procesos en los que diferentes especies químicas se mezclan para formar medicamentos, detergentes, pinturas, aceites, combustibles, ... Esto ocurre porque cuando dos fases se ponen en contacto, tienden a intercambiar sus componentes hasta que la composición de cada una de las fase alcanza un valor constante; cuando esto ocurre se dice que se ha llegado al equilibrio de fases. Las composiciones de las fases en equilibrio son frecuentemente muy diferentes entre sí, y dependen de diversas variables como la temperatura, la presión, la naturaleza química y las concentraciones de las diferentes sustancias en cada fase. El objetivo de la Termodinámica de Equilibrios Entre Fases (T.E.E.F.) es tratar de establecer las relaciones que se cumplen entre las diferentes variables termodinámicas cuando se establece el equilibrio de fases. 
En vista de la multitud de ejemplos propuestos, es fácil percibir que la T.E.E.F. es una materia de importancia decisiva en muchas ramas de la ciencia, por ejemplo en Química, Biología y Física. La descripción y la predicción del comportamiento del equilibrio de fluidos puros y sus mezclas es quizás uno de los objetivos más desafiantes y significativos en el límite entre la física y la química física. Esto se debe especialmente al gran número de procesos químicos que ocurren en la fase líquida.

Desde el punto de vista práctico, el desarrollo de teorías sobre el equilibrio de fases es de vital importancia en ciencias químicas, ingeniería química y en las industrias petrolíferas y petroquímicas ya que en la obtención de productos químicos ocurren muchos procesos que involucran la separación de fases en contacto distintas: extracción, adsorción, destilación, lixiviación, absorción, etc. [1]. De acuerdo con Gmehling [2] estos procesos pueden suponer un coste de hasta el $80 \%$ del presupuesto total de una planta química. Por otra parte, la aplicación de la T.E.E.F. a la biotecnología puede ayudar a la compresión de la formación de biomoléculas, por ejemplo del plegamiento de las proteínas [3]. En particular, el comportamiento de las disoluciones acuosas de proteínas es de gran importancia en el estudio de una gran variedad de enfermedades entre las que se pueden citar [4] la aparición de cataratas ${ }^{*}$, anemia falciforme 0 Alzheimer.

Desde el punto de vista teórico, una descripción exacta de los fluidos y sus mezclas es interesante en ciencias físicas debido principalmente a las dos razones que a continuación se exponen [5]:

a) Primera, y que constituye la motivación principal para el desarrollo de esta Tesis Doctoral, sirve para caracterizar las fuerzas que actúan entre las moléculas del fluido que se estudia y las relaciones que existen entre dichas fuerzas y la estructura del mismo, es decir, entre dichas fuerzas y la ordenación espacial del conjunto de moléculas que forma el fluido. Aunque el estudio de mezclas líquidas no es la manera más directa de obtener esta información, ya que se pueden realizar estudios espectroscópicos de dímeros gaseosos o de scattering de haces moleculares, además de obtener datos experimentales sobre de las propiedades de transporte [6]-[7], la utilización de las mezclas de líquidos para este propósito ha adquirido gran relevancia gracias al avance de sus estudios teóricos sufrido en las últimas décadas.

La Física Estadística sirve como puente de unión entre los modelos microscópicos, los cuales describen adecuadamente las fuerzas entre las moléculas, y las propiedades macroscópicas dadas por la Termodinámica. Así, partiendo de una correcta determinación experimental de las propiedades termodinámicas (macroscópicas) podemos obtener información a nivel microscópico en lo que a la estructura de los líquidos y sus mezclas e interacciones entre sus moléculas se refiere gracias al proceso de inferencia estadística que es esencialmente la física estadística [8].

b) Segunda, aparición de fenómenos que no están presentes en las sustancias puras, como los nuevos tipos de equilibrio entre fases que aparecen como

\footnotetext{
* En un ojo sano hay una fase líquida estable correspondiente a la disolución acuosa de la proteína $\gamma$ cristalino. Cuando el ojo envejece, la concentración de esta proteína cambia y puede exceder la concentración de saturación, de modo que aparece en el ojo una nueva fase líquida cuyas propiedades ópticas interfieren gravemente en la visión. La precipitación de la segunda fase puede evitarse si se añade una pequeña cantidad del tripéptido glutatión, que forma un complejo soluble con el $\gamma$-cristalino de manera que se mantiene éste en la disolución.
} 
consecuencia del número extra de grados de libertad introducidos por la posibilidad de variar las proporciones de los componentes.

Por lo visto hasta aquí, el estudio de las mezclas entre líquidos es un campo de investigación suficientemente amplio como para justificar los miles de trabajos que se publican cada año sobre el tema. En concreto, en la presente memoria se han estudiado las propiedades termodinámicas asociadas a mezclas binarias de no electrolitos formadas por algunas aminas y algunas cetonas. Concretamente, en lo que respecta a sus propiedades volumétricas se ha procedido, mediante un densímetro y analizador de la velocidad del sonido Anton Paar DSA 5000, a la determinación experimental en función de la composición, a presión atmosférica y a las temperaturas $293.15,298.15$ y $303.15 \pm 0.01 \mathrm{~K}$ de las densidades y velocidades de propagación del sonido, y al posterior cálculo de los volúmenes de exceso, compresibilidades isoentrópicas de exceso y coeficiente de expansión térmica a presión constante de exceso de las mezclas binarias líquidas siguientes:

$$
\begin{aligned}
& \text { piridina }+\left\{\begin{array}{l}
\text { 2-propanona } \\
2 \text {-butanona } \\
2 \text {-pentanona } \\
2 \text {-heptanona }
\end{array} \quad \text { anilina }+\left\{\begin{array}{l}
\text { 2-propanona } \\
2 \text {-butanona } \\
2 \text {-pentanona } \\
2 \text {-heptanona }
\end{array}\right.\right. \\
& N \text {-metilanilina }+\left\{\begin{array}{l}
\text { 2-propanona } \\
\text { 2-butanona } \\
\text { 2-pentanona } \\
2 \text {-heptanona }
\end{array} \quad \text { di- } n \text {-propilamina }+\left\{\begin{array}{l}
\text { 2-propanona } \\
\text { 2-butanona } \\
\text { 2-heptanona }
\end{array}\right.\right. \\
& N, N, N \text {-trietilamina }+\left\{\begin{array}{l}
\text { 2-propanona } \\
\text { 2-butanona } \\
\text { 2-heptanona }
\end{array} \quad \text { di- } n \text {-butilamina }+\left\{\begin{array}{l}
\text { 2-propanona } \\
\text { 2-butanona } \\
\text { 2-heptanona }
\end{array}\right.\right. \\
& N \text {-metilanilina }+\left\{\begin{array}{l}
\text { heptano } \\
\text { ciclohexano } \\
\text { tolveno }
\end{array}\right.
\end{aligned}
$$

Respecto sus propiedades térmicas, con la ayuda de un microcalorímetro Tian-Calvet se han obtenido como función de la composición, a presión atmosférica y $298.15 \pm 0.01 \mathrm{~K}$ las entalpías de exceso de las siguientes mezclas:

$$
\text { anilina }+\left\{\begin{array}{l}
\text { 2-propanona } \\
2 \text {-butanona } \\
2 \text {-pentanona } \\
2 \text {-heptanona }
\end{array} \quad N \text {-metilanilina }+\left\{\begin{array}{l}
2 \text {-propanona } \\
2 \text {-butanona } \\
2 \text {-pentanona } \\
2 \text {-heptanona }
\end{array}\right.\right.
$$




$$
\text { di- } n \text {-propilamina }+\left\{\begin{array}{l}
\text { 2-propanona } \\
2 \text {-butanona } \\
2 \text {-pentanona } \\
2 \text {-heptanona }
\end{array} \quad N, N, N \text {-trietilamina }+\left\{\begin{array}{l}
\text { 2-propanona } \\
2 \text {-butanona } \\
\text { 2-pentanona } \\
\text { 2-heptanona }
\end{array}\right.\right.
$$$$
\text { di- } n \text {-butilamina }\left\{\begin{array}{l}
\text { 2-propanona } \\
\text { 2-butanona } \\
2 \text {-pentanona } \\
\text { 2-heptanona }
\end{array} \quad N \text {-metilanilina }+\left\{\begin{array}{l}
\text { heptano } \\
\text { octano } \\
\text { decano } \\
\text { ciclohexano } \\
\text { tolveno }
\end{array}\right.\right.
$$

Así mismo, se han determinado experimentalmente las entalpías de exceso del sistema ternario formado por 1-propanol $+N, N, N$-trietilamina +2-butanona a $298.15 \mathrm{~K}$.

La definición de las magnitudes mencionadas, los métodos más extendidos para la determinación experimental de las mismas y los resultados obtenidos para los sistemas considerados se presentan en los Capítulos 2, 3 y 4 de esta memoria.

La elección de los sistemas arriba citados y no de otros para el estudio de sus propiedades termodinámicas no es fruto de la casualidad y ni siquiera se argumenta en la importancia industrial, biológica, fisiológica... de los mismos, la cual se expone en el Anexo B, sino que se debe a la búsqueda de la caracterización sistemática de las fuerzas intermoleculares iniciada por Kehiaian [9]-[10] dentro del llamado proyecto T.O.M. (Thermodynamics of Organic Mixtures). La idea básica es la siguiente: si bien en las mezclas de líquidos muy sencillos $\left(\mathrm{N}_{2}, \mathrm{CO}_{2}, \mathrm{CH}_{4}, \ldots\right)$ las interacciones son puramente dispersivas, consecuencia de las fluctuaciones cuánticas instantáneas en las densidades de carga de las moléculas y que generan fuerzas atractivas de tipo London, en la mayoría de las mezclas reales se hayan presentes igualmente otros tipos de interacciones inter e intramoleculares (dipolares, transferencia de carga, puentes de hidrógeno, etc.) que aumentan considerablemente su complejidad. Esto nos obliga a realizar un estudio comparativo del comportamiento termodinámico de las diversas mezclas líquidas que existen, trabajando en series homólogas donde se aumenta progresivamente la complejidad (momento dipolar, estructura, etc.) de las moléculas puestas en juego. Así puede conocerse el peso relativo sobre el efecto global de cada una de las contribuciones elementales que se generan en el proceso de mezcla, y el predominio en cada conjunto de sistemas de un tipo de interacciones $u$ otro. El valor absoluto de dichas interacciones nos es desconocido por el momento, lo cual no supone ningún obstáculo para que en primera aproximación podamos conocer la importancia relativa que poseen cada una de ellas en la descripción del comportamiento de los sistemas de nuestro interés.

El objetivo del proyecto T.O.M. es realizar una comparación sistemática de los principales grupos funcionales orgánicos en base a la idea mencionada arriba de caracterizar las contribuciones relativas de cada tipo de interacción. Se estudian sistemas en orden creciente de la complejidad de su estructura molecular e interacciones intermoleculares. En dicho proyecto se utiliza como sustrato teórico que racionalice dicho comportamiento las teorías de contribución de grupos (ASOG, UNIFAC, DISQUAC,...) ajustando en cada caso los parámetros de interacción 
característicos de la relación entre cada par de grupos funcionales. En los métodos de contribución de grupos una molécula se divide en grupos funcionales o segmentos, y las interacciones entre moléculas se consideran como sumas ponderadas adecuadamente de las interacciones grupo-grupo. Por tanto, para un componente multifuncional en una mezcla los métodos de contribución de grupos suponen que cada grupo funcional se comporta independientemente de la molécula a la que pertenece. Así, a partir del estudio de ciertos sistemas clave, una vez ajustados los parámetros de interacción de pares de grupos funcionales, pueden calcularse las interacciones molécula-molécula (y en consecuencia, las propiedades termodinámicas) de otros sistemas para los que no existen datos experimentales. La ventaja de este procedimiento es que el número de grupos funcionales distintos es menor que el número moléculas, disminuyendo así la cantidad de datos experimentales necesarios para caracterizar a los distintos sistemas.

En concreto, el objetivo del subproyecto NCO del proyecto T.O.M. es el estudio sistemático de las propiedades termodinámicas de las moléculas con los grupos funcionales amino, $-\mathrm{NR}_{2}$, y carbonilo, $-\mathrm{C}=\mathrm{O}$, además de los grupos alifáticos de cadena lineal $-\mathrm{CH}_{2}$ y $-\mathrm{CH}_{3}$. Con estos segmentos es posible formar las sustancias base siguientes:

a) n-alcanos:

$$
\mathrm{CH}_{3}-\left[\mathrm{CH}_{2}\right]_{\mathrm{n}}-\mathrm{CH}_{3}
$$

b) Aminas primarias, secundarias y terciarias:

$$
\mathrm{R}-\mathrm{NH}_{2}
$$<smiles>\smiles</smiles>$[ R] N[R] }$

c) Cetonas:<smiles>[R]C([R])=O</smiles>

d) Amidas primarias, secundarias y terciarias:<smiles>[R]C(N)=O</smiles><smiles>[R]NC([R])=O</smiles>

e) Amino-cetonas:<smiles>[R]N([R])[R]</smiles>

$$
\text { ' }
$$


A partir de las sustancias base a), b) y c) se pueden formar las tres clases de sistemas:

a) Amina+alcano.

b) Cetona+alcano.

c) Amina+cetona.

Del estudio de al menos un "sistema clave" perteneciente a cada una de estas clases se podrán caracterizar las interacciones intermoleculares que se producen en todo este conjunto de mezclas líquidas, ya que el estudio de un solo sistema perteneciente a cada una de esas clases permite definir los parámetros de interacción entre los diferentes grupos funcionales involucrados. Por tanto, del conocimiento de dichos parámetros de interacción se podrían deducir, al menos a priori, las propiedades de otros sistemas de la misma clase, así como de otras que contuviesen a los mismos grupos funcionales, por ejemplo: aldehído + alcano, amina + amina, etc. Desafortunadamente, es frecuente encontrar que los resultados obtenidos para los sistemas clave no se pueden extrapolar directamente a mezclas con moléculas más complejas debido a la aparición de efectos intramoleculares, como los efectos de proximidad que aparecen cuando en la misma molécula están presentes dos o más grupos funcionales, lo cual se ha demostrado, por ejemplo, en los estudios concernientes a los grupos hidroxilo y éter, $-\mathrm{OH}$ y $-\mathrm{O}-$, y a los grupos carbonilo y éter, - $\mathrm{CO}$ y - $\mathrm{O}$-, ampliamente desarrollados por el grupo de investigación G.E.T.E.F. dentro de los subproyectos $\mathrm{OHO}$ y $\mathrm{OCO}$ en el caso de las celosolvas (hidroxiéteres) y los carbonatos, respectivamente [1 1]-[23]. En esos casos, los datos experimentales no pueden reproducirse correctamente a partir de los parámetros de interacción de los grupos básicos, por lo que debe procederse a la determinación experimental de los mismos, realizando un reajuste de los parámetros de interacción. El grupo funcional debe redefinirse y será uno más grande que incluye a los otros subgrupos, aunque se debe tener cuidado ya que si el nuevo grupo funcional es muy grande en comparación con las distancias moleculares promedio, su potencial de interacción puede ser tan complejo que ninguna teoría pudiese describirla convenientemente, y en este caso ningún modelo de contribución de grupos sería aplicable [10].

Dentro del subproyecyo NCO, el presente trabajo puede verse como la continuación natural de los realizados por Villa [25] sobre la caracterización de las mezclas de aminas secundarias con alcanos, Riesco [26] sobre la caracterización de las mezclas de aminas terciarias con alcanos y Kehiaian y Grolier [27]-[28] sobre la caracterización de las mezclas de cetonas con alcanos en términos de teorías de contribución de grupos basados en los datos obtenidos anteriormente por sus colaboradores [29]-[33]. Aquí se presenta un estudio sistemático de las mezclas arriba citadas de aminas con cetonas, el cual se ha completado con el análisis de algunas mezclas de alcanos con moléculas que contienen también a los grupos $\mathrm{N}$ y CO (amidas y amino-cetonas). La comparación entre los datos experimentales de estas mezclas con los valores teóricos calculados a partir de los parámetros de interacción de los sistemas clave ha permitido determinar los posibles efectos intramoleculares, dando continuidad al subproyecto NCO de acuerdo con el esquema habitual de trabajo seguido por el grupo de investigación G.E.T.E.F. (véase la llustración 1-1). 


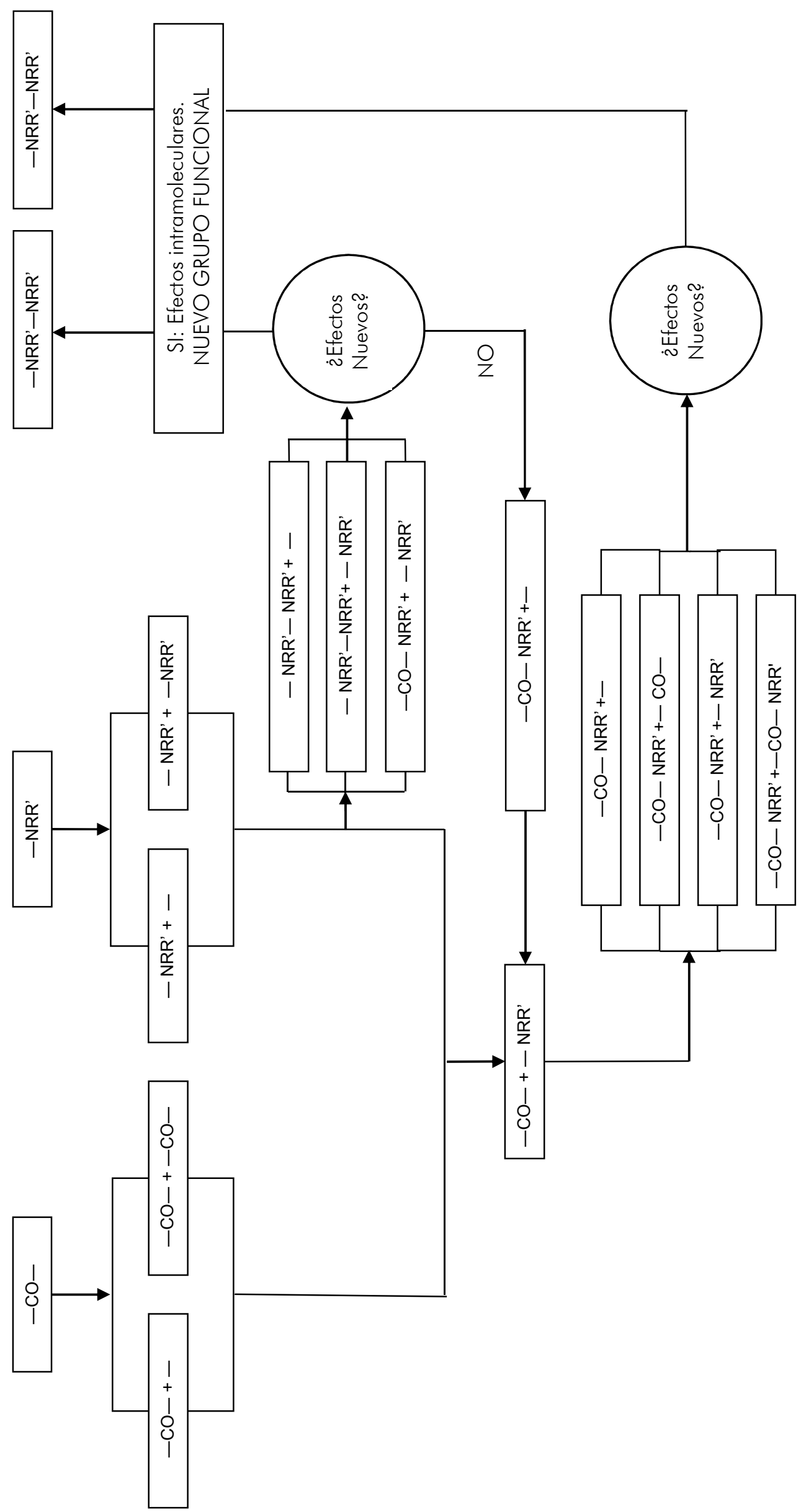

Ilustración 1-1: Subproyecto NCO del proyecto T.O.M. 
CAPÍTULO 1 


\section{BIBLIOGRAFÍA}

[1]: Prausnitz, J. M., Lichtenthaler, R.N., Gomes de Azevedo, E., "Termodinámica Molecular de los Equilibrios Entre Fases", $3^{a}$ edición, Prentice Hall (2000).

[2]: Gmehling, J., UNIFAC - ein wichtiges Werkzeug für die chemische Industrie, Software-Entwicklung in der Industrie, Springer Verlag (1987).

[3]: Prausnitz, J.M.; Fluid Phase Equlib., 53 (1989), 439.

[4]: Prausnitz, J.M.; Pure and Appl. Chem., 75(7) (2003), 859.

[5]: Rowlinson J.S., Swinton, F.L.; "Liquids and Liquids Mixtures", Butterworths Monographs in Chemistry (1982).

[6]: Maitland, G.C.; Rugby, M.; Smith, E.B.; Wakeham, W.A., "Intermolecular Forces"; Oxford University Press, Oxford (1981)

[7]: Israelachvili, J.N.; "Intermolecular and Surface Forces", Elsevier, $3^{\text {rd }}$ edition (2011).

[8]: Jaynes, E.T.; "Where do we stand on maximum entropy?. The Maximum Entropy Formalism"; Levine, R., Tribus, M. (eds.), M.I.T. Press, Cambridge, MA (1979).

[9]: Kehiaian, H.V.; Ber. Bunsenges. Phys. Chem., 81 (1977), 908.

[10]: Kehiaian, H.V.; Fluid Phase Equilib., 13 (1983), 243.

[11]: Cobos Hernández, J.C.; "Estudio termodinámico de mezclas líquidas de alcoxietanoles con disolventes orgánicos". Tesis Doctoral. Facultad de Ciencias. Universidad de Valladolid (1987).

[12]: Villamañán, M.A.; "Estudio termodinámico de mezclas líquidas alcohol+éter". Tesis Doctoral. Departamento de Física Fundamental. Facultad de Ciencias. Universidad de Valladolid (1979).

[13]: García de la Fuente, I.; "Estudio termodinámico de mezclas líquidas de carbonatos con disolventes orgánicos". Tesis Doctoral. Departamento de Física Aplicada II. Facultad de Ciencias. Universidad de Valladolid, 1987.

[14]: González, J.A.; García de la Fuente, I.; Cobos, J.C.; Casanova, C.; Ber. Bunsenges. Phys. Chem., 95 (1991), 1658.

[15]: González, J.A.; García de la Fuente, I.; Fernández Martínez, J.M.; Cobos, J.C.; Can. J. Chem., 74 (1996), 1815. 
[16]: Kehiaian, H.V.; González, J.A.; García de la Fuente, I.; Escarda, R.; Cobos, J.C.; Casanova, C.; Fluid Phase Equilib., 69 (1991), 91.

[17]: Serna Gutiérrez, A.; "Determinación de los $V_{m}^{E}$ de los sistemas n-alcohol+mono y poliéteres mediante un densímetro de oscilación mecánica". Tesis de Licenciatura. Departamento de Física Aplicada II. Facultad de Ciencias. Universidad de Valladolid (1994).

[18]: Arroyo Maestu, F.J.; "Estudio experimental de los volúmenes de exceso de mezclas líquidas de n-heptanol y n-nonanol con poliéteres lineales a 298.15K". Tesis de Licenciatura. Departamento de Física Aplicada II. Facultad de Ciencias. Universidad de Valladolid (1997).

[19]: Carmona, F.J.; "Estudio experimental de los volúmenes de exceso de mezclas líquidas de metanol y etanol con poliéteres lineales a 298.15K". Tesis de Licenciatura. Departamento de Física Aplicada II. Facultad de Ciencias. Universidad de Valladolid (1994).

[20]: Carmona, F.J.; "Carmona, F.J.; "Estudio termodinámico de mezclas de alcoxietaanoles con hidrocarburos o éteres". Tesis Doctoral. Departamento de Física Aplicada II. Facultad de Ciencias. Universidad de Valladolid (2001).

[21]: Romero Albillos, T.; "Estudio experimental de los volúmenes de exceso en sistemas binarios formados por cetonas con éteres lineales a 298.15K". Tesis de Licenciatura. Departamento de Termodinámica y Física Aplicada. Facultad de Ciencias. Universidad de Valladolid (2000).

[22]: Villa Vallejo, S.; "Estudio experimental de los $V_{m}^{E}$ de sistemas binarios formados por 1-propanol y 1-hexanol con éteres lineales a 298.15K". Tesis de Licenciatura. Departamento de Termodinámica y Física Aplicada. Facultad de Ciencias. Universidad de Valladolid (1999).

[23]: Mozo Ruiz, I.; "Estudio experimental y teórico de mezclas binarias de n-butan-1-ol, di-n-butiléter y celosolvas". Tesis Doctoral. Departamento de Física Aplicada. Facultad de Ciencias. Universidad de Valladolid (2010).

[24]: Kehiaian, H.V.; Fluid Phase Equilib., 13 (1983), 243.

[25]: Villa Vallejo, S. "Contribución experimental y teórica al estudio de las propiedades termodinámicas de las mezclas líquidas formadas por aminas y alcanos o 1 -alcoholes". Tesis Doctoral. Departamento de Termodinámica y Física Aplicada. Facultad de Ciencias. Universidad de Valladolid (2003).

[26]: Riesco Fernández, N.; "Contribución experimental y teórica al estudio de las propiedades termodinámicas de las mezclas binarias entre compuestos orgánicos". Tesis Doctoral. Departamento de Termodinámica y Física Aplicada. Facultad de Ciencias. Universidad de Valladolid (2003).

[27]: Kehiaian, H.V.; Grolier, J.P. E.; Kechavarz, M.R.; Benson, G.C.; Fluid Phase Equilib., 5(3-4) (1981), 159-189.

[28]: Kehiaian, H.V.; Grolier, J.P. E.; Kechavarz, M.R.; Benson, G.C.; Kiyohara, O.; Handa, Y. P.; Fluid Phase Equilib., 7(2) (1981), 95. 
[29]: Kiyohara, O.; Benson, G.C.; Grolier, J.P. E.; J. Chem. Thermodyn., 9(4) (1977), 315.

[30]: Grolier, J.P. E.; Kiyohara, O.; Benson, G.C.; J. Chem. Thermodyn., 9(7) (1977), 697.

[31]: Kiyohara, O.; Handa, Y.P.; Benson, G.C.; J. Chem. Thermodyn., 11 (5) (1979), 453.

[32]: Urdaneta, O.; Hamam, S.; Handa, Y.P.; Benson, G.C.; J. Chem. Thermodyn., $11(9)$ (1979) 851.

[33]: Urdaneta, O.; Handa, Y.P.; Benson, G.C.; J. Chem. Thermodyn., 11 (9) (1979), 857.

[34]: Funke, H.; Wetzel, M.; Heintz, A.; Pure and Appl. Chem., 61, (1989), 1429.

[35]: Bertagnolli, H. ; Engelhardt, T. ; Chieux, P. ; Ber. Bunsenges. Phys. Chem., 90, (1986), 512.

[36]: González, J.A.; García de la Fuente, I.; Cobos, J.C.; Fluid Phase Equilib., 168, (2000), 31.

[37]: González, J.A.; Mozo, I.; García de la Fuente, I.; Cobos, J.C.; Can. J. Chem., 83, (2005), 1812.

[38]: González, J.A.; Mozo, I.; García de la Fuente, I.; Cobos, J.C.; Thermochim. Acta, 441, (2006), 53.

[39]: González, J.A.; Domanska, U.; Zawadzki, M.; J. Chem. Thermodyn., 40, (2008), 1261. 
CAPÍTULO 1 
CAPITULO 2

\section{Termodinámica aplicada a los sistemas mono y multicomponentes}

\footnotetext{
"We who are in the field of Thermodynamics and Thermochemistry are most fortunate in working in a science that is basic to the behaviour of all matter in the Universe" Rossini, F.D.; "Excursion in Chemical Thermodynamics, from the Past into the Future"; IUPAC Thermodynamics and Thermochemistry Symposium, Lund 1963.
}

\footnotetext{
Desto que este trabajo se enmarca en el contexto del estudio Termodinámico de mezclas líquidas de no electrolitos, es necesaria la exposición de los aspectos de la Termodinámica específicos para este tipo de sistemas, introduciendo conceptos y expresiones que aparecerán en posteriores capítulos. De eso trata este capítulo.
}

\subsection{CONCEPTOS INICIALES EN TERMODINÁMICA}

\subsubsection{Variables termodinámicas. Ecuaciones de estado}

El tipo más importante y sencillo de sistema termodinámico es el formado por un número finito de partes homogéneas en las que se subdivide el mismo, llamadas fases. En rigor, también habría que considerar las superficies de separación, que son fases no homogéneas y son de capital importancia por ejemplo para la explicación de la existencia de estados metaestables en las proximidades de una transición de fase. No obstante, en el desarrollo que se va a exponer, los fenómenos superfaciales no serán tenidos en cuenta.

El estado de un sistema está determinado por el valor de los atributos, parámetros o propiedades macroscópicas que son característicos del mismo. Según Bazarov [1], estas propiedades o parámetros macroscópicos pueden clasificarse según el siguiente criterio:

a) Externas, si dependen solamente de las posiciones de las partículas externas al sistema: $a_{\mathrm{i}}=a_{\mathrm{i}}\left(R_{1}, \ldots, R_{\mathrm{ne}}\right), \mathrm{i}=1, \ldots, \mathrm{c}$; por ejemplo el volumen de un gas, la presión atmosférica que actúa sobre un sistema, el campo eléctrico aplicado sobre el sistema, ... 
b) Internas, si dependen de las posiciones y velocidades de las partículas del sistema además de hacerlo de las partículas externas, o como consecuencia de a), de los parámetros externos: $b_{\mathrm{j}}=b_{\mathrm{j}}\left(r_{1}, \ldots, r_{\mathrm{n}} ; \dot{r}_{1}, \ldots, \dot{r}_{\mathrm{n}} ; a_{1}, \ldots, a_{\mathrm{c}}\right), \mathrm{j}=1, \ldots \mathrm{m}$; por ejemplo, el volumen y la presión interna de un líquido o un sólido (que dependen de la cohesión de las moléculas), la densidad, la energía, la entropía, la temperatura, la polarizabilidad, la magnetización, la composición, ...

Entre los diferentes parámetros que caracterizan el estado del sistema puede haber ligaduras de modo que el número de parámetros independientes sea menor que el número total de parámetros. El conjunto de parámetros macroscópicos independientes de un sistema determina el estado del mismo.

Los parámetros macroscópicos independientes de la historia del sistema y que están perfectamente definidas por el estado actual del sistema se llaman parámetros de estado.

El principio cero o segundo postulado de la Termodinámica establece la existencia de un parámetro interno, llamado temperatura, que expresa el estado de movimiento interno de un sistema en equilibrio por lo que los parámetros internos no son independientes. De hecho establece que todos los parámetros internos son funciones de los parámetros externos y la temperatura (c+1 variables): $b_{\mathrm{j}}=b_{\mathrm{j}}\left(a_{1}, \ldots, a_{\mathrm{c}} ; T\right)$. Esto justifica la existencia de las ecuaciones de estado: si $b_{\mathrm{j}}=A_{\mathrm{j}}$ ó $U$, entonces

$$
\begin{array}{ll}
A_{\mathrm{j}}=A_{\mathrm{j}}\left(a_{1}, \ldots, a_{\mathrm{c}} ; T\right) & \text { (Ec. térmica de estado, E.T.E.) } \\
U=U\left(a_{1}, \ldots, a_{\mathrm{c}} ; T\right) & \text { (Ec. energética de estado, E.E.E.) }
\end{array}
$$

donde $A_{\mathrm{j}}$ es la fuerza generalizada asociada al parámetro externo $a_{\mathrm{j}}$ y $U$ es la energía interna, que es la suma de las energías potenciales de interacción y de las energías cinéticas (respecto el centro de masas del sistema) de todas las individualidades que componen el sistema. El segundo principio de la Termodinámica demuestra que la E.E.E. y cada una de las E.T.E. estás conectadas mediante una ecuación en derivadas parciales.

El segundo postulado puede reformularse si a partir de la E.E.E. se expresa la temperatura en función de los parámetros externos y $U$ : la temperatura y todos los parámetros internos son función de los parámetros externos y de $U$. Si en lugar de la E.E.E. se utilizase alguna de las E.T.E., en lugar de $U$, se elegiría el parámetro interno correspondiente y obtendríamos otra formulación del postulado.

Si las ecuaciones de estado del sistema se conociesen, entonces todas las propiedades termodinámicas del sistema estarían determinadas. Sin embargo, la obtención de las ecuaciones de estado está fuera del alcance de los métodos de la Termodinámica; solamente pueden determinarse mediante datos volumétricos experimentales o de manera muy limitada mediante modelos estadísticos de la estructura microscópica de la materia. Por lo tanto la Termodinámica necesita completarse con resultados experimentales y/o con la Mecánica Estadística. Esto se refleja en el guión seguido en esta Tesis Doctoral, pues como se ha dejado entrever en el capitulo de Introducción, en posteriores capítulos se mostrarán los datos experimentales obtenidos para ciertas propiedades macroscópicas de los sistemas que nos son de interés y se ajustarán, a esos datos, los parámetros de interacción molecular correspondientes al modelo estadístico empleado, con el objetivo de obtener información a nivel microscópico. De 
los resultados así obtenidos y de la aplicación de las ecuaciones de la Termodinámica, podrán estimarse nuevas propiedades macroscópicas del sistema.

Otra posible clasificación de las propiedades macroscópicas es la siguiente [2]:

a) Independientes del tamaño del sistema: son aquellas cuyo valor no se altera al subdividir el sistema inicial en varios subsistemas. Se llaman también variables intensivas. En una fase homogénea, son continuas y no dependen de la cantidad de sustancia de la fase tomada para medirla. A veces, se define una propiedad intensiva como aquella que no depende del número de partículas, lo que puede llevar a cierta ambigüedad [3]. Ello se ilustra en el siguiente ejemplo. Consideremos $n$ moles de un gas ideal ocupando un recipiente de volumen $V$ a la temperatura $T$. La presión viene dada por la ley de los gases ideales:

$$
P=\frac{n \cdot R \cdot T}{V}
$$

siendo $R$ la constante universal de los gases. La ecuación anterior sugiere que la derivada de la presión respecto el número de moles no es cero sino:

$$
\left(\frac{\partial P}{\partial n}\right)_{T, V}=\frac{R \cdot T}{V}>0
$$

lo que es lógico ya que si en un recipiente de volumen fijo se le añade más cantidad de gas, la presión en su interior aumenta. No obstante, al ser la presión una variable intensiva no debe depender de la cantidad de sustancia, por lo que nos topamos con una posible contradicción. Ella no es tal si se tiene en cuenta que en la ecuación (2.4) al considerar que $n$ cambia, en realidad se está modificando el sistema. Para no errar de este modo, debe considerarse explícitamente que la cantidad total de gas es constante y que en (2.4) $n$ representa el número de moles que se escogen del total para medir $P$. Así, si cogemos una cantidad fija de gas, por ejemplo un mol, la presión es:

$$
P=\frac{R \cdot T}{V_{\mathrm{m}}}
$$

Luego su dependencia con $n$ es nula:

$$
\left(\frac{\partial P}{\partial n}\right)_{V_{\mathrm{m}}, T}=0
$$

Una propiedad intensiva se puede expresar como una función homogénea de grado 0 . Una función $f$ de las variables intensivas $y_{1}, \ldots, y_{\mathrm{m}}$ y el resto no intensivas $x_{1}, \ldots, x_{\mathrm{n}}$ se dice que es homogénea de grado $\mathrm{k}$ respecto de las $x_{\mathrm{i}}^{\prime}$ s si:

$$
f\left(\alpha \cdot x_{1}, \ldots, \alpha \cdot x_{n} ; y_{1}, \ldots, y_{m}\right)=\alpha^{\mathrm{k}} \cdot f\left(x_{1}, \ldots, x_{n} ; y_{1}, \ldots, y_{m}\right)
$$

Luego, una propiedad es intensiva si:

$$
f\left(\alpha \cdot x_{1}, \ldots, \alpha \cdot x_{n} ; y_{1}, \ldots, y_{m}\right)=f\left(x_{1}, \ldots, x_{n} ; y_{1}, \ldots, y_{m}\right)
$$

Las funciones homogéneas de grado $k$ verifican el teorema de Euler:

$$
k \cdot f\left(x_{1}, \ldots, x_{n} ; y_{1}, \ldots, y_{m}\right)=\sum_{\mathrm{i}=1}^{\mathrm{n}} x_{i} \cdot\left(\frac{\partial f}{\partial x_{i}}\right)_{x_{j} \neq x_{i}, y_{1}, \ldots y_{m}}
$$


b) Dependientes del tamaño del sistema, o de la cantidad de sustancia del sistema. $\mathrm{Si}$ esta dependencia es tal que al dividir el sistema en diferentes subsistemas independientes (es decir, que no se mezclan), el valor de la propiedad del sistema es igual a la suma de los valores de la propiedad de cada subsistema, es decir, la propiedad del total es igual a la suma de las propiedades de sus partes independientes, se dice que las magnitudes es aditiva. Si la propiedad en cuestión además de ser aditiva, puede expresarse como una función homogénea de grado 1 en sus variables dependientes del tamaño del sistema, se dice que es extensiva:

$$
\begin{gathered}
f=\sum_{\mathrm{i}=1}^{\mathrm{c}} f_{\mathrm{i}} \\
f\left(\alpha \cdot x_{1}, \ldots, \alpha \cdot x_{n} ; y_{1}, \ldots, y_{m}\right)=\alpha \cdot f\left(x_{1}, \ldots, x_{n}, y_{1}, \ldots, y_{m}\right)
\end{gathered}
$$

donde c es el número de partes independientes del sistema.

Esta definición termodinámica de variable extensiva es compatible con su definición estadística [4, pp 18], la cual se debe emplear siempre que el grado de rigor requerido para el estudio en cuestión sea elevado.

No obstante, cuando existe interacción entre los subsistemas que forman el sistema (es decir, se mezclan), por lo general la propiedad total no será igual a la suma de las propiedades de las diferentes partes, es decir, ya no se cumple la aditividad (por ello existen las propiedades de mezcla como entropía de mezcla, volumen de mezcla, etc.) Por lo tanto, el que una magnitud dependa de la cantidad de sustancia no implica que sea extensiva en el sentido en como aquí se ha definido (aditiva y homogénea de grado 1). Para conseguir que la propiedad sea extensiva, debe definirse una nueva partición del sistema total tal que sus entidades elementales sean independientes.

\subsubsection{Primer principio de la Termodinámica}

La primera ley de la Termodinámica establece la relación cuantitativa entre calor y trabajo basada en los estudios de Clausius [5], Joule [6], Kelvin [7] y en el fondo es un enunciado del principio de conservación de la energía para un sistema aislado. Si consideramos un sistema cerrado (uno que no puede intercambiar materia con el exterior) que realiza un proceso infinitesimal en el que puede intercambiar energía con el exterior en forma de calor y /o trabajo, el primer principio se puede expresar mediante la ecuación siguiente:

$$
\mathrm{d} U=\delta Q-\delta W
$$

donde $\mathrm{d} U$ es el cambio en la energía interna del sistema durante el proceso y $\delta Q$ y $\delta W$ son las cantidades de energía que atraviesan los límites que delimitan al sistema en forma de calor intercambiado con el exterior y de trabajo realizado por o sobre el sistema, respectivamente. En la ecuación (2.12) está implícito el convenio de signos según el cual $\delta Q>0$ si energía en forma de calor es absorbida por el sistema y $\delta W>0$ si trabajo es realizado por el sistema sobre el exterior. En cualquier caso, si el flujo neto de energía es hacia el sistema, su energía interna debe aumentar. 


\subsubsection{Segundo principio de la Termodinámica}

El segundo principio de la termodinámica establece que para sistemas en equilibrio térmico, es decir, térmicamente homogéneos ${ }^{*}$, abiertos o cerrados que realizan un proceso infinitesimal, existe una magnitud extensiva respecto de las partes independientes que forman el sistema [9, pp 125], llamada entropía, que depende solamente del estado actual del sistema y tal que su variación está relacionada con el calor intercambiado entre el sistema y el exterior mediante la relación siguiente:

$$
\mathrm{d} S \geq \frac{\delta Q}{T}
$$

$T$ es la temperatura absoluta. La igualdad ocurre si el proceso es reversible, mientras que la desigualdad es válida si el proceso que realiza el sistema es irreversible. Si los subsistemas en los que se ha dividido el sistema total no son independientes sino que interaccionan entre sí, la entropía ya no será extensiva pues deja de ser aditiva. En este caso habría que redefeinir los subsistemas de tal modo que fuesen independientes. Esto es muy importante, pues la aplicación de los métodos que se exponen en este capítulo depende fundamentalmente de que la entropía sea extensiva; en el fondo, de la elección de la partición del sistema total en subsistemas o fases independientes.

El cambio en la entropía del sistema se escribe como la suma de dos términos [10]:

a) Uno debido a la transferencia de entropía hacia o desde el exterior en virtud del calor intercambiado a través de las paredes que delimitan al sistema: $\mathrm{d} S_{\mathrm{e}}=\delta Q / T$. Este término puede ser positivo, negativo o nulo.

b) Otro debido a la producción de entropía en el interior del sistema debido a que el mismo está realizando un proceso irreversible en un tiempo finito, como por ejemplo reacciones químicas, reacciones nucleares, cambios de fase, mezcla de varios componentes, etc.: $\mathrm{d} S_{\mathrm{i}}=\delta Q_{\mathrm{i}} / T \geq 0$.

Entonces,

$$
\mathrm{d} S=\mathrm{d} S_{\mathrm{e}}+\mathrm{d} S_{\mathrm{i}}=\frac{\delta Q}{T}+\frac{\delta Q_{\mathrm{i}}}{T}
$$

Clausius llamó a $\delta Q_{\mathrm{i}}$ calor no compensado, aunque no es un nombre adecuado [1 1].

\subsubsection{Cambios físico-químicos. Potencial químico. Afinidad}

Sea un sistema multicomponente y /o multifásico que no intercambia materia con el exterior y en el que ocurre una reacción química, un cambio de fase o cualquier cambio físico-químico que pueda describirse mediante la relación estequiométrica siguiente:

$$
\sum_{A} v_{A} \cdot A \Leftrightarrow \sum_{B} v_{B} \cdot B
$$

donde $v_{A}$ representa el número de moléculas del componente químico $\mathrm{A}$ que desaparece y origina $v_{\mathrm{B}}$ moléculas del componente B. Se llama afinidad $A$ del cambio físico-químico (2.15) a la magnitud:

\footnotetext{
* En rigor, es difícil encontrar sistemas en la naturaleza que estén en equilibrio térmico, por lo que normalmente se hace referencia al equilibrio térmico local. Así, por ejemplo en el estudio termodinámico del Universo, el cual no se encuentra en equilibrio térmico, deben tomarse precauciones [8 Cap. 4].
} 


$$
A=\sum_{\mathrm{B}} \mathrm{v}_{\mathrm{B}} \cdot \mu_{\mathrm{B}}-\sum_{\mathrm{A}} \mathrm{v}_{\mathrm{A}} \cdot \mu_{\mathrm{A}}
$$

donde $\mu_{\mathrm{i}}$ es el potencial químico molecular del componente químico i, que es la función de Gibbs molar parcial de dicho componente, como se verá en la sección 2.3.2.

Se define extensión al proceso de reacción y se denota $\xi$ a una magnitud tal que su variación de $\xi$ a $\xi+\mathrm{d} \xi$ implica que $v_{\mathrm{A}} \cdot \mathrm{d} \xi$ moléculas de $\mathrm{A}$ desaparecen para formar $v_{\mathrm{B}} \cdot \mathrm{d} \xi$ moléculas de $\mathrm{B}$.

De Donder [12] demostró que el término de producción de entropía $\mathrm{d} S_{\mathrm{i}}$ asociado a un proceso físico-químico del tipo descrito por (2.15) puede escribirse como función de $A$ y $\xi$ mediante la relación:

$$
T \cdot \mathrm{d} S_{\mathrm{i}}=\delta Q_{\mathrm{i}}=A \cdot \mathrm{d} \xi \geq 0
$$

La igualdad sólo es válida si el proceso es reversible, o si no ocurre ningún proceso del tipo (2.15) porque el sistema se encuentre en equilibrio físico-químico.

Sea un sistema de $\mathrm{n}$ componentes, $\pi$ fases y en el que se producen $\mathrm{R}$ reacciones químicas diferentes. Se puede obtener la siguiente ecuación [1 1 pp 75], [13 pp 63-67]:

$$
\sum_{\rho=1}^{\mathrm{R}} A_{\rho} \cdot \mathrm{d} \xi_{\rho}=-\sum_{k=1}^{\mathrm{n}} \sum_{\alpha=1}^{\pi} \sum_{\rho=1}^{\mathrm{R}} \mu_{\mathrm{k}}^{\alpha} \cdot \mathrm{d} N_{\mathrm{k}, \rho}^{\alpha}
$$

donde $A_{\rho}$ es la afinidad de la reacción $\rho, \xi_{\rho}$ es la extensión al cambio asociada a la reacción $\rho, \mu_{k}^{\alpha}$ es el potencial químico por partícula de la especie química k-ésima en la fase $\alpha$ y $\mathrm{d} N_{\mathrm{k}, \rho}^{\alpha}$ es el cambio en el número de partículas de la especie química k-ésima que está en la fase $\alpha$ participando en la reacción $\rho$.

La ecuación (2.18) puede simplificarse si en el sistema no se producen reacciones químicas y solamente se producen cambios de fase, sin más que eliminar los subíndices $\rho$. Por otra parte, en condiciones de equilibrio termodinámico, los potenciales químicos de cada sustancia en las diferentes fases deben ser iguales $\left(\mu_{\mathrm{k}}^{\alpha}=\mu_{\mathrm{k}}^{\beta}=\ldots=\mu_{\mathrm{k}}^{\pi}\right)$ siempre que haya equilibrio térmico e hidrostático, como se detallará en las ecuaciones (2.75)(2.77), luego la ecuación anterior puede rescribirse como:

$$
A \cdot \mathrm{d} \xi=-\sum_{\mathrm{k}=1}^{\mathrm{n}} \sum_{\alpha=1}^{\pi} \mu_{\mathrm{k}} \cdot \mathrm{d} N_{\mathrm{k}}^{\alpha}=-\sum_{\mathrm{k}=1}^{\mathrm{n}} \mu_{\mathrm{k}} \cdot \mathrm{d} N_{\mathrm{k}} \geq 0
$$

Donde $N_{\mathrm{k}}$ es el número total de partículas del componente k-ésimo, sin importar la fase en la que se encuentre:

$$
\mathrm{d} N_{\mathrm{k}}=\sum_{\alpha=1}^{\pi} \mathrm{d} N_{\mathrm{k}}^{\alpha}
$$

De (2.19), (2.17) y (2.14) se deduce que el calor intercambiado durante el proceso es:

$$
\delta Q=T \cdot \mathrm{d} S+\sum_{\mathrm{k}=1}^{\mathrm{n}} \mu_{\mathrm{k}} \cdot \mathrm{d} N_{\mathrm{k}} \geq 0
$$

El número total de partículas del sistema cerrado es: 


$$
N=\sum_{k=1}^{\mathrm{n}} N_{k}
$$

\subsubsection{Ecuación fundamental de la Termodinámica. Potenciales termodinámicos}

De acuerdo con Bazarov [1, pp 12], el trabajo realizado por o sobre el sistema es igual al cambio de energía del sistema debido a la variación de los parámetros externos $a_{1}, \ldots, a_{\mathrm{c}}$. Se define la fuerza generalizada $A_{\mathrm{i}}$ asociada a la variación infinitesimal del parámetro externo $a_{\mathrm{i}}$ de tal modo que el trabajo realizado por o sobre el sistema sea:

$$
\delta W=\sum_{\mathrm{i}=1}^{\mathrm{c}} A_{\mathrm{i}} \cdot \mathrm{d} a_{\mathrm{i}}
$$

Supongamos que en la región del espacio ocupada por el sistema los campos gravitatorio, eléctrico y magnético son homogéneos y nulos, que no hay fenómenos interfaciales y que el único trabajo que se puede realizar por o sobre el sistema en cada fase es de tipo hidrostático e igual a $-P \cdot \mathrm{d} V$. En caso contrario, las ecuaciones correspondientes para cada tipo de trabajo se pueden consultar, por ejemplo, en el libro de Sychev [14]. Al sustituir (2.21) en (2.12) se obtiene la ecuación:

$$
\mathrm{d} U=T \cdot \mathrm{d} S-P \cdot \mathrm{d} V+\sum_{\mathrm{k}=1}^{\mathrm{n}} \mu_{\mathrm{k}} \cdot \mathrm{d} N_{\mathrm{k}}
$$

Esta ecuación es válida para sistemas cerrados, que realizan procesos reversibles $\left(\sum_{\mathrm{k}=1}^{\mathrm{n}} \mu_{\mathrm{k}} \cdot \mathrm{d} N_{\mathrm{k}}=0\right)$ o irreversibles $\left(\sum_{\mathrm{k}=1}^{\mathrm{n}} \mu_{\mathrm{k}} \cdot \mathrm{d} N_{\mathrm{k}}<0\right)$.

Si el sistema es abierto, puede intercambiar materia con el exterior a través de las paredes que lo limitan, y en consecuencia, energía asociada a dicha materia. Por lo tanto, a la ecuación (2.24) se le debe añadir un término que representa este intercambio:

$$
\mathrm{d} U=T \cdot \mathrm{d} S-P \cdot \mathrm{d} V+\sum_{\mathrm{k}=1}^{\mathrm{n}} \mu_{\mathrm{k}} \cdot \mathrm{d} N_{\mathrm{k}}+\sum_{\mathrm{j}=1}^{\mathrm{n}} \mu_{\mathrm{j}} \cdot \mathrm{d} N_{\mathrm{j}}
$$

donde $\mathrm{d} N_{\mathrm{k}}$ es el cambio en el número de partículas de la especie química k-ésima debido a que se producen procesos irreversibles en el interior del sistema y $\mathrm{d} N_{\mathrm{j}}$ representa el intercambio de materia con el exterior de la especie química j-ésima. La cantidad:

$$
\sum_{\mathrm{j}=1}^{\mathrm{n}} \mu_{\mathrm{j}} \cdot \mathrm{d} N_{\mathrm{j}}
$$

puede ser positiva o negativa, en función de como sea el intercambio de materia con el exterior. El calor intercambiado sigue siendo el dado por (2.21).

La ecuación (2.25) se puede rescribir como sigue:

$$
\mathrm{d} U=T \cdot \mathrm{d} S-P \cdot \mathrm{d} V+\sum_{\mathrm{i}=1}^{\mathrm{n}} \mu_{\mathrm{i}} \cdot \mathrm{d} N_{\mathrm{i}}
$$

\footnotetext{
*Esta ecuación se podría haber escrito en términos de la afinidad $A$ y la extensión al proceso de reacción $\xi$, lo cual es muy útil en el caso de que se produzcan reacciones químicas en el sistema.
} 
donde $\mathrm{d} N_{\mathrm{i}}$ representa el cambio infinitesimal de cantidad de partículas de la especie química i-ésima. Ahora el calor ya no es $T \cdot \mathrm{d} S+\sum_{\mathrm{i}=1}^{\mathrm{n}} \mu_{\mathrm{i}} \cdot \mathrm{d} N_{\mathrm{i}}$ puesto que en este sumatorio no sólo se está teniendo en cuenta el cambio en el número de partículas debido a procesos físico-químicos sino también la transferencia de masa con el exterior. La ecuación (2.27) es válida para sistemas abiertos o cerrados, que realizan procesos reversibles o irreversibles.

El número total de partículas del sistema es:

$$
N=\sum_{i=1}^{\mathrm{n}} N_{i}
$$

De la ecuación (2.27) se puede deducir que el conocimiento de $U$ en función de las variables $S, V$ y $N_{\mathrm{i}}$ implica el conocimiento de toda la información termodinámica del sistema pues a partir de las derivadas parciales de $U$ respecto de dichas variables, se obtienen las propiedades térmicas del sistema (ecuaciones de estado):

$$
\begin{gathered}
T\left(S, V, N_{\mathrm{i}}, \mathrm{i}=1, \ldots, \mathrm{n}\right)=\left(\frac{\partial U}{\partial S}\right)_{V, N_{\mathrm{i}}, \mathrm{i}=1, \ldots, \mathrm{n}} \quad \text { (E.E.E.) } \\
P\left(S, V, N_{\mathrm{i}}, \mathrm{i}=1, \ldots, \mathrm{n}\right)=\left(\frac{\partial U}{\partial V}\right)_{S, N_{\mathrm{i}}, \mathrm{i}=1, \ldots \mathrm{n}} \quad \text { (E.T.E.) } \\
\mu_{\mathrm{k}}\left(S, V, N_{\mathrm{i}}, \mathrm{i}=1, \ldots, \mathrm{n}\right)=\left(\frac{\partial U}{\partial N_{\mathrm{k}}}\right)_{S, V, N_{\mathrm{j}}, \mathrm{j} \neq \mathrm{k}} \mathrm{k}=1, \ldots, \mathrm{n} \quad \text { (E.M.E.) }
\end{gathered}
$$

y de las segundas derivadas se obtienen las propiedades caloríficas. Por esta razón, a la ecuación (2.27) se la llama ecuación fundamental de la termodinámica o ecuación de Gibbs y a $U$ se le llama función característica en las variables naturales $S, V, N_{\mathrm{i}}$, $\mathrm{i}=1$, ...n que definen el estado extensivo del sistema. Todas las funciones características son funciones univaluadas del estado del sistema. De ellas, las que tienen unidades de energía reciben el nombre de potenciales termodinámicos.

La función $U$ cumple el teorema de Schwartz para funciones de varias variables con segundas derivadas parciales continuas. Por lo tanto, se da la igualdad de las derivadas cruzadas, lo que permite obtener las diferentes relaciones de Maxwell [15]. Por ejemplo,

$$
\left(\frac{\partial T}{\partial V}\right)_{S, N_{\mathrm{i}}, \mathrm{i}=1, \ldots, \mathrm{n}}=-\left(\frac{\partial P}{\partial S}\right)_{V, N_{\mathrm{i}}, \mathrm{i}=1, \ldots, \mathrm{n}}
$$

La transformada de Legendre permite definir otros potenciales termodinámicos que tengan $P$ como variable natural en vez de $V ; T$ como variable natural en vez de $S$; o bien $T$ y $P$ como variables naturales en lugar de $S$ y $V$; etc. La transformada de Legendre consiste en restar a $U$ el producto del par conjugado variable intensivaextensiva [15], [16]. Las transformadas de Legendre más sencillas son las siguientes:

$$
\begin{aligned}
& H=U-V \cdot\left(\frac{\partial U}{\partial V}\right)_{S, N_{\mathrm{i}}, \mathrm{i}=1, \ldots, \mathrm{n}}=U+P \cdot V \\
& F=U-S \cdot\left(\frac{\partial U}{\partial S}\right)_{V, N_{\mathrm{i}}, \mathrm{i}=1, \ldots, \mathrm{n}}=U-T \cdot S
\end{aligned}
$$




$$
G=U-S \cdot\left(\frac{\partial U}{\partial S}\right)_{V, N_{\mathrm{i}}, \mathrm{i}=1, \ldots, \mathrm{n}}-V \cdot\left(\frac{\partial U}{\partial V}\right)_{S, N_{\mathrm{i}}, \mathrm{i}=1, \ldots, \mathrm{n}}=U-T \cdot S+P \cdot V
$$

que definen la entalpía $H$, la energía libre de Helmholtz $F$ y la entalpía libre de Gibbs $G^{*}$. Se pueden definir más potenciales termodinámicos si se introducen alguno o todos los potenciales químicos como variables naturales, o si se consideran los trabajos de tipo gravitatorio, eléctrico, superficial,..., lo que permite introducir como nuevas variables naturales el potencial gravitatorio, el potencial eléctrico, la tensión superficial, ...[15].

De las ecuaciones (2.33)-(2.35) se obtienen las ecuaciones de Gibbs-Helmholtz, que relacionan los diferentes potenciales termodinámicos. Por ejemplo:

$$
\begin{aligned}
& \frac{H}{T^{2}}=-\left(\frac{\partial(G / T)}{\partial T}\right)_{P, N_{\mathrm{i}}, \mathrm{i}=1, \ldots, \mathrm{n}} \\
& \frac{H}{V^{2}}=-\left(\frac{\partial(G / V)}{\partial V}\right)_{S, N_{\mathrm{i}}, \mathrm{i}=1, \ldots, \mathrm{n}}
\end{aligned}
$$

Las ecuaciones fundamentales para los demás potenciales termodinámicos se obtienen al diferenciar las ecuaciones (2.33), (2.34) y (2.35) y sustituir en la ecuación (2.27):

$$
\begin{gathered}
\mathrm{d} H=T \cdot \mathrm{d} S+V \cdot \mathrm{d} P+\sum_{\mathrm{i}=1}^{\mathrm{n}} \mu_{\mathrm{i}} \cdot \mathrm{d} N_{\mathrm{i}} \\
\mathrm{d} F=-S \cdot \mathrm{d} T-P \cdot \mathrm{d} V+\sum_{\mathrm{i}=1}^{\mathrm{n}} \mu_{\mathrm{i}} \cdot \mathrm{d} N_{\mathrm{i}} \\
\mathrm{d} G=-S \cdot \mathrm{d} T+V \cdot \mathrm{d} P+\sum_{\mathrm{i}=1}^{\mathrm{n}} \mu_{\mathrm{i}} \cdot \mathrm{d} N_{\mathrm{i}}
\end{gathered}
$$

Se pueden establecer relaciones análogas a las (2.29)-(2.31). En el caso de la función de Gibbs, estas son las siguientes:

$$
\begin{gathered}
S\left(T, P, N_{\mathrm{i}} ; \mathrm{i}=1, \ldots, \mathrm{n}\right)=-\left(\frac{\partial G}{\partial T}\right)_{P, N_{\mathrm{i}}, \mathrm{i}=1, \ldots, \mathrm{n}} \\
V\left(T, P, N_{\mathrm{i}} ; \mathrm{i}=1, \ldots, \mathrm{n}\right)=\left(\frac{\partial G}{\partial P}\right)_{T, N_{\mathrm{i}}, \mathrm{i}=1, \ldots, \mathrm{n}} \\
\mu_{\mathrm{i}}\left(T, P, N_{\mathrm{i}} ; \mathrm{i}=1, \ldots, \mathrm{n}\right)=\left(\frac{\partial G}{\partial N_{\mathrm{i}}}\right)_{P, T, N_{\mathrm{j}}, \mathrm{j} \neq \mathrm{i}}
\end{gathered}
$$

Todos los potenciales termodinámicos son funciones extensivas (respecto de las partes independientes en las que se subdivide el sistema) en sus variables extensivas, luego verifican el teorema de Euler. La aplicación de éste a cada potencial termodinámico permite obtener la forma integral de cada ecuación fundamental:

\footnotetext{
* No existe un convenio claro en el nombre ni en el símbolo de los potenciales termodinámicos, aunque Alberty [15] ha intentado poner solución al problema. Por ejemplo, la energía libre de Gibbs ha recibido muchos nombres: potencial termodinámico total, función de Gibbs, energía libre de Gibbs, función de Gibbs, potencial de Gibbs,... Otro ejemplo es la entalpía, que fue llamada por Planck y Gibbs [17] función calor a presión constante.
} 


$$
\begin{gathered}
U\left(S, V, N_{\mathrm{i}} ; \mathrm{i}=1, \ldots, \mathrm{n}\right)=T \cdot S-P \cdot V+\sum_{\mathrm{i}=1}^{\mathrm{n}} \mu_{\mathrm{i}} \cdot N_{\mathrm{i}} \\
H\left(S, P, N_{\mathrm{i}} ; \mathrm{i}=1, \ldots, \mathrm{n}\right)=T \cdot S+\sum_{\mathrm{i}=1}^{\mathrm{n}} \mu_{\mathrm{i}} \cdot N_{\mathrm{i}} \\
F\left(T, V, N_{\mathrm{i}} ; \mathrm{i}=1, \ldots, \mathrm{n}\right)=-P \cdot V+\sum_{\mathrm{i}=1}^{\mathrm{n}} \mu_{\mathrm{i}} \cdot N_{\mathrm{i}} \\
G\left(T, P, N_{\mathrm{i}} ; \mathrm{i}=1, \ldots, \mathrm{n}\right)=\sum_{\mathrm{i}=1}^{\mathrm{n}} \mu_{\mathrm{i}} \cdot N_{\mathrm{i}}
\end{gathered}
$$

Se observa que $G$ es el único potencial termodinámico que está escrito como la suma de $\mathrm{n}$ términos, siendo el término j-ésimo una función del componente j-ésimo. Si el sistema fuese monocomponente se tendría $\mu=G / N$, luego en este caso el potencial químico es el potencial de Gibbs por partícula. Si la cantidad de sustancia se expresa en número de moles, entonces $\mu$ es el potencial de Gibbs molar: $\mu=G_{m}$.

Las ecuaciones (2.33)-(2.35) definen transformadas de Legendre de $U$ parciales. La transformada de Legendre de $U$ total define el potencial cero, dado por:

$$
\begin{gathered}
0_{U}=U-S \cdot\left(\frac{\partial U}{\partial S}\right)_{V, N_{\mathrm{i}}, \mathrm{i}=1, \ldots, \mathrm{n}}-V \cdot\left(\frac{\partial U}{\partial V}\right)_{S, N_{\mathrm{i}}, \mathrm{i}=1, \ldots, \mathrm{n}}-\sum_{\mathrm{i}=1}^{\mathrm{n}} N_{\mathrm{i}} \cdot\left(\frac{\partial U}{\partial N_{\mathrm{i}}}\right)_{T, S, N_{\mathrm{j}}, \mathrm{j} \neq \mathrm{i}}= \\
=U-T \cdot S+P \cdot V-\sum_{\mathrm{i}=1}^{\mathrm{n}} \mu_{\mathrm{i}} \cdot N_{\mathrm{i}}=0
\end{gathered}
$$

Su diferencial también es cero:

$$
\mathrm{d} 0_{U}=-S \cdot \mathrm{d} T+V \cdot \mathrm{d} P-\sum_{\mathrm{i}=1}^{\mathrm{n}} N_{\mathrm{i}} \cdot \mathrm{d} \mu_{\mathrm{i}}=0
$$

A la ecuación (2.49) se le conoce como ecuación de Gibbs-Duhem. Todas las variables naturales del potencial $0_{U}$ son propiedades intensivas del sistema, por lo que la ecuación de Gibbs-Duhem refleja el importante hecho de que no todas las propiedades intensivas del sistema son independientes. Así, para un sistema hidrostático, monofásico y con $n$ componentes, solamente existen $n+1$ propiedades intensivas independientes. La ecuación de Gibbs-Duhem puede verse como un precursor de la regla de las fases de Gibbs, según la cual el número de variables intensivas independientes de un sistema en el que solamente es posible que aparezca trabajo de tipo hidrostático y en el que no hay reacciones químicas es $f=2+\mathrm{n}-\pi^{*}$.

En representación entrópica, la ecuación fundamental se obtiene al despejar d $S$ en la ecuación (2.27):

$$
\mathrm{d} S=\frac{\mathrm{d} U}{T}+\frac{P}{T} \cdot \mathrm{d} V-\sum_{\mathrm{i}=1}^{\mathrm{n}} \frac{\mu_{\mathrm{i}}}{T} \cdot \mathrm{d} N_{\mathrm{i}}
$$

\footnotetext{
${ }^{*} f$ se llama número de grados de libertad del sistema. Si en el sistema aparecen $\mathrm{R}$ reacciones químicas independientes y el trabajo realizado por o sobre el sistema puede llevarse a cabo de $c$ formas diferentes, entonces el número de grados de libertad es $f=1+c+\mathrm{n}-\pi-\mathrm{R}$. El número de variables independientes que definen el estado extensivo del sistema es $D=f+\pi=1+c+\mathrm{n}-\mathrm{R}$.
} 
$S$ en función de sus variables naturales $U, V$ y $N_{\mathrm{i}}, \mathrm{i}=1, \ldots, \mathrm{n}$, no es un potencial termodinámico, pues tiene dimensiones de energía temperatura ${ }^{-1}$. No obstante, si que es una función característica ya que su conocimiento permite obtener toda la información termodinámica del sistema a partir de sus derivadas parciales [18]. Se pueden definir otras funciones características en la representación entrópica a partir de las transformadas de Legendre de $S$. Por ejemplo, la función de Massieu se define como:

$$
\Psi\left(\frac{1}{T}, V, N_{\mathrm{i}}, \mathrm{i}=1, \ldots, \mathrm{n}\right)=S-U \cdot\left(\frac{\partial S}{\partial U}\right)_{V, N_{\mathrm{i}}, \mathrm{i}=1, \ldots, \mathrm{n}}=S-\frac{U}{T}=-\frac{F}{T}
$$

y la función de Planck es:

$$
\begin{aligned}
\eta\left(\frac{1}{T}, \frac{P}{T}, N_{\mathrm{i}}, \mathrm{i}=1, \ldots, \mathrm{n}\right) & =S-U \cdot\left(\frac{\partial S}{\partial U}\right)_{V, N_{\mathrm{i}}, \mathrm{i}=1, \ldots, \mathrm{n}}-V \cdot\left(\frac{\partial S}{\partial V}\right)_{U, N_{\mathrm{i}}, \mathrm{i}=1, \ldots, \mathrm{n}}= \\
& =S-\frac{U}{T}-\frac{P \cdot V}{T}=-\frac{G}{T}
\end{aligned}
$$

Las funciones características de la representación entrópica son importantes pues además de dar las condiciones de equilibrio termodinámico de un sistema aislado (2.75)-(2.77), están relacionadas con el logaritmo natural de la función de partición del conjunto estadístico considerado [19]. Por ejemplo, si el sistema es aislado su estado macroscópico está perfectamente definido por las variables $U, V$ y $N_{\mathrm{i}}, \mathrm{i}=1, \ldots$. n y la mencionada relación es la ecuación básica del conjunto microcanónico deducida en 1877 por Boltzmann :

$$
S=k_{\mathrm{B}} \cdot \ln \Omega
$$

con $\Omega$ el número de microestados compatibles con las ligaduras macroscópicas del sistema.

Si el estado macroscópico del sistema estuviese definido por las variables $1 / T, V$ y $N_{\mathrm{i}}$, $\mathrm{i}=1, \ldots \mathrm{n}$, la mencionada relación sería:

$$
\psi=k_{\mathrm{B}} \cdot \ln Z
$$

con $Z$ la función de partición del conjunto canónico. En general, se tendrá una ecuación del tipo:

$$
\text { ( función característica entrópica })=k_{\mathrm{B}} \cdot \ln (\text { función de partición })
$$

El paso de un conjunto estadístico a otro de la ecuación (2.55) se consigue haciendo la transformada de Legendre de la función característica entrópica y la transformada de Laplace de la función de partición, respecto las variables adecuadas.

\subsubsection{Procesos en sistemas cerrados}

La dirección de los procesos naturales que realizan los sistemas puede establecerse en términos de las ecuaciones fundamentales para los diferentes potenciales termodinámicos y es consecuencia del segundo principio de la termodinámica. En general, esta dirección depende de cómo el sistema interacciona con su entorno. En

\footnotetext{
*Explícitamente, esta expresión fue escrita por primera vez por Planck. Véase la referencia [20].
} 
efecto, en virtud de las ecuaciones (2.27), (2.38), (2.39), (2.40) y (2.50), teniendo en cuenta las ecuaciones (2.17) y (2.19) se obtiene que en los diferentes procesos:

$$
\begin{array}{ll}
\mathrm{d} U \leq 0 & \text { (procesos infinitesimales a } S, V \text { y } N_{\mathrm{i}} \text { ctes.) } \\
\mathrm{d} H \leq 0 & \text { (procesos infinitesimales a } S, P \text { y } N_{\mathrm{i}} \text { ctes.) } \\
\mathrm{d} F \leq 0 & \text { (procesos infinitesimales a } T, V \text { y } N_{\mathrm{i}} \text { ctes.) } \\
\mathrm{d} G \leq 0 & \text { (procesos infinitesimales a } T, P \text { y } N_{\mathrm{i}} \text { ctes.) } \\
\mathrm{d} S \geq 0 & \text { (procesos infinitesimales a } U, V \text { y } N_{\mathrm{i}} \text { ctes.) }
\end{array}
$$

Las igualdades se cumplen cuando el proceso es reversible o cuando el sistema está en equilibrio, mientras que la desigualdad se da cuando el sistema realiza un proceso irreversible. Para procesos finitos basta con cambiar el símbolo de la diferencial d por el de un cambio finito $\Delta$ :

$$
\begin{aligned}
\Delta U \leq 0 & \text { (procesos finitos a } \left.S, V \text { y } N_{\mathrm{i}} \text { ctes. }\right) \\
\Delta H \leq 0 & \text { (procesos finitos a } \left.S, P \text { y } N_{\mathrm{i}} \text { ctes. }\right) \\
\Delta F \leq 0 & \text { (procesos finitos a } \left.T, V \text { y } N_{\mathrm{i}} \text { ctes. }\right) \\
\Delta G \leq 0 & \text { (procesos finitos a } \left.T, P \text { y } N_{\mathrm{i}} \text { ctes. }\right) \\
\Delta S \geq 0 & \text { (procesos finitos a } \left.U, V \text { y } N_{\mathrm{i}} \text { ctes. }\right)
\end{aligned}
$$

De las relaciones que existen entre los potenciales termodinámicos y de las condiciones que cumplen los diferentes procesos, (2.56)-(2.65), se pueden encontrar las condiciones de cambio que deben satisfacer los potenciales termodinámicos cuando el proceso realizado por el sistema no mantiene constantes a sus variables naturales. Así, como consecuencia de la ecuación (2.39) se tiene que la entropía es igual a:

$$
S=S\left(T, V, N_{\mathrm{i}}, \mathrm{i}=1, \ldots, \mathrm{n}\right)=-\left(\frac{\partial F}{\partial T}\right)_{V, N_{\mathrm{i}}, \mathrm{i}=1, \ldots, \mathrm{n}}
$$

en este caso, $S$ no está escrita como función de sus variables naturales luego no es una función característica. En un proceso a $T$ y $V$ constantes, $\Delta F \leq 0$, luego la variación de entropía en dicho proceso será:

$$
\Delta S=-\left(\frac{\partial \Delta F}{\partial T}\right)_{V, N_{\mathrm{i}}, \mathrm{i}=1, \ldots, \mathrm{n}} \geq 0
$$

Este resultado significa que en un proceso a $T$ y $V$ constantes, la variación de entropía es siempre mayor o igual a cero.

La ecuación (2.40) da la entropía en función de $T, P$ y $n_{\mathrm{i}}$ :

$$
S=S\left(T, P, N_{\mathrm{i}}, \mathrm{i}=1, \ldots, \mathrm{n}\right)=-\left(\frac{\partial G}{\partial T}\right)_{P, N_{\mathrm{i}}, \mathrm{i}=1, \ldots, \mathrm{n}}
$$

$S$ no es una función característica por no estar descrita en sus variables naturales. En un proceso a $T$ y $P$ constantes, $\Delta G \leq 0$, luego la variación de entropía en dicho proceso será igual a:

$$
\Delta S=-\left(\frac{\partial \Delta G}{\partial T}\right)_{P, N_{\mathrm{i}}, \mathrm{i}=1, \ldots, \mathrm{n}} \geq 0
$$


Este resultado significa que en un proceso a $T$ y $P$ constantes, el cambio en la entropía es siempre mayor o igual a cero.

Por ejemplo, en el caso de una mezcla ideal de gases ideales se encuentra [21] que:

A $T$ y $V$ constantes, $\Delta_{\mathrm{m}} S=0 \rightarrow$ es un proceso reversible.

A $T$ y $P$ constantes, $\Delta_{\mathrm{m}} S=-\mathrm{R} \cdot \sum_{\mathrm{i}=1}^{\mathrm{n}} x_{\mathrm{i}} \cdot \ln x_{\mathrm{i}}>0 \rightarrow$ es un proceso irreversible.

Este resultado podría parecer ambiguo ya que al ser la entropía una función de estado, su variación no depende del proceso seguido, sino solamente de los estados inicial y final. Por lo tanto, en los proceso a $V$ y $T$ constantes, y a $P$ y $T$ constantes, $\Delta_{\mathrm{m}} S$ debería ser la misma. Para aclarar esta aparente ambigüedad, que no es tal, hay que tener en cuenta que en la mezcla a $V$ y $T$ constantes el estado final es diferente al estado final al que se llega en la mezcla a $P$ y $T$ constantes. Además, en la mezcla ideal (sistemas que no interactúan), la entropía es extensiva respecto de sus variables extensivas, como el volumen, pero no en sus variables intensivas, como la presión; por lo tanto, la entropía es aditiva en la variable $V$ y no en la variable $P$. Para ver esto con mayor claridad, supongamos que antes de mezclarse los gases, éstos tienen entropía dada por $S_{1}^{0}\left(V, U_{1}^{0}, N_{1}\right)$ y $S_{2}^{0}\left(V, U_{2}^{0}, N_{2}\right)$. La entropía del sistema $S^{0}=S_{1}^{0}+S_{2}^{0}$. Si se mezclan los gases a $V$ y $T$ constante, la presión en el interior del sistema aumentará, $U$ será constante (en un gas ideal es función solamente de la temperatura, el ser ésta constante para todo el proceso, $U$ también lo será; por ello la dependencia de la entropía del gas ideal con $U$ se puede sustituir por la dependencia en $T$ ) y el número de partículas de cada componente es constante. Por lo tanto, la entropía de cada gas en la mezcla será igual a la que tenía antes de mezclarse ya que depende de las variables naturales $V, U_{\mathrm{i}}$ y $N_{\mathrm{i}}$ las cuales se han mantenido constantes durante el proceso: $S_{1}=S_{1}^{0}$ y $S_{2}=S_{2}^{0}$, luego $S=S_{1}+S_{2}=S^{0}$, por lo que $\Delta_{\mathrm{m}} S=S-S^{0}=0$, lo que implica que el proceso de mezcla de gases ideales a $V$ y $T$ constantes es reversible. En el estado final, $S(V, T, N)=$ $S_{1}(V, T, N)+S_{2}(V, T, N)$, o sea $S$ es aditiva pues es extensiva respecto $V, U(T)$ y $N$.

Por otra parte, si la mezcla se realiza a $P$ y $T$ constantes, para mantener la presión constante el volumen que ocupan éstos en el estado final necesariamente debe ser mayor que en el estado inicial. Por lo tanto, la entropía de cada gas es ahora $S_{1}\left(V_{f}, U_{1}^{0}, N_{1}\right)$ y $S_{2}\left(V_{f}, U_{2}^{0}, N_{2}\right)$, que por ser $V_{f} \neq V$, entonces $S_{1} \neq S_{1}^{0}$ y $S_{2} \neq S_{2}^{0}$, de modo que $S=S_{1}+S_{2} \neq S^{0}$, por lo que, $\Delta_{\mathrm{m}} S=S-S^{0} \neq 0$; de hecho, se encuentra que la entropía de mezcla es $\Delta_{\mathrm{m}} S=-\mathrm{R} \cdot \sum_{\mathrm{i}=1}^{\mathrm{n}} N_{\mathrm{i}} \cdot \ln x_{\mathrm{i}}>0$, lo que implica que la mezcla de gases ideales a $P$ y $T$ constantes es irreversible. En el estado final, $S(P, T, N) \neq S_{1}(P, T, N)+$ $S_{2}(P, T, N)$, o sea, $S$ no es aditiva en la variable $P$, pues $P$ no es extensiva.

\subsubsection{Equilibrio en sistemas aislados}

Las condiciones de cambio de los potenciales termodinámicos para los diferentes procesos de un sistema dan lugar a las condiciones de equilibrio termodinámico. En efecto, cuando un sistema llega al equilibrio termodinámico, el proceso que estaba realizando cesa y las condiciones (2.61)-(2.65) implican que:

$U$ es un mínimo si $S, V$ y $N_{\mathrm{i}}$ son constantes. 
$H$ es un mínimo si $S, P$ y $N_{\mathrm{i}}$ son constantes.

$F$ es un mínimo si $T, V$ y $N_{\mathrm{i}}$ son constantes.

$G$ es un mínimo si $T, P$ y $N_{\mathrm{i}}$ son constantes.

$S$ es un máximo si $U, V$ y $N_{\text {i }}$ son constantes. (Sistema aislado)

Para el resto de funciones características se obtienen relaciones similares.

De las diferentes maneras en las que un sistema termodinámico puede interaccionar con su entorno, el estudio de las condiciones de equilibrio de un sistema aislado tiene gran importancia práctica, aunque en la naturaleza ningún sistema es aislado. Por (2.74) sabemos que en este tipo de sistemas su entropía es máxima en el equilibrio. Puesto que $U, V$ y $N_{\text {i }}$ son aditivas, y si el sistema está particionado en $\pi$ fases independientes y tiene $\mathrm{n}$ componentes, las condiciones de equilibrio entre las diferentes fases son:

$$
\begin{aligned}
& \frac{1}{T^{1}}=\frac{1}{T^{2}}=\ldots=\frac{1}{T^{\pi}} \quad \text { (equilibrio térmico) } \\
& \frac{P^{1}}{T^{1}}=\frac{P^{2}}{T^{2}}=\ldots=\frac{P^{\pi}}{T^{\pi}} \quad \text { (equilibrio hidrostático) } \\
& \begin{array}{l}
\frac{\mu_{1}^{1}}{T^{1}}=\frac{\mu_{1}^{2}}{T^{2}}=\ldots=\frac{\mu_{1}^{\pi}}{T^{\pi}} \\
\text { (equilibrio másico) }
\end{array} \\
& \frac{\mu_{\mathrm{n}}^{1}}{T^{1}}=\frac{\mu_{\mathrm{n}}^{2}}{T^{2}}=\ldots=\frac{\mu_{\mathrm{n}}^{\pi}}{T^{\pi}}
\end{aligned}
$$

$\mathrm{Si}$ se dan los tres tipos de equilibrio, se dice que el sistema está en equilibrio termodinámico. Si no se cumplen todas las condiciones, se dice que el sistema está en equilibrio parcial, ya sea térmico, hidrostático o másico, o alguna combinación de ellos.

Las condiciones de equilibrio que aparecen en los libros clásicos de Termodinámica $\left(T^{1}=T^{2}=\ldots=T^{\pi} ; P^{1}=P^{2}=\ldots=P^{\pi} ; \mu_{1}^{1}=\mu_{1}^{2}=\ldots=\mu_{1}^{\pi} ; \ldots ; \mu_{\mathrm{n}}^{1}=\mu_{\mathrm{n}}^{2}=\ldots=\mu_{\mathrm{n}}^{\pi}\right)$ se deducen de la representación energética y no son del todo correctas, ya que, por (2.75)-(2.77), si no se verifica el equilibrio térmico, ni las presiones ni los potenciales químicos de las diferentes fases serán, en general, iguales.

En todas las ecuaciones que se han presentado en esta sección se ha supuesto que se cumplen las condiciones de equilibrio termodinámico (2.75)-(2.77).

\subsection{SISTEMAS MONOCOMPONENTES}

\subsubsection{Actividad absoluta}

En muchas aplicaciones resulta más útil trabajar con la actividad absoluta que con el potencial químico. Para la sustancia i-ésima de un sistema multicomponente ella se define como: 


$$
\lambda_{\mathrm{i}}=\exp \left(\frac{\mu_{\mathrm{i}}}{k_{\mathrm{B}} \cdot T}\right)
$$

lvego:

$$
\mu_{\mathrm{i}}=k_{\mathrm{B}} \cdot T \cdot \ln \left(\lambda_{\mathrm{i}}\right)
$$

donde $\mu_{\mathrm{i}}$ es el potencial químico por molécula de la sustancia i. Para cantidades por mol, basta con multiplicar por el número de avogadro:

$$
\mu_{\mathrm{i}}^{*}=R \cdot T \cdot \ln \left(\lambda_{\mathrm{i}}\right)
$$

En este caso, $\mu_{\mathrm{i}}^{*}$ es el potencial químico por mol. A partir de ahora, nos referiremos a esta cantidad, suprimiendo el superíndice * para simplificar la notación.

Tanto los potenciales termodinámicos como las condiciones de equilibrio pueden escribirse en función de la actividad absoluta en lugar de en función del potencial químico [10]. Por ejemplo, para un sistema monocomponente con dos fases que está en equilibrio térmico, la condición de equilibrio másico es : $\lambda_{i}^{\alpha}=\lambda_{i}^{\beta}$.

\subsubsection{Ecuaciones de estado de gases puros}

Como se explica en la sección 2.1.1., el estado termodinámico de un sistema queda definido si se conoce su ecuación de estado. Para los gases existe un número creciente de ecuaciones de estado, siendo la mayor parte de ellas empíricas o semiempíricas. Una importante colección de ellas pueden consultarse en el libro de Walas [22]. De todas, la ecuación más sencilla es la propuesta por Clapeyron [23] que aúna las leyes de Boyle y Charles-Gay Lussac, conocida como ley de los gases ideales:

$$
P=\frac{n \cdot R \cdot T}{V}=\rho \cdot k_{\mathrm{B}} \cdot T
$$

donde $n$ es el número de moles del gas que ocupan el volumen $V$ a temperatura $T$ y presión $P, \rho=N / V$ es la densidad de partículas, $N$ es el número total de moléculas del gas, y $k_{\mathrm{B}}$ la constante de Boltzmann. El modelo de gas ideal se basa en suponer que no hay energía potencial de interacción entre las moléculas del gas. A pesar de su sencillez, este modelo tiene gran importancia teórica, debida fundamentalmente a dos razones:

a) Todos los gases reales se comportan en muchos aspectos, aunque no en todos (véase [24] pp 33), igual que los ideales en el límite de bajas presiones, pues en esas condiciones, la distancia intermolecular se hace, en el límite, infinita lo que se traduce en la ausencia de interacción entre moléculas.

b) Históricamente se ha observado con frecuencia que la descripción cuantitativa de la naturaleza se puede conseguir más fácilmente idealizando los procesos de interés, es decir, estableciendo un modelo simplificado que describa a grandes rasgos el comportamiento esencial aunque se desprecien ciertos detalles. El comportamiento real se deduce del ideal mediante la aplicación de ciertas correcciones, de modo que las propiedades de las sustancias reales pueden expresarse convenientemente como desviaciones respecto la idealidad. En este sentido se definen las magnitudes residuales, de las cuales no vamos a decir nada aquí. El interesado puede consultar por ejemplo el libro de Smith y Van Ness [25, pp 179]. 
De (2.81) puede obtenerse fácilmente la función de Gibbs en función de la presión para un gas ideal puro. Sustituyendo dicha ecuación en (2.42) para un sistema monocomponente y monofásico e integrando a temperatura constante se tiene:

$$
G(T, P)-G\left(T, P^{+}\right)=n \cdot R \cdot T \cdot \ln \frac{P}{P^{+}}
$$

Para un mol de sustancia, $n=1, \mu=G_{\mathrm{m}}$, la ecuación anterior se rescribe como:

$$
\mu(T, P)=\mu\left(T, P^{+}\right)+R \cdot T \cdot \ln \frac{P}{P^{+}}
$$

$P^{+}$es la presión de un estado de referencia escogido arbitrariamente para hacer la integración. El resto de ecuaciones del gas ideal se obtienen fácilmente: la entropía de (2.41), la entalpía de $H=G-P \cdot V$ y el volumen de (2.42). De (2.78) y (2.83) se obtiene la actividad absoluta:

$$
\lambda=\frac{P}{P^{+}} \cdot e^{\frac{\mu\left(T, P^{+}\right)}{R \cdot T}}=\frac{P}{P^{+}} \cdot \lambda^{+}
$$

La ecuación que mejor se ajusta a los datos volumétricos de un gas es la ecuación del virial, deducida empíricamente por Thiesen [26] y mejorada por Onnes [27], según la cual el volumen se expresa como un desarrollo en serie de potencias de la presión:

$$
V=\frac{R \cdot T}{P}+\mathrm{B}(T)+\mathrm{C}(T) \cdot P+\mathrm{D}(T) \cdot P^{2}+\ldots
$$

donde $\mathrm{B}(T), \mathrm{C}(T), \mathrm{D}(T), \ldots$ dependen de la temperatura y de la naturaleza química del gas y se llaman segundo, tercer, cuarto,... coeficientes del virial y se determinan al ajustar los datos a la anterior ecuación. En el libro de Van Ness y Abbott [24, pp 119] se da un método para ello. El número de términos que se deben tomar dependerá de la precisión que se desee tener. Si se toman todos los términos ad infinitum, la ecuación es exacta, y representa el comportamiento de los gases con total exactitud. No obstante, solamente se han desarrollados métodos experimentales para calcular los primeros coeficientes del virial. Sin embargo, para presiones de hasta algunos cientos de atmósferas es suficiente con tomar hasta el tercer coeficiente del virial, y para presiones de unas pocas atmósferas basta con tomar hasta el segundo coeficiente del virial. A presiones bajas, se trunca la serie en el primer término, obteniéndose la ecuación del gas ideal.

Ursell [28] obtuvo esta ecuación mediante consideraciones estadísticas a partir del estudio de las fuerzas intermoleculares, de manera que los coeficientes del virial pueden estimarse a partir del potencial de interacción molecular [29] sin necesidad de realizar medidas $P V T$. De hecho, el segundo coeficiente del virial representa interacciones entre pares de moléculas vecinas; el tercer coeficiente del virial, entre tríos de moléculas vecinas, etc. Una vez conocidos los coeficientes del virial, el volumen de un gas en función de la presión puede predecirse por medio de la ecuación (2.85), la cual dejaría de ser una mera ecuación de ajuste de los datos experimentales para convertirse en una ecuación capaz de predecir el comportamiento de los gases reales. Sin embargo, el desconocimiento del potencial de interacción molecular debido a la no integrabilidad 
de las ecuaciones del movimiento del problema mecánico de tres o más cuerpos no permite el cálculo de los cuartos y superiores coeficientes del virial. En rigor, ni siquiera se puede calcular el tercer coeficiente del virial pues hay que introducir la hipótesis de que el potencial es aditivo por pares [31, pp 104]. Por lo tanto, esta ecuación sólo es de interés en un rango de presiones de hasta aproximadamente la mitad de la presión crítica ya que cuanto mayor sea la presión más cercanas están unas moléculas de otras y las interacciones entre 4, 5, ... moléculas vecinas son importantes, razón por la que se deben tener en cuenta el cuarto y superiores coeficientes del virial.

Si los campos de fuerza son centrales, el segundo coeficiente del virial es [32]:

$$
\mathrm{B}=-\frac{N_{\mathrm{A}}}{2} \cdot \int_{0}^{\mathrm{R}}\left(e^{-W(r) / k_{\mathrm{B}} \cdot T}-1\right) \cdot 4 \cdot \pi \cdot r^{2} \cdot \mathrm{d} r
$$

donde $N_{\mathrm{A}}$ es el número de avogadro, $W$ es la energía potencial de interacción entre dos moléculas, que depende de la posición relativa entre ambas y debe introducirse como una función conocida, lo cual es bastante discutible; $\mathrm{R}$ es una distancia intermolecular tal que si $r>\mathrm{R}$, entonces $W \approx 0$; por ejemplo, si se consideran las moléculas como esferas duras impenetrables de radio $\sigma$ cuyo potencial de interacción viene descrito por:

$$
W(r)= \begin{cases}\infty & r<\sigma \\ 0 & r>\sigma\end{cases}
$$

$R$ sería igual a $\sigma$, y el segundo coeficiente del virial, $\mathrm{B}=2 \cdot \pi \cdot N_{\mathrm{A}} \cdot \sigma^{3} / 3 \cdot$ Otros modelos para el potencial $W$ se pueden encontrar en la literatura [31, Cap. 4]-[33], por ejemplo el potencial de pozo cuadrado finito, de Lennard-Jones, de Kiyohara, etc.

$\mathrm{Si}$ las fuerzas no son centrales, la dependencia de $W$ con los ángulos complica el problema muchísimo (véase la ecuación (4.2)).

Otra desventaja de esta ecuación es que, al contrario que otras ecuaciones de estado como la de Van der Waals [34] o de Redlich-Kwong [35], no es capaz de representar al estado líquido ni la coexistencia entre las fases líquida y vapor, por las razones que se explican en la sección 6.2.

La generalización de esta ecuación a mezclas es muy sencilla. Para ello, es necesario definir unas reglas de mezcla que pueden consultarse por ejemplo en las referencias [24, pp 134] y [31, pp 105].

Conocidos los coeficientes del virial, de (2.85) y (2.42) se obtiene la función de Gibbs en función de la presión para un gas real puro:

$$
\begin{gathered}
\mu(T, P)=\mu\left(T, P^{+}\right)+R \cdot T \cdot \ln \frac{P}{P^{+}}+\mathrm{B}(T) \cdot P-\mathrm{B}(T) \cdot P^{+}+\mathrm{C}(T) \cdot P^{2}-\mathrm{C}(T) \cdot\left(P^{+}\right)^{2}+\ldots= \\
=\mu^{+}+R \cdot T \cdot \ln \frac{P}{P^{+}}+\mathrm{B}(T) \cdot P+\mathrm{C}(T) \cdot P^{2}+\ldots
\end{gathered}
$$

donde $\mu^{+}=\mu\left(T, P^{+}\right)-\mathrm{B}(T) \cdot P^{+}-\mathrm{C}(T) \cdot\left(P^{+}\right)^{2}-\ldots$

\footnotetext{
*El problema mecánico de tres o más cuerpos no tiene solución analítica, como demostró Poincaré en 1889 en el trabajo que le llevó a ganar el premio ofrecido por el Rey Oscar II de Suecia y Noruega con motivo de su 60 aniversario [30].
} 
La actividad absoluta es:

$$
\lambda=e^{\frac{\mu^{+}}{R \cdot T}} \cdot \frac{P}{P^{+}} \cdot e^{\frac{\mathrm{B} \cdot P+\mathrm{C} \cdot P^{2}+\ldots}{R \cdot T}}=\lambda^{+} \cdot \frac{P}{P^{+}} \cdot e^{\frac{\mathrm{B} \cdot P+\mathrm{C} \cdot P^{2}+\ldots}{R \cdot T}}
$$

Si en (2.88) y (2.89) se hace $\mathrm{B}(T)=\mathrm{C}(T)=\ldots=0$, se recuperan las ecuaciones del gas ideal.

\subsubsection{Fugacidad de sustancias puras}

Como se ha dicho, la importancia del modelo de gas ideal radica en que es un modelo simple con el cual se obtiene el comportamiento de los gases reales haciendo las correcciones necesarias. Así es útil comparar el comportamiento de los gases reales con el de los gases ideales. Con este propósito, Lewis [36] introdujo el concepto de una presión ficticia llamada fugacidad, de manera que las ecuaciones de los gases reales se pueden escribir de la misma forma que las de los gases ideales. En palabras de Hildebrand [37], la fugacidad mide la tendencia de una sustancia a escapar de la fase en la que se encuentra. En el caso de que esa fase sea una mezcla, mide la tendencia de la sustancia a escapar de la mezcla, es decir, la miscibilidad. La fugacidad $p^{*}$ de un gas real se define por las dos propiedades siguientes:

$$
\begin{aligned}
& \frac{p^{*}}{\lambda}=\text { cte. } \text { si } T=\text { cte. } \\
& \lim _{P \rightarrow 0} \frac{p^{*}}{P}=1 \text { si } T=\text { cte. }
\end{aligned}
$$

Así, para un gas real descrito por la ecuación del virial se encuentra que $p^{*}=P^{+} \cdot \lambda / \lambda^{+}$, de manera que se tiene una ecuación análoga a la (2.84) válida para el gas ideal:

$$
\lambda=\frac{p^{*}}{P^{+}} \cdot \lambda^{+}
$$

En el caso del gas ideal, la fugacidad es igual a la presión. Por tanto, la fugacidad es una presión efectiva que sirve como corrección a las desviaciones de las leyes de los gases ideales.

De (2.79) y (2.92), se tiene que:

$$
\mu=\mu^{+}+R \cdot T \cdot \ln \frac{p^{*}}{P^{+}}
$$

De (2.93) pueden obtenerse el resto de funciones termodinámicas. Por ejemplo,

$$
V_{\mathrm{m}}=R \cdot T \cdot\left(\frac{\partial \ln p^{*}}{\partial P}\right)_{T}
$$

La simplificación que se obtiene empleando la fugacidad es sólo aparente, puesto si se desean obtener los resultados cuantitativos se debe expresar $p^{*}$ en función de la presión, es decir, se debe conocer la ecuación de estado.

Para un sistema monocomponente que consta de una fase condensada en equilibrio con su vapor, la fugacidad de la fase condensada se define como igual a la fugacidad de la fase vapor con la que se encuentra en equilibrio. Así, si el sistema estuviese formado por varias fases condensadas, todas ellas tendrían la misma fugacidad. 


\subsection{SISTEMAS DE VARIOS COMPONENTES: MEZCLAS}

A partir de ahora vamos a estudiar aquellos sistemas que tienen más de un componente y a los que nos referiremos con el nombre de mezclas o disoluciones. En concreto, nos referiremos al estudio de mezclas líquidas de no electrolitos, excluyendo así el estudio de las sales fundidas y de las mezclas de diferentes especies iónicas.

\subsubsection{Propiedad molar}

Para una mezcla de n componentes, a cada magnitud extensiva $X$ le corresponde una intensiva $X_{\mathrm{m}}$ sin más que dividir $X$ por el número total de moles:

$$
X_{\mathrm{m}}=\frac{X}{\sum_{\mathrm{i}=1}^{\mathrm{n}} n_{\mathrm{i}}}
$$

Así definimos entropía molar media, $S_{\mathrm{m}}$, función de Gibbs molar media, $G_{\mathrm{m}}, \ldots$

Una propiedad muy importante de las propiedades molares o propiedades molares medias es que se siguen cumpliendo las relaciones existentes entre $U, S, G, H, U, F, \ldots$ (por ejemplo, las ecuaciones (2.29)-(2.31), o las (2.41)-(2.43)).

\subsubsection{Propiedad molar parcial}

El potencial termodinámico $G$ tiene una importante propiedad y es que de sus variables naturales solamente las $n_{i}$ son variables extensivas. Por tanto, como consecuencia del teorema de Euler, ecuación (2.9), G puede escribirse como la suma (2.47), en donde se observa que el potencial por molécula es:

$$
\mu_{\mathrm{i}}=\left(\frac{\partial G}{\partial N_{\mathrm{i}}}\right)_{T, P, N_{\mathrm{i}}, \text { i } \neq \mathrm{j}}
$$

A partir de ahora nos referiremos al potencial químico por mol. Para ello, basta con multiplicar (2.47) por $N_{\mathrm{A}}$ :

$$
\mu_{\mathrm{i}}^{*}=\left(\frac{\partial G}{\partial n_{\mathrm{i}}}\right)_{T, P, n_{\mathrm{i}}, i \neq \mathrm{j}}
$$

Para simplificar la notación, a partir de ahora se eliminará el superíndice *

Por analogía, para una magnitud extensiva $X$ cualquiera, expresada como una función de $T, P, n_{\mathrm{i}} ; \mathrm{i}=1, \ldots, \mathrm{n}$ :

$$
\mathrm{d} X=\left(\frac{\partial X}{\partial T}\right)_{P, n_{\mathrm{i}} \mathrm{i}=1, \ldots, \mathrm{n}} \cdot \mathrm{d} T+\left(\frac{\partial X}{\partial P}\right)_{T, n_{\mathrm{i}}, \mathrm{i}=1, \ldots, \mathrm{n}} \cdot \mathrm{d} P+\sum_{\mathrm{i}=1}^{\mathrm{n}}\left(\frac{\partial X}{\partial n_{\mathrm{i}}}\right)_{T, P, n_{\mathrm{j}, j \neq \mathrm{i}}} \cdot \mathrm{d} n_{\mathrm{i}}
$$

se define $X_{\mathrm{i}}$ como:

$$
X_{\mathrm{i}}=\left(\frac{\partial X}{\partial n_{\mathrm{i}}}\right)_{T, P, n_{\mathrm{j}}, \mathrm{j} \neq \mathrm{i}}
$$


de manera que:

$$
X=\sum_{\mathrm{i}=1}^{\mathrm{n}} n_{\mathrm{i}} \cdot X_{\mathrm{i}}
$$

y:

$$
X_{\mathrm{m}}=\sum_{\mathrm{i}=1}^{\mathrm{n}} x_{\mathrm{i}} \cdot X_{\mathrm{i}}
$$

A $X_{\mathrm{i}}$ se le llama propiedad molar parcial de $X$ del componente $\mathrm{i}$ en la mezcla. Las propiedades parciales son magnitudes intensivas. Aunque suponiendo que la propiedad molar parcial $X_{\mathrm{i}}$ es el valor de la propiedad $X$ de la especie i-ésima cuando ésta existe en la mezcla siempre se puede llegar a conclusiones correctas, esto no es del todo preciso pues cada especie en una disolución no existe como una entidad aislada con propiedades independientes.

$X$ puede ser cualquier magnitud, siempre que sea extensiva: $G, V, U, H, S, F, \ldots$ En el caso de $G$ es fácil ver que como consecuencia de la ecuación (2.96) el potencial químico es el potencial de Gibbs molar parcial: $\mu_{\mathrm{i}}=G_{\mathrm{i}}$.

Como las propiedades molares parciales se obtienen por derivación, las relaciones entre las diferentes funciones se mantienen, igual que ocurre en el caso de las magnitudes molares medias:

$$
\begin{gathered}
F_{\mathrm{i}}=U_{\mathrm{i}}-T \cdot S_{\mathrm{i}} \\
H_{\mathrm{i}}=U_{\mathrm{i}}+P \cdot V_{\mathrm{i}} \\
G_{\mathrm{i}}=U_{\mathrm{i}}-T \cdot S_{\mathrm{i}}+P \cdot V_{\mathrm{i}} \\
S_{\mathrm{i}}=-\left(\frac{\partial G_{\mathrm{i}}}{\partial T}\right)_{P, n_{\mathrm{j}}, \mathrm{j}=1, \ldots, \mathrm{n}} \\
V_{\mathrm{i}}=\left(\frac{\partial G_{\mathrm{i}}}{\partial P}\right)_{T, n_{\mathrm{j}}, \mathrm{j}=1, \ldots, \mathrm{n}}
\end{gathered}
$$

Esta analogía entre las relaciones que cumplen las funciones termodinámicas de sistemas monocomponentes y las que cumplen las propiedades parciales es válida solamente si dichas relaciones son lineales en el sentido algebraico.

Para entender el significado de propiedad molar parcial, consideremos por ejemplo el volumen. La ecuación (2.100) en este caso es:

$$
V=\sum_{\mathrm{i}=1}^{\mathrm{n}} n_{\mathrm{i}} \cdot V_{\mathrm{i}}
$$

Supongamos que añadimos, a $P, T$ y $n_{\mathrm{i}}\left(\neq n_{\mathrm{j}}\right)$ constantes, una cantidad $\Delta n_{\mathrm{j}}$ de la sustancia j-ésima a la mezcla, lo suficientemente pequeña como para suponer que la mezcla se hace a composición constante. En ese caso, el volumen de la misma es:

$$
V=\sum_{\mathrm{i}=1, \neq \mathrm{j}}^{\mathrm{n}} n_{\mathrm{i}} \cdot V_{\mathrm{i}}+\lim _{\Delta n_{\mathrm{j}} \rightarrow 0}\left(n_{\mathrm{j}}+\Delta n_{\mathrm{j}}\right) \cdot V_{\mathrm{j}}
$$


lo que significa que el volumen de la mezcla se ha incrementado en $\Delta V=\lim _{\Delta n_{j} \rightarrow 0} \Delta n_{\mathrm{j}} \cdot V_{\mathrm{j}}$. Luego,

$$
V_{\mathrm{j}}=\lim _{\Delta n_{\mathrm{j}} \rightarrow 0} \frac{\Delta V}{\Delta n_{\mathrm{j}}}=\left(\frac{\partial V}{\partial n_{\mathrm{j}}}\right)_{T, P, n_{i}}
$$

Por lo tanto, el volumen molar parcial del componente j-ésimo en la mezcla representa el ritmo del cambio de volumen de la mezcla con $n_{\mathrm{j}}$ a $T, P$ y $n_{\mathrm{i}}$ constantes. Lo mismo ocurre si en vez de considerar volúmenes se considera cualquier otra magnitud.

En una mezcla binaria, $1=x_{1}+x_{2}$ luego, si $x_{2}=x$, de (2.101) se tiene que para una propiedad molar media cualquiera se cumple la ecuación siguiente:

$$
X_{\mathrm{m}}=x_{1} \cdot X_{1}+x_{2} \cdot X_{2}=(1-x) \cdot X_{1}+x \cdot X_{2}
$$

Diferenciando esta expresión:

$$
\mathrm{d} X_{\mathrm{m}}=(1-x) \cdot \mathrm{d} X_{1}+x \cdot \mathrm{d} X_{2}+\left(X_{2}-X_{1}\right) \cdot \mathrm{d} x
$$

Dividiendo (2.98) por $n_{1}+n_{2}$ y comparando con (2.111), se tiene la ecuación de GibbsDuhem:

$$
0=\left(\frac{\partial X_{\mathrm{m}}}{\partial T}\right)_{P, n_{\mathrm{i}}} \cdot \mathrm{d} T+\left(\frac{\partial X_{\mathrm{m}}}{\partial P}\right)_{P, T, n_{\mathrm{i}}, \mathrm{i}=1, \ldots, \mathrm{n}} \cdot \mathrm{d} P-\sum_{\mathrm{i}=1}^{2} x_{\mathrm{i}} \cdot \mathrm{d} X_{\mathrm{i}}
$$

Para $T$ y $P$ constantes, la ecuación anterior se transforma en la siguiente:

$$
(1-x) \cdot \mathrm{d} X_{1}+x \cdot \mathrm{d} X_{2}=0^{*}
$$

Que ciertas funciones $\chi_{i}$ satisfagan la ecuación (2.100):

$$
X=\sum_{\mathrm{i}=1}^{\mathrm{n}} n_{\mathrm{i}} \cdot \chi_{\mathrm{i}}
$$

no quiere decir que éstas sean propiedades molares parciales. De hecho, si no cumplen la ecuación de Gibbs Duhem (2.113) a $P$ y $T$ constantes, no serán propiedades molares parciales en el sentido de la ecuación (2.99).

El estado intensivo de un sistema binario monofásisco está determinado por $2+2-1=3$ variables independientes, consecuencia de la ecuación de Gibbs-Duhem. Por lo tanto, al ser las propiedades molares parciales propiedades intensivas, ellas son función de tres variables, $X_{\mathrm{i}}=X_{\mathrm{i}}(T, P, x)$, luego:

$$
\mathrm{d} X_{i}=\left(\frac{\partial X_{\mathrm{i}}}{\partial T}\right)_{P, x_{1}, x_{2}} \cdot \mathrm{d} T+\left(\frac{\partial X_{\mathrm{i}}}{\partial P}\right)_{T, x_{1}, x_{2}} \cdot \mathrm{d} P+\sum_{\mathrm{j}=1}^{2}\left(\frac{\partial X_{\mathrm{i}}}{\partial x_{\mathrm{j}}}\right)_{T, P, x_{\mathrm{k}} \neq x_{\mathrm{j}}} \cdot \mathrm{d} x_{\mathrm{j}}
$$

donde $x_{1}=1-x_{2}=1-x$. A $T$ y $P$ constantes se tiene que:

\footnotetext{
* En algunos libros se llama ecuación de Gibbs-Duhem a $P$ y $T$ constantes para la propiedad molar parcial $X_{i}$. Esta ecuación es importante ya que si se dispone de datos experimentales de $X_{i}$ en un amplio rango de composiciones, se puede comprobar la consistencia termodinámica de los datos gracias a ella. Esta ecuación demuestra que no pueden imponerse expresiones arbitrarias para la dependencia con la composición de $X_{1}$ y $X_{2}$ : dada una expresión para uno de ellos, la otra se obtiene a partir de (2.113).
} 


$$
\mathrm{d} X_{i}=\sum_{\mathrm{j}=1}^{2}\left(\frac{\partial X_{\mathrm{i}}}{\partial x_{\mathrm{j}}}\right)_{T, P, x_{\mathrm{k}} \neq x_{\mathrm{j}}} \cdot \mathrm{d} x_{\mathrm{j}}=2 \cdot\left(\frac{\partial X_{\mathrm{i}}}{\partial x}\right)_{T, P} \cdot \mathrm{d} x
$$

Sustituyendo en (2.113):

$$
2 \cdot\left[(1-x) \cdot\left(\frac{\partial X_{1}}{\partial x}\right)_{T, P}+x \cdot\left(\frac{\partial X_{2}}{\partial x}\right)_{T, P}\right] \cdot \mathrm{d} x=0
$$

Esta ecuación implica que si la pendiente de $X_{2}$ es horizontal para cierta concentración $x$, entonces la pendiente de $X_{1}$ en esa misma concentración será horizontal. En otras palabras, $X_{1}$ y $X_{2}$ tienen extremos relativos en los mismos valores de composición.

El comportamiento de las propiedades molares parciales en mezclas diluidas se puede examinar gracias a la ecuación (2.117). Si se elimina $2 \cdot \mathrm{d} x$ y se rescribe como:

$$
\left(\frac{\partial X_{1}}{\partial x}\right)_{T, P} /\left(\frac{\partial X_{2}}{\partial x}\right)_{T, P}=-\frac{x}{(1-x)}
$$

En las regiones de concentración diluida:

a) Si $1-x \rightarrow 1, x \rightarrow 0$, entonces

$$
\left(\frac{\partial X_{1}}{\partial x}\right)_{T, P}=0
$$

o bien toma un valor finito no nulo, en cuyo caso $\left(\frac{\partial X_{2}}{\partial x}\right)_{T, P} \rightarrow \pm \infty$.

b) Si $1-x \rightarrow 0, x \rightarrow 1$, entonces

$$
\left(\frac{\partial X_{2}}{\partial x}\right)_{T, P}=0
$$

o bien toma un valor finito no nulo en cuyo caso $\left(\frac{\partial X_{1}}{\partial x}\right)_{T, P} \rightarrow \pm \infty$.

En los casos en que la derivada es cero, $X_{\mathrm{i}}$ tiende al valor $X_{\mathrm{i}}^{0}$ con pendiente horizontal cuando $x_{\mathrm{i}} \rightarrow 1$, como se observa en la llustración 2-1. Esto se cumple en la mayoría de las magnitudes de mezclas de no electrolitos, excepto para $G, F, S$ como se verá unos párrafos más adelante.

La ecuación (2.117) también implica que, dado que $x$ y 1-x siempre son cantidades positivas, las pendientes deben ser de signo contrario.

Derivando respecto $x$ la ecuación (2.110) a $T$ y $P$ constantes, se tiene que:

$$
\left(\frac{\partial X_{\mathrm{m}}}{\partial x}\right)_{T, P}=-X_{1}+(1-x) \cdot\left(\frac{\partial X_{1}}{\partial x}\right)_{T, P}+X_{2}+x \cdot\left(\frac{\partial X_{2}}{\partial x}\right)_{T, P}
$$

De (2.117), se tiene:

$$
\left(\frac{\partial X_{\mathrm{m}}}{\partial x}\right)_{T, P}=-X_{1}+X_{2}
$$


Multiplicando esta expresión por $x$ y restando el resultado a la (2.110) se tiene que:

$$
X_{\mathrm{m}}-x \cdot\left(\frac{\partial X_{\mathrm{m}}}{\partial x}\right)_{T, P}=X_{1}
$$

Multiplicando la expresión (2.120) por $(1-x)$ y sumando el resultado a la (2.110) se tiene que:

$$
X_{\mathrm{m}}+(1-x) \cdot\left(\frac{\partial X_{\mathrm{m}}}{\partial x}\right)_{T, P}=X_{2}
$$

Las ecuaciones (2.121) y (2.122) se pueden generalizar para el caso de más componentes [38 pp 90]:

$$
X_{\mathrm{i}}=X_{\mathrm{m}}-\sum_{\mathrm{j} \neq \mathrm{i}}^{\mathrm{n}} x_{\mathrm{j}} \cdot\left(\frac{\partial X_{\mathrm{m}}}{\partial x_{\mathrm{j}}}\right)_{T, P, x_{\mathrm{k} \neq j, \mathrm{i}}}
$$

Estas expresiones dan un significado geométrico a las propiedades molares parciales, como se explica a continuación. En la llustración 2-1 se representan para la mezcla anilina (1) + 2-butanona (2), a $298.15 \mathrm{~K}$ el volumen molar y los volúmenes molares parciales en función de la composición. Fijemos ésta en $x=0.3064$, y sea $X_{\mathrm{m}}$, el valor del volumen molar para dicha composición, lo que se representa por el punto $P$ de la figura. En la misma se observa la igualdad entre segmentos $\overline{B A} \equiv \overline{B O^{\prime}}-\overline{A O^{\prime}}$, donde $A$ es el punto de corte de la línea perpendicular al eje de las abscisas en $x=1$ y la recta tangente a la curva $X_{\mathrm{m}}$ en el punto $P$; $B$ es el punto de corte de la recta paralela al eje de abscisas que pasa por el punto $P$ y la línea perpendicular al eje de las abscisas en $x=1 ; O$ ' es el punto del eje de abscisas en que $x=1$. La pendiente de la recta tangente a la curva en el punto $P$ es:

$$
\operatorname{tg} \alpha=\left(\frac{\partial X_{\mathrm{m}}}{\partial x}\right)_{T, P}=-\frac{\overline{B A}}{\overline{B P}}
$$

Ahora bien, de la gráfica se observa que $X_{\mathrm{m}}=\overline{O^{\prime} B}=\overline{B A}+\overline{A O^{\prime}} ; \overline{B P}=1-x$ y $\overline{P B^{\prime}}=x$, donde $B^{\prime}$ es el punto de corte de la recta paralela al eje de abscisas que pasa por el punto $P$ y el eje de ordenadas. Por tanto,

$$
\overline{B A}=-\overline{B P} \cdot\left(\frac{\partial X_{\mathrm{m}}}{\partial x}\right)_{T, P}=-(1-x) \cdot\left(\frac{\partial X_{\mathrm{m}}}{\partial x}\right)_{T, P}=X_{\mathrm{m}}-\overline{A O^{\prime}}
$$

Luego,

$$
\overline{A O^{\prime}}=X_{\mathrm{m}}+(1-x) \cdot\left(\frac{\partial X_{\mathrm{m}}}{\partial x}\right)_{T, P}
$$

que coincide justamente con $X_{2}$ como se refleja en la ecuación (2.122). Esto quiere decir que el valor $X_{2}$ de la propiedad molar parcial del componente (2) en una mezcla cuya concentración del componente 2 es $x$ viene dado por el punto de corte de la recta perpendicular al eje de abscisas en $x=x_{2}=1$ y la recta tangente a la curva en el punto $P$. Mediante un razonamiento similar se puede encontrar que la intersección de la recta tangente a la curva en $P$ con el eje de ordenadas da el valor de $X_{1}$. 


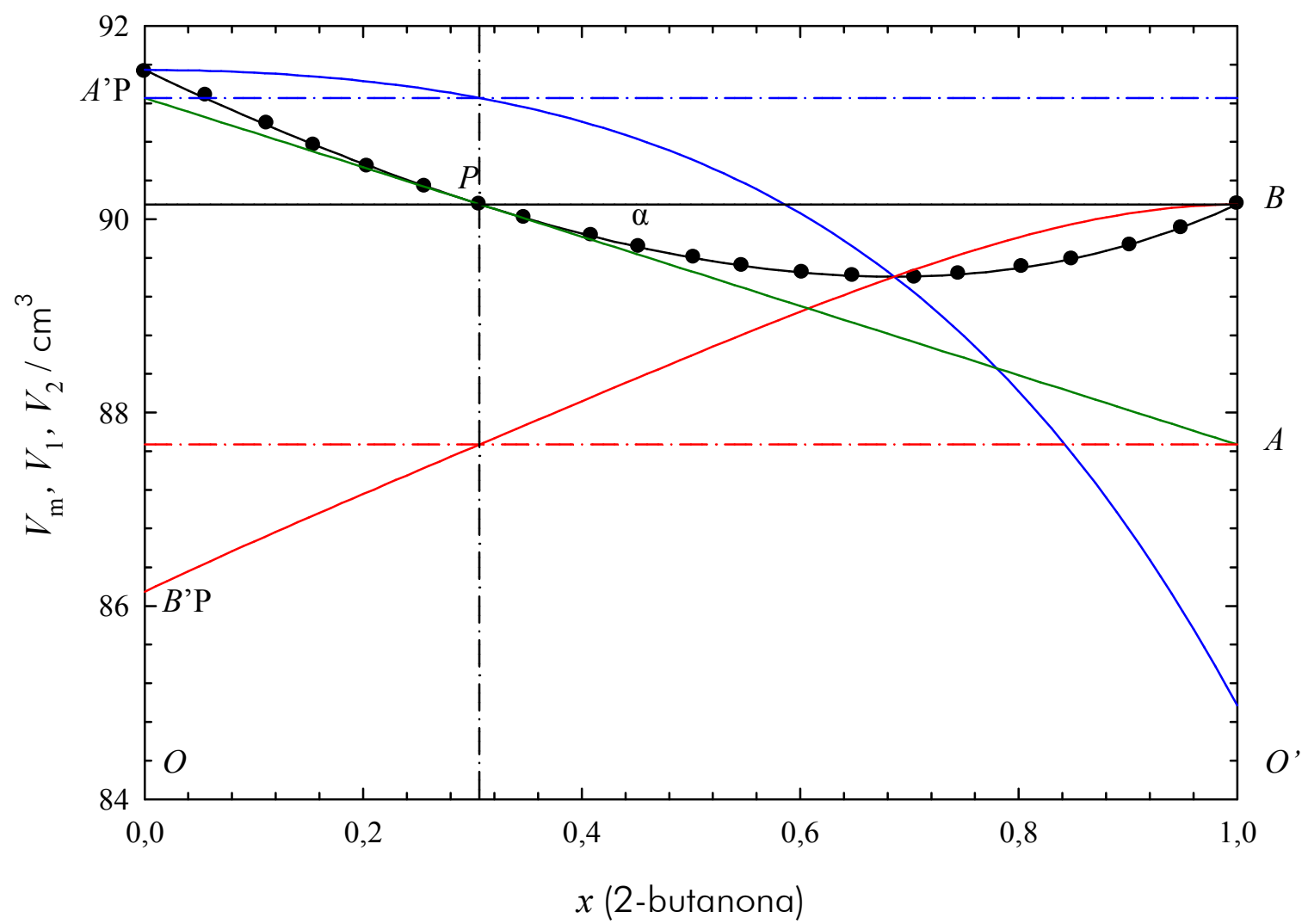

Ilustración 2-1: Volumen molar y volúmenes molares parciales a $298.15 \mathrm{~K}$ y presión atmosférica de la mezcla anilina (1) + 2-butanona (2) en función de la composición de la 2-butanona. • datos experimentales de $V_{\mathrm{m}}$; - polinomio de orden cuatro al que se han ajustado esos datos; $-V_{1} ;-V_{2}$; recta tangente a $V_{\mathrm{m}}$ que pasa por el punto $\mathrm{P} ;-\cdot-$ rectas paralelas a los ejes que pasan por el punto $\mathrm{P}$; -.- recta paralela al eje de abscisas y que corta a $V_{1}$ justo en el valor del volumen molar parcial de la anilina en $x=0.3064$; -- - recta paralela al eje de abscisas y que corta a $V_{2}$ justo en el valor del volumen molar parcial de la 2-butanona en $x=0.3064$.

Este método gráfico para encontrar las propiedades molares parciales de una mezcla binaria, debido a Bakhuis-Roozeboom [39], ha sido criticado por Rowlinson [38]. Según él, el mejor método para obtener las propiedades molares parciales de una mezcla es a partir de las funciones de exceso.

En la figura se observa muy bien cómo las propiedades molares parciales no son propiedades de los componentes puros sino de la mezcla, ya que los puntos de corte van variando con la composición $x$. Si por el contrario, no dependiesen de la composición, serían propiedades de los componentes puros; Así, dadas $X_{1}^{0}$ y $X_{2}^{0}$ las propiedades $X$ molares de los componentes puros 1 y 2 , se tendría $X_{\mathrm{m}}=x_{1} \cdot X_{1}^{0}+x_{2} \cdot X_{2}^{0}$ luego $X_{\mathrm{m}}=(1-x) \cdot X_{1}^{0}+x \cdot X_{2}^{0}=X_{1}^{0}+x \cdot\left(X_{2}^{0}-X_{1}^{0}\right)$, lo que indica una relación lineal entre $X_{\mathrm{m}}$ y $x$. Esta linealidad aparece en algunos casos, por ejemplo:

a) Si $M_{\mathrm{m}}$ es la masa molar de la mezcla y $M_{1}^{0}$ y $M_{2}^{0}$ las masas molares de los componentes puros 1 y 2 , el principio de conservación de la masa establece la relación:

$$
M_{\mathrm{m}}=M_{1}^{0}+x \cdot\left(M_{2}^{0}-M_{1}^{0}\right)
$$


b) En el caso de mezclas ideales, si $p^{*}$ representa la fugacidad de la mezcla y $p_{1}^{* 0}$ y $p_{2}^{* 0}$ las fugacidades de los componentes puros 1 y 2 , se tiene la ley de Lewis-Randall:

$$
p^{*}=p_{1}^{* 0}+x \cdot\left(p_{2}^{* 0}-p_{1}^{* 0}\right)
$$

c) Si las fases de vapor pueden considerarse gases ideales, la ley de Lewis-Randall se transforma en la ley de Raoult sin más que sustituir las fugacidades por las presiones.

En la llustración 2-1 también se observa que las curvas $V_{\mathrm{m}}, V_{1}$ y $V_{2}$ se cortan en el mínimo de $V_{\mathrm{m}}$. Esta característica se puede extender a cualquier magnitud $X_{\mathrm{m}}$.

La ecuación de Gibbs-Duhem en la forma (2.117) para $X_{\mathrm{i}}=G_{\mathrm{i}}=\mu_{\mathrm{i}}$ puede escribirse en términos de la actividad absoluta, definida por la ecuación (2.78):

$$
(1-x) \cdot\left(\frac{\partial \ln \lambda_{1}}{\partial x}\right)_{T, P}+x \cdot\left(\frac{\partial \ln \lambda_{2}}{\partial x}\right)_{T, P}=0
$$

\subsubsection{Actividad relativa. Fugacidad en mezclas}

En el estudio de mezclas, la comparación de las funciones termodinámicas de las mismas con las de las sustancias puras que las forman constituye un método recurrente. Así, la cantidad $\mu_{\mathrm{i}}-\mu_{\mathrm{i}}^{0}$ adquiere vital importancia, donde $\mu_{\mathrm{i}}^{0}$ es el potencial químico de la sustancia i-esima en el estado de referencia de gas puro si la mezcla es de gases o en estado de líquido puro si la mezcla fuese de líquidos, a la misma presión y temperatura de la mezcla. Consideremos solamente mezclas líquidas. De acuerdo con esta definición, el estado de referencia 0 a veces puede tener significado abstracto ya que normalmente la presión de vapor de la mezcla es diferente que la de alguno de sus componentes puros, por lo que puede suceder que a la $P$ y $T$ de la mezcla, dichos componentes puros no se encuentren en estado líquido. Entonces $\mu_{\mathrm{i}}^{0}$ puede referirse a estados líquidos extrapolados a presiones por debajo de la presión de vapor, lo que carece de sentido físico. Afortunadamente, el cambio de $\mu_{\mathrm{i}}^{0}$ con la presión es pequeño en líquidos cerca de su punto triple, por lo que la extrapolación necesaria para obtener $\mu_{\mathrm{i}}^{0}$ es casi despreciable por debajo de 3 bares. Ello justifica el definir un estado de líquido puro a la misma $P$ y $T$ de la mezcla a presiones menores de 3 bares.

Lewis [40] definió la actividad relativa $a_{\mathrm{i}}$ del componente i-ésimo en la mezcla:

$$
a_{\mathrm{i}}=\lambda_{\mathrm{i}} / \lambda_{\mathrm{i}}^{0}
$$

Por lo que la diferencia $\mu_{\mathrm{i}}-\mu_{\mathrm{i}}^{0}$ se puede escribir como:

$$
\mu_{\mathrm{i}}-\mu_{\mathrm{i}}^{0}=R \cdot T \cdot \ln \frac{\lambda_{\mathrm{i}}}{\lambda_{\mathrm{i}}^{0}}=R \cdot T \cdot \ln a_{\mathrm{i}}
$$

La fugacidad en un sistema multicomponente gaseoso se define de manera similar a como se hace para el caso de gases puros. Dada la presión parcial $p_{\mathrm{i}}$ del gas i-ésimo en una mezcla de n gases distintos a la presión $P$ :

$$
p_{\mathrm{i}}=x_{\mathrm{i}} \cdot P=\frac{n_{\mathrm{i}}}{\sum_{\mathrm{j}=1}^{\mathrm{n}} n_{\mathrm{j}}} \cdot P
$$


se define la fugacidad del componente i-ésimo en la mezcla gaseosa $p_{\mathrm{i}}^{* \mathrm{G}}$ como una presión ficticia que satisface las dos condiciones siguientes:

$$
\begin{aligned}
& \frac{p_{\mathrm{i}}^{*_{\mathrm{G}}}}{\lambda_{\mathrm{i}}^{\mathrm{G}}}=\text { cte. si } T=\text { cte. } \\
& \lim _{P \rightarrow 0} \frac{p_{\mathrm{i}}^{*_{\mathrm{G}}}}{p_{\mathrm{i}}}=1 \text { si } T=\text { cte. }
\end{aligned}
$$

El superíndice $\mathrm{G}$ indica que los componentes están en fase gaseosa.

La fugacidad de la mezcla $p^{*}$ se define a partir de:

$$
\begin{gathered}
\frac{p^{*}}{\lambda}=\text { cte. si } T=\text { cte. } \\
\lim _{P \rightarrow 0} \frac{p^{*}}{P}=1
\end{gathered}
$$

Existen ciertas similitudes entre la fugacidad de una mezcla y de un compuesto puro:

a) A presiones bajas la mezcla de gases reales se puede considerar una mezcla ideal de gases ideales y sustituir la fugacidad por la presión parcial.

b) La introducción del concepto de fugacidad transforma las ecuaciones de mezclas de gases reales en las ecuaciones de mezcla de gases ideales, sin más que sustituir $p_{\text {i }}$ por $p_{\mathrm{i}}^{* \mathrm{G}}$. Sin embargo, esta simplificación es aparente puesto que para obtener las propiedades del sistema debe conocerse la fugacidad en función de la presión, además de la composición, lo que implica el conocimiento de la ecuación de estado del sistema. Sea una mezcla de fases condensadas, en equilibrio con su vapor, que será una mezcla de los vapores de las diferentes sustancias. Se define la fugacidad del componente i-ésimo en la mezcla condensada como igual a la fugacidad de la misma sustancia en la fase vapor con la que se encuentra en equilibrio, por tanto en la mezcla todas las sustancias tienen la misma fugacidad sin importar la fase en la que estén. Por ejemplo, si la mezcla es líquida y el superíndice $\mathrm{L}$ representa la fase líquida, entonces $p_{\mathrm{i}}^{{ }_{\mathrm{L}}}=p_{\mathrm{i}}^{{ }_{\mathrm{G}} \mathrm{G}}$. De la definición de fugacidad para una fase condensada, de (2.133) y de la condición de equilibrio entre fases a igual presión y temperatura, $\lambda_{\mathrm{i}}^{\alpha}=\lambda_{\mathrm{i}}^{\beta}$, se deduce que el cociente $p_{i}^{*} / \lambda_{i}$ no depende de la fase considerada. En conclusión el equilibrio entre fases se puede escribir en términos de la fugacidad: $p_{\mathrm{i}}^{* \mathrm{~L}}=p_{\mathrm{i}}^{* \mathrm{G}}=\ldots$ Para dos fases cualesquiera,

$$
\frac{p_{i}^{* \alpha}}{p_{i}^{* \beta}}=\frac{\lambda_{i}^{\alpha}}{\lambda_{i}^{\beta}}
$$

Si una fase es la mezcla líquida y la otra fase es la sustancia i-ésima pura en estado líquido a igual presión y temperatura que la mezcla, la ecuación (2.137) es:

$$
\frac{p_{\mathrm{i}}^{*}}{p_{\mathrm{i}}^{* 0}}=\frac{\lambda_{\mathrm{i}}}{\lambda_{\mathrm{i}}^{0}}=a_{\mathrm{i}}
$$


en donde ya no se hace uso de los superíndices $L \circ G$ ya que se ha visto que las actividades absolutas y las fugacidades de un componente en una mezcla no dependen de la fase en que se encuentre. Por esta razón es frecuente encontrar en la bibliografía que a la actividad relativa se le llame fugacidad relativa [41].

$\operatorname{De}(2.131)$ y $(2.138)$,

$$
\mu_{\mathrm{i}}-\mu_{\mathrm{i}}^{0}=R \cdot T \cdot \ln \frac{p_{\mathrm{i}}^{*}}{p_{\mathrm{i}}^{* 0}}
$$

Esta ecuación permite hacer una traslación conceptual desde las variables termodinámicas a las variables físicas, ya que la fugacidad es una presión corregida cuya desviación con el caso ideal se debe a las fuerzas que actúan entre las moléculas.

Si los gases se consideran ideales, sus fugacidades son iguales a las presiones parciales; diferenciando la ecuación (2.138) se tiene que $d \ln \lambda_{\mathrm{i}}=d \ln p_{\mathrm{i}}=d \ln a_{\mathrm{i}}$, luego la ecuación de Gibbs-Duhem (2.129) se puede escribir como:

$$
(1-x) \cdot\left(\frac{\partial \ln p_{1}}{\partial x}\right)_{T, P}+x \cdot\left(\frac{\partial \ln p_{2}}{\partial x}\right)_{T, P}=0
$$

que es la conocida ecuación de Duhem-Margules [42] para una mezcla binaria e implica que si en el entorno de cierta composición la fugacidad de uno de los componentes es constante, la del otro también lo será.

\subsubsection{Coeficiente de fugacidad}

La fugacidad se define en términos de una magnitud adimensional llamada coeficiente de fugacidad:

$$
\varphi_{\mathrm{i}}=p_{\mathrm{i}}^{*} / p_{\mathrm{i}}=p_{\mathrm{i}}^{*} /\left(x_{\mathrm{i}} \cdot P\right)
$$

como puede apreciarse en la ecuación (2.134). Por tanto, el problema de encontrar la fugacidad, o por (2.139), el potencial químico, es el mismo que el de encontrar el coeficiente de fugacidad. A temperatura y composición constantes, se puede demostrar la siguiente relación, si se toma como estado de referencia el estado de gas ideal [31]:

$$
R \cdot T \cdot \ln \varphi_{\mathrm{i}}=R \cdot T \cdot \ln \frac{p_{\mathrm{i}}^{*}}{x_{\mathrm{i}} \cdot P}=\int_{0}^{P}\left(V_{\mathrm{i}}-\frac{R T}{P}\right) \cdot \mathrm{d} P
$$

En una mezcla de gases ideales, $\varphi_{\mathrm{i}}=1$. Valores diferentes a 1 representan desviaciones respecto el comportamiento ideal, por lo que $\varphi_{i}$ es una magnitud que sirve para medir dichas desviaciones.

Para un sistema monocomponente se tiene,

$$
R \cdot T \cdot \ln \varphi=\int_{0}^{P} \frac{Z-1}{P} \cdot \mathrm{d} P
$$

donde $Z$ es el factor de compresibilidad:

$$
Z=\frac{P V_{\mathrm{m}}}{R T}
$$


Existe un extenso conjunto de ecuaciones a temperatura y composición constantes a partir de las cuales se pueden calcular todas las propiedades termodinámicas del sistema [43]-[45]. Dichas ecuaciones son generales, exactas y simples. Por ejemplo,

$$
\begin{gathered}
U=\int_{0}^{P}\left[V-T \cdot\left(\frac{\partial V}{\partial T}\right)_{P, n_{1}, \ldots, n_{\mathrm{n}}}\right] \cdot \mathrm{d} P-P \cdot V+\sum_{\mathrm{i}=1}^{\mathrm{n}} n_{\mathrm{i}} \cdot H_{\mathrm{i}}^{0} \\
G=\int_{0}^{P}\left[V-\frac{R \cdot T \cdot \sum_{\mathrm{i}=1}^{\mathrm{n}} n_{\mathrm{i}}}{P}\right] \cdot \mathrm{d} P+R \cdot T \cdot \sum_{\mathrm{i}=1}^{\mathrm{n}} n_{\mathrm{i}} \cdot \ln x_{\mathrm{i}} \cdot P+\sum_{\mathrm{i}=1}^{\mathrm{n}} n_{\mathrm{i}} \cdot\left(H_{\mathrm{i}}^{0}-T \cdot S_{\mathrm{i}}^{0}\right)
\end{gathered}
$$

El conocimiento de $\mu_{\mathrm{i}}, p_{\mathrm{i}}^{*}, \varphi_{\mathrm{i}}$ (ecuación (2.142)) ó $G$ (ecuación (2.146)) permite encontrar todas las propiedades termodinámicas del sistema respecto el estado de gas ideal a 1 bar de presión y misma temperatura y composición que la mezcla, siempre que se disponga información sobre el comportamiento volumétrico en la forma de ecuación de estado:

$$
V=V\left(T, P, n_{1}, \ldots, n_{\mathrm{n}}\right)
$$

Para evaluar las integrales que aparecen en las ecuaciones (2.142)-(2.146), la dependencia de $V$ con la presión debe conocerse no solamente a la presión $P$, sino en todo el rango de presiones desde 0 hasta $P$.

Frecuentemente las propiedades volumétricas de los fluidos se expresan mediante ecuaciones explícitas en la presión:

$$
P=P\left(T, V, n_{1}, \ldots, n_{\mathrm{n}}\right)
$$

En esos casos es más conveniente calcular las propiedades termodinámicas en función de las variables $V$ y $T$ :

$$
\begin{array}{r}
R \cdot T \cdot \ln \varphi_{\mathrm{i}}=R \cdot T \cdot \ln \frac{p_{\mathrm{i}}^{*}}{x_{\mathrm{i}} \cdot P}=\int_{V}^{\infty}\left[\left(\frac{\partial P}{\partial n_{\mathrm{i}}}\right)_{T, V, n_{\mathrm{j}} \neq n_{\mathrm{i}}}-\frac{R T}{V}\right] \cdot \mathrm{d} V-R \cdot T \cdot \ln Z \\
U=\int_{V}^{\infty}\left[P-T \cdot\left(\frac{\partial P}{\partial T}\right)_{V, n_{1}, \ldots, n_{\mathrm{n}}}\right] \cdot \mathrm{d} V+\sum_{\mathrm{i}=1}^{\mathrm{n}} n_{\mathrm{i}} \cdot U_{\mathrm{i}}^{0} \\
G=\int_{V}^{\infty}\left[P-\frac{R \cdot T \cdot \sum_{\mathrm{i}=1}^{\mathrm{n}} n_{\mathrm{i}}}{V}\right] \cdot \mathrm{d} V-R \cdot T \cdot \sum_{\mathrm{i}=1}^{\mathrm{n}} n_{\mathrm{i}} \cdot \ln \frac{V}{n_{\mathrm{i}} \cdot R \cdot T}+P \cdot V+\sum_{\mathrm{i}=1}^{\mathrm{n}} n_{\mathrm{i}} \cdot\left(U_{\mathrm{i}}^{0}-T \cdot S_{\mathrm{i}}^{0}\right)
\end{array}
$$

En los conjuntos de ecuaciones (2.142) - (2.146) y (2.149) - (2.151) nos volvemos a topar con la importancia de conocer la ecuación de estado (2.147) ó (2.148) para poder encontrar la información termodinámica. Por ejemplo, en el caso de gases se puede aplicar la ecuación de estado del virial (2.85). Para una mezcla binaria que contiene moléculas de las especies i y j, el segundo coeficiente del desarrollo del virial, $\mathrm{B}$, debe reflejar las interacciones entre pares de moléculas iguales $\mathrm{i}-\mathrm{i}$ y $\mathrm{j}-\mathrm{j}$ y distintas $\mathrm{i}-\mathrm{j}$. Dados los parámetros independientes de la composición $\mathrm{B}_{\mathrm{kk}}$, el segundo coeficiente del virial del componente $\mathrm{k}=\mathrm{i}$ ó $\mathrm{j}$ puro que depende del potencial molecular $W_{\mathrm{kk}}(\vec{r})$ en la 
forma dada por la ecuación (2.86) si los campos de fuerza son centrales y $\mathrm{B}_{\mathrm{ij}}$, el segundo coeficiente del virial de la interacción $\mathrm{i}-\mathrm{j}$ que depende del potencial de interacción entre moléculas diferentes $W_{\mathrm{ij}}(\vec{r})$, el cual en caso de ser central permite escribir:

$$
\mathrm{B}_{\mathrm{ij}}=-\frac{N_{\mathrm{A}}}{2} \cdot \int_{0}^{\mathrm{R}}\left(e^{-W_{\mathrm{ij}}(r) / k_{B} \cdot T}-1\right) \cdot 4 \cdot \pi \cdot r^{2} \cdot \mathrm{d} r
$$

entonces se puede adoptar la siguiente regla de mezcla para el segundo coeficiente del virial de la mezcla binaria, el cual es función cuadrática de las fracciones molares [46]:

$$
\mathrm{B}=x_{\mathrm{i}}^{2} \cdot \mathrm{B}_{\mathrm{ii}}+x_{\mathrm{j}}^{2} \cdot \mathrm{B}_{\mathrm{jj}}+2 \cdot x_{\mathrm{i}} \cdot x_{\mathrm{j}} \cdot \mathrm{B}_{\mathrm{ij}}
$$

Existen reglas de mezcla para el tercer coeficiente del virial, el cual es función cúbica de las fracciones molares. No obstante, la aplicabilidad de la ecuación de estado del virial a mezclas tiene las mismas limitaciones que para el caso de sistemas monocomponentes, debidas a la no existencia de solución analítica del problema de los 3 ó más cuerpos.

Como se dijo en la sección 2.1.1. la Termodinámica solamente presenta relaciones generales entre las propiedades macroscópicas, sin ocuparse de las predicciones cuantitativas de las mismas. El conocimiento de la ecuación de estado general que da cuenta de las diferentes fases y las transiciones entre ellas debe obtenerse de métodos ajenos a la Termodinámica:

a) A partir de los datos sobre las propiedades volumétricas de los sistemas.

b) A partir de una teoría estadística microscópica que sea capaz de predecir los datos volumétricos estableciendo relaciones entre estas propiedades macroscópicas y las fuerzas intermoleculares a través de la función de partición. No obstante esto no es tan sencillo, debido en parte a la no integrabilidad del problema de tres o más cuerpos, es decir, el desconocimiento de los potenciales de interacción, por lo que la Física Estadística resulta insuficiente para muchas aplicaciones prácticas, como se explica en el Capítulo 6.

\subsubsection{Mezcla ideal. Leyes de Lewis - Randall, Raoult y Henry.}

Como se ha visto, el cálculo de fugacidades a partir de las ecuaciones obtenidas por el método de la ecuación de estado es de carácter general pudiéndose aplicar tanto a fases gaseosas como condensadas. Sin embargo, no es útil debido al desconocimiento de las propiedades volumétricas, por lo que se emplea un método alternativo en el que las propiedades de las mezclas reales se expresan mediante las desviaciones que presentan respecto de una mezcla hipotética llamada mezcla ideal. Estas desviaciones, llamadas funciones de exceso, se definen como funciones de $P, T$ y la composición. Una vez más, el comportamiento de los sistemas reales se trata como una corrección del caso ideal, como se discutió en la sección 2.2.2.

Así pues, este procedimiento introduce las propiedades de la mezcla ideal en la descripción termodinámica de mezclas reales como estándares convenientes del comportamiento normal. El concepto de mezcla ideal no se limita a las mezclas de dos o más componentes líquidos, sino que también se utiliza en mezclas gaseosas y sólidas. En su definición existe falta de precisión debida a que hace uso de propiedades de sólidos, líquidos o gases extrapolados a rangos de presión y temperatura en los que 
tales fases pueden no existir, de la misma manera que ocurre en lo referente a lo expuesto en la sección 2.3.3.; no obstante, estas imprecisiones carecen de importancia en el caso de mezclas puramente líquidas, y son totalmente despreciables a presiones bajas en el caso de mezclas líquidas de gases o sólidos en disolventes líquidos como ya se explicó en dicha sección.

Antes de definir convenientemente qué se entiende por mezcla ideal, se debe introducir el concepto de magnitud de mezcla: dada una mezcla de varios componentes cuya composición viene dada por $n_{1}, \ldots n_{\mathrm{n}}$, para una propiedad $X$ se define la propiedad de mezcla $\Delta X$ como la diferencia entre el valor de esta propiedad en la mezcla y el correspondiente a los componentes puros, en la misma proporción y condiciones de presión y temperatura que la mezcla:

$$
\Delta X=X-\sum_{\mathrm{i}=1}^{\mathrm{n}} n_{\mathrm{i}} \cdot X_{\mathrm{i}}^{0}=\sum_{\mathrm{i}=1}^{\mathrm{n}} n_{\mathrm{i}} \cdot\left(X_{\mathrm{i}}-X_{\mathrm{i}}^{0}\right)=\sum_{\mathrm{i}=1}^{\mathrm{n}} n_{\mathrm{i}} \cdot \Delta X_{\mathrm{i}}
$$

donde $X_{\mathrm{i}}^{0}$ es la propiedad $X$ de la sustancia i-ésima en estado de referencia 0 de líquido puro a la misma presión y temperatura de la mezcla, como se definió en la sección 2.3.3. $\Delta X_{\mathrm{i}}$ es la propiedad molar parcial de mezcla de la sustancia i-ésima, luego cumple la ecuación (2.123). En el caso de mezcla binaria:

$$
\Delta_{\mathrm{m}} X_{\mathrm{i}}=\Delta_{\mathrm{m}} X_{\mathrm{m}}-x_{\mathrm{j}} \cdot\left(\frac{\partial \Delta_{\mathrm{m}} X_{\mathrm{m}}}{\partial x_{\mathrm{j}}}\right)_{T, P}
$$

Donde, $X_{\mathrm{m}}=X / n$ y $\Delta X_{\mathrm{m}}=\Delta X / n$. El que las propiedades de mezcla así definidas no sean nulas implica la interacción entre los componentes de la mezcla, es decir, que la propiedad $X$ no es aditiva. No obstante, la definición de propiedad molar parcial es un artilugio matemático que permite expresar una función que no es aditiva como si lo fuese, asegurando la aplicación de las ecuaciones de la Termodinámica.

Como se vio en la sección 2.3.2, $\Delta X_{1}$ y $\Delta X_{2}$ son las intersecciones de la curva tangente a $\Delta X_{\mathrm{m}}$ con el eje de ordenadas y el eje $x=1$ respectivamente. En el límite $x_{\mathrm{i}}=1, \Delta X_{\mathrm{i}}=0, \mathrm{y}$ en el límite $x_{\mathrm{i}}=0, \Delta X_{\mathrm{i}}=\Delta X_{\mathrm{i}}^{\infty}$, el valor a dilución infinita.

La mezcla ideal se define como aquella en la que a $P$ y $T$ constantes, la función de Gibbs molar viene dada por la ecuación:

$$
G_{\mathrm{m}}^{\mathrm{id}}=\sum_{\mathrm{i}=1}^{\mathrm{n}} x_{\mathrm{i}} \cdot \mu_{\mathrm{i}}^{\mathrm{id}}=\sum_{\mathrm{i}=1}^{\mathrm{n}} x_{\mathrm{i}} \cdot\left(\mu_{\mathrm{i}}^{0}+R \cdot T \cdot \ln x_{\mathrm{i}}\right)
$$

Luego, la función de Gibbs molar de mezcla de la mezcla ideal a $P$ y $T$ constantes es:

$$
\Delta G_{\mathrm{m}}^{\mathrm{id}}=G_{\mathrm{m}}^{\mathrm{id}}-\sum_{\mathrm{i}=1}^{\mathrm{n}} x_{\mathrm{i}} \cdot \mu_{\mathrm{i}}^{0}=R \cdot T \cdot \sum_{\mathrm{i}=1}^{\mathrm{n}} x_{\mathrm{i}} \cdot \ln x_{\mathrm{i}}
$$

Como $x_{\mathrm{i}}<1 \forall$ i entonces $\Delta G_{\mathrm{m}}^{\mathrm{id}}<0$ en todo el rango de composiciones, igual que ocurre en el caso de mezcla de gases ideales como se adelantó en la sección 2.1.6.; como consecuencia de las condiciones de equilibrio y estabilidad termodinámica, la mezcla ideal de líquidos es un estado más estable en todo el rango de concentraciones que el correspondiente a tener ambos líquidos en fases separadas, lo que se corresponde con la miscibilidad total. 
En la mezcla ideal a $V$ y $T$ constantes, $U, S, F, G, \ldots$ de mezcla son cero, luego las correspondientes magnitudes molares son aditivas: $X_{m(V, T)}^{i d}=\sum_{\mathrm{i}=1}^{\mathrm{n}} x_{\mathrm{i}} \cdot X_{\mathrm{i}}^{0}$.

Aunque el comportamiento de las mezclas reales dista del ideal, se debe prestar atención a este tipo de mezclas por varias razones:

a) Desde el punto de vista físico y matemático son las mezclas más sencillas.

b) En términos de la Física Estadística se puede predecir que el comportamiento de mezclas de sustancias muy parecidas es similar al de las mezclas ideales, como ocurre en la mezcla de isótopos, excepto del helio.

c) Experimentalmente se han encontrado mezclas casi ideales como $\mathrm{C}_{6} \mathrm{H}_{6}+\mathrm{BrC}_{6} \mathrm{H}_{5}$.

d) Las propiedades de las mezclas reales se expresan mediante las desviaciones que presentan respecto de la mezcla ideal.

De $(2.156)$ se obtienen las funciones de mezcla ideal a $P$ y $T$ constantes. La entropía de la mezcla ideal es:

$$
\begin{gathered}
S_{\mathrm{m}}^{\mathrm{id}}=-\left(\frac{\partial G_{\mathrm{m}}^{\mathrm{id}}}{\partial T}\right)_{P, x_{\mathrm{i}}, \mathrm{i} \neq \mathrm{j}}=-\sum_{\mathrm{i}=1}^{\mathrm{n}} x_{\mathrm{i}} \cdot\left(\frac{\partial \mu_{\mathrm{i}}^{0}}{\partial T}\right)_{P, x_{\mathrm{i}}, \mathrm{i} \neq \mathrm{j}}-R \cdot \sum_{\mathrm{i}=1}^{\mathrm{n}} x_{\mathrm{i}} \cdot \ln x_{\mathrm{i}}= \\
=\sum_{\mathrm{i}=1}^{\mathrm{n}} x_{\mathrm{i}} \cdot S_{\mathrm{i}}^{0}-R \cdot \sum_{\mathrm{i}=1}^{\mathrm{n}} x_{\mathrm{i}} \cdot \ln x_{\mathrm{i}}
\end{gathered}
$$

Luego la entropía de mezcla de la mezcla ideal a $P$ y $T$ constantes es:

$$
\Delta S_{\mathrm{m}}^{\mathrm{id}}=-R \cdot \sum_{\mathrm{i}=1}^{\mathrm{n}} x_{\mathrm{i}} \cdot \ln x_{\mathrm{i}}>0
$$

En una mezcla ideal a $P$ y $T$ constantes la entropía aumenta, lo que quiere decir que la mezcla ideal es un proceso espontáneo irreversible.

La entalpía de la mezcla ideal es aditiva ya que:

$$
\begin{aligned}
H_{\mathrm{m}}^{\mathrm{id}}= & G_{\mathrm{m}}^{\mathrm{id}}+T \cdot S_{\mathrm{m}}^{\mathrm{id}}=G_{\mathrm{m}}^{\mathrm{id}}-T \cdot\left(\frac{\partial G_{\mathrm{m}}^{\mathrm{id}}}{\partial T}\right)_{P, x_{\mathrm{i}}, \mathrm{i} \neq \mathrm{j}}= \\
& =\sum_{\mathrm{i}=1}^{\mathrm{n}} x_{\mathrm{i}} \cdot\left(\mu_{\mathrm{i}}^{0}+S_{\mathrm{i}}^{0} \cdot T\right)=\sum_{\mathrm{i}=1}^{\mathrm{n}} x_{\mathrm{i}} \cdot H_{\mathrm{i}}^{0}
\end{aligned}
$$

luego la entalpía de mezcla de la mezcla ideal a $P$ y $T$ constantes es cero: $\Delta H_{\mathrm{m}}^{\mathrm{id}}=0$.

El volumen de la mezcla ideal es aditivo:

$$
V_{\mathrm{m}}^{\mathrm{id}}=\left(\frac{\partial G_{\mathrm{m}}}{\partial P}\right)_{T, x_{\mathrm{i}}, \mathrm{i} \neq \mathrm{j}}=\sum_{\mathrm{i}=1}^{\mathrm{n}} x_{\mathrm{i}} \cdot\left(\frac{\partial \mu_{\mathrm{i}}^{0}}{\partial P}\right)_{T, x_{\mathrm{i}}, \mathrm{i} \neq \mathrm{j}}=\sum_{\mathrm{i}=1}^{\mathrm{n}} x_{\mathrm{i}} \cdot V_{\mathrm{i}}^{0}
$$

luego el volumen de mezcla de la mezcla ideal a $P$ y $T$ constantes es cero: $\Delta V_{\mathrm{m}}^{\text {id }}=0$. La energía interna de la mezcla ideal es aditiva, pues:

$$
\Delta U_{\mathrm{m}}^{\mathrm{id}}=\Delta H_{\mathrm{m}}^{\mathrm{id}}-\Delta\left(P \cdot V_{\mathrm{m}}^{\mathrm{id}}\right)=0
$$


la energía interna de mezcla de la mezcla ideal a $P$ y $T$ constantes es cero.

Las capacidades caloríficas a presión constante $C_{P}^{\text {id }}$ y a volumen constante $C_{V}^{\text {id }}$, el coeficiente de expansión térmica a presión constante $\alpha_{P}^{\text {id }}$ y los coeficientes de compresibilidad isotermo $\kappa_{T}^{\text {id }}$ e isoentrópico $\kappa_{S}^{\text {id }}$ de la mezcla ideal se calculan a partir de las propiedades de los líquidos puros según las ecuaciones propuestas por Benson y Kiyohara [47]:

$$
\begin{gathered}
C_{P}^{\text {id }}=\sum_{\mathrm{i}=1}^{\mathrm{n}} x_{\mathrm{i}} \cdot C_{P, \mathrm{i}}^{0} \\
\alpha_{P}^{\mathrm{id}}=\sum_{\mathrm{i}=1}^{\mathrm{n}} \Phi_{\mathrm{i}} \cdot \alpha_{P, \mathrm{i}}^{0} \\
\kappa_{T}^{\mathrm{id}}=\sum_{\mathrm{i}=1}^{\mathrm{n}} \Phi_{\mathrm{i}} \cdot \kappa_{T, \mathrm{i}}^{0} \\
\kappa_{S}^{\mathrm{id}}=\kappa_{T}^{\mathrm{id}}-\frac{T \cdot V_{m}^{\mathrm{id}} \cdot\left(\alpha_{P}^{\mathrm{id}}\right)^{2}}{C_{P}^{\mathrm{id}}} \\
C_{V}^{\mathrm{id}}=C_{P}^{\mathrm{id}}-\frac{T \cdot V_{\mathrm{m}}^{\mathrm{id}} \cdot\left(\alpha_{P}^{\mathrm{id}}\right)^{2}}{\kappa_{T}^{\mathrm{id}}}=\frac{C_{P}^{\mathrm{id}} \cdot \kappa_{S}^{\mathrm{id}}}{\kappa_{T}^{\mathrm{id}}}
\end{gathered}
$$

donde $\Phi_{\mathrm{i}}$ es la fracción de volumen del componente i-ésimo:

$$
\Phi_{\mathrm{i}}=\frac{x_{\mathrm{i}} \cdot V_{\mathrm{i}}^{0}}{\sum_{\mathrm{j}=1}^{\mathrm{n}} x_{\mathrm{j}} \cdot V_{\mathrm{j}}^{0}}
$$

El potencial químico de la sustancia i-ésima es:

$$
\mu_{\mathrm{i}}^{\mathrm{id}}=G_{\mathrm{m}}^{\mathrm{i}, \mathrm{id}}=\left(\frac{\partial G^{\mathrm{id}}}{\partial n_{\mathrm{i}}}\right)_{T, P, n_{\mathrm{i}}, \mathrm{i} \neq \mathrm{j}}=\left(\frac{\partial G_{\mathrm{m}}^{\mathrm{id}}}{\partial x_{\mathrm{i}}}\right)_{T, P, x_{\mathrm{i}}, \mathrm{i} \neq \mathrm{j}}=\mu_{\mathrm{i}}^{0}+R \cdot T \cdot \ln x_{\mathrm{i}}
$$

Entropía molar parcial:

$$
S_{\mathrm{i}}^{\mathrm{id}}=\left(\frac{\partial S_{\mathrm{m}}^{\mathrm{id}}}{\partial x_{\mathrm{i}}}\right)_{T, P, x_{\mathrm{i}}, \mathrm{i} \neq \mathrm{j}}=-\left(\frac{\partial \mu_{\mathrm{i}}^{0}}{\partial T}\right)_{P, x_{\mathrm{i}}, \mathrm{i} \neq \mathrm{j}}-R \cdot \ln x_{\mathrm{i}}=S_{\mathrm{i}}^{0}-R \cdot \ln x_{\mathrm{i}}
$$

Entalpía molar parcial:

$$
H_{\mathrm{i}}^{\mathrm{id}}=\mu_{\mathrm{i}}^{0}-T \cdot\left(\frac{\partial \mu_{\mathrm{i}}^{0}}{\partial T}\right)_{P, x_{\mathrm{i}}, \mathrm{i} \neq \mathrm{j}}=H_{\mathrm{i}}^{0}
$$

Volumen molar parcial:

$$
V_{\mathrm{i}}^{\mathrm{id}}=\left(\frac{\partial \mu_{\mathrm{i}}^{0}}{\partial P}\right)_{T, x_{\mathrm{i}}, \mathrm{i \neq j}}=V_{\mathrm{i}}^{0}
$$

Potencial de Helmholtz molar parcial:

$$
F_{\mathrm{i}}^{\mathrm{id}}=\mu_{\mathrm{i}}^{0}+R \cdot T \cdot \ln x_{\mathrm{i}}-P \cdot V_{\mathrm{i}}^{0}
$$


De las ecuaciones (2.169) a (2.173) se deduce que en el límite a dilución infinita, las propiedades molares parciales de la sustancia i-ésima en la mezcla ideal son:

$$
\mu_{\mathrm{i}}^{\mathrm{id} \infty}=-\infty, S_{\mathrm{i}}^{\mathrm{id} \infty}=+\infty, F_{\mathrm{i}}^{\mathrm{id} \infty}=-\infty ; H_{\mathrm{i}}^{\mathrm{id} \infty}=H_{i}^{0} ; V_{\mathrm{i}}^{\mathrm{id} \infty}=V_{i}^{0}
$$

Las tres primeras igualdades se mantienen tanto para las propiedades de mezcla no ideales [24, pp 200] como para las propiedades molares parciales de mezcla, ideal o no:

$$
\Delta \mu_{\mathrm{i}}^{\infty}=-\infty, \Delta S_{\mathrm{i}}^{\infty}=+\infty, \Delta F_{\mathrm{i}}^{\infty}=-\infty
$$

En estas circunstancias, de las ecuaciones (2.123) y (2.155) se tiene que $\left(\frac{\partial X_{\mathrm{m}}}{\partial x_{\mathrm{j}}}\right)_{T, P}= \pm \infty$ y $\left(\frac{\partial \Delta X_{\mathrm{m}}}{\partial x_{\mathrm{j}}}\right)_{T, P}= \pm \infty$ para $X_{\mathrm{m}}=\mu, S_{\mathrm{m},} F_{\mathrm{m}}$. Esto implica que cuando $X_{\mathrm{m}}$ representa a una de estas tres magnitudes, las gráficos $X_{\mathrm{m}}=X_{\mathrm{m}}\left(x_{\mathrm{i}}\right)$ y $\Delta X_{\mathrm{m}}=\Delta X_{\mathrm{m}}\left(x_{\mathrm{i}}\right)$ terminan en los extremos $x_{\mathrm{i}}=0$ y $x_{\mathrm{i}}=1$ con pendientes infinitas, es decir, los ejes verticales representan las rectas tangentes a dichas curvas. Además, se puede demostrar que cuando $x_{\mathrm{i}} \rightarrow 0,\left(\frac{\partial X_{\mathrm{i}}}{\partial x_{\mathrm{i}}}\right)_{T, P}= \pm \infty$, lo que implica que $X_{\mathrm{i}} \rightarrow \infty$ con pendiente infinita.

Si $X$ no representa ninguna de las magnitudes $\mu, S, F$ no aparecen divergencias hacia el infinito.

De (2.131) y (2.169) se tiene que para la mezcla ideal, la actividad relativa de la sustancia i-ésima en la mezcla es igual a su fracción molar:

$$
a_{\mathrm{i}}=x_{\mathrm{i}}
$$

luego, de (2.139), la mezcla ideal cumple la ley de Lewis-Randall [41] en todos los rangos de composición, presión y temperatura:

$$
p_{\mathrm{i}}^{*}=p_{\mathrm{i}}^{* 0} \cdot x_{\mathrm{i}}
$$

$\mathrm{Si}$ los vapores se consideran gases ideales, la fugacidad de la sustancia en fase condensada es igual a la presión parcial de su vapor, de modo que la ley de LewisRandall se transforma en la ley de Raoult [48], [49]:

$$
p_{\mathrm{i}}=p_{\mathrm{i}}^{0} \cdot x_{\mathrm{i}}
$$

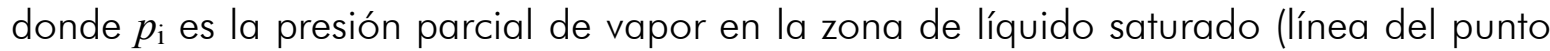
de burbuja). Para una mezcla binaria en la que $x=x_{2}$, la presión de la mezcla es:

$$
P=p_{1}^{0}+x \cdot\left(p_{2}^{0}-p_{1}^{0}\right)
$$

es una línea recta en $x$ con pendiente $p_{2}^{0}-p_{1}^{0}$ y ordenada en el origen $p_{1}^{0}$.

En algunas ocasiones la ley de Raoult se toma como definición de mezcla ideal, lo cual no es cierto ya que la ley de Raoult es resultado de la definición de mezcla ideal siempre que los vapores se puedan considerar gases ideales. De hecho, una mezcla ideal de líquidos en equilibrio con la mezcla de sus vapores, considerada mezcla ideal de gases imperfectos, muestra desviaciones negativas respecto la ley de Raoult. Por el contrario, una mezcla que satisface la ley de Raoult no será, en general, una mezcla ideal en el sentido de (2.156), ya que algunas de estas mezclas como la benceno + 
dicloruro de etileno tiene valores positivos de $\Delta G_{m}^{i d}$ en algún intervalo de la composición, lo que indica inmiscibilidad.

Se podrían encontrar mezclas binarias en las que se verificase en todo el rango de composiciones la ley de Lewis-Randall (o en el caso de que los vapores fuesen gases ideales, la ley de Raoult) para uno de los componentes, por ejemplo, para el componente 1 de una mezcla. En estas condiciones, $x_{1}=a_{1}$, y $\mathrm{d} \ln x_{1}=\mathrm{d} \ln a_{1}$; de la ecuación de Gibbs-Duhem en la forma (2.140) se tiene que $\mathrm{d} \ln x_{2}=\mathrm{d} \ln a_{2}$ lo que implica la proporcionalidad siguiente en todo el espectro de composiciones:

$$
p_{2}=k \cdot x_{2}
$$

que es la conocida ley de Henry [50]. Este resultado puede resumirse como sigue: en una mezcla binaria, siempre que para un componente se verifica en todo el rango de composiciones la ley de Lewis-Randall (o de Raoult), para el otro componente se verifica la ley de Henry en todo el rango de composición, y ello es consecuencia de la ecuación de Gibbs-Duhem, por lo que no se restringe a disoluciones ideales. En el caso de que la mezcla fuese ideal, $k=1$.

Se puede demostrar que en cualquier mezcla, ideal o real, se verifican las ligaduras siguientes:

$$
\begin{aligned}
& \lim _{x_{\mathrm{i}} \rightarrow 0}\left(\frac{p_{\mathrm{i}}^{*}}{x_{\mathrm{i}}}\right)=k_{\mathrm{i}} \\
& \lim _{x_{i} \rightarrow 1}\left(\frac{p_{\mathrm{i}}^{*}}{x_{\mathrm{i}}}\right)=p_{\mathrm{i}}^{* 0}
\end{aligned}
$$

es decir, en toda disolución, se cumple la Ley de Henry para el soluto diluido, y la ley de Lewis-Randall para el disolvente.

A veces es conveniente considerar una mezcla ideal como aquella que verifica la ley de Henry para uno de sus componentes. Esto es adecuado cuando se trabaja con disoluciones en las que uno o más de sus componentes no existen como líquido puro estable en las condiciones de presión y temperatura de la mezcla, por ejemplo en las mezclas líquidas de sólidos y/o gases disueltos en un disolvente líquido [24, pp 231].

\subsubsection{Mezcla real. Funciones de exceso. Coeficiente de actividad}

El comportamiento de las disoluciones reales se aleja de la idealidad debido a las interacciones entre las moléculas de los componentes que la forman. Las propiedades de las mezclas reales se expresan como desviaciones respecto de la mezcla ideal, viniendo estas desviaciones dadas por las funciones de exceso que se definen como funciones de $P, T$ y la composición, y fueron introducidas por primera vez en 1938 [51]. Dada la propiedad molar $X_{\mathrm{m}}$, se define su exceso como $X_{\mathrm{m}}^{\mathrm{E}}$ :

$$
X_{\mathrm{m}}^{\mathrm{E}}=X_{\mathrm{m}}-X_{\mathrm{m}}^{\mathrm{id}}=X_{\mathrm{m}}-\sum_{\mathrm{i}=1}^{\mathrm{n}} x_{\mathrm{i}} \cdot X_{\mathrm{i}}^{0}-\left(X_{\mathrm{m}}^{\mathrm{id}}-\sum_{\mathrm{i}=1}^{\mathrm{n}} x_{\mathrm{i}} \cdot X_{\mathrm{i}}^{0}\right)=\Delta X_{\mathrm{m}}-\Delta X_{\mathrm{m}}^{\mathrm{id}}=\Delta X_{\mathrm{m}}^{\mathrm{E}}
$$

Donde $\Delta X_{\mathrm{m}}$ y $\Delta X_{\mathrm{m}}^{\mathrm{id}}$ son las funciones de mezcla a $P$ y $T$ constantes de la mezcla real e ideal, respectivamente. Entonces, $X_{\mathrm{m}}^{\mathrm{E}}=\Delta X_{\mathrm{m}}^{\mathrm{E}}$, es decir la propiedad de exceso coincide 
con el exceso de la propiedad de mezcla. De acuerdo con esta definición y con (2.160), $(2.161)$ y $(2.162)$ :

$$
\begin{gathered}
H_{\mathrm{m}}^{\mathrm{E}}=\Delta H_{\mathrm{m}} \\
V_{\mathrm{m}}^{\mathrm{E}}=\Delta V_{\mathrm{m}} \\
U_{\mathrm{m}}^{\mathrm{E}}=\Delta U_{\mathrm{m}}
\end{gathered}
$$

Por esta razón se habla indistintamente de entalpía de mezcla y de exceso, de volumen de mezcla y de exceso, o de energía interna de mezcla y de exceso. No ocurre lo mismo para $G_{\mathrm{m}}^{\mathrm{E}}$ y $S_{\mathrm{m}}^{\mathrm{E}}$, pues por $(2.157)$ y $(2.159)$ se tiene que:

$$
\begin{gathered}
G_{\mathrm{m}}^{\mathrm{E}}=\Delta G_{\mathrm{m}}-R \cdot T \cdot \sum_{\mathrm{i}=1}^{\mathrm{n}} x_{\mathrm{i}} \cdot \ln x_{\mathrm{i}} \\
S_{\mathrm{m}}^{\mathrm{E}}=\Delta S_{\mathrm{m}}+R \cdot \sum_{\mathrm{i}=1}^{\mathrm{n}} x_{\mathrm{i}} \cdot \ln x_{\mathrm{i}}
\end{gathered}
$$

En el caso de los coeficientes térmicos también se pueden definir las propiedades de exceso. En concreto, para el coeficiente de compresibilidad isoentrópica se tiene:

$$
\kappa_{S}^{\mathrm{E}}=\kappa_{S}-\left(\kappa_{T}^{\mathrm{id}}-\frac{T \cdot V_{\mathrm{m}}^{\mathrm{id}} \cdot\left(\alpha_{P}^{\mathrm{id}}\right)^{2}}{C_{P}^{\mathrm{id}}}\right)
$$

Las propiedades de exceso siguen manteniendo las relaciones típicas entre las magnitudes termodinámicas. Por ejemplo,

$$
\begin{gathered}
S_{\mathrm{m}}^{\mathrm{E}}=-\left(\frac{\partial G_{\mathrm{m}}^{\mathrm{E}}}{\partial T}\right)_{P, n_{1}, \ldots, n_{\mathrm{n}}} \\
H_{\mathrm{m}}^{\mathrm{E}}=G_{\mathrm{m}}^{\mathrm{E}}-T \cdot\left(\frac{\partial G_{\mathrm{m}}^{\mathrm{E}}}{\partial T}\right)_{P, n_{1}, \ldots, n_{\mathrm{n}}} \\
V_{\mathrm{m}}^{\mathrm{E}}=\left(\frac{\partial G_{\mathrm{m}}^{\mathrm{E}}}{\partial P}\right)_{T, n_{1}, \ldots, n_{\mathrm{n}}} \\
U_{\mathrm{m}}^{\mathrm{E}}=G_{\mathrm{m}}^{\mathrm{E}}-T \cdot\left(\frac{\partial G_{\mathrm{m}}^{\mathrm{E}}}{\partial T}\right)_{P, n_{1}, \ldots, n_{\mathrm{n}}}+\left(\frac{\partial G_{\mathrm{m}}^{\mathrm{E}}}{\partial P}\right)_{T, n_{1}, \ldots, n_{\mathrm{n}}}
\end{gathered}
$$

Los potenciales químicos de exceso juegan un papel especial dentro de este formalismo ya que a partir de ellos se pueden conocer el resto de funciones de exceso [52]. Se define el potencial químico de exceso de la sustancia i-ésima como la función de Gibbs de exceso molar parcial de dicha sustancia:

$$
\mu_{\mathrm{i}}^{\mathrm{E}}=\left(\frac{\partial G_{\mathrm{m}}^{\mathrm{E}}}{\partial n_{\mathrm{i}}}\right)_{T, P, n_{\mathrm{j}} \mathrm{j} \neq \mathrm{i}}
$$

Para una mezcla binaria se cumplen las ecuaciones (2.121) y (2.122): 


$$
\begin{gathered}
\mu_{1}^{\mathrm{E}}=G_{\mathrm{m}}^{\mathrm{E}}-x \cdot\left(\frac{\partial G_{\mathrm{m}}^{\mathrm{E}}}{\partial x}\right)_{T, P} \\
\mu_{2}^{\mathrm{E}}=G_{\mathrm{m}}^{\mathrm{E}}+(1-x) \cdot\left(\frac{\partial G_{\mathrm{m}}^{\mathrm{E}}}{\partial x}\right)_{T, P}
\end{gathered}
$$

donde $G_{\mathrm{m}}^{\mathrm{E}}=\mu^{\mathrm{E}}$.

En lugar de los potenciales químicos de exceso, es más práctico emplear el cociente de la fugacidad del componente en la mezcla entre la fugacidad de dicho componente en el estado de referencia. Por ello Brönsted [53] definió el coeficiente de actividad $f_{\mathrm{i}}$ :

$$
f_{\mathrm{i}}=\frac{p_{\mathrm{i}}^{*}}{p_{\mathrm{i}}^{* 0} \cdot x_{\mathrm{i}}}=\frac{a_{\mathrm{i}}}{x_{\mathrm{i}}}
$$

donde $f_{i}=1$ si la mezcla es ideal. En el caso de mezcla de gases, el estado de referencia es el estado de gas ideal puro, mientras que en mezclas líquidas se toma como estado de referencia el del líquido puro a la misma presión y temperatura de la mezcla, el cual puede ser ficticio como ya se ha discutido unas líneas más arriba. En cualquier caso, el conocimiento del coeficiente de actividad implica el de la actividad relativa, y éste el del coeficiente de fugacidad, luego del potencial químico.

Se puede demostrar fácilmente que:

$$
\mu_{\mathrm{i}}^{\mathrm{E}}=R \cdot T \cdot \ln f_{\mathrm{i}}
$$

lvego,

$$
G_{\mathrm{m}}^{\mathrm{E}}=\mu^{\mathrm{E}}=R \cdot T \cdot \sum_{\mathrm{i}=1}^{\mathrm{n}} x_{\mathrm{i}} \cdot \ln f_{\mathrm{i}}
$$

Esta ecuación es importante ya que relaciona el potencial de Gibbs de exceso con los coeficientes de actividad.

El resto de propiedades de exceso molares parciales son:

$$
\begin{gathered}
V_{\mathrm{i}}^{\mathrm{E}}=\left(\frac{\partial V_{\mathrm{m}}^{\mathrm{E}}}{\partial n_{\mathrm{i}}}\right)_{T, P, x_{\mathrm{i}}, \mathrm{i} \neq \mathrm{j}}=R \cdot T \cdot\left(\frac{\partial \ln f_{\mathrm{i}}}{\partial P}\right)_{T, x_{\mathrm{i}}, \mathrm{i} \neq \mathrm{j}} \\
H_{\mathrm{i}}^{\mathrm{E}}=\left(\frac{\partial H_{\mathrm{m}}^{\mathrm{E}}}{\partial n_{\mathrm{i}}}\right)_{T, P, x_{\mathrm{i}}, \mathrm{i} \neq \mathrm{j}}=-R \cdot T^{2} \cdot\left(\frac{\partial \ln f_{\mathrm{i}}}{\partial T}\right)_{P, x_{\mathrm{i}}, \mathrm{i} \neq \mathrm{j}} \\
S_{\mathrm{i}}^{\mathrm{E}}=\left(\frac{\partial S_{\mathrm{m}}^{\mathrm{E}}}{\partial n_{\mathrm{i}}}\right)_{T, P, x_{\mathrm{i}}, \mathrm{i} \neq \mathrm{j}}=-R \cdot T \cdot\left(\frac{\partial \ln f_{\mathrm{i}}}{\partial T}\right)_{P, x_{\mathrm{i}}, \mathrm{i} \neq \mathrm{j}}-R \cdot \ln \left(x_{\mathrm{i}} \cdot f_{\mathrm{i}}\right)
\end{gathered}
$$

Se encuentra experimentalmente que $f_{\mathrm{i}}$ es finito para mezclas de no electrolitos en todo el rango de composiciones. De esta manera el comportamiento de las propiedades de la disolución está unificado mediante las funciones de exceso, ya que la naturaleza general de $X_{\mathrm{m}}^{\mathrm{E}}$ es la misma, sea cual sea la propiedad $X$, sin tener que distinguir entre diferentes comportamientos correspondientes a cuando $X$ es $G, F$ y $S$, o cuando es $U, V$, $H, C_{V}, C_{P} \ldots$ Como consecuencia, la utilización de las funciones de exceso elimina la aparición de infinitos en las regiones de dilución, mostrados unos párrafos más arriba. 
Las funciones de exceso y las funciones de mezcla a volumen constante a veces son más interesantes que a presión constante. Para ellas se tiene:

$$
G_{\mathrm{m}, V}^{\mathrm{E}}-F_{\mathrm{m}, V}^{\mathrm{E}}=H_{\mathrm{m}, V}^{\mathrm{E}}-U_{\mathrm{m}, V}^{\mathrm{E}}=P V_{\mathrm{m}, V}^{\mathrm{E}}=0
$$

Las propiedades de exceso a presión y volumen constante están relacionadas de manera aproximada [37], [38], [54], [55]:

$$
G_{\mathrm{m}}^{\mathrm{E}}-G_{\mathrm{m}, V}^{\mathrm{E}}=G_{\mathrm{m}}^{\mathrm{E}}-F_{\mathrm{m}, V}^{\mathrm{E}}=\frac{1}{2} \cdot \frac{1}{V_{\mathrm{m}} \cdot \kappa_{T}} \cdot\left(V_{\mathrm{m}}^{\mathrm{E}}\right)^{2}
$$

Donde los subíndices $V$ hacen referencia a que la mezcla se produce a volumen constante; hasta ahora no se había escrito ningún subíndice de esta índole ya que siempre se habían considerado a presión constante, omitiendo el subíndice $P$; $V_{\mathrm{m}}$ es el volumen molar de la mezcla; $\kappa_{T}$ es el coeficiente de compresibilidad isoterma de la mezcla. También se obtiene la aproximación siguiente:

$$
\begin{gathered}
T \cdot S_{\mathrm{m}}^{\mathrm{E}}-T \cdot S_{\mathrm{m}, V}^{\mathrm{E}}=H_{\mathrm{m}}^{\mathrm{E}}-H_{\mathrm{m}, V}^{\mathrm{E}}=H_{\mathrm{m}}^{\mathrm{E}}-U_{\mathrm{m}, V}^{\mathrm{E}}= \\
=\left[T \cdot\left(\frac{\partial P}{\partial T}\right)_{V}\right]_{P=0} \cdot V_{\mathrm{m}}^{\mathrm{E}}+o\left(V_{\mathrm{m}}^{\mathrm{E}}\right)^{2} \approx T \cdot \frac{\alpha}{\kappa_{T}} \cdot V_{\mathrm{m}}^{\mathrm{E}}=\left(P+P_{\mathrm{int}}\right) \cdot V_{\mathrm{m}}^{\mathrm{E}}
\end{gathered}
$$

De modo que la diferencia entre entropías de exceso, así como entre la energía interna y la entalpía de exceso es del orden de $V_{\mathrm{m}}^{\mathrm{E}}$ y depende de la suma de la presión y la presión interna, mientras que la diferencia entre las funciones de Gibbs de exceso y la de Helmholtz es del orden de $\left(V_{\mathrm{m}}^{\mathrm{E}}\right)^{2}$. Esto hace que, si bien la diferencia es pequeña en el caso de $G_{\mathrm{m}}^{\mathrm{E}}-F_{\mathrm{m}, V}^{\mathrm{E}}$, puede ser significativa en el caso de $T \cdot S_{\mathrm{m}}^{\mathrm{E}}-T \cdot S_{\mathrm{m}, V}^{\mathrm{E}}$ ó $H_{\mathrm{m}}^{\mathrm{E}}-H_{\mathrm{m}, V}^{\mathrm{E}}$.

Como ejemplo cuantitativo, considérense los siguientes valores de una mezcla binaria típica a composición equimolar [56]:

$$
\begin{gathered}
G_{\mathrm{m}}^{\mathrm{E}}=200 \mathrm{~J} \cdot \mathrm{mol}^{-1} \quad H_{\mathrm{m}}^{\mathrm{E}}=500 \mathrm{~J} \cdot \mathrm{mol}^{-1} \quad T \cdot S_{\mathrm{m}}^{\mathrm{E}}=300 \mathrm{~J} \cdot \mathrm{mol}^{-1} \\
V_{\mathrm{m}}^{\mathrm{E}}=1 \mathrm{~cm}^{3} \cdot \mathrm{mol}^{-1} \quad V_{\mathrm{m}}=100 \mathrm{~cm}^{3} \cdot \mathrm{mol}^{-1} \quad k_{T}=10^{-3} \mathrm{MPa}^{-1} \quad T \cdot \frac{\alpha}{k_{T}}=300 \mathrm{MPa}
\end{gathered}
$$

Estos valores dan los siguientes resultados para las diferencias entre las funciones de exceso a presión y a volumen constante:

$$
\begin{gathered}
G_{\mathrm{m}}^{\mathrm{E}}-G_{\mathrm{m}, V}^{\mathrm{E}}=G_{\mathrm{m}}^{\mathrm{E}}-F_{\mathrm{m}, V}^{\mathrm{E}}=5 \mathrm{~J} \cdot \mathrm{mol}^{-1} \Rightarrow G_{\mathrm{m}}^{\mathrm{E}} \approx G_{\mathrm{m}, V}^{\mathrm{E}} \quad \circ \text { bien } \quad G_{\mathrm{m}}^{\mathrm{E}} \approx F_{\mathrm{m}, V}^{\mathrm{E}} \\
T \cdot S_{\mathrm{m}}^{\mathrm{E}}-T \cdot S_{\mathrm{m}, V}^{\mathrm{E}}=H_{\mathrm{m}}^{\mathrm{E}}-H_{\mathrm{m}, V}^{\mathrm{E}}=H_{\mathrm{m}}^{\mathrm{E}}-U_{\mathrm{m}, V}^{\mathrm{E}}=300 \mathrm{~J} \cdot \mathrm{mol}^{-1} \Rightarrow\left\{\begin{array}{c}
H_{\mathrm{m}, V}^{\mathrm{E}}=U_{\mathrm{m}, V}^{\mathrm{E}}=200 \mathrm{~J} \cdot \mathrm{mol}^{-1} \\
T \cdot S_{\mathrm{m}, V}^{\mathrm{E}}=0 \mathrm{~J} \cdot \mathrm{mol}^{-1}
\end{array}\right.
\end{gathered}
$$

Resulta que la energía y la entropía de exceso de una mezcla tienen valores muy diferentes si se considera el proceso de mezcla a volumen total constante en lugar de a presión constante. Sólo las magnitudes a presión constante pueden medirse directamente, mientras que las magnitudes a volumen constante se calculan indirectamente a través de las ecuaciones anteriores. 


\subsubsection{Coeficiente de actividad a partir de funciones de exceso. Ecuaciones de ajuste}

Como se ha dicho reiteradamente a lo largo de este capítulo, toda la información termodinámica del sistema vendrá dada si se conocen los potenciales químicos, lo que es semejante a conocer la actividad, fugacidad, coeficiente de fugacidad, $\ldots \circ G_{\mathrm{m}}^{\mathrm{E}}$ (por la ecuación (2.197)). Por eso necesitamos tener funciones analíticas de las variables independientes $\left(P, T, x_{\mathrm{i}}\right)$ que sean aplicables a las ecuaciones en las que aparecen dichas funciones, que como sabemos, deben obtenerse por métodos ajenos a la Termodinámica. Así, en el caso de las funciones de exceso, las expresiones matemáticas propuestas pueden agruparse en tres clases bien diferenciadas [57]:

a) Ecuaciones procedentes de modelos: se parte de una teoría estructural de forma que los parámetros que figuran en la representación posean un significado físico completo (teoría de red, teoría de Flory, métodos de contribución de grupos, etc.)

b) Ecuaciones semiempíricas: en este caso las funciones analíticas incluyen parámetros con significado físico o sujetos a algún tipo de correlación, para así poder predecir las funciones de exceso (desarrollo de Whol, teorías de Wilson, NRTL...)

c) Ecuaciones empíricas: las funciones analíticas son puramente algebraicas del tipo $X=X\left(P=\right.$ cte., $T=$ cte., $\left.x_{\mathrm{i}}\right)$, y no atribuyen significado físico alguno a los parámetros utilizados en el ajuste.

En lo siguiente se expondrán algunas representaciones puramente empíricas, ya que cobrarán gran importancia en los capítulos posteriores.

Son muchas las ecuaciones del tipo c) propuestas por diversos autores para ajustar los datos experimentales de las funciones termodinámicas de exceso. En los trabajos de Scatchard [58], Handa \& Benson [59] y Gordon \& Hornibrook [60] se estudia la aplicabilidad de muchas de ellas. Quizás la ecuación de ajuste de este tipo más importante sea la llamada de Redlich-Kister, que procede de la teoría de disoluciones regulares pero que se amplió empíricamente y por ello se considera un ajuste del tipo c).

Guggenheim [61] sugirió que la energía libre de Gibbs molar de exceso de una mezcla binaria puede representarse mediante la expresión polinómica siguiente:

$$
G_{\mathrm{m}}^{\mathrm{E}}=x_{1} \cdot x_{2} \cdot\left[\mathrm{A}_{0}+\mathrm{A}_{1} \cdot\left(x_{1}-x_{2}\right)+\mathrm{A}_{2} \cdot\left(x_{1}-x_{2}\right)^{2}+\ldots\right]
$$

siendo $\mathrm{A}_{0}, \mathrm{~A}_{1}, \mathrm{~A}_{2}, \ldots$ parámetros dependientes de la presión y la temperatura. La expresión (2.204) satisface el requisito que debe cumplir toda función de exceso en sus extremos de composición:

$$
G_{\mathrm{m}}^{\mathrm{E}}\left(x_{1}=0\right)=G_{\mathrm{m}}^{\mathrm{E}}\left(x_{2}=0\right)=0
$$

Scatchard y Hamer [62] compararon los desarrollos en serie de potencias de las fracciones molares y las fracciones de volumen. Redlich y Kister [63] demostraron que con esta ecuación muchos sistemas multicomponentes se pueden tratar con gran exactitud al añadir las expresiones de todos los pares binarios que los componen. Además, obtuvieron expresiones similares para los coeficientes de actividad:

$$
\ln f_{1}=\left(1-x_{1}\right)^{2} \cdot\left[\mathrm{A}_{0}{ }^{\prime}+\mathrm{A}_{1}{ }^{\prime} \cdot\left(4 \cdot x_{1}-1\right)+\mathrm{A}_{2}{ }^{\prime} \cdot\left(2 \cdot x_{1}-1\right) \cdot\left(6 \cdot x_{1}-1\right)+\ldots\right]
$$




$$
\ln f_{2}=x_{1}^{2} \cdot\left[\mathrm{A}_{0}^{\prime}-\mathrm{A}_{1}^{\prime} \cdot\left(3-4 \cdot x_{1}\right)+\mathrm{A}_{2}^{\prime} \cdot\left(1-2 \cdot x_{1}\right) \cdot\left(5-6 \cdot x_{1}\right)+\ldots\right]
$$

donde $\mathrm{A}_{\mathrm{i}}^{\prime}=\mathrm{A}_{\mathrm{i}} / R \cdot T, \mathrm{i}=0,1,2, \ldots$

Originariamente a las ecuaciones (2.206) y (2.207) se las llamó de Redlich- Kister (R-K). Pero con el paso de los años la generalización para cualquier función termodinámica de exceso de la ecuación de ajuste propuesta por Guggenheim pasó a conocerse también como ecuación R-K, hecho no justificado, aunque probablemente se deba a que fueron Redlich y Kister quienes recomendaron la extensión del polinomio propuesto por Guggenheim a sistemas multicomponentes [64].

La ecuación (2.204) puede emplearse para cualquier función de exceso:

$$
X_{\mathrm{m}}^{\mathrm{E}}=x_{1} \cdot x_{2} \cdot \sum_{\mathrm{i}=0}^{\mathrm{n}} \mathrm{A}_{\mathrm{i}} \cdot\left(x_{1}-x_{2}\right)^{\mathrm{i}}=(1-x) \cdot x \cdot \sum_{\mathrm{i}=0}^{\mathrm{n}} \mathrm{A}_{\mathrm{i}} \cdot(1-2 \cdot x)^{\mathrm{i}}
$$

Sobre la elección de un número $\mathrm{n}$ apropiado de coeficientes para representar el conjunto de datos experimentales se hablará en el Anexo A.

El ajuste dado por la ecuación (2.208) puede aplicarse a cualquier función que nunca toma valores infinitos y que no tiene derivadas infinitas. Proporciona una gran calidad de representación en la mayoría de los casos incluso para curvas con forma de S. Sin embargo esta expansión en serie de potencias de la fracción molar no siempre es la mejor representación de las funciones de exceso [58]:

a) Cuando existe una notable asimetría respecto la fracción equimolar, la calidad del ajuste se reduce considerablemente.

b) Falla para $\Delta G_{\mathrm{m}}$ debido a que esta función tiene pendiente infinita en concentraciones nulas de ambos componentes, y debe expresarse como un número constante de veces la fracción de volumen de los componentes, más que como un desarrollo en serie de potencias del tipo representado por la ecuación (2.208).

c) Falla para $G_{\mathrm{m}}^{\mathrm{E}}, V_{\mathrm{m}}^{\mathrm{E}}$ o cualquier función similar $X_{\mathrm{m}}^{\mathrm{E}}$ de disoluciones diluidas de electrolitos. No obstante la teoría de Debye para disoluciones de electrolitos permite calcular un término electrostático $X_{\mathrm{m}}^{\mathrm{D}}$ tal que la cantidad $X_{\mathrm{m}}^{\mathrm{E}}-X_{\mathrm{m}}^{\mathrm{D}}$ puede tratarse de la misma manera que $X_{\mathrm{m}}^{\mathrm{E}}$ para disoluciones de no electrolitos.

Una ventaja evidente de este ajuste es que a partir de él se obtiene fácilmente el valor de la función ajustada para la fracción equimolar:

$$
X_{\mathrm{m}}^{\mathrm{E}}\left(x_{1}=x_{2}=0.5\right)=\frac{\mathrm{A}_{0}}{4}
$$

Cuando los volúmenes molares de ambos componentes puros no difieren en más del $10 \%$ y no hay interacciones específicas entre sus moléculas, suele encontrarse que las funciones $X_{\mathrm{m}}^{\mathrm{E}}=X_{\mathrm{m}}^{\mathrm{E}}\left(x_{1}\right)$ y $X_{\mathrm{m}}^{\mathrm{E}}=X_{\mathrm{m}}^{\mathrm{E}}\left(\Phi_{1}\right)$ son casi simétricas respecto de $x_{1}=0.5 \circ$ bien $\Phi_{1}=0.5$ respectivamente, donde $X_{\mathrm{m}}^{\mathrm{E}}\left(\Phi_{1}\right)$ se obtiene de (2.208) sustituyendo $x_{1}$ por la fracción de volumen del componente 1 definida por la ecuación (2.168).

Por el contrario, si los volúmenes molares difieren considerablemente, la posición del extremo relativo suele desplazarse de $x_{1}=0.5$, aunque suele mantenerse simétrica 
respecto $\Phi_{1}=0.5$ en la representación $X_{\mathrm{m}}^{\mathrm{E}}=X_{\mathrm{m}}^{\mathrm{E}}\left(\Phi_{1}\right)$, por lo que en estos casos suele tomarse esta última representación.

Los coeficientes pares representan curvas simétricas respecto la fracción equimolar y tienden a ensanchar o estrechar una curva en forma de parábola, mientras que los coeficientes impares generan asimetría de la curva respecto la composición equimolar, inclinándola hacia la izquierda o hacia la derecha.

En la llustración 2-2 se representan las contribuciones al ajuste de una función de exceso cualquiera $X_{\mathrm{m}}{ }^{\mathrm{E}}$ a una ecuación del tipo (2.208) de los tres primeros términos del desarrollo en serie. Se observa que el extremo relativo (máximo o mínimo) disminuye en valor absoluto al aumentar el número de términos que se toman para el ajuste.

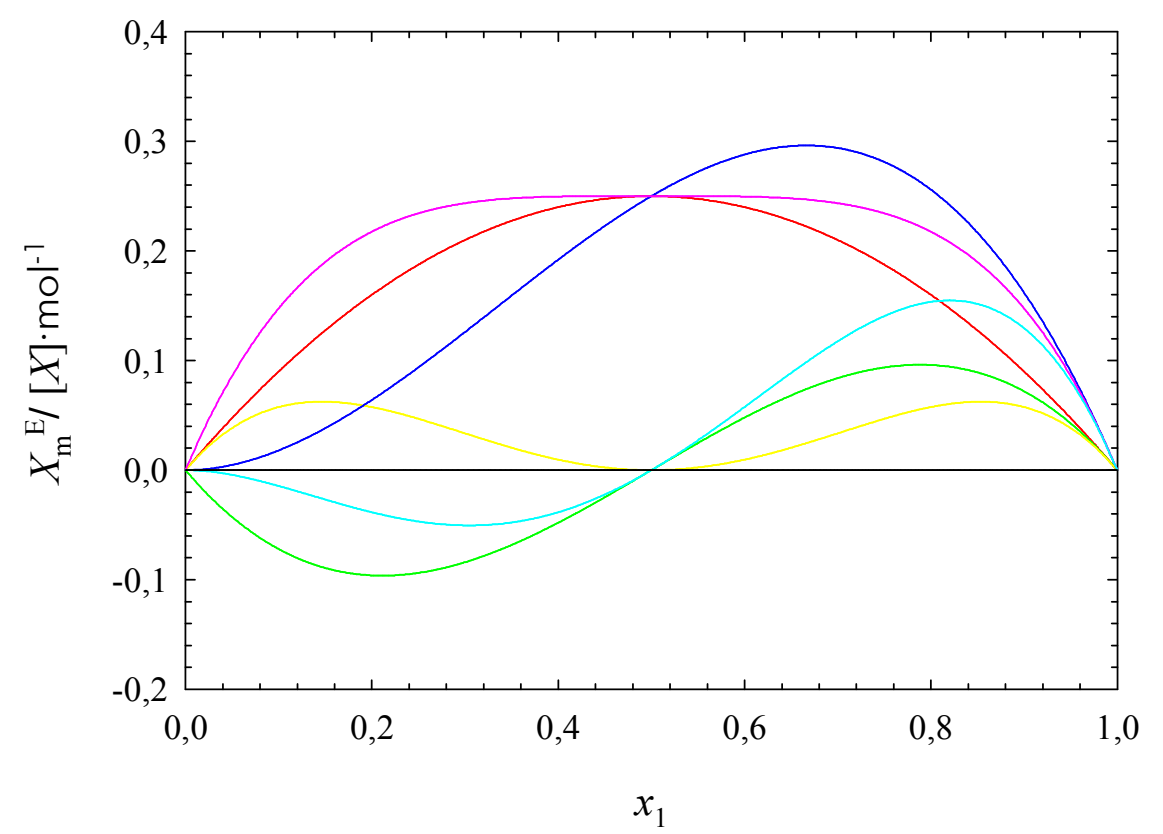

Ilustración 2-2: contribución de los tres primeros coeficientes de la ecuación de ajuste (2.208) para $X_{\mathrm{m}}^{\mathrm{E}}:-\mathrm{A}_{0}=1, \mathrm{~A}_{1}=\mathrm{A}_{2}=0 ;-\mathrm{A}_{0}=\mathrm{A}_{2}=0, \mathrm{~A}_{1}=1 ;-\mathrm{A}_{0}=\mathrm{A}_{1}=0, \mathrm{~A}_{2}=1 ;-\mathrm{A}_{0}=\mathrm{A}_{1}=1, \mathrm{~A}_{2}=0 ;-\mathrm{A}_{0}=\mathrm{A}_{2}=$ $1, \mathrm{~A}_{1}=0 ;-\mathrm{A}_{0}=0, \mathrm{~A}_{1}=\mathrm{A}_{2}=1$.

Los valores de los coeficientes se determinan por el método de los mínimos cuadrados, suponiendo que las fracciones molares están libres de error accidental y que los resultados para $X_{\mathrm{m}}^{\mathrm{E}}$ tienen iguales pesos estadísticos [59], a no ser que así lo justifiquen las condiciones experimentales. Para decidir acertadamente si deben o no considerarse los pesos estadísticos, se debe verificar la homogeneidad $u$ heterogeneidad de las dispersiones estándar generales de reproductibilidad de los datos de la función de exceso que se van a ajustar [65]. Esto se hizo con todos los datos que se presentan en este trabajo, utilizando el criterio de Kokren, puesto que el número de mediciones de las ordenadas para diferentes abscisas es el mismo.

Durante la Discussion of the Faraday Society en 1953, Korvezee [66] sugirió que las gráficas que representan las propiedades de exceso divididas por el producto de las fracciones molares, $X_{\mathrm{m}}^{\mathrm{E}} /\left(x_{1} \cdot x_{2}\right)$ son preferibles a las gráficas convencionales de las propiedades de exceso, $X_{\mathrm{m}}^{\mathrm{E}}$, lo que equivale a ajustar $X_{\mathrm{m}}^{\mathrm{E}}$ con pesos estadísticos 
$1 /\left(x_{1} \cdot x_{2}\right)^{2}$ para cada valor de $X_{\mathrm{m}}^{\mathrm{E}}$. Ello tiende a mejorar el ajuste en las regiones de concentración diluida $x \rightarrow 0$ ó $x \rightarrow 1$. El ajuste de $X_{\mathrm{m}}^{\mathrm{E}} /\left(x_{1} \cdot x_{2}\right)$ es muy sensible a la precisión de los datos, por lo que este tipo de ajuste es muy bueno cuando los datos son de calidad. En caso contrario la gráfica refleja claramente errores en el ajuste [67]. Además este tipo de ajuste es interesante ya que los valores de $X_{\mathrm{m}}^{\mathrm{E}} /\left(x_{1} \cdot x_{2}\right)$ en $x=1$ y en $x=0$ dan lugar a las propiedades de exceso molares parciales a dilución infinita:

$$
\begin{aligned}
& X_{1}^{\mathrm{E} \infty}=\frac{X_{\mathrm{m}}^{\mathrm{E}}}{x_{1} \cdot x_{2}}, \quad(x=1) \\
& X_{2}^{\mathrm{E} \infty}=\frac{X_{\mathrm{m}}^{\mathrm{E}}}{x_{1} \cdot x_{2}}, \quad(x=0)
\end{aligned}
$$

La ecuación R-K no solo proporciona un método adecuado del ajuste de los datos experimentales a una función analítica, sino que también sirve como una primera clasificación de los diferentes tipos de mezclas líquidas, ya que el número de parámetros necesarios para representar la función de exceso es una indicación de la complejidad aparente de la mezcla, siempre que la calidad de los datos sea buena:

a) Mezcla ideal: $\mathrm{A}_{\mathrm{i}}=0 \forall \mathrm{i}$.

b) Mezcla simple: $\mathrm{A}_{0} \neq 0, \mathrm{~A}_{\mathrm{i}}=0 \forall \mathrm{i} \neq 0$. Son mezclas de líquidos cuyas moléculas son apolares y tienen tamaño y forma similar, por ejemplo, c- $\mathrm{C}_{6} \mathrm{H}_{12}+\mathrm{CCl}_{4}$. Las ecuaciones (2.206) y (2.207) se transforma en las ecuaciones de Margules de dos subíndices:

$$
\begin{aligned}
& \ln f_{1}=\frac{\mathrm{A}_{0}}{R \cdot T} \cdot x_{2}{ }^{2} \\
& \ln f_{2}=\frac{\mathrm{A}_{0}}{R \cdot T} \cdot x_{1}^{2}
\end{aligned}
$$

c) $\mathrm{A}_{0}, \mathrm{~A}_{1} \neq 0, \mathrm{~A}_{\mathrm{i}}=0 \quad \forall \mathrm{i} \neq 0,1$. Son mezclas de líquidos cuyas moléculas son apolares y tienen tamaños diferentes. Las ecuaciones resultantes para los coeficientes de actividad serían las ecuaciones de Margules de tres subíndices.

d) $\quad A_{i} \neq 0 \mathrm{i}=2,3, \ldots$ Estas mezclas son más complejas y en ellas aparecen efectos de solvatación, formación de complejos, puentes de hidrógeno, etc.

La mayoría de las mezclas de interés en ingeniería química son de complejidad intermedia, requiriendo dos o tres coeficientes de ajuste R-K. No obstante, la clasificación de las disoluciones según el número de parámetros necesarios es algo arbitraria. Cuando se obtiene un buen ajuste de los datos con dos parámetros, no se puede concluir que la mezcla sea simple, pues puede aparecer cancelación de términos. Además, la calidad de los datos experimentales es un factor clave para la elección de uno u otro número de coeficientes.

A veces se emplea una forma equivalente de la función dada en la ecuación (2.208):

$$
X_{\mathrm{m}}^{\mathrm{E}}=x_{1} \cdot x_{2} \cdot \sum_{\mathrm{j}=0}^{\mathrm{n}-1} v_{\mathrm{j}} \cdot x_{1}{ }^{\mathrm{j}}
$$

Puesto que las funciones de ajuste a las que nos estamos refiriendo son todas empíricas, no es esencial que los parámetros obtenidos mediante el método de los mínimos 
cuadrados constituya un conjunto completo de coeficientes consecutivos del polinomio. Por ejemplo, Visser et al [68] encontraron que en algunos casos se obtiene un mejor ajuste con un polinomio de grado 10 en la ecuación (2.214) con los coeficientes $v_{2}=v_{9}=0$ que usando un polinomio del tipo (2.208) ó (2.214) con un gran número de coeficientes consecutivos. La ecuación (2.214) es apropiada para ajustar resultados experimentales que no guardan simetría respecto la fracción equimolar, mientras que (2.208), como ya se ha dicho, es muy apropiada en el caso de que exista dicha simetría. No obstante, las dos representaciones son totalmente equivalentes y sus coeficientes están relacionados mediante la ecuación:

$$
v_{\mathrm{j}}=\left[\frac{(-2)^{\mathrm{j}}}{\mathrm{j} !}\right] \cdot \sum_{\mathrm{k}=\mathrm{j}}^{\mathrm{n}} \frac{A_{\mathrm{k}} \cdot \mathrm{k} !}{\mathrm{k}-\mathrm{j}) !}
$$

Ambos ajustes pueden generalizarse para representar $X_{\mathrm{m}}^{\mathrm{E}}$ como función de la temperatura así como también de la concentración, si se considera que los coeficientes $v_{j}$ son funciones polinómicas de la temperatura $T$ :

$$
v_{\mathrm{j}}(T)=\sum_{\mathrm{l}=0}^{\mathrm{n}} v_{\mathrm{j} 1} \cdot T^{2}
$$

Normalmente una dependencia lineal o cuadrática es suficiente. Por tanto, el número de coeficientes a calcular por el método de los mínimos cuadrados se multiplica por un factor de 2 ó 3 respectivamente.

Las funciones asimétricas respecto la fracción equimolar también se pueden representar como formas polinómicas en la raíz cuadrada de la fracción molar de uno de los componentes:

$$
X_{\mathrm{m}}^{\mathrm{E}}=x_{1} \cdot x_{2} \cdot \sum_{\mathrm{j}=0}^{\mathrm{n}-1} \eta_{\mathrm{j}} \cdot x_{1}^{\mathrm{j} / 2}
$$

o como cociente de polinomios [69]:

$$
X_{\mathrm{m}}^{\mathrm{E}}=x_{1} \cdot x_{2} \cdot \frac{\sum_{\mathrm{j}=1}^{\mathrm{n}} \theta_{\mathrm{j}} \cdot\left(1-2 \cdot x_{1}\right)^{\mathrm{j}-1}}{1+\sum_{\mathrm{k}=0}^{\mathrm{p}} \lambda_{\mathrm{k}} \cdot\left(1-2 \cdot x_{1}\right)^{\mathrm{k}+1}}
$$

La representación (2.218) es útil cuando el denominador no es cero para todo $x_{1}$ entre 0 y 1 . Un conocido ejemplo de la utilización de ésta le recomiendan Myers \& Scott [71].

Christiansen y Fredenslund [72] propusieron expresiones alternativas para el ajuste de las funciones de exceso basándose en series de polinomios ortogonales. Las expresiones que se obtienen tienen el mérito de que la adición de términos extra para mejorar el ajuste de los datos experimentales tiene un efecto menor sobre la magnitud que los términos anteriores. La expresión propuesta es:

$$
G_{\mathrm{m}}^{\mathrm{E}}=\sum_{\mathrm{i}=2}^{\mathrm{N}} \xi_{\mathrm{i}} \cdot P_{\mathrm{i}}\left(x_{1}, x_{2}\right)
$$

donde las $\xi_{\mathrm{i}}$ dependen de la temperatura y los polinomios $P_{\mathrm{i}}\left(x_{1}, x_{2}\right)$ cumplen las condiciones de ortogonalidad: 


$$
\int_{0}^{1} P_{\mathrm{i}} \cdot P_{\mathrm{j}}=\delta_{\mathrm{ij}}
$$

Los tres primeros polinomios:

$$
\begin{gathered}
P_{2}=x_{1} \cdot x_{2} \\
P_{3}=x_{1} \cdot x_{2} \cdot\left(1-2 \cdot x_{1}\right) \\
P_{4}=x_{1} \cdot x_{2} \cdot\left(1-\frac{14}{3} \cdot x_{1}+\frac{14}{3} \cdot x_{1}^{2}\right)
\end{gathered}
$$

El resto de polinomios pueden obtenerse mediante la ecuación de recurrencia:

$$
P_{\mathrm{N}}=\left[(2 \mathrm{~N}-1) \cdot\left(1-2 \cdot x_{1}\right) \cdot P_{\mathrm{N}-1}-(\mathrm{N}-3) \cdot P_{\mathrm{N}-2}\right] /(\mathrm{N}+2)
$$


CAPITULO 2 


\section{BIBLIOGRAFÍA}

[1]: Bazarov, I.P.; "Thermodynamics" Pergamon Press (1964).

[2]: M. W. Zemansky y R. H. Dittmann; "Calor y Termodinámica”, McGraw-Hill (1966).

[3]: O'Connell, J. P.; Haile, J. M.; "Thermodynamics, Fundamentals for Applications", Cambridge University Press (2005).

[4]: Dunning-Davies, J.; Phys. Letters, 94A(8) (1983), 346.

[5]: Clausius, R.; Ann. Phys. Chem. (Leipzig), 155(3) (1850), 368.

[6]: Joule, J.P.; Brit. Assoc. Rep., trans. Chemical Sect, (1845), 31.

[7]: Thomson, W; "On the dynamical theory of heat; with numerical results deduced from Mr. Joule's equivalent of a thermal unit and M. Regnault's observations on steam" Math. and Phys. Papers vol. 1 (1851), 175-183.

[8]: Brodianski V.M.; "Móvil Perpetuo, antes y ahora", Editorial MIR (1990).

[9]: Landsberg, P.T.; "Thermodynamics and Statistical Mechanics", Oxford University Press, (1978).

[10]: Prigogine, I; "Introducción a la Termodinámica de los Procesos Irreversibles", Selecciones Científicas, Madrid (1974).

[11]: Prigogine, I; Defay, R.; "Chemical Thermodynamics", Longmans, $3^{\text {rd }}$ edition (1965).

[12]: De Donder Th., Bull. Ac. Roy. Belg. (Cl. Sc.) (5) 7, 197, 205, (1922)

[13]: Guggenheim, E. A.; "Termodinámica para químicos y físicos", Editorial Tecnos (1970).

[14]: Sychev, V. V.; "Complex Thermodynamic Systems", traducido del ruso "Slozhnye Termodinamicheskie Sistemy", 3rd (1982), Import Pubn.

[15]: Alberty, R. A.; J. Chem. Thermodyn. (Technical Report), 34 (2002), 1787.

[16]: Callen, H.B; "Thermodynamics and an Introduction to Thermostatics"; Wiley, New York (1985).

[17]: Planck, M.; "Treatise On Thermodynamics"; London: Longmans, Green, and Company, traducido del alemán "Vorlesungen über Thermodynamik"; Leipzig: Verlag Von Veit \& Company. $5^{a}$ edición (1917). 
[18]: Bazarov, I. P.; "Equivocaciones y Errores en la Termodinámica"; Editorial URSS, Traducido de la segunda edición rusa (2003).

[19]: Planes, A.; Vives, E.; J. Statistical Phys., 106, 3/4 (2002), 827.

[20]: Brush, S.G., "The Kind of Motion We Call Heat", Vol 2, Noth-Holland, Amsterdam (1976).

[21]: Guggenheim, E. A.; "Mixtures, The Theory of the Equilibrium Properties of Some Simple Classes of Mixtures Solutions and Alloys", Oxford (1952).

[22]: Walas, S. M.; "Phase Equilibrium in Chemical Engineering", Butterworth Publishers (1985).

[23]: Clapeyron, E.; Journal de l'École Polytechnique XIV (1834), 153.

[24]: Van Ness, H.C.; Abbot, M.M.; "Classical Thermodynamics of Noneletrolyte Solutions- With Applications to Phase Equilibria", McGraw-Hill (1952).

[25]: Smith, J.M.; Van Ness, H.C.; "Introducción a la Termodinámica en Ingeniería Química", 4 ed., McGraw-Hill (1988).

[26]: Thiessen; Ann. Phys. 24 (1885), 467.

[27]: Onnes, K.; Konink. Akad. Wetens, 273, (1912).

[28]: Ursell, H. D.; Proc. Cambridge Philos. Soc, 23 (1927), 685.

[29]: Hayden, J. G., O'Conell, P.O.; Ind. Eng. Chem., Process Des. Dev., 14(3) (1975), 209.

[30]: H. Poincaré, "Sur le probléme des trois corps et les equations de la dynamique", withdrawn version 1889 and Acta Mathematica 13 (1890), 1-270.

[31]: Prausnitz, J. M., Lichtenthaler, R.N., Gomes de Azevedo, E., "Termodinámica Molecular de los Equilibrios Entre Fases", $3^{a}$ edición, Prentice Hall (2000).

[32]: Mayer, J.E.; Mayer, M. G.; "Statistical Mechanics", Capítulo XIII, Wiley (1940).

[33]: Israelachvili, J.N.; "Intermolecular and Surface Forces", $3^{a}$ ed., Elsevier (2011).

[34]: Van der Waals, J. D.; "Over de Continuiteit van den Gas- en Vloeistoftoestand"; Leiden (1873).

[35]: Redlich, Otto; Kwong, J. N. S.; Chem. Rev., 44(1) (1949), 233.

[36]: Lewis, G.N.; Proc. Amer. Acad. Arts Sci., 37(3) (1901), 49; Z. Phys. Chem. 38 (1901), 205.

[37]: Hildebrand, J.H.; Scott, R.L., "Regular Solutions", Prentice-Hall (1962).

[38]: Rowlinson J.S., Swinton, F.L.; "Liquids and Liquids Mixtures", Butterworths Monographs in Chemistry (1982).

[39]: Bakhuis-Roozeboom, H.W.; "Die Heterogenen Glichgewichte", II-1, Braunschweig (1904), 288.

[40]: Lewis, G.N.; J. Amer. Chem. Soc., 35 (1913), 17.

[41]: Lewis, G.N.; Randall, M.; "Thermodynamics and the Free Energiy of Chemical Substances", McGraw-Hill, New York (1923), 255. 
[42]: Margules, S.B.; Akad. Wiss. Wien, 104 (1845), 1258.

[43]: Beattie, J.A.; Stockmayer, W.H.; "Treatise on Phyisical Chemistry" (1942), Princeton: Van Nostrand.

[44]: Beattie, J.A.; Chem. Rev., 44, 141.

[45]: Beattie, J.A., "Thermodynamics and Physics of Matter", Princeton University Press (1955).

[46]: Wilhelm, E.; Thermochim. Acta, 69 (1983), 1.

[47]: Benson, G.C.; Kiyohara, O.; J. Chem. Thermodyn., 11 (1979), 1061.

[48]: Raoult, F.M.; Comptes Rendu, 104 (1887), 1430.

[49]: Raoult, F.M.; Z. Phys. Chem., 2 (1888), 353.

[50]: Henry; Phil. Trans. Roy. Soc. (London), 29 (1803), 274.

[51]: Scatchard, G.; Raymond,C.; J. Amer. Chem. Soc., 60 (1938), 1283.

[52]: Gayé, J.B.; "Curso sobre el Formalismo y los Métodos de la Termodinámica"; Editorial Reverté, Barcelona (1998).

[53]: Brönsted, N.; J. Chem. Soc., 119, (1921), 574.

[54]: Scott, R.L.; J. Phys. Chem., Ithaca, 64, (1960), 1241.

[55]: Scatchard, G.; Trans. Faraday Soc., 33, (1937), 160.

[56]: Mozo Ruiz, I.; "Estudio experimental y teórico de mezclas binarias de n-butan-1-ol, di-n-butiléter y celosolvas". Tesis Doctoral. Departamento de Física Aplicada. Facultad de Ciencias. Universidad de Valladolid (2010).

[57]: Serna Gutiérrez, A.; "Determinación de los $V_{m}^{E}$ de los sistemas n-alcohol+mono y poliéteres mediante un densímetro de oscilación mecánica". Tesis de Licenciatura. Departamento de Física Aplicada II. Facultad de Ciencias. Universidad de Valladolid, (1994).

[58]: Scatchard, G.; Chem. Rev., 44 (1949), 7.

[59]: Handa, Y.P.; Benson, G.C.; Fluid Phase Equilib., 3, (1979), 185.

[60]: Gordon, A.R.; Hornibrook, E.J.; Can. J. Res., 24B (1946), 263.

[61]: Guggenheim, E.A.; Trans. Faraday Soc., 33 (1937), 151.

[62]: Scatchard, G.; Hamer, W.J.; J. Amer. Chem. Soc., 57 (1935), 1805.

[63]: O. Redlich, A.T. Kister, Ind. Eng. Chem., 40(2) (1948), 345

[64]: Ganguly, J.; "Thermodynamics in Earth and Planetary Sciences", Springer (2008), 257.

[65]: Spiridonov, V.P.; Lopatkin, A.A.; "Tratamiento Matemático de Datos FísicoQuímicos", MIR (1973).

[66]: Korvezee, A.E.; Discuss. Faraday Soc., 15 (1953), 255.

[67]: Wisniak, J.; Ind. Eng. Chem. Res., 32 (1993), 240.

[68]: De Visser, C.; Perron, G.; Desnoyers, J.E.; J. Chem. Eng. Data, 22, (1977), 74. 
CAPITULO 2

[69]: Marsh, K.N.; J. Chem. Thermodyn., 9 (1977), 719.

[70]: Marsh, K.N.; Chemical Thermodynamics, vol 2. The Chemical Society, London. (1978).

[71]: Myers, D.B.; Scott, R.L.; Ind. Eng. Chem., 55 (1963), 43.

[72]: Christiansen, L.J.; Fredenslund, A.; A. I. Ch. E. J., 21 (1975), 49. 


\title{
Medida de volúmenes y compresibilidades isoentrópicas de
}

exceso

\begin{abstract}
"Of the various thermodynamic functions for the mixing process, the volume change $\Delta \mathrm{V}^{\mathrm{M}}$ on mixing at constant presure (which is the same as the excess volume $\mathrm{V}^{\mathrm{E}}$ ) is one of the most interesting, yet certainly still one of the least understood" Hildebrand, J.H.; Scott, R.L.; "Regular Solutions", Prentice-Hall, Englewood Cliffs, N. J., 1962.
\end{abstract}

B asta con repasar brevemente la bibliografía especializada para ser conscientes de que el cambio de volumen que ocurre al mezclar diferentes sustancias líquidas es un fenómeno que ha levantado el interés de físicos y químicos desde hace mucho tiempo. Por ejemplo, podemos encontrar que en 1873 Fouvre y Valson [1] observaron que el volumen de una disolución de sales halogenadas de amonio en agua es mayor que la suma de los volúmenes de la sal y el agua por separado. Pronto se encontró que los cambios de volumen y de temperatura que ocurren al mezclar líquidos están estrechamente relacionados [2],[3].

Ahora se sabe que el cambio de volumen que se produce al mezclar dos líquidos se debe a la combinación de diversos factores [4]:

a) Diferencia de tamaño entre las moléculas de cada componente.

b) Diferencia de forma entre las moléculas de cada componente.

c) Cambios estructurales tales como cambios en la correlación de las orientaciones de las moléculas.

d) Diferencia en las interacciones entre moléculas iguales y diferentes.

e) Formación de nuevas especies químicas.

La complejidad asociada al origen del volumen de exceso junto con la facilidad con la que puede medirse con elevada precisión hacen que sea una herramienta útil para comprobar las teorías de mezclas líquidas. Además, los datos de $V_{\mathrm{m}}^{\mathrm{E}}$ son necesarios tanto para la conversión de funciones termodinámicas de exceso a presión constante o 
a volumen constante (véanse las ecs. 2.202 y 2.203) como para la determinación de la composición a partir de la medida de la densidad de las mezclas.

Por su parte, la compresibilidad expresa la variación del volumen cuando se ejerce una presión externa. Si el proceso transcurre a temperatura constante se habla de compresibilidad isoterma:

$$
\kappa_{T}=-\left(\frac{1}{V}\right) \cdot\left(\frac{\partial V}{\partial P}\right)_{T}
$$

La compresibilidad isoterma de un líquido es un parámetro termodinámico muy importante, tanto en la teoría como en la práctica [5]. Por ejemplo, se necesita para calcular la pendiente Debye-Hückel de la ecuación de Redlich-Rosenfeld, que se utiliza en la determinación de los volúmenes molares parciales de electrolitos en mezclas a dilución infinita a partir de las medidas de los volúmenes molares aparentes de concentraciones finitas [6].

A pesar de su importancia, no se ha desarrollado ningún método para la medida directa de $\kappa_{T}$ que resulte satisfactorio, pues hasta ahora todos ellos son muy tediosos. No obstante, las técnicas para determinar la compresibilidad isoentrópica:

$$
\kappa_{S}=-\left(\frac{1}{V}\right) \cdot\left(\frac{\partial V}{\partial P}\right)_{S}
$$

están más desarrolladas. A partir de $\kappa_{S}$ se puede obtener $\kappa_{T}$ ya que ambas magnitudes están relacionadas en términos de la capacidad calorífica a presión constante, $C_{p}$, y del coeficiente de dilatación térmica a presión constante $\alpha_{P}=(\partial \ln V / \partial \mathrm{T})_{P}$ mediante la ecuación:

$$
\kappa_{T}=\kappa_{S}+\frac{T \cdot V \cdot \alpha_{P}^{2}}{C_{P}}
$$

Además $\kappa_{S}$ es de gran interés en Física, Química, Ingeniería y Biología [7]-[12].

La compresibilidad isoentrópica de exceso:

$$
\kappa_{S}^{\mathrm{E}}=\kappa_{S}-\kappa_{S}^{\mathrm{id}}
$$

es un parámetro que proporciona información útil e importante, dependiendo del tipo de interacción y del comportamiento de las moléculas en las mezclas.

\subsection{MEDIDA DEL VOLUMEN DE EXCESO}

Sin pérdida de generalidad se puede decir que $V_{\mathrm{m}}^{\mathrm{E}}$ de una mezcla líquida se puede determinar mediante dos métodos bien diferenciados:

a) Indirectos. Consisten en determinar la constante dieléctrica o la densidad de los líquidos puros y de su mezcla, cuya composición se conoce de antemano.

b) Directos. El cambio en el volumen cuando se produce la mezcla se puede observar directamente mediante un dispositivo llamado dilatómetro. 
Hay muchos factores que afectan a la precisión y exactitud de la medida del $V_{\mathrm{m}}^{\mathrm{E}}$ : i) presencia de gases disueltos en el líquido, lo que hace indispensable desgasificarlo (en un baño de ultrasonidos por ejemplo); ii) la temperatura debe mantenerse aproximadamente constante durante el tiempo necesario para levar a cabo la medida; etc.

En medidas directas, basta con que la precisión en la composición no sea mayor que la precisión en $V_{\mathrm{m}}^{\mathrm{E}}$. En medidas indirectas basadas en la determinación de la densidad, la precisión en la composición es proporcional a la diferencia entre las densidades de ambos componentes y la precisión en la densidad: una diferencia de $0.1 \mathrm{~g}^{\circ} \mathrm{cm}^{-3}$ en la densidad de los componentes puros para una precisión de $1 \cdot 10^{-5} \mathrm{~g} \cdot \mathrm{cm}^{-3}$ en la medida de la densidad se corresponde con una precisión de $\pm 1 \cdot 10^{-4}$ en la fracción molar.

\subsubsection{Métodos indirectos}

La medida de los volúmenes de exceso de una mezcla de líquidos puede realizarse a partir del conocimiento de la constante dieléctrica $\varepsilon$, ya que, definida la función de Clausius-Mossotti (C-M):

$$
\operatorname{\Re }_{\mathrm{i}}(\rho, T)=\frac{\varepsilon_{\mathrm{i}}-1}{\varepsilon_{\mathrm{i}}+2} \cdot V_{\mathrm{i}}
$$

el volumen de exceso puede escribirse como [13]:

$$
V_{\mathrm{m}}^{\mathrm{E}}=\sum_{\mathrm{i}=1}^{\mathrm{n}} x_{\mathrm{i}} \cdot \Re_{\mathrm{i}} \cdot\left[\left(\frac{\varepsilon+2}{\varepsilon-1}\right)-\left(\frac{\varepsilon_{\mathrm{i}}+2}{\varepsilon_{\mathrm{i}}-1}\right)\right]
$$

La ventaja de este procedimiento es que $\varepsilon$ puede medirse fácilmente en amplios rangos de $P$ y $T$ utilizando aparatos relativamente sencillos. No obstante, este método sólo es válido para un número limitado de casos ya que la ecuación (3.6) es resultado de suponer que para la mezcla la función C-M es la suma de las funciones individuales de cada componente puro $\operatorname{or}(\rho, T)=\sum_{\mathrm{i}=1}^{\mathrm{n}} x_{\mathrm{i}} \cdot \operatorname{Or}_{\mathrm{i}}(\rho, T)$ (es decir, su exceso es cero) lo que es aceptable solamente si las moléculas de los líquidos que se mezclan son pequeñas y apolares.

En el caso más general, sin importar la forma de las moléculas, el tamaño, naturaleza química, etc los volúmenes de exceso de una mezcla se puede determinar a partir de las densidades de la mezcla y de los líquidos puros y de la composición. Para una mezcla binaria,

$$
V_{\mathrm{m}}^{\mathrm{E}}=V_{\mathrm{m}}-\sum_{\mathrm{i}=1}^{2} x_{\mathrm{i}} \cdot V_{\mathrm{i}}^{0}=\frac{x_{1} \cdot M_{1}^{0}+x_{2} \cdot M_{2}^{0}}{\rho}-\left(\frac{x_{1} \cdot M_{1}^{0}}{\rho_{1}}+\frac{x_{2} \cdot M_{2}^{0}}{\rho_{2}}\right)
$$

donde $x_{\mathrm{i}}, M_{\mathrm{i}}^{0}$ y $\rho_{\mathrm{i}}$ son la fracción molar, la masa molar y la densidad del componente puro i, y $\rho$ es la densidad de la mezcla.

La densidad de un líquido generalmente se mide por comparación con la densidad de otro líquido tomado como patrón de densidad. Brown \& Lane [14] recomiendan el uso de agua, mercurio, 2,2,4-trimetilpentano, ciclohexano y transbiciclo $(4,4,6)$ decano como patrones de densidad, aunque Girard [15], Harvey et all [1 6] y Fujii [17] solamente 
recomiendan el uso de agua y mercurio.

\subsubsection{Agua como patrón de densidad}

Las primeras medidas fiables de la densidad del agua se hicieron a finales del siglo XIX en el Bureau Internacional des Poids et Mesures (BIPM) mediante métodos de balanza hidrostática. Timmermans [18] cita un gran número de medidas de la densidad del agua hechas entre finales del siglo XIX y principios del siglo XX.

La densidad del agua depende de diversos factores que deberán o no considerarse dependiendo del grado de exactitud deseado:

- Efecto isotópico: El efecto que provocan los diferentes isótopos sobre la densidad no es trivial; ello se refleja en que los promedios anuales de la composición isotópica del agua precipitada varían en diferentes lugares de la corteza terrestre y sin embargo su efecto sobre la densidad relativa del agua como mucho es de $5 \cdot 10^{-6}$.

Existen principalmente cuatro especies de moléculas de agua: $\mathrm{H}_{2}^{16} \mathrm{O}, \mathrm{H}_{2}^{17} \mathrm{O}, \mathrm{H}_{2}^{18} \mathrm{O}$ y $\mathrm{HD}^{16} \mathrm{O}$. La composición isotópica de una muestra de agua se describe por los cocientes:

$$
R_{\mathrm{D}}=\frac{n(\mathrm{D})}{n(\mathrm{H})} \quad R_{17}=\frac{n\left({ }^{17} \mathrm{O}\right)}{n\left({ }^{16} \mathrm{O}\right)} \quad R_{18}=\frac{n\left({ }^{18} \mathrm{O}\right)}{n\left({ }^{16} \mathrm{O}\right)}
$$

donde $\frac{n(\mathrm{X})}{n(\mathrm{Y})}$ se determina mediante espectrometría de masas y es la cantidad de sustancia relativa del núclido $X$ respecto del núclido $Y$, correspondiente éste último a la molécula más normal de agua, $\mathrm{H}_{2}^{16} \mathrm{O}$.

Para comparar la composición isotópica de diferentes muestras de agua sobre una base de confianza, Craig [19] definió el SMOW (Standard Mean Ocean Water), que además sirve como patrón de densidad, tal y como recomiendan Girard y Menaché [20]. La muestra original de SMOW se conserva en la Agencia Internacional de Energía Atómica, que es responsable de su distribución en pequeñas cantidades a laboratorios especializados en espectrometría. La determinación de la composición isotópica de una muestra cualquiera de agua se determina por comparación con la de la muestra de $S M O W$. Los resultados de esta comparación se expresan mediante las diferencias relativas $\delta \mathrm{D}, \delta^{17} \mathrm{O}, \delta^{18} \mathrm{O}$ que se calculan como:

$$
\begin{aligned}
\delta \mathrm{D} & =\left[\frac{R_{\mathrm{D}}(\text { muestra })}{R_{\mathrm{D}}(\text { SMOW })}-1\right] \cdot 10^{3} \\
\delta^{17} \mathrm{O} & =\left[\frac{R_{17}(\text { muestra })}{R_{17}(\text { SMOW })}-1\right] \cdot 10^{3} \\
\delta^{18} \mathrm{O} & =\left[\frac{R_{18}(\text { muestra })}{R_{18}(\text { SMOW })}-1\right] \cdot 10^{3}
\end{aligned}
$$

La corrección de la densidad viene dada por:

$$
\rho(\text { muestra })-\rho(S M O W)=\left(0.233 \cdot \delta^{18} \mathrm{O}+0.0166 \cdot \delta \mathrm{D}\right) \pm 1 \mathrm{~g} \cdot \mathrm{m}^{-3}
$$


Diferentes valores de la densidad del SMOW pueden encontrarse en la bibliografía. A $293.15 \mathrm{~K}$ y $101325 \mathrm{KPa}$, se han encontrado los valores $999.975 \mathrm{Kg} \cdot \mathrm{m}^{-3}$ [21], 999.9736 $\mathrm{Kg} \cdot \mathrm{m}^{-3}[22]$ y $999.9757 \mathrm{Kg} \cdot \mathrm{m}^{-3}$ [23].

- Efectos térmicos. Tanaka et al [24] midieron la densidad del SMOW entre $273.15 \mathrm{~K}$ y $313.15 \mathrm{~K}$ obteniendo la relación siguiente entre la densidad y la temperatura:

$$
\rho_{\text {SMOW }}(T)=a_{5} \cdot\left[1-\frac{\left(T+a_{1}\right)^{2} \cdot\left(T+a_{2}\right)}{a_{3} \cdot\left(T+a_{4}\right)}\right]
$$

siendo $T$ la temperatura expresada en unidades de la Escala Internacional de Temperatura Absoluta ITS-90.

Existe una ecuación de estado desarrollada por Wagner y Pru $\beta$ [25] para las propiedades termodinámicas del agua válida desde $251.2 \mathrm{~K}$ hasta $1273.15 \mathrm{~K}$ y desde 209.9 MPa hasta $1 \mathrm{GPa}$.

\subsubsection{Mercurio como patrón de densidad}

La importancia de tener un valor lo más exacto posible de la densidad del mercurio no sólo radica en que éste sirva como patrón de densidad, sino que además es una cantidad indispensable para obtener el patrón de presión, ya que el mercurio se utiliza como fluido manométrico.

La medida absoluta de la densidad del mercurio se realizó mediante pesada hidrostática y picnometría entre 1957 y 1961 [26], [27]. A 293.15 K y 101325 KPa su valor es $13545.854 \mathrm{Kg} \cdot \mathrm{m}^{-3}$.

Igual que en el caso del agua, la densidad del mercurio está afectada por diversos factores:

- Efecto isotópico. El mercurio está compuesto por isótopos de números másicos $196,198,199,200,201,202$ y 204. Variaciones de $0.005 \%$ en la abundancia de uno o dos isótopos pueden cambiar la densidad del mercurio en $10^{-2} \mathrm{Kg} \cdot \mathrm{m}^{-3}$.

- Efecto de las impurezas. En la Tabla 3-1 se muestran diferentes valores de fracción másica de distintas impurezas que producirían un cambio en la densidad del mercurio igual a $10^{-2} \mathrm{Kg} \cdot \mathrm{m}^{-3}$.

Tabla 3-1: efecto sobre la densidad del mercurio de pequeñas cantidades de impurezas

\begin{tabular}{cccc}
\hline Metal & $10^{-6}$ en fracción de masa & Metal & $10^{-6}$ en fracción de masa \\
\hline $\mathrm{Pt}$ & 2.7 & $\mathrm{Sn}$ & 1.1 \\
$\mathrm{Au}$ & 3.4 & $\mathrm{Fe}$ & 1.4 \\
$\mathrm{Zn}$ & 1.5 & $\mathrm{Na}$ & 0.13 \\
$\mathrm{Cu}$ & 1.9 & $\mathrm{Ca}$ & 0.13 \\
$\mathrm{~Pb}$ & 4.3 & $\mathrm{Al}$ & 0.25 \\
\hline
\end{tabular}

Estos datos permiten apreciar que pequeñas cantidades de impurezas producen cambios considerables en la densidad del mercurio, por lo que éste debe purificarse antes de determinar su densidad, tratándolo con ácido nítrico diluido, seguido de un aclarado con agua y una destilación a presión reducida, de unos $3 \mathrm{KPa}$ y en presencia de aire limpio [15]. 


\subsubsection{Otros líquidos como patrones de densidad}

Hay sustancias cuya densidad ha sido determinada en numerosas ocasiones y por diferentes experimentadores, siendo las diferencias entre los distintos resultados menores que la precisión obtenible hoy en día. En estos casos, pueden tomarse dichas sustancias como patrones de densidad, como ocurre con las siguientes: $n$-hexano, $n$ heptano, $n$-octano, 2,2,4-trimetilpentano (isooctano), $n$-nonano, ciclohexano, benceno, metilciclohexano, trans-decahidronaftaleno, tolveno y transbiciclo $(4,4,6)$ decano [15].

\subsubsection{Medida de la densidad de líquidos y sus mezclas}

La densidad de los líquidos y de sus mezclas puede determinarse mediante varios métodos, muchos de los cuales se tratan con profundidad en multitud de trabajos [28][31]. En lo siguiente se exponen los principales:

\section{a) Densímetros basados en el principio de Arquímedes}

Existen diferentes tipos de densímetros basados en la aplicación de este principio:

- Densímetros de balanza hidrostática. Una plomada esférica o cilíndrica se suspende por el extremo de un alambre fino de platino que en el otro extremo está sujeto a una balanza. El líquido cuya densidad quiere determinarse se sitúa en una celda termostatada debajo del brazo de la balanza del que pende el alambre fino de manera que la plomada queda inmersa completamente en el fluido. Se pesa la plomada antes y después de sumergirse en el líquido; teniendo en cuenta el empuje del aire sobre el alambre, sobre la plomada y sobre el líquido, y despreciando los efectos debidos a la parte del alambre sumergida en el líquido, y a la tensión superficial, se tiene que la densidad del líquido es:

$$
\rho=\frac{\Delta m}{V}+\rho_{\mathrm{a}}
$$

donde $\rho$ y $\rho_{\mathrm{a}}$ son respectivamente las densidades del líquido y el aire (determinada ésta previamente), $\Delta m$ es la diferencia de la lectura de la balanza entre ambas etapas y $V$ es el volumen de la plomada. Los efectos debidos a la parte del alambre sumergida en el líquido y a la tensión superficial pueden introducirse en la ecuación anterior como correcciones a la misma [15].

A veces, en vez de la balanza se utiliza un resorte de cristal de cuarzo [32]-[34].

- Densímetros de flotador magnético y de suspensión magnética. Son muy útiles cuando el efecto de la tensión superficial entre el alambre y el líquido no es despreciable. La boya es un cuerpo hueco de cristal que contiene un imán o un núcleo de hierro dulce y que se sumerge en una celda termostatada y conteniendo al líquido. Encima de la celda se coloca un solenoide que mantiene a la boya en una posición fija, gracias al campo magnético que crea cuando por él circula una corriente.

La fuerza magnética que debe aplicarse para el equilibrio de las fuerzas que actúan sobre la boya depende de la diferencia entre las densidades de la boya, $\rho_{b}$, y el líquido, $\rho$ :

$$
F_{\mathrm{m}}=V \cdot g \cdot\left(\rho_{\mathrm{b}}-\rho\right)
$$

donde $V$ es el volumen sumergido de la boya y $g$ es el valor de la gravedad en el lugar donde se está efectuando la medida. La fuerza magnética se puede determinar 
mediante la corriente que circula por el solenoide o mediante una balanza [35], [36].

Los densímetros de flotador magnético se dividen en dos grupos, dependiendo del sentido en el que la fuerza magnética actúa: hacia abajo, densímetros de flotador magnético propiamente dichos; hacia arriba, densímetros de suspensión magnética. Este último tipo es más adecuado para medir densidades en condiciones extremas de presión y temperatura.

Este tipo de densímetros puede utilizarse en condiciones de presión y temperatura variables. Hasta ahora, se han utilizado [37]-[41] rangos de presión desde 1 atm hasta $120 \mathrm{MPa}$ y de temperatura desde $90 \mathrm{~K}$ hasta $473 \mathrm{~K}$.

- Densímetros de doble plomada. Gracias a la rigurosa aplicación del principio de Arquímedes todos los efectos perturbadores que reducirían la exactitud con la que se determina la densidad (fuerzas de empuje, fuerzas de tensión superficial, incertidumbre de la balanza, ...) se compensan automáticamente. Incluso la adsorción del fluido en la superficie de contacto con las plomadas se compensa, gracias a que ambos tienen igual superficie.

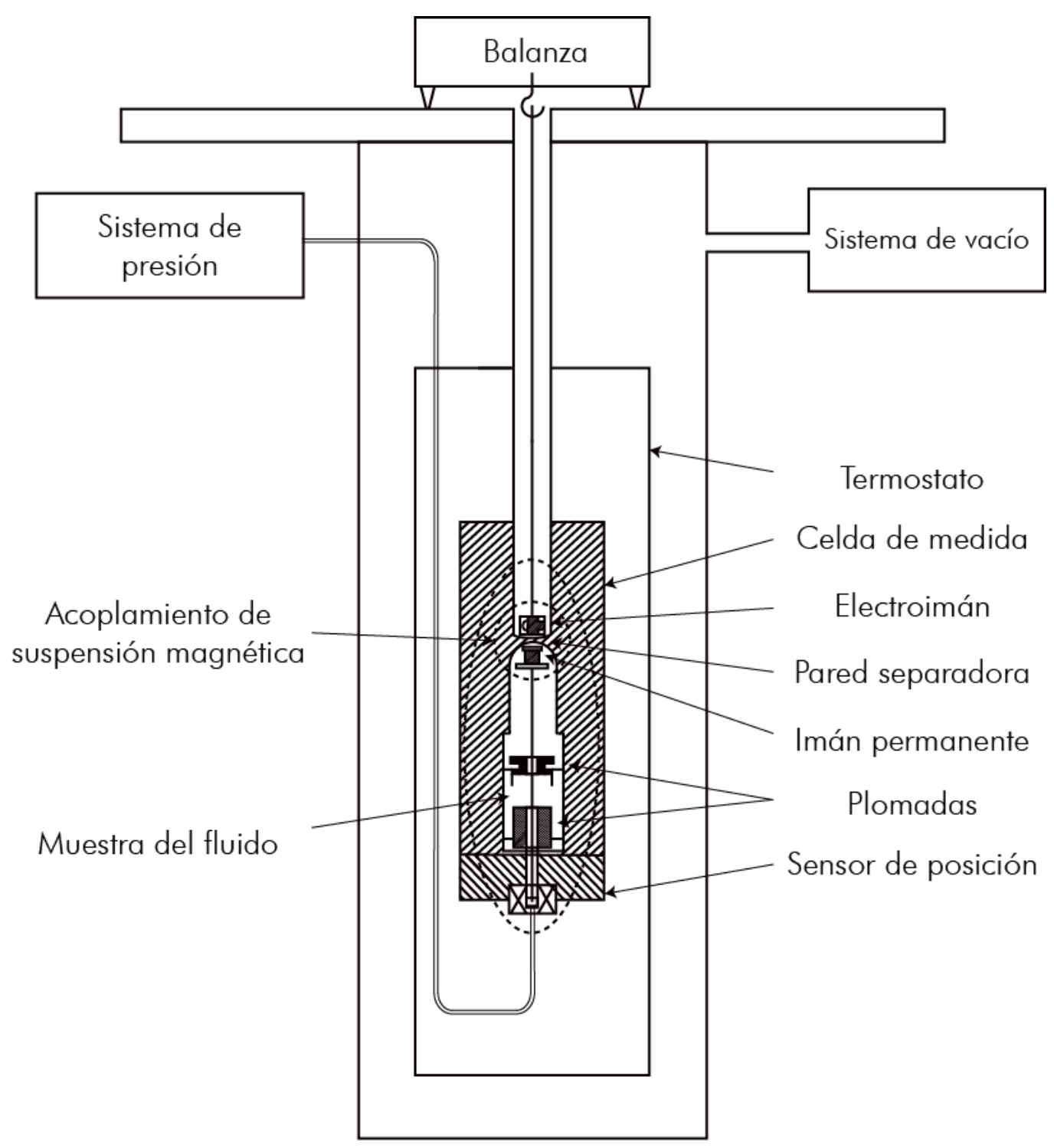

Ilustración 3-1: esquema de un densímetro de doble plomada 
En lugar de utilizar una sola plomada, se utilizan dos de igual masa y área, pero volumen diferente. En el diseño original [42], [43] cuyo esquema aparece en la Ilustración 3-1 una de las plomadas es una esfera de cuarzo cubierta por una fina capa de oro y otra es un disco sólido de oro. Ambas plomadas se sitúan sobre unos soportes conectados ambos a una balanza mediante un fino alambre vía suspensión magnética, de manera que la diferencia de masa aparente entre los plomos $\Delta m^{*}=m_{D}^{*}-m_{S}^{*}$, ambos inmersos en el fluido, puede medirse de manera muy precisa. La densidad del líquido es:

$$
\rho=\frac{\Delta m^{*}-\Delta m_{v}}{V_{S}-V_{D}}
$$

donde $\Delta m_{v}=m_{D}-m_{S}$ es la diferencia residual de las masas de ambos plomos que se determina al vaciar la celda de medida. $S$ se refiere a la esfera y $D$ al disco.

McLinden \& Lösch [44] han diseñado recientemente un densímetro de este tipo con algunas mejoras respecto del diseño original entre las que se incluyen una mayor resolución en la densidad (incertidumbre en la densidad de $\pm 0.001 \%$ ), un mayor rango de presión $(1 \mathrm{KPa}$ a $12 \mathrm{MPa}$ ) y un diseño más compacto que requiere menos cantidad de líquido y reduce los gradientes de temperatura entre las diferentes partes del aparato.

- Densímetros de una plomada. En la mayoría de aplicaciones, la enorme precisión en un amplio rango de densidad característica de los densímetros de doble plomada es menos importante que la complejidad de su diseño. Para estos casos se han diseñado aparatos más simples con sólo una plomada que conservan algunos de los rasgos de los densímetros de doble plomada [45]. Con ellos se han logrado incertidumbres de $\pm 0.002 \%$ tanto para fluidos puros y mezclas, excepto para gases de baja densidad cuya incertidumbre resultó ser menor de $20 \mathrm{Kg} \cdot \mathrm{m}^{-3}$, en rangos de presión 0.2 a $30 \mathrm{MPa}$, de temperatura 233 a $523 \mathrm{~K}$ y de densidad 2 a $2000 \mathrm{Kg} \cdot \mathrm{m}^{-3}$ [46], [47].

b) Densímetros basados en la retrodispersión de ultrasonidos

La presión de ultrasonidos retrodispersada por un alambre depende de la densidad del fluido en el que las ondas se propagan, por lo que se puede medir ésta analizando el espectro de la presión de ultrasonidos retrodispersados. En el diseño original [48] se utilizaron alambres de cobre y nylon, un transductor de presión plano trabajando con frecuencias de $1.1 \mathrm{MHz}$ con un ancho de banda de $600 \mathrm{KHz}$, y un emisor de ultrasonidos excitado por pulsos de $20 \mathrm{~V}$ con duración de 500 ns.

La fuente principal de error se debe a la colocación relativa entre el alambre y el transductor. Otras fuentes de error son la incertidumbre en la medida del diámetro del alambre, en la medida de la longitud de onda, en la resolución del convertidor analógico-digital y en la localización del alambre en relación con el haz de ultrasonidos.

\section{c) Método de la columna equilibrada}

Útil para medir densidades de líquidos volátiles [49], se puede conseguir gran exactitud con pequeñas cantidades de líquido. Se basan en que la presión hidrostática de una columna de un líquido es proporcional a su altura y densidad. El aparato consiste en un tubo con forma de $U$ en el que se emplaza el líquido de densidad desconocida. En uno de los brazos del tubo se aplica presión y se mide la diferencia de altura del menisco de ambos brazos, $\Delta h$, comparándose con la diferencia de altura $\Delta h_{0}$ cuando en el tubo hay 
un líquido de densidad conocida, $\rho_{0}$. La densidad del líquido problema es:

$$
\rho=\rho_{0} \cdot \frac{\Delta h}{\Delta h_{0}}
$$

Las diferencias de tensión superficial entre las dos columnas pueden producir errores significativos así como impurezas en el líquido o el tubo.

\section{d) Picnometría}

Es el método más sencillo para determinar la densidad y consiste en determinar el peso de un líquido que ocupa el volumen conocido de un recipiente llamado picnómetro. La determinación geométrica del volumen del picnómetro es impracticable, por lo que éste se obtiene previamente con un líquido patrón de densidad.

El actual catálogo de picnómetros es amplísimo. Todos se fabrican de un cristal resistente con pequeño coeficiente de expansión térmica como el Lyrex, Vycer, cuarzo fundido, Pyrex (vidrio borosilicatado)... En la llustración 3-2 se presentan algunos diseños cuya precisión, exactitud, ventajas e inconvenientes vienen perfectamente explicados en el libro de Baver \& Levin [29].

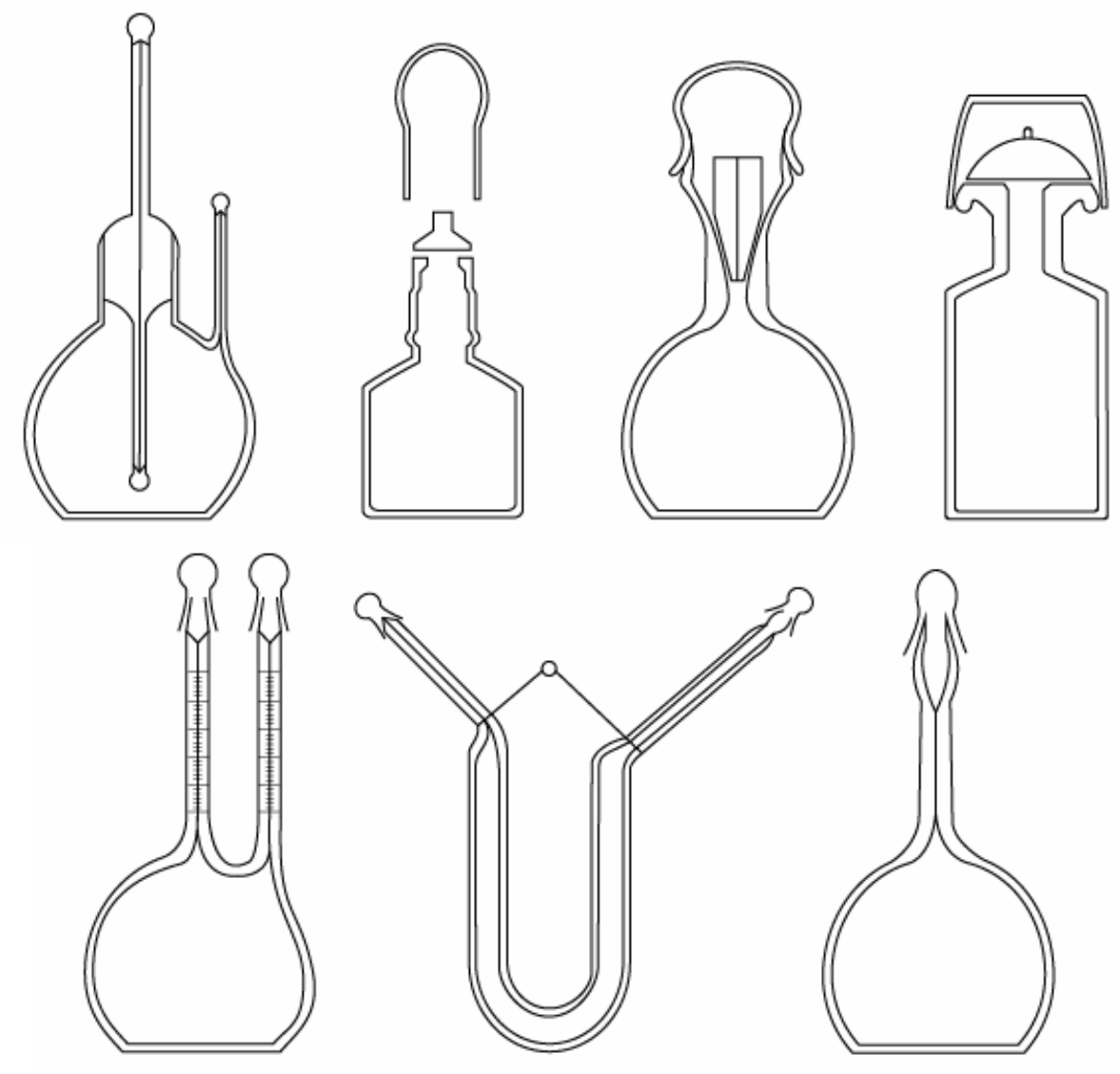

Ilustración 3-2: algunos picnómetros típicos

Independientemente de su diseño, los picnómetros tienen los siguientes rasgos comunes:

- Constan de un tapón de vidrio esmerilado para evitar que los líquidos con mayor presión de vapor se volatilizen.

- Constan de una marca que define el volumen del picnómetro. El nivel del líquido 
en consideración debe colocarse al ras de dicha marca, lo cual supone la mayor fuente de error asociado a la picnometría.

- $\quad$ El peso del picnómetro cambia apreciablemente en función del líquido adsorbido.

- Se debe tener en cuenta el empuje del aire sobre el picnómetro cuando se mide su peso en la balanza.

- El volumen del picnómetro puede cambiar si se somete a cambios de $P$ y $T$.

- El picnómetro debe limpiarse tanto por dentro como por fuera, dejándolo en contacto una noche con mezcla sulfocrómica $\left(\mathrm{K}_{2} \mathrm{Cr}_{2} \mathrm{O}_{7}+\mathrm{H}_{2} \mathrm{SO}_{4}\right.$ concentrado) y después con agua destilada para eliminar el ácido adsorbido.

- $\quad$ Si se mide la densidad de un líquido volátil, en el picnómetro aparecerán dos fases: el líquido y su vapor mezclado con aire. En estos casos debe hacerse una la corrección específica [29].

\section{e) Método de la gota que cae}

Este método es rápido y requiere pequeñas cantidades de líquido [50]. Una gota esférica de líquido cae a través de un recipiente que contiene otro líquido en el cual es inmiscible. Al alcanzar el equilibrio de las fuerzas de empuje, rozamiento y gravedad que actúan en la gota, ella tendrá velocidad constante, dada por la ley de Stokes:

$$
v=k \cdot\left(\rho-\rho_{0}\right)
$$

Midiendo el tiempo que la gota tarda en recorrer una distancia dada, se obtiene un valor preciso de la densidad de la gota, habiendo hecho una calibración previa con líquidos patrón para obtener el coeficiente $k$ y la densidad $\rho_{0}$ del líquido que llena el recipiente.

Este método presenta varios inconvenientes: el rango de densidad que se puede medir es pequeño, ya que la diferencia de densidad relativa entre la gota y el líquido que llena el recipiente debe ser menor de 0.05 ; pueden surgir dificultades al buscar un medio líquido no miscible con la gota; se requiere mejor control de temperatura que para cualquier otro método diferencial, para minimizar los efectos de las corrientes de convección; el método de medición de la velocidad debe ser muy sotisficado.

Este método es muy apropiado para la rápida detección de pequeños cambios de concentración [29]. Por ejemplo, se ha utilizado en centros de salud para detectar cambios en la composición de la sangre de los pacientes.

\section{f) Densímetros de oscilación mecánica}

La variación de la frecuencia de oscilación de un cuerpo con la masa de éste es la base para el diseño de una amplia gama de densímetros de precisión. Los diferentes densímetros de este tipo se clasifican en función de la forma que tenga el elemento o elementos oscilantes: densímetros de tubo en $U$, de cilindro vibrante, de horquilla, de tubo recto, de doble tubo, de Coriolis,... (ver la llustración 3-3). De todos ellos, el más común es el densímetro de tubo en $U$; de hecho, se lleva utilizando más de 45 años con excelentes resultados. Se ha llegado a emplear en condiciones extremas (50 MPa y $723 \mathrm{~K}$ ). El pequeño tamaño del tubo permite que pueda fabricarse con aleaciones ricas en platino, pudiendo trabajar con materiales corrosivos. No obstante, debe tenerse cuidado si aparecen fases sólidas, ya que el tubo puede obstruirse irreversiblemente.

Un densímetro de tubo vibrante consiste en un tubo hueco y forma de U ó V (esta forma 
permite definir un plano de vibración y así evitar el riesgo de vibración elíptica con frecuencia de resonancia ambigua) con finas paredes de cristal o metal, firmemente sujeto a un bloque que está a su vez sujeto a una gran masa que aísla al tubo de cualquier perturbación mecánica externa

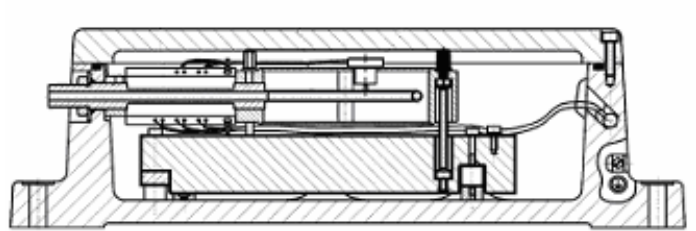

a)

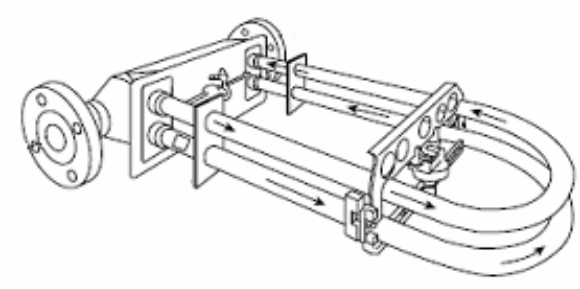

c)

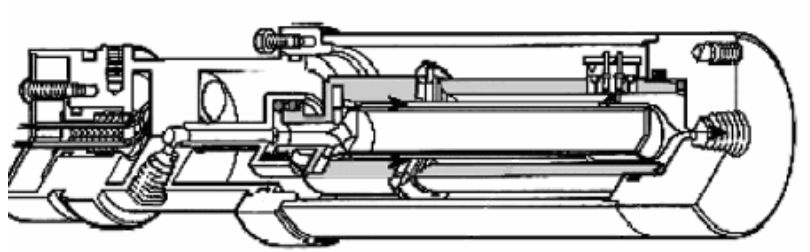

b)

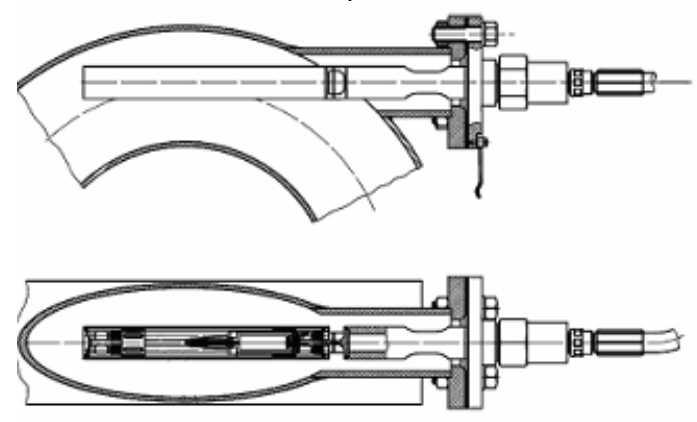

d)

Ilustración 3-3: diferentes tipos de densímetros de oscilación mecánica: a), densímetro de tubo en U; b), densímetro de cilindro vibrante para gases; c), densímetro de Coriolis; d), densímetro de doble tubo

El conjunto completo está inmerso en un baño termoestático para conseguir temperaturas estables. El tubo se llena con el líquido de interés y se excita electrónicamente haciendo que vibre perpendicular a su plano gracias a un campo electromagnético, llevándose el sistema a la resonancia. Aparece pues un concepto fundamental en el que se basan este tipo de densímetros y que no es otro sino la resonancia. Es conocido por todos que un sistema mecánico cuyo comportamiento viene dado por una ecuación diferencial de tipo oscilador armónico amplifica sus oscilaciones cuando es excitado por una fuerza externa periódica cuya frecuencia coincide con la frecuencia natural del sistema (frecuencia característica del oscilador armónico simple equivalente). En esta situación, los fenómenos disipativos pierden importancia y el sistema se comporta de forma estacionaria.
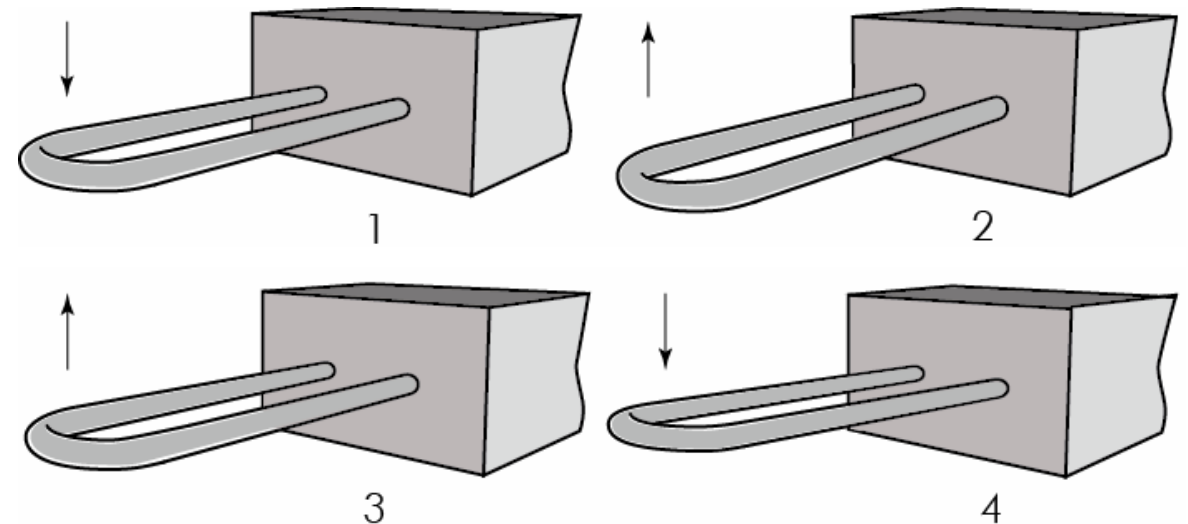

Ilustración 3-4: esquema en el que se puede visualizar la manera en la que vibra el tubo en forma de $U$ de un densímetro de oscilación mecánica 
La manera en cómo vibra el tubo se entiende mejor si se observa la llustración 3-4. En 1, el tubo esta en posición horizontal, y por acción del campo magnético se desplaza hacia abajo hasta alcanzar la posición de máxima amplitud, 2. Entonces el tubo sube hasta alcanzar de nuevo la posición horizontal 3, la sobrepasa y llega a la de máxima amplitud 4. Tras ello el tubo comienza a bajar hasta llegar a la posición 1 y el ciclo se repite hasta que el campo magnético cesa. La frecuencia de oscilación del tubo depende de su masa total y, por tanto de la densidad del líquido contenido en el tubo.

Las características: 1) plano de oscilación perfectamente definido; 2) resonancia son fundamentales para el diseño de este tipo de aparatos ya que nos aseguran la reproducibilidad de la situación física que constituye el principio de medida.

En los densímetros de tubo vibrante hay dos elementos magnéticos: una bobina de excitación, que hace vibrar al tubo, al tener éste solidario un imán permanente; otra bobina, sobre la que se induce una corriente debida al flujo de campo electromagnético variable debido a que otro imán solidario al tubo se mueve. Esta corriente inducida pasa por un amplificador, y se utiliza para registrar la frecuencia de oscilación del tubo, en términos de un contador digital conectado a un cristal de cuarzo. Los elementos magnéticos pueden montarse en dos configuraciones:

- Un imán permanente crea un campo que actúa sobre dos barras fijas al tubo, conectadas a los circuitos de excitación y de adquisición de datos ([51], Ilustración 3-5).
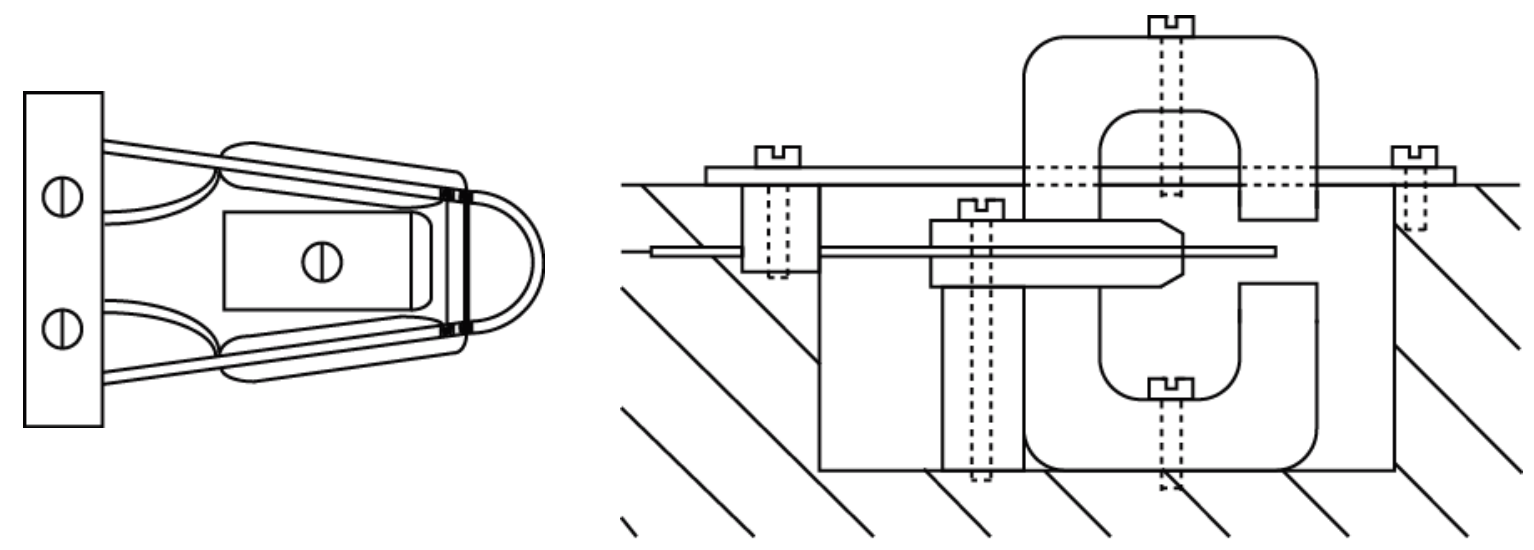

llustración 3-5: planta y perfil de un densímetro de tubo en U con imán permanente en posición estática

- Los imanes permanentes están sujetos al tubo y se mueven en los ejes de las bobinas de excitación e inducida respectivamente ([52], Ilustración 3-6).

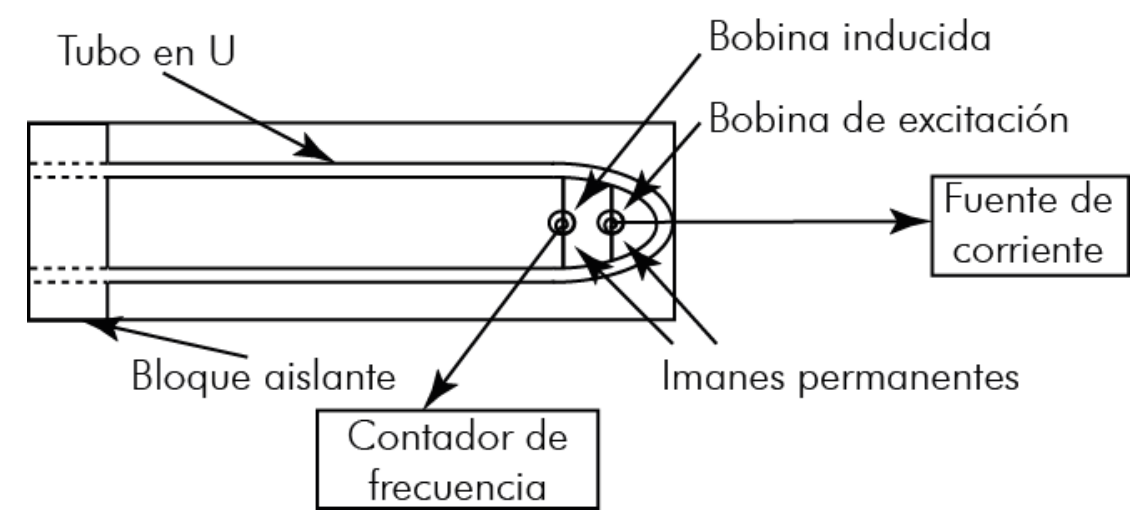

Ilustración 3-6: esquema de un densímetro de tubo en $U$ con los elementos magnéticos solidarios al tubo 
El incremento de la masa del cuerpo vibrante debida a los imanes permanentes que van sujetos al tubo reduce la sensibilidad del aparato.

Esta segunda configuración es la que se corresponde con la del densímetro que se ha utilizado para obtener los volúmenes de exceso que aparecen en este trabajo. Por ello vamos a exponer su funcionamiento de manera detallada.

En el primer diseño de Kratky et al [52], [53], en vez de un tubo con forma de U ó V lleno del líquido en cuestión, se tiene un elemento oscilador con forma de $V$ que sujeta en su extremo una esfera hueca de cristal llena del líquido del cual se desea conocer su densidad, como se observa llustración 3-7.

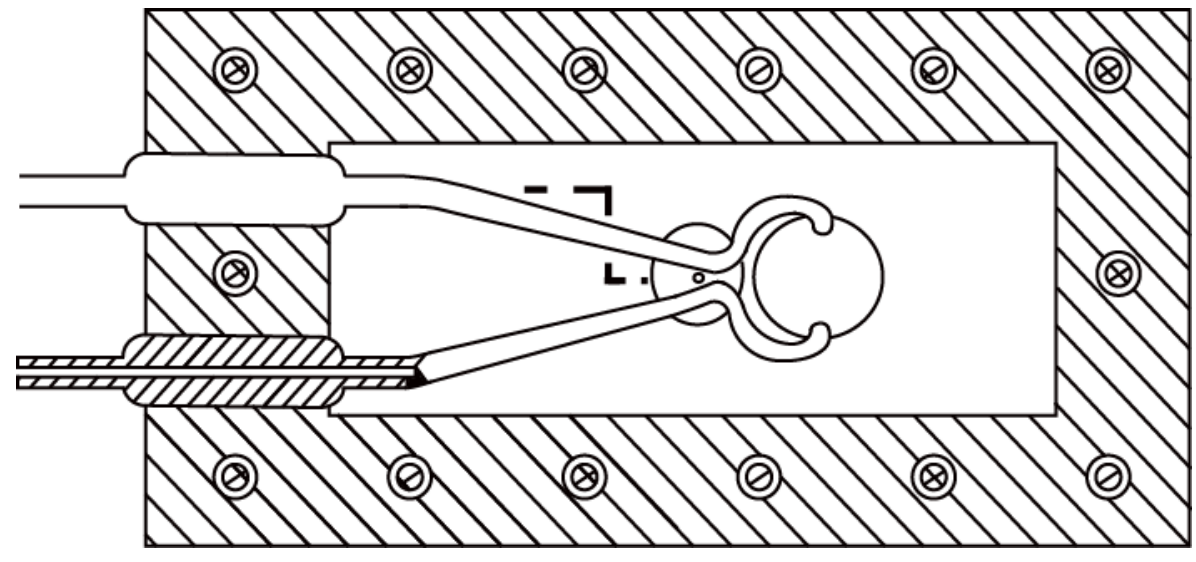

Ilustración 3-7: primer diseño de densímetro de tubo en $\vee$ de Kratky et al

La frecuencia de resonancia de un oscilador de este tipo es [53]:

$$
\omega^{2}=\frac{\int_{0}^{l} E \cdot I \cdot \ddot{a} \cdot\left(\frac{\mathrm{d}^{2} y}{\mathrm{~d} x^{2}}\right)^{2} \cdot \mathrm{d} x}{\int_{0}^{l} F_{1} \cdot \rho_{1} \cdot y^{2} \cdot \mathrm{d} x+\int_{0}^{l} F_{2} \cdot y^{2} \cdot \mathrm{d} x+M \cdot y^{2}+\rho \cdot V \cdot y^{2}}
$$

donde, $\omega$ es la frecuencia angular de resonancia; $x$ es la posición a lo largo del eje del vibrador; $E$ es el módulo de elasticidad en la posición $x ; V$ es el volumen de la esfera de cristal; $I \cdot \ddot{a}$ es el momento de inercia en la posición $x ; y$ es la altura del oscilador en la posición $x ; F_{1}$ es la sección transversal del elemento de cristal en la posición $x ; F_{2}$ es la sección transversal del líquido en la posición $x ; \rho_{1}$ es la densidad del cristal; $\rho$ es la densidad del líquido; $M$ es la masa de la esfera vacía; $l$ es la longitud del oscilador en la dirección $x$.

Aunque se desconoce $y=y(x)$, se puede suponer que las integrales de la ecuación (3.18) son constantes. Entonces, dicha ecuación se puede simplificar para llegar a esta otra:

$$
\omega^{2}=\frac{C}{D+\rho \cdot F}=\frac{1}{G+\rho \cdot H}
$$

o bien, como $\omega=2 \cdot \pi / \tau$,

$$
\rho=A+B \cdot \tau^{2}
$$

Como pudo demostrar Stabinger [54], puede aplicarse el modelo de oscilador armónico amortiguado y forzado, obteniendo las mismas ecuaciones que las dadas por 
el método anterior. Lo interesante de este modelo es que puede aplicarse a elementos oscilantes como el tubo de la llustración 3-6 que representa un densímetro de tubo en U moderno.

El calibrado del aparato consiste en la determinación de los valores de los coeficientes $A$ y $B$, lo cual no se hace a partir de la geometría del tubo, su módulo de Young, etc., sino del ajuste a una línea recta por mínimos cuadrados de la densidad de algunos líquidos patrón y el correspondiente valor del cuadrado del periodo de oscilación del tubo cuando éste se llena con cada uno de dichos líquidos.

El valor de los coeficientes $A$ y $B$ no es constante sino que fluctúa debido principalmente a tres factores:

- Variaciones de temperatura, pues las propiedades mecánicas de los materiales con los que el densímetro está construido son funciones de ella.

- Variaciones en la presión de trabajo, puesto que ésta puede variar el volumen interno del tubo.

- Fatiga de los materiales por el uso continuado del aparato.

En este tipo de densímetros, el control de la temperatura de la muestra es muy importante para obtener medidas precisas, por lo que se suelen dotar de un sistema de control que consiste en asignar dos temperaturas como puntos fijos, uno superior y otro inferior, para cada temperatura de trabajo de tal manera que ésta se encuentre en el intervalo definido por ambos. Se montan células Peltier que trabajan de manera que si la temperatura del fluido supera el punto superior, se enfría hasta que la temperatura esté entre los puntos superior e inferior. Si la temperatura del fluido está por debajo del punto inferior, se calentará hasta que su temperatura permanezca en dicho intervalo. Por tanto, la temperatura no permanece constante sino que fluctúa entre los dos valores inferior y superior, siendo mayor la precisión en el control de temperatura cuanto más pequeño sea el intervalo comprendido entre los dos puntos fijos.

La determinación de la densidad con este tipo de aparatos solamente es apropiada si todo el fluido sigue el movimiento de la superficie del elemento vibrante de la misma manera, lo cual no sucede en tres casos específicos:

- Si la velocidad de la superficie del elemento vibrante es comparable con la velocidad de propagación del sonido en el fluido, el fluido ejercerá diferentes fuerzas de inercia provocando la aparición de diferentes frecuencias en el oscilador causando error en la medida.

- $\quad$ Si el movimiento de la superficie del elemento vibrante crea un flujo en el interior del fluido que cambia la viscosidad del mismo, pueden aparecer fuerzas de inercia que originan frecuencias de oscilación ambiguas, causando error en la medida. El efecto es mayor cuanto mayor sea la variación de la viscosidad.

- Medidas en fluidos altamente heterogéneos: si los diferentes constituyentes de una mezcla heterogénea difieren considerablemente en su comportamiento de flujo y en su densidad, se moverán unos respecto a otros cuando el tubo vibra. Esto, igual que en los otros dos casos, origina fuerzas de inercia en el tubo causando error en la medida.

Se debe tener especial cuidado cuando se forman fases sólidas en el tubo ya que la masa efectiva del mismo aumentará, disminuyendo el volumen ocupado por el fluido y causando desviaciones en el comportamiento del tubo, lo que requiere una limpieza a 
fondo del tubo Si se trabaja con fluido abrasivos la masa del tubo y su constante de elasticidad cambian, siendo necesaria una nueva calibración o bien reemplazar los elementos del densímetro que se hayan dañado.

\section{g) Densímetros pseudo-isócoros}

Éste método es muy fácil de automatizar y permite un manejo seguro de fluidos peligrosos puesto que estos permanecen estáticos en un recipiente todo el tiempo [55][59]. Con este método se busca medir la densidad de un líquido a diferentes presiones y temperaturas y con una pequeña cantidad del mismo, ya que se utiliza una sola muestra para cubrir el rango deseado de $P$ y $T$.

Una celda estanca se sumerge en un baño termoestático y se llena con el líquido cuya densidad se quiere determinar. El líquido puede comprimirse gracias a un compresor. Cuando la temperatura del baño es estable, se miden ésta y la presión en la celda. Después, se aumenta la temperatura y se espera a obtener el nuevo equilibrio, midiendo otra vez la presión y la temperatura. Se repite el proceso hasta que se haya cubierto el rango de $P$ y $T$ deseado. La densidad del fluido se determina a partir del volumen del interior de la celda y de la cantidad de fluido en su interior. El volumen cambia según sea su coeficiente de expansión térmica y de deformación mecánica bajo presión debido a las variaciones de $P$ y $T$ por lo que la densidad será diferente en cada punto de equilibrio $(P, T)$.

\section{h) Volumetría de fuelle}

Un volúmetro de fuelle es un tipo particular de piezómetro en el que el fluido a medir está contenido dentro de una celda, hermética para evitar que la muestra se contamine. La celda, o una parte de ella, es un fuelle flexible capaz de transmitir la presión aplicada al fluido con pérdidas de presión insignificantes. Se mide el movimiento lineal del fuelle para determinar la compresión del fluido causado por la presión aplicada. Conocido el alargamiento del fuelle a una presión de referencia y con un líquido patrón de densidad dados, se puede obtener la densidad de un líquido problema a partir del alargamiento producido en el fuelle a la presión deseada [30].

La exactitud de este método depende del diseño de la celda y del método empleado para determinar el movimiento del fuelle.

Existe gran cantidad de diseños, los cuales pueden clasificarse según el método operativo y método de construcción. Además, se han cubierto amplios rangos de presión (hasta $1200 \mathrm{MPa}$ ) y temperatura (de $80 \mathrm{~K}$ hasta $450 \mathrm{~K}$ ) [60]-[62].

\section{i) Métodos interferométricos}

En el sistema internacional de unidades (SI), la unidad de densidad $\mathrm{kg} \cdot \mathrm{m}^{3}$ se deriva de las dos unidades básicas de masa $(\mathrm{kg})$ y de longitud (m). Así, cuando se trata de determinar el valor absoluto de la densidad, su medida debe obtenerse a partir del estándar de masa definido por el prototipo internacional de kilogramo y del estándar de longitud referido como 1650763.73 veces la longitud de onda en el vacío de la radiación naranja del átomo del ${ }^{86} \mathrm{Kr}$. En la práctica, la medida absoluta de la densidad consiste en la determinación de la masa y el volumen de un objeto sólido, cuyo volumen se puede relacionar con la longitud patrón con buena precisión. En cuanto a la forma del objeto, normalmente se escoge un cubo porque su volumen puede obtenerse, en principio, a partir de la medida de las distancias entra las caras opuestas 
del cubo, mediante interferometría óptica. Este fue el método empleado por el National Physical Laboratory (NPL, Teddington, UK) en 1957 para la determinación de la densidad del mercurio, donde se utilizó un cubo de carburo de tungsteno y un interferómetro de Michelson con una fuente de luz cuya longitud de onda se conocía con gran exactitud [26]. La densidad del mercurio se determinó por pesado hidrostático del cubo de carburo de tungsteno inmerso en el mercurio, midiendo la fuerza de empuje del mercurio sobre el cubo con una balanza analítica.

Las esquinas del cubo son susceptibles a ser dañadas, y por eso no se recomienda el empleo de esta forma. En su lugar, se utilizan esferas, cuyo volumen se puede determinar a partir del radio, que se toma como el valor promedio del medido en todas las orientaciones de la esfera [63].

Para una mayor profundización en la determinación de la densidad absoluta, técnicas interferométricas empleadas para la medida de la longitud, etc., consúltese la referencia [30].

\subsubsection{Métodos directos}

Los aparatos que permiten medir directamente $V_{\mathrm{m}}^{\mathrm{E}}$ se llaman dilatómetros y básicamente se clasifican en dos tipos de diseños:

\subsubsection{Dilatómetros de tipo batch}

Consisten en un recipiente de vidrio con forma de $\mathrm{U}$ o $\mathrm{V}$ más o menos modificada. La parte del fondo que une las dos ramas se encuentra inicialmente llena de mercurio (u otro líquido adecuado), cuya misión es separar físicamente los dos componentes que van a ser mezclados, los cuales se introducen en la parte superior de cada una de las ramas del dilatómetro. Una vez alcanzado el equilibrio térmico, la mezcla se produce cuando se mece o se hace rotar el dilatómetro permitiendo que los dos líquidos puros se pongan en contacto directo. El aparato consta de uno o varios capilares de precisión de manera que el cambio de volumen que acompaña a la mezcla se mide mediante la lectura del nivel de los meniscos de mercurio en los capilares, lo que se lleva a cabo con la ayuda de catetómetros de precisión. En todos los diseños existentes se ha tenido el cuidado de que sea siempre el mercurio el que esté en contacto directo con el ambiente, a fin de evitar la evaporación selectiva de los componentes que forman la mezcla. Para confirmar que ésta se ha producido de forma completa, se mece o se rota el aparato hasta obtener un nivel de mercurio constante en los capilares.

En la llustración 3-8 se representan los aparatos diseñados por Keyes \& Hildebrand [64], con el que se obtuvo una precisión igual a $\pm 0.003 \mathrm{~cm}^{3} \cdot \mathrm{mol}^{-1}$ y por Duncan, Sheridan y Swinton [65] el cual necesita solamente entre 0.2 y $0.8 \mathrm{~cm}^{3}$ de cada líquido (desgasificado) para realizar la mezcla y con el que consiguieron una precisión de $\pm 0.002 \mathrm{~cm}^{3} \cdot \mathrm{mol}^{-1}$ ó $\pm 0.5 \%$ en $V_{\mathrm{m}}^{\mathrm{E}}$. En éste último diseño, los capilares se pueden cambiar para acomodar el instrumento a los diferentes valores del volumen de exceso.

Muchos otros diseños se exponen en los trabajos de Battino [66] y de Handa \& Benson [4]. Por ejemplo, se citan los aparatos de Bellemans [67], Brown \& Smith [68], Zamyatnin [69], etc.

Stookey et al [70] realizaron un detallado análisis de los errores en la medida que aparecen en estos dilatómetros, teniendo en cuenta el efecto de la pureza de los 
componentes químicos, el error en la pesada de los mismos, variaciones con la temperatura, lectura del catetómetro y compresibilidad de los líquidos. Las fugas por evaporación de los componentes volátiles durante su inyección en cada rama parecen ser la principal fuente de error de este tipo de aparatos, aunque existen diseños que minimizan este efecto.
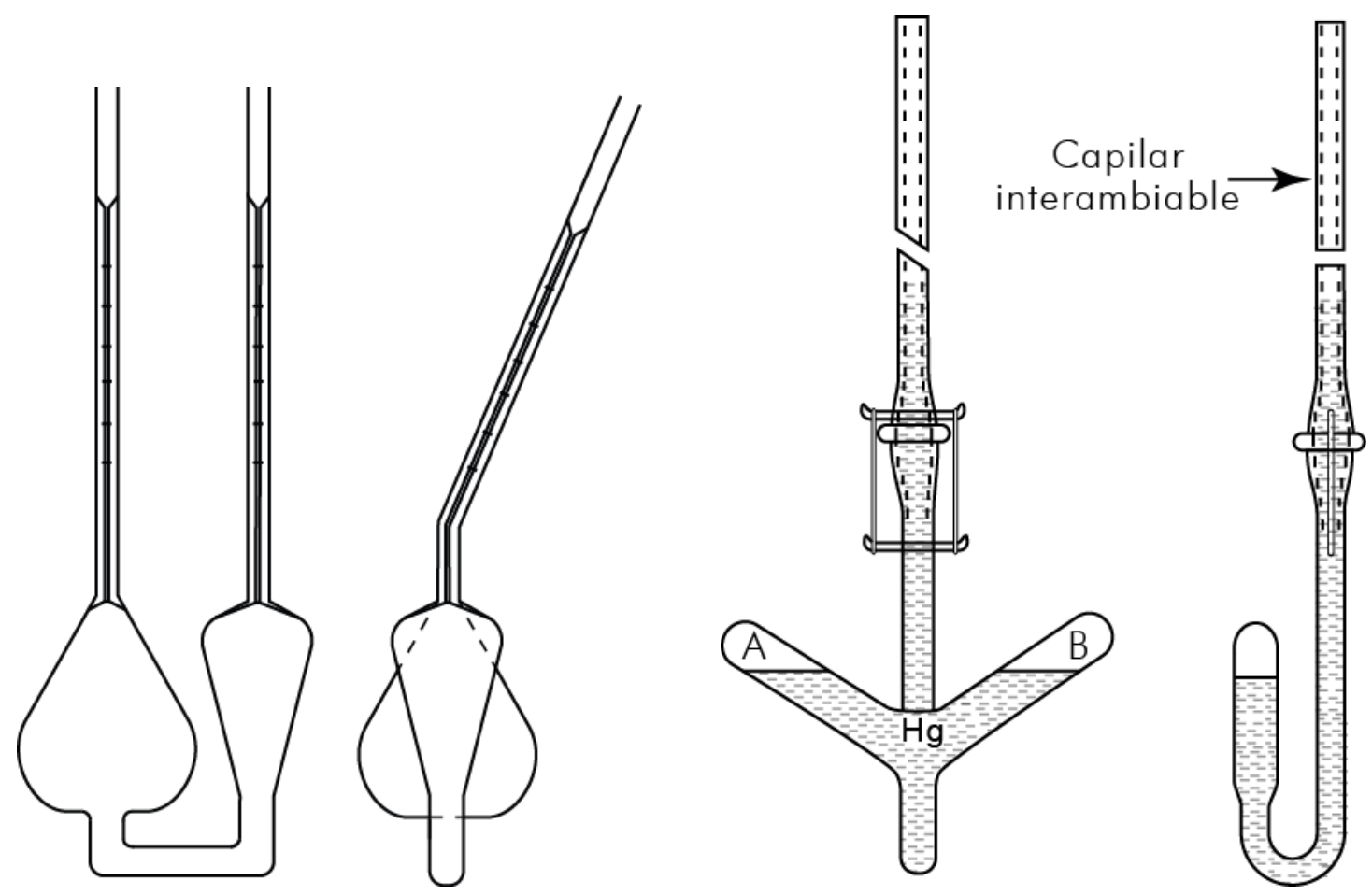

Ilustración 3-8: dilatómetros de Keyes \& Hildebrand (izquierda) y de Duncan et al (derecha)

\subsubsection{Dilatómetros de dilución}

Basados en la idea inicial de los dilatómetros de tipo batch de separar los componentes puros antes de efectuar la mezcla por medio de un líquido inmiscible con ambos. Estos aparatos requieren al menos dos cargas para hacer las medidas en todo el rango de fracciones molares. Sobre un líquido puro dado, se va añadiendo el otro de manera continua (dilatómetros de dilución sucesiva) o incremental (dilatómetros de dilución continua).

El principio de operación del aparato se entiende mejor si nos fijamos en la llustración 3-9. El aparato se encuentra en un baño termostático. La cámara de mezcla $C$ está inicialmente cargada con el componente puro A y el mercurio. Un grifo lubricado abre el paso a la cámara de reserva $R$ donde el componente $B$ está confinado encima del mercurio. Se hace lectura del nivel de mercurio en el capilar calibrado $D$ con respecto la marca F. Cuando se abre S, el mercurio de $C$ fuerza a parte del componente B a pasar a través de E y llegar a la cámara $C$. $S$ se cierra, la mezcla comienza, y se produce un cambio en el nivel del mercurio en el capilar D. Sucesivos incrementos de B se van aplicando de un modo similar para determinar directamente el volumen de exceso como función de la composición a una temperatura.

Bottomley \& Scott [71] y Tanaka et al. [72] estudiaron el efecto de la grasa del grifo. Cuando éste se abre, el mercurio arrastra parte de la grasa de manera que el volumen del sistema se ve afectado. Ambos estudios demostraron que no sólo el efecto 
contaminante de la grasa de la llave sobre el mercurio que pasa a su través cambia el volumen, sino que la propia operación de girar el grifo puede aumentar o disminuir el volumen del contenido de la celda de mezcla y estimaron que cada vez que se gira el grifo el volumen cambia $0.00005 \mathrm{~cm}^{3}$, lo que puede producir un error importante en $V_{\mathrm{m}}^{\mathrm{E}}$.
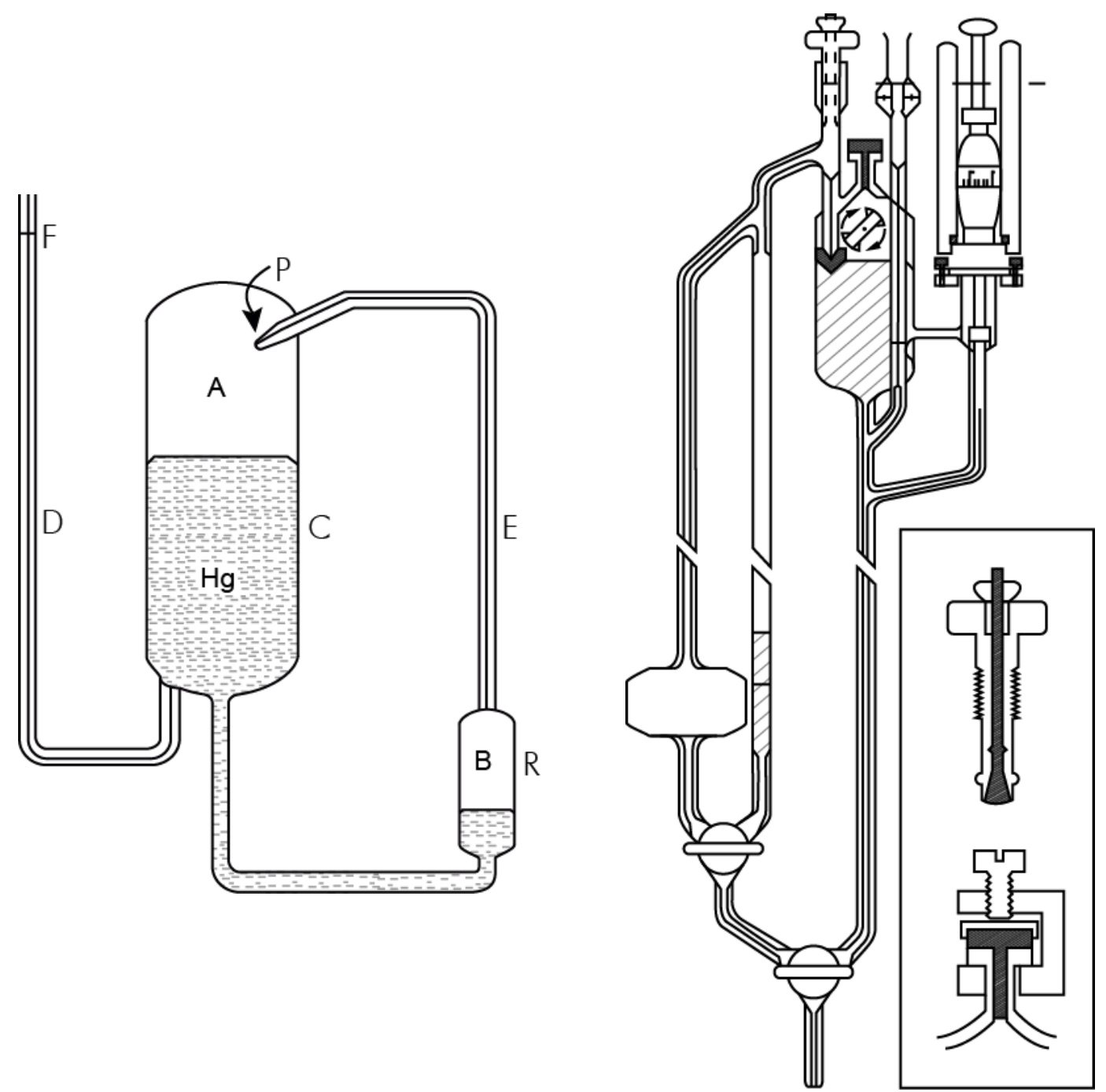

Ilustración 3-9: esquema general de un dilatómetro de dilución contínua (izquierda); dilatómetro de Tanaka et al (derecha)

Los dilatómetros de dilución han ido sufriendo numerosas modificaciones: variación de la cantidad de A que está inicialmente presente y las cantidades incrementales de B que se van añadiendo; incorporación de capilares $D$ intercambiables (diferentes diámetros internos para cubrir un mayor rango de volúmenes de exceso); separación de los dos componentes en el punto $P$, etc. Los dilatómetros más primitivos necesitaban celdas de mezcla grandes (entre 250 y $600 \mathrm{~cm}^{3}$ ) para obtener buena precisión al detectar los cambios de volumen [73]. A partir de los años 1960, el desarrollo de los dilatómetros se enfocó al diseño de celdas de mezcla más pequeñas, con menor número de grifos (o ninguno), y a facilitar el uso del aparato, mientras que al mismo tiempo se trataba de mejorar la precisión de las medidas de $V_{\mathrm{m}}^{\mathrm{E}}$. Entre estos diseños están los de Stokes et al. [74], Bottomley \& Scott [71], Kumaran \& McGlashan [75], Chareyron \& Clechet [76]. 
Otra desventaja de este tipo de dilatómetros es que no miden cambios de volumen a presión constante ya que los cambios de altura del menisco de mercurio en el capilar producen en la celda de mezcla un cambio de presión, que debido a la compresibilidad de los líquidos y la elasticidad del cristal, puede cambiar el volumen aparente del contenido en la celda en un $1.5 \%$. Aunque se aplica una corrección a este efecto, lo mejor es eliminar esta fuente de error, lo cual consiguieron Tanaka et al [72] al modificar el aparato de Chareyron \& Clechet (Ilustración 3-9).

Al igual que ocurre en los dilatómetros batch, las fugas por evaporación de los componentes volátiles durante su inyección también son una fuente de error en los dilatómetros de dilución.

En conclusión, los resultados que se obtienen con los dilatómetros de dilución se ajustan muy bien y proporcionan desviaciones estándar muy pequeñas, pero tienen el inconveniente de que cada operación de dilución está acompañada por una acumulación de errores, debidos principalmente al efecto contaminante de la grasa y del giro de la llave de paso. No obstante, son muy rápidos y los únicos dilatómetros capaces de estudiar las regiones de concentración diluida; además, si se pueden evitar los grifos y hacerlos trabajar a presión constante, son el medio más directo y rápido de obtener el volumen de exceso.

\subsection{MEDIDA DE LA COMPRESIBILIDAD ISOENTRÓPICA DE EXCESO}

La compresibilidad isoentrópica de exceso se determina a partir de la compresibilidad isoentrópica de la mezcla y la de la mezcla ideal, de acuerdo con la ecuación (2.187) procedente del modelo de Benson \& Kiyohara de mezcla ideal:

$$
\kappa_{S}^{\mathrm{E}}=\kappa_{S}-\left(\kappa_{T}^{\mathrm{id}}-\frac{T \cdot V_{\mathrm{m}}^{\mathrm{id}} \cdot\left(\alpha_{P}^{\mathrm{id}}\right)^{2}}{C_{P}^{\mathrm{id}}}\right)
$$

Donde $\kappa_{T}^{\text {id }}, V_{\mathrm{m}}^{\text {id }}, \alpha_{P}^{\text {id }}$ y $C_{P}^{\text {id }}$ se calculan a partir de las propiedades de los componentes puros de acuerdo con las reglas de mezcla ideal (2.161) y (2.164)-(2.166). Por lo tanto, para determinar la compresibilidad isoentrópica de exceso, es esencial el conocimiento de $V_{\mathrm{i}}^{0}, \alpha_{P, \mathrm{i}}^{0}, C_{P, \mathrm{i}}^{0}$ y $\kappa_{T, \mathrm{i}}^{0}$ y además de un método que permita obtener $\kappa_{S}$ de la mezcla.

Como ocurre en el caso de la medida de los volúmenes de exceso, la compresibilidad isoentrópica de exceso puede medirse directa o indirectamente:

\subsubsection{Medida indirecta de $\kappa_{S}$}

Una onda sonora que se propaga en un fluido sufre un proceso adiabático bajo ciertas condiciones mediante el cual se puede obtener importante información termodinámica del fluido. Existen tres métodos para obtener dicha información: utilizar los datos de la velocidad del sonido para ajustar los parámetros de una expansión de tipo virial o de una ecuación empírica de estado para la presión asumiendo una forma funcional [78][82]; si hay varias aproximaciones de la variación de alguna propiedad termodinámica y se procede a la integración de los datos de la velocidad del sonido empleando relaciones termodinámicas [83]-[86]; utilizar métodos numéricos en los que todas las propiedades termodinámicas se derivan de la velocidad del sonido sin aproximaciones 
ni suposiciones sobre su forma funcional [86]-[90]. Por ejemplo, la compresibilidad isoentrópica de un fluido se puede determinar a partir de su densidad $\rho$ y de la velocidad de propagación del sonido en su interior $u$ mediante la ecuación de NewtonLaplace:

$$
\kappa_{S}=\frac{1}{\rho \cdot u^{2}}
$$

El desarrollo de aparatos que miden la velocidad de propagación del sonido en medios fluidos es bastante reciente, si bien ha alcanzado un grado tal de precisión que los datos obtenidos son interesantes no sólo en los estudios fundamentales de químicafísica, biofísica y bioquímica [91]-[93], sino también en ingeniería química, preparación de emulsiones y coloides, industria farmacéutica, procesado de comidas y bebidas, etc [94]-[96].

Los métodos experimentales de la medida de la velocidad del sonido en líquidos y la teoría de operación de esas técnicas han sido revisadas por McSkimm [97], Papadakis [98], Heydeman [99], Trusler [100], Kaatze et al [101] y Leroy [102]. A continuación se presentan los tres métodos principales.

\subsubsection{Método interferométrico}

Consiste esencialmente en provocar una onda estacionaria dentro de una columna de fluido. Un reflector se coloca de forma paralela al transmisor a una distancia dada del mismo. Al variar la distancia se percibe en el transductor las variaciones de presión que distan entre sí media longitud de onda. Es un método análogo al tubo de Kundt [103].

\subsubsection{El método óptico}

Se basa en que una onda sonora que se transmite en un medio produce alternativamente regiones de mayor y menor presión. Este fenómeno puede aprovecharse para utilizarlo como una red de difracción. A través de los distintos ángulos en los cuales aparecen los distintos órdenes de difracción se puede hallar la longitud de la onda.

\subsubsection{El método del pulso}

Prestaremos más atención a este método por ser el que se ha empleado para la determinación de $\kappa_{S}$ de todos los sistemas estudiados en este trabajo.

Los aparatos que se basan en este método constan de un transductor consistente en un platillo de cristal de cuarzo que actúa tanto de receptor como de emisor, localizado dentro de una cavidad (en nuestro caso de unos $5 \mathrm{~mm}$ y de acero inoxidable) que se llena del fluido de interés. El cristal de cuarzo genera cientos de pulsos por segundo que viajan en todas las direcciones hasta llegar a unos reflectores idénticos, paralelos al cristal de cuarzo, y que tienen sección cónica para dispersar los pulsos de sonido que puedan atravesar el reflector [104]. Los pulsos reflejados o ecos vuelven al transductor. Para entender el funcionamiento del aparato supongamos que en el cristal de cuarzo se genera solamente un pulso y que cuando éste es emitido, llega hasta el reflector más lejano, que se encuentra a distancia $L_{1}$, en el cual se refleja y vuelve al transductor. Un segundo pulso viaja hasta el otro reflector más cercano, a distancia $L_{2}$, de manera que el eco del primer pulso y el del segundo llegan al transductor en el mismo instante. La diferencia de fase entre ambos ecos es: 


$$
\Delta \phi=2 \cdot \pi \cdot v \cdot \Delta t
$$

donde $\Delta t$ es el intervalo de tiempo que transcurre entre la emisión del primer pulso y del segundo:

$$
\Delta t=\frac{2 \cdot\left(L_{1}-L_{2}\right)}{u}=\frac{2 \cdot \Delta L}{u}
$$

$\Delta L$ se obtiene mediante la calibración del aparato con líquidos patrón de la velocidad del sonido. La frecuencia de los pulsos se varía hasta que ambos ecos se cancelan (interferencia destructiva), lo que sucede si la diferencia de fase es:

$$
\Delta \phi=2 \cdot \pi \cdot(\mathrm{n}+1)
$$

siendo $\mathrm{n}$ un entero. La cancelación completa ocurre cuando la amplitud del segundo eco es igual a la del primero en el momento en el que vuelven al transductor. Las frecuencias en las que esto ocurre vienen dadas por:

$$
\mathrm{v} \cdot \Delta t=\mathrm{n}+0.5
$$

n se determina a partir de la diferencia de frecuencia entre dos cancelaciones sucesivas. Finalmente, la velocidad del sonido está dada por (3.23).

En las ecuaciones anteriores $u$ es velocidad de grupo [105] mientras que en la ecuación (3.21) es velocidad de fase, que coinciden si no hay absorción; por ello es necesario hacer un barrido en frecuencias y determinar si se produce ésta. No obstante, no es una hipótesis disparatada suponer que no hay absorción, lo que ahorra mucho trabajo.

Existen limitaciones en la aplicabilidad de este método: el pulso no debe contener longitudes de onda muy diferentes para que en la transformada de Fourier no aparezcan muchos términos; la longitud de la cavidad debe ser suficientemente grande como para evitar que se formen ondas estacionarias en su interior y lo suficientemente pequeña como para evitar que el pulso se atenúe antes de llegar al receptor.

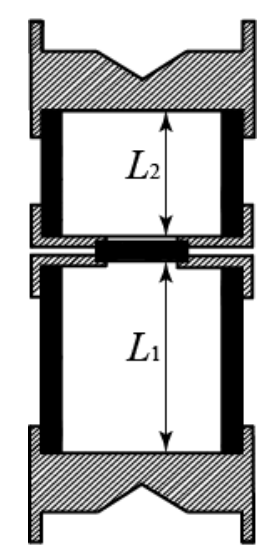

Ilustración 3-10: esquema de una celda para la medición de la velocidad del sonido en un fluido según el método del pulso

\subsubsection{Medida directa de $\kappa_{S}$}

La determinación directa de la compresibilidad isoentrópica se suele llevar a cabo en condiciones de alta presión, ya que sus valores son pequeños, del orden de $10^{-4}$ a $10^{-6}$ $\mathrm{Pa}^{-1}$. El montaje puede verse en la llustración 3-11. El líquido se encierra en un vaso de cristal equipado con un tubo con forma de $U$ y que consta de dos contactos eléctricos 
(electrodos) [106]. Una columna de mercurio actúa como pistón y de su posición depende que haya o no contacto eléctrico entre los dos electrodos. Todo el aparato está encerrado en una bomba de presión, llena de un líquido apropiado y conectado a una bomba hidráulica. Cuando se aplica presión sobre el mercurio, su nivel cambia y se rompe el contacto. El experimento se repite tras la adición de una cantidad conocida de mercurio. La presión necesaria para romper el contacto se determina nuevamente y la compresibilidad se calcula como el cociente de la presión adicional entre el volumen de mercurio añadido. Se deben aplicar correcciones por la compresibilidad del mercurio (que se puede obtener con el mismo método).

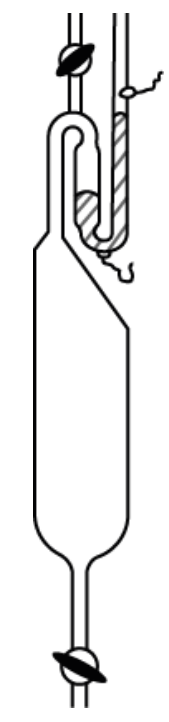

Ilustración 3-11: esquema del aparato para la medida directa de $\kappa_{S}$

\subsection{RESULTADOS EXPERIMENTALES}

Para las mezclas estudiadas en este trabajo, los valores de densidad y velocidad del sonido a partir de los cuales se calcularon los volúmenes de exceso, las diferencias de compresibilidad isoentrópica y otras magnitudes derivadas como el coeficiente de expansión térmica a presión constante de exceso, se obtuvieron mediante un densímetro-analizador del sonido Anton Paar DSA 5000, el cual cuenta con dos celdas conectadas en serie que se llenan con la misma muestra de líquido (Ilustración 3-12).

\section{Celda de densidad}
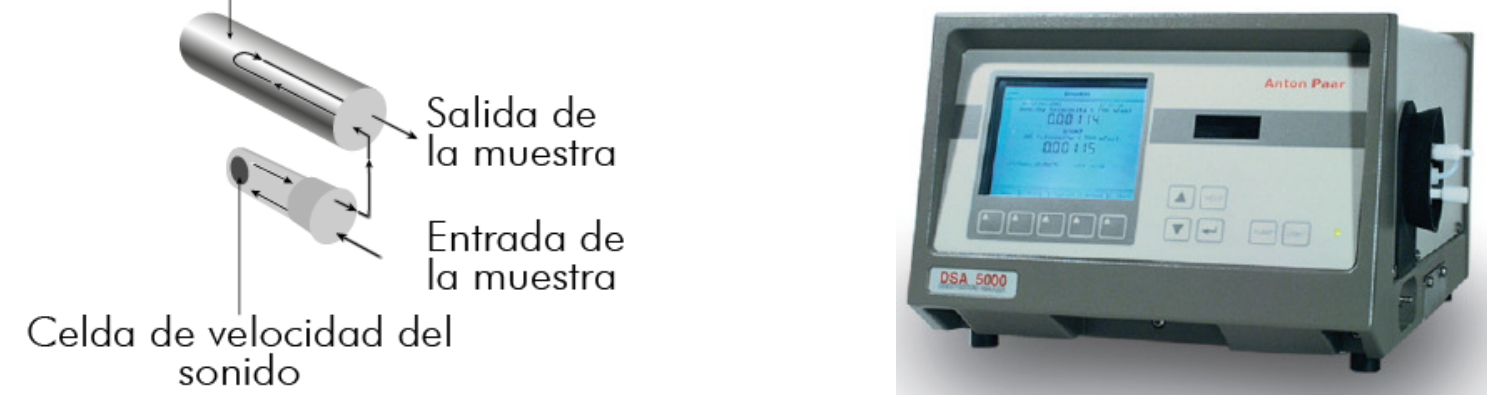

Ilustración 3-12: densímetro-analizador del sonido Anton Paar DSA 5000 
Se necesitan tan sólo $2 \mathrm{ml}$ para cargar el aparato. En una de las celdas se mide la densidad mediante un tubo vibrante en forma de $U$ de cristal Pyrex, basado en el diseño de Kratky et al [52]-[54] explicado en la sección 3.1.1.4, mientras que en la otra celda, de acero inoxidable, se determina la velocidad de propagación del sonido mediante el método del pulso, suponiendo que no se produce absorción. El equipo es capaz de medir densidades entre 0 y $3 \mathrm{~g} \cdot \mathrm{cm}^{-3}$ y velocidades del sonido entre 1000 y $2000 \mathrm{~m} \cdot \mathrm{s}^{-1}$, con precisión $\pm 5 \cdot 10^{-6} \mathrm{~g} \cdot \mathrm{cm}^{-3} \mathrm{y} \pm 0.1 \mathrm{~m} \cdot \mathrm{s}^{-1}$ respectivamente.

Ambas celdas se encuentran en el interior de un tubo cilíndrico de doble pared recubierto de cobre. El espacio comprendido entre el oscilador, la celda de sonido y la pared interna del cilindro está lleno de un gas de elevada conductividad térmica, cuya misión es facilitar un rápido equilibrio térmico entre la muestra inyectada y un líquido termostático que circula por la doble pared del cilindro.

La corriente inducida en el bobinado secundario es amplificada y se utiliza para registrar la frecuencia de oscilación del tubo mediante un contador de cristal de cuarzo. No obstante, para compensar los errores derivados por el efecto de la viscosidad de la muestra se coloca un circuito cambiador de fase en el circuito amplificador. Observando los efectos del cambio de fase sobre la frecuencia de oscilación del tubo, se puede medir la viscosidad de la muestra, y el sistema podrá compensar los errores debidos a ésta.

Para el control de la temperatura de la muestra, una sonda de resistencia de platino Pt-100, situada dentro del tubo cilíndrico, mide la temperatura de dicho tubo, suponiendo que es la misma que de ambas celdas, con una precisión de $\pm 0.001 \mathrm{~K}$. Esta sonda sirve también para controlar la corriente que circula por una célula Peltier que está en contacto con el tubo recubierto por cobre. El aparato consta de un microprocesador Intel 8052 AH-BASIC que almacena información acerca de un conjunto de puntos fijos de temperatura, sobre los que se va a comparar la temperatura que detecta la sonda de Platino en la manera que se explicó en la sección 3.1.1.4f. La temperatura detectada por la sonda de platino es comparada con los puntos fijos definidos para dicha temperatura por un microcontrolador que además calcula la diferencia de potencial que debe aplicarse a la célula Peltier para llevar la temperatura al valor deseado. Esta información sobre la diferencia de potencial necesaria se lleva a un amplificador de potencia que actúa sobre la célula Peltier con una señal positiva o negativa, según se necesite enfriar o calentar [107], y que permite al aparato cubrir el rango de temperatura de $273.15 \mathrm{~K}$ hasta $343.15 \mathrm{~K}$.

Por defecto el DSA 5000 utiliza las constantes de calibrado obtenidas a partir del ajuste de dos puntos (agua y aire) a una línea recta para calcular $\rho$ y $u$. No obstante, cuantos más puntos se tomen en la calibración, mejores serán los valores de las constantes de calibrado, y por tanto más fiables serán los resultados obtenidos. Por ello, el DSA 5000 del laboratorio del G.E.T.E.F. está conectado a un ordenador mediante puerto serie y con el entorno de programación VEE de Agilent de manera que se pueden tomar los datos del periodo de oscilación $\tau$ y del tiempo que tarda el pulso en recorrer la celda de sonido $\Delta t$ y a partir de ellos y de un calibrado con 6 puntos fijos, obtener $\rho$ y $u$ de la muestra.

Las celdas deben lavarse con un disolvente apropiado según los líquidos utilizados (en nuestro caso, etanol, y después 2-propanona), y secarse mediante un chorro de aire. El dispositivo cuenta con una bomba que proporciona dicho chorro, el cual se aplica 
durante 5 minutos. Pasado ese tiempo, se puede volver a cargar el aparato con el líquido a medir. Ya que las capacidades caloríficas de los líquidos suelen ser elevadas, se requieren unos 25 minutos para que las celdas llenas del líquido estén en equilibrio térmico con el tubo cilíndrico. El criterio seguido para determinar si se alcanza el equilibrio térmico es poner el aparato a medir en el mismo momento en el que se inyecta el líquido y esperar a que las desviaciones estándar de los últimos valores de $\tau$ y $\Delta t$ sean menores a un valor predefinido $\left(10^{-6} \mathrm{~ms}\right.$ y $0.02 \mathrm{~ms}$ respectivamente), tomando como valores de $\tau$ y $\Delta t$ los promedios de esos 20 valores.

\subsubsection{Calibración del DSA 5000}

Para calibrar el DSA 5000 se seleccionaron ciertos líquidos puros cuyos valores de la densidad $\rho$ y la velocidad del sonido $u$ son bien conocidos: $n$-heptano, 2,2,4trimetilpentano, octano, ciclohexano, benceno y agua, y se dan en la Tabla 3-2.

Tabla 3-2: valores de referencia de la densidad, $\rho$, y de la velocidad de propagación del sonido, $u$, a presión atmosférica y temperatura $T$, de los compuestos utilizados para calibrar el Anton Paar DSA-5000. ' [108];

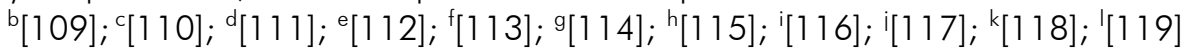

\begin{tabular}{|c|c|c|c|c|}
\hline Propiedad & $T / \mathrm{K}$ & Heptano & Isooctano & Octano \\
\hline \multirow[t]{3}{*}{$\rho / \mathrm{g} \cdot \mathrm{cm}^{3}$} & 293.15 & $0.68375^{a}$ & $0.691959^{b}$ & $0.70267^{a}$ \\
\hline & 298.15 & $0.67946^{a}$ & $0.687849^{b}$ & $0.69862^{a}$ \\
\hline & 303.15 & $0.67519^{a}$ & $0.683711^{b}$ & $0.69445^{c}$ \\
\hline \multirow[t]{4}{*}{$u / \mathrm{m}^{-\mathrm{s}^{-1}}$} & 293.15 & $1152.20^{d}$ & $1102.2^{f}$ & $1193^{h}$ \\
\hline & 298.15 & $1129.92^{e}$ & $1081.28^{\mathrm{g}}$ & $1171.768^{a}$ \\
\hline & 303.15 & $1108.99^{d}$ & $1061.6^{c}$ & $1151.6^{i}$ \\
\hline & & Ciclohexano & Benceno & Agua \\
\hline \multirow[t]{3}{*}{$\rho / \mathrm{g} \cdot \mathrm{cm}^{3}$} & 293.15 & $0.778583^{b}$ & $0.879^{a}$ & $0.9982058^{a}$ \\
\hline & 298.15 & $0.773896^{b}$ & $0.87360^{a}$ & $0.9970474^{a}$ \\
\hline & 303.15 & $0.769172^{b}$ & $0.86829^{a}$ & $0.9956504^{a}$ \\
\hline \multirow[t]{3}{*}{$u / \mathrm{m}^{-\mathrm{s}^{-1}}$} & 293.15 & $1279.32^{i}$ & $1322.95^{k}$ & $1482.32^{\prime}$ \\
\hline & 298.15 & $1253.789^{b}$ & $1299.109^{a}$ & $1496.69^{\prime}$ \\
\hline & 303.15 & $1230.00^{i}$ & $1275.70^{k}$ & $1509.12^{g}$ \\
\hline
\end{tabular}

En el caso de la densidad, el calibrado se realiza a partir de la determinación del periodo de resonancia $\tau$ de dichos líquidos. Los pares de puntos $\left(\tau^{2}, \rho\right)$ se ajustan a la ecuación (3.20) mediante el método de los mínimos cuadrados, obteniéndose los valores de los coeficientes $A$ y $B$, que como sabemos dependen de la temperatura.

En el caso de la velocidad del sonido, al explicar el método del pulso se ha visto que $u$ se calcula como el cociente entre la distancia recorrida por el pulso y el tiempo necesario para recorrer dicha distancia como se deduce de la ecuación (3.23). Por lo tanto, la ecuación de ajuste sería:

$$
u=\frac{2 \cdot \Delta L}{\Delta t}=\frac{L_{\text {celda }}}{\Delta t}
$$


Sin embargo es necesaria hacer una corrección a dicha ecuación para tener en cuenta el error inducido en la medida de $\Delta t$ debido a que los tiempos de respuesta propios de los elementos electrónicos del aparato son finitos. La relación entre $u$ y $\Delta t$ utilizada es la siguiente:

$$
u=\frac{L_{\text {celda }}}{a^{\prime}+b^{\prime} \cdot \Delta t}
$$

$L_{\text {celda }}$ básicamente es la longitud de la celda de acero inoxidable cuya dependencia con la temperatura viene dada en términos de su coeficiente de dilatación térmica. La ecuación anterior se puede rescribir como:

$$
u=\frac{1}{a+b \cdot \Delta t}
$$

de modo que:

$$
u^{-1}=a+b \cdot \Delta t
$$

Los coeficientes $a$ y $b$ son función de la temperatura, y se obtienen mediante el método de los mínimos cuadrados al hacer la regresión lineal entre los pares de datos $\left(\Delta t, u^{-1}\right)$.

Como hemos visto los valores de los coeficientes $A, B, a$ y $b$ son función de la temperatura, por lo que el calibrado del aparato debe hacerse para cada temperatura de trabajo (en nuestro caso, 293.15 K, 298.15 K y $303.15 \mathrm{~K}$ ).

En la Tabla 3-3 se reflejan los valores de los coeficientes que aparecen en las ecuaciones (3.20) y (3.29) para $T=293.15$ K, 298.15 K y 303.15 K y presión atmosférica, junto con sus errores y el coeficiente de correlación.

\begin{tabular}{|c|c|c|c|c|c|}
\hline$A / \mathrm{g} \cdot \mathrm{cm}^{-3}$ & $B / \mathrm{g}^{\prime} \mathrm{cm}^{-3} \cdot \mathrm{ms}^{-2}$ & $r^{2}$ & $a / \mathrm{m}^{-1} \cdot \mathrm{s}$ & $\mathrm{b} / \mathrm{m}^{-1}$ & $r^{2}$ \\
\hline \multicolumn{6}{|c|}{$293.15 \mathrm{~K}$} \\
\hline $\begin{array}{l}-1.059513 \\
\left( \pm 1.1 \cdot 10^{-5}\right)\end{array}$ & $\begin{array}{c}0.2418447 \\
\left( \pm 1.3 \cdot 10^{-6}\right)\end{array}$ & 0.99999971 & $\begin{array}{c}-0.0001057 \\
\left( \pm 1.8 \cdot 10^{-6}\right)\end{array}$ & $\begin{array}{c}0.0003912 \\
\left( \pm 8 \cdot 10^{-7}\right)\end{array}$ & 0.99998469 \\
\hline \multicolumn{6}{|c|}{$298.15 \mathrm{~K}$} \\
\hline $\begin{array}{l}-1.059412 \\
\left( \pm 1.1 \cdot 10^{-5}\right)\end{array}$ & $\begin{array}{l}0.2418254 \\
\left( \pm 1.2 \cdot 10^{-6}\right)\end{array}$ & 0.99999972 & $\begin{array}{c}-0.0001058 \\
\left( \pm 5 \cdot 10^{-7}\right)\end{array}$ & $\begin{array}{c}0.0003913 \\
\left( \pm 2 \cdot 10^{-7}\right)\end{array}$ & 0.99999875 \\
\hline \multicolumn{6}{|c|}{$303.15 \mathrm{~K}$} \\
\hline $\begin{array}{l}-1.059390 \\
\left( \pm 1.0 \cdot 10^{-5}\right)\end{array}$ & $\begin{array}{l}0.2418146 \\
\left( \pm 1.2 \cdot 10^{-6}\right)\end{array}$ & 0.99999962 & $\begin{array}{c}-0.0001031 \\
\left( \pm 1.0 \cdot 10^{-6}\right)\end{array}$ & $\begin{array}{c}0.0003899 \\
\left( \pm 4 \cdot 10^{-7}\right)\end{array}$ & 0.99999544 \\
\hline
\end{tabular}

Tabla 3-3: valores de los coeficientes de calibración del Anton Paar DSA 5000 con sus errores, y el coeficiente de correlación, a presión atmosférica. Fecha de la calibración: 21/6/2009

En la llustración 3-13 se representan los puntos ( $\tau^{2}$ experimental, $\rho$ de referencia) y la regresión lineal de los mismos, para $T=298.15 \mathrm{~K}$, mientras que en la llustración 3-14 se representan los puntos ( $\Delta t$ experimental, $u^{-1}$ de referencia) y la regresión lineal de los mismos, para $T=298.15 \mathrm{~K}$. Las rectas de ajuste responden a los parámetros dados en la Tabla 3-3 a la misma temperatura.

En la referencia [120] se pueden consultar los datos numéricos y las gráficas correspondientes a cada uno de los ajustes para las tres temperaturas de trabajo. 


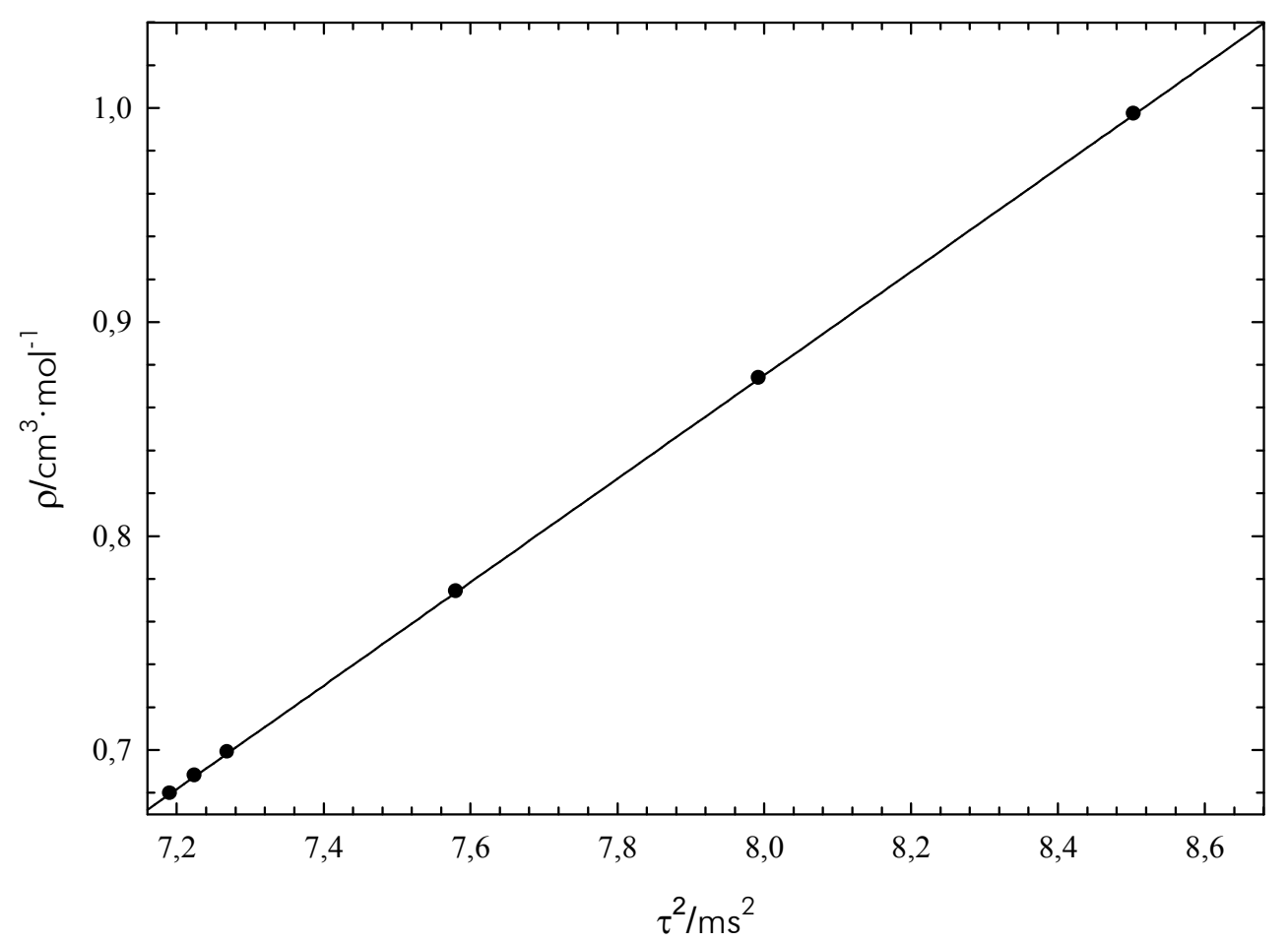

Ilustración 3-13: calibrado de la densidad a $298.15 \mathrm{~K}$ y presión atmosférica. Densidad de los líquidos de referencia, $\rho$, en función del cuadrado del periodo de oscilación del tubo en $U$ del Anton Paar DSA 5000, $\tau^{2}$.

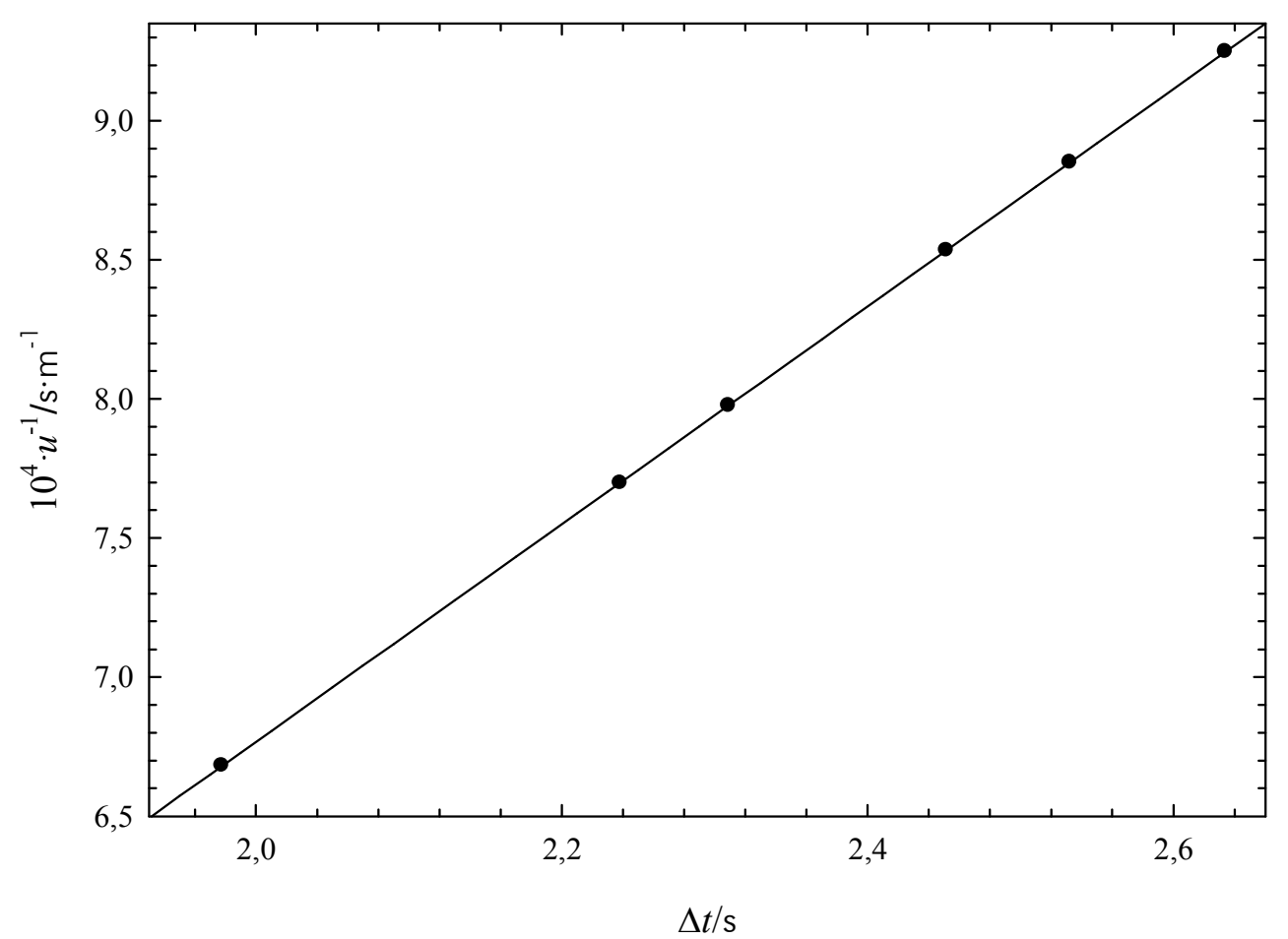

Ilustración 3-14: calibrado de la velocidad del sonido a 298.15 K y presión atmosférica. Inverso de la velocidad del sonido de los líquidos de referencia, $u^{-1}$, en función del tiempo de viaje del pulso, $\Delta t$. 
Además de las variaciones con la temperatura y la presión, la fatiga de los materiales por el uso continuado del aparato es otro factor que influye en el valor de los coeficientes, como se refleja en la sección 3.1.1.4 g). Para mitigar estos efectos, es habitual utilizar una modificación de las ecuaciones (3.20) y (3.29). En concreto, para la densidad se utiliza un líquido de referencia (isooctano) de manera que la ecuación (3.20) se transforma en la siguiente:

$$
\rho-\rho_{\text {iso }}=B \cdot\left(\tau^{2}-\tau_{\text {iso }}^{2}\right)
$$

donde $\rho_{\text {iso }}$ es la densidad del isooctano y $\tau_{\text {iso }}$ el periodo de oscilación del tubo cuando está lleno de dicho líquido.

En el caso de la velocidad del sonido, la transformación que sufre la ecuación (3.29) es la siguiente:

$$
u^{-1}=u_{\text {iso }}^{-1}+b \cdot\left(\Delta t-\Delta t_{\text {iso }}\right)
$$

donde $u_{\text {iso }}$ es la velocidad de propagación del sonido en el isooctano y $\Delta t_{\text {iso }}$ el tiempo que tarda el pulso en viajar por la celda llena de dicho líquido.

Mientras que lo ideal sería trabajar con las ecuaciones (3.20) y (3.29) calibrando el aparato cada vez que se utiliza, es decir, a diario (lo cual es una tarea impracticable debido al número limitado de horas de un jornada laboral, (:)), en su lugar se trabaja con las ecuaciones (3.30) y (3.31), donde $\tau_{\text {iso }}$ y $\Delta t_{\text {iso }}$ se miden a diario. La experiencia del grupo de investigación G.E.T.E.F. ha permitido observar que la constante de calibrado que es más sensible al paso del tiempo es la ordenada en el origen. La utilización de las ecuaciones (3.30) y (3.31) elimina la influencia de los cambios temporales de la ordenada en el origen en la ecuación de trabajo del aparato, lo que permite que no haya que calibrar tan a menudo. A pesar de todo, en los cuatro años durante los que se utilizó el DSA 5000 para obtener los datos que se presentan en este trabajo, se realizaron varias calibraciones, de las cuales aquí solamente se han expuesto los resultados obtenidos para la calibración realizada el 21/6/2009, a modo de ejemplo.

Los volúmenes de exceso se calculan mediante la ecuación (3.7). En ella aparecen:

a) la densidad, que se obtiene a través de la ecuación (3.30) como se ha explicado.

b) la masa molar de las sustancias consideradas, que se obtiene a partir de la tabla de masas atómicas [121] y la fórmula química de dichas sustancias.

c) la fracción molar, la cual se determina por pesada a partir de la masa de cada componente en la mezcla y sus masas molares. La masa de cada componente en la mezcla se obtiene gracias una balanza de precisión A\&D Instruments HR-202. El error estimado para la fracción molar es menor de \pm 0.0001 . El protocolo de pesada de cada componente se detalla a continucación:

- Estimación de los volúmenes de cada uno de los componentes a mezclar, para obtener un volumen total de unos $9 \mathrm{~cm}^{3}$ con la fracción molar aproximada deseada.

- Pesada de la celda de mezcla vacía.

- Pesada de la celda con el primer componente: se retira el tapón de la celda y se introduce el primer componente en su interior. Rápidamente se cierra la celda y se repite la pesada. 
- Pesada de la celda con la mezcla: se retira el tapón de la celda y se introduce el segundo componente en su interior. Rápidamente se cierra la celda y se repite la pesada.

La pesada en una balanza, cuando las necesidades de precisión lo requieran, debe ser corregida por efecto del empuje, lo que es función de las densidades del aire y de las pesas de calibrado. Las ecuaciones correspondientes a dicha corrección pueden consultarse en la bibliografía [122].

Para calcular la compresibilidad isoentrópica de exceso se hace uso de la ecuación (2.166), en la que aparecen:

a) $\kappa_{S}$, que se obtiene a partir de $u, \rho$ y la ecuación (3.21).

b) $\quad \kappa_{T}^{\text {id }}$, que se obtiene a partir de $\kappa_{T, i}^{0}$, la fracción de volumen y la ecuación (2.165).

c) $\quad V_{m}^{i d}$, que se obtiene a partir de $V_{0}^{i}$, la fracción molar y la ecuación (2.161).

d) $\alpha_{P}^{i d}$, que se obtiene a partir de $\alpha_{P, i}^{0}$, la fracción de volumen y la ecuación (2.164).

e) $\quad C_{P}^{i d}$, que se obtiene a partir de $C_{P}^{i d}$, la fracción molar y la ecuación (2.163).

Para llevar a cabo estos cálculos se necesita conocer:

$\kappa_{T, i}^{0}$, que se obtiene a partir de $\kappa_{S, i}^{0}$ mediante la ecuación:

$$
\kappa_{T, \mathrm{i}}^{0}=\kappa_{S, \mathrm{i}}^{0}+T \cdot V_{\mathrm{i}}^{0} \cdot \frac{\left(\alpha_{P, \mathrm{i}}^{0}\right)^{2}}{C_{P, \mathrm{i}}^{0}}
$$

$V_{i}^{0}$, que se determina a partir de la densidad y la masa molar de los componentes puros:

$$
V_{\mathrm{i}}^{0}=M_{0}^{\mathrm{i}} / \rho_{\mathrm{i}}^{0}
$$

$C_{P, \mathrm{i}}^{0}$, que se toma de la bibliografía.

$\kappa_{S, \mathrm{i}}^{0}$, que se obtiene a partir de $u_{\mathrm{i}}^{0}$ y de $\rho_{\mathrm{i}}^{0}$ utilizando la ecuación (3.21).

$\alpha_{P, \mathrm{i}}^{0}$, que se determina a partir de la densidad una vez que se ha medido ésta como función de la temperatura, gracias a la ecuación:

$$
\alpha_{P, \mathrm{i}}^{0}=\frac{1}{V_{\mathrm{i}}^{0}} \cdot\left(\frac{\partial V_{\mathrm{i}}^{0}}{\partial T}\right)_{P}=-\frac{1}{\rho_{\mathrm{i}}^{0}} \cdot\left(\frac{\partial \rho_{\mathrm{i}}^{0}}{\partial T}\right)_{P}
$$

En algunas ocasiones no hay disponibles datos experimentales de $C_{P, \mathrm{i}}^{0}$, lo que no permite obtener $\kappa_{S}^{\mathrm{E}}$ de la manera arriba indicada. En esos casos se trabaja con el cambio en la compresibilidad isoentrópica $\Delta \kappa_{S}$, definido como:

$$
\Delta \kappa_{S}=\kappa_{S}-\sum_{\mathrm{i}=1}^{\mathrm{n}} \kappa_{S, \mathrm{i}}^{0} \cdot \Phi_{\mathrm{i}}
$$

Las diferencias entre $\kappa_{S}^{E}$ y $\Delta \kappa_{S}$ aparecen perfectamente explicadas en la literatura [123]. Se define el coeficiente de expansión térmica de exceso:

$$
\alpha_{P}^{\mathrm{E}}=\alpha_{P}-\alpha_{P}^{\mathrm{id}}
$$

donde $\alpha_{P}^{\text {id }}$ viene dada por la ecuación (2.164).

Otra magnitud importante es la velocidad del sonido de exceso:

donde

$$
u^{\mathrm{E}}=u-u^{\mathrm{id}}
$$




$$
u^{\text {id }}=\left(\kappa_{S}^{\text {id }} \cdot \rho^{\text {id }}\right)^{-1 / 2}=\left(\kappa_{T}^{\text {id }}-\frac{T \cdot V_{\mathrm{m}}^{\text {id }} \cdot\left(\alpha_{P}^{\text {id }}\right)^{2}}{C_{P}^{\text {id }}}\right)^{-1 / 2} \cdot\left(\frac{\sum_{\mathrm{i}=1}^{\mathrm{n}} x_{\mathrm{i}} \cdot M_{\mathrm{i}}^{0}}{\sum_{\mathrm{i}=1}^{\mathrm{n}} x_{\mathrm{i}} \cdot V_{\mathrm{i}}^{0}}\right)^{-1 / 2}
$$

Igual que ocurre en el caso de $\kappa_{S}^{E}$, si algún dato no está disponible, se define la diferencia de velocidad del sonido como:

$$
\Delta u=u-\sum_{\mathrm{i}=1}^{\mathrm{n}} u_{\mathrm{i}}^{0} \cdot x_{\mathrm{i}}
$$

\subsubsection{Sistema test}

Para comprobar la fiabilidad del calibrado, se midieron los volúmenes de exceso y las compresibilidades isoentrópicas de exceso de la mezcla ciclohexano (1) + benceno (2) y se compararon los resultados obtenidos con los de la bibliografía. En la Tabla 3-4 se presentan los datos de los líquidos puros.

Tabla 3-4: características a 298.15 K y presión atmosférica de los compuestos puros utilizados en la medida del sistema ciclohexano + benceno para testar el calibrado del Anton Paar DSA 5000: densidad,

$\rho$, compresibilidad isoentrópica, $\kappa_{S}$, coeficiente de dilatación isobárico, $\alpha_{P}$, volumen molar, $V_{\mathrm{m}}, \mathrm{y}$

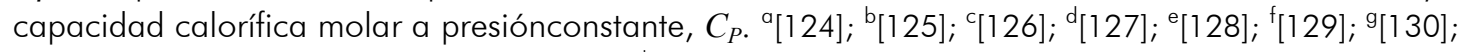

\begin{tabular}{|c|c|c|c|c|}
\hline & \multicolumn{2}{|c|}{$c-\mathrm{C}_{6} \mathrm{H}_{12}$} & \multicolumn{2}{|c|}{$\mathrm{C}_{6} \mathrm{H}_{6}$} \\
\hline Procedencia & \multicolumn{2}{|c|}{$\begin{array}{c}\text { Fluka puriss } \\
\text { p.a. } \geq 99.5 \%(G C)\end{array}$} & \multicolumn{2}{|c|}{$\begin{array}{c}\text { Fluka puriss } \\
\text { p.a. } \geq 99.5 \%(\mathrm{GC})\end{array}$} \\
\hline \multirow[t]{2}{*}{$\mathrm{H}_{2} \mathrm{O} / \mathrm{ppm}$} & \multicolumn{2}{|c|}{11} & \multicolumn{2}{|c|}{13} \\
\hline & Exp & Lit & Exp. & Lit \\
\hline \multirow[t]{4}{*}{$\rho / \mathrm{g} \cdot \mathrm{cm}^{3}$} & 0.773964 & $0.77385^{a}$ & 0.873584 & $0.87365^{\circ}$ \\
\hline & & $0.77387^{b}$ & & $0.8736^{\mathrm{e}}$ \\
\hline & & $0.773845^{c}$ & & $0.87367^{\dagger}$ \\
\hline & & $0.773877^{d}$ & & $0.87352^{\mathrm{c}}$ \\
\hline \multirow[t]{4}{*}{$\kappa_{S} / \mathrm{TPa}^{-1}$} & 822.19 & $822.02^{a}$ & 678.66 & $678.26^{a}$ \\
\hline & & $821.3^{\mathrm{e}}$ & & $677.6^{\mathrm{e}}$ \\
\hline & & $822.79^{f}$ & & $678.45^{f}$ \\
\hline & & $821.95^{\mathrm{g}}$ & & $678.51^{\mathrm{g}}$ \\
\hline$\alpha_{P} / 10^{-3} \mathrm{~K}^{-1}$ & & $1.220^{\mathrm{h}}$ & & $1.213^{h}$ \\
\hline$V_{\mathrm{m}} / \mathrm{cm}^{-3} \cdot \mathrm{mol}^{-1}$ & 108.738 & & 89.416 & \\
\hline$C_{P} / \mathrm{J} \cdot \mathrm{mol}^{-1} \cdot \mathrm{K}^{-1}$ & & $156.01^{i}$ & & 135.760 \\
\hline
\end{tabular}
h[108]; '[1 18];

En las llustraciones 3-15 y 3-16 aparecen los resultados experimentales obtenidos para los volúmenes de exceso y las compresibilidades isoentrópicas de exceso del sistema test, dados en la Tabla 3-6, junto con la curva resultante del ajuste de esos resultados a la ecuación de Redlich-Kister (2.208), cuyos coeficientes se dan en la Tabla 3-5.

Tabla 3-5: coeficientes de los ajustes a la ecuación Redlich-Kister (2.208) de los datos experimentales obtenidos para el sistema ciclohexano(1)+benceno(2) a $T=298.15 \mathrm{~K}$ y presión atmosférica

\begin{tabular}{ccccc}
\hline Propiedad & $A_{0}$ & $A_{1}$ & $A_{2}$ & $\sigma$ \\
\hline$V_{\mathrm{m}}^{\mathrm{E}} / \mathrm{cm}^{-3} \cdot \mathrm{mol}^{-1}$ & $2.612 \pm 0.004$ & $0.097 \pm 0.011$ & & 0.003 \\
$\kappa_{S}^{\mathrm{E}} / \mathrm{TPa}^{-1}$ & $100.90 \pm 0.11$ & $8.5 \pm 0.2$ & $3.8 \pm 0.5$ & 0.071 \\
\hline
\end{tabular}




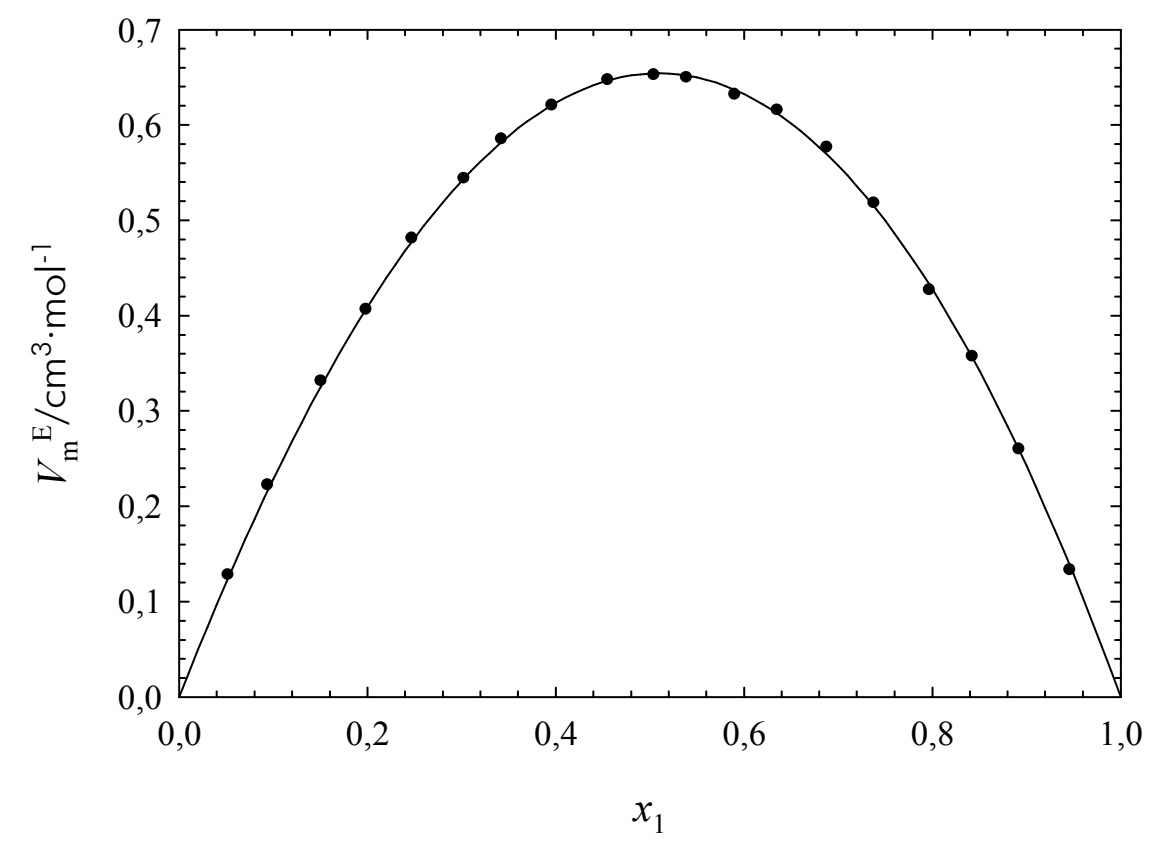

Ilustración 3-15: $V_{\mathrm{m}}{ }^{\mathrm{E}}$ a $298.15 \mathrm{~K}$ y presión atmosférica del sistema ciclohexano(1)+benceno(2): • puntos experimentales; - ajuste a una ecuación Redlich-Kister con los coeficientes de la Tabla 3-5.

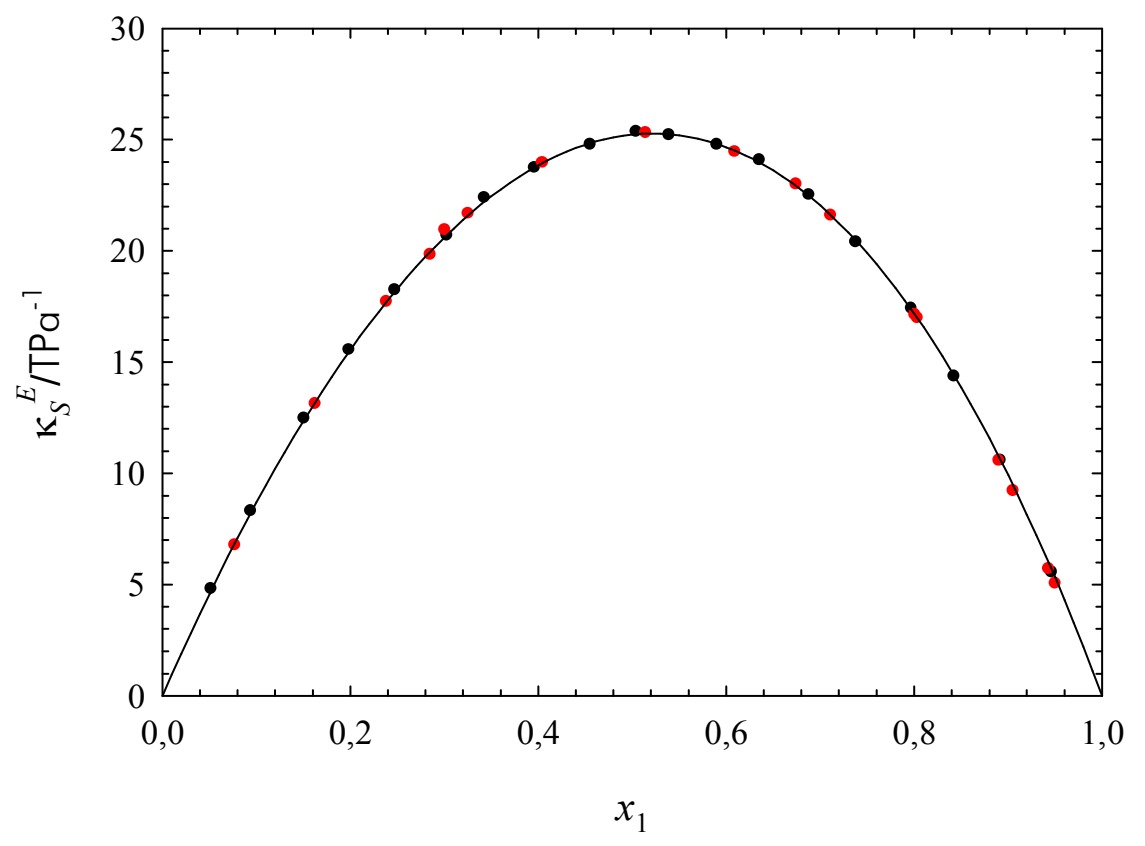

llustración 3-16: $\kappa_{S}^{\mathrm{E}}$ a $298.15 \mathrm{~K}$ y presión atmosférica del sistema ciclohexano(1)+benceno(2): • puntos experimentales; • puntos experimentales de Tamura et al [124]; - ajuste a una ecuación Redlich-Kister con los coeficientes de la Tabla 3-5 
Tabla 3-6: volúmenes molares de exceso $V_{\mathrm{m}}^{\mathrm{E}}$, densidades $\rho$ y compresibilidades isoentrópicas de exceso $\kappa_{S}^{\mathrm{E}}$ a $T=298.15 \mathrm{~K}$ y presión atmosférica del sistema ciclohexano(1) + benceno(2)

\begin{tabular}{cccccccc}
\hline$x_{1}$ & $\rho / \mathrm{g} \cdot \mathrm{cm}^{-3}$ & $V_{\mathrm{m}}^{\mathrm{E}} / \mathrm{cm}^{3} \cdot \mathrm{mol}^{-1}$ & $\kappa_{S}^{\mathrm{E}} / \mathrm{TPa}^{-1}$ & $x_{1}$ & $\rho / \mathrm{g} \cdot \mathrm{cm}^{-3}$ & $V_{\mathrm{m}}^{\mathrm{E}} / \mathrm{cm}^{3} \cdot \mathrm{mol}^{-1}$ & $\kappa_{S}^{\mathrm{E}} / \mathrm{TPa}^{-1}$ \\
\hline 0.0526 & 0.866057 & 0.1275 & 4.79 & 0.5397 & 0.809786 & 0.6490 & 25.18 \\
0.0949 & 0.860235 & 0.2215 & 8.28 & 0.5907 & 0.805086 & 0.6311 & 24.75 \\
0.1516 & 0.852748 & 0.3306 & 12.45 & 0.6358 & 0.801019 & 0.6147 & 24.05 \\
0.1992 & 0.846760 & 0.4058 & 15.52 & 0.6887 & 0.796495 & 0.5760 & 22.49 \\
0.2481 & 0.840775 & 0.4805 & 18.22 & 0.7386 & 0.792465 & 0.5175 & 20.37 \\
0.3035 & 0.834329 & 0.5432 & 20.67 & 0.7977 & 0.787952 & 0.4263 & 17.39 \\
0.3432 & 0.829834 & 0.5844 & 22.37 & 0.8432 & 0.784531 & 0.3566 & 14.34 \\
0.3967 & 0.824059 & 0.6197 & 23.71 & 0.8925 & 0.781040 & 0.2593 & 10.56 \\
0.4561 & 0.818989 & 0.6465 & 24.76 & 0.9469 & 0.777400 & 0.1328 & 5.53 \\
0.5048 & 0.813098 & 0.6518 & 25.33 & & & & \\
\hline
\end{tabular}

Los $V_{\mathrm{m}}^{\mathrm{E}}$ del sistema ciclohexano + benceno se han determinado frecuentemente lo que le convierte en el más apropiado para comprobar la bondad del calibrado del aparato empleado para la medida de $V_{\mathrm{m}}^{\mathrm{E}}$. Handa y Benson [4] realizaron una excelente revisión de las medidas del mismo a $298.15 \mathrm{~K}$ y presión atmosférica. Tomando la ecuación de Redlich-Kister que ellos obtienen al ajustar 164 datos correspondientes a medidas realizadas por Stokes et al [74], Tanaka et al [72] y Kumaran y McGlashan [75]:

$$
V_{\mathrm{m}}^{\mathrm{E}}=(1-x) \cdot x \cdot\left[2.60360-0.09339 \cdot(1-2 \cdot x)+0.04179 \cdot(1-2 \cdot x)^{2}\right]
$$

en la llustración 3-17 se representan las diferencias entre los datos que se presentan en este trabajo y en otros, con los recomendados por Handa y Benson.

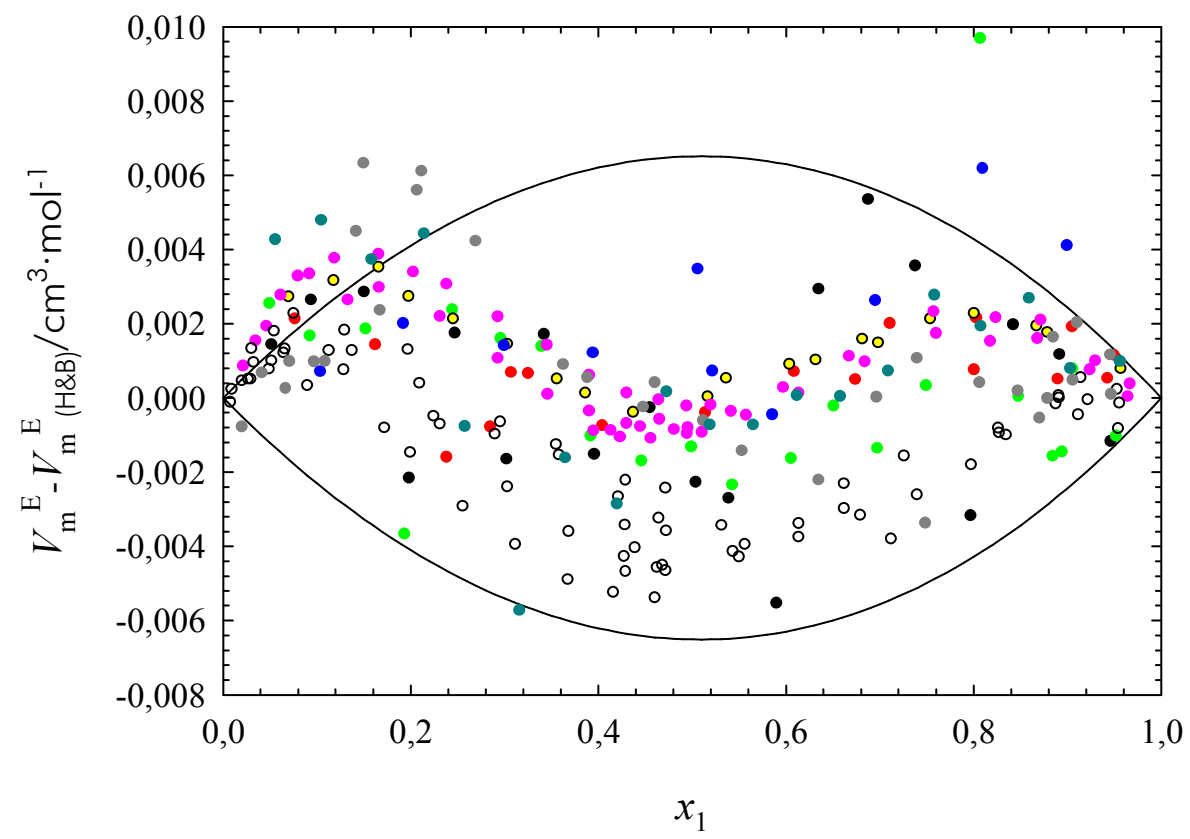

Ilustración 3-17: Diferencias obtenidas en la medida de $V_{\mathrm{m}}{ }^{\mathrm{E}}$ a $T=298.15 \mathrm{~K}$ y presión atmosférica para el sistema test ciclohexano(1)+benceno(2) con respecto Handa y Benson [4]: • este trabajo; • Weeks [38]; - Tanaka [72]; ○ Stokes [74]; • Tamura [1 11]; • Goates [125]; - Takenaka [126];•Villa [127]; • Mozo

$$
[130] ; - \pm 1 \% V_{\mathrm{m}}^{\mathrm{E}}(\mathrm{H \& B})
$$


Como se observa, las diferencias entre los valores obtenidos y los valores de Handa y Benson son menores del $1 \%$, y del mismo orden de magnitud que las obtenidas por el resto de autores.

Respecto a $\kappa_{S}^{\mathrm{E}}$, los datos a $T=298.15 \mathrm{~K}$ y presión atmosférica se comparan con los obtenidos por Tamura et al [124] en la llustración 3-16, y se observa la gran semejanza existente entre los dos conjuntos de datos.

A la vista de los resultados obtenidos para el sistema test, de ahora en adelante consideraremos que la calibración del Anton Paar DSA 5000 es lo suficientemente buena como para garantizar que los datos obtenidos tienen la exactitud deseada.

\subsubsection{Mezclas cetona + amina y $N$-metilanilina + alcano}

Con la ayuda del DSA-5000, en este trabajo se han determinado, a $293.15 \mathrm{~K}, 298.15 \mathrm{~K}$ y $303.15 \mathrm{~K}$ y presión atmosférica, las densidades, las velocidades del sonido, los volúmenes de exceso, las compresibilidades isoentrópicas de exceso y los coeficientes de expansión térmica a presión constante de exceso de las mezclas binarias que se propusieron en el Capítulo 1, en el marco del subproyecto NCO del proyecto T.O.M. Para ello se han utilizado algunos de los compuestos puros que aparecen en la Tabla 3-7 donde se detalla su pureza (medida por cromatografía de gases) y contenido en agua, determinado por el método de Karl-Fischer con un Mettler DL35 Karl-Fischer Tritator [131], excepto para la di-n-propilamina, di- $n$-butilamina y $N, N, N$-trietilamina por no disponer del tritante adecuado para tales compuestos.

Sin llevar a cabo ningún otro procedimiento de purificación que el realizado por el fabricante, a parte de la desgasificación por ultrasonidos por los motivos dados en la sección 3.1, en la manipulación y almacenamiento de las sustancias de trabajo se tuvo especial cuidado dirigido a minimizar la contaminación de las mismas, debida a varios factores: oxidación e hidratación de los compuestos debido al contacto con el aire (por ejemplo, se observó que la densidad de las aminas aumentaba rápidamente, debido a que las aminas de bajo peso molecular forman hidratos estables con agua [108, página 1061]); aparición de trazas de plásticos debido al contacto con envases y tapones de plástico; etc. Para ello se tomaron las siguientes medidas:

- Todos los compuestos fueron almacenados en frascos de vidrio, sustituyendo su tapón convencional de plástico por un septum hermético, lo que evita la inclusión de aire atmosférico dentro del frasco cada vez que se toma una muestra de la sustancia. Para evitar el vacío, los compuestos se mantuvieron en una atmósfera inerte de $\mathrm{N}_{2}$.

- Las aminas son fotosensibles, lo cual se manifiesta en la aparición de una coloración amarillenta. Por ello fueron protegidas de la luz con lámina de aluminio.

- Todos los compuestos se almacenaron con tamiz molecular $4 \AA$ a excepción de la 2-propanona en la que se detectó un incremento en su densidad cuando era almacenada tanto con tamiz $3 \AA$ y $4 \AA$, a pesar de las indicaciones dadas en la bibliografía [108 pp. 952], [132 pp30 y 90]. Aunque Coetzee \& Siao [133] defienden el uso de drierita ( $\mathrm{CaSO}_{4}$ anhidro) como desecante más apropiado, se tomó la opción de no emplear ninguno, observando así un valor de la densidad de la 2-propanona estable durante varias semanas. Anecdóticamente, después de obtener las propiedades termodinámicas de las mezclas con 2-propanona, se encontró en la literatura [134, pp 159] que el tamiz molecular no puede utilizarse con la misma ya que sirve como 
catalizador para la formación de 4-hidroxi-4-metil-penta-2-ona (alcohol de diacetona) y metil-isobutenil-cetona (óxido mesitílico), por lo que la medida tomada de almacenar la acetona en ausencia de tamiz molecular está completamente justificada.

- Cuando fue posible se utilizaron jeringuillas de vidrio para la manipulación de los compuestos. También es recomendable sustituir los tapones de plástico por tapones de corcho envueltos en papel de aluminio, aunque hay que tener cuidado pues determinados compuestos como la bencilamina y el ácido propanoico disuelven el aluminio [132, pp3].

Tabla 3-7: características de los compuestos puros empleados para la determinación de las propiedades de exceso de las mezclas binarias de interés propuestas en el Capítulo 1

\begin{tabular}{cccc}
\hline Compuesto & Procedencia & Pureza $\%$ & $\mathrm{H}_{2} \mathrm{O} / \mathrm{ppm}$ \\
\hline 2-propanona & Fluka / Sigma-Aldrich & $\geq 99.5 / \geq 99.8$ & \\
2-butanona & Fluka & $\geq 99.5$ & \\
2-pentanona & Fluka & $\geq 99$ & \\
2-heptanona & Sigma-Aldrich & $\geq 99$ & $1.4 \cdot 10^{-4}$ \\
piridina & Riedel de Haën & $\geq 99.5$ & $1.5 \cdot 10^{-4}$ \\
anilina & Riedel de Haën & $\geq 99.5$ & $2.6 \cdot 10^{-4}$ \\
$N$-metilanilina & Fluka & $\geq 98.0$ & $1.6 \cdot 10^{-4}$ \\
di- $n$-propilamina & Aldrich & $>99 \%$ & \\
di- $n$-butilamina & Aldrich & $\geq 99.5$ & \\
$N, N$, $N$-trietilamina & Sigma-Aldrich / Fluka & $\geq 99.5 / \geq 99.5$ & \\
ciclohexano & Fluka & $\geq 99.5$ & $4.4 \cdot 10^{-5}$ \\
$n$-heptano & Fluka & $\geq 99.5$ & $2.0 \cdot 10^{-5}$ \\
$n$-octano & Sigma-Aldrich & $\geq 99$ & $3.8 \cdot 10^{-5}$ \\
tolueno & Fluka & $\geq 99.7$ & $1.1 \cdot 10^{-4}$ \\
1 -propanol & Fluka & $\geq 99.5$ & \\
\hline
\end{tabular}

En la Tabla 3-8 se comparan los valores experimentales con los hallados en la literatura, a 298.15 y presión atmosférica, de la densidad, la velocidad del sonido, el coeficiente de expansión térmica a presión constante y los coeficientes de compresibilidad adiabática e isoterma de los compuestos puros utilizados. También se muestran los valores de la capacidad calorífica a presión constante encontrados en la literatura. Cabe resaltar la gran concordancia existente, como es de esperar, entre los datos experimentales y los de la bibliografía. Los resultados obtenidos de las propiedades de los componentes puros a 293.15 K y 303.15 K, además de una comparación más exhaustiva de dichos resultados con la literatura pueden consultarse en las referencias [135]-[142].

Las Tablas 3-9 a la 3-80 presentan los siguientes datos correspondientes a los diferentes sistemas medidos a presión atmosférica:

a) Las fracciones molares del componente (1), $x_{1}$, y los volúmenes molares de exceso, $V_{\mathrm{m}}^{\mathrm{E}}$, a $293.15 \mathrm{~K}, 298.15 \mathrm{~K}$ y $303.15 \mathrm{~K}$. 
b) Las fracciones molares del componente (1), $x_{1}$, la velocidades del sonido de exceso $u^{\mathrm{E}}$, los coeficientes de expansión térmica a presión constante de exceso, $\alpha_{P}^{\mathrm{E}}, \mathrm{y}$ las compresibilidades isoentrópicas de exceso, $\kappa_{S}^{\mathrm{E}}$, a $298.15 \mathrm{~K}$.

c) Los coeficientes del ajuste de los datos de todas estas magnitudes a la ecuación Redlich-Kister (2.208) junto con las desviaciones estándar de dichos ajustes.

Dichas tablas están acompañadas por diversas gráficas (llustración 3-18 a la 3-41) en las que se representan en función de la composición del componente 1, $x_{1}$, las magnitudes siguientes:

a) Los volúmenes molares de exceso, $V_{\mathrm{m}}^{\mathrm{E}}$, y las curvas resultantes del ajuste a la ecuación R-K (2.208), $V_{\mathrm{m}}^{\mathrm{E}}=V_{\mathrm{m}}^{\mathrm{E}}\left(x_{1}\right)$, a $293.15(\bullet-), 298.15(\mathbf{\Delta}-)$ y $303.15 \mathrm{~K}(\mathbf{\square}-)$.

b) Los volúmenes molares de exceso reducidos, $\frac{V_{\mathrm{m}}^{\mathrm{E}}}{x_{1} \cdot\left(1-x_{1}\right)}=g\left(x_{1}\right)$, y la curva resultante del ajuste a la ecuación R-K de forma reducida, a $298.15 \mathrm{~K}$.

c) Los volúmenes molares parciales de exceso de cada uno de los componentes que forman la mezcla, $V_{\mathrm{m}, 1}^{\mathrm{E}}=V_{\mathrm{m}, 1}^{\mathrm{E}}\left(x_{1}\right)(-), V_{\mathrm{m}, 2}^{\mathrm{E}}=V_{\mathrm{m}, 2}^{\mathrm{E}}\left(x_{1}\right)(--)$, calculados de acuerdo con las ecuaciones (2.121) y (2.122) y el ajuste $\mathrm{R}-\mathrm{K}, V_{\mathrm{m}}^{\mathrm{E}}=V_{\mathrm{m}}^{\mathrm{E}}\left(x_{1}\right)$, a $298.15 \mathrm{~K}$.

d) Las compresibilidades isoentrópicas de exceso, $\kappa_{S}^{\mathrm{E}}$, y las curvas resultantes del ajuste a la ecuación R-K (2.208), $\kappa_{S}^{\mathrm{E}}=\kappa_{S}^{\mathrm{E}}\left(x_{1}\right)$, a $298.15 \mathrm{~K}(\bullet-)$.

El procedimiento de cálculo de todas estas magnitudes ya se ha explicado en la sección 3.3.1. No obstante, la determinación de los coeficientes de expansión térmica a presión constante de la mezcla y de los componentes puros $\alpha_{P}$ y $\alpha_{P}^{0}$, necesarios para la determinación de $\alpha_{P}^{\mathrm{E}}$, debe aclararse: tales magnitudes se calculan a partir de la ecuación (3.34). Si bien la dependencia de la densidad con la temperatura nos es desconocida, en su lugar, disponemos de datos a presión constante de la densidad para las temperaturas $293.15 \mathrm{~K}, 298.15 \mathrm{~K}$ y $303.15 \mathrm{~K}$, lo que permite ajustar a una recta mediante el método de los mínimos cuadrados el conjunto de estos tres datos $(\rho, T)$, y obtener la pendiente $(\partial \rho / \partial T)_{P}$ necesaria para determinar $\alpha_{P}$ a $298.15 \mathrm{~K}$, sin más que dividir dicha pendiente por el valor de $\rho$ a esa temperatura. 
Tabla 3-8: Características a $298.15 \mathrm{~K}$ y presión atmosférica de los compuestos puros utilizados. [ [143]; b[144]; [ [108]; d[145]; e[146]; f[147]; 9[1 48]; h[calculado a partir de los datos de [108] y de la ecuación (3.21) o bien de la ecuación (3.3)]; i[149]; '[150]; $[151] ;$ '[152]; ${ }^{\mathrm{m}}[153] ;{ }^{\mathrm{n}}[154] ;{ }^{\circ}[155] ;{ }^{\mathrm{p}}[156] ;{ }^{\mathrm{a}}[157]$; r[158]; ${ }^{\mathrm{s}}[159] ;{ }^{\mathrm{t}}[160] ;{ }^{\mathrm{u}}[161] ;{ }^{\mathrm{v}}[162] ;{ }^{\mathrm{w}}[163] ;{ }^{\mathrm{x}}[164] ;{ }^{\mathrm{y}}[165] ;{ }^{\mathrm{r}}[166] ;{ }^{\mathrm{ac}}[167]$

\begin{tabular}{|c|c|c|c|c|c|c|c|c|c|c|c|c|c|c|c|c|c|}
\hline U & $\ddot{\Xi}$ & 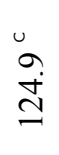 & ڤั) & $\vec{\infty}$ & \begin{tabular}{l}
\multirow{H}{n}{} \\
$\stackrel{i}{\sim}$ \\
$\stackrel{\sim}{\sim}$
\end{tabular} & $\stackrel{n}{\ddot{n}}$ & $\begin{array}{l}c \\
\overrightarrow{\dot{J}}\end{array}$ & $\frac{\vec{c}}{\overrightarrow{0}}$ & 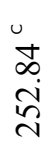 & $\begin{array}{l}\sigma \\
\tilde{\sigma} \\
\tilde{n}\end{array}$ & $\underset{\stackrel{+}{*}}{\vec{v}}$ & \begin{tabular}{l}
$>$ \\
\multirow{2}{n}{} \\
$i n$ \\
$n$
\end{tabular} & 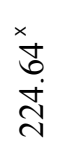 & & & \multicolumn{2}{|l|}{$\begin{array}{l}\text { No } \\
\text { aे. } \\
\text { na }\end{array}$} \\
\hline 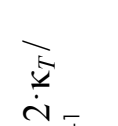 & $\ddot{\Xi}$ & $\stackrel{+}{\sim}$ & $\stackrel{\infty}{\infty}=$ & $\tilde{\sigma}$ & in & & $\stackrel{\varepsilon}{\underset{f}{\sigma}}$ & & $\begin{array}{l}\sigma \\
\infty \\
=\end{array}$ & $\begin{array}{l}\sigma \\
\hat{\sigma} \\
\hat{\sigma}\end{array}$ & 孛 & $\begin{array}{l}\text { U } \\
\text { I }\end{array}$ & $\begin{array}{l}\text { O } \\
\text { 巳 }\end{array}$ & & & $\stackrel{u}{\bar{n}}$ & \\
\hline $\begin{array}{l}H \\
\underline{\Sigma}\end{array}$ & 这 & $\frac{n}{n}$ & $\stackrel{g}{\stackrel{n}{\Xi}}$ & $\begin{array}{l}\hat{8} \\
\dot{0} \\
\dot{\delta}\end{array}$ & $\begin{array}{l}\hat{\alpha} \\
\dot{\alpha} \\
\alpha\end{array}$ & $\stackrel{+}{\circ}$ & $\stackrel{\vec{s}}{\dot{q}}$ & $\begin{array}{l}\vec{\sim} \\
\tilde{n}\end{array}$ & $\begin{array}{l}\vec{\sigma} \\
\stackrel{\vec{\sigma}}{\Xi}\end{array}$ & 苍 & $\stackrel{\circ}{\stackrel{一}{\dot{J}}}$ & 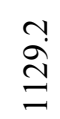 & 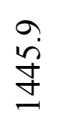 & & & $\frac{n}{a}$ & \\
\hline$\underline{\square}$ & $\ddot{\Xi}$ & 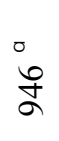 & $\begin{array}{l}\infty \\
\infty \\
\infty\end{array}$ & $\stackrel{0}{\circ}$ & $\stackrel{2}{2}$ & & $\begin{array}{l}u \\
\infty \\
\infty\end{array}$ & & $\begin{array}{l}\sigma \\
\text { 品 }\end{array}$ & $\begin{array}{l}\sigma \\
\stackrel{\sigma}{\infty}\end{array}$ & $\stackrel{+}{\Xi}$ & $\begin{array}{l}\text { Oo } \\
\dot{\tilde{H}} \\
\text { J }\end{array}$ & $\begin{array}{l}3 \\
\vdots \\
\infty \\
i \\
\end{array}$ & & & 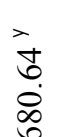 & \\
\hline $\begin{array}{l}\dot{0}- \\
\dot{H} \\
\underline{y}\end{array}$ & 这 & $\begin{array}{l}\text { ते } \\
\text { ఏँ }\end{array}$ & $\underset{\infty}{\stackrel{\infty}{\infty}}$ & $\begin{array}{l}\stackrel{a}{+} \\
\stackrel{\circ}{n} \\
\infty\end{array}$ & $\stackrel{\infty}{\stackrel{n}{n}}$ & ñ & $\overrightarrow{\dot{\delta}}$ & $\begin{array}{l}\vec{\sigma} \\
\vec{\sigma}\end{array}$ & 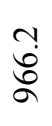 & $\begin{array}{l}n \\
\infty \\
\infty \\
\infty \\
\infty\end{array}$ & 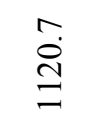 & $\begin{array}{l}\underset{N}{\mathbb{N}} \\
\underset{\infty}{n}\end{array}$ & $\begin{array}{l}\stackrel{n}{n} \\
\stackrel{n}{=}\end{array}$ & & & $\frac{\tilde{b}}{\dot{\infty}}$ & \\
\hline 8 & 芦 & 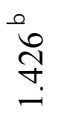 & $\stackrel{0}{\tilde{m}}$ & 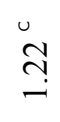 & $\tilde{\sigma}_{0}$ & $\stackrel{0}{0}$ & $\begin{array}{l}u \\
0 \\
\infty \\
\infty \\
0\end{array}$ & $\begin{array}{l}0 \\
\infty \\
0\end{array}$ & 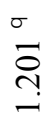 & $\begin{array}{l}\sigma \\
\hat{\sigma} \\
\hat{\sigma}\end{array}$ & $\stackrel{+}{\stackrel{+}{ت}}$ & तิ & 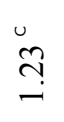 & & & $\stackrel{\infty}{\circ}$ & \\
\hline $\begin{array}{l}+ \\
8 \\
8\end{array}$ & 这 & $\stackrel{n}{\stackrel{n}{q}}$ & $\bar{n}$ & $\stackrel{\infty}{\stackrel{\infty}{=}}$ & $\stackrel{\circ}{\circ}$ & $\stackrel{0}{0}$ & $\begin{array}{l}n \\
\infty \\
0\end{array}$ & 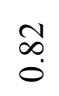 & $\stackrel{\mathbb{J}}{\stackrel{I}{-}}$ & ô. & సે & $\underset{\sim}{\tilde{Z}}$ & ָี & & & $\stackrel{\text { ọ }}{.}$ & \\
\hline & 芦 & $\begin{array}{l}0 \\
0 \\
0 \\
0 \\
= \\
=\end{array}$ & $\stackrel{\circ}{\sigma}$ & $\stackrel{0}{\stackrel{m}{I}}$ & $\stackrel{r}{\stackrel{0}{0}}$ & & 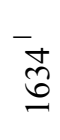 & & $\begin{array}{l}\sigma \\
\infty \\
\stackrel{\infty}{=}\end{array}$ & 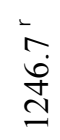 & $\stackrel{\sigma}{\cong}$ & 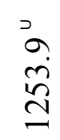 & $\begin{array}{l}3^{3} \\
\infty \\
\stackrel{\Xi}{\Xi}\end{array}$ & & & 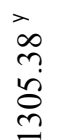 & \\
\hline & 这 & $\stackrel{\nabla}{\stackrel{0}{0}}$ & $\stackrel{\circ}{\stackrel{a}{\Xi}}$ & $\begin{array}{l}\beth \\
\Xi \\
\Xi\end{array}$ & 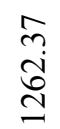 & $\begin{array}{l}\hat{\sigma} \\
\dot{\sigma}\end{array}$ & $\begin{array}{l}0 \\
\infty \\
\delta \\
\hat{\sigma}\end{array}$ & $\begin{array}{l}+ \\
\stackrel{d}{0} \\
\stackrel{n}{n}\end{array}$ & 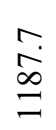 & $\stackrel{\infty}{\stackrel{\infty}{\Xi}}$ & $\exists$ & $\begin{array}{l}0 \\
\stackrel{n}{n} \\
\stackrel{-}{J}\end{array}$ & $\begin{array}{l}\stackrel{0}{\grave{\Xi}} \\
\Xi\end{array}$ & & & 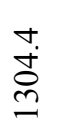 & \\
\hline & $\ddot{\Xi}$ & \begin{tabular}{l}
0 \\
$i n$ \\
\multirow{2}{*}{} \\
$\infty$ \\
0 \\
0
\end{tabular} & 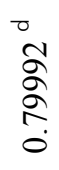 & $\begin{array}{l}u \\
i n \\
\infty \\
0 \\
0\end{array}$ & $\begin{array}{l}0 \\
\equiv \\
\infty \\
0\end{array}$ & $\begin{array}{l}- \\
0 \\
\infty \\
\vdots \\
0 \\
0\end{array}$ & $\frac{\bar{J}}{\overline{0}}$ & 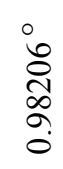 & $\begin{array}{l}e \\
0 \\
n \\
m \\
\stackrel{n}{0} \\
0\end{array}$ & $\begin{array}{l}a \\
n \\
n \\
n \\
o\end{array}$ & $\begin{array}{l}\infty \\
\stackrel{\infty}{a} \\
\stackrel{0}{0}\end{array}$ & 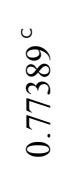 & 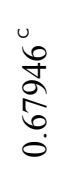 & 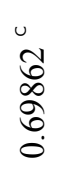 & $\begin{array}{l}u \\
\tilde{n} \\
\tilde{\delta} \\
\tilde{0}\end{array}$ & 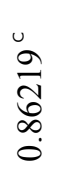 & $\begin{array}{l}8 \\
0 \\
12 \\
2 \\
2 \\
0 \\
0\end{array}$ \\
\hline $\begin{array}{ll}H & 0 \\
a & 0\end{array}$ & 官 & $\begin{array}{l}\infty \\
\infty \\
\infty \\
\infty \\
\infty \\
\vdots \\
0\end{array}$ & 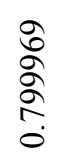 & $\begin{array}{l}\tilde{N} \\
n \\
0 \\
\infty \\
0 \\
0\end{array}$ & $\begin{array}{l}\infty \\
\equiv \\
\equiv \\
0 \\
0\end{array}$ & $\begin{array}{l}0 \\
\stackrel{n}{0} \\
\infty \\
\vdots \\
0\end{array}$ & $\begin{array}{l}\text { o } \\
\stackrel{+}{ \pm} \\
\vdots \\
0\end{array}$ & 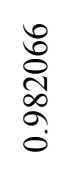 & $\begin{array}{l}0 \\
0 \\
0 \\
m \\
0 \\
0\end{array}$ & $\begin{array}{l}\hat{n} \\
i n \\
i n \\
0\end{array}$ & 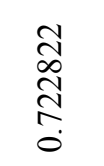 & $\underset{\substack{\infty \\
\multirow{2}{*}{}}}{\stackrel{\infty}{0}}$ & 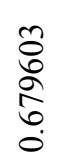 & & & $\begin{array}{l}\stackrel{2}{2} \\
\text { तु } \\
\infty \\
0\end{array}$ & $\begin{array}{l}0 \\
2 \\
2 \\
2 \\
0\end{array}$ \\
\hline & & $\begin{array}{l}\text { O } \\
0 \\
0 \\
\frac{1}{0} \\
\frac{0}{0} \\
\frac{0}{1} \\
\stackrel{1}{1}\end{array}$ & $\begin{array}{l}0 \\
\frac{0}{c} \\
0 \\
\frac{c}{0} \\
\frac{1}{3} \\
\frac{0}{1} \\
\dot{d}\end{array}$ & 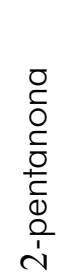 & $\begin{array}{l}0 \\
\frac{0}{0} \\
\frac{0}{0} \\
\frac{0}{0} \\
\frac{0}{d} \\
\frac{d}{1} \\
d\end{array}$ & 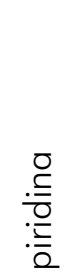 & 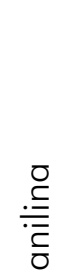 & 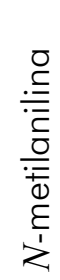 & 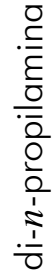 & 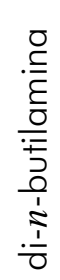 & 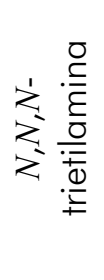 & $\begin{array}{l}O \\
\frac{O}{0} \\
0 \\
\mathbb{D} \\
\frac{D}{O} \\
\frac{O}{U} \\
U\end{array}$ & $\begin{array}{l}0 \\
\frac{1}{0} \\
\frac{0}{0} \\
0 \\
0 \\
\frac{1}{1} \\
\frac{1}{2}\end{array}$ & $\begin{array}{l}0 \\
\frac{0}{0} \\
\frac{0}{U} \\
0 \\
\stackrel{1}{1}\end{array}$ & $\begin{array}{l}0 \\
\frac{0}{0} \\
0 \\
0 \\
0 \\
1 \\
=\end{array}$ & 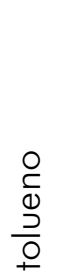 & $\begin{array}{l}\overline{0} \\
\frac{1}{0} \\
\frac{0}{0} \\
0 \\
\frac{0}{1} \\
\frac{1}{1}\end{array}$ \\
\hline
\end{tabular}


Tabla 3-9: volúmenes molares de exceso del sistema anilina (1) + 2-propanona (2)

\begin{tabular}{|c|c|c|c|c|c|}
\hline$x_{1}$ & $V_{\mathrm{m}}^{\mathrm{E}} / \mathrm{cm}^{3} \cdot \mathrm{mol}^{-1}$ & $x_{1}$ & $V_{\mathrm{m}}^{\mathrm{E}} / \mathrm{cm}^{3} \cdot \mathrm{mol}^{-1}$ & $x_{1}$ & $V_{\mathrm{m}}^{\mathrm{E}} / \mathrm{cm}^{3} \cdot \mathrm{mol}^{-1}$ \\
\hline \multicolumn{6}{|c|}{$293.15 \mathrm{~K}$} \\
\hline 0.0525 & -0.3046 & 0.4170 & -1.169 & 0.6984 & -0.8513 \\
\hline 0.0955 & -0.5220 & 0.4503 & -1.160 & 0.7510 & -0.7314 \\
\hline 0.1503 & -0.7433 & 0.5092 & -1.120 & 0.7926 & -0.6142 \\
\hline 0.1954 & -0.8819 & 0.5687 & -1.052 & 0.8433 & -0.4681 \\
\hline 0.2535 & -1.024 & 0.6168 & -0.9842 & 0.8922 & -0.3316 \\
\hline 0.3089 & -1.118 & 0.6546 & -0.9303 & 0.9478 & -0.1611 \\
\hline 0.3554 & -1.153 & & & & \\
\hline \multicolumn{6}{|c|}{$298.15 \mathrm{~K}$} \\
\hline 0.0455 & -0.2750 & 0.4078 & -1.215 & 0.6961 & -0.8805 \\
\hline 0.1049 & -0.5816 & 0.4499 & -1.203 & 0.7516 & -0.7492 \\
\hline 0.1467 & -0.7530 & 0.4965 & -1.181 & 0.8022 & -0.6111 \\
\hline 0.1918 & -0.9146 & 0.5415 & -1.142 & 0.8461 & -0.4898 \\
\hline 0.2478 & -1.048 & 0.5886 & -1.081 & 0.8912 & -0.3486 \\
\hline 0.3007 & -1.145 & 0.6495 & -0.9826 & 0.9431 & -0.1850 \\
\hline 0.3501 & -1.190 & & & & \\
\hline \multicolumn{6}{|c|}{$303.15 \mathrm{~K}$} \\
\hline 0.0506 & -0.3342 & 0.3963 & -1.269 & 0.6976 & -0.8977 \\
\hline 0.0986 & -0.5938 & 0.4498 & -1.261 & 0.7601 & -0.7470 \\
\hline 0.1433 & -0.7897 & 0.5021 & -1.225 & 0.8074 & -0.6104 \\
\hline 0.1922 & -0.9626 & 0.5497 & -1.183 & 0.8525 & -0.4805 \\
\hline 0.2452 & -1.111 & 0.6053 & -1.087 & 0.8998 & -0.3311 \\
\hline 0.3025 & -1.197 & 0.6525 & -0.9716 & 0.9485 & -0.1691 \\
\hline 0.3536 & -1.247 & & & & \\
\hline
\end{tabular}

Tabla 3-10: velocidades del sonido de exceso, coeficientes de expansión térmica a $P=$ cte. de exceso, y compresibilidades isoentrópicas de exceso del sistema anilina (1) + 2-propanona (2)

\begin{tabular}{cccccccc}
\hline$x_{1}$ & $u^{\mathrm{E}} / \mathrm{m} \cdot \mathrm{s}^{-1}$ & $\alpha_{\mathrm{P}}^{\mathrm{E}} / 10^{-6} \mathrm{~K}^{-1}$ & $\kappa_{S}^{\mathrm{E}} / \mathrm{TPa}^{-1}$ & $x_{1}$ & $u^{\mathrm{E}} / \mathrm{m} \cdot \mathrm{s}^{-1}$ & $\alpha_{\mathrm{P}}^{\mathrm{E}} / 10^{-6} \mathrm{~K}^{-1}$ & $\kappa_{S}^{\mathrm{E}} / \mathrm{TPa}^{-1}$ \\
\hline 0.0455 & 24.1 & -33.77 & -40.17 & 0.5415 & 156.0 & -87.00 & -135.71 \\
0.1049 & 52.8 & -67.19 & -81.16 & 0.5886 & 154.4 & -77.89 & -126.55 \\
0.1467 & 71.1 & -84.25 & -103.18 & 0.6495 & 148.5 & -65.46 & -112.54 \\
0.1918 & 89.2 & -97.66 & -121.87 & 0.6961 & 140.4 & -55.68 & -100.14 \\
0.2478 & 108.6 & -107.53 & -137.58 & 0.7516 & 126.6 & -44.26 & -83.96 \\
0.3007 & 124.2 & -111.56 & -146.83 & 0.8022 & 110.2 & -34.13 & -68.26 \\
0.3501 & 135.7 & -111.2 & -150.56 & 0.8461 & 92.6 & -25.78 & -53.97 \\
0.4078 & 146.3 & -107.09 & -150.71 & 0.8912 & 70.7 & -17.60 & -38.63 \\
0.4499 & 151.5 & -101.96 & -147.91 & 0.9431 & 40.4 & -8.84 & -20.43 \\
0.4965 & 155.0 & -94.91 & -142.74 & & & & \\
\hline
\end{tabular}

Tabla 3-11: coeficientes y desviaciones estándar del ajuste a la ecuación R-K de las diferentes propiedades de exceso del sistema anilina (1) + 2-propanona (2)

\begin{tabular}{ccccccc}
\hline$T / \mathrm{K}$ & Propiedad & $A_{0}$ & $A_{1}$ & $A_{2}$ & $A_{3}$ & $\sigma$ \\
\hline 293.15 & $V_{\mathrm{m}}^{\mathrm{E}} / \mathrm{cm}^{3} \cdot \mathrm{mol}^{-1}$ & -4.541 & 1.58 & -0.34 & & 0.008 \\
298.15 & $V_{\mathrm{m}}^{\mathrm{E}} / \mathrm{cm}^{3} \cdot \mathrm{mol}^{-1}$ & -4.731 & 1.614 & -0.31 & & 0.005 \\
& $u^{\mathrm{E}} / \mathrm{m}^{-1}$ & 620.66 & 88.3 & 40.7 & 28.6 & 0.10 \\
& $\alpha_{\mathrm{p}}^{\mathrm{E}} / 10^{-6} \mathrm{~K}^{-1}$ & -377.64 & 337.6 & -113.8 & & 0.11 \\
& $\kappa_{S}^{\mathrm{E}} / \mathrm{TPa}^{-1}$ & -569.04 & 278.8 & -98.9 & 27.8 & 0.09 \\
303.15 & $V_{\mathrm{m}}^{\mathrm{E}} / \mathrm{cm}^{3} \cdot \mathrm{mol}^{-1}$ & -4.905 & 1.85 & -0.42 & & 0.008 \\
\hline
\end{tabular}



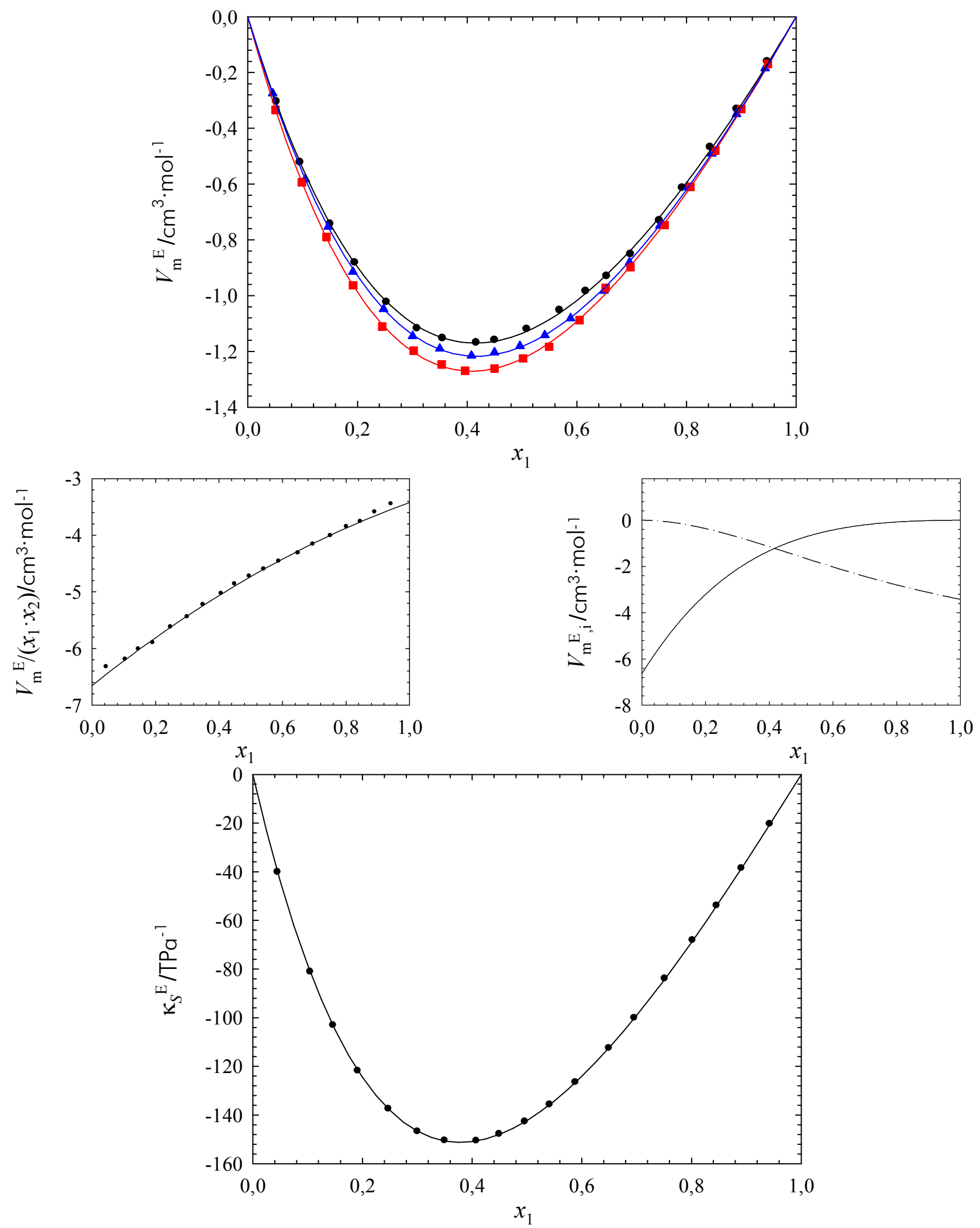

llustración 3-18: volúmenes molares de exceso $V_{\mathrm{m}}^{\mathrm{E}}$, volúmenes molares de exceso reducidos $V_{\mathrm{m}}^{\mathrm{E}} /\left(x_{1} \cdot x_{2}\right)$, volúmenes molares parciales de exceso $V_{\mathrm{m}, \mathrm{i}}^{\mathrm{E}}$ y compresibilidades isoentrópicas de exceso $\kappa_{S}^{\mathrm{E}}$ del sistema anilina (1) +2 -propanona (2) 
Tabla 3-12: volúmenes molares de exceso del sistema anilina (1) + 2-butanona (2)

\begin{tabular}{|c|c|c|c|c|c|}
\hline$x_{1}$ & $V_{\mathrm{m}}^{\mathrm{E}} / \mathrm{cm}^{3} \cdot \mathrm{mol}^{-1}$ & $x_{1}$ & $V_{\mathrm{m}}^{\mathrm{E}} / \mathrm{cm}^{3} \cdot \mathrm{mol}^{-1}$ & $x_{1}$ & $V_{\mathrm{m}}^{\mathrm{E}} / \mathrm{cm}^{3} \cdot \mathrm{mol}^{-1}$ \\
\hline \multicolumn{6}{|c|}{$293.15 \mathrm{~K}$} \\
\hline 0.0524 & -0.3025 & 0.3880 & -1.206 & 0.6957 & -0.9143 \\
\hline 0.0973 & -0.5139 & 0.4557 & -1.212 & 0.7457 & -0.8029 \\
\hline 0.1507 & -0.7431 & 0.4952 & -1.195 & 0.7948 & -0.6538 \\
\hline 0.1919 & -0.8772 & 0.5470 & -1.151 & 0.8424 & -0.5028 \\
\hline 0.2246 & -0.9697 & 0.5941 & -1.101 & 0.8878 & -0.3132 \\
\hline 0.2956 & -1.109 & 0.6438 & -1.016 & 0.9420 & -0.1639 \\
\hline 0.3520 & -1.189 & & & & \\
\hline \multicolumn{6}{|c|}{$298.15 \mathrm{~K}$} \\
\hline 0.0511 & -0.3197 & 0.3981 & -1.253 & 0.6936 & -0.9548 \\
\hline 0.0981 & -0.5614 & 0.4533 & -1.258 & 0.7437 & -0.8367 \\
\hline 0.1513 & -0.7778 & 0.4974 & -1.233 & 0.7962 & -0.6992 \\
\hline 0.1970 & -0.9208 & 0.5477 & -1.191 & 0.8453 & -0.5500 \\
\hline 0.2549 & -1.072 & 0.5911 & -1.134 & 0.8882 & -0.3801 \\
\hline 0.2952 & -1.165 & 0.6526 & -1.033 & 0.9440 & -0.1670 \\
\hline 0.3519 & -1.222 & & & & \\
\hline \multicolumn{6}{|c|}{$303.15 \mathrm{~K}$} \\
\hline 0.0534 & -0.3274 & 0.3956 & -1.299 & 0.6936 & -0.9736 \\
\hline 0.1012 & -0.5788 & 0.4463 & -1.297 & 0.7493 & -0.8030 \\
\hline 0.1519 & -0.7966 & 0.5090 & -1.262 & 0.7950 & -0.6424 \\
\hline 0.1921 & -0.9332 & 0.5530 & -1.230 & 0.8515 & -0.4610 \\
\hline 0.2284 & -1.043 & 0.5860 & -1.182 & 0.8903 & -0.3320 \\
\hline 0.2962 & -1.185 & 0.6574 & -1.057 & 0.9418 & -0.1799 \\
\hline 0.3566 & -1.265 & & & & \\
\hline
\end{tabular}

Tabla 3-13: velocidades del sonido de exceso, coeficientes de expansión térmica a $P=$ cte. de exceso, y compresibilidades isoentrópicas de exceso del sistema anilina (1) + 2-butanona (2)

\begin{tabular}{cccccccc}
\hline$x_{1}$ & $u^{\mathrm{E}} / \mathrm{m} \cdot \mathrm{s}^{-1}$ & $\alpha_{\mathrm{P}}^{\mathrm{E}} / 10^{-6} \mathrm{~K}^{-1}$ & $\kappa_{S}^{\mathrm{E}} / \mathrm{TPa}^{-1}$ & $x_{1}$ & $u^{\mathrm{E}} / \mathrm{m} \cdot \mathrm{s}^{-1}$ & $\alpha_{\mathrm{P}}^{\mathrm{E}} / 10^{-6} \mathrm{~K}^{-1}$ & $\kappa_{S}^{\mathrm{E}} / \mathrm{TPa}^{-1}$ \\
\hline 0.0511 & 19.9 & -27.78 & -30.72 & 0.5477 & 126.1 & -80.88 & -108.67 \\
0.0981 & 37.0 & -40.65 & -54.06 & 0.5911 & 125.9 & -76.98 & -102.98 \\
0.1513 & 54.8 & -47.37 & -74.99 & 0.6526 & 122.3 & -63.97 & -92.82 \\
0.1970 & 68.7 & -50.42 & -89.03 & 0.6936 & 117.5 & -50.41 & -84.78 \\
0.2549 & 84.6 & -54.39 & -102.50 & 0.7437 & 108.9 & -29.43 & -73.72 \\
0.2952 & 94.7 & -58.27 & -109.46 & 0.7962 & 96.1 & -4.79 & -60.78 \\
0.3519 & 106.0 & -64.76 & -114.72 & 0.8453 & 80.4 & 16.73 & -47.57 \\
0.3981 & 113.8 & -70.85 & -116.76 & 0.8882 & 63.1 & 30.26 & -34.99 \\
0.4533 & 120.7 & -77.40 & -116.13 & 0.9440 & 35.1 & 31.31 & -17.81 \\
0.4974 & 124.2 & -80.65 & -113.48 & & & & \\
\hline
\end{tabular}

Tabla 3-14: coeficientes y desviaciones estándar del ajuste a la ecuación R-K de las diferentes propiedades de exceso del sistema anilina (1) + 2-butanona (2)

\begin{tabular}{ccccccc}
\hline$T / \mathrm{K}$ & Propiedad & $A_{0}$ & $A_{1}$ & $A_{2}$ & $A_{3}$ & $\sigma$ \\
\hline 293.15 & $V_{\mathrm{m}}^{\mathrm{E}} / \mathrm{cm}^{3} \cdot \mathrm{mol}^{-1}$ & -4.79 & 1.40 & & & 0.018 \\
298.15 & $V_{\mathrm{m}}^{\mathrm{E}} / \mathrm{cm}^{3} \cdot \mathrm{mol}^{-1}$ & -4.985 & 1.38 & & & 0.016 \\
& $u^{\mathrm{E}} /{\mathrm{m} \cdot \mathrm{s}^{-1}}^{\mathrm{E}}$ & 497.3 & 120.3 & 49.5 & 31 & 0.12 \\
& $\alpha_{\mathrm{p}}^{\mathrm{E}} / 10^{-6} \mathrm{~K}^{-1}$ & -322.94 & -85.2 & 430.5 & 927.8 & 0.09 \\
& $\kappa_{S}^{\mathrm{E}} / \mathrm{TPa}^{-1}$ & -453.5 & 155.1 & -40.64 & 9 & 0.16 \\
303.15 & $V_{\mathrm{m}}^{\mathrm{E}} / \mathrm{cm}^{3} \cdot \mathrm{mol}^{-1}$ & -5.135 & 1.35 & 0.39 & 0.85 & 0.009 \\
\hline
\end{tabular}



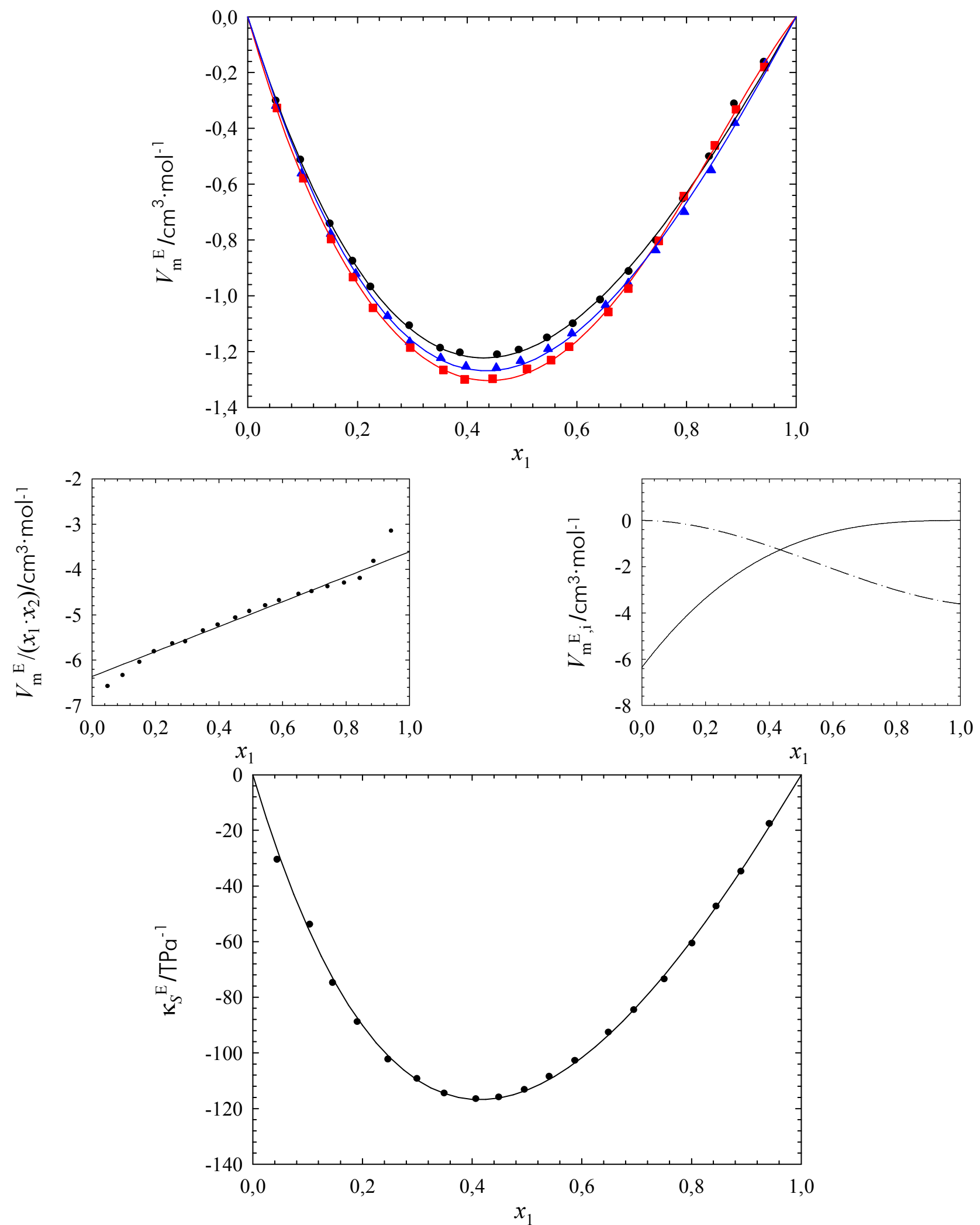

Ilustración 3-19: volúmenes molares de exceso $V_{\mathrm{m}}^{\mathrm{E}}$, volúmenes molares de exceso reducidos $V_{\mathrm{m}}^{\mathrm{E}} /\left(x_{1} \cdot x_{2}\right)$, volúmenes molares parciales de exceso $V_{\mathrm{m}, \mathrm{i}}^{\mathrm{E}}$ y compresibilidades isoentrópicas de exceso $\kappa_{S}^{\mathrm{E}}$ del sistema anilina (1) +2 -butanona (2) 
Tabla 3-15: volúmenes molares de exceso del sistema anilina (1) + 2-pentanona (2)

\begin{tabular}{|c|c|c|c|c|c|}
\hline$x_{1}$ & $V_{\mathrm{m}}^{\mathrm{E}} / \mathrm{cm}^{3} \cdot \mathrm{mol}^{-1}$ & $x_{1}$ & $V_{\mathrm{m}}^{\mathrm{E}} / \mathrm{cm}^{3} \cdot \mathrm{mol}^{-1}$ & $x_{1}$ & $V_{\mathrm{m}}^{\mathrm{E}} / \mathrm{cm}^{3} \cdot \mathrm{mol}^{-1}$ \\
\hline \multicolumn{6}{|c|}{$293.15 \mathrm{~K}$} \\
\hline 0.0587 & -0.2684 & 0.3974 & -1.031 & 0.6987 & -0.7669 \\
\hline 0.1105 & -0.4668 & 0.4548 & -1.039 & 0.7443 & -0.6728 \\
\hline 0.1614 & -0.6289 & 0.4951 & -1.024 & 0.8075 & -0.5267 \\
\hline 0.2055 & -0.7473 & 0.5485 & -0.9789 & 0.8543 & -0.4074 \\
\hline 0.2505 & -0.8443 & 0.5919 & -0.9362 & 0.9046 & -0.2719 \\
\hline 0.3032 & -0.9322 & 0.6487 & -0.8525 & 0.9489 & -0.1471 \\
\hline 0.3539 & -1.005 & & & & \\
\hline \multicolumn{6}{|c|}{$298.15 \mathrm{~K}$} \\
\hline 0.0610 & -0.2579 & 0.3945 & -1.087 & 0.7007 & -0.8060 \\
\hline 0.1110 & -0.5046 & 0.4489 & -1.119 & 0.7487 & -0.7084 \\
\hline 0.1607 & -0.6664 & 0.4995 & -1.103 & 0.8061 & -0.5651 \\
\hline 0.2057 & -0.7973 & 0.5493 & -1.057 & 0.8503 & -0.4431 \\
\hline 0.2559 & -0.9149 & 0.5942 & -1.003 & 0.9052 & -0.2858 \\
\hline 0.3037 & -1.000 & 0.6464 & -0.9232 & 0.9554 & -0.1374 \\
\hline 0.3523 & -1.065 & & & & \\
\hline \multicolumn{6}{|c|}{$303.15 \mathrm{~K}$} \\
\hline 0.0730 & -0.3726 & 0.4025 & -1.198 & 0.7024 & -0.8927 \\
\hline 0.1087 & -0.5176 & 0.4511 & -1.199 & 0.7462 & -0.7821 \\
\hline 0.1604 & -0.7122 & 0.4988 & -1.190 & 0.8048 & -0.6201 \\
\hline 0.2043 & -0.8445 & 0.5530 & -1.148 & 0.8511 & -0.4681 \\
\hline 0.2571 & -0.9800 & 0.5901 & -1.099 & 0.9102 & -0.2883 \\
\hline 0.2958 & -1.0492 & 0.6475 & -1.005 & 0.9518 & -0.1553 \\
\hline 0.3457 & -1.1538 & & & & \\
\hline
\end{tabular}

Tabla 3-16: velocidades del sonido de exceso, coeficientes de expansión térmica a $P=$ cte. de exceso, y compresibilidades isoentrópicas de exceso del sistema anilina (1) + 2-pentanona (2)

\begin{tabular}{cccccccc}
\hline$x_{1}$ & $u^{\mathrm{E}} / \mathrm{m} \cdot \mathrm{s}^{-1}$ & $\alpha_{\mathrm{P}}^{\mathrm{E}} / 10^{-6} \mathrm{~K}^{-1}$ & $\kappa_{S}^{\mathrm{E}} / \mathrm{TPa}^{-1}$ & $x_{1}$ & $u^{\mathrm{E}} / \mathrm{m} \cdot \mathrm{s}^{-1}$ & $\alpha_{\mathrm{P}}^{\mathrm{E}} / 10^{-6} \mathrm{~K}^{-1}$ & $\kappa_{S}^{\mathrm{E}} / \mathrm{TPa}^{-1}$ \\
\hline 0.0610 & 17.0 & -25.10 & -24.55 & 0.5493 & 107.6 & -159.33 & -94.04 \\
0.1110 & 31.5 & -48.05 & -43.64 & 0.5942 & 108.6 & -153.47 & -90.11 \\
0.1607 & 44.4 & -70.48 & -58.49 & 0.6464 & 107.6 & -142.25 & -83.90 \\
0.2057 & 55.6 & -90.23 & -69.93 & 0.7007 & 103.4 & -125.88 & -75.33 \\
0.2559 & 67.1 & -110.57 & -80.21 & 0.7487 & 97.5 & -108.33 & -66.73 \\
0.3037 & 77.0 & -127.56 & -87.67 & 0.8061 & 86.1 & -84.16 & -54.45 \\
0.3523 & 86.0 & -141.81 & -93.12 & 0.8503 & 73.7 & -64.17 & -43.75 \\
0.3945 & 92.6 & -151.19 & -95.86 & 0.9052 & 53.3 & -39.02 & -29.05 \\
0.4489 & 99.9 & -159.14 & -97.66 & 0.9554 & 28.3 & -17.17 & -14.24 \\
0.4995 & 104.7 & -161.59 & -96.87 & & & & \\
\hline
\end{tabular}

Tabla 3-17: coeficientes y desviaciones estándar del ajuste a la ecuación R-K de las diferentes propiedades de exceso del sistema anilina (1) + 2-pentanona (2)

\begin{tabular}{ccccccc}
\hline$T / \mathrm{K}$ & Propiedad & $A_{0}$ & $A_{1}$ & $A_{2}$ & $A_{3}$ & $\sigma$ \\
\hline 293.15 & $V_{\mathrm{m}}^{\mathrm{E}} / \mathrm{cm}^{3} \cdot \mathrm{mol}^{-1}$ & -4.080 & 1.006 & 0.22 & & 0.005 \\
298.15 & $V_{\mathrm{m}}^{\mathrm{E}} / \mathrm{cm}^{3} \cdot \mathrm{mol}^{-1}$ & -4.384 & 1.05 & 0.38 & & 0.010 \\
& $u^{\mathrm{E}} / \mathrm{m}^{-1}$ & 418.0 & 154.5 & 70.4 & 54 & 0.2 \\
& $\alpha_{\mathrm{P}}^{\mathrm{E}} / 10^{-6} \mathrm{~K}^{-1}$ & -646.06 & -2.1 & 278.8 & 19.2 & 0.10 \\
& $\kappa_{S}^{\mathrm{E}} / \mathrm{TPa}^{-1}$ & -387.6 & 66.7 & & 0.3 \\
303.15 & $V_{\mathrm{m}}^{\mathrm{E}} / \mathrm{cm}^{3} \cdot \mathrm{mol}^{-1}$ & -4.762 & 1.07 & 0.49 & & 0.008 \\
\hline
\end{tabular}



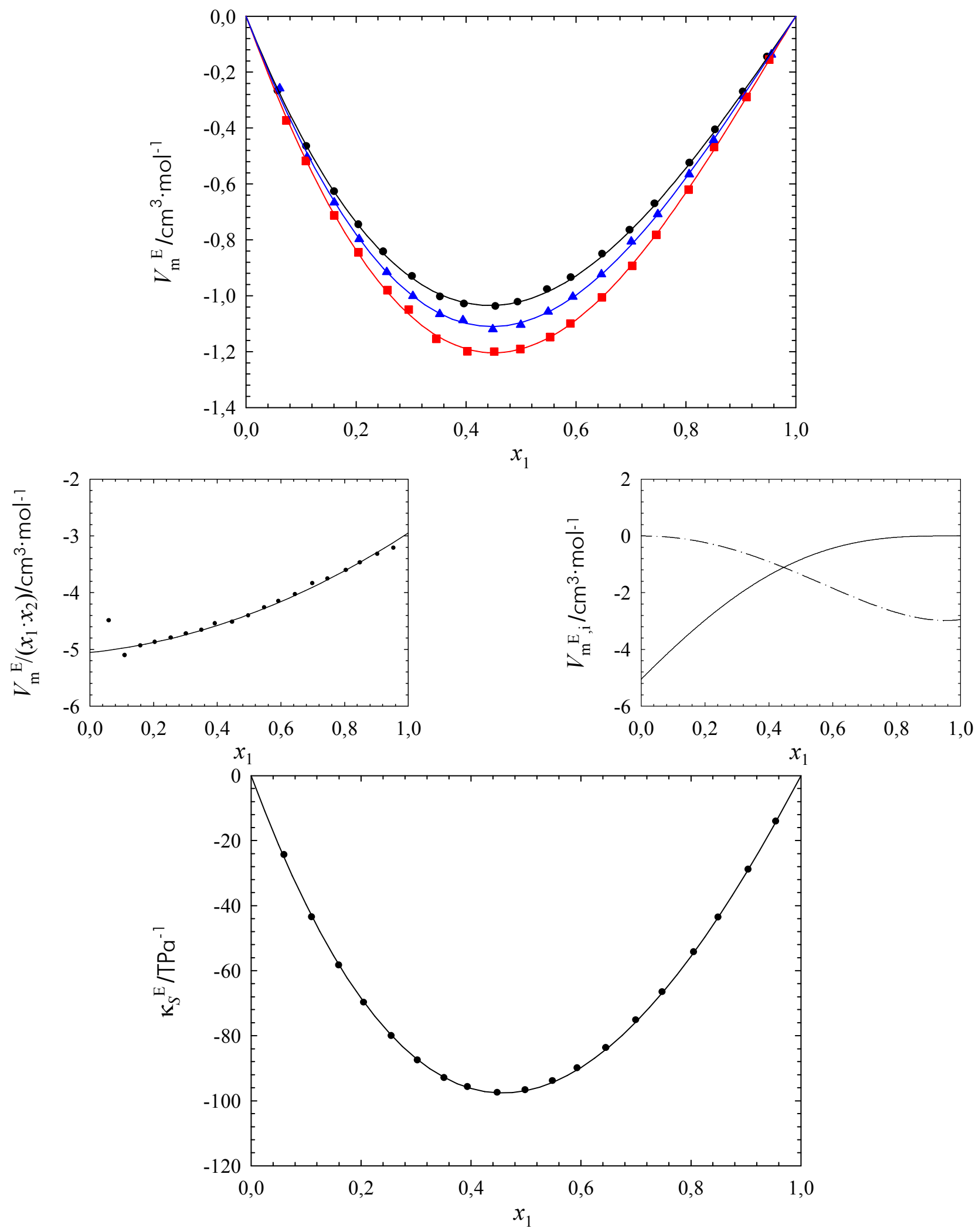

Ilustración 3-20: volúmenes molares de exceso $V_{\mathrm{m}}^{\mathrm{E}}$, volúmenes molares de exceso reducidos $V_{\mathrm{m}}^{\mathrm{E}} /\left(x_{1} \cdot x_{2}\right)$, volúmenes molares parciales de exceso $V_{\mathrm{m}, \mathrm{i}}^{\mathrm{E}}$ y compresibilidades isoentrópicas de exceso $\kappa_{S}^{\mathrm{E}}$ del sistema anilina (1) +2 -pentanona (2) 
Tabla 3-18: volúmenes molares de exceso del sistema anilina (1) + 2-heptanona (2)

\begin{tabular}{|c|c|c|c|c|c|}
\hline$x_{1}$ & $V_{\mathrm{m}}^{\mathrm{E}} / \mathrm{cm}^{3} \cdot \mathrm{mol}^{-1}$ & $x_{1}$ & $V_{\mathrm{m}}^{\mathrm{E}} / \mathrm{cm}^{3} \cdot \mathrm{mol}^{-1}$ & $x_{1}$ & $V_{\mathrm{m}}^{\mathrm{E}} / \mathrm{cm}^{3} \cdot \mathrm{mol}^{-1}$ \\
\hline \multicolumn{6}{|c|}{$293.15 \mathrm{~K}$} \\
\hline 0.0522 & -0.2120 & 0.3974 & -0.8355 & 0.6957 & -0.6671 \\
\hline 0.0989 & -0.3576 & 0.4467 & -0.8443 & 0.7394 & -0.5963 \\
\hline 0.1460 & -0.4808 & 0.4836 & -0.8456 & 0.7855 & -0.5213 \\
\hline 0.1970 & -0.6134 & 0.5261 & -0.8326 & 0.8430 & -0.4041 \\
\hline 0.2493 & -0.6893 & 0.5892 & -0.7912 & 0.8882 & -0.3056 \\
\hline 0.2999 & -0.7575 & 0.6383 & -0.7243 & 0.9364 & -0.1688 \\
\hline 0.3589 & -0.8133 & & & & \\
\hline \multicolumn{6}{|c|}{$298.15 \mathrm{~K}$} \\
\hline 0.0533 & -0.2173 & 0.4036 & -0.8530 & 0.6936 & -0.6705 \\
\hline 0.0967 & -0.3526 & 0.4570 & -0.8696 & 0.7425 & -0.6022 \\
\hline 0.1485 & -0.4939 & 0.4908 & -0.8666 & 0.7867 & -0.5162 \\
\hline 0.1959 & -0.6046 & 0.5463 & -0.8401 & 0.8399 & -0.4111 \\
\hline 0.2416 & -0.6855 & 0.5998 & -0.8036 & 0.8885 & -0.3012 \\
\hline 0.2943 & -0.7685 & 0.6362 & -0.7445 & 0.9381 & -0.1659 \\
\hline 0.3401 & -0.8168 & & & & \\
\hline \multicolumn{6}{|c|}{$303.15 \mathrm{~K}$} \\
\hline 0.0526 & -0.2198 & 0.4128 & -0.8741 & 0.6949 & -0.6927 \\
\hline 0.0982 & -0.3667 & 0.4580 & -0.8874 & 0.7461 & -0.6148 \\
\hline 0.1457 & -0.4954 & 0.5035 & -0.8767 & 0.7939 & -0.5181 \\
\hline 0.1946 & -0.6149 & 0.5517 & -0.8603 & 0.8437 & -0.4153 \\
\hline 0.2454 & -0.7130 & 0.5921 & -0.8309 & 0.8913 & -0.2997 \\
\hline 0.2922 & -0.7811 & 0.6408 & -0.7579 & 0.9390 & -0.1640 \\
\hline 0.3488 & -0.8435 & & & & \\
\hline
\end{tabular}

Tabla 3-19: velocidades del sonido de exceso, coeficientes de expansión térmica a $P=$ cte. de exceso, y compresibilidades isoentrópicas de exceso del sistema anilina (1) + 2-heptanona (2)

\begin{tabular}{cccccccc}
\hline$x_{1}$ & $u^{\mathrm{E}} / \mathrm{m} \cdot \mathrm{s}^{-1}$ & $\alpha_{\mathrm{P}}^{\mathrm{E}} / 10^{-6} \mathrm{~K}^{-1}$ & $\kappa_{S}^{\mathrm{E}} / \mathrm{TPa}^{-1}$ & $x_{1}$ & $u^{\mathrm{E}} / \mathrm{m} \cdot \mathrm{s}^{-1}$ & $\alpha_{\mathrm{P}}^{\mathrm{E}} / 10^{-6} \mathrm{~K}^{-1}$ & $\kappa_{S}^{\mathrm{E}} / \mathrm{TPa}^{-1}$ \\
\hline 0.0533 & 9.4 & 1.66 & -12.32 & 0.5463 & 72.4 & -31.26 & -63.33 \\
0.0967 & 16.7 & 0.73 & -21.13 & 0.5998 & 74.8 & -29.20 & -61.95 \\
0.1485 & 25.2 & -2.51 & -30.70 & 0.6362 & 75.2 & -26.53 & -59.88 \\
0.1959 & 32.7 & -6.85 & -38.50 & 0.6936 & 74.5 & -21.09 & -55.59 \\
0.2416 & 39.6 & -11.64 & -44.98 & 0.7425 & 72.1 & -15.46 & -50.70 \\
0.2943 & 47.0 & -17.46 & -51.30 & 0.7867 & 67.7 & -9.89 & -44.93 \\
0.3401 & 53.0 & -22.15 & -55.72 & 0.8399 & 59.5 & -3.59 & -36.61 \\
0.4036 & 60.4 & -27.45 & -60.21 & 0.8885 & 48.1 & 0.93 & -27.45 \\
0.4570 & 65.8 & -30.43 & -62.58 & 0.9381 & 31.1 & 3.17 & -16.31 \\
0.4908 & 68.7 & -31.39 & -63.36 & & & & \\
\hline
\end{tabular}

Tabla 3-20: coeficientes y desviaciones estándar del ajuste a la ecuación R-K de las diferentes propiedades de exceso del sistema anilina (1) + 2-heptanona (2)

\begin{tabular}{ccccccccc}
\hline$T / \mathrm{K}$ & Propiedad & $A_{0}$ & $A_{1}$ & $A_{2}$ & $A_{3}$ & $A_{4}$ & $A_{5}$ & $\sigma$ \\
\hline 293.15 & $V_{\mathrm{m}}^{\mathrm{E}} / \mathrm{cm}^{3} \cdot \mathrm{mol}^{-1}$ & -3.348 & 0.62 & -0.25 & & & & 0.007 \\
298.15 & $V_{\mathrm{m}}^{\mathrm{E}} / \mathrm{cm}^{3} \cdot \mathrm{mol}^{-1}$ & -3.443 & 0.64 & & & & 0.007 \\
& $u^{\mathrm{E}} / \mathrm{m}^{-1}$ & 277.55 & 150.2 & 76.0 & 49 & 43 & 21 & 0.06 \\
& $\alpha_{\mathrm{P}}^{\mathrm{E}} / 10^{-6} \mathrm{~K}^{-1}$ & -126.10 & -21.7 & 216.3 & 46.3 & & 0.06 \\
303.15 & $\kappa_{S}^{\mathrm{E}} / \mathrm{TPa}^{-1}$ & -253.73 & -18.1 & -4.7 & -6.23 & -8 & & 0.07 \\
\hline
\end{tabular}



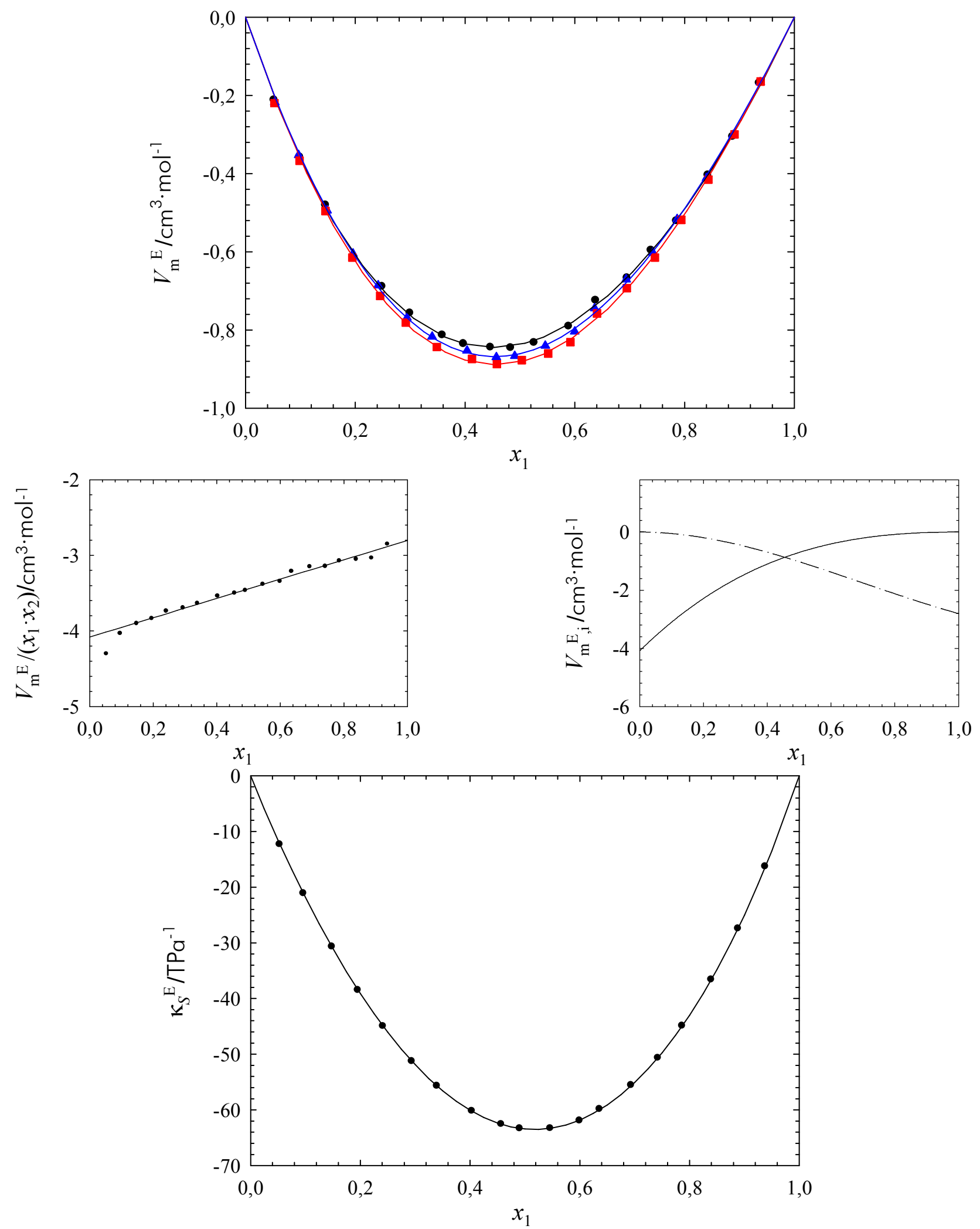

llustración 3-21: volúmenes molares de exceso $V_{\mathrm{m}}^{\mathrm{E}}$, volúmenes molares de exceso reducidos $V_{\mathrm{m}}^{\mathrm{E}} /\left(x_{1} \cdot x_{2}\right)$, volúmenes molares parciales de exceso $V_{\mathrm{m}, \mathrm{i}}^{\mathrm{E}}$ y compresibilidades isoentrópicas de exceso $\kappa_{S}^{\mathrm{E}}$ del sistema anilina (1) +2 -heptanona (2) 
Tabla 3-21: volúmenes molares de exceso del sistema $N$-metilanilina (1) +2 -propanona (2)

\begin{tabular}{|c|c|c|c|c|c|}
\hline$x_{1}$ & $V_{\mathrm{m}}^{\mathrm{E}} / \mathrm{cm}^{3} \cdot \mathrm{mol}^{-1}$ & $x_{1}$ & $V_{\mathrm{m}}^{\mathrm{E}} / \mathrm{cm}^{3} \cdot \mathrm{mol}^{-1}$ & $x_{1}$ & $V_{\mathrm{m}}^{\mathrm{E}} / \mathrm{cm}^{3} \cdot \mathrm{mol}^{-1}$ \\
\hline \multicolumn{6}{|c|}{$293.15 \mathrm{~K}$} \\
\hline 0.0452 & -0.1564 & 0.4035 & -0.6994 & 0.7048 & -0.5209 \\
\hline 0.0947 & -0.3047 & 0.4511 & -0.6971 & 0.7490 & -0.4576 \\
\hline 0.1437 & -0.4247 & 0.5019 & -0.6888 & 0.8084 & -0.3664 \\
\hline 0.1980 & -0.5245 & 0.5522 & -0.6619 & 0.8506 & -0.2955 \\
\hline 0.2528 & -0.5948 & 0.5961 & -0.6344 & 0.8968 & -0.2094 \\
\hline 0.2982 & -0.6508 & 0.6445 & -0.5953 & 0.9435 & -0.1177 \\
\hline 0.3515 & -0.6849 & & & & \\
\hline \multicolumn{6}{|c|}{$298.15 \mathrm{~K}$} \\
\hline 0.0512 & -0.1884 & 0.3996 & -0.7371 & 0.7081 & -0.5456 \\
\hline 0.1014 & -0.3377 & 0.4437 & -0.7343 & 0.7497 & -0.4773 \\
\hline 0.1482 & -0.4516 & 0.5052 & -0.7256 & 0.8004 & -0.3971 \\
\hline 0.1991 & -0.5449 & 0.5480 & -0.6986 & 0.8553 & -0.2985 \\
\hline 0.2494 & -0.6268 & 0.6076 & -0.6516 & 0.9016 & -0.2105 \\
\hline 0.3038 & -0.6904 & 0.6489 & -0.6199 & 0.9560 & -0.0971 \\
\hline 0.3527 & -0.7266 & & & & \\
\hline \multicolumn{6}{|c|}{$303.15 \mathrm{~K}$} \\
\hline 0.0516 & -0.2065 & 0.3533 & -0.7715 & 0.7166 & -0.5653 \\
\hline 0.1023 & -0.3691 & 0.4051 & -0.7909 & 0.7558 & -0.4829 \\
\hline 0.1531 & -0.4966 & 0.5096 & -0.7704 & 0.8063 & -0.4108 \\
\hline 0.2007 & -0.5956 & 0.5540 & -0.7526 & 0.8476 & -0.3337 \\
\hline 0.2576 & -0.6836 & 0.6009 & -0.7029 & 0.9066 & -0.2135 \\
\hline 0.3072 & -0.7384 & 0.6643 & -0.6316 & 0.9539 & -0.1079 \\
\hline
\end{tabular}

Tabla 3-22: velocidades del sonido de exceso, coeficientes de expansión térmica a $P=$ cte. de exceso, y compresibilidades isoentrópicas de exceso del sistema $N$-metilanilina (1) + 2-propanona (2)

\begin{tabular}{cccccccc}
\hline$x_{1}$ & $u^{\mathrm{E}} / \mathrm{m} \cdot \mathrm{s}^{-1}$ & $\alpha_{\mathrm{P}}^{\mathrm{E}} / 10^{-6} \mathrm{~K}^{-1}$ & $\kappa_{S}^{\mathrm{E}} / \mathrm{TPa}^{-1}$ & $x_{1}$ & $u^{\mathrm{E}} / \mathrm{m} \cdot \mathrm{s}^{-1}$ & $\alpha_{\mathrm{P}}^{\mathrm{E}} / 10^{-6} \mathrm{~K}^{-1}$ & $\kappa_{S}^{\mathrm{E}} / \mathrm{TPa}^{-1}$ \\
\hline 0.0512 & 19.8 & -26.30 & -32.34 & 0.5480 & 101.1 & -79.97 & -91.51 \\
0.1014 & 37.0 & -47.23 & -56.69 & 0.6076 & 97.7 & -71.02 & -82.84 \\
0.1482 & 51.1 & -62.79 & -73.81 & 0.6489 & 94.0 & -64.07 & -76.19 \\
0.1991 & 64.3 & -75.58 & -87.21 & 0.7081 & 86.1 & -53.12 & -65.36 \\
0.2494 & 75.6 & -84.65 & -96.46 & 0.7497 & 78.2 & -45.01 & -56.75 \\
0.3038 & 85.5 & -90.62 & -102.29 & 0.8004 & 67.1 & -35.09 & -46.09 \\
0.3527 & 92.4 & -92.94 & -104.40 & 0.8553 & 52.4 & -24.49 & -33.93 \\
0.3996 & 97.0 & -92.68 & -103.80 & 0.9016 & 38.0 & -15.97 & -23.36 \\
0.4437 & 100.2 & -90.63 & -101.86 & 0.9560 & 18.3 & -6.69 & -10.58 \\
0.5052 & 101.8 & -85.28 & -96.68 & & & & \\
\hline
\end{tabular}

Tabla 3-23: coeficientes y desviaciones estándar del ajuste a la ecuación R-K de las diferentes propiedades de exceso del sistema $N$-metilanilina (1) + 2-propanona (2)

\begin{tabular}{|c|c|c|c|c|c|c|}
\hline$T / \mathrm{K}$ & Propiedad & $A_{0}$ & $A_{1}$ & $A_{2}$ & $A_{3}$ & $\sigma$ \\
\hline 293.15 & $V_{\mathrm{m}}^{\mathrm{E}} / \mathrm{cm}^{3} \cdot \mathrm{mol}^{-1}$ & -2.760 & 0.754 & -0.22 & & 0.004 \\
\hline \multirow[t]{4}{*}{298.15} & $V_{\mathrm{m}}^{\mathrm{E}} / \mathrm{cm}^{3} \cdot \mathrm{mol}^{-1}$ & -2.908 & 0.801 & -0.19 & & 0.004 \\
\hline & $u^{\mathrm{E}} / \mathrm{m} \cdot \mathrm{s}^{-1}$ & 407.00 & 13.5 & 14.8 & & 0.11 \\
\hline & $\alpha_{\mathrm{P}}^{\mathrm{E}} / 10^{-6} \mathrm{~K}^{-1}$ & -343.32 & 211.97 & -10.1 & & 0.05 \\
\hline & $\kappa_{S}^{\mathrm{E}} / \mathrm{TPa}^{-1}$ & -388.4 & 204.4 & -83.9 & 31 & 0.14 \\
\hline 303.15 & $V_{\mathrm{m}}^{\mathrm{E}} / \mathrm{cm}^{3} \cdot \mathrm{mol}^{-1}$ & -3.106 & 0.907 & -0.20 & & 0.005 \\
\hline
\end{tabular}



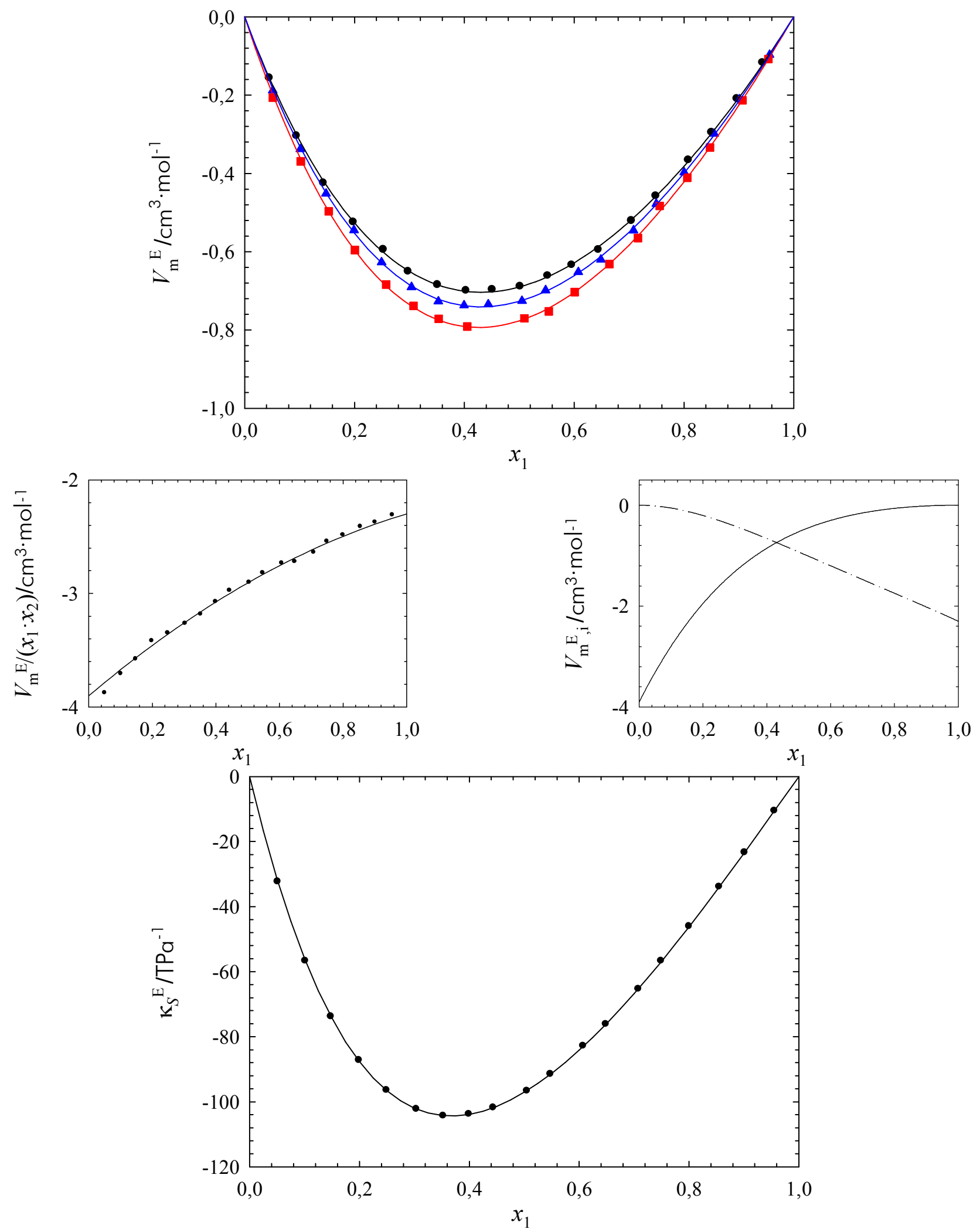

Ilustración 3-22: volúmenes molares de exceso $V_{\mathrm{m}}^{\mathrm{E}}$, volúmenes molares de exceso reducidos $V_{\mathrm{m}}^{\mathrm{E}} /\left(x_{1} \cdot x_{2}\right)$, volúmenes molares parciales de exceso $V_{\mathrm{m}, \mathrm{i}}^{\mathrm{E}}$ y compresibilidades isoentrópicas de exceso $\kappa_{S}^{\mathrm{E}}$ del sistema $N$-metilanilina (1) +2 -propanona (2) 
Tabla 3-24: volúmenes molares de exceso del sistema $N$-metilanilina (1) + 2-butanona (2)

\begin{tabular}{|c|c|c|c|c|c|}
\hline$x_{1}$ & $V_{\mathrm{m}}^{\mathrm{E}} / \mathrm{cm}^{3} \cdot \mathrm{mol}^{-1}$ & $x_{1}$ & $V_{\mathrm{m}}^{\mathrm{E}} / \mathrm{cm}^{3} \cdot \mathrm{mol}^{-1}$ & $x_{1}$ & $V_{\mathrm{m}}^{\mathrm{E}} / \mathrm{cm}^{3} \cdot \mathrm{mol}^{-1}$ \\
\hline \multicolumn{6}{|c|}{$293.15 \mathrm{~K}$} \\
\hline 0.0475 & -0.1415 & 0.4041 & -0.6421 & 0.6982 & -0.4888 \\
\hline 0.1019 & -0.2925 & 0.4492 & -0.6519 & 0.7462 & -0.4313 \\
\hline 0.1508 & -0.3749 & 0.5093 & -0.6464 & 0.7942 & -0.3659 \\
\hline 0.2062 & -0.4688 & 0.5565 & -0.6256 & 0.8436 & -0.2859 \\
\hline 0.2511 & -0.5203 & 0.6062 & -0.5814 & 0.8867 & -0.2140 \\
\hline 0.3471 & -0.6126 & 0.6531 & -0.5388 & 0.9373 & -0.1220 \\
\hline \multicolumn{6}{|c|}{$298.15 \mathrm{~K}$} \\
\hline 0.0528 & -0.1697 & 0.4008 & -0.6667 & 0.7033 & -0.5070 \\
\hline 0.1007 & -0.2918 & 0.4529 & -0.6678 & 0.7411 & -0.4612 \\
\hline 0.1530 & -0.4009 & 0.5050 & -0.6593 & 0.7924 & -0.3850 \\
\hline 0.1993 & -0.4866 & 0.5523 & -0.6495 & 0.8434 & -0.2977 \\
\hline 0.2537 & -0.5624 & 0.6027 & -0.6117 & 0.8885 & -0.2205 \\
\hline 0.3079 & -0.6281 & 0.6529 & -0.5683 & 0.9240 & -0.1540 \\
\hline \multicolumn{6}{|c|}{$303.15 \mathrm{~K}$} \\
\hline 0.0476 & -0.1599 & 0.4016 & -0.6998 & 0.6939 & -0.5407 \\
\hline 0.1035 & -0.3135 & 0.4464 & -0.7015 & 0.7409 & -0.4795 \\
\hline 0.1503 & -0.4237 & 0.4975 & -0.6939 & 0.7964 & -0.3983 \\
\hline 0.1953 & -0.4991 & 0.5528 & -0.6670 & 0.8419 & -0.3172 \\
\hline 0.2457 & -0.5773 & 0.5978 & -0.6449 & 0.8877 & -0.2330 \\
\hline 0.3031 & -0.6432 & 0.6487 & -0.5925 & 0.9359 & -0.1386 \\
\hline 0.3511 & -0.6921 & & & & \\
\hline
\end{tabular}

Tabla 3-25: velocidades del sonido de exceso, coeficientes de expansión térmica a $P=$ cte. de exceso, y compresibilidades isoentrópicas de exceso del sistema $N$-metilanilina (1) +2 -butanona (2)

\begin{tabular}{cccccccc}
\hline$x_{1}$ & $u^{\mathrm{E}} / \mathrm{m} \cdot \mathrm{s}^{-1}$ & $\alpha_{\mathrm{P}}^{\mathrm{E}} / 10^{-6} \mathrm{~K}^{-1}$ & $\kappa_{S}^{\mathrm{E}} / \mathrm{TPa}^{-1}$ & $x_{1}$ & $u^{\mathrm{E}} / \mathrm{m} \cdot \mathrm{s}^{-1}$ & $\alpha_{\mathrm{P}}^{\mathrm{E}} / 10^{-6} \mathrm{~K}^{-1}$ & $\kappa_{S}^{\mathrm{E}} / \mathrm{TPa}^{-1}$ \\
\hline 0.0528 & 14.0 & -19.51 & -21.13 & 0.5523 & 77.1 & -46.20 & -68.76 \\
0.1007 & 25.2 & -32.38 & -36.11 & 0.6027 & 75.7 & -43.22 & -64.06 \\
0.1530 & 36.4 & -42.21 & -49.25 & 0.6529 & 72.9 & -40.06 & -58.54 \\
0.1993 & 45.4 & -48.04 & -58.42 & 0.7033 & 68.1 & -36.52 & -51.91 \\
0.2537 & 54.6 & -52.02 & -66.31 & 0.7411 & 63.5 & -33.65 & -46.52 \\
0.3079 & 62.4 & -53.79 & -71.66 & 0.7924 & 55.3 & -29.22 & -38.43 \\
0.4008 & 71.7 & -52.8 & -74.70 & 0.8434 & 45.2 & -24.02 & -29.73 \\
0.4529 & 74.9 & -50.96 & -73.96 & 0.8885 & 34.5 & -18.59 & -21.65 \\
0.5050 & 76.7 & -48.59 & -71.76 & 0.9240 & 24.9 & -13.58 & -15.02 \\
\hline
\end{tabular}

Tabla 3-26: coeficientes y desviaciones estándar del ajuste a la ecuación R-K de las diferentes propiedades de exceso del sistema $N$-metilanilina (1) +2 -butanona (2)

\begin{tabular}{ccccccc}
\hline$T / \mathrm{K}$ & Propiedad & $A_{0}$ & $A_{1}$ & $A_{2}$ & $A_{3}$ & $\sigma$ \\
\hline 293.15 & $V_{\mathrm{m}}^{\mathrm{E}} / \mathrm{cm}^{3} \cdot \mathrm{mol}^{-1}$ & -2.561 & 0.55 & & & 0.007 \\
298.15 & $V_{\mathrm{m}}^{\mathrm{E}} / \mathrm{cm}^{3} \cdot \mathrm{mol}^{-1}$ & -2.677 & 0.604 & & & 0.005 \\
& $u^{\mathrm{E}} /{\mathrm{m} \cdot \mathrm{s}^{-1}}^{\mathrm{E}}$ & 306.83 & 43.9 & 12.0 & & 0.11 \\
& $\alpha_{\mathrm{p}}^{\mathrm{E}} / 10^{-6} \mathrm{~K}^{-1}$ & -195.56 & 98.1 & -122.0 & 11.5 & 0.05 \\
& $\kappa_{S}^{\mathrm{E}} / \mathrm{TPa}^{-1}$ & -288.5 & 108.0 & -30.5 & 10 & 0.13 \\
303.15 & $V_{\mathrm{m}}^{\mathrm{E}} / \mathrm{cm}^{3} \cdot \mathrm{mol}^{-1}$ & -2.782 & 0.645 & -0.11 & & 0.004 \\
\hline
\end{tabular}



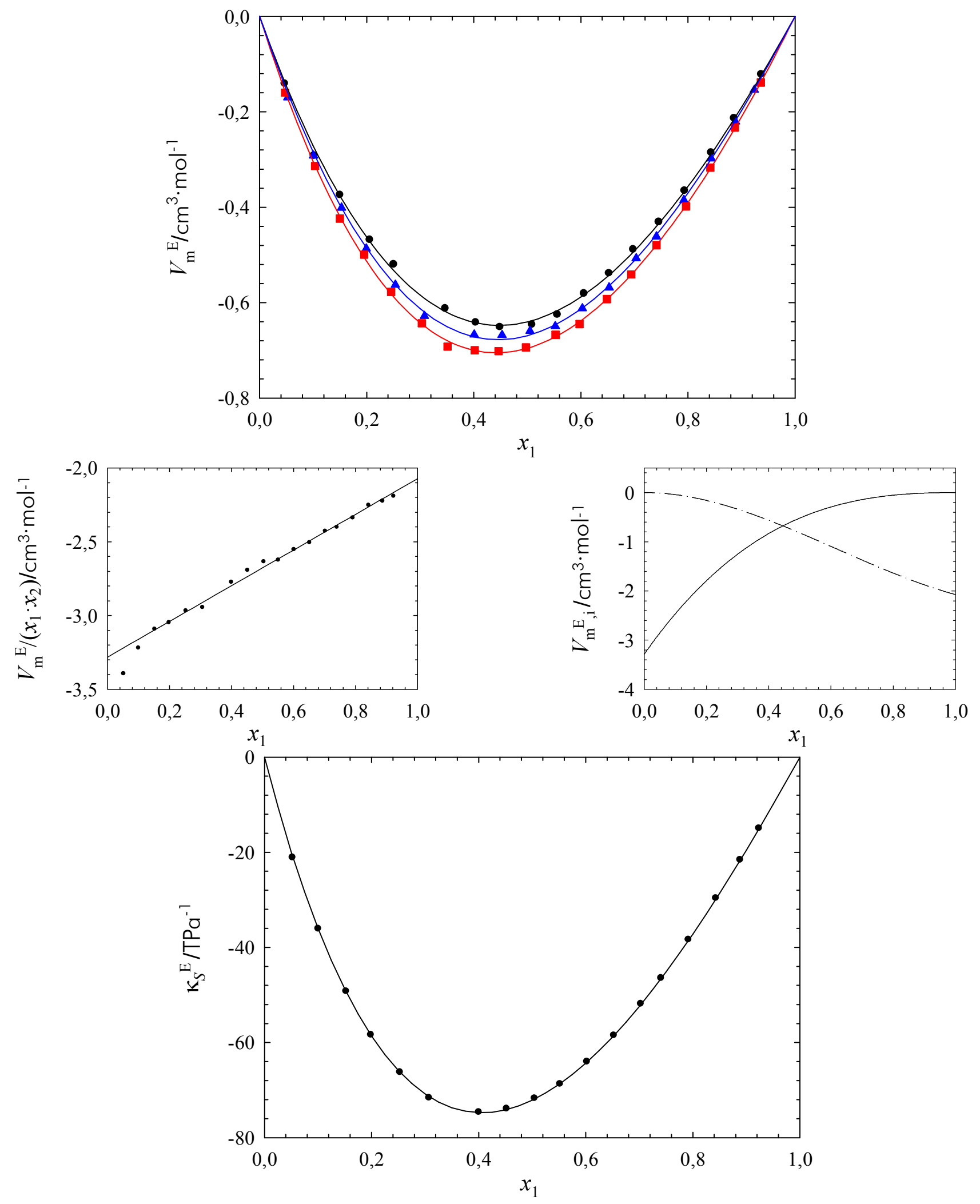

Ilustración 3-23: volúmenes molares de exceso $V_{\mathrm{m}}^{\mathrm{E}}$, volúmenes molares de exceso reducidos $V_{\mathrm{m}}^{\mathrm{E}} /\left(x_{1} \cdot x_{2}\right)$, volúmenes molares parciales de exceso $V_{\mathrm{m}, \mathrm{i}}^{\mathrm{E}}$ y compresibilidades isoentrópicas de exceso $\kappa_{S}^{\mathrm{E}}$ del sistema $N$-metilanilina (1) +2 -butanona (2) 
Tabla 3-27: volúmenes molares de exceso del sistema $N$-metilanilina (1) + 2-pentanona (2)

\begin{tabular}{|c|c|c|c|c|c|}
\hline$x_{1}$ & $V_{\mathrm{m}}^{\mathrm{E}} / \mathrm{cm}^{3} \cdot \mathrm{mol}^{-1}$ & $x_{1}$ & $V_{\mathrm{m}}^{\mathrm{E}} / \mathrm{cm}^{3} \cdot \mathrm{mol}^{-1}$ & $x_{1}$ & $V_{\mathrm{m}}^{\mathrm{E}} / \mathrm{cm}^{3} \cdot \mathrm{mol}^{-1}$ \\
\hline \multicolumn{6}{|c|}{$293.15 \mathrm{~K}$} \\
\hline 0.0536 & -0.1679 & 0.3974 & -0.6585 & 0.6919 & -0.5263 \\
\hline 0.1021 & -0.2802 & 0.4522 & -0.6682 & 0.7645 & -0.4336 \\
\hline 0.1590 & -0.3711 & 0.4966 & -0.6589 & 0.8005 & -0.3809 \\
\hline 0.2037 & -0.4644 & 0.5463 & -0.6395 & 0.8557 & -0.2876 \\
\hline 0.2561 & -0.5488 & 0.5953 & -0.6164 & 0.9041 & -0.2001 \\
\hline 0.3042 & -0.5998 & 0.6483 & -0.5712 & 0.9528 & -0.1021 \\
\hline 0.3471 & -0.6303 & & & & \\
\hline \multicolumn{6}{|c|}{$298.15 \mathrm{~K}$} \\
\hline 0.0584 & -0.1742 & 0.3948 & -0.6811 & 0.6943 & -0.5471 \\
\hline 0.1106 & -0.3043 & 0.4520 & -0.6923 & 0.7509 & -0.4711 \\
\hline 0.1615 & -0.4142 & 0.4986 & -0.6838 & 0.8020 & -0.3937 \\
\hline 0.2051 & -0.4913 & 0.5456 & -0.6685 & 0.8558 & -0.2999 \\
\hline 0.2616 & -0.5765 & 0.5963 & -0.6403 & 0.9000 & -0.2169 \\
\hline 0.3015 & -0.6158 & 0.6502 & -0.5931 & 0.9493 & -0.1123 \\
\hline 0.3470 & -0.6524 & & & & \\
\hline \multicolumn{6}{|c|}{$303.15 \mathrm{~K}$} \\
\hline 0.0589 & -0.1843 & 0.3944 & -0.7075 & 0.6928 & -0.5654 \\
\hline 0.1041 & -0.3003 & 0.4503 & -0.7286 & 0.7470 & -0.4884 \\
\hline 0.1565 & -0.4196 & 0.4925 & -0.7121 & 0.8050 & -0.4000 \\
\hline 0.2024 & -0.5022 & 0.5487 & -0.6929 & 0.8553 & -0.3111 \\
\hline 0.2515 & -0.5896 & 0.5939 & -0.6628 & 0.9008 & -0.2204 \\
\hline 0.2994 & -0.6405 & 0.6490 & -0.6094 & 0.9486 & -0.1193 \\
\hline 0.3539 & -0.6869 & & & & \\
\hline
\end{tabular}

Tabla 3-28: velocidades del sonido de exceso, coeficientes de expansión térmica a $P=$ cte. de exceso, y compresibilidades isoentrópicas de exceso del sistema $N$-metilanilina (1) +2 -pentanona (2)

\begin{tabular}{cccccccc}
\hline$x_{1}$ & $u^{\mathrm{E}} / \mathrm{m} \cdot \mathrm{s}^{-1}$ & $\alpha_{\mathrm{P}}^{\mathrm{E}} / 10^{-6} \mathrm{~K}^{-1}$ & $\kappa_{S}^{\mathrm{E}} / \mathrm{TPa}^{-1}$ & $x_{1}$ & $u^{\mathrm{E}} / \mathrm{m} \cdot \mathrm{s}^{-1}$ & $\alpha_{\mathrm{P}}^{\mathrm{E}} / 10^{-6} \mathrm{~K}^{-1}$ & $\kappa_{S}^{\mathrm{E}} / \mathrm{TPa}^{-1}$ \\
\hline 0.0584 & 12.3 & -12.09 & -17.77 & 0.5456 & 70.7 & -40.8 & -64.35 \\
0.1106 & 22.5 & -21.06 & -30.95 & 0.5963 & 70.7 & -38.21 & -61.20 \\
0.1615 & 31.8 & -28.24 & -41.68 & 0.6502 & 68.9 & -34.65 & -56.48 \\
0.2051 & 39.0 & -33.20 & -49.16 & 0.6943 & 66.1 & -31.25 & -51.79 \\
0.2616 & 47.6 & -38.16 & -56.91 & 0.7509 & 60.4 & -26.27 & -44.53 \\
0.3015 & 52.9 & -40.60 & -60.87 & 0.8020 & 53.0 & -21.35 & -37.03 \\
0.3470 & 58.2 & -42.50 & -64.12 & 0.8558 & 42.8 & -15.80 & -28.14 \\
0.3948 & 62.9 & -43.54 & -66.29 & 0.9000 & 32.3 & -11.07 & -20.20 \\
0.4520 & 67.2 & -43.49 & -67.03 & 0.9493 & 17.9 & -5.62 & -10.57 \\
0.4986 & 69.5 & -42.50 & -66.20 & & & & \\
\hline
\end{tabular}

Tabla 3-29: coeficientes y desviaciones estándar del ajuste a la ecuación R-K de las diferentes propiedades de exceso del sistema $N$-metilanilina (1) +2 -pentanona (2)

\begin{tabular}{ccccccc}
\hline$T / \mathrm{K}$ & Propiedad & $A_{0}$ & $A_{1}$ & $A_{2}$ & $A_{3}$ & $\sigma$ \\
\hline 293.15 & $V_{\mathrm{m}}^{\mathrm{E}} / \mathrm{cm}^{3} \cdot \mathrm{mol}^{-1}$ & -2.641 & 0.44 & & 0.007 \\
298.15 & $V_{\mathrm{m}}^{\mathrm{E}} / \mathrm{cm}^{3} \cdot \mathrm{mol}^{-1}$ & -2.747 & 0.454 & & 0.0018 \\
& $u^{\mathrm{E}} / \mathrm{m}^{-1}$ & 278.15 & 75.5 & 23.1 & 9.3 & 0.04 \\
& $\alpha_{\mathrm{p}}^{\mathrm{E}} / 10^{-6} \mathrm{~K}^{-1}$ & -169.95 & 58.02 & 1.34 & 0.019 & 0.04 \\
303.15 & $\kappa_{S}^{\mathrm{E}} / \mathrm{TPa}^{-1}$ & -264.85 & 57.57 & -8.5 & 0.004 \\
\hline
\end{tabular}



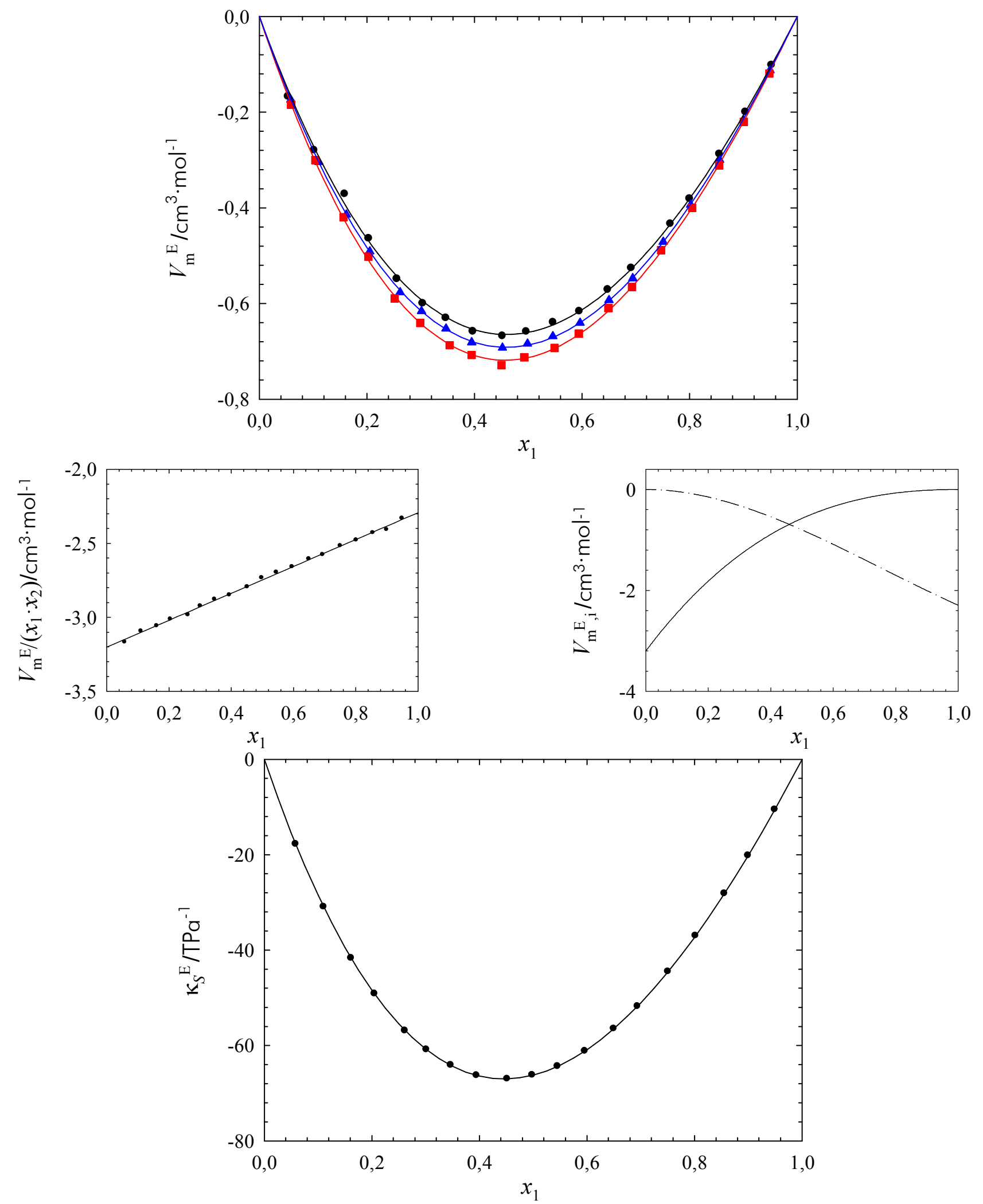

llustración 3-24: volúmenes molares de exceso $V_{\mathrm{m}}^{\mathrm{E}}$, volúmenes molares de exceso reducidos $V_{\mathrm{m}}^{\mathrm{E}} /\left(x_{1} \cdot x_{2}\right)$, volúmenes molares parciales de exceso $V_{\mathrm{m}, \mathrm{i}}^{\mathrm{E}}$ y compresibilidades isoentrópicas de exceso $\kappa_{S}^{\mathrm{E}}$ del sistema $N$-metilanilina (1) +2 -pentanona (2) 
Tabla 3-30: volúmenes molares de exceso del sistema $N$-metilanilina (1) + 2-heptanona (2)

\begin{tabular}{|c|c|c|c|c|c|}
\hline$x_{1}$ & $V_{\mathrm{m}}^{\mathrm{E}} / \mathrm{cm}^{3} \cdot \mathrm{mol}^{-1}$ & $x_{1}$ & $V_{\mathrm{m}}^{\mathrm{E}} / \mathrm{cm}^{3} \cdot \mathrm{mol}^{-1}$ & $x_{1}$ & $V_{\mathrm{m}}^{\mathrm{E}} / \mathrm{cm}^{3} \cdot \mathrm{mol}^{-1}$ \\
\hline \multicolumn{6}{|c|}{$293.15 \mathrm{~K}$} \\
\hline 0.0532 & -0.1060 & 0.3903 & -0.4829 & 0.6965 & -0.3942 \\
\hline 0.0981 & -0.1842 & 0.4373 & -0.4911 & 0.7462 & -0.3511 \\
\hline 0.1488 & -0.2618 & 0.5103 & -0.4904 & 0.7993 & -0.2889 \\
\hline 0.2014 & -0.3362 & 0.5488 & -0.4857 & 0.8488 & -0.2295 \\
\hline 0.2503 & -0.3849 & 0.6040 & -0.4559 & 0.8995 & -0.1568 \\
\hline 0.2951 & -0.4322 & 0.6493 & -0.4341 & 0.9413 & -0.0933 \\
\hline 0.3397 & -0.4556 & & & & \\
\hline \multicolumn{6}{|c|}{$298.15 \mathrm{~K}$} \\
\hline 0.0506 & -0.1092 & 0.4023 & -0.5046 & 0.7013 & -0.4024 \\
\hline 0.1011 & -0.1996 & 0.4535 & -0.5143 & 0.7525 & -0.3573 \\
\hline 0.1501 & -0.2761 & 0.5051 & -0.5145 & 0.7964 & -0.3046 \\
\hline 0.1977 & -0.3405 & 0.5548 & -0.4993 & 0.8476 & -0.2435 \\
\hline 0.2494 & -0.4050 & 0.6006 & -0.4763 & 0.8968 & -0.1714 \\
\hline 0.3096 & -0.4490 & 0.6552 & -0.4442 & 0.9417 & -0.1002 \\
\hline 0.3536 & -0.4791 & & & & \\
\hline \multicolumn{6}{|c|}{$303.15 \mathrm{~K}$} \\
\hline 0.0544 & -0.1186 & 0.4041 & -0.5152 & 0.7073 & -0.4111 \\
\hline 0.1004 & -0.2018 & 0.4531 & -0.5230 & 0.7863 & -0.3293 \\
\hline 0.1542 & -0.2887 & 0.5040 & -0.5264 & 0.7990 & -0.3091 \\
\hline 0.2035 & -0.3565 & 0.5514 & -0.5156 & 0.8481 & -0.2482 \\
\hline 0.2469 & -0.4067 & 0.5998 & -0.4897 & 0.8964 & -0.1764 \\
\hline 0.3045 & -0.4589 & 0.6520 & -0.4565 & 0.9420 & -0.1026 \\
\hline 0.3584 & -0.4960 & & & & \\
\hline
\end{tabular}

Tabla 3-31: velocidades del sonido de exceso, coeficientes de expansión térmica a $P=$ cte. de exceso, y compresibilidades isoentrópicas de exceso del sistema $N$-metilanilina (1) +2 -heptanona (2)

\begin{tabular}{cccccccc}
\hline$x_{1}$ & $u^{\mathrm{E}} / \mathrm{m} \cdot \mathrm{s}^{-1}$ & $\alpha_{\mathrm{P}}^{\mathrm{E}} / 10^{-6} \mathrm{~K}^{-1}$ & $\kappa_{S}^{\mathrm{E}} / \mathrm{TPa}^{-1}$ & $x_{1}$ & $u^{\mathrm{E}} / \mathrm{m} \cdot \mathrm{s}^{-1}$ & $\alpha_{\mathrm{P}}^{\mathrm{E}} / 10^{-6} \mathrm{~K}^{-1}$ & $\kappa_{S}^{\mathrm{E}} / \mathrm{TPa}^{-1}$ \\
\hline 0.0506 & 6.0 & -4.17 & -7.71 & 0.5548 & 47.0 & -20.65 & -41.83 \\
0.1011 & 11.8 & -7.62 & -14.65 & 0.6006 & 47.7 & -20.64 & -40.79 \\
0.1501 & 17.1 & -10.43 & -20.66 & 0.6552 & 47.6 & -20.29 & -38.66 \\
0.1977 & 22.1 & -12.71 & -25.82 & 0.7013 & 46.2 & -19.57 & -35.95 \\
0.2494 & 27.3 & -14.83 & -30.75 & 0.7525 & 43.6 & -18.34 & -32.24 \\
0.3096 & 32.6 & -16.72 & -35.19 & 0.7964 & 39.9 & -16.72 & -28.18 \\
0.3536 & 36.2 & -17.89 & -37.86 & 0.8476 & 33.9 & -14.16 & -22.64 \\
0.4023 & 39.8 & -18.96 & -40.12 & 0.8968 & 25.9 & -10.77 & -16.33 \\
0.4535 & 42.9 & -19.79 & -41.51 & 0.9417 & 16.3 & -6.77 & -9.74 \\
0.5051 & 45.4 & -20.38 & -42.17 & & & & \\
\hline
\end{tabular}

Tabla 3-32: coeficientes y desviaciones estándar del ajuste a la ecuación R-K de las diferentes propiedades de exceso del sistema $N$-metilanilina (1) +2 -heptanona (2)

\begin{tabular}{cccccccc}
\hline$T / \mathrm{K}$ & Propiedad & $A_{0}$ & $A_{1}$ & $A_{2}$ & $A_{3}$ & $A_{4}$ & $\sigma$ \\
\hline 293.15 & $V_{\mathrm{m}}^{\mathrm{E}} / \mathrm{cm}^{3} \cdot \mathrm{mol}^{-1}$ & -1.974 & 0.226 & 0.091 & & & 0.002 \\
298.15 & $V_{\mathrm{m}}^{\mathrm{E}} / \mathrm{cm}^{3} \cdot \mathrm{mol}^{-1}$ & -2.036 & 0.227 & & & 0.003 \\
& $u^{\mathrm{E}} / \mathrm{m}^{-1}$ & 180.75 & 83.5 & 32.0 & 17.3 & 9.4 & 0.04 \\
& $\alpha_{\mathrm{P}}^{\mathrm{E}} / 10^{-6} \mathrm{~K}^{-1}$ & -81.20 & -18.09 & -29.79 & -3.9 & & 0.02 \\
& $\kappa_{S}^{\mathrm{E}} / \mathrm{TPa}^{-1}$ & -168.40 & -9.14 & & & 0.05 \\
303.15 & $V_{\mathrm{m}}^{\mathrm{E}} / \mathrm{cm}^{3} \cdot \mathrm{mol}^{-1}$ & -2.095 & 0.220 & 0.058 & & & 0.002 \\
\hline
\end{tabular}



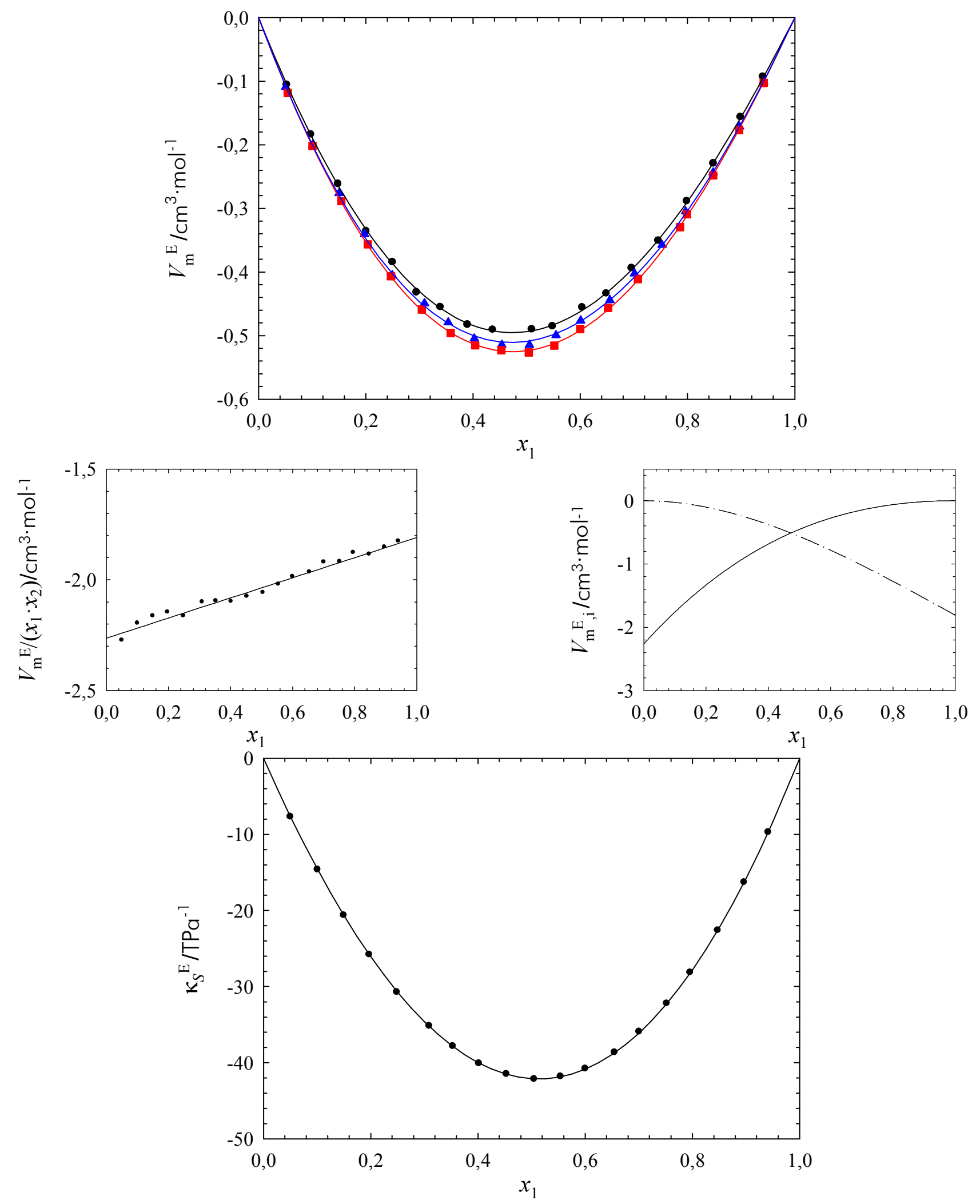

Ilustración 3-25: volúmenes molares de exceso $V_{\mathrm{m}}^{\mathrm{E}}$, volúmenes molares de exceso reducidos $V_{\mathrm{m}}^{\mathrm{E}} /\left(x_{1} \cdot x_{2}\right)$, volúmenes molares parciales de exceso $V_{\mathrm{m}, \mathrm{i}}^{\mathrm{E}}$ y compresibilidades isoentrópicas de exceso $\kappa_{S}^{\mathrm{E}}$ del sistema $N$-metilanilina (1) +2 -heptanona (2) 
Tabla 3-33: volúmenes molares de exceso del sistema piridina (1) + 2-propanona (2)

\begin{tabular}{|c|c|c|c|c|c|}
\hline$x_{1}$ & $V_{\mathrm{m}}^{\mathrm{E}} / \mathrm{cm}^{3} \cdot \mathrm{mol}^{-1}$ & $x_{1}$ & $V_{\mathrm{m}}^{\mathrm{E}} / \mathrm{cm}^{3} \cdot \mathrm{mol}^{-1}$ & $x_{1}$ & $V_{\mathrm{m}}^{\mathrm{E}} / \mathrm{cm}^{3} \cdot \mathrm{mol}^{-1}$ \\
\hline \multicolumn{6}{|c|}{$293.15 \mathrm{~K}$} \\
\hline 0.0544 & -0.0652 & 0.3575 & -0.2290 & 0.7032 & -0.1766 \\
\hline 0.0981 & -0.1041 & 0.4031 & -0.2332 & 0.7781 & -0.1369 \\
\hline 0.1431 & -0.1428 & 0.4481 & -0.2351 & 0.7957 & -0.1319 \\
\hline 0.1934 & -0.1802 & 0.4809 & -0.2319 & 0.8464 & -0.0993 \\
\hline 0.2496 & -0.2028 & 0.5949 & -0.2117 & 0.8993 & -0.0648 \\
\hline 0.2970 & -0.2181 & 0.6463 & -0.1952 & 0.9423 & -0.0418 \\
\hline \multicolumn{6}{|c|}{$298.15 \mathrm{~K}$} \\
\hline 0.0544 & -0.0666 & 0.4060 & -0.2570 & 0.7040 & -0.1850 \\
\hline 0.1045 & -0.1177 & 0.4552 & -0.2568 & 0.7556 & -0.1598 \\
\hline 0.1544 & -0.1575 & 0.4964 & -0.2488 & 0.7977 & -0.1356 \\
\hline 0.1951 & -0.1844 & 0.5484 & -0.2393 & 0.8514 & -0.0997 \\
\hline 0.2482 & -0.2125 & 0.6035 & -0.2268 & 0.9031 & -0.0661 \\
\hline 0.3029 & -0.2349 & 0.6456 & -0.2062 & 0.9486 & -0.0338 \\
\hline 0.3546 & -0.2535 & & & & \\
\hline \multicolumn{6}{|c|}{$303.15 \mathrm{~K}$} \\
\hline 0.0557 & -0.0727 & 0.4035 & -0.2804 & 0.7071 & -0.2001 \\
\hline 0.1054 & -0.1259 & 0.4588 & -0.2759 & 0.7576 & -0.1707 \\
\hline 0.1525 & -0.1697 & 0.5030 & -0.2683 & 0.8017 & -0.1457 \\
\hline 0.2509 & -0.2306 & 0.5503 & -0.2575 & 0.8537 & -0.1106 \\
\hline 0.3088 & -0.2607 & 0.5952 & -0.2463 & 0.9046 & -0.0743 \\
\hline 0.3406 & -0.2746 & 0.6478 & -0.2233 & 0.9507 & -0.0401 \\
\hline
\end{tabular}

Tabla 3-34: velocidades del sonido de exceso, coeficientes de expansión térmica a $P=$ cte. de exceso, y compresibilidades isoentrópicas de exceso del sistema piridina (1) + 2-propanona (2)

\begin{tabular}{cccccccc}
\hline$x_{1}$ & $u^{\mathrm{E}} / \mathrm{m} \cdot \mathrm{s}^{-1}$ & $\alpha_{\mathrm{P}}^{\mathrm{E}} / 10^{-6} \mathrm{~K}^{-1}$ & $\kappa_{S}^{\mathrm{E}} / \mathrm{TPa}^{-1}$ & $x_{1}$ & $u^{\mathrm{E}} / \mathrm{m} \cdot \mathrm{s}^{-1}$ & $\alpha_{\mathrm{P}}^{\mathrm{E}} / 10^{-6} \mathrm{~K}^{-1}$ & $\kappa_{S}^{\mathrm{E}} / \mathrm{TPa}^{-1}$ \\
\hline 0.0544 & 10.6 & -7.74 & -17.40 & 0.5484 & 56.9 & -47.80 & -61.95 \\
0.1045 & 19.5 & -15.60 & -30.58 & 0.6035 & 56.0 & -43.68 & -58.19 \\
0.1544 & 27.4 & -23.47 & -41.20 & 0.6456 & 54.1 & -39.37 & -54.32 \\
0.1951 & 33.2 & -29.62 & -48.27 & 0.7040 & 50.3 & -32.39 & -48.11 \\
0.2482 & 39.8 & -36.87 & -55.39 & 0.7556 & 45.5 & -25.48 & -41.61 \\
0.3029 & 45.5 & -43.02 & -60.56 & 0.7977 & 40.4 & -19.65 & -35.60 \\
0.3546 & 50.1 & -47.40 & -63.91 & 0.8514 & 32.3 & -12.48 & -27.19 \\
0.4060 & 53.3 & -49.92 & -65.25 & 0.9031 & 22.9 & -6.48 & -18.39 \\
0.4552 & 55.7 & -50.74 & -65.42 & 0.9486 & 13.0 & -2.44 & -9.99 \\
0.4964 & 56.6 & -50.09 & -64.26 & & & & \\
\hline
\end{tabular}

Tabla 3-35: coeficientes y desviaciones estándar del ajuste a la ecuación R-K de las diferentes propiedades de exceso del sistema piridina (1) + 2-propanona (2)

\begin{tabular}{ccccccc}
\hline$T / \mathrm{K}$ & Propiedad & $A_{0}$ & $A_{1}$ & $A_{2}$ & $A_{3}$ & $\sigma$ \\
\hline 293.15 & $V_{\mathrm{m}}^{\mathrm{E}} / \mathrm{cm}^{3} \cdot \mathrm{mol}^{-1}$ & -0.922 & 0.271 & -0.096 & & 0.002 \\
298.15 & $V_{\mathrm{m}}^{\mathrm{E}} / \mathrm{cm}^{3} \cdot \mathrm{mol}^{-1}$ & -1.003 & 0.296 & & 0.002 \\
& $u^{\mathrm{E}} / \mathrm{m}^{-1}$ & 226.70 & 32.2 & 11.9 & & 0.06 \\
& $\alpha_{\mathrm{P}}^{\mathrm{E}} / 10^{-6} \mathrm{~K}^{-1}$ & -200.10 & 59.7 & 124.7 & -6.1 & 0.04 \\
303.15 & $\kappa_{S}^{\mathrm{E}} / \mathrm{TPa}^{-1}$ & -256.76 & 69.8 & -18.5 & 5.3 & 0.08 \\
\hline
\end{tabular}



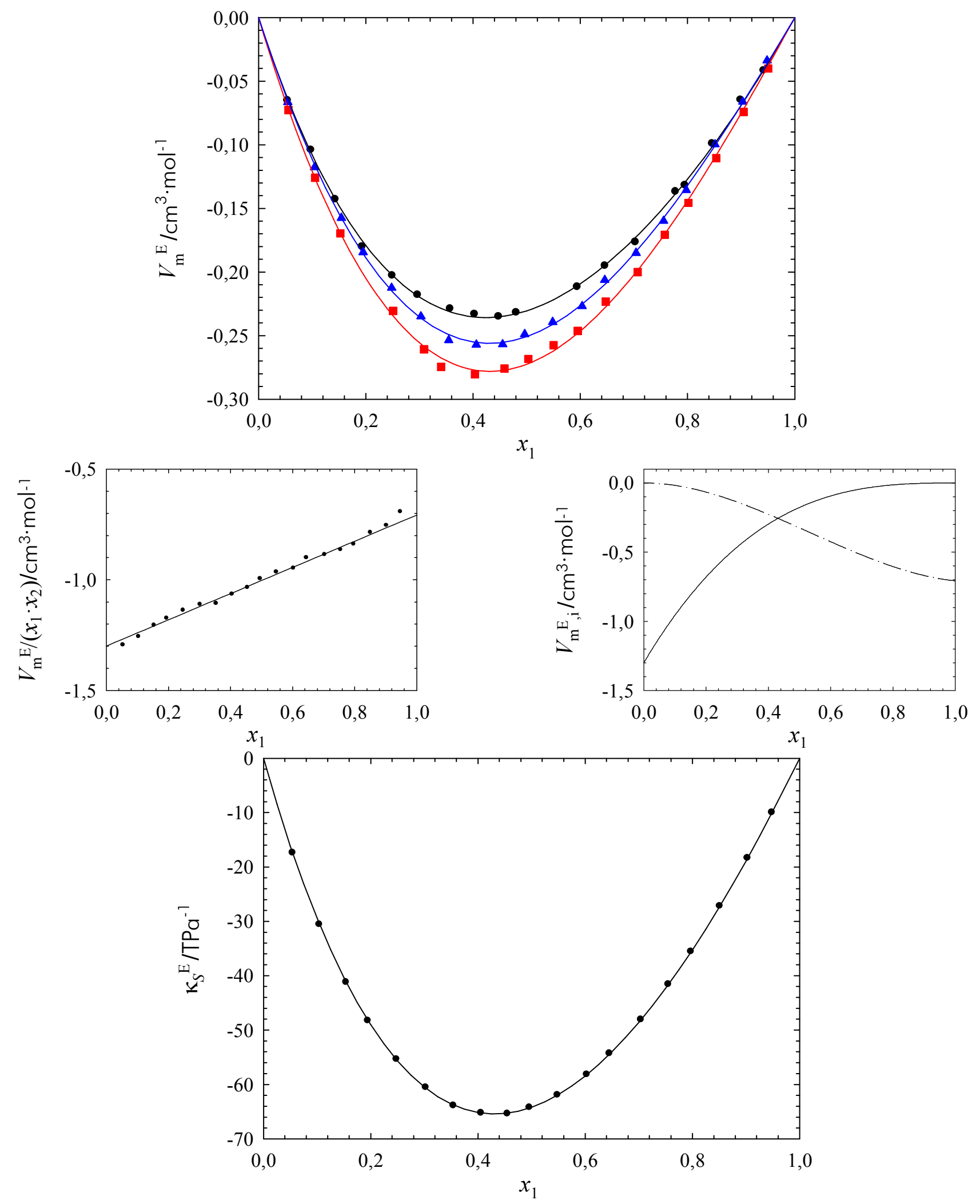

Ilustración 3-26: volúmenes molares de exceso $V_{\mathrm{m}}^{\mathrm{E}}$, volúmenes molares de exceso reducidos $V_{\mathrm{m}}^{\mathrm{E}} /\left(x_{1} \cdot x_{2}\right)$, volúmenes molares parciales de exceso $V_{\mathrm{m}, \mathrm{i}}^{\mathrm{E}}$ y compresibilidades isoentrópicas de exceso $\kappa_{S}^{\mathrm{E}}$ del sistema piridina (1) +2 -propanona (2) 
Tabla 3-36: volúmenes molares de exceso del sistema piridina (1) + 2-butanona (2)

\begin{tabular}{|c|c|c|c|c|c|}
\hline$x_{1}$ & $V_{\mathrm{m}}^{\mathrm{E}} / \mathrm{cm}^{3} \cdot \mathrm{mol}^{-1}$ & $x_{1}$ & $V_{\mathrm{m}}^{\mathrm{E}} / \mathrm{cm}^{3} \cdot \mathrm{mol}^{-1}$ & $x_{1}$ & $V_{\mathrm{m}}^{\mathrm{E}} / \mathrm{cm}^{3} \cdot \mathrm{mol}^{-1}$ \\
\hline \multicolumn{6}{|c|}{$293.15 \mathrm{~K}$} \\
\hline 0.0541 & -0.0531 & 0.3997 & -0.2456 & 0.7072 & -0.1852 \\
\hline 0.1093 & -0.1165 & 0.4539 & -0.2465 & 0.7521 & -0.1609 \\
\hline 0.1450 & -0.1409 & 0.5038 & -0.2460 & 0.7927 & -0.1412 \\
\hline 0.2481 & -0.2017 & 0.5465 & -0.2381 & 0.8443 & -0.1098 \\
\hline 0.2733 & -0.2131 & 0.6008 & -0.2257 & 0.8990 & -0.0739 \\
\hline 0.3052 & -0.2235 & 0.6466 & -0.2136 & 0.9416 & -0.0437 \\
\hline 0.3575 & -0.2380 & & & & \\
\hline \multicolumn{6}{|c|}{$298.15 \mathrm{~K}$} \\
\hline 0.0475 & -0.0581 & 0.4084 & -0.2603 & 0.6990 & -0.1985 \\
\hline 0.1056 & -0.1139 & 0.4528 & -0.2611 & 0.7527 & -0.1740 \\
\hline 0.1475 & -0.1488 & 0.5006 & -0.2558 & 0.8048 & -0.1419 \\
\hline 0.1971 & -0.1842 & 0.5506 & -0.2479 & 0.8490 & -0.1117 \\
\hline 0.2437 & -0.2122 & 0.5959 & -0.2342 & 0.8991 & -0.0786 \\
\hline 0.3049 & -0.2410 & 0.6441 & -0.2225 & 0.9483 & -0.0418 \\
\hline \multicolumn{6}{|c|}{$303.15 \mathrm{~K}$} \\
\hline 0,0629 & $-0,0821$ & 0,4033 & $-0,2769$ & 0,6925 & $-0,2169$ \\
\hline 0.1072 & -0.1269 & 0.4518 & -0.2784 & 0.7511 & -0.1849 \\
\hline 0.1524 & -0.1654 & 0.5041 & -0.2718 & 0.7944 & -0.1587 \\
\hline 0.2048 & -0.2008 & 0.5506 & -0.2643 & 0.8380 & -0.1279 \\
\hline 0.2554 & -0.2303 & 0.5984 & -0.2567 & 0.8830 & -0.0947 \\
\hline 0.3028 & -0.2492 & 0.6494 & -0.2363 & 0.9411 & -0.0516 \\
\hline 0.3516 & -0.2668 & & & & \\
\hline
\end{tabular}

Tabla 3-37: velocidades del sonido de exceso, coeficientes de expansión térmica a $P=$ cte. de exceso, y compresibilidades isoentrópicas de exceso del sistema piridina (1) +2 -butanona (2)

\begin{tabular}{cccccccc}
\hline$x_{1}$ & $u^{\mathrm{E}} / \mathrm{m} \cdot \mathrm{s}^{-1}$ & $\alpha_{\mathrm{P}}^{\mathrm{E}} / 10^{-6} \mathrm{~K}^{-1}$ & $\kappa_{S}^{\mathrm{E}} / \mathrm{TPa}^{-1}$ & $x_{1}$ & $u^{\mathrm{E}} / \mathrm{m} \cdot \mathrm{s}^{-1}$ & $\alpha_{\mathrm{P}}^{\mathrm{E}} / 10^{-6} \mathrm{~K}^{-1}$ & $\kappa_{S}^{\mathrm{E}} / \mathrm{TPa}^{-1}$ \\
\hline 0.0475 & 6.5 & -6.37 & -9.92 & 0.5506 & 44.4 & -31.11 & -47.41 \\
0.1056 & 13.8 & -13.07 & -20.20 & 0.5959 & 44.2 & -29.90 & -45.66 \\
0.1475 & 18.7 & -17.27 & -26.58 & 0.6441 & 43.3 & -28.17 & -43.09 \\
0.1971 & 24.1 & -21.53 & -33.07 & 0.6990 & 41.0 & -25.48 & -39.15 \\
0.2437 & 28.7 & -24.86 & -38.20 & 0.7527 & 37.5 & -22.24 & -34.30 \\
0.3049 & 33.9 & -28.26 & -43.22 & 0.8048 & 32.6 & -18.48 & -28.62 \\
0.4084 & 40.4 & -31.45 & -47.91 & 0.8490 & 27.4 & -14.87 & -23.14 \\
0.4528 & 42.4 & -31.92 & -48.62 & 0.8991 & 20.1 & -10.38 & -16.26 \\
0.5006 & 43.8 & -31.81 & -48.48 & 0.9483 & 11.3 & -5.54 & -8.76 \\
\hline
\end{tabular}

Tabla 3-38: coeficientes y desviaciones estándar del ajuste a la ecuación R-K de las diferentes propiedades de exceso del sistema piridina (1) + 2-butanona (2)

\begin{tabular}{ccccccc}
\hline$T / \mathrm{K}$ & Propiedad & $A_{0}$ & $A_{1}$ & $A_{2}$ & $A_{3}$ & $\sigma$ \\
\hline 293.15 & $V_{\mathrm{m}}^{\mathrm{E}} / \mathrm{cm}^{3} \cdot \mathrm{mol}^{-1}$ & -0.982 & 0.209 & & & 0.002 \\
298.15 & $V_{\mathrm{m}}^{\mathrm{E}} / \mathrm{cm}^{3} \cdot \mathrm{mol}^{-1}$ & -1.032 & 0.225 & & & 0.002 \\
& $u^{\mathrm{E}} /{\mathrm{m} \cdot \mathrm{s}^{-1}}^{\mathrm{E}}$ & 174.97 & 43.3 & 13.8 & 5.8 & 0.04 \\
& $\alpha_{\mathrm{P}}^{\mathrm{E}} / 10^{-6} \mathrm{~K}^{-1}$ & -127.34 & 15.26 & 1.18 & & 0.02 \\
& $\kappa_{S}^{\mathrm{E}} / \mathrm{TPa}^{-1}$ & -194.03 & 22.3 & -4.7 & & 0.06 \\
303.15 & $V_{\mathrm{m}}^{\mathrm{E}} / \mathrm{cm}^{3} \cdot \mathrm{mol}^{-1}$ & -1.102 & 0.232 & & & 0.002 \\
\hline
\end{tabular}



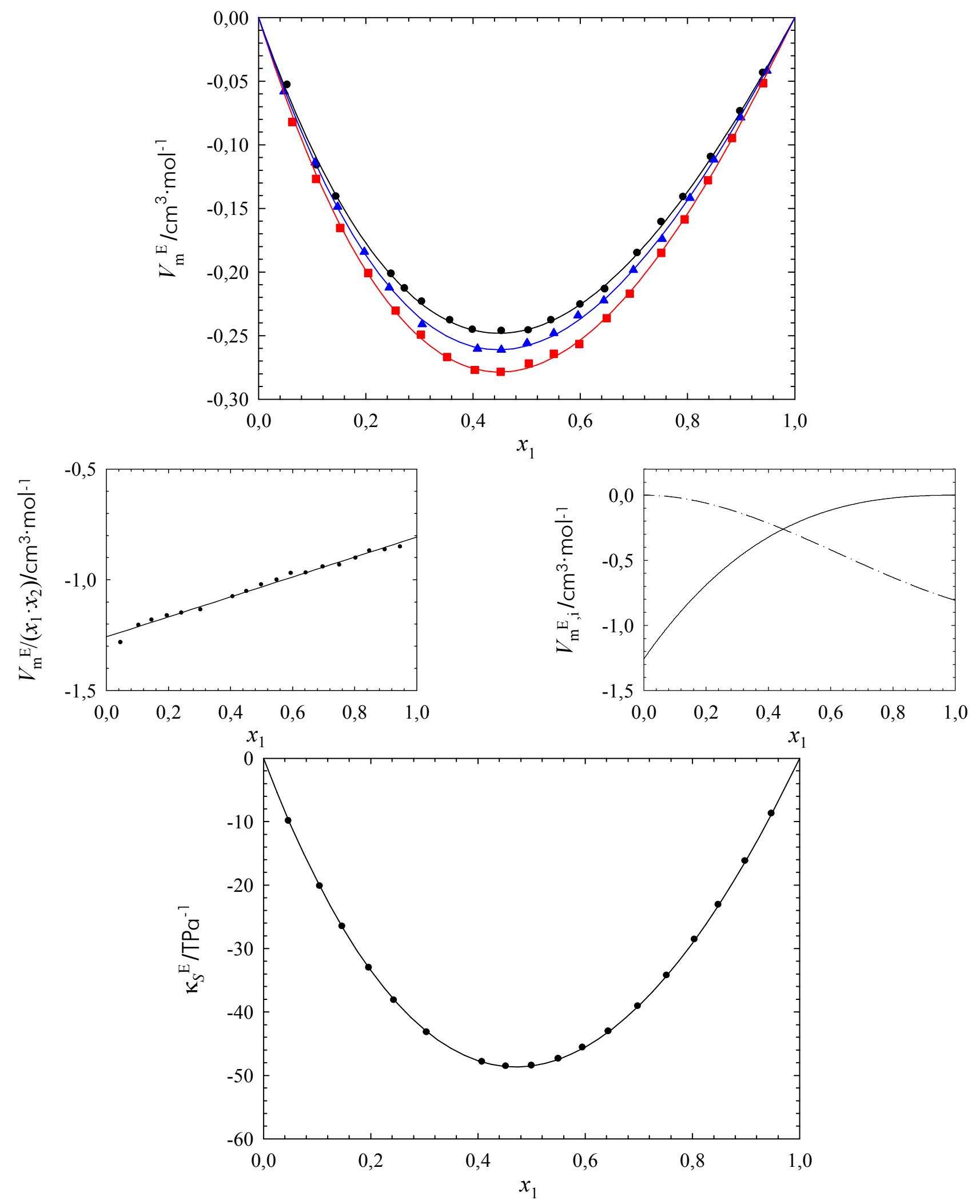

Ilustración 3-27: volúmenes molares de exceso $V_{\mathrm{m}}^{\mathrm{E}}$, volúmenes molares de exceso reducidos $V_{\mathrm{m}}^{\mathrm{E}} /\left(x_{1} \cdot x_{2}\right)$, volúmenes molares parciales de exceso $V_{\mathrm{m}, \mathrm{i}}^{\mathrm{E}}$ y compresibilidades isoentrópicas de exceso $\kappa_{S}^{\mathrm{E}}$ del sistema piridina (1) +2 -butanona (2) 
Tabla 3-39: volúmenes molares de exceso del sistema piridina (1) + 2-pentanona (2)

\begin{tabular}{|c|c|c|c|c|c|}
\hline$x_{1}$ & $V_{\mathrm{m}}^{\mathrm{E}} / \mathrm{cm}^{3} \cdot \mathrm{mol}^{-1}$ & $x_{1}$ & $V_{\mathrm{m}}^{\mathrm{E}} / \mathrm{cm}^{3} \cdot \mathrm{mol}^{-1}$ & $x_{1}$ & $V_{\mathrm{m}}^{\mathrm{E}} / \mathrm{cm}^{3} \cdot \mathrm{mol}^{-1}$ \\
\hline \multicolumn{6}{|c|}{$293.15 \mathrm{~K}$} \\
\hline 0.0763 & -0.0779 & 0.3955 & -0.2185 & 0.6977 & -0.1782 \\
\hline 0.1130 & -0.0992 & 0.4519 & -0.2252 & 0.7425 & -0.1599 \\
\hline 0.1517 & -0.1269 & 0.4940 & -0.2257 & 0.8000 & -0.1282 \\
\hline 0.2020 & -0.1640 & 0.5486 & -0.2207 & 0.8525 & -0.1016 \\
\hline 0.2546 & -0.1779 & 0.5965 & -0.2078 & 0.9015 & -0.0693 \\
\hline 0.3011 & -0.1962 & 0.6429 & -0.1976 & 0.9482 & -0.0361 \\
\hline 0.3572 & -0.2122 & & & & \\
\hline \multicolumn{6}{|c|}{$298.15 \mathrm{~K}$} \\
\hline 0.0668 & -0.0716 & 0.3952 & -0.2283 & 0.6994 & -0.1842 \\
\hline 0.1214 & -0.1074 & 0.4454 & -0.2331 & 0.7485 & -0.1634 \\
\hline 0.1532 & -0.1293 & 0.4974 & -0.2331 & 0.7967 & -0.1399 \\
\hline 0.2047 & -0.1594 & 0.5494 & -0.2293 & 0.8531 & -0.1071 \\
\hline 0.2600 & -0.1864 & 0.5993 & -0.2138 & 0.9016 & -0.0747 \\
\hline 0.3046 & -0.2050 & 0.6450 & -0.2039 & 0.9519 & -0.0385 \\
\hline 0.3545 & -0.2233 & & & & \\
\hline \multicolumn{6}{|c|}{$303.15 \mathrm{~K}$} \\
\hline 0.0579 & -0.0636 & 0.4039 & -0.2372 & 0.7428 & -0.1745 \\
\hline 0.1107 & -0.1031 & 0.4470 & -0.2391 & 0.7975 & -0.1441 \\
\hline 0.1629 & -0.1404 & 0.4994 & -0.2393 & 0.8523 & -0.1102 \\
\hline 0.2062 & -0.1657 & 0.6013 & -0.2223 & 0.9026 & -0.0765 \\
\hline 0.3079 & -0.2133 & 0.6496 & -0.2100 & 0.9493 & -0.0404 \\
\hline 0.3523 & -0.2259 & 0.7011 & -0.1911 & & \\
\hline
\end{tabular}

Tabla 3-40: velocidades del sonido de exceso, coeficientes de expansión térmica a $P=$ cte. de exceso, y compresibilidades isoentrópicas de exceso del sistema piridina (1) + 2-pentanona (2)

\begin{tabular}{cccccccc}
\hline$x_{1}$ & $u^{\mathrm{E}} / \mathrm{m} \cdot \mathrm{s}^{-1}$ & $\alpha_{\mathrm{P}}^{\mathrm{E}} / 10^{-6} \mathrm{~K}^{-1}$ & $\kappa_{S}^{\mathrm{E}} / \mathrm{TPa}^{-1}$ & $x_{1}$ & $u^{\mathrm{E}} / \mathrm{m} \cdot \mathrm{s}^{-1}$ & $\alpha_{\mathrm{P}}^{\mathrm{E}} / 10^{-6} \mathrm{~K}^{-1}$ & $\kappa_{S}^{\mathrm{E}} / \mathrm{TPa}^{-1}$ \\
\hline 0.0668 & 7.0 & -1.97 & -10.09 & 0.5494 & 38.7 & -14.68 & -41.42 \\
0.1214 & 12.1 & -3.52 & -16.86 & 0.5993 & 39.1 & -15.00 & -40.37 \\
0.1532 & 15.1 & -4.50 & -20.55 & 0.6450 & 38.9 & -15.07 & -38.81 \\
0.2047 & 19.6 & -6.11 & -25.94 & 0.6994 & 37.5 & -14.67 & -35.95 \\
0.2600 & 24.0 & -7.84 & -30.82 & 0.7485 & 35.2 & -13.84 & -32.48 \\
0.3046 & 27.3 & -9.21 & -34.17 & 0.7967 & 31.7 & -12.52 & -28.20 \\
0.3545 & 30.7 & -10.69 & -37.24 & 0.8531 & 26.0 & -10.26 & -22.04 \\
0.3952 & 33.0 & -11.74 & -39.07 & 0.9016 & 19.4 & -7.62 & -15.74 \\
0.4454 & 35.5 & -12.92 & -40.65 & 0.9519 & 10.6 & -4.14 & -8.22 \\
0.4974 & 37.5 & -13.93 & -41.48 & & & & \\
\hline
\end{tabular}

Tabla 3-41: coeficientes y desviaciones estándar del ajuste a la ecuación R-K de las diferentes propiedades de exceso del sistema piridina (1) + 2-pentanona (2)

\begin{tabular}{cccccccc}
\hline$T / \mathrm{K}$ & Propiedad & $A_{0}$ & $A_{1}$ & $A_{2}$ & $A_{3}$ & $A_{4}$ & $\sigma$ \\
\hline 293.15 & $V_{\mathrm{m}}^{\mathrm{E}} / \mathrm{cm}^{3} \cdot \mathrm{mol}^{-1}$ & -0.895 & 0.135 & & & & 0.003 \\
298.15 & $V_{\mathrm{m}}^{\mathrm{E}} / \mathrm{cm}^{3} \cdot \mathrm{mol}^{-1}$ & -0.928 & 0.115 & & & & 0.003 \\
& $u^{\mathrm{E}} / \mathrm{m}^{-1}$ & 150.18 & 61.2 & 19.6 & 7.2 & 7.0 & 0.04 \\
& $\alpha_{\mathrm{p}}^{\mathrm{E}} / 10^{-6} \mathrm{~K}^{-1}$ & -55.84 & -33.38 & -4.6 & & & 0.006 \\
& $\kappa_{S}^{\mathrm{E}} / \mathrm{TPa}^{-1}$ & -165.80 & -12.1 & -3.0 & & & 0.03 \\
303.15 & $V_{\mathrm{m}}^{\mathrm{E}} / \mathrm{cm}^{3} \cdot \mathrm{mol}^{-1}$ & -0.958 & 0.113 & & & & 0.0018 \\
\hline
\end{tabular}



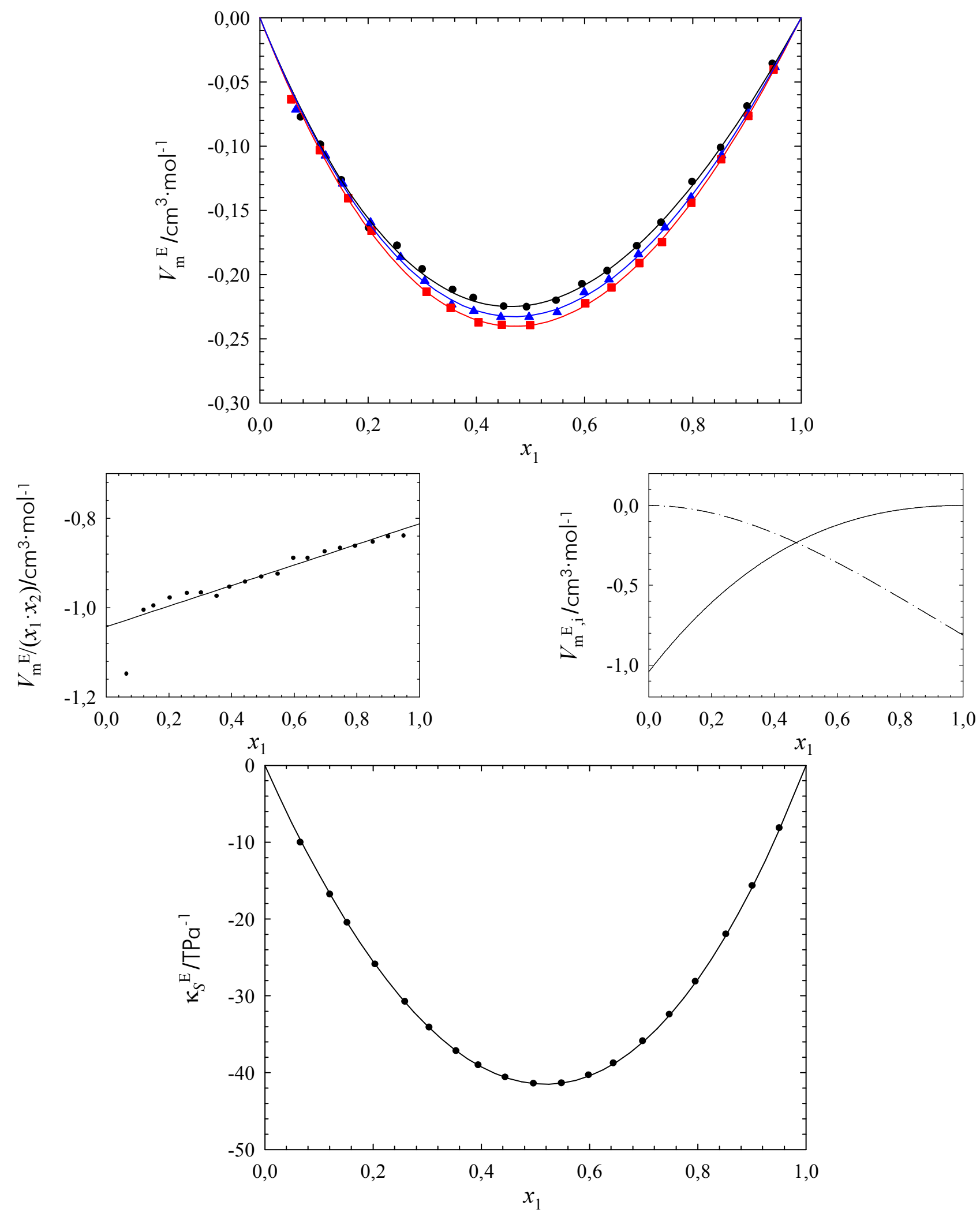

llustración 3-28: volúmenes molares de exceso $V_{\mathrm{m}}^{\mathrm{E}}$, volúmenes molares de exceso reducidos $V_{\mathrm{m}}^{\mathrm{E}} /\left(x_{1} \cdot x_{2}\right)$, volúmenes molares parciales de exceso $V_{\mathrm{m}, \mathrm{i}}^{\mathrm{E}}$ y compresibilidades isoentrópicas de exceso $\kappa_{S}^{\mathrm{E}}$ del sistema piridina (1) +2 -pentanona (2) 
Tabla 3-42: volúmenes molares de exceso del sistema piridina (1) + 2-heptanona (2)

\begin{tabular}{|c|c|c|c|c|c|}
\hline$x_{1}$ & $V_{\mathrm{m}}^{\mathrm{E}} / \mathrm{cm}^{3} \cdot \mathrm{mol}^{-1}$ & $x_{1}$ & $V_{\mathrm{m}}^{\mathrm{E}} / \mathrm{cm}^{3} \cdot \mathrm{mol}^{-1}$ & $x_{1}$ & $V_{\mathrm{m}}^{\mathrm{E}} / \mathrm{cm}^{3} \cdot \mathrm{mol}^{-1}$ \\
\hline \multicolumn{6}{|c|}{$293.15 \mathrm{~K}$} \\
\hline 0.0415 & -0.0162 & 0.3458 & -0.0633 & 0.6929 & -0.0622 \\
\hline 0.1016 & -0.0286 & 0.3977 & -0.0657 & 0.7478 & -0.0538 \\
\hline 0.1449 & -0.0370 & 0.4545 & -0.0688 & 0.7960 & -0.0450 \\
\hline 0.1793 & -0.0429 & 0.4895 & -0.0700 & 0.8471 & -0.0377 \\
\hline 0.2449 & -0.0529 & 0.5413 & -0.0701 & 0.8926 & -0.0277 \\
\hline 0.3001 & -0.0577 & 0.6458 & -0.0649 & 0.9425 & -0.0172 \\
\hline \multicolumn{6}{|c|}{$298.15 \mathrm{~K}$} \\
\hline 0.0556 & -0.0205 & 0.3981 & -0.0704 & 0.6958 & -0.0625 \\
\hline 0.1044 & -0.0309 & 0.4456 & -0.0728 & 0.7479 & -0.0565 \\
\hline 0.1472 & -0.0387 & 0.4947 & -0.0739 & 0.7968 & -0.0486 \\
\hline 0.1944 & -0.0456 & 0.5456 & -0.0733 & 0.8457 & -0.0398 \\
\hline 0.2500 & -0.0543 & 0.6006 & -0.0709 & 0.8966 & -0.0293 \\
\hline 0.2953 & -0.0596 & 0.6458 & -0.0663 & 0.9447 & -0.0145 \\
\hline 0.3329 & -0.0646 & & & & \\
\hline \multicolumn{6}{|c|}{$303.15 \mathrm{~K}$} \\
\hline 0.0484 & -0.0213 & 0.3344 & -0.0673 & 0.6985 & -0.0619 \\
\hline 0.1004 & -0.0307 & 0.3966 & -0.0721 & 0.7495 & -0.0555 \\
\hline 0.1442 & -0.0382 & 0.4392 & -0.0754 & 0.7976 & -0.0509 \\
\hline 0.1981 & -0.0514 & 0.4886 & -0.0760 & 0.8466 & -0.0406 \\
\hline 0.2537 & -0.0571 & 0.6047 & -0.0716 & 0.8951 & -0.0313 \\
\hline 0.2964 & -0.0634 & 0.6497 & -0.0685 & 0.9448 & -0.0135 \\
\hline
\end{tabular}

Tabla 3-43: velocidades del sonido de exceso, coeficientes de expansión térmica a $P=$ cte. de exceso, y compresibilidades isoentrópicas de exceso del sistema piridina (1) + 2-heptanona (2)

\begin{tabular}{cccccccc}
\hline$x_{1}$ & $u^{\mathrm{E}} / \mathrm{m} \cdot \mathrm{s}^{-1}$ & $\alpha_{\mathrm{P}}^{\mathrm{E}} / 10^{-6} \mathrm{~K}^{-1}$ & $\kappa_{S}^{\mathrm{E}} / \mathrm{TPa}^{-1}$ & $x_{1}$ & $u^{\mathrm{E}} / \mathrm{m} \cdot \mathrm{s}^{-1}$ & $\alpha_{\mathrm{P}}^{\mathrm{E}} / 10^{-6} \mathrm{~K}^{-1}$ & $\kappa_{S}^{\mathrm{E}} / \mathrm{TPa}^{-1}$ \\
\hline 0.0556 & 2.5 & -0.88 & -3.15 & 0.5456 & 21.3 & -3.97 & -21.61 \\
0.1044 & 4.7 & -1.57 & -5.80 & 0.6006 & 22.2 & -3.50 & -21.93 \\
0.1472 & 6.6 & -2.16 & -7.97 & 0.6458 & 22.6 & -3.08 & -21.74 \\
0.1944 & 8.7 & -2.78 & -10.31 & 0.6958 & 22.6 & -2.69 & -21.07 \\
0.2500 & 11.1 & -3.46 & -12.87 & 0.7479 & 21.9 & -2.40 & -19.74 \\
0.2953 & 12.9 & -3.91 & -14.73 & 0.7968 & 20.4 & -2.25 & -17.73 \\
0.3329 & 14.5 & -4.21 & -16.25 & 0.8457 & 17.9 & -2.21 & -15.04 \\
0.3981 & 16.9 & -4.49 & -18.45 & 0.8966 & 14.0 & -2.12 & -11.28 \\
0.4456 & 18.5 & -4.48 & -19.78 & 0.9447 & 8.7 & -1.62 & -6.67 \\
0.4947 & 20.0 & -4.31 & -20.83 & & & & \\
\hline
\end{tabular}

Tabla 3-44: coeficientes y desviaciones estándar del ajuste a la ecuación R-K de las diferentes propiedades de exceso del sistema piridina (1) + 2-heptanona (2)

\begin{tabular}{|c|c|c|c|c|c|c|c|c|}
\hline$T / \mathrm{K}$ & Propiedad & $A_{0}$ & $A_{1}$ & $A_{2}$ & $A_{3}$ & $A_{4}$ & $A_{5}$ & $\sigma$ \\
\hline 293.15 & $V_{\mathrm{m}}^{\mathrm{E}} / \mathrm{cm}^{3} \cdot \mathrm{mol}^{-1}$ & -0.2813 & -0.021 & 0.02 & 0.063 & -0.10 & & 0.0010 \\
\hline \multirow[t]{4}{*}{298.15} & $V_{\mathrm{m}}^{\mathrm{E}} / \mathrm{cm}^{3} \cdot \mathrm{mol}^{-1}$ & -0.2953 & 0.008 & 0.042 & -0.14 & -0.128 & 0.25 & 0.0005 \\
\hline & $u^{\mathrm{E}} / \mathrm{m} \cdot \mathrm{s}^{-1}$ & 80.58 & 53.62 & 27.0 & 15.3 & 8.0 & & 0.02 \\
\hline & $\alpha_{\mathrm{P}}^{\mathrm{E}} / 10^{-6} \mathrm{~K}^{-1}$ & -17.130 & 11.13 & 13.09 & -20.4 & -27.42 & -5.1 & 0.006 \\
\hline & $\kappa_{S}^{\mathrm{E}} / \mathrm{TPa}^{-1}$ & -83.78 & -35.40 & -10.9 & -3.4 & -2.6 & & 0.02 \\
\hline 303.15 & $V_{\mathrm{m}}^{\mathrm{E}} / \mathrm{cm}^{3} \cdot \mathrm{mol}^{-1}$ & -0.303 & -0.004 & 0.03 & 0.04 & -0.13 & & 0.002 \\
\hline
\end{tabular}



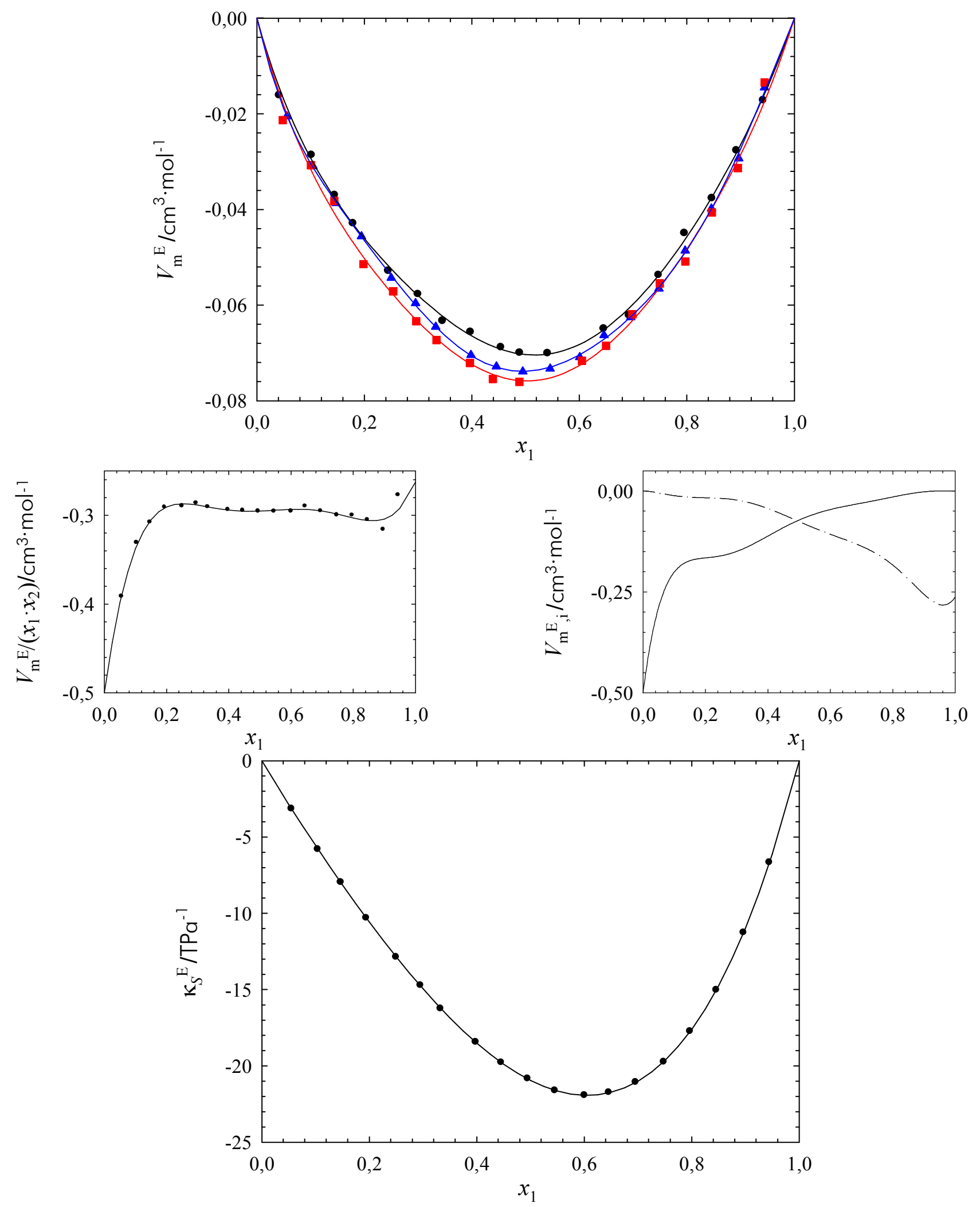

Ilustración 3-29: volúmenes molares de exceso $V_{\mathrm{m}}^{\mathrm{E}}$, volúmenes molares de exceso reducidos $V_{\mathrm{m}}^{\mathrm{E}} /\left(x_{1} \cdot x_{2}\right)$, volúmenes molares parciales de exceso $V_{\mathrm{m}, \mathrm{i}}^{\mathrm{E}}$ y compresibilidades isoentrópicas de exceso $\kappa_{S}^{\mathrm{E}}$ del sistema piridina (1) +2 -heptanona (2) 
Tabla 3-45: volúmenes molares de exceso del sistema di- $n$-propilamina (1) + 2-propanona (2)

\begin{tabular}{|c|c|c|c|c|c|}
\hline$x_{1}$ & $V_{\mathrm{m}}^{\mathrm{E}} / \mathrm{cm}^{3} \cdot \mathrm{mol}^{-1}$ & $x_{1}$ & $V_{\mathrm{m}}^{\mathrm{E}} / \mathrm{cm}^{3} \cdot \mathrm{mol}^{-1}$ & $x_{1}$ & $V_{\mathrm{m}}^{\mathrm{E}} / \mathrm{cm}^{3} \cdot \mathrm{mol}^{-1}$ \\
\hline \multicolumn{6}{|c|}{$293.15 \mathrm{~K}$} \\
\hline 0.0540 & 0.0643 & 0.4072 & 0.2433 & 0.6925 & 0.1832 \\
\hline 0.1004 & 0.1091 & 0.4557 & 0.2440 & 0.7477 & 0.1574 \\
\hline 0.1504 & 0.1607 & 0.5049 & 0.2409 & 0.7883 & 0.1360 \\
\hline 0.2053 & 0.1879 & 0.5552 & 0.2316 & 0.8333 & 0.1126 \\
\hline 0.2511 & 0.2099 & 0.5967 & 0.2199 & 0.8857 & 0.0788 \\
\hline 0.2993 & 0.2278 & 0.6433 & 0.2034 & 0.9319 & 0.0474 \\
\hline 0.3489 & 0.2354 & & & & \\
\hline \multicolumn{6}{|c|}{$298.15 \mathrm{~K}$} \\
\hline 0.0557 & 0.0644 & 0.4055 & 0.2432 & 0.7012 & 0.1780 \\
\hline 0.1035 & 0.1131 & 0.4498 & 0.2458 & 0.7482 & 0.1547 \\
\hline 0.1552 & 0.1624 & 0.5082 & 0.2432 & 0.7919 & 0.1299 \\
\hline 0.2037 & 0.1889 & 0.5512 & 0.2328 & 0.8374 & 0.1010 \\
\hline 0.2556 & 0.2120 & 0.6073 & 0.2177 & 0.8913 & 0.0637 \\
\hline 0.3076 & 0.2307 & 0.6527 & 0.2011 & 0.9459 & 0.0246 \\
\hline 0.3534 & 0.2389 & & & & \\
\hline \multicolumn{6}{|c|}{$303.15 \mathrm{~K}$} \\
\hline 0.0514 & 0.0611 & 0.4041 & 0.2618 & 0.6920 & 0.2007 \\
\hline 0.1007 & 0.1152 & 0.4473 & 0.2616 & 0.7406 & 0.1758 \\
\hline 0.1487 & 0.1568 & 0.4999 & 0.2571 & 0.7993 & 0.1431 \\
\hline 0.2060 & 0.1998 & 0.5501 & 0.2503 & 0.8364 & 0.1193 \\
\hline 0.2507 & 0.2265 & 0.5966 & 0.2364 & 0.8875 & 0.0839 \\
\hline 0.3041 & 0.2458 & 0.6463 & 0.2193 & 0.9345 & 0.0472 \\
\hline 0.3513 & 0.2565 & & & & \\
\hline
\end{tabular}

Tabla 3-46: velocidades del sonido de exceso, coeficientes de expansión térmica a $P=$ cte. de exceso, y compresibilidades isoentrópicas de exceso del sistema di- $n$-propilamina (1) + 2-propanona (2)

\begin{tabular}{cccccccc}
\hline$x_{1}$ & $u^{\mathrm{E}} / \mathrm{m} \cdot \mathrm{s}^{-1}$ & $\alpha_{\mathrm{P}}^{\mathrm{E}} / 10^{-6} \mathrm{~K}^{-1}$ & $\kappa_{S}^{\mathrm{E}} / \mathrm{TPa}^{-1}$ & $x_{1}$ & $u^{\mathrm{E}} / \mathrm{m} \cdot \mathrm{s}^{-1}$ & $\alpha_{\mathrm{P}}^{\mathrm{E}} / 10^{-6} \mathrm{~K}^{-1}$ & $\kappa_{S}^{\mathrm{E}} / \mathrm{TPa}^{-1}$ \\
\hline 0.0557 & -3.9 & -0.21 & 7.14 & 0.5512 & -10.4 & 15.68 & 19.28 \\
0.1035 & -6.5 & 1.26 & 12.07 & 0.6073 & -9.7 & 14.32 & 17.96 \\
0.1552 & -8.5 & 3.88 & 16.05 & 0.6527 & -8.9 & 12.75 & 16.38 \\
0.2037 & -10.0 & 6.53 & 18.75 & 0.7012 & -7.9 & 10.70 & 14.45 \\
0.2556 & -11.0 & 9.42 & 20.60 & 0.7482 & -6.8 & 8.52 & 12.42 \\
0.3076 & -11.5 & 12.05 & 21.63 & 0.7919 & -5.8 & 6.42 & 10.47 \\
0.3534 & -11.8 & 13.90 & 22.12 & 0.8374 & -4.5 & 4.31 & 8.17 \\
0.4055 & -11.8 & 15.38 & 22.07 & 0.8913 & -3.1 & 2.12 & 5.47 \\
0.4498 & -11.6 & 16.11 & 21.73 & 0.9459 & -1.5 & 0.52 & 2.58 \\
0.5082 & -11.0 & 16.23 & 20.56 & & & & \\
\hline
\end{tabular}

Tabla 3-47: coeficientes y desviaciones estándar del ajuste a la ecuación R-K de las diferentes propiedades de exceso del sistema di- $n$-propilamina (1) +2 -propanona (2)

\begin{tabular}{cccccccc}
\hline$T / \mathrm{K}$ & Propiedad & $A_{0}$ & $A_{1}$ & $A_{2}$ & $A_{3}$ & $A_{4}$ & $\sigma$ \\
\hline 293.15 & $V_{\mathrm{m}}^{\mathrm{E}} / \mathrm{cm}^{3} \cdot \mathrm{mol}^{-1}$ & 0.960 & -0.286 & 0.077 & & & 0.003 \\
298.15 & $V_{\mathrm{m}}^{\mathrm{E}} / \mathrm{cm}^{3} \cdot \mathrm{mol}^{-1}$ & 0.971 & -0.252 & -0.02 & -0.2 & & 0.003 \\
& $u^{\mathrm{E}} / \mathrm{m}^{-1}$ & -44.56 & 20.9 & -9.7 & 5.3 & & 0.06 \\
& $\alpha_{\mathrm{P}}^{\mathrm{E}} / 10^{-6} \mathrm{~K}^{-1}$ & 64.92 & -8.9 & -69.6 & 20.9 & -9.8 & 0.04 \\
& $\kappa_{S}^{\mathrm{E}} / \mathrm{TPa}^{-1}$ & 83.13 & -40.8 & 16.4 & -9.3 & & 0.11 \\
303.15 & $V_{\mathrm{m}}^{\mathrm{E}} / \mathrm{cm}^{3} \cdot \mathrm{mol}^{-1}$ & 1.043 & -0.279 & & & 0.002 \\
\hline
\end{tabular}



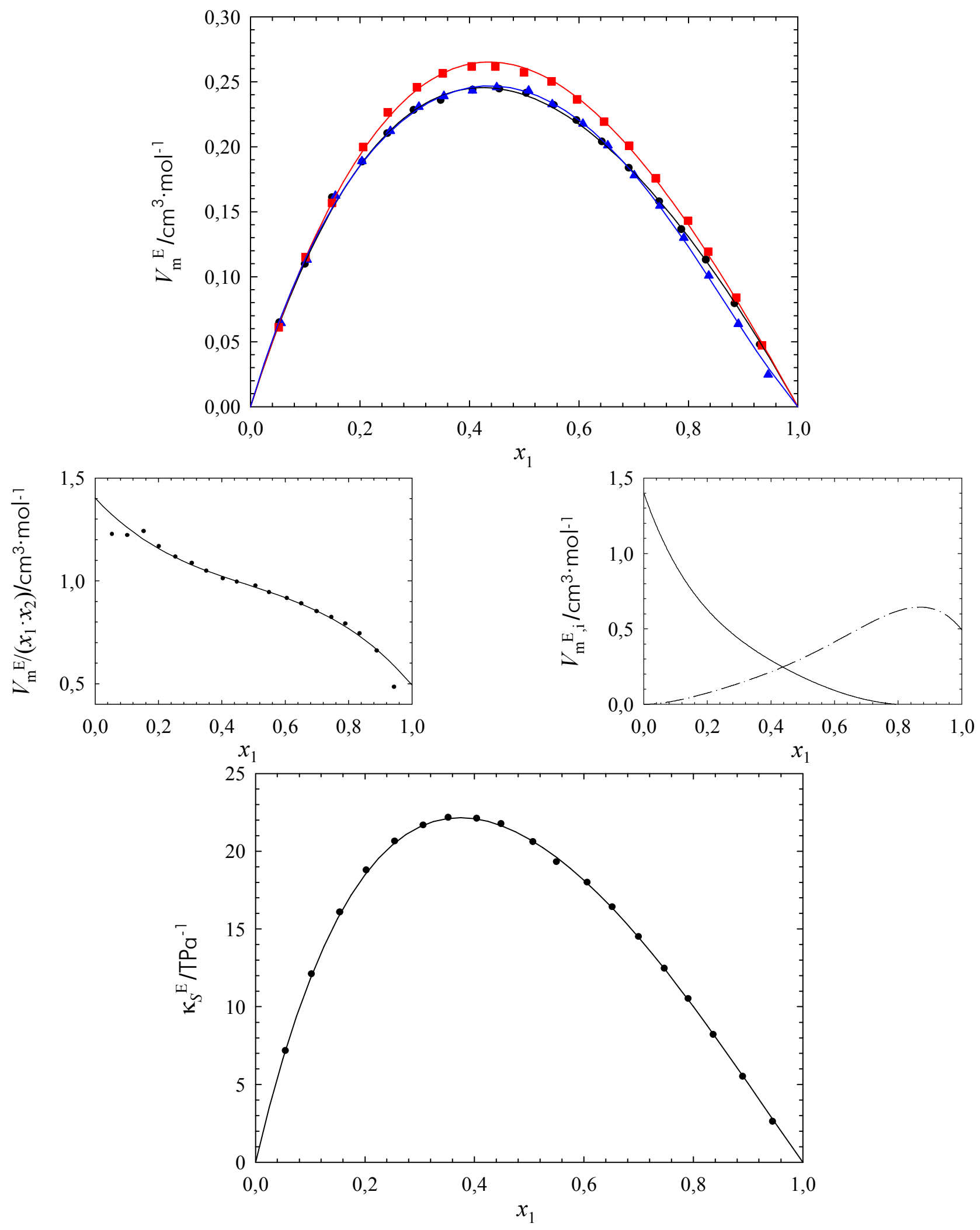

Ilustración 3-30: volúmenes molares de exceso $V_{\mathrm{m}}^{\mathrm{E}}$, volúmenes molares de exceso reducidos $V_{\mathrm{m}}^{\mathrm{E}} /\left(x_{1} \cdot x_{2}\right)$, volúmenes molares parciales de exceso $V_{\mathrm{m}, \mathrm{i}}^{\mathrm{E}}$ y compresibilidades isoentrópicas de exceso $\kappa_{S}^{\mathrm{E}}$ del sistema di- $n$-propilamina (1) +2 -propanona (2) 
Tabla 3-48: volúmenes molares de exceso del sistema di-n-propilamina (1) + 2-butanona (2)

\begin{tabular}{|c|c|c|c|c|c|}
\hline$x_{1}$ & $V_{\mathrm{m}}^{\mathrm{E}} / \mathrm{cm}^{3} \cdot \mathrm{mol}^{-1}$ & $x_{1}$ & $V_{\mathrm{m}}^{\mathrm{E}} / \mathrm{cm}^{3} \cdot \mathrm{mol}^{-1}$ & $x_{1}$ & $V_{\mathrm{m}}^{\mathrm{E}} / \mathrm{cm}^{3} \cdot \mathrm{mol}^{-1}$ \\
\hline \multicolumn{6}{|c|}{$293.15 \mathrm{~K}$} \\
\hline 0.0521 & 0.0270 & 0.3986 & 0.1356 & 0.7017 & 0.1225 \\
\hline 0.0990 & 0.0493 & 0.4449 & 0.1388 & 0.7541 & 0.1118 \\
\hline 0.1427 & 0.0679 & 0.4932 & 0.1424 & 0.8018 & 0.1006 \\
\hline 0.1991 & 0.0924 & 0.5505 & 0.1417 & 0.8503 & 0.0878 \\
\hline 0.2445 & 0.1069 & 0.5988 & 0.1371 & 0.8979 & 0.0690 \\
\hline 0.3032 & 0.1205 & 0.6496 & 0.1301 & 0.9461 & 0.0429 \\
\hline 0.3432 & 0.1278 & & & & \\
\hline \multicolumn{6}{|c|}{$298.15 \mathrm{~K}$} \\
\hline 0.0529 & 0.0253 & 0.4008 & 0.1357 & 0.7042 & 0.1232 \\
\hline 0.0959 & 0.0504 & 0.4461 & 0.1423 & 0.7554 & 0.1139 \\
\hline 0.1443 & 0.0662 & 0.4994 & 0.1433 & 0.7999 & 0.1012 \\
\hline 0.1952 & 0.0892 & 0.5375 & 0.1446 & 0.8491 & 0.0874 \\
\hline 0.2469 & 0.1055 & 0.5997 & 0.1387 & 0.8973 & 0.0706 \\
\hline 0.3013 & 0.1153 & 0.6455 & 0.1340 & 0.9408 & 0.0496 \\
\hline 0.3526 & 0.1296 & & & & \\
\hline \multicolumn{6}{|c|}{$303.15 \mathrm{~K}$} \\
\hline 0.0552 & 0.0289 & 0.3919 & 0.1394 & 0.6902 & 0.1294 \\
\hline 0.1042 & 0.0549 & 0.4446 & 0.1454 & 0.7405 & 0.1179 \\
\hline 0.1487 & 0.0710 & 0.4846 & 0.1486 & 0.7854 & 0.1044 \\
\hline 0.1968 & 0.0940 & 0.5430 & 0.1492 & 0.8894 & 0.0716 \\
\hline 0.2483 & 0.1101 & 0.5869 & 0.1429 & 0.9012 & 0.0670 \\
\hline 0.2959 & 0.1236 & 0.6370 & 0.1378 & 0.9342 & 0.0546 \\
\hline 0.3343 & 0.1325 & & & & \\
\hline
\end{tabular}

Tabla 3-49: velocidades del sonido de exceso, coeficientes de expansión térmica a $P=$ cte. de exceso, y compresibilidades isoentrópicas de exceso del sistema di- $n$-propilamina (1) +2 -butanona (2)

\begin{tabular}{cccccccc}
\hline$x_{1}$ & $u^{\mathrm{E}} / \mathrm{m} \cdot \mathrm{s}^{-1}$ & $\alpha_{\mathrm{P}}^{\mathrm{E}} / 10^{-6} \mathrm{~K}^{-1}$ & $\kappa_{S}^{\mathrm{E}} / \mathrm{TPa}^{-1}$ & $x_{1}$ & $u^{\mathrm{E}} / \mathrm{m} \cdot \mathrm{s}^{-1}$ & $\alpha_{\mathrm{P}}^{\mathrm{E}} / 10^{-6} \mathrm{~K}^{-1}$ & $\kappa_{S}^{\mathrm{E}} / \mathrm{TPa}^{-1}$ \\
\hline 0.0529 & -1.1 & 2.64 & 1.86 & 0.5997 & -4.1 & 3.63 & 7.67 \\
0.0959 & -1.7 & 2.19 & 2.97 & 0.6455 & -3.9 & 2.25 & 7.33 \\
0.1443 & -2.6 & 0.85 & 4.48 & 0.7042 & -3.4 & 0.51 & 6.46 \\
0.1952 & -3.4 & 0.07 & 5.96 & 0.7554 & -3.1 & -0.48 & 5.91 \\
0.2469 & -3.8 & 0.23 & 6.78 & 0.7999 & -2.7 & -0.73 & 5.11 \\
0.3526 & -4.4 & 2.86 & 7.92 & 0.8491 & -2.2 & -0.23 & 4.26 \\
0.4008 & -4.5 & 4.19 & 8.14 & 0.8973 & -1.6 & 0.73 & 3.14 \\
0.4994 & -4.5 & 5.35 & 8.30 & 0.9408 & -1.0 & 1.37 & 1.89 \\
\hline
\end{tabular}

Tabla 3-50: coeficientes y desviaciones estándar del ajuste a la ecuación R-K de las diferentes propiedades de exceso del sistema di- $n$-propilamina (1) + 2-butanona (2)

\begin{tabular}{cccccccc}
\hline$T / \mathrm{K}$ & Propiedad & $A_{0}$ & $A_{1}$ & $A_{2}$ & $A_{3}$ & $A_{4}$ & $\sigma$ \\
\hline 293.15 & $V_{\mathrm{m}}^{\mathrm{E}} / \mathrm{cm}^{3} \cdot \mathrm{mol}^{-1}$ & 0.562 & -0.018 & 0.127 & 0.21 & & 0.0016 \\
298.15 & $V_{\mathrm{m}}^{\mathrm{E}} / \mathrm{cm}^{3} \cdot \mathrm{mol}^{-1}$ & 0.576 & 0.006 & -0.04 & 0.20 & 0.27 & 0.002 \\
& $u^{\mathrm{E}} /{\mathrm{m} \cdot \mathrm{s}^{-1}}^{\mathrm{E}}$ & -17.99 & 4.3 & -2.3 & -3.3 & & 0.07 \\
& $\alpha_{\mathrm{P}}^{\mathrm{E}} / 1 \mathrm{O}^{-6} \mathrm{~K}^{-1}$ & 21.8 & -3.5 & -138 & -7 & 201 & 0.18 \\
303.15 & $\kappa_{S}^{\mathrm{E}} / \mathrm{TPa}^{-1}$ & 33.0 & -6.3 & 3.6 & 7.4 & & 0.11 \\
\hline
\end{tabular}



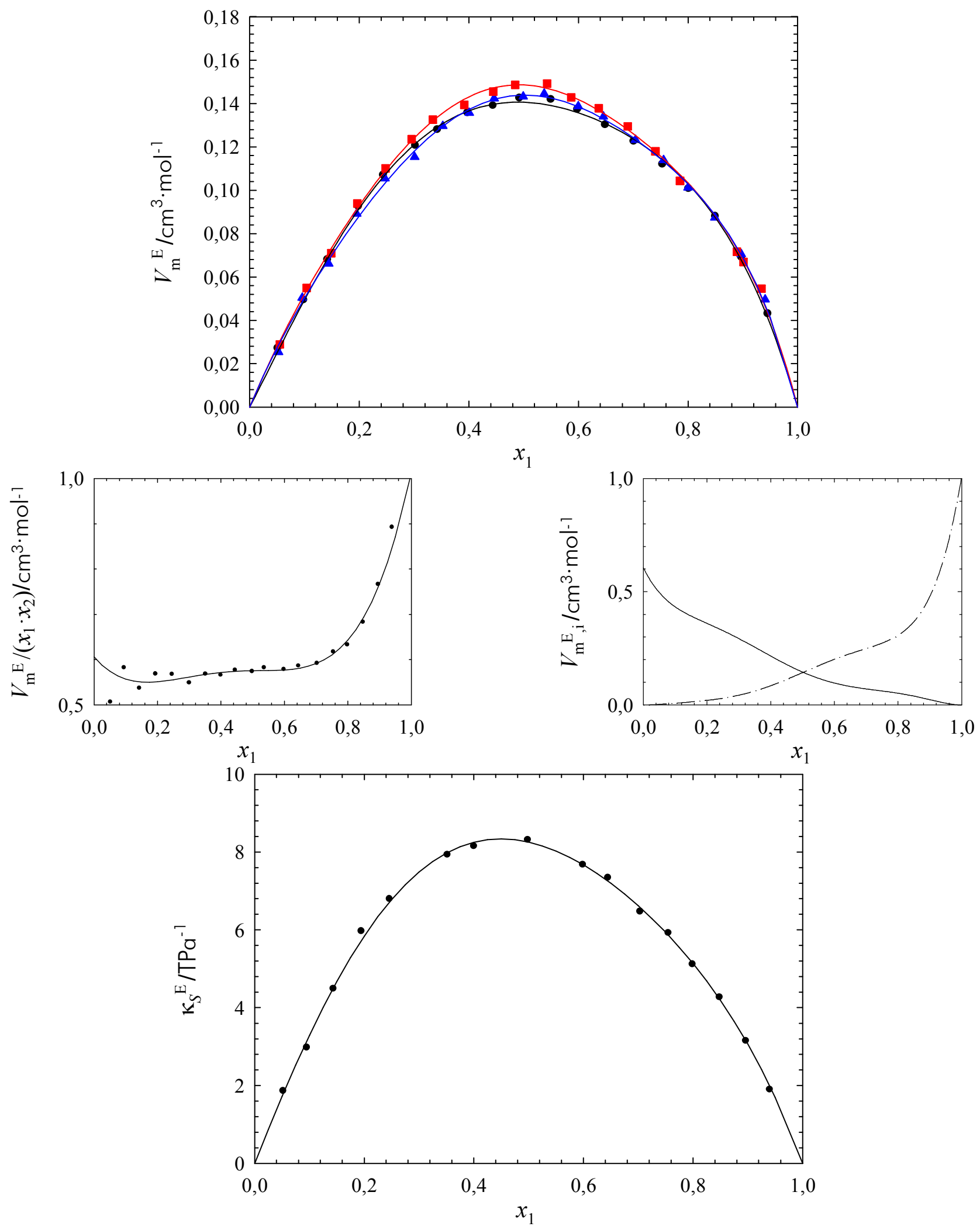

llustración 3-31: volúmenes molares de exceso $V_{\mathrm{m}}^{\mathrm{E}}$, volúmenes molares de exceso reducidos $V_{\mathrm{m}}^{\mathrm{E}} /\left(x_{1} \cdot x_{2}\right)$, volúmenes molares parciales de exceso $V_{\mathrm{m}, \mathrm{i}}^{\mathrm{E}}$ y compresibilidades isoentrópicas de exceso $\kappa_{S}^{\mathrm{E}}$ del sistema di- $n$-propilamina (1) +2 -butanona (2) 
Tabla 3-51: volúmenes molares de exceso del sistema di- $n$-propilamina (1) + 2-heptanona (2)

\begin{tabular}{|c|c|c|c|c|c|}
\hline$x_{1}$ & $V_{\mathrm{m}}^{\mathrm{E}} / \mathrm{cm}^{3} \cdot \mathrm{mol}^{-1}$ & $x_{1}$ & $V_{\mathrm{m}}^{\mathrm{E}} / \mathrm{cm}^{3} \cdot \mathrm{mol}^{-1}$ & $x_{1}$ & $V_{\mathrm{m}}^{\mathrm{E}} / \mathrm{cm}^{3} \cdot \mathrm{mol}^{-1}$ \\
\hline \multicolumn{6}{|c|}{$293.15 \mathrm{~K}$} \\
\hline 0.0520 & -0.0280 & 0.4506 & -0.0822 & 0.7474 & -0.0710 \\
\hline 0.1007 & -0.0423 & 0.5070 & -0.0833 & 0.7987 & -0.0630 \\
\hline 0.2385 & -0.0647 & 0.5421 & -0.0838 & 0.8441 & -0.0546 \\
\hline 0.3104 & -0.0727 & 0.6508 & -0.0809 & 0.8955 & -0.0419 \\
\hline 0.3522 & -0.0772 & 0.7064 & -0.0775 & 0.9397 & -0.0300 \\
\hline 0.4106 & -0.0798 & & & & \\
\hline \multicolumn{6}{|c|}{$298.15 \mathrm{~K}$} \\
\hline 0.0472 & -0.0291 & 0.3564 & -0.0832 & 0.7038 & -0.0741 \\
\hline 0.1007 & -0.0486 & 0.3997 & -0.0858 & 0.7474 & -0.0664 \\
\hline 0.1452 & -0.0574 & 0.4573 & -0.0877 & 0.800 & -0.0596 \\
\hline 0.1939 & -0.0658 & 0.5522 & -0.0868 & 0.8443 & -0.0498 \\
\hline 0.2582 & -0.0732 & 0.6150 & -0.0846 & 0.8952 & -0.0389 \\
\hline 0.3100 & -0.0787 & 0.6564 & -0.0798 & 0.9479 & -0.0215 \\
\hline \multicolumn{6}{|c|}{$303.15 \mathrm{~K}$} \\
\hline 0.0503 & -0.0328 & 0.3580 & -0.0897 & 0.7049 & -0.0855 \\
\hline 0.1007 & -0.0488 & 0.4109 & -0.0956 & 0.7503 & -0.0798 \\
\hline 0.1436 & -0.0572 & 0.4558 & -0.0973 & 0.7995 & -0.0693 \\
\hline 0.2006 & -0.0707 & 0.5496 & -0.0975 & 0.8421 & -0.0581 \\
\hline 0.2587 & -0.0781 & 0.6144 & -0.0955 & 0.8946 & -0.0441 \\
\hline 0.3130 & -0.0841 & 0.6524 & -0.0905 & 0.9422 & -0.0273 \\
\hline
\end{tabular}

Tabla 3-52: velocidades del sonido de exceso, coeficientes de expansión térmica a $P=$ cte. de exceso, y compresibilidades isoentrópicas de exceso del sistema di-n-propilamina (1) + 2-heptanona (2)

\begin{tabular}{cccccccc}
\hline$x_{1}$ & $u^{\mathrm{E}} / \mathrm{m} \cdot \mathrm{s}^{-1}$ & $\alpha_{\mathrm{P}}^{\mathrm{E}} / 10^{-6} \mathrm{~K}^{-1}$ & $\kappa_{S}^{\mathrm{E}} / \mathrm{TPa}^{-1}$ & $x_{1}$ & $u^{\mathrm{E}} / \mathrm{m} \cdot \mathrm{s}^{-1}$ & $\alpha_{\mathrm{P}}^{\mathrm{E}} / 10^{-6} \mathrm{~K}^{-1}$ & $\kappa_{S}^{\mathrm{E}} / \mathrm{TPa}^{-1}$ \\
\hline 0.0472 & 1.1 & -2.62 & -1.48 & 0.6150 & 5.0 & -8.28 & -7.89 \\
0.1007 & 2.3 & -4.56 & -3.12 & 0.6564 & 4.8 & -7.37 & -7.53 \\
0.1452 & 2.9 & -5.66 & -4.02 & 0.7038 & 4.4 & -6.09 & -7.05 \\
0.1939 & 3.7 & -6.60 & -5.17 & 0.7474 & 3.9 & -4.75 & -6.39 \\
0.2582 & 4.3 & -7.61 & -6.14 & 0.8000 & 3.4 & -3.07 & -5.56 \\
0.3100 & 4.8 & -8.32 & -6.92 & 0.8443 & 2.7 & -1.71 & -4.60 \\
0.3564 & 5.1 & -8.87 & -7.42 & 0.8952 & 2.0 & -0.46 & -3.38 \\
0.4573 & 5.4 & -9.54 & -8.10 & 0.9479 & 0.9 & 0.21 & -1.60 \\
0.5522 & 5.3 & -9.18 & -8.11 & & & & \\
\hline
\end{tabular}

Tabla 3-53: coeficientes y desviaciones estándar del ajuste a la ecuación R-K de las diferentes propiedades de exceso del sistema di- $n$-propilamina (1) + 2-heptanona (2)

\begin{tabular}{cccccccc}
\hline$T / \mathrm{K}$ & Propiedad & $A_{0}$ & $A_{1}$ & $A_{2}$ & $A_{3}$ & $A_{4}$ & $\sigma$ \\
\hline 293.15 & $V_{\mathrm{m}}^{\mathrm{E}} / \mathrm{cm}^{3} \cdot \mathrm{mol}^{-1}$ & -0.337 & -0.046 & -0.05 & 0.07 & -0.24 & 0.001 \\
298.15 & $V_{\mathrm{m}}^{\mathrm{E}} / \mathrm{cm}^{3} \cdot \mathrm{mol}^{-1}$ & -0.3547 & 0 & 0 & 0.12 & -0.29 & 0.0007 \\
& $u^{\mathrm{E}} / \mathrm{m}^{-1}$ & 21.62 & -2.10 & 1.3 & & 0.05 & 0.009 \\
& $\alpha_{\mathrm{p}}^{\mathrm{E}} / 10^{-6} \mathrm{~K}^{-1}$ & -38.12 & 6.15 & 26.5 & 35.57 & -15.0 & 0.07 \\
303.15 & $\kappa_{S}^{\mathrm{E}} / \mathrm{TPa}^{-1}$ & -32.67 & -1.5 & -2.5 & & & 0.26 \\
\hline
\end{tabular}



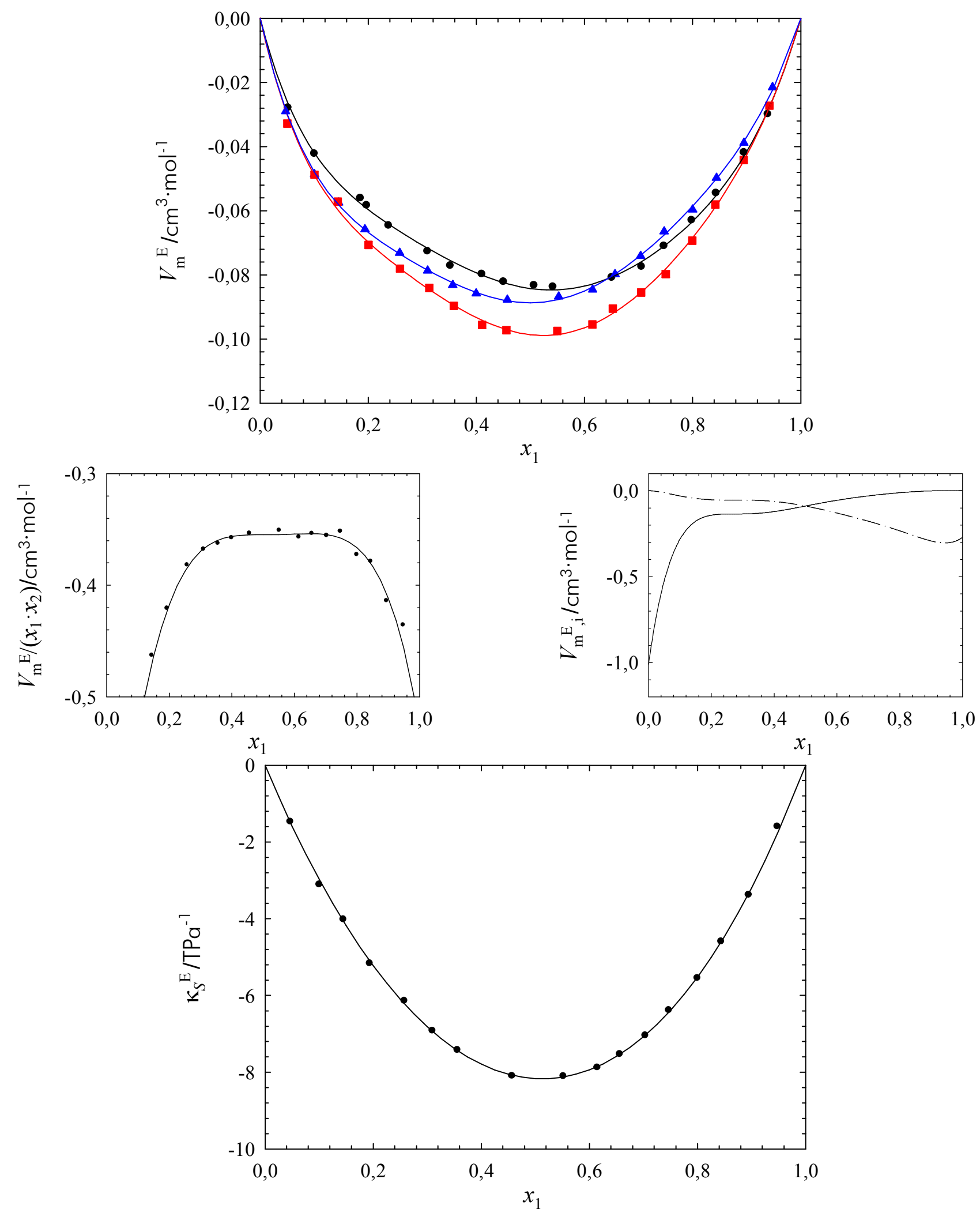

llustración 3-32: volúmenes molares de exceso $V_{\mathrm{m}}^{\mathrm{E}}$, volúmenes molares de exceso reducidos $V_{\mathrm{m}}^{\mathrm{E}} /\left(x_{1} \cdot x_{2}\right)$, volúmenes molares parciales de exceso $V_{\mathrm{m}, \mathrm{i}}^{\mathrm{E}}$ y compresibilidades isoentrópicas de exceso $\kappa_{S}^{\mathrm{E}}$ del sistema di- $n$-propilamina (1) +2 -heptanona (2) 
Tabla 3-54: volúmenes molares de exceso del sistema di-n-butilamina (1) +2 -propanona (2)

\begin{tabular}{|c|c|c|c|c|c|}
\hline$x_{1}$ & $V_{\mathrm{m}}^{\mathrm{E}} / \mathrm{cm}^{3} \cdot \mathrm{mol}^{-1}$ & $x_{1}$ & $V_{\mathrm{m}}^{\mathrm{E}} / \mathrm{cm}^{3} \cdot \mathrm{mol}^{-1}$ & $x_{1}$ & $V_{\mathrm{m}}^{\mathrm{E}} / \mathrm{cm}^{3} \cdot \mathrm{mol}^{-1}$ \\
\hline \multicolumn{6}{|c|}{$293.15 \mathrm{~K}$} \\
\hline 0.0497 & 0.1066 & 0.4066 & 0.4178 & 0.6973 & 0.3085 \\
\hline 0.0948 & 0.1897 & 0.4577 & 0.4189 & 0.7376 & 0.2826 \\
\hline 0.1458 & 0.2611 & 0.5037 & 0.4059 & 0.7787 & 0.2443 \\
\hline 0.2047 & 0.3140 & 0.5510 & 0.3873 & 0.8355 & 0.1936 \\
\hline 0.2544 & 0.3650 & 0.6108 & 0.3627 & 0.8933 & 0.1303 \\
\hline 0.3005 & 0.3904 & 0.6487 & 0.3424 & 0.9383 & 0.0738 \\
\hline 0.3387 & 0.4044 & & & & \\
\hline \multicolumn{6}{|c|}{$298.15 \mathrm{~K}$} \\
\hline 0.0480 & 0.0934 & 0.4004 & 0.4304 & 0.6990 & 0.3121 \\
\hline 0.0965 & 0.1894 & 0.4493 & 0.4294 & 0.7463 & 0.2754 \\
\hline 0.1444 & 0.2731 & 0.4984 & 0.4188 & 0.7901 & 0.2407 \\
\hline 0.1995 & 0.3346 & 0.5418 & 0.4003 & 0.8502 & 0.1808 \\
\hline 0.2471 & 0.3743 & 0.5914 & 0.3768 & 0.8908 & 0.1353 \\
\hline 0.3001 & 0.4046 & 0.6437 & 0.3471 & 0.9421 & 0.0664 \\
\hline 0.3480 & 0.4220 & & & & \\
\hline \multicolumn{6}{|c|}{$303.15 \mathrm{~K}$} \\
\hline 0.0482 & 0.1150 & 0.3996 & 0.4412 & 0.7023 & 0.3304 \\
\hline 0.0934 & 0.1903 & 0.4514 & 0.4398 & 0.7602 & 0.2789 \\
\hline 0.1512 & 0.2919 & 0.5018 & 0.4321 & 0.7970 & 0.2392 \\
\hline 0.1998 & 0.3462 & 0.5440 & 0.4134 & 0.8441 & 0.1926 \\
\hline 0.2491 & 0.3779 & 0.6045 & 0.3934 & 0.8928 & 0.1369 \\
\hline 0.3497 & 0.4303 & 0.6559 & 0.3593 & 0.9437 & 0.0733 \\
\hline
\end{tabular}

Tabla 3-55: velocidades del sonido de exceso, coeficientes de expansión térmica a $P=$ cłe. de exceso, y compresibilidades isoentrópicas de exceso del sistema di-n-butilamina (1) +2 -propanona (2)

\begin{tabular}{cccccccc}
\hline$x_{1}$ & $u^{\mathrm{E}} / \mathrm{m} \cdot \mathrm{s}^{-1}$ & $\alpha_{\mathrm{P}}^{\mathrm{E}} / 10^{-6} \mathrm{~K}^{-1}$ & $\kappa_{S}^{\mathrm{E}} / \mathrm{TPa}^{-1}$ & $x_{1}$ & $u^{\mathrm{E}} / \mathrm{m} \cdot \mathrm{s}^{-1}$ & $\alpha_{\mathrm{P}}^{\mathrm{E}} / 10^{-6} \mathrm{~K}^{-1}$ & $\kappa_{S}^{\mathrm{E}} / \mathrm{TPa}^{-1}$ \\
\hline 0.0480 & -5.1 & 7.68 & 9.43 & 0.5418 & -13.4 & 14.82 & 22.80 \\
0.0965 & -9.1 & 13.22 & 16.80 & 0.5914 & -12.4 & 13.45 & 20.89 \\
0.1444 & -12.0 & 16.84 & 22.06 & 0.6437 & -11.3 & 12.01 & 18.76 \\
0.1995 & -14.0 & 18.98 & 25.54 & 0.6990 & -9.9 & 10.49 & 16.25 \\
0.2471 & -15.1 & 19.76 & 27.37 & 0.7463 & -8.5 & 9.14 & 13.82 \\
0.3001 & -15.8 & 19.78 & 28.22 & 0.7901 & -7.3 & 7.87 & 11.79 \\
0.3480 & -15.9 & 19.28 & 28.18 & 0.8502 & -5.4 & 5.95 & 8.58 \\
0.4004 & -15.6 & 18.35 & 27.39 & 0.8908 & -3.9 & 4.53 & 6.27 \\
0.4493 & -15.1 & 17.26 & 26.27 & 0.9421 & -2.1 & 2.50 & 3.23 \\
0.4984 & -14.4 & 16.01 & 24.71 & & & & \\
\hline
\end{tabular}

Tabla 3-56: coeficientes y desviaciones estándar del ajuste a la ecuación R-K de las diferentes propiedades de exceso del sistema di-n-butilamina (1) +2 -propanona (2)

\begin{tabular}{ccccccc}
\hline$T / \mathrm{K}$ & Propiedad & $A_{0}$ & $A_{1}$ & $A_{2}$ & $A_{3}$ & $\sigma$ \\
\hline 293.15 & $V_{\mathrm{m}}^{\mathrm{E}} / \mathrm{cm}^{3} \cdot \mathrm{mol}^{-1}$ & 1.628 & -0.488 & 0.20 & & 0.003 \\
298.15 & $V_{\mathrm{m}}^{\mathrm{E}} / \mathrm{cm}^{3} \cdot \mathrm{mol}^{-1}$ & 1.670 & -0.549 & 0.21 & & 0.005 \\
& $u^{\mathrm{E}} / \mathrm{m}^{-1}$ & -57.19 & 34.4 & -23.1 & 7.8 & 0.06 \\
& $\alpha_{\mathrm{P}}^{\mathrm{E}} / 10^{-6} \mathrm{~K}^{-1}$ & 63.62 & -52.6 & 53.9 & -19.5 & 0.05 \\
& $\kappa_{S}^{\mathrm{E}} / \mathrm{TPa}^{-1}$ & 98.19 & -69.8 & 46.5 & -15.4 & 0.12 \\
303.15 & $V_{\mathrm{m}}^{\mathrm{E}} / \mathrm{cm}^{3} \cdot \mathrm{mol}^{-1}$ & 1.727 & -0.529 & 0.24 & & 0.005 \\
\hline
\end{tabular}



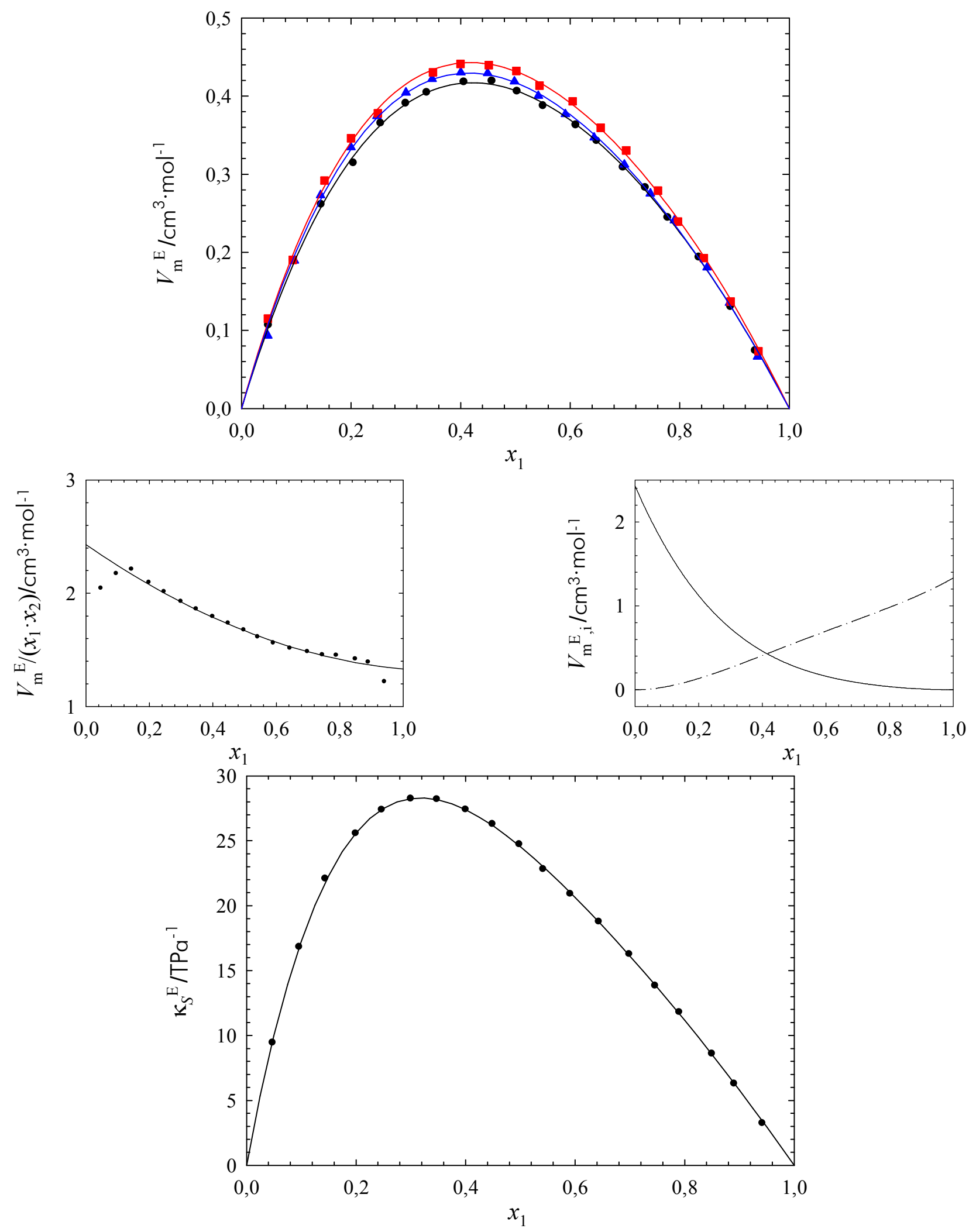

Ilustración 3-33: volúmenes molares de exceso $V_{\mathrm{m}}^{\mathrm{E}}$, volúmenes molares de exceso reducidos $V_{\mathrm{m}}^{\mathrm{E}} /\left(x_{1} \cdot x_{2}\right)$, volúmenes molares parciales de exceso $V_{\mathrm{m}, \mathrm{i}}^{\mathrm{E}}$ y compresibilidades isoentrópicas de exceso $\kappa_{S}^{\mathrm{E}}$ del sistema di- $n$-butilamina $(1)+2$-propanona (2) 
Tabla 3-57: volúmenes molares de exceso del sistema di- $n$-butilamina (1) + 2-butanona (2)

\begin{tabular}{|c|c|c|c|c|c|}
\hline$x_{1}$ & $V_{\mathrm{m}}^{\mathrm{E}} / \mathrm{cm}^{3} \cdot \mathrm{mol}^{-1}$ & $x_{1}$ & $V_{\mathrm{m}}^{\mathrm{E}} / \mathrm{cm}^{3} \cdot \mathrm{mol}^{-1}$ & $x_{1}$ & $V_{\mathrm{m}}^{\mathrm{E}} / \mathrm{cm}^{3} \cdot \mathrm{mol}^{-1}$ \\
\hline \multicolumn{6}{|c|}{$293.15 \mathrm{~K}$} \\
\hline 0.0487 & 0.0555 & 0.3978 & 0.2538 & 0.6944 & 0.2087 \\
\hline 0.0999 & 0.1094 & 0.4447 & 0.2594 & 0.7461 & 0.1884 \\
\hline 0.1402 & 0.1474 & 0.4995 & 0.2560 & 0.8032 & 0.1585 \\
\hline 0.1978 & 0.1857 & 0.5455 & 0.2497 & 0.8512 & 0.1286 \\
\hline 0.2446 & 0.2113 & 0.5895 & 0.2427 & 0.8848 & 0.1047 \\
\hline 0.2964 & 0.2306 & 0.6436 & 0.2283 & 0.9432 & 0.0528 \\
\hline 0.3446 & 0.2454 & & & & \\
\hline \multicolumn{6}{|c|}{$298.15 \mathrm{~K}$} \\
\hline 0.0518 & 0.0636 & 0.3964 & 0.2611 & 0.6888 & 0.2164 \\
\hline 0.0978 & 0.1124 & 0.4545 & 0.2672 & 0.7397 & 0.1942 \\
\hline 0.1544 & 0.1637 & 0.4982 & 0.2645 & 0.7966 & 0.1631 \\
\hline 0.2022 & 0.1979 & 0.5580 & 0.2601 & 0.8375 & 0.1393 \\
\hline 0.2527 & 0.2257 & 0.6063 & 0.2458 & 0.8962 & 0.0973 \\
\hline 0.2997 & 0.2406 & 0.6477 & 0.2323 & 0.9319 & 0.0664 \\
\hline 0.3486 & 0.2533 & & & & \\
\hline \multicolumn{6}{|c|}{$303.15 \mathrm{~K}$} \\
\hline 0.0473 & 0.0613 & 0.3995 & 0.2763 & 0.6910 & 0.2280 \\
\hline 0.1005 & 0.1222 & 0.4598 & 0.2820 & 0.7407 & 0.2047 \\
\hline 0.1544 & 0.1693 & 0.5043 & 0.2805 & 0.7926 & 0.1780 \\
\hline 0.1995 & 0.2017 & 0.5434 & 0.2746 & 0.8334 & 0.1506 \\
\hline 0.2514 & 0.2319 & 0.5955 & 0.2621 & 0.8902 & 0.0969 \\
\hline 0.2996 & 0.2534 & 0.6449 & 0.2503 & 0.9379 & 0.0551 \\
\hline 0.3507 & 0.2671 & & & & \\
\hline
\end{tabular}

Tabla 3-58: velocidades del sonido de exceso, coeficientes de expansión térmica a $P=$ cte. de exceso, y compresibilidades isoentrópicas de exceso del sistema di- $n$-butilamina (1) + 2-butanona (2)

\begin{tabular}{cccccccc}
\hline$x_{1}$ & $u^{\mathrm{E}} / \mathrm{m} \cdot \mathrm{s}^{-1}$ & $\alpha_{\mathrm{P}}^{\mathrm{E}} / 10^{-6} \mathrm{~K}^{-1}$ & $\kappa_{S}^{\mathrm{E}} / \mathrm{TPa}^{-1}$ & $x_{1}$ & $u^{\mathrm{E}} / \mathrm{m} \cdot \mathrm{s}^{-1}$ & $\alpha_{\mathrm{P}}^{\mathrm{E}} / 10^{-6} \mathrm{~K}^{-1}$ & $\kappa_{S}^{\mathrm{E}} / \mathrm{TPa}^{-1}$ \\
\hline 0.0518 & -2.9 & 3.28 & 4.81 & 0.5580 & -8.2 & 14.73 & 13.39 \\
0.0978 & -4.8 & 6.25 & 8.04 & 0.6063 & -7.4 & 13.04 & 12.12 \\
0.1544 & -6.6 & 9.68 & 11.08 & 0.6477 & -7.0 & 11.46 & 11.41 \\
0.2022 & -7.6 & 12.19 & 12.88 & 0.6888 & -6.5 & 9.76 & 10.46 \\
0.2527 & -8.4 & 14.32 & 14.17 & 0.7397 & -5.7 & 7.59 & 9.09 \\
0.2997 & -8.8 & 15.70 & 14.69 & 0.7966 & -4.5 & 5.19 & 7.23 \\
0.3486 & -9.0 & 16.64 & 15.01 & 0.8375 & -3.9 & 3.61 & 6.18 \\
0.3964 & -9.1 & 17.01 & 15.10 & 0.8962 & -2.6 & 1.77 & 4.25 \\
0.4545 & -8.8 & 16.79 & 14.55 & 0.9319 & -1.8 & 0.88 & 2.90 \\
0.4982 & -8.6 & 16.11 & 14.18 & & & & \\
\hline
\end{tabular}

Tabla 3-59: coeficientes y desviaciones estándar del ajuste a la ecuación R-K de las diferentes propiedades de exceso del sistema di- $n$-butilamina (1) +2 -butanona (2)

\begin{tabular}{ccccccc}
\hline$T / \mathrm{K}$ & Propiedad & $A_{0}$ & $A_{1}$ & $A_{2}$ & $A_{3}$ & $\sigma$ \\
\hline 293.15 & $V_{\mathrm{m}}^{\mathrm{E}} / \mathrm{cm}^{3} \cdot \mathrm{mol}^{-1}$ & 1.024 & -0.141 & 0.159 & & 0.0017 \\
298.15 & $V_{\mathrm{m}}^{\mathrm{E}} / \mathrm{cm}^{3} \cdot \mathrm{mol}^{-1}$ & 1.058 & -0.168 & 0.161 & 0.0018 \\
& $u^{\mathrm{E}} / \mathrm{m}^{-1}$ & -34.4 & 15.7 & -10.3 & 0.06 & 0.04 \\
& $\alpha_{\mathrm{P}}^{\mathrm{E}} / 10^{-6} \mathrm{~K}^{-1}$ & 64.42 & -40.0 & -30.5 & 10.6 & 0.11 \\
& $\kappa_{S}^{\mathrm{E}} / \mathrm{TPa}^{-1}$ & 56.43 & -28.2 & 17.9 & & 0.003 \\
\hline
\end{tabular}



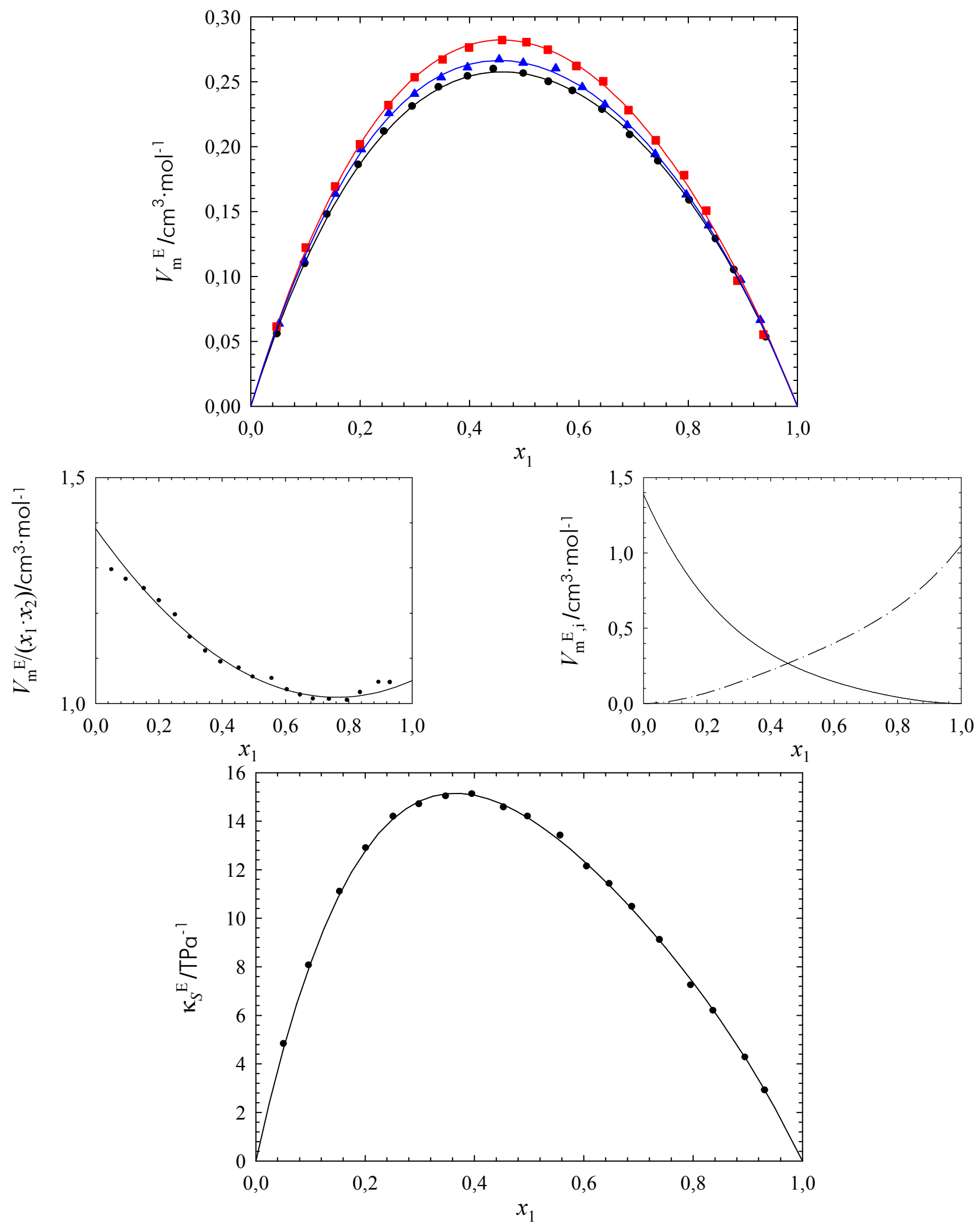

Ilustración 3-34: volúmenes molares de exceso $V_{\mathrm{m}}^{\mathrm{E}}$, volúmenes molares de exceso reducidos $V_{\mathrm{m}}^{\mathrm{E}} /\left(x_{1} \cdot x_{2}\right)$, volúmenes molares parciales de exceso $V_{\mathrm{m}, \mathrm{i}}^{\mathrm{E}}$ y compresibilidades isoentrópicas de exceso $\kappa_{S}^{\mathrm{E}}$ del sistema di- $n$-butilamina (1) +2 -butanona (2) 
Tabla 3-60: volúmenes molares de exceso del sistema di- $n$-butilamina (1) +2 -heptanona (2)

\begin{tabular}{cccccc}
\hline$x_{1}$ & $V_{\mathrm{m}}^{\mathrm{E}} / \mathrm{cm}^{3} \cdot \mathrm{mol}^{-1}$ & $x_{1}$ & $V_{\mathrm{m}}^{\mathrm{E}} / \mathrm{cm}^{3} \cdot \mathrm{mol}^{-1}$ & $x_{1}$ & $V_{\mathrm{m}}^{\mathrm{E}} / \mathrm{cm}^{3} \cdot \mathrm{mol}^{-1}$ \\
\hline \multicolumn{7}{c}{$293.15 \mathrm{~K}$} \\
\hline 0.0982 & -0.0012 & 0.4906 & 0.0089 & 0.7939 & 0.0176 \\
0.2489 & -0.0011 & 0.5507 & 0.0102 & 0.8367 & 0.0175 \\
0.3560 & 0.0042 & 0.7394 & 0.0164 & 0.9360 & 0.0104 \\
\hline \multicolumn{7}{c}{$298.15 \mathrm{~K}$} \\
0.0512 & -0.0019 & 0.3518 & 0.0024 & 0.6387 & 0.0168 \\
0.1014 & -0.0038 & 0.4504 & 0.0076 & 0.7903 & 0.0253 \\
0.2013 & -0.0034 & 0.5501 & 0.0114 & 0.8893 & 0.0241 \\
0.2491 & -0.0011 & 0.5951 & 0.0141 & 0.9376 & 0.0202 \\
\hline \multicolumn{7}{c}{$303.15 \mathrm{~K}$} \\
0.1016 & -0.0163 & 0.3522 & 0.0049 & 0.7413 & 0.0329 \\
0.1480 & -0.0150 & 0.5014 & 0.0158 & 0.8395 & 0.0324 \\
0.2530 & -0.0105 & 0.5904 & 0.0240 & 0.8878 & 0.0268 \\
\hline
\end{tabular}

Tabla 3-61: velocidades del sonido de exceso, coeficientes de expansión térmica a $P=$ cłe. de exceso, y compresibilidades isoentrópicas de exceso del sistema di- $n$-butilamina (1) +2 -heptanona (2)

\begin{tabular}{cccccccc}
\hline$x_{1}$ & $u^{\mathrm{E}} / \mathrm{m} \cdot \mathrm{s}^{-1}$ & $\alpha_{\mathrm{p}}^{\mathrm{E}} / 10^{-6} \mathrm{~K}^{-1}$ & $\kappa_{S}^{\mathrm{E}} / \mathrm{TPa}^{-1}$ & $x_{1}$ & $u^{\mathrm{E}} / \mathrm{m} \cdot \mathrm{s}^{-1}$ & $\alpha_{\mathrm{p}}^{\mathrm{E}} / 10^{-6} \mathrm{~K}^{-1}$ & $\kappa_{S}^{\mathrm{E}} / \mathrm{TPa}^{-1}$ \\
\hline 0.0512 & & -8.46 & & 0.5951 & -0.6 & 6.99 & 0.96 \\
0.1014 & -0.1 & -10.23 & 0.12 & 0.6387 & -0.7 & 8.12 & 1.05 \\
0.2013 & -0.2 & -6.21 & 0.24 & 0.7419 & 0.0 & 9.76 & \\
0.2491 & -0.3 & -3.55 & 0.35 & 0.7897 & -0.7 & & 1.18 \\
0.3518 & -0.4 & 0.84 & 0.53 & 0.8403 & -0.8 & & 1.21 \\
0.4504 & -0.5 & 3.46 & 0.69 & 0.8893 & -0.7 & 6.91 & 1.11 \\
0.5501 & -0.6 & 5.82 & 0.88 & 0.9376 & -0.5 & 4.11 & 0.88 \\
\hline
\end{tabular}

Tabla 3-62: coeficientes y desviaciones estándar del ajuste a la ecuación R-K de las diferentes propiedades de exceso del sistema di- $n$-butilamina (1) +2 -heptanona (2)

\begin{tabular}{cccccccc}
\hline$T / \mathrm{K}$ & Propiedad & $A_{0}$ & $A_{1}$ & $A_{2}$ & $A_{3}$ & $A_{4}$ & $\sigma$ \\
\hline 293.15 & $V_{\mathrm{m}}^{\mathrm{E}} / \mathrm{cm}^{3} \cdot \mathrm{mol}^{-1}$ & 0.036 & 0.078 & 0.02 & 0.05 & 0.05 & 0.001 \\
298.15 & $V_{\mathrm{m}}^{\mathrm{E}} / \mathrm{cm}^{3} \cdot \mathrm{mol}^{-1}$ & 0.0383 & 0.092 & 0.045 & 0.158 & 0.12 & 0.0007 \\
& $u^{\mathrm{E}} / \mathrm{m}^{-1}$ & -2.25 & -1.89 & -0.5 & -3.1 & -4.3 & 0.02 \\
& $\alpha_{\mathrm{P}}^{\mathrm{E}} / 10^{-6} \mathrm{~K}^{-1}$ & 18.5 & 44.7 & 32 & 110 & -148 & 0.11 \\
& $\kappa_{S}^{\mathrm{E}} / \mathrm{TPa}^{-1}$ & 3.25 & 3.2 & 1.2 & 5.3 & 6.7 & 0.03 \\
303.15 & $V_{\mathrm{m}}^{\mathrm{E}} / \mathrm{cm}^{3} \cdot \mathrm{mol}^{-1}$ & 0.0651 & 0.155 & 0.081 & 0.209 & -0.17 & 0.0005 \\
\hline
\end{tabular}



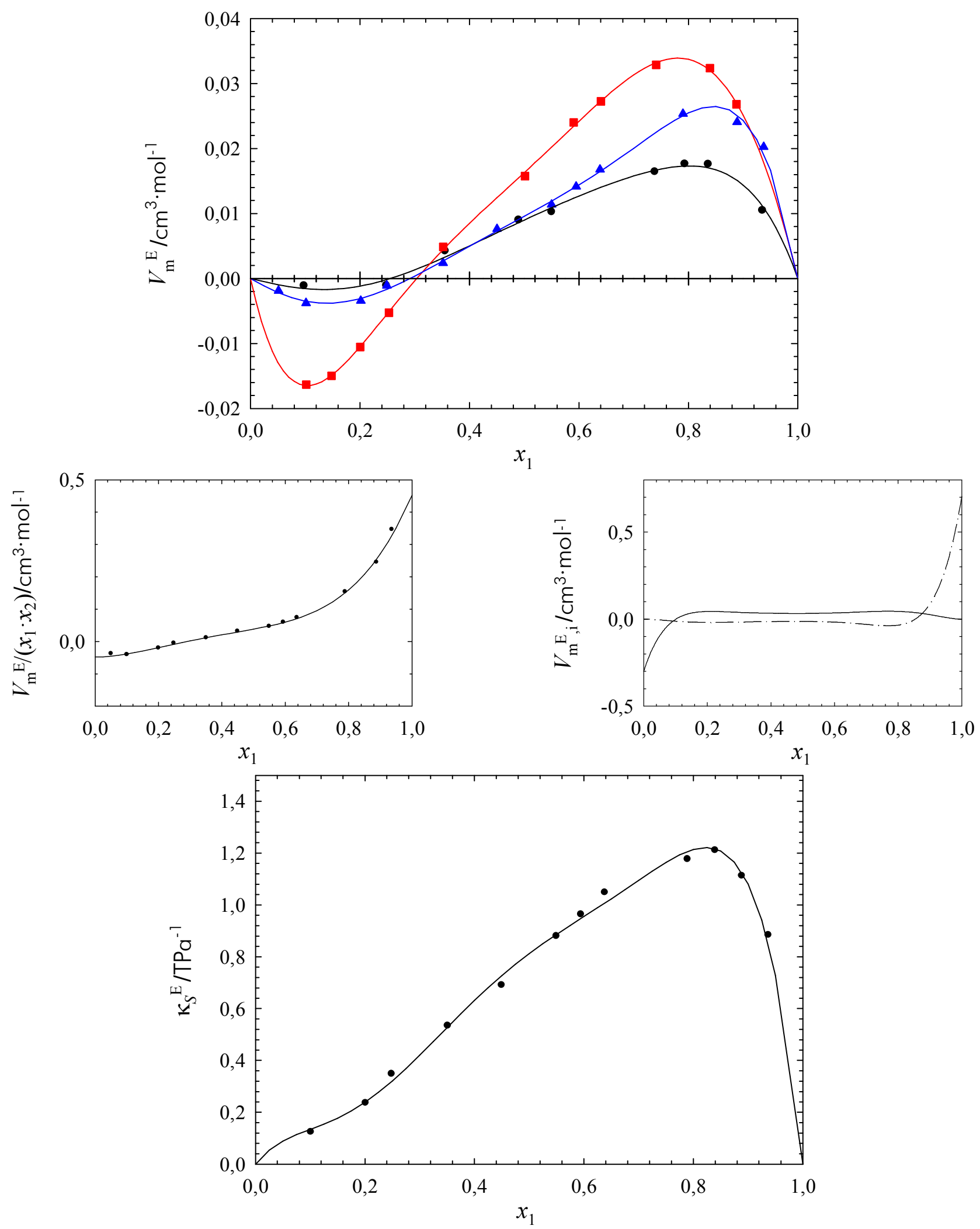

Ilustración 3-35: volúmenes molares de exceso $V_{\mathrm{m}}^{\mathrm{E}}$, volúmenes molares de exceso reducidos $V_{\mathrm{m}}^{\mathrm{E}} /\left(x_{1} \cdot x_{2}\right)$, volúmenes molares parciales de exceso $V_{\mathrm{m}, \mathrm{i}}^{\mathrm{E}}$ y compresibilidades isoentrópicas de exceso $\kappa_{S}^{\mathrm{E}}$ del sistema di- $n$-butilamina $(1)+2$-heptanona (2) 
Tabla 3-63: volúmenes molares de exceso del sistema $N, N, N$-trietilamina (1) + 2-propanona (2)

\begin{tabular}{|c|c|c|c|c|c|}
\hline$x_{1}$ & $V_{\mathrm{m}}^{\mathrm{E}} / \mathrm{cm}^{3} \cdot \mathrm{mol}^{-1}$ & $x_{1}$ & $V_{\mathrm{m}}^{\mathrm{E}} / \mathrm{cm}^{3} \cdot \mathrm{mol}^{-1}$ & $x_{1}$ & $V_{\mathrm{m}}^{\mathrm{E}} / \mathrm{cm}^{3} \cdot \mathrm{mol}^{-1}$ \\
\hline \multicolumn{6}{|c|}{$293.15 \mathrm{~K}$} \\
\hline 0.0485 & 0.0571 & 0.3954 & 0.3597 & 0.7070 & 0.3209 \\
\hline 0.1081 & 0.1417 & 0.4484 & 0.3738 & 0.7472 & 0.2962 \\
\hline 0.1520 & 0.2003 & 0.5060 & 0.3756 & 0.8106 & 0.2456 \\
\hline 0.1960 & 0.2372 & 0.5502 & 0.3746 & 0.8406 & 0.2205 \\
\hline 0.2491 & 0.2764 & 0.5931 & 0.3692 & 0.8921 & 0.1587 \\
\hline 0.2979 & 0.3141 & 0.6631 & 0.3473 & 0.9401 & 0.0897 \\
\hline 0.3463 & 0.3365 & & & & \\
\hline \multicolumn{6}{|c|}{$298.15 \mathrm{~K}$} \\
\hline 0.0499 & 0.0766 & 0.3942 & 0.3859 & 0.7014 & 0.3515 \\
\hline 0.1113 & 0.1620 & 0.4454 & 0.4018 & 0.7538 & 0.3200 \\
\hline 0.1470 & 0.2054 & 0.4976 & 0.4053 & 0.8042 & 0.2770 \\
\hline 0.1982 & 0.2600 & 0.5489 & 0.4044 & 0.8456 & 0.2342 \\
\hline 0.2491 & 0.3048 & 0.6022 & 0.3923 & 0.8935 & 0.1718 \\
\hline 0.2988 & 0.3391 & 0.6418 & 0.3762 & 0.9389 & 0.1011 \\
\hline 0.3451 & 0.3644 & & & & \\
\hline \multicolumn{6}{|c|}{$303.15 \mathrm{~K}$} \\
\hline 0.0504 & 0.0692 & 0.3466 & 0.3979 & 0.6526 & 0.4133 \\
\hline 0.0972 & 0.1532 & 0.3964 & 0.4179 & 0.7014 & 0.3898 \\
\hline 0.1519 & 0.2241 & 0.4501 & 0.4327 & 0.7812 & 0.3262 \\
\hline 0.2036 & 0.2770 & 0.4779 & 0.4378 & 0.8411 & 0.2659 \\
\hline 0.2557 & 0.3310 & 0.5486 & 0.4372 & 0.8810 & 0.2063 \\
\hline 0.2977 & 0.3670 & 0.5996 & 0.4315 & 0.9293 & 0.1358 \\
\hline
\end{tabular}

Tabla 3-64: velocidades del sonido de exceso, coeficientes de expansión térmica a $P=$ cte. de exceso, y compresibilidades isoentrópicas de exceso del sistema $N, N, N$-trietilamina (1) + 2-propanona (2)

\begin{tabular}{cccccccc}
\hline$x_{1}$ & $u^{\mathrm{E}} / \mathrm{m} \cdot \mathrm{s}^{-1}$ & $\alpha_{\mathrm{P}}^{\mathrm{E}} / 10^{-6} \mathrm{~K}^{-1}$ & $\kappa_{S}^{\mathrm{E}} / \mathrm{TPa}^{-1}$ & $x_{1}$ & $u^{\mathrm{E}} / \mathrm{m} \cdot \mathrm{s}^{-1}$ & $\alpha_{\mathrm{P}}^{\mathrm{E}} / 10^{-6} \mathrm{~K}^{-1}$ & $\kappa_{S}^{\mathrm{E}} / \mathrm{TPa}^{-1}$ \\
\hline 0.0499 & -3.3 & 9.12 & 6.53 & 0.5489 & -12.8 & 52.82 & 28.76 \\
0.1113 & -6.6 & 22.09 & 13.41 & 0.6022 & -12.2 & 50.61 & 27.63 \\
0.1470 & -8.2 & 29.34 & 16.78 & 0.6418 & -11.4 & 48.73 & 25.97 \\
0.1982 & -10.2 & 38.51 & 21.17 & 0.7014 & -10.6 & 45.59 & 24.09 \\
0.2491 & -11.5 & 45.69 & 24.35 & 0.7538 & -9.2 & 42.31 & 21.15 \\
0.2988 & -12.5 & 50.68 & 26.64 & 0.8042 & -7.6 & 38.21 & 17.54 \\
0.3451 & -13.0 & 53.61 & 28.21 & 0.8456 & -6.4 & 33.78 & 14.92 \\
0.3942 & -13.4 & 55.16 & 29.33 & 0.8935 & -4.6 & 26.81 & 10.68 \\
0.4454 & -13.5 & 55.38 & 29.88 & 0.9389 & -2.7 & 17.68 & 6.28 \\
0.4976 & -13.3 & 54.44 & 29.69 & & & & \\
\hline
\end{tabular}

Tabla 3-65: coeficientes y desviaciones estándar del ajuste a la ecuación R-K de las diferentes propiedades de exceso del sistema $N, N, N$-trietilamina (1) + 2-propanona (2)

\begin{tabular}{ccccccc}
\hline$T / \mathrm{K}$ & Propiedad & $A_{0}$ & $A_{1}$ & $A_{2}$ & $A_{3}$ & $\sigma$ \\
\hline 293.15 & $V_{\mathrm{m}}^{\mathrm{E}} / \mathrm{cm}^{3} \cdot \mathrm{mol}^{-1}$ & 1.511 & 0.085 & 0.09 & & 0.004 \\
298.15 & $V_{\mathrm{m}}^{\mathrm{E}} / \mathrm{cm}^{3} \cdot \mathrm{mol}^{-1}$ & 1.622 & 0.089 & 0.174 & & 0.003 \\
& $u^{\mathrm{E}} / \mathrm{m}^{-1}$ & -53.21 & 12.2 & -8.2 & & 0.09 \\
& $\alpha_{\mathrm{P}}^{\mathrm{E}} / 10^{-6} \mathrm{~K}^{-1}$ & 218.9 & -54 & 59 & 148 & 0.3 \\
& $\kappa_{S}^{\mathrm{E}} / \mathrm{TPa}^{-1}$ & 118.6 & -16.4 & 10.4 & & 0.2 \\
303.15 & $V_{\mathrm{m}}^{\mathrm{E}} / \mathrm{cm}^{3} \cdot \mathrm{mol}^{-1}$ & 1.767 & 0.10 & 0.16 & 0.18 & 0.004 \\
\hline
\end{tabular}



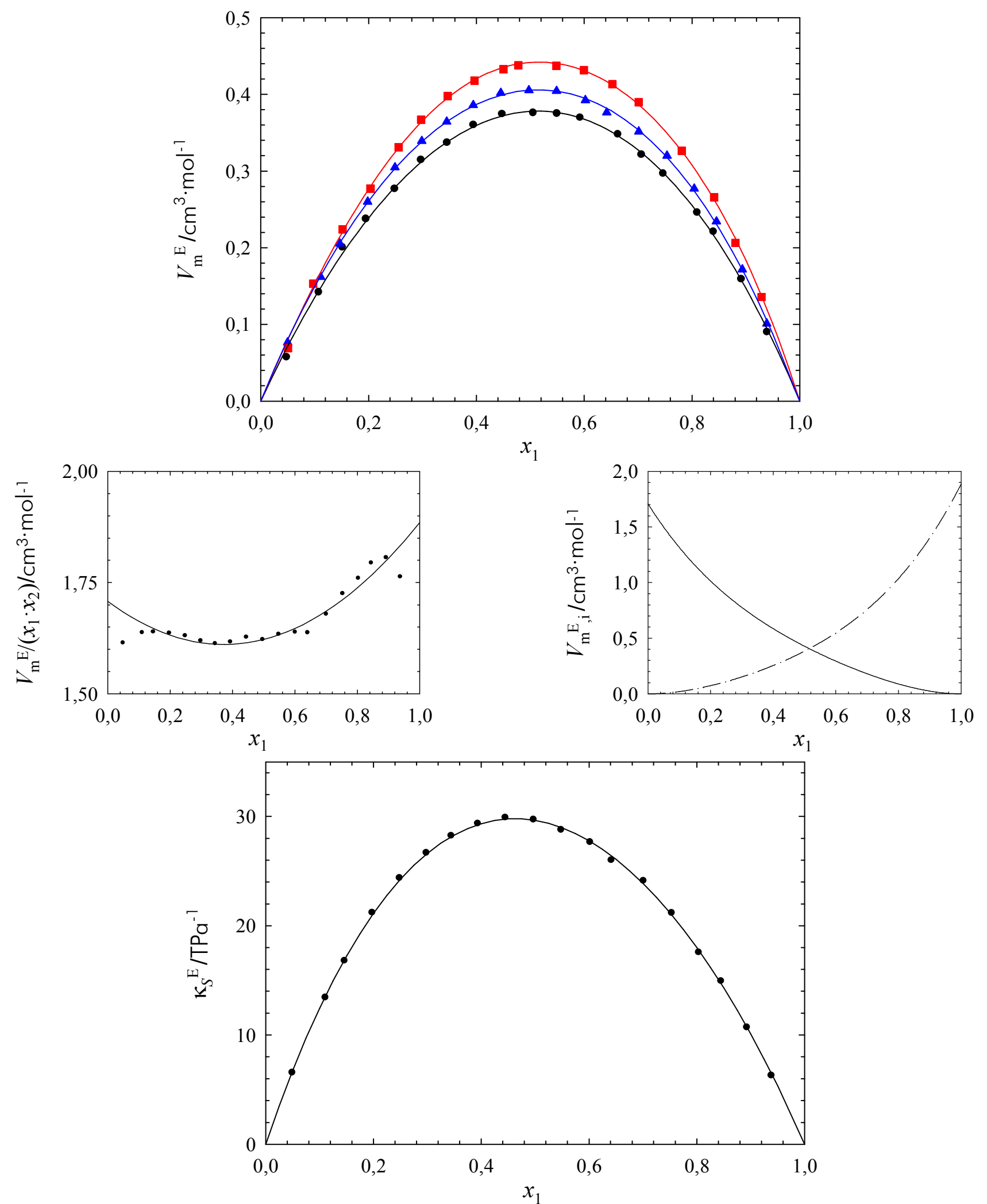

Ilustración 3-36: volúmenes molares de exceso $V_{\mathrm{m}}^{\mathrm{E}}$, volúmenes molares de exceso reducidos $V_{\mathrm{m}}^{\mathrm{E}} /\left(x_{1} \cdot x_{2}\right)$, volúmenes molares parciales de exceso $V_{\mathrm{m}, \mathrm{i}}^{\mathrm{E}}$ y compresibilidades isoentrópicas de exceso $\kappa_{S}^{\mathrm{E}}$ del sistema $N, N, N$-trietilamina (1) +2 -propanona (2) 
Tabla 3-66: volúmenes molares de exceso del sistema $N, N, N$-trietilamina (1) +2 -butanona (2)

\begin{tabular}{|c|c|c|c|c|c|}
\hline$x_{1}$ & $V_{\mathrm{m}}^{\mathrm{E}} / \mathrm{cm}^{3} \cdot \mathrm{mol}^{-1}$ & $x_{1}$ & $V_{\mathrm{m}}^{\mathrm{E}} / \mathrm{cm}^{3} \cdot \mathrm{mol}^{-1}$ & $x_{1}$ & $V_{\mathrm{m}}^{\mathrm{E}} / \mathrm{cm}^{3} \cdot \mathrm{mol}^{-1}$ \\
\hline \multicolumn{6}{|c|}{$293.15 \mathrm{~K}$} \\
\hline 0.0549 & 0.0278 & 0.3900 & 0.1348 & 0.6859 & 0.1666 \\
\hline 0.0981 & 0.0421 & 0.4428 & 0.1439 & 0.7314 & 0.1617 \\
\hline 0.1414 & 0.0608 & 0.4946 & 0.1545 & 0.7782 & 0.1495 \\
\hline 0.1943 & 0.0795 & 0.5364 & 0.1606 & 0.8373 & 0.1324 \\
\hline 0.2397 & 0.0967 & 0.5846 & 0.1655 & 0.8831 & 0.0985 \\
\hline 0.2949 & 0.1125 & 0.6451 & 0.1683 & 0.9316 & 0.0665 \\
\hline 0.3481 & 0.1238 & & & & \\
\hline \multicolumn{6}{|c|}{$298.15 \mathrm{~K}$} \\
\hline 0.0544 & 0.0248 & 0.3961 & 0.1455 & 0.6797 & 0.1755 \\
\hline 0.1079 & 0.0424 & 0.4299 & 0.1536 & 0.7390 & 0.1668 \\
\hline 0.1563 & 0.0628 & 0.4894 & 0.1633 & 0.7819 & 0.1595 \\
\hline 0.1977 & 0.0838 & 0.5393 & 0.1712 & 0.8420 & 0.1382 \\
\hline 0.2396 & 0.0991 & 0.5841 & 0.1751 & 0.8851 & 0.1146 \\
\hline 0.2878 & 0.1188 & 0.6395 & 0.1777 & 0.9291 & 0.0823 \\
\hline 0.3400 & 0.1340 & & & & \\
\hline \multicolumn{6}{|c|}{$303.15 \mathrm{~K}$} \\
\hline 0.0500 & 0.0207 & 0.3949 & 0.1513 & 0.6898 & 0.1807 \\
\hline 0.1013 & 0.0431 & 0.4348 & 0.1594 & 0.7361 & 0.1724 \\
\hline 0.1458 & 0.0625 & 0.4919 & 0.1724 & 0.7899 & 0.1607 \\
\hline 0.2055 & 0.0871 & 0.5500 & 0.1802 & 0.8357 & 0.1430 \\
\hline 0.2370 & 0.1070 & 0.6131 & 0.1851 & 0.8851 & 0.1164 \\
\hline 0.2913 & 0.1208 & 0.6498 & 0.1850 & 0.9314 & 0.0819 \\
\hline 0.3439 & 0.1366 & & & & \\
\hline
\end{tabular}

Tabla 3-67: velocidades del sonido de exceso, coeficientes de expansión térmica a $P=$ cte. de exceso, y compresibilidades isoentrópicas de exceso del sistema $N, N, N$-trietilamina (1) + 2-butanona (2)

\begin{tabular}{cccccccc}
\hline$x_{1}$ & $u^{\mathrm{E}} / \mathrm{m} \cdot \mathrm{s}^{-1}$ & $\alpha_{\mathrm{P}}^{\mathrm{E}} / 10^{-6} \mathrm{~K}^{-1}$ & $\kappa_{S}^{\mathrm{E}} / \mathrm{TPa}^{-1}$ & $x_{1}$ & $u^{\mathrm{E}} / \mathrm{m} \cdot \mathrm{s}^{-1}$ & $\alpha_{\mathrm{P}}^{\mathrm{E}} / 10^{-6} \mathrm{~K}^{-1}$ & $\kappa_{S}^{\mathrm{E}} / \mathrm{TPa}^{-1}$ \\
\hline 0.0544 & 0.5 & -4.52 & -0.55 & 0.5393 & -0.9 & 12.19 & 3.16 \\
0.1079 & 0.9 & -3.42 & -0.99 & 0.5841 & -1.1 & 11.34 & 3.63 \\
0.1563 & 1.1 & -0.03 & -1.12 & 0.6395 & -1.4 & 10.53 & 4.17 \\
0.1977 & 0.8 & 3.51 & -0.50 & 0.6797 & -1.5 & 10.21 & 4.40 \\
0.2396 & 0.7 & 6.88 & -0.28 & 0.7390 & -1.6 & 10.18 & 4.45 \\
0.2878 & 0.5 & 10.11 & 0.31 & 0.7819 & -1.7 & 10.41 & 4.62 \\
0.3400 & 0.2 & 12.44 & 0.90 & 0.8420 & -1.5 & 10.47 & 4.19 \\
0.3961 & 0.0 & 13.56 & 1.31 & 0.8851 & -1.4 & 9.82 & 3.67 \\
0.4299 & -0.3 & 13.67 & 1.86 & 0.9291 & -1 & 7.90 & 2.73 \\
0.4894 & -0.6 & 13.08 & 2.60 & & & & \\
\hline
\end{tabular}

Tabla 3-68: coeficientes y desviaciones estándar del ajuste a la ecuación R-K de las diferentes propiedades de exceso del sistema $N, N, N$-trietilamina (1) +2 -butanona (2)

\begin{tabular}{ccccccc}
\hline$T / \mathrm{K}$ & Propiedad & $A_{0}$ & $A_{1}$ & $A_{2}$ & $A_{3}$ & $\sigma$ \\
\hline 293.15 & $V_{\mathrm{m}}^{\mathrm{E}} / \mathrm{cm}^{3} \cdot \mathrm{mol}^{-1}$ & 0.624 & 0.318 & 0.218 & & 0.002 \\
298.15 & $V_{\mathrm{m}}^{\mathrm{E}} / \mathrm{cm}^{3} \cdot \mathrm{mol}^{-1}$ & 0.664 & 0.273 & 0.223 & 0.27 & 0.0017 \\
& $u^{\mathrm{E}} / \mathrm{m}^{-1}$ & -2.72 & -11.1 & 0.7 & -5.6 & 0.06 \\
& $\alpha_{\mathrm{P}}^{\mathrm{E}} / 10^{-6} \mathrm{~K}^{-1}$ & 52.9 & -35 & -28 & 197 & 0.3 \\
& $\kappa_{S}^{\mathrm{E}} / \mathrm{TPa}^{-1}$ & 10.84 & 22.7 & 4.0 & 11.3 & 0.12 \\
303.15 & $V_{\mathrm{m}}^{\mathrm{E}} / \mathrm{cm}^{3} \cdot \mathrm{mol}^{-1}$ & 0.692 & 0.296 & 0.205 & 0.22 & 0.002 \\
\hline
\end{tabular}



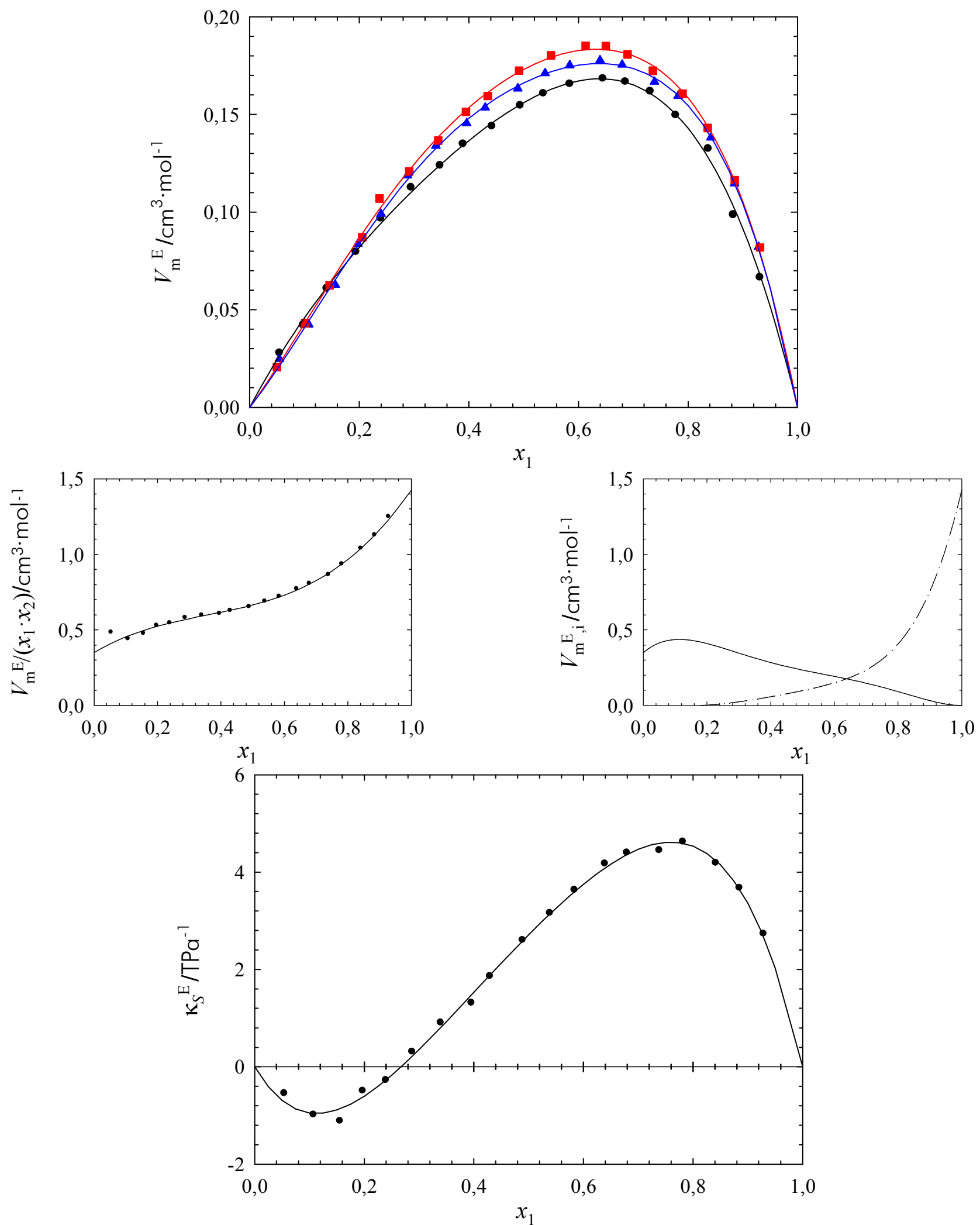

llustración 3-37: volúmenes molares de exceso $V_{\mathrm{m}}^{\mathrm{E}}$, volúmenes molares de exceso reducidos $V_{\mathrm{m}}^{\mathrm{E}} /\left(x_{1} \cdot x_{2}\right)$, volúmenes molares parciales de exceso $V_{\mathrm{m}, \mathrm{i}}^{\mathrm{E}}$ y compresibilidades isoentrópicas de exceso $\kappa_{S}^{\mathrm{E}}$ del sistema $N, N, N$-trietilamina (1) +2 -butanona (2) 
Tabla 3-69: volúmenes molares de exceso del sistema $N, N, N$-trietilamina (1) + 2-heptanona (2)

\begin{tabular}{|c|c|c|c|c|c|}
\hline$x_{1}$ & $V_{\mathrm{m}}^{\mathrm{E}} / \mathrm{cm}^{3} \cdot \mathrm{mol}^{-1}$ & $x_{1}$ & $V_{\mathrm{m}}^{\mathrm{E}} / \mathrm{cm}^{3} \cdot \mathrm{mol}^{-1}$ & $x_{1}$ & $V_{\mathrm{m}}^{\mathrm{E}} / \mathrm{cm}^{3} \cdot \mathrm{mol}^{-1}$ \\
\hline \multicolumn{6}{|c|}{$293.15 \mathrm{~K}$} \\
\hline 0.0493 & -0.0469 & 0.3922 & -0.2128 & 0.6909 & -0.1737 \\
\hline 0.0992 & -0.0782 & 0.4423 & -0.2146 & 0.7464 & -0.1524 \\
\hline 0.1483 & -0.1093 & 0.4978 & -0.2127 & 0.7923 & -0.1321 \\
\hline 0.2032 & -0.1486 & 0.5379 & -0.2087 & 0.8415 & -0.1064 \\
\hline 0.2510 & -0.1714 & 0.5957 & -0.1998 & 0.8939 & -0.0854 \\
\hline 0.2999 & -0.1863 & 0.6386 & -0.1884 & 0.9364 & -0.0409 \\
\hline 0.3396 & -0.1970 & & & & \\
\hline \multicolumn{6}{|c|}{$298.15 \mathrm{~K}$} \\
\hline 0.0538 & -0.0471 & 0.3897 & -0.2057 & 0.6925 & -0.1704 \\
\hline 0.0986 & -0.0810 & 0.4455 & -0.2093 & 0.7402 & -0.1495 \\
\hline 0.1424 & -0.1113 & 0.4968 & -0.2092 & 0.7932 & -0.1296 \\
\hline 0.1969 & -0.1495 & 0.5452 & -0.2045 & 0.8381 & -0.1070 \\
\hline 0.2516 & -0.1728 & 0.5919 & -0.1963 & 0.8950 & -0.0798 \\
\hline 0.3006 & -0.1874 & 0.6422 & -0.1821 & 0.9426 & -0.0322 \\
\hline 0.3421 & -0.1967 & & & & \\
\hline \multicolumn{6}{|c|}{$303.15 \mathrm{~K}$} \\
\hline 0.0497 & -0.0444 & 0.3464 & -0.1984 & 0.6914 & -0.1680 \\
\hline 0.0993 & -0.0738 & 0.4017 & -0.2087 & 0.7394 & -0.1511 \\
\hline 0.1511 & -0.1097 & 0.4958 & -0.2144 & 0.7932 & -0.1263 \\
\hline 0.1912 & -0.1390 & 0.5447 & -0.2119 & 0.8398 & -0.1094 \\
\hline 0.2548 & -0.1727 & 0.5918 & -0.2016 & 0.8944 & -0.0878 \\
\hline 0.2969 & -0.1886 & 0.6417 & -0.1860 & 0.9388 & -0.0563 \\
\hline
\end{tabular}

Tabla 3-70: velocidades del sonido de exceso, coeficientes de expansión térmica a $P=$ cte. de exceso, y compresibilidades isoentrópicas de exceso del sistema $N, N, N$-trietilamina (1) + 2-heptanona (2)

\begin{tabular}{cccccccc}
\hline$x_{1}$ & $u^{\mathrm{E}} / \mathrm{m} \cdot \mathrm{s}^{-1}$ & $\alpha_{\mathrm{P}}^{\mathrm{E}} / 10^{-6} \mathrm{~K}^{-1}$ & $\kappa_{S}^{\mathrm{E}} / \mathrm{TPa}^{-1}$ & $x_{1}$ & $u^{\mathrm{E}} / \mathrm{m} \cdot \mathrm{s}^{-1}$ & $\alpha_{\mathrm{P}}^{\mathrm{E}} / 10^{-6} \mathrm{~K}^{-1}$ & $\kappa_{S}^{\mathrm{E}} / \mathrm{TPa}^{-1}$ \\
\hline 0.0538 & 4.2 & 2.12 & -5.59 & 0.5452 & 16.6 & 2.11 & -28.44 \\
0.0986 & 7.2 & 3.68 & -9.79 & 0.5919 & 15.9 & 4.05 & -27.82 \\
0.1424 & 9.7 & 4.37 & -13.55 & 0.6422 & 14.8 & 5.73 & -26.52 \\
0.1969 & 12.4 & 3.89 & -17.76 & 0.6925 & 13.5 & 6.24 & -24.79 \\
0.2516 & 14.4 & 2.43 & -21.22 & 0.7402 & 11.9 & 5.25 & -22.41 \\
0.3006 & 15.7 & 0.83 & -23.74 & 0.7932 & 10.0 & 2.17 & -19.23 \\
0.3421 & 16.5 & -0.35 & -25.45 & 0.8381 & 8.3 & -1.63 & -16.40 \\
0.3897 & 17.1 & -1.13 & -27.00 & 0.8950 & 5.6 & -6.43 & -11.41 \\
0.4455 & 17.2 & -0.94 & -28.09 & 0.9426 & 3.0 & -7.53 & -6.21 \\
0.4968 & 17.0 & 0.29 & -28.50 & & & & \\
\hline
\end{tabular}

Tabla 3-71: coeficientes y desviaciones estándar del ajuste a la ecuación R-K de las diferentes propiedades de exceso del sistema $N, N, N$-trietilamina (1) + 2-heptanona (2)

\begin{tabular}{cccccccc}
\hline$T / \mathrm{K}$ & Propiedad & $A_{0}$ & $A_{1}$ & $A_{2}$ & $A_{3}$ & $A_{4}$ & $\sigma$ \\
\hline 293.15 & $V_{\mathrm{m}}^{\mathrm{E}} / \mathrm{cm}^{3} \cdot \mathrm{mol}^{-1}$ & -0.853 & 0.086 & & & 0.004 \\
298.15 & $V_{\mathrm{m}}^{\mathrm{E}} / \mathrm{cm}^{3} \cdot \mathrm{mol}^{-1}$ & -0.841 & 0.115 & & & 0.004 \\
& $u^{\mathrm{E}} / \mathrm{m} \cdot \mathrm{s}^{-1}$ & 68.26 & -14.3 & 3.3 & & & 0.06 \\
& $\alpha_{\mathrm{P}}^{\mathrm{E}} / 10^{-6} \mathrm{~K}^{-1}$ & 1.62 & 66.51 & 135.3 & -216.6 & 255.4 & 0.03 \\
303.15 & $\kappa_{S}^{\mathrm{E}} / \mathrm{TPa}^{-1}$ & -114.53 & -4.9 & & & & 0.15 \\
\hline
\end{tabular}



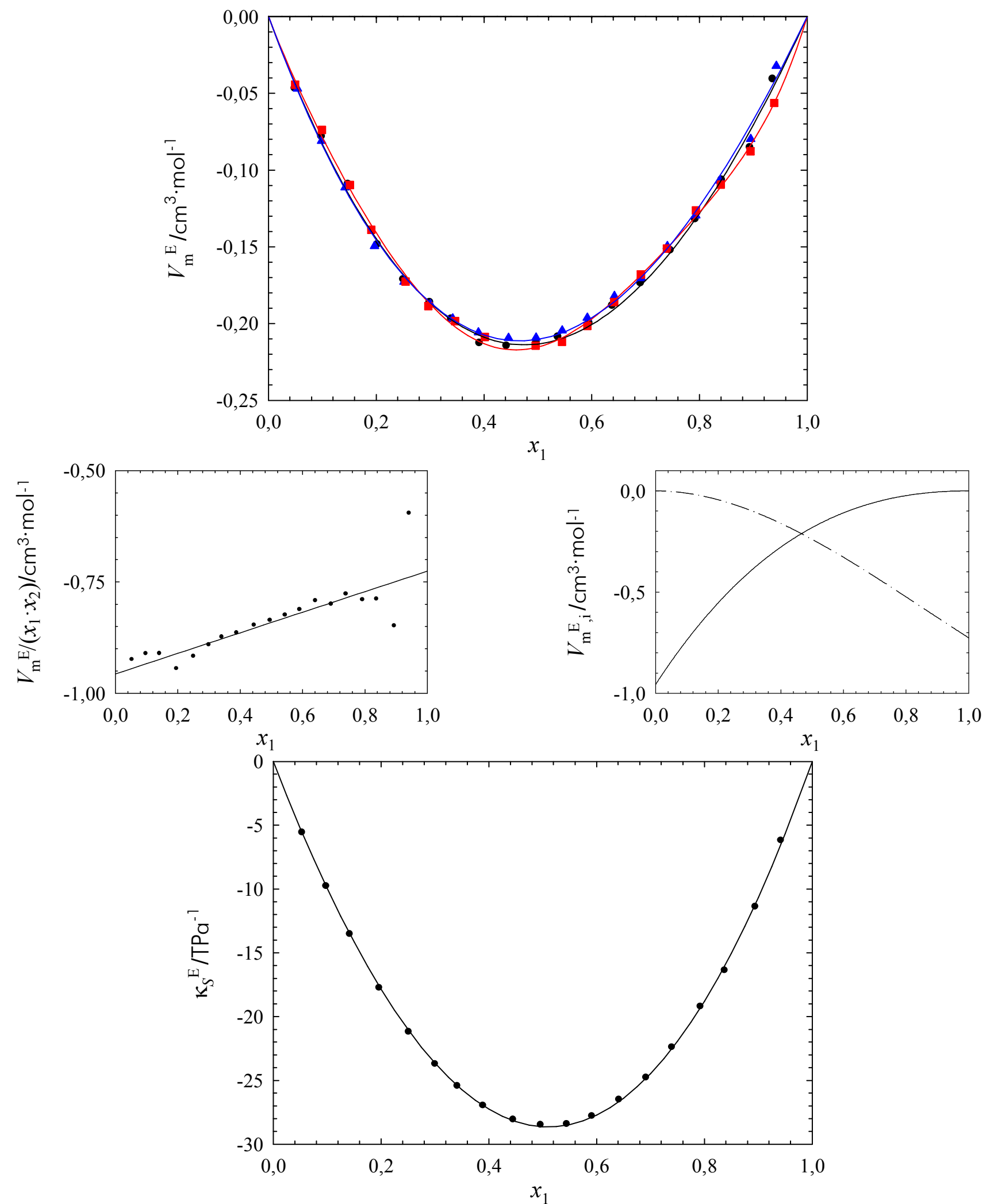

Ilustración 3-38: volúmenes molares de exceso $V_{\mathrm{m}}^{\mathrm{E}}$, volúmenes molares de exceso reducidos $V_{\mathrm{m}}^{\mathrm{E}} /\left(x_{1} \cdot x_{2}\right)$, volúmenes molares parciales de exceso $V_{\mathrm{m}, \mathrm{i}}^{\mathrm{E}}$ y compresibilidades isoentrópicas de exceso $\kappa_{S}^{\mathrm{E}}$ del sistema $N, N, N$-trietilamina (1) +2 -heptanona (2) 
Tabla 3-72: volúmenes molares de exceso del sistema $N$-metilanilina (1) $+n$-heptano (2)

\begin{tabular}{|c|c|c|c|c|c|}
\hline$x_{1}$ & $V_{\mathrm{m}}^{\mathrm{E}} / \mathrm{cm}^{3} \cdot \mathrm{mol}^{-1}$ & $x_{1}$ & $V_{\mathrm{m}}^{\mathrm{E}} / \mathrm{cm}^{3} \cdot \mathrm{mol}^{-1}$ & $x_{1}$ & $V_{\mathrm{m}}^{\mathrm{E}} / \mathrm{cm}^{3} \cdot \mathrm{mol}^{-1}$ \\
\hline \multicolumn{6}{|c|}{$293.15 \mathrm{~K}$} \\
\hline 0.0540 & 0.0325 & 0.3944 & -0.1772 & 0.7024 & -0.2306 \\
\hline 0.1084 & 0.0189 & 0.4485 & -0.1984 & 0.7457 & -0.2196 \\
\hline 0.1536 & 0.0020 & 0.4953 & -0.2125 & 0.7858 & -0.2043 \\
\hline 0.2000 & -0.0349 & 0.5355 & -0.2220 & 0.8459 & -0.1717 \\
\hline 0.2556 & -0.0864 & 0.5990 & -0.2345 & 0.8952 & -0.1274 \\
\hline 0.2976 & -0.1151 & 0.6436 & -0.2365 & 0.9491 & -0.0684 \\
\hline 0.3473 & -0.1439 & & & & \\
\hline \multicolumn{6}{|c|}{$298.15 \mathrm{~K}$} \\
\hline 0.0577 & 0.0308 & 0.3955 & -0.1895 & 0.6995 & -0.2465 \\
\hline 0.1072 & 0.0052 & 0.4559 & -0.2107 & 0.7491 & -0.2270 \\
\hline 0.1473 & -0.0157 & 0.5030 & -0.2298 & 0.7964 & -0.2130 \\
\hline 0.2055 & -0.0625 & 0.5431 & -0.2385 & 0.8446 & -0.1838 \\
\hline 0.2595 & -0.1013 & 0.5956 & -0.2497 & 0.8949 & -0.1345 \\
\hline 0.3033 & -0.1335 & 0.6441 & -0.2509 & 0.9465 & -0.0730 \\
\hline 0.3401 & -0.1487 & & & & \\
\hline \multicolumn{6}{|c|}{$303.15 \mathrm{~K}$} \\
\hline 0.0615 & 0.0282 & 0.3979 & -0.1869 & 0.7026 & \\
\hline 0.1111 & 0.0074 & 0.4513 & -0.2281 & 0.7475 & -0.2508 \\
\hline 0.1548 & -0.0206 & 0.5003 & -0.2420 & 0.7954 & -0.2277 \\
\hline 0.2069 & -0.0621 & 0.5583 & -0.2654 & 0.8446 & -0.1920 \\
\hline 0.2501 & -0.0935 & 0.5988 & -0.2792 & 0.8917 & -0.1479 \\
\hline 0.2964 & -0.1198 & 0.6470 & -0.2739 & 0.9404 & -0.0876 \\
\hline 0.3480 & -0.1572 & & & & \\
\hline
\end{tabular}

Tabla 3-73: velocidades del sonido de exceso, coeficientes de expansión térmica a $P=$ cte. De exceso, y compresibilidades isoentrópicas de exceso del sistema $N$-metilanilina (1) $+n$-heptano (2)

\begin{tabular}{cccccccc}
\hline$x_{1}$ & $u^{\mathrm{E}} / \mathrm{m} \cdot \mathrm{s}^{-1}$ & $\alpha_{\mathrm{P}}^{\mathrm{E}} / 10^{-6} \mathrm{~K}^{-1}$ & $\kappa_{S}^{\mathrm{E}} / \mathrm{TPa}^{-1}$ & $x_{1}$ & $u^{\mathrm{E}} / \mathrm{m} \cdot \mathrm{s}^{-1}$ & $\alpha_{\mathrm{P}}^{\mathrm{E}} / 10^{-6} \mathrm{~K}^{-1}$ & $\kappa_{S}^{\mathrm{E}} / \mathrm{TPa}^{-1}$ \\
\hline 0.0577 & 10.8 & -5.01 & -20.99 & 0.5431 & 77.7 & -25.86 & -97.68 \\
0.1072 & 19.5 & -9.92 & -36.74 & 0.5956 & 82.9 & -30.40 & -97.41 \\
0.1473 & 26.1 & -11.72 & -47.77 & 0.6441 & 86.9 & -31.78 & -95.33 \\
0.2055 & 35.1 & -11.03 & -61.54 & 0.6995 & 90.0 & -29.61 & -90.65 \\
0.2595 & 40.8 & -8.64 & -68.83 & 0.7491 & 90.2 & -24.88 & -83.69 \\
0.3033 & 48.8 & -7.18 & -79.01 & 0.7964 & 87.7 & -19.53 & -74.74 \\
0.3401 & 53.6 & -7.00 & -83.88 & 0.8446 & 80.5 & -14.85 & -62.47 \\
0.3955 & 60.6 & -9.48 & -90.01 & 0.8949 & 66.2 & -11.96 & -46.18 \\
0.4559 & 67.8 & -15.30 & -94.51 & 0.9465 & 41.4 & -9.74 & -25.62 \\
0.5030 & 73.3 & -21.12 & -96.82 & & & & \\
\hline
\end{tabular}

Tabla 3-74: coeficientes y desviaciones estándar del ajuste a la ecuación R-K de las diferentes propiedades de exceso del sistema $N$-metilanilina (1) $+n$-heptano (2)

\begin{tabular}{cccccccccc}
\hline$T / \mathrm{K}$ & Propiedad & $A_{0}$ & $A_{1}$ & $A_{2}$ & $A_{3}$ & $A_{4}$ & $A_{5}$ & $A_{6}$ & $\sigma$ \\
\hline 293.15 & $V_{\mathrm{m}}^{\mathrm{E}} / \mathrm{cm}^{3} \cdot \mathrm{mol}^{-1}$ & -0.861 & -0.565 & 0.14 & -0.75 & -0.58 & & 0.003 \\
298.15 & $V_{\mathrm{m}}^{\mathrm{E}} / \mathrm{cm}^{3} \cdot \mathrm{mol}^{-1}$ & -0.909 & -0.59 & -0.03 & -0.61 & 0.69 & & 0.004 \\
& $u^{\mathrm{E}} / \mathrm{m}^{-1}$ & 292.2 & 227 & 211 & 145 & 79 & & 0.5 & 0.03 \\
& $\alpha_{\mathrm{P}}^{\mathrm{E}} / 10^{-6} \mathrm{~K}^{-1}$ & -82.99 & -250.8 & -31.5 & 861.7 & 35 & -776 & -109 & 0.7 \\
& $\kappa_{S}^{\mathrm{E}} / \mathrm{TPa}^{-1}$ & -387.4 & -73 & -81 & & & & 0.004 \\
\hline
\end{tabular}



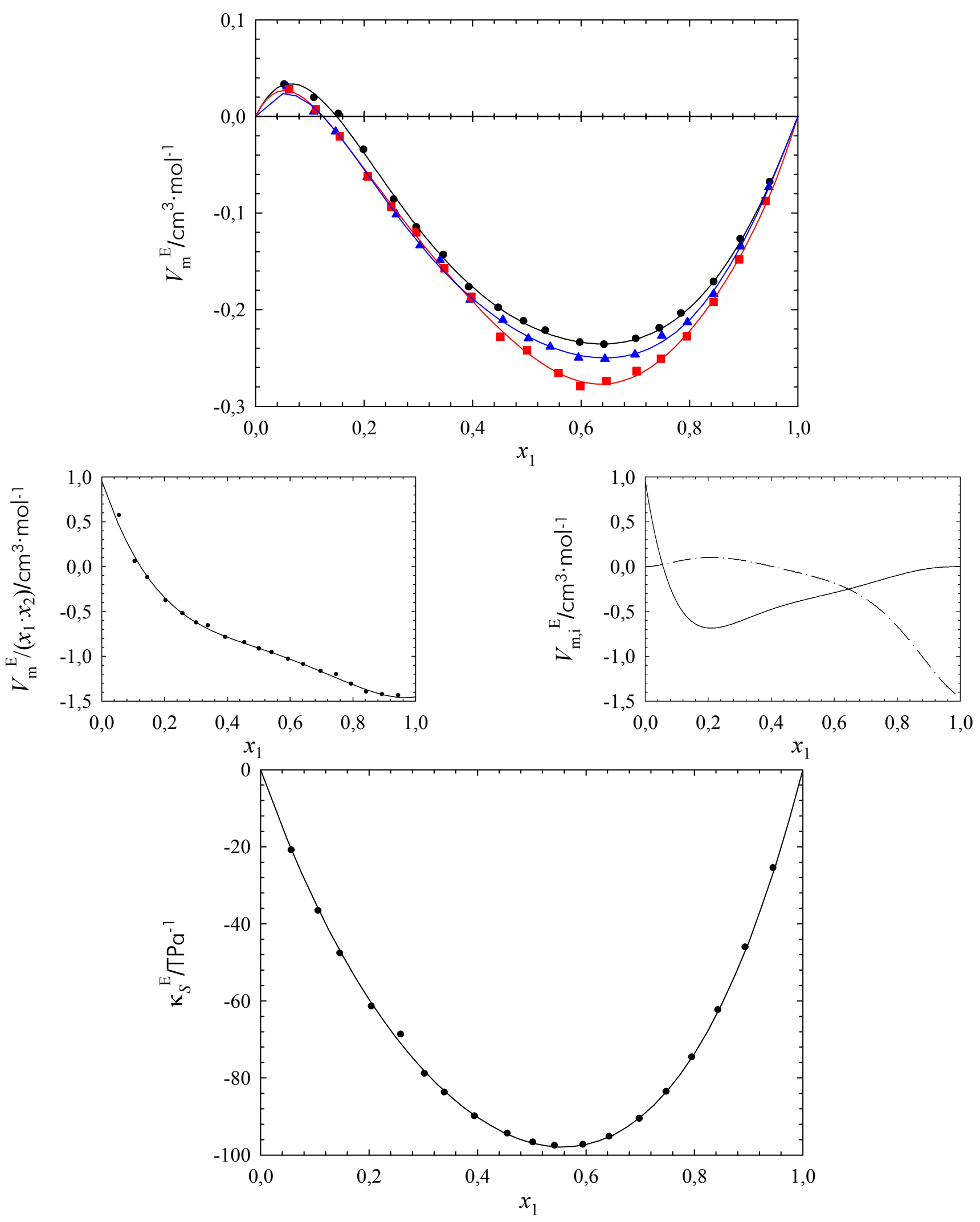

llustración 3-39: volúmenes molares de exceso $V_{\mathrm{m}}^{\mathrm{E}}$, volúmenes molares de exceso reducidos $V_{\mathrm{m}}^{\mathrm{E}} /\left(x_{1} \cdot x_{2}\right)$, volúmenes molares parciales de exceso $V_{\mathrm{m}, \mathrm{i}}^{\mathrm{E}}$ y compresibilidades isoentrópicas de exceso $\kappa_{S}^{\mathrm{E}}$ del sistema $N$-metilanilina (1) $+n$-heptano (2) 
Tabla 3-75: volúmenes molares de exceso del sistema $N$-metilanilina (1) + ciclohexano (2)

\begin{tabular}{|c|c|c|c|c|c|}
\hline$x_{1}$ & $V_{\mathrm{m}}^{\mathrm{E}} / \mathrm{cm}^{3} \cdot \mathrm{mol}^{-1}$ & $x_{1}$ & $V_{\mathrm{m}}^{\mathrm{E}} / \mathrm{cm}^{3} \cdot \mathrm{mol}^{-1}$ & $x_{1}$ & $V_{\mathrm{m}}^{\mathrm{E}} / \mathrm{cm}^{3} \cdot \mathrm{mol}^{-1}$ \\
\hline \multicolumn{6}{|c|}{$293.15 \mathrm{~K}$} \\
\hline 0.0516 & 0.2089 & 0.4049 & 0.5020 & 0.6931 & 0.3422 \\
\hline 0.1047 & 0.3369 & 0.4478 & 0.4976 & 0.7564 & 0.2817 \\
\hline 0.1522 & 0.4076 & 0.4972 & 0.4719 & 0.7917 & 0.2474 \\
\hline 0.2023 & 0.4523 & 0.5444 & 0.4498 & 0.8372 & 0.1968 \\
\hline 0.2482 & 0.4869 & 0.5942 & 0.4198 & 0.8900 & 0.1366 \\
\hline 0.3018 & 0.5059 & 0.6445 & 0.3813 & 0.9446 & 0.0693 \\
\hline 0.3462 & 0.5075 & & & & \\
\hline \multicolumn{6}{|c|}{$298.15 \mathrm{~K}$} \\
\hline 0.0673 & 0.2568 & 0.3981 & 0.4950 & 0.6939 & 0.3206 \\
\hline 0.1073 & 0.3444 & 0.4479 & 0.4866 & 0.7530 & 0.2722 \\
\hline 0.1609 & 0.4226 & 0.5055 & 0.4584 & 0.7950 & 0.2264 \\
\hline 0.2065 & 0.4600 & 0.5537 & 0.4285 & 0.8347 & 0.1775 \\
\hline 0.2536 & 0.4889 & 0.5968 & 0.4012 & 0.8896 & 0.1216 \\
\hline 0.2963 & 0.4993 & 0.6482 & 0.3654 & 0.9399 & 0.0682 \\
\hline 0.3554 & 0.5048 & & & & \\
\hline \multicolumn{6}{|c|}{$303.15 \mathrm{~K}$} \\
\hline 0.0581 & 0.2286 & 0.3974 & 0.5004 & 0.6956 & 0.3206 \\
\hline 0.1038 & 0.3340 & 0.4510 & 0.4884 & 0.7501 & 0.2681 \\
\hline 0.1557 & 0.4137 & 0.5005 & 0.4689 & 0.7995 & 0.2187 \\
\hline 0.2015 & 0.4605 & 0.5512 & 0.4343 & 0.8447 & 0.1631 \\
\hline 0.2526 & 0.4903 & 0.5922 & 0.4080 & 0.8956 & 0.1138 \\
\hline 0.2975 & 0.4971 & 0.6575 & 0.3643 & 0.9411 & 0.0633 \\
\hline 0.3479 & 0.5067 & & & & \\
\hline
\end{tabular}

Tabla 3-76: velocidades del sonido de exceso, coeficientes de expansión térmica a $P=$ cte. De exceso, y compresibilidades isoentrópicas de exceso del sistema $N$-metilanilina (1) + ciclohexano (2)

\begin{tabular}{cccccccc}
\hline$x_{1}$ & $u^{\mathrm{E}} / \mathrm{m} \cdot \mathrm{s}^{-1}$ & $\alpha_{\mathrm{P}}^{\mathrm{E}} / 10^{-6} \mathrm{~K}^{-1}$ & $\kappa_{S}^{\mathrm{E}} / \mathrm{TPa}^{-1}$ & $x_{1}$ & $u^{\mathrm{E}} / \mathrm{m} \cdot \mathrm{s}^{-1}$ & $\alpha_{\mathrm{P}}^{\mathrm{E}} / 10^{-6} \mathrm{~K}^{-1}$ & $\kappa_{S}^{\mathrm{E}} / \mathrm{TPa}^{-1}$ \\
\hline 0.0673 & 6.1 & -4.91 & -5.82 & 0.5537 & 40.0 & -10.54 & -32.86 \\
0.1073 & 9.6 & -2.66 & -9.40 & 0.5968 & 41.4 & -11.36 & -32.85 \\
0.1609 & 14.1 & -0.47 & -13.98 & 0.6482 & 41.7 & -13.79 & -31.59 \\
0.2065 & 18.1 & -0.92 & -17.90 & 0.6939 & 41.3 & -17.15 & -30.05 \\
0.2536 & 21.9 & -3.12 & -21.35 & 0.7530 & 39.1 & -21.57 & -26.82 \\
0.2963 & 25.2 & -5.85 & -24.24 & 0.7950 & 36.3 & -23.45 & -23.86 \\
0.3554 & 29.4 & -9.09 & -27.52 & 0.8347 & 32.5 & -23.04 & -20.53 \\
0.3981 & 32.3 & -10.48 & -29.55 & 0.8896 & 25.0 & -17.63 & -14.83 \\
0.4479 & 35.2 & -10.86 & -31.18 & 0.9399 & 15.5 & -8.55 & -8.62 \\
0.5055 & 38.1 & -10.55 & -32.50 & & & & \\
\hline
\end{tabular}

Tabla 3-77: coeficientes y desviaciones estándar del ajuste a la ecuación R-K de las diferentes propiedades de exceso del sistema $N$-metilanilina (1) + ciclohexano (2)

\begin{tabular}{ccccccccc}
\hline$T / \mathrm{K}$ & Propiedad & $A_{0}$ & $A_{1}$ & $A_{2}$ & $A_{3}$ & $A_{4}$ & $A_{5}$ & $\sigma$ \\
\hline 293.15 & $V_{\mathrm{m}}^{\mathrm{E}} / \mathrm{cm}^{3} \cdot \mathrm{mol}^{-1}$ & 1.896 & -0.86 & 0.49 & -0.84 & 0.74 & & 0.004 \\
298.15 & $V_{\mathrm{m}}^{\mathrm{E}} / \mathrm{cm}^{3} \cdot \mathrm{mol}^{-1}$ & 1.847 & -1.00 & 0.50 & -0.46 & 0.70 & -0.6 & 0.003 \\
& $u^{\mathrm{E}} / \mathrm{m}^{-1}$ & 151.7 & 91.7 & 42.7 & 11.8 & & -0.0 & 0.08 \\
& $\alpha_{\mathrm{P}}^{\mathrm{E}} / 10^{-6} \mathrm{~K}^{-1}$ & -42.33 & 14.3 & -84.4 & -637.1 & -23.5 & 742.0 & 0.03 \\
& $\kappa_{S}^{\mathrm{E}} / \mathrm{TPa}^{-1}$ & -129.8 & -31.0 & 3.1 & -4.0 & 9 & & 0.08 \\
303.15 & $V_{\mathrm{m}}^{\mathrm{E}} / \mathrm{cm}^{3} \cdot \mathrm{mol}^{-1}$ & 1.869 & -0.94 & 0.40 & -0.93 & 0.73 & 0.003 \\
\hline
\end{tabular}



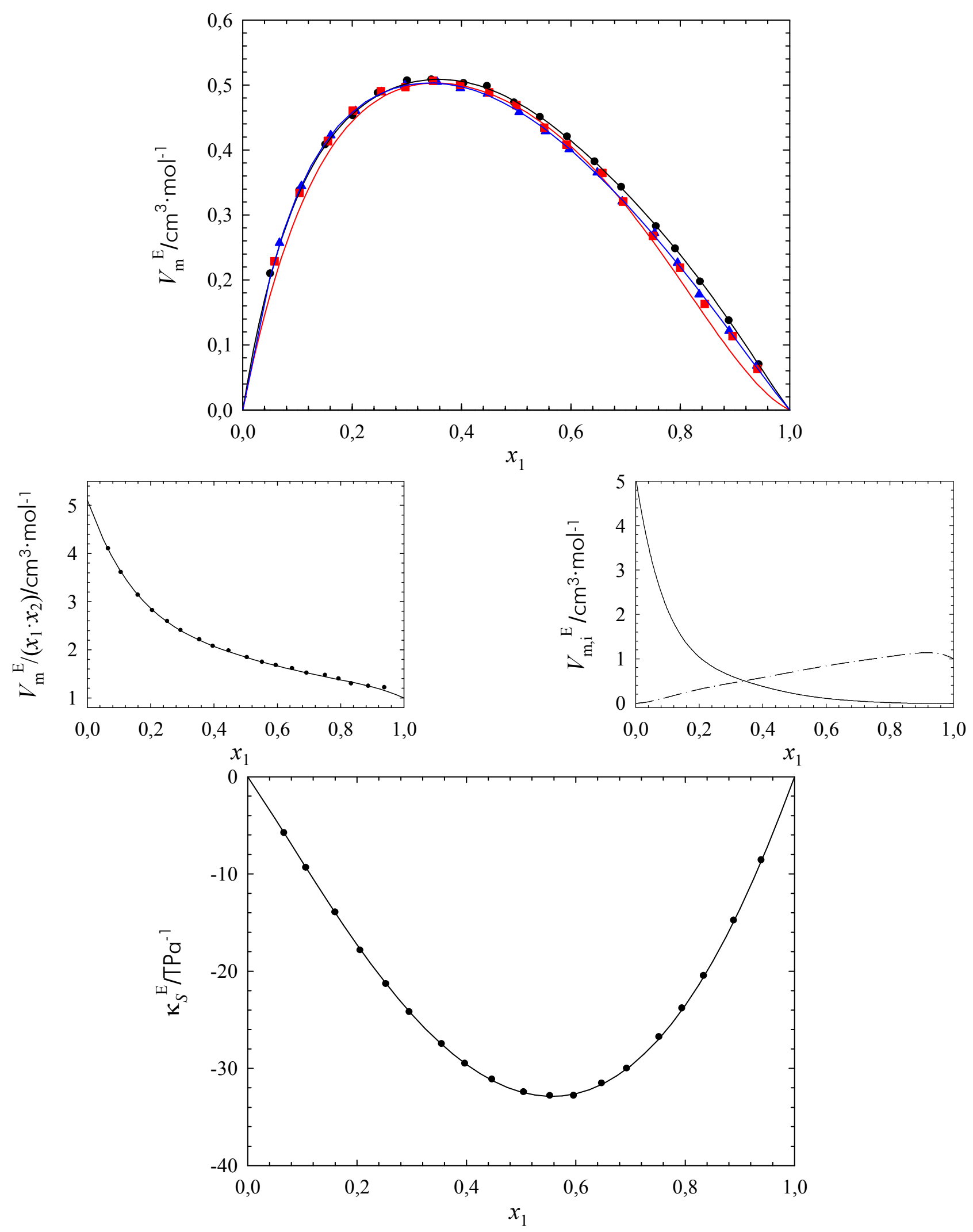

Ilustración 3-40: volúmenes molares de exceso $V_{\mathrm{m}}^{\mathrm{E}}$, volúmenes molares de exceso reducidos $V_{\mathrm{m}}^{\mathrm{E}} /\left(x_{1} \cdot x_{2}\right)$, volúmenes molares parciales de exceso $V_{\mathrm{m}, \mathrm{i}}^{\mathrm{E}}$ y compresibilidades isoentrópicas de exceso $\kappa_{S}^{\mathrm{E}}$ del sistema $N$-metilanilina (1) + ciclohexano (2) 
Tabla 3-78: volúmenes molares de exceso del sistema $N$-metilanilina (1) + tolveno (2)

\begin{tabular}{|c|c|c|c|c|c|}
\hline$x_{1}$ & $V_{\mathrm{m}}^{\mathrm{E}} / \mathrm{cm}^{3} \cdot \mathrm{mol}^{-1}$ & $x_{1}$ & $V_{\mathrm{m}}^{\mathrm{E}} / \mathrm{cm}^{3} \cdot \mathrm{mol}^{-1}$ & $x_{1}$ & $V_{\mathrm{m}}^{\mathrm{E}} / \mathrm{cm}^{3} \cdot \mathrm{mol}^{-1}$ \\
\hline \multicolumn{6}{|c|}{$293.15 \mathrm{~K}$} \\
\hline 0.0541 & -0.0391 & 0.3898 & -0.1986 & 0.6894 & -0.1744 \\
\hline 0.0986 & -0.0748 & 0.4460 & -0.2040 & 0.7385 & -0.1581 \\
\hline 0.1502 & -0.1065 & 0.4872 & -0.2064 & 0.7823 & -0.1386 \\
\hline 0.1989 & -0.1325 & 0.5426 & -0.2059 & 0.8377 & -0.1108 \\
\hline 0.2473 & -0.1537 & 0.5859 & -0.2001 & 0.8834 & -0.0834 \\
\hline 0.2982 & -0.1747 & 0.6441 & -0.1889 & 0.9299 & -0.0528 \\
\hline 0.3500 & -0.1873 & & & & \\
\hline \multicolumn{6}{|c|}{$298.15 \mathrm{~K}$} \\
\hline 0.0532 & -0.0417 & 0.3900 & -0.2046 & 0.6868 & -0.1827 \\
\hline 0.0998 & -0.0725 & 0.4434 & -0.2109 & 0.7407 & -0.1622 \\
\hline 0.1518 & -0.1131 & 0.4900 & -0.2138 & 0.7823 & -0.1437 \\
\hline 0.1906 & -0.1341 & 0.5415 & -0.2120 & 0.8378 & -0.1143 \\
\hline 0.2452 & -0.1600 & 0.5792 & -0.2081 & 0.8844 & -0.0861 \\
\hline 0.2887 & -0.1777 & 0.6432 & -0.1960 & 0.9340 & -0.0516 \\
\hline 0.3417 & -0.1918 & & & & \\
\hline \multicolumn{6}{|c|}{$303.15 \mathrm{~K}$} \\
\hline 0.0530 & -0.0416 & 0.3919 & -0.2123 & 0.6846 & -0.1901 \\
\hline 0.0982 & -0.0777 & 0.4478 & -0.2203 & 0.7398 & -0.1686 \\
\hline 0.1501 & -0.1159 & 0.4918 & -0.2221 & 0.7782 & -0.1519 \\
\hline 0.1929 & -0.1381 & 0.5391 & -0.2193 & 0.8420 & -0.1139 \\
\hline 0.2456 & -0.1654 & 0.5886 & -0.2145 & 0.8790 & -0.0929 \\
\hline 0.2837 & -0.1829 & 0.6395 & -0.2036 & 0.9340 & -0.0533 \\
\hline 0.3444 & -0.2026 & & & & \\
\hline
\end{tabular}

Tabla 3-79: velocidades del sonido de exceso, coeficientes de expansión térmica a $P=$ cte. De exceso, y compresibilidades isoentrópicas de exceso del sistema $N$-metilanilina (1) + tolveno (2)

\begin{tabular}{cccccccc}
\hline$x_{1}$ & $u^{\mathrm{E}} / \mathrm{m} \cdot \mathrm{s}^{-1}$ & $\alpha_{\mathrm{P}}^{\mathrm{E}} / 10^{-6} \mathrm{~K}^{-1}$ & $\kappa_{S}^{\mathrm{E}} / \mathrm{TPa}^{-1}$ & $x_{1}$ & $u^{\mathrm{E}} / \mathrm{m} \cdot \mathrm{s}^{-1}$ & $\alpha_{\mathrm{P}}^{\mathrm{E}} / 10^{-6} \mathrm{~K}^{-1}$ & $\kappa_{S}^{\mathrm{E}} / \mathrm{TPa}^{-1}$ \\
\hline 0.0532 & 7.0 & -2.49 & -7.38 & 0.5415 & 38.5 & -12.37 & -29.90 \\
0.0998 & 12.5 & -4.47 & -12.73 & 0.5792 & 38.5 & -12.05 & -29.06 \\
0.1518 & 18.1 & -6.53 & -17.97 & 0.6432 & 37.3 & -11.17 & -26.94 \\
0.1906 & 21.7 & -7.85 & -21.09 & 0.6868 & 35.6 & -10.33 & -24.92 \\
0.2452 & 26.3 & -9.44 & -24.69 & 0.7407 & 32.7 & -9.05 & -21.97 \\
0.2887 & 29.4 & -10.48 & -26.89 & 0.7823 & 29.6 & -7.90 & -19.28 \\
0.3417 & 32.6 & -11.45 & -28.83 & 0.8378 & 24.3 & -6.15 & -15.18 \\
0.3900 & 35.0 & -12.09 & -30.06 & 0.8844 & 18.7 & -4.52 & -11.29 \\
0.4434 & 36.9 & -12.48 & -30.61 & 0.9340 & 11.6 & -2.65 & -6.71 \\
0.4900 & 38.1 & -12.56 & -30.57 & & & & \\
\hline
\end{tabular}

Tabla 3-80: coeficientes y desviaciones estándar del ajuste a la ecuación R-K de las diferentes propiedades de exceso del sistema $N$-metilanilina (1) + tolveno (2)

\begin{tabular}{|c|c|c|c|c|c|c|}
\hline$T / \mathrm{K}$ & Propiedad & $A_{0}$ & $A_{1}$ & $A_{2}$ & $A_{3}$ & $\sigma$ \\
\hline 293.15 & $V_{\mathrm{m}}^{\mathrm{E}} / \mathrm{cm}^{3} \cdot \mathrm{mol}^{-1}$ & -0.8273 & 0.013 & 0.015 & & 0.0012 \\
\hline \multirow[t]{4}{*}{298.15} & $V_{\mathrm{m}}^{\mathrm{E}} / \mathrm{cm}^{3} \cdot \mathrm{mol}^{-1}$ & -0.8543 & 0.014 & & & 0.0016 \\
\hline & $u^{\mathrm{E}} / \mathrm{m} \cdot \mathrm{s}^{-1}$ & 152.83 & 28.71 & 14.5 & & 0.04 \\
\hline & $\alpha_{\mathrm{P}}^{\mathrm{E}} / 10^{-6} \mathrm{~K}^{-1}$ & -50.19 & 3.99 & 4.94 & & 0.015 \\
\hline & $\kappa_{S}^{\mathrm{E}} / \mathrm{TPa}^{-1}$ & -121.91 & 18.0 & -6.8 & 3.8 & 0.04 \\
\hline 303.15 & $V_{\mathrm{m}}^{\mathrm{E}} / \mathrm{cm}^{3} \cdot \mathrm{mol}^{-1}$ & -0.8895 & 0.018 & 0.021 & & 0.0012 \\
\hline
\end{tabular}



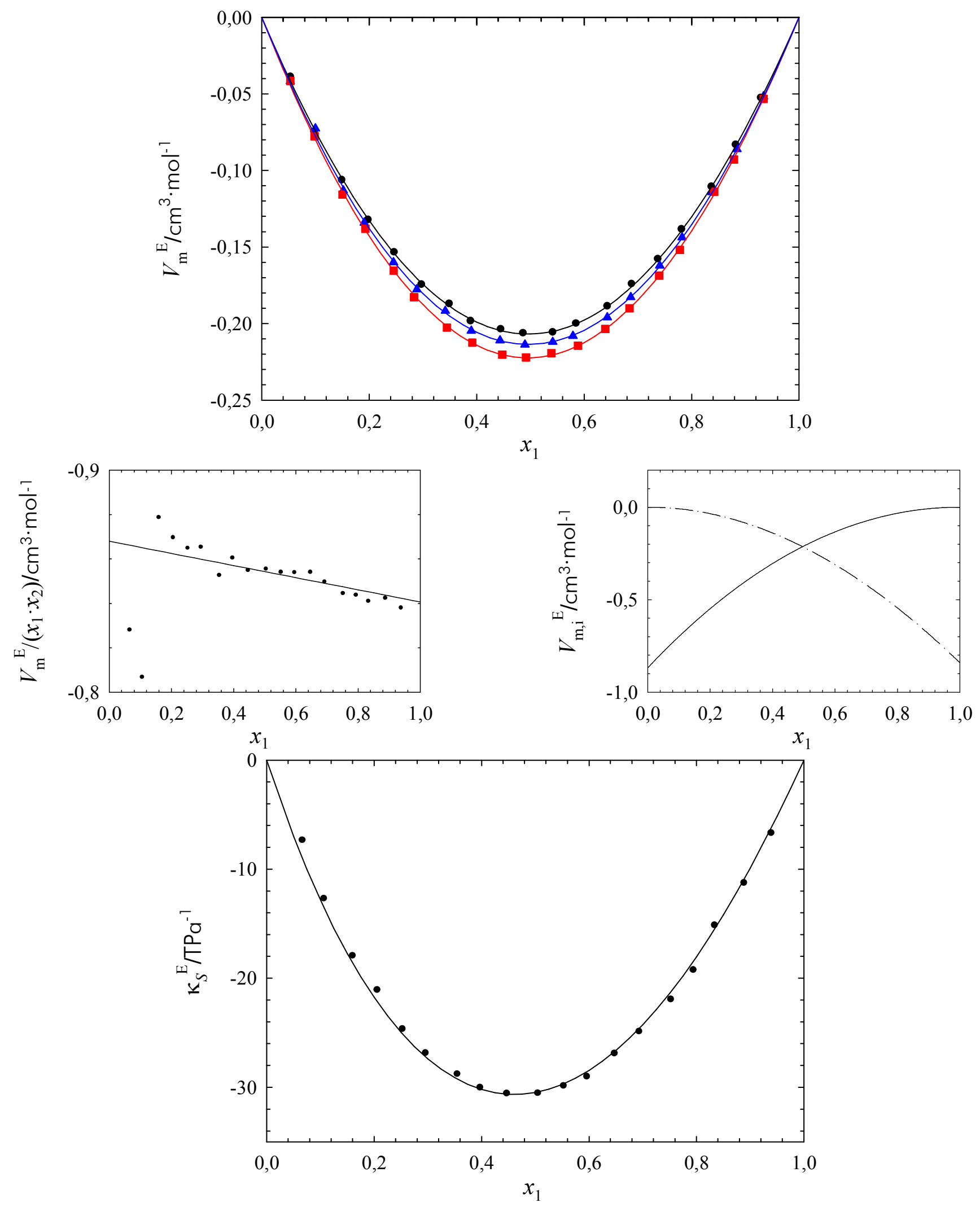

Ilustración 3-41: volúmenes molares de exceso $V_{\mathrm{m}}^{\mathrm{E}}$, volúmenes molares de exceso reducidos $V_{\mathrm{m}}^{\mathrm{E}} /\left(x_{1} \cdot x_{2}\right)$, volúmenes molares parciales de exceso $V_{\mathrm{m}, \mathrm{i}}^{\mathrm{E}}$ y compresibilidades isoentrópicas de exceso $\kappa_{S}^{\mathrm{E}}$ del sistema $N$-metilanilina (1) + tolveno (2) 
CAPITULO 3 


\section{BIBLIOGRAFÍA}

[1]: Fouvre; Valson; Comptes Rendu, 77 (1873), 802.

[2]: E. G. Guthrie; Phil. Mag., 18(5) (1884), 495.

[3]: Patterson; Montgomerie; J. Chem. Soc., 95 (1909), 1136.

[4]: Handa, Y. P.; Benson, G.C.; Fluid Phase Equilib., 3 (1979), 185.

[5]: Chao, K. C.; Robinson, R. L.; Adv. Chem. Ser. Vol. 182, Am. Chem. Soc., Washington (1979); Perry, R. H. and Green, D.

[6]: Millero, F. J.; Chem. Rev., 71 (1971), 147.

[7]: lqbal, M.; Verrall, R. E.; J. Phys. Chem., 91 (1987), 967.

[8]: Millero, F. J.; Lo Surdo, A.; Shin, C.; J. Phys. Chem., 87 (1978), 784.

[9]: Hedwig, G.R.; J. Chem. Thermodyn., 23 (1991), 123.

[10]: Hedwig, G.R.; Hoiland, H.; J. Sol. Chem., 20 (1991), 1113.

[1 1]: Khoshkbarchi, M. K.; Vera, J. H.; Ind. Eng. Chem. Res., 35 (1996), 2735.

[12]: Natarajan, M.; Wadi, R. K.; Gaur, H. C.; J. Chem. Eng. Data, 35 (1990), 87.

[13]: Singh, S. P.; Miller, R.C.; J. Chem. Thermodyn., 10 (1978), 741.

[14]: Brown, I.; Lane, J.E.; Pure and Appl. Chem., 45 (1) (1976), 1.

[15]: Girard, G.; "Recommended Reference Materials For the Realization of PhysicoChemical Properties" ed. por Marsh, K.N.; Capítulo 2, Blackwell Science; 1987.

[16]: Harvey, A. H.; Span, R.; Fujii, K; Tanaka, M; Davis, R. S.; Metrologia, 46 (2009), 196.

[17]: Fujii, K; Metrologia, 41 (2004), S1-S15.

[18]: "The Physico-Chemical Constants of Binary Systems in Concentrated Solutions", Interscience, New York, N. Y.; Vol. 1 , 2, 1959; Vol 3, 4, 1960.

[19]: Craig, H.; Science, 133 (1961), 1833.

[20]: Girard, G.; Menache, M.; Acad. Sci. Paris, 274B (1972), 377.

[21]: Menaché, M.; Girard, G.; Metrologia, 1973, 9, 62-8.

[22]: Patterson, J; Morris, E.C.; Metrologia, 31 (1994), 277. 
[23]: Masui, R.; Fujii, K.; Takenaka, M.; Metrologia, 32 (1995/96), 333.

[24]: Tanaka, M.; Girard, G.; Davis, R.; Peuto, A.; Bignell, N.; Metrologia, 38 (2001), 301.

[25]: Wagner, W.; Pruß, A.; J. Phys. Chem. Ref. Data, 31 (2002), 387.

[26]: Cook, A. H.; Stone, N. W. B.; Phil. Trans. R. Soc. Lond. A, 250 (1957), 279.

[27]: Cook, A. H.; Phil. Trans. R. Soc. Lond. A, 254 (1961), 125.

[28]: "Experimental Thermodynamics Vol II, Experimental Thermodynamics of non reacting Fluids"; Edited by Le Neindre, B.; Vodar, B.; Eds. For IUPAC, Butterworths, London (1975).

[29]: Baver, E.; Lewin, S. Z.; "Techniques of Chemistry, Vol I: Physical Methods of Chemistry, Part IV, Determination of Mass, Transport and Electrical-Magnetic Properties", Weissberger ed.; Wiley-Interscience, New York (1972).

[30]: "Experimental Thermodynamics Vol VI, Measurement of the Thermodynamic Properties of Single Phases"; edited by Goodwin, A.R.H.; Marsh, K.N.; Wakeham, W.A.; Eds for IUPAC; Elsevier, Amsterdam (2003).

[31]: Cobos Hernández, J.C.; "Estudio termodinámico de mezclas líquidas de alcoxietanoles con disolventes orgánicos". Tesis Doctoral. Facultad de Ciencias. Universidad de Valladolid (1987).

[32]: Goldman, K.; Scrase, N. G.; Physica, 44 (1969), 555.

[33]: McClune, C. R.; Cryogenics, 16 (1976), 289.

[34]: Griger, A.F.; Steele, W. A.; Rev. Sci. Instr., 37 (1966), 51.

[35]: Bignell, N.; Meas. Sci. Technol., 17 (2006), 2574.

[36]: Beams, J. W.; Clarke, A. M.; Rev. Sci. Instr., 33 (1962), 750.

[37]: Millero, F. J.; Knox, J. H.; Emmet, R. T. ; J. Sol. Chem., 1 (1972), 173.

[38]: Weeks, J. A.; Benson, G.C.; J. Chem. Thermodyn., 5 (1973), 107.

[39]: Haynes, W. H.; Hiza, M. J.; Frederick, N. V.; Rev. Sci. Instr., 47 (1976), 1237.

[40]: Masui, R.; Haynes, W.M.; Chang, R.F.; Davis, H.A.; Levelt Sengers, J.M.H.; Rev.Sci. Instr., 55 (1984), 1132.

[41]: Fujii, K.; Takenaka, M.; Nara, K.; Rev. Sci. Instr., 59 (1988), 2539.

[42]: Kleinrham, R.; Wagner, W.; Fortschr.-Ber. VDI, Reiche 3, VDI-Verlag, Düsseldorf, Nr. 92 (1984).

[43]: Kleinrham, R.; Wagner, W. J.; Chem. Thermodyn., 18 (1986), 739.

[44]: Mark O.; McLinden, A.; Cornelia Lösch-Will; J. Chem. Thermodyn., 39 (2007), 507.

[45]: Brachthäuser, K.; Kleinrahm, R.; Lösch, H.W.; Wagner, W.; Entwicklung eines neven Dichtemeßverfahrens und Aufbau einer Hochtemperatur-HochdruckDichtemeßanlage. Fortschr.-Ber. VDI, Reihe 8, Nr. 371, VDI-Verlag, Düsseldorf (1993).

[46]: Klimeck, J; Dissertation, Ruhr-Universitat, Bochum (1997). 
[47]: Klimeck, J. ; Kleinrahm, R; Wagner, W. J. ; Chem. Thermodyn.

[48]: Mathieu, J. ; Schweitzer, P.; Meas. Sci. Technol., 15 (2004), 869.

[49]: Ciochina, J. Z.; Anal. Chem., 107 (1936), 108.

[50]: Barbour, H. G. ; Hamilton, W. F. ; J. Biol. Chem., 69 (1926), 625.

[51]: Albert, H. J. ; Wood, R. H.; Rev. Sci. Instr., 55 (1984), 589.

[52]: Kratky, O. ; Leopold, H. ; Stabinger, H. ; Z. Angew. Physik, 27 (1969), 273.

[53]: Kratky, O. ; Leopold, H. ; Stabinger, H. Z.; United States Patent number 3523446.

[54]: Stabinger, H.; Dissertation, Technische Hochschule Graz, (1969).

[55]: Straty, G.C.; Palavra, A.M.F. ; J. Res. Natl. Bur. Stand., 89 (1984), 375.

[56]: Linsky, D. ; Levelt Sengers, J.M.H. ; Davis, H.A. ; Rev. Sci. Instr., 58 (1987), 817.

[57]: Magee, J.W.; Ely, J.F. ; Int. J. Thermophys., 9 (1988), 547.

[58]: Mourato, M.F.B. ; Calado, J.C.; Palavra, A.M.F.; High-Temp. High Press., 31 (1999), 91.

[59]: Fenghour, A. ; Wakeham, W.A. ; Ferguson, D. ; Scott, A.C. ; Watson, J.T.R. ; J. Chem. Thermodyn., 25 (1993), 831.

[60]: Bridgman, P.W., Proc. Am. Acad. Arts Sci., 66 (1931), 185.

[61]: Blagoi, Y.P. and Sorokin, V.A., Russ. J. Phys. Chem., 42 (1968), 290.

[62]: Hocq, H., Bur, Y. and Berro, C., Int. Electron J. Phys-Chem. Data, 1 (1995), 79.

[63]: Bowman, H.A., Schoonover, R.M. and Carroll, C.L., J. Res. Natl. Bur. Stand. A, 78 (1974), 13.

[64]: Keyes, B. ; Hildebrand, J. H. ; J. Amer. Chem. Soc., 39 (1917), 2126.

[65]: Duncan, W. A.; Sheridan, J. P.; Swinton; F.L. Trans. Faraday Soc., 62 (1966), 1090.

[66]: Battino, R.; Chem. Rev., $71(1), 1971,5$.

[67]: Bellemans; A. Bull. Soc. Chim. Belges, 66 (1957), 636.

[68]: Brown, I. ; Smith, F.; Aust. J. Chem., 15 (1962), 1.

[69]: Zamyatnin, A.A. ; Russ. J. Phys. Chem., 45 (1971), 567.

[70]: Stookey, D. J. ; Sallak, H. M. ; Smith, B. D. ; J. Chem. Thermodyn., 5 (1973), 741.

[71]: Bottomley, G. A. ; Scott, R. L. ; J. Chem. Thermodyn., 6 (1974), 973.

[72]: Tanaka, R. ; Kiyohara. O. ; D’Arcy, P. J. ; Benson, G. C.; Can. J. Chem., 53 (1975), 2262.

[73]: Wirth, H. E. ; Lindstrom, R. E. ; Johnson, J. N.; J. Phys. Chem., 67 (1963), 2339.

[74]: Stokes, R. H. ; Levien, B. J. ; Marsh, K. N.; J. Chem. Thermodyn., 2 (1970), 43.

[75]: Kumaran, M. K.; McGlashan, M. L.; J. Chem. Thermodyn., 9 (1977), 259.

[76]: Chareyron, R. ; Clechet, P. ; Bull. Soc. Chim. Fr., 8 (1971), 2853. 
[77]: Esper, G. ; Lemming, W.; Beckermann, W.; Kohler, F.; Fluid Phase Equilib., 105 (1995), 173.

[78]: Hozumi, T.; Ichikawa, T.; Sato, H.; Watanabe, K.; Int. J. Thermophys., 20 (1999), 1677.

[79]: Shabani, M.R.; Riazi, M.R.; Shaban, H.I.; Can. J. Chem. Eng., 76 (1998), 281.

[80]: Hurly, J.J.; Int. J. Thermophys., 21 (2000), 805.

[81]: Benedetto, G.; Gavioso, R.M.; Spagnolo, R.; Grigiante, M.; Int. J. Thermophys., $22(2001), 1073$.

[82]: Davis, L.A.; Gordon, R.B.; J. Chem. Phys., 46 (1967), 2650.

[83]: Daridon, J.L.; Lagourette, B.; Xans, P.; Fluid Phase Equilib., 100 (1994), 269.

[84]: Dutour, S.; Daridon, J.L.; Lagourette, B.; Int. J. Thermophys., 21 (2000), 173.

[85]: Pires, P.F.; Esperanca, J.M.S.S.; Guedes, H.J.R.; J. Chem. Eng. Data, 45 (2000), 496.

[86]: Trusler, J.P.M.; Zarari, M.; J. Chem. Thermodyn., 24 (1992), 973.

[87]: Estrada-Alexanders, A.F.; Trusler, J.P.M.; Zarari, M.P.; Int. J. Thermophys., 16(3) (1995), 663.

[88]: Dayton, T.C.; Beyerlein, S.W.; Goodwin, A.R.H.; J. Chem. Thermodyn., 31 (1999), 847.

[89]: Estrada-Alexanders, A.F.; Trusler, J.P.M.; Int. J. Thermophys., 17 (1996), 1325.

[90]: Estrada-Alexanders, A.F.; Trusler, J.P.M.; J. Chem. Thermodyn., 29 (1997), 991.

[91]: Douheret, G.; Davis, M. I. ; Høiland, H.; J. Mol. Liq., 80 (1999), 1.

[92]: Pfeiffer, H.; Heremans, K.; Chem. Phys. Lett., 361 (2002), 226.

[93]: Smirnovas, V.; Winter, R.; Funck, T.; Dzwolak, W.; J. Phys. Chem. B, 109(2005), 19043.

[94]: Mosquera, V. V.; del Rio J. M.; Attwood, D.; Garcia, M.; Jones, M. N.; Prieto, G.; Suarez, M. J.; Sarmiento, F.; J. Colloid Interf. Sci., 206 (1998), 66.

[95]: Povey, M. .J W.; Pharma. Sci. Technol. Today, 3(2000), 373.

[96]: Benedito, J.; Carcel, J. A.; Gonzalez, R.; Mulet, A.; Ultrasonics, 40 (2002), 19.

[97]: McSkimin, H.J.; "Physical Acoustics", Vol I, Part A, Ch. 4., W.P. Mason ed., Academic Press, New York (1964).

[98]: Papadakis, E.P.; "Physical Acoustics: Principles and Methods", Vol XII, Ch. 5., W.P. Mason and R.N. Thurston eds., Academic Press, New York (1976).

[99]: Heydeman, P.; "Physical Acoustics: Principles and Methods", Vol VIII, Ch. 4., W.P.

Mason and R.N. Thurston eds., Academic Press, New York (1971).

[100]: Trusler, J.P.M.; "Physical Acoustics and Metrology of Fluids", Adam-Hilger, Bristol (1991).

[101]: Kaatze, U.; Hushcha, T.; Eggers, F.; J. Sol. Chem., 29 (2000), 299. 
[102]: Leroy, C.C.; "Handbook of Elastic Properties of Solids, Liquids, and Gases", Vol IV, Elastic Properties of Fluids: Liquids and Gases, Ch. 2., M. Levy, H.E. Bass and R.R. Stern eds.-in-chief, M. Levy, R. Raspet and D. Sinha, vol. eds., L. Furr, tech. ed., V. Keppens, supervising ed., Academic Press, New York (2001).

[103]: Kundt, A.; "Acoustic experiments", The London, Edinburgh and Dublin Philosophical Magazine and Journal of Science, Vol XXXV (1868), 41.

[104]: J.P.M. Trusler; "Physical Acoustics and Metrology of Fluids", Adam Hilger, Bristol, England (1991).

[105]: Kaatze, U.; Eggers, F.; Lautscham, K.; Meas. Sci. Technol., 19 (2008), 062001.

[106]: Ten Seldam, C.A.; Biswas, S.N.; J. Chem. Phys., 94 (1991), 2130.

[107]: Leopold, H.; Stabinger, H.; United States Patent, patent ner 4,838,084; 13 / 6 / 1989 "DENSITY MEASURING INSTRUMENT".

[108]: Riddick, J. A.; Bunger, W. B., Sakano, T. K. "Organic Solvents, Physical properties and methods of purification", $4^{\mathrm{a}}$ ed. Techniques of Chemistry Volume II, a Wiley Interscience Publication.

[109]: "Recommended Reference Materials for the Realization of Physicochemical Properties", Edited by Marsh, K. N.; International Union Of Pure And Applied Chemistry.

[1 10]: Nath, J.; J. Chem. Thermodyn., 30 (1998), 885.

[ $\left.\begin{array}{lll}1 & 1 & 1\end{array}\right]$ : Tamura, K.; Ohomuro, K.; Murakami, S.; J. Chem. Thermodyn., 16 (1984), 121.

[1 12]: Dzida, M.; Ernst, S.; J. Chem. Eng. Data, 48 (2003), 1453.

[113]: Nath, J.; J. Chem. Thermodyn., 29 (1997), 853.

[1 14]: Treszczanowicz, A.J.; Handa, Y.P.; Benson, G.C.; J. Chem. Thermodyn., 14 (1982), 871.

[1 15]: Rodríguez, A.; Canosa, J.; Tojo, J.; J. Chem. Thermodyn., 35 (2003), 1321.

[1 16]: Orge, B.; Rodríguez, A.; Canosa, J.; Marino, G.; Iglesias, M.; Tojo, J.; J. Chem. Eng. Data., 44 (1999), 1041.

[1 17]: Nishimoto, M.; Tabata, S.; Tamura, K.; Murakami, S.; Fluid Phase Equilib., 136 (1997), 235.

[1 18]: Tamura, K.; Murakami, S.; J. Chem. Thermodyn., 16 (1984), 33.

[1 19]: Doia, H.; Tamura, K.; J. Chem. Thermodyn., 32 (2000), 729.

[120]: Alonso, I.; "Estudio de los volúmenes de exceso y compresibilidades isoentrópicas de sistemas cetona + amina", Trabajo de Fin de Máster. Facultad de Ciencias. Universidad de Valladolid (2009).

[121]: IUPAC. "Atomic weights of the elements 1995 (IUPAC technical report)". Pure Appl. Chem., 1996, 68, 2339-2359.

[122]: Riesco Fernández, N.; "Contribución experimental y teórica al estudio de las propiedades termodinámicas de las mezclas binarias entre compuestos orgánicos". Tesis Doctoral. Departamento de Termodinámica y Física Aplicada. Facultad de Ciencias. Universidad de Valladolid (2003). 
[123]: Douhéret, G.; Davis, M.I.; Reis, J.C.R.; Fluid Phase Equilib., 231 (2005), 246.

[124]: Tamura, K.; Ohomuro, O.; Murakami, S.; J. Chem. Thermodyn., 15 (1983), 859.

[125]: Goates, J. R.; Ott, J. B.; Moellmer, J. F.; J. Chem. Thermodyn., 9 (1977), 249.

[126]: Takenaka, M.; Tanaka, R.; Murakami, S.; J. Chem. Thermodyn., 12 (1980), 849.

[127]: Villa Vallejo, S.; "Contribución experimental y teórica al estudio de las propiedades termodinámicas de mezclas líquidas formadas por aminas y alcanos 0 1 -alcoholes". Tesis doctoral (2003), 21-23.

[128]: Domínguez, M.; Pardo, J.; Santafe, J.; Royo, F. M.; Urieta, J. S.; Fluid Phase Equilib., 118 (1996), 227.

[129]: Junquera, E.; Tardajos, G.; Aicart, E.; J. Chem. Thermodyn., 20 (1988), 1461.

[130]: Mozo Ruiz, I.; "Estudio experimental y teórico de mezclas binarias de n-butan-1ol, di-n-butiléter y celosolvas". Tesis Doctoral. Departamento de Física Aplicada. Facultad de Ciencias. Universidad de Valladolid (2010).

[131]: Mitchell, J.; Smith, D.M.; "Aquametry", 2nd Ed., J. Wiley \& Sons, New York (1980).

[132]: Armarego, W.L.F.; Chai, C.L.L.; "Purification of Laboratory Chemicals", 6" Ed., Butterworth-Heinemann (2009).

[133]: Coetzee, J.F.; Siao, Wei-San; Inorg. Chem., 2 (1963), 14.

[134]: Stokes, R. H. en el capítulo 7 de "Experimental Thermodynamics Vol IV, Solution Calorimetry"; Edited by Marsh, K. N.; O'Hare, P. A. G.; Eds. For IUPAC, Blackwell Scientific Publications, Oxford (1994).

[135]: Alonso, I.; Alonso, V.; Mozo, I.; García de la Fuente, I.; González, J. A.; Cobos, J.C.; J. Chem. Eng. Data, 55 (2010), 2505.

[136]: Alonso, I.; Alonso, V.; Mozo, I.; García de la Fuente, I.; González, J. A.; Cobos, J.C.; J. Mol. Liq., 155 (2010), 109.

[137]: Alonso, I.; Mozo, I.; García de la Fuente, I.; González, J. A.; Cobos, J.C.; J. Chem. Eng. Data, 55 (2010), 5400.

[138]: Alonso, I.; Mozo, I.; García de la Fuente, I.; González, J. A.; Cobos, J.C.; Thermochim. Acta, 512 (2011), 86.

[139]: Alonso, I.; Mozo, I.; García de la Fuente, I.; González, J. A.; Cobos, J.C.; J. Sol. Chem., 40 (2011), 2057.

[140]: González, J. A.; Alonso, I.; Alonso, V.; Mozo, I.; García de la Fuente, I.; Cobos, J.C.; J. Chem. Thermodyn.; 43 (2011), 1506.

[141]: Alonso, I.; Mozo, I.; García de la Fuente, I.; González, J. A.; Cobos, J.C.; J. Mol. Liq., 160 (2011), 180.

[142]: Alonso, I.; García de la Fuente, I.; González, J. A.; Cobos, J.C.; J. Chem. Eng. Data, 58 (2013), 1697.

[143]: Papaioannou, D.; Ziakas, D.; Panayiotou, C; J. Chem. Eng. Data., 36 (1988), 35. 
[144]: Gomes Azevedo, R.; Szydlowski, J.; Pires, P.-F.; Esperança, J.M.S.S.; Guedes, H.J.R.; Rebelo, L.P.N.; J. Chem. Thermodyn., 36 (2004), 211.

[145]: Lee, L.-S.; Chuang, M.-L.; J. Chem. Eng. Data, 42 (1997), 850.

[146]: González, B.; Domínguez, A.; Tojo, J.; J. Chem. Thermodyn., 38 (2006), 707.

[147]: Grolier, J.-P.E.; Benson, G.C.; Picker, P.; J. Chem. Eng. Data, 20 (1975), 243.

[148]: Rubio, R.G.; Renuncio, J.A.; Díaz Peña, M.; J. Chem Thermodyn., 15 (1983), 779.

[149]: Brocos, P.; Piñeiro, A.; Bravo, R.; Amigo, A.; Roux, A.-H.; Roux-Desgranges, G.; J. Chem. Eng. Data, 47 (2002), 351.

[150]: Bakshi, M.S., Kaur, G.; J. Chem. Eng. Data., 42 (1997), 298.

[151]: Laínez, A.; Rodrigo, M.M.; Wilhelm, E.; Grolier, J.-P.E.; J. Sol. Chem., 21 (1992), 49.

[152]: Takagi, T.; J. Chem. Thermodyn., 12 (1980), 277.

[153]: Su. L., Wang, H.; J. Chem. Thermodyn., 41 (2009), 315.

[154]: Lesbats, C., Lichanot, A.; Thermochim. Acta, 109 (1987), 317.

[155]: Nakanishi, K., Touhara, H.; J. Chem. Thermodyn., 18 (1986), 657.

[156]: Lampreia, I.M.S.; Barbosa, E.F.G.; Fluid Phase Equilib., 71 (1992), 125.

[157]: Oswal, S.L.; Oswal, P.; Gardas, R.L.; Patel, S.G.; Shinde, R.G.; Fluid Phase Equilib., 216 (2004), 33.

[158]: Pal, A.; Gaba, R.; Sharma, S.; J. Chem. Eng. Data, 53 (2008), 1643.

[159]: Letcher, T.M.; J. Chem. Thermodyn., 4 (1972), 159.

[160]: Goralski, P.;Wasiak, M.; Bald, A.; J. Chem. Eng. Data, 47 (2002), 83.

[161]: Malek, N. I., ljardar, S.P., Oswal, S. B.; Thermochim. Acta, 539 (2012), 71.

[162]: Saito, A. Tanaka, R.; J. Chem. Thermodyn., 20 (1988), 859.

[163]: Treszczanowicz, A.J., Halpin, C.J., Benson, G.C.; J. Chem. Eng. Data, 27 (1982), 321.

[164]: Andreoli-Ball, L.; Patterson, D.; Costas, M.; Caceres-Alonso, M.; J. Chem. Soc., Faraday Trans., 84 (1988), 3991.

[165]: Al-Kandary, J.A., Al-Jimaz, A.S., Abdul-Latif, A.-H.M.; J. Chem. Eng. Data, 51 (2006), 2074.

[166]: Grolier, J.-P.E., Roux-Desgranges, G., Berkane, M., Jimenez, E., Wilhelm, E.; J. Chem. Thermodyn., 25 (1993), 41.

[167]: Bai, T.-C.; Yao, J.; Han, S.-J.; Fluid Phase Equilib., 152 (1998), 283. 
CAPITULO 3 


\title{
CAPITULO 4
}

\section{Medida de las entalpías de exceso}

\begin{abstract}
"One property that is present in nearly all chemical and physical processes is enthalpy change. The heat effects as processes proceed can be used to study a wide spectrum of chemical and physical phenomena. Thus, calorimetry is useful in that it uses heat production (or absorption) as a probe to study and analyze processes."

Izatt, R. M.; Redd, E. H.; Christensen, J. J.; Thermochimica Acta, 64 (1983) 355-372
\end{abstract}

$\mathrm{E}$ n el estudio de las mezclas de líquidos, las entalpías de exceso (o de mezcla) son muy importantes, aunque no más que, por ejemplo, los volúmenes de exceso. Por ello la mayoría de las medidas sobre las entalpías de mezcla se han publicado junto con otras propiedades de exceso.

El estudio de las entalpías de exceso de disoluciones líquidas es de gran interés por diferentes razones [1]:

a) Construcción de tablas termoquímicas para la aplicación directa de los datos en ellas reflejados.

b) Sirve como test de teorías que intentan predecir las propiedades de las mezclas a partir de los datos de los componentes puros. Por ejemplo, los datos de $H_{\mathrm{m}}^{\mathrm{E}}$ de algunas mezclas de alcanos se han utilizado para chequear la validez de las teorías de red de mezclas de moléculas de diferente tamaño.

c) Proporcionan datos para la evaluación de los parámetros de interacción entre especies distintas, es decir, para el estudio de las fuerzas intermoleculares. La entalpía de exceso de una mezcla binaria de líquidos surge, al igual que otras funciones de exceso, como consecuencia de reemplazar interacciones entre moléculas iguales presentes en los líquidos puros (tipos 1-1 y 2-2) por interacciones entre moléculas diferentes, presentes en la mezcla (1-2).

Precisamente uno de los problemas no resueltos más importante de la Física es el conocimiento de la energía de interacción entre pares de moléculas como función de la distancia entre ambas y de su orientación relativa. Una manera de abordar el problema es mediante el estudio de la dependencia con la temperatura del segundo coeficiente 
del virial de un gas puro diluido:

$$
B(T)=\lim _{V \rightarrow \infty}\left[\frac{V}{n} \cdot\left(\frac{P \cdot V}{n \cdot R \cdot T}-1\right)\right]
$$

que está relacionado con el potencial de interacción por pares $w\left(r, \theta_{1}, \theta_{2}, \varphi_{2}-\varphi_{1}\right)$ mediante la ecuación siguiente:

$$
B(T)=-\frac{N_{A}}{4} \cdot \int_{0}^{R} \int_{0}^{2 \cdot \pi} \int_{-1}^{1} \int_{-1}^{1}\left(e^{-w / k_{B} \cdot T}-1\right) \cdot r^{2} \cdot \mathrm{d} \cos \theta_{1} \cdot \mathrm{d} \cos \theta_{2} \cdot \mathrm{d}\left(\varphi_{2}-\varphi_{1}\right) \cdot \mathrm{d} r
$$

que se reduce a la (2.86) si las moléculas tienen simetría esférica.

Los valores de $B$ se obtienen a partir de las medidas $P V T$, o a partir de los datos de las entalpías de vaporización junto con los de las presiones de vapor [2], [3]. Los valores de $(B-T \cdot \mathrm{d} B / \mathrm{d} T)$ se pueden obtener a partir de las medidas del coeficiente de JouleThomson isotermo $(\partial H / \partial P)_{T}$ hechas por calorimetría de flujo, o combinando los valores de los coeficientes de Joule-Thomson isoentálpicos $(\partial T / \partial P)_{H}$ junto con los de las capacidades caloríficas a presión constante $C_{P}$. Los valores de $T^{2} \cdot \mathrm{d}^{2} B / \mathrm{d} T^{2}$ se pueden obtener a partir de medidas de calorimetría de flujo de la dependencia con la presión de la capacidad calorífica, $\left(\partial C_{P} / \partial P\right)_{T}$.

En el caso de una mezcla binaria de gases diluidos, el segundo coeficiente del virial es:

$$
B(T, x)=(1-x)^{2} \cdot B_{11}(T)+2 \cdot x \cdot(1-x) \cdot B_{12}(T)+x^{2} \cdot B_{22}(T)
$$

de manera que cada $B_{\mathrm{ij}}$ se puede medir y está relacionado con su correspondiente $w_{\mathrm{ij}}$ mediante una relación análoga a la (4.2). La entalpía de mezcla para este sistema es $[4]:$

$$
H_{\mathrm{m}}^{\mathrm{E}}=x \cdot(1-x) \cdot\left(\delta_{12}-T \cdot \mathrm{d} \delta_{12} / \mathrm{d} T\right) \cdot P
$$

donde $\delta_{12}=2 \cdot B_{12}-B_{11}-B_{22}$. Así, a partir de los datos de entalpías de exceso de mezclas de gases diluidos y de los datos $P V T$ de los líquidos puros se puede calcular $B_{12}$ el cual está relacionado con el potencial entre pares de moléculas distintas $w_{12}$. La ecuación (4.4) se puede rescribir como:

$$
H_{\mathrm{m}}^{\mathrm{E}}=\Delta H_{1-1}+\Delta H_{2-2}+\Delta H_{1-2}
$$

donde $\Delta H_{\mathrm{i}-\mathrm{i}}$ es la contribución (positiva) a la entalpía de exceso debida a la ruptura de interacciones entre pares de moléculas iguales del tipo i, y $\Delta H_{\mathrm{i}-\mathrm{j}}$ es la contribución (negativa) a la entalpía de exceso debida a la formación de interacciones entre pares de moléculas diferentes.

Las propiedades de los gases densos, los líquidos, y sus mezclas, también serán función de los potenciales de interacción entre moléculas pero de una manera más complicada que para los gases diluidos, ya que deben tenerse en cuenta las interacciones entre tres, cuatro,... moléculas. No obstante, diversos autores [5]-[10] han venido utilizando una ecuación similar a la (4.5):

$$
H_{\mathrm{m}}^{\mathrm{E}}=\Delta H_{1-1}+\Delta H_{2-2}+\Delta H_{1-2}+\Delta H_{s t r}
$$

siendo en este caso $\Delta H_{\mathrm{i}-\mathrm{i}}$ la contribución (positiva) a la entalpía de exceso debida a la ruptura de interacciones entre dos, tres,... moléculas del tipo i; $\Delta H_{1-2}$ la contribución 
(negativa) a la entalpía de exceso debido a la formación de interacciones entre dos, tres,... moléculas de las sustancias químicas 1 y 2 . Si alguno de los dos componentes en estado puro está muy estructurado, como ocurre en el caso de los alcoholes, el agua, las aminas primarias, etc., la entrada del otro componente produce efectos energéticos (exotérmicos o endotérmicos, dependiendo de cada caso particular) debidos a la reorganización del primero en torno a las moléculas del segundo; por ello, a la ecuación (4.5) se le ha añadido el término $\Delta H_{\text {str }}$ que da cuenta de estos cambios estructurales. Por lo tanto, $H_{\mathrm{m}}^{\mathrm{E}}$ da cuenta no solamente de los efectos interaccionales (energéticos), sino también de los estructurales (entrópicos). Para restringirse solamente a los efectos energéticos es más oportuno considerar $U_{m, V}^{\mathrm{E}}$ obtenida a partir de la ecuación (2.203).

Estos resultados se pueden emplear en la región de dilución infinita (baja concentración de uno de los componentes, por ejemplo el componente 1). En dicha región, cada molécula de soluto (1) está completamente rodeada por moléculas del disolvente (2), de manera que las interacciones 1 - 1 han desaparecido. Los efectos energéticos debidos a cambios estructurales en el disolvente se pueden despreciar también. En particular, en el caso de las mezclas de 1-alcohol (1) + n-alcano o cicloalcano (2) Van Ness [11], Woycicka [12] y Stokes [13] demostraron que $H_{\mathrm{m}, 1}^{\mathrm{E}, \infty}$ proporciona el valor de la energía de la interacción 1-1. Este método ha sido ampliado por Letcher \& Bricknell [9] para cualquier componente asociado, de modo que en una mezcla componente (1) + componente (2) la energía de interacción 1-2 viene dada por:

$$
\Delta H_{1-2}\left(x_{1}=0\right)=H_{m, 1}^{E, \infty}(1+2)-H_{m, 1}^{E, \infty}(1+\text { alcano })-H_{m, 1}^{E, \infty}(2+\text { alcano })
$$

en donde, la molécula del tipo 1 se encuentra rodeada por las moléculas del disolvente (2). Es decir, $\Delta H_{1-2}\left(x_{1}=0\right)$ no da cuenta de la fuerza de interacción de pares de moléculas, sino de la molécula 1 con un medio homogéneo formado por varias moléculas del tipo 2. En el estudio de la otra zona de dilución $\left(x_{2}=0\right)$ se obtendría la fuerza de interacción de una molécula del tipo 2 con un medio homogéneo de varias moléculas del tipo 1.

Para que esta estimación sea correcta, los valores de las entalpías molares parciales deben obtenerse midiéndolas directamente, puesto que si se calculan a partir de las entalpías de mezcla (la cual se ajusta a la ecuación R-K (2.208)) vía las ecuaciones (2.121), (2.122) se tiene una grave pérdida de precisión inherente al proceso de diferenciación [14]. Además, los valores calculados de $H_{\mathrm{m}, 1}^{\mathrm{E}, \infty}$ son muy susceptibles a los valores de los parámetros de la ecuación R-K, lo que implica que la estimación de $H_{\mathrm{m}, 1}^{\mathrm{E}, \infty}$ a partir de datos de las entalpías de mezcla en intervalos de concentraciones medias no es fiable. De hecho, las medidas particularmente minuciosas de algunas mezclas con 1-hexanol a dilución infinita reflejan este hecho [15].

El estudio de la dependencia con la temperatura de $C_{P}^{\mathrm{E}}=\left(\partial H_{\mathrm{m}}^{\mathrm{E}} / \partial T\right)_{P}$ también es importante. Es una fuente potencialmente rica de información acerca de la formación de complejos en mezclas líquidas [1] por enlace puente de hidrógeno, complejos de transferencia de carga o interacciones dipolo-dipolo. 
La dependencia con la presión:

$$
\left(\frac{\partial H_{\mathrm{m}}^{\mathrm{E}}}{\partial P}\right)_{T}=V_{\mathrm{m}}^{\mathrm{E}}-T \cdot\left(\frac{\partial V_{\mathrm{m}}^{\mathrm{E}}}{\partial T}\right)_{P}=V_{\mathrm{m}}^{\mathrm{E}} \cdot\left(1-T \cdot \alpha_{P}\right)
$$

también es una cantidad interesante. Además, la ecuación (4.8) sirve como test de consistencia de los resultados una vez que se tienen datos de $H_{\mathrm{m}}^{\mathrm{E}}$ a diferentes presiones y de $V_{\mathrm{m}}^{\mathrm{E}}$ a diferentes temperaturas [16]-[18].

\subsection{TÉCNICAS EXPERIMENTALES PARA LA MEDIDA DE LA ENTALPÍA DE EXCESO}

En principio, al igual que en el caso de las medidas de los volúmenes de exceso, los valores de $H_{\mathrm{m}}^{\mathrm{E}}$ se pueden obtener mediante dos métodos diferenciados:

a) Indirectos. La entalpía de exceso se puede calcular a partir de datos de la función de Gibbs de exceso.

b) Directos. La entalpía de exceso de una mezcla a presión constante es igual al calor producido o absorbido por el sistema durante el proceso de mezcla (calor de mezcla). Este calor se puede controlar mediante algún elemento eléctrico, de manera que si la caída de potencial a través de dicho elemento es $V$ y la corriente que circula por él es $i$, el calor dado al sistema en un intervalo de tiempo $t$ es $V \cdot i \cdot t$. Como $V, i$ y $t$ son mensurables, puede calcularse el calor de mezcla $H_{\mathrm{m}}^{\mathrm{E}}$.

\subsubsection{Métodos indirectos}

En principio, los valores de las entalpías de exceso se pueden obtener a partir de la ecuación de Gibbs-Helmholtz (2.189):

$$
H_{m}^{\mathrm{E}}=G_{m}^{\mathrm{E}}-T \cdot\left(\frac{\partial G_{m}^{\mathrm{E}}}{\partial T}\right)_{P, n_{1}, \ldots n_{n}}
$$

a partir de los valores experimentales de $G_{m}^{\mathrm{E}}$ obtenidos en experimentos de equilibrio líquido-vapor a varias temperaturas [19]-[21]. Sin embargo, los valores así obtenidos de $H_{\mathrm{m}}^{\mathrm{E}}$ son notablemente inapropiados incluso en el caso de medidas de equilibrio líquido-vapor de la más alta precisión, principalmente debido a dos factores [22]:

a) El pequeño intervalo de temperaturas en el que se hacen los experimentos de equilibrio líquido-vapor. Raramente la medida de $G_{m}^{\mathrm{E}}$ se hace en un intervalo mayor del $15 \%$ de la temperatura absoluta, lo que es debido a dificultades técnicas y de diseño del aparato. Por ejemplo [23], si $G_{m}^{\mathrm{E}}$ se ha determinado con un error de $2 \%$ en el rango de temperatura comprendido entre $T$ y $1.1 \cdot T$, al utilizar la ecuación (2.189) para calcular $H_{\mathrm{m}}^{\mathrm{E}}$ a $1.05 \cdot T$, se cometerá un error en su determinación igual al $15 \%$, simplemente por el hecho de tener que derivar $G_{m}^{\mathrm{E}}$, pues como resultado de la teoría de de errores, cada diferenciación conduce a una pérdida de exactitud de un orden de magnitud [24]. De hecho, si se vuelve ha derivar para obtener $C_{P}^{\mathrm{E}}$, el valor obtenido no podría, ni siquiera, tener el signo correcto. 
b) La necesidad de hacer correcciones en la fase de vapor por la desviación de su comportamiento respecto el gas ideal, siendo la corrección mayor cuanto mayor sea la temperatura de trabajo.

\subsubsection{Métodos directos}

Las razones expuestas no hacen sino enfatizar la importancia de la determinación directa de las entalpías de exceso, y es aquí donde entra en juego la calorimetría.

Existen numerosas revisiones dedicadas a los principios básicos de la calorimetría, sus diferentes métodos, y aplicaciones. Por ejemplo, en el libro de Skinner [25] y la serie de libros "Experimental Thermodynamics" [26]-[29] se dan los métodos clásicos para la determinación de las capacidades caloríficas de sustancias en fase gaseosa, líquida y sólida, calorimetría de mezclas de líquidos reaccionantes y no reaccionantes, calorimetría de combustión, etc. Ranzdio [30] hace una amplia selección de los instrumentos más novedosos, hasta 2002, dando numerosas aplicaciones; Brown et al. [31] analizan la calorimetría de absorción en superficies de cristales simples. Korchagina [32] hace una notable discusión sobre las tendencias actuales en el desarrollo de la calorimetría de combustión. Rogez [33] presenta los desarrollos recientes en calorimetría de altas temperaturas, con aplicaciones a la industria metalúrgica. Renuncio et al. [34] hacen un exhaustivo estudio sobre las técnicas de calorimetría en las regiones crítica y supercrítica desarrolladas hasta 1999. Gmelin [35] presenta los progresos recientes en calorimetría de bajas temperaturas. Wadsö [36] presenta un resumen de algunos problemas bastante actuales en calorimetría isoterma.

Las técnicas calorimétricas han ido evolucionando de modo que puede trabajarse con gases, líquidos, polímeros y sus mezclas, para medir capacidades caloríficas y calores de mezcla, en amplios intervalos de presión y temperatura. Los resultados se han utilizado sobre todo para el estudio de transiciones de fase [37], de sistemas gaseosos, de absorción de gases en líquidos, de mezclas de no electrolitos, de sistemas biológicos [38] y farmacéuticos [39], investigaciones acerca del origen del sabor en los whiskies [40], catálisis [41], etc.

\subsubsection{Clasificación de las diferentes técnicas calorimétricas}

Todo calorímetro consiste en esencia en un recipiente calorimétrico donde se produce el fenómeno a estudiar, y un sistema de detección que permite comparar los efectos térmicos ligados a los procesos que intervienen durante el calibrado del aparato y la medida propiamente dicha.

Se han hecho numerosos intentos para organizar en grupos o clases de características particulares a los diferentes calorímetros existentes [26]-[30], [42]-[46]. Sin embargo, a veces resulta complicado o incluso imposible encuadrar alguno de ellos dentro de un determinado grupo en un sistema de clasificación sencillo, ya que el significado de los términos que se utilizan para definir un calorímetro suele ser vago, además de que los criterios utilizados para distinguir los diferentes tipos de calorímetros son extremadamente numerosos y suelen dar lugar a clases que se solapan. Por ejemplo, los calorímetros de Hielo de Lavoisier [47] o de Bunsen [48], típicamente considerados calorímetros isotermos, algunas veces se han clasificado como adiabáticos, no sin la apropiada justificación [49], [50]. Otro ejemplo lo podemos encontrar en el microcalorímetro Tian-Calvet, el cual puede clasificarse como isotermo, cuasi-isotermo, de compensación de potencia, de barrido diferencial (cuando se programan rampas en 
temperatura), de conducción térmica, etc. [44]. Por otra parte, el elevadísimo número de artículos que se pueden encontrar en la bibliografía con las palabras "calorimeter" o "calorimetry" en su título refleja la gran diversidad de las técnicas calorimétricas existentes, lo que no hace sino agravar el problema. Concretamente, a día de 25 de noviembre de 2013, se pueden encontrar en la ISI WEB of SCIENCE 17879 artículos con alguna de estas dos palabras en su título. La revisión de todos ellos para su posterior clasificación se antoja imposible. Por todo esto resulta obvio el que cualquier sistema de clasificación que quiera cubrir el campo entero de la calorimetría tienda a ser muy sotisficado y bastante poco práctico para su utilización. Cuando se utilizan los principios termodinámicos de intercambio de calor o la manera en cómo se forma la señal energética como base para una posible clasificación, un gran número de calorímetros estarán caracterizados satisfactoriamente por los grupos de dicho sistema de clasificación, mientras que muchos otros necesitarán explicaciones adicionales. Por lo tanto, se debe tener presente que cualquier sistema de clasificación de las diferentes técnicas calorimétricas no es definitivo. A pesar de todo, una clasificación resulta útil para mostrar los principios básicos de la calorimetría, y debe basarse en los instrumentos existentes, estando abierto a posibles desarrollos futuros. De entre todas las clasificaciones que se encuentran en la literatura, existen dos que abarcan con la menor ambigüedad la mayoría de los aparatos existentes:

a) Según el método para la manipulación de las mezclas líquidas utilizadas [43].

Existen cuatro métodos generales para manipular las mezclas en un calorímetro:

- Calorimetría de dilución o tritación o TET (Thermometric Enthalpy Titration, [52]). Consiste en ir inyectando un líquido (titrante o reactivo) en el recipiente calorimétrico que se encuentra parcialmente ocupado por el otro o por una mezcla de ambos.

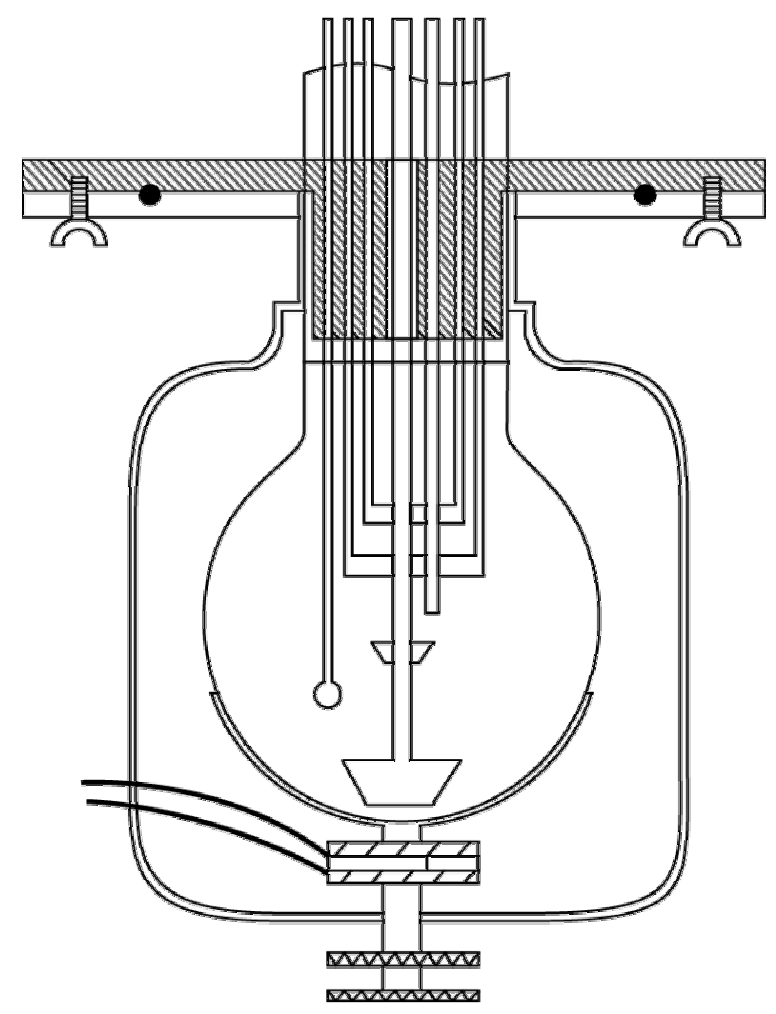

Ilustración 4-1: calorímetro de dilución isoterma de Christiansen et al [60] 
La inyección puede ser continua (dilución continua) o incremental (dilución sucesiva). Para la introducción del líquido se pueden utilizar pistones, buretas termostatadas o jeringuillas automáticas de precisión.

Los efectos térmicos producidos en el interior del recipiente calorimétrico se correlacionan con la cantidad de líquido añadido, o con el tiempo si la dilución se realiza de manera continua y el flujo de entrada del líquido es conocido.

Aunque una revisión de las técnicas de dilución más importantes puede encontrarse en la bibliografía [28], son dignos de mención los calorímetros isotermos de dilución sucesiva de Van Ness et al [53]-[55] y su versión modificada por Stokes et al [56]-[59], y los calorímetros isotermos de dilución continua de Christiansen et al. [60, Ilustración 4-1], [61].

La ventaja más notable de esta técnica es que se necesita muy pocas manipulaciones para cubrir todo el rango de composición, por lo que es muy rápida. Sin embargo, presenta varias desventajas: cada medida depende de la anterior, lo que conlleva un error que se va aumentando medida tras medida; dependiendo del diseño, puede haber fase de vapor que influya negativamente en los resultados; la temperatura de la mezcla y del componente que se va añadiendo puede no ser exactamente la misma, lo que implica un efecto térmico no deseado [62].

- Calorimetría de inyección directa o DIE (Direct Injection Enthalpimetry, [63]). Consiste en la inyección rápida y de una sola vez, de una cierta cantidad de un líquido o reactivo en el recipiente calorimétrico, que, como en el caso anterior, se encuentra parcialmente ocupado por el otro componente, y la subsiguiente medida del flujo térmico o cambio de temperatura causado durante el proceso de mezcla.

La principal ventaja de este tipo de calorímetros es que son baratos en comparación con otros, ya que la composición de las mezclas se determina mediante medios convencionales (pesada, por ejemplo), que no requieren la complejidad de los sistemas de inyección continua, flujo, etc.

La desventaja más notable es la existencia de fase de vapor en el recipiente calorimétrico, la cual exige hacer una corrección. Además son muy lentos.

- Calorimetría de flujo [63], [64]. Se caracteriza por una configuración del recipiente calorimétrico en el que dos o más reactivos fluyen mediante bombeo externo hasta una cámara de mezcla donde se mezclan o reaccionan. Posteriormente recorren todo el recipiente calorimétrico y salen del mismo como un único producto que se almacena en un recipiente exterior al calorímetro que sirve de sumidero del líquido sobrante. El calor producido o absorbido es registrado como un cambio de temperatura en el producto de la mezcla, o como flujo térmico entre el recipiente calorimétrico y una pila calorífica o célula Peltier que lo rodea.

El número de Reynolds para la mayoría de los calorímetros de flujo sugiere que en los tubos rectos el flujo es laminar. Como la mezcla de los líquidos es más efectiva cuando el flujo es turbulento, la mayoría de este tipo de calorímetros incorporan dispositivos para romper el flujo laminar, como alambres metálicos o hilos anudados de Nylon.

Pratt [65] fue el primero en describir un aparato de este tipo. Él medía, con un termómetro de mercurio, el cambio de temperatura debido a la mezcla, y fue capaz de obtener entalpías de dilución de disoluciones acuosas de electrolitos y no electrolitos. 
Un aparato más moderno fue diseñado por Keyes et al. [66], que combinaron la variación de temperatura medida con un termómetro de resistencia de platino, con medidas de la capacidad calorífica para obtener la entalpía de neutralización de la reacción $\mathrm{HCl}(\mathrm{aq})+\mathrm{NaOH}(\mathrm{aq})$. Actualmente esta técnica ha adquirido un elevado grado de desarrollo y el número de aplicaciones, especialmente en biología y química orgánica, ha crecido considerablemente. Los diseños modernos se han dirigido a cubrir un amplio rango de presión y temperatura. Como ejemplo de estos se pueden citar los calorímetros de flujo de Rose \& Storvick [67], Monk \& Wadsö [68], McGlashan \& Stoeckli [69], Christensen et al. [70]-[73] (en su primera versión, para temperaturas desde $273.15 \mathrm{~K}$ hasta $343.15 \mathrm{~K}$ y presiones hasta $40 \mathrm{MPa}$; en la versión de 1987 llegaba hasta temperaturas de trabajo de $773 \mathrm{~K}$ y presiones de $40.5 \mathrm{MPa}$ ), de Picker et al [74][76], de Wormald et al (para temperaturas hasta $698 \mathrm{~K}$ y presiones hasta $15 \mathrm{MPa}$ [77]), de Gopal et al [78] (para gases licuados a temperaturas del nitrógeno líquido), Guan et al [79] y Oakes et al [80], de Castro Gómez (para temperaturas entre $200 \mathrm{~K}$ y $500 \mathrm{~K}$ y presiones hasta $20 \mathrm{MPa}[81])$, etc.

Las principales ventajas son: ausencia de la fase vapor; tiempos de respuesta muy cortos, y como consecuencia, rapidez; son los únicos que por el momento permiten trabajar en la zona de altas temperaturas.

Se debe tener especial cuidado con la aparición de burbujas en los conductos, las cuales causan desequilibrios en el caudal, impidiendo la correcta medición del efecto en estudio. Además, si los líquidos son muy viscosos, existen problemas de circulación y mezcla, y el rozamiento con las paredes de los conductos produce efectos térmicos residuales. Otra fuente de error proviene de la falta de bombas que produzcan un flujo lo suficientemente estable.

- Calorimetría de conjunto (batch). En este tipo de calorímetros, no se introduce ninguna masa externa en el recipiente calorimétrico en el transcurso de la medición.

Una configuración típica que se encuentra en este tipo de aparatos consiste en una cápsula de vidrio que contiene uno de los dos líquidos, e inmersa en el otro, el cual ocupa el resto del recipiente calorimétrico. Mediante un mecanismo adecuado la cápsula se rompe, y se produce la mezcla de ambos líquidos.

Otra configuración bastante común consiste en construir el recipiente calorimétrico de manera que los líquidos están separados por mercurio. La separación se puede anular, por ejemplo, por rotación del recipiente, lo que permite la mezcla de los componentes.

Este tipo de aparatos presenta una serie de ventajas: requieren pequeñas cantidades de sustancia para llevar a cabo la medida; no necesitan corrección por fase de vapor, ya que ésta no existe; la agitación continuada en el interior del recipiente calorimétrico asegura la completa mezcla de los líquidos, por lo que se pueden estudiar sistemas que presenten zonas de inmiscibilidad.

Dependiendo de la elección de un diseño u otro, estos dispositivos presentan varias desventajas, siendo las más comunes: el aparato debe reensamblarse después de cada operación ya que normalmente el medio de separación de ambos líquidos se rompe para realizar la mezcla; en algunos casos, los líquidos se separan mediante mercurio, que puede reaccionar con algunos líquidos, originando así la aparición de sustancias no deseadas; son lentos; el elemento agitador puede producir calentamiento, siendo necesaria una corrección. 
En la referencia [28 pp 198] puede consultarse una amplia descripción de esta técnica y de los diseños más importantes enfocados a trabajar con bajas temperaturas. Quizás el diseño más memorable sea el calorímetro isotermo de Larkin \& McGlashan [82], utilizado por diversos autores entre los que se encuentran Díaz Peña y Martín [83].

b) Según la magnitud del flujo térmico en su interior.

Esta segunda clasificación se basa esencialmente en el criterio utilizado por Calvet y Prat [84] y aceptado por otros autores [42], [51], [85] de clasificar a los diferentes calorímetros según la magnitud del flujo térmico $\delta Q / \mathrm{d} t$ en su interior. Para estos autores, un calorímetro está formado por tres elementos: un contenedor o recipiente calorimétrico, una cavidad y el entorno. El recipiente calorimétrico, en el que ocurre el efecto térmico a estudiar, está situado dentro de la cavidad, la cual está llena de intercambiadores térmicos entre aquel y el entorno. Se llama recinto interno a las paredes del recipiente calorimétrico, y recinto externo a las paredes del entorno.

El flujo térmico producido dentro del calorímetro viene dado por la ley de Newton del enfriamiento:

$$
\frac{\delta Q}{\mathrm{~d} t}=p \cdot\left(T_{i}-T_{e}\right)
$$

donde $T_{\mathrm{i}}$ y $T_{\mathrm{e}}$ son las temperaturas del recinto interno y externo respectivamente, y $p$ es el coeficiente de transferencia de calor. Esta ecuación es aproximada y tiene aceptable exactitud cuando las temperaturas que la definen son uniformes en todo el recinto correspondiente y su diferencia es pequeña.

La clasificación se basa en los diferentes valores que puede tomar $p$. Así se pueden distinguir los siguientes tipos de calorímetro:

- Valores nulos o muy pequeños de $p$ : calorímetro adiabático. En estos calorímetros, el flujo térmico es despreciable y no hay pérdidas de calor. En teoría, son muy similares a los calorímetros isoperiféricos, con la diferencia de que en los adiabáticos, el "escudo" isotermo que rodea al recipiente calorimétrico se mantiene a la misma temperatura que éste. Así, cualquier gradiente de temperatura entre el recipiente calorimétrico y su entorno es eliminado.

Este fue el método utilizado por primera vez por Person [86] en su calorímetro "with cancelled heat losses", y más tarde por otros [87], [88]. Numerosos diseños diferentes de calorímetros adiabáticos se pueden encontrar en la bibliografía [44]. No obstante, es conveniente aclarar que los calorímetros adiabáticos con buena precisión son muy sotisficados y complejos, habiendo en todo el mundo unos pocos repartidos en los laboratorios más importantes.

Los calorímetros adiabáticos permiten la determinación muy precisa del comportamiento en diferentes temperaturas de los fenómenos en estudio, sobre todo a bajas temperaturas, donde la isotermia es difícil de conseguir.

La principal desventaja se debe a las fugas térmicas, que dependen de las capacidades caloríficas de los materiales con los que está construido el aparato, y son mayores cuanto mayor sea la temperatura.

- Valores grandes de $p$ : calorímetro isotermo. Todo el calor producido en el calorímetro es transferido rápidamente al entorno, de manera que los recintos interno y 
externo se mantienen a temperatura constante, que se puede conseguir, bien mediante el contacto del conjunto con alguna sustancia que está llevando a cabo un cambio de fase (calorímetros de Lavoisier [47], Bunsen [48], Dewar [89] ó Jessup [90]), o bien mediante la incorporación un sistema eléctrico de compensación de potencia formado por células Peltier y calentadores de potencia variable (calorímetros de Van Ness et al de dilución [53]-[55], de dilución de Stokes et al [56]-[59], de Christensen et al. de dilución [60][61] y de flujo que cubren amplios rangos de presión y temperatura [70][73] y de otros muchos autores [91]-[102]). La célula Peltier refrigera el recipiente a ritmo constante mientras que el calentador se acciona adecuadamente y de forma intermitente, para mantener el recipiente y su contenido a temperatura constante. Cuando ocurre un proceso exotérmico, se produce calor, lvego se necesita una menor potencia de calentamiento para equilibrar el enfriamiento continuo de la célula Peltier. Por el contrario, cuando ocurre un proceso endotérmico, se necesita mayor potencia de calentamiento.

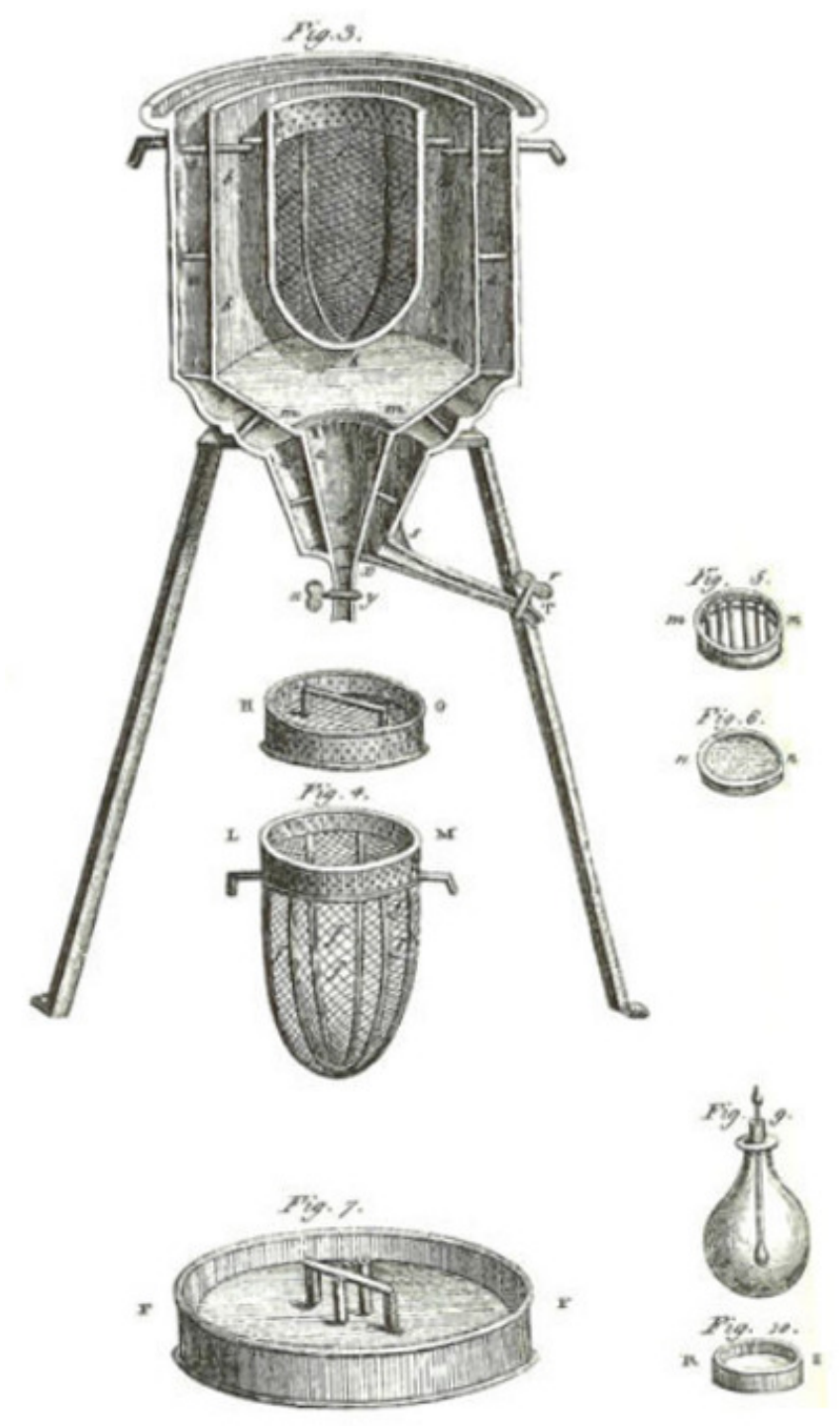

Ilustración 4-2: calorímetro de Lavoissier tal y como viene dibujado en la referencia [47]

La principal ventaja de este tipo de calorímetros es que no son necesarias correcciones por fugas térmicas (dependientes de la capacidad calorífica) pues la temperatura no cambia. 
- Valores moderados de $p$. En este tipo de calorímetros, el intercambio de calor entre el recipiente calorimétrico y el entorno está favorecido activamente por medios físicos [25 p 201], y el flujo de calor entre los recintos externo e interno se controla en todo momento. Dentro de este grupo, se pueden definir los siguientes subgrupos:

Calorímetros de conducción térmica. También llamados calorímetros de flujo de calor. Desarrollados originalmente por Calvet [84] y adoptados por un gran número de investigadores [68], [103]-[121], se caracterizan por poseer un recipiente calorimétrico en contacto térmico con un disipador de calor que lo rodea. El contacto térmico se consigue situando termopares o cualquier dispositivo termoeléctrico, conectados en serie, entre ambos elementos. Al conjunto disipador + elementos termoeléctricos se le llama termopila. Cuando ocurre cualquier proceso dentro del recipiente calorimétrico que conlleva la absorción o producción de calor, se produce un gradiente de temperatura entre el recipiente y el disipador gracias al cual aparece un pequeño voltaje en cada elemento termoeléctrico, el cual, por la conexión en serie de todos ellos, se suma y en total adquiere un valor significativo que se puede monitorizar y correlacionar, gracias a una calibración previa, con el calor producido o absorbido en el recipiente calorimétrico.

A veces a este tipo de aparatos se les da el nombre de calorímetros de conducción isotermos. Esta designación es inapropiada ya que, si bien es verdad que los cambios de temperatura en el recipiente calorimétrico se minimizan debido a la conducción térmica a través de la termopila, por lo que el término cuasi-isotermo podría ser adecuado, sin embargo si estos calorímetros operasen de modo isotermo, no podría haber ningún gradiente de temperatura que causase la aparición de voltaje en la termopila, no pudiéndose medir la producción o absorción de calor en el proceso que ocurre dentro del recipiente calorimétrico.

Normalmente se utilizan asegurando la constancia de la temperatura del recinto exterior y haciendo que el efecto térmico generado en el proceso estudiado sea transmitido por conducción desde el recipiente calorimétrico hasta el recinto exterior, evacuándose así el calor producido y midiendo su magnitud durante dicho proceso.

El aparato con el que se han obtenido las entalpías de exceso que se muestran a lo largo de este capítulo corresponde a este grupo, razón por la cual la descripción del mismo se detallará unas páginas más abajo.

La principal ventaja es que la ausencia de líquidos intercambiadores que reciban el calor desarrollado en la cámara de mezcla hace que las medidas sean más directas y tengan menor error sistemático.

Calorímetros isoperiféricos. A veces llamados calorímetros con "escudo" isotermo, se caracterizan por poseer un recipiente calorimétrico tipo Dewar con un entorno a temperatura constante, que consiste generalmente en agua termostatada. El recipiente calorimétrico se diseña lo más adiabático posible, de forma que se minimice el flujo térmico con su entorno. La medida de la energía puesta en juego se determina mediante la variación de temperatura en el mismo, y se correlaciona con la capacidad calorífica del recipiente y de su contenido. En este tipo de calorímetros, se deben realizar correcciones por fugas térmicas que surgen de su no total adiabatismo, y por efectos térmicos no debidos al proceso estudiado. 
Los calorímetros de este tipo más conocidos son los de Favre \& Silbermann [122], Berthelot ([123], Ilustración 4-3) Thomsen [124], Nernst [125], Armstrong [126], Abramowitz et al. [127], Eckman \& Rossini [128], Battino \& Marsh [129], etc [44].

Una ventaja obvia de este método es la simplicidad de la funcionalidad del escudo térmico, el cual puede controlarse manual o automáticamente. Otra ventaja es la relativa independencia con las condiciones variables externas que actúan sobre el escudo, ya que éste se mantiene isotermo.

Por el contrario, las correcciones por fugas térmicas pueden llegar a ser importantes.

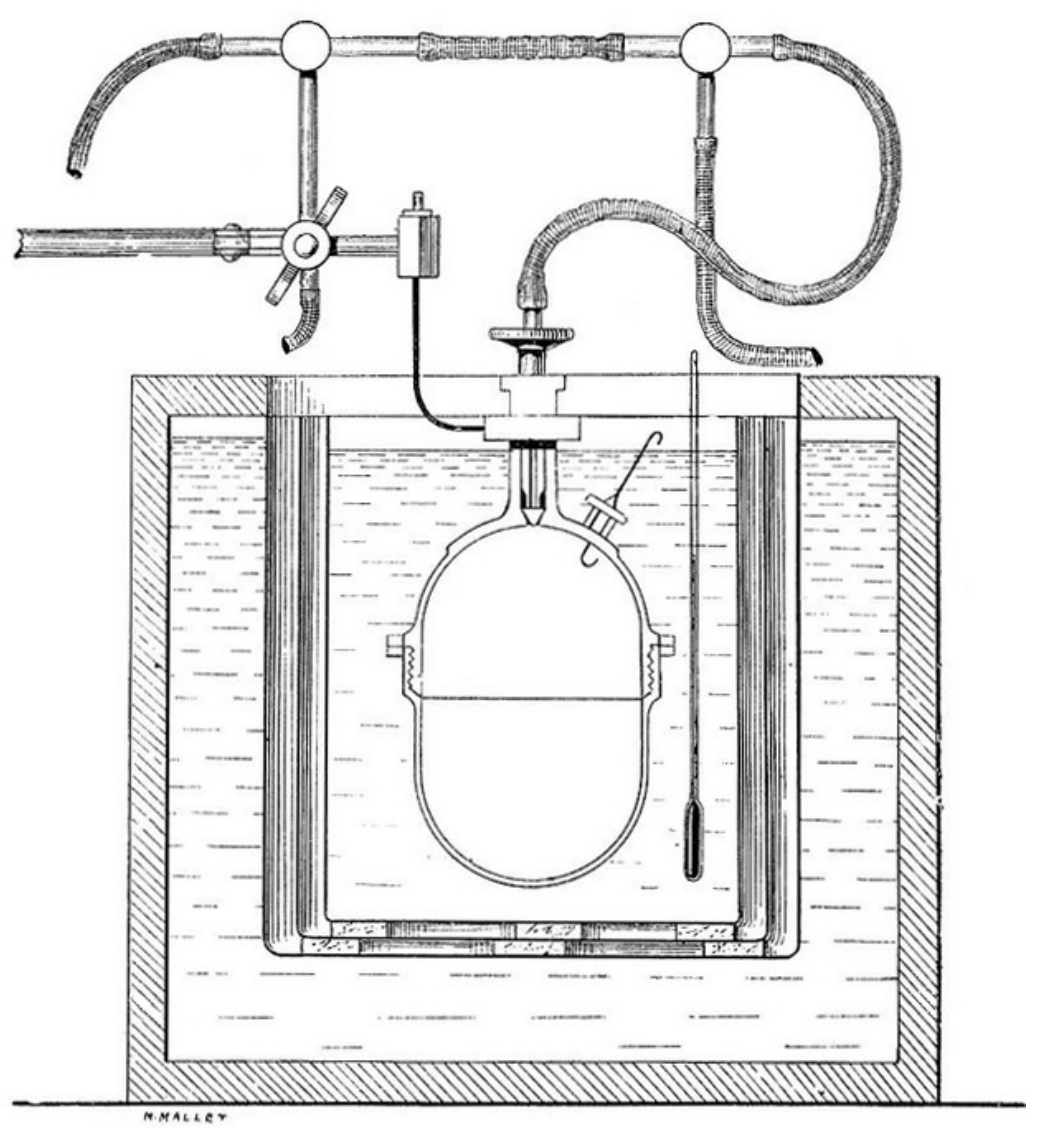

Ilustración 4-3: calorímetro de Berthelot tal y como viene dibujado en la referencia [123]

El gran número de los sistemas de clasificación de las diferentes técnicas calorimétricas disponibles en la literatura hace que, además de los ya mencionados, sea conveniente presentar otros dos de ellos [46]:

c) Según la técnica empleada para la medida de los cambios de entalpía y de la potencia calorífica desarrollada que se produce en el recipiente calorimétrico durante el proceso a estudiar.

Esta clasificación distingue entre dos grupos de calorímetros bien diferenciados, con varios subgrupos:

- Modo de funcionamiento estático: isotermos, isoperiféricos, adiabáticos.

- Modo de funcionamiento dinámico: barrido en temperatura (differential scanning calorimetry, DSC [63], [130], [131], barrido en temperatura del entorno, barrido en presión [132], barrido en el volumen, calorimetría fotoacústica [30, pp 176], 
calorimetría AC (la capacidad calorífica se mide con flujos de calor oscilantes de frecuencia variable [133]), barrido modulado en temperatura [134], [135], etc.

d) Según el principio de construcción del calorímetro:

- Calorímetros simples. Solamente hay un elemento calorimétrico, por ello son muy sensibles a las perturbaciones externas, razón por la que su sistema regulador de temperatura debe ser muy sotisficado.

- Calorímetros diferenciales. Propuesto por Joule en 1845, suele utilizarse en calorímetros de conducción térmica sobre todo si se necesita alta precisión en procesos lentos. En este tipo de aparatos se dispone simétricamente de dos recipientes calorimétricos iguales (uno de referencia y otro en el que ocurre el proceso a estudiar), de manera que ambos se ven igualmente afectados por las perturbaciones externas. Los dos recipientes se encuentran conectados en oposición, de modo que la señal de salida de ambos recipientes es restada una a la otra y así los efectos térmicos debidos a las perturbaciones son eliminados. Con este montaje, se consigue disminuir el error debido a fugas de calor, cuando los calores medidos son pequeños, por lo que se aplica comúnmente en microcalorimetría. Como ejemplos representativos, se pueden citar el calorímetro de Tian-Calvet [84], utilizado por el grupo de investigación del G.E.T.E.F., que será descrito en la sección 4.1.1.3, el de Grolier [136] basado en un calorímetro de flujo capaz de cubrir un rango de temperaturas hasta $573 \mathrm{~K}$ y presiones hasta 30 $\mathrm{MPa}$, etc.

Las principales desventajas se encuentran en el esfuerzo extra que requiere la construcción de un segundo recipiente calorimétrico y en la necesidad de comprobar que ambos recipientes tienen idénticas características térmicas.

\subsubsection{Calorímetros más usuales para la determinación $H_{\mathrm{m}}^{\mathrm{E}}$}

Antes de comenzar con el estudio del comportamiento calorimétrico de un sistema dado, deben considerarse ciertos aspectos relativos a la elección de uno u otro tipo de calorímetro. No obstante, la gran variedad de modelos disponibles no permite discernir si una alternativa será mejor que otra, aunque si se pueden establecer ciertos criterios para su elección:

a) Según la elección del método y diseño del calorímetro. En la sección anterior ya se han enumerado las principales ventajas y desventajas de cada uno de ellos.

- Adiabático, isoperiférico, isotermo de cambio de fase o de compensación eléctrica de potencia, de conducción térmica.

- Montaje simple o diferencial.

- Barrido o no en temperatura y/o presión.

b) Según las características operacionales del calorímetro:

- Estabilidad, nivel de ruido, sensibilidad, resolución, exactitud, precisión,...

- Rango de presión y temperatura de trabajo deseado.

- Comportamiento dinámico: tiempo de respuesta, capacidad calorífica del recipiente calorimétrico, etc.

c) Según el tipo de proceso que se va a estudiar: 
- Combustión, reacción, mezcla, cambio de fase, etc.

d) Propiedades físicas de la muestra y las propiedades térmicas que se van a considerar.

e) Cantidad disponible de la muestra a analizar.

f) Modo de manipulación de los líquidos: dilución, inyección directa, flujo,...

A parte de las consideraciones inherentes a cualquier tipo de medida calorimétrica enumeradas en la anterior lista, en la medida de las entalpías de exceso se debe tener especial cuidado con determinados aspectos, como explica McGlashan [1]:

a) Reducir o incluso eliminar la fase de vapor en el recipiente calorimétrico, ya que la entalpía de vaporización contribuye a un valor erróneo de la entalpía de mezcla. Como se ha dejado entrever en la sección 4.1.1.1, si en el recipiente calorimétrico hay espacio suficiente para la formación de la fase vapor, la entalpía de vaporización puede ocasionar un error sistemático en la determinación de las entalpías de mezcla. En ese caso debe llevarse a cabo una corrección.

b) Tener la certeza de la mezcla completa de los componentes.

c) Asegurarse de que la mezcla se realiza en las condiciones deseadas: $V=$ cte., $P=$ cte., $T=$ cte., etc. Así, por ejemplo, en el calorímetro de Larkin \& McGlashan [82], la cantidad que se mide es $U_{m}^{E}$ y no $H_{m}^{E}$. Si la presión cambia durante el proceso de mezcla, se producirá un error en la medida. Además, aunque ésta sea la misma antes y después de la mezcla, su valor debe conocerse ya que la dependencia de la entalpía con la presión no es nula (véase la ecuación (4.8)).

d) Comprobar la no contaminación de los líquidos en cuestión.

Habida cuenta de todas estas consideraciones, a continuación se citan los principales métodos empleados por varios autores para la determinación de las entalpías de exceso.

a) Numerosos calorímetros han sido diseñados para la medida de entalpías de exceso de gases condensados, a temperaturas lejanas de la ambiente, entre los que nombramos como ejemplo los de tipo batch ideados por Jeener [137], Staveley et al [138], Beenakker et al [139], y el isotermo de flujo de Wormald et al [140]-[142].

b) También se ha producido un vigoroso desarrollo en las técnicas calorimétricas para la determinación de entalpías de mezcla a altas temperaturas de sales y metales fundidos. Por ejemplo, Murgulescu \& Marchidan [143] obtuvieron los resultados para las mezclas de $\mathrm{BrPb}+\mathrm{CIPb}$ a $833.15 \mathrm{~K}$ y $823.15 \mathrm{~K}$, y de $\mathrm{KCl}+\mathrm{KBr}$ a $1083.15 \mathrm{~K}$ y 1163.15 K, con un calorímetro similar al diseñado por Witting et al [144], [145]. Zubkov et al [146] determinan los calores de mezcla a dilución infinita de Níquel puro y circonio + aluminio con un calorímetro isoperiférico.

c) En cuanto a la medida de las entalpías de exceso de mezclas de líquidos no electrolitos, el número de diferentes calorímetros utilizados es muy amplio:

- Entre los calorímetros de dilución sucesiva isotermos, el de Van Ness et al es [53]-[55] el más importante, y en su tiempo representó un antes y un después en la calorimetría de las mezclas líquidas, pues resolvió de un plumazo los problemas que por aquél entonces eran típicos de este campo de investigación: fue el primero en incorporar un refrigerador Peltier junto con un sistema de calefacción para el control de 
la temperatura del recipiente calorimétrico; aseguraba la total y rápida mezcla de los líquidos; con sólo dos manipulaciones podía cubrir el rango completo de concentración, siendo así un método muy rápido; los errores debido a la vaporización durante la mezcla son eliminados ya que no existe espacio para la aparición del vapor. Dos versiones del diseño de Van Ness fueron utilizadas con éxito en Canadá por Benson et al [147], [148] y en Australia por el equipo de Stokes et al [56]-[59], siendo la principal diferencia entre el último y el de Van Ness la manera en cómo se evitaba la aparición de la fase vapor. Mientras que en el calorímetro de Van Ness, la mezcla se produce en un recipiente calorimétrico de volumen variable pues está cerrado herméticamente con una tapa con una junta tórica siendo la tapa corrediza, en el primer diseño de Stokes et al [56] la mezcla se realiza en un recipiente de volumen fijo en el que se sitúa el primer componente y el espacio sobrante es ocupado por mercurio (véase la llustración 4-4). Cuando se produce la mezcla, el segundo componente desplaza al mercurio. En diseños posteriores se evitó el uso del mercurio además de por razones de salud, porque es una sustancia que puede reaccionar con algunos líquidos, y que amalgama la mayoría de los metales, lo que encarece la construcción del aparato pues deben utilizarse materiales como acero inoxidable, níquel, tungsteno y nicrom [28 capítulo 7].

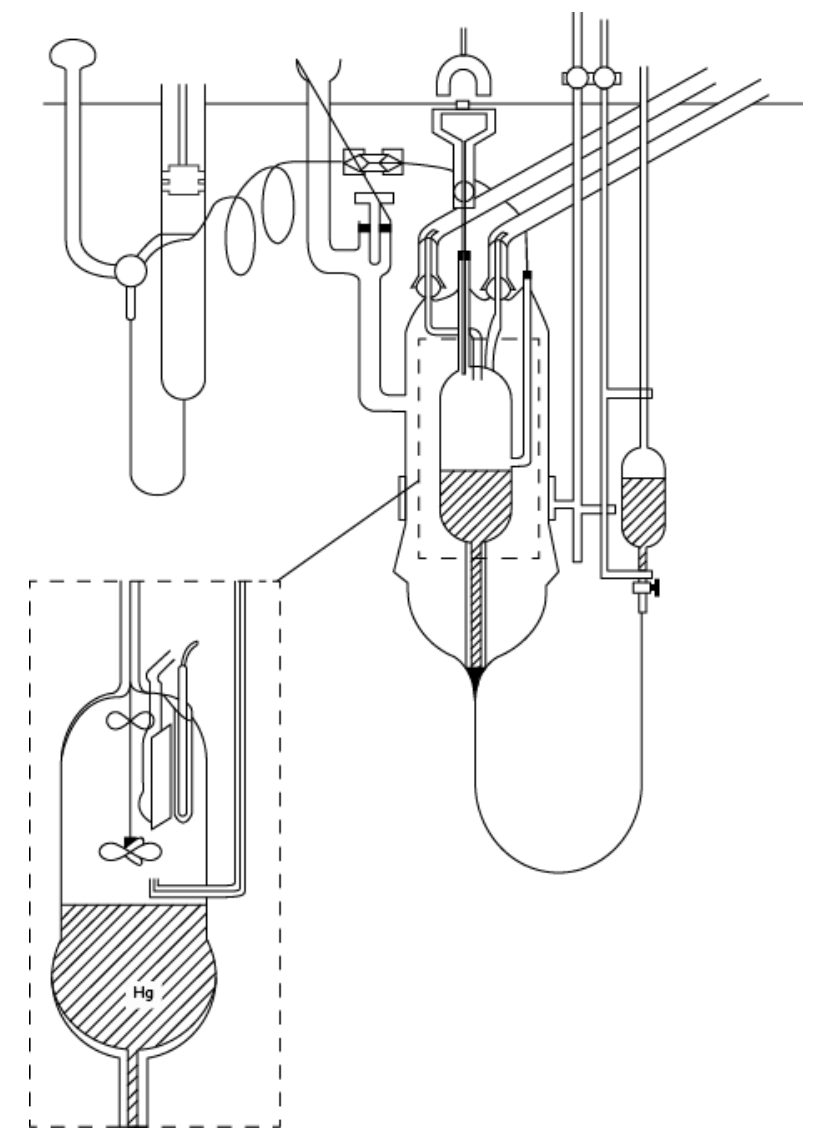

llustración 4-4: calorímetro de desplazamiento isotermo de Stokes et al [56]

- De entre los calorímetros de tipo batch, se pueden citar los adiabáticos de Benson \& Benson [149] y de Bennett \& Benson (véase la referencia [150] y la llustración 4-5), y el isotermo de Larkin \& McGlashan [82]. 


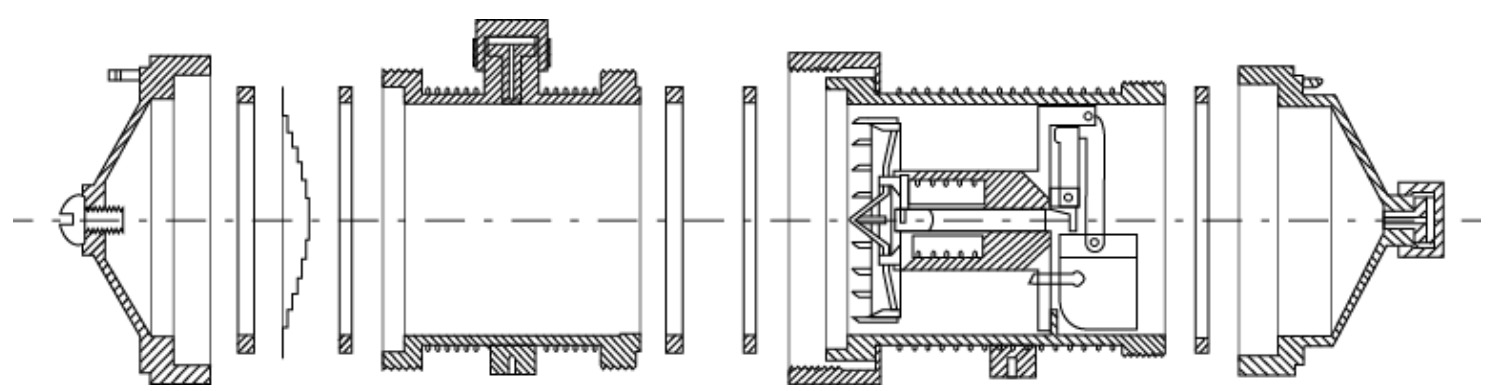

Ilustración 4-5: corte transversal de la celda de mezcla del calorímetro de Bennet \& Benson [150]

- Los calorímetros de dilución continua a veces se han utilizado para determinar entalpías de exceso, no obteniéndose buenos resultados debido a que la presencia de fase de vapor es común en este tipo de aparatos [60], [61]. Una excepción notable es el calorímetro de sobreflujo de Becker et al [151].

- En lo que respecta a los calorímetros de flujo, entre los primeros utilizados para medir entalpías de exceso de mezclas líquidas destacan los de Rose \& Storvick [67] McGlashan \& Stoeckli [69] y Picker [74]-[76] que fue mejorado por Grolier et al [152][155]. Como se ha dicho, por razones técnicas estos son los únicos calorímetros con los que se puede trabajar en la zonas de temperatura y/o presión elevados; por mencionar algunos ejemplos, citamos los diseños clásicos de Christensen et al [70], [73], y los más actuales de Huemer et al [156] y de Diamali et al [157].

Heintz \& Lichtenhalter [17], Ott et al [158] y Grolier \& Randzio [159] han estudiado el efecto de la presión sobre las propiedades termodinámicas de mezclas binarias. A temperaturas muy por debajo de la crítica de alguno de los dos componentes, la dependencia con la presión de $H_{\mathrm{m}}^{\mathrm{E}}$ es muy pequeña $\left(\partial H_{\mathrm{m}}^{\mathrm{E}} / \partial P\right)_{T}<<1$; sin embargo, a temperaturas cercanas a la crítica de uno o ambos componentes, el efecto de la presión es mayor. Así, para la mezcla ciclohexano $+n$-decano a temperatura ambiente, el máximo de $H_{\mathrm{m}}^{\mathrm{E}}$ varía de 15.3 a $37.4 \mathrm{~J} \cdot \mathrm{mol}^{-1}$ para presiones desde 0.1 hasta $48.5 \mathrm{MPa}$, mientras que el máximo de $H_{\mathrm{m}}^{\mathrm{E}}$ para el sistema etano + etanol a $298.15 \mathrm{~K}$ cambia de

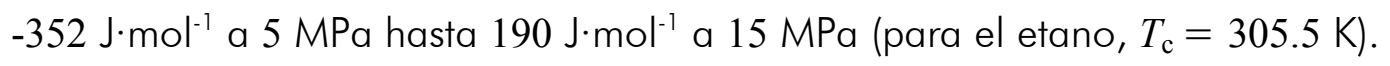

La dependencia de $H_{\mathrm{m}}^{\mathrm{E}}$ con la temperatura, a presión constante, suele ser pequeña aunque en ocasiones como cuando ocurren interacciones específicas tales como enlaces puente de hidrógeno, puede ser grande. Por ejemplo, se ha encontrado que en la mezcla etanol + agua a $5 \mathrm{MPa}, H_{\mathrm{m}}^{\mathrm{E}}$ cambia de $-790 \mathrm{~J} \cdot \mathrm{mol}^{-1}$ a $298.15 \mathrm{~K}^{2}$ hasta 1140 $\mathrm{J} \cdot \mathrm{mol}^{-1}$ a $473.15 \mathrm{~K}$. A temperaturas intermedias, $H_{\mathrm{m}}^{\mathrm{E}}$ tiene forma de $\mathrm{M}$, con dos puntos de corte en el eje de las abscisas, dos mínimos en la región $H_{\mathrm{m}}^{\mathrm{E}}<0$ y un máximo en la zona $H_{\mathrm{m}}^{\mathrm{E}}>0$.

- Los calorímetros de conducción térmica más usuales en la determinación de las entalpías de exceso de mezclas líquidas son los basados en los diseños de Tian \& Calvet [84], Benzinger \& Kitzinger [1 10] y Wadsö [68], éste último utilizado muy a menudo para la determinación de $H_{\mathrm{m}}^{\mathrm{E}}$ de disoluciones acuosas de moléculas con interés biológico. Mientras que los dos últimos son calorímetros de flujo, el Calvet suele utilizar el método de inyección directa para la formación de las mezclas, de la manera en cómo lo ha hecho el autor de esta memoria para la determinación de las entalpías 
de exceso que en este capítulo se presentan. Sin embargo, existen modificaciones que permiten utilizarlo en configuraciones tipo batch [160] y como un calorímetro de flujo [161]-[163], lo que lo convierte en un calorímetro apropiado para ser utilizado en condiciones de alta temperatura y presión [164], [165]. También se ha ampliado a métodos isoperiféricos y de barrido de temperatura [166]. Quizás la manera más comprensible de entender su aceptación por la comunidad investigadora queda plasmada en el éxito que ha tenido su comercialización por la casa SETARAM (antes DAM), Francia, Lyon [167]. Entre sus modelos destacan el microcalorímetro MS 80, que es prácticamente el mismo que con el que se obtuvieron los datos de las entalpías de exceso de esta Tesis Doctoral; el calorímetro de bajas temperaturas BT 2.15, que gracias a su refrigeración por nitrógeno líquido permite alcanzar temperaturas de hasta $77.15 \mathrm{~K}$; el calorímetro de altas temperaturas HT 1000; el calorímetro de flujo C 80, cuyas principales limitaciones son: i) aunque se pueden determinar $H_{\mathrm{m}}^{\mathrm{E}}$ a altas presiones siempre y cuando se disponga del recipiente calorimétrico adecuado, las mezclas deben realizarse a presión atmosférica; ii) no pueden mezclarse líquidos cuyas presiones de vapor sean muy diferentes; iii) aparición de efectos residuales debidos a la fase de vapor. Estas limitaciones han sido superadas por Huemer et al [156] añadiendo una bomba de precisión termostatada y un globo de Viton presurizado con nitrógeno que evita la aparición de vapor; etc.

\subsubsection{Calorímetro Tian-Calvet}

El calorímetro que se ha utilizado para la determinación de las entalpías de exceso que aparecen en este trabajo es del tipo Tian-Calvet [84]. Según los sistemas de clasificación discutidos en la sección 4.1.1.1 es un "calorímetro diferencial de conducción térmica, cuasi-isotermo y de inyección directa". Originalmente, el de Tian era un calorímetro simple con un solo recipiente calorimétrico. Fue construido 7 metros por debajo de un sótano con el objetivo de proporcionar una temperatura exterior estable. Su elemento calorimétrico constaba de una celda de mezcla rodeada de un conjunto de termopares en los que se produce por efecto Seebeck un voltaje debido a los efectos caloríficos que ocurren dentro de la celda. Se supone que el calor generado dentro de la celda es transferido completamente a los termopares, por lo que se ignoran los efectos de conducción desde el elemento calorimétrico y el resto del calorímetro, radiación y convección por el aire en la región contigua a la celda, los cuales han sido recientemente estimados mediante cálculos por el método de los elementos finitos [168]. En torno a 1922 [169] Tian introdujo módulos Peltier para compensar eléctricamente los efectos térmicos producidos en el interior del recipiente calorimétrico. Así, su elemento microcalorimétrico tenía dos pilas termoeléctricas, la que servía para medir el flujo de calor por efecto Seebeck y otra que formaba parte del sistema de control de temperatura y que servía para refrigerar gracias al efecto Peltier. De este modo el calorímetro podía trabajar de manera cuasi-isoterma ya que el calor liberado puede ser compensado por enfriamiento gracias a la termopila Peltier. En 1948 el Catedrático de la Facultad de Ciencias de Marsella y director del Instituto de Microcalorimétric et Thermogénese del C.N.R.S, E. Calvet, modificó el calorímetro de Tian colocando las termopilas en una disposición con simetría radial y realizando un montaje diferencial para minimizar la influencia de las perturbaciones externas sobre las experiencias a realizar [170]. El sistema así creado se hace sensiblemente isotermo. De hecho, la estabilidad de la temperatura del recinto interno (o entorno de los elementos calorimétricos), constituido por un bloque calorimétrico metálico de geometría 
previamente determinada, permite esperar fluctuaciones mínimas (gracias al termostato de recintos múltiples), de forma que si posee estabilidad del orden de $0.001 \mathrm{~K}$, permite detectar efectos de $1 \mu \mathrm{W}$ en el recipiente calorimétrico. Ello le hace muy adecuado para medidas de entalpías de exceso de mezclas líquidas a temperatura constante, que son las de interés en este trabajo.

\section{a) Descripción del microcalorímetro Tian-Calvet}

La construcción del calorímetro Tian-Calvet del laboratorio del grupo de investigación G.E.T.E.F. fue llevada a cabo en la Universidad de Santiago de Compostela por la profesora Dra. M. I. Paz-Andrade*, bajo la petición del grupo de Termodinámica del Departamento de Física Fundamental de la Facultad de Ciencias de la Universidad de Valladolid, que por aquel entonces no poseía ningún equipo experimental que permitiese la determinación de alguna de las propiedades termodinámicas de disoluciones líquidas. Por ello se vio obligado a colaborar con los laboratorios de Marseille y Clermont-Ferrand hasta que la construcción del calorímetro finalizó a principios del año 1978.

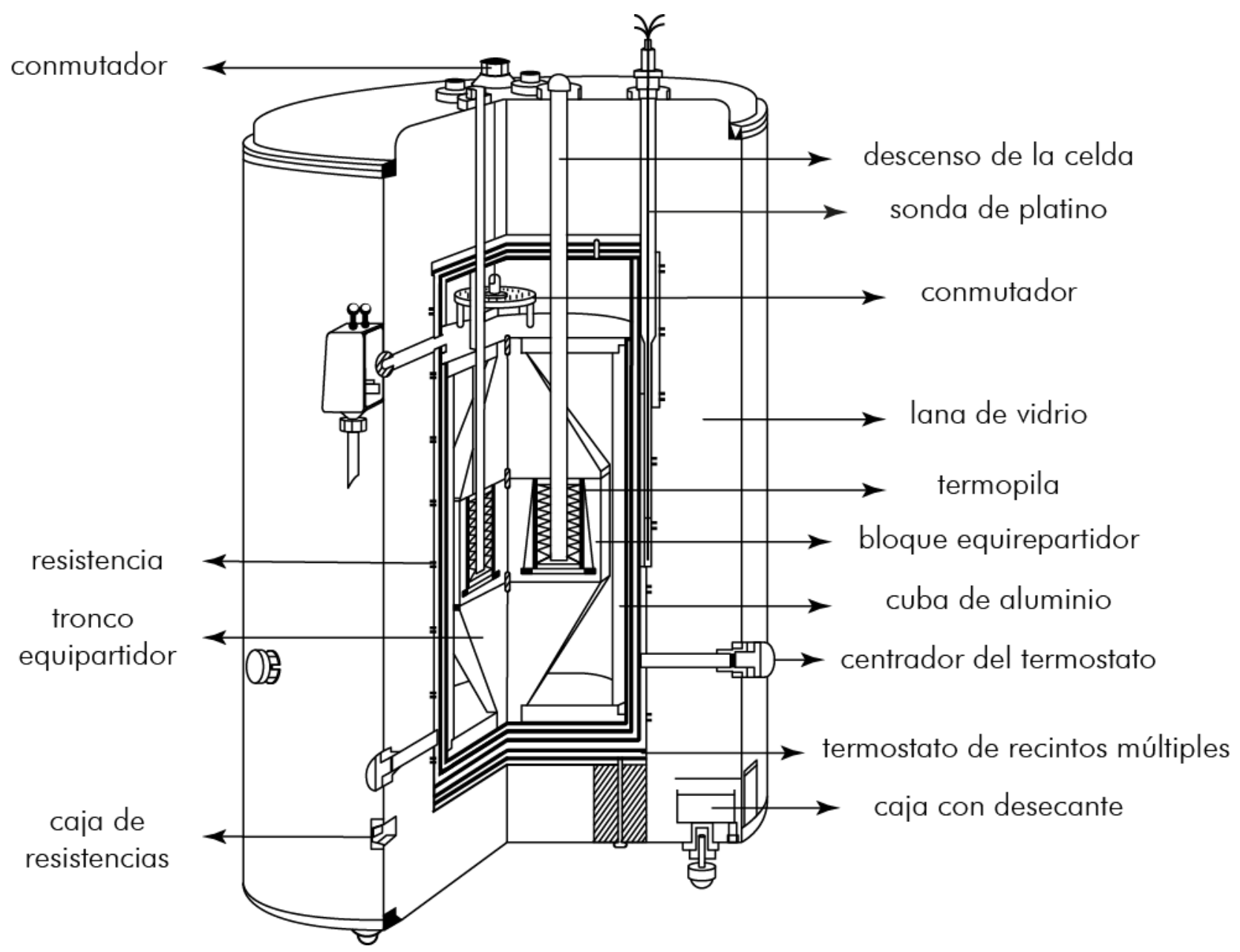

Ilustración 4-6: corte transversal de un calorímetro Tian-Calvet

Una vez acabado, el microcalorímetro fue trasladado al laboratorio de Termodinámica de la Facultad de Ciencias de la Universidad de Valladolid. En ese momento, J. C.

\footnotetext{
* La profesora Paz-Andrade realizó parte de su Tesis doctoral con el Prof. E. Calvet, por lo que disponía de experiencia más que suficiente para poder llevar a cabo dicho cometido.
} 
Cobos se encargó de la puesta a punto y calibración del aparato. Los detalles de la metodología utilizada así como de los resultados obtenidos para cumplir dicho cometido pueden encontrarse en su trabajo de Licenciatura [171]. En posteriores proyectos se informatizó el sistema de adquisición de datos, se mejoraron el sistema de control y de monitorización de temperatura, se mejoró el protocolo de calibración, se diseñaron diferentes recipientes calorimétricos, etc. [172]-[174].

Aunque Cobos [51] describió con total exactitud las diferentes partes del calorímetro Tian-Calvet, el autor de este trabajo considera necesaria la breve exposición de las mismas ya que algunas de ellas, sobre todo aquellas relativas a los periféricos de control, han sufrido diversas modificaciones con el paso de los años.

En la llustración 4-6 se muestra un corte transversal del calorímetro, lo que facilita situar cada una de las piezas que a continuación se detallan, de afuera a dentro:

- Carcasa: el conjunto del calorímetro está contenido en un cilindro exterior de chapa de hierro, con unas ruedas que facilitan su desplazamiento.

- Capa de lana de vidrio: esta capa actúa como aislante térmico.

- Resistencias calefactoras: son dos resistencias de unos $120 \Omega$ aproximadamente cada una, enrolladas helicoidalmente en sentidos opuestos, para evitar efectos inductivos, en torno al termostato de recintos múltiples. Este par de resistencias van al exterior a través de dos cables coaxiales etiquetados como calefacción directa y calefacción controlada.

- Resistencia termométrica de platino: es una resistencia de platino tipo SP 683 (DIN 43760) de la Sociedad Española de Metales Preciosos, cuya resistencia es $100 \Omega$ a $273.15 \mathrm{~K}$ y $138.5 \Omega$ a $373.15 \mathrm{~K}$ y está recubierta por una capa de plástico para evitar su degeneración. Se encuentra situada entre el termostato de recintos múltiples y la capa de lana de vidrio. El sistema de control de temperatura utiliza esta resistencia.

- Termostato de recintos múltiples: está formado por un primer recinto interno de aluminio de $3 \mathrm{~cm}$ de espesor. Rodeando a esta primera capa se disponen de 5 a 10 recintos concéntricos de aluminio separados por una capa de amianto de unos milímetros de espesor, que actúa como aislante térmico, con lo que se consigue una temperatura constante (con fluctuaciones de $0.001 \mathrm{~K}$ ) durante largos periodos de tiempo. Este recinto multicapas tiene la finalidad de distribuir cualquier perturbación térmica que provenga del exterior y de que la temperatura en ambos elementos microcalorimétricos sea la misma.

- Troncoconos equipartidores: los únicos puntos de contacto entre el termostato de recintos múltiples y el interior del calorímetro son las bases menores de un par de troncoconos de aluminio. Con ello se pretende que toda perturbación térmica que haya podido atravesar el termostato de recintos múltiples, solamente pueda alcanzar el interior del calorímetro a través de estos troncoconos.

- Lentes térmicas: situadas en las bases mayores del par de troncoconos se encuentran dos piezas en forma de lente de acero inoxidable, mal conductor térmico comparado con el aluminio. Estas lentes de acero tienen la forma adecuada para funcionar como colimadores de las perturbaciones térmicas que han alcanzado las bases menores de los troncoconos. De esta forma se logra que toda perturbación que 
haya alcanzado el interior del calorímetro sea distribuida uniformemente sobre las bases mayores de los troncoconos.

- Cilindro principal: entre las bases mayores de los troncoconos se sitúa un cilindro de aluminio. Este cilindro contiene el par de elementos calorimétricos.

- Elementos calorimétricos o microcalorimétricos: es la pieza clave sobre la que se basa el sistema de detección en un calorímetro Tian-Calvet y que se muestra en la Ilustración 4-7.
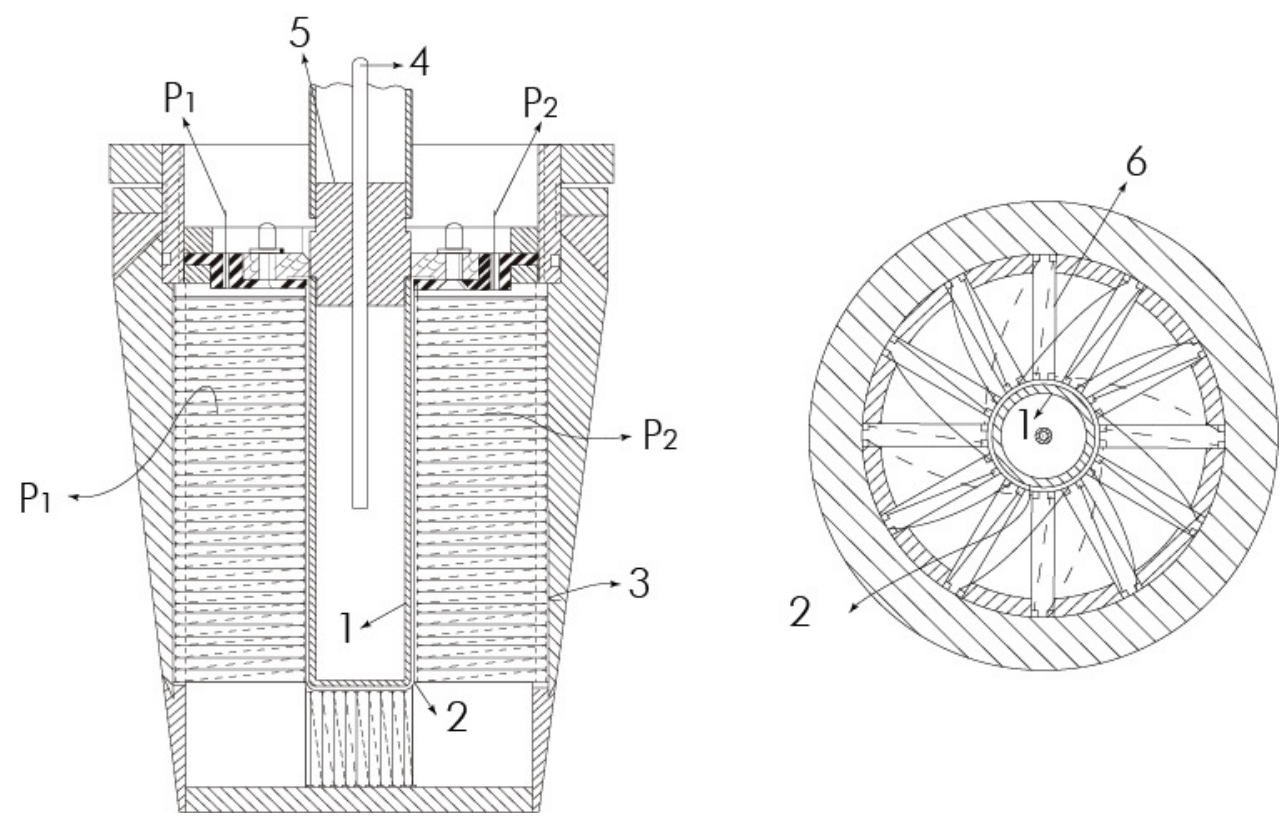

Ilustración 4-7: Cortes transversal (izq.) y en planta (drcha.) de un elemento microcalorimétrico [175]: celda de mezcla (1); recinto interno (2); recinto externo (3); aguja de la jeringuilla (4); tapón de teflón de la celda (5); montura de las termopilas (6); termopila efecto Seebeck $\left(P_{1}\right)$; termopila efecto Peltier $\left(P_{2}\right)$.

Las diferentes partes de las que consta un elemento calorimétrico son las siguientes:

Celda de mezcla: es un recipiente cilíndrico metálico con un tapón roscado de teflón, donde se produce el proceso que se va a estudiar. Se sitúa dentro del recinto interno. Por comodidad, la celda es extraíble, pues así el experimento puede prepararse en el exterior del calorímetro.

Recinto interno: es un cilindro delgado formado por una lámina de plata y una capa de mica de $0.01 \mathrm{~mm}$ de espesor. La lámina de plata estará en contacto directo con la celda de mezcla, mientras que la lámina de mica estará en contacto con la galleta de termopares. Una capa tan fina de mica aísla eléctrica pero no térmicamente, garantizándose así que las galletas de termopares no entren en cortocircuito. El recinto interno está preparado para contener una celda cilíndrica de $16.65 \pm 0.05 \mathrm{~mm}$ de diámetro y $82.0 \pm 0.5 \mathrm{~mm}$ de altura. Como las galletas de termopares solamente alcanzan una altura de $70 \mathrm{~mm}$, el resto del espacio $(12 \mathrm{~mm})$ queda reservado para los sistemas de aislamiento térmico (tapón de teflón) que siempre poseen las celdas en su parte superior.

Recinto externo: rodea las galletas de termopares y permanece en contacto directo con el cilindro principal que lo mantiene a temperatura constante. 
Galletas de termopares, pilas termoeléctricas o termopilas: formadas por un conjunto de termopares regularmente distribuidos que ligan los recintos interno y externo. Cuando se produce un efecto térmico en el recinto interno, la diferencia de temperatura con el recinto externo dará lugar, por efecto Seebeck, a una fuerza electromotriz entre los extremos de los termopares. Este fenómeno durará hasta que la temperatura de ambos recintos se iguale por conducción térmica a través de los termopares.

Los termopares de este calorímetro son de cromel-constantán (cromel: $90 \mathrm{Ni}, 10 \mathrm{Cr}$; constantán: $57 \mathrm{Cu}-43 \mathrm{Ni}$ ), permiten alcanzar temperaturas de trabajo de $473 \mathrm{~K}$ y tienen un poder termoeléctrico de $67 \cdot 10^{-6} \mathrm{~V} \cdot \mathrm{K}^{-1}$. El número de estos y sus dimensiones están calculados para obtener la máxima sensibilidad, así como la menor inercia térmica posible. Las galletas de termopares están formadas por 496 termopares agrupados en dos series, una serie de 372, denominada termopila detectora y una serie de 124, denominada termopila compensadora. Combinando convenientemente ambas termopilas mediante un conmutador ad hoc situado en la parte superior del calorímetro puede variarse la sensibilidad de detección del aparato: pequeña sensibilidad, que corresponde al empleo de la termopila compensadora como termopila detectora (124 termopares); mediana sensibilidad, que se corresponde al empleo de la termopila detectora solamente (372 termopares); gran sensibilidad, que se corresponde al uso de ambas termopilas conectadas en serie para el sistema de detección (496 termopares). Por tanto, se puede utilizar $1 / 4,3 / 4$ ó 1 de la sensibilidad nominal del aparato en nuestras experiencias. De esta manera podemos asignar al aparato las especificaciones siguientes, determinadas por Cobos [51]:

Tabla 4-1: Características básicas de los termopares en el calorímetro Calvet

\begin{tabular}{ccc}
\hline $\mathrm{N}^{\circ}$ de termopares & Sensibilidad $/ \mu \mathrm{V} \cdot \mathrm{mW}^{-1}$ & Resistencia base \\
\hline 124 & 15 & 11 \\
372 & 45 & 33 \\
496 & 60 & 44 \\
\hline
\end{tabular}

El conmutador tiene seis posiciones, que en sentido contrario a las agujas del reloj son: 0 termopares, 124 termopares, 0 termopares, 372 termopares, 0 termopares y 496 termopares. La razón que llevó a dividir los termopares en dos conjuntos fue la posibilidad de medir capacidades caloríficas, para lo cual es necesario usar una parte de los termopares como células Peltier, como se ha explicado al comienzo de la sección 4.1.1.3. En cualquier caso, en la presente tesis no se han medido capacidades caloríficas y siempre se han usado los 496 termopares.

En conclusión, un elemento microcalorimétrico consta de dos termopilas que conectan entre sí los recintos externo e interno. A parte de esta conexión a través de los hilos de termopares, ambos recintos deben de estar aislados entre sí. La termopila detectora se conecta a un voltímetro situado en el exterior del calorímetro. La fuerza electromotriz que se forma en cada instante es proporcional al flujo de calor intercambiado entre los dos recintos, y por tanto a la diferencia de temperatura entre ambos. La otra termopila se puede conectar a una fuente de tensión o de corriente mediante unos terminales situados en el exterior del calorímetro para compensar o descompensar, por efecto Peltier, la liberación del calor producido en la celda de mezcla en el caso de que se deseen efectuar medidas de capacidades caloríficas. La manera de operar en este caso se describe en la referencia [51].

- Salidas: los siguientes elementos son visibles en el exterior del calorímetro: 
Cable coaxial etiquetado como salida Seebeck.

Cable coaxial etiquetado como salida Peltier.

Cable coaxial etiquetado como calefacción directa.

Cable coaxial etiquetado como calefacción controlada.

Conmutador que permite seleccionar la sensibilidad del calorímetro.

Cuatro hilos de la resistencia termométrica de platino.

Acceso al elemento calorimétrico laboratorio.

Acceso al elemento calorimétrico referencia.

- Montaje diferencial: como se ha dicho, la principal modificación que hizo el profesor Calvet al calorímetro de Tian fue el montaje diferencial: se montan dos elementos calorimétricos como el descrito anteriormente, denominados elemento calorimétrico laboratorio y elemento calorimétrico referencia. Como en cualquier calorímetro diferencial, ambos se encuentran conectados en oposición, esto es, las salidas de sus termopilas están conectadas de tal forma que cualquier perturbación térmica detectada en el elemento calorimétrico referencia se resta de la salida detectada en el elemento calorimétrico laboratorio, lográndose eliminar la mayoría de las perturbaciones térmicas que llegan al calorímetro del exterior. Téngase en cuenta, además, que los troncoconos equipartidores distribuyen uniformemente las perturbaciones térmicas sobre ambos elementos calorimétricos.

Para conseguir que el montaje funcione correctamente, ambos elementos calorimétricos deben montarse en las mismas condiciones de intercambio térmico con un mismo recinto externo, para lo cual las capacidades caloríficas de los recintos internos y las celdas de medida de ambos elementos deben ser muy parecidas. Por esta razón, cuando se estudia la mezcla de dos líquidos, en la celda de referencia se debe situar un líquido o una mezcla cuya capacidad calorífica sea similar a la de la mezcla que se pretende estudiar en el elemento calorimétrico laboratorio. Al estudiar dicha mezcla, el elemento microcalorimétrico de referencia permanece a la misma temperatura que el termostato, mientras que en el elemento microcalorimétrico laboratorio se produce un pequeño cambio de temperatura debido al proceso de mezcla

- Sistema monitor de la temperatura: tradicionalmente la medida de la temperatura del calorímetro se realizaba introduciendo un termómetro en el recinto interno de los elementos calorimétricos [51]. Esto significaba que durante la realización de las medidas no era posible hacer un seguimiento de la temperatura, sino que había que dejar de medir, introducir el termómetro y esperar a que se alcanzara el equilibrio térmico. La introducción de una sonda Pt-100 en el interior del calorímetro, conectada a un multímetro digital Fluke 8842A, evita esta situación, de modo que se puede hacer un seguimiento constante de la temperatura, como explican Riesco y Mozo [172]-[173]. Como es obvio, ya que lo que se busca es la medida de la temperatura a la que se encuentra el recinto externo, la sonda no se encuentra en el recinto interno, sino en los troncoconos. La gran homogeneidad de la temperatura en el interior del calorímetro permite que se pueda considerar esta como la temperatura a la cual la mezcla se produce. Recientemente, Alonso [174] ha mejorado el sistema de monitorización de temperatura, como consecuencia de las graves variaciones en las condiciones de trabajo debidas al traslado del laboratorio del G.E.T.E.F. desde la antigua Facultad de 
Ciencias en la C/Doctor Mergelina hasta su nuevo emplazamiento en el Campus Miguel Delibes.

- Sistema de control de la temperatura: se utilizó el ideado por Riesco [172] y mejorado por Mozo [173]. Para evitar cambios bruscos en la potencia que alimenta las resistencias calefactoras, se modificó su sistema de control respecto del utilizado por Cobos [51]. La salida digital para el control de dicha potencia se cambió por una analógica (por medio de un controlador Eurotherm 2416 conectado a una fuente Agilent 6644A). De este modo, si se quiere dar, por ejemplo, un $50 \%$ de la potencia calefactora máxima, efectivamente se da tal potencia de forma continua, en vez de suministrar el $100 \%$ de la potencia durante un periodo de tiempo, y posteriormente un $0 \%$ durante el siguiente intervalo de tiempo (de forma discontinua). En contra de lo esperado, esto no supuso ninguna mejora en la estabilidad de la temperatura del calorímetro, de modo que se sigue manteniendo la misma configuración que en trabajos anteriores [51], [171], [172]. Gracias al sistema de resistencias calefactoras y al aire acondicionado instalado en la sala del laboratorio, que mantiene su temperatura a $293.15 \pm 0.5 \mathrm{~K}$, se logra una estabilidad en la temperatura del microcalorímetro mejor que $0.005 \mathrm{~K}$ durante una semana, y desde luego mejor que esto en el tiempo que dura la medida del calor de mezcla. Recientemente, tanto esta estabilidad como el algoritmo de control del Eurotherm 2416 se han mejorado como consecuencia de la modificación del sistema de control de temperatura realizada por Alonso [174], por las mismas razones que por las que se cambió el sistema de monitorización de temperatura.

\section{b) Teoría de funcionamiento del calorímetro Tian-Calvet}

A continuación se desarrolla un modelo matemático simplificado que da cuenta del funcionamiento del calorímetro Tian-Calvet. El lector interesado en estudios más profundos puede acudir a las referencias [42], [84] y [176]-[183]. En dicho modelo matemático se supone que se verifican las siguientes hipótesis:

- La temperatura de los recintos interno y externo son uniformes en todo momento, lo que es equivalente a admitir que ambos recintos poseen conductividad térmica infinita, algo en realidad imposible. En realidad hay heterogeneidad en la temperatura, lo que se llama desequilibrio térmico interno, que puede obviarse si se considera que el recinto interno tiene una capacidad calorífica aparente superior a su capacidad calorífica real.

En lo que sigue, $T_{\mathrm{i}}$ representa la temperatura en el recinto interno y $T_{\mathrm{e}}$ la temperatura en el recinto externo.

- El flujo de calor entre el recinto interno y externo obedece la ley de Fourier. La ecuación (4.10) expresa la ley de Fourier en su forma diferencial para el flujo de calor instantáneo entre dos secciones de área $A$, separadas una distancia $\mathrm{d} x$ y con una diferencia de temperaturas $\mathrm{d} T$.

$$
\frac{\mathrm{d} Q_{x}}{\mathrm{~d} t}=-k \cdot A \cdot \frac{\mathrm{d} T}{\mathrm{~d} x}
$$

donde $k$ es la conductividad térmica de la región en la cual el flujo de calor se propaga.

El resultado de aplicar la ley de Fourier a la descripción del flujo térmico entre los recintos interno y externo del calorímetro es la ley de enfriamiento de Newton: 


$$
\frac{\mathrm{d} Q}{\mathrm{~d} t}=p \cdot\left(T_{\mathrm{i}}-T_{\mathrm{e}}\right)
$$

donde $p$ es el coeficiente de transferencia térmica y da cuenta de la linealidad del proceso.

- El calor necesario para elevar la temperatura del recinto interno viene expresada por la ecuación siguiente:

$$
\mathrm{d} Q=C_{P} \cdot \mathrm{d} T
$$

siendo $C_{P}$ la capacidad calorífica aparente a presión constante del recinto interno. Puesto que se asume que la temperatura del recinto externo $T_{\mathrm{e}}$ es constante, para simplificar la notación se ha escrito $\mathrm{d} T$ en lugar de $\mathrm{d} T_{\mathrm{i}}$.

- La diferencia de temperaturas entre los recintos interno y externo está correlacionada con la fuerza electromotriz $V$ creada en cada termopila mediante la expresión matemática del efecto Seebeck:

$$
V=n \cdot e \cdot\left(T_{\mathrm{i}}-T_{\mathrm{e}}\right)
$$

donde $e$ es la fuerza electromotriz generada en cada termopar y $n$ es el número total de éstos en la termopila.

Una vez introducidas las hipótesis básicas sobre las que se apoya el modelo, se puede afirmar que la potencia calorífica, $\mathrm{d} Q / \mathrm{d} t$, generada o disipada, según sea el proceso exotérmico o endotérmico, en la celda de mezcla de un elemento microcalorimétrico se invierte en dos procesos:

- Parte de la potencia térmica se pierde por la aparición de un flujo térmico desde el recinto interno hasta el externo consecuencia de la diferencia de temperatura entre ambos. Dicho flujo térmico es $p \cdot\left(T_{\mathrm{i}}-T_{\mathrm{e}}\right)$.

- La parte de potencia térmica que no se pierde es la causante de la elevación de la temperatura del recinto interno. Dicha potencia térmica es $C_{P} \cdot(\mathrm{d} T / \mathrm{d} t)$.

Si el calorímetro utilizase el sistema de compensación, además de la potencia calorífica $\mathrm{d} Q / \mathrm{d} t$ debería tenerse en cuenta la potencia térmica generada por efecto Peltier $\mathrm{d} Q \% / \mathrm{d} t$. No obstante, en este trabajo este término es cero pues, como ya se ha comentado, no se ha utilizado el sistema de compensación Peltier y el calorímetro Calvet ha trabajado en su configuración de máxima sensibilidad.

Por lo dicho, se puede escribir:

$$
\frac{\mathrm{d} Q}{\mathrm{~d} t}+\frac{\mathrm{d} Q^{\prime}}{\mathrm{d} t}=\frac{\mathrm{d} Q}{\mathrm{~d} t}=p \cdot\left(T_{\mathrm{i}}-T_{\mathrm{e}}\right)+C_{P} \cdot \frac{\mathrm{d} T}{\mathrm{~d} t}
$$

ecuación que puede rescribirse en función de la fuerza electromotriz $V$ generada en las galletas de termopares (ecuación diferencial del microcalorímetro Tian):

$$
\frac{\mathrm{d} Q}{\mathrm{~d} t}=\frac{p \cdot V}{n \cdot e}+\frac{C_{P}}{n \cdot e} \cdot \frac{\mathrm{d} V}{\mathrm{~d} t}=C \cdot V+C^{\prime} \cdot \frac{\mathrm{d} V}{\mathrm{~d} t}
$$

donde $C$ es la constante de calibrado del calorímetro y engloba las constantes que aparecen en las leyes de Fourier y del efecto Seebeck, y $C^{\prime}$ es otra constante que 
engloba la capacidad calorífica y las constantes que aparecen en la formulación del efecto Seebeck.

Como se explicará unas páginas más adelante, uno de los métodos de calibrado posibles consiste en suministrar una potencia constante al recinto interno de manera que el calorímetro alcance el régimen estacionario. En estas condiciones los términos $\mathrm{d} T / \mathrm{d} t$ y $\mathrm{d} V / \mathrm{d} t$ son nulos y las ecuaciones (4.14) y (4.15) se reducen a las siguientes:

$$
\begin{gathered}
\frac{\mathrm{d} Q}{\mathrm{~d} t}=p \cdot\left(T_{\mathrm{i}}-T_{\mathrm{e}}\right) \\
\frac{\mathrm{d} Q}{\mathrm{~d} t}=C \cdot V
\end{gathered}
$$

La ecuación (4.17) es la ecuación diferencial del microcalorímetro Tian en régimen estacionario.

Fuera del régimen estacionario, en el intervalo de tiempo comprendido entre los instantes $t_{1}$ y $t_{2}$ el calor total disipado o generado en la celda de mezcla (es decir, el calor de mezcla) viene dado por la ecuación integral del microcalorímetro Tian:

$$
Q=C \cdot \int_{t_{1}}^{t_{2}} V \cdot \mathrm{d} t+C^{\prime} \cdot\left(V_{2}-V_{1}\right)
$$

en donde se ha supuesto que $C$ y $C^{\prime}$ permanecen constantes durante el intervalo de integración. Para evitar la evaluación de la constante $C^{\prime}$ en la ecuación (4.18) el intervalo de integración se escoge de forma que $V_{2}=V_{1}$, lo que se conoce como recuperación de la línea base. En estas condiciones la citada ecuación se reduce a la siguiente:

$$
Q=C \cdot \int_{t_{1}}^{t_{2}} V \cdot \mathrm{d} t
$$

Hasta ahora las ecuaciones obtenidas se refieren a un único elemento microcalorimétrico. Sin embargo, como se ha dicho, Calvet dotó al calorímetro de Tian de un montaje diferencial para disminuir los efectos debidos a las perturbaciones térmicas externas. Así, el calorímetro Tian-Calvet consta de dos elementos calorimétricos montados en oposición llamados referencia y laboratorio respectivamente. En lo que sigue se expondrán las ecuaciones correspondientes al montaje diferencial.

Restando las ecuaciones (4.15) aplicadas a los elementos calorimétricos laboratorio y referencia se obtiene la ecuación que describe la diferencia entre los flujos térmicos en ambos (ecuación diferencial del microcalorímetro Tian-Calvet):

$$
\frac{\mathrm{d} Q_{\mathrm{L}}}{\mathrm{d} t}-\frac{\mathrm{d} Q_{\mathrm{R}}}{\mathrm{d} t}=\left(C_{\mathrm{L}} \cdot V_{\mathrm{L}}-C_{\mathrm{R}} \cdot V_{\mathrm{R}}\right)+\left(C_{\mathrm{L}}^{\prime} \cdot \frac{\mathrm{d} V_{\mathrm{L}}}{\mathrm{d} t}-C_{\mathrm{R}}^{\prime} \cdot \frac{\mathrm{d} V_{\mathrm{R}}}{\mathrm{d} t}\right)
$$

donde los subíndices $\mathrm{L}$ y $\mathrm{R}$ indican las propiedades de los elementos calorimétricos laboratorio y referencia, respectivamente. No se ha considerado la utilización del sistema de compensación Peltier. Además, se ha supuesto que ambos elementos calorimétricos son diferentes por lo que sus constantes $C$ y $C^{\prime}$ son distintos. En caso contrario, es decir, suponiendo que las galletas de termopares y la capacidad calorífica 
del recinto interno en ambos elementos calorimétricos son iguales, la expresión anterior se reduce a:

$$
\frac{\mathrm{d} Q_{\mathrm{L}}}{\mathrm{d} t}-\frac{\mathrm{d} Q_{\mathrm{R}}}{\mathrm{d} t}=C \cdot V+C^{\prime} \cdot \frac{\mathrm{d} V}{\mathrm{~d} t}
$$

donde $V=V_{\mathrm{L}}-V_{\mathrm{R}}$ es la fuerza electromotriz medida en un montaje diferencial.

La ecuación anterior se simplifica en condiciones de régimen estacionario, ya que el término $\mathrm{d} V / \mathrm{d} t$ es nulo. En ese caso, se tiene la ecuación diferencial de Tian-Calvet de un calorímetro diferencial en régimen estacionario:

$$
\frac{\mathrm{d} Q_{\mathrm{L}}}{\mathrm{d} t}-\frac{\mathrm{d} Q_{\mathrm{R}}}{\mathrm{d} t}=C \cdot V
$$

A esta ecuación se puede llegar a partir de la (4.20) bajo una hipótesis ligeramente menos restrictivas que para llegar a la ecuación (4.21): basta con suponer que $C_{\mathrm{L}}=C_{\mathrm{R}}=C$ sin importar como sean las constantes $C$ '. Es decir, las termopilas de ambos elementos calorimétricos se consideran similares, obviando si las capacidades caloríficas de sus recintos internos son similares.

Fuera del régimen estacionario, en el intervalo de tiempo comprendido entre los instantes $t_{1}$ y $t_{2}$ el calor total disipado o generado en la celda de mezcla (calor de mezcla corregido de las perturbaciones térmicas externas) viene dado por la ecuación integral del microcalorímetro Tian-Calvet:

$$
Q_{\mathrm{L}}-Q_{\mathrm{R}}=\int_{t_{1}}^{t_{2}}\left(C_{\mathrm{L}} \cdot V_{\mathrm{L}}-C_{\mathrm{R}} \cdot V_{\mathrm{R}}\right) \cdot \mathrm{d} t+\left[C_{\mathrm{L}}^{\prime} \cdot\left(V_{\mathrm{L}, 2}-V_{\mathrm{L}, 1}\right)-C_{\mathrm{R}}^{\prime} \cdot\left(V_{\mathrm{R}, 2}-V_{\mathrm{R}, 1}\right)\right]
$$

Cuando las galletas de termopares y la capacidad calorífica de los recintos internos de ambos elementos calorimétricos son parecidas, la ecuación anterior se reduce a:

$$
Q_{\mathrm{L}}-Q_{\mathrm{R}}=C \cdot \int_{t_{1}}^{t_{2}} V \cdot \mathrm{d} t+C^{\prime} \cdot\left(V_{2}-V_{1}\right)
$$

en donde se ha supuesto que $C$ y $C^{\prime}$ permanecen constantes durante el intervalo de integración. Como se hizo en el caso del montaje simple, para evitar la evaluación de la constante $C$ ' en la ecuación (4.24) el intervalo de integración se escoge de forma que $V_{2}=V_{1}$, es decir, recuperando la línea base. En estas condiciones la citada ecuación se transforma en la ecuación integral de un microcalorímetro Tian-Calvet integrador:

$$
Q_{\mathrm{L}}-Q_{\mathrm{R}}=C \cdot \int_{t_{1}}^{t_{2}} V \cdot \mathrm{d} t
$$

En el caso más general en el que las termopilas no son idénticas o similares, la ecuación anterior se vería modificada:

$$
Q_{\mathrm{L}}-Q_{\mathrm{R}}=\int_{t_{1}}^{t_{2}}\left(C_{\mathrm{L}} \cdot V_{\mathrm{L}}-C_{\mathrm{R}} \cdot V_{\mathrm{R}}\right) \cdot \mathrm{d} t
$$

c) Procedimiento de medida 
A diferencia del procedimiento de medida establecido por Cobos [51], se ha trabajado con jeringuillas de plástico (Becton-Dickinson Discardit II (PP+PE) de $5 \mathrm{ml}$ y libres de PVC), que a diferencia de las de vidrio, mantienen la posición de su émbolo, de manera que se evita el goteo indeseado del segundo componente sobre el primero y el uso de silicona. Además se ha trabajado con las celdas de mezcla diseñadas por Mozo [173], que sustituyen a las celdas antiguas. Como Mozo explica [173], las anteriores celdas eran de vidrio esmerilado y su fragilidad ha sido responsable de que el número de éstas se haya reducido drásticamente con el paso de los años. Las nuevas celdas se diseñaron en acero inoxidable, cerradas en su parte superior por unos tapones de teflón roscados que también fueron diseñados a medida, siguiendo las pautas sugeridas por McGlashan [25]. Los tapones tienen un agujero que pasa por su eje para permitir el paso de una aguja metálica de inyección. Se pudo comprobar que todos los tapones de teflón taladrados con una broca de menos de $2 \mathrm{~mm}$ de diámetro se desbordaban en el momento de la inyección, lo cual es debido probablemente a la condensación de los vapores de la mezcla en las paredes del agujero. Se diseñaron dos tamaños de celda (10 y $6 \mathrm{ml}$ ), de modo que la corrección a la entalpía de mezcla debida a la evaporización cuando la composición de alguno de los componentes es pequeña se reduce si se utiliza la celda de $6 \mathrm{ml}$.

El procedimiento a seguir en cada medida es el siguiente:

- Estimación del volumen de cada uno de los componentes a mezclar para obtener un volumen total aproximado igual a 10 ó $6 \mathrm{~cm}^{3}$, dependiendo de la celda de mezcla utilizada. Esta estimación se realiza suponiendo que la mezcla es ideal.

- Pesada de la celda de mezcla vacía: se cierra la celda de mezcla con el tapón de teflón, sellando su agujero con un recorte de cinta aislante.

- Pesada de la celda con el primer componente. Se retira el recorte de cinta aislante y se introduce el primer componente en el interior de la celda. Rápidamente se vuelve a sellar el agujero y se repite la pesada. En este momento, parte de este componente se evapora sin salir de la celda. Siempre que sea posible el líquido menos denso debe ser el primero en ser introducido en la celda * para favorecer la mezcla por efecto gravitatorio.

- Se llena la jeringuilla con el segundo componente, evitando que gotee. Por ello, la aguja de inyección debe limpiarse con papel secante. Una vez limpia, se retira nuevamente el pedazo de cinta aislante que sella el tapón de teflón y se introduce la jeringuilla, evitando en todo momento que empiece a gotear.

El conjunto celda metálica + jeringuilla se une a una caña de plástico hueca de unos $50 \mathrm{~cm}$ de longitud, mediante un tapón cilíndrico de teflón con rosca por sus dos bases, estando una de las roscas atornillada al tapón de la celda metálica y la otra a la caña. Este conjunto celda metálica + jeringuilla + caña de plástico se introduce en el interior del Calvet y se espera aproximadamente una hora a que el sistema alcance el equilibrio térmico, dado por la recuperación de la línea base. Entonces, una segunda caña maciza se introduce en el interior de la primera y se empuja suavemente el émbolo de la jeringuilla, produciéndose la mezcla. En este momento, no solamente el líquido

\footnotetext{
* Si uno de los componentes es muy volátil, éste debe situarse en la jeringuilla, aún siendo el menos denso, pues aparecen grandes errores en la medida de $H_{\mathrm{m}}^{\mathrm{E}}$ cuando el primer líquido en la celda es el más volátil, como ocurre en las mezclas de $n$-hexano + ciclohexano si el primer líquido es el $n$-hexano.
} 
inyectado desplaza parte del vapor presente en la celda, sino que también parte del líquido inyectado se evaporará, por lo que al final debe tenerse en cuenta una corrección de la composición debida a estos efectos de evaporización. El sistema se deja evolucionar hasta que los efectos térmicos hayan cesado, es decir, hasta que se recupera de nuevo la línea base. Si el émbolo se empuja de manera brusca, parte de la mezcla puede escaparse de la celda, lo que se manifiesta en que la línea base se recupera de forma más lenta de lo habitual, y por efecto de la evaporación, lo hace en torno a un valor diferente al inicial.

- Recuperada línea base, se procede a extraer la celda del interior del calorímetro. Se retira la caña de plástico y la jeringuilla, y rápidamente se sella el agujero del tapón de teflón con el mismo pedazo de cinta aislante que previamente había sido usado. Finalmente se pesa la celda con la mezcla y se calcula la fracción molar de cada uno de los componentes, teniendo en cuenta las correcciones por empuje y por la evaporización de los componentes [172]. Se ha estimado que el error para la fracción molar es menor de \pm 0.0001 .

Debido al montaje diferencial del Calvet, es necesario introducir en el elemento calorimétrico referencia una celda cuya capacidad calorífica sea similar a la que se vaya a introducir en el elemento calorimétrico laboratorio. Por ello, la celda referencia se llena con una mezcla de composición aproximadamente equimolar de los mismos componentes que se inyectarán en la celda laboratorio. Si no se quiere cambiar el contenido de la celda de referencia durante el tiempo que lleva la determinación de las entalpías de exceso de un sistema (una semana aproximadamente), se debe evitar la fuga de los vapores cerrando herméticamente dicha celda colocando una goma inerte entre los dos tapones de teflón.

\subsection{RESULTADOS EXPERIMENTALES}

\subsubsection{Calibración del microcalorímetro Tian-Calvet}

La calibración del microcalorímetro Calvet se puede realizar siguiendo dos métodos similares basados en controlar la potencia suministrada al elemento calorimétrico laboratorio y medir la fuerza electromotriz en la salida del microcalorímetro.

\section{a) Método estático:}

Se suministra potencia constante para que el calorímetro alcance el régimen estacionario. La ecuación (4.22) permite determinar la constante de calibrado $C$ como el cociente entre la potencia térmica suministrada a la celda de calibración y el voltaje generado por efecto Seebeck cuando se ha alcanzado el régimen estacionario:

$$
C=\left[\frac{\frac{d Q_{\mathrm{L}}}{d t}-\frac{d Q_{\mathrm{R}}}{d t}}{V}\right]=\frac{P}{V}
$$

\section{b) Método dinámico:}

Se suministra potencia durante un pequeño intervalo de tiempo y se basa en la ecuación (4.25) que permite calcular la constante del Calvet como el cociente de dos integrales: la integral de la potencia térmica suministrada y la integral de la potencia generada por 
efecto Seebeck. El intervalo de integración se escoge de forma que se recupere la línea base como se ha explicado más arriba. Riesco [172] reportó que los valores estimados del error de la constante de calibración obtenida por el método dinámico es más de 30 veces superior al error obtenido por el método estático. La principal fuente de error se debía a que el multímetro que utilizó para medir el voltaje Seebeck rechazaba las medidas cuando el voltaje varía rápidamente, además de que el ordenador empleado no era lo suficientemente potente como para registrar todos los datos que recibía. Actualmente, ambos dispositivos se han cambiado y probablemente la calibración dinámica proporcione mejores resultados. No obstante, en el presente trabajo sólo se ha realizado el calibrado estático

Ambos procedimientos de calibración pueden implementarse con el mismo equipo, formado esencialmente por tres módulos controlados mediante un sistema informático:

a) Módulo encargado de suministrar la potencia deseada al elemento calorimétrico laboratorio durante el intervalo de tiempo deseado. Este módulo consta de los siguientes elementos:

- Dos celdas metálicas de calibración, fabricadas en 1979 por Cobos. Su diseño fue clave a la hora de establecer el procedimiento de calibración. Cobos discute en sus tesis doctoral [51] el efecto de la geometría de las resistencias en el interior de las celdas de calibración. No son satisfactorias aquellas celdas en las que la potencia térmica se libere de forma puntual, como ya había sido puesto de manifiesto por el $\mathrm{E}$. Calvet [84]. El diseño de Cobos consta de un cilindro de acero inoxidable, cerrado por su base y que posee un tapón roscado de teflón en su parte superior. Su interior contiene otro cilindro hueco de acero inoxidable y alrededor del mismo se encuentra enrollada una resistencia de hilo de manganina (la variación de la resistencia de la manganina con la temperatura es despreciable) y que termina en un montaje de 4 hilos con cables de plata embutidos en plástico para protegerlos y aislarlos. En la actualidad, solamente una de las dos celdas de calibración continúa en funcionamiento, la que posee una resistencia de $390 \Omega$ aproximadamente. Esta celda se coloca en el elemento calorimétrico laboratorio, mientras que la otra celda se introduce como testigo simétrico en el elemento calorimétrico referencia.

- Fuente de alimentación Agilent 6644A. Tiene un interfaz GP-IB (IEEE-488.1) que permite gobernarla remotamente. Así, una aplicación informática desarrollada en el entorno de programación Agilent VEE controla la potencia que este instrumento suministra a la celda de calibración situada en el elemento calorimétrico laboratorio, así como el intervalo de tiempo durante el que dicha potencia es suministrada.

b) Módulo para la monitorización de la potencia térmica suministrada durante la calibración, formado por los siguientes aparatos:

- Multímetro Philips PM2534. Se utiliza para visualizar el voltaje aplicado sobre la resistencia de la celda de calibración. Dispone de un interfaz GP-IB (IEEE-488) (IEC$625 / 1)$ que permite la configuración y el control remotos. Su exactitud en un año es $0.015 \%+51 \mu \mathrm{V}$. Para los voltajes utilizados en el calibrado, se ha encontrado que el error es siempre menor que $2.2 \mathrm{mV}$.

- Multímetro Agilent 34410A. Se utiliza para monitorizar la corriente que circula por la resistencia de la celda de calibración. Dispone de un interfaz GP-IB que permite la configuración y el control remotos. Su exactitud en noventa días para corrientes entre 
$1 \mathrm{~mA}$ y $10 \mathrm{~mA}$ es mayor que $0.03 \%+0.002 \mathrm{~mA}$, y para corrientes entre $10 \mathrm{~mA}$ y $100 \mathrm{~mA}$ es mayor que $0.03 \%+0.005 \mathrm{~mA}$. Para las corrientes utilizadas en el calibrado, se ha encontrado que el error es menor que $0.016 \mathrm{~mA}$.

La razón de utilizar el multímetro de Philips para medir voltajes y el Agilent para medir corrientes es resultado de estimar la exactitud y la precisión de todas las combinaciones posibles que permiten monitorizar el suministro de $1 \mathrm{~W}$ durante $180 \mathrm{~s}$ a la celda de calibración, como explica Riesco [172]. Por otra parte, el error sistemático en la determinación de la potencia disipada en la celda de calibración se reduce si el montaje empleado para ello responde a la configuración en la que se mide directamente la caída de potencial en sus extremos, como discute Riesco [172]. Se ha estimado que el error en la determinación de la potencia térmica nunca es mayor que $0.29 \mathrm{~mW}$.

c) Módulo para la monitorización de la potencia de salida generada por efecto Seebeck. Este módulo es común al sistema de medición, y de hecho es el único que se necesita para la determinación de las entalpías de exceso una vez que el aparato se ha calibrado. Esta formado únicamente por un multímetro Keithley 2000 de 6 1/2 dígitos con un interfaz GP-IB (IEEE-488), y con exactitud en el rango de voltaje de $100 \mathrm{nV}$ a $1 \mathrm{kV}$ igual a $0.002 \%$. Anteriormente la función de este aparato la cumplía un multímetro Philips PM2534, pero al observar que rechazaba las medidas cuando el voltaje variaba rápidamente, algo no aceptable para la precisión buscada ya que esta situación ocurre cuando se empiezan a mezclar los componentes y también cuando se realiza un calibrado dinámico, fue sustituido por el Keithley 2000. Teniendo en cuenta que la sensibilidad del Calvet es igual a $60 \mu \mathrm{V} / \mathrm{mW}$ y que durante el calibrado la potencia nunca es mayor que $0.5 \mathrm{~W}$, el voltaje máximo de calibración será menor a $30 \mathrm{mV}$. En esas condiciones se obtiene una cota superior para el error en el voltaje Seebeck igual a $0.6 \mu \mathrm{V}$. El programa de control recoge los datos registrados por este multímetro y los almacena en un archivo. Además, calcula los parámetros de una regresión lineal con las 100 últimas medidas del voltaje generado por efecto Seebeck en función del tiempo. La pendiente de esta regresión lineal $B$ y su desviación estándar $\sigma$ permiten estimar si se ha recuperado la línea base. El criterio escogido para la recuperación de la línea base es que se satisfagan las siguientes condiciones:

$$
\begin{gathered}
|B| \leq 1.5 \cdot 10^{-9} \mathrm{~V} \cdot \mathrm{s}^{-1} \\
|\sigma| \leq 1.0 \cdot 10^{-6} \mathrm{~V}
\end{gathered}
$$

El procedimiento seguido en la calibración estática es el siguiente: el programa de control ordena a la fuente de alimentación proporcionar a la celda metálica una corriente de $5 \mathrm{~mA}$ aproximadamente, manteniéndola estable durante unas 4 horas. En estas condiciones, el calorímetro funciona en régimen estacionario y la ecuación (4.27) es aplicable, donde $P$ es la potencia generada en la celda e igual al producto del voltaje y la corriente en la misma, registrados por los multímetros Philips PM2534 y Agilent 34410A respectivamente; $V$ es el voltaje generado por efecto Seebeck en las termopilas y registrado en el multímetro Keithley 2000. Una vez pasadas esas 4 horas, el programa de control ordena a la fuente de alimentación elevar la corriente de la celda a $10 \mathrm{~mA}$ aproximadamente, durante otras 4 horas; se aplica nuevamente la ecuación (4.22) obteniéndose un valor diferente de $C$. El proceso se repite hasta que la corriente en la fuente es de unos $35 \mathrm{~mA}$. El valor válido que se toma para $C$ es la media ponderada de los valores obtenidos en cada tramo. 
El error relativo de la constante del Calvet $C$ viene dado por la ecuación:

$$
\varepsilon(C)=\frac{\Delta C}{C}=\frac{1}{V} \cdot \Delta V+\frac{1}{P} \cdot \Delta P=\varepsilon(V)+\varepsilon(P)
$$

Como se ha dicho anteriormente, se estimó que el error de la potencia de calibración es inferior a $0.29 \mathrm{~mW}$ y que en el voltaje Seebeck es inferior a $0.6 \mu \mathrm{V}$. Con estos datos se podría estimar una cota superior del error cometido al calcular la constante del Calvet en la calibración estática; no obstante, se ha optado por determinar exactamente el error y así poder comparar los resultados obtenidos con los de Riesco [172], ya que después de la presentación de sus resultados, varios periféricos de control del microcalorímetro se han reemplazado por otros nuevos y es deseable comprobar en que grado ello ha mejorado la precisión del equipo.

En la llustración 4-8 se observa cómo la corriente aplicada en la celda y el voltaje generado por efecto Seebeck evolucionan durante el tiempo que dura la calibración.

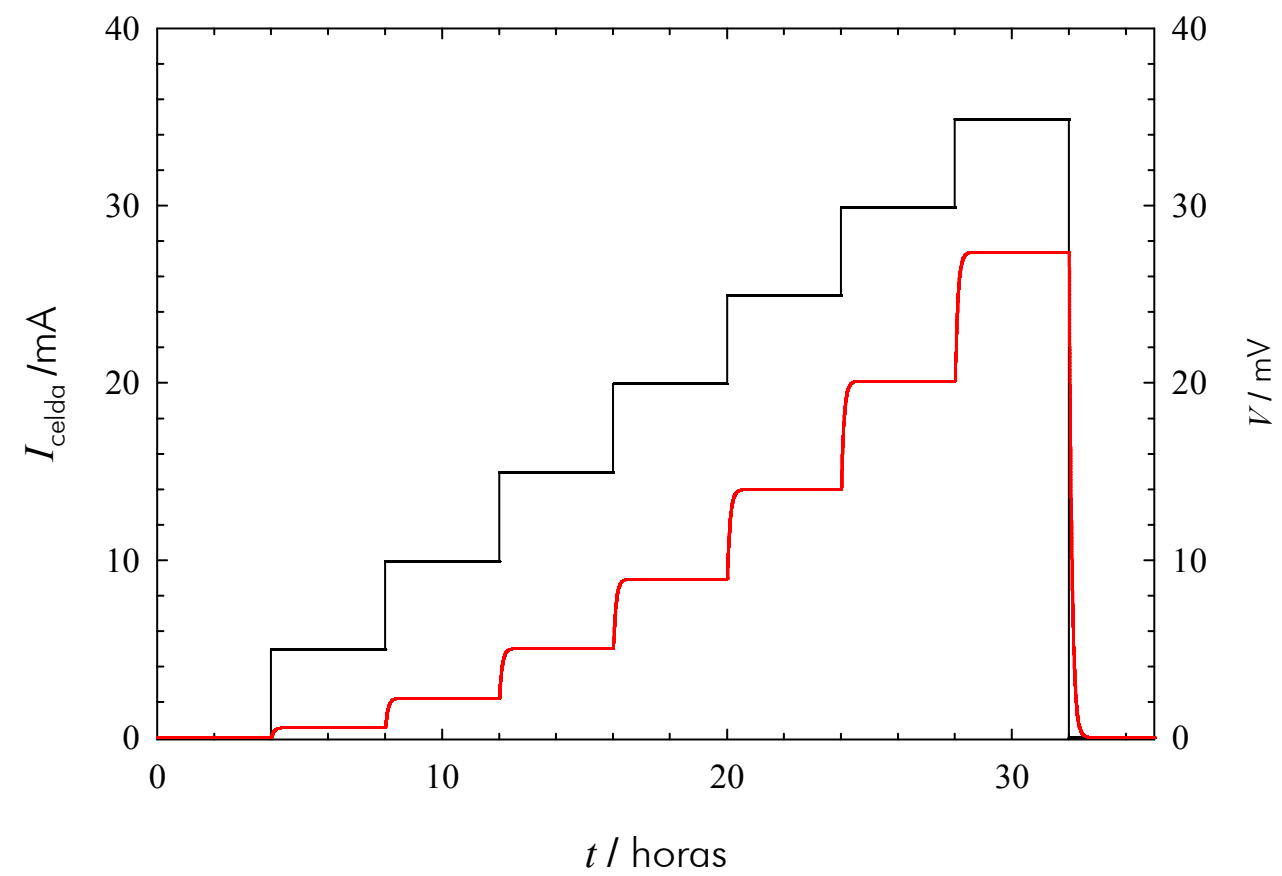

Ilustración 4-8: calibrado del Calvet: — intensidad de corriente en la celda metálica; — voltaje generado por efecto Seebeck

En la Tabla 4-2 se presentan los valores medios obtenidos en cada tramo para la corriente en la celda, el voltaje generado por efecto Seebeck, la potencia de calibración, y la constante de calibración, junto con su error. Basándonos en la ecuación (4.28) y dicha tabla, se observa que la mayor contribución al error en la determinación de la constante de calibrado viene dada por la incertidumbre en la determinación de la potencia. A pesar de ello, la utilización del multímetro Agilent 34410A para registrar la corriente ha mejorado esta incertidumbre comparada con la obtenida por Riesco [172]. Además, la incertidumbre en la medida de $V$ también se ha mejorado con la utilización del Keithley 2000. Véase el Cuadro 1.5 de la referencia [172]. 
Tabla 4-2: resultados obtenidos para la calibración estática.

\begin{tabular}{cccccccc}
\hline$I \pm 0.016 / \mathrm{mA}$ & $V / \mathrm{mV}$ & $\varepsilon(V) \%$ & $P / \mathrm{mW}$ & $\varepsilon(P) \%$ & $C / \mathrm{W} \cdot \mathrm{V}^{-1}$ & $\Delta C / \mathrm{W} \cdot \mathrm{V}^{-1}$ & $\varepsilon(C) \%$ \\
\hline 4.978 & 0.560 & 0.002 & 9.725 & 0.09 & 17.440 & 0.016 & 0.09 \\
9.944 & 2.23 & 0.002 & 38.82 & 0.07 & 17.440 & 0.012 & 0.07 \\
14.935 & 5.02 & 0.002 & 87.56 & 0.08 & 17.440 & 0.014 & 0.08 \\
19.929 & 8.94 & 0.002 & 155.90 & 0.07 & 17.440 & 0.013 & 0.07 \\
24.917 & 14.0 & 0.002 & 243.73 & 0.07 & 17.430 & 0.012 & 0.07 \\
29.872 & 20.1 & 0.002 & 350.4 & 0.06 & 17.430 & 0.011 & 0.06 \\
34.856 & 27.4 & 0.002 & 477.2 & 0.06 & 17.430 & 0.011 & 0.06 \\
\hline
\end{tabular}

El valor de la constante de calibrado que en lo que sigue se tomará como verdadero es el promedio de todos los que se escriben en la Tabla 4-2:

$$
C=17.40 \pm 0.013 \mathrm{~mW} \cdot \mathrm{mV}^{-1} \quad \varepsilon(C)=0.09 \%
$$

\subsubsection{Sistema test}

Una vez calibrado el aparato, se pueden determinar las entalpías de exceso de cualquier sistema. El calor total liberado por una mezcla se determina multiplicando la constante de calibrado del Calvet $C$ por la integral del voltaje Seebeck durante el intervalo de tiempo en el que la mezcla absorbe o cede calor al elemento microcalorimétrico laboratorio $\int V \cdot \mathrm{d} t$, como se observa en la ecuación (4.25). Dicho intervalo de tiempo viene determinado por el instante inicial $t_{1}$ en el que los componentes en la celda se empiezan a mezclar y se rompe la línea base, y el instante final $t_{2}$ en el que la línea base se recupera según el criterio dado en la sección 0 . Como el proceso se realiza a presión constante, el calor liberado es igual a la entalpía de mezcla (o de exceso), por lo que la entalpía molar de exceso es:

$$
H_{\mathrm{m}}^{\mathrm{E}, 0}=\frac{C \cdot \int_{t_{1}}^{t_{2}} V \cdot d t}{n}
$$

donde $n$ es el número de moles totales presentes en la mezcla.

Como se dijo en la sección 4.1.1.1, la principal desventaja los calorímetros de inyección directa es que uno o los dos componentes de la celda pueden evaporarse. En caso de que esto suceda, el calor total medido $\int V \cdot \mathrm{d} t$ es la suma de las entalpía de mezcla y de las entalpías de evaporización. Por lo tanto, el valor dado por (4.29) no es exactamente el de la entalpía molar de exceso y por eso se ha puesto el superíndice 0. Dicho valor debe corregirse para tener en cuenta los efectos de evaporización de los componentes. Además, como se ha explicado al exponer el procedimiento de medida, sección 4.1.1.3 c), también es necesario corregir la composición de la mezcla final. Para ver la importancia de estos efectos, McGlashan [25] muestra algunos casos en los que el calor de evaporación es del mismo orden que la entalpía de mezcla.

Las correcciones aplicadas en este trabajo son las desarrolladas por C. Casanova y expuestas en las tesis de Cobos [51] y Riesco [172]. Si designamos por $H_{\mathrm{v}}$ la corrección a la entalpía molar, entonces: 


$$
H_{\mathrm{m}}^{\mathrm{E}}=\frac{C \cdot \int_{t_{1}}^{t_{2}} V \cdot d t}{n}+H_{\mathrm{v}}
$$

En todas las mezclas consideradas (aquellas presentadas en el Capítulo 1) esta corrección ha resultado ser menor que el $4 \%$ del valor de la entalpía de mezcla. No obstante, en los sistemas cetona + piridina en la región de elevada composición en la piridina la corrección por la fase de vapor puede llegar a ser del orden del $40 \%$ (por ejemplo, en el sistema 2 -propanona $(1)+$ piridina $(2)$, es igual al $26 \%$ para $x_{1}=0.1137$ ). Por esta razón y porque los valores estimados de $H_{\mathrm{m}}^{\mathrm{E}}$ de estas disoluciones son del orden del error de escala del aparato, no se consideraron estas mezclas. Quizá la calorimetría de flujo aporte una manera apropiada de determinar estas entalpías, ya que en los calorímetros de flujo la fase de vapor es despreciable o incluso nula.

El error relativo de la entalpía de exceso viene dado por la ecuación:

$$
\varepsilon\left(H_{\mathrm{m}}^{\mathrm{E}}\right)=\frac{\Delta H_{\mathrm{m}}^{\mathrm{E}}}{H_{\mathrm{m}}^{\mathrm{E}}}=\varepsilon(C)+\varepsilon\left(\int_{t_{1}}^{t_{2}} V \cdot d t\right)+\varepsilon(n)+\frac{\Delta H_{\mathrm{v}}}{H_{\mathrm{m}}^{\mathrm{E}}}
$$

En esta expresión se observa la contribución a $\varepsilon\left(H_{\mathrm{m}}^{\mathrm{E}}\right)$ de cuatro términos:

a) $\quad \varepsilon(n)$, error en la determinación del número de moles. Riesco [172] demuestra que este error es siempre menor de $\pm 0,017 \%$.

b) $\varepsilon\left(\int_{t_{1}}^{t_{2}} V \cdot d t\right)$ el error debido a la integración del voltaje Seebeck, que se calcula de la manera siguiente:

Supóngase que se han medido los puntos $\left(V_{1}, t_{1}\right),\left(V_{2}, t_{2}\right), \ldots,\left(V_{\mathrm{N}}, t_{\mathrm{N}}\right)$ del voltaje Seebeck que se desea integrar en el intervalo de tiempo $\left[t_{1}, t_{\mathrm{N}}\right]$. Se calcula una cota inferior de esta integral por la expresión $\operatorname{mín}\left\{V_{2}, V_{1}\right\} \cdot\left(t_{2}-t_{1}\right)+\operatorname{mín}\left\{V_{3}, V_{2}\right\} \cdot\left(t_{3}-t_{2}\right)+\ldots+$ $\operatorname{mín}\left\{V_{\mathrm{N}}, V_{\mathrm{N}-1}\right\} \cdot\left(t_{\mathrm{N}}-t_{\mathrm{N}-1}\right)$ y una cota superior por la expresión máx $\left\{V_{2}, V_{1}\right\} \cdot\left(t_{2}-t_{1}\right)+$ máx $\left\{V_{3}, V_{2}\right\} \cdot\left(t_{3}-t_{2}\right)+\ldots+\operatorname{máx}\left\{V_{\mathrm{N}}, V_{\mathrm{N}-1}\right\} \cdot\left(t_{\mathrm{N}}-t_{\mathrm{N}-1}\right)$. Se asume que el valor de la integral viene dado por la semisuma de ambas cotas y el error por la diferencia entre esta semisuma y una de las cotas. El error asociado a esta forma de integración será tanto mayor cuanto mayor sea la diferencia entre la cota superior e inferior, lo que equivale a decir que será mayor cuanto mayor sea la pendiente de la curva que dibuja los datos experimentales, como ocurre en el momento en el que los componentes se empiezan a mezclar o cuando se realiza la calibración dinámica del aparato. No obstante, si el multímetro registrador de $V$ tiene velocidad de muestreo muy alta, los intervalos $\left[t_{1}, t_{2}\right],\left[t_{2}, t_{3}\right], \ldots,\left[t_{\mathrm{N}-1} t_{\mathrm{N}}\right]$ pueden tomarse pequeños y el error en la integración será pequeño también. Esta es una razón más por la que la sustitución del multímetro Philips PM2534 por el Keithley 2000 ha contribuido positivamente a la evolución del calorímetro Tian-Calvet del G.E.T.E.F..

c) $\varepsilon(C)$, error de la constante de calibrado. Como se ha probado es igual $\pm 0,013$ $\mathrm{mW} \cdot \mathrm{mV}^{-1}(0.09 \%)$. 
d) $\Delta H_{\mathrm{v}} / H_{\mathrm{m}}^{\mathrm{E}}$ representa la contribución al error de la entalpía debido a la evaporización. En todos los sistemas medidos esta contribución era muy pequeña por lo que ha sido ignorada.

Falta tener en cuenta otra contribución a $\varepsilon\left(H_{\mathrm{m}}^{\mathrm{E}}\right)$, de tipo sistemático y que está relacionada con el hecho de que el calibrado podría haberse realizado de forma descuidada, sin haber tenido en cuenta algún efecto, con alguno de los componentes del sistema de calibración en mal estado o contaminado, etc. Para evaluar de algún modo este error sistemático, se determinan las entalpías de exceso de uno o varios sistemas test y se comparan con los datos reportados en la bibliografía, de la misma manera en cómo se hizo en el caso de la determinación de los volúmenes de exceso y compresibilidades isoentrópicas de exceso (véase la sección 3.3.2.). McGlashan [1] da algunos criterios para la elección del sistema test adecuado:

a) Las presiones de vapor de los componentes deben ser lo suficientemente distintas como para que los errores debidos a la presencia de fase vapor sean detectables.

b) Las densidades de ambos componentes deben ser lo suficientemente diferentes como para que los errores debidos a que la mezcla no es completa sean detectables.

c) Las capacidades caloríficas de exceso $C_{P}^{\mathrm{E}}$ no deben ser tan grandes como para necesitar medidas de la temperatura con precisión mejor que $0.05 \mathrm{~K}$.

d) Los pares de líquidos deben escogerse de modo que $H_{\mathrm{m}}^{\mathrm{E}}\left(x_{1}=0.5\right)$ sea del orden de $+100,-100,+1000$ y $-1000 \mathrm{~J} \cdot \mathrm{mol}-1$, para así cubrir un rango muy representativo de entalpías de mezcla.

Wadsö \& Goldberg [184] discuten los principios de la microcalorimetría isoterma y proponen una terminología estándar y unos sistemas de calibración y test. En particular, para el caso de mezclas entre líquidos orgánicos se citan las referencias [185] y [186] y se proponen las siguientes mezclas orgánicas como sistemas test en la medición de entalpías de exceso: ciclohexano $+n$-hexano; 1,4-dioxano + tetraclorometano; metanol + agua; etanol + agua. No obstante, por razones de seguridad y de salud se desaconseja el uso del dioxano, el tetraclorometano y el metanol, por lo que los sistemas 1,4-dioxano + tetraclorometano y metanol + agua no se utilizaron como sistema test. Tampoco se utilizó el sistema etanol + agua, pues no se disponía en el laboratorio de agua con la pureza suficiente como para ser utilizada en cualquier test de calidad. Desafortunadamente, tampoco se pudo utilizar el sistema ciclohexano + $n$-hexano debido a problemas con la pureza del $n$-hexano*. Por estas razones se desecharon los sistemas propuestos por Wadsö \& Goldberg y se consideró como criterio para la elección del sistema test el mismo que se utilizó en el caso de los volúmenes de exceso: un sistema test apropiado es aquél cuyas $H_{\mathrm{m}}^{\mathrm{E}}$ hayan sido determinadas en un gran número de ocasiones. Por ello, se escogieron como sistemas test las mezclas ciclohexano (1) + benceno (2) y ciclohexano (1) + 2,2,4-trimetilpentano (isooctano) (2). En las Tablas 3-4 y $4-3$ se presentan los datos de los líquidos puros utilizados.

\footnotetext{
* Incluso tras haber solicitado a las casas comerciales muestras de mayor pureza, el análisis de la densidad (que puede ser utilizado como criterio de pureza) reveló que no era lo suficientemente puro.
} 
Tabla 4-3: características a 298.15 K y presión atmosférica del 2,2,4-trimetilpentano, utilizado para comprobar el calibrado del microcalorímetro Tian-Calvet. Densidad, $\rho .{ }^{a}[187] ;{ }^{b}[185]$;

\begin{tabular}{ccc}
\hline & $\left(\mathrm{CH}_{3}\right)_{3} \mathrm{CCH}_{2} \mathrm{CH}\left(\mathrm{CH}_{3}\right)_{2}$ \\
\hline Procedencia & \multicolumn{2}{c}{ Fluka puriss } \\
& \multicolumn{2}{c}{ p.a. $\geq 99.5 \%(\mathrm{GC})$} \\
\hline$\rho / \mathrm{g} \cdot \mathrm{cm}^{3}$ & Exp & Lit \\
& 0.687852 & $0.68781^{\mathrm{a}}$ \\
& & $0.687849^{\mathrm{b}}$ \\
\hline
\end{tabular}

En la Tabla 4-4 aparecen los resultados experimentales obtenidos para los sistemas test: fracción molar del primer componente, entalpía de exceso, errores absoluto y relativo de las entalpías de exceso y corrección por el efecto de la evaporización. En las ilustraciones 4-9 y 4-10 se representan los resultados de $H_{\mathrm{m}}^{\mathrm{E}}$ frente a $x_{1}$ junto con la curva resultante del ajuste a la ecuación de Redlich-Kister (2.208), cuyos coeficientes se dan en la Tabla 4-5. En las ilustraciones también se han representado los resultados obtenidos por otros autores, observándose que la coincidencia con los datos obtenidos es muy buena.

Tabla 4-4: Entalpías molares de exceso $H_{\mathrm{m}}^{\mathrm{E}}$, error absoluto $\Delta H_{\mathrm{m}}^{\mathrm{E}}$, error relativo $\varepsilon\left(H_{\mathrm{m}}^{\mathrm{E}}\right)$ y corrección por fase de vapor de los sistemas test a $T=298,15 \mathrm{~K}$ y presión atmosférica

\begin{tabular}{|c|c|c|c|c|}
\hline$x_{1}$ & $H_{\mathrm{m}}^{\mathrm{E}} / \mathrm{J} \cdot \mathrm{mol}^{-1}$ & $\Delta H_{\mathrm{m}}^{\mathrm{E}} / \mathrm{J} \cdot \mathrm{mol}^{-1}$ & $\varepsilon\left(H_{\mathrm{m}}^{\mathrm{E}}\right) \%$ & $\begin{array}{c}\text { Corrección de } \\
\text { vapor } \%\end{array}$ \\
\hline \multicolumn{5}{|c|}{ ciclohexano (1) + 2,2,4-trimetilpentano (2) } \\
\hline 0.1069 & 338.6 & 1.3 & 0.4 & 1.1 \\
\hline 0.2179 & 555 & 2 & 0.4 & 0.7 \\
\hline 0.3014 & 680 & 3 & 0.4 & 0.6 \\
\hline 0.4045 & 786 & 3 & 0.3 & 0.5 \\
\hline 0.5000 & 814 & 3 & 0.4 & 0.5 \\
\hline 0.5991 & 764 & 3 & 0.3 & 0.5 \\
\hline 0.6955 & 668 & 2 & 0.3 & 0.6 \\
\hline 0.8012 & 504.0 & 1.8 & 0.3 & 0.8 \\
\hline 0.8934 & 295 & 1 & 0.3 & 1.3 \\
\hline \multicolumn{5}{|c|}{ ciclohexano (1) + isooctano (2) } \\
\hline 0.1026 & 69.5 & 0.4 & 0.5 & 1.7 \\
\hline 0.2119 & 130.8 & 0.5 & 0.4 & 0.6 \\
\hline 0.3065 & 161.2 & 0.7 & 0.4 & 0.4 \\
\hline 0.4030 & 180.7 & 0.7 & 0.4 & 3 \\
\hline 0.4989 & 186.3 & 0.7 & 0.4 & 3 \\
\hline 0.5957 & 169.0 & 0.7 & 0.4 & 3 \\
\hline 0.6974 & 141.1 & 0.5 & 0.4 & 4 \\
\hline 0.7935 & 104.8 & 0.4 & 0.4 & 5 \\
\hline 0.8913 & 57.8 & 0.3 & 0.5 & 9 \\
\hline
\end{tabular}




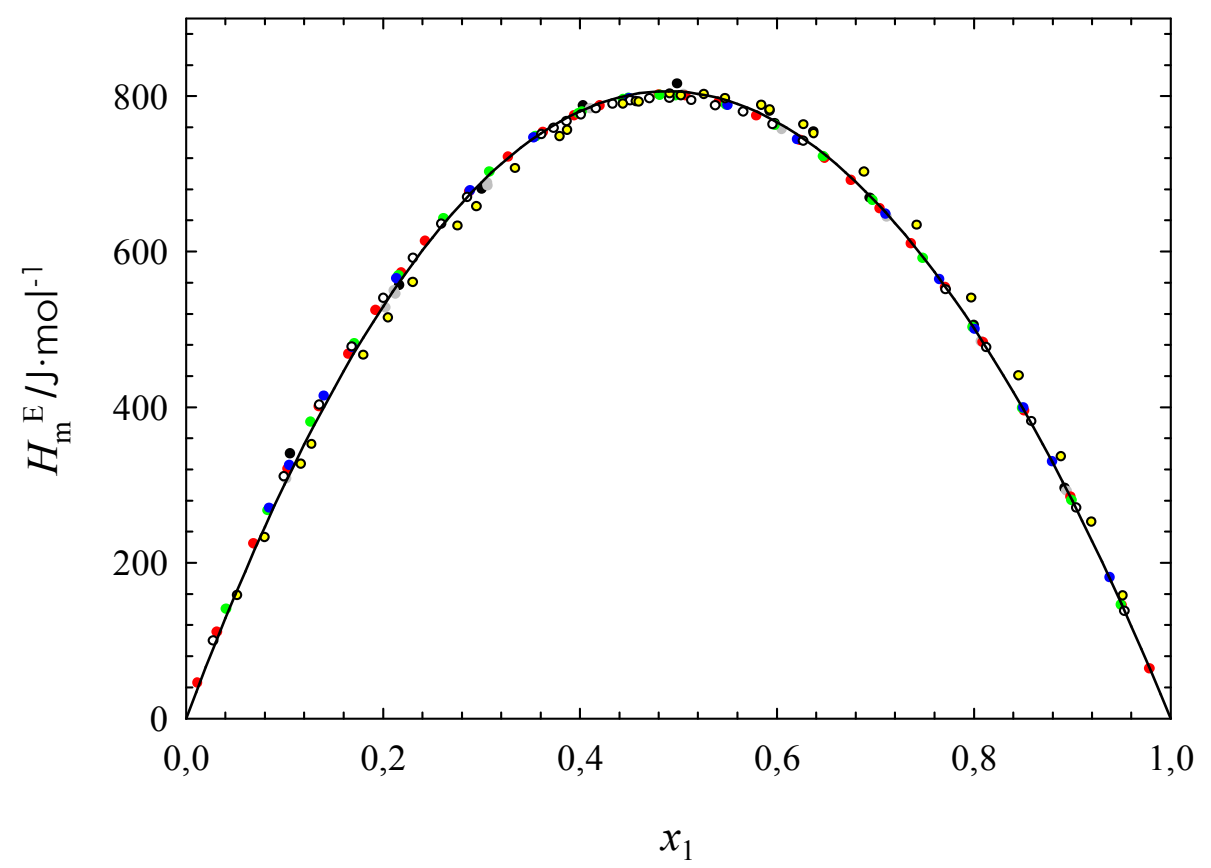

Ilustración 4-9: Entalpías molares de exceso a $T=298.15 \mathrm{~K}$ y presión atmosférica para el sistema test ciclohexano (1) + benceno (2):• este trabajo; • Mozo [173]; • Stokes [56]; • Nagata [188]; • Ellioł [189]; o Touhara [190]; $\bigcirc$ Murakami [148]; - curva del ajuste a la ecuación R-K con los coeficientes de la Tabla 4-5

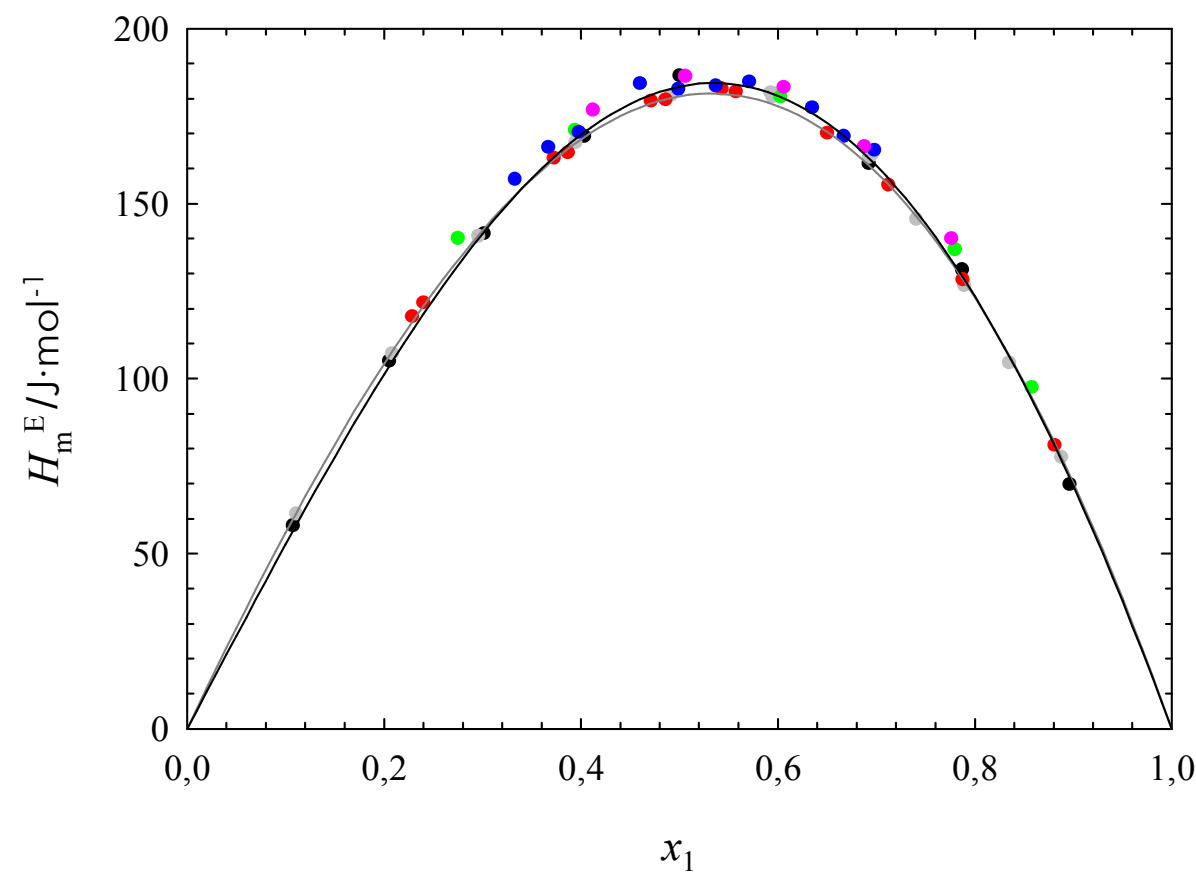

Ilustración 4-10: Entalpías molares de exceso a $T=298.15 \mathrm{~K}$ y presión atmosférica para el sistema test ciclohexano (1) + 2,2,4-trimetilpentano (2): • este trabajo; • Mozo [173]; • Heintz [191]; • Te Lam [192]; - Grolier [193]; - Heintz [194];- curva del ajuste a la ecuación R-K según los parámetros de Wang et al [195]; — curva del ajuste a la ecuación R-K con los coeficientes de la Tabla 4-5 
Tabla 4-5: coeficientes de los ajustes a la ecuación Redlich-Kister (2.208) de los datos experimentales obtenidos para los sistemas test a $T=298.15 \mathrm{~K}$ y presión atmosférica

\begin{tabular}{|c|c|c|c|c|}
\hline Sistema & $A_{0} / \mathrm{J} \cdot \mathrm{mol}^{-1}$ & $A_{1} / \mathrm{J} \cdot \mathrm{mol}^{-1}$ & $A_{2} / \mathrm{J} \cdot \mathrm{mol}^{-1}$ & $\sigma / \mathrm{J} \cdot \mathrm{mol}^{-1}$ \\
\hline $\begin{array}{c}\text { ciclohexano }(1)+ \\
\text { benceno }(2)\end{array}$ & $3223 \pm 17$ & $-150 \pm 50$ & & 10 \\
\hline $\begin{array}{c}\text { ciclohexano }(1)+ \\
2,2,4 \text {-trimetilpentano }(2)\end{array}$ & $734 \pm 4$ & $-116 \pm 9$ & $-90 \pm 20$ & 1.9 \\
\hline
\end{tabular}

A la vista de los resultados obtenidos para los dos sistemas test se puede confirmar que la calibración del microcalorímetro Tian-Calvet es lo suficientemente buena como para garantizar que los datos obtenidos con su error son exactos.

\subsubsection{Mezclas binarias amina + cetona y $\mathrm{N}$-metilanilina + alcano}

Con la ayuda del microcalorímetro Tian-Calvet, en este trabajo se han determinado a $298.15 \mathrm{~K}$ y presión atmosférica las entalpías de exceso de las mezclas binarias amina + cetona y $\mathrm{N}$-metilanilina + alcano que se propusieron en el Capítulo 1, en el marco del subproyecto NCO del proyecto T.O.M. Como se ha escrito en la sección 0, los sistemas cetona + piridina fueron excluidos del estudio puesto que los valores estimados de $H_{\mathrm{m}}^{\mathrm{E}}$ son del orden del error de escala del aparato, y la corrección debida a la fase de vapor puede llegar a ser del $40 \%$ para esos sistemas, mientras que para los sistemas propuestos la corrección por fase de vapor es, a lo sumo, igual al $9 \%$ del valor de la entalpía molar.

Las propiedades de los compuestos puros utilizados son las que aparecen en las Tablas 3-7 y 3-8 de la sección 3.3.3. En dicha sección ya se explicó el método de manipulación de dichas sustancias y por eso no es necesario repetirlo ahora.

En todos los casos se ha encontrado que el error relativo de la entalpía de mezcla es menor o igual a $0.4 \%$, por lo que en el peor de los casos, el error absoluto es $7 \mathrm{~J} \cdot \mathrm{mol}^{-1}$; por ello los resultados se escriben redondeados a la unidad de $\mathrm{J} \cdot \mathrm{mol}^{-1}$. En lo que sigue debe tenerse esto en mente pues las entalpías de exceso no se escribirán con su error con el objetivo de simplificar la presentación de los datos.

De la Tabla 4-6 a la Tabla 4-75 se presentan los siguientes datos correspondientes a los diferentes sistemas medidos a presión atmosférica:

a) Las fracciones molares del componente (1), $x_{1}$, las entalpías molares de exceso, $H_{\mathrm{m}}^{\mathrm{E}}$ y las energías internas de exceso a volumen constante, $U_{\mathrm{m}, V}^{\mathrm{E}}$, calculadas a partir de la ecuación (2.203):

$$
U_{\mathrm{m}, V}^{\mathrm{E}}=H_{\mathrm{m}}^{\mathrm{E}}-T \cdot \frac{\alpha_{P}}{\kappa_{T}} \cdot V_{\mathrm{m}}^{\mathrm{E}}
$$

a $298.15 \mathrm{~K}$ y presión atmosférica de las mezclas consideradas. En la ecuación anterior, se utilizan los volúmenes de exceso y los coeficientes de expansión térmica dados en el Capítulo 3. La ausencia de datos del volumen de exceso para los sistemas di- $n$ propilamina (1), di- $n$-butilamina (1) $\circ N, N, N$-trietilamina (1) +2 -pentanona (2) y $N$ metilanilina (1) $+n$-octano (2) o $n$-decano (2) es responsable de que para dichos sistemas no se hayan calculado los valores $\operatorname{de} U_{\mathrm{m}, V}^{\mathrm{E}}$. Los coeficientes de compresibilidad isoterma fueron calculados mediante la ecuación: 


$$
\kappa_{T}=\kappa_{S}+T \cdot V_{\mathrm{m}} \cdot \frac{\alpha_{P}^{2}}{C_{P, \mathrm{~m}}}
$$

donde $V_{\mathrm{m}}=V_{\mathrm{m}}^{\mathrm{id}}+V_{\mathrm{m}}^{\mathrm{E}}$ y se supone que $C_{P, \mathrm{~m}}=C_{P, \mathrm{~m}}^{\mathrm{id}}=\sum_{i=1}^{n} x_{\mathrm{i}} \cdot C_{P, \mathrm{i}}^{0}$ ya que no se dispone de datos para las capacidades caloríficas de las mezclas estudiadas.

b) Los coeficientes del ajuste de los datos obtenidos para todas estas magnitudes a la ecuación Redlich-Kister (2.208) junto con las desviaciones estándar de dichos ajustes.

Dichas tablas están acompañadas por diversas gráficas, desde la llustración 4- 11 a la Ilustración 4-35 en las cuales se representan como función de la composición del componente 1, $x_{1}$, las magnitudes siguientes:

a) Las entalpías molares de exceso, $H_{\mathrm{m}}^{\mathrm{E}}(\bullet)$, las energías internas de exceso a volumen constante, $U_{\mathrm{m}, V}^{\mathrm{E}}(\mathbf{\Lambda})$, y las curvas resultantes del ajuste a la ecuación R-K (2.208), $H_{\mathrm{m}}^{\mathrm{E}}=H_{\mathrm{m}}^{\mathrm{E}}\left(x_{1}\right)(-), U_{\mathrm{m}, V}^{\mathrm{E}}=U_{\mathrm{m}, V}^{\mathrm{E}}\left(x_{1}\right)(-)$ a $298.15 \mathrm{~K}$ y presión atmosférica.

b) Las entalpías molares de exceso reducidas, $\frac{H_{\mathrm{m}}^{\mathrm{E}}}{x_{1} \cdot\left(1-x_{1}\right)}=g\left(x_{1}\right)$ y la curva resultante del ajuste a la ecuación R-K de forma reducida.

c) Las entalpías molares parciales de exceso de cada uno de los componentes que forman la mezcla, $H_{\mathrm{m}, 1}^{\mathrm{E}}=H_{\mathrm{m}, 1}^{\mathrm{E}}\left(x_{1}\right)(-), H_{\mathrm{m}, 2}^{\mathrm{E}}=H_{\mathrm{m}, 2}^{\mathrm{E}}\left(x_{1}\right)(-\cdot-)$, calculados de acuerdo con las ecuaciones $(2.121)$ y $(2.122)$ y el ajuste $\mathrm{R}-\mathrm{K}, H_{\mathrm{m}}^{\mathrm{E}}=H_{\mathrm{m}}^{\mathrm{E}}\left(x_{1}\right)$, a $298.15 \mathrm{~K}$. 
4.2. RESULTADOS EXPERIMENTALES

Esta página está en blanco intencionadamente 
Tabla 4-6: entalpías molares de exceso del sistema anilina (1) + 2-propanona (2)*

\begin{tabular}{cccccc}
\hline$x_{1}$ & $H_{\mathrm{m}}^{\mathrm{E}} / \mathrm{J} \cdot \mathrm{mol}^{-1}$ & $x_{1}$ & $H_{\mathrm{m}}^{\mathrm{E}} / \mathrm{J} \cdot \mathrm{mol}^{-1}$ & $x_{1}$ & $H_{\mathrm{m}}^{\mathrm{E}} / \mathrm{J} \cdot \mathrm{mol}^{-1}$ \\
\hline 0.0117 & -64 & 0.1182 & -515 & 0.8903 & -381 \\
0.0208 & -109 & 0.1196 & -521 & 0.9046 & -332 \\
0.0305 & -155 & 0.1955 & -816 & 0.9075 & -320 \\
0.0410 & -203 & 0.3009 & -1076 & 0.9104 & -317 \\
0.0509 & -246 & 0.3985 & -1219 & 0.9203 & -281 \\
0.0606 & -288 & 0.4918 & -1245 & 0.9301 & -247 \\
0.0700 & -329 & 0.4986 & -1243 & 0.9397 & -214 \\
0.0788 & -364 & 0.5893 & -1165 & 0.9496 & -179 \\
0.0897 & -411 & 0.6948 & -965 & 0.9599 & -145 \\
0.0977 & -430 & 0.7855 & -746 & 0.9697 & -109 \\
0.0999 & -446 & 0.8897 & -376 & 0.9797 & -75 \\
0.1035 & -456 & 0.8902 & -371 & 0.9894 & -40 \\
0.1113 & -489 & & & & \\
\hline
\end{tabular}

Tabla 4-7: energías molares de exceso a $V=$ cte. del sistema anilina (1) + 2-propanona (2)

\begin{tabular}{cccccc}
\hline$x_{1}$ & $U_{\mathrm{m}, V}^{\mathrm{E}} / \mathrm{J} \cdot \mathrm{mol}^{-1}$ & $x_{1}$ & $U_{\mathrm{m}, V}^{\mathrm{E}} / \mathrm{J} \cdot \mathrm{mol}^{-1}$ & $x_{1}$ & $U_{\mathrm{m}, V}^{\mathrm{E}} / \mathrm{J} \cdot \mathrm{mol}^{-1}$ \\
\hline 0.0455 & -118 & 0.4078 & -691 & 0.6961 & -528 \\
0.1049 & -258 & 0.4499 & -706 & 0.7516 & -444 \\
0.1467 & -350 & 0.4965 & -702 & 0.8022 & -359 \\
0.1918 & -435 & 0.5415 & -684 & 0.8461 & -278 \\
0.2478 & -533 & 0.5886 & -651 & 0.8912 & -196 \\
0.3007 & -602 & 0.6495 & -586 & 0.9431 & -100 \\
0.3501 & -655 & & & & \\
\hline
\end{tabular}

Tabla 4-8: coeficientes y desviaciones estándar del ajuste a la ecuación R-K de las diferentes propiedades de exceso del sistema anilina (1) +2 -propanona (2)

\begin{tabular}{ccccc}
\hline Propiedad & $A_{0}$ & $A_{1}$ & $A_{2}$ & $\sigma$ \\
\hline$H_{\mathrm{m}}^{\mathrm{E}} / \mathrm{J} \cdot \mathrm{mol}^{-1}$ & -4972 & 700 & 820 & 8 \\
$U_{\mathrm{m}, V}^{\mathrm{E}} / \mathrm{J} \cdot \mathrm{mol}^{-1}$ & -2796 & 477 & 690 & 2 \\
\hline
\end{tabular}

* Las entalpías de exceso de este sistema en las regiones de baja concentración fueron determinadas con el objetivo de desarrollar un procedimiento de medida que fuese válido para la obtención de las entalpías de exceso de mezclas ternarias, el cual se describe en la sección 0. Además, de todas las mezclas consideradas en este trabajo, esta es la única para la que se encontraron datos en la bibliografía, los cuales se representan en la llustración 4-11 junto con los datos obtenidos que se dan en la Tabla 4-6. 

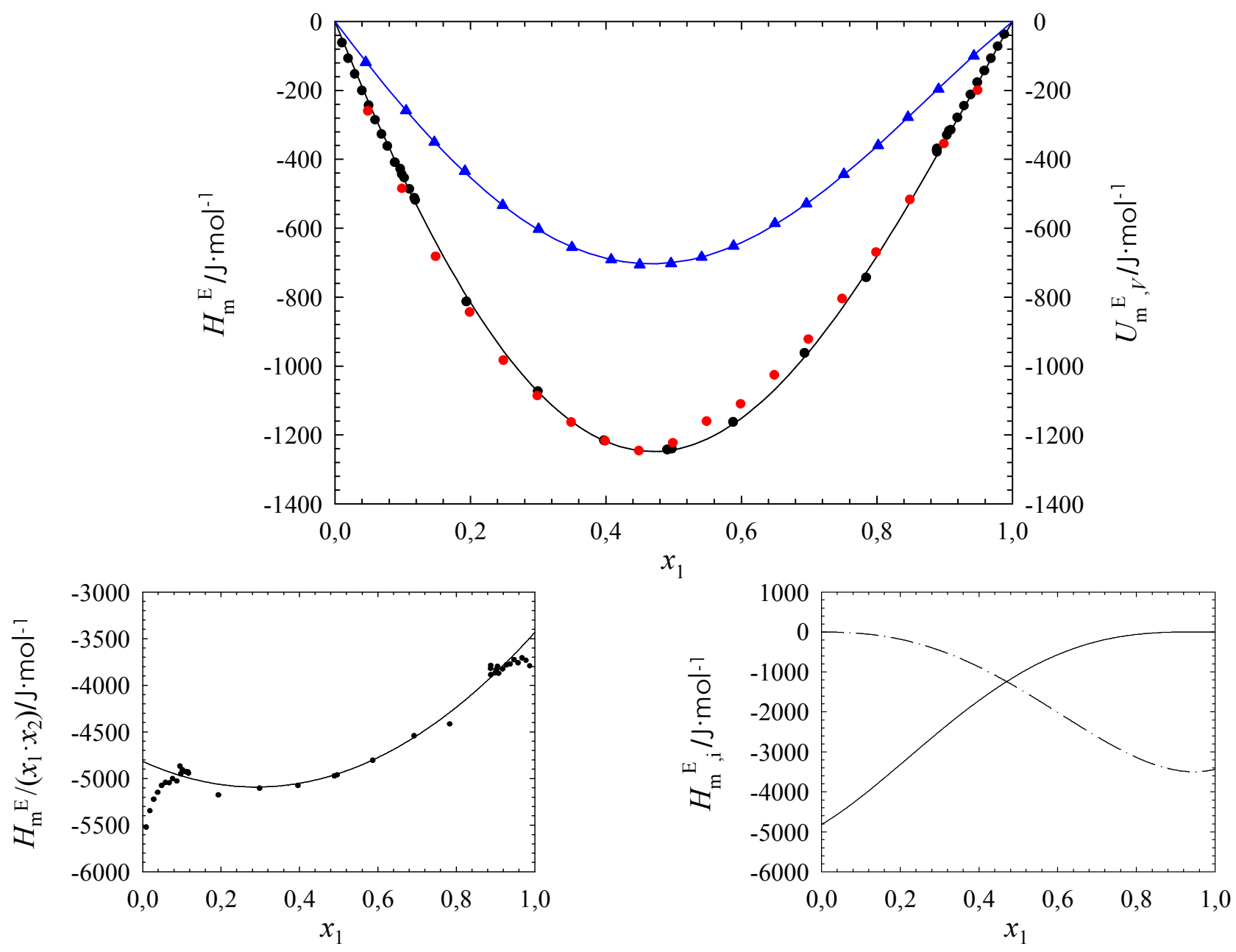

Ilustración 4-11: entalpías molares de exceso $H_{\mathrm{m}}^{\mathrm{E}}$, energías internas de exceso a volumen constante $U_{\mathrm{m}, V}^{\mathrm{E}}$, entalpías molares de exceso reducidos $H_{\mathrm{m}}^{\mathrm{E}} /\left(x_{1} \cdot x_{2}\right)$ y entalpías molares parciales de exceso $H_{\mathrm{m}, \mathrm{i}}^{\mathrm{E}}$ del sistema anilina (1) + 2-propanona (2); • datos de Nagata \& Ksiazczak [196] 
Tabla 4-9: entalpías molares de exceso del sistema anilina (1) + 2-butanona (2)

\begin{tabular}{cccc}
\hline$x_{1}$ & $H_{\mathrm{m}}^{\mathrm{E}} / \mathrm{J} \cdot \mathrm{mol}^{-1}$ & $x_{1}$ & $H_{\mathrm{m}}^{\mathrm{E}} / \mathrm{J} \cdot \mathrm{mol}^{-1}$ \\
\hline 0.0982 & -469 & 0.5884 & -1075 \\
0.1936 & -814 & 0.6879 & -879 \\
0.2991 & -1071 & 0.7901 & -648 \\
0.3914 & -1171 & 0.8435 & -499 \\
0.4894 & -1173 & 0.8844 & -378 \\
\hline
\end{tabular}

Tabla 4-10: energías molares de exceso a $V=$ cte. del sistema anilina (1) + 2-butanona (2)

\begin{tabular}{cccccc}
\hline$x_{1}$ & $U_{\mathrm{m}, V}^{\mathrm{E}} / \mathrm{J} \cdot \mathrm{mol}^{-1}$ & $x_{1}$ & $U_{\mathrm{m}, V}^{\mathrm{E}} / \mathrm{J} \cdot \mathrm{mol}^{-1}$ & $x_{1}$ & $U_{\mathrm{m}, V}^{\mathrm{E}} / \mathrm{J} \cdot \mathrm{mol}^{-1}$ \\
\hline 0.0511 & -154 & 0.3981 & -639 & 0.6936 & -426 \\
0.0981 & -277 & 0.4533 & -636 & 0.7437 & -353 \\
0.1513 & -396 & 0.4974 & -623 & 0.7962 & -272 \\
0.1970 & -480 & 0.5477 & -591 & 0.8453 & -199 \\
0.2549 & -556 & 0.5911 & -553 & 0.8882 & -153 \\
0.2952 & -587 & 0.6526 & -483 & 0.9440 & -90 \\
0.3519 & -628 & & & & \\
\hline
\end{tabular}

Tabla 4-11: coeficientes y desviaciones estándar del ajuste a la ecuación R-K de las diferentes propiedades de exceso del sistema anilina (1) + 2-butanona (2)

\begin{tabular}{ccccc}
\hline Propiedad & $A_{0}$ & $A_{1}$ & $A_{2}$ & $\sigma$ \\
\hline$H_{\mathrm{m}}^{\mathrm{E}} / \mathrm{J} \cdot \mathrm{mol}^{-1}$ & -4660 & 1150 & 300 & 300 \\
$U_{\mathrm{m}, V}^{\mathrm{E}} / \mathrm{J} \cdot \mathrm{mol}^{-1}$ & -2470 & 1070 & 290 & 7 \\
\hline
\end{tabular}



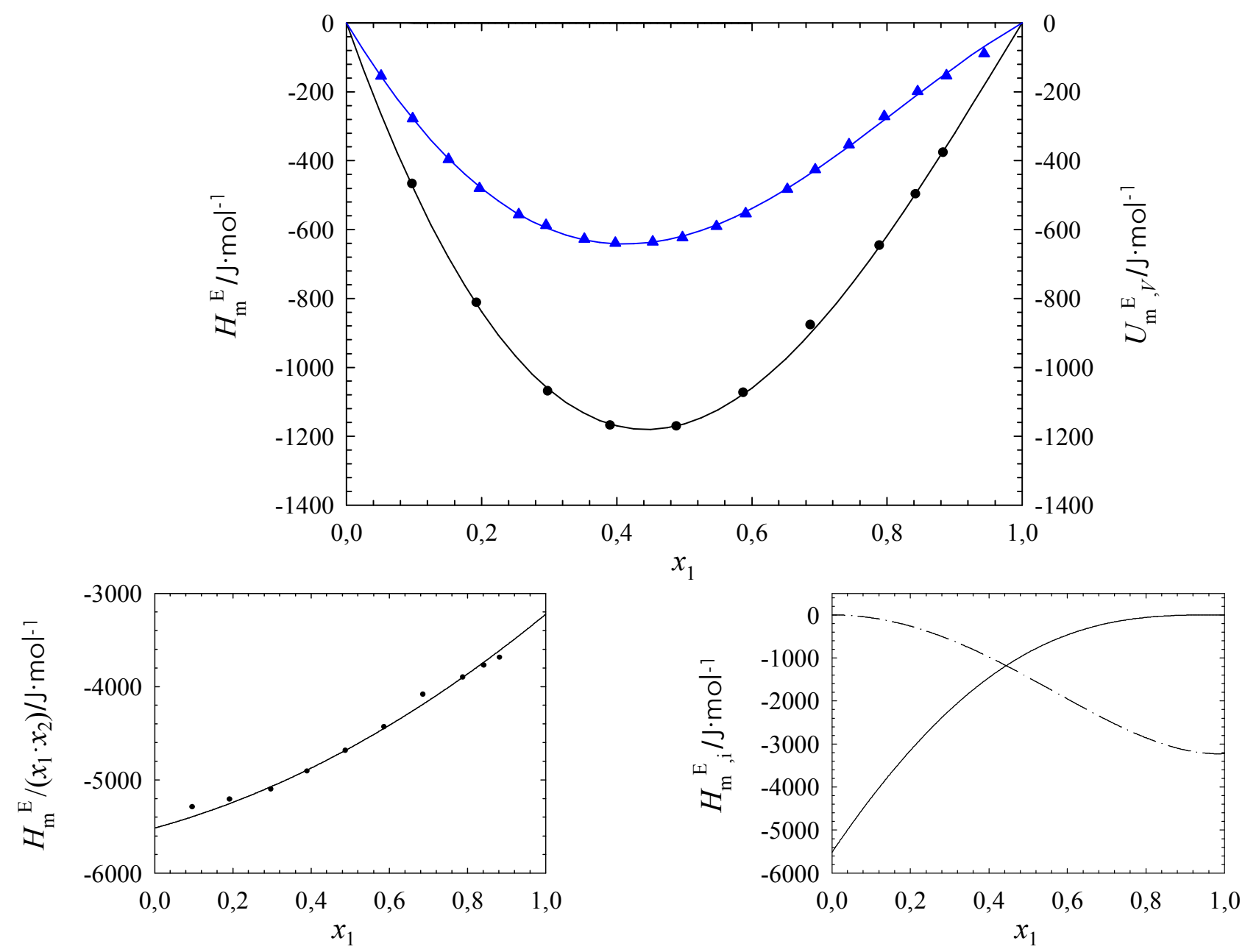

Ilustración 4-12: entalpías molares de exceso $H_{\mathrm{m}}^{\mathrm{E}}$, energías internas de exceso a volumen constante $U_{\mathrm{m}, V}^{\mathrm{E}}$, entalpías molares de exceso reducidos $H_{\mathrm{m}}^{\mathrm{E}} /\left(x_{1} \cdot x_{2}\right)$ y entalpías molares parciales de exceso $H_{\mathrm{m}, \mathrm{i}}^{\mathrm{E}}$ del sistema anilina (1) +2 -butanona (2) 
Tabla 4-12: entalpías molares de exceso del sistema anilina (1) + 2-pentanona (2)

\begin{tabular}{cccc}
\hline$x_{1}$ & $H_{\mathrm{m}}^{\mathrm{E}} / \mathrm{J} \cdot \mathrm{mol}^{-1}$ & $x_{1}$ & $H_{\mathrm{m}}^{\mathrm{E}} / \mathrm{J} \cdot \mathrm{mol}^{-1}$ \\
\hline 0.1005 & -432 & 0.4933 & -1028 \\
0.2021 & -737 & 0.5927 & -926 \\
0.2892 & -940 & 0.6920 & -766 \\
0.3912 & -1051 & 0.7948 & -511 \\
0.3951 & -1049 & 0.8887 & -293 \\
0.4912 & -1030 & & \\
\hline
\end{tabular}

Tabla 4-13: energías molares de exceso a $V=$ cte. del sistema anilina (1) + 2-pentanona (2)

\begin{tabular}{cccccc}
\hline$x_{1}$ & $U_{\mathrm{m}, V}^{\mathrm{E}} / \mathrm{J} \cdot \mathrm{mol}^{-1}$ & $x_{1}$ & $U_{\mathrm{m}, V}^{\mathrm{E}} / \mathrm{J} \cdot \mathrm{mol}^{-1}$ & $x_{1}$ & $U_{\mathrm{m}, V}^{\mathrm{E}} / \mathrm{J} \cdot \mathrm{mol}^{-1}$ \\
\hline 0.0610 & -181 & 0.3945 & -636 & 0.7007 & -383 \\
0.1110 & -291 & 0.4489 & -621 & 0.7487 & -311 \\
0.1607 & -400 & 0.4995 & -597 & 0.8061 & -229 \\
0.2057 & -480 & 0.5493 & -561 & 0.8503 & -169 \\
0.2559 & -550 & 0.5942 & -515 & 0.9052 & -100 \\
0.3037 & -596 & 0.6464 & -451 & 0.9554 & -43 \\
0.3523 & -623 & & & & \\
\hline
\end{tabular}

Tabla 4-14: coeficientes y desviaciones estándar del ajuste a la ecuación R-K de las diferentes propiedades de exceso del sistema anilina (1) + 2-pentanona (2)

\begin{tabular}{cccccc}
\hline Propiedad & $A_{0}$ & $A_{1}$ & $A_{2}$ & $A_{3}$ & $\sigma$ \\
\hline$H_{\mathrm{m}}^{\mathrm{E}} / \mathrm{J} \cdot \mathrm{mol}^{-1}$ & -4115 & 1340 & 540 & -300 & 10 \\
$U_{\mathrm{m}, V}^{\mathrm{E}} / \mathrm{J} \cdot \mathrm{mol}^{-1}$ & -2394 & 1300 & 480 & -230 & 3 \\
\hline
\end{tabular}



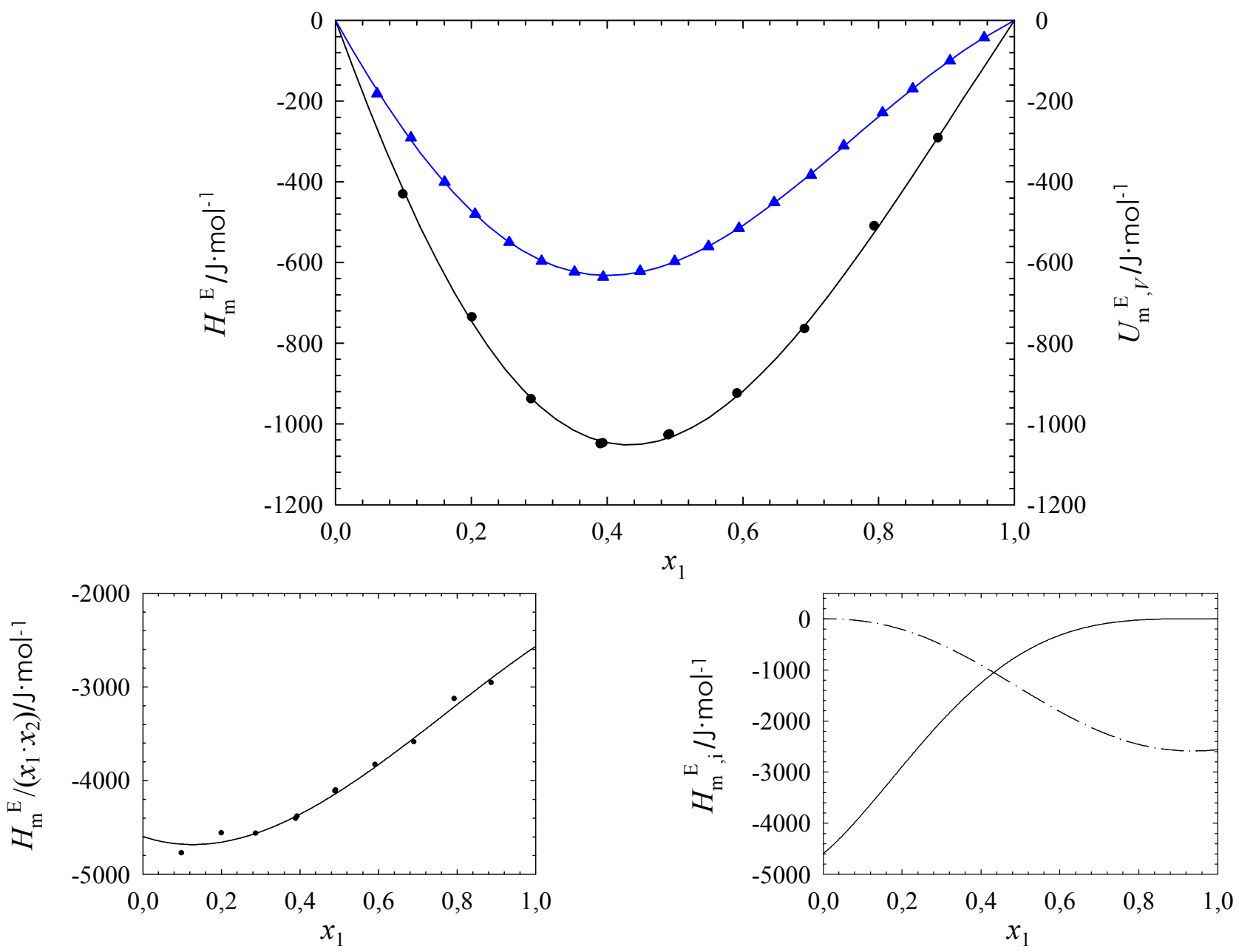

Ilustración 4-13 entalpías molares de exceso $H_{\mathrm{m}}^{\mathrm{E}}$, energías internas de exceso a volumen constante $U_{\mathrm{m}, V}^{\mathrm{E}}$, entalpías molares de exceso reducidos $H_{\mathrm{m}}^{\mathrm{E}} /\left(x_{1} \cdot x_{2}\right)$ y entalpías molares parciales de exceso $H_{\mathrm{m}, \mathrm{i}}^{\mathrm{E}}$ del sistema anilina (1) + 2-pentanona (2) 
Tabla 4-15: entalpías molares de exceso del sistema anilina (1) + 2-heptanona (2)

\begin{tabular}{cccc}
\hline$x_{1}$ & $H_{\mathrm{m}}^{\mathrm{E}} / \mathrm{J} \cdot \mathrm{mol}^{-1}$ & $x_{1}$ & $H_{\mathrm{m}}^{\mathrm{E}} / \mathrm{J} \cdot \mathrm{mol}^{-1}$ \\
\hline 0.1028 & -346 & 0.4446 & -724 \\
0.1519 & -472 & 0.4904 & -691 \\
0.1521 & -471 & 0.5477 & -647 \\
0.2066 & -565 & 0.5948 & -588 \\
0.2966 & -699 & 0.6918 & -449 \\
0.3974 & -740 & 0.7821 & -294 \\
0.3976 & -744 & 0.8876 & -131 \\
\hline
\end{tabular}

Tabla 4-16: energías molares de exceso a $V=$ cte. del sistema anilina (1) + 2-heptanona (2)

\begin{tabular}{cccccc}
\hline$x_{1}$ & $U_{\mathrm{m}, V}^{\mathrm{E}} / \mathrm{J} \cdot \mathrm{mol}^{-1}$ & $x_{1}$ & $U_{\mathrm{m}, V}^{\mathrm{E}} / \mathrm{J} \cdot \mathrm{mol}^{-1}$ & $x_{1}$ & $U_{\mathrm{m}, V}^{\mathrm{E}} / \mathrm{J} \cdot \mathrm{mol}^{-1}$ \\
\hline 0.0533 & -119 & 0.4036 & -404 & 0.6936 & -144 \\
0.0967 & -206 & 0.4570 & -374 & 0.7425 & -85 \\
0.1485 & -289 & 0.4908 & -349 & 0.7867 & -43 \\
0.1959 & -345 & 0.5463 & -301 & 0.8399 & 1 \\
0.2416 & -385 & 0.5998 & -242 & 0.8885 & 26 \\
0.2943 & -408 & 0.6362 & -210 & 0.9381 & 26 \\
0.3401 & -415 & & & & \\
\hline
\end{tabular}

Tabla 4-17: coeficientes y desviaciones estándar del ajuste a la ecuación R-K de las diferentes propiedades de exceso del sistema anilina (1) + 2-heptanona (2)

\begin{tabular}{ccccc}
\hline Propiedad & $A_{0}$ & $A_{1}$ & $A_{2}$ & $\sigma$ \\
\hline$H_{\mathrm{m}}^{\mathrm{E}} / \mathrm{J} \cdot \mathrm{mol}^{-1}$ & -2767 & 1570 & 450 & 6 \\
$U_{\mathrm{m}, V}^{\mathrm{E}} / \mathrm{J} \cdot \mathrm{mol}^{-1}$ & -1375 & 1654 & 531 & 3 \\
\hline
\end{tabular}



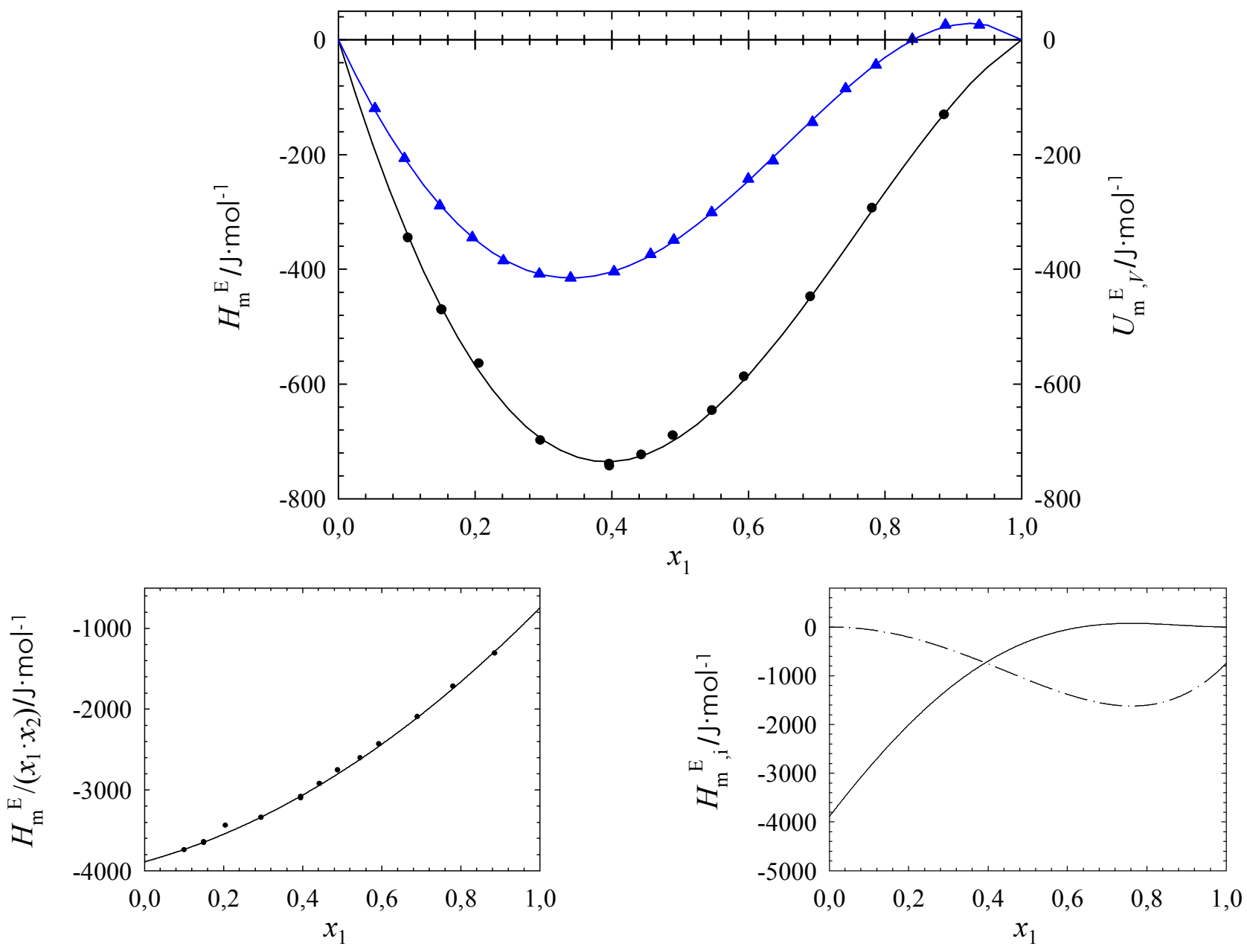

Ilustración 4-14: entalpías molares de exceso $H_{\mathrm{m}}^{\mathrm{E}}$, energías internas de exceso a volumen constante $U_{\mathrm{m}, V}^{\mathrm{E}}$, entalpías molares de exceso reducidos $H_{\mathrm{m}}^{\mathrm{E}} /\left(x_{1} \cdot x_{2}\right)$ y entalpías molares parciales de exceso $H_{\mathrm{m}, \mathrm{i}}^{\mathrm{E}}$ del sistema anilina (1) + 2-heptanona (2) 
Tabla 4-18: entalpías molares de exceso del sistema $N$-metilanilina (1) +2 -propanona (2)

\begin{tabular}{cccc}
\hline$x_{1}$ & $H_{\mathrm{m}}^{\mathrm{E}} / \mathrm{J} \cdot \mathrm{mol}^{-1}$ & $x_{1}$ & $H_{\mathrm{m}}^{\mathrm{E}} / \mathrm{J} \cdot \mathrm{mol}^{-1}$ \\
\hline 0.0988 & -204 & 0.5911 & -666 \\
0.1945 & -381 & 0.6809 & -598 \\
0.2903 & -525 & 0.7684 & -500 \\
0.3925 & -622 & 0.8714 & -335 \\
0.4914 & -672 & & \\
\hline
\end{tabular}

Tabla 4-19: energías molares de exceso a $V=$ cte. del sistema $N$-metilanilina (1) + 2-propanona (2)

\begin{tabular}{cccccc}
\hline$x_{1}$ & $U_{\mathrm{m}, V}^{\mathrm{E}} / \mathrm{J} \cdot \mathrm{mol}^{-1}$ & $x_{1}$ & $U_{\mathrm{m}, V}^{\mathrm{E}} / \mathrm{J} \cdot \mathrm{mol}^{-1}$ & $x_{1}$ & $U_{\mathrm{m}, V}^{\mathrm{E}} / \mathrm{J} \cdot \mathrm{mol}^{-1}$ \\
\hline 0.0512 & -49 & 0.3996 & -325 & 0.7081 & -354 \\
0.1014 & -91 & 0.4437 & -353 & 0.7497 & -328 \\
0.1482 & -147 & 0.5052 & -364 & 0.8004 & -287 \\
0.1991 & -186 & 0.5480 & -374 & 0.8553 & -244 \\
0.2494 & -236 & 0.6076 & -380 & 0.9016 & -194 \\
0.3038 & -257 & 0.6489 & -359 & 0.9560 & -112 \\
0.3527 & -297 & & & & \\
\hline
\end{tabular}

Tabla 4-20: coeficientes y desviaciones estándar del ajuste a la ecuación R-K de las diferentes propiedades de exceso del sistema $N$-metilanilina (1) +2 -propanona (2)

\begin{tabular}{cccccc}
\hline Propiedad & $A_{0}$ & $A_{1}$ & $A_{2}$ & $A_{3}$ & $\sigma$ \\
\hline$H_{\mathrm{m}}^{\mathrm{E}} / \mathrm{J} \cdot \mathrm{mol}^{-1}$ & -2672 & -340 & & & 8 \\
$U_{\mathrm{m}, V}^{\mathrm{E}} / \mathrm{J} \cdot \mathrm{mol}^{-1}$ & -1447 & -450 & -200 & -350 & 8 \\
\hline
\end{tabular}



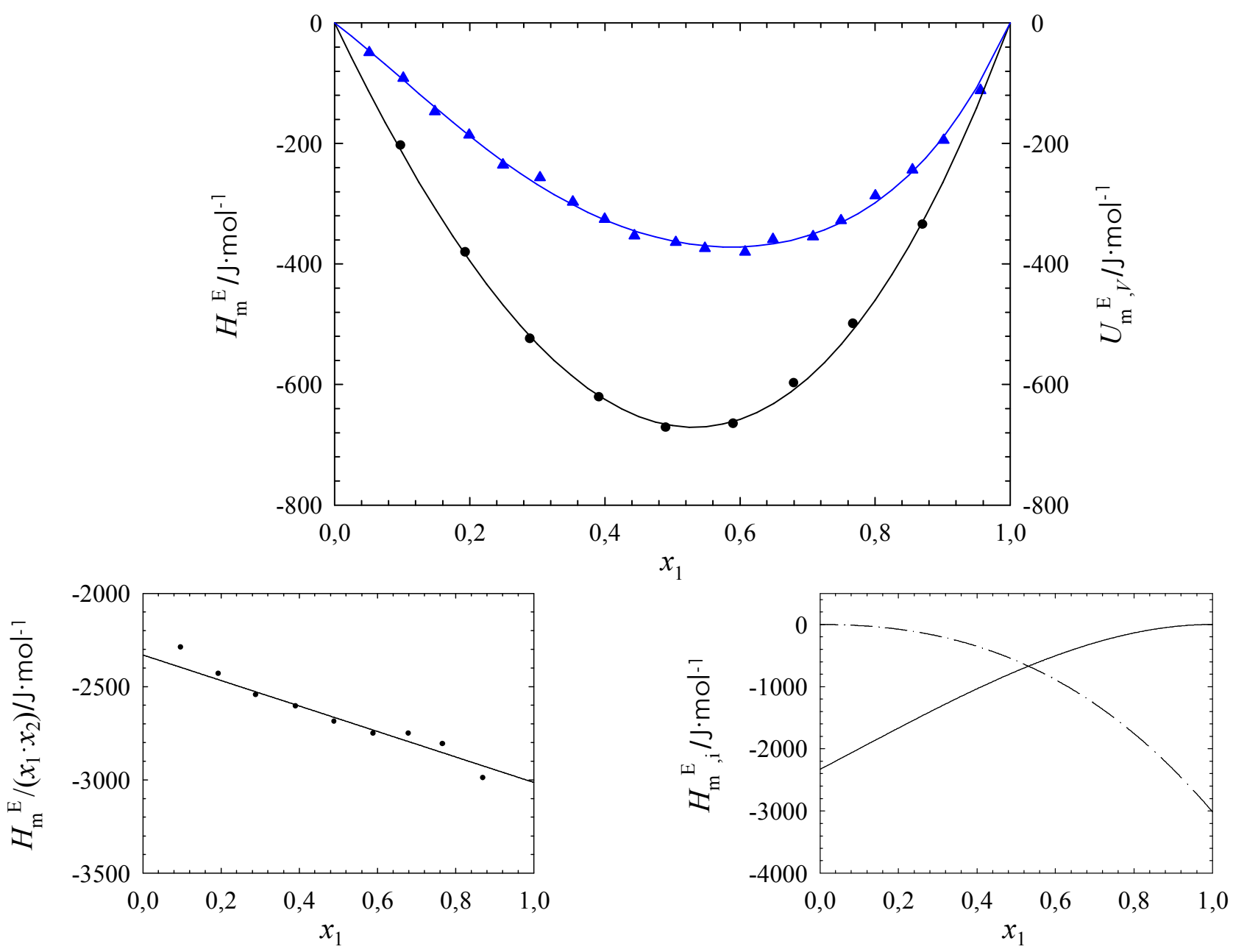

Ilustración 4-15: entalpías molares de exceso $H_{\mathrm{m}}^{\mathrm{E}}$, energías internas de exceso a volumen constante $U_{\mathrm{m}, V}^{\mathrm{E}}$, entalpías molares de exceso reducidos $H_{\mathrm{m}}^{\mathrm{E}} /\left(x_{1} \cdot x_{2}\right)$ y entalpías molares parciales de exceso $H_{\mathrm{m}, \mathrm{i}}^{\mathrm{E}}$ del sistema $N$-metilanilina (1) +2 -propanona (2) 
Tabla 4-21: entalpías molares de exceso del sistema $N$-metilanilina (1) + 2-butanona (2)

\begin{tabular}{cccc}
\hline$x_{1}$ & $H_{\mathrm{m}}^{\mathrm{E}} / \mathrm{J} \cdot \mathrm{mol}^{-1}$ & $x_{1}$ & $H_{\mathrm{m}}^{\mathrm{E}} / \mathrm{J} \cdot \mathrm{mol}^{-1}$ \\
\hline 0.0970 & -280 & 0.5907 & -781 \\
0.1972 & -506 & 0.6813 & -676 \\
0.2945 & -672 & 0.7717 & -540 \\
0.3909 & -779 & 0.8639 & -356 \\
0.4910 & -816 & & \\
\hline
\end{tabular}

Tabla 4-22: energías molares de exceso a $V=$ cte. del sistema $N$-metilanilina (1) +2 -butanona (2)

\begin{tabular}{cccccc}
\hline$x_{1}$ & $U_{\mathrm{m}, V}^{\mathrm{E}} / \mathrm{J} \cdot \mathrm{mol}^{-1}$ & $x_{1}$ & $U_{\mathrm{m}, V}^{\mathrm{E}} / \mathrm{J} \cdot \mathrm{mol}^{-1}$ & $x_{1}$ & $U_{\mathrm{m}, V}^{\mathrm{E}} / \mathrm{J} \cdot \mathrm{mol}^{-1}$ \\
\hline 0.0528 & -93 & 0.4008 & -521 & 0.7033 & -449 \\
0.1007 & -178 & 0.4529 & -541 & 0.7411 & -390 \\
0.1530 & -267 & 0.5050 & -544 & 0.7924 & -325 \\
0.1993 & -333 & 0.5523 & -533 & 0.8434 & -258 \\
0.2537 & -407 & 0.6027 & -520 & 0.8885 & -192 \\
0.3079 & -439 & 0.6529 & -478 & 0.9240 & -81 \\
\hline
\end{tabular}

Tabla 4-23: coeficientes y desviaciones estándar del ajuste a la ecuación R-K de las diferentes propiedades de exceso del sistema $N$-metilanilina (1) + 2-butanona (2)

\begin{tabular}{ccccc}
\hline Propiedad & $A_{0}$ & $A_{1}$ & $A_{2}$ & $\sigma$ \\
\hline$H_{\mathrm{m}}^{\mathrm{E}} / \mathrm{J} \cdot \mathrm{mol}^{-1}$ & -3248 & 140 & 330 & 6 \\
$U_{\mathrm{m}, V}^{\mathrm{E}} / \mathrm{J} \cdot \mathrm{mol}^{-1}$ & -2190 & 80 & 500 & 14 \\
\hline
\end{tabular}



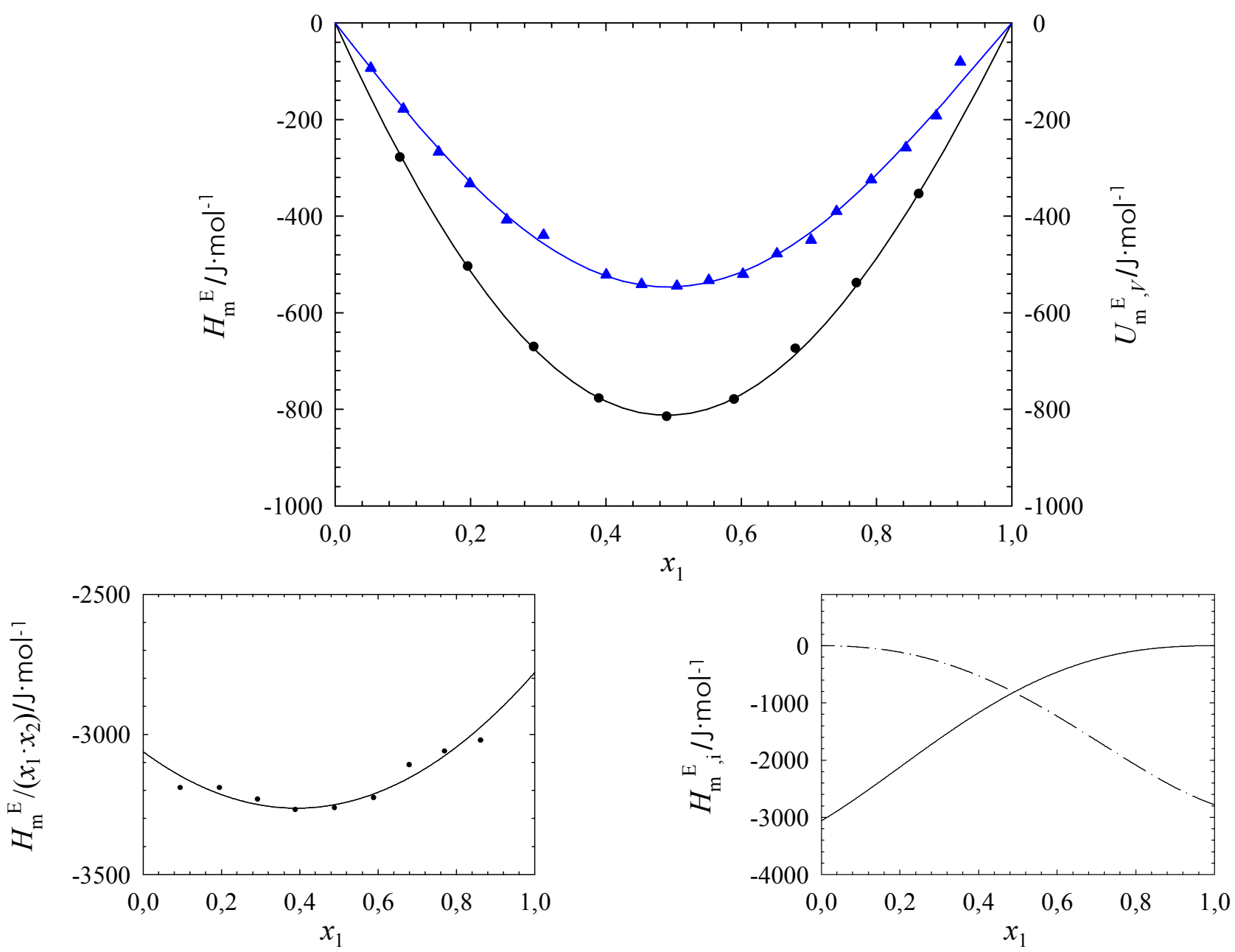

Ilustración 4-16: entalpías molares de exceso $H_{\mathrm{m}}^{\mathrm{E}}$, energías internas de exceso a volumen constante $U_{\mathrm{m}, V}^{\mathrm{E}}$, entalpías molares de exceso reducidos $H_{\mathrm{m}}^{\mathrm{E}} /\left(x_{1} \cdot x_{2}\right)$ y entalpías molares parciales de exceso $H_{\mathrm{m}, \mathrm{i}}^{\mathrm{E}}$ del sistema $N$-metilanilina (1) +2 -butanona (2) 
Tabla 4-24: entalpías molares de exceso del sistema $N$-metilanilina (1) + 2-pentanona (2)

\begin{tabular}{cccc}
\hline$x_{1}$ & $H_{\mathrm{m}}^{\mathrm{E}} / \mathrm{J} \cdot \mathrm{mol}^{-1}$ & $x_{1}$ & $H_{\mathrm{m}}^{\mathrm{E}} / \mathrm{J} \cdot \mathrm{mol}^{-1}$ \\
\hline 0.0969 & -292 & 0.5940 & -793 \\
0.1942 & -529 & 0.6761 & -693 \\
0.2956 & -707 & 0.7778 & -528 \\
0.3943 & -807 & 0.8703 & -332 \\
0.4917 & -837 & & \\
\hline
\end{tabular}

Tabla 4-25: energías molares de exceso a $V=$ cte. del sistema $N$-metilanilina (1) + 2-pentanona (2)

\begin{tabular}{cccccc}
\hline$x_{1}$ & $U_{\mathrm{m}, V}^{\mathrm{E}} / \mathrm{J} \cdot \mathrm{mol}^{-1}$ & $x_{1}$ & $U_{\mathrm{m}, V}^{\mathrm{E}} / \mathrm{J} \cdot \mathrm{mol}^{-1}$ & $x_{1}$ & $U_{\mathrm{m}, V}^{\mathrm{E}} / \mathrm{J} \cdot \mathrm{mol}^{-1}$ \\
\hline 0.0584 & -124 & 0.3948 & -550 & 0.6943 & -441 \\
0.1106 & -225 & 0.4520 & -564 & 0.7509 & -376 \\
0.1615 & -312 & 0.4986 & -565 & 0.8020 & -308 \\
0.2051 & -378 & 0.5456 & -552 & 0.8558 & -229 \\
0.2616 & -447 & 0.5963 & -525 & 0.9000 & -160 \\
0.3015 & -489 & 0.6502 & -484 & 0.9493 & -82 \\
0.3470 & -526 & & & & \\
\hline
\end{tabular}

Tabla 4-26: coeficientes y desviaciones estándar del ajuste a la ecuación R-K de las diferentes propiedades de exceso del sistema $N$-metilanilina (1) +2 -pentanona (2)

\begin{tabular}{ccccc}
\hline Propiedad & $A_{0}$ & $A_{1}$ & $A_{2}$ & $\sigma$ \\
\hline$H_{\mathrm{m}}^{\mathrm{E}} / \mathrm{J} \cdot \mathrm{mol}^{-1}$ & -3340 & 295 & 370 & 3 \\
$U_{\mathrm{m}, V}^{\mathrm{E}} / \mathrm{J} \cdot \mathrm{mol}^{-1}$ & -2254.4 & 311 & 352 & 0.6 \\
\hline
\end{tabular}



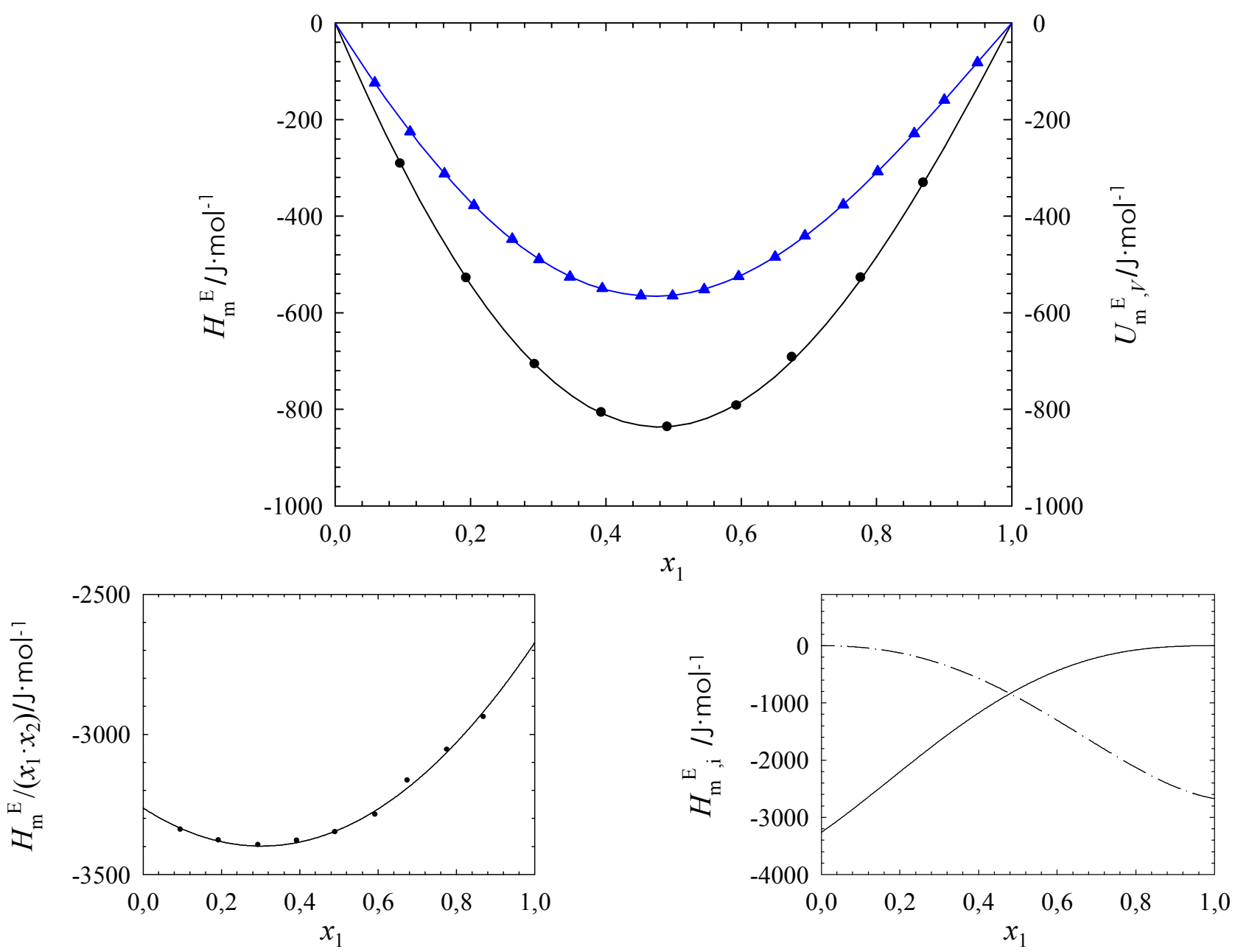

Ilustración 4-17: entalpías molares de exceso $H_{\mathrm{m}}^{\mathrm{E}}$, energías internas de exceso a volumen constante $U_{\mathrm{m}, V}^{\mathrm{E}}$, entalpías molares de exceso reducidos $H_{\mathrm{m}}^{\mathrm{E}} /\left(x_{1} \cdot x_{2}\right)$ y entalpías molares parciales de exceso $H_{\mathrm{m}, \mathrm{i}}^{\mathrm{E}}$ del sistema $N$-metilanilina (1) +2 -pentanona (2) 
Tabla 4-27: entalpías molares de exceso del sistema $N$-metilanilina (1) + 2-heptanona (2)

\begin{tabular}{cccc}
\hline$x_{1}$ & $H_{\mathrm{m}}^{\mathrm{E}} / \mathrm{J} \cdot \mathrm{mol}^{-1}$ & $x_{1}$ & $H_{\mathrm{m}}^{\mathrm{E}} / \mathrm{J} \cdot \mathrm{mol}^{-1}$ \\
\hline 0.1025 & -284 & 0.5924 & -691 \\
0.1963 & -489 & 0.6922 & -578 \\
0.2937 & -641 & 0.7867 & -415 \\
0.3936 & -728 & 0.8781 & -248 \\
0.4971 & -741 & & \\
\hline
\end{tabular}

Tabla 4-28: energías molares de exceso a $V=$ cte. del sistema $N$-metilanilina (1) +2 -heptanona (2)

\begin{tabular}{cccccc}
\hline$x_{1}$ & $U_{\mathrm{m}, V}^{\mathrm{E}} / \mathrm{J} \cdot \mathrm{mol}^{-1}$ & $x_{1}$ & $U_{\mathrm{m}, V}^{\mathrm{E}} / \mathrm{J} \cdot \mathrm{mol}^{-1}$ & $x_{1}$ & $U_{\mathrm{m}, V}^{\mathrm{E}} / \mathrm{J} \cdot \mathrm{mol}^{-1}$ \\
\hline 0.0506 & -110 & 0.4023 & -545 & 0.7013 & -395 \\
0.1011 & -212 & 0.4535 & -551 & 0.7525 & -330 \\
0.1501 & -300 & 0.5051 & -542 & 0.7964 & -273 \\
0.1977 & -374 & 0.5548 & -521 & 0.8476 & -199 \\
0.2494 & -438 & 0.6006 & -492 & 0.8968 & -131 \\
0.3096 & -499 & 0.6552 & -442 & 0.9417 & -71 \\
0.3536 & -528 & & & & \\
\hline
\end{tabular}

Tabla 4-29: coeficientes y desviaciones estándar del ajuste a la ecuación R-K de las diferentes propiedades de exceso del sistema $N$-metilanilina (1) + 2-heptanona (2)

\begin{tabular}{ccccc}
\hline Propiedad & $A_{0}$ & $A_{1}$ & $A_{2}$ & $\sigma$ \\
\hline$H_{\mathrm{m}}^{\mathrm{E}} / \mathrm{J} \cdot \mathrm{mol}^{-1}$ & -2966 & 517 & 490 & 3 \\
$U_{\mathrm{m}, V}^{\mathrm{E}} / \mathrm{J} \cdot \mathrm{mol}^{-1}$ & -2179.1 & 568 & 474 & 1.2 \\
\hline
\end{tabular}



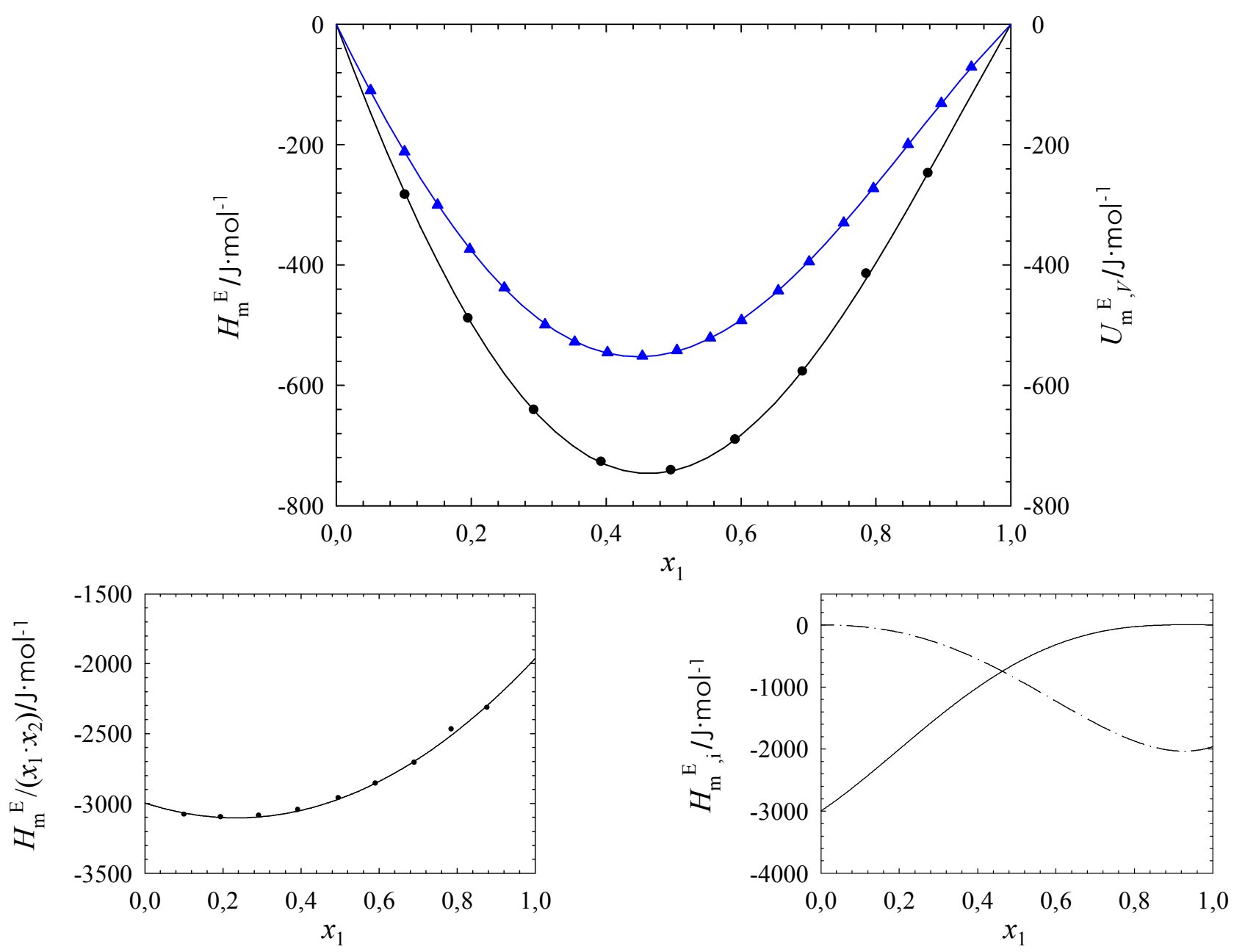

Ilustración 4-18: entalpías molares de exceso $H_{\mathrm{m}}^{\mathrm{E}}$, energías internas de exceso a volumen constante $U_{\mathrm{m}, V}^{\mathrm{E}}$, entalpías molares de exceso reducidos $H_{\mathrm{m}}^{\mathrm{E}} /\left(x_{1} \cdot x_{2}\right)$ y entalpías molares parciales de exceso $H_{\mathrm{m}, \mathrm{i}}^{\mathrm{E}}$ del sistema $N$-metilanilina (1) +2 -heptanona (2) 
Tabla 4-30: entalpías molares de exceso del sistema di- $n$-propilamina (1) + 2-propanona (2)

\begin{tabular}{cccc}
\hline$x_{1}$ & $H_{\mathrm{m}}^{\mathrm{E}} / \mathrm{J} \cdot \mathrm{mol}^{-1}$ & $x_{1}$ & $H_{\mathrm{m}}^{\mathrm{E}} / \mathrm{J} \cdot \mathrm{mol}^{-1}$ \\
\hline 0.1063 & 273 & 0.5821 & 616 \\
0.2076 & 471 & 0.6773 & 541 \\
0.3036 & 585 & 0.7652 & 434 \\
0.3982 & 631 & 0.8488 & 296 \\
0.4932 & 642 & & \\
\hline
\end{tabular}

Tabla 4-31: energías molares de exceso a $V=$ cte. del sistema di- $n$-propilamina (1) + 2-propanona (2)

\begin{tabular}{cccccc}
\hline$x_{1}$ & $U_{\mathrm{m}, V}^{\mathrm{E}} / \mathrm{J} \cdot \mathrm{mol}^{-1}$ & $x_{1}$ & $U_{\mathrm{m}, V}^{\mathrm{E}} / \mathrm{J} \cdot \mathrm{mol}^{-1}$ & $x_{1}$ & $U_{\mathrm{m}, V}^{\mathrm{E}} / \mathrm{J} \cdot \mathrm{mol}^{-1}$ \\
\hline 0.0557 & 133 & 0.4055 & 566 & 0.7012 & 456 \\
0.1035 & 232 & 0.4498 & 574 & 0.7482 & 405 \\
0.1552 & 322 & 0.5082 & 570 & 0.7919 & 350 \\
0.2037 & 397 & 0.5512 & 559 & 0.8374 & 287 \\
0.2556 & 461 & 0.6073 & 531 & 0.8913 & 203 \\
0.3076 & 511 & 0.6527 & 499 & 0.9459 & 108 \\
0.3534 & 543 & & & & \\
\hline
\end{tabular}

Tabla 4-32: coeficientes y desviaciones estándar del ajuste a la ecuación R-K de las diferentes propiedades de exceso del sistema di- $n$-propilamina (1) + 2-propanona (2)

\begin{tabular}{cccc}
\hline Propiedad & $A_{0}$ & $A_{1}$ & $\sigma$ \\
\hline$H_{\mathrm{m}}^{\mathrm{E}} /{\mathrm{J} \cdot \mathrm{mol}^{-1}}^{\mathrm{E}} / \mathrm{J} \cdot \mathrm{mol}^{-1}$ & 2592 & -380 & 6 \\
$U_{\mathrm{m}, V}^{\mathrm{n}}$ & 2289.2 & -273 & 1.3 \\
\hline
\end{tabular}



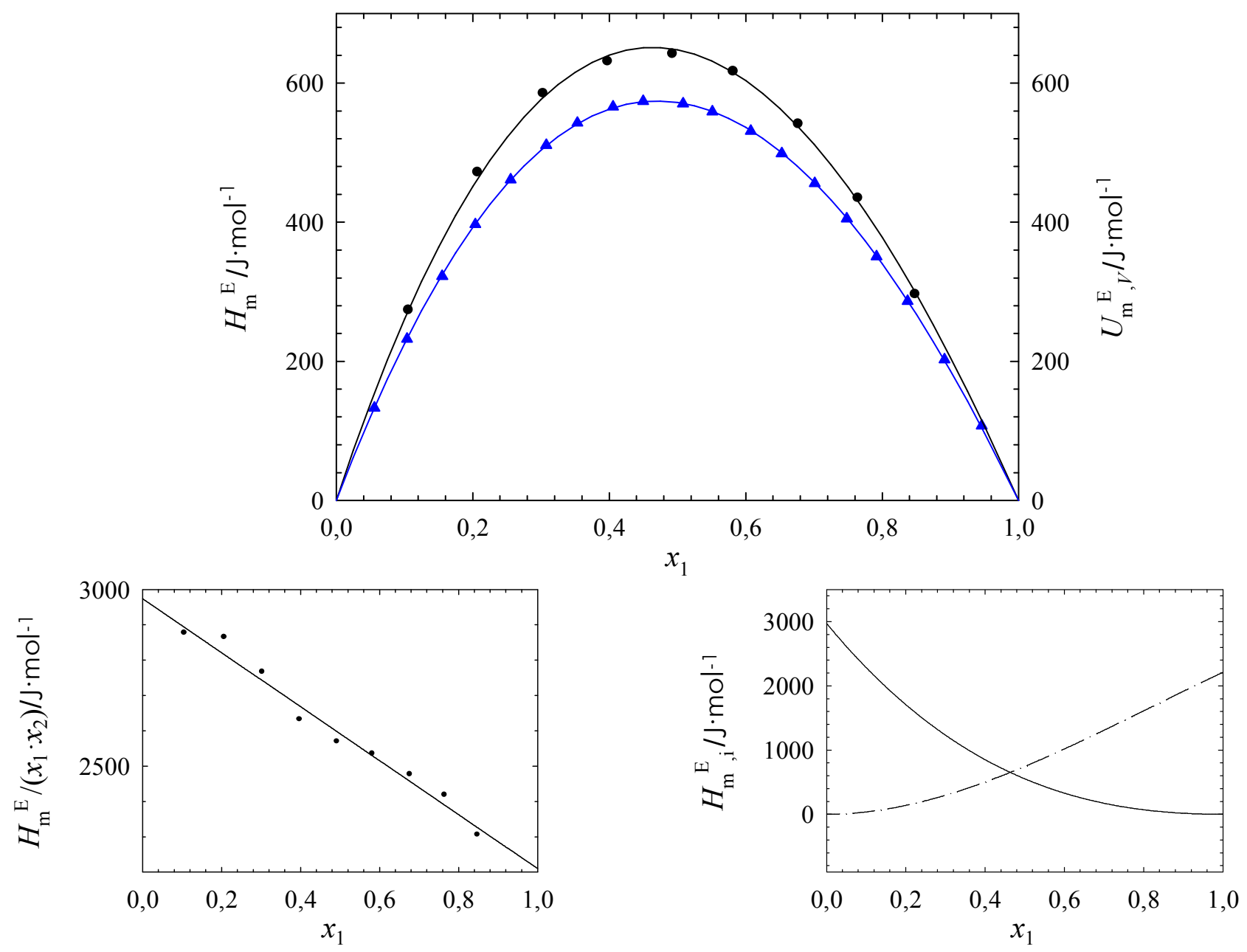

llustración 4-19: entalpías molares de exceso $H_{\mathrm{m}}^{\mathrm{E}}$, energías internas de exceso a volumen constante $U_{\mathrm{m}, V}^{\mathrm{E}}$, entalpías molares de exceso reducidos $H_{\mathrm{m}}^{\mathrm{E}} /\left(x_{1} \cdot x_{2}\right)$ y entalpías molares parciales de exceso $H_{\mathrm{m}, \mathrm{i}}^{\mathrm{E}}$ del sistema di- $n$-propilamina (1) +2 -propanona (2) 
Tabla 4-33: entalpías molares de exceso del sistema di- $n$-propilamina (1) + 2-butanona (2)

\begin{tabular}{cccc}
\hline$x_{1}$ & $H_{\mathrm{m}}^{\mathrm{E}} / \mathrm{J} \cdot \mathrm{mol}^{-1}$ & $x_{1}$ & $H_{\mathrm{m}}^{\mathrm{E}} / \mathrm{J} \cdot \mathrm{mol}^{-1}$ \\
\hline 0.1042 & 155 & 0.5459 & 396 \\
0.1978 & 253 & 0.5908 & 392 \\
0.3016 & 334 & 0.6833 & 356 \\
0.3858 & 370 & 0.7647 & 299 \\
0.4950 & 397 & 0.8559 & 204 \\
\hline
\end{tabular}

Tabla 4-34: energías molares de exceso a $V=$ cte. del sistema di- $n$-propilamina (1) + 2-butanona (2)

\begin{tabular}{cccccc}
\hline$x_{1}$ & $U_{\mathrm{m}, V}^{\mathrm{E}} / \mathrm{J} \cdot \mathrm{mol}^{-1}$ & $x_{1}$ & $U_{\mathrm{m}, V}^{\mathrm{E}} / \mathrm{J} \cdot \mathrm{mol}^{-1}$ & $x_{1}$ & $U_{\mathrm{m}, V}^{\mathrm{E}} / \mathrm{J} \cdot \mathrm{mol}^{-1}$ \\
\hline 0.0529 & 73 & 0.4008 & 337 & 0.7042 & 303 \\
0.0959 & 123 & 0.4461 & 347 & 0.7554 & 271 \\
0.1443 & 177 & 0.4994 & 353 & 0.7999 & 236 \\
0.1952 & 222 & 0.5375 & 352 & 0.8491 & 190 \\
0.2469 & 261 & 0.5997 & 343 & 0.8973 & 137 \\
0.3013 & 297 & 0.6455 & 329 & 0.9408 & 82 \\
0.3526 & 320 & & & & \\
\hline
\end{tabular}

Tabla 4-35: coeficientes y desviaciones estándar del ajuste a la ecuación R-K de las diferentes propiedades de exceso del sistema di- $n$-propilamina (1) +2 -butanona (2)

\begin{tabular}{cccccc}
\hline Propiedad & $A_{0}$ & $A_{1}$ & $A_{2}$ & $A_{3}$ & $\sigma$ \\
\hline$H_{\mathrm{m}}^{\mathrm{E}} / \mathrm{J}_{\mathrm{mol}^{-1}}^{\mathrm{E}}$ & 1592 & 63 & 120 & & 4 \\
$U_{\mathrm{m}, V}^{\mathrm{N}} / \mathrm{J} \cdot \mathrm{mol}^{-1}$ & 1413.2 & 69 & 80 & -59 & 0.9 \\
\hline
\end{tabular}



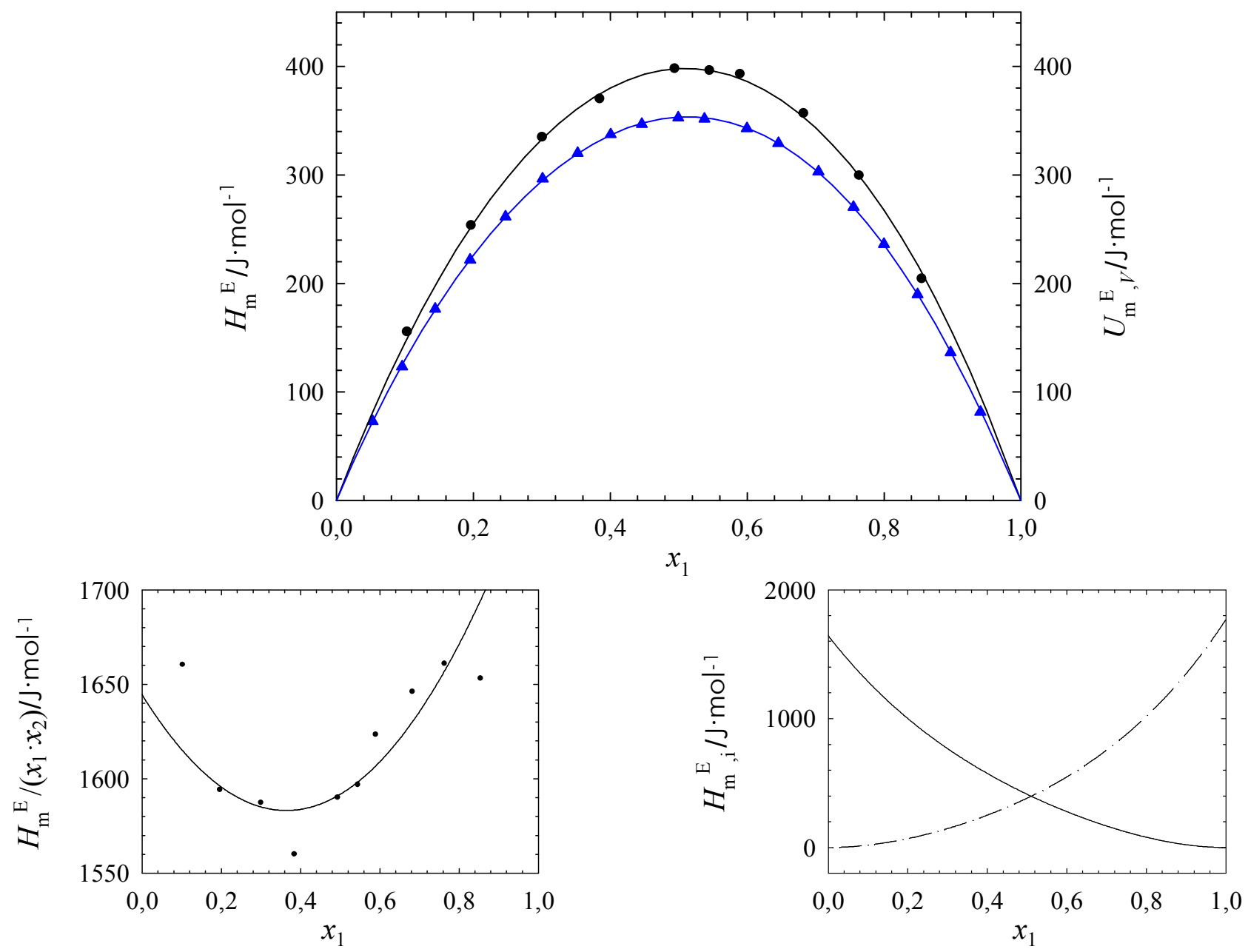

Ilustración 4-20: entalpías molares de exceso $H_{\mathrm{m}}^{\mathrm{E}}$, energías internas de exceso a volumen constante $U_{\mathrm{m}, V}^{\mathrm{E}}$, entalpías molares de exceso reducidos $H_{\mathrm{m}}^{\mathrm{E}} /\left(x_{1} \cdot x_{2}\right)$ y entalpías molares parciales de exceso $H_{\mathrm{m}, \mathrm{i}}^{\mathrm{E}}$ del sistema di- $n$-propilamina (1) +2 -butanona (2) 
Tabla 4-36: entalpías molares de exceso del sistema di-n-propilamina (1) + 2-pentanona (2)

\begin{tabular}{cccc}
\hline$x_{1}$ & $H_{\mathrm{m}}^{\mathrm{E}} / \mathrm{J} \cdot \mathrm{mol}^{-1}$ & $x_{1}$ & $H_{\mathrm{m}}^{\mathrm{E}} / \mathrm{J} \cdot \mathrm{mol}^{-1}$ \\
\hline 0.1056 & 87 & 0.5922 & 284 \\
0.1981 & 154 & 0.6821 & 267 \\
0.3098 & 217 & 0.7810 & 228 \\
0.3988 & 260 & 0.8693 & 160 \\
0.5023 & 281 & & \\
\hline
\end{tabular}

Tabla 4-37: coeficientes y desviaciones estándar del ajuste a la ecuación R-K de las diferentes propiedades de exceso del sistema di- $n$-propilamina (1) +2 -pentanona (2)

\begin{tabular}{ccccc}
\hline Propiedad & $A_{0}$ & $A_{1}$ & $A_{2}$ & $\sigma$ \\
\hline$H_{\mathrm{m}}^{\mathrm{E}} / \mathrm{J} \cdot \mathrm{mol}^{-1}$ & 1123 & 304 & 80 & 3 \\
\hline
\end{tabular}



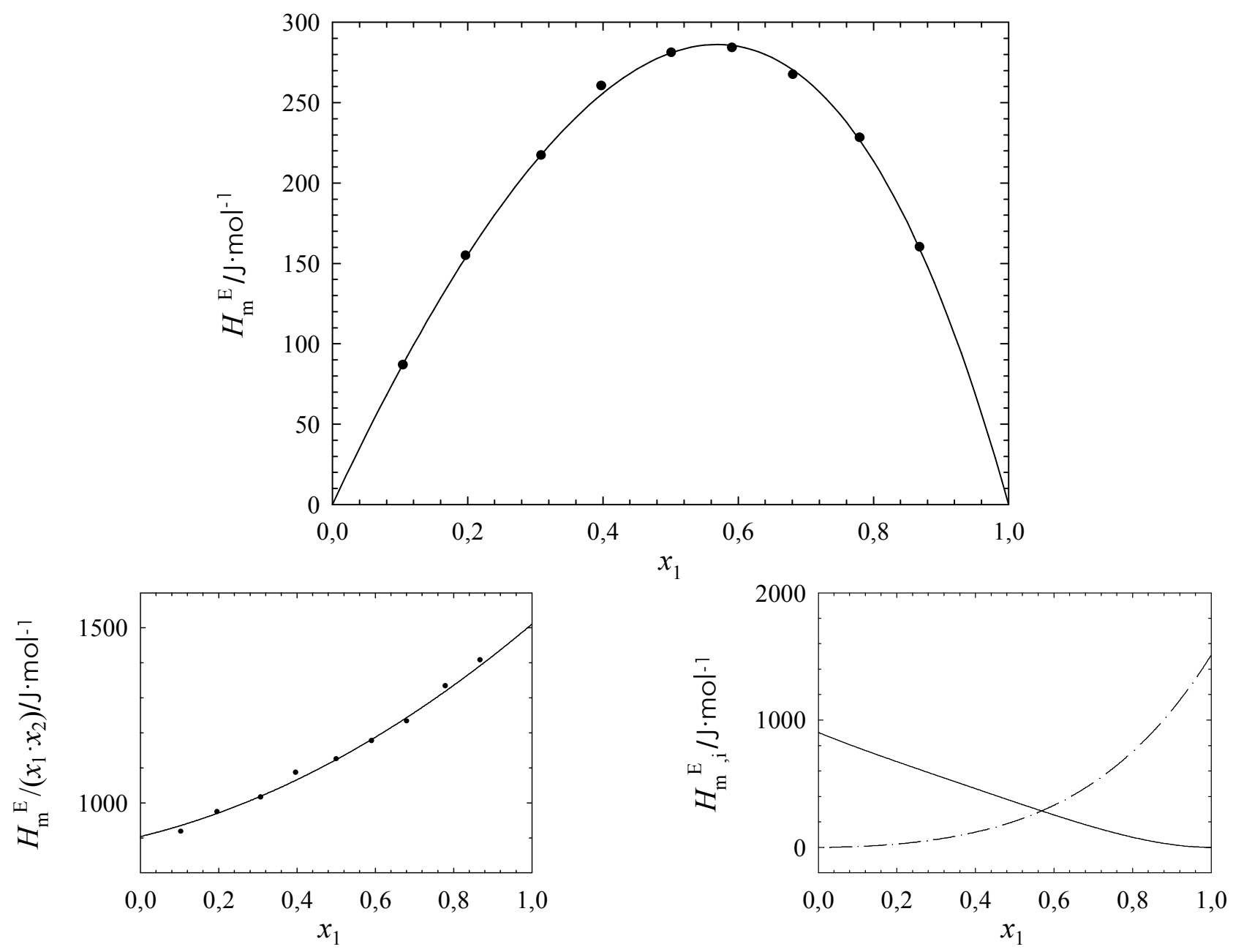

llustración 4-21: entalpías molares de exceso $H_{\mathrm{m}}^{\mathrm{E}}$, entalpías molares de exceso reducidos $H_{\mathrm{m}}^{\mathrm{E}} /\left(x_{1} \cdot x_{2}\right)$ y entalpías molares parciales de exceso $H_{\mathrm{m}, \mathrm{i}}^{\mathrm{E}}$ del sistema di-n-propilamina (1) +2 -pentanona (2) 
Tabla 4-38: entalpías molares de exceso del sistema di- $n$-propilamina (1) + 2-heptanona (2)

\begin{tabular}{cccc}
\hline$x_{1}$ & $H_{\mathrm{m}}^{\mathrm{E}} / \mathrm{J} \cdot \mathrm{mol}^{-1}$ & $x_{1}$ & $H_{\mathrm{m}}^{\mathrm{E}} / \mathrm{J} \cdot \mathrm{mol}^{-1}$ \\
\hline 0.1145 & 47 & 0.6017 & 170 \\
0.2120 & 88 & 0.6872 & 159 \\
0.3072 & 118 & 0.7868 & 137 \\
0.4034 & 143 & 0.8796 & 96 \\
0.4993 & 162 & & \\
\hline
\end{tabular}

Tabla 4-39: energías molares de exceso a $V=$ cte. del sistema di- $n$-propilamina (1) +2 -heptanona (2)

\begin{tabular}{cccccc}
\hline$x_{1}$ & $U_{\mathrm{m}, V}^{\mathrm{E}} / \mathrm{J} \cdot \mathrm{mol}^{-1}$ & $x_{1}$ & $U_{\mathrm{m}, V}^{\mathrm{E}} / \mathrm{J} \cdot \mathrm{mol}^{-1}$ & $x_{1}$ & $U_{\mathrm{m}, V}^{\mathrm{E}} / \mathrm{J} \cdot \mathrm{mol}^{-1}$ \\
\hline 0.0472 & 30 & 0.3564 & 158 & 0.7474 & 170 \\
0.1007 & 59 & 0.4573 & 182 & 0.8000 & 151 \\
0.1452 & 80 & 0.5522 & 193 & 0.8443 & 128 \\
0.1939 & 101 & 0.6150 & 194 & 0.8952 & 96 \\
0.2582 & 126 & 0.6564 & 190 & 0.9479 & 53 \\
0.3100 & 144 & 0.7038 & 182 & & \\
\hline
\end{tabular}

Tabla 4-40: coeficientes y desviaciones estándar del ajuste a la ecuación R-K de las diferentes propiedades de exceso del sistema di- $n$-propilamina (1) + 2-heptanona (2)

\begin{tabular}{ccccc}
\hline Propiedad & $A_{0}$ & $A_{1}$ & $A_{2}$ & $\sigma$ \\
\hline$H_{\mathrm{m}}^{\mathrm{E}} / \mathrm{J} \cdot \mathrm{mol}^{-1}$ & 643 & 262 & 72 & 1.7 \\
$U_{\mathrm{m}, V}^{\mathrm{E}} / \mathrm{J} \cdot \mathrm{mol}^{-1}$ & 751.7 & 244 & 125 & 1.0 \\
\hline
\end{tabular}



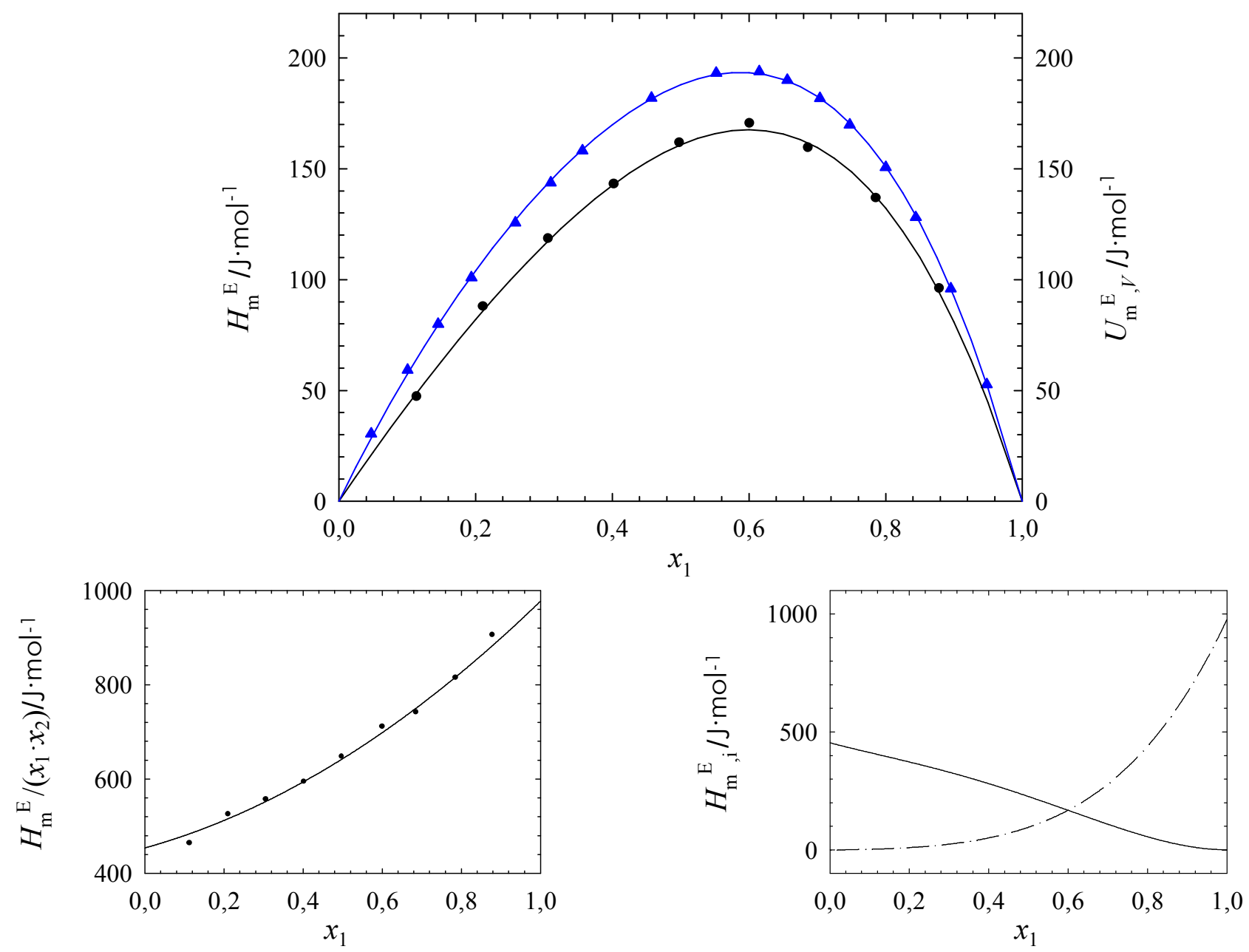

llustración 4-22: entalpías molares de exceso $H_{\mathrm{m}}^{\mathrm{E}}$, energías internas de exceso a volumen constante $U_{\mathrm{m}, V}^{\mathrm{E}}$, entalpías molares de exceso reducidos $H_{\mathrm{m}}^{\mathrm{E}} /\left(x_{1} \cdot x_{2}\right)$ y entalpías molares parciales de exceso $H_{\mathrm{m}, \mathrm{i}}^{\mathrm{E}}$ del sistema di- $n$-propilamina (1) +2 -heptanona (2) 
Tabla 4-41: entalpías molares de exceso del sistema di- $n$-butilamina (1) + 2-propanona (2)

\begin{tabular}{cccc}
\hline$x_{1}$ & $H_{\mathrm{m}}^{\mathrm{E}} / \mathrm{J} \cdot \mathrm{mol}^{-1}$ & $x_{1}$ & $H_{\mathrm{m}}^{\mathrm{E}} / \mathrm{J} \cdot \mathrm{mol}^{-1}$ \\
\hline 0.1101 & 442 & 0.6088 & 863 \\
0.2103 & 692 & 0.6999 & 757 \\
0.2999 & 853 & 0.7900 & 598 \\
0.3980 & 922 & 0.8913 & 346 \\
0.4977 & 936 & & \\
\hline
\end{tabular}

Tabla 4-42: energías molares de exceso a $V=$ cte. del sistema di- $n$-butilamina (1) +2 -propanona (2)

\begin{tabular}{cccccc}
\hline$x_{1}$ & $U_{\mathrm{m}, V}^{\mathrm{E}} / \mathrm{J} \cdot \mathrm{mol}^{-1}$ & $x_{1}$ & $U_{\mathrm{m}, V}^{\mathrm{E}} / \mathrm{J} \cdot \mathrm{mol}^{-1}$ & $x_{1}$ & $U_{\mathrm{m}, V}^{\mathrm{E}} / \mathrm{J} \cdot \mathrm{mol}^{-1}$ \\
\hline 0.0480 & 181 & 0.4004 & 793 & 0.6990 & 661 \\
0.0965 & 331 & 0.4493 & 806 & 0.7463 & 595 \\
0.1444 & 453 & 0.4984 & 805 & 0.7901 & 521 \\
0.1995 & 571 & 0.5418 & 793 & 0.8502 & 403 \\
0.2471 & 652 & 0.5914 & 766 & 0.8908 & 310 \\
0.3001 & 720 & 0.6437 & 723 & 0.9421 & 178 \\
0.3480 & 763 & & & & \\
\hline
\end{tabular}

Tabla 4-43: coeficientes y desviaciones estándar del ajuste a la ecuación R-K de las diferentes propiedades de exceso del sistema di-n-butilamina (1) + 2-propanona (2)

\begin{tabular}{ccccc}
\hline Propiedad & $A_{0}$ & $A_{1}$ & $A_{2}$ & $\sigma$ \\
\hline$H_{\mathrm{m}}^{\mathrm{E}} / \mathrm{J} \cdot \mathrm{mol}^{-1}$ & 3733 & -540 & 500 & 6 \\
$U_{\mathrm{m}, V}^{\mathrm{E}} / \mathrm{J} \cdot \mathrm{mol}^{-1}$ & 3218 & -356 & 417 & 1.5 \\
\hline
\end{tabular}



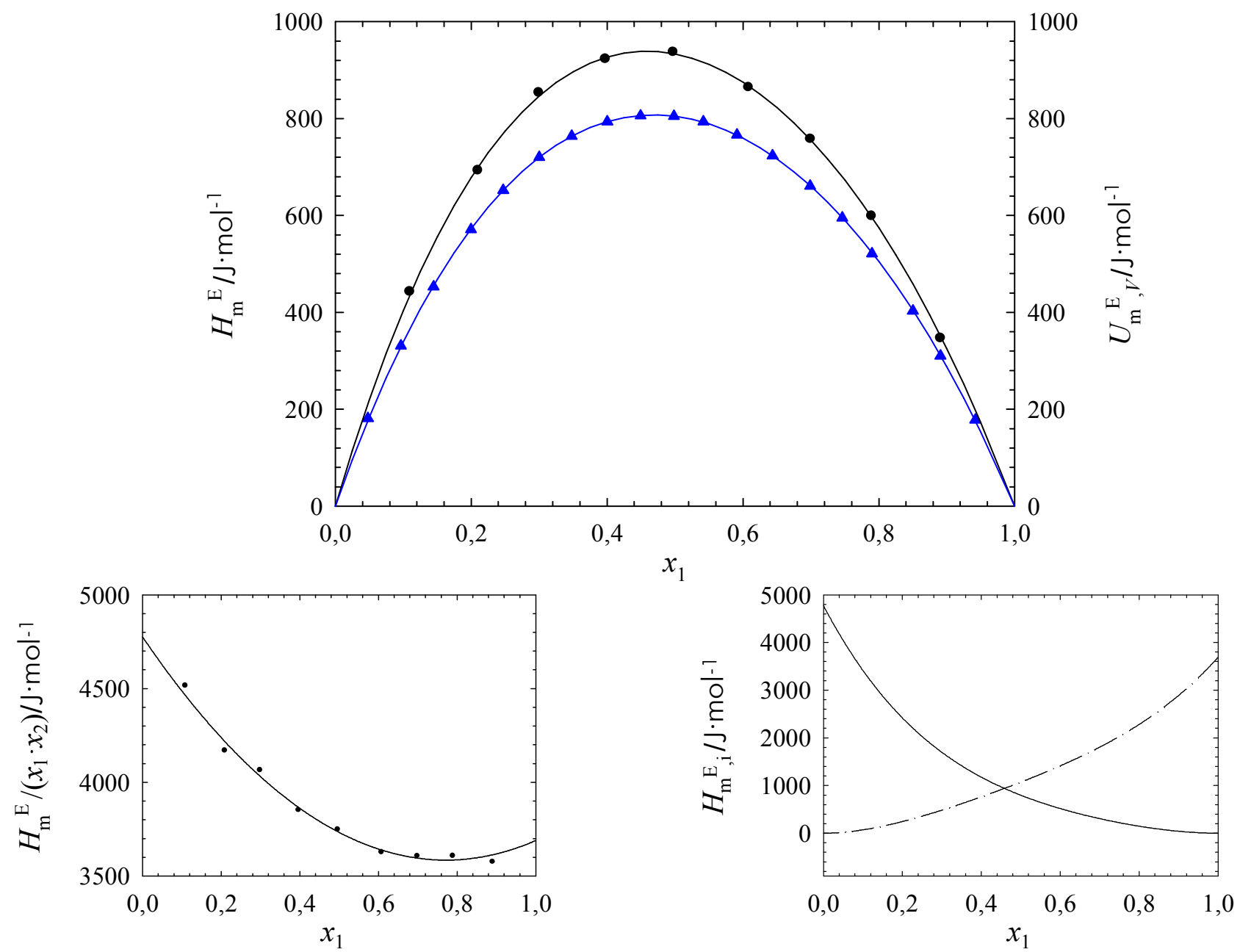

Ilustración 4-23: entalpías molares de exceso $H_{\mathrm{m}}^{\mathrm{E}}$, energías internas de exceso a volumen constante $U_{\mathrm{m}, V}^{\mathrm{E}}$, entalpías molares de exceso reducidos $H_{\mathrm{m}}^{\mathrm{E}} /\left(x_{1} \cdot x_{2}\right)$ y entalpías molares parciales de exceso $H_{\mathrm{m}, \mathrm{i}}^{\mathrm{E}}$ del sistema di- $n$-butilamina (1) +2 -propanona (2) 
Tabla 4-44: entalpías molares de exceso del sistema di- $n$-butilamina (1) + 2-butanona (2)

\begin{tabular}{cccc}
\hline$x_{1}$ & $H_{\mathrm{m}}^{\mathrm{E}} / \mathrm{J} \cdot \mathrm{mol}^{-1}$ & $x_{1}$ & $H_{\mathrm{m}}^{\mathrm{E}} / \mathrm{J} \cdot \mathrm{mol}^{-1}$ \\
\hline 0.1142 & 274 & 0.6001 & 593 \\
0.2078 & 425 & 0.6930 & 529 \\
0.2985 & 536 & 0.7846 & 417 \\
0.3978 & 601 & 0.8802 & 270 \\
0.4956 & 619 & & \\
\hline
\end{tabular}

Tabla 4-45: energías molares de exceso a $V=$ cte. del sistema di- $n$-butilamina (1) + 2-butanona (2)

\begin{tabular}{cccccc}
\hline$x_{1}$ & $U_{\mathrm{m}, V}^{\mathrm{E}} / \mathrm{J} \cdot \mathrm{mol}^{-1}$ & $x_{1}$ & $U_{\mathrm{m}, V}^{\mathrm{E}} / \mathrm{J} \cdot \mathrm{mol}^{-1}$ & $x_{1}$ & $U_{\mathrm{m}, V}^{\mathrm{E}} / \mathrm{J} \cdot \mathrm{mol}^{-1}$ \\
\hline 0.0518 & 113 & 0.3964 & 517 & 0.6888 & 463 \\
0.0978 & 201 & 0.4545 & 532 & 0.7397 & 418 \\
0.1544 & 292 & 0.4982 & 536 & 0.7966 & 355 \\
0.2022 & 357 & 0.5580 & 528 & 0.8375 & 299 \\
0.2527 & 414 & 0.6063 & 512 & 0.8962 & 206 \\
0.2997 & 458 & 0.6477 & 491 & 0.9319 & 142 \\
0.3486 & 493 & & & & \\
\hline
\end{tabular}

Tabla 4-46: coeficientes y desviaciones estándar del ajuste a la ecuación R-K de las diferentes propiedades de exceso del sistema di- $n$-butilamina (1) +2 -butanona (2)

\begin{tabular}{ccccc}
\hline Propiedad & $A_{0}$ & $A_{1}$ & $A_{2}$ & $\sigma$ \\
\hline$H_{\mathrm{m}}^{\mathrm{E}} / \mathrm{J} \cdot \mathrm{mol}^{-1}$ & 2479 & -94 & 220 & 4 \\
$U_{\mathrm{m}, V}^{\mathrm{E}} / \mathrm{J} \cdot \mathrm{mol}^{-1}$ & 2144.9 & -26.5 & 160 & 0.6 \\
\hline
\end{tabular}



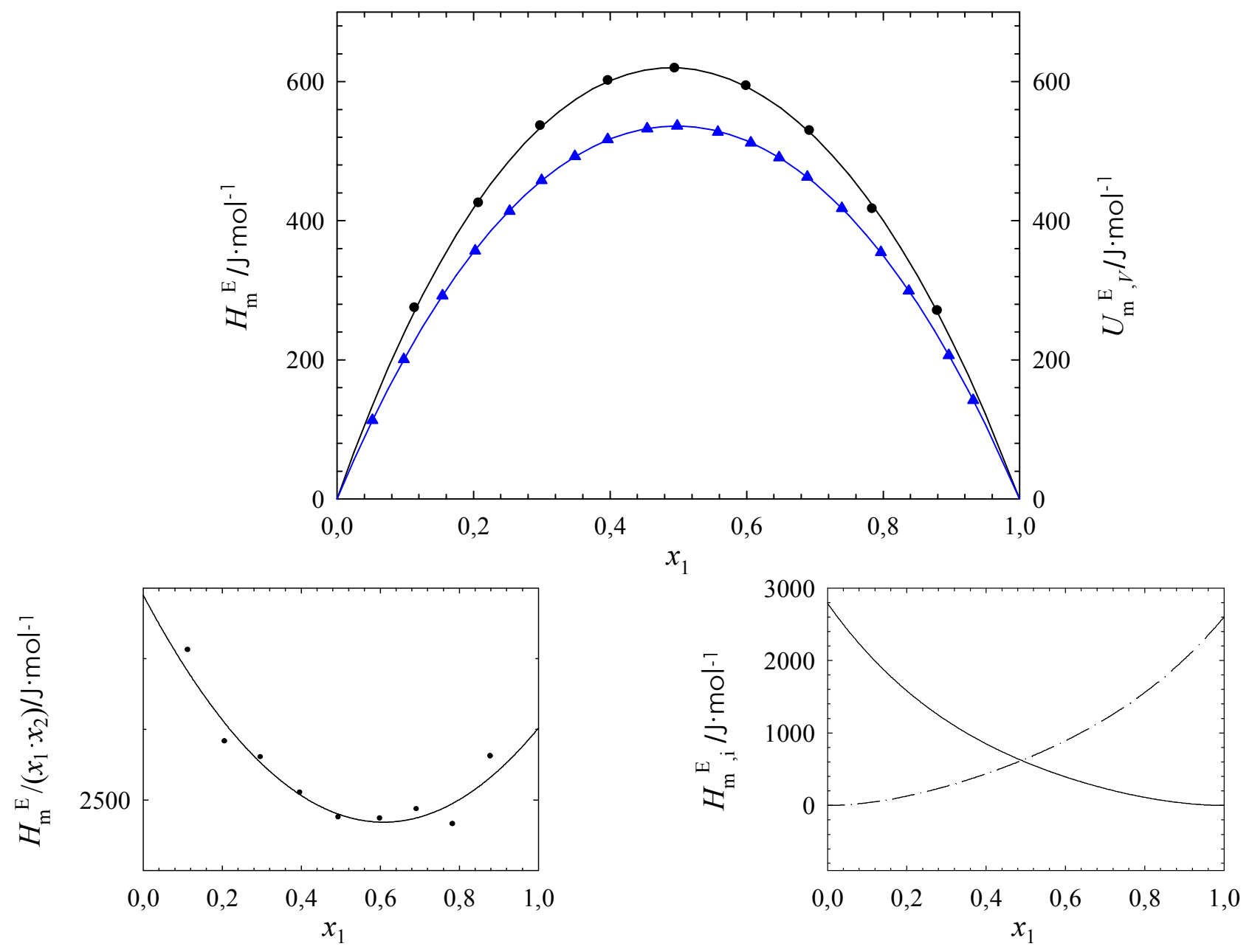

llustración 4-24: entalpías molares de exceso $H_{\mathrm{m}}^{\mathrm{E}}$, energías internas de exceso a volumen constante $U_{\mathrm{m}, V}^{\mathrm{E}}$, entalpías molares de exceso reducidos $H_{\mathrm{m}}^{\mathrm{E}} /\left(x_{1} \cdot x_{2}\right)$ y entalpías molares parciales de exceso $H_{\mathrm{m}, \mathrm{i}}^{\mathrm{E}}$ del sistema di- $n$-butilamina (1) +2 -butanona (2) 
Tabla 4-47: entalpías molares de exceso del sistema di- $n$-butilamina (1) +2 -pentanona (2)

\begin{tabular}{cccc}
\hline$x_{1}$ & $H_{\mathrm{m}}^{\mathrm{E}} / \mathrm{J} \cdot \mathrm{mol}^{-1}$ & $x_{1}$ & $H_{\mathrm{m}}^{\mathrm{E}} / \mathrm{J} \cdot \mathrm{mol}^{-1}$ \\
\hline 0.1111 & 170 & 0.6052 & 437 \\
0.2106 & 286 & 0.6999 & 392 \\
0.3072 & 380 & 0.7881 & 319 \\
0.3973 & 427 & 0.8848 & 203 \\
0.4944 & 451 & & \\
\hline
\end{tabular}

Tabla 4-48: coeficientes y desviaciones estándar del ajuste a la ecuación R-K de las diferentes propiedades de exceso del sistema di- $n$-butilamina (1) +2 -pentanona (2)

\begin{tabular}{cccc}
\hline Propiedad & $A_{0}$ & $A_{1}$ & $\sigma$ \\
\hline$H_{\mathrm{m}}^{\mathrm{E}} / \mathrm{J} \cdot \mathrm{mol}^{-1}$ & 1816 & 143 & 4 \\
\hline
\end{tabular}



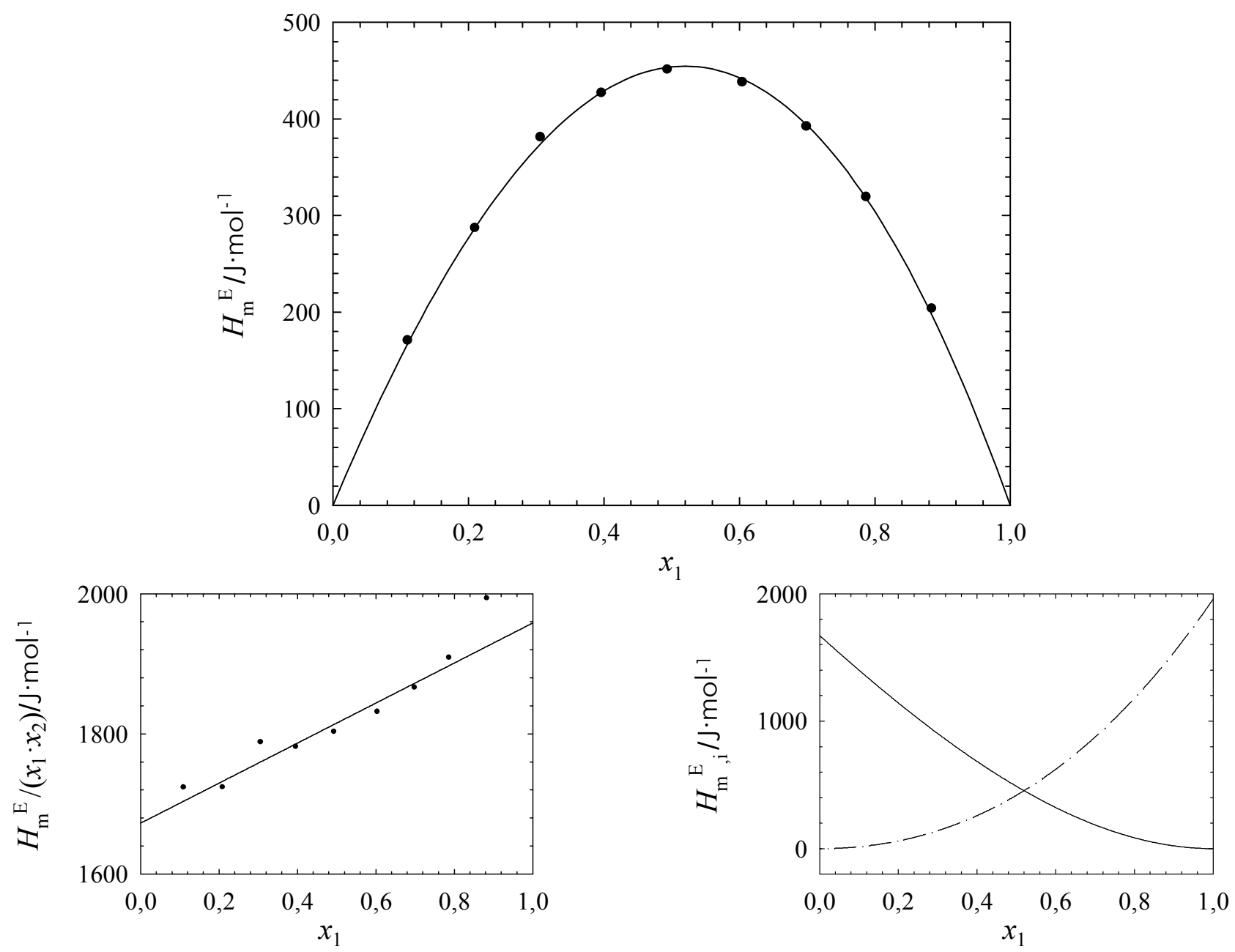

llustración 4-25: entalpías molares de exceso $H_{\mathrm{m}}^{\mathrm{E}}$, entalpías molares de exceso reducidos $H_{\mathrm{m}}^{\mathrm{E}} /\left(x_{1} \cdot x_{2}\right)$ y entalpías molares parciales de exceso $H_{\mathrm{m}, \mathrm{i}}^{\mathrm{E}}$ del sistema di-n-butilamina (1) +2 -pentanona (2) 
Tabla 4-49: entalpías molares de exceso del sistema di- $n$-butilamina (1) +2 -heptanona (2)

\begin{tabular}{cccc}
\hline$x_{1}$ & $H_{\mathrm{m}}^{\mathrm{E}} / \mathrm{J} \cdot \mathrm{mol}^{-1}$ & $x_{1}$ & $H_{\mathrm{m}}^{\mathrm{E}} / \mathrm{J} \cdot \mathrm{mol}^{-1}$ \\
\hline 0.1114 & 86 & 0.5962 & 278 \\
0.2189 & 164 & 0.7053 & 256 \\
0.3057 & 217 & 0.8017 & 208 \\
0.4038 & 254 & 0.8891 & 138 \\
0.5087 & 278 & & \\
\hline
\end{tabular}

Tabla 4-50: energías molares de exceso a $V=$ cte. del sistema di- $n$-butilamina (1) +2 -heptanona (2)

\begin{tabular}{cccccc}
\hline$x_{1}$ & $U_{\mathrm{m}, V}^{\mathrm{E}} / \mathrm{J} \cdot \mathrm{mol}^{-1}$ & $x_{1}$ & $U_{\mathrm{m}, V}^{\mathrm{E}} / \mathrm{J} \cdot \mathrm{mol}^{-1}$ & $x_{1}$ & $U_{\mathrm{m}, V}^{\mathrm{E}} / \mathrm{J} \cdot \mathrm{mol}^{-1}$ \\
\hline 0.1014 & 84 & 0.4504 & 265 & 0.7897 & 215 \\
0.2013 & 154 & 0.5501 & 278 & 0.8403 & 179 \\
0.2491 & 182 & 0.5951 & 277 & 0.8893 & 127 \\
0.3518 & 233 & 0.6387 & 270 & 0.9376 & 76 \\
\hline
\end{tabular}

Tabla 4-51: coeficientes y desviaciones estándar del ajuste a la ecuación R-K de las diferentes propiedades de exceso del sistema di- $n$-butilamina (1) + 2-heptanona (2)

\begin{tabular}{ccccc}
\hline Propiedad & $A_{0}$ & $A_{1}$ & $A_{2}$ & $\sigma$ \\
\hline$H_{\mathrm{m}}^{\mathrm{E}} / \mathrm{J} \cdot \mathrm{mol}^{-1}$ & 1108 & 295 & 49 & 3 \\
$U_{\mathrm{m}, V}^{\mathrm{E}} / \mathrm{J} \cdot \mathrm{mol}^{-1}$ & 1105 & 273 & & 3 \\
\hline
\end{tabular}



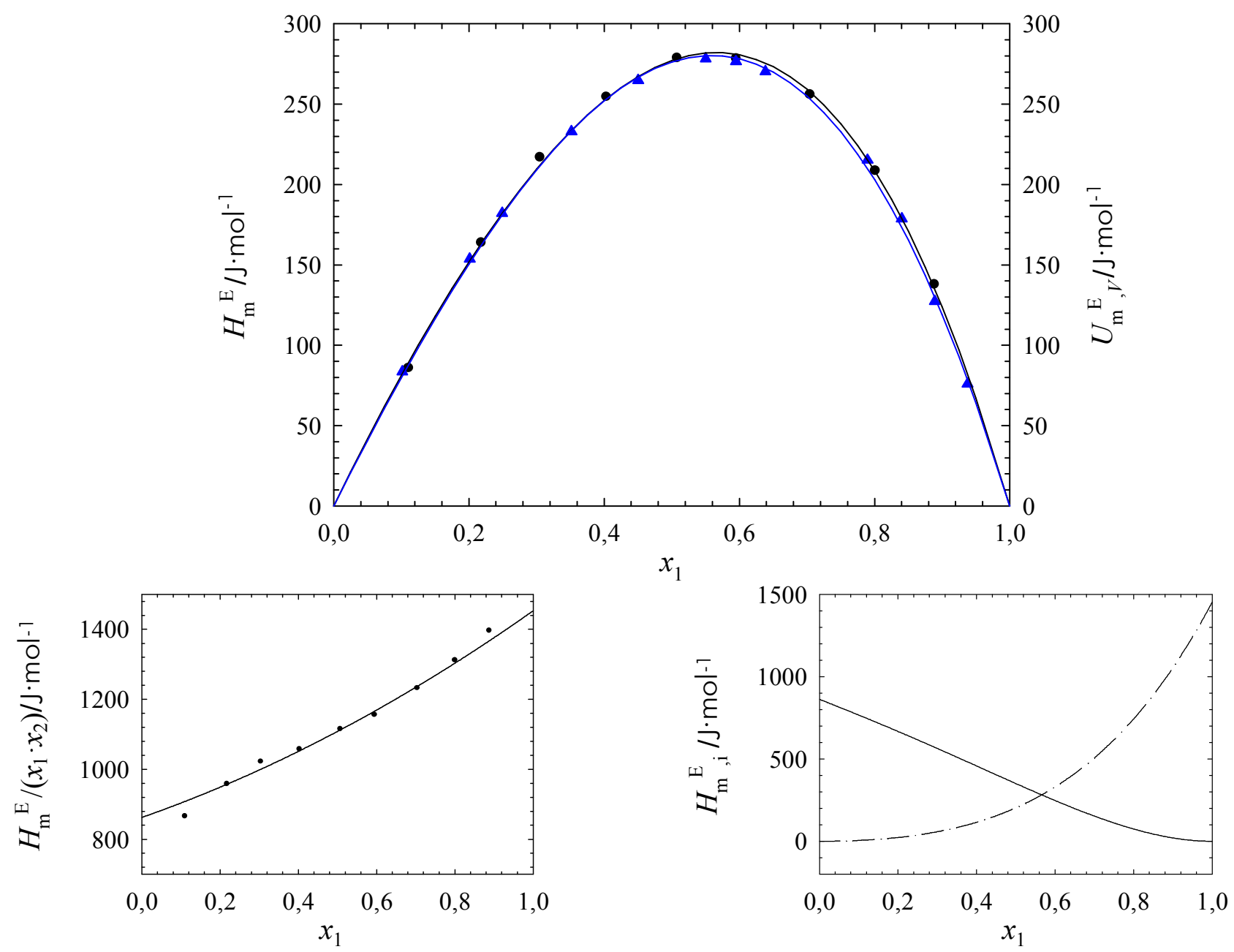

Ilustración 4-26: entalpías molares de exceso $H_{\mathrm{m}}^{\mathrm{E}}$, energías internas de exceso a volumen constante $U_{\mathrm{m}, V}^{\mathrm{E}}$, entalpías molares de exceso reducidos $H_{\mathrm{m}}^{\mathrm{E}} /\left(x_{1} \cdot x_{2}\right)$ y entalpías molares parciales de exceso $H_{\mathrm{m}, \mathrm{i}}^{\mathrm{E}}$ del sistema di- $n$-butilamina (1) +2 -heptanona (2) 
Tabla 4-52: entalpías molares de exceso del sistema $N, N, N$-trietilamina (1) +2 -propanona (2)

\begin{tabular}{cccc}
\hline$x_{1}$ & $H_{\mathrm{m}}^{\mathrm{E}} / \mathrm{J} \cdot \mathrm{mol}^{-1}$ & $x_{1}$ & $H_{\mathrm{m}}^{\mathrm{E}} / \mathrm{J} \cdot \mathrm{mol}^{-1}$ \\
\hline 0.1108 & 446 & 0.6123 & 1028 \\
0.2016 & 674 & 0.7023 & 931 \\
0.3005 & 908 & 0.7881 & 767 \\
0.4042 & 1011 & 0.8828 & 509 \\
0.4967 & 1069 & & \\
\hline
\end{tabular}

Tabla 4-53: energías molares de exceso a $V=$ cte. del sistema $N, N, N$-trietilamina (1) +2 -propanona (2)

\begin{tabular}{cccccc}
\hline$x_{1}$ & $U_{\mathrm{m}, V}^{\mathrm{E}} / \mathrm{J} \cdot \mathrm{mol}^{-1}$ & $x_{1}$ & $U_{\mathrm{m}, V}^{\mathrm{E}} / \mathrm{J} \cdot \mathrm{mol}^{-1}$ & $x_{1}$ & $U_{\mathrm{m}, V}^{\mathrm{E}} / \mathrm{J} \cdot \mathrm{mol}^{-1}$ \\
\hline 0.0499 & 173 & 0.3942 & 908 & 0.7014 & 846 \\
0.1113 & 366 & 0.4454 & 932 & 0.7538 & 762 \\
0.1470 & 485 & 0.4976 & 947 & 0.8042 & 663 \\
0.1982 & 592 & 0.5489 & 948 & 0.8456 & 555 \\
0.2491 & 706 & 0.6022 & 936 & 0.8935 & 425 \\
0.2988 & 792 & 0.6418 & 895 & 0.9389 & 241 \\
0.3451 & 855 & & & & \\
\hline
\end{tabular}

Tabla 4-54: coeficientes y desviaciones estándar del ajuste a la ecuación R-K de las diferentes propiedades de exceso del sistema $N, N, N$-trietilamina (1) + 2-propanona (2)

\begin{tabular}{ccccc}
\hline Propiedad & $A_{0}$ & $A_{1}$ & $A_{2}$ & $\sigma$ \\
\hline$H_{\mathrm{m}}^{\mathrm{E}} / \mathrm{J} \cdot \mathrm{mol}^{-1}$ & 4250 & 270 & 640 & 13 \\
$U_{\mathrm{m}, V}^{\mathrm{E}} / \mathrm{J} \cdot \mathrm{mol}^{-1}$ & 3816 & 330 & 430 & 8 \\
\hline
\end{tabular}



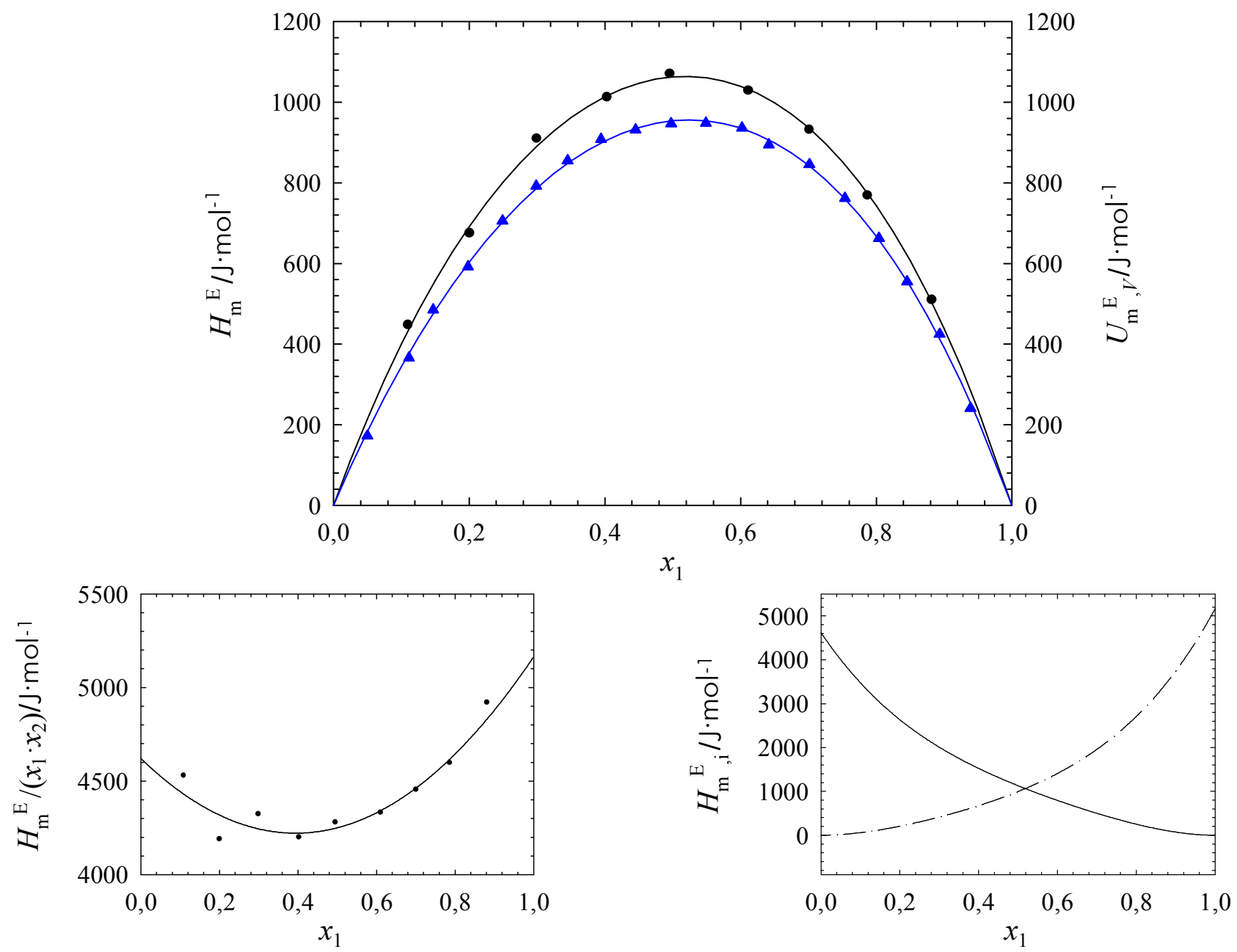

Ilustración 4-27: entalpías molares de exceso $H_{\mathrm{m}}^{\mathrm{E}}$, energías internas de exceso a volumen constante $U_{\mathrm{m}, V}^{\mathrm{E}}$, entalpías molares de exceso reducidos $H_{\mathrm{m}}^{\mathrm{E}} /\left(x_{1} \cdot x_{2}\right)$ y entalpías molares parciales de exceso $H_{\mathrm{m}, \mathrm{i}}^{\mathrm{E}}$ del sistema $N, N, N$-trietilamina (1) +2 -propanona (2) 
Tabla 4-55: entalpías molares de exceso del sistema $N, N, N$-trietilamina (1) + 2-butanona (2)

\begin{tabular}{cccc}
\hline$x_{1}$ & $H_{\mathrm{m}}^{\mathrm{E}} / \mathrm{J} \cdot \mathrm{mol}^{-1}$ & $x_{1}$ & $H_{\mathrm{m}}^{\mathrm{E}} / \mathrm{J} \cdot \mathrm{mol}^{-1}$ \\
\hline 0.1089 & 258 & 0.5906 & 735 \\
0.2107 & 455 & 0.7022 & 682 \\
0.3018 & 608 & 0.7876 & 570 \\
0.4042 & 700 & 0.8875 & 367 \\
0.5033 & 746 & & \\
\hline
\end{tabular}

Tabla 4-56: energías molares de exceso a $V=$ cte. del sistema $N, N, N$-trietilamina (1) +2 -butanona (2)

\begin{tabular}{cccccc}
\hline$x_{1}$ & $U_{\mathrm{m}, V}^{\mathrm{E}} / \mathrm{J} \cdot \mathrm{mol}^{-1}$ & $x_{1}$ & $U_{\mathrm{m}, V}^{\mathrm{E}} / \mathrm{J} \cdot \mathrm{mol}^{-1}$ & $x_{1}$ & $U_{\mathrm{m}, V}^{\mathrm{E}} / \mathrm{J} \cdot \mathrm{mol}^{-1}$ \\
\hline 0.0544 & 102 & 0.3961 & 661 & 0.6797 & 631 \\
0.1079 & 235 & 0.4299 & 684 & 0.7390 & 574 \\
0.1563 & 318 & 0.4894 & 697 & 0.7819 & 503 \\
0.1977 & 400 & 0.5393 & 700 & 0.8420 & 427 \\
0.2396 & 492 & 0.5841 & 692 & 0.8851 & 329 \\
0.2878 & 562 & 0.6395 & 665 & 0.9291 & 188 \\
0.3400 & 615 & & & & \\
\hline
\end{tabular}

Tabla 4-57: coeficientes y desviaciones estándar del ajuste a la ecuación R-K de las diferentes propiedades de exceso del sistema $N, N, N$-trietilamina (1) +2 -butanona (2)

\begin{tabular}{cccccc}
\hline Propiedad & $A_{0}$ & $A_{1}$ & $A_{2}$ & $A_{3}$ & $\sigma$ \\
\hline$H_{\mathrm{m}}^{\mathrm{E}} / \mathrm{J} \cdot \mathrm{mol}^{-1}$ & 2982 & 400 & 330 & 460 & 6 \\
$U_{\mathrm{m}, V}^{\mathrm{E}} / \mathrm{J} \cdot \mathrm{mol}^{-1}$ & 2821 & 170 & -80 & 570 & 11 \\
\hline
\end{tabular}



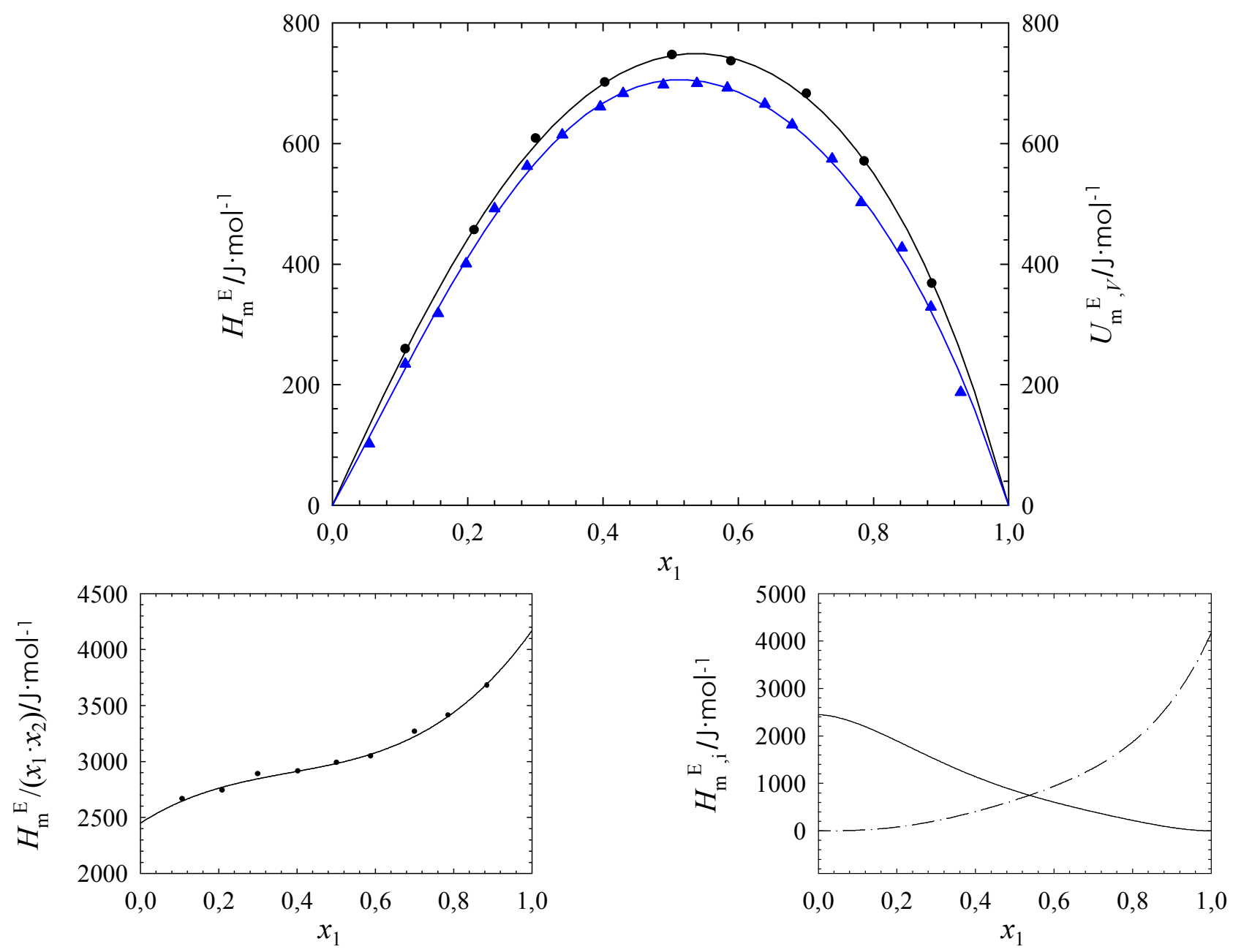

Ilustración 4-28: entalpías molares de exceso $H_{\mathrm{m}}^{\mathrm{E}}$, energías internas de exceso a volumen constante $U_{\mathrm{m}, V}^{\mathrm{E}}$, entalpías molares de exceso reducidos $H_{\mathrm{m}}^{\mathrm{E}} /\left(x_{1} \cdot x_{2}\right)$ y entalpías molares parciales de exceso $H_{\mathrm{m}, \mathrm{i}}^{\mathrm{E}}$ del sistema $N, N, N$-trietilamina (1) +2 -butanona (2) 
Tabla 4-58: entalpías molares de exceso del sistema $N, N, N$-trietilamina (1) +2 -pentanona (2)

\begin{tabular}{cccc}
\hline$x_{1}$ & $H_{\mathrm{m}}^{\mathrm{E}} / \mathrm{J} \cdot \mathrm{mol}^{-1}$ & $x_{1}$ & $H_{\mathrm{m}}^{\mathrm{E}} / \mathrm{J} \cdot \mathrm{mol}^{-1}$ \\
\hline 0.1135 & 178 & 0.5750 & 596 \\
0.2162 & 336 & 0.7077 & 548 \\
0.3067 & 449 & 0.8027 & 448 \\
0.4077 & 534 & 0.8907 & 300 \\
0.5057 & 585 & & \\
\hline
\end{tabular}

Tabla 4-59: coeficientes y desviaciones estándar del ajuste a la ecuación R-K de las diferentes propiedades de exceso del sistema $N, N, N$-trietilamina (1) +2 -pentanona (2)

\begin{tabular}{cccccc}
\hline Propiedad & $A_{0}$ & $A_{1}$ & $A_{2}$ & $A_{3}$ & $\sigma$ \\
\hline$H_{\mathrm{m}}^{\mathrm{E}} / \mathrm{J} \cdot \mathrm{mol}^{-1}$ & 2336 & 610 & 150 & 350 & 2 \\
\hline
\end{tabular}



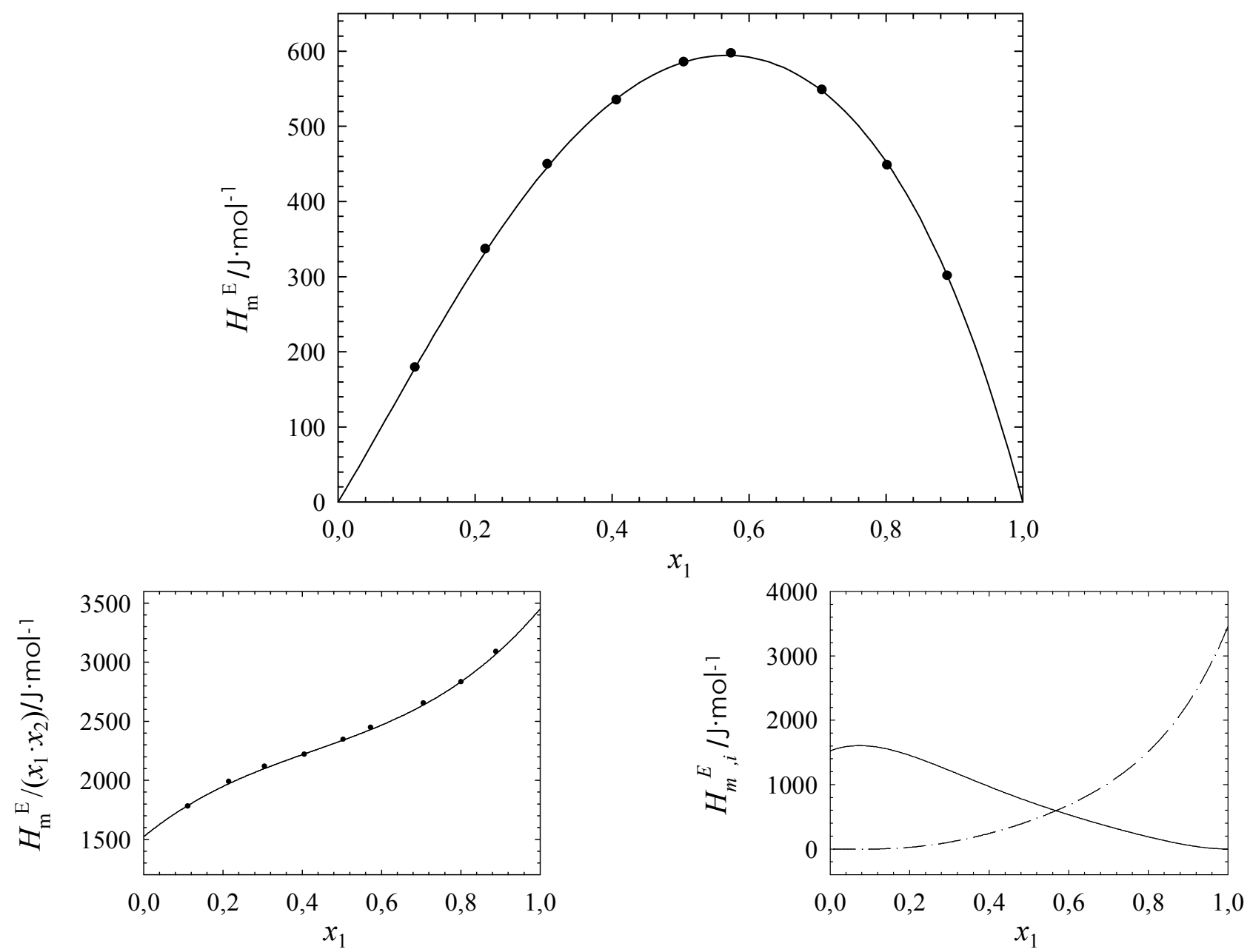

llustración 4-29: entalpías molares de exceso $H_{\mathrm{m}}^{\mathrm{E}}$, entalpías molares de exceso reducidos $H_{\mathrm{m}}^{\mathrm{E}} /\left(x_{1} \cdot x_{2}\right)$ y entalpías molares parciales de exceso $H_{\mathrm{m}, \mathrm{i}}^{\mathrm{E}}$ del sistema $N, N, N$-trietilamina (1) +2-pentanona (2) 
Tabla 4-60: entalpías molares de exceso del sistema $N, N, N$-trietilamina (1) + 2-heptanona (2)

\begin{tabular}{cccc}
\hline$x_{1}$ & $H_{\mathrm{m}}^{\mathrm{E}} / \mathrm{J} \cdot \mathrm{mol}^{-1}$ & $x_{1}$ & $H_{\mathrm{m}}^{\mathrm{E}} / \mathrm{J} \cdot \mathrm{mol}^{-1}$ \\
\hline 0.1292 & 119 & 0.5973 & 432 \\
0.2196 & 216 & 0.7061 & 412 \\
0.3104 & 291 & 0.8014 & 344 \\
0.4092 & 374 & 0.8954 & 231 \\
0.5080 & 419 & & \\
\hline
\end{tabular}

Tabla 4-61: energías molares de exceso a $V=$ cte. del sistema $N, N, N$-trietilamina (1) +2 -heptanona (2)

\begin{tabular}{cccccc}
\hline$x_{1}$ & $U_{\mathrm{m}, V}^{\mathrm{E}} / \mathrm{J} \cdot \mathrm{mol}^{-1}$ & $x_{1}$ & $U_{\mathrm{m}, V}^{\mathrm{E}} / \mathrm{J} \cdot \mathrm{mol}^{-1}$ & $x_{1}$ & $U_{\mathrm{m}, V}^{\mathrm{E}} / \mathrm{J} \cdot \mathrm{mol}^{-1}$ \\
\hline 0.0538 & 60 & 0.3421 & 385 & 0.6422 & 486 \\
0.0986 & 129 & 0.3897 & 428 & 0.6925 & 469 \\
0.1424 & 179 & 0.4455 & 453 & 0.7402 & 432 \\
0.1969 & 234 & 0.4968 & 475 & 0.8950 & 249 \\
0.2516 & 293 & 0.5452 & 488 & 0.9426 & 140 \\
0.3006 & 343 & 0.5919 & 493 & & \\
\hline
\end{tabular}

Tabla 4-62: coeficientes y desviaciones estándar del ajuste a la ecuación R-K de las diferentes propiedades de exceso del sistema trietilamina (1) + 2-heptanona (2)

\begin{tabular}{ccccc}
\hline Propiedad & $A_{0}$ & $A_{1}$ & $A_{2}$ & $\sigma$ \\
\hline$H_{\mathrm{m}}^{\mathrm{E}} / \mathrm{J} \cdot \mathrm{mol}^{-1}$ & 1656 & 800 & 110 & 7 \\
$U_{\mathrm{m}, V}^{\mathrm{E}} / \mathrm{J} \cdot \mathrm{mol}^{-1}$ & 1910 & 720 & 100 & 6 \\
\hline
\end{tabular}



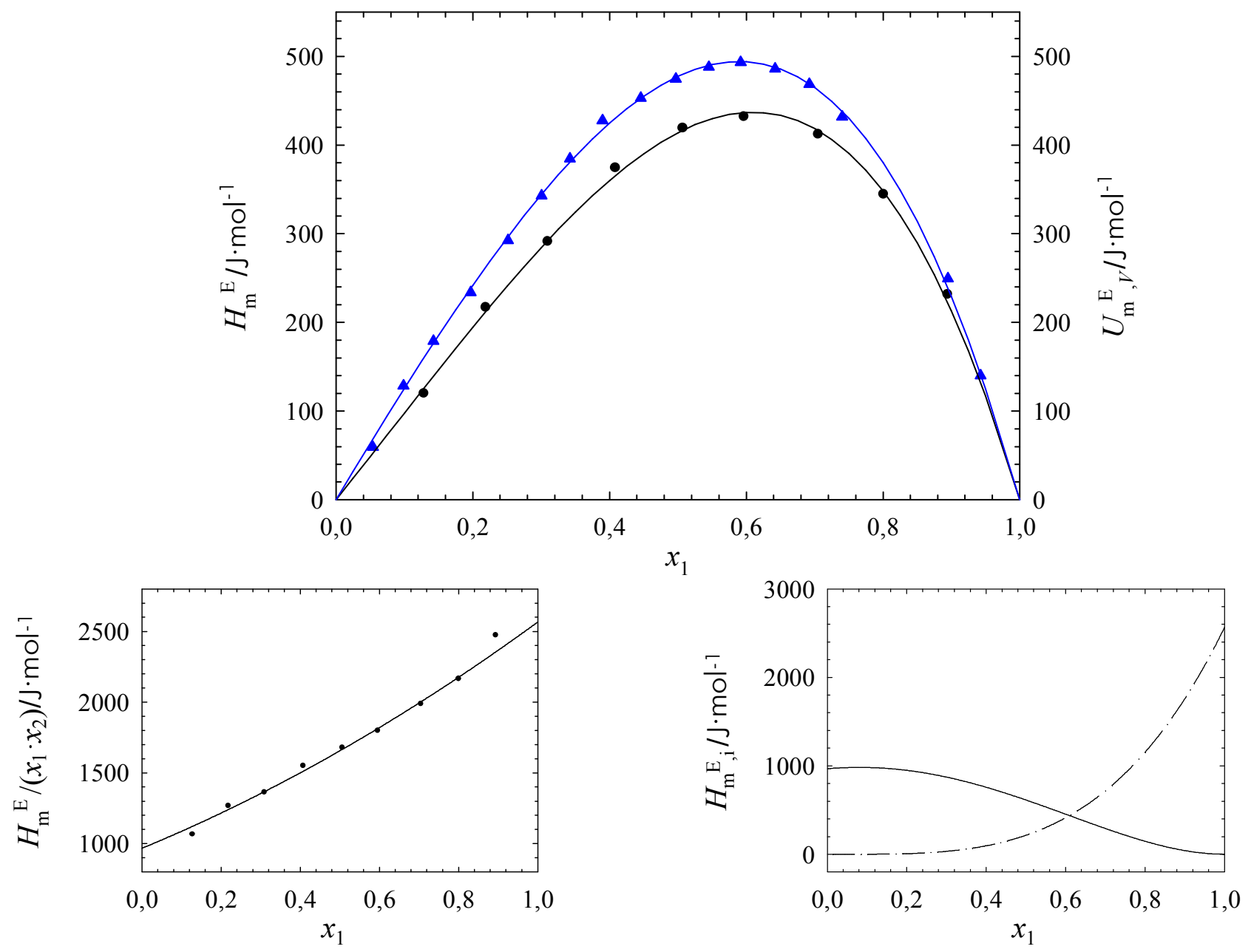

Ilustración 4-30: entalpías molares de exceso $H_{\mathrm{m}}^{\mathrm{E}}$, energías internas de exceso a volumen constante $U_{\mathrm{m}, V}^{\mathrm{E}}$, entalpías molares de exceso reducidos $H_{\mathrm{m}}^{\mathrm{E}} /\left(x_{1} \cdot x_{2}\right)$ y entalpías molares parciales de exceso $H_{\mathrm{m}, \mathrm{i}}^{\mathrm{E}}$ del sistema $N, N, N$-trietilamina (1) +2 -heptanona (2) 
Tabla 4-63: entalpías molares de exceso del sistema $N$-metilanilina (1) + heptano (2)

\begin{tabular}{cccc}
\hline$x_{1}$ & $H_{\mathrm{m}}^{\mathrm{E}} / \mathrm{J} \cdot \mathrm{mol}^{-1}$ & $x_{1}$ & $H_{\mathrm{m}}^{\mathrm{E}} / \mathrm{J} \cdot \mathrm{mol}^{-1}$ \\
\hline 0.1020 & 818 & 0.5982 & 1600 \\
0.1982 & 1292 & 0.6934 & 1439 \\
0.2968 & 1539 & 0.7941 & 1126 \\
0.3935 & 1654 & 0.8938 & 679 \\
0.4983 & 1676 & & \\
\hline
\end{tabular}

Tabla 4-64: energías molares de exceso a $V=$ cte. del sistema $N$-metilanilina (1) + heptano (2)

\begin{tabular}{cccccc}
\hline$x_{1}$ & $U_{\mathrm{m}, V}^{\mathrm{E}} / \mathrm{J} \cdot \mathrm{mol}^{-1}$ & $x_{1}$ & $U_{\mathrm{m}, V}^{\mathrm{E}} / \mathrm{J} \cdot \mathrm{mol}^{-1}$ & $x_{1}$ & $U_{\mathrm{m}, V}^{\mathrm{E}} / \mathrm{J} \cdot \mathrm{mol}^{-1}$ \\
\hline 0.0577 & 512 & 0.3955 & 1717 & 0.6995 & 1509 \\
0.1072 & 856 & 0.4559 & 1748 & 0.7491 & 1370 \\
0.1473 & 1076 & 0.5030 & 1749 & 0.7964 & 1207 \\
0.2055 & 1325 & 0.5431 & 1733 & 0.8446 & 999 \\
0.2595 & 1491 & 0.5956 & 1689 & 0.8949 & 730 \\
0.3033 & 1590 & 0.6441 & 1621 & 0.9465 & 401 \\
0.3401 & 1651 & & & & \\
\hline
\end{tabular}

Tabla 4-65: coeficientes y desviaciones estándar del ajuste a la ecuación R-K de las diferentes propiedades de exceso del sistema $N$-metilanilina (1) + heptano (2)

\begin{tabular}{ccccccc}
\hline Propiedad & $A_{0}$ & $A_{1}$ & $A_{2}$ & $A_{3}$ & $A_{4}$ & $\sigma$ \\
\hline$H_{\mathrm{m}}^{\mathrm{E}} / \mathrm{J} \cdot \mathrm{mol}^{-1}$ & 6704 & -630 & 2190 & -840 & & 7 \\
$U_{\mathrm{m}, V}^{\mathrm{E}} / \mathrm{J} \cdot \mathrm{mol}^{-1}$ & 6998 & -353 & 2290 & -637 & -170 & 1.2 \\
\hline
\end{tabular}



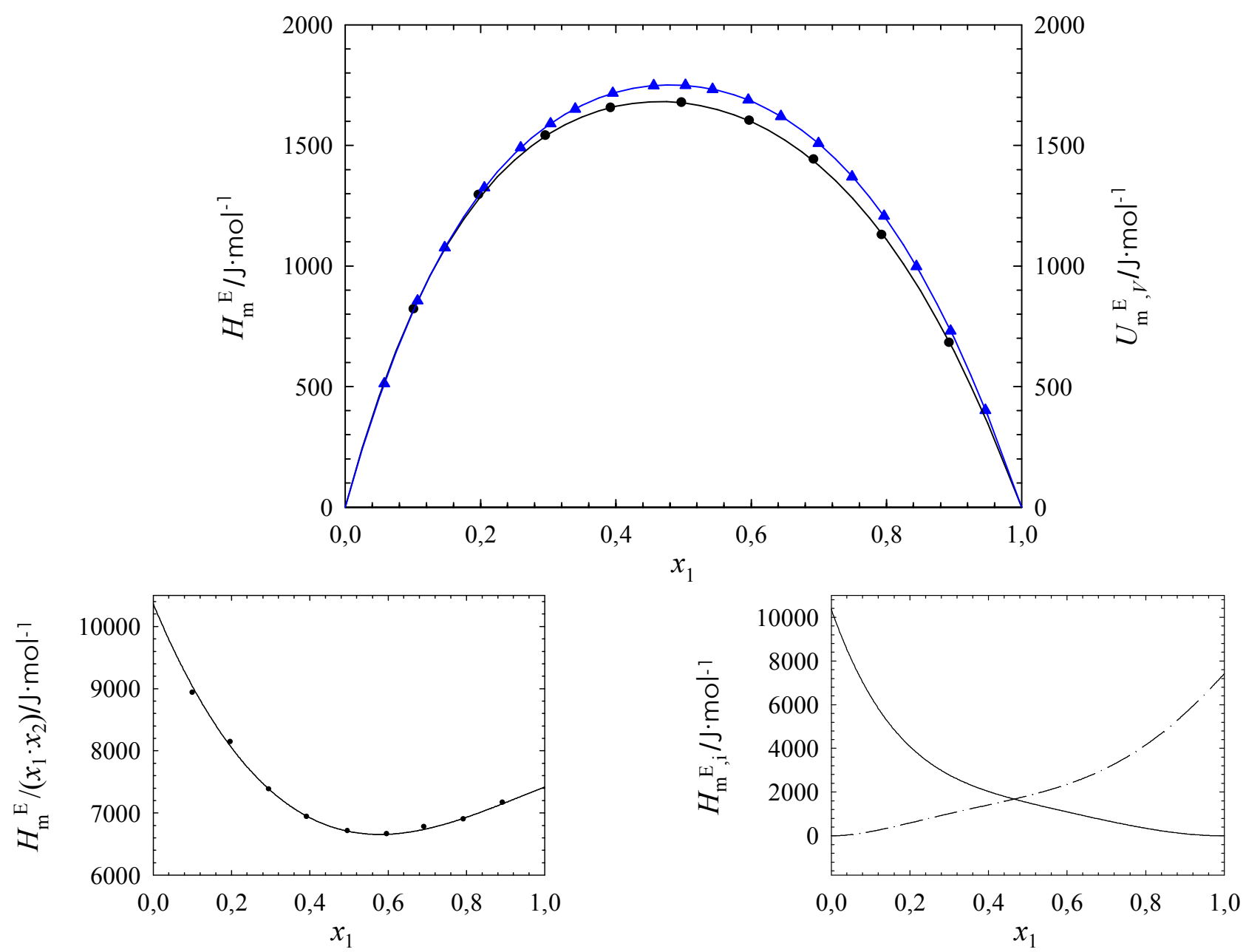

llustración 4-31: entalpías molares de exceso $H_{\mathrm{m}}^{\mathrm{E}}$, energías internas de exceso a volumen constante $U_{\mathrm{m}, V}^{\mathrm{E}}$, entalpías molares de exceso reducidos $H_{\mathrm{m}}^{\mathrm{E}} /\left(x_{1} \cdot x_{2}\right)$ y entalpías molares parciales de exceso $H_{\mathrm{m}, \mathrm{i}}^{\mathrm{E}}$ del sistema $N$-metilanilina (1) + heptano (2) 
Tabla 4-66: entalpías molares de exceso del sistema $N$-metilanilina (1) + octano (2)

\begin{tabular}{cccc}
\hline$x_{1}$ & $H_{\mathrm{m}}^{\mathrm{E}} / \mathrm{J} \cdot \mathrm{mol}^{-1}$ & $x_{1}$ & $H_{\mathrm{m}}^{\mathrm{E}} / \mathrm{J} \cdot \mathrm{mol}^{-1}$ \\
\hline 0.1045 & 853 & 0.5965 & 1694 \\
0.2002 & 1346 & 0.6933 & 1510 \\
0.3030 & 1624 & 0.7910 & 1192 \\
0.4117 & 1755 & 0.8890 & 769 \\
0.4956 & 1774 & & \\
\hline
\end{tabular}

Tabla 4-67: coeficientes y desviaciones estándar del ajuste a la ecuación R-K de las diferentes propiedades de exceso del sistema $N$-metilanilina (1) + octano (2)

\begin{tabular}{ccccc}
\hline Propiedad & $A_{0}$ & $A_{1}$ & $A_{2}$ & $\sigma$ \\
\hline$H_{\mathrm{m}}^{\mathrm{E}} / \mathrm{J} \cdot \mathrm{mol}^{-1}$ & 7070 & -840 & 2160 & 12 \\
\hline
\end{tabular}



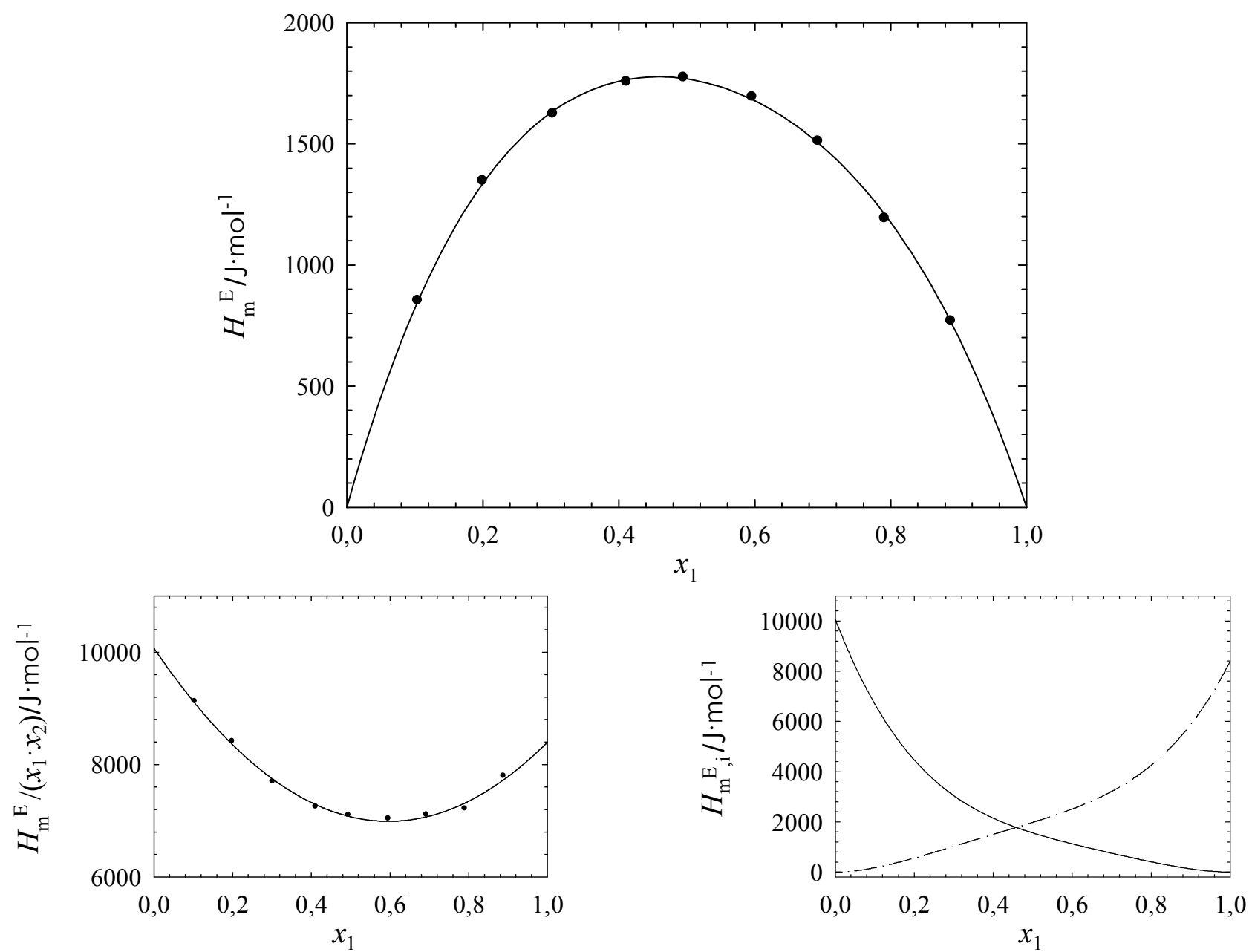

Ilustración 4-32: entalpías molares de exceso $H_{\mathrm{m}}^{\mathrm{E}}$, energías internas de exceso a volumen constante $U_{\mathrm{m}, V}^{\mathrm{E}}$, entalpías molares de exceso reducidos $H_{\mathrm{m}}^{\mathrm{E}} /\left(x_{1} \cdot x_{2}\right)$ y entalpías molares parciales de exceso $H_{\mathrm{m}, \mathrm{i}}^{\mathrm{E}}$ del sistema $N$-metilanilina (1) + octano (2) 
Tabla 4-68: entalpías molares de exceso del sistema $N$-metilanilina (1) + decano (2)

\begin{tabular}{cccc}
\hline$x_{1}$ & $H_{\mathrm{m}}^{\mathrm{E}} / \mathrm{J} \cdot \mathrm{mol}^{-1}$ & $x_{1}$ & $H_{\mathrm{m}}^{\mathrm{E}} / \mathrm{J} \cdot \mathrm{mol}^{-1}$ \\
\hline 0.1015 & 881 & 0.5963 & 1840 \\
0.2010 & 1422 & 0.6951 & 1651 \\
0.2993 & 1730 & 0.7934 & 1321 \\
0.3848 & 1875 & 0.8899 & 857 \\
0.4870 & 1918 & & \\
\hline
\end{tabular}

Tabla 4-69: coeficientes y desviaciones estándar del ajuste a la ecuación R-K de las diferentes propiedades de exceso del sistema $N$-metilanilina (1) + decano (2)

\begin{tabular}{ccccc}
\hline Propiedad & $A_{0}$ & $A_{1}$ & $A_{2}$ & $\sigma$ \\
\hline$H_{\mathrm{m}}^{\mathrm{E}} / \mathrm{J} \cdot \mathrm{mol}^{-1}$ & 7654 & -590 & 2380 & 7 \\
\hline
\end{tabular}



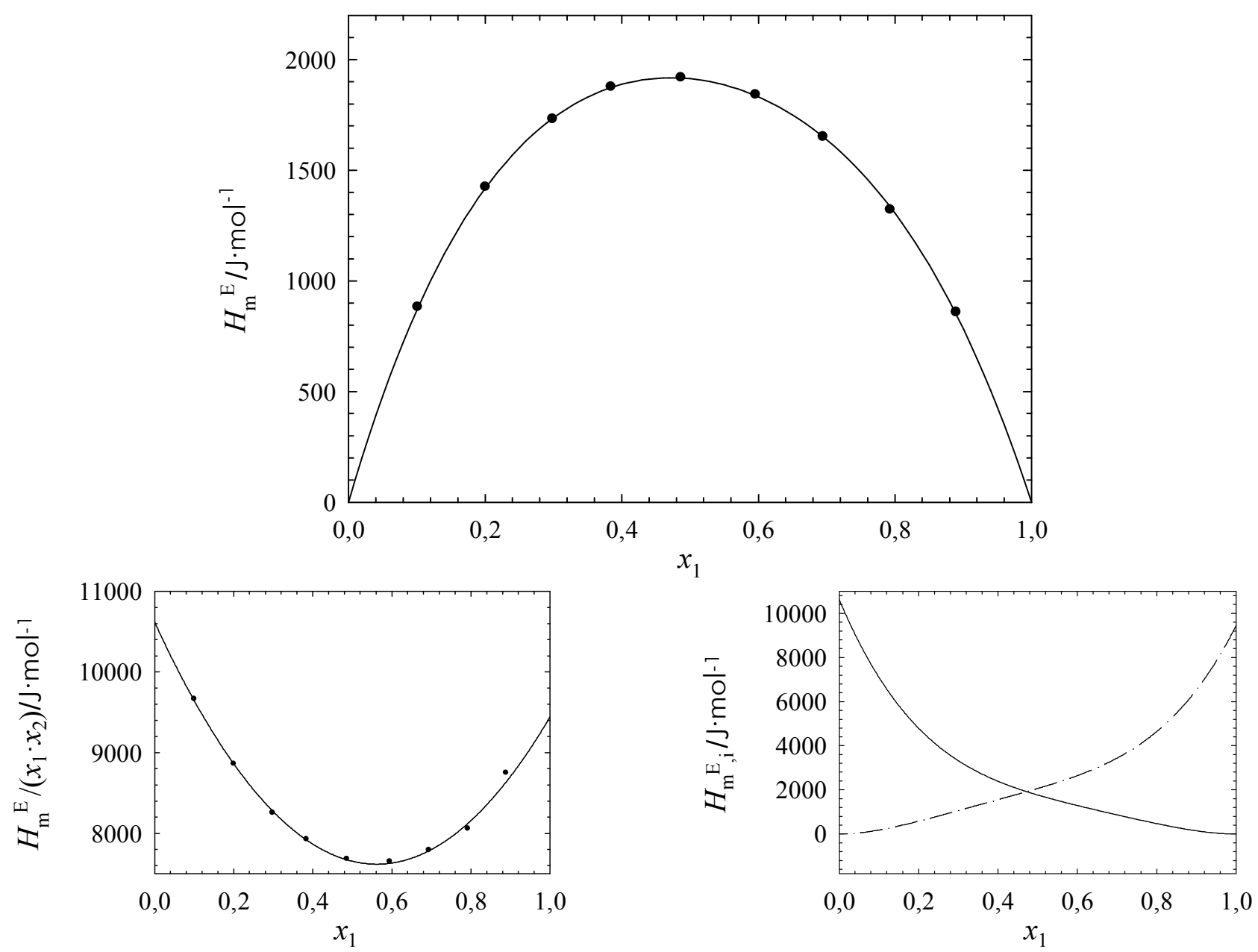

llustración 4-33: entalpías molares de exceso $H_{\mathrm{m}}^{\mathrm{E}}$, energías internas de exceso a volumen constante $U_{\mathrm{m}, V}^{\mathrm{E}}$, entalpías molares de exceso reducidos $H_{\mathrm{m}}^{\mathrm{E}} /\left(x_{1} \cdot x_{2}\right)$ y entalpías molares parciales de exceso $H_{\mathrm{m}, \mathrm{i}}^{\mathrm{E}}$ del sistema $N$-metilanilina (1) + decano (2) 
Tabla 4-70: entalpías molares de exceso del sistema $N$-metilanilina (1) + ciclohexano (2)

\begin{tabular}{cccc}
\hline$x_{1}$ & $H_{\mathrm{m}}^{\mathrm{E}} / \mathrm{J} \cdot \mathrm{mol}^{-1}$ & $x_{1}$ & $H_{\mathrm{m}}^{\mathrm{E}} / \mathrm{J} \cdot \mathrm{mol}^{-1}$ \\
\hline 0.0978 & 826 & 0.5934 & 1538 \\
0.1987 & 1296 & 0.6935 & 1308 \\
0.2934 & 1521 & 0.7953 & 972 \\
0.4002 & 1611 & 0.8938 & 568 \\
0.4937 & 1628 & & \\
\hline
\end{tabular}

Tabla 4-71: energías molares de exceso a $V=$ cte. del sistema $N$-metilanilina (1) + ciclohexano (2)

\begin{tabular}{cccccc}
\hline$x_{1}$ & $U_{\mathrm{m}, V}^{\mathrm{E}} / \mathrm{J} \cdot \mathrm{mol}^{-1}$ & $x_{1}$ & $U_{\mathrm{m}, V}^{\mathrm{E}} / \mathrm{J} \cdot \mathrm{mol}^{-1}$ & $x_{1}$ & $U_{\mathrm{m}, V}^{\mathrm{E}} / \mathrm{J} \cdot \mathrm{mol}^{-1}$ \\
\hline 0.0673 & 531 & 0.3981 & 1445 & 0.6939 & 1187 \\
0.1073 & 769 & 0.4479 & 1454 & 0.7530 & 1028 \\
0.1609 & 1010 & 0.5055 & 1441 & 0.7950 & 893 \\
0.2065 & 1162 & 0.5537 & 1409 & 0.8347 & 750 \\
0.2536 & 1275 & 0.5968 & 1361 & 0.8896 & 521 \\
0.2963 & 1351 & 0.6482 & 1279 & 0.9399 & 289 \\
0.3554 & 1417 & & & & \\
\hline
\end{tabular}

Tabla 4-72: coeficientes y desviaciones estándar del ajuste a la ecuación R-K de las diferentes propiedades de exceso del sistema $N$-metilanilina (1) + ciclohexano (2)

\begin{tabular}{ccccccc}
\hline Propiedad & $A_{0}$ & $A_{1}$ & $A_{2}$ & $A_{3}$ & $A_{4}$ & $\sigma$ \\
\hline$H_{\mathrm{m}}^{\mathrm{E}} / \mathrm{J} \cdot \mathrm{mol}^{-1}$ & 6470 & -1110 & 1710 & -1700 & & 15 \\
$U_{\mathrm{m}, V}^{\mathrm{E}} / \mathrm{J} \cdot \mathrm{mol}^{-1}$ & 5774 & -874 & 1560 & -1403 & -210 & 1.2 \\
\hline
\end{tabular}



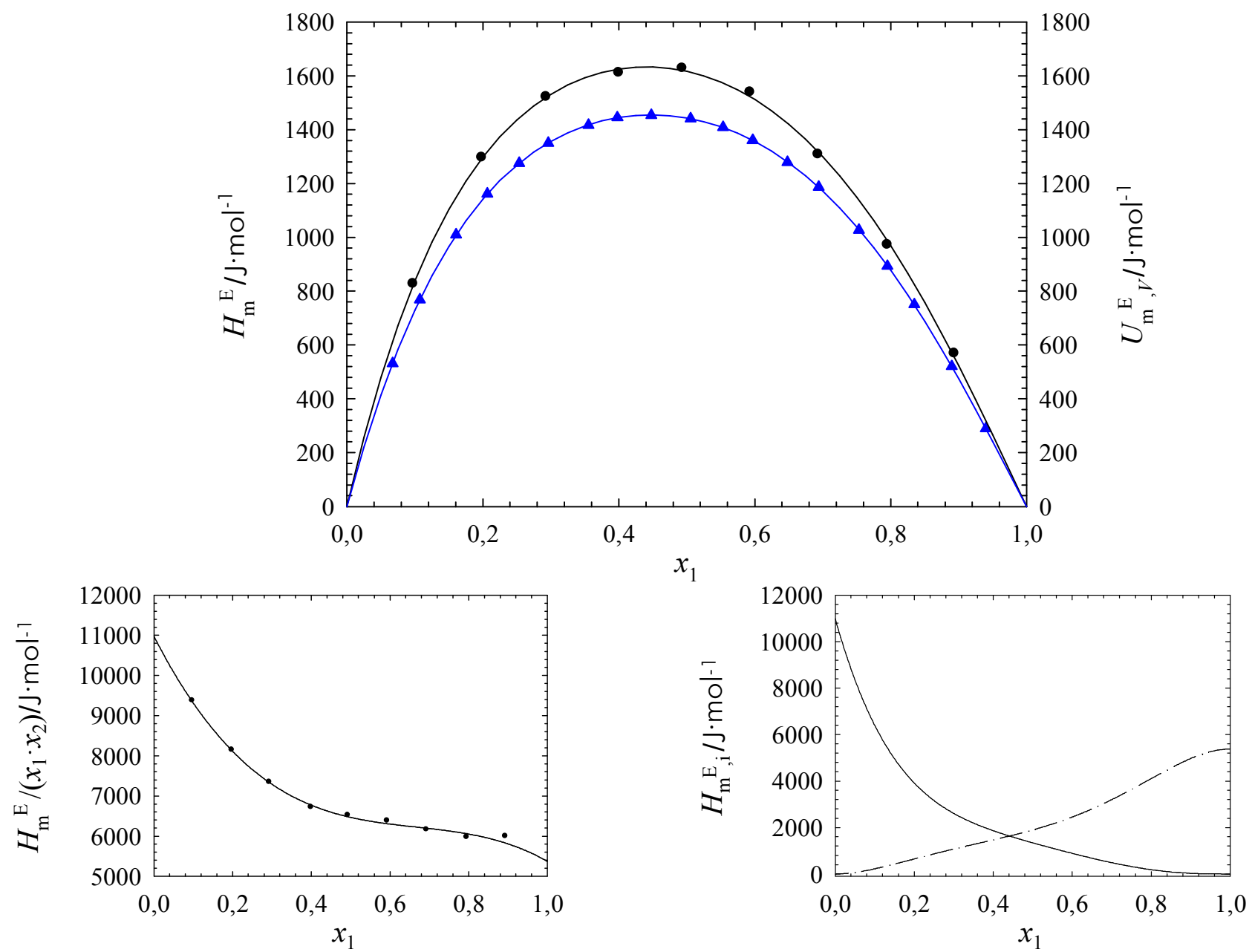

Ilustración 4-34: entalpías molares de exceso $H_{\mathrm{m}}^{\mathrm{E}}$, energías internas de exceso a volumen constante $U_{\mathrm{m}, V}^{\mathrm{E}}$, entalpías molares de exceso reducidos $H_{\mathrm{m}}^{\mathrm{E}} /\left(x_{1} \cdot x_{2}\right)$ y entalpías molares parciales de exceso $H_{\mathrm{m}, \mathrm{i}}^{\mathrm{E}}$ del sistema $N$-metilanilina (1) + ciclohexano (2) 
Tabla 4-73: entalpías molares de exceso del sistema $N$-metilanilina (1) + tolveno (2)

\begin{tabular}{cccc}
\hline$x_{1}$ & $H_{\mathrm{m}}^{\mathrm{E}} / \mathrm{J} \cdot \mathrm{mol}^{-1}$ & $x_{1}$ & $H_{\mathrm{m}}^{\mathrm{E}} / \mathrm{J} \cdot \mathrm{mol}^{-1}$ \\
\hline 0.0991 & 224 & 0.5922 & 465 \\
0.1956 & 368 & 0.6882 & 387 \\
0.2952 & 459 & 0.7892 & 287 \\
0.3990 & 500 & 0.8851 & 166 \\
0.4974 & 497 & & \\
\hline
\end{tabular}

Tabla 4-74: energías molares de exceso a $V=$ cte. del sistema $N$-metilanilina (1) + tolveno (2)

\begin{tabular}{cccccc}
\hline$x_{1}$ & $U_{\mathrm{m}, V}^{\mathrm{E}} / \mathrm{J} \cdot \mathrm{mol}^{-1}$ & $x_{1}$ & $U_{\mathrm{m}, V}^{\mathrm{E}} / \mathrm{J} \cdot \mathrm{mol}^{-1}$ & $x_{1}$ & $U_{\mathrm{m}, V}^{\mathrm{E}} / \mathrm{J} \cdot \mathrm{mol}^{-1}$ \\
\hline 0.0532 & 145 & 0.3900 & 580 & 0.6868 & 471 \\
0.0998 & 251 & 0.4434 & 589 & 0.7407 & 411 \\
0.1518 & 352 & 0.4900 & 586 & 0.7823 & 359 \\
0.1906 & 412 & 0.5415 & 572 & 0.8378 & 279 \\
0.2452 & 481 & 0.5792 & 554 & 0.8844 & 205 \\
0.2887 & 523 & 0.6432 & 510 & 0.9340 & 120 \\
0.3417 & 558 & & & & \\
\hline
\end{tabular}

Tabla 4-75: coeficientes y desviaciones estándar del ajuste a la ecuación R-K de las diferentes propiedades de exceso del sistema $N$-metilanilina (1) + tolveno (2)

\begin{tabular}{cccccc}
\hline Propiedad & $A_{0}$ & $A_{1}$ & $A_{2}$ & $A_{3}$ & $\sigma$ \\
\hline$H_{\mathrm{m}}^{\mathrm{E}} / \mathrm{J} \cdot \mathrm{mol}^{-1}$ & 1989 & -460 & 100 & -150 & 3 \\
$U_{\mathrm{m}, V}^{\mathrm{E}} / \mathrm{J} \cdot \mathrm{mol}^{-1}$ & 2338.0 & -420 & 95 & -142 & 0.6 \\
\hline
\end{tabular}



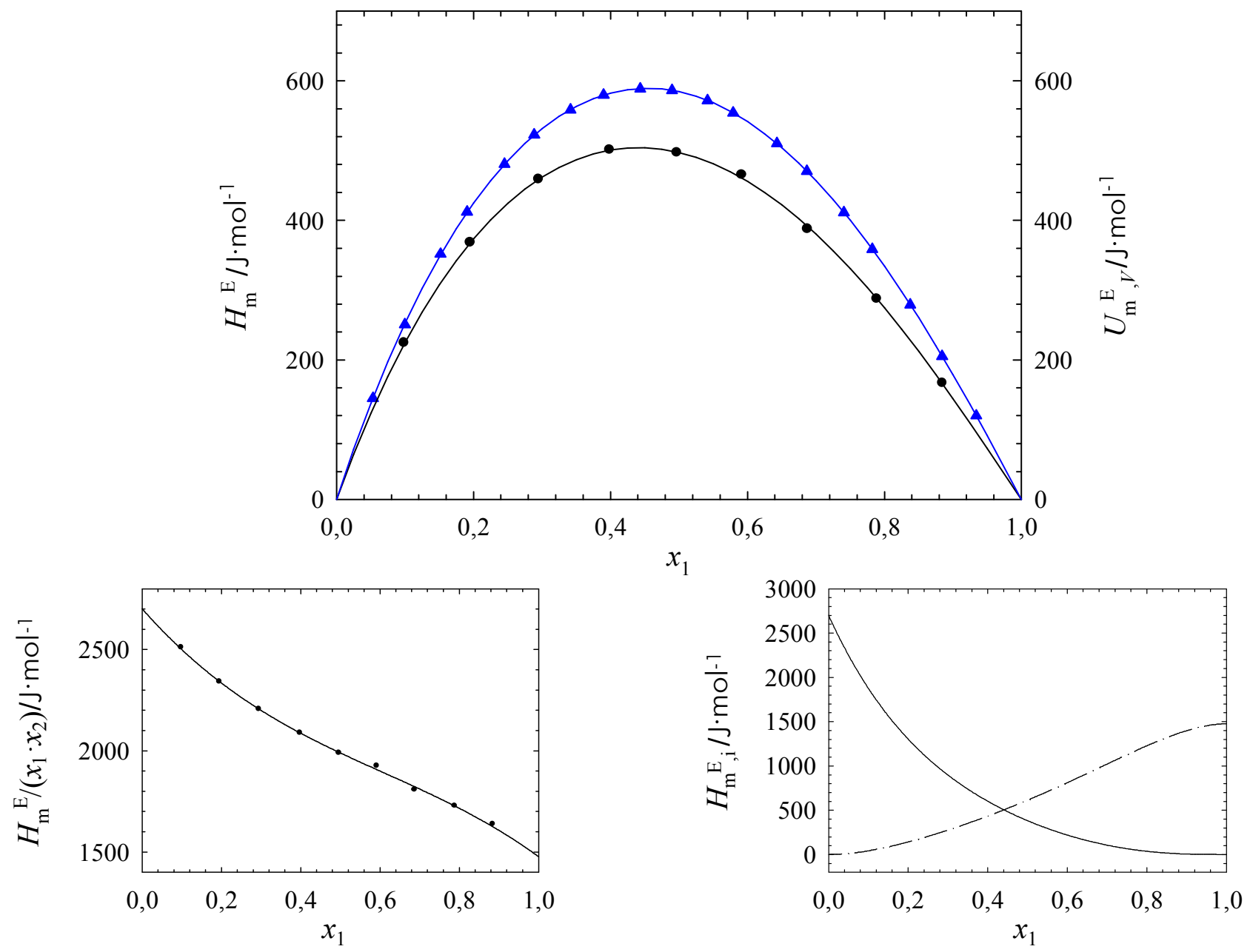

llustración 4-35: entalpías molares de exceso $H_{\mathrm{m}}^{\mathrm{E}}$, energías internas de exceso a volumen constante $U_{\mathrm{m}, V}^{\mathrm{E}}$, entalpías molares de exceso reducidos $H_{\mathrm{m}}^{\mathrm{E}} /\left(x_{1} \cdot x_{2}\right)$ y entalpías molares parciales de exceso $H_{\mathrm{m}, \mathrm{i}}^{\mathrm{E}}$ del sistema $N$-metilanilina (1) + tolveno (2) 


\subsubsection{Mezcla 1-propanol (1) $+N, N, N$-trietilamina (2) +2 -butanona (3)}

En esta sección se dan los datos obtenidos para las entalpías de exceso del sistema 1-propanol (1) $+N, N, N$-trietilamina (2) +2 -butanona (3). El método para la medición de dichos datos es diferente al empleado en el caso de mezclas binarias.

En primer lugar deben estimarse los volúmenes a mezclar de cada uno de los componentes para cubrir el rango deseado de composiciones. La determinación de las entalpías de exceso de un sistema ternario se realiza mezclando uno de los componentes con una mezcla de los otros dos (llamada "disolución madre"), siendo constante en todo momento el cociente entre las composiciones de estos dos últimos componentes, lo cual debe tenerse en cuenta para todos los cálculos. En nuestro caso, se hicieron tres disoluciones madre $N, N, N$-trietilamina $\left(2^{\prime}\right)+2$-butanona $\left(3^{\prime}\right)$, cuyos cocientes entre composiciones fueron:

$$
\frac{x_{2^{\prime}}}{x_{3^{\prime}}}=1.0249 \quad \frac{x_{2^{\prime}}}{x_{3^{\prime}}}=2.9529 \quad \frac{x_{2^{\prime}}}{x_{3^{\prime}}}=0.3264
$$

Dichos cocientes se mantienen en la mezcla ternaria, ya que en ella ni se añade cantidad de materia del componente 2 ni del 3.

Teniendo en cuenta que es necesario determinar la entalpía de exceso de la "disolución madre" $H_{\mathrm{m}, 2^{\prime} 3^{\prime}}^{\mathrm{E}}$ para determinar las entalpías de exceso del sistema ternario $H_{\mathrm{m}, 123}^{\mathrm{E}}$, en segundo lugar se debe determinar dicho valor, lo que se consigue mediante el procedimiento estándar para la medida de $H_{\mathrm{m}}^{\mathrm{E}}$ de mezclas binarias. En dicho procedimiento la mezcla se produce en la celda del calorímetro, por lo que es imposible medir el calor de mezcla de la disolución madre directamente ya que esta se ha preparado fuera del calorímetro. Por ello, se determinan las entalpías de exceso de unas cuantas mezclas de composición tan parecida a la madre como se pueda, se ajustan los datos obtenidos a una ecuación R-K y de dicho ajuste se obtiene el valor de la entalpía de mezcla a la composición exacta de la disolución madre.

El siguiente paso es ir añadiendo cantidades de 1-propanol a la "disolución madre" e ir midiendo la entalpía de exceso $H_{\mathrm{m}, \psi}^{\mathrm{E}}$ de la mezcla pseudibinaria resultante.

Finalmente se calcula la entalpía de exceso de la mezcla ternaria con composición $x_{2}, x_{3}$ $\left(x_{1}=1-x_{2}-x_{3}\right)$ como la suma siguiente [197]:

$$
H_{\mathrm{m}, 123}^{\mathrm{E}}=H_{\mathrm{m}, \psi}^{\mathrm{E}}+\left(x_{2}+x_{3}\right) \cdot H_{\mathrm{m}, 2^{\prime} 3^{\prime}}^{\mathrm{E}}
$$

La ecuación anterior es exacta y no incluye ninguna aproximación. Los datos así obtenidos junto con la fracción molar del 1-propanol se muestran en la Tabla 4-76 y se representan en la llustración 4-38.

Tras probar con diferentes ecuaciones de ajuste discutidas en las referencias [198][200], se comprobó que la ecuación que proporciona el mejor ajuste a los datos experimentales obtenidos es:

$$
H_{\mathrm{m}, 123}^{\mathrm{E}}=H_{\mathrm{m}, 12}^{\mathrm{E}}+H_{\mathrm{m}, 13}^{\mathrm{E}}+H_{\mathrm{m}, 23}^{\mathrm{E}}+x_{2} \cdot x_{3} \cdot\left(1-x_{2}-x_{3}\right) \cdot \Delta_{123}
$$

donde

$$
\Delta_{123}=R \cdot T \cdot\left(B_{0}-B_{1} \cdot x_{3}-B_{2} \cdot x_{2}-B_{3} \cdot x_{3}^{2}-B_{4} \cdot x_{2}^{2}-B_{5} \cdot x_{3} \cdot x_{2}-B_{6} \cdot x_{3}^{3}\right)
$$


siendo $R=8.314 \mathrm{~J} \cdot \mathrm{K}^{-1} \cdot \mathrm{mol}^{-1}, T=298.15 \mathrm{~K}$, los coeficientes $B_{\mathrm{i}}$ se dan en la Tabla $4-77 \mathrm{y}$ :

$$
H_{\mathrm{m}, \mathrm{ij}}^{\mathrm{E}}=x_{\mathrm{i}} \cdot x_{\mathrm{j}} \cdot \sum_{\mathrm{k}=0}^{\mathrm{n}} A_{\mathrm{k}}(\mathrm{i}, \mathrm{j}) \cdot\left(x_{\mathrm{i}}-x_{\mathrm{j}}\right)^{k}
$$

son los polinomios resultantes del ajuste a la ecuación R-K de los datos de las tres mezclas binarias: 1 -propanol (1) $+N, N, N$-trietilamina (2), 1-propanol (1) +2 -butanona (2) y $N, N, N$-ttrietilamina (1) + 2-butanona (2), los cuales aparecen en la Tabla 4-78.

Tabla 4-76: entalpías molares de exceso del sistema 1-propanol (1) $+N, N, N$-trietilamina (2) +2 -butanona (3)

\begin{tabular}{|c|c|c|c|c|c|}
\hline$x_{1}$ & $H_{\mathrm{m}, 123}^{\mathrm{E}} / \mathrm{J} \cdot \mathrm{mol}^{-1}$ & $x_{1}$ & $H_{\mathrm{m}, 123}^{\mathrm{E}} / \mathrm{J} \cdot \mathrm{mol}^{-1}$ & $x_{1}$ & $H_{\mathrm{m}, 123}^{\mathrm{E}} / \mathrm{J} \cdot \mathrm{mol}^{-1}$ \\
\hline \multicolumn{6}{|c|}{$x_{2} / x_{3}=2.9529\left(x_{2}{ }^{\prime}=0.7470, x_{3}{ }^{\prime}=0.2530\right)$} \\
\hline 0.0944 & 259.44 & 0.4057 & -691.18 & 0.6997 & -876.72 \\
\hline 0.1453 & 37.67 & 0.4563 & -771.41 & 0.7488 & -813.31 \\
\hline 0.1920 & -132.70 & 0.4996 & -851.80 & 0.7911 & -718.77 \\
\hline 0.2225 & -229.85 & 0.5535 & -897.06 & 0.8362 & -619.16 \\
\hline 0.2709 & -371.47 & 0.5963 & -926.60 & 0.8872 & -477.38 \\
\hline 0.3094 & -470.97 & 0.6479 & -908.90 & 0.9336 & -313.23 \\
\hline 0.3651 & -626.16 & & & & \\
\hline \multicolumn{6}{|c|}{$x_{2} / x_{3}=1.0249\left(x_{2}{ }^{\prime}=0.5061, x_{3}{ }^{\prime}=0.4939\right)$} \\
\hline 0.0691 & 520.91 & 0.3971 & -190.76 & 0.6960 & -335.73 \\
\hline 0.1133 & 392.88 & 0.4524 & -279.25 & 0.7487 & -309.61 \\
\hline 0.1672 & 254.72 & 0.5031 & -311.56 & 0.8002 & -259.09 \\
\hline 0.2211 & 124.02 & 0.5533 & -344.08 & 0.8403 & -227.75 \\
\hline 0.2653 & 21.59 & 0.6027 & -346.61 & 0.8914 & -168.09 \\
\hline 0.3111 & -63.36 & 0.6451 & -376.71 & 0.9410 & -104.99 \\
\hline 0.3610 & -140.19 & & & & \\
\hline \multicolumn{6}{|c|}{$x_{2} / x_{3} 0.3264\left(x_{2}^{\prime}=0.2461, x_{3}^{\prime}=0.7539\right)$} \\
\hline 0.0769 & 482.08 & 0.4031 & 397.79 & 0.6947 & 335.90 \\
\hline 0.1301 & 457.90 & 0.4547 & 392.59 & 0.7399 & 312.76 \\
\hline 0.1697 & 441.32 & 0.5030 & 388.38 & 0.7939 & 270.60 \\
\hline 0.2225 & 425.41 & 0.5479 & 380.48 & 0.8313 & 238.94 \\
\hline 0.2723 & 412.46 & 0.5922 & 373.37 & 0.8811 & 181.97 \\
\hline 0.3157 & 405.22 & 0.6467 & 358.51 & 0.9332 & 106.88 \\
\hline 0.3598 & 399.37 & & & & \\
\hline
\end{tabular}

Tabla 4-77: coeficientes y desviaciones estándar del ajuste a la ecuación (4.34), teniendo en cuenta (4.35), de las entalpías molares de exceso del sistema 1-propanol (1) $+N, N, N$-trietilamina (2) +2 -butanona (3)

\begin{tabular}{cccccc}
\hline$B_{0}$ & $B_{1}$ & $B_{2}$ & $B_{3}$ & $B_{4}$ & $\sigma$ \\
\hline-5.4 & 0.2 & -8.0 & 3.0 & 6.4 & 14 \\
\hline
\end{tabular}

Tabla 4-78: coeficientes y desviaciones estándar del ajuste a la ecuación R-K de las entalpías molares de exceso de los sistemas binarios 1-propanol (1) $+N, N, N$-trietilamina (2), 1-propanol (1) +2 -butanona (2) y $N, N, N$-trietilamina (1) +2 -butanona $(2) ;{ }^{1}[198] ;{ }^{2}[199] ;{ }^{3}$ Tabla $4-57$

\begin{tabular}{cccccc}
\hline Sistema & $A_{0} / \mathrm{J} \cdot \mathrm{mol}^{-1}$ & $A_{1} / \mathrm{J} \cdot \mathrm{mol}^{-1}$ & $A_{2} / \mathrm{J} \cdot \mathrm{mol}^{-1}$ & $A_{3} / \mathrm{J} \cdot \mathrm{mol}^{-1}$ & $\sigma / \mathrm{J} \cdot \mathrm{mol}^{-1}$ \\
\hline $\begin{array}{c}\text { 1-propanol (1) + } \\
N, N, N \text {-trietilamina (2) }\end{array}$ & -5700 & -2230 & 398 & -2100 & 12.5 \\
$\begin{array}{c}\text { 1-propanol (1)+ } \\
\text { 2-butanona (2) }\end{array}$ & 5006 & -213 & 778 & & 1.4 \\
$\begin{array}{c}N, N, N \text {-trietilamina (1) } \\
+2 \text {-butanona (2) }\end{array}$ & 2982 & 400 & 330 & 460 & 6 \\
\hline
\end{tabular}


En las mezclas ternarias es costumbre representar las curvas de entalpía molar de exceso constante en función de la composición de cada uno de los componentes en el denominado triángulo de Gibbs. Para obtener dichas curvas se escribió un algoritmo que resolvía la ecuación (4.34) en $x_{1}$ para valores fijos de $H^{\mathrm{E}}{ }_{\mathrm{m}, 123}$ y del cociente $x_{2} x_{3}$, el cual fue ejecutado en el entorno de programación Wolfram Mathematica. Los resultados obtenidos se representan en la llustración 4-36.

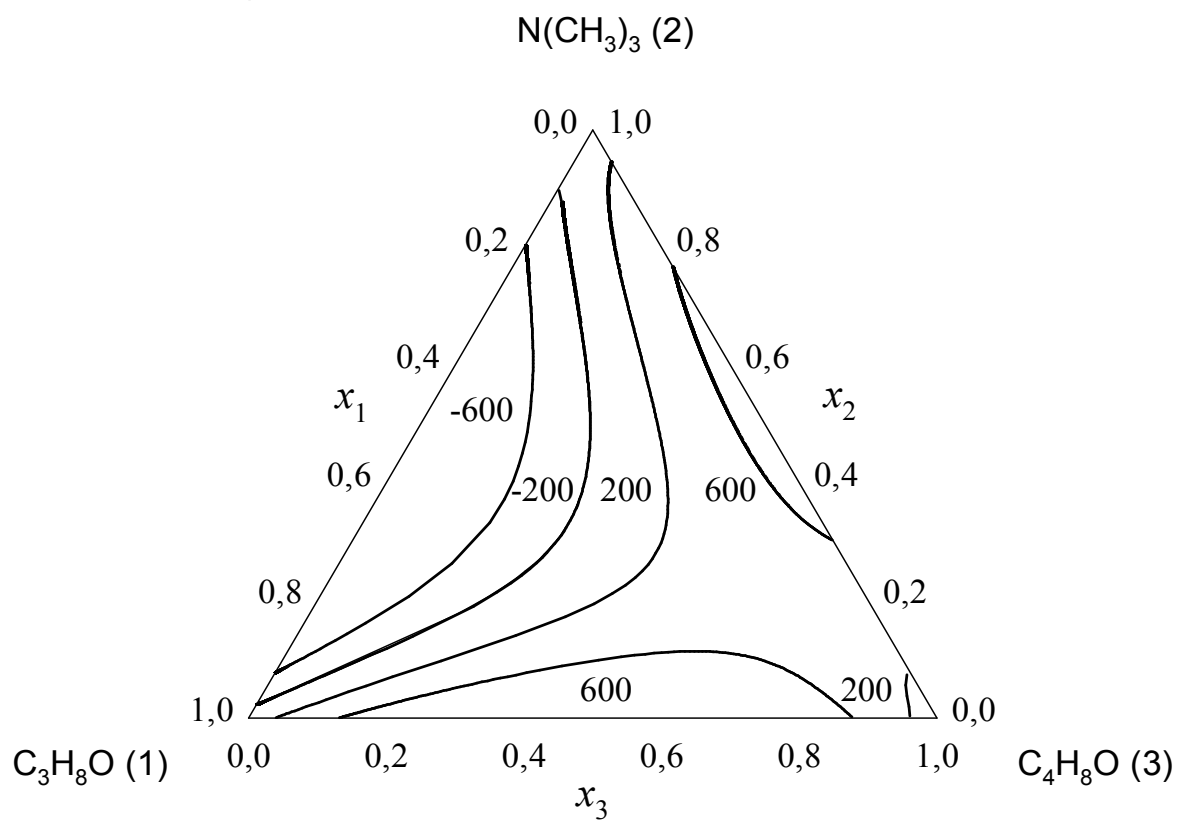

Ilustración 4-36: curvas de entalpía molar de mezcla constante, $H_{\mathrm{m}, 123}^{\mathrm{E}}$ a $298.15 \mathrm{~K}$ para el sistema ternario 1-propanol, $\mathrm{C}_{3} \mathrm{H}_{8} \mathrm{O}$, (1) $+N, N, N$-trietilamina, $\mathrm{N}\left(\mathrm{CH}_{3}\right)_{3},(2)+2$-butanona, $\mathrm{C}_{4} \mathrm{H}_{8} \mathrm{O}$, (3)

En la llustración siguiente se representan las diferentes composiciones de las mezclas para las cuales se determinaron las entalpías de exceso.

$$
\mathrm{N}\left(\mathrm{CH}_{3}\right)_{3}(2)
$$

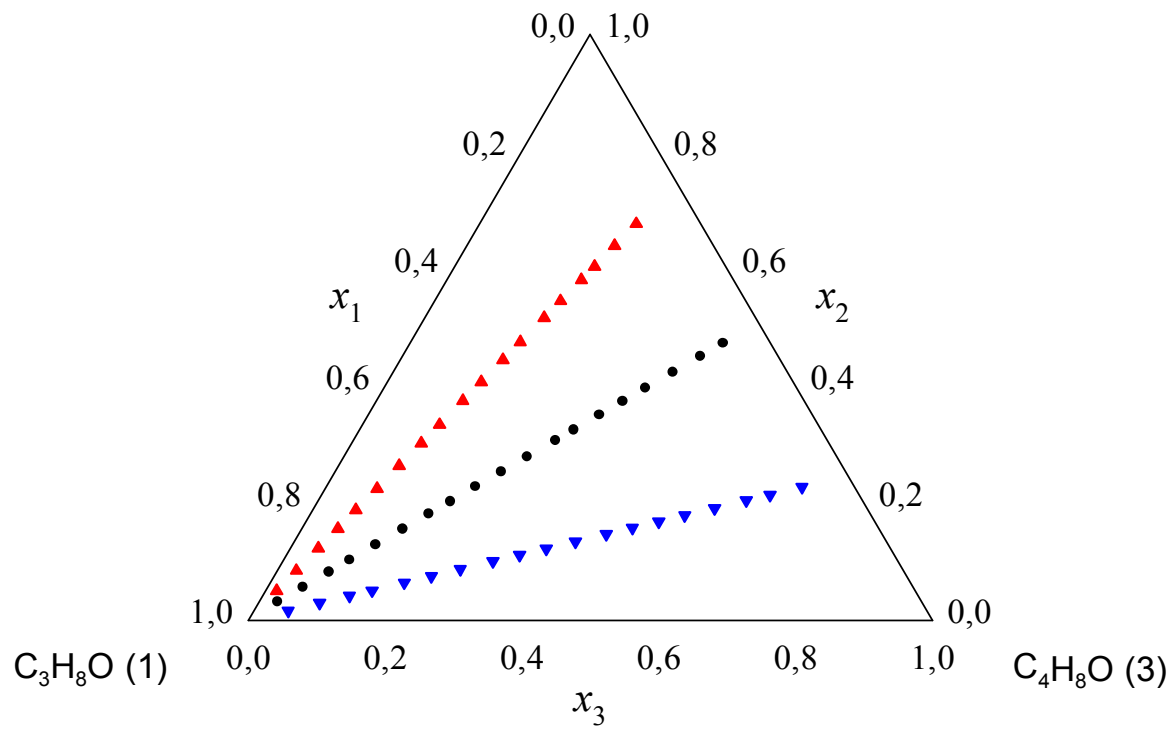

Ilustración 4-37: composición de las mezclas ternarias del sistema 1-propanol, $\mathrm{C}_{3} \mathrm{H}_{8} \mathrm{O}$, (1) + $N, N, N$-trietilamina, $\mathrm{N}\left(\mathrm{CH}_{3}\right)_{3},(2)+2$-butanona, $\mathrm{C}_{4} \mathrm{H}_{8} \mathrm{O}$, (3) para las cuales se determinaron sus entalpías de exceso: $\Delta x_{2} / x_{3}=2.9529 ; \bullet x_{2} / x_{3}=1.0249 ; \boldsymbol{\nabla} x_{2} / x_{3} 0.3264$ 


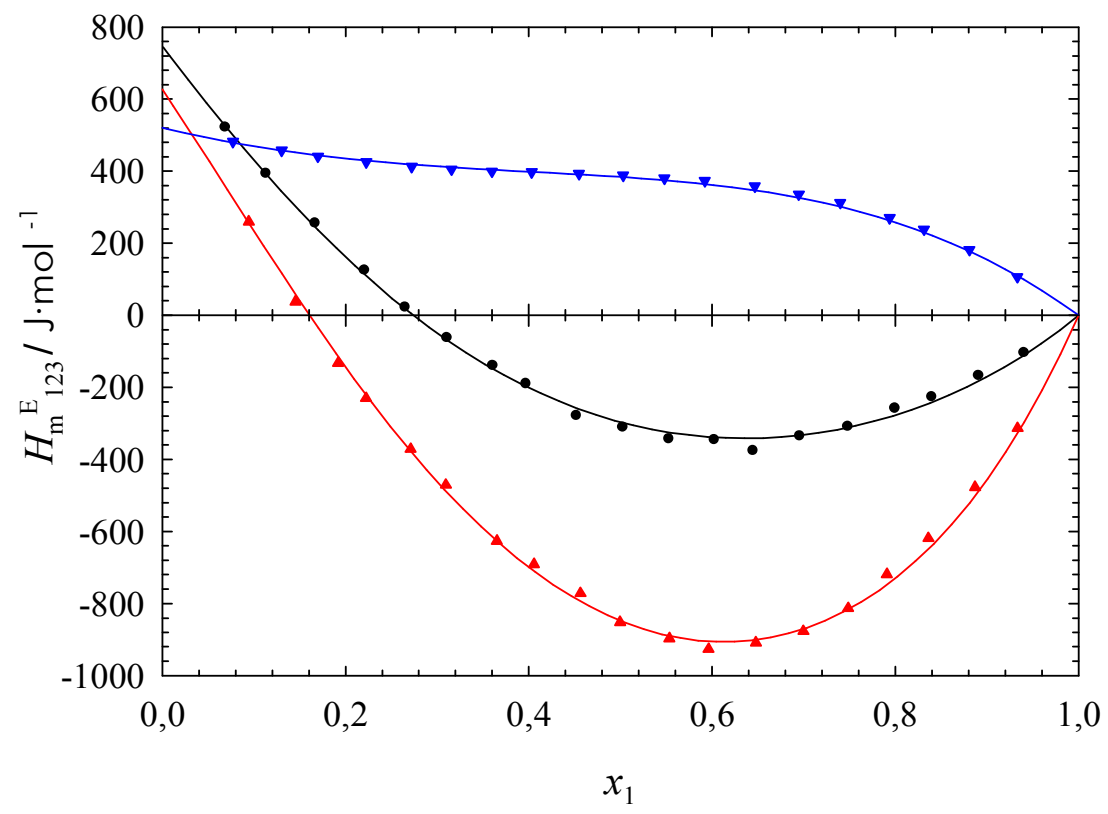

Ilustración 4-38: representación pseudobinaria de los conjuntos de datos de las entalpías molares de exceso $H^{\mathrm{E}}{ }_{\mathrm{m}, 123}$ del sistema 1-propanol, $\mathrm{C}_{3} \mathrm{H}_{8} \mathrm{O},(1)+N, N$, $N$-trietilamina, $\mathrm{N}\left(\mathrm{CH}_{3}\right)_{3}$, (2) + 2-butanona,

$\mathrm{C}_{4} \mathrm{H}_{8} \mathrm{O}$, (3) junto con las curvas del ajuste a la ecuación (4.34) en función de la composición del

1 -propanol $x_{1}$ a $298.15 \mathrm{~K}$ para los cocientes: $\Delta-x_{2} / x_{3}=2.9529 ; \bullet-x_{2} / x_{3}=1.0249 ; \boldsymbol{\nabla}-x_{2} / x_{3}=0.3264$.

\subsubsection{Comentario}

En el planteamiento del subproyecto NCO se consideró en primer momento el estudio de los siguientes sistemas, además de los presentados en el Capítulo 1 de esta memoria:

$$
\begin{aligned}
& \text { hexilamina }+\left\{\begin{array}{l}
2 \text {-propanona } \\
2 \text {-butanona } \\
2 \text {-pentanona } \\
2 \text {-heptanona }
\end{array} \quad \text { butilamina }+\left\{\begin{array}{l}
2 \text {-propanona } \\
2 \text {-butanona } \\
2 \text {-pentanona } \\
2 \text {-heptanona }
\end{array}\right.\right. \\
& \text { ciclohexilamina }+\left\{\begin{array}{l}
2 \text {-propanona } \\
2 \text {-butanona } \\
2 \text {-pentanona } \\
2 \text {-heptanona }
\end{array} \quad \text { bencilamina }+\left\{\begin{array}{l}
2 \text {-propanona } \\
2 \text {-butanona } \\
2 \text {-pentanona } \\
2 \text {-heptanona }
\end{array}\right.\right.
\end{aligned}
$$

El orden cronológico seguido para la obtención de los resultados que se presentan a lo largo de esta Tesis Doctoral fue, primero la determinación de los volúmenes de exceso, y segundo la determinación de las entalpías de exceso. Cuando se llegó a los sistemas arriba indicados aparecieron serias dificultades al intentar obtener ambas magnitudes: primero, cuando se intentaron medir los $V_{\mathrm{m}}^{\mathrm{E}}$ el valor de la frecuencia de resonancia del tubo en $U$ no era estable y no daba un valor fiable de la densidad de la mezcla (como se ha visto en la sección 3.1.1.4, si los constituyentes de una mezcla heterogénea difieren considerablemente en su comportamiento de flujo y en su densidad, se 
moverán unos respecto a otros cuando el tubo vibra, originando fuerzas de inercia y en consecuencia, frecuencias de oscilación ambiguas causantes del error en la medida. En este caso, se tenía la mezcla del producto de la reacción de los dos componentes, junto con la disolución de dichos componentes); segundo, cuando se intentaron medir las $H_{\mathrm{m}}^{\mathrm{E}}$ el termograma que se obtiene al representar el voltaje por efecto Seebeck en función del tiempo para estas mezclas es muy diferente al obtenido en el resto de sistemas considerados en el Capítulo 1, extendiéndose durante un intervalo de tiempo unas 10 veces mayor (15 horas frente a las 1.5 horas aproximadas habituales). Estos resultados hicieron pensar la posibilidad de que las sustancias mezcladas reaccionaban entre sí, lo que quedó corroborado al analizar muestras de estas mezclas mediante espectroscopia infrarroja, como se ve en el Apéndice C. 


\section{BIBLIOGRAFÍA}

[1]: McGlashan, M. L.; IUPAC Symposium on Thermodynamics and Thermochemistry, Lund (1963). Plenary Lectures, 157-169.

[2]: McGlashan, M. L.; Potter, D. J. B.; Proc. Roy. Soc. (London), A267 (1962), 478.

[3]: Curtiss, C.F.; Hirschfelder, J. O.; J. Chem. Phys., 10 (1942), 491.

[4]: Wormald, C.J.; J. Chem. Thermodyn., 9 (1977), 901.

[5]: Villamañan, M.A.; Casanova, C.; Roux, A.H.; Grolier, J.P.E.; J. Chem. Thermodyn., 14 (1982), 251.

[6]: Diogo, H.P.; Minas da Piedade, M.E.; Moura Ramos, J.J.; Simoni, J.A.; Martinho Simoes, J.A.; J. Chem. Edu., 70 (9) (1993), A227.

[7]: Letcher, T.M.; Govender, P.U.; J. Chem. Eng. Data, 40 (1995), 997.

[8]: Letcher, T.M.; Govender, P.U.; J. Chem. Eng. Data, 40 (1995), 1097.

[9]: Letcher, T.M.; Bricknell, B.C.; J. Chem. Eng. Data, 41 (1996), 166.

[10]: Calvo, E.; Brocos, P.; Piñeiro, A.; Pintos, M.; Amigo, A.; Bravo, R.; Roux, A.H.; Roux-Desgranges, G.; J. Chem. Eng. Data, 44 (1999), 948.

[1 1]: Van Ness, H. C.; Van Winkle, J.; Richtol, H. H.; Hollinger, H. B.; J. Phys. Chem., 71 (1967), 1483.

[12]: Woycicka, M. K.; Recko, W. M.; Bull. Acad. Pol. Sci., Ser. Sci. Chem., 20 (1972), 783.

[13]: Stokes, R. H.; Adamson, M.; J. Chem. Soc., Faraday Trans. 1, 71 (1975), 1707.

[14]: Trampe, D.M.; Eckert, C.A.; J. Chem. Eng. Data, 36 (1991), 112.

[15]: Hepler, L.G.; Pure and Appl. Chem., 55(3) (1983), 493.

[16]: Christensen, J. J.; Hansen, L. D.; Eatough, D. J.; Izatt, R. M.; Hart, R. M.; Rev. Sci. Instr., 52, (1981) 1226.

[17]: Heintz, A.; Lichtenthaler, R. N.; Ber. Bunsenges. Phys. Chem., 83 (1979), 853.

[18]: Goates, J. R.; Ott, J.B.; Moellmer, J.F.; J. Chem. Thermodyn., 9 (1977), 249.

[19]: Villa Vallejo, S.; "Contribución experimental y teórica al estudio de las propiedades termodinámicas de mezclas líquidas formadas por aminas y alcanos 01 alcoholes". Tesis doctoral (2003), 21-23. 
[20]: Prausnitz, J. M., Lichtenthaler, R.N., Gomes de Azevedo, E., "Termodinámica Molecular de los Equilibrios Entre Fases", $3^{a}$ edición, Prentice Hall (2000).

[21]: Barker, J. A.; Aust. J. Chem., 6 (1953), 207.

[22]: Hildebrand, J. H.; Scott, R. L.; "Regular Solutions", Prentice-Hall, Englewood Cliffs, N. J. (1962).

[23]: Rowlinson J. S., Swinton, F. L.; "Liquids and Liquids Mixtures", Butterworths Monographs in Chemistry (1982).

[24]: Van Ness, H. C.; Ind. Eng. Chem., 59 (1967), 33.

[25]: "Experimental Thermochemistry, Vol II"; ed. by Skinner, H. A.; Eds. For IUPAC; Interscience, New York (1962).

[26]: "Experimental Thermodynamics Vol I, Calorimetry of Non-reacting Systems"; edited by McCullough, J. P.; Scott, D. W.; Eds for IUPAC; Butterworths, Londres (1968).

[27]: "Experimental Thermodynamics Vol II, Experimental Thermodynamics of non reacting Fluids"; Edited by Le Neindre, B.; Vodar, B.; Eds. For IUPAC, Butterworths, London (1975).

[28]: "Experimental Thermodynamics Vol IV, Solution Calorimetry"; Edited by Marsh, K. N.; O'Hare, P. A. G.; Eds. For IUPAC, Blackwell Scientific Publications, Oxford (1994).

[29]: "Experimental Thermodynamics Vol VI, Measurement of the Thermodynamic Properties of Single Phases"; edited by Goodwin, A.R.H.; Marsh, K.N.; Wakeham, W.A.; Eds for IUPAC; Elsevier, Amsterdam (2003).

[30]: Randzio, S. L.; Annu. Rep. Prog. Chem., Sect. C, 98 (2002), 157.

[31]: Brown, W. A.; Kose, R.; King, D. A.; Chem. Rev., 98 (1998), 797.

[32]: Korchagina, E. N.; Meas. Tech., 41 (1998), 1057.

[33]: Rogez, J.; Rev. Metall.-Paris, 95 (1998), 1047.

[34]: Renuncio, J. A. R.; Cabañas, A.; Pando, C.; Pure and Appl. Chem., 71 (1999), 1197.

[35]: Gmelin, E.; J. Therm. Anal. Calorim., 56 (1999), 655.

[36]: Wadsö, I.; J. Therm. Anal. Calorim., 64 (2001), 75.

[37]: "Investigation of Phase Transitions by Temperature-Modulated Calorimetry", Thermochim. Acta, (1999), 330. Special issue, ed. Schick, C.; Höhne, G. W. H.

[38]: Jelesarov, I.; Bosshard, H. R.; J. Mol. Recognit., 12 (1999), 3.

[39]: "New Advances in Pharmaceutical Thermal Analysis", Thermochim. Acta, (2001) 380. Special issue, ed. Craig, D. M.; Thomson, K.

[40]: Lee, K. Y. M.; Paterson, A.; Piggott, J. R.; Richardson, G. D.; J. Inst. Brew., 107 (2001), 287.

[41]: "Calorimetry in Catalysis"; Thermochim. Acta, (1998), 312. Special issue, ed. Philips, J.

[42]: Tanaka, S.; Thermochim. Acta, 61 (1983), 147.

[43]: Izatt, R. M.; Redd, E. H.; Christensen, J. J.; Thermochim. Acta, 64, (1983), 355. 
[44]: Rouquerol, J.; Zielenkiewicz, W.; Thermochim. Acta, 109 (1986), 121.

[45]: Höhne, G. W. H.; Hemminger, W. F.; Flammersheim, H. -J.; "Differential Scanning Calorimetry", Springer (2003).

[46]: Hemminger, W. W.; Höhne, G.; "Calorimetry- Fundamentals and Practice"; Verlag Chemi; Weinheim (1984), 5 - 19.

[47]: Lavoisier, A. L.; de Laplace, P. S.; "Memoire sur la Chaleur"; C.R. Academic Royale des Sciences (1783).

[48]: Bunsen, R.W.; Ann. Phys., 141 (1870), 1.

[49]: Sunner, S.; Wadsö, I.; Sci. Tools, 13 (1966), 1.

[50]: Kubaschewski, O.; Evans, E.; "La Thermochimie en Metallurgic", Paris (1964), $110-113$.

[51]: Cobos Hernández, J.C.; "Estudio termodinámico de mezclas líquidas de alcoxietanoles con disolventes orgánicos". Tesis Doctoral. Departamento de Física Aplicada II. Facultad de Ciencias. Universidad de Valladolid (1987).

[52]: Barthel, J.; "Thermometric Titrations", Wiley, New York (1975), 56-76.

[53]: Mrazek, R.V.; Van Ness, H.C.; Am. Inst. Chem. Eng. J., 7 (1961), 190.

[54]: Savini, G.C.; Winterhalter, D.R.; Kovach, L.H.; Van Ness, H.C.; J. Chem. Eng. Data, 11 (1966), 40.

[55]: Winterhalter, D.R.; Van Ness, H.C.; J. Chem. Eng. Data, 11 (1966), 189.

[56]: Stokes, R.H.; Marsh, K.N.; Tomlins, R.P.; J. Chem. Thermodyn., 1 (1969), 211.

[57]: Ewing, M.B.; Marsh, K.N.; Stokes, R.H.; Tuxford, C.W.; J. Chem. Thermodyn., 2 (1970), 751.

[58]: Stokes, R.H.; J. Chem. Thermodyn., 18 (1986), 359.

[59]: Stokes, R.H.; J. Chem. Thermodyn., 20 (1988), 1349.

[60]: Christensen, J. J.; Johnston, H. D.; Izatt, R. M.; Rev. Sci. Instr., 39 (1968), 1356.

[61]: Christensen, J. J.; Gardner, J. W.; Eatough, D. J.; lzatt, R. M.; Watts, P. J.; Hart, R. M.; Rev. Sci. Instr., 44 (1973), 481.

[62]: Rodríguez de Rivera, M.; Socorro, F.; Matos, J.S.; Int. J. Mol. Sci., 10 (2009), 2911.

[63]: Schifreen, R.S.; "Comprehensive Analytical Chemistry", Vol. XII, Part B, Ed. by Svehla, G. ; Wilson and Wilson's, Elsevier, Amsterdam (1982), 53-74.

[64]: Spink, C. H.; Wadsö, I.; "Methods of Biochemical Analysis", Vol. 23, in D. Glick (Ed.), New York (1976), 1-160.

[65]: Pratt, F. R.; J. Franklin Inst., 185 (1918), 663.

[66]: Keyes, F. G.; Gillespie, L. J.; Mitsukuri, S.; J. Amer. Chem. Soc., 44 (1922), 707.

[67]: Rose, V. C.; Storvick, T. S.; J. Chem. Eng. Data, 11 (1966), 143.

[68]: Monk, P.; Wadsö, I.; Acta Chem. Stand., 22 (1968), 1842.

[69]: McGlashan, M. L.; Stoeckli, J.F.; J. Chem. Thermodyn., 1 (1969), 589. 
[70]: Christensen, J. J.; Hansen, L. D.; Eatough, D. J.; Izatt, R. M.; Hart, R. M.; Rev. Sci. Instr., 47 (1976), 730.

[71]: Christensen, J. J.; Hansen, L. D.; Izatt, R. M.; Eatough, D. J.; Hart, R. M.; Rev. Sci. Instr., 52 (1981), 1226.

[72]: Christensen, J. J.; Izatt, R. M.; Thermochim. Acta, 73 (1984), 117.

[73]: Christensen, J. J.; Brown, P.R.; Izatt, R. M.; Thermochim.a Acta, 99 (1986), 159.

[74]: Picker, P.; Jolicoeur, C.; Desnoyers, J. E.; J. Chem. Thermodyn., 1 (1969), 469.

[75]: Picker, P.; "La Microcalorimétrie Dynamique et ses Applications"; Colloques Internationaux du C.N.R.S., n 201: "Thermochimie". Marseille, 30 junio a 2 de julio de 1971. Editions du C.N.R.S. París (1972), 161.

[76]: Picker, P.; Can. Res. Develop., 7 (1974), 11.

[77]: Wormald, C.J.; Yerlett, T.K., J. Chem. Thermodyn., 17 (1985), 1171.

[78]: Gopal, P.; Zollweg, J. A.; Streett, W. B.; Rev. Sci. Instr., 60 (1989), 2720.

[79]: Guan, Y. H.; Kemp, R. B.; Thermochim. Acta, 349 (2000), 163.

[80]: Oakes, C. S.; Pitzer, K. S.; Sterner, S. M.; Geochim. Cosmochim. Acta, 62 (1998), 1133.

[81]: Castro-Gomez, R.C., Hall, K.R., Holste, J.C., Gammon, B.E.; Marsh, K.N., J. Chem. Thermodyn., 22 (1990), 269.

[82]: Larkin, J. A.; McGlashan, M. L.; J. Chem. Soc., 666 (1961), 3425.

[83]: Díaz-Peña, M.; Fernández Martín, F.; An. R.S.E.F. y Q.; Ser. B, 59 (1963), 323.

[84]: Calvet, E.; Prat, H.; "Microcalorimétrie. Applications Physico-Chimiques et Biologiques", Masson et Cie. Paris (1956).

[85]: Paz Andrade, M. I. ; "Concepto, Método, Programa y Fuentes de la Microcalorimetría", Santiago de Compostela (1981).

[86]: Person, C. C.; Ann. Chim. Phys., 21 (1847), 295.

[87]: Richards, T. W.; Henderson, L. J.; Forbes, G. S.; Proc. Am. Acad. Sci., 41 (1905), 1.

[88]: Swietoslawski, W.; "Microcalorimetry", Reinhold, New York (1946).

[89]: Dewar, J.; Proc. R. Sot. (London) Ser. A, 74 (1904), 122.

[90]: Jessup, R. S.; J. Res. Natl. Bur. Stand., 55 (1955), 317.

[91]: Kanbour, F.; Joncich, M. J.; Rev. Sci. Instr., 38 (1967), 913.

[92]: Sykes, C.; Proc. Roy. Soc. (London), Ser. A, 148 (1935), 422.

[93]: Ter Minassian, L.; Milliou, F.; J. Phys. E, 16 (1983), 450.

[94]: Eyraud, C.; C.R. Acad. Sci., 238 (1954), 1511.

[95]: Speros, D.; Woodhouse, R. L.; J. Phys. Chem., 67 (1963), 2164 ; Nature (London), 197 (1963), 1261.

[96]: Ohlmeyer, P.; Z. Naturforsch., 1 (1946), 30. 
[97]: Kiselev, W. F.; Kiselev, A. V.; Mikos, N. N.; Muttik, G. G.; Shtcherbakova; Zh. Fiz. Chim., 5 (1949), 577; 31 (1957), 1111.

[98]: Dzhigit, O. M.; Kiselev, A. V.; Muttik, G. G.; J. Phys. Chem., 66 (1962), 2127.

[99]: Pankratiev, J. D.; Kinet. Katal., 10(I) (1969), 191; 9(4) (1969), 420.

[100]: Zielenkiewicz, W.; Chajn, J.; Proc. 1st Natl. Conf. on Calorimetry, Zakopane (1973), Poland.

[101]: Wittig, F. E.; Schilling, W.; Z. Elektrochem. Ber. Bunsenges. Phys. Chem., 65 (1961), 1.

[102]: Hansen, L. D.; Hart, R. H.; Chen, D. M.; Gibbard, H. F.; Rev. Sci. Instr., 53(4) (1982), 503.

[103]: Petit, J. L.; Sicard, L.; Eyraud, L.; C.R. Acad. Sci., 252 (1961), 1741.

[104]: Kleppa, O. J.; J. Phys. Chem., 64 (1960), 1937.

[105]: Mraw, S. C.; Kleppa, O. J.; J. Chem. Thermodyn., 16 (1984), 865.

[106]: Radenac, A.; Chesneau, C.; Rapin, M.; Rev. Int. Hautes Temp. Refract., 7 (1970), 230.

[107]: Barberi, P.; Comptes Rendus Journees de Calorimetric et ATD, Mulhouse, 3-4 Mai 1971.

[108]: Wadsö, I.; Acta Chem. Stand., (1968), 927.

[109]: Suurkuusk, J.; Wadsö, I.; Chem. Ser., 20 (1982), 155.

[1 10]: Benzinger, T. H.; Kitzinger, C.; "Temperature-lts Measurement and Control in Science and Industry", Reinhold, New York (1963) Vol. 3, Part 3, Chap. 5.

[1 11 1]: Reggiani, J. C.; Cudey, G.; Bernard, J.; J. Chim. Phys., 70 (1973), 292.

[1 12]: Capelli, R.; Ferro, R.; Borsese, A.; Thermochim. Acta, 10 (1974), 13.

[1 13]: Kaminski, M.; Zielenkiewicz, W.; Cem. Concr. Res., 12 (1982), 549.

[114]: Rouquerol, J.; "Thermochimie", Paris, CNRS (1972), 537-545; Thermochim. Acta, 96 (1985), 377.

[1 15]: Denoyel, R.; Rouquerol, F.; Rouquerol, J.; "Adsorption from Solution" Ed. by Ottewill, R. H.; Rochester, C. H.; Smith, A. L.; Academic Press, London (1983), 225.

[1 16]: Zielenkiewicz, W.; Bull. Acad. Polon. Sci., Ser. Sci. Chim., 21 (1973), 333.

[1 17]: Wertenstein, L.; J. Phys. Radium, 1 (1920), 126.

[1 18]: Evans, W. J.; McCourtney, E. J.; Carney, W. B.; Anal. Chem., 40 (1968), 262.

[1 19]: Becker, F.; Malicke, A.; Z. Phys. Chem., Neve Folge., 55 (1967), 280; 61 (1968), 192.

[120]: Tenoutasse, N.; Rev. Mater. Constr. Trav. Publics, 622-623 (1967), 265; 655 (1970), 98.

[121]: Stein, H. N.; J. Appl. Chem., 11 (1961), 474; 13 (1963), 228.

[122]: Favre, P. A.; Silbermann, J. T.; Ann. Chim. Phys., 34 (1852), 357. 
[123]: Berthelot, M. ; "Essai de Mecanique Chimique Fondie sur la Thermochimie", Dunod, Paris (1879).

[124]: Thomsen, J. ; "Thermochemische Untersuchungen", 4 Barth, Leipzig (1882), 188.

[125]: Nernst, W.; Ann. Phys. (Leipzig), 36 (1911), 395.

[126]: Armstrong, L. D.; Can. J. Res., Sect. A, 28 (1950), 44.

[127]: Abramowitz, S.; Domalski, E. S.; Chumey, K. L.; Ledford, A. E.; Ryan, R. V.; Reilly, M. L.; Nato ASI Ser., Ser. C, 119 (1984), 61.

[128]: Eckman, J. R.; Rossini, F. D.; J. Res. Natl. Bur. Stand., 3 (1929), 597.

[129]: Battino, R.; Marsh, K. N.; Aust. J. Chem., 33 (1980), 1997.

[130]: Garn, P. D.; "Thermoanalytical Methods of Investigation", Academic Press Inc., New York (1965).

[131]: Privalov, P. L.; "Biological Microcalorimetry", Ed. by Beezen, A. E.; Academic Press, London (1980), 413-451.

[132]: Kujawa, P.; Winnik, F. M.; "Macromolecules", 34 (2001), 4130.

[133]: Garnier, P.R.; Salamon, M.B., Phys. Rev. Letters., 27 (1971), 1523.

[134]: Hatta, I.; Katayama, N., J. Thermal Anal., 54 (1998), 577.

[135]: Hatta, I., Thermochim. Acta, 272 (1996), 49.

[136]: Grolier, J. P. E.; Pure and Appl. Chem., 69(11) (1997), 2231.

[137]: Jenner, J.; Rev. Sci. Instr., 28 (1957) 263.

[138]: Pool, R.A.H.; Staveley, L.A.K.; Trans. Faraday. Soc., 53 (1957), 1168.

[139]: Knobler, C.M.; van Heijningen, R.J.J.; Beenakker, J.J.M.; Physica, 27 (1961), 296.

[140]: Wormald, C.J.; Lewis, K.L.; Mosedale, S.; J. Chem. Thermodyn., 9 (1977), 27.

[141]: Wormald, C.J.; J. Chem. Thermodyn., 9 (1977), 901.

[142]: Wormald, C.J. Colling, C.N.; J. Chem. Thermodyn. 15 (1983), 725.

[143]: Murgulescu, I.G.; Marchidan, D.I.; Rev. Roumaine Chim., 9 (1964), 793.

[144]: Witting, F.E.; Huber, F.; Z. Elektrochem., 60 (1956), 1181.

[145]: Witting, F.E.; Gehring, E.; Z. Naturforsch., 18a (1963), 351.

[146]: Zubkov, A.A; Turchanin, A.A; Tomilin, I.A; Industrial Laboratory, 61(9) (1995), 544.

[147]: Pope, A.E.; Pflug, H.D.; Dacre, B.; Benson, G.C.; Can. J. Chem., 45 (1967), 2665.

[148]: Murakami, S.; Benson, G.C.; J. Chem. Thermodyn., 1 (1969), 559.

[149]: Benson, G.C.; Benson, G.W.; Rev. Sci. Instr., 26 (1965), 477.

[150]: Bennett, J.E.; Benson, G.C.; Can. J. Chem., 43 (1965), 1912.

[151]: Becker, F.; Kiefer, M. Z.; Naturforsch, Ser A., 24 (1969), 7. 
[152]: Grolier, J.P.E.; Benson, G.C.; Picker, P.; J. Chem. Thermodyn., 7 (1975), 89.

[153]: Grolier, J.P.E.; Inglese, A.; Can. J. Chem., 54 (1976), 1952.

[154]: Grolier, J.P.E.; Thermochim. Acta, 16 (1976), 27.

[155]: Inglese, A.; Grolier, J.P.E.; Thermochim. Acta, 18 (1977), 257.

[156]: Huemer, H.; Platzer, E.; Rehak, K.; Thermochim. Acta, 231 (1994), 21.

[157]: Diamali, E.; Turner, P.J.; Murray, Jr., R.C.; Cobble, J.W.; Review Of Scientific Instruments, 81, 075105 (2010).

[158]: Ott, J.B.; Stouffer, C.E.; Cornett, G.V.; Woodfield, B.F.; Wirthlin, R.C.; Christensen, J.J.; Deiters, U.K.; J. Chem. Thermodyn., 18 (1986), 1.

[159]: Grolier, J.-P.E.; Randzio, S.L.; Fluid Phase Equilib., 133 (1997), 35.

[160]: Bares, D.; Soulie, M.; Metzger, J.; J. Chem. Phys. Physicochim. Biol., 70 (1973), 1531.

[161]: Kechavarz, R.; Dubes, J.P.; Tachoire, H.; Thermochim. Acta, 53 (1982), 39.

[162]: Sarmiento Escalona, F.; Tesis Doctoral, Universidad de Santiago de Compostela (1982).

[163]: Solinas, V.; Ferino, I.; Catalysis Today, 41, (1998), 179.

[164]: Busey, R.H.; Holmes, H.F.; Mesmer, R.E.; J. Chem. Thermodyn., 16 (1984), 343.

[165]: Jones, D.E.G.; Handa, P.; Feng, H.; J. Ther. Anal. Calor., 53(1) (1998), 3.

[166]: Hemminger, W. W.; Höhne, G.; "Grundlagen der Kalorimetrie", Verlag Chemie, Weinheim (1979).

[167]: "Handbook Of Thermal Analysis And Calorimetry" Series Editor Gallagher, P. K.; Volume 1 "Principles And Practice" Ed. Brown, M. E.; Amsterdam, Elsevier (1998) 618.

[168]: Vilchiz, L.E.; Pacheco-Vega, A.; Handy, B. E.; Thermochim. Acta, 439, (2005), 110.

[169]: Tian, A.; Bull. Soc. Chim. France Ser. 4, 33 (1923), 427.

[170]: Calvet, E.; C.R. Acad. Sci. (Paris), 226 (1948), 1702.

[171]: Cobos Hernández, J.C.; "Montaje y puesta a punto de un microcalorímetro TianCalvet". Trabajo de Licenciatura. Departamento de Física Fundamental. Facultad de Ciencias. Universidad de Valladolid (1979).

[172]: Riesco Fernández, N.; "Contribución experimental y teórica al estudio de las propiedades termodinámicas de las mezclas binarias entre compuestos orgánicos". Tesis Doctoral. Departamento de Termodinámica y Física Aplicada. Facultad de Ciencias. Universidad de Valladolid (2003).

[173]: Mozo Ruiz, I.; "Estudio experimental y teórico de mezclas binarias de n-butan-1ol, di-n-butiléter y celosolvas". Tesis Doctoral. Departamento de Física Aplicada. Facultad de Ciencias. Universidad de Valladolid (2010).

[174]: Alonso Gómez, V.; Tesis Doctoral. En preparación.

[175]: Calvet, E.; "Calorimeter"; United States Patent Office; 3.059.471, (1957), Ser. No. 641.223. 
[176]: Rojas, E.; "Sistemática de un microcalorímetro y análisis armónico de la respuesta". Master's thesis, Tesis Doctoral. Universidad de Barcelona (1971).

[177]: Rojas, E.; Torra, V.; Navarro, J.; Anales de Física, 67 (1971), 359.

[178]: Navarro, J.; Cesari, E.; Torra, V.; Mcqueron, J.L.; Dubes, J.P.; Tachoire, H.; Thermochim. Acta, 52 (1982), 175.

[179]: Navarro, J.; Torra, V.; Cesari, E.; Mcqueron, J.L.; Prost, R.; Dubes, J.P.; Tachoire, H.; Mise, H.; Bulletin de la Societe Chimique de France, 12 (1982), 149.

[180]: Navarro, J.; Torra, V.; Cesari, E.; Mcqueron, J.L.; Prost, R.; Dubes, J.P.; Tachoire, H.; Mise, H.; Bulletin de la Societe Chimique de France, 12 (1982), 154.

[181]: Laville, G.; Comptes rendus de l'Académie des sciences, 240 (1955), 1060.

[182]: Laville, G.; Comptes rendus de l'Académie des sciences, 240 (1955), 1195.

[183]: Roux, R.; Tachoire, H.; Comptes rendus de l'Académie de sciences, 261 (1965), 331.

[184]: Wadsö, I.; Goldberg, R.N.; Pure and Appl. Chem., 73(10) (2001), 1625.

[185]: Marsh, K.N.; "Recommended Reference Materials for the Realization of Physicochemical Properties". Blackwell Scientific Publications. Oxford (1987).

[186]: Sabbah, R.; An Xu-wu; Chickos, J.S.; Planas Leitão, M. L.; Roux, M.V.; Torres, L.A.; Thermochim. Acta, 331 (1999), 93. Special Issue.

[187]: Riddick, J. A.; Bunger, W. B., Sakano, T. K. "Organic Solvents, Physical properties and methods of purification", $4^{\mathrm{a}}$ ed. Techniques of Chemistry Volume II, a Wiley Interscience Publication.

[188]: Nagata, I.; Kazuma, K.; J. Chem. Eng. Data, 22(7) (1977), 79.

[189]: Elliott, K.; Wormald, C.J.; J. Chem.Thermodyn., 8 (1976), 881.

[190]: Touhara, H.; Ikeda, M.; Nakanishi, K.; Watanabe, N.; J. Chem. Thermodyn., 7 (1975), 887.

[191]: Heintz, A.; Lichtenthaler, R. N.; Ber. Bunsenges. Phys. Chem., 81 (1977), 921.

[192]: Te Lam, V.; Picker, P.; Patterson, D.; Tancréde, P.; J. Chem. Soc. Faraday Trans. II, 70 (1974), 1465.

[193]: Grolier, J.P.E.; International DATA series, Series A-Selected data on mixtures, 3 (1974), 220.

[194]: Heintz, A.; Lichtenthaler, R. N.; Ber. Bunsenges. Phys. Chem., 84 (1980), 727.

[195]: Wang, Z.; Peng, D.-Y.; Benson, G.C.; Lub, B.C.-Y.; J. Chem. Thermodyn., 32 (2000), 261.

[196]: Nagata, I.; Ksiazczak, A.; J. Chem. Thermodyn., 27 (1995), 1235.

[197]: Verdes, P.V.; Mato, M.M.; Salgado, J.; Legido, J.L.; Paz Andrade, M.I.; Fluid Phase Equilib., 232 (2005), 16.

[198]: Bender, M.; Hauser, J.; Heintz, A.; Ber. Bunsenges. Phys. Chem., 95(7) (1991), 801.

[199]: Tamura, K.; Nagata, I.; J. Chem. Thermodyn., 23 (1991), 359. 
[200]: Zhou, L.; Wang, Y.; Zhang, Y.; Shi, J.; Benson, G.C.; Lu, B.C.-Y.; Thermochim. Acta, 189 (1991), 57. 
CAPITULO 4 


\section{CAPITULO 5}

\section{Interpretación de los resultados experimentales}

"What does a fish know about the water in which he swims all his life?"

Einstein, A.

- n este capítulo se intenta dar una perspectiva más amplia de los datos que aparecen en los dos capítulos anteriores y discutir el comportamiento sistemático de los sistemas involucrados.

\subsection{DESVIACIONES RESPECTO DEL COMPORTAMIENTO IDEAL}

El modelo de mezcla ideal está limitado por su propia definición ya que es aplicable a moléculas lo suficientemente parecidas como para estar bajo los mismos campos de fuerza cuando se encuentran en estado puro y en la mezcla. Como se ha visto en el Capítulo 2, en dichas mezclas las fugacidades cumplen la ley de Lewis-Randall. Por otra parte, en mezclas de moléculas diferentes que no forman complejos supramoleculares, la ley se cumple para el disolvente cuando su composición tiende a la unidad, mientras que para los solutos a dilución infinita se cumple la ley de Henry. Esto significa que en los extremos de composición la mezcla es ideal y que los líquidos son miscibles. Sin embargo, en composiciones intermedias los valores de las fugacidades se desvían en mayor o menor medida de los dados por la ley de Lewis-Randall. Estas desviaciones pueden ser positivas o negativas (negativas especialmente cuando se forman complejos entre las sustancias que se mezclan [1]). Si la ley de Lewis-Randall es tomada como definición de mezcla ideal, valores no nulos de $V_{\mathrm{m}}^{\mathrm{E}}$ y $/ \mathrm{o} H_{\mathrm{m}}^{\mathrm{E}}$ significan desviaciones respecto del comportamiento ideal, lo que sugiere que las interacciones entre las moléculas y los efectos de tamaño y forma de las mismas son importantes. $V_{\mathrm{m}}^{\mathrm{E}}>0$ suele estar asociado con absorción de calor $\left(H_{\mathrm{m}}^{\mathrm{E}}>0\right)$ y desviaciones positivas respecto la ley de Lewis-Randall, mientras que $V_{\mathrm{m}}^{\mathrm{E}}<0$ 
suele estar asociado con liberación de calor $\left(H_{\mathrm{m}}^{\mathrm{E}}<0\right)$ y desviaciones negativas [2]. Como sabemos, las desviaciones respecto de la idealidad originan las funciones termodinámicas de exceso. Dichas funciones pueden examinarse considerando la naturaleza de los campos de fuerza actuando en las moléculas, las diferencias de tamaño y forma de los componentes de las mezclas, etc. [3]-[14]. Por ello, los parámetros siguientes son fundamentales en la discusión acerca del comportamiento de las mezclas:

a) Presión interna: da idea del "ambiente molecular" sobre una molécula, y es la fuerza que junto con la presión externa se opone a la presión térmica debida a la energía cinética de las moléculas [1]. Es la responsable del fenómeno de tensión superficial, de que los gases y vapores rellenen el recipiente que los contiene, de que los líquidos no llenen el recipiente que lo contiene pero adopten su forma y de que los sólidos ni llenen el recipiente ni adopten su forma (en otras palabras, de que los sólidos y los líquidos estén autocondensados, es decir, de que no se expandan sin límite si la presión externa es nula). La definición de presión interna procede de la relación puente:

$$
T \cdot\left(\frac{\partial P}{\partial T}\right)_{V, N_{\mathrm{i}}}=P+\left(\frac{\partial U}{\partial V}\right)_{T, N_{\mathrm{i}}}=\left(\frac{\partial U}{\partial V}\right)_{S, N_{\mathrm{i}}}+\left(\frac{\partial U}{\partial V}\right)_{T, N_{\mathrm{i}}}=P+P_{\mathrm{int}}
$$

de donde se deduce que:

$$
P_{\text {int }}=T \cdot \frac{\alpha_{P}}{\kappa_{T}}-P
$$

Como sabemos, $\kappa_{T}$ se calcula mediante la ecuación:

$$
\kappa_{T}=\kappa_{S}+\frac{T \cdot \alpha_{P}^{2} \cdot V_{\mathrm{m}}}{C_{P, \mathrm{~m}}}
$$

Los valores de $C_{P, \mathrm{~m}}$ pueden ser experimentales o calculados mediante la ecuación 2.163. En el caso de mezclas, las caracterizadas por fuertes interacciones entre moléculas diferentes están descritas por valores positivos y grandes de $P_{\text {int }}^{\mathrm{E}}$, mientras que valores negativos son propios de mezclas en las que las interacciones entre las moléculas iguales son más importantes. $P_{\text {int }}^{\mathrm{E}}$ se define como sigue:

$$
P_{\text {int }}^{\mathrm{E}}=P_{\text {int }}-P_{\text {int }}^{\mathrm{id}}=P_{\text {int }}-\left(T \cdot \frac{\alpha_{P}^{\mathrm{id}}}{\kappa_{T}^{\mathrm{id}}}-P\right)
$$

Se puede demostrar que en una mezcla ideal, el valor de la presión interna en todo el rango de presión y temperatura es independiente de la composición, luego es igual a la de las sustancias puras. Por tanto, cuando se mezclan líquidos cuyas presiones internas en estado puro no son iguales, la mezcla no será ideal. Por esta razón, entre otras, el concepto de presión interna es idóneo para explicar desviaciones respecto la idealidad.

En la literatura aparecen diferentes métodos para calcular $P_{\text {int }}$ [15], [16], así como sus valores en diferentes gases, líquidos, polímeros, sales fundidas y mezclas líquidas [1 7].

En los gases y vapores, donde la energía cinética de traslación es dominante en el comportamiento molecular, la presión de vapor es mucho mayor que la presión interna, de modo que la última puede despreciarse, es decir, suponer que las fuerzas de interacción intermoleculares atractivas de corto alcance tienen un efecto despreciable, lo que da origen al concepto de gas ideal. Consecuentemente los gases rellenan el 
recipiente que los contiene, siendo el volumen de un gas una variable externa, según la clasificación de Bazarov [18]. Esto no evita la existencia de fuerzas repulsivas de muy corto alcance que dan cuenta del tamaño molecular, pues como consecuencia del principio de exclusión de Pauli, los orbitales moleculares no se pueden superponer.

En la aproximación propuesta por van der Waals, se introduce una corrección al modelo de gas ideal suponiendo que existen fuerzas de interacción intermoleculares atractivas de corto alcance, las cuales explican la licuefacción de los vapores y la aparición de fase líquida. Para un fluido de este tipo, la presión interna es:

$$
P_{\text {int }}=\frac{a}{V^{2}}
$$

En el caso de las fases condensadas la presión interna es mucho mayor que la presión de vapor, de modo que son las fuerzas inter e intramoleculares las que justifican el comportamiento macroscópico de las mismas. Por consiguiente, la estructura de los líquidos está relacionada con el balance entre las fuerzas atractivas cuyo efecto promedio se refleja en la presión interna y las fuerzas repulsivas que dan cuenta del tamaño de las moléculas. En particular, los modelos de líquido o sólido incompresibles dependen esencialmente de que dichas fuerzas repulsivas impidan el colapso del sistema y les hacen insensible a los cambios de presión. El volumen en las fases condensadas es una variable interna, y de ahí que los líquidos no llenen el recipiente que los contiene pero adopten su forma y los sólidos ni lo llenen ni adopten su forma.

b) Naturaleza polar de las moléculas y capacidad de formación de complejos supramoleculares mediante mecanismos de transferencia de carga o mediante enlaces puente de hidrógeno. Así, la mezcla de dos líquidos cuyas presiones internas son similares, pero cuyas moléculas, al menos de uno de los líquidos, forman complejos o interacciones dipolares fuertes, no será ideal. Las sustancias que en estado puro están asociadas al mezclarse con disolventes inertes (como los $n$-alcanos) ven rota su asociación, desapareciendo por completo a dilución infinita. Como la asociación es el resultado de enlaces direccionales cuya ruptura es endotérmica, la mayoría de estas mezclas muestran desviaciones positivas respecto de la idealidad, e incluso puede aparecer inmiscibilidad. Sin embargo, si el disolvente no es inerte, sino muy polar y /o asociado o incluso apolar y no asociado pero muy polarizable, aparecen interacciones entre moléculas diferentes. Puesto que la formación de éstas es un proceso exotérmico, las desviaciones positivas de la idealidad debidas a la ruptura de las interacciones entre el compuesto asociado se compensan por contribuciones negativas debidas a la formación de nuevas interacciones, de modo que el efecto total puede originar fuertes desviaciones negativas respecto la ley de Lewis-Randall [19], desviaciones positivas, o incluso verificarse por compensación de efectos, dependiendo de cada caso particular.

Las interacciones dipolares no están dadas por el momento dipolar de las moléculas, $\mu$, sino por su polarizabilidad, $\alpha$, y su momento dipolar efectivo $\bar{\mu}$ (véase el Capítulo 6):

$$
\bar{\mu}=\sqrt{\frac{\mu^{2} \cdot N_{A}}{4 \cdot \pi \cdot \varepsilon_{0} \cdot V_{\mathrm{m}} \cdot k_{\mathrm{B}} \cdot T}}
$$

donde $V_{\mathrm{m}}$ es el volumen molar, $N_{A}$ es el número de Avogadro, $k_{\mathrm{B}}$ es la constante de Boltzmann, $\varepsilon_{0}$ es la constante dieléctrica del vacío y $T$ es la temperatura.

Otra magnitud útil para examinar los cambios relativos en las fuerzas intermoleculares 
de compuestos homomorfos es $\mathrm{D} H_{\mathrm{v}}$, la diferencia entre la entalpía estándar de vaporización $\Delta H_{\mathrm{v}}$ del componente a estudiar y la de su alcano homomorfo [20] a 298.15 K. En la Tabla $5-1$ se muestran $\mu, \bar{\mu}$ y $\mathrm{D} H_{\mathrm{v}}$ de diferentes aminas y cetonas de interés.

Tabla 5-1: Momento dipolar, $\mu$, momento dipolar efectivo a $298.15 \mathrm{~K}, \bar{\mu}$, y $\mathrm{D} H_{\mathrm{v}}$ para diferentes sustancias. ${ }^{a}[21]$; ${ }^{\mathrm{b}}[22] ;{ }^{\mathrm{c}}[23] ;{ }^{\mathrm{d}}[24] ;{ }^{\mathrm{e}}[25] ;{ }^{\mathrm{f}}[26] ;{ }^{\mathrm{g}}[27] ;{ }^{\mathrm{h}}[28] ;{ }^{\mathrm{i}}[5]-[13]$

\begin{tabular}{|c|c|c|c|}
\hline & $\mu / D$ & $\bar{\mu}$ & $\mathrm{D} H_{\mathrm{v}} / \mathrm{kJ} \cdot \mathrm{mol}^{-1}$ \\
\hline \multicolumn{4}{|c|}{ amina lineal primaria } \\
\hline$N$-metilamina & $1.3^{a}$ & 0.748 & \\
\hline$N$-etilamina & $1.3^{a}$ & 0.609 & \\
\hline$N$-propilamina & $1.3^{a}$ & 0.546 & \\
\hline$N$-butilamina & $1.3^{a}$ & 0.499 & \\
\hline \multicolumn{4}{|c|}{ amina lineal secundaria } \\
\hline di-n-metilamina & $1^{b}$ & 0.459 & \\
\hline di- $n$-etilamina & $1.1^{\mathrm{b}}$ & 0.412 & \\
\hline di- $n$-propilamina & $1^{b}$ & 0.326 & $3.49^{d}$ \\
\hline di-n-butilamina & $1.1^{\mathrm{b}}$ & 0.322 & $3.01^{\mathrm{h}}$ \\
\hline \multicolumn{4}{|c|}{ Tri- $n$-alquilamina } \\
\hline$N, N, N$-trimetilamina & $0.8^{c}$ & 0.315 & \\
\hline$N, N, N$-trietilamina & $0.9^{a}$ & 0.291 & \\
\hline$N, N, N$-tripropilamina & $0.74^{\mathrm{c}}$ & 0.205 & \\
\hline$N, N, N$-tributilamina & $0.8^{\mathrm{c}}$ & 0.198 & \\
\hline \multicolumn{4}{|c|}{ amina aromática } \\
\hline anilina & $1.51^{d}$ & 0.19 & $17.844^{d}$ \\
\hline$N$-metilanilina & $1.70^{\mathrm{e}}$ & 0.20 & $10.85^{d}$ \\
\hline piridina & $2.37^{d}$ & 0.32 & $6.567^{d}$ \\
\hline \multicolumn{4}{|c|}{ cetona } \\
\hline 2-propanona & $2.69^{f}$ & $1.12^{\mathrm{g}}$ & \\
\hline 2-butanona & $2.76^{f}$ & $1.11^{\mathrm{g}}$ & $13.77^{i}$ \\
\hline 2-pentanona & $2.7^{\dagger}$ & $1.00^{\mathrm{g}}$ & $11.97^{i}$ \\
\hline 3-pentanona & $2.82^{f}$ & $1.04^{\mathrm{g}}$ & $12.9^{i}$ \\
\hline 2-hexanona & $2.66^{f}$ & $0.914^{g}$ & $11.58^{i}$ \\
\hline 2-heptanona & $2.59^{f}$ & $0.836^{\mathrm{g}}$ & $10.67^{i}$ \\
\hline
\end{tabular}

Ignorando efectos estructurales se puede estimar la energía de interacción entre dos moléculas diferentes, $\Delta H_{1-2}$, de una mezcla binaria $1+2$ (veáse el Capitulo 4):

$$
\Delta H_{1-2}=H_{m, 1}^{E, \infty}(1+2)-H_{m, 1}^{E, \infty}(1+\text { alcano })-H_{m, 1}^{E, \infty}(2+\text { alcano })
$$

En ausencia de datos experimentales, los valores de $H_{\mathrm{m}, 1}^{\mathrm{E}, \infty}$ se estiman mediante el ajuste de $H_{\mathrm{m}}^{\mathrm{E}}$ a la ecuación R-K a concentraciones intermedias. Este procedimiento no es muy preciso, como se ha explicado en el Capítulo 4. En la Tabla 5-2 se escriben los valores de $H_{\mathrm{m}, 1}^{\mathrm{E}, \infty}$ y $\Delta H_{1-2}$ de diferentes sistemas, suponiendo como es habitual en teorías de asociación, que $H_{\mathrm{m}, 1}^{\mathrm{E}, \infty}$ es independiente del alcohol. 
Tabla 5-2: Entalpías molares parciales a dilución infinita, $H_{m, 1}^{E, \infty}$, a $298.15 \mathrm{~K}$, y energía de interacción 1-2, $\Delta H_{1-2}$,

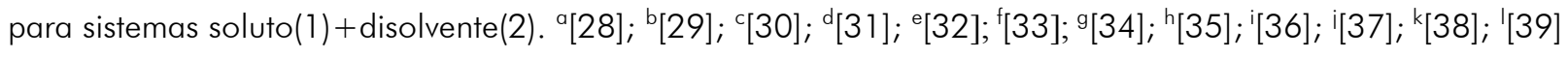

\begin{tabular}{|c|c|c|}
\hline & $H_{m, 1}^{E, \infty} / \mathrm{kJ} \cdot \mathrm{mol}^{-1}$ & $\Delta H_{1-2} / \mathrm{kJ} \cdot \mathrm{mol}^{-1}$ \\
\hline benceno $+n$-heptano & $3.06^{a}$ & \\
\hline 2-propanona $+n$-heptano & $8.34^{\mathrm{b}}$ & \\
\hline 2-butanona $+n$-heptano & $7.47^{\mathrm{c}}$ & \\
\hline 2-pentanona $+n$-heptano & $6.35^{c}$ & \\
\hline 3-pentanona $+n$-heptano & $5.9^{c}$ & \\
\hline 2-heptanona $+n$-heptano & $5.58^{\mathrm{d}}$ & \\
\hline di-n-propilamina $+n$-heptano & $1.96^{\mathrm{e}}$ & \\
\hline di- $n$-butilamina $+n$-heptano & $1.54^{\mathrm{e}}$ & \\
\hline$N, N, N$-trietilamina $+n$-heptano & $0.42^{\mathrm{e}}$ & \\
\hline$N, N$-dimetiletilamina $+n$-heptano & $1.23^{f}$ & \\
\hline$N, N$-dietilmetilamina $+n$-heptano & $0.94^{f}$ & \\
\hline anilina $+n$-heptano & $15.00^{\mathrm{g}}$ & \\
\hline$N$-metilanilina $+n$-heptano & $10.07^{\mathrm{g}}$ & \\
\hline piridina $+n$-heptano & $8.74^{\mathrm{h}}$ & \\
\hline 1-alcohol $+n$-heptano & $23.2^{\mathrm{g}}$ & \\
\hline 2-propanona + benceno & 8.91 & $-2.49^{\prime}$ \\
\hline 2-butanona + benceno & 8.21 & $-2.32^{\prime}$ \\
\hline 2-pentanona + benceno & 7.27 & $-2.14^{\prime}$ \\
\hline 3-pentanona + benceno & 6.82 & $-2.14^{\prime}$ \\
\hline anilina + benceno & $4.5^{9}$ & -13.6 \\
\hline$N$-metilanilina + benceno & $3.1^{9}$ & -10 \\
\hline 2-propanona + tolveno & & $-2.01^{\prime}$ \\
\hline 2-butanona + tolveno & & $-1.91^{\prime}$ \\
\hline 2-pentanona + tolveno & & $-1.80^{\prime}$ \\
\hline metanol + anilina & $0.07^{\mathrm{g}}$ & -38.1 \\
\hline 1-butanol + anilina & $6^{9}$ & -32.2 \\
\hline metanol $+N$-metilanilina & $2.5^{\mathrm{g}}$ & -30.7 \\
\hline 1-butanol $+N$-metilanilina & $7.9^{\mathrm{g}}$ & -25.3 \\
\hline di-n-propilamina +2 -propanona & $2.97^{i}$ & -8.08 \\
\hline di- $n$-propilamina +2 -butanona & $1.64^{\mathrm{i}}$ & -7.79 \\
\hline di- $n$-propilamina +2 -pentanona & $0.90^{i}$ & -7.41 \\
\hline di- $n$-propilamina +2 -heptanona & $0.45^{i}$ & -7.09 \\
\hline di- $n$-butilamina +2 -propanona & $4.77^{i}$ & -5.86 \\
\hline di- $n$-butilamina +2 -butanona & $2.79^{i}$ & -6.22 \\
\hline di- $n$-butilamina +2 -pentanona & $1.67^{\mathrm{i}}$ & -6.22 \\
\hline di- $n$-butilamina +2 -heptanona & $0.86^{\mathrm{i}}$ & -6.26 \\
\hline$N, N, N$-trietilamina +2 -propanona & $4.58^{i}$ & -4.93 \\
\hline$N, N, N$-trietilamina +2 -butanona & $2.45^{\mathrm{i}}$ & -5.44 \\
\hline$N, N, N$-trietilamina +2 -pentanona & $1.53^{i}$ & -5.24 \\
\hline$N, N, N$-trietilamina +2 -heptanona & $0.91^{i}$ & -5.09 \\
\hline$N, N$-dimetiletilamina +2 -propanona & $2.75^{i}$ & -7.57 \\
\hline$N, N$-dietilmetilamina + 2-propanona & $3.01^{i}$ & -7.02 \\
\hline anilina +2 -propanona & $-5.24^{k}$ & -28.68 \\
\hline anilina +2 -butanona & $-5.52^{k}$ & -27.99 \\
\hline anilina +2 -pentanona & $-4.60^{k}$ & -25.95 \\
\hline anilina +2 -heptanona & $-3.89^{k}$ & -24.47 \\
\hline$N$-metilanilina +2 -propanona & $-2.33^{k}$ & -21.49 \\
\hline$N$-metilanilina +2 -butanona & $-3.06^{k}$ & -20.60 \\
\hline$N$-metilanilina +2 -pentanona & $-3.26^{k}$ & -19.69 \\
\hline$N$-metilanilina +2 -heptanona & $-3.00^{k}$ & -18.65 \\
\hline
\end{tabular}


c) Forma de las moléculas. Un alcano ramificado tienen menor punto de ebullición que su isómero lineal, disminuyendo a medida que aumenta la ramificación, lo cual se debe a que cuanto mayor sea ésta más se aproxima su forma a la de una esfera, reduciendo su superficie de interacción y la atracción entre las moléculas. Existen otras propiedades afectadas por la ramificación: por ejemplo, los datos espectroscópicos sugieren la presencia de orden orientacional entre $n$-alcanos puros de cadena larga, que no existe en alcanos ramificados de forma aproximadamente esférica. La mezcla de líquidos con diferentes grados de orden ocasiona un descenso de éste, y por tanto una contribución positiva a la entropía y la entalpía de mezcla, lo que explica los efectos Patterson y Wilhelm, explicados en la sección 5.3.3.

d) Tamaño de las moléculas. El principio de equipartición de la energía de la Física Estadística clásica garantiza que, en el caso de átomos y agregados moleculares donde existen pocos grados de libertad internos (moléculas pequeñas), toda la agitación térmica se emplea principalmente en la traslación del centro de masas, mientras que en el caso de agregados moleculares con muchos grados de libertad intramoleculares, la energía disponible para trasladar la molécula como un todo es mucho menor. Eso hace que, en una serie homóloga, las sustancias cuyas entidades elementales sean pequeñas sean gases (interacciones débiles), las intermedias sean líquidas (interacciones intermedias) y las mayores sean sólidas (interacciones fuertes). Todo ello para unas condiciones normales de temperatura y presión. Esto explica claramente cómo la noción intuitiva de que a baja temperatura y alta presión las entidades elementales se organizan en forma de cristales mientras que, a alta temperatura y baja presión lo hacen en forma de vapores, deba matizarse con cuidado atendiendo al tipo de agregados moleculares en estudio.

En definitiva, en base a la polaridad, la capacidad de formación de complejos supramoleculares y a las diferencias de presión interna, forma y tamaño de las moléculas, se puede explicar cualitativamente el comportamiento de las mezclas. De manera simple, denotando por NP a los líquidos simples apolares, por $\mathrm{P}$ a los líquidos simples polares y por AS a los líquidos complejos (véase la segunda clasificación de los líquidos que se da en la sección 6.2), existen los siguientes tipos de mezclas binarias:

a) NP+NP: si las presiones internas son similares, y las moléculas tienen tamaño y forma similares, la mezcla es ideal. Por el contrario, si las presiones internas son diferentes aparecerán desviaciones positivas respecto la idealidad. Pueden aparecer dos fases si las desviaciones son muy acusadas. Esto puede explicarse cualitatitavemente teniendo en cuenta que en este tipo de mezclas solamente aparecen fuerzas de tipo dispersivo y que el potencial de interacción dispersivo entre pares de moléculas distintas [40], [41] es menor que el promedio aritmético de los potenciales de interacción entre pares de moléculas iguales, lo que da lugar a un debilitamiento de la cohesión [42 pp 80]. Esto ocurre por ejemplo en las mezclas benceno + heptano y ciclohexano + hexadecano.

b) P+P: cuando la diferencia entre la polaridad sea grande, independientemente de la presión interna, hay desviaciones positivas respecto de la idealidad. Sin embargo, cuando ambos tipos de molécula son muy polares, puede haber desviaciones negativas o cumplirse la ley de Lewis-Randall por la compensación de efectos.

c) P+AP: en general aparecen desviaciones positivas. Sin embargo, si la molécula no polar es polarizable, aparecen interacciones dipolo permanente-dipolo inducido que contribuyen de manera negativa a las desviaciones respecto de la idealidad. 
d) AP+AS: desviaciones positivas respecto la idealidad. Pueden aparecer dos fases si las desviaciones son muy acusadas. Si la molécula apolar es muy polarizable, pueden aparecen desviaciones negativas como en la mezcla benceno + hexafluorobenceno.

e) P+AS: puede haber desviaciones negativas, positivas, o compensación de efectos.

f) AS+AS: desviaciones positivas y negativas respecto la idealidad, aunque lo más normal es que sean negativas, como en el sistema etanol + agua. Puede que se cumpla la ley de Lewis-Randall por compensación de efectos.

Esto permite interpretar cualitativamente las mezclas de nuestro interés, cetonas + aminas, cuyos valores de las entalpías y volúmenes de exceso son los reflejadas en las tablas y gráficas de los Capítulos 3 y 4.

\subsection{MARCO DE REFERENCIA DE ESTE TRABAJO}

Antes de realizar el análisis sistemático de los datos presentados en los Capítulos 3 y 4 , es conveniente recordar que el trabajo se ha realizado en el seno de un grupo de investigación (G.E.T.E.F.) dedicado desde hace muchos años al estudio de la termodinámica de mezclas líquidas y aleaciones. Si bien el grupo ha sabido diversificar sus líneas de investigación, sin duda alguna la mayor parte de sus labores se han encaminado a contribuir al Proyecto T.O.M. (Thermodynamics of Organic Mixtures).

El proyecto T.O.M., propuesto por Kehiaian [43], [44], consiste en un intento de análisis sistemático de las mezclas de grupos orgánicos caracterizando las contribuciones de cada uno de ellos. Se basa pues, en las mismas ideas que los métodos de contribución de grupos y su propósito, al sistematizar el trabajo, es el de optimizar los esfuerzos invertidos en el estudio de estas mezclas y conseguir de forma eficiente la caracterización de todos los compuestos orgánicos.

Dentro del proyecto T.O.M., el presente trabajo, dedicado al estudio de aminas y cetonas, es una contribución al desarrollo del subproyecto NCO. Este subproyecto se encarga de la caracterización de los grupos funcionales amino, $-\mathrm{NR}_{2}$, y carbonilo, $-\mathrm{C}=\mathrm{O}$. En la llustración 1.1 puede verse el esquema de trabajo. Se pretende caracterizar las interacciones de tales grupos y ver si tales parámetros de interacción son válidos en todas las configuraciones posibles. Es decir, siguiendo el esquema de la llustración 1.1, nos preguntamos si la caracterización del grupo amino y del grupo carbonilo cuando éstos están en moléculas diferentes es suficiente para conocer su comportamiento cuando ambos grupos están en la misma molécula. En caso afirmativo, supondrá un gran avance pues hace innecesaria la caracterización experimental de esos sistemas (por ejemplo, algunas amino-cetonas). En caso negativo, debe definirse un nuevo grupo funcional para caracterizar el conjunto formado (por ejemplo, amidas).

Dentro del subproyecyo NCO, el presente trabajo puede verse como la continuación natural de los realizados por Villa [45] sobre la caracterización de las mezclas de aminas secundarias con alcanos, Riesco [46] sobre la caracterización de las mezclas de aminas terciarias y alcanos y Kehiaian y Grolier [47]-[48] sobre la caracterización de las mezclas de cetonas con alcanos en términos de teorías de contribución de grupos, basados en los datos obtenidos anteriormente por sus colaboradores [31], [49]-[51]. Aquí se presenta un tratamiento sistemático de los resultados experimentales obtenidos 
para las mezclas de aminas con cetonas, que ha sido completado con el de mezclas de amino-cetonas y alcanos para la determinación de los posibles efectos intramoleculares, y puede compararse con estudios ya realizados sobre las mezclas de amidas con alcanos [52], siguiendo el esquema habitual de trabajo (Ilustración 1-1).

En las siguientes secciones se explican las características de las propiedades termodinámicas obtenidas para las mezclas amina + cetona aquí consideradas (esquemas de llaves del Capítulo 1), así como de las mezclas amina o cetona + alcano.

\subsection{MEZCLAS AMINA + ALCANO}

Los efectos que se producen en una mezcla no pueden considerarse aisladamente sin tener en cuenta las características principales de los componentes que la forman. Las mezclas de cetonas con aminas son la resultante global de las propiedades de las moléculas de cetona, de amina y su mutua interacción cuando ambas se encuentran en disolución líquida.

La gran complejidad de las interacciones en fase condensada impide conocer su valor absoluto, obligándonos a trabajar mediante un método comparativo, donde se estudia el comportamiento termodinámico de series homólogas de sistemas en los que se aumenta progresivamente la complejidad (momento dipolar, tamaño, forma, etc.) de las moléculas puestas en juego. Así puede conocerse el peso relativo sobre el efecto global de cada una de las contribuciones elementales que se generan en el proceso de mezcla y, por tanto, el predominio en cada conjunto de sistemas de un tipo de interacción u otro.

Los líquidos de referencia, en este campo de trabajo, son los $n$-alcanos. Sin ser fluidos simples* en el sentido que se da a este término en las teorías más fundamentales (véase la sección 6.3), son lo suficientemente "sencillos" como para servir de base en el proceso comparativo. Así, para explicar el comportamiento termodinámico de las funciones de exceso de las mezclas de amina + cetona, se ha de considerar previamente el de las mezclas del tipo cetona $+n$-alcano y amina $+n$-alcano, de acuerdo con el esquema habitual de trabajo (llustración 1.1). Con ello pueden tipificarse por separado lo que ocurre cuando las cetonas y las aminas pasan a formar parte de disoluciones líquidas. Las mezclas en estudio, por diferencia, nos permitirán caracterizar su interacción mutua.

\subsubsection{Amina lineal primaria $+n$-alcano}

A continuación se realiza el estudio de las funciones termodinámicas de exceso de algunos sistemas amina lineal primaria $+n$-alcano considerados en otros trabajos. La Tabla 5-3 muestra la entalpía de exceso a fracción equimolar para tales sistemas:

Tabla 5-3: $H_{\mathrm{m}}^{\mathrm{E}}\left(x_{1}=0.5\right) / \mathrm{J} \cdot \mathrm{mol}^{-1}$ en mezclas amina primaria $(1)+n$-alcano (2) a $298.15 \mathrm{~K} \cdot{ }^{\mathrm{a}}[53]{ }^{;}{ }^{\mathrm{b}}[54]{ }^{\mathrm{c}}[55]$; ${ }^{\mathrm{d}}[56] ;$; $[57]$

\begin{tabular}{cccc}
\hline & $N$-butilamina & $N$-hexilamina & $N$-octilamina \\
\hline$n$-hexano & $1014^{\mathrm{a}}$ & $935^{\mathrm{e}}$ & \\
$n$-heptano & $1321^{\mathrm{b}}$ & $1064^{\mathrm{c}, \mathrm{d}}$ & $864^{\mathrm{c}, \mathrm{d}}$ \\
$n$-decano & $1278^{\mathrm{c}, \mathrm{d}}$ & $1211^{\mathrm{c}, \mathrm{d}}$ & $995^{\mathrm{c}, \mathrm{d}}$ \\
$n$-hexadecano & $1445^{\mathrm{c} d}$ & $1513^{\mathrm{c}, \mathrm{d}}$ & $1289^{\mathrm{c}, \mathrm{d}}$ \\
\hline
\end{tabular}


En estos sistemas, $H_{\mathrm{m}}^{\mathrm{E}}>0$ en todo el rango de concentración, debido a la ruptura, por parte de los alcanos, de los puentes de hidrógeno existentes entre las moléculas de amina. La Tabla 5-3 muestra que para una amina primaria dada, $H_{\mathrm{m}}^{\mathrm{E}}$ aumenta con la longitud de la cadena del $n$-alcano. En otras palabras, la contribución positiva debida a la ruptura, por parte del $n$-alcano, de los puentes de hidrógeno entre las moléculas de la amina primaria aumenta con la longitud del $n$-alcano, debido a su mayor superficie alifática. En cambio, el comportamiento de $H_{\mathrm{m}}^{\mathrm{E}}$ fijado el $n$-alcano es tal que disminuye al aumentar el tamaño de la amina, lo que indica que su asociación disminuye del mismo modo. El momento dipolar efectivo varía de forma similar, como se observa de la Tabla 5-1: $\bar{\mu}(N$-metilamina $)>\bar{\mu}$ ( $N$-etilamina $)>\bar{\mu}(N$-propilamina $)>\bar{\mu}$ ( $N$-butilamina $)$. Esto indica que las interacciones dipolares son más fuertes cuanto menor es el tamaño de la amina primaria.

El mismo comportamiento se observa en mezclas 1-alcohol $+n$-alcano. Sin embargo, $H_{\mathrm{m}}^{\mathrm{E}}$ es menor que en las mezclas amina primaria $+n$-alcano, a pesar de que la energía de las interacciones amina-amina $\left(\approx-13 \mathrm{~kJ} \cdot \mathrm{mol}^{-1}\right)$ es mucho menor que la alcohol-alcohol ( $\left.\approx-25 \mathrm{~kJ} \mathrm{~mol}^{-1}\right)$ [58]-[60]. Ello se debe a que para los $n$-alcanos es más probable romper los enlaces más débiles (amina-amina) que los más fuertes (alcoholalcohol). Esto también explica que las curvas $H_{\mathrm{m}}^{\mathrm{E}}(x)$ sean más simétricas en disoluciones con amina primaria.

Desde el punto de vista entrópico, en las mezclas amina primaria $+n$-alcano, $T \cdot S_{\mathrm{m}}^{\mathrm{E}}>0$ en todo el rango de composición, lo que sugiere que incluso en concentraciones bajas de $n$-alcano la estructura asociada con la amina se destruye.

Puede efectuarse un estudio de $V_{\mathrm{m}}^{\mathrm{E}}$ de las mezclas amina primaria $+n$-alcano, similar al realizado para $H_{\mathrm{m}}^{\mathrm{E}}$. Valores positivos de $V_{\mathrm{m}}^{\mathrm{E}}$ (Tabla 5 -4) revelan que las interacciones dipolares y la ruptura de los puentes de hidrógeno entre las moléculas de la amina cuando ésta es mezclada con un $n$-alcano son importantes. Valores negativos de $V_{\mathrm{m}}^{\mathrm{E}}$ indican la mayor importancia de los efectos estructurales y de volumen libre. La contribución estructural depende en gran medida de las diferencias de tamaño entre las moléculas. Así, la capacidad de acomodación del alcano en la estructura de la amina aumenta cuanto mayor sea el tamaño de la molécula de amina y menor el de la molécula del alcano, lo que contribuye negativamente al volumen de exceso.

Tabla 5-4: $V_{\mathrm{m}}^{\mathrm{E}}\left(x_{1}=0.5\right) / \mathrm{cm}^{3} \cdot \mathrm{mol}^{-1}$ en mezclas amina primaria (1) + alcano (2) a $298.15 \mathrm{~K} .{ }^{\mathrm{a}}[22]{ }^{\mathrm{b}}[61]{ }^{\mathrm{c}}[62]$

\begin{tabular}{cccccc}
\hline & $N$-butilamina & $N$-pentilamina & $N$-hexilamina & $N$-heptilamina & $N$-octilamina \\
\hline$n$-hexano & $0.414^{\mathrm{a}}$ & $0.295^{\mathrm{c}}$ & $0.131^{\mathrm{c}}$ & $-0.027^{\mathrm{c}}$ & $-0.131^{\mathrm{c}}$ \\
$n$-heptano & $0.6973^{\mathrm{a}}$ & $0.497^{\mathrm{c}}$ & $0.331^{\mathrm{c}}$ & $0.219^{\mathrm{c}}$ & $0.076^{\mathrm{c}}$ \\
& & & $0.330^{\mathrm{b}}$ & & \\
$n$-octano & $0.864^{\mathrm{b}}$ & $0.604^{\mathrm{c}}$ & $0.451^{\mathrm{c}}$ & $0.347^{\mathrm{c}}$ & $0.232^{\mathrm{c}}$ \\
& & & $0.469^{\mathrm{b}}$ & & \\
$n$-nonano & $0.861^{\mathrm{b}}$ & & & & \\
$n$-decano & $0.902^{\mathrm{b}}$ & $0.734^{\mathrm{b}}$ & $0.610^{\mathrm{b}}$ & $0.533^{\mathrm{b}}$ & $0.437^{\mathrm{b}}$ \\
$n$-dodecano & & & $0.645^{\mathrm{b}}$ & & \\
ciclohexano & $0.8217^{\mathrm{a}}$ & $0.8312^{\mathrm{c}}$ & $0.7559^{\mathrm{c}}$ & $0.734^{\mathrm{c}}$ & $0.697^{\mathrm{c}}$ \\
\hline
\end{tabular}


$V_{\mathrm{m}}^{\mathrm{E}}$ varía de la misma forma que $H_{\mathrm{m}}^{\mathrm{E}}$, es decir, aumenta con la longitud del $n$-alcano y disminuye al aumentar la longitud de la amina.

Como se ha visto, cuando la mezcla está formada por moléculas de distinto tamaño la contribución negativa debida a la acomodación intersticial (efectos estructurales) puede predominar, dando lugar a valores negativos de $V_{\mathrm{m}}^{\mathrm{E}}$. Los efectos estructurales también son responsables de las formas sigmoides de $V_{\mathrm{m}}^{\mathrm{E}}=V_{\mathrm{m}}^{\mathrm{E}}(x)$, como ocurre en las mezclas heptilamina $+n$-hexano y octilamina $+n$-hexano $\circ n$-heptano. $S i$ el tamaño de las moléculas presentes en la mezcla es similar, predomina la contribución positiva debido a la ruptura de los puentes de hidrógeno y a las interacciones dipolares durante el proceso de mezclado, siendo el volumen de exceso positivo en todo el rango de concentración.

Aunque no han sido encontrados en la literatura valores experimentales de $H_{\mathrm{m}}^{\mathrm{E}}$ para los sistemas amina primaria + ciclohexano, los valores de $V_{\mathrm{m}}^{\mathrm{E}}$ muestran inequívocamente la mayor capacidad de este alcano, en comparación con los lineales, para romper los enlaces puente de hidrógeno presentes entre las moléculas de amina. Además, la molécula de ciclohexano, al ser más plana, se acomoda peor intersticialmente en la estructura de la amina. Por tanto, $V_{\mathrm{m}}^{\mathrm{E}}(n$-hexano $)<V_{\mathrm{m}}^{\mathrm{E}}$ (ciclohexano). Un comportamiento similar se ha encontrado en mezclas 1 -alcohol $+n$-alcano o cicloalcano [63]-[65].

\subsubsection{Amina lineal secundaria $+n$-alcano}

La Tabla 5-5 muestra los valores de $H_{\mathrm{m}}^{\mathrm{E}}$ de algunas mezclas amina lineal secundaria $+n$ alcano a fracción equimolar y $298.15 \mathrm{~K}$. Como puede comprobarse, tales mezclas son endotérmicas, de modo que la ruptura de los puentes de hidrógeno y de las interacciones dipolares predominan sobre otro tipo de efectos. A una temperatura dada, fijada la amina, $H_{\mathrm{m}}^{\mathrm{E}}$ aumenta a medida que lo hace el tamaño del alcano, como en el caso de los sistemas amina primaria $+n$-alcano. Para un $n$-alcano dado se observa que $H_{\mathrm{m}}^{\mathrm{E}}$ (di-n-etilamina $)>H_{\mathrm{m}}^{\mathrm{E}}$ (di- $n$-propilamina $)>H_{\mathrm{m}}^{\mathrm{E}}$ (di- $n$-butilamina), es decir, $H_{\mathrm{m}}^{\mathrm{E}}$ es mayor cuanto menor es el tamaño de la amina. Por lo tanto, la asociación de la amina es menor cuanto mayor su tamaño. Nótese que el momento dipolar efectivo varía de la misma forma (Tabla 5-1), es decir, las interacciones dipolo-dipolo son más intensas en las mezclas que contienen aminas más cortas. El mismo comportamiento se ha encontrado en los sistemas amina primaria $+n$-alcano (ver la sección 5.3.1).

Tabla 5-5: $H_{\mathrm{m}}^{\mathrm{E}}\left(x_{1}=0.5\right) / \mathrm{J} \cdot \mathrm{mol}^{-1}$ en sistemas amina secundaria (1) + n-alcano (2) a $298.15 \mathrm{~K} .{ }^{\mathrm{a}}[54] ;{ }^{\mathrm{b}}[66] ;{ }^{\mathrm{c}}[67]$ ${ }^{\mathrm{d}}[32]$

\begin{tabular}{|c|c|c|c|}
\hline & di- $n$-etilamina (DEA) & di-n-propilamina (DPA) & di-n-butilamina (DBA) \\
\hline$n$-hexano & & $429^{b}$ & $279^{c}$ \\
\hline$n$-heptano & $742^{a}$ & $456^{\mathrm{b}}$ & $317^{d}$ \\
\hline$n$-octano & & $473^{b}$ & \\
\hline$n$-nonano & & $492^{b}$ & \\
\hline$n$-decano & & $524^{b}$ & \\
\hline
\end{tabular}

El comportamiento entrópico también es similar al de mezclas amina lineal primaria + 
alcano, es decir, $T \cdot S_{\mathrm{m}}^{\mathrm{E}}>0$ en todo el rango de concentración, lo que implica destrucción de la estructura asociada con la amina.

El comportamiento volumétrico se refleja en la Tabla 5-6, donde se observa que $V_{\mathrm{m}}^{\mathrm{E}}>0$ para todo este tipo de mezclas, es decir, la contribución positiva es mayor que la debida a los efectos estructurales y de volumen libre, excepto en el caso de la mezcla di-n-butilamina $+n$-hexano, en la que $V_{\mathrm{m}}^{\mathrm{E}}<0$ lo que se debe a que la contribución negativa ocasionada por la diferencia de tamaño entre los componentes de la mezcla predominar sobre la contribución positiva debida a la ruptura de los puentes de hidrógeno de la amina.

Para un $n$-alcano dado, $V_{\mathrm{m}}^{\mathrm{E}}$ y $H_{\mathrm{m}}^{\mathrm{E}}$ varían de igual forma, es decir, $V_{\mathrm{m}}^{\mathrm{E}}$ (di-n-etilamina) $>$ $V_{\mathrm{m}}^{\mathrm{E}}($ di-n-propilamina $)>V_{\mathrm{m}}^{\mathrm{E}}$ (di-n-butilamina). Es decir, la variación del volumen de exceso es paralela a la de la contribución interaccional a esta magnitud, mayor para las mezclas con di-n-etilamina debido a su mayor autoasociación y más elevado momento dipolar.

Los efectos estructurales también son importantes en las mezclas de di- $n$-etilamina $\circ \mathrm{N}$ butilmetilamina con $n$-alcanos, pues en las primeras $V_{\mathrm{m}}^{\mathrm{E}}$ disminuye con la longitud del alcano cuando éste es más largo que el $n$-heptano, mientras que en las segundas $V_{\mathrm{m}}^{\mathrm{E}}$ aumenta con el tamaño del alcano, siendo casi constante a partir del $n$-decano.

Las diferencias de tamaño entre las moléculas presentes en la mezcla también sirven para caracterizar la simetría de las curvas $V_{\mathrm{m}}^{\mathrm{E}}=V_{\mathrm{m}}^{\mathrm{E}}(x)$. Así, mientras que el desplazamiento de $V_{\mathrm{m}}^{\mathrm{E}}(x)$ hacia la zona rica en $n$-alcano en el sistema $N$ butilmetilamina $+n$-hexano puede atribuirse a la autoasociación de la amina, en el sistema $N$-butilmetilamina $+n$-hexadecano el desplazamiento de la curva $V_{\mathrm{m}}^{\mathrm{E}}=V_{\mathrm{m}}^{\mathrm{E}}(x)$ hacia la zona de alta concentración de la amina (componente más pequeño) sugiere la existencia de efectos de volumen libre.

Las mezclas con ciclohexano en lugar de un alcano lineal están acompañadas de un aumento en $V_{\mathrm{m}}^{\mathrm{E}}$. Este mismo comportamiento se encuentra en los sistemas 1-alcohol o amina primaria + ciclohexano, tal y como se ha visto en la sección 5.3.1.

Tabla 5-6: $V_{\mathrm{m}}^{\mathrm{E}}\left(x_{1}=0.5\right) / \mathrm{cm}^{3} \cdot \mathrm{mol}^{-1}$ en sistemas amina secundaria (1) + alcano (2) a $298.15 \mathrm{~K} .{ }^{\mathrm{a}}[68] ;{ }^{\mathrm{b}}[22] ;{ }^{\mathrm{c}}[61]$; d[69]

\begin{tabular}{ccccc}
\hline & di- $n$-etilamina (DEA) & $N$-butilmetilamina & di- $n$-propilamina (DPA) di- $n$-butilamina (DBA) \\
\hline$n$-pentano & $0.458^{\mathrm{a}}$ & & & \\
$n$-hexano & $0.612^{\mathrm{b}}$ & $0.320^{\mathrm{d}}$ & $0.146^{\mathrm{d}}$ & $-0.185^{\mathrm{d}}$ \\
$n$-heptano & $0.658^{\mathrm{b}}$ & & $0.259^{\mathrm{d}}$ & $0.052^{\mathrm{d}}$ \\
& $0.659^{\mathrm{c}}$ & & $0.268^{\mathrm{c}}$ & \\
$n$-octano & $0.590^{\mathrm{c}}$ & $0.479^{\mathrm{d}}$ & $0.308^{\mathrm{d}}$ & \\
$n$-decano & & $0.520^{\mathrm{d}}$ & & \\
$n$-dodecano & $0.526^{\mathrm{c}}$ & $0.524^{\mathrm{d}}$ & & \\
$n$-tetradecano & & $0.516^{\mathrm{d}}$ & & $0.530^{\mathrm{d}}$ \\
$n$-hexadecano & & $0.517^{\mathrm{d}}$ & & \\
ciclohexano & $0.676^{\mathrm{d}}$ & $0.693^{\mathrm{d}}$ & $0.503^{\mathrm{d}}$ & \\
\hline
\end{tabular}




\subsubsection{Tri- $n$-alquilamina $+n$-alcano}

Las aminas terciarias no presentan asociación por puentes de hidrógeno y sus momentos dipolares son menores que los de las aminas primarias o secundarias (véase la Tabla 5-1). Por lo tanto las interacciones dipolares no son muy fuertes y el comportamiento de los sistemas amina terciaria $+n$-alcano es prácticamente ideal, como delatan los pequeños valores de las entalpías y volúmenes de exceso de estas mezclas, que se se muestran en las tablas 5.7 y 5.8 , a fracción equimolar y $298.15 \mathrm{~K}$.

Tabla 5-7: $H_{\mathrm{m}}^{\mathrm{E}}\left(x_{1}=0.5\right) / J \cdot \mathrm{mol}^{-1}$ en mezclas amina terciaria (1) + n-alcano (2) a $298.15 \mathrm{~K} .{ }^{\mathrm{a}}[70] ;{ }^{\mathrm{b}}[71] ;{ }^{\mathrm{c}}[54] ;$ ${ }^{\mathrm{d}}[32] ;{ }^{\mathrm{e}}[\mathrm{72}]$

\begin{tabular}{cccc}
\hline & $N, N, N$-trietilamina (TEA) & $N, N, N$-tripropilamina (TPA) & $N, N, N$-tributilamina (TBA) \\
\hline$n$-pentano & $60^{\mathrm{a}}$ & $-5^{\mathrm{b}}$ & $-18^{\mathrm{e}}$ \\
$n$-hexano & $82^{\mathrm{b}}$ & & $-3^{\mathrm{e}}$ \\
$n$-heptano & $84^{\mathrm{c}}$ & $47^{\mathrm{d}}$ & $20^{\mathrm{d}}$ \\
$n$-octano & $106^{\mathrm{b}}$ & $37^{\mathrm{b}}$ & $34^{\mathrm{e}}$ \\
$n$-decano & $136^{\mathrm{b}}$ & & $70^{\mathrm{e}}$ \\
$n$-dodecano & $201^{\mathrm{b}}$ & $118^{\mathrm{b}}$ & $119^{\mathrm{e}}$ \\
$n$-tetradecano & $258^{\mathrm{b}}$ & & $181^{\mathrm{e}}$ \\
$n$-hexadecano & $324^{\mathrm{b}}$ & $225^{\mathrm{b}}$ & $227^{\mathrm{e}}$ \\
\hline
\end{tabular}

La Tabla 5-7 muestra que en este tipo de sistemas, fijado el $n$-alcano, la variación de $H_{\mathrm{m}}^{\mathrm{E}}$ con la longitud de la amina es la misma que para los sistemas amina primaria $\mathrm{O}$ secundaria $+n$-alcano, es decir, disminuye al aumentar el tamaño de la amina: $H_{\mathrm{m}}^{\mathrm{E}}(N, N, N$-trietilamina $)>H_{\mathrm{m}}^{\mathrm{E}}(N, N, N$-tripropilamina $)>H_{\mathrm{m}}^{\mathrm{E}}(N, N, N$-tributilamina $)$. Ello es debido a que las interacciones dipolares son más fuertes en mezclas que contienen $N, N, N$-trietilamina, como delatan los valores de $\bar{\mu}$ (Tabla 5-1), que varían siguiendo la secuencia: $\bar{\mu}(N, N, N$-trietilamina $)>\bar{\mu}(N, N, N$-tripropilamina $)>\bar{\mu}(N, N, N$-tributilamina $)$.

Por otro lado, fijada la amina terciaria, la $H_{\mathrm{m}}^{\mathrm{E}}$ es mayor cuanto mayor es el tamaño del n-alcano (Tabla 5-7). Este incremento es superior al esperado en el caso de alcanos muy largos, probablemente debido a la existencia de un cierto orden orientacional en dichos alcanos, que es destruido cuando se mezclan con moléculas globulares. Este efecto, conocido como efecto Patterson [76], es responsable de una contribución endotérmica extra a $H_{\mathrm{m}}^{\mathrm{E}}$. El efecto contrario, llamado efecto Wilhelm [77]-[79], aparece cuando alcanos largos se mezclan con moléculas planas. En estos casos, existe una contribución exotérmica extra en $H_{\mathrm{m}}^{\mathrm{E}}$ debida a la creación de algún tipo de orden.

Los valores negativos de las entalpías de exceso de las mezclas $N, N, N$-tripropilamina $+n$ pentano y $N, N, N$-tributilamina $+n$-pentano $o+n$-hexano son llamativos, sobre todo teniendo en cuenta que al mezclar un alcano lineal con una amina terciaria, el $n$-alcano únicamente debería debilitar las interacciones dipolares presentes entre las molécula de amina, originando un efecto endotérmico (positivo). Este comportamiento puede explicarse teniendo en cuenta que la entalpía de exceso es la suma de dos contribuciones: una puramente energética dada por la energía interna de exceso a volumen constante, $U_{\mathrm{m}, V}^{\mathrm{E}}$, que da cuenta de los efectos interaccionales y otra debida a la ecuación térmica de estado, que da cuenta de los efectos estructurales. En efecto, de la ecuación (2.203) se obtiene la siguiente relación: 


$$
H_{\mathrm{m}}^{\mathrm{E}} \approx U_{\mathrm{m}, V}^{\mathrm{E}}+T \cdot \frac{\alpha_{P}}{\kappa_{T}} \cdot V_{\mathrm{m}}^{\mathrm{E}}=U_{\mathrm{m}, V}^{\mathrm{E}}+\left(P+P_{\mathrm{int}}\right) \cdot V_{\mathrm{m}}^{\mathrm{E}}
$$

Cuando los datos del coeficiente de dilatación térmica $\alpha_{P}$ y de la compresibilidad isoterma $\kappa_{T}$ no se estén disponibles, dichos valores se consideran iguales a los de la mezcla ideal (ecuaciones 2.164 y 2.165 ):

$$
\begin{aligned}
& \alpha_{P}^{\text {id }}=\sum_{\mathrm{i}=1}^{\mathrm{n}} \phi_{\mathrm{i}} \cdot \alpha_{P, \mathrm{i}}^{0} \\
& \kappa_{T}^{\mathrm{id}}=\sum_{\mathrm{i}=1}^{\mathrm{n}} \phi_{\mathrm{i}} \cdot \kappa_{T, \mathrm{i}}^{0}
\end{aligned}
$$

Esta aproximación está justificada cuando $\alpha_{P}^{\mathrm{E}}$ y $\kappa_{T}^{\mathrm{E}}$ tienen valores relativamente pequeños.

Sabiendo esto, la contribución debida a la ecuación de estado para el sistema $N, N, N$ tributilamina $+n$-hexano es $-102 \mathrm{~J} \cdot \mathrm{mol}^{-1}$, valor suficientemente negativo como para hacer que $H_{\mathrm{m}}^{\mathrm{E}}$ sea negativa a pesar de la contribución energética positiva (véase la Tabla 5-7).

Tabla 5-8: $V_{\mathrm{m}}^{\mathrm{E}}\left(x_{1}=0.5\right) / \mathrm{cm}^{3} \cdot \mathrm{mol}^{-1}$ en sistemas amina terciarias (1) + alcano (2) a $298.15 \mathrm{~K} .{ }^{\mathrm{a}}[74] ;{ }^{\mathrm{b}}[22] ; \mathrm{c}[75]$

\begin{tabular}{cccc}
\hline & $N, N, N$-trietilamina & $N, N, N$-tripropilamina & $N, N, N$-tributilamina \\
\hline$n$-hexano & $0.0698^{a}$ & $-0.1738^{\mathrm{c}}$ & $-0.3838^{\mathrm{a}}$ \\
$n$-heptano & $0.1078^{\mathrm{b}}$ & $-0.0406^{\mathrm{b}}$ & $-0.1941^{\mathrm{b}}$ \\
$n$-octano & $0.1184^{\mathrm{a}}$ & $0.0145^{\mathrm{c}}$ & $-0.0700^{\mathrm{a}}$ \\
$n$-decano & $0.0717^{\mathrm{a}}$ & $0.0498^{\mathrm{c}}$ & $0.0411^{\mathrm{a}}$ \\
$n$-dodecano & & $0.0401^{\mathrm{c}}$ & $0.0814^{\mathrm{a}}$ \\
$n$-tetradecano & $-0.0544^{\mathrm{a}}$ & & $0.1095^{\mathrm{a}}$ \\
$n$-hexadecano & $-0.0979^{\mathrm{a}}$ & $0.0043^{\mathrm{c}}$ & $0.1190^{\mathrm{a}}$ \\
ciclohexano & $0.2641^{\mathrm{b}}$ & $0.3620^{\mathrm{b}}$ & $0.2803^{\mathrm{b}}$ \\
\hline
\end{tabular}

En mezclas con $N, N, N$-trietilamina, $V_{\mathrm{m}}^{\mathrm{E}}$ aumenta hasta el $n$-octano, donde los efectos estructurales y de volumen libre comienzan a cobrar importancia. Esto justifica también la asimetría de las curvas hacia la zona de composición rica en el componente de menor tamaño. La contribución negativa al volumen de exceso puede incluso superar a la contribución positiva resultado de la debilitación de las interacciones dipolares aminaamina, lo que explica los valores negativos de $V_{\mathrm{m}}^{\mathrm{E}}$ en las mezclas $N, N, N$-trietilamina + $n$-tetradecano o $n$-hexadecano. Estos efectos, en sistemas con $N, N, N$-tripropilamina, son detectados a partir del $n$-dodecano. En cambio, en los sistemas $N, N, N$-tributilamina + $n$-alcano, $V_{\mathrm{m}}^{\mathrm{E}}$ aumenta con el tamaño del $n$-alcano. Los efectos de volumen libre, sin embargo, se observan claramente cuando la mezcla incluye alcanos de pequeño tamaño, como $n$-hexano o $n$-heptano. Un aumento en el tamaño del $n$-alcano hace que la contribución negativa, consecuencia de los efectos estructurales, sea menos importante que la contribución positiva debido a efectos interaccionales.

\subsubsection{Amina aromática + alcano}

Las mezclas de anilina, $\mathrm{N}$-metilanilina y piridina con alcanos están caracterizadas por la ruptura de las fuertes interacciones entre las moléculas de la amina. Así lo sugieren los siguientes resultados experimentales: 
a) En tales sistemas hay zonas de inmiscibilidad, de modo que la curva de coexistencia líquido-líquido tiene una temperatura de disolución crítica superior (UCST), $T_{\mathrm{c}}$. Por ejemplo, $T_{\mathrm{c}}$ (anilina $+n$-hexano) $=342.7 \mathrm{~K}[80], T_{\mathrm{c}}$ (anilina $+n$-heptano) $=343.11 \mathrm{~K}[81]$; $T_{\mathrm{c}}(N$-metilanilina $+n$-heptano $)=273.15 \mathrm{~K}[82], T_{\mathrm{c}}(N$-metilanilina + tetradecano $)=286.85$ $\mathrm{K}$ [34], $T_{\mathrm{c}}(N$-metilanilina $+n$-hexadecano $)=292.43 \mathrm{~K}[34] ; T_{\mathrm{c}}($ piridina $+n$-hexano $)=$ $252.2 \mathrm{~K}, T_{\mathrm{c}}$ (piridina $+n$-heptano) $=255.2 \mathrm{~K}$ [83]. Si se tiene en cuenta que la anilina, la $N$-metilanilina y la piridina son aminas primaria, secundaria y terciaria respectivamente, formando las dos primeras complejos mediante enlaces puente de hidrógeno, las temperaturas críticas dadas revelan que las interacciones amina-amina disminuyen en el orden anilina $>N$-metilanilina $>$ piridina. La fuerza de las mencionadas interacciones también pueden evaluarse en términos de $\mathrm{D} H_{\mathrm{v}}$ (Tabla 5-1). Por el contrario, el momento dipolar efectivo cambia de modo inverso: $\bar{\mu}$ (anilina) $<\bar{\mu}(N$-metilanilina $)<\bar{\mu}$ (piridina) (Tabla 5-1). Ello implica que las interacciones por el puente de hidrógeno en las aminas primarias y secundarias predominan sobre las interacciones dipolares, o bien que la polarizabilidad de la anilina es mayor que en las otras aminas aromáticas y originando mayores interacciones dipolares en las disoluciones con anilina. Es decir, el momento dipolar efectivo no es una panacea que sirva para caracterizar totalmente las interacciones dipolares. De hecho, mayor $T_{\mathrm{c}}$ indica inequívocamente interacciones dipolares más intensas.

La existencia de zonas de inmiscibilidad en mezclas sustancia polar + alcano es propia de mezclas caracterizadas por interacciones dipolares entre las moléculas de la sustancia polar (en este caso la amina). Debe tenerse en cuenta que los enlaces de tipo puente de hidrógeno son fundamentalmente de tipo dipolar con cierto carácter covalente.

b) Valores altos de $H_{\mathrm{m}}^{\mathrm{E}}$ en mezclas con $n$-alcanos. Dichos valores son mucho mayores que en las mezclas amina lineal $+n$-alcano, lo que implica que las interacciones entre las moléculas de las aminas aromáticas son mucho mayores que entre las aminas lineales, como muestran los valores de $H_{\mathrm{m}, 1}^{\mathrm{E}, \infty}$ en las mezclas con $n$-heptano (Tabla 5-2) y el estudio del espectro de absorción infrarrojo de la $N$-metilanilina, el cual indica que ella forma enlaces de hidrógeno mucho más intensos que las aminas lineales secundarias [84].

Para un alcano dado, $H_{\mathrm{m}}^{\mathrm{E}}$ varía de la misma manera que $\mathrm{D} H_{\mathrm{v}}$. Por ejemplo, para las mezclas con ciclohexano, $H_{\mathrm{m}}^{\mathrm{E}}$ (anilina) $>H_{\mathrm{m}}^{\mathrm{E}}\left(N\right.$-metilanilina) $>H_{\mathrm{m}}^{\mathrm{E}}$ (piridina) (véase la Tabla 5-9). Esto confirma que las interacciones entre aminas aromáticas son más débiles siguiendo la secuencia: primaria $>$ secundaria $>$ terciaria, de manera similar a como ocurre en las aminas lineales, como se ha dejado entrever en las secciones 5.3.1 a 5.3.3.

Tabla 5-9: $H_{\mathrm{m}}^{\mathrm{E}}\left(x_{1}=0.5\right) / \mathrm{J} \cdot \mathrm{mol}^{-1}$ en sistemas amina aromática (1) + alcano (2) a $\left.\left.298.15 \mathrm{~K}^{\circ}{ }^{\mathrm{a}}[85]\right]^{;}{ }^{\mathrm{b}}[28]\right]^{;}{ }^{\mathrm{c}}[34]$; d[86]; ${ }^{[}[73] ;{ }^{f}[87]$

\begin{tabular}{cccc}
\hline & anilina & $N$-metilanilina & piridina \\
\hline$n$-hexano & & & $1539^{\mathrm{e}}$ \\
$n$-heptano & & $1675^{\mathrm{c}}$ & $1730^{\mathrm{e}}$ \\
$n$-octano & $1774^{\mathrm{c}}$ & $1757^{\mathrm{e}}$ \\
$n$-nonano & & $1843^{\mathrm{e}}$ \\
$n$-decano & & $1914^{\mathrm{c}}$ & $1945^{\mathrm{e}}$ \\
ciclohexano & $1868^{\mathrm{a}}(T=308.15 \mathrm{~K})$ & $1617^{\mathrm{c}}$ & $1438^{\mathrm{f}}$ \\
tolueno & $1009^{\mathrm{b}}$ & $497^{\mathrm{c}}$ & \\
benceno & $751^{\mathrm{b}}$ & $457^{\mathrm{d}}$ & \\
\hline
\end{tabular}


En los sistemas anilina o $N$-metilanilina + benceno o tolveno, la contribución positiva a $H_{\mathrm{m}}^{\mathrm{E}}$ por la ruptura de interacciones entre moléculas iguales es mayor que la contribución negativa debida a las interacciones entre moléculas diferentes, siendo ésta mayor en las mezclas con anilina, lo que está en consonancia con los valores de $\Delta H_{1-2}$ en la Tabla 5-2.

Como sabemos, $H_{\mathrm{m}}^{\mathrm{E}}$ no refleja únicamente efectos interaccionales, sino que también está determinada por efectos estructurales, pues se puede escribir como la suma de una contribución puramente energética dada por $U_{\mathrm{m}, V}^{\mathrm{E}}$ y otra debida a la ecuación térmica de estado (ecuación (5.7)). Las diferencias entre $H_{\mathrm{m}}^{\mathrm{E}}$ y $U_{\mathrm{m}, V}^{\mathrm{E}}$ permiten discutir los efectos estructurales y los puramente energéticos. En las mezclas $N$-metilanilina $+n$-heptano, ciclohexano y tolveno estas diferencias son $\approx 5 \%, \approx 11 \%$ y $\approx 18 \%$ respectivamente, lo que significa que la importancia de los efectos estructurales sigue la secuencia tolveno $>$ ciclohexano $>n$-heptano.

c) Aunque no se han encontrado datos de los volúmenes de exceso para las mezclas de anilina con alcanos, debido a que a temperatura ambiente no son miscibles (recuérdese que $T_{\mathrm{c}}$ (anilina $+n$-hexano $)=342.7 \mathrm{~K}, T_{\mathrm{c}}$ (anilina $+n$-heptano $)=343.11 \mathrm{~K}$ ), sí se dispone de los mismos en algunas mezclas tipo piridina $\circ \mathrm{N}$-metilanilina + alcano. En las mezclas de piridina + alcano, los valores positivos de los volúmenes de exceso revelan que la ruptura de las interacciones amina-amina es predominante sobre otros efectos que contribuyen negativamente a $V_{\mathrm{m}}^{\mathrm{E}}$, excepto para la mezcla con $n$-hexano, en donde dichos efectos son predominantes. En las mezclas de $N$-metilanilina + alcano, los volúmenes de exceso son mucho menores, e incluso negativos, lo que implica que los efectos estructurales son relevantes. De hecho, los valores negativos de $\alpha_{P}^{\mathrm{E}}$ y $\kappa_{S}^{\mathrm{E}}$ (véase la Tabla 3.73), típicos de sistemas en los que los efectos estructurales son importantes, así lo demuestran. En la mezcla con $n$-heptano, la curva $V_{\mathrm{m}}^{\mathrm{E}}=V_{\mathrm{m}}^{\mathrm{E}}(x)$ está desplazada hacia las mayores concentraciones de la amina, el componente más pequeño (Ilustración 3.39), lo que también es típico de aquellos sistemas en los que aparecen efectos de volumen libre, como en la mezcla $N$-butilmetilamina $+n$-hexadecano, como ya se ha comentado.

Tabla 5-10: $V_{\mathrm{m}}^{\mathrm{E}}\left(x_{1}=0.5\right) / \mathrm{cm}^{3} \cdot \mathrm{mol}^{-1}$ en sistemas amina aromática (1) + alcano (2) a $298.15 \mathrm{~K}$. ${ }^{\mathrm{a}}[88] ;{ }^{\mathrm{b}}[73]$

\begin{tabular}{ccc}
\hline & $N$-metilanilina & piridina \\
\hline$n$-hexano & $-0.227^{\mathrm{a}}$ & $-0.020^{\mathrm{b}}$ \\
$n$-heptano & & $0.240^{\mathrm{b}}$ \\
$n$-octano & & $0.430^{\mathrm{b}}$ \\
$n$-nonano & $0.580^{\mathrm{b}}$ \\
$n$-decano & $0.690^{\mathrm{b}}$ \\
ciclohexano & & \\
tolueno & $0.462^{\mathrm{a}}$ & \\
\hline
\end{tabular}

El intercambio de un alcano lineal por un hidrocarburo aromático se traduce en la disminución de $H_{\mathrm{m}}^{\mathrm{E}}$ y en valores negativos de $V_{\mathrm{m}}^{\mathrm{E}}$, lo que revela que en sistemas de este tipo, la contribución a $H_{\mathrm{m}}^{\mathrm{E}}$ y $V_{\mathrm{m}}^{\mathrm{E}}$ debida a la formación de interacciones dipolo-dipolo inducido entre moléculas diferentes es importante, pues el anillo bencénico es muy polarizable. 
Fijada la amina, $H_{\mathrm{m}}^{\mathrm{E}}$ y $V_{\mathrm{m}}^{\mathrm{E}}$ aumentan con la longitud del $n$-alcano, lo que se debe a la mayor facilidad de los alcanos largos para romper las interacciones amina-amina, debido a su mayor superficie alifática.

Los valores positivos encontrados para la magnitud $\left(\partial H_{\mathrm{m}}^{\mathrm{E}} / \partial P\right)_{T}=V_{\mathrm{m}}^{\mathrm{E}}-T \cdot\left(\partial V_{\mathrm{m}}^{\mathrm{E}} / \partial T\right)_{P}$ en los sistemas $N$-metilanilina $+n$-heptano $\left(0.79 \mathrm{~cm}^{3} \cdot \mathrm{mol}^{-1}\right), N$-metilanilina + ciclohexano $\left(0.67 \mathrm{~cm}^{3} \cdot \mathrm{mol}^{-1}\right)$ y $N$-metilanilina + tolueno $\left(0.26 \mathrm{~cm}^{3} \cdot \mathrm{mol}^{-1}\right)$ indican que la ruptura de las interacciones entre las moléculas iguales es el efecto interaccional dominante cuando aumenta la presión. El menor valor de $\left(\partial H_{\mathrm{m}}^{\mathrm{E}} / \partial P\right)_{T}$ en el caso del tolueno vuelve a poner de manifiesto que en las mezclas con hidrocarburos aromáticos, la contribución debida a la formación de interacciones entre moléculas diferentes es importante. Esto también puede explicar que las mezclas con $n$-heptano sean menos compresibles que las de tolveno, pues como se observa en las Tablas 3.73 y 3.79 y las llustraciones 3.39 y 3.41 , se verifica la desigualdad $\kappa_{S}^{\mathrm{E}}(N$-metilanilina $+n$-heptano $)<\kappa_{S}^{\mathrm{E}}(N$-metilanilina + tolveno $)$.

\subsection{MEZCLAS CETONA + ALCANO}

La Tabla 5-11 muestra los valores de las entalpías de exceso a $x_{1}=0.5$ y $298.15 \mathrm{~K}$ obtenidos de la literatura para algunas mezclas del tipo cetona (1) + alcano (2).

Tabla 5-11: $H_{\mathrm{m}}^{\mathrm{E}}\left(x_{1}=0.5\right)$ en mezclas cetona (1) + alcano (2) a $298.15 \mathrm{~K} .{ }^{\mathrm{a}}[89] ;{ }^{\mathrm{b}}[90] ;{ }^{\mathrm{c}}[91] ;{ }^{\mathrm{e}}[92] ;{ }^{\mathrm{d}}[93] ; \mathrm{f}[94] ;$ g[95]; ${ }^{h}[49] ;$ i $[96] ;$ i $[97] ;{ }^{k}[98]$

\begin{tabular}{ccccccc}
\hline & 2-propanona & 2-butanona & 2-pentanona & 3-pentanona & 2-hexanona & 2-heptanona \\
\hline$n$-pentano & & $1159.7^{f}$ & $966.1^{f}$ & $921.0^{f}$ & & \\
$n$-hexano & $1599^{\mathrm{a}}$ & $1252.4^{f}$ & $1040.3^{f}$ & $999^{f}$ & $949^{\mathrm{i}}$ & \\
$n$-heptano & $1704^{\mathrm{b}}$ & $1338.1^{\mathrm{g}}$ & $1135.0^{f}$ & $1079.3^{f}$ & $978^{\mathrm{i}}$ & $861^{\mathrm{k}}$ \\
$n$-octano & & $1408.2^{f}$ & $1202.2^{f}$ & $1140.7^{f}$ & $1130^{\mathrm{i}}$ & \\
$n$-nonano & & & & & $1200^{\mathrm{i}}$ & \\
$n$-decano & $1967^{\mathrm{c}}$ & $1544.5^{f}$ & $1334.9^{\mathrm{f}}$ & $1261.5^{f}$ & $1260^{\mathrm{i}}$ & \\
tolueno & $242^{\mathrm{d}}$ & $-22^{\mathrm{d}}$ & $-116^{\mathrm{d}}$ & $-157^{\mathrm{i}}$ & & \\
benceno & $146^{\mathrm{e}}$ & $-50^{\mathrm{h}}$ & $-74^{\mathrm{e}}$ & $-129^{\mathrm{i}}$ & & \\
\hline
\end{tabular}

En primer lugar, se estudian las mezclas del tipo cetona $+n$-alcano. En comparación con las aminas, las cetonas poseen mayores $\bar{\mu}$, como se puede apreciar en la Tabla 5-1. Por lo tanto, puede afirmarse que las interacciones dipolo-dipolo son mucho más intensas en el caso de cetonas que en el de aminas [95], lo que es consistente con los más elevados valores de $H_{\mathrm{m}}^{\mathrm{E}}$ de las mezclas cetonas $+n$-alcanos y con la existencia de una región de inmiscibilidad en el sistema 2 -propanona $+n$-heptano $\left(T_{\mathrm{c}}=245.22 \mathrm{~K}\right.$ [107]).

En segundo lugar, se estudian las mezclas cetona + hidrocarburo aromático. Al sustituir un $n$-alcano por un hidrocarburo aromático en las mezclas con cetona, se produce una gran disminución en $H_{\mathrm{m}}^{\mathrm{E}}$. De hecho, dichos valores pueden ser negativos. Ello es consecuencia de la formación de complejos por transferencia de carga o de interacciones dipolo-dipolo inducido entre las moléculas distintas, debido a la gran polaridad de los anillos bencénicos. 
Los datos de $\Delta H_{1-2}$ de la Tabla 5-2 muestran que para un alcano fijo, las interacciones cetona-hidrocarburo aromático disminuyen con la longitud de la cetona, lo que está de acuerdo con la variación que sigue el momento dipolar efectivo: $\bar{\mu}(2$-propanona) > $\bar{\mu}(2$-butanona $)>\bar{\mu}(2$-pentanona $)>\bar{\mu}$ (2-hexanona) $>\bar{\mu}$ (2-heptanona) (Tabla 5-1), debida al impedimento estérico del grupo carbonilo en cetonas de cadena larga.

Como sabemos, para tener en cuenta los efectos energéticos y estructurales es conveniente calcular $U_{\mathrm{m} . V}^{\mathrm{E}}$ (ecuación (5.7)). Los cálculos pertinentes demuestran que la contribución estructural es importante, tanto que en ocasiones $H_{\mathrm{m}}^{\mathrm{E}}$ y $U_{\mathrm{m} . V}^{\mathrm{E}}$ tienen diferentes signos. No obstante, $H_{\mathrm{m}}^{\mathrm{E}}$ y $U_{\mathrm{m} . V}^{\mathrm{E}}$ varían con la longitud de la cetona de igual modo.

Por otra parte, fijada la cetona, puede verse que las interacciones cetona-hidrocarburo aromático varían de la forma: $\mid \Delta H_{1-2}$ (benceno) $|>| \Delta H_{1-2}$ (tolveno) $\mid$. Esto se debe a que la molécula de tolveno es menos polarizable que la del benceno, debido al mayor apantallamiento estérico del anillo aromático en el tolveno.

En la Tabla 5-12 se presentan los valores de $V_{\mathrm{m}}^{\mathrm{E}}$ a $x_{1}=0.5$ y a $298.15 \mathrm{~K}$ obtenidos de la literatura para algunas mezclas del tipo cetona + alcano.

Tabla 5-12: $V_{\mathrm{m}}^{\mathrm{E}}\left(x_{1}=0.5\right)$ para sistemas cetona (1) + alcano (2) a $298.15 \mathrm{~K} .{ }^{\mathrm{a}}[100] ;{ }^{\mathrm{b}}[101] ;{ }^{\mathrm{c}}[102] ;{ }^{\mathrm{d}}[103]$; e[104]; f[105]; 9[97]; h[106];

\begin{tabular}{cccccc}
\hline & 2-propanona & 2-butanona & 2-pentanona & 3-pentanona & 2-hexanona \\
\hline$n$-hexano & & & & & $0.154^{\mathrm{g}}$ \\
$n$-heptano & $0.9975^{\mathrm{a}}$ & $0.7325^{\mathrm{a}}$ & $0.550^{\mathrm{a}}$ & $0.5117^{\mathrm{f}}$ & $0.3745^{\mathrm{h}}$ \\
$n$-octano & & & & $0.516^{\mathrm{g}}$ \\
$n$-nonano & & & & $0.594^{\mathrm{g}}$ \\
$n$-decano & & $0.9523^{\mathrm{c}}$ & & $0.642^{\mathrm{g}}$ \\
$n$-undecano & & & & $0.695^{\mathrm{g}}$ \\
$n$-dodecano & & $0.9956^{\mathrm{c}}$ & & $0.714^{\mathrm{g}}$ \\
$n$-tetradecano & & $1.0408^{\mathrm{c}}$ & & $0.741^{\mathrm{g}}$ \\
tolueno & $-0.161^{\mathrm{b}}$ & $-0.121^{\mathrm{d}}$ & $-0.286^{\mathrm{d}}$ & & \\
benceno & $-0.070^{\mathrm{b}}$ & $-0.123^{\mathrm{e}}$ & & & \\
\hline
\end{tabular}

Los volúmenes de exceso en las mezclas con $n$-alcanos son positivos, como se aprecia en la Tabla 5-12, ya que predomina la contribución procedente de la rotura de las interacciones dipolo-dipolo en el proceso de mezcla sobre los efectos estructurales y de volumen libre.

Respecto a las mezclas con un hidrocarburo aromático, se produce una gran disminución en $V_{\mathrm{m}}^{\mathrm{E}}$, al igual que en $H_{\mathrm{m}}^{\mathrm{E}}$. De hecho, los valores de $V_{\mathrm{m}}^{\mathrm{E}}$ son negativos, lo que fortalece la hipótesis de la formación de complejos por transferencia de carga o de interacciones dipolo-dipolo inducido entre las moléculas distintas. Además, los efectos estructurales también pueden contribuir a los valores negativos de $V_{\mathrm{m}}^{\mathrm{E}}$.

Los datos de las Tablas 5.11 y 5.12 muestran que para un alcano dado, tanto $H_{\mathrm{m}}^{\mathrm{E}}$ como $V_{\mathrm{m}}^{\mathrm{E}}$ decrecen con la longitud de la cadena de la cetona. Con toda probabilidad, esto se debe al debilitamiento de las interacciones dipolo-dipolo al aumentar la longitud de la cadena de la cetona, pues el apantallamiento estérico del grupo 
carbonilo disminuye la polaridad de la cetona (Tabla 5-1). La disminución que se observa en $H_{\mathrm{m}}^{\mathrm{E}}$ cuando se pasa de la forma asimétrica a la simétrica en cetonas isómeras, se debe a efectos estructurales.

Para una cetona dada, tanto $H_{\mathrm{m}}^{\mathrm{E}}$ como $V_{\mathrm{m}}^{\mathrm{E}}$ crecen con la longitud de la cadena del n-alcano, debido a la facilidad de los alcanos más largos de romper las interacciones cetona-cetona.

Los valores de $C_{P, \mathrm{~m}}^{\mathrm{E}}$ para este tipo de mezclas son moderados o bajos [39]. Por ejemplo, $2.9 \mathrm{~J} \cdot \mathrm{mol}^{-1} \cdot \mathrm{K}^{-1}$ (2-propanona $+n$-heptano), $3.7 \mathrm{~J} \cdot \mathrm{mol}^{-1} \cdot \mathrm{K}^{-1}$ (2-propanona $+n$-hexadecano), $1.8 \mathrm{~J} \cdot \mathrm{mol}^{-1} \cdot \mathrm{K}^{-1}$ (2-butanona $+n$-heptano). Estos valores son típicos de disoluciones caracterizadas por interacciones dipolares. Una importante característica de estos sistemas es la forma de $C_{P, \mathrm{~m}}^{\mathrm{E}}=C_{P, \mathrm{~m}}^{\mathrm{E}}(x)$, que puede tener forma de $\mathrm{S}$ (2-butanona $+n$ heptano [108]) o de W (2-propanona ó 2-butanona + n-dodecano [109]). El último caso se ha interpretado en términos de la distribución no al azar de las moléculas del sistema (véase el Capítulo 6 y las citas bibliográficas allí indicadas), que es más importante en sistemas cercanos a un UCST. Esto hace que $C_{P, \mathrm{~m}}^{\mathrm{E}}$ dependa fuertemente de la temperatura y de la longitud del alcano.

\subsection{MEZCLAS AMINA + CETONA}

Estudiadas las propiedades de los sistemas amina $+n$-alcano y cetona $+n$-alcano, el estudio de las mezclas del tipo amina + cetona resulta más sencillo.

Como se ha visto en secciones anteriores, en estado puro las aminas primarias y secundarias forman complejos supramoleculares mediante puentes de hidrógeno del tipo $\mathrm{N}-\mathrm{H} \cdots \mathrm{H}$. Además, tanto en las aminas como en las cetonas en estado puro hay presentes fuertes interacciones tipo dipolo-dipolo. Al producirse la mezcla, las fuertes interacciones intermoleculares entre los grupos funcionales amino y carbonilo hacen que exista una gran contribución negativa a la entalpía y volumen de exceso, en algunos casos superior a la contribución positiva debida a la ruptura de las interacciones entre moléculas iguales. Asimismo, la polaridad del grupo carbonilo influye considerablemente en la magnitud de las funciones de exceso cuando las cetonas se mezclan con aminas (moléculas también polares) respecto a los valores encontrados cuando se mezclan cetonas ó aminas con alcanos. En la sección 5.4 se ha visto que cuando una cetona se mezcla con un alcano, predomina la contribución positiva a la entalpía y al volumen de exceso debido a la ruptura de las interacciones dipolo-dipolo en el proceso de mezcla. Las interacciones dipolares son precisamente la causa de que en la mezcla piridina + 2-propanona, aun siendo la piridina una amina terciaria, no presentando puentes de hidrógeno, el volumen de exceso sea negativo, aunque inferior en valor absoluto al observado en las mezclas con anilina y $\mathrm{N}$ metilanilina. Este valor se debe a que la contribución negativa debida a la formación de interacciones dipolo-dipolo entre moléculas distintas es mayor que la contribución positiva debida a la ruptura de interacciones entre moléculas iguales.

En las Tablas $5-13$ y 5-14 se muestran los valores de $H_{\mathrm{m}}^{\mathrm{E}}, U_{\mathrm{m} . V}^{\mathrm{E}}$ y $V_{\mathrm{m}}^{\mathrm{E}}$ de mezclas amina aromática + cetona, amina lineal secundaria + cetona y tri- $n$-alquilamina + cetona. 
Tabla 5-13: $H_{\mathrm{m}}^{\mathrm{E}}\left(x_{1}=0.5\right)$ y $U_{\mathrm{m}, V}^{\mathrm{E}}\left(x_{1}=0.5\right)$ para sistemas amina (1) + cetona (2) a $298.15 \mathrm{~K} .{ }^{\mathrm{a}}[121]$

\begin{tabular}{ccccccccc}
\hline & \multicolumn{2}{c}{ 2-propanona } & \multicolumn{2}{c}{ 2-butanona } & \multicolumn{2}{c}{ 2-pentanona } & \multicolumn{2}{c}{ 2-heptanona } \\
\hline & $H_{\mathrm{m}}^{\mathrm{E}}$ & $U_{\mathrm{m}, V}^{\mathrm{E}}$ & $H_{\mathrm{m}}^{\mathrm{E}}$ & $U_{\mathrm{m}, V}^{\mathrm{E}}$ & $H_{\mathrm{m}}^{\mathrm{E}}$ & $U_{\mathrm{m}, V}^{\mathrm{E}}$ & $H_{\mathrm{m}}^{\mathrm{E}}$ & $U_{\mathrm{m}, V}^{\mathrm{E}}$ \\
\hline anilina & -1237 & -693 & -1164 & -618 & -1029 & -599 & -692 & -344 \\
$N$-metilanilina & -668 & -362 & -812 & -546 & -835 & -564 & -741 & -545 \\
di- $n$-propilamina & 648 & 572 & 398 & 353 & 281 & & 161 & 188 \\
di- $n$-butilamina & 933 & 805 & 620 & 536 & 454 & & 277 & 276 \\
$N, N, N$-trietilamina & 1063 & 954 & 746 & 705 & 747 & 706 & 414 & 478 \\
$N, N, N$-dimetiletilamina & $690^{\mathrm{a}}$ & & & & & & & \\
$N, N, N$-dietilmetilamina & $930^{\mathrm{a}}$ & & & & & & & \\
\hline
\end{tabular}

Tabla 5-14: $V_{\mathrm{m}}^{\mathrm{E}}\left(x_{1}=0.5\right)$ para sistemas amina $(1)+$ cetona $(2)$ a $298.15 \mathrm{~K}$.

\begin{tabular}{ccccc}
\hline & 2-propanona & 2-butanona & 2-pentanona & 2-heptanona \\
\hline anilina & -1.1828 & -1.2463 & -1.0960 & -0.8607 \\
$N$-metilanilina & -0.7269 & -0.6692 & -0.6869 & -0.5091 \\
piridina & -0.2508 & -0.2581 & -0.2319 & -0.0738 \\
di- $n$-propilamina & 0.2429 & 0.1439 & & -0.0887 \\
di- $n$-butilamina & 0.4175 & 0.2644 & & 0.0096 \\
$N, N, N$-trietilamina & 0.4056 & 0.1661 & 0.1674 & -0.2103 \\
\hline
\end{tabular}

\subsubsection{Amina aromática + cetona}

En las mezclas con amina aromática, los valores negativos de $H_{\mathrm{m}}^{\mathrm{E}}, V_{\mathrm{m}}^{\mathrm{E}}, \kappa_{S}^{\mathrm{E}}{ }^{*} \mathrm{y}$ $\left(\partial V_{\mathrm{m}}^{\mathrm{E}} / \partial T\right)_{P}^{\dagger}$ y positivos de $u^{\mathrm{E}} \ddagger$ implican la existencia de fuertes interacciones entre moléculas diferentes. Ello está de acuerdo con los altos valores de $\left|\Delta H_{1-2}\right|$ de estas mezclas (Tabla 5-2). Por ejemplo, la energía de interacción anilina-2-propanona $\left|\Delta H_{1-2}\right|=28.68 \mathrm{~kJ} \cdot \mathrm{mol}^{-1}$ es incluso mayor que la energía de interacción alcohol-alcohol $\left|\Delta H_{1-2}\right|=23.2 \mathrm{~kJ} \cdot \mathrm{mol}^{-1}$.

$H_{\mathrm{m}}^{\mathrm{E}}, \kappa_{S}^{\mathrm{E}}$, y $V_{\mathrm{m}}^{\mathrm{E}}$ disminuyen en la secuencia anilina $<$ (amina primaria) $<N$-metilanilina (amina secundaria) < piridina (amina terciaria), mientras que $u_{S}^{\mathrm{E}}$ lo hace justo al contrario. Esto significa que las interacciones entre las moléculas diferentes son mayores en el caso de la anilina, debido a su mayor capacidad para formar enlaces de hidrógeno con el átomo de oxígeno de la cetona por ser una amina primaria, como así lo justifican los valores de $\Delta H_{1-2}$. Por ejemplo, en $\mathrm{kJ} \cdot \mathrm{mol}^{-1}$, se tiene para $\left|\Delta H_{1-2}\right|$ lo

${ }^{*}$ Valores muy negativos de $\kappa_{S}^{\mathrm{E}}$ indican que la mezcla es menos compresible que la correspondiente mezcla ideal.

$\dagger\left(\partial V_{\mathrm{m}}^{\mathrm{E}} / \partial T\right)_{P}<0$ significa que las interacciones entre moléculas distintas son mayores a mayor temperatura, es decir, que los dos componentes se mezclan mejor a temperaturas superiores. Esto puede interpretarse en términos de la disminución del volumen molar de la formación de complejos, que sobrecompensa la disminución de la formación de complejos y se ha encontrado en mezclas amina + triclorometano [1 10], [1 1111$]$.

* Si $V_{\mathrm{m}}^{\mathrm{E}}<0$, las moléculas están más juntas y por ello el sonido se propaga mejor por el medio, es decir, la velocidad del sonido es mayor $\left(u^{\mathrm{E}}>0\right)$ 
siguiente: $($ anilina +2 -propanona $)=28.68>(N$-metilanilina +2 -propanona $)=21.69$; (anilina +2 -butanona $)=27.99>(N$-metilanilina +2 -butanona $)=20.60$; etc. (véase la Tabla 5-2). Los valores más negativos de $H_{\mathrm{m}}^{\mathrm{E}}$ para la anilina pueden atribuirse, además, a otros dos efectos adicionales: i) un menor número de interacciones aminacetona es formado en los casos de la $N$-metilanilina y la piridina debido a que en estas moléculas el grupo amino está más apantallado estéricamente; ii) una mayor contribución positiva a $H_{\mathrm{m}}^{\mathrm{E}}$ en las mezclas con $N$-metilanilina debida a la ruptura de las interacciones cetona-cetona es consecuencia de la mayor superficie alifática de esta amina, lo que hace más probable la ruptura de dichas interacciones.

En las mezclas con 2-propanona o 2-butanona, el mínimo de las curvas $H_{\mathrm{m}}^{\mathrm{E}}, V_{\mathrm{m}}^{\mathrm{E}}$ y $\kappa_{S}^{\mathrm{E}}$ está desplazado hacia composiciones mayores en la cetona (el componente más pequeño), lo que es propio de sistemas en los que los efectos de volumen libre y estructurales, debidos a grandes diferencias de tamaño de las moléculas, son importantes. En las mezclas con 2-pentanona ó 2-heptanona, las curvas son más simétricas, lo que sugiere que dichos efectos son menores.

Por otra parte, las diferencias entre $H_{\mathrm{m}}^{\mathrm{E}}$ y $U_{\mathrm{m} . V}^{\mathrm{E}}$ (entre $35 \%$ y $45 \%$ ) remarcan la importancia de la contribución de la ecuación de estado a $H_{\mathrm{m}}^{\mathrm{E}}$, relacionada con los grandes y negativos valores de $V_{\mathrm{m}}^{\mathrm{E}}$ para estos sistemas, es decir, con los efectos estructurales.

Respecto a la variación con la longitud de la cetona, en el caso de las mezclas con anilina se encuentra que $H_{\mathrm{m}}^{\mathrm{E}}, U_{\mathrm{m} . V}^{\mathrm{E}}, V_{\mathrm{m}}^{\mathrm{E}}$ y $\kappa_{S}^{\mathrm{E}}$ cambian aproximadamente como sigue: 2 -propanona $<2$-butanona $<2$-pentanona $<2$-heptanona, mientras que $u^{\mathrm{E}}$ lo hace en la secuencia inversa (véanse las llustraciones 5-1 y 5-2). Todo ello es consistente con el hecho de que las interacciones amina-cetona son mayores cuanto más corta es la cetona, lo que es consecuencia de que en cetonas de cadena larga el grupo carbonilo se halla apantallado. El mismo comportamiento se observa en las mezclas con piridina para las propiedades determinadas $\left(V_{\mathrm{m}}^{\mathrm{E}}, \kappa_{S}^{\mathrm{E}}\right.$ y $\left.u^{\mathrm{E}}\right)$. Por el contrario, en los sistemas con $N$-metilanilina, el comportamiento es similar para todas las funciones de exceso excepto para $H_{\mathrm{m}}^{\mathrm{E}}$ y $U_{\mathrm{m} . V}^{\mathrm{E}}$. Estas funciones de exceso varían como sigue: 2-propanona > 2-heptanona $>2$-butanona $>2$-pentanona. $H_{\mathrm{m}}^{\mathrm{E}}$ y $U_{\mathrm{m} . V}^{\mathrm{E}}$ de las mezclas alcanonitrilo + 2-alcanona varían de la forma análoga. Esto puede ser debido a la ruptura de las interacciones 2-heptanona-2-heptanona favorecida por la existencia de la superficie alifática en la $N$-metilanilina. Puede verse (Ilustración 5-1, Tabla 5-13) que a partir de la 2-butanona, $U_{\mathrm{m} . V}^{\mathrm{E}}$ es prácticamente constante para las mezclas con $N$-metilanilina, lo que significa que las diferentes contribuciones a dicha magnitud se compensan.

El parámetro de interacción molecular, $\chi=\left(u / u^{\text {id }}\right)^{2}-1$ se puede utilizar para estimar la no idealidad del sistema [112]-[115], de manera que fuertes desviaciones respecto la idealidad están caracterizados por valores distintos de cero. Así mismo, la presión interna también permite hacer un estudio de las interacciones entre diferentes moléculas [1 16], [1 17], como se ha discutido al comienzo de este Capítulo. En la Tabla 5-15 se muestran los valores calculados de las presiones internas y los parámetros de interacción molecular en las mezclas amina + cetona de interés en este trabajo. En ella 


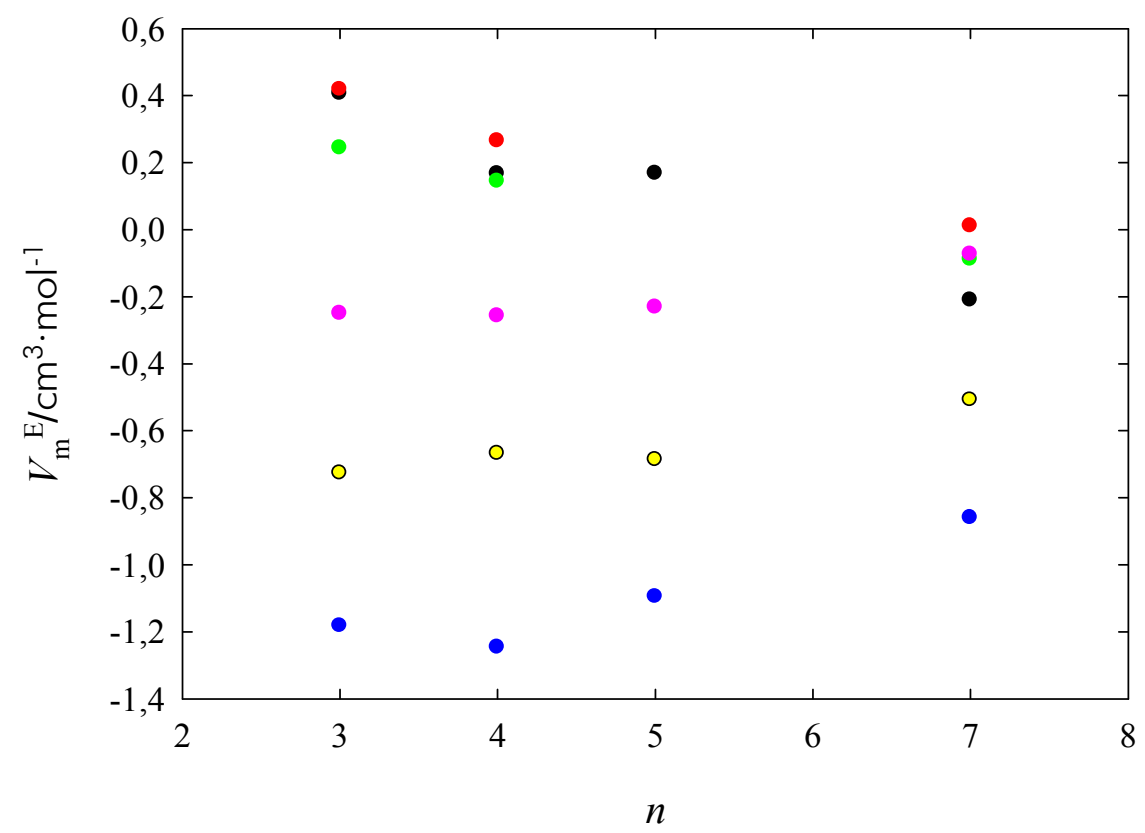

Ilustración 5-1: $V_{\mathrm{m}}^{\mathrm{E}}\left(x_{1}=0.5\right)$ a $T=298.15 \mathrm{~K}$ para sistemas amina (1)+cetona (2) en función de la longitud de la cetona, $n$ : $\bullet$ anilina, o N-metilanilina, $\bullet$ piridina, $\bullet$ DPA, $\bullet$ DBA, $\bullet$ TEA

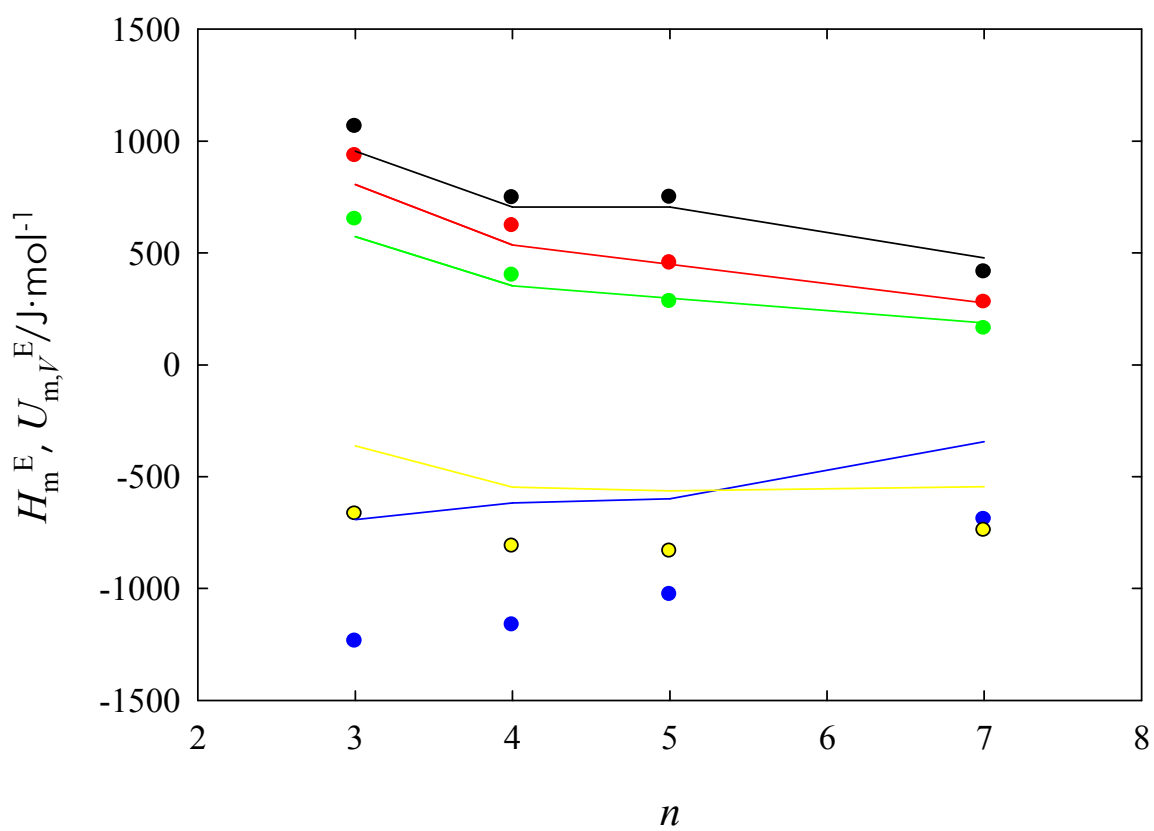

Ilustración 5-2: $H_{\mathrm{m}}^{\mathrm{E}}\left(x_{1}=0.5\right)$ y $U_{\mathrm{m}, V}^{\mathrm{E}}\left(x_{1}=0.5\right)$ a $T=298.15 \mathrm{~K}$ para mezclas amina (1)+cetona (2) en función de la longitud de la cetona, $n\left(\right.$ círculos, $H_{\mathrm{m}}^{\mathrm{E}} ;$ líneas, $\left.U_{\mathrm{m}, V}^{\mathrm{E}}\right): \bullet$ anilina, o $\mathrm{N}$-metilanilina, $\bullet \mathrm{DPA}, \bullet$ $\mathrm{DBA}, \bullet \mathrm{TEA}$ 
puede verse que para una cetona dada, la variación tanto de $P_{\text {int }}$ como de $\chi$ es como sigue: anilina $>N$-metilanilina $>$ piridina, lo que vuelve a incidir en el hecho, discutido previamente, de que en las disoluciones con anilina las interacciones entre moléculas diferentes son más fuertes. En dicha Tabla también se observa que para una amina aromática dada, tanto $P_{\text {int }}$ como $\chi$ cambian con la longitud de la cetona como sigue: 2-propanona $>2$-butanona $>2$-pentanona $>2$-heptanona, lo que está de acuerdo con las conclusiones anteriormente expuestas, según las cuales las interacciones entre moléculas diferentes son más importantes cuanto menor es el tamaño de la cetona.

Como se ve en la Tabla 5-15, ambas magnitudes son mayores en las mezclas con aminas aromáticas que en mezclas con aminas lineales, lo que es debido a que en las primeras las interacciones amina-cetona son más fuertes.

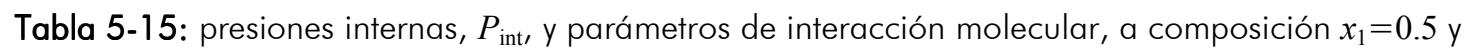
298.15 K para mezclas amina (1)+cetona (2)

\begin{tabular}{ccc}
\hline Sistema & $P_{\text {int }} / \mathrm{MPa}$ & $\chi$ \\
\hline anilina +2 -propanona & 459.1 & 0.257 \\
anilina +2 -butanona & 439.9 & 0.198 \\
anilina+2-pentanona & 393.8 & 0.165 \\
anilina+2-heptanona & 403.7 & 0.106 \\
$N$-metilanilina+2-propanona & 417.7 & 0.164 \\
$N$-metilanilina+2-butanona & 407.2 & 0.119 \\
$N$-metilanilina+2-pentanona & 394.8 & 0.108 \\
$N$-metilanilina+2-heptanona & 384.1 & 0.068 \\
piridina+2-propanona & 392.6 & 0.094 \\
piridina+2-butanona & 384.4 & 0.070 \\
piridina+2-pentanona & 375.3 & 0.061 \\
piridina+2-heptanona & 366.1 & 0.031 \\
di- $n$-propilamina+2-propanona & 309.7 & -0.019 \\
di- $n$-propilamina+2-butanona & 314.4 & -0.008 \\
di- $n$-propilamina +2 -heptanona & 314.9 & 0.009 \\
di- $n$-butilamina +2 -propanona & 307.1 & -0.023 \\
di- $n$-butilamina +2 -butanona & 312.8 & -0.014 \\
di- $n$-butilamina +2 -heptanona & 292.9 & -0.0009 \\
$N, N, N$-trietilamina +2 -propanona & 285.6 & -0.023 \\
$N, N, N$-trietilamina+2-butanona & 290.3 & -0.001 \\
$N, N, N$-trietilamina+2-heptanona & 297.3 & 0.029 \\
\hline
\end{tabular}

\subsubsection{Amina no aromática + cetona}

En mezclas con aminas no aromáticas (DPA, DBA y TEA), $H_{\mathrm{m}}^{\mathrm{E}}>0$, lo que quiere decir que la contribución positiva debida a la ruptura de interacciones entre moléculas iguales es más importante que la debida a la creación de interacciones entre moléculas diferentes. Así lo demuestran los valores de $\Delta H_{1-2}$ de la Tabla 5-2. La gran diferencia observada en $\Delta H_{1-2}$ para las mezclas con aminas aromáticas y aminas lineales secundarias revela la importancia de los efectos aromáticos sobre las propiedades termodinámicas y que las interacciones amina-cetona son mucho mayores en las disoluciones con aminas aromáticas.

Excepto en los sistemas DPA ó TEA + 2-heptanona, los valores positivos de $V_{\mathrm{m}}^{\mathrm{E}}, \kappa_{S}^{\mathrm{E}}$ y 
$\left(\partial V_{\mathrm{m}}^{\mathrm{E}} / \partial T\right)_{P}$ para las mezclas DPA ó DBA + cetona también sustentan el que las interacciones entre moléculas iguales sean más importantes que entre moléculas distintas y que los efectos estructurales. Nótese que la disminución en el volumen de exceso en las mezclas con 2-propanona cuando se sustituye el heptano por la DPA, dos disolventes de tamaño similar, confirma la existencia de interacciones amina-cetona antes citada.

En el caso de las mezclas DPA ó TEA + 2-heptanona, $V_{\mathrm{m}}^{\mathrm{E}},\left(\partial V_{\mathrm{m}}^{\mathrm{E}} / \partial T\right)_{P}$ y $\kappa_{S}^{\mathrm{E}}<0$ lo que significa que en estas mezclas las contribuciones negativas debidas a los efectos estructurales y a las interacciones entre moléculas diferentes son mayores que la contribución positiva debida a la ruptura de interacciones entre moléculas similares. En la mezcla DBA + 2-heptanona, las diferentes contribuciones están compensadas de modo que $V_{\mathrm{m}}^{\mathrm{E}}$ es muy pequeño.

Para una cetona dada, $H_{\mathrm{m}}^{\mathrm{E}}$ varía de la forma siguiente: anilina $<N$-metilanilina $<\mathrm{DPA}$ $<$ DBA $<$ TEA. Esta variación puede atribuirse a: i) las interacciones cetona-amina son más importantes en el orden: anilina $>N$-metilanilina $>$ DPA $>$ DBA, es decir, $\left|\Delta H_{1-2}\right|$ aumenta del mismo modo (ver Tabla 5-2); ii) el número de interacciones entre moléculas diferentes es mayor cuando hay involucradas moléculas aromáticas; iii) el grupo amino está más apantallado estéricamente en la DBA que en la DPA. Por tanto, un menor número de interacciones amina-cetona se crea durante el proceso de mezclado con DBA. El mismo efecto (contribución positiva) es originado por el mayor número de interacciones cetona-cetona que la DBA es capaz de destruir con respecto a la DPA, como consecuencia de su mayor superficie alifática.

Excluyendo las mezclas con TEA, la variación de $V_{\mathrm{m}}^{\mathrm{E}}$ es similar a la encontrada para $H_{\mathrm{m}}^{\mathrm{E}}$ : anilina $<N$-metilanilina $<\mathrm{DPA}<\mathrm{DBA}$, por lo que la explicación de esta tendencia para $V_{\mathrm{m}}^{\mathrm{E}}$ es la misma que para $H_{\mathrm{m}}^{\mathrm{E}}$. Sin embargo, los sistemas con TEA deben tratarse a parte. En las mezclas TEA + 2-propanona ó 2-butanona, $V_{\mathrm{m}}^{\mathrm{E}}$ es mayor que en las mezclas con DPA, lo que puede explicarse como sigue: en las aminas terciarias el grupo amino está más apantallado que en las secundarias, por lo que las contribuciones negativas debidas a la creación de interacciones entre moléculas distintas y positivas debidas a la destrucción de interacciones amina-amina son mayores en las mezclas con DPA. Sin embargo, el valor mayor de $V_{\mathrm{m}}^{\mathrm{E}}$ en las disoluciones con TEA indica que ambas contribuciones están más compensados en las mezclas con DPA. Por otra parte, en la mezcla TEA + 2-heptanona, $V_{\mathrm{m}}^{\mathrm{E}}$ es menor que en las mezclas con DPA y DBA, lo que es consecuencia de la aparición de efectos estructurales importantes.

En los sistemas con 2-pentanona ó 2-heptanona, el extremo de las curvas $H_{\mathrm{m}}^{\mathrm{E}}, V_{\mathrm{m}}^{\mathrm{E}}$ y $\kappa_{S}^{\mathrm{E}}$ está desplazado hacia composiciones mayores en la amina, el componente más pequeño, lo que es propio de sistemas en los que los efectos de volumen libre, relativos a las grandes diferencias de tamaño de las moléculas, son importantes. En las mezclas con 2-propanona ó 2-butanona, las curvas son más simétricas, lo que quiere decir que los efectos de volumen libre son menores. 
Por otra parte, las diferencias entre $H_{\mathrm{m}}^{\mathrm{E}}$ y $U_{\mathrm{m} . V}^{\mathrm{E}}$ son del $15 \%$ o menores, mucho más pequeñas que en las disoluciones con aminas aromáticas. Los valores de la Tabla 5-13 permiten dilucidar que la contribución a $H_{\mathrm{m}}^{\mathrm{E}}$ de la ecuación de estado no es tan importante.

Respecto a la variación con la longitud de la cetona, $H_{\mathrm{m}}^{\mathrm{E}}, U_{\mathrm{m} . V}^{\mathrm{E}}, V_{\mathrm{m}}^{\mathrm{E}}$ y $\kappa_{S}^{\mathrm{E}}$ varían aproximadamente como sigue: 2-propanona $>2$-butanona $>2$-pentanona $>$ 2-heptanona, mientras que $u^{\mathrm{E}}$ lo hace en sentido inverso (véanse las llustraciones 5-1 y 5-2). Este comportamiento es similar al encontrado en mezclas con $n$-heptano. Por el contrario, $\Delta H_{1-2}$ (amina-cetona) es aproximadamente constante en las series homólogas consideradas. Este comportamiento se puede explicar en base a los siguientes puntos clave: i) en las cetonas de cadena larga, en las que el grupo carbonilo está más apantallado estéricamente, la formación de interacciones entre moléculas diferentes es más difícil y da lugar a menores contribuciones negativas; ii) el mayor apantallamiento del grupo carbonilo en las cetonas más largas también es el responsable de que la contribución positiva debida a las interacciones cetona-cetona sea menor que en las cetonas de cadena corta (lo que concuerda con el comportamiento de las mezclas cetona $+n$-heptano). Sin embargo, esta contribución positiva ii) es mayor que la negativa i); iii) los efectos estructurales y de volumen libre son importantes, sobre todo en las mezclas con 2-heptanona en las que los valores de $V_{\mathrm{m}}^{\mathrm{E}}$ son negativos o las curvas son muy asimétricas.

En la Tabla 5-15 se muestran los valores de las presiones internas y los parámetros de interacción molecular de las mezclas amina + cetona. Puede comprobarse que para una cetona dada (excepto la 2 -heptanona), la variación tanto de $P_{\text {int }}$ como de $\chi$ es como sigue: DPA > DBA > TEA, lo que es consistente con el hecho de que la contribución negativa debida a la formación de interacciones entre moléculas diferentes sea mayor que la contribución positiva debida a la ruptura de interacciones entre moléculas iguales en ese orden.

En mezclas con 2-heptanona, la variación es: $P_{\text {int }}(\mathrm{DPA})>P_{\text {int }}($ TEA $)>P_{\text {int }}(\mathrm{DBA}) ; \chi(\mathrm{TEA})$ $>\chi(\mathrm{DPA})>\chi(\mathrm{DBA})$, mientras que el volumen de exceso cambia siguiendo el orden siguiente: $V_{\mathrm{m}}^{\mathrm{E}}(\mathrm{TEA})<V_{\mathrm{m}}^{\mathrm{E}}(\mathrm{DPA})<0<V_{\mathrm{m}}^{\mathrm{E}}(\mathrm{DBA})$.

En la Tabla 5-15 también se observa que para una amina lineal dada, tanto $P_{\text {int }}$ como $\chi$ cambian con la longitud de la cetona de manera inversa al caso de las mezclas con aminas aromáticas: 2-propanona $<2$-butanona $<2$-pentanona $<2$-heptanona, excepto en las mezclas DBA + 2-heptanona. Ello implica la existencia de efectos estructurales y está de acuerdo con las interpretaciones anteriores.

El mayor valor tanto de $P_{\text {int }}$ como de $\chi$ en las mezclas con aminas aromáticas respecto de las mezclas con aminas lineales confirma nuevamente que en las primeras las interacciones amina-cetona son más fuertes.

En la Tabla 5-16 se encuentran los valores calculados de $P_{\text {int }}^{\mathrm{E}}$ para los sistemas DPA, DBA y TEA + cetona. Puede comprobarse que los valores de $P_{\text {int }}^{\mathrm{E}}$ son negativos 0 débilmente positivos, lo que vuelve a resaltar el hecho de que en estas mezclas las interacciones entre moléculas iguales son importantes. Además, se observa que para una amina dada $P_{\text {int }}^{\mathrm{E}}$ aumenta con la longitud de la cetona, de modo que dichas 
interacciones son menos importantes cuanto más larga es la cetona. Es llamativo el alto valor de $P_{\text {int }}^{\mathrm{E}}$ para la mezcla TEA +2 -heptanona, que revela una vez más que los efectos estructurales en esta disolución son importantes.

Tabla 5-16: $P_{\text {int }}^{\mathrm{E}}$ a composición equimolar y $298.15 \mathrm{~K}$ para algunos sistemas amina (1) + cetona (2)

\begin{tabular}{cc}
\hline Sistema & $P_{\text {int }}^{\mathrm{E}} / \mathrm{MPa}$ \\
\hline di- $n$-propilamina +2 -propanona & -3.22 \\
di- $n$-propilamina +2 -butanona & -1.50 \\
di- $n$-propilamina +2 -heptanona & 0.85 \\
di- $n$-butilamina +2 -propanona & -4.46 \\
di- $n$-butilamina +2 -butanona & -1.59 \\
di- $n$-butilamina +2 -heptanona & 0.59 \\
$N, N, N$-trietilamina +2 -propanona & -3.80 \\
$N, N, N$-trietilamina +2 -butanona & 0.90 \\
$N, N, N$-trietilamina +2 -heptanona & 7.22 \\
\hline
\end{tabular}

\subsubsection{Tri- $n$-alquilamina + cetona}

Aunque en la sección anterior se ha tratado de comparar los resultados obtenidos para las disoluciones con TEA y las aminas secundarias, un estudio más sistemático de las mezclas TEA + cetona exige la comparación de éstas con otras en las que se encuentren diferentes aminas terciarias. Por esa razón, se han escogido de la bibliografía algunos datos relevantes para algunas mezclas $N, N$-dimetiletilamina ó $N, N$ dietilmetilamina + cetona (véanse las Tablas 5-1,5-2 y 5-13). En todas ellas, $H_{\mathrm{m}}^{\mathrm{E}}>0$, disminuyendo con el incremento de la longitud de la cetona, mientras que los correspondientes valores de $\Delta H_{1-2}$ son prácticamente independientes de dicha longitud. Por lo tanto, $H_{\mathrm{m}}^{\mathrm{E}}$ decrece como consecuencia de que la contribución positiva debida a las interacciones cetona-cetona disminuye también cuando la longitud de la cetona se hace mayor, debido a que el grupo carbonilo se encuentra más estéricamente qpantallado.

Por otra parte, $\left|\Delta H_{1-2}\right|$ es mayor en los sistemas $N, N$-dimetiletilamina ó $N, N$ dietilmetilamina +2 -propanona que en la mezcla con TEA, lo que significa que la intensidad de las interacciones N-CO depende de la longitud de las cadenas alquílicas unidas al grupo amino, más estéricamente apantallado en la TEA que en la $N, N$ dimetiletilamina y en la $N, N$-dietilmetilamina. Tanto los valores de $\mathrm{D} H_{\mathrm{v}}$ como los de $H_{\mathrm{m}}^{\mathrm{E}}$ para las mezclas con $n$-heptano son consistentes con este resultado: en $\mathrm{kJ} \cdot \mathrm{mol}^{-1}$, se tiene para $\mathrm{D} H_{\mathrm{v}}$ la secuencia siguiente: 3.3 (N,N-dimetiletilamina) $>1.5(N, N$ -

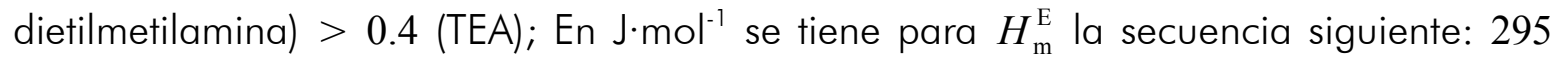
$(N, N$-dimetiletilamina $)>224(N, N$-dietilmetilamina $)>112$ (TEA).

Además, $\left|\Delta H_{1-2}\right|$ varía en el orden: anilina $>N$-metilanilina $>N, N$-dimetiletilamina $>$ $N, N$-dietilmetilamina $>$ TEA, lo que significa que las interacciones entre moléculas distintas son mayores cuando las aminas más polares entran en juego, particularmente las aminas aromáticas.

En mezclas con TEA, $H_{\mathrm{m}}^{\mathrm{E}}$ y $V_{\mathrm{m}}^{\mathrm{E}}$ varían según la secuencia 2-propanona $>2$-butanona $>$ 2 -heptanona. Ello significa, una vez más, que la contribución positiva debida a la ruptura 
de interacciones entre moléculas iguales es menor para las cetonas más largas.

Como ya se ha dicho en la sección anterior, el valor negativo de $V_{\mathrm{m}}^{\mathrm{E}}$ de la mezcla TEA +2 -heptanona es consecuencia de la importancia de los efectos estructurales en dicha mezcla. El sistema di-n-propilamina + 2-heptanona se comporta de manera similar, poseyendo también valores negativos de $V_{\mathrm{m}}^{\mathrm{E}}$. Sin embargo, tales valores son más negativos que para la disolución con TEA, lo que indica que los efectos estructurales son más importantes en las mezclas con TEA, probablemente debido a la forma globular de dicha amina, como se ha dicho.

Las diferencias del $15 \%$ entre $H_{\mathrm{m}}^{\mathrm{E}}$ y $U_{V}^{\mathrm{E}}$ en la mezcla TEA + 2-heptanona confirman la importancia de la contribución debida a la ecuación de estado.

\subsection{MEZCLAS CON AMINO-CETONAS}

La investigación de los datos termodinámicos de estas mezclas (véase la Tabla 5-17) permite establecer ciertas características: i) la inclusión de un grupo amino en la estructura de la cetona da lugar a interacciones más intensas entre las moléculas de la amino-cetona resultante. Así lo confirman los elevados valores de $\mathrm{D} H_{\mathrm{v}}$ de estas sustancias. Por ejemplo, $\mathrm{D} H_{\mathrm{v}}\left(1\right.$-dimetilamino-2-propanona) $=13.7 \mathrm{~kJ} \cdot \mathrm{mol}^{-1}$, $\mathrm{D} H_{\mathrm{v}}$ (4-metil-2-pentanona) $=10.7 \mathrm{~kJ} \cdot \mathrm{mol}^{-1}$; ii) $H_{\mathrm{m}}^{\mathrm{E}}$ de los sistemas amino-cetona + alcano disminuye cuando la separación entre los grupos amino y carbonilo aumenta (Tabla 5-17), lo que revela el debilitamiento de las interacciones dipolares entre las moléculas de la amino-cetona; iii) el aumento de la longitud de las cadenas alifáticas unidas al átomo de nitrógeno origina una disminución de $H_{\mathrm{m}}^{\mathrm{E}}$. Ello es consistente con el valor de $\mathrm{D} H_{\mathrm{v}}$ para el sistema 1-dietilamino-2-propanona $\left(8 \mathrm{~kJ} \cdot \mathrm{mol}^{-1}\right)$, valor mucho menor que el encontrado para la mezcla 1-dimetilamino-2-propanona $\left(13.7 \mathrm{~kJ} \cdot \mathrm{mol}^{-1}\right)$. Este comportamiento es similar al observado en las mezclas amina terciaria + 2-propanona. Por otra parte, $H_{\mathrm{m}}^{\mathrm{E}}$ de las mezclas con 1-dietilamino-2-propanona ó 5-dimetilamino-2-pentanona es similar, lo que significa que los efectos de proximidad en mezclas con la primera amino-cetona están compensadas por fuertes efectos estéricos relacionados con los grupos alquilo unidos al átomo de nitrógeno; iv) las amidas están caracterizadas por el grupo amida $\mathrm{N}-\mathrm{CO}$, es decir no existe separación entre los grupos $\mathrm{N}$ y $\mathrm{CO}$, lo que origina fuertes interacciones entre las moléculas de amida (mayores que entre las moléculas de la amino-cetona de tamaño similar) y desviaciones grandes y positivas respecto de la Ley de Lewis-Randall en las mezclas con alcanos. Este resultado está refrendado por los elevados valores de $\mathrm{DH}$ para $N, N$-dimetilacetamida) [118], por la existencia de lagunas de miscibilidad a temperaturas elevadas $(309.8 \mathrm{~K}$ para la mezcla $n$-heptano $+N$, $N$-dimetilacetamida [119]), y por valores altos y positivos de $H_{\mathrm{m}}^{\mathrm{E}}$ en las mezclas con alcanos $\left(1687 \mathrm{~J} \cdot \mathrm{mol}^{-1}\right.$ para la mezcla $N, N$ dimetilacetamida + ciclohexano [120]), mayores que en las mezclas de amino-cetonas con alcanos (ver la Tabla 5-17). 
Tabla 5-17: $H_{\mathrm{m}}^{\mathrm{E}}\left(x_{1}=0.5\right)$ para sistemas amino-cetona (1) + alcano (2) a $298.15 \mathrm{~K} .{ }^{a}[121]$

\begin{tabular}{cccc}
\hline & 1-dimetilamino-2-propanona & 5-dimetilamino-2-pentanona & 1-dietilamino-2-propanona \\
\hline$n$-hexano & $1066^{a}$ & $721^{a}$ & $752^{a}$ \\
$n$-heptano & $1159^{a}$ & $782^{a}$ & $829^{a}$ \\
$n$-dodecano & $1501^{a}$ & $1040^{a}$ & $1085^{a}$ \\
$n$-hexadecano & $1674^{a}$ & $1206^{a}$ & $1243^{a}$ \\
\hline
\end{tabular}

\subsection{MEZCLAS 1-PROPANOL + N,N,N-TRIETILAMINA + 2-BUTANONA}

Los sistemas binarios 1-propanol +2 -butanona y TEA +2 -butanona están descritos por valores positivos de $H_{\mathrm{m}}^{\mathrm{E}}\left(1251 \mathrm{~J} \cdot \mathrm{mol}^{-1}\right.$ [122] y $745 \mathrm{~J} \cdot \mathrm{mol}^{-1}$ respectivamente), mientras que el sistema 1-propanol + TEA por $H_{\mathrm{m}}^{\mathrm{E}}=-1425 \mathrm{~J} \cdot \mathrm{mol}^{-1}$ [123]. En la mezcla ternaria 1propanol (1) + TEA (2) + 2-butanona (3) se encuentran valores positivos y negativos de $H_{\mathrm{m}}^{\mathrm{E}}$, dependiendo del cociente $x_{2} / x_{3}$ (véase la Tabla 4-76 y las Ilustraciones 4-36, 4-38 y 5-3). Así, para $x_{2} / x_{3}=0.3264$ la contribución dominante a $H_{\mathrm{m}}^{\mathrm{E}}$ procede de la ruptura de las interacciones entre moléculas diferentes. Para este valor de $x_{2} / x_{3} H_{\mathrm{m}}^{\mathrm{E}}$ cambia débilmente con $x_{1}$ en un amplio rango de fracción molar, lo que implica una cierta compensación entre las diferentes contribuciones a $H_{\mathrm{m}}^{\mathrm{E}}$ en dicha región. En contraste, las interacciones entre moléculas diferentes son predominantes para los valores 1.0249 y 2.9529 del cociente $x_{2} / x_{3}$. Para tales cocientes, la misma dependencia con la concentración de $H_{\mathrm{m}}^{\mathrm{E}}$ se ha encontrado en diversos sistemas ternarios [124]-[126].

En la llustración 5-3 se comparan los resultados de $H_{\mathrm{m}}^{\mathrm{E}}$ para la mezcla ternaria y las correspondientes mezclas binarias. Se observa que al añadir 2-butanona a la mezcla 1propanol + TEA, los valores de $H_{\mathrm{m}}^{\mathrm{E}}$ aumentan. Esto se debe a: i) ruptura de las interacciones amina-amina por la cetona; ii) ruptura de las interacciones cetona-cetona.

Por otra parte, al añadir TEA a la mezcla 1-propanol + 2-butanona, ó 1-propanol a la mezcla TEA + 2-butanona, los valores de $H_{\mathrm{m}}^{\mathrm{E}}$ disminuyen. Esto puede explicarse en términos de las fuertes interacciones alcohol-amina que se forman en la mezcla, como muestra el elevado valor $\Delta H_{1-2}=-31.5 \mathrm{~kJ} \cdot \mathrm{mol}^{-1}$ para el sistema 1-propanol + TEA [60].

En la llustración 5-4 se compara $H_{\mathrm{m}}^{\mathrm{E}}$ de los sistemas ternarios 1-propanol (1) + TEA (2) +2 -butanona (3), 1-propanol (1) + TEA (2) $+n$-heptano (3) [123] y 1 -propanol (1) + benceno (2) + 2-butanona (3) [122] para valores similares de $x_{2} / x_{3}$. La mezcla 1-propanol (1) + TEA (2) + 2-butanona (3) tiene mayores valores de $H_{\mathrm{m}}^{\mathrm{E}}$ que la mezcla con $n$-heptano, lo que se puede explicar en términos similares a los anteriores: i) rupura de la asociación del alcohol por parte de la cetona; ii) ruptura de las interacciones cetona-cetona. Esto es consistente con los valores de $H_{\mathrm{m}}^{\mathrm{E}}$ de las mezclas binarias: 1251

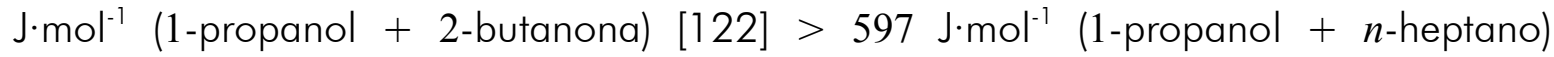
[127]. En contraste, nuestro sistema ternario tiene menores valores de $H_{\mathrm{m}}^{\mathrm{E}}$ que la mezcla con benceno. Esto se pueden explicar en términos similares a los anteriores: al añadir TEA a la mezcla 1-propanol + 2-butanona se forman fuertes interacciones alcohol-amina en la mezcla contribuyendo negativamente a $H_{\mathrm{m}}^{\mathrm{E}}$. 


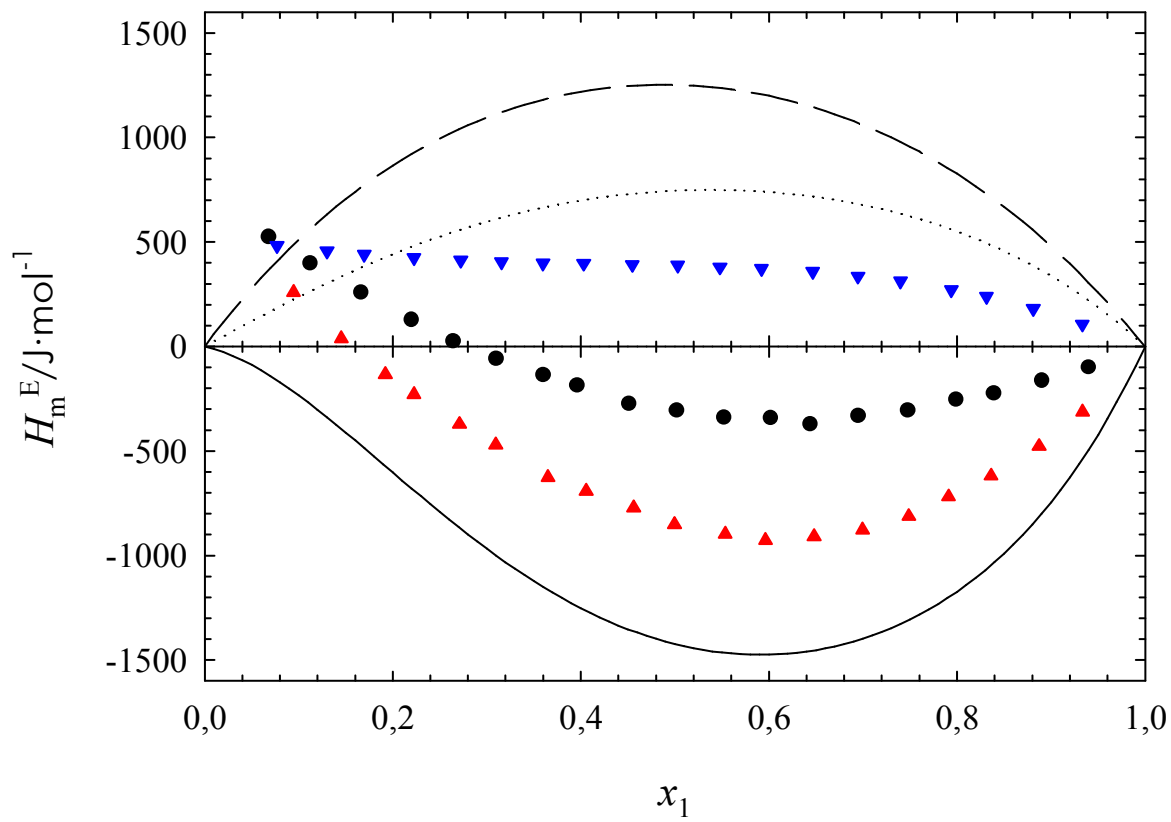

llustración 5-3: $H_{\mathrm{m}}^{\mathrm{E}}$ en función de la fracción molar a $298.15 \mathrm{~K}$ para el sistema ternario 1-propanol (1) + $N, N, N$-trietilamina (2) +2 -butanona (3) para los cocientes $\Delta x_{2} / x_{3}=2.9529 ; \bullet x_{2} / x_{3}=1.0249 ; \boldsymbol{v} x_{2} / x_{3}$ $=0.3264$ y para las mezclas binarias relacionadas: línea continua, 1 -propanol (1) $+N, N, N$-trietilamina (2); línea de puntos, $N, N, N$-trietilamina (1) +2 -butanona (2); línea discontinua, 1-propanol (1) +2 -butanona (2).

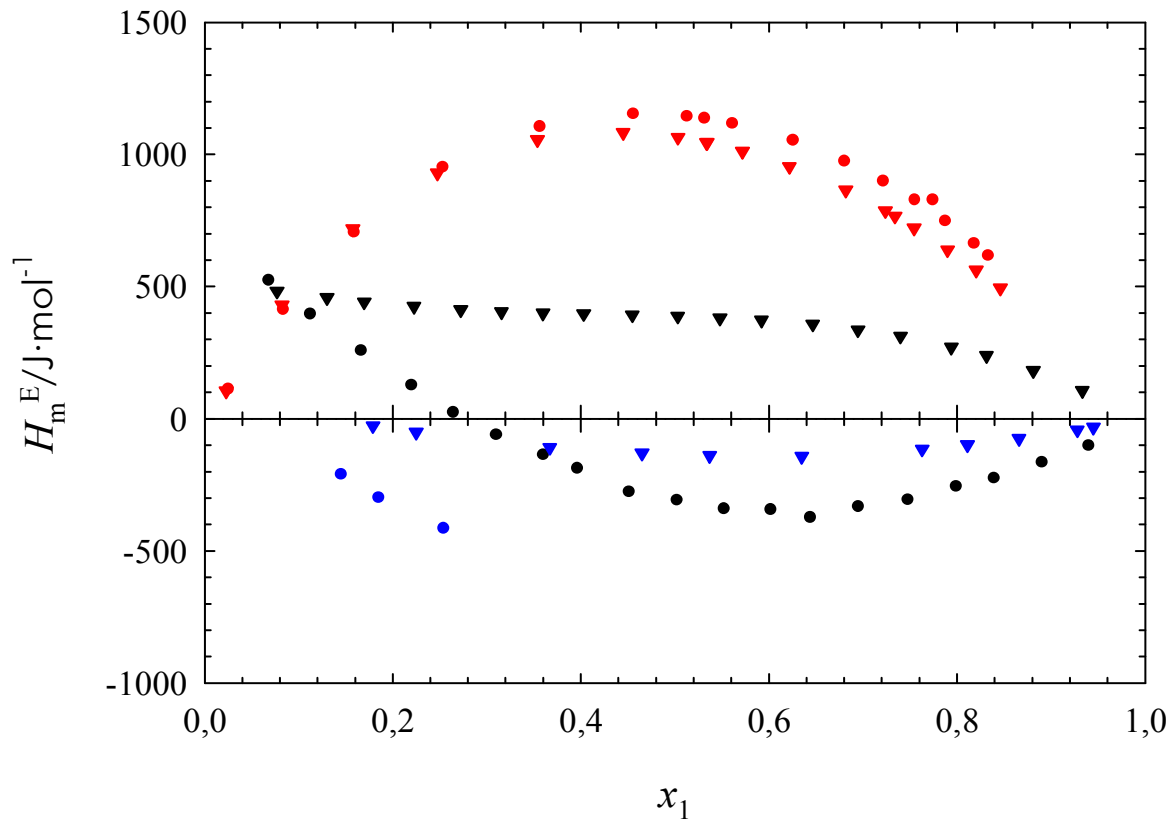

Ilustración 5-4: $H_{\mathrm{m}}^{\mathrm{E}}$ en función de la fracción molar a $298.15 \mathrm{~K}$ para los sistemas ternario 1-propanol (1) + $N, N, N$-trietilamina (2) + 2-butanona (3) $\left(\bullet x_{2} / x_{3}=1.0249 ; \mathbf{v} x_{2} / x_{3}=0.3264\right), 1$-propanol $(1)+N, N, N$ trietilamina (2) $+n$-heptano (3) $\left(\bullet x_{2} / x_{3}=0.986 ; \nabla x_{2} / x_{3}=0.303\right)$ y 1 -propanol $(1)+$ benceno 2$)+n$-heptano (3) $\left(\bullet x_{2} / x_{3}=1.00 ; \nabla x_{2} / x_{3}=0.333\right)$ 


\section{BIBLIOGRAFÍA}

[1]: Hildebrand, J.H.; J. Amer. Chem. Soc., 38 (8) (1916), 1452.

[2]: Hildebrand, J.H.; J. Amer. Chem. Soc., 43 (1921), 500.

[3]: Rowlinson, J.S., Swinton, F.L.; "Liquids and Liquids Mixtures", Butterworths Monographs in Chemistry (1982).

[4]: Wilhelm, E.; Laínez, A.; Grolier, J.P.E.; Fluid Phase Equilib., 49 (1989), 233.

[5]: Kehiaian, H.V.; Grolier, J.-P.E.; Benson, G.C.; J. Chem. Phys., 75 (1978), 1031.

[6]: González, J.A.; García, I.; Cobos, J.C.; Casanova, C., Ber. Bunsenges. Phys. Chem., 95 (1991), 1658;

[7]: González, J.A.; García, I.; Cobos, J.C.; Casanova, C.; Fluid Phase Equilib., 78 (1992), 61.

[8]: González, J.A.; García, I.; Cobos, J.C.; Fluid Phase Equilib., 123 (1996), 39.

[9]: Hofman, T.; Casanova, C.; Ber. Bunsenges. Phys. Chem., 100 (1996), 490.

[10]: González, J.A.; García, I.; Cobos, J.C.; Ber. Bunsenges. Phys. Chem., 101 (1997), 219.

[1 1]: González, J.A.; García, I.; Cobos, J.C.; Fluid Phase Equilib., 154 (1999), 11.

[12]: González, J.A.; García, I.; Cobos, J.C.; Phys. Chem. Chem. Phys., 1 (1999), 275.

[13]: González, J.A.; Carmona, F.J.; Riesco, N.; García de la Fuente, I.; Cobos, J.C.; Phys. Chem. Chem. Phys., 2 (2000), 2587.

[14]: González, J.A.; García de la Fuente, I.; Cobos, J.C.; Fluid Phase Equilib., 168, (2000), 31.

[15]: Hildebrand, J.H.; J. Amer. Chem. Soc., 41 (1919), 1067.

[16]: Bagley, E.B.; Nelson, T.P.; Barlow, J.W.; Chen, S-A.; I\&EC Fundamentals, 9 (1) (1970), 93.

[17]: Marcus, Y.; Chem. Rev., 113 (2013), 6536.

[18]: Bazarov, I.P.; "Thermodynamics" Pergamon Press (1964).

[19]: Marsh, K.N.; Pure and Appl. Chem., 55 (3) (1983), 467. 
[20]: Kehiaian, H.V.; Tiné, M.R.; Lepori, L.; Matteoli, E.; Marongiu, B.; Fluid Phase Equilib., 46 (1989), 131.

[21]: Reid, R.C.; Praustniz, J.M.; Poling, B.E.; "The Properties of Gases and Liquids" $4^{\text {th }}$ edn.; McGraw-Hill; US (1995).

[22]: Letcher, T.M.; J. Chem. Thermodyn., 4 (1972), 159.

[23]: McClellan, A.L.; "Tables of Experimental Dipole Moments", Rahara Enterprises, El Cerrito, CA, 1974.

[24]: Riddick, J. A.; Bunger, W. B., Sakano, T. K. "Organic Solvents, Physical properties and methods of purification", $4^{\mathrm{a}}$ ed. Techniques of Chemistry Volume II, a Wiley Interscience Publication.

[25]: Ellison, H.R.; Meyer, B.W.; J. Phys. Chem., 74 (2) (1970), 3861.

[26]: Hide, D.R.; Kehiaian, H.V.; "CRC Handbook of Thermophysical and Thermochemical Data", CRC press. Boca de Ratón. Florida (1994).

[27]: McLellan, A.L.; "Tables of experimental dipoles moments". Vols 2,3, Rahara Enterpriser. El Cerrito, California. (1974).

[28]: Gonzalez, J.A.; Mozo, I.; García de la Fuente, I.; Cobos, J.C.; Can. J. Chem., 83 (2005), 1812.

[29]: Trampe, D.M.; Eckert, C.A.; J. Chem. Eng. Data., 36 (1991), 112.

[30]: Kiyohara, O.; Handa, Y.P.; Benson, G.C.; J. Chem. Thermodyn., 11 (5) (1979), 453.

[31]: Urdaneta, O.; Handa, Y.P.; Benson, G.C.; J. Chem. Thermodyn., 11 (9) (1979), 857.

[32]: Matteoli, E.; Gianni, P.; Lepori, L.; Fluid Phase Equilib., 306 (201 1), 234.

[33]: Ferino, I.; Marongiu, B.; Solinas, V.; Torraza, S.; Kehiaian, H.V.; Fluid Phase Equilib., 9 (1982), 49.

[34]: González, J.A.; Alonso, I.; Alonso-Tristán, C.; García de la Fuente, I.; Cobos, J.C.; J. Chem. Thermodyn., 56 (2013), 89.

[35]: Kechavarz, R.; Dubés, J.P.; Tachoire, H.; Int. DATA Ser., Sel. Data Mixtures, Ser. A, $1992(1), 12$.

[36]: González, J.A.; Alonso, I.; García De La Fuente, I.; Cobos, J.C.; Fluid Phase Equilib., 343 (2013), 1.

[37]: González, J.A.; Alonso, I.; García De La Fuente, I.; Cobos, J.C.; Fluid Phase Equilib., 356 (2013), 117.

[38]: Alonso, I.; Mozo, I.; García de la Fuente, I.; González, J.A.; Cobos, J.C.; J. Chem. Eng. Data, 56 (2011), 3236.

[39]: González, J.A.; Alonso, I.; Alonso-Tristán, C.; García de la Fuente, I.; Cobos, J.C.; Fluid Phase Equilib., 337 (2013), 125.

[40]: Eisenschitz, R., London, F.; Z. Physik, 60 (1930), 491.

[41]: London, F.; Z. Physik, 63 (1930), 245. 
[42]: Hildebrand, J.H.; Scott, R.L.; "Regular Solutions", Prentice-Hall, Inc., Englewood Cliffs, New Jersey (1962).

[43]: Kehiaian, H.V.; Fluid Phase Equilib., 13 (1983), 243.

[44]: Kehiaian, H.V.; Pure and Appl. Chem., 57 (1985), 15.

[45]: Villa Vallejo, S. "Contribución experimental y teórica al estudio de las propiedades termodinámicas de las mezclas líquidas formadas por aminas y alcanos o 1 -alcoholes". Tesis Doctoral. Departamento de Termodinámica y Física Aplicada. Facultad de Ciencias. Universidad de Valladolid (2003).

[46]: Riesco Fernández, N.; "Contribución experimental y teórica al estudio de las propiedades termodinámicas de las mezclas binarias entre compuestos orgánicos". Tesis Doctoral. Departamento de Termodinámica y Física Aplicada. Facultad de Ciencias. Universidad de Valladolid (2003).

[47]: Kehiaian, H.V.; Grolier, J.P. E.; Kechavarz, M.R.; Benson, G.C.; Fluid Phase Equilib., 5(3-4) (1981), 159-189.

[48]: Kehiaian, H.V.; Grolier, J.P. E.; Kechavarz, M.R.; Benson, G.C.; Kiyohara, O.; Handa, Y. P.; Fluid Phase Equilib., 7(2) (1981), 95.

[49]: Kiyohara, O.; Benson, G.C.; Grolier, J.P. E.; J. Chem. Thermodyn., 9(4) (1977), 315.

[50]: Grolier, J.P. E.; Kiyohara, O.; Benson, G.C.; J. Chem. Thermodyn., 9(7) (1977), 697.

[51]: Urdaneta, O.; Hamam, S.; Handa, Y.P.; Benson, G.C.; J. Chem. Thermodyn., $11(9)$ (1979) 851.

[52]: Gonzalez, J.A.; Cobos, J.C.; García de la Fuente, I.; Fluid Phase Equilib., 224 (2004), 169.

[53]: Murakami, S.; Fujishiro, R.; Bull. Chem. Soc. Jpn., 39 (1966), 720.

[54]: Letcher, T.M.; Bayles, J.W.; J. Chem. Eng. Data, 16 (1971), 266.

[55]: Pfestorf, R.; Kucenbecker, D.; Quitzsch, K.; Z. Phys. Chem-Leipzig, 263 (1982), 233.

[56]: Velasco, I.; Fernández, J.; Otín, S.; Kehiaian, H.V.; Fluid Phase Equilib., 69 (1991), 15.

[57]: Kern, M. ; Abello, L.; Cáceres, D.; Pannetier, G.; Bull. Soc. Chim. France, 11 (1970), 3849.

[58]: Heintz, A.; Papaioannou, D.; Termochim. Acta, 310 (1998), 69.

[59]: Heintz, A.; Naicker, P.K.; Verevkin, S.P.; Pfestorf, R.; Ber. Bunsenges Phys. Chem., 102 (1998), 953.

[60]: Villa, S.; Riesco, N.; García de la Fuente, I.; González, J.A.; Cobos, J.C.; Fluid Phase Equilib., 216 (2004), 123.

[61]: Kasparek, M.; Cibulka, I.; Hnedkovsky, L.; J. Chem. Thermodyn., 28 (1996), 595.

[62]: Nagata, I.; Tamura, K.; Ksiazczak, A.; J. Chem. Thermodyn., 28 (1996), 551.

[63]: Marsh, K.N.; Burfitt, C.; J. Chem. Thermodyn., 7 (1975), 955. 
[64]: Kiyohara, O.;. Benson, G.C; Treszczanowicz, A.; J. Chem. Eng. Data, 26 (1981), 263.

[65]: Mascato, E.; Mosteiro, L.; Piñeiro, M.M.; García, J.; Iglesias, T.P.; Legido, J.L.; J. Chem. Eng. Data, 45 (2000), 1154.

[66]: Sarmiento, F.; Bravo, R.; Paz Andrade, M.I.; Kechavarz, R.; Dubes, J.P.; Tachoire, H.; Thermochim. Acta, 68 (1983), 273.

[67]: Velasco, I.; Otín, S.; Gutierrez Losa, C.; J. Chim. Phys., 75 (1978), 706.

[68]: McLure, I.A.; James, J.W.; J. Chem. Thermodyn., 11 (1979), 507.

[69]: Villa, S.; González, J.A.; García de la Fuente, I.; Riesco, N.; Cobos, J.C.; J. Sol. Chem., 31 (12) (2002), 1019.

[70]: Philippe, R.; Delmas, G.; Hong, P.N.; Can. J. Chem., 57 (1979), 517.

[71]: Philippe, R.; Delmas, G.; Select. Data on Mixtures Int. Data Ser.A (1983), 154.

[72]: Philippe, R., Delmas, G.; Select. Data on Mixtures Int. Data Ser.A (1983), 51.

[73]: Kasprzycka-Guttman, T.; Kurcinska, H.; J. Sol. Chem., 18 (8) (1989), 727.

[74]: Riesco, N.; Villa, S.; González, J.A.; García de la Fuente, I.; Cobos, J.C.; Fluid Phase Equilib., 202 (2002), 345.

[75]: Riesco, N.; Villa, S.; González, J.A.; García de la Fuente, I.; Cobos, J.C.; Phys. Chem. Liq., 41 (2003), 309.

[76]: Costas, M.; Patterson, D.; Thermochim. Acta, 120 (1987), 161.

[77]: Wilhelm, E.; Inglese, A.; Roux, A. H.;. Grolier, J.-P.E.; Fluid Phase Equilib., 34 (1987), 49.

[78]: Battacharyya, S.N.; Costas, M.; Patterson, D.; Tra, H.-V.; Fluid Phase Equilib., 20 (1985) , 27.

[79]: Wilhelm, E.; Ber. Bunsenges. Phys. Chem., 81 (1977), 1150.

[80]: Ochi, K.; Momose, M.; Kojima, K.; Can. J. Chem., 71 (1993), 982.

[81]: Matsuda, H.; Ochi, K.; Kojima, K.; J. Chem. Eng. Data, 48 (2003), 184.

[82]: Francis, A.W.; "Critical Solution Temperatures", in: "Advances in Chemistry Series", American Chemical Society, Washington DC (1961).

[83]: Dahamni, G.; Ait-Kaci, A.; J. Therm. Anal., 44 (1995), 385.

[84]: Bernard-Houplain, M.C.; Belanger, G.; Sandorfy, C.; J. Chem. Phys., 57 (1972), 530.

[85]: Woycicki, W.; J. Chem. Thermodyn., 18 (1986), 317.

[86]: Pannetier, G.; Abello, L.; Bull. Soc. Chim. Fr., (1965), 2048.

[87]: Díez, D.; Ruiz, B.; Royo, F.M.; Gutiérrez Losa, C.; J. Chem. Thermodyn., 17 (1985), 371.

[88]: Alonso, I.; García de la Fuente, I.; González, J.A.; Cobos, J.C.; J. Chem. Eng. Data, 58 (2013), 1697.

[89]: Murakami, S.; Amaya, K.; Fujishiro, R.; Bull. Chem. Soc. Jpn., 37 (1964), 1776. 
[90]: Hanson, D.O.; Van Winkle, M.; J.Chem. Eng. Data., 5 (1960), 30.

[91]: Messow, U.; Doyé, U.; Kuntzsch, S.; Kuchenbecker, D.; Z. Phys. Chem. (Leipzig), 259 (1977), 90.

[92]: Marongiu, B.; Dernini, S.; Lepori, L. Matteoli, E. ; Thermocim. Acta, 149 (1989), 111.

[93]: Francesconi, R.; Comelli, F.; Thermochim. Acta, 216 (1993), 35.

[94]: Kiyohara, O.; Benson, G.C; Handa, Y.P.; J. Chem. Thermodyn., 11 (1979), 453.

[95]: Kiyohara, O.; Benson, G.C.; Grolier, J.P.E.; J. Chem. Thermodyn., 9 (1977), 315.

[96]: Tamura, K.; Murakami, S.; Fujishiro, R.; J. Chem. Thermodyn., 7 (1975), 1089.

[97]: Legido Soto, J.L.; Tesis Doctoral. Universidad de Santiago de Compostela (1987).

[98]: Dusart, O.; Pierkarski, S.; Grolier, J.P.E.; J. Chim. Phys., 74 (1979), 433.

[99]: Benson, G.C; Handa, Y.P.; Int. Data Series., (1980), 66.

[100]: Dusart, O.; Pierkarski, S.; Pierkarski, S.; Villard, A.; J. Chim. Phys., 73 (1976), 837.

[101]: Nath, J.; Dixit, A.P.; J. Chem. Eng. Data, 28 (1983), 190.

[102]: Grolier, J.P.E.; Benson, G.C.; Can. J. Chem., 62 (1984), 949.

[103]: Francesconi, R.; Comeli, F.; Thermochim. Acta, 228 (1993), 61.

[104]: Grguric, I.R.; Serbanovic, S.P.; Kijevcanin, M.L.; Tasic, A.Z.; Diordjevic, B.D.; Thermochim. Acta, 412 (2004), 25.

[105]: Ohomuro, K.; Tamura, K.; Murakami, S.; J. Chem. Thermodyn., 19 (1987), 163.

[106]: Ortega, J.; Paz Andrade, M.I.; Rodríguez-Núñez, E.; Jiménez, E.; Can. J. Chem., 63 (1985), 3354.

[107]: Spinolo, G.; Riccardi, R.; Int. DATA Ser., Sel. Data Mixtures, Ser. A, 5 (1977), 91.

[108]: Grolier, J.-P.E.; Benson, G.C.; Can. J. Chem., 62 (1984), 949.

[109]: Saint Victor, M.-E.; Patterson, D.; Fluid Phase Equilib., 38 (1987), 237.

[1 10]: Magalhaes, J. G.; Torres, R. B.; Volpe, P. L. O.; J. Chem. Thermodyn., 40 (2008), 1402.

[1 11 1]: Chand, A.; Handa, Y. P.; Fenby, D. V.; J. Chem. Thermodyn., 7 (1975), 401.

[1 12]: Das, A.K.; Jha, B.L.; J. Mol. Liq., 50 (1981), 155.

[1 13]: Mehta, S.K.; Chauhan, R.K; Dewan, R.K.; J. Chem. Soc. Faraday Trans., 92 (1996), 1167.

[1 14]: Mehra, R.; Z. Phys. Chem., 219 (2005), 425.

[1 15]: González, J.A.; Mozo, I.; García de la Fuente, I.; Cobos, J.C.; Riesco, N.; Phys. Chem. Liq., 46 (2008), 390.

[1 16]: Duck, M.J.; Aust. J. Chem., 28 (1975), 1643.

[1 17]: Marczak, W.; Kielek, K.; Int. J. Thermophys., 31 (2010), 85. 
[1 18]: González, J.A.; Alonso, I.; García De La Fuente, I.; Cobos, J.C.; Fluid Phase Equilib., 356 (2013), 117.

[1 19]: Lobos, J.; Mozo, I.; Fernández Regúlez, M.; González, J.A.; García de la Fuente, I.; Cobos, J.C.; J. Chem. Eng. Data, 51 (2006), 623.

[120]: Ukibe, H.; Tanaka, R.; Murakami, S.; Fujishiro, R.; J. Chem. Thermodyn., 6 (1974), 201.

[121]: Ferino, I.; Marongiu, B.; Solinas, V.; Torraza, S.; Int. DATA Ser., Sel. Data Mixtures Ser., A 1 (1985), 52.

[122]: Tamura, K.; Nagata, I.; J. Chem. Thermodyn., 23 (1991), 359.

[123]: Bender, M.; Hauser, J.; Heintz, A.; Ber. Bunsenges. Phys. Chem., 95 (1991), 801.

[124]: Zhu, S ; Shen, S. ; Benson, G.C. ; Lu, B.C.-Y.; J. Sol. Chem., 22, (1993), 1073.

[125]: Ramalho, R.S.; Ruel, M.; Can. J. Chem. Eng., 46 (1968), 467.

[126]: González, J.A.; Mozo, I.; García de la Fuente, I.; Cobos, J.C.; Ind, Eng. Chem. Res., 43 (2004), 7622.

[127]: Keller, M.; Schnabel, S.; Heintz, A.; Fluid Phase Equilib., 110 (1995), 231. 


\title{
CAPITULO 6
}

\section{Teorías de líquidos y disoluciones de no electrolitos}

\begin{abstract}
"Given the success of Ludwig Boltzmann's statistical approach in explaining the observed irreversible behavior of macroscopic systems . . . , it is quite surprising that there is still so much confusion about the problem of irreversibility."

Lebowitz, J.L.; Phys. Today 46(9), 32 (1993), 41

"Boltzmann's ideas are as controversial today, as they were more than hundred years ago, yet they are still defended (Lebowitz 1993). Boltzmann's H-Theorem is based on the unjustifiable assumption that the motions of particles are uncorrelated before collision."

Primas, H.; Mind and Matter 1(1), 81 (2003), 41
\end{abstract}

"Pero esta es la eterna historia: lo que ocurrió en la antigüedad con los estoicos hoy sigue ocurriendo tan pronto como una filosofía empieza a creer en sí misma. Se forja un mundo a su imagen y semejanza; no podría ser de otra manera"

Nietzsche, F.; "Jenseits von Gut und Böse. Vorspiel einer Philosophie der Zukunft" (1886).

$\mathrm{L}$

a Termodinámica es el estudio de las relaciones entre ciertas propiedades macroscópicas (las variables de estado) de un sistema en equilibrio. Si nuestra curiosidad va más allá de estas relaciones formales y si sentimos la necesidad de comprender la conexión entre los valores observados de las variables termodinámicas y las propiedades microscópicas del agregado molecular que es el sistema bajo estudio, hay que acudir a la Física Estadística en la formulación tradicional. Por lo tanto, la Física Estadística tradicional proporciona la teoría molecular de las propiedades macroscópicas de los sistemas termodinámicos. En este capítulo se trata de dar una visión general de la Física Estadística así como de presentar los diferentes modelos teóricos de los líquidos y de sus mezclas.

\subsection{EL PROBLEMA DE LOS SISTEMAS CON MUCHAS INDIVIDUALIDADES}

La mayoría de los sistemas de interés científico constan de un gran número de individualidades o elementos constitutivos (electrones, iones, átomos, moléculas, neuronas, células, ordenadores, mamíferos,...), que presentan interacciones entre ellos. En dichos sistemas hay involucrados un gran número de grados de libertad. Por ejemplo, una porción macroscópica de un cristal contiene unos $10^{23}$ electrones, siendo cada coordenada de cada electrón un grado de libertad. En contraste, la mayoría de los métodos teóricos solamente funcionan cuando hay una variable independiente, es decir, un solo grado de libertad. Por ejemplo, la función de ondas de un electrón $\psi(x, y, z)$ es infinitamente más fácil de calcular cuando es posible hacer una separación de variables en la ecuación de Schrödinger. Aún así, es obviamente desesperanzador tratar de computar la función de ondas de $10^{23}$ electrones sin llevar a cabo un número 
extraordinario de simplificaciones. Afortunadamente, el carácter intensivo o extensivo de los observables físicos permite reducir enormemente estos aproximadamente $10^{23}$ grados de libertad, es decir, permite reconstruir las propiedades de los sistemas macroscópicos a partir de una muestra reducida de los mismos. La distancia máxima para la que el comportamiento de un elemento consitutivo del sistema está influido (correlacionado) por el comportamiento de otro elemento se llama longitud de correlación, $\xi$. En otras palabras, es el tamaño mínimo al cual se puede reducir un sistema sin cambiar cualitativamente sus propiedades. Por lo tanto, el estudio de un sistema macroscópico se puede reducir al estudio de una región del tamaño de su longitud de correlación [1], lo que disminuye drásticamente el número de grados de libertad a considerar.

El comportamiento de los sistemas es cualitativamente diferente en función del tamaño de $\xi$. En las situaciones más favorables (desde el punto de vista matemático) $\xi$ es del orden de una o dos distancias elementales, lo que reduce enormemente el número de grados de libertad a considerar (entendiendo distancia elemental como la distancia entre dos elementos constitutivos adyacentes del sistema: por ejemplo, distancia entre dos átomos, entre dos moléculas, entre dos neuronas, etc.). En estos casos el comportamiento del sistema está determinado principalmente por el tipo de interacciones entre sus elementos constituyentes y por la fuerzas de las ligaduras correspondientes, dando lugar a un comportamiento simple del mismo. Así, por ejemplo en el campo de la Física de particulas, el sistema vendrá definido en términos del hamiltoniano de cada partícula. En estos casos la Mecánica aporta una amplia variedad de métodos para calcular las propiedades locales del sistema: expansiones de tipo virial, métodos perturbativos, ecuaciones de Hartree-Fock, etc. Implícitamente todos estos métodos asumen que las propiedades macroscópicas globales pueden obtenerse a partir de las propiedades de pequeños clusters de átomos (hipótesis reduccionista). Son métodos aproximados pues, de nuevo en el ejemplo de la Física de partículas, ni siquiera un cluster de sólo tres átomos puede resolverse sin llevar a cabo las simplificaciones pertinentes, pues las ecuaciones de movimiento son no integrables, ya que el problema de los tres o más cuerpos no tiene solución o dicha solución es caótica.

En muchos otros sistemas de interés, como los gases, los líquidos, los sólidos, los ecosistemas, la economía, la sociedad, el cerebro, etc. la longitud de correlación es mucho mayor, siendo muy grande el número de grados de libertad a considerar. En estos casos, ni la Mecánica, ni los cálculos perturbativos, ni otros similares proporcionan la solución completa del problema. ¿̇Por qué la computación, digamos, de todas las ecuaciones de movimiento de un conjunto de partículas no da lugar a la descripción macroscópica de éste? La respuesta está en que el individuo deja de tener sentido fuera del medio del cual depende y tiene común dependencia. La complejidad que surge de las interacciones entre las unidades constitutivas del sistema no es en general ni simple ni reducible. La respuesta macroscópica a las ligaduras externas a las que se ve sometido ocasiona la emergencia de una superestructura en el sistema, una estructura a gran escala que, en absoluto, está impuesta por las leyes dinámicas o por las interaciones a nivel local [2]. Para la descripción del comportamiento colectivo o cooperativo de estos sistemas complejos son necesarios conceptos completamente nuevos respecto de los que describen habitualmente el estado microscópico de los sistemas simples. En lugar de hablar de unidades constituyentes, en el sistema global se habla de parámetro de orden, llamado así porque determina el grado de organización 
de dichas unidades. Así, el comportamiento de un conjunto de moléculas o átomos está determinado no sólo por las interacciones entre ellas (el hamiltoniano juega un papel secundario), sino por la estructura que adquiere dicho sistema. Los métodos de la Mecánica no son válidos para describir el estado del sistema y se hace necesaria la utilización de otros métodos, macroscópicos. Por lo tanto, el comportamiento de tales sistemas no puede comprenderse a partir de la extrapolación de las propiedades de unas pocas partículas. Por ejemplo, en el estudio de las ondas transversales en una cuerda, aunque se pudiese resolver la ecuación de Schrödinger de cada átomo de la cuerda, ello no daría opción a imaginar que macroscópicamente el conjunto se mueve de forma sinusoidal con una longitud de onda dada, que es precisamente el parámetro de orden. Otro ejemplo claro es el estudio del cerebro: la descripción de todas las reacciones electroquímicas entre las neuronas no permite explicar el funcionamiento de éste [3]. Por lo tanto, la hipótesis reduccionista falla. El sistema adquiere nuevas propiedades derivadas de los cambios de escala y de complejidad, las llamadas propiedades emergentes [4], [5]. En cada nivel de complejidad se deben definir nuevas entidades elementales y leyes que rijan el comportamiento de los sistemas [6]. El gran problema de las Ciencias es que no se conoce la conexión entre el comportamiento individual y el cooperativo, es decir, se desconoce el mecanismo por el cual la asociación de varios individuos da lugar a la emergencia de nuevas propiedades y de la estructura macroscópica del sistema.

En el estudio de líquidos, disoluciones y aleaciones, nos encontramos precisamente ante un sistema formado por un gran número de átomos que interaccionan entre sí con intensidad intermedia entre el caso de los gases (interacciones suficientemente débiles como para que no exista estructura) y de los cristales (interacciones suficientemente fuertes como para que dicha estructura exista). La complejidad del estudio de la fase líquida reside precisamente en su carácter intermedio entre las fases gaseosa y sólida. Las ideas de los párrafos anteriores pueden aplicarse a este tipo de sistemas. Ellas sugieren que, en aquellas mezclas donde las moléculas posean pocos grados de libertad, los efectos energéticos son los que determinan el comportamiento del sistema, mientras que la organización juega un papel secundario (en el caso límite $T \cdot S_{\mathrm{m}}^{\mathrm{E}}=0$ y toda la contribución a $G_{\mathrm{m}}^{\mathrm{E}}$ se debe a efectos energéticos $H_{\mathrm{m}}^{\mathrm{E}}$ ). No obstante, cuando las entidades elementales tienen muchos grados de libertad, los efectos entrópicos (cooperativos) claramente sobrepasan a los efectos energéticos (en el caso límite $H_{\mathrm{m}}^{\mathrm{E}}=0$ y toda la contribución a $G_{\mathrm{m}}^{\mathrm{E}}$ se debe a efectos entrópicos $T \cdot S_{\mathrm{m}}^{\mathrm{E}}$ : modelo de mezclas atérmicas).

Todavía no se ha llegado a dar una definición universalmente aceptada de complejidad. En un principio se buscó definir dicho concepto como sigue: se supone que un sistema puede describirse mediante una secuencia de datos. Dada dicha secuencia, hemos de preguntarnos si existen un conjunto de datos iniciales y un programa de ordenador que permitan obtenerla. La complejidad del sistema vendrá dada por la longitud de dicho programa. Naturalmente, ésta depende de la arquitectura del ordenador, es decir, del tipo de computadora, por lo que para poder comparar la longitud de diferentes programas es necesario utilizar un computador universal, la llamada máquina de Turing. En dicha máquina, los datos iniciales y el programa se reducen al mínimo. Por lo tanto, la mínima longitud de un programa y de los datos iniciales forman una medida de la complejidad del sistema, la llamada entropía de complejidad algorítmica [7]. Sin 
embargo esta definición tiene un inconveniente: el problema de encontrar un conjunto de datos iniciales y un programa mínimos no puede resolverse de manera universal, es decir, no existe un algoritmo universal que resuelva este problema. Sólo pueden desarrollarse dichos algoritmos en casos particulares [8].

El estudio de los sistemas complejos en Física ha sido abordado tradicionalmente mediante la Termodinámica y la Física Estadística del equilibrio en la formulación tradicional, desarrollada principalmente por Boltzmann y Gibbs [9], [10]. Mientras que la primera es una ciencia fenomenológica, que ignora la constitución microscópica de la materia y tiene un carácter tautológico, la segunda trata de explicar de forma reduccionista (es decir, postulando la existencia de átomos o moléculas que forman el sistema y sin tener en cuenta la formación de estructuras jerárquicas emergentes de mayor escala que las contiene y que no pueden subdividirse sin más en dichos átomos - moléculas constituyentes) las propiedades macroscópicas del sistema en estudio; además se fundamenta en hipótesis teóricas cuya validez hoy en día siguen siendo objeto de controversia. En las últimas décadas han surgido nuevos tratamientos teóricos entre los que destacan la Teoría de Sistemas [11] y la Sinergética [3], [8], que tratan no solamente los sistemas físicos en equilibrio, sino también sistemas físicos lejos del equilibrio termodinámico como los fluidos en régimen turbulento, el láser, etc., y sistemas químicos, biológicos, económicos, sociales,... La Sinergética busca una formulación de leyes universales válidas para los sistemas complejos, los cuales pueden formar estructuras funcionales, espaciales o temporales, en términos de la autoorganización. Como resultado de esta búsqueda, la atención está dirigida a cambios cualitativos macroscópicos. Este punto de vista permite ver como evidente el que las leyes de la Termodinámica sean deducibles a partir de las leyes de la Sinergética.

Por su parte, el problema básico en la Física Estadistica del equilibrio tradicional formulada mediante el conjunto canónico es el cálculo de la suma de estados, es decir, de la función de partición:

$$
Z=\sum_{\mathrm{i}=1}^{\Omega} e^{-\beta \cdot \varepsilon_{\mathrm{i}}}
$$

Desafortunadamente, salvo en el caso de partículas que no interactúan entre sí o en modelos de interacción de campo promedio, este cálculo es tremendamente complicado. De hecho, sólo se han resuelto exactamente modelos muy idealizados de elementos interactuntes en una y dos dimensiones, los cuales pueden clasificarse en los cuatro grupos siguientes [12]:

a) Modelos unidimensionales [13]-[22].

b) Modelos de dimensión infinita, entendiendo diménsión no el sentido de la dimensión del espacio, sino del número de vecinos próximos a un elemento en una red [12].

c) Modelos esféricos [23]-[25].

d) Modelos de red bidimensional como el modelo de Ising, modelo ferroeléctrico, modelos de 8 vértices, y modelo de 3 espines [26]-[28].

En definitiva, la Física Estadística tradicional no ha llegado ni llegará a aplicarse con éxito a ningún modelo real. No es la teoría definitiva mediante la cual las características 
del sistema global se obtienen a partir de la información microscópica, no sólo por la dificultad del cálculo de la función de partición, sino también por las razones siguientes:

a) La resolución del problema mecánico intrínseco de las ecuaciones del movimiento es prohibitivamente complicado. Esto se debe, en primer lugar, a que la mayoría de los sistemas mecánicos son muy sensibles a las condiciones iniciales. Una pequeña incertidumbre en las posiciones y /o velocidades iniciales de los elementos del sistema, cuyos valores exactos no son conocidos por el Principio de incertidumbre de Heisenberg, produce cambios en la configuración futura del sistema, que genera horizontes espaciales y temporales (los llamados horizontes de Lyapunov) por encima de los cuales el sistemas es intrínsecamente impredecible, siendo más acusada esta impredecibilidad cuanto mayor sea la incertidumbre en las condiciones iniciales y/o cuanto más lejos se quiera ir en el tiempo, lo que se llama caos. Por lo tanto el futuro es impredecible y probabilista [29]. Las interacciones entre los constituyentes del sistema hacen más probable la presencia de caos. Esto introduce serios problemas técnicos en la resolución de las ecuaciones del movimiento de los elementos individuales. En segundo lugar, el problema mecánico de tres o más cuerpos interactuando no es integrable, como demostró Poincaré [30]. En consecuencia, ni siquiera la implementación de caos determinista de manera rigurosa en los resultados de las ecuaciones de movimiento resuelve el problema [31]-[34].

b) La hipótesis ergódica, piedra angular de la Física Estadística en la metodología tradicional, es falsa. Según Boltzmann, en un sistema de partículas aislado del exterior (donde no hay intercambio de energía) y que no presenta pérdidas internas de energía, visitará con el paso del tiempo todas las configuraciones posibles desde el punto de vista energético; es decir, todas las configuraciones de velocidades y posiciones de las partículas con la energía correcta serán observadas siempre que se espere el tiempo suficiente. En otras palabras, el punto representativo del sistema en el espacio de las fases recorre todas las configuraciones permitidas con el trascurso del tiempo. Como la energía es constante, el sistema volverá incansablemente a pasar tan cerca como se quiera de cualquier configuración permitida, es decir, existe eterno retorno. Ello es consecuencia de que las fuerzas entre las moléculas son invariantes respecto la inversión temporal, y por el Teorema de Noether, existe una cantidad que se conserva, que es precisamente la energía. Esto es lo que se llama hipótesis ergódica. Sin embargo, la vida cotidiana demuestra que los sistemas reales no son aislados y llevan a cabo procesos que son irreversibles, es decir, presentan pérdidas de energía. Para explicarlos, o bien se da por falsa la hipótesis ergódica, de forma que se suponga que existen estructuras disipativas para las cuales la invariancia bajo inversión temporal se rompe, o bien se da por hecho que los tiempos de retorno a una situación inicial son larguísimos (mayores que la edad del Universo). No obstante, si el sistema no es demasiado complejo, aún existiendo estructuras disipativas o larguísimos tiempos de retorno es posible que éste vuelva a estados cercanos a los anteriores.

c) En sistemas muy complejos es posible la irrupción de un ser inteligente que manipule la configuración del sistema a su antojo, así como de mecanismos reguladores que tiendan a eliminar los efectos de dicho ser, al menos en parte. En tales sistemas la evolución temporal tiene sentido único, es decir, la historia no se repite. Por lo tanto, en estos sistemas la hipótesis ergódica es categóricamente falsa.

d) La Física Estadística tradicional es capaz, en parte, de explicar el comportamiento de algunas de las variables que caracterizan el estado macroscópico 
de un gas, gracias a las propiedades de simetría asociadas a alguna ley Física. Por ejemplo, en un sistema aislado, en el que las variables energía, $E$, volumen, $V$, y número de partículas, $N$, son constantes, éstas pueden explicarse a partir de la simetría temporal de las leyes dinámicas del movimiento, el concepto de la ruptura de simetría en el contexto del Teorema de Goldstone y simetrías Gauge dentro del contexto de la teoría de partículas fundamentales, respectivamente [35, Cap. 21]. Por lo tanto, para la explicación de todas las propiedades macroscópicas emergentes es necesario encontrar todas las simetrías presentes en el sistema, lo cual sólo se ha conseguido en algunos sistemas como en gases ideales y en el orto y para hidrógeno. En el resto de casos, como en cuásares, agujeros negros, estrellas de neutrones, (en los que las componentes del momento angular y lineal son un ejemplo de las nuevas propiedades macroscópicas, es decir, emergentes a tener en cuenta y que aparecen como resultado de las simetrías espaciales de las leyes de movimiento bajo transformaciones traslacionales y rotacionales), gases de gluones,... queda mucho trabajo por hacer en este sentido. $Y$ es que el Teorema de incompletitud de Gödel*, juega un papel muy relevante, pues en este contexto implica que siempre habrá simetrías nuevas a considerar, es decir siempre existirán propiedades de los sistemas que no pueden explicarse a partir de la Física Estadística tradicional, ni de ninguna otra teoría. Existen infinitas simetrías. Este razonamiento permite echar por tierra cualquier búsqueda de teoría del todo ${ }^{\dagger}$. Es digna de reconocimiento la siguiente cita de Hawking [36]:

"Some people will be very disappointed if there is not an ultimate theory, that can be formulated as a finite number of principles. I used to belong to that camp, but I have changed my mind."

e) Es un hecho constatado empíricamente que existe ruptura espontánea de la simetría en la naturaleza: el aumento de la complejidad y funcionalidad de un sistema está relacionado con niveles incrementales de simetrías rotas. Esto quiere decir que en cada nivel de complejidad existen leyes que dejan de ser válidas (se rompe la simetría) al pasar al siguiente nivel. Así, aún suponiendo que todas las simetrías en un nivel de complejidad dado fuesen conocidas (lo cual, por cierto, es imposible por el Teorema de Gödel), al pasar al siguiente nivel aparecerán simetrías nuevas, que son las responsables de la emergencia de nuevas propiedades del sistema, las propiedades emergentes. Si conociésemos el mecanismo de ruptura y aparición de las simetrías el problema estaría parcialmente resuelto. No obstante, lo cierto es que a día de hoy dicho mecanismo está muy lejos de ser comprendido. La Física Estadística tradicional, vista como la teoría cinética de los gases, explica muy bien el comportamiento de los gases reales (salvo por el desconocimiento de las fuerzas de interacción, es decir, por la no integrabilidad de las ecuaciones de movimiento). Cuando se intenta explicar el fenómeno de la condensación, es decir, los cambios de fase, deja de funcionar puesto

\footnotetext{
* Teorema de incompletitud de Gödel: una vez fijadas las reglas de inferencia y un número finito de axiomas cualesquiera, que pueden definir una teoría, siempren existirán proposiciones formuladas de manera precisa para las cuales no es demostrable que sean verdaderas o falsas. Y aúnque estas proposiciones se adopten como nuevos axiomas de la teoría, seguirán existiendo proposiciones no falsables.

† Lo más parecido a una teoría del todo debería girar en torno a la invarianza de los exponentes críticos en cada clase de universalidad, lo que permite tratar por igual a sistemas de diferentes niveles de complejidad que se encuentran en un estado autocrítico.
} 
que en la nueva fase aparecen propiedades emergentes (por ejemplo, viscosidad y orden a corto alcanze en los líquidos, dureza y estructura en los sólidos), es decir, nuevas simetrías. Esencialmente por ello la teoría de Yang-Lee [37], [38] de los cambios de fase fracasó, ya que un cambio de fase representa un cambio en la escala de complejidad, una ruptura de la simetría, la cual no se tiene en cuenta en dicha teoría. Por el contrario, la única teoría que a día de hoy es capaz de predecir valores de los exponentes críticos con exactitud aceptable es el método del grupo de renormalización, pues dicho método emplea el concepto de invarianza bajo cambio de escala (simetría de escala). Cuando dicha simetría se rompe, se produce un cambio de fase.

Los inconvenientes arriba enumerados hacen necesaria la evolución de la Física Estadística tradicional hacia una nueva que supere los mismos. Con este objetivo se han desarrollado la Física Estadística del equilibrio para sistemas caóticos [39], la Física Estadística del no equilibrio [40], [41], etc., que superan algunos de los anteriores inconvenientes, pero no todos. De acuerdo con Cobos [comunicación personal], existe otra manera de proceder que elimina por completo los inconvenientes citados. Este punto de vista consiste en considerar a la Física Estadística como un proceso de inferencia ${ }^{*}$ estadística que permite estudiar el comportamiento de un conjunto de entidades elementales que jerárquicamente describen el sistema en estudio en lo que respecta a como interaccionan unas con otras y a como se estructuran para dar lugar a los distintos estados de agregación y fases características en que aparece la materia. Para definir correctamente qué se entiende por entidad elemental se deben recordar las cuatro etapas del esquema de trabajo general de la Física Estadística:

a) Definición del macroestado del sistema a caracterizar.

b) Elaboración de un modelo de realidad del mismo (comportamiento microscópico del sistema).

c) Cálculo de la función de estructura característica del conjunto estadístico de Gibbs asociado al macroestado en estudio: número de microestados $(\Omega)$, función de partición (Z), . . .

d) Cálculos correspondientes:

- Relación entre el potencial termodinámico natural y la función de estructura: $S=$ $\mathrm{k}_{\mathrm{B}} \cdot \ln \Omega, \Psi=-F / T=\mathrm{k}_{\mathrm{B}} \cdot \ln \mathrm{Z}, \ldots$

- Primeras derivadas del potencial termodinámico natural: ecuaciones térmica, energética y másica de estado

- Segundas derivadas: funciones respuesta (coeficientes térmicos, energéticos y másicos- fluctuaciones)

Se define entidad elemental como la unidad estructural más pequeña que, introducida en la etapa b) para elaborar el modelo de realidad, permite resolver el problema. En otras palabras, que transforma el sistema complejo donde los grados de libertad están acoplados entre sí, en un nuevo problema donde las entidades elementales tienen sus

\footnotetext{
* Por inferencia se entiende: i) razonaminto deductivo siempre que haya disponible la suficiente información que permita hacerlo. Si un problema puede resolverse mediante razonamiento deductivo, la teoría de probabilidad no es necesaria; ii) razonamiento inductivo o plausible, cundo la información necesaria no está disponibe, como ocurre en la mayoría de las situaciones reales [48].
} 
grados de libertad desacoplados. Es decir, que convierte el problema mecánico de tres o más cuerpos en un problema tipo gas ideal o de campo promedio. En el conjunto canónico, la función de partición de las $N$ entidades elementales indistinguibles no interactuantes que constituyen el sistema en estudio es:

$$
Z=\sum_{\mathrm{i}=1}^{\Omega} e^{-\beta \cdot \varepsilon_{\mathrm{i}}}=\sum_{\mathrm{i}=1}^{\Omega} e^{-\beta \cdot \sum_{\mathrm{j}=1}^{N} \mathrm{e}_{\mathrm{j}}^{\mathrm{i}}}=\sum_{\mathrm{i}=1}^{\Omega} \prod_{\mathrm{j}=1}^{N} e^{-\beta \cdot \mathrm{e}_{\mathrm{j}}^{\mathrm{j}}}=\frac{\prod_{\mathrm{j}=1}^{N}\left(Z_{\mathrm{j}}\right)}{N !}=\frac{\left(Z_{1}\right)^{N}}{N !}=e^{-F / k_{B} \cdot T}
$$

Por lo tanto, se trabaja con individualidades que han sido definidas como tales a fin de ser independientes. En definitiva, se reduce el estudio a modelos de campo promedio (se supera el inconveniente a). Si los sistemas estudiados están en estado de equilibrio, se superan los inconvenientes b) y c). Si las propiedades emergentes propias de cada fase se incluyen ad hoc por medio de un modelo, como por ejemplo la introducción del concepto de estructura en un sólido mediante la adopción de un modelo de red, y si se supone que no hay cambios de fase, se superan d) y e). De esta manera se puede hacer plausible el estudio de los líquidos y de cualquier otro sistema mediante la Física Estadística.

La verdad es que ninguna de las tres mencionadas aproximaciones (caos, no equilibrio, Cobos) aporta la solución definitiva en cuanto a la conexión entre lo microscópico y lo macroscópico. De hecho, el actual estado del conocimiento está muy lejos de conseguir dicha conexión.

La formulación más coherente que se ha conseguido a día de hoy de la Física Estadística está basada en la teoría de la información [42] y en el principio de máxima entropía [43]-[47]. De este modo la Física Estadística no adquiere el carácter de teoría Física respecto su carácter predictivo, sino que se convierte en una técnica de inferencia estadística de validez universal, y tiene sus bases en: primero, el teorema central del límite, que establece que el tratamiento estadístico de las propiedades de los sistemas complejos formados por un gran número de individualidades (límite termodinámico) es insensible a los detalles estructurales o dinámicos de dichas individualidades, es decir, el tratamiento estadístico de un sistema complejo es universal; segundo, el principio de crecimiento de la entropía en sistemas aislados, el cual es un resultado de la Termodinámica ampliamente aceptado. Por lo tanto, la entropía de un sistema termodinámico definida como la ignorancia o la incertidumbre que un observador tiene acerca del estado microscópico de dicho sistema cuando solamente dispone de la información proporcionada por las variables macroscópicas que definen el estado termodinámico de dicho sistema, juega un papel fundamental. Esta definición de entropía la desliga claramente del concepto de desorden, y permite que en determinados procesos aumente el orden sin violar la segunda ley de la Termodinámica. Sin embargo, ningún sistema de la naturaleza es aislado, pues en general todos ellos intercambian energía, materia, información, etc. con el entorno, por lo que en cada proceso no será la entropía la cantidad que sea máxima sino la correspondiente función característica entrópica compatible con las ligaduras macroscópicas impuestas al sistema. La distribución de probabilidades en el equilibrio es la que maximiza dicha función. Tal distribución de probabilidades se puede obtener mediante el método de los multiplicadores de Lagrange, una vez que se conoce la información macroscópica disponible. La probabilidad debe entenderse en el sentido bayesiano y no como una frecuencia. Así, estas probabilidades ya no representan, como en la Física Estadística 
tradicional, la fracción de tiempo que un sistema pasa en un determinado estado cuántico, sino que determina el grado de conocimiento que el observador tiene acerca del microestado del sistema. En consecuencia, las fluctuaciones de las magnitudes termodinámicas no están asociadas a cambios instantáneos debidos a variaciones en los estados cuánticos de las partículas, sino a la imprecisión del observador en la determinación de dichas magnitudes, de acuerdo con la información macroscópica de que dispone. Sorprendentemente, esta manera de enfocar la Física Estadística permite obtener las mismas relaciones formales entre las diferentes propiedades termodinámicas, funciones de partición, fluctuaciones,... que se obtienen a partir de la Física Estadística tradicional.

A pesar de todo lo dicho, la Física Estadística tradicional ocupó un lugar privilegiado en la Física durante mucho tiempo, pues la idea de obtener información macroscópica a partir de la microscópica es muy atractiva. En ese tiempo se formularon gran cantidad de teorías moleculares de líquidos, disoluciones y aleaciones, cuyo objetivo principal era explicar y predecir el comportamiento macroscópico en función de las entidades elementales que las forman. Así se pretende relacionar el tamaño, la forma, la flexibilidad, los momentos multipolares y la polarizabilidad de esas entidades elementales con las magnitudes termodinámicas que, en el equilibrio, describen el sistema termodinámico multifásico y multicomponente que son las mezclas fluidas y aleaciones. Dichas teorías siguen utilizándose a día de hoy. En todas ellas, aún suponiendo que el sistema está en estado de equilibrio termodinámico y que no se producen cambios de fase, persiste el gran problema de la no integrabilidad de las ecuaciones del movimiento. Por lo tanto, en dichas teorías aparecen términos energéticos que son consecuencia de las interacciones entre las moléculas y que son desconocidas. Para hacer operativas dichas teorías, tales parámetros energéticos se deben considerar como parámetros ajustables a los datos experimentales, de modo que sea la información a nivel microscópico la que se infiera a partir de la información macroscópica. En otras palabras, se pierde el total poder de predicción de dichas teorías. Por esta razón es esencial tener datos experimentales fiables y reducir el número de parámetros ajustables al mínimo, pues como Fermi declaró a von Neumann [49]:

"With four parameters I can fit an elephant, and with five I can make him wiggle his trunk"

Para acabar esta sección, volvamos a la discusión acerca de la longitud de correlación de los sistemas complejos, y consideremos el caso límite en que ésta sea máxima, es decir, al caso en el que las individualidades del sistema están muy fuertemente correlacionadas. Esta situación se puede encontrar en metales con impurezas magnéticas (problema de Kondo), el agua y en las transiciones de fase, por ejemplo. En dichas circunstancias, $\xi$ es infinita, solamente limitada por el tamaño total del sistema:

$$
\xi \sim\left|T-T_{c}\right|^{-0}
$$

donde $v$ es un exponente crítico (positivo). La divergencia de $\xi$ en los puntos críticos $\left(T=T_{c}\right)$ implica que dos elementos distantes influyen el uno sobre el otro, lo que está asociado con la emergencia de clusters muy grandes es decir, de estructuras macroscópicas.

El estudio de las transiciones de fase es muy importante. Kadanoff propuso las reglas generales que permiten el cálculo de los exponentes críticos de un sistema sin necesidad 
de conocer exactamente su función de partición, poniendo énfasis en las configuraciones del sistema, en su aspecto y en cómo la invariancia de escala puede ser utilizada para la extracción de los exponentes críticos, lo que llevó a Wilson a la posterior formulación matemática del método del grupo de renormalización (RG, del acrónimo en inglés de Renormalization Group) [1], [50]-[54]. De acuerdo con este método, la suma de estados en $Z$ se evalúa en pasos sucesivos, con un hamiltoniano renormalizado que se define en cada uno de los pasos. En principio, el método permite resolver el problema de manera exacta, pero ello sería tanto o más difícil que calcular $Z$ directamente, por lo que para hacer operativo al método, deben realizarse algunas aproximaciones, a pesar de las cuales, se obtienen resultados muy buenos. La renormalización en el espacio real sólo es aplicable a modelos de red que poseen simetría discreta de escala es decir, invariancia sobre cambios de escala, la cual fortalece la validez de las descripciones realizadas con modelos en espacio o tiempo discreto cuando el sistema evoluciona cerca de un punto crítico. Por lo tanto, el método RG elimina los detalles microscópicos del sistema, proporcionando no obstante resultados casi exactos. Así, los modelos ideales de fenómenos críticos deben verse desde la perspectiva de exactitud. No es necesario tener en cuenta los detalles de la descripción del modelo, ya que la aparición de estructuras a gran escala los borra por completo. La prueba más contundente de ello es la diversidad de sistemas que pueden describirse mediante los mismos exponentes críticos. Así se llega al concepto de Universalidad de los exponentes críticos, que se refiere a la independencia de los exponentes críticos respecto de los detalles del hamiltoniano, es decir, de las interacciones locales y de los elementos constitutivos del sistema, dependiendo solamente de la dimensión del espacio y de la simetría del modelo; así, $v$ es igual a 0.625 en 3-d. De este modo, las propiedades descritas mediante los exponentes críticos son universales, lo que permite la asociación de cada tipo de transición con una clase de universalidad diferente, siendo finito el número de tales clases, por lo que en principio se pueden clasificar diferentes fenómenos como miembros de diferentes clases de universalidad [55]. Por esta razón, a pesar de que el modelo de Ising 3-d es el modelo tridimensional más simple, la hipótesis de Universalidad lo dota de gran importancia pues ella asegura que siempre que la dimensionalidad del sistema se mantenga, los exponentes críticos obtenidos para este modelo son los mismos que para cualquier sistema tridimensional con la misma simetría. Es destacable que aunque el modelo de Ising 3-d se ha estudiado de manera extensiva, aún no ha sido resuelto, y de hecho se ha sugerido que no existe solución exacta del mismo [56], aunque sí existen resultados aproximados muy buenos.

Por lo tanto, en el caso extremo de máxima correlación entre las individualidades del sistema, es decir, en los estados autocríticos y las transiciones de fase, en los que las interacciones entre los constituyentes del sistema son bestiales, los detalles microscópicos del sistema no son importantes en absoluto. Así, carecen de importancia características tales como el número de vecinos próximos o si la interacción entre los constituyentes es de tipo puente de hidrógeno o mediante estados de espin. La emergencia de una estructura que ocupa toda la escala espacial del sistema, el fenómeno cooperativo que aparece a escala mucho mayor que la del elemento individual, los hace irrelevantes.

La hipótesis de Universalidad hace que el estudio de los fenómenos críticos sea interesante no sólo en Física, sino también en otras ramas de la Ciencia. El estudio de 
la historia de la evolución y de la sociedad muestra que los cambios profundos que han ocurrido en las formas de vida, los ecosistemas y las civilizaciones pueden considerarse transiciones de fase. La historia de la humanidad está marcada por eventos cruciales, tales como el descubrimiento de América en 1492, que provocó transformaciones a escala planetaria en la ecología, economía y la cultura. Otros eventos ocurridos en el sistema Tierra, como los impactos sufridos por asteroides, también modificaron profundamente el curso de la evolución. De estos ejemplos puede intuirse que los cambios cualitativos están siempre asociados a eventos raros, inesperados, ejecutados por la acción de un agente externo que irrumpe en el sistema, del mismo modo que para observar una transición crítica en un fluido hay que "empujar" al fluido ha dicho estado mediante el control de ciertos parámetros (temperatura y presión). Esta intuición es equivocada, pues en la naturaleza existen sistemas críticos autorganizdos, que están fuera del equilibrio, altamente disipativos, los cuales parece ser que han sido empujados a dicho estado crítico mediante algún mecanismo interno (dinámica intrínseca, interacción y relación con el medio, etc.). Tómese como ejemplo lo que sucedió hace 6000 años en el norte de África, en la región donde actualmente se encuentra el desierto del Sáhara: múltiples remanentes indican que por aquél entonces, esta región era húmeda y estaba cubierta de vegetación, ríos, mamíferos y asentaciones humanas. El proceso de desertificación fue inicialmente lento y gradual. Sin embargo en algún momento el ecosistema colapsó rápidamente. El Sáhara verde se convirtió en un desierto.

En definitiva, pueden describirse transiciones entre estados alternados no sólo en el contexto de la Física sino en otros muchos tipos de sistemas. Los sistemas complejos presentan todos estos tipos de fenómenos. Tales transiciones pueden afectar, a veces dramáticamente, a los patrones moleculares de la actividad genética dentro de las células, patrones de comportamiento colectivo en hormigas, al éxito o al fracaso de la propagación de epidemias, etc. Así, en un sentido amplio podemos entender por fase como cada uno de los patrones de organización. Cuando un parámetro dado es ajustado y cruza un cierto umbral, se observa un cambio en la organización del sistema o en su dinámica. El estudio de la complejidad es, a grandes rasgos, una búsqueda de los principios prevalecentes autoorganizados, fenómenos emergentes y la definición de sus fases potenciales [3], [4], [57]-[66]. Tales transiciones de fase son colectivas por naturaleza y resultan de las interacciones que tienen lugar entre las individualidades.

\subsection{LÍQUIDOS Y DISOLUCIONES}

De acuerdo con lo dicho en la sección anterior, en sistemas complejos los detalles de las individualidades como por ejemplo las interacciones locales (dadas por el hamiltoniano) son irrelevantes puesto que el sistema se organiza adquiriendo una estructura determinada (entropía) por las ligaduras macroscópicas externas, por lo que el tratamiento Estadístico tradicional de, por ejemplo, los líquidos, las disoluciones y las aleaciones debe mirarse con reservas, pues sólo es rigurosamente válido en el caso ideal o en modelos de campo promedio, siendo esencial la definición de entidad elemental. No obstante, es útil para hacer una primera clasificación de los sistemas, suponer que su comportamiento está condicionado por el carácter de las interacciones que se dan entre sus elementos constitutivos, lo que puede especificarse suponiendo que dichas interacciones pueden describirse en términos de una función de las coordenadas 
de todos sus elementos constitutivos (electrones, iones, átomos, moléculas,... partículas en general) de manera que los grados de libertad de todos ellos estén acoplados. Por lo tanto, una discusión de los grados de libertad presentes en el sistema permite hacer una vaga clasificación de los mismos en ideales y no ideales. Para entender dicha disusión, es primordial entender la mecánica de una molécula aislada.

El hamiltoniano de la ecuación de Schrödinger independiente del tiempo de una molécula aislada (etiquetada por $\gamma$ ) es:

$$
H_{\gamma}=T_{\gamma}+V_{\gamma}
$$

donde $T_{\gamma}$ es el operador energía cinética que incluye todas las posibles traslaciones, rotaciones y vibraciones de los núcleos y los electrones. El operador energía potencial $V_{\gamma}$ contiene básicamente la contribución de las interacciones electrostáticas de tipo electrón-electrón, núcleo-núcleo y electrón-núcleo, y términos de segundo orden como el acoplamiento spin-órbita, spin-spin, el término de contacto de Fermi, etc. El problema puede simplificarse enormemente teniendo en cuenta el hecho de que las masas de los núcleos de los átomos son muy grandes comparadas con la masa de los electrones [67]. Gracias a esta diferencia entre las masas, las velocidades del movimiento de los núcleos en la molécula son pequeñas respecto de las velocidades de los electrones. Esto ofrece la posibilidad de estudiar el movimiento electrónico y nuclear separadamente, suponiendo que los núcleos están en reposo respecto de los electrones y situados a distancias dadas unos de otros, lo que se llama aproximación adiabática o de BornOppenheimer [68]. Es decir, el hamiltoniano molecular se puede separar en dos partes: una que depende solamente de las coordenadas nucleares y que contiene la energía cinética de los núcleos y la energía potencial de interacción núcleo-núcleo y electrónnúcleo (hamiltoniano nuclear) y otra que contiene el resto de términos, que depende tanto de las coordenadas nucleares, que aparecen como parámetros, como de las electrónicas, de manera que la ecuación de Schrödinger molecular se desdobla en una ecuación para los núcleos y otra para los electrones, apareciendo en ésta última las coordenadas nucleares como parámetros.

Dando por válida la aproximación de Born-Oppenheimer, el siguiente paso consiste en el estudio del hamiltoniano nuclear. Para ello, es fundamental la determinación de la energía cinética de todos los núcleos que forman parte de la molécula, por lo que es necesaria la descripción de la misma en unas coordenadas dadas. Mientras que los tres operadores posición $\vec{R}_{\gamma} \equiv\left(\mathrm{X}_{\gamma}, \mathrm{Y}_{\gamma}, \mathrm{Z}_{\gamma}\right)$ son el análogo cuántico a las coordenadas que describen el movimiento del centro de masas de la molécula respecto de un sistema fijo en el espacio O'X'Y'Z', los tres operadores $\vec{\Omega}_{\gamma} \equiv\left(\theta_{\gamma}, \Phi_{\gamma}, \chi_{\gamma}\right)$ son análogos a los tres ángulos de Euler clásicos que describen la orientación en el espacio respecto del sistema O'X'Y'Z' de un segundo sistema de referencia Oxyz solidario a la molécula y cuyo origen coincide con el centro de masas de la misma. En otras palabras, $\vec{\Omega}_{\gamma}$ da cuenta de la orientación de la molécula cuando ésta rota como un todo respecto del sistema de referencia fijo en el espacio, es decir, del movimiento de libración. Si la molécula fuese rígida, la descripción de su movimiento en términos de estos operadores sería suficiente. Sin embargo la realidad es bien distinta: en primer lugar, los núcleos atómicos pueden vibrar respecto de sus posiciones de equilibrio, siendo necesarios un total de $3 \cdot 1_{\gamma^{-}}-6\left(3 \cdot 1_{\gamma^{-}}\right.$

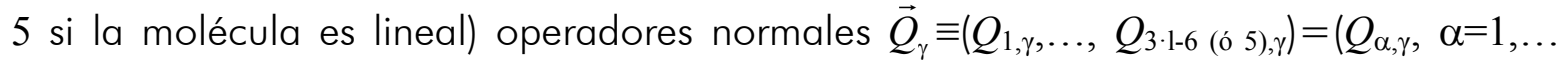


$3 \cdot 1_{\gamma}-6$ (ó 5$)$ ), análogos a las coordenadas normales clásicas que dan la posición de los átomos en vibración respecto de sus posiciones de equilibrio en el sistema de ejes rotante, siendo $1_{\gamma}$ el número de núcleos de la molécula $\gamma$ [69], [70]; en segundo lugar, las rotaciones de los núcleos también deben ser tenidas en cuenta, lo que se hace en términos de los operadores de spin nuclear $\vec{S}_{\gamma}$, que son función del número de nucleones en los núcleos. Por si fuese poco, también es necesaria la consideración de otro conjunto de operadores $\vec{\xi}_{\gamma}$ análogo al conjunto de coordenadas clásicas que describe las posibles rotaciones alrededor de algunos enlaces interatómicos, responsables de la isomería conformacional, efecto muy importante en polímeros y que explica la estructura en forma de hélice de dichas macromoléculas [71]-[73].

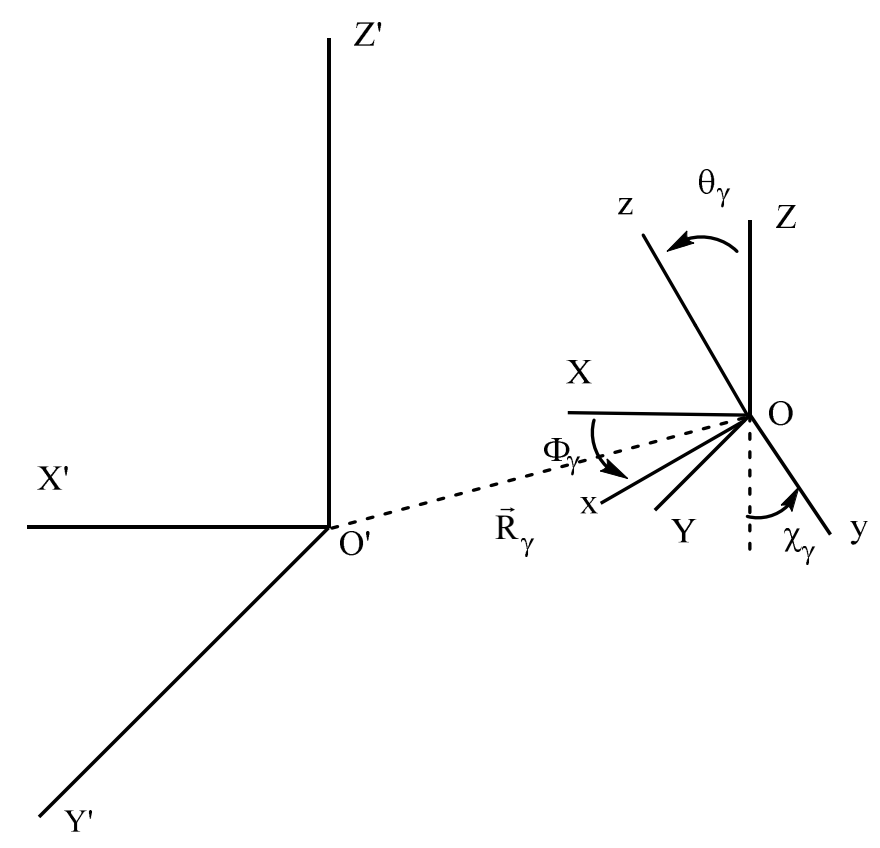

llustración 6-1: sistemas de referencia fijo y solidario a la molécula. Ángulos de Euler

Teniendo todo esto en cuenta, es posible separar los diferentes tipos de movimiento. Si los ejes principales de inercia de la molécula coinciden con los ejes $\mathrm{x}, \mathrm{y}, \mathrm{z}$, el hamiltoniano nuclear se escribe:

$$
\begin{aligned}
H_{\mathrm{n}, \gamma}=T_{\mathrm{cdm}, \gamma} & +\frac{1}{2} \cdot\left[\frac{\left(L_{x}-l_{x}\right)^{2}}{I_{x}^{0}}+\frac{\left(L_{y}-l_{y}\right)^{2}}{I_{y}^{0}}+\frac{\left(L_{z}-l_{z}\right)^{2}}{I_{z}^{0}}\right]_{\gamma}+ \\
+ & \frac{1}{2} \cdot \sum_{\alpha_{\gamma}=1}^{3 \cdot l_{\gamma}-6(\dot{o} s)} P_{\alpha_{\gamma}}^{2}+T_{\mathrm{spin}, \gamma}+T_{\mathrm{conf}, \gamma}+V_{\mathrm{n}, \gamma}
\end{aligned}
$$

donde $T_{\mathrm{cdm}, \gamma}$ es el operador energía cinética de traslación del centro de masas, el término entre corchetes es el operador de energía rotación-vibración $\left(L_{\mathrm{i}}\right.$ es la componente $\mathrm{i}$ del operador momento angular, $l_{\mathrm{i}}$ es la componente del operador momento angular vibracional en el eje i e $I_{i}^{0}$ es el momento principal de inercia del eje $\mathrm{i}$ en las posiciones de vibración de equilibrio), $P_{\alpha, \gamma}$ es el operador momento canónico conjugado asociado al operador $Q_{\alpha, \gamma}, T_{\text {spin, } \gamma}$ es el operador energía cinética de spin nuclear, $T_{\text {conf, } \gamma}$ es el operador de energía cinética de rotación conformacional y $V_{\mathrm{n}, \gamma}$ es 
el operador energía potencial de interacción nuclear, que incluye las interacciones entre núcleos y entre núcleos y electrones, ignorando los términos de segundo orden.

En conclusión el hamiltoniano molecular puede escribirse:

$$
\begin{gathered}
H_{\gamma}=H_{\mathrm{n}, \gamma}+H_{\mathrm{e}, \gamma}=T_{\mathrm{cdm}, \gamma}+T_{\mathrm{rot}-\mathrm{vib}, \gamma}+T_{\mathrm{spin}, \gamma}+T_{\mathrm{conf}, \gamma}+T_{\mathrm{vib}, \gamma}+T_{\mathrm{e}, \gamma}+V_{\mathrm{e}, \gamma}+V_{\mathrm{n}, \gamma}= \\
=T_{\mathrm{cdm}, \gamma}+T_{\mathrm{rot}-\mathrm{vib}, \gamma}+T_{\mathrm{rot} \text { int }, \gamma}+T_{\mathrm{vib}, \gamma}+T_{\mathrm{e}, \gamma}+V_{\gamma}=T_{\mathrm{cdm}, \gamma}+H_{\mathrm{int}, \gamma}
\end{gathered}
$$

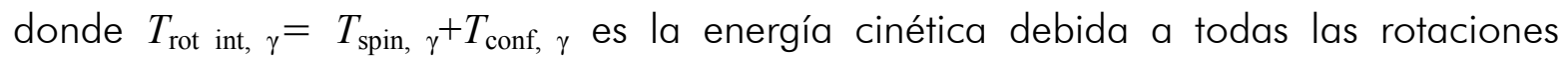
internas, y $V_{\gamma}=V_{\mathrm{e}, \gamma}+V_{\mathrm{n}, \gamma}$ depende de las coordenadas de los núcleos y electrones de la molécula. Se ha definido el hamiltoniano de los grados de libertad internos de la molécula como la cantidad: $H_{\text {int, } \gamma}=T_{\text {rot-vib, } \gamma}+T_{\text {rot int, } \gamma}+T_{\text {vib, } \gamma}+T_{\mathrm{e}, \gamma}+V_{\gamma}$, de modo que el hamiltoniano de la molécula se puede escribir como la contribución de la traslación de su centro de masas y de los grados internos de libertad. En rigor, no son todos grados de libertad internos, pues no lo son las rotaciones de la molécula como un todo.

Estudiado el caso de una molécula aislada, es más fácil comprender la primitiva clasificación de los sistemas complejos atediendo a la naturaleza de sus grados de libertad. Así, en un sistema de $N$ moléculas se supone que existe un hamiltoniano total igual a:

$$
H=-\sum_{\gamma=1}^{N} \frac{\hbar^{2}}{2 \cdot m_{\gamma}} \cdot \vec{\nabla}_{\gamma}^{2}+W\left(\vec{R}_{1}, \ldots \vec{R}_{N} ; \vec{\Omega}_{1}, \ldots, \vec{\Omega}_{N} ; \vec{Q}_{1}, \ldots \vec{Q}_{N} ; \vec{S}_{1}, \ldots \vec{S}_{N} ; \vec{\xi}_{1}, \ldots \vec{\xi}_{N}\right)
$$

El primer término es el operador energía cinética, que incluye todas las posibles traslaciones, rotaciones, vibraciones,... de las $N$ moléculas y por lo general no es una suma del tipo $\sum H_{\gamma}$, siendo $H_{\gamma}$ el dado en (6.6) El segundo término es el operador de energía de interacción intermolecular, que en general depende de las posiciones de los centros de masas de los núcleos y los electrones, orientaciones de las moléculas, coordenadas normales,... las cuales pueden no ser independientes entre sí, es decir, los diferentes grados de libertad pueden estar acopladas. Así, en función de la forma de la energía de interacción intermolecular $W$, se puede hacer la siguiente clasificación de los sistemas físicos:

a) Sistema ideal: la energía de interacción es despreciable. En este caso, los grados de libertad de las moléculas permanecen invariantes respecto del caso de las moléculas libres. A este grupo pertenecen sistemas como los gases perfectos, la mezcla de gases perfectos, las mezclas diluidas de no electrolitos, el cristal ideal de Einstein (visto como gas ideal cuántico degenerado de bosones independientes, los fonones), etc. En estos casos, el hamiltoniano total del sistema es separable con respecto las coordenadas de las moléculas individuales, de manera que se puede limitar la discusión a la ecuación de Schrödinger de cada molécula [74]:

$$
H=\sum_{\gamma=1}^{N} H_{\gamma}=\sum_{\gamma=1}^{N}\left(T_{\mathrm{cdm}, \gamma}+H_{\mathrm{int}, \gamma}\right)=T_{\mathrm{cdm}}+H_{\mathrm{int}}
$$

En este caso,

$$
T_{\mathrm{cdm}}=\sum_{\gamma=1}^{N} T_{\mathrm{cdm}, \gamma}=\sum_{\gamma=1}^{N} \frac{P_{\mathrm{cdm}, \gamma}^{2}}{2 \cdot m_{\gamma}}
$$


La solución al problema estadístico es trivial ya que la función de partición del sistema, suponiendo que las $N$ moléculas son indistinguibles (luego $m=m_{\gamma}=$ cte.) y que la aproximación semiclásica* es válida, es igual a:

$$
Z=\frac{Z_{1}^{N}}{N !}=\frac{\left(Z_{\mathrm{t}, 1} \cdot Z_{\mathrm{int}, 1}\right)^{N}}{N !}
$$

donde,

$$
Z_{\mathrm{t}, 1}=\frac{\left(2 \cdot \pi \cdot m \cdot k_{\mathrm{B}} \cdot T\right)^{3 / 2} \cdot V}{h^{3}}=\Lambda^{-3} \cdot V
$$

$\Lambda=h \cdot\left(2 \cdot \pi \cdot m \cdot k_{\mathrm{B}} \cdot T\right)^{-1 / 2}$ es la longitud de onda de de Broglie asociada a la molécula de masa $m$.

Este tipo de sistemas carecen de estructura alguna pues no hay correlación entre las moléculas. De hecho, $\xi=0$.

b) Sistema no ideal, colectivo o cooperativo: como sabemos, en este tipo de sistemas las interacciones son tan importantes que el estado de cada constituyente depende del estado del resto, y es prácticamente imposible tratar cada uno de ellos como independiente. Las propiedades de las individualidades son irrelevantes debido a la aparición de un mecanismo que otorga estructura al sistema. En este caso no tiene sentido hablar del hamiltoniano de cada molécula. Los líquidos, los cristales vistos como un conjunto de átomos o moléculas y las sustancias ferromagnéticas son ejemplos típicos de este tipo de sistemas.

La diferencia fundamental entre los sistemas no ideales y los sistemas ideales es pues que en los primeros los elementos constitutivos no son independientes, y ni siquiera se puede hablar de hamiltoniano. No obstante, antes de aceptar esto, lo cual tiene que ver con el concepto de emergencia de las propiedades de un sistema macroscópico, el punto de vista predominante era que dicho hamiltoniano existe; así, es común hablar de acoplamiento de los grados de libertad moleculares debido al potencial de interacción, el cual puede no ser total, lo que puede simplificar el problema enormemente. Sólo en este contexto es comprensible la clasificación de los líquidos en relación al análisis del comportamiento de sus grados de libertad. Dicha clasificación está reflejada en las monografías de Fowler \& Guggenheim y Prigogine \& Defay $[77$ pp 319], [78, pp 409]:

- Líquidos normales: son aquellos cuyos grados de libertad internos de las moléculas no están afectados por su ambiente molecular, es decir, no dependen de la densidad; por lo tanto, no se alteran al pasar de la fase vapor a la fase líquida ni al pasar de líquidos puros a mezclas. Así, las contribuciones al hamiltoniano debidas a los grados de libertad internos son iguales para gases, líquidos puros y mezclas. Por el contrario, las coordenadas que dan cuenta de las traslaciones de los centros de masa

\footnotetext{
*En la aproximación semiclásica, la separación entre niveles de energía es mucho menor que $k_{\mathrm{B}} \cdot T$, de modo que la energía puede considerarse una variable continua. Esto ocurre cuando las temperaturas son lo suficientemente altas, o bien, las densidades lo suficientemente bajas. En estas condiciones, el número de partículas es mucho menor que el número de niveles energéticos accesibles, por lo que los números de ocupación de los diferentes niveles energéticos vienen descritos por la estadística de Maxwell-Boltzmann, [76, pp 159, 187]. Aunque los estudios del hidrógeno y del helio líquidos no pueden realizarse en el ámbito de esta aproximación, se supone que para el resto de líquidos es adecuada.
} 
de las moléculas sí están afectados por la densidad. Para este tipo de sistemas, el hamiltoniano total es:

$$
H=\sum_{\gamma=1}^{N} H_{\mathrm{int}, \gamma}+T_{\mathrm{cdm}}
$$

donde $H_{\text {int, } \gamma}$ es el mismo que en (6.6) y:

$$
T_{\mathrm{cdm}}=\sum_{\gamma=1}^{N} \frac{P_{\mathrm{cdm}, \gamma}^{2}}{2 \cdot m_{\gamma}}+W\left(\vec{R}_{1}, \ldots \vec{R}_{N}\right)
$$

Como consecuencia de la separabilidad (6.12) del hamiltoniano, la función de partición del sistema se puede factorizar. En la aproximación semiclásica,

$$
\begin{gathered}
Z=Z_{\mathrm{t}} \cdot Z_{\text {int }}=Z_{\mathrm{t}} \cdot Z_{\text {int, } 1}^{N}= \\
=\left[\frac{1}{N !} \cdot \Lambda^{-3 \cdot N} \cdot \int \ldots \int e^{-W\left(\vec{R}_{1}, \ldots, \vec{R}_{N}\right) / k_{\mathrm{B}} T} \mathrm{~d} \vec{R}_{1} \cdot \ldots \cdot \mathrm{d} \vec{R}_{N}\right] \cdot Z_{\text {int, } 1}^{N}=\Lambda^{-3 \cdot N} \cdot Z_{\text {int }} \cdot \Omega(T)
\end{gathered}
$$

Donde se ha definido la función de partición configuracional:

$$
\Omega(T)=\frac{1}{N !} \cdot \int \ldots \int e^{-W\left(\vec{R}_{1}, \ldots, \vec{R}_{N}\right) / k_{\mathrm{B}} T} \mathrm{~d} \vec{R}_{1} \cdot \ldots \cdot \mathrm{d} \vec{R}_{N}
$$

La factorización de $Z$ permite escribir las funciones termodinámicas del sistema como la suma de diferentes términos. Por ejemplo, la capacidad calorífica a volumen constante es:

$$
C_{V}=C_{V, \Lambda}+C_{V, \text { int }}+C_{V, \Omega}
$$

Donde $C_{V, \Lambda}$ es la contribución debida a longitud de onda de de Broglie; $C_{V \text {,int }}$ es la contribución debida a la libración de las moléculas, a la vibración de los enlaces interatómicos, a los movimientos electrónicos, de spin nuclear y a rotaciones conformacionales; $C_{V, \Omega}$ es la contribución debida a las interacciones entre moléculas, lo que se llama contribución configuracional.

En mezclas de moléculas de los tipos A, B,..., la función de partición es:

$$
\begin{gathered}
Z=\prod_{\mathrm{i}}\left[\Lambda_{\mathrm{i}}^{-3 \cdot N_{\mathrm{i}}} \cdot\left(Z_{\text {int }}\right)_{\mathrm{i}} \cdot \Omega(T)\right]= \\
=\prod_{\mathrm{i}}\left[\Lambda_{\mathrm{i}}^{-3 \cdot N_{\mathrm{i}}} \cdot\left(Z_{\mathrm{int}}\right)_{\mathrm{i}} \cdot \frac{1}{N_{\mathrm{i}} !}\right] \cdot \int \ldots \int e^{-W\left(\vec{R}_{\mathrm{I}_{\mathrm{A}}}, \ldots, \vec{R}_{N_{\mathrm{A}}}, \vec{R}_{\mathrm{l}_{\mathrm{B}}}, \ldots, \vec{R}_{N_{\mathrm{B}}}, \ldots\right) / k_{\mathrm{B}} T} \prod_{\mathrm{i}}\left(\mathrm{d} \vec{R}_{\mathrm{l}_{\mathrm{i}}} \cdot \ldots \cdot \mathrm{d} \vec{R}_{N_{\mathrm{i}}}\right)
\end{gathered}
$$

Es inmediato ver de (6.17) que $\Omega(T)$ en mezclas no depende únicamente de la posición de las moléculas sino también de qué tipo de molécula $A, B, \ldots$ está en cada posición del espacio, es decir, $W$ cambia si las posiciones de un par de moléculas de especies químicas diferentes se intercambian. No obstante, existen dos simplificaciones teóricas importantes para $\Omega(T)$ que dan lugar a los tipos de mezcla siguientes:

Mezcla ideal: los potenciales de interacción de las especies moleculares diferentes son iguales. En este caso $W$ es insensible a los cambios en la asignación de cada especie a un punto del espacio, de modo que las diferentes especies químicas están distribuidas aleatoriamente en todo el espacio. Pese a que la mezcla de isótopos 
se ha considerado típicamente ideal, se sabe que la mezcla $\mathrm{He}^{3}+\mathrm{He}^{4}$ tiene lagunas de miscibilidad.

Mezcla aleatoria o al azar: si $W$ se sustituye por su valor promedio sobre todas las asignaciones de las moléculas a las $N_{\mathrm{A}}+N_{\mathrm{B}}+\ldots$ posiciones de cada configuración. En una mezcla aleatoria, una molécula no muestra preferencia alguna a la hora de escoger a sus vecinos y por tanto todas las posibles distribuciones de las moléculas son igualmente probables. La hipótesis de mezcla aleatoria descansa sobre fundamentos físicos poco realistas, y de hecho sólo es estrictamente válida en el caso de mezclas de moléculas con tamaño similar (luego $V_{\mathrm{m}}^{\mathrm{E}}=0$ ). El empaquetamiento de los líquidos impide ver como sensata la suposición de que moléculas de especies químicas distintas puedan intercambiarse aleatoriamente si tienen distinto tamaño, pues una molécula pequeña en una red no puede intercambiarse por otra más grande, ya que no hay sitio para la segunda. En rigor, sólo una mezcla ideal puede ser completamente al azar.

Como consecuencia de todo lo dicho, al estudiar las funciones de mezcla y exceso de disoluciones de líquidos normales sólo las integrales configuracionales son importantes. Por ejemplo, la energía molar libre de Helmholtz de mezcla es,

$$
\Delta_{\mathrm{m}} F_{\mathrm{m}}=-k_{\mathrm{B}} \cdot T \cdot \ln \Omega_{\mathrm{m}}-\left(-k_{\mathrm{B}} \cdot T \cdot \sum_{\mathrm{i}} x_{\mathrm{i}} \cdot \ln \Omega_{\mathrm{i}}^{0}\right)
$$

donde $\Omega_{\mathrm{m}}(T)$ es la función de partición configuracional de un mol de mezcla y $\Omega_{\mathrm{i}}{ }^{0}(T)$ es la misma función para un mol de sustancia pura. La energía molar libre de Helmholtz de exceso es:

$$
F_{\mathrm{m}}^{\mathrm{E}}=\Delta_{\mathrm{m}} F_{\mathrm{m}}-\Delta_{\mathrm{m}} F_{\mathrm{m}}(\text { ideal })=k_{\mathrm{B}} \cdot T \cdot\left[-\ln \Omega_{\mathrm{m}}+\sum_{\mathrm{i}} x_{\mathrm{i}} \cdot\left(\ln \Omega_{\mathrm{i}}^{0}-\ln x_{\mathrm{i}}\right)\right]
$$

Las interacciones posibles entre las moléculas de los líquidos normales son fuertes repulsiones a distancias cortas, que dan cuenta del tamaño de las moléculas y son debidas a que el solapamiento de los orbitales moleculares está prohibido por el principio de exclusión de Pauli (suponiendo que no hay reacciones químicas) y atracciones a distancias largas debidas a las fuerzas dispersivas o de tipo London entre dipolos instantáneos. Los dipolos instantáneos existen en todos los tipos de molécula, y son creados por las fluctuaciones en las posiciones de los núcleos y de los electrones en los orbitales moleculares, las cuales originan descompensaciones en la distribución de las cargas de las moléculas.

- Líquidos asociados: en este tipo de líquidos existen fuerzas que acoplan los grados de libertad internos y de traslación de los centros de masas. Por ejemplo, las moléculas polares se orientan dependiendo de la posición de las moléculas vecinas, del mismo modo que un imán se alinea con otro cercano. También puede ocurrir que las vibraciones atómicas estén afectadas por la cercanía de otras moléculas [79]. Así mismo, las rotaciones respecto los enlaces en moléculas flexibles pueden depender de la proximidad de otras moléculas, dando lugar a efectos conformacionales (por ejemplo en mezclas con 1,2-dibromometano se ha observado que si se añade ciclohexano la fracción molar relativa de los rotámeros tipo gauche disminuye, mientras que aumenta si se añade benceno). En rigor, no solo la orientación de las moléculas, las vibraciones atómicas y las rotaciones internas, sino también los movimientos electrónicos y las rotaciones nucleares dependen del ambiente molecular. En definitiva, los líquidos 
asociados son aquellos cuyos grados de libertad internos de las moléculas dependen de la densidad, es decir, cambian al pasar de la fase vapor a la fase líquida (y del líquido puro a la mezcla). Esto ha sido refrendado por al análisis de los espectros de absorción roto-vibracional, que demuestran que en sustancias que interactúan mediante fuerzas dipolares fuertes y en los que aparecen complejos supramoleculares (por la formación de puentes de hidrógeno [80], [81] o por transferencias de carga) los niveles de energía dependen del ambiente molecular. Lo mismo se encuentra en el estudio de moléculas muy polares en las que el momento dipolar está en la periferia, lo que se llama efecto aromático, que explica la polarizabilidad local y es importante en la aparición de efectos conformacionales [82]. Por lo tanto, la definición clásica de líquido asociado como aquél que puede formar complejos es errónea ya que incluye un tipo de líquido más amplio.

El acoplamiento entre los grados de libertad internos y de traslación de los centros de masas no permite escribir el hamiltoniano como en la ecuación (6.12). Por lo tanto, la factorización (6.14) de la función de partición no es posible, y en consecuencia las funciones de mezcla y de exceso dependen no solo de los grados de libertad intermoleculares sino también de los intramoleculares, pues éstos últimos no se cancelan al calcular las funciones de mezcla.

Las fuerzas dipolares fuertes son la consecuencia de interacciones tipo dipolo permanente-dipolo permanente, propias de aquellas moléculas cuya distribución de cargas es asimétrica. Este tipo de fuerzas tiene a alinear los dipolos, por eso se dice que son fuerzas orientacionales. Una molécula polar aislada está caracterizada por su momento dipolar eléctrico permanente $\mu$. Sin embargo, cuando la molécula no está aislada, las interacciones entre las moléculas no están completamente descritas por $\mu$, en primer lugar porque la agitación térmica tiende a destruir los efectos orientacionales (alineación de los dipolos). Por ello, la molécula tiene un momento dipolar efectivo $\bar{\mu}$ :

$$
\bar{\mu}=\sqrt{\frac{\mu^{2} \cdot N_{A}}{4 \cdot \pi \cdot \varepsilon_{0} \cdot V_{\mathrm{m}} \cdot k_{\mathrm{B}} \cdot T}}
$$

donde $V_{\mathrm{m}}$ es el volumen molar.

En segundo lugar, el campo eléctrico creado por el resto de moléculas induce un dipolo debido a que la molécula tiene cierta polarizabilidad, $\alpha$. Aparecen así fuerzas del tipo dipolo permanente-dipolo inducido, que en general son menos importantes que las interacciones dipolo permanente-dipolo permanente (en ocasiones pueden ser muy grandes, como es el caso de moléculas aromáticas en las que los electrones $\pi$ tienen gran movilidad, es decir, la molécula tiene mucha polarizabilidad). En conclusión, $\mu$ no caracteriza el verdadero comportamiento eléctrico de la molécula, sino la polarizabilidad, $\alpha$, y el momento dipolar efectivo, $\bar{\mu}$.

El tratamiento exacto de las fuerzas dipolares es muy complejo debido a la anisotropía de los campos de fuerzas, es decir, a la dependencia con no sólo las distancias sino también los ángulos. Los grados de libertad de rotación de la molécula como un todo están acoplados con los grados de libertad de traslación de centro de masas, lo cual se nota en la forma de $W$. El efecto orientacional es mayor cuanto mayor sea el carácter polar de las moléculas, y por tanto, en estas disoluciones la hipótesis de mezcla al azar no es válida, lo que introduce problemas combinatoriales muy difíciles. Sin embargo, las fuerzas dipolares pueden tratarse mediante una aproximación basada en que mientras 
que las fuerzas dispersivas pueden aparecer en cualquier tipo de molécula, ya que las fluctuaciones en la distribución de las cargas siempre pueden ocurrir, siendo la magnitud de dichas fuerzas importante y y por tanto no pueden ignorarse, las contribuciones de las fuerzas de inducción no suelen ser mayores al 7\%, y de los dipolos permanentes a temperaturas cercanas a la temperatura ambiente son menores al $5 \%$ (para valores del momento dipolar menores de 1 D), disminuyendo a medida que aumenta la temperatura pues la agitación térmica tiende a desalinear los dipolos. Por esta razón, en sustancias en las que las fuerzas dipolares son lo suficientemente débiles comparadas con las dispersivas, las rotaciones de las moléculas pueden considerarse prácticamente libres, y el grado de desorden es tal que se puede considerar distribución al azar. En este caso los efectos macroscópicos pueden tratarse mediante un promedio de las interacciones dipolares sobre todas las posibles orientaciones de las moléculas, es decir, más que tratar con la función energía $W\left(\vec{R}_{1}, \ldots \vec{R}_{N}, \vec{\Omega}_{1}, \ldots, \vec{\Omega}_{N}\right)$, se trata con una función promedio de todas las posibles orientaciones, en la que la dependencia con la temperatura no es de extrañar, ya que a medida que ésta aumenta el grado de orden desaparece: $\tilde{W}\left(\vec{R}_{1}, \ldots \vec{R}_{N} ; T\right)$, que es una energía libre. En estos casos el tratamiento es similar al de los líquidos normales.

En los líquidos que presentan enlaces de hidrógeno, los modos de vibración de los átomos están tan fuertemente acoplados que casi se forman enlaces químicos verdaderos entre ellos. En estos líquidos no tiene sentido hablar de moléculas, pues éstas se unen fuertemente formando complejos o unidades supramoleculares (dímeros, trímeros, tetrámeros,...). El tamaño de las unidades supramoleculares depende del número de enlaces efectivos que pueda realizar cada molécula: si es uno, serán dímeros; si es dos, serán anillos cerrados o cadenas abiertas; si es tres o más, se deberá considerar el líquido como un todo. Tanto la presión como el movimiento térmico disminuyen el número de enlaces efectivos, es decir el grado de asociación. Por ello, todo líquido que en condiciones normales forma complejos, no lo hace en las proximidades del punto crítico. Estos líquidos, de los cuales el agua es un ejemplo típico, tienen coeficientes de expansión anómalos, alta viscosidad, altos puntos de ebullición, grandes calores latentes de cambio de estado y baja tensión superficial. Son susceptibles de permanecer en estados metaestables y de formar vidrios.

Cuando se discuten aspectos relacionados con sustancias que presentan enlaces de hidrógeno, es útil hablar de autoasociación o simplemente asociación para referirse a la formación de complejos por la unión de moléculas del mismo tipo y de solvatación, cuando las moléculas que forman el complejo son diferentes.

Un enlace puente de hidrógeno intermolecular se forma entre una molécula donadora de hidrógeno -AH y un sitio receptor rico en electrones $\mathrm{B}$. La entidad $\mathrm{A}$ es un átomo $\mathrm{O}$ conjunto de átomos más electronegativo que el hidrógeno, mientras que el sitio B puede ser un átomo más electronegativo que el hidrógeno, un enlace doble o triple o un hidrocarburo aromático. Ejemplos de fuertes donadores de hidrógeno son el fluoruro de hidrógeno $(\mathrm{HF})$, el agua $\left(\mathrm{H}_{2} \mathrm{O}\right)$, el peróxido de hidrógeno $\left(\mathrm{H}_{2} \mathrm{O}_{2}\right)$, los alcoholes $(\mathrm{R}-\mathrm{OH})$, los ácidos carboxílicos $(\mathrm{R}-\mathrm{COOH})$, el amoniaco $\left(\mathrm{NH}_{3}\right)$ y las aminas primarias $\left(\mathrm{R}-\mathrm{NH}_{2}\right)$ y secundarias $\left(\mathrm{R}_{2}-\mathrm{NH}\right)$. En cada una de estas moléculas, uno o más átomos de hidrógeno están unidos a un átomo de un elemento muy electronegativo ( $\mathrm{F}, \mathrm{O}$ ó $\mathrm{N}$ ). Los ácidos halogenados, $\mathrm{HCl}, \mathrm{HBr}$ y $\mathrm{HI}$, también son donadores de hidrógeno, como lo son algunas pocas especies que contienen el enlace $\mathrm{C}-\mathrm{H}$. Sin embargo, la diferencia de 
electronegatividad entre el carbono y el hidrógeno no es grande por lo que la capacidad de estas especies para actuar como donador de hidrógeno sólo es posible cuando el átomo de carbono está unido a otro átomos altamente electronegativos o a sitios ricos en electrones, como ocurre en el caso del cloroformo $\left(\mathrm{Cl}_{3} \mathrm{CH}\right)$, diclorometano $\left(\mathrm{Cl}_{2} \mathrm{CH}_{2}\right)$ y cianuro de hidrógeno $(\mathrm{NCH})$. Por otra parte, elementos altamente electronegativos sirven como sitios receptores de hidrógeno, por ejemplo, $\mathrm{HF}, \mathrm{H}_{2} \mathrm{O}, \mathrm{H}_{2} \mathrm{O}_{2}$, $\mathrm{R}-\mathrm{OH}, \mathrm{R}-\mathrm{COOH}, \mathrm{NH}_{3}, \mathrm{R}-\mathrm{NH}_{2}, \mathrm{R}_{2}-\mathrm{NH}$ y $\mathrm{NCH}$. Como estas especies también son donadoras de hidrógeno, todas ellas están (auto)-asociadas. Los aldehídos (R-OCH), las cetonas (R$O C R)$, los éteres (R-O-R), los ésteres (R-OCO-R) y las aminas terciarias $\left(R_{3} N\right)$ también son receptores de hidrógeno, luego pueden solvatarse con sustancias donadoras. Se habla de solvatación "pura"cuando ninguna de las especies que forman el complejo está asociada (por ejemplo, acetona + cloroformo). Sin embargo, puede ocurrir solvatación entre dos sustancias asociadas (etanol y agua), entre un asociado y un donador de hidrógeno (etanol y cloroformo) y entre un asociado y un receptor de hidrógeno (etanol y acetona) [83].

Por otra parte, se ha encontrado evidencia experimental de la formación de complejos entre hidrocarburos aromáticos (por ejemplo, el benceno) y ciertos compuestos polares no donadores de hidrógeno (piridina, cetonas y aldehídos), por lo que la interacción no puede ser de tipo enlace puente de hidrógeno. Por ello al complejo resultante se le llama complejo de transferencia de carga [84].

El tratamiento riguroso de este tipo de líquidos por medios de la Física Estadística tradicional es inviable. Aunque se pensó que esto era consecuencia de la dificultad asociada a la dependencia de $W$ con las coordenadas normales, los grados de libertad rotacionales y con el resto de grados de libertad internos que pudiesen estar acoplados, lo cierto es que todas estas discusiones son erróneas porque no se puede hablar de hamiltoniano, ni de grados de libertad de las moléculas. Los líquidos que forman complejos son un caso particular de sistemas cooperativos que interaccionan fuertemente, en los que no tiene sentido hablar del individuo, y en los que aparece una superestructura (unidad supramolecular) que es consecuencia del comportamiento colectivo. Por ello, es imposible aplicar la Física Estadística tradicional a los líquidos que forman complejos. En consecuencia, la anterior clasificación de los sistemas en ideales y no ideales y de los líquidos en normales y asociados no es apropiada pues es fruto de una discusión en torno a los grados de libertad moleculares, es decir, está basada en conceptos intrínsecos a las individualidades que componen el sistema, los cuales dejan de tener sentido en sistemas que interaccionan fuertemente, como sabemos. Tal es el caso del agua, los ácidos carboxílicos, los alcoholes, las aminas primarias, etc. Además, el apelativo de líquido normal puede inducir a pensar que los líquidos asociados son anormales, raros. No obstante, 己̇es acaso rara el agua, siendo componte mayoritario del planeta Tierra y de los organismos vivos? Por lo tanto, es necesaria una mejor clasificación de los sistemas con muchas partículas, más coherente. Aquí se propone la siguiente:

a) Sistemas ideales: en ellos las interacciones son nulas. La función de partición es el producto de las funciones de partición asociadas a cada unidad constiutiva del sistema, es decir:

$$
Z=\frac{Z_{1}^{N}}{N !}=\frac{\left(Z_{\mathrm{t}, 1} \cdot Z_{\mathrm{int}, 1}\right)^{N}}{N !}
$$


En este tipo de sistemas la entidad elemental que permite resolver la función de partición es la unidad constitutiva del sistema: por ejemplo, la molécula en el caso de gases perfectos, mezclas de gases perfectos o mezclas diluidas de no electrolitos, o el fonón en el caso de cristales ideales. Respecto a la clasificación anterior, esto no cambia.

b) Sistemas no ideales. La función de partición no puede escribirse como (6.21). No obstante, en el caso de los líquidos puede hacerse la distinción siguiente:

- Líquidos simples: son aquellos en los que aún tiene sentido hablar de molécula. En estos casos, la función de partición puede escribirse como:

$$
Z=\Lambda^{-3 \cdot N} \cdot Z_{\text {int }} \cdot \Omega(T)
$$

donde $Z_{\text {int }}$ no se refiere completamente a los grados internos de libertad puesto que en este término están incluidas las rotaciones y vibraciones de la molécula como un todo. La experiencia ha demostrado que esta es una buena aproximación en aquellos sistemas en los que hay presentes fuerzas de tipo dispersivo, y/o fuerzas dipolares.

En este tipo de sistemas la entidad elemental es la molécula, situada dentro de un campo de fuerzas central efectivo que es el promedio debido a las interacciones con el resto de moléculas y que es función de la temperatura si las fuerzas no son solamente de tipo central, lo que incluye las fuerzas entre dipolos. Es cierto que los potenciales de interacción entre las moléculas no son necesariamente centrales, pero también es cierto que los métodos de cálculo actuales sólo son viables para el caso de las fuerzas centrales. Por ello, se busca sustituir los campos de fuerza reales no centrales por campos de fuerza centrales efectivos, dependientes de la temperatura. De hecho, una de las grandes preguntas que deben contestarse en el futuro es:

¿Hasta qué punto puede sustituirse el efecto de las fuerzas no centrales que actúan entre las moléculas que forman una mezcla por otros potenciales centrales efectivos dependientes de la temperatura que actúan entre los posibles agregados moleculares de orden superior que constituirían las entidades elementales necesarias para describir de forma teóricamente rigurosa las disoluciones?

- Líquidos complejos o emergentes: la función de partición no puede factorizarse como en (6.21) ni como (6.22). En su lugar, la única forma de calcularla es a partir de la función de Massieu (macroscópica):

$$
Z=e^{\frac{\psi}{k_{\mathrm{B}}}}=e^{\frac{-F}{k_{\mathrm{B}} \cdot T}}
$$

A esta clase pertenecen aquellos líquidos cuyas moléculas interaccionan fuertemente, mediante puentes de hidrógeno o complejos de transferencia de carga por ejemplo. Las interacciones son tan fuertes que el sistema está autoorganizado. No tiene sentido hablar de las moléculas individualmente, sino que debe tratarse la muestra de líquido como un todo. No obstante, cuando la asociación entre moléculas es parcial, es decir, cuando el número de enlaces efectivos es menor que tres, es una buena estrategia a seguir definir como entidad elemental a la unidad supramolecular (dímero, trímero, tetrámero,...) correspondiente. Ello permite en primera instancia hacer un tratamiento aproximado de este tipo de líquidos idéntico al de los líquidos simples. Y otra posibilidad es suponer que existe una mezcla de líquidos simples, las llamadas especies verdaderas, cuyas unidades elementales (dímeros, trímeros, tetrámeros,...) están en equilibrio químico. Por ejemplo, en una mezcla binaria, además de los componentes nominales 
(monómeros $A$ y $B$ ) están presentes diferentes especies verdaderas $A_{i} B_{j}, A_{i}$ y $B_{j}$. En esta aproximación se supone que cada especie verdadera, es decir, cada unidad supramolecular, tiene bien definida su función de partición de los grados internos de libertad, en las que la dependencia angular se ha sustituido por una dependencia respecto de la temperatura, es decir, en los que los potenciales reales se sustituyen por potenciales efectivos dependientes de la temperatura. No obstante, hay que tener en cuentas las limitaciones de este tipo de tratamiento debidas a la consideración de un equilibrio químico entre sustancias. Por otra parte, si el número de enlaces efectivos es tres o mayor, como ocurre en el agua, las aminas primarias, etc., la unidad elemental es todo el líquido. Existe correlación casi total, $\xi$ es del orden del tamaño total del sistema. El sistema está autoorganizado, y su comportamieto es parecido al de un fluido en su punto crítico. En consecuencia, el sistema no puede describirse en términos microscópicos, sino que debe hacerse en virtud de nuevas propiedades que emergen, definidas de acuerdo con el tamaño del sistema. Así, este tipo de sustancias nunca se podrá tratar mediante el tratamiento mecánico-estadístico tradicional. En su lugar, se debe buscar un patrón de comportamiento macroscópico que las incluyan en una de las clases de universalidad de los exponentes cíticos y que haga su estudio comparable al de sistemas de otras ramas como las biológicas, sociales, económicas,...

Por todo lo dicho, en el estudio de cualquier líquido es fundamental el estudio de los líquidos simples.

Para concluir esta sección, cabe destacar que el tratamiento de un conjunto de partículas que interactúan en la formulación tradicional de la Física Estadística conduce de manera directa al método de los clusters de Ursell-Mayer [85]. En su forma original el método es aplicable en el límite semiclásico a moléculas con simetría esférica y cuyo potencial de interacción molecular es aditivo por pares:

$$
W\left(\vec{R}_{1}, \ldots \vec{R}_{N}\right)=\sum_{1 \leq i \leq j \leq N} u\left(R_{\mathrm{ij}}\right)
$$

donde $u\left(R_{\mathrm{ij}}\right)$ es el potencial de interacción entre las moléculas i y j, el cual depende de la distancia entre ambas $R_{\mathrm{ij}}$. Las interacciones entre tres, cuatro,... moléculas se pueden incluir mediante técnicas perturbativas. La hipótesis de aditividad por pares del potencial de interacción está justificada estrictamente solamente en gases nobles y sus mezclas, aunque también es ampliamente aceptada en el caso de los gases reales pocos densos. Posteriormente, el método se generalizó a sistemas cuánticos con moléculas no esféricas y cuyo potencial de interacción no es aditivo por pares [86]. En cualquier caso, el principal resultado que se obtiene es que el producto $P \cdot V$ puede escribirse como la serie de potencias siguiente:

$$
P \cdot V=k_{B} \cdot T \cdot \sum_{\mathrm{j} \geq 1} b_{\mathrm{j}} \cdot z^{\mathrm{j}}
$$

donde $z=V^{-1} \cdot Z_{1} \cdot \mathrm{e}^{-\mu / k_{\mathrm{B}} \cdot T}, Z_{1}$ es la función de partición de una partícula, y los coeficientes $b_{\mathrm{j}}$ están relacionados con los coeficientes de la expansión del virial [87], [88].

El método tiene los inconvenientes de que introduce el potencial intermolecular como una función conocida a priori (inconveniente que se arrastra en todos los modelos de la Física Estadística tradicional, pues el problema de 3 ó más cuerpos no tiene solución) y de que solamente pueden calcularse los primeros coeficientes del virial, consecuencia también de la no integrabilidad de las ecuaciones de movimiento. Además el método de 
los clusters tiene las limitaciones siguientes: primero, se supone que la serie (6.25) converge; segundo, los coeficientes $b_{\mathrm{j}}$ son independientes del volumen. El cumplimiento de ambas restricciones está garantizado en los gases solamente, como es de esperar puesto que el método de los clusters básicamente consiste en la aplicación estricta de la Física Estadística tradicional a un sistema de partículas que interactúan (es la teoría cinética de los gases mejorada). Por lo tanto, no es capaz de explicar el fenómeno de la condensación ni la fases líquida o sólida [89], [90] puesto que en el salto de nivel complejidad que es esencialmente el cambio de fase, aparecen propiedades emergentes. Por eso la teoría de Yang Lee no funciona. Por ejemplo, en el caso de los cristales aparece una estructura que es consecuencia de la simetría de traslación de la red. Esta estructura debe introducirse ad hoc, es decir, como por arte de magia, sin estar justificada en absoluto por las ecuaciones del movimiento, lo que se consigue introduciendo un modelo de realidad. De hecho, la única opción plausible para el tratamiento de líquidos y sólidos pasa por la construcción de un modelo para el sistema completo, que introduzca de manera ad hoc las propiedades emergentes, es decir, las nuevas simetrías asociadas con el nuevo nivel de complejidad y que además permita calcular la integral de configuración con razonable exactitud. Mientras que en el caso de los sólidos esta simetría es clara (simetría traslacional de una red), en los líquidos no lo es. Esta es la razón por la que el estudio de líquidos es tan complicado.

Sin embargo, quiénes no conocen la noción de complejidad asociada a los sistemas físicos de muchas individualidades en interacción, han intentado comprender el comportamiento de dichos sistemas mediante alguna de las dos posibilidades siguientes:

a) Obtención de las funciones de distribución molecular y a partir de ellas proceder al cálculo de las funciones termodinámicas. Este método es similar al del los clusters, excepto en que el cálculo de la función de partición se sustituye por el de las funciones de distribución molecular. Por tanto este procedimiento no resuelve el problema pues no es capaz de introducir las propiedades emergentes. Sin embargo, a bajas densidades (en el sentido de que la correlación entre las moléculas es muy pequeña, luego lejos de los puntos críticos) es decir, cuando el comportamiento de los gases y los líquidos es similar (y no confundir con igual, lo que ocurre en los puntos críticos) se pueden obtener resultados apreciablemente buenos.

b) Simulación por ordenador [91]. Principalmente existen dos técnicas a considerar: dinámica molecular (MD) y el método de Monte-Carlo (MC). La primera se basa en la creencia de que la manera directa de resolver el problema de muchas partículas que interactúan pasa por resolver la ecuación de Schrödinger para todas ellas:

$$
\left[-\sum_{\mathrm{i}=1}^{N} \frac{\hbar^{2}}{2 \cdot m_{\mathrm{i}}} \cdot \nabla_{\mathrm{i}}^{2}+W\left(\vec{r}_{1}, \ldots, \vec{r}_{\mathrm{N}}\right)\right] \psi=\frac{\hbar}{i} \cdot \frac{\partial \psi}{\partial t}
$$

- las ecuaciones de Newton, Euler-Lagrange, Hamilton o alguna similar. Como el problema de los tres y más cuerpos no tiene solución analítica, el problema debe resolverse numéricamente, lo cual consume gran cantidad de tiempo computacional. Además, como el comportamiento colectivo de un sistema de varios elementos que interactúan es independiente del hamiltoniano, el método impide obtener las propiedades termodinámicas ni ninguna propiedad emergente, por lo que carece de interés a nivel macroscópico. 
El método $M C$ consiste en evaluar las propiedades de equilibrio del sistema en el conjunto canónico, reduciendo el espacio de configuraciones a un conjunto arbitrario de puntos, lo que se consigue generando cadenas markovianas homogéneas tales que cuando la longitud de la cadena tienda a infinito, el promedio sobre la cadena de una función del estado configuracional (es decir, de las posiciones y velocidades de las partículas) sea igual al promedio estadístico calculado habitualmente en términos de la función de partición [92, sec.16.9 $\alpha$ ]. Los principales inconvenientes de este método son: primero, se introduce el potencial de interacción como una función conocida a priori; segundo, la naturaleza de los cálculos involucrados en el método $M C$ restringe el número de partículas que puede considerarse (para los computadores más rápidos es del orden de $10^{3}$ ), por lo que se hace necesaria la extrapolación de las propiedades de estos sistemas finitos a los sistemas macroscópicos $\left(\approx 10^{23}\right.$ partículas), la cual solo es válida cuando el valor de la longitud de correlación del sistema lo permita.

En las secciones 6.3 y 6.4 se exponen la primera de estas dos posibilidades, así como los modelos de disoluciones y líquidos más utilizados.

\subsection{FUNCIONES DE DISTRIBUCIÓN MOLECULAR}

Siempre que las limitaciones inherentes a la naturaleza de los líquidos en estudio y a la aplicabilidad de la Física Estadística lo permitan, es decir, siempre que se estén estudiando líquidos a muy baja densidad, la estructura y las propiedades de dichos líquidos en estado de equilibrio termodinámico pueden describirse por medio de las funciones de distribución molecular, como se ha dicho en la sección anterior. Para definir éstas de manera rigurosa es imprescindible conocer la configuración de cada una de las moléculas presentes en el fluido. Aunque en el caso más general, es necesaria la consideración de las coordenadas que dan cuenta de las rotaciones internas, vibraciones de los átomos respecto de sus posiciones de equilibrio, movimientos electrónicos, etc. para simplificar la notación se supondrá en lo siguiente que las moléculas son rígidas, por lo que basta con considerar el conjunto de 6 coordenadas $\vec{X}_{\gamma}=\left(\vec{R}_{\gamma}, \vec{\Omega}_{\gamma}\right)$. Por tanto, la configuración del sistema de $N$ moléculas viene dada por el conjunto de $6 \cdot N$ coordenadas $\vec{X}^{N}=\left(\vec{X}_{1}, \ldots, \vec{X}_{N}\right)$.

A $V, T$ y $N$ constantes, la Física Estadística permite obtener la distribución de probabilidad del sistema utilizando la colectividad canónica:

$$
P(E)=\frac{e^{-\beta E}}{Z}
$$

$E$ es la energía del sistema en una configuración dada, luego es función de $\vec{X}^{N}$. Como consecuencia, $P(E)=P\left(\vec{X}^{N}\right)^{*}$. Ahora no se hace uso de la probabilidad en el sentido de Jaynes pues no representa el estado de conocimiento de información del observador acerca del microestado del sistema, sino que representa la probabilidad de que la molécula se encuentre en un estado de cierta energía.

\footnotetext{
* Al trabajar en el conjunto canónico, tanto $P\left(\vec{X}^{\mathrm{N}}\right)$ como el resto de funciones, dependen de $T$ y $\rho=N / V$ aunque para simplificar la notación no se escriba explícitamente.
} 
Supongamos que las $N$ moléculas son idénticas, es decir, que el sistema es monocomponente. La probabilidad de que una molécula específica, por ejemplo la 1 , se encuentre en el volumen $S$ (es decir, el vector $\vec{R}_{1}$ se encuentra en la región $S$ ), sea cual sea su orientación, es:

$$
P_{1}(S)=\int_{\vec{R}_{1} \in S} \int P\left(\vec{X}^{N}\right) \cdot \mathrm{d} \vec{X}^{N}
$$

Puesto que todas las partículas son iguales, $P_{1}(S)$ coincide con la fracción de moléculas en la región $S$ respecto del total:

$$
P_{1}(S)=x(S)=\frac{N(S)}{N}
$$

Se define la función $A_{1}\left(\vec{R}_{1}, S\right)$ por:

$$
A_{1}\left(\vec{R}_{1}, S\right)=\left\{\begin{array}{cc}
1, \text { si } & \vec{R}_{1} \in S \\
0, \text { si } & \vec{R}_{1} \notin S
\end{array}\right\}=\int_{S} \delta\left(\vec{R}_{1}-\vec{R}^{\prime}\right) \cdot \mathrm{d} \vec{R}^{\prime}
$$

Si el volumen $S$ es infinitesimalmente pequeño e igual a $\mathrm{d} \vec{R}^{\prime} *$, el número de moléculas en dicha región es:

$$
\begin{aligned}
N\left(\mathrm{~d} \vec{R}^{\prime}\right)=N \cdot P_{1}\left(\mathrm{~d} \vec{R}^{\prime}\right)= & N \cdot \int_{\vec{R}_{1} \in \mathrm{d} \vec{R}^{\prime}} \int P\left(\vec{X}^{N}\right) \cdot \mathrm{d} \vec{X}^{N}=N \cdot \int \ldots \int A_{1}\left(\vec{R}_{1}, \mathrm{~d} \vec{R}^{\prime}\right) \cdot P\left(\vec{X}^{N}\right) \cdot \mathrm{d} \vec{X}^{N}= \\
& =N \cdot \mathrm{d} \vec{R}^{\prime} \int \ldots \int \delta\left(\vec{R}_{1}-\vec{R}^{\prime}\right) \cdot P\left(\vec{X}^{N}\right) \cdot \mathrm{d} \vec{X}^{N}
\end{aligned}
$$

Se define la función espacial de distribución general de singletes moleculares en la región en $\vec{R}^{\prime}$ como:

$$
\rho^{(1)}\left(\vec{R}^{\prime}\right)=\frac{N\left(\mathrm{~d} \vec{R}^{\prime}\right)}{\mathrm{d} \vec{R}^{\prime}}=N \cdot \int \ldots \int \delta\left(\vec{R}_{1}-\vec{R}^{\prime}\right) \cdot P\left(\vec{X}^{N}\right) \cdot \mathrm{d} \vec{X}^{N}=N \cdot P^{(1)}\left(\vec{R}^{\prime}\right)
$$

que es independiente de la orientación de las moléculas, es decir, de $\vec{\Omega}_{\mathrm{i}}$. Las ecuaciones $(6.31)$ y $(6.32)$ permiten interpretar $P^{(1)}\left(\vec{R}^{\prime}\right) \cdot \mathrm{d} \vec{R}^{\prime}$ como igual a $P_{1}\left(\mathrm{~d} \vec{R}^{\prime}\right)$, la probabilidad de encontrar a una molécula específica, por ejemplo la 1, en la región entre $\vec{R}^{\prime}$ y $\vec{R}^{\prime}+\mathrm{d} \vec{R}^{\prime}$. Como los eventos \{encontrar una molécula específica entre $\vec{R}^{\prime}$ y $\left.\vec{R}^{\prime}+\mathrm{d} \vec{R}^{\prime}\right\}$ y $\left\{\right.$ encontrar otra molécula específica entre $\vec{R}^{\prime \prime}$ y $\left.\vec{R}^{\prime \prime}+\mathrm{d} \vec{R}^{\prime \prime}\right\}$ son disjuntos, entonces:

$$
\int_{S} P^{(1)}\left(\vec{R}^{\prime}\right) \cdot \mathrm{d} \vec{R}^{\prime}
$$

es igual a la probabilidad de encontrar una molécula específica, por ejemplo la 1 , en la región $S$, es decir, es igual a $P_{1}(S)$. Así, $P^{(1)}\left(\vec{R}^{\prime}\right)$ es una función densidad de probabilidad y se llama función espacial de distribución específica de singletes moleculares.

\footnotetext{
* Para simplificar la notación se ha utilizado $\mathrm{d} \vec{R}$ indistintamente para referirse a un elemento diferencial de volumen $4 \cdot \pi \cdot R^{2} \cdot \mathrm{d} R$ y a un vector infinitesimal de desplazamiento, luego debe prestarse atención a la hora de interpretar su significado según convenga.
} 
A $\rho^{(1)}\left(\vec{R}^{\prime}\right)$ también se le puede atribuir un significado probabilístico ya que $\rho^{(1)}\left(\vec{R}^{\prime}\right) \cdot \mathrm{d} \vec{R}^{\prime}$ es igual a la probabilidad de encontrar una molécula cualquiera en la región entre $\vec{R}^{\prime}$ y $\vec{R}^{\prime}+\mathrm{d} \vec{R}^{\prime}$ [93]. No obstante, los eventos \{encontrar una molécula cualquiera en $\vec{R}^{\prime}$ y $\left.\vec{R}^{\prime}+\mathrm{d} \vec{R}^{\prime}\right\}$ y $\left\{\right.$ encontrar una molécula cualquiera entre $\vec{R}^{\prime \prime}$ y $\left.\vec{R}^{\prime \prime}+\mathrm{d} \vec{R}^{\prime \prime}\right\}$ no son disjuntos, por lo que la integral:

$$
\int_{S} \rho^{(1)}\left(\vec{R}^{\prime}\right) \cdot \mathrm{d} \vec{R}^{\prime}
$$

no es igual a la probabilidad de encontrar una molécula cualquiera en la región $S$. Por tanto, $\rho^{(1)}\left(\vec{R}^{\prime}\right)$ no es una función densidad de probabilidad y por ello es preferible utilizarla con un significado de densidad local, ya que $N\left(\mathrm{~d} \vec{R}^{\prime}\right)=\rho^{(1)}\left(\vec{R}^{\prime}\right) \cdot \mathrm{d} \vec{R}^{\prime}$ es el número de moléculas en la región $\vec{R}^{\prime}$ y $\vec{R}^{\prime}+\mathrm{d} \vec{R}^{\prime}$ y la integral (6.34) es igual al número de moléculas en la región $S$. Si la región se extiende a todo el volumen del sistema, el resultado de la integral será igual al número total de moléculas del mismo. En fluidos homogéneos e isótropos es igual a la densidad global:

$$
\rho^{(1)}\left(\vec{R}^{\prime}\right)=\rho=\frac{N}{V}=\text { cte. }
$$

$V$ es el volumen total del sistema. Esta igualdad no es general ya que el número de partículas en una región está influenciado por la presencia de un tipo u otro de molécula.

Se define la función espacial específica de distribución por pares $P^{(2)}\left(\vec{R}^{\prime}, \vec{R}^{\prime \prime}\right)$ como una función densidad de probabilidad tal que $P^{(2)}\left(\vec{R}^{\prime}, \vec{R}^{\prime \prime}\right) \cdot \mathrm{d} \vec{R}^{\prime} \cdot \mathrm{d} \vec{R}^{\prime \prime}$ representa la probabilidad de que una molécula específica, por ejemplo la 1 , se encuentre entre $\vec{R}^{\prime}$ y $\vec{R}^{\prime}+\mathrm{d} \vec{R}^{\prime}$ y simultáneamente otra molécula específica distinta, por ejemplo la 2 , se encuentre entre $\vec{R}^{\prime \prime}$ y $\vec{R}^{\prime \prime}+\mathrm{d} \vec{R}^{\prime \prime}$. Por tanto,

$$
\begin{gathered}
P^{(2)}\left(\vec{R}^{\prime}, \vec{R}^{\prime \prime}\right) \mathrm{d} \vec{R}^{\prime} \cdot \mathrm{d} \vec{R}^{\prime \prime}=\int_{\substack{\vec{R}_{1} \in d \vec{R}^{\prime} \\
\vec{R}_{R} \in d \vec{R}^{\prime \prime}}} \int P\left(\vec{X}^{N}\right) \cdot \mathrm{d} \vec{X}^{N}= \\
=\int \ldots \int P\left(\vec{X}^{N}\right) \cdot \delta\left(\vec{R}_{1}-\vec{R}^{\prime}\right) \cdot \delta\left(\vec{R}_{2}-\vec{R}^{\prime \prime}\right) \cdot \mathrm{d} \vec{X}^{N}
\end{gathered}
$$

Para definir la función espacial general de distribución por pares, se han de considerar todas las posibilidades, es decir, el evento \{cualquier molécula entre $\vec{R}^{\prime}$ y $\vec{R}^{\prime}+\mathrm{d} \vec{R}^{\prime}$ y al mismo tiempo cualquier otra molécula distinta entre $\vec{R}^{\prime \prime}$ y $\left.\vec{R}^{\prime \prime}+\mathrm{d} \vec{R}^{\prime \prime}\right\}$, que es la unión de $N \cdot(N-1)$ eventos del tipo \{molécula específica, por ejemplo la i, se encuentre entre $\vec{R}^{\prime}$ y $\vec{R}^{\prime}+\mathrm{d} \vec{R}^{\prime}$ y simultáneamente otra molécula específica, por ejemplo la $\mathrm{j}$, se encuentre entre $\vec{R}^{\prime \prime}$ y $\left.\vec{R}^{\prime \prime}+\mathrm{d} \vec{R}^{\prime \prime}\right\}$. La probabilidad del primer evento es igual a la suma de las probabilidades de todos los eventos del segundo tipo ya que todos ellos son disjuntos al ser los elementos de volumen $\mathrm{d} \vec{R}^{\prime}$ y $\mathrm{d} \vec{R}^{\prime \prime}$ lo suficientemente pequeños como para que al mismo tiempo sólo puedan contener una molécula. Entonces, se define la función espacial general de distribución por pares $\rho^{(2)}\left(\vec{R}^{\prime}, \vec{R}^{\prime \prime}\right)$ de modo que: 


$$
\begin{gathered}
\rho^{(2)}\left(\vec{R}^{\prime}, \vec{R}^{\prime \prime}\right) \cdot \mathrm{d} \vec{R}^{\prime} \cdot \mathrm{d} \vec{R}^{\prime \prime}=N \cdot(N-1) \cdot P^{(2)}\left(\vec{R}^{\prime}, \vec{R}^{\prime \prime}\right) \mathrm{d} \vec{R}^{\prime} \cdot \mathrm{d} \vec{R}^{\prime \prime}= \\
\quad=\int \ldots \int P\left(\vec{X}^{N}\right) \cdot \sum_{\substack{i=1 \\
i \neq j}}^{N} \sum_{j=1}^{N} \delta\left(\vec{R}_{\mathrm{j}}-\vec{R}^{\prime}\right) \cdot \delta\left(\vec{R}_{\mathrm{j}}-\vec{R}^{\prime \prime}\right) \cdot \mathrm{d} \vec{X}^{N}
\end{gathered}
$$

es la probabilidad de que haya una molécula cualquiera entre $\vec{R}^{\prime}$ y $\vec{R}^{\prime}+\mathrm{d} \vec{R}^{\prime}$ y al mismo tiempo cualquier otra molécula diferente entre $\vec{R}^{\prime \prime}$ y $\vec{R}^{\prime \prime}+\mathrm{d} \vec{R}^{\prime \prime}$. Esta cantidad también es igual al número de pares de moléculas ocupando los elementos de volumen $\mathrm{d} \vec{R}^{\prime}$ y $\mathrm{d} \vec{R}^{\prime \prime}$. De hecho, el número total de pares de moléculas contenido en el volumen total del sistema es:

$$
\iint_{V} \rho_{V}^{(2)}\left(\vec{R}^{\prime}, \vec{R}^{\prime \prime}\right) \cdot \mathrm{d} \vec{R}^{\prime} \cdot \mathrm{d} \vec{R}^{\prime \prime}=N \cdot(N-1)
$$

Cuando el sistema no presenta interacciones, o bien cuando la separación entre las dos moléculas consideradas es muy grande en comparación con el tamaño molecular, la probabilidad de que una molécula cualquiera se encuentre entre $\vec{R}^{\prime}$ y $\vec{R}^{\prime}+\mathrm{d} \vec{R}^{\prime}$ es independiente de que cualquier otra molécula se encuentre entre $\vec{R}^{\prime \prime}$ y $\vec{R}^{\prime \prime}+\mathrm{d} \vec{R}^{\prime \prime}$. En estos casos puede escribirse:

$$
\rho^{(2)}\left(\vec{R}^{\prime}, \vec{R}^{\prime \prime}\right) \mathrm{d} \vec{R}^{\prime} \cdot \mathrm{d} \vec{R}^{\prime \prime}=\left[\rho^{(1)}\left(\vec{R}^{\prime}\right) \cdot \mathrm{d} \vec{R}^{\prime}\right] \cdot\left[\rho^{(1)}\left(\vec{R}^{\prime \prime}\right) \cdot \mathrm{d} \vec{R}^{\prime \prime}\right]
$$

es decir, es posible realizar la factorización $\rho^{(2)}\left(\vec{R}^{\prime}, \vec{R}^{\prime \prime}\right)=\rho^{(1)}\left(\vec{R}^{\prime}\right) \cdot \rho^{(1)}\left(\vec{R}^{\prime \prime}\right)$ y se dice que las densidades locales no están correlacionadas. Este resultado es falso si hay presentes interacciones entre las moléculas, por lo que la probabilidad de que una molécula se encuentre en una región está influenciada por la localización de otra molécula en otra posición cercana. En estos casos, la desviación respecto de la factorización de $\rho^{(2)}\left(\vec{R}^{\prime}, \vec{R}^{\prime \prime}\right)$ como el producto de $\rho^{(1)}\left(\vec{R}^{\prime}\right)$ y $\rho^{(1)}\left(\vec{R}^{\prime \prime}\right)$ se mide en términos de la función de correlación por pares, $g\left(\vec{R}^{\prime}, \vec{R}^{\prime \prime}\right)$, definida de modo que:

$$
\rho^{(2)}\left(\vec{R}^{\prime}, \vec{R}^{\prime \prime}\right)=\rho^{(1)}\left(\vec{R}^{\prime}\right) \cdot \rho^{(1)}\left(\vec{R}^{\prime \prime}\right) \cdot g\left(\vec{R}^{\prime}, \vec{R}^{\prime \prime}\right)
$$

Lejos del punto crítico, dos moléculas a distancias mucho mayores que el tamaño molecular no interaccionan, es decir, no están correlacionadas. Por tanto, se cumple la condición:

$$
\lim _{\left\|\vec{R}^{\prime}-\vec{R}^{\prime \prime}\right\| \rightarrow \infty} g\left(\vec{R}^{\prime}, \vec{R}^{\prime \prime}\right)=1
$$

En el caso de fluidos homogéneos e isótropos, la función de correlación por pares depende solamente de la distancia entre las moléculas $R=\left\|\vec{R}^{\prime \prime}-\vec{R}^{\prime}\right\|$. Si se sitúa una de las moléculas en el origen de coordenadas, la posición de la otra vendrá dada por la cantidad $R$. Al expresar la función $g$ como dependiente de la coordenada $R$, se obtiene la función de distribución radial $g(R)$.

Se pueden definir funciones de distribución de orden $h$-ésimo $\rho^{(h)}\left(\vec{R}^{\prime}, \vec{R}^{\prime \prime}, \ldots, \vec{R}^{h}\right)$, siendo $\rho^{(h)}\left(\vec{R}^{\prime}, \vec{R}^{\prime \prime}, \ldots, \vec{R}^{h}\right) \cdot \mathrm{d} \vec{R}^{\prime} \cdot \ldots \cdot \mathrm{d} \vec{R}^{h}$ la probabilidad de encontrar simultáneamente una molécula cualquiera entre $\vec{R}^{\prime}$ y $\vec{R}^{\prime}+\mathrm{d} \vec{R}^{\prime}$, otra molécula cualquiera diferente entre $\vec{R}^{\prime \prime}$ y $\vec{R}^{\prime \prime}+$ 
$\mathrm{d} \vec{R}^{\prime \prime}, \ldots$, y una molécula $h$-ésima diferente entre $\vec{R}^{h}$ y $\vec{R}^{h}+\mathrm{d} \vec{R}^{h}$. Es fácil ver que se cumple la siguiente relación de recurrencia:

$$
\int \rho^{(h)}\left(\vec{R}^{\prime}, \vec{R}^{\prime \prime}, \ldots, \vec{R}^{h}\right) \cdot \mathrm{d} \vec{R}^{h}=[N-(h-1)] \cdot \rho^{(h-1)}\left(\vec{R}^{\prime}, \vec{R}^{\prime \prime}, \ldots, \vec{R}^{h-1}\right)
$$

Para entender el significado de la función de correlación, discutiremos cuatro casos:

a) Gas ideal: $g(R)=1-1 / N \approx 1$ cuando $N \rightarrow \infty$. Este comportamiento refleja la ausencia de correlación en el gas ideal, consecuencia de la ausencia de interacciones. Este caso permite dilucidar una propiedad general de la función de distribución radial, independientemete del tipo de sistema: puede escribirse como la suma de dos contribuciones, una debida a las interacciones (en este caso son nulas y el término correspondiente es 1) y otra debida a la condición $N=$ cte. por estar trabajando en el conjunto canónico (término $-1 / N$ ). Así, en el conjunto gran canónico se encuentra que para el gas ideal, $\bar{g}(R)=1$.

b) Fluido simple* moderadamente denso. En este contexto, por simple no se debe entender la clase de líquidos que aparece en la clasificación dada en la sección 6.2, sino un tipo de líquido cuyas moléculas son pequeñas y tienen simetría esférica (por ello se escribe el asterisco). La interacción está descrita con suficiente exactitud por fuerzas centrales de corto alcance entre dos cuerpos. A este grupo pertenecen los gases nobles*, así como algunos líquidos no polares de pequeña masa molecular como $\mathrm{CO}, \mathrm{N}_{2}, \mathrm{O}_{2}$ y $\mathrm{CH}_{4}$. Al existir simetría esférica, está asegurada la isotropía. Si además el fluido es homogéneo, la integral (6.38) se puede escribir:

$$
\begin{gathered}
N \cdot(N-1)=\int_{V} \int_{V} \rho^{(2)}\left(\vec{R}^{\prime}, \vec{R}^{\prime \prime}\right) \cdot \mathrm{d} \vec{R}^{\prime} \cdot \mathrm{d} \vec{R}^{\prime \prime}=\int_{V} \int_{V} \rho^{2} \cdot g(R) \cdot \mathrm{d} \vec{R}^{\prime} \cdot \mathrm{d} \vec{R}^{\prime \prime}= \\
=\frac{N^{2}}{V^{2}} \cdot \int_{V} \int_{V} g(R) \cdot \mathrm{d} \vec{R}^{\prime} \cdot \mathrm{d} \vec{R}^{\prime \prime}=\frac{N^{2}}{V} \cdot \int_{V} g(R) \cdot \mathrm{d} \vec{R}^{\prime \prime}=\frac{N^{2}}{V} \cdot \int_{0}^{\infty} g(R) \cdot 4 \cdot \pi \cdot R^{2} \cdot \mathrm{d} R
\end{gathered}
$$

Por lo tanto,

$$
\rho \cdot \int_{0}^{\infty} g(R) \cdot 4 \cdot \pi \cdot R^{2} \cdot \mathrm{d} R=N-1
$$

es el número de moléculas total, menos la molécula que se considera como origen de coordenadas. Aunque el sistema es finito, en el límite superior de esta integral se ha escrito $\infty$ lo cual puede hacerse ya que a cierta distancia $g(R)$ es uno (recuérdese la condición (6.41)). La cantidad

$$
N_{\mathrm{c}}(r)=4 \cdot \pi \cdot \rho \cdot \int_{0}^{r} g(R) \cdot R^{2} \cdot \mathrm{d} R
$$

es el número de moléculas contenidas en una esfera de radio $r$, con centro en la molécula considerada como origen, sin contar ésta última. Es decir, es el número de vecinos de una molécula dada dentro de una esfera de radio $r$, lo que se llama número de coordinación de partículas computado para una esfera de radio $r$.

La integral (6.44) es justamente $\int_{V} \rho^{(1)}(R) \cdot \mathrm{d} \vec{R}-1$. Por lo tanto, en un elemento diferencial de volumen se tiene:

\footnotetext{
*A excepción del helio, si la aproximación semiclásica se está utilizando.
} 


$$
\rho^{(1)}(R) \cdot \mathrm{d} \vec{R}=\rho \cdot g(R) \cdot \mathrm{d} \vec{R}
$$

Luego,

$$
g(R)=\frac{\rho^{(1)}(R)}{\rho}
$$

La ecuación (6.47) permite interpretar a la función de distribución radial como el factor por el cual la densidad local se desvía de la densidad global, es decir, es una medida de la composición local de la mezcla.

La función de distribución radial de un fluido simple* es similar a la que se observa en la Ilustración 6-2. Cuando $R \rightarrow 0, g(R) \rightarrow 0$, pues las moléculas son impenetrables por debajo de cierta distancia y en consecuencia $\rho^{(2)}$ debe ser cero; cuando $R \rightarrow \infty, g(R) \rightarrow 1$, pues a distancias grandes respecto el tamaño molecular no hay interacción, como se ha dicho anteriormente. A distancias del orden de los primeros diámetros moleculares $(R \approx$ $\sigma, 2 \cdot \sigma, 3 \cdot \sigma, \ldots$,$) aparecen máximos, lo que refleja la tendencia de las moléculas a$ empaquetarse en esferas equidistantes en torno a una molécula dada. Esto explica que la distribución de moléculas, a pesar de ser aleatoria, presenta cierto grado de orden, lo que se llama estructura local del fluido, en contraste con el orden de largo alcance típico de los cristales. Si la densidad del fluido disminuyera hasta el límite nulo, $g(R)$ presentaría sólo un pico en $r=\sigma^{*}$.
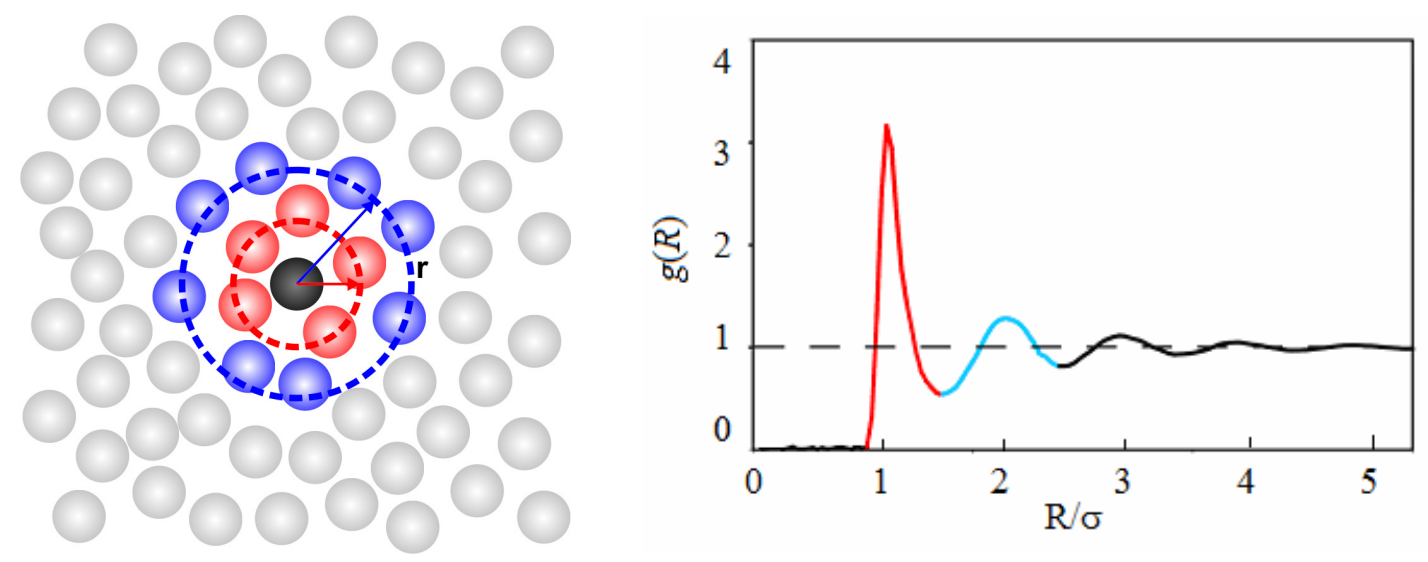

Ilustración 6-2: izq.: dos primeras esferas de solvatación en torno a una molécula dada. dcha.: función de distribución radial de un líquido simple en función del cociente entre la distancia al centro de una molécula dada y el diámetro molecular.

c) Fluido molecular: a este grupo pertenecen todos los fluidos del mundo real exceptuando los gases nobles. Son moléculas que no tienen simetría esférica. En este caso se deben tener en cuenta las orientaciones moleculares a la hora de definir la función de distribución por pares. En este caso $g\left(\vec{R}^{\prime}, \vec{R}^{\prime \prime}\right)$ se obtiene como el promedio de una función más general, $g\left(\vec{X}^{\prime}, \vec{X}^{\prime \prime}\right)$, respecto de todas las orientaciones de ambas moléculas:

$$
g\left(\vec{R}^{\prime}, \vec{R}^{\prime \prime}\right)=\frac{1}{8 \cdot \pi^{2}} \cdot \iint g\left(\vec{X}^{\prime}, \vec{X}^{\prime \prime}\right) \cdot \mathrm{d} \Omega^{\prime} \cdot \mathrm{d} \Omega^{\prime \prime}
$$

\footnotetext{
* Por tanto, aunque en un gas real cuando su densidad tiende a cero su ecuación de estado es la del gas ideal, hay algún diferencia por ejemplo la concerniente a la forma de $g(R)$.
} 
Al contrario que en fluidos simples* y en gases ideales, en fluidos moleculares, homogéneos e isótropos la función de correlación por pares no puede expresarse como función únicamente de una coordenada. Por ello resulta útil definir funciones de correlación entre diferentes pares de sitios de dentro la molécula. Estos sitios pueden ser los centros de los núcleos atómicos, o cualquier lugar de la molécula escogido arbitrariamente. La función de correlación de pares sitio-sitio $g_{\alpha \beta}\left(R_{\alpha \beta}\right)$ así definida es proporcional a la densidad de probabilidad de encontrar el sitio $\beta$ de alguna molécula a una distancia $R_{\alpha \beta}$ del sitio $\alpha$ de alguna otra molécula. Para un fluido molecular puro, homogéneo e isótropo, se define como [94, cap. 3]:

$$
g_{\alpha \beta}\left(R_{\alpha \beta}\right)=\frac{1}{\rho^{2}} \cdot\left\langle\delta\left(\vec{R}_{\mathrm{i} \alpha}-\vec{R}_{\mathrm{ci}}-\vec{R}_{\mathrm{ci} \alpha}\right) \cdot \delta\left(\vec{R}_{\mathrm{j} \beta}-\vec{R}_{\mathrm{cj}}-\vec{R}_{\mathrm{cj} \beta}\right)\right\rangle
$$

donde $\vec{R}_{\mathrm{i} \alpha}$ y $\vec{R}_{\mathrm{ci}}$ son las posiciones del sitio $\alpha$ y del centro de la molécula $\mathrm{i}$ respectivamente, con respecto a un sistema de coordenadas arbitrario y $R_{\alpha \beta}$ es $\left\|\vec{R}_{\mathrm{i} \alpha}-\vec{R}_{\mathrm{j} \beta}\right\|$. El promedio se hace sobre todos los $\vec{R}_{c i \alpha}{ }^{\prime}$ s y todos los $\vec{R}_{\mathrm{ci}}{ }^{\prime}$ 's, considerando a $\vec{R}_{\mathrm{i} \alpha}$ como un parámetro fijo. Nótese que la función de distribución radial es un tipo particular de función de correlación de pares de sitios, siendo éstos los centros nucleares de dos moléculas monoatómicas diferentes.

Para una molécula que contiene $s$ sitios existe un total de $s \cdot(s+1) / 2$ funciones $g_{\alpha \beta}\left(R_{\alpha \beta}\right)$, pues $g_{\alpha \beta}=g_{\beta \alpha}$. Sin embargo, si en la molécula hay presente algún tipo de simetría, algunas de estas funciones pueden ser idénticas. Por ejemplo, las moléculas $\mathrm{N}_{2}$ y $\mathrm{CO}$ tienen ambas dos sitios. Sin embargo, en la primera por ser homonuclear sólo existe una función sitio-sitio, $g_{\mathrm{NN}}$, mientras que en la segunda existes tres: $g_{\mathrm{OO}}, g_{\mathrm{Cc}}$ y $g_{\mathrm{CO}}$.

La llustración 6-3 representa las tres funciones de correlación de pares sitio-sitio $g_{\alpha \beta}$ de una molécula aislada de agua a $298.15 \mathrm{~K}$, las cuales son muy diferentes unas de otras. Solamente $g_{00}$ se parece a la función de distribución radial de un líquido simple.

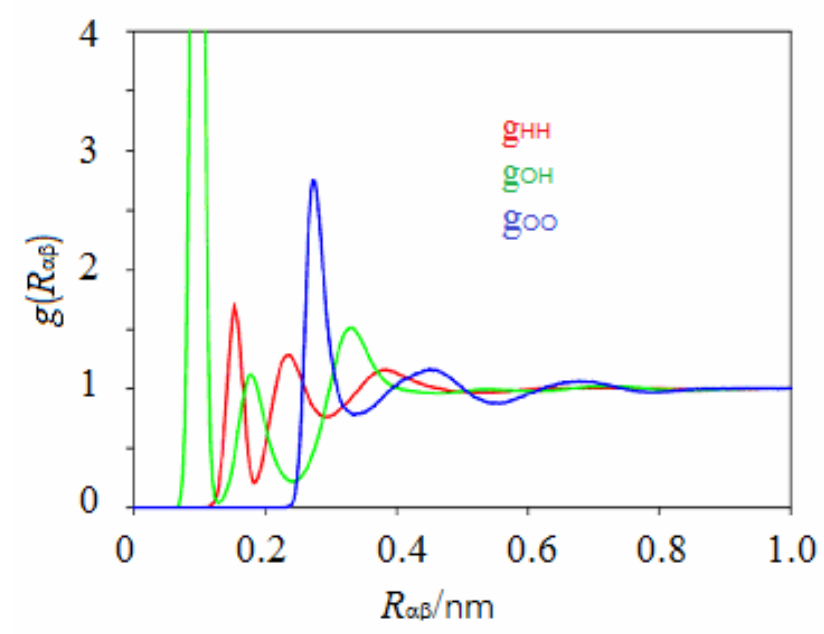

llustración 6-3: funciones de correlación por pares del agua

Las funciones $g_{\alpha \beta}$ pueden obtenerse a partir de $g\left(\vec{X}^{\prime}, \vec{X}^{\prime \prime}\right)$; sin embargo la recíproca no es cierta, pues $g\left(\vec{X}^{\prime}, \vec{X}^{\prime \prime}\right)$ contiene más información que las $g_{\alpha \beta}$. 
d) Mezclas: las funciones de distribución se definen de manera análoga al caso de los sistemas monocomponentes, siendo la única complicación de tipo notacional. Supongamos una mezcla binaria, donde $N_{\mathrm{A}}$ es el número de moléculas de la especie A, y $N_{\mathrm{B}}$ el de la especie B. La configuración del sistema está dada por $\vec{X}^{N}=\vec{X}^{N_{\mathrm{A}}+N_{\mathrm{B}}}=\left(\vec{X}_{1_{\mathrm{A}}}, \ldots, \vec{X}_{N_{\mathrm{A}}}, \vec{X}_{1_{\mathrm{B}}}, \ldots, \vec{X}_{N_{\mathrm{B}}}\right)$. En el caso general de fluidos moleculares, se define la función orientacional de distribución de singletes de la especie i en la mezcla de modo similar al caso de una sustancia pura:

$$
\rho_{\mathrm{i}}^{(1)}\left(\vec{X}^{\prime}\right)=n_{\mathrm{i}} \cdot \int \ldots \int \delta\left(\vec{X}_{1}^{\mathrm{i}}-\vec{X}^{\prime}\right) \cdot P\left(\vec{X}^{N}\right) \cdot \mathrm{d} \vec{X}^{N}
$$

La densidad local de moléculas del tipo i en $\vec{R}^{\prime}$ es el promedio de $\rho_{\mathrm{i}}^{(1)}\left(\vec{X}^{\prime}\right)$ en todas las posibles orientaciones:

$$
\rho_{\mathrm{i}}^{(1)}\left(\vec{R}^{\prime}\right)=\int \rho_{\mathrm{i}}^{(1)}\left(\vec{X}^{\prime}\right) \cdot \mathrm{d} \vec{\Omega}^{\prime}=\frac{N_{\mathrm{i}}}{V}=\rho_{\mathrm{i}}
$$

Las dos últimas igualdades se cumplen en fluidos homogéneos. $\rho_{\mathrm{i}}^{(1)}\left(\vec{R}^{\prime}\right) \cdot \mathrm{d} \vec{R}^{\prime}$ es igual a la probabilidad de encontrar una molécula cualquiera de la especie i en la región entre $\vec{R}^{\prime}$ y $\vec{R}^{\prime}+\mathrm{d} \vec{R}^{\prime}$.

Similarmente se puede definir las funciones orientacionales de distribución por pares para las cuatro posibilidades: AA, AB, BA, BB. Por ejemplo, para pares de moléculas iguales:

$$
\rho_{\mathrm{ii}}^{(2)}\left(\vec{X}^{\prime}, \vec{X}^{\prime \prime}\right)=N_{\mathrm{i}} \cdot\left(N_{\mathrm{i}}-1\right) \cdot \int \ldots \int \delta\left(\vec{X}_{1}^{\mathrm{i}}-\vec{X}^{\prime}\right) \cdot \delta\left(\vec{X}_{2}^{\mathrm{i}}-\vec{X}^{\prime \prime}\right) \cdot P\left(\vec{X}^{N}\right) \cdot \mathrm{d} \vec{X}^{N}
$$

Para moléculas diferentes:

$$
\rho_{\mathrm{ij}}^{(2)}\left(\vec{X}^{\prime}, \vec{X}^{\prime \prime}\right)=N_{\mathrm{A}} \cdot N_{\mathrm{B}} \cdot \int \ldots \int \delta\left(\vec{X}_{1}^{\mathrm{i}}-\vec{X}^{\prime}\right) \cdot \delta\left(\vec{X}_{2}^{\mathrm{j}}-\vec{X}^{\prime \prime}\right) \cdot P\left(\vec{X}^{N}\right) \cdot \mathrm{d} \vec{X}^{N}
$$

Las condiciones de normalización son:

$$
\begin{gathered}
\int \rho_{\mathrm{ii}}^{(2)}\left(\vec{X}^{\prime}, \vec{X}^{\prime \prime}\right) \cdot \mathrm{d} \vec{X}^{\prime} \cdot \mathrm{d} \vec{X}^{\prime \prime}=N_{\mathrm{i}} \cdot\left(N_{\mathrm{i}}-1\right) \\
\int \rho_{\mathrm{ij}}^{(2)}\left(\vec{X}^{\prime}, \vec{X}^{\prime \prime}\right) \cdot \mathrm{d} \vec{X}^{\prime} \cdot \mathrm{d} \vec{X}^{\prime \prime}=N_{\mathrm{A}} \cdot N_{\mathrm{B}}
\end{gathered}
$$

(6.54) es el número total de pares de la misma especie. (6.55) es el número de pares AB.

En una mezcla binaria se define la función de correlación por pares $g_{\mathrm{ij}}\left(\vec{X}^{\prime}, \vec{X}^{\prime \prime}\right)$ que depende de $T, V$ y la fracción molar, por:

$$
\rho_{\mathrm{ij}}^{(2)}\left(\vec{X}^{\prime}, \vec{X}^{\prime \prime}\right)=\rho_{\mathrm{i}}^{(1)}\left(\vec{X}^{\prime}\right) \cdot \rho_{\mathrm{j}}^{(1)}\left(\vec{X}^{\prime}\right) \cdot g_{\mathrm{ij}}\left(\vec{X}^{\prime}, \vec{X}^{\prime \prime}\right)
$$

y se define la función de correlación espacial $g_{\mathrm{ij}}\left(\vec{R}^{\prime}, \vec{R}^{\prime \prime}\right)$ por:

$$
g_{\mathrm{ij}}\left(\vec{R}^{\prime}, \vec{R}^{\prime \prime}\right)=\frac{\int \cdot \mathrm{d} \vec{\Omega}^{\prime \prime} \cdot \int g_{\mathrm{ij}}\left(\vec{X}^{\prime}, \vec{X}^{\prime \prime}\right) \cdot \mathrm{d} \vec{\Omega}^{\prime}}{\left(8 \cdot \pi^{2}\right)^{2}}
$$


En mezclas de fluidos homogéneos e isótropos $g_{\mathrm{ij}}\left(\vec{R}^{\prime}, \vec{R}^{\prime \prime}\right)$ depende sólo de $R=\left\|\vec{R}^{\prime \prime}-\vec{R}^{\prime}\right\|$, luego $g_{\mathrm{AB}}(R)=g_{\mathrm{BA}}(R)$. Cuando $R \rightarrow \infty, g_{\mathrm{ij}}\left(\vec{R}^{\prime}, \vec{R}^{\prime \prime}\right) \rightarrow 1$. En estos casos, el primer pico está a una distancia igual al promedio del diámetro molecular del par $\mathrm{i}-\mathrm{j}, \sigma_{\mathrm{ij}}=\left(\sigma_{\mathrm{ii}}+\sigma_{\mathrm{jj}}\right) / 2$, lo que es debido al predominio de la interacción directa entre i y j a esta distancia. Sin embargo, las posiciones del resto de picos no están determinadas por las interacciones directas, puesto que las fuerzas intermoleculares disminuyen con la distancia, sino por la correlación indirecta debida al ambiente del par i-j.

\subsubsection{Propiedades termodinámicas en función de la función de distribución radial}

En un sistema de $N$ moléculas iguales en condiciones de volumen y temperatura constantes, la energía interna del mismo puede calcularse a partir de la función de partición, teniendo en cuenta que $F=-\mathrm{k}_{\mathrm{B}} \cdot T \cdot \ln \mathrm{Z}$ y la ecuación de Gibbs -Helmholtz:

$$
U=-T^{2} \cdot\left(\frac{\partial(F / T)}{\partial T}\right)_{V}
$$

En líquidos simples, de acuerdo con nuestra clasificación en líquidos simples y complejos, está permitida la separación de la función de partición (6.22). Así, mientras que los grados de libertad internos y $\Lambda$ contribuyen a la energía interna con un término:

$$
k_{\mathrm{B}} \cdot T^{2} \cdot\left[\frac{\partial \ln \left(\Lambda^{-3 \cdot N} \cdot Z_{\mathrm{int}}\right)}{\partial T}\right]_{V}=U_{\Lambda}+U_{\mathrm{int}}
$$

la contribución de la función de partición configuracional es precisamente el valor promedio de la energía de interacción entre las moléculas:

$$
U(\mathrm{CONF})=\langle W\rangle=\int \ldots \int P\left(\vec{X}^{N}\right) \cdot W\left(\vec{X}^{N}\right) \cdot \mathrm{d} \vec{X}^{N}
$$

es decir, $U=U_{\Lambda}+U_{\text {int }}+\langle W\rangle$. Recuérdese que $P\left(\vec{X}^{N}\right)$ está dada por la ecuación (6.27).

La expresión para $\langle W\rangle$ puede escribirse en términos de la función de distribución por pares si la energía de interacción es aditiva por pares, es decir, si:

$$
W\left(\vec{X}^{N}\right)=\frac{1}{2} \cdot \sum_{\mathrm{i} \neq \mathrm{j}} u\left(\vec{X}_{\mathrm{i}}, \vec{X}_{\mathrm{j}}\right)
$$

En este caso,

$$
\langle W\rangle=\frac{1}{2} \cdot \int \ldots \int P\left(\vec{X}^{N}\right) \cdot \sum_{\mathrm{i} \neq \mathrm{j}} u\left(\vec{X}_{\mathrm{i}}, \vec{X}_{\mathrm{j}}\right) \cdot \mathrm{d} \vec{X}^{N}=\frac{1}{2} \cdot \int \mathrm{d} \vec{X}^{\prime} \int \rho^{(2)}\left(\vec{X}^{\prime}, \vec{X}^{\prime \prime}\right) \cdot u\left(\vec{X}^{\prime}, \vec{X}^{\prime \prime}\right) \cdot \mathrm{d} \vec{X}^{\prime \prime}
$$

En la última igualdad se han cambiado los índices i y j por 'y " para reflejar el hecho de que no nos referimos a dos moléculas en particular.

En el caso de mezclas, la generalización es trivial. Para el caso binario, siendo la configuración del sistema $\vec{X}^{N}=\vec{X}^{N_{\mathrm{A}}+N_{\mathrm{B}}}=\left(\vec{X}_{1_{\mathrm{A}}}, \ldots, \vec{X}_{N_{\mathrm{A}}}, \vec{X}_{1_{\mathrm{B}}}, \ldots, \vec{X}_{N_{\mathrm{B}}}\right)$ y suponiendo que la energía de interacción es aditiva por pares, es decir, que:

$$
W\left(\vec{X}^{N}\right)=\frac{1}{2} \cdot \sum_{\mathrm{k}=1}^{N_{\mathrm{A}}} u_{\mathrm{AA}}\left(\vec{X}_{k}, \vec{X}_{l}\right)+\frac{1}{2} \cdot \sum_{\mathrm{k}=1}^{N_{\mathrm{B}}} u_{\mathrm{BB}}\left(\vec{X}_{k}, \vec{X}_{l}\right)+\sum_{k=1}^{N_{\mathrm{A}}} \sum_{l=1}^{N_{\mathrm{B}}} u_{\mathrm{AB}}\left(\vec{X}_{k}, \vec{X}_{l}\right)
$$


Se tiene que la energía interna es:

$$
U=\sum_{\mathrm{i}=\mathrm{A}}^{\mathrm{B}}\left(U_{\mathrm{t}, \mathrm{i}}+U_{\mathrm{int,i}}\right)+\frac{1}{2} \cdot \sum_{\mathrm{i}=\mathrm{A}}^{\mathrm{B}} \sum_{\mathrm{j}=\mathrm{A}}^{\mathrm{B}} \int \mathrm{d} \vec{X}^{\prime} \int \rho_{\mathrm{ij}}^{(2)}\left(\vec{X}^{\prime}, \vec{X}^{\prime \prime}\right) \cdot u_{\mathrm{ij}}\left(\vec{X}^{\prime}, \vec{X}^{\prime \prime}\right) \cdot \mathrm{d} \vec{X}^{\prime \prime}
$$

El caso de fluidos simples* es muy sencillo puesto que al ser las moléculas simetrícamente esféricas, el potencial de interacción es función de las distancias entre las moléculas, $W\left(\vec{R}^{\mathrm{N}}\right)=\sum_{\mathrm{i} \neq \mathrm{j}} u\left(R_{\mathrm{ij}}\right) / 2$, y la función de distribución por pares se reduce al producto del cuadrado de la densidad por la función de distribución radial. En este caso la expresión de $\langle W\rangle$ es:

$$
\langle W\rangle=\frac{1}{2} \cdot N \cdot \rho \cdot \int_{0}^{\infty} u(R) \cdot g(R) \cdot 4 \cdot \pi \cdot R^{2} \cdot \mathrm{d} R
$$

Para el resto de propiedades termodinámicas (potencial químico, presión,...) pueden obtenerse las correspondientes ecuaciones que las relacionan con las funciones de correlación (fluidos moleculares [93, cap. 3]) o función de distribución radial (fluidos simples* [95], [96, cap 17]). Por ejemplo, en fluidos simples* puros la presión es:

$$
P=k_{B} \cdot T \cdot \rho-\frac{\rho^{2}}{6} \cdot \int_{0}^{\infty} R \cdot \frac{\partial u(R)}{\partial R} \cdot g(R) \cdot 4 \cdot \pi \cdot R^{2} \cdot \mathrm{d} R
$$

mientras que en mezclas de $\mathrm{n}$ fluidos simples*:

$$
P=\sum_{\mathrm{i}=1}^{\mathrm{n}} k_{B} \cdot T \cdot \rho_{\mathrm{i}}-\frac{1}{6} \cdot \sum_{\mathrm{i}, \mathrm{j}=1}^{\mathrm{n}} \rho_{\mathrm{i}} \cdot \rho_{\mathrm{j}} \cdot \int_{0}^{\infty} R \cdot \frac{\partial u_{\mathrm{ij}}(R)}{\partial R} \cdot g_{\mathrm{ij}}(R) \cdot 4 \cdot \pi \cdot R^{2} \cdot \mathrm{d} R
$$

En el caso de fluidos moleculares en los que el potencial intermolecular es una función de la separación entre sitios $\alpha$ y $\beta$ de diferentes moléculas, también se pueden encontrar ecuaciones relativamente simples que relacionan las funciones $g_{\alpha \beta}$ con las propiedades termodinámicas. Por ejemplo, para $\langle W\rangle$ se tiene:

$$
\langle W\rangle=\frac{1}{2} \cdot N \cdot \rho \cdot \sum_{\alpha, \beta} \int_{0}^{\infty} u_{\alpha \beta}\left(R_{\alpha \beta}\right) \cdot g_{\alpha \beta}\left(R_{\alpha \beta}\right) \cdot 4 \cdot \pi \cdot R_{\alpha \beta}^{2} \cdot \mathrm{d} R_{\alpha \beta}
$$

Ecuaciones para otras propiedades termodinámicas pueden encontrarse en la bibliografía [97]-[99].

\subsubsection{Determinación de la función de correlación}

El potencial del método desarrollado en la sección anterior radica en que a partir de la energía de interacción molecular y de las funciones de correlación por pares se pueden calcular todas las propiedades termodinámicas del sistema. Es decir, a partir de la información microscópica se puede obtener información a nivel global. No obstante, esto presenta las serias limitaciones discutidas en los primeros párrafos de este capítulo. Así, entre otros inconvenientes, cabe recordar que la no integrabilidad del problema de tres o más cuerpos influye en el desconocimiento acerca de la forma del potencial de interacción. Además, la hipótesis de aditividad por pares del potencial de interacción sólo es estrictamente válida para el caso de bajas densidades. Por su parte, la función de correlación sólo puede obtenerse mediante alguna de las tres posibilidades siguientes: 
a) Experimentos de difracción de rayos $X$ y de neutrones. La función radial de distribución de los fluidos simples* se puede determinar mediante la difracción de un haz monocromático de rayos $X$ [100] o de neutrones [101] con longitud de onda de unos $0.1 \mathrm{~nm}$, sabiendo que el patrón de difracción surge de la interferencia de los haces dispersados entre diferentes núcleos (caso de los neutrones) u orbitales electrónicos (caso de rayos X). De hecho, es la transformada de Fourier del factor de estructura estático [102].

La obtención de las funciones de correlación en fluidos moleculares a través de los patrones de difracción de rayos $X$ y de neutrones es mucho más complicada puesto que la dispersión se debe tanto a grados de libertad intermoleculares como intramoleculares. Además, los patrones de difracción resultan de la interferencia de los haces dispersados entre pares de sitios diferentes, luego estos experimentos permiten determinar las funciones $g_{\alpha \beta}$ en lugar de $g\left(\vec{X}^{\prime}, \vec{X}^{\prime \prime}\right) \circ g\left(\vec{R}^{\prime}, \vec{R}^{\prime \prime}\right)$. Los mejores resultados se han obtenido para $\mathrm{N}_{2}, \mathrm{Br}_{2}, \mathrm{CS}_{2}$ y $\mathrm{H}_{2} \mathrm{O}$. No obstante, excepto en el caso de moléculas homonucleares en las cuales $g_{\alpha \beta}$ contiene las correlaciones inter e intramolecular, en el resto de moléculas ni siquiera el conocimiento de todas las funciones $g_{\alpha \beta}$ permite obtener información tan completa acerca de la estructura del líquido como la proporcionada por $g\left(\vec{X}^{\prime}, \vec{X}^{\prime \prime}\right)$, por lo que el problema no se resuelve completamente.

b) Simulación por ordenador. Esta posibilidad la descartamos por todo lo dicho en la sección 6.1.

c) Resolución analítica o numérica de un conjunto de tantas ecuaciones integrodiferenciales como número de moléculas contenga el sistema. Esta posibilidad presenta tres inconvenientes importantes: primero, el número de ecuaciones a resolver es muy elevado, lo cual consume mucho tiempo de computación, en caso de ser resolubles; segundo, en las ecuaciones está presente el potencial de interacción, cuya forma es desconocida; tercero, debido a la naturaleza del conjunto de ecuaciones, éstas no pueden resolverse a no ser que se adopten ciertas hipótesis acerca de la forma de las funciones de distribución molecular, como el principio de superposición de Kirkwood [103] (que lleva a la ecuación de Yvon-Bogoliubov-Born-Green-Kirkwood, YBBGK [103][106]), la aproximación HNC (por su acrónimo en inglés Hyper-Netted Chain [107][109]), la aproximación de Percus y Yevick [110], la aproximación RISM (por su acrónimo en inglés Reference Interaction Site Model [1 111$]$ ), etc. Aunque estos métodos dan resultados aceptablemente buenos en el caso de fluidos simples*, y sobre todo para el modelo de potencial de esferas duras, para fluidos moleculares, dada la complejidad de las ecuaciones integro-diferenciales, el método preferido es el estudio de un fluido de esferas duras (entre las que solamente hay fuerzas repulsivas a corto alcance) en el que se van introduciendo los efectos de fuerzas atractivas de largo alcance, asimetría de los campos de fuerzas, etc. mediante cálculos perturbativos. En cuanto al estudio de mezclas, aunque éste presenta el inconveniente de que se deben asumir reglas de mezcla para el tamaño y el potencial intermoleculares, arbitrarias hasta cierto punto [112]-[116], se ha intentado proceder de manera similar al caso de fluidos puros: primero, se resuelve la ecuación de Percus-Yevick para mezclas binarias de esferas duras de tamaño similar [1 17]; segundo, se aplican métodos perturbativos [1 18]-[120]. En este sentido son dignos de mencionar los métodos SAFT (Statistical Associated Fluid Theory) y el PHSC (Perturbed Hard-Sphere Chain). SAFT [121], [122] se ha aplicado con relativo éxito a la descripción de las propiedades termodinámicas de fluidos puros y 
mezclas con moléculas grandes, pequeñas, polidispersivas, asociadas y no asociadas, en ciertos rangos de presión y temperatura (pues las hipótesis físicas en las que se basa son válidas a presiones altas y temperaturas relativamente altas). PHSC [123] es una generalización de la ecuación de Percus-Yevick para cadenas de esferas rígidas. Se ha aplicado con bastante notoriedad a fluidos simples, polímeros y sus mezclas, en ausencia de asociación y en los mismos rangos de presión y temperatura en los que el método SAFT funciona. En la bibliografía se puede encontrar amplios resúmenes de estos métodos [124]-[127].

En conclusión, el elevado número de aproximaciones necesarias para obtener la función de correlación por pares hacen del método presentado en la sección 6.3.1 inadecuado para el estudio de los líquidos, exceptuando el caso de los fluidos simples* a densidades bajas en los que la función de distribución radial puede determinarse experimentalmente de manera exacta; no obstante, incluso en estos casos se deben hacer suposiciones acerca de la forma del potencial de interacción molecular. Por todas estas razones, a pesar del enorme esfuerzo realizado en esta dirección, la aplicación de estos métodos a la caracterización de los líquidos reales y sus mezclas está muy limitada y será ignorada por nuestra parte.

\subsubsection{Teoría de Kirkwood-Buff e inversión de Ben-Naim}

En esta sección se presenta la teoría de mezclas de Kirkwood-Buff [128], una teoría que no hace hipótesis alguna acerca de la forma del potencial de interacción intermolecular (de hecho no se necesita el conocimiento explícito de $W$ ) ni de la forma de las funciones de distribución molecular. Es aplicable tanto a sistemas cuánticos como clásicos a densidades bajas.

En primer lugar se desarrolla la teoría tal y como fue concebida originalmente. Sin embargo, los resultados así obtenidos sólo se pueden aplicar si las funciones de distribución por pares son conocidas, por lo que persisten los problemas discutidos en la sección anterior.

En la segunda parte, se invierte la teoría, como lo hizo Ben-Naim [129]. La inversión de la teoría de Kirkwood-Buff permite obtener información local a partir de las propiedades globales, o en otras palabras, obtener información a nivel microscópico a partir de la información termodinámica, esta última disponible gracias a la experimentación, en lugar de intentar predecir el comportamiento macroscópico del sistema a partir de información microscópica, como otros métodos han buscado.

\subsubsection{Teoría de Kirkwood Buff}

Sea un sistema abierto de $N$ componentes, en condiciones de $T, V$ y $\vec{\mu}=\left(\mu_{1}, \ldots, \mu_{N}\right)$ constantes. Las condiciones de normalización de las funciones de distribución molecular son:

$$
\begin{gathered}
\int_{V} \bar{\rho}_{\mathrm{i}}^{(1)}\left(\vec{X}^{\prime}\right) \cdot \mathrm{d} \vec{X}^{\prime}=\left\langle N_{\mathrm{i}}\right\rangle \\
\int_{V} \int_{V} \bar{\rho}_{\mathrm{ij}}^{(2)}\left(\vec{X}^{\prime}, \vec{X}^{\prime \prime}\right) \cdot \mathrm{d} \vec{X}^{\prime} \cdot \mathrm{d} \vec{X}^{\prime \prime}=\left\langle N_{\mathrm{i}} \cdot N_{\mathrm{j}}\right\rangle-\delta_{\mathrm{ij}}\left\langle N_{\mathrm{i}}\right\rangle=\left\{\begin{array}{c}
\left\langle N_{\mathrm{i}} \cdot N_{\mathrm{j}}\right\rangle, \text { si } \mathrm{i} \neq \mathrm{j} \\
\left\langle N_{\mathrm{i}} \cdot\left(N_{\mathrm{i}}-1\right)\right\rangle, \text { si } \mathrm{i}=\mathrm{j}
\end{array}\right.
\end{gathered}
$$


En este caso, es necesario trabajar con funciones de distribución gran canónicas [93], lo que se hace notar por la línea escrita encima de $\rho_{\mathrm{i}}^{(1)}$ y $\rho_{\mathrm{ij}}^{(2)}$. La dependencia es con $\vec{X}$ y no con $\vec{R}$, luego el estudio no se limita al caso de moléculas con simetría esférica.

El resultado de restar a (6.70) el producto de (6.69) por (6.69) es:

$$
\begin{gathered}
\int_{V} \int_{V} \bar{\rho}_{\mathrm{ij}}^{(2)}\left(\vec{X}^{\prime}, \vec{X}^{\prime \prime}\right) \cdot \mathrm{d} \vec{X}^{\prime} \cdot \mathrm{d} \vec{X}^{\prime \prime}-\int_{V} \bar{\rho}_{\mathrm{i}}^{(1)}\left(\vec{X}^{\prime}\right) \cdot \mathrm{d} \vec{X}^{\prime} \cdot \int_{V} \bar{\rho}_{\mathrm{j}}^{(1)}\left(\vec{X}^{\prime \prime}\right) \cdot \mathrm{d} \vec{X}^{\prime \prime}= \\
=\left\langle N_{\mathrm{i}} \cdot N_{\mathrm{j}}\right\rangle-\delta_{\mathrm{ij}} \cdot\left\langle N_{\mathrm{i}}\right\rangle-\left\langle N_{\mathrm{i}}\right\rangle \cdot\left\langle N_{\mathrm{j}}\right\rangle
\end{gathered}
$$

En fluidos homogéneos e isótropos cuya densidad global es $\rho_{\mathrm{i}}=\left\langle N_{\mathrm{i}}\right\rangle / V$, se tiene:

$$
\begin{gathered}
\bar{\rho}_{\mathrm{i}}^{(1)}\left(\vec{X}^{\prime}\right)=\frac{\rho_{\mathrm{i}}}{8 \cdot \pi^{2}} \\
\bar{\rho}_{\mathrm{ij}}^{(2)}\left(\vec{X}^{\prime}, \vec{X}^{\prime \prime}\right)=\frac{\rho_{\mathrm{i}} \cdot \rho_{\mathrm{j}} \cdot \bar{g}_{\mathrm{ij}}^{(2)}\left(\vec{X}^{\prime}, \vec{X}^{\prime \prime}\right)}{\left(8 \cdot \pi^{2}\right)^{2}}
\end{gathered}
$$

La integración en todas las orientaciones posibles de las moléculas permite definir funciones espaciales de distribución molecular y función de correlación espacial (recuérdese la ecuación (6.48)). Teniendo esto en cuenta y los resultados (6.72) y (6.73), la ecuación (6.71) se simplifica bastante para fluidos homogéneos:

$$
(6.71)=\rho_{\mathrm{i}} \cdot \rho_{\mathrm{j}} \cdot \int_{V} \int_{V}\left[\bar{g}_{\mathrm{ij}}\left(\vec{R}^{\prime}, \vec{R}^{\prime \prime}\right)-1\right] \cdot \mathrm{d} \vec{R}^{\prime} \cdot \mathrm{d} \vec{R}^{\prime \prime}
$$

La cantidad $\bar{G}_{\mathrm{ij}}=\int_{V}\left[\bar{g}_{\mathrm{ij}}\left(\vec{R}^{\prime}, \vec{R}^{\prime \prime}\right)-1\right] \cdot \mathrm{d} \vec{R}^{\prime}$ se llama integral de Kirkwood-Buff (KBI). En el caso de fluidos homogéneos e isótropos:

$$
\bar{G}_{\mathrm{ij}}=\int_{0}^{\infty}\left[\bar{g}_{\mathrm{ij}}(R)-1\right] \cdot 4 \cdot \pi \cdot R^{2} \cdot \mathrm{d} R
$$

Aunque el sistema es finito, en el límite superior de esta integral se ha escrito $\infty$ lo cual puede hacerse ya que a cierta distancia $\bar{g}(R)$ es 1 (recuérdese la condición (6.41)). Es decir, a partir de cierta distancia $k \cdot \sigma_{\mathrm{ij}}$ la integral se anula. De hecho, se puede escribir como la suma de dos contribuciones, una negativa y otra positiva [130]:

$$
\bar{G}_{\mathrm{ij}}=-\int_{0}^{\sigma_{i j}} 4 \cdot \pi \cdot R^{2} \cdot \mathrm{d} R+\int_{\sigma_{i j}}^{k \cdot \sigma_{i j}}\left[\bar{g}_{\mathrm{ij}}(R)-1\right] \cdot 4 \cdot \pi \cdot R^{2} \cdot \mathrm{d} R=-V_{\mathrm{ij}}+I_{\mathrm{ij}}
$$

donde $\sigma_{\mathrm{ij}}$ es la distancia de máximo acercamiento entre las moléculas i y j, y es tal que a distancias menores, $\overline{\mathrm{g}}_{\mathrm{ij}}=0$ (en moléculas esféricas es el diámetro promedio $\sigma_{\mathrm{ij}}=\left(\sigma_{\mathrm{ii}}+\sigma_{\mathrm{jj}}\right) / 2$ ). $V_{\mathrm{ij}}$ es esencialmente un volumen y es casi independiente de la composición. $I_{\mathrm{ij}}$ es la contribución positiva, y mide un tipo de atracción total entre las dos moléculas i y j.

Como $\rho_{\mathrm{i}} \cdot \bar{g}_{\mathrm{ij}}(R) \cdot 4 \cdot \pi \cdot R^{2} \cdot \mathrm{d} R$ es el número promedio de moléculas del tipo $\mathrm{i}$ en una corteza esférica de espesor $\mathrm{d} R$ a una distancia $R$ del centro de una molécula del tipo $\mathrm{j}, \mathrm{y}$ $\rho_{\mathrm{i}} \cdot 4 \cdot \pi \cdot R^{2} \cdot \mathrm{d} R$ es el número promedio de moléculas del tipo i en una corteza esférica de espesor $\mathrm{d} R$ a una distancia $R$ del centro de la esfera sin que en el centro de la misma 
esté situada una molécula del tipo $\mathrm{j}$. Entonces, la cantidad $\rho_{\mathrm{i}} \cdot\left[\bar{g}_{\mathrm{ij}}(R)-1\right] \cdot 4 \cdot \pi \cdot R^{2} \cdot \mathrm{d} R$ es el exceso o la deficiencia en el número promedio de moléculas del tipo i en la corteza esférica a distancia $R$ de la molécula del tipo j en comparación con el número obtenido eliminando la condición de que la molécula j esté situada en el centro de la esfera, es decir, en comparación con el promedio global [129], [131]. $\Delta N_{\mathrm{ij}}=\rho_{\mathrm{i}} \cdot \bar{G}_{\mathrm{ij}}$ representa el promedio del exceso o la deficiencia total de moléculas del tipo i en torno a una molécula del tipo j, luego es una medida de la composición local. Entonces, $\bar{G}_{\mathrm{ij}}$ da una medida de ese exceso por unidad de densidad, lo que expresa información acerca de la afinidad de las moléculas del tipo i hacia las moléculas del tipo j y viceversa. No obstante, la cantidad $\rho_{\mathrm{i}} \cdot \bar{G}_{\mathrm{ij}}$ no da valores nulos de $\Delta N_{\mathrm{ij}}$ para mezclas ideales, como debería de ser [132], [133]. Por esta razón algunos autores han propuesto que $\Delta N_{\mathrm{ij}}$ debe calcularse respecto de un estado de referencia [132]-[137], lo que ha dado lugar a fuertes enemistades como está plasmado en las referencias [138][142]. El carácter expositivo que se está dando aquí no permite dilucidar qué punto de vista es el correcto.

A pesar de que la literatura está plagada de errores acerca del signo de $\bar{G}_{\mathrm{ij}}$, lo cierto es que puede ser tanto positiva como negativa. De hecho, $\bar{G}_{\mathrm{ij}}>0$ representa, como se ha dicho, el exceso de moléculas del tipo i que rodean a otra del tipo j, lo que sugiere la existencia de interacciones atractivas $\mathrm{i}-\mathrm{j}$. Por el contrario, $\bar{G}_{\mathrm{ij}}<0$ significa que las interacciones $\mathrm{i}-\mathrm{i}$ son preferidas a las interacciones cruzadas.

Para fuerzas de corto alcance, las $\mathrm{KBI}^{\prime}$ s pueden expandirse en serie de potencias en la concentración de los solutos, estando, a dilución infinita del disolvente, las funciones de distribución de conjuntos de $h$ moléculas incluidas en los coeficientes de la expansión en serie. La expansión resultante para la presión osmótica es idéntica a la obtenida en la teoría de disoluciones de McMillan \& Mayer [143].

Gracias a (6.71) y (6.74), se puede escribir la siguiente ecuación que relaciona las fluctuaciones cruzadas en el número de partículas de las diferentes especies y las KBI's:

$$
\bar{G}_{\mathrm{ij}}=V \cdot\left(\frac{\left\langle N_{\mathrm{i}} \cdot N_{\mathrm{j}}\right\rangle-\left\langle N_{\mathrm{i}}\right\rangle \cdot\left\langle N_{\mathrm{j}}\right\rangle}{\left\langle N_{\mathrm{i}}\right\rangle \cdot\left\langle N_{\mathrm{j}}\right\rangle}-\frac{\delta_{\mathrm{ij}}}{\left\langle N_{\mathrm{j}}\right\rangle}\right)
$$

La gran función de partición del sistema es:

$$
\Xi(T, V, \vec{\mu})=\sum Z(T, V, \vec{N}) \cdot e^{\beta \cdot \vec{\mu} \cdot \vec{N}}
$$

El número promedio de moléculas de la especie i es:

$$
\left\langle N_{\mathrm{i}}\right\rangle=\frac{1}{\Xi} \cdot \sum n_{\mathrm{i}} \cdot Z(T, V, \vec{N}) \cdot e^{\beta \cdot \vec{\mu} \cdot \vec{N}}=k_{B} \cdot T \cdot\left[\frac{\partial \ln \Xi(T, V, \vec{\mu})}{\partial \mu_{\mathrm{i}}}\right]_{T, V, \vec{\mu}_{\mathrm{i}}^{\prime}}
$$

donde $\vec{\mu}_{\mathrm{i}}^{\prime}$ es $\vec{\mu}$ excluyendo $\mu_{\mathrm{i}}$. Derivando (6.79) respecto $\mu_{\mathrm{j}}$ se tiene:

$$
B_{\mathrm{ij}} \cdot V=k_{\mathrm{B}} \cdot T \cdot\left[\frac{\partial \ln \left\langle N_{\mathrm{i}}\right\rangle}{\partial \mu_{\mathrm{j}}}\right]_{T, V, \overrightarrow{\mu_{\mathrm{i}}}}=\left\langle N_{\mathrm{i}} \cdot N_{\mathrm{j}}\right\rangle-\left\langle N_{\mathrm{i}}\right\rangle \cdot\left\langle N_{\mathrm{j}}\right\rangle
$$

Sabiendo que todas las ecuaciones son simétricas respecto el intercambio de los índices i y j, y combinando (6.77) con (6.80) se tiene: 


$$
B_{\mathrm{ij}}=k_{\mathrm{B}} \cdot T \cdot\left[\frac{\partial \ln \rho_{\mathrm{i}}}{\partial \mu_{\mathrm{j}}}\right]_{T, V, \overrightarrow{\mathrm{\mu}}_{\mathrm{i}}^{\prime}}=\frac{\left\langle N_{\mathrm{i}} \cdot N_{\mathrm{j}}\right\rangle-\left\langle N_{\mathrm{i}}\right\rangle \cdot\left\langle N_{\mathrm{j}}\right\rangle}{V}=\bar{G}_{\mathrm{ij}} \cdot \rho_{\mathrm{i}} \cdot \rho_{\mathrm{j}}+\delta_{\mathrm{ij}} \cdot \rho_{\mathrm{i}}
$$

Esta ecuación es de importancia en sistemas osmóticos. En ella aparecen derivadas respecto de los potenciales químico a $T, V$ y $\vec{\mu}_{\mathrm{i}}^{\prime}$ constantes. No obstante, de mayor interés son las derivadas a $T$ y $P$ constante. Haciendo las transformaciones necesarias y teniendo en cuenta la definición de volumen molar parcial:

$$
V_{i}=\left(\frac{\partial V}{\partial N_{\mathrm{i}}}\right)_{T, P, N_{\mathrm{j}} \mathrm{i} \neq \mathrm{j}}=\left(\frac{\partial \mu_{\mathrm{i}}}{\partial P}\right)_{T, P, N}
$$

y la definición de la cantidad*:

$$
\mu_{\mathrm{ij}}:=\left(\frac{\partial \mu_{\mathrm{i}}}{\partial N_{\mathrm{j}}}\right)_{T, P, \overrightarrow{\mathrm{N}}_{\mathrm{i}}^{\prime}}=\left(\frac{\partial \mu_{\mathrm{i}}}{\partial N_{\mathrm{j}}}\right)_{T, V, \vec{N}_{\mathrm{i}}^{\prime}}-\frac{V_{\mathrm{i}} \cdot V_{\mathrm{j}}}{V \cdot \kappa_{T}}
$$

se obtienen explícitamente $N^{2}+N+1$ ecuaciones que permiten encontrar las $N^{2}$ cantidades $\mu_{\mathrm{ij}}, N$ cantidades $V_{\mathrm{i}}$ y $\kappa_{T}$. El resultado es:

$$
\begin{gathered}
\kappa_{T}=\frac{\operatorname{det} B}{k_{\mathrm{B}} \cdot T \cdot \sum_{\mathrm{i}, \mathrm{j}} \rho_{\mathrm{i}} \cdot \rho_{\mathrm{j}} \cdot B^{\mathrm{ij}}} \\
V_{\mathrm{j}}=\frac{\sum_{\mathrm{i}} \rho_{\mathrm{i}} \cdot B^{\mathrm{ij}}}{\sum_{\mathrm{i}, \mathrm{k}} \rho_{\mathrm{i}} \cdot \rho_{\mathrm{k}} \cdot B^{\mathrm{ik}}} \\
\mu_{\mathrm{ij}}=\frac{k_{\mathrm{B}} \cdot T}{V \cdot \operatorname{det} B} \cdot \frac{\sum_{\mathrm{k}, 1} \rho_{\mathrm{k}} \cdot \rho_{1} \cdot\left(B^{\mathrm{ij}} \cdot B^{\mathrm{kl}}-B^{\mathrm{ik}} \cdot B^{\mathrm{jl}}\right)}{\sum_{\mathrm{k}, \mathrm{l}} \rho_{\mathrm{k}} \cdot \rho_{1} \cdot B^{\mathrm{kl}}}
\end{gathered}
$$

Estos son los resultados de la teoría de Kirkwood-Buff. Debe tenerse en cuenta que las ecuaciones obtenidas son válidas para sistemas abiertos, luego las funciones de correlación a considerar para calcular las $\mathrm{KBI}^{\prime}$ s son las correspondientes al conjunto gran canónico. det $B$ es el determinante de la matriz $B$, cuyos elementos $B_{\mathrm{ij}}$ están definidos por la ecuación (6.81) y $B^{\mathrm{ij}}$ es el cofactor del elemento $B_{\mathrm{ij}}$ en el determinante. Se obtiene eliminando la fila y la columna que contienen a $B_{\mathrm{ij}}$ en el determinante de $B$ y multiplicando el resultado por $(-1)^{\mathrm{i}+\mathrm{j}}$. En las ecuaciones (6.84)-(6.86) aparecen las funciones de distribución radial $\bar{g}_{\mathrm{ij}}(R) \sin$ haber prestado atención a la forma de las moléculas. Por ello, en el caso de que las moléculas no tengan simetría esférica, $\bar{g}_{\mathrm{ij}}(R)$ debe entenderse como el promedio sobre todas las orientaciones (ecuación (6.57)). En el caso de un fluido puro las anteriores ecuaciones se reducen a las siguientes ${ }^{\dagger}$ :

${ }^{*} \vec{N}_{\mathrm{i}}^{\prime}$ es $\vec{N}_{\mathrm{i}}$ excluyendo $N_{\mathrm{i}}$

† La ecuación (6.87) se llama ecuación de la compresibilidad, obtenida por primera vez en 1914 por Ornstein y Zernike [144]. 


$$
\begin{gathered}
\kappa_{T}=\frac{\rho \cdot G+1}{k_{\mathrm{B}} \cdot T \cdot \rho} \\
V_{\mathrm{i}}=\frac{1}{\rho} \\
\left(\frac{\partial \mu}{\partial N}\right)_{T, P}=0 \\
\left(\frac{\partial \mu}{\partial N}\right)_{T, V}=\frac{k_{\mathrm{B}} \cdot T}{V \cdot\left(\rho+\rho^{2} \cdot G\right)}
\end{gathered}
$$

Y en una mezcla binaria a:

$$
\begin{gathered}
\kappa_{T}=\frac{\zeta}{k_{\mathrm{B}} \cdot T \cdot \eta} \\
V_{\mathrm{i}}=\frac{1+\rho_{\mathrm{j}} \cdot\left(\bar{G}_{\mathrm{jj}}-\bar{G}_{\mathrm{ij}}\right)}{\eta} \\
\mu_{\mathrm{ii}}=\frac{\rho_{\mathrm{j}} \cdot k_{\mathrm{B}} \cdot T}{\rho_{\mathrm{i}} \cdot V \cdot \eta} \\
\mu_{\mathrm{ij}}=\mu_{\mathrm{ji}}=-\frac{k_{\mathrm{B}} \cdot T}{V \cdot \eta}
\end{gathered}
$$

donde:

$$
\begin{gathered}
\eta=\rho_{\mathrm{A}}+\rho_{\mathrm{B}}+\rho_{\mathrm{A}} \cdot \rho_{\mathrm{B}} \cdot\left(\bar{G}_{\mathrm{AA}}+\bar{G}_{\mathrm{BB}}-2 \cdot \bar{G}_{\mathrm{AB}}\right)>0 \\
\zeta=1+\rho_{\mathrm{A}} \cdot \bar{G}_{\mathrm{AA}}+\rho_{\mathrm{B}} \cdot \bar{G}_{\mathrm{BB}}+\rho_{\mathrm{A}} \cdot \rho_{\mathrm{B}} \cdot\left(\bar{G}_{\mathrm{AA}}+\bar{G}_{\mathrm{BB}}-\bar{G}_{\mathrm{AB}}^{2}\right)>0
\end{gathered}
$$

\subsubsection{Inversión de la teoría de Kirkwood-Buff}

La teoría de Kirkwood-Buff se formuló originalmente para obtener cantidades termodinámicas a partir de las funciones de distribución molecular. Esta formulación es útil siempre y cuando las funciones de correlación $g_{\mathrm{ij}}\left(\vec{R}^{\prime}, \vec{R}^{\prime \prime}\right)$ sean conocidas, lo cual solamente es cierto en el caso de fluidos simples en los cuales se determinan directamente mediante experimentos de difracción, como sabemos. Por el contrario, la inversión de la teoría de Kirkwood-Buff, es decir, la evaluación de las KBI's a partir de los datos de $\kappa_{T}, V_{\mathrm{i}}$ y $\mu_{\mathrm{ij}}$ proporciona una poderosa herramienta para la investigación de las características del ambiente local de las moléculas de cada especie en la mezcla, aunque debe tenerse en cuenta que la evaluación de las cantidades $\bar{G}_{\mathrm{ij}}$ a partir de los datos termodinámicos experimentales presenta ciertas dificultades [145]-[149].

Aunque el procedimiento de inversión puede llevarse a cabo para cualquier mezcla de $\mathrm{n}$ componentes, aquí se expondrá el caso binario por ser de mayor interés. Las $N^{2}+N+1=7$ ecuaciones que determinan las cantidades $\kappa_{T}, V_{\mathrm{i}}$ y $\mu_{\mathrm{ij}}$ no son todas independientes puesto que se cumplen las relaciones siguientes:

$$
\begin{aligned}
& \rho_{A} \cdot \mu_{A A}+\rho_{B} \cdot \mu_{A B}=0 \\
& \rho_{B} \cdot \mu_{B B}+\rho_{A} \cdot \mu_{A B}=0
\end{aligned}
$$




$$
\rho_{\mathrm{A}} \cdot V_{\mathrm{A}}+\rho_{\mathrm{B}} \cdot V_{\mathrm{B}}=1
$$

Por lo tanto sólo 4 de las 7 ecuaciones entre las cantidades termodinámicas y las $\mathrm{KBI}^{\prime} \mathrm{s}$ son independientes. De hecho, como $\mu_{\mathrm{AB}}=\mu_{\mathrm{BA}}$, sólo quedan 3 ecuaciones para las 3 cantidades $\bar{G}_{\mathrm{AA}}, \bar{G}_{\mathrm{BB}}$ y $\bar{G}_{\mathrm{AB}}=\bar{G}_{\mathrm{BA}}$ :

$$
\begin{gathered}
\bar{G}_{\mathrm{AB}}=k_{\mathrm{B}} \cdot T \cdot \kappa_{T}-\rho \cdot V_{\mathrm{A}} \cdot V_{\mathrm{B}} / D \\
\bar{G}_{\mathrm{AA}}=k_{\mathrm{B}} \cdot T \cdot \kappa_{T}-\frac{1}{\rho_{\mathrm{A}}}+\frac{\rho_{\mathrm{B}} \cdot \rho \cdot V_{\mathrm{B}}^{2}}{\rho_{\mathrm{A}} \cdot D} \\
\bar{G}_{\mathrm{BB}}=k_{\mathrm{B}} \cdot T \cdot \kappa_{T}-\frac{1}{\rho_{\mathrm{B}}}+\frac{\rho_{\mathrm{A}} \cdot \rho \cdot V_{\mathrm{A}}^{2}}{\rho_{\mathrm{B}} \cdot D}
\end{gathered}
$$

donde $\rho=\rho_{\mathrm{A}}+\rho_{\mathrm{B}}$ es la densidad total de partículas y $D=\left[x_{\mathrm{A}} \cdot\left(\partial \mu_{\mathrm{A}} / \partial x_{\mathrm{A}}\right)_{T, P}\right] / k_{\mathrm{B}} \cdot T$. Estas ecuaciones se pueden expresar en una forma más condensada:

$$
\bar{G}_{\mathrm{ij}}=k_{B} \cdot T \cdot \kappa_{T}-\frac{\delta_{\mathrm{ij}}}{\rho_{\mathrm{i}}}+\rho \cdot k_{B} \cdot T \cdot \frac{\left(1-\rho_{\mathrm{i}} \cdot V_{\mathrm{i}}\right) \cdot\left(1-\rho_{\mathrm{j}} \cdot V_{\mathrm{j}}\right)}{\rho_{\mathrm{i}} \cdot \rho_{\mathrm{j}} \cdot \mu_{\mathrm{ij}}}
$$

Las ecuaciones (6.100)-(6.103) permiten obtener $\bar{G}_{\mathrm{ij}}$ a partir de datos puramente termodinámicos. El papel de $\kappa_{T}$ en el calculo es tan pequeño que el uso de $\kappa_{S}$ o de $\kappa_{T}^{\text {id }}$ (ecuación 2.165) en su lugar está justificado [149]-[151]. El mayor inconveniente está en determinar $\mu_{\mathrm{ij} j}$ lo cual puede conseguirse a partir de la segunda derivada de la energía de Gibbs de exceso obtenida mediante datos de equilibrio líquido-vapor o a partir de la presión de vapor de uno de los componentes. Cuando estos datos no están disponibles, se puede hacer una estimación de las cantidades $\mu_{\mathrm{ij}}$ mediante modelos teóricos (UNIQUAC, DISQUAC, etc). Debido a la complejidad de este tipo de experimentos y a que los modelos teóricos no dan resultados exactos, los valores calculados de $\bar{G}_{\mathrm{ij}}$ poseen errores considerables. Existe un cálculo alternativo de las cantidades $\bar{G}_{\mathrm{ij}}$ utilizando datos procedentes de la dispersión Rayleigh de la luz [145]. Lamentablemente, hay pocos datos disponibles en la literatura que permitan hacer este cálculo.

Es útil definir el grado de similitud entre dos componentes $\Delta_{\mathrm{AB}}$ :

$$
\Delta_{\mathrm{AB}}=\bar{G}_{\mathrm{AA}}+\bar{G}_{\mathrm{BB}}-2 \cdot \bar{G}_{\mathrm{AB}}
$$

Esta magnitud sirve para cuantificar la desviación respecto del comportamiento ideal, pues es condición necesaria y suficiente para que una mezcla sea ideal que $\Delta_{\mathrm{AB}}=0$ [130]. En ese caso, $\bar{G}_{\mathrm{AB}}=\left(\bar{G}_{\mathrm{AA}}+\bar{G}_{\mathrm{BB}}\right) / 2$ en todo el rango de concentraciones.

De los valores de $\bar{G}_{\mathrm{ij}}$ no puede inferirse nada acerca del modo microscópico en el que ocurre la agrupación de moléculas del tipo i en torno a una molécula central del tipo j, puesto que esta información, transportada por $\bar{g}_{\mathrm{ij}}$, se pierde en el proceso de integración. No obstante, puede obtenerse información acerca de las interacciones i-i, i-j y j-j a partir de las KBl's utilizando un mismo conjunto de datos experimentales. Así, en función de la dependencia de $\bar{G}_{\mathrm{ij}}$ con $x_{\mathrm{B}}$, se puede distinguir tres tipos de comportamientos:

a) $\bar{G}_{\mathrm{ij}}$ es función monótona creciente. En este caso el comportamiento de la mezcla se aproxima al de una mezcla ideal, para la cual $\bar{G}_{\mathrm{AB}}=\left(\bar{G}_{\mathrm{AA}}+\bar{G}_{\mathrm{BB}}\right) / 2$. Esto significa que 
a parte del efecto debido a los diferentes volúmenes molares, no hay ningún cambio debido a las correlaciones estructurales con respecto a los componentes puros, ni a la asociación ${ }^{*}$ fuerte de moléculas iguales o solvatación entre moléculas diferentes.

b) $\bar{G}_{\mathrm{AA}}$ y $\bar{G}_{\mathrm{BB}}$ presentan máximos y $\bar{G}_{\mathrm{AB}}$ mínimos. Esto significa que en torno a la concentración de los máximos, existe una gran tendencia entre moléculas del mismo tipo a agruparse, habiendo entonces fuertes interacciones atractivas entre ellas. En contraste, la disminución de $\bar{G}_{\mathrm{AB}}$ con el aumento de $x_{\mathrm{B}}$ cuando $\bar{G}_{\mathrm{AB}}$ tiende a su valor mínimo sugiere que algún cambio en la estructura de la esfera de solvatación, probablemente una pérdida de solvatación A-B, acompaña al aumento de la asociación B-B cuando aumenta la concentración de B. La inversión de este comportamiento, que ocurre tras haber alcanzado el mínimo, es decir, cuando $\bar{G}_{\mathrm{AB}}$ empieza a crecer, y la suave tendencia de $\bar{G}_{\mathrm{BB}}$ hacia el valor del componente B puro sugieren que la interacción directa B-B por contacto comienza a aparecer.

c) $\bar{G}_{\mathrm{AA}}$ y $\bar{G}_{\mathrm{BB}}$ presentan mínimos y $\bar{G}_{\mathrm{AB}}$ máximos. Esto significa que en torno a la concentración de los mínimos, la agrupación de moléculas del mismo tipo disminuye, lo que contrasta con el aumento de $\bar{G}_{\mathrm{AB}} \operatorname{con} x_{\mathrm{B}}$ cuando $\bar{G}_{12}$ tiende a su valor máximo, lo que sugiere que la solvatación A-B aumenta.

El concepto de composición local [152] es de enorme importancia en el cálculo de las propiedades termodinámicas de los fluidos y de sus mezclas, pues sirve como una herramienta conveniente para describir los procesos de solvatación y las desviaciones del comportamiento de las mezclas respecto del ideal [153]. Este concepto ha demostrado que incluso a bajas densidades las interacciones preferenciales es decir, la mezcla no al azar, son importantes [154]. Además ha desencadenado el desarrollo de nuevas líneas de investigación como por ejemplo el estudio de la dependencia de las reglas de mezcla con la densidad [155]. Pues bien, la teoría de disoluciones de Kirkwood-Buff permite desarrollar un método para calcular la composición local [156]. En efecto, recordemos que $\rho_{\mathrm{i}} \cdot \bar{g}_{\mathrm{ij}}(R) \cdot 4 \cdot \pi \cdot R^{2} \cdot \mathrm{d} R$ es el número promedio de moléculas del tipo i en una corteza esférica de espesor $\mathrm{d} R$ a una distancia $R$ del centro de una molécula del tipo j, luego:

$$
N_{\mathrm{ij}}=\int_{0}^{k \cdot \sigma_{i j}} \rho_{\mathrm{i}} \cdot \bar{g}_{\mathrm{ij}}(R) \cdot 4 \cdot \pi \cdot R^{2} \cdot \mathrm{d} R=x_{i} \cdot \rho \cdot \int_{0}^{k \cdot \sigma_{i j}} \bar{g}_{\mathrm{ij}}(R) \cdot 4 \cdot \pi \cdot R^{2} \cdot \mathrm{d} R
$$

es el número promedio de moléculas del tipo i en una esfera de radio $k \cdot \sigma_{i j}$ y centro en una molécula del tipo $\mathrm{j}$, donde $x_{\mathrm{i}}$ es la fracción molar del componente $\mathrm{i}$ de la mezcla y $k \cdot \sigma_{i j}=R_{c}$ es el mismo parámetro que aparece en la ecuación (6.76) y es la distancia a partir de la cual $\bar{g}_{\text {ij }}$ es uno, es decir, la distancia a partir de la cual $\bar{G}_{\mathrm{ij}}$ es cero, lo que define la llamada esfera de solvatación. En otras palabras,

$$
\int_{R_{c}}^{\infty}\left[\bar{g}_{\mathrm{ij}}(R)-1\right] \cdot 4 \cdot \pi \cdot R^{2} \cdot \mathrm{d} R=0
$$

Recuérdese que $\rho_{\mathrm{i}} \cdot \bar{G}_{\mathrm{ij}}$ representa el promedio del exceso o de la deficiencia total de moléculas del tipo i en torno a una molécula del tipo j sobre el promedio global, luego:

\footnotetext{
* En el contexto de la teoría de Kirkwood-Buff, además de las interacciones de tipo puente de hidrógeno y formación de complejos de transferencia de carga, por asociación y solvatación también se entiende interacciones de tipo físico entre moléculas iguales o distintas, respectivamente.
} 


$$
\rho_{\mathrm{i}} \cdot \bar{G}_{\mathrm{ij}}=N_{\mathrm{ij}}-N_{\mathrm{ij}}^{0}
$$

donde,

$$
N_{\mathrm{ij}}^{0}=\int_{0}^{R_{c}} \rho_{\mathrm{i}} \cdot 4 \cdot \pi \cdot R^{2} \cdot \mathrm{d} R=x_{i} \cdot \rho \cdot \int_{0}^{R_{c}} 4 \cdot \pi \cdot R^{2} \cdot \mathrm{d} R=\rho_{\mathrm{i}} \cdot \frac{4}{3} \cdot \pi \cdot R_{c}^{3}=\rho_{\mathrm{i}} \cdot V_{c}
$$

es el número promedio de moléculas del tipo i dentro de una esfera de radio $R_{c}$ y volumen $V_{c}$ centrada en un punto aleatorio del fluido, sin una molécula del tipo $\mathrm{j}$ en dicho centro.

La fracción molar local $x_{\mathrm{ij}}$ de moléculas i en torno a una j se define por:

$$
x_{\mathrm{ij}}=\frac{N_{\mathrm{ij}}}{\sum_{\mathrm{i}} N_{\mathrm{ij}}}
$$

la cual, por (6.105) se escribe:

$$
x_{\mathrm{ij}}=\frac{x_{\mathrm{i}} \cdot \rho \cdot \bar{G}_{\mathrm{ij}}+N_{i j}^{0}}{N_{i j}^{0}+N_{j j}^{0}+\rho \cdot\left(x_{\mathrm{i}} \cdot \bar{G}_{\mathrm{ij}}+x_{\mathrm{j}} \cdot \bar{G}_{\mathrm{jj}}\right)}
$$

Definiendo $\varepsilon=V_{c}^{-1}$ y teniendo en cuenta (6.108), se reescribe la ecuación (6.110):

$$
x_{\mathrm{ij}}=\frac{x_{\mathrm{i}} \cdot\left(\varepsilon \cdot \bar{G}_{\mathrm{ij}}+1\right)}{1+\varepsilon \cdot \sum_{i} x_{\mathrm{i}} \cdot \bar{G}_{\mathrm{ij}}}
$$

La comparación de los resultados obtenidos mediante esta ecuación con los obtenidos por medio de un modelo de composición local sirve como test de dicho modelo. La principal dificultad es la determinación de la distancia $R_{c}$ [156]. Por ejemplo, Zielkiewicz [157] propone que $R_{c}=3 \cdot \sigma_{\mathrm{j}}$, siendo $\sigma_{\mathrm{j}}$ el radio de la molécula j que está en el centro de la esfera de solvatación.

La desviación de la fracción molar local respecto la global es un parámetro importante:

$$
\delta_{\mathrm{ij}}=x_{\mathrm{ij}}-x_{\mathrm{i}}
$$

Si se hace una expansión en serie de potencias en torno a $\varepsilon=0$, a los primeros términos se les llama coeficientes de solvatación preferencial $\delta_{\mathrm{ij}}^{0}$ [153]. En mezclas binarias son:

$$
\begin{aligned}
& \delta_{\mathrm{ii}}^{0}=x_{\mathrm{i}} \cdot x_{\mathrm{j}}\left(\bar{G}_{\mathrm{ii}}-\bar{G}_{\mathrm{ij}}\right) \\
& \delta_{\mathrm{ij}}^{0}=x_{\mathrm{i}} \cdot x_{\mathrm{j}}\left(\bar{G}_{\mathrm{ij}}-\bar{G}_{\mathrm{jj}}\right)
\end{aligned}
$$

Así, truncando el desarrollo en serie de potencias de $\delta_{\mathrm{ij}}$ en el primer término, se tiene:

$$
x_{\mathrm{ij}}=\delta_{\mathrm{ij}}+x_{\mathrm{i}}=\delta_{\mathrm{ij}}^{0} \cdot \varepsilon+x_{\mathrm{i}}=\frac{\delta_{\mathrm{ij}}^{0}}{V_{c}}+x_{i}
$$

Los valores de $\delta_{\mathrm{ij}}^{0}$ reflejan cambios en la fracción molar local de la especie i en torno a la molécula central de tipo j respecto la fracción molar global. Valores pequeños de $\delta_{\mathrm{ij}}^{0}$ $\left(x_{\mathrm{ij}} \approx x_{\mathrm{i}}\right)$ revelan que la molécula del tipo i se incorpora dentro de la estructura polimérica 
de las moléculas del tipo $\mathrm{j}$, lo que concuerda con la existencia de complejos de asociación i-j. La estructura de la mezcla, en este caso, está próxima a la de una mezcla al azar. En caso contrario, por ejemplo si $x_{\mathrm{ii}}>x_{\mathrm{i}}$, existen interacciones preferenciales entre las moléculas del tipo i.

Para concluir, se debe tener en cuenta que la magnitud de $\delta_{\mathrm{ij}}^{0}$ depende principalmente de dos factores: la energía de las interacciones intermoleculares y las diferencias entre los tamaños moleculares.

\subsection{MODELOS DE LIQQUIDOS Y DISOLUCIONES}

La construcción de un modelo para el sistema que se pretende investigar se lleva a cabo mediante la adopción a priori de ciertas hipótesis ad hoc acerca de la distribución y movimientos de las partículas, de forma que el espacio de las configuraciones se reduce a aquella porción que produce las principales contribuciones a $\Omega(T)$, eliminando el resto de configuraciones. El éxito de este procedimiento viene determinado esencialmente por la elección de las configuraciones que se retienen en el modelo. La adopción de dichas hipótesis se realiza a partir de consideraciones teóricas o resultados experimentales, que son responsables de la forma de la función de partición configuracional.

Un modelo estadístico perfecto debería ser capaz de expresar la energía libre de Helmholtz y las funciones derivadas de la misma en términos de energías de interacción independientes de la temperatura. Sin embargo, este objetivo nunca se ha alcanzado en los modelos propuestos, ni siquiera para los más sencillos, lo que es debido a que ni siquiera el campo de fuerzas en torno a la molécula orgánica más simple (el metano), y mucho menos en moléculas más complejas, tiene simetría esférica perfecta. Por tanto, las fuerzas de interacción molecular dependen no sólo de las distancias sino también de los ángulos. Debido a las dificultades físicas y matemáticas que surgen al intentar operar con la energía de interacción, normalmente se toma un conjunto reducido de estados microscópicos, cada uno de ellos siendo en realidad un promedio de los estados microscópicos reales. Por ello, los parámetros de interacción de las ecuaciones resultantes son cantidades pre-promediadas, no representando energía sino energías libres, por lo que no debe resultar extraño que ellos tengan algún tipo de dependencia con la temperatura, como se ha visto en la sección 6.1.

Por todo lo dicho, es obvio que ningún modelo del estado líquido o de disoluciones proporciona una respuesta absolutamente satisfactoria. En consecuencia puede encontrarse una gran cantidad de modelos en la bibliografía [92], [124], [158]-[161], que proporcionan resultados más o menos buenos dentro de los límites de aplicabilidad de los mismos. Puesto que es difícil percibir las consecuencias de las aproximaciones introducidas con la adopción del modelo empleado, se requiere una detallada discusión de los casos individuales así como la búsqueda del mayor número posible de pruebas de consistencia interna del modelo.

La teoría del estado líquido más antigua es la de van der Waals [162], cuya característica más notable es que los estados gaseoso y líquido están descritos por la misma ecuación de estado. La extensión al caso de mezclas fue desarrollada por van Laar [163]. La teoría de van Laar supone que a $P$ y $T$ constantes la mezcla se produce sin cambio de volumen y con entropía de mezcla igual a la ideal, es decir, $V_{\mathrm{m}}^{\mathrm{E}}=S_{\mathrm{m}}^{\mathrm{E}}=0$, 
luego $G_{\mathrm{m}}^{\mathrm{E}}=U_{\mathrm{m}}^{\mathrm{E}}$. Utilizando unas reglas de mezcla específicas y suponiendo que las propiedades volumétricas de los fluidos y de la mezcla vienen dadas por la ecuación de van der Waals (las mayores fuentes de error de esta teoría residen en ambas hipótesis) se pueden obtener los coeficientes de actividad. En el caso binario,

$$
\ln f_{\mathrm{i}}=\frac{A_{\mathrm{i}}}{\left(1+\frac{A_{\mathrm{i}}}{A_{\mathrm{j}}} \cdot \frac{x_{\mathrm{i}}}{x_{\mathrm{j}}}\right)^{2}}
$$

donde $A_{\mathrm{i}}$ y $A_{\mathrm{j}}$ están relacionados con las constantes que aparecen en la ecuación de van der Waals. Los coeficientes de actividad predichos por esta teoría nunca son menores que la unidad, por lo que las desviaciones respecto la ley de Raoult (que es el estado de mezcla ideal escogido por la teoría) siempre son positivas, razón por la que esta teoría solamente es aplicable al caso de mezclas de sustancias apolares (fuerzas dispersivas).

Como se ha dicho, lo importante de las teorías de van der Waals y van Laar es que tratan a los líquidos como gases muy densos, altamente comprimidos, lo que proporcionó un primer punto de partida para el desarrollo de posteriores tratamientos teóricos basados en la búsqueda de una ecuación de estado, y da lugar a las llamadas teorías de van der Waal generalizadas [164], por ejemplo la ecuación de estado de Flory. Este tipo de tratamiento está de acuerdo con el hecho experimental, por todos conocido, de que en sustancias monocomponentes es posible, mediante las transformaciones adecuadas de volumen y temperatura, pasar de la fase líquida a la gaseosa sin que se produzca una transición de fase de primer orden. De hecho, en el punto crítico ambas fases son indistinguibles y por eso se las llama indistintamente fases fluidas. En contraste, no se ha encontrado un procedimiento similar por el cual se alcance la fase cristalina a partir de las fases fluidas, pues siempre es necesario traspasar la zona de coexistencia cristal-fluido. Por su parte, los patrones de difracción de rayos $X$ y de difracción de neutrones sugieren que los sólidos cristalinos están caracterizados por tener estructura total (lo que permite estudiarlos en términos de modelos de red, en los que el número de vecinos próximo a un sitio de la red, $z$, es constante) que no poseen los gases (desestructura total) ni los líquidos, sobre todo en las proximidades del punto crítico, [165, pp 229]. No obstante, a pesar de los cambios estructurales instantáneos e incesantes, el análisis de las funciones de distribución radial y similares lejos del punto crítico (véase la llustración 6-2) sugiere que los líquidos tienen un orden a corto alcance que no poseen los gases, es decir, tienen cierto grado de empaquetamiento, lo que es consecuencia de que los líquidos son más densos que los gases (el volumen accesible para cada molécula es del orden de su volumen; por ello la distribución de las moléculas de un líquido en el recipiente que lo contiene es mucho más compacta que en un gas) y de las fuerzas repulsivas debidas a la no superposición de los orbitales moleculares. Ello justifica un segundo punto de partida para la descripción de la fase líquida, contraria a la dada por van der Waals: el tratamiento de un líquido lejos de los puntos críticos como una red cuasi-cristalina [166]. Existe otra razón empírica que justifica la utilización de los modelos reticulares [167], y es el hecho de que en las fases condensadas la presión interna es mucho mayor que la presión de vapor.

A parte de los modelos tipo van der Waals y cuasicristalinos, existe una tercera posibilidad, una aproximación basada en el tratamiento perturbativo de primer orden de 
una mezcla ideal que es independiente de cualquier modelo del estado líquido. Es lo que se conoce por teoría conformacional, la cual se ha desarrollado tanto para líquidos puros como para mezclas y en base a ella se han discutido efectos importantes, por ejemplo los efectos Patterson y Wilhelm [168]-[178].

Antes de exponer los modelos de interés sobre los líquidos y sus mezclas, es necesario tener en cuenta que aunque a primera vista la teoría concerniente a mezclas líquidas parece ser la generalización de la teoría de los líquidos puros para sistemas multicomponentes, ello no es del todo cierto por las siguientes razones:

a) Hay diferentes aspectos físicos relevantes y tipos de información experimental disponible en el caso de líquidos puros y en el caso de mezclas. En el caso de las disoluciones, las propiedades más importantes son las funciones de exceso, que se definen, como sabemos, en función de un estado de referencia, el cual puede no existir en el caso de los líquidos puros. El estado de referencia puede ser escogido como aquél en el que todos los componentes de la disolución se encuentran puros, o bien como una disolución infinitamente diluida en todos sus componentes excepto en uno. La existencia de un estado de referencia físicamente aceptable plantea la cuestión de si son posibles algunas reglas generales que deban aplicarse al acceder a dicho estado. La respuesta a esta pregunta la dan las llamadas "leyes asintóticas para disoluciones infinitamente diluidas". Estas reglas son muy importantes en las aplicaciones prácticas de la Termodinámica Fenomenológica. Todo el formalismo que se ha desarrollado para la discusión de mezclas en fases condensadas por medio de la Termodinámica Fenomenológica se basa en estas leyes límite. La teoría exacta de las leyes límite, que se formula usando el método de los clusters de Mayer [92 cap. 17.2], se basa en la hipótesis de que las fuerzas de interacción son de corto alcance, de forma que su validez está limitada a las mezclas de no electrolitos.

b) Las teorías existentes para líquidos puros son meras aproximaciones. El que una aproximación sea válida para un líquido puro no implica que lo sea para una mezcla (y viceversa).

c) El conocimiento de las fuerzas de interacción molecular es muy limitado. A pesar de que existen algunas conexiones razonables entre la teoría cuántica de las interacciones y la teoría estadística, la verdad es que ni siquiera en el caso más simple, mezcla binaria, la base teórica de la interacción entre diferentes moléculas es suficiente como para evitar introducir reglas empíricas y parámetros ajustables (Lorentz, Berthelot, etc.). La complejidad del problema crece con la complejidad de las moléculas involucradas ya que pueden aparecen interacciones entre moléculas distintas que no ocurrían entre moléculas del mismo tipo: enlaces puente de hidrógeno, fuerzas dipolo permanente-dipolo inducido entre moléculas polares y apolares (que son consecuencia de que el campo eléctrico de la molécula polar induce un dipolo en la apolar ya que la polarizabilidad de ésta no es nula), etc.

d) El problema de la estructura del estado líquido juega un papel menor en el caso de las mezclas. Ello se debe a que en el cálculo de las propiedades de exceso respecto del estado de referencia se cancela gran parte de la información relativa a la estructura.

\subsubsection{Principios básicos de los modelos de celdas}

Cuando se utilizan los llamados modelos de celdas se debe tener presente que como consecuencia de que la Física Estadística en la formulación tradicional sólo es aplicable 
estrictamente a líquidos simples, la función de partición es separable de acuerdo con la ecuación (6.22).

Las premisas en las que se basan dichos modelos son las siguientes:

a) Se trata al líquido como si cada molécula se moviese en torno a un nudo de la red, en una región llamada celda cuyo centro es dicho nudo. El tamaño de la celda está determinado por el potencial de interacción promedio con los vecinos más próximos, y puede que también con los segundos y terceros vecinos. Mientras que en un cristal cada molécula está rodeada por un número fijo de vecinos más próximos (número de coordinación $z$ ), en el caso de los líquidos este número es constante solo en promedio y propio del tipo de red. Así, en una red cúbica simple, $z=6$; red cúbica centrada en el cuerpo, $z=8$; red cúbica centrada en las caras, $z=12$. En el último caso se dice que la red tiene máximo empaquetamiento, pues por razones geométricas es imposible encontrar números de coordinación mayores de 12. Aunque existen fluctuaciones en torno a este valor promedio, no son significativas y por ello se considera que las relaciones geométricas de cada molécula con sus vecinos próximos son similares a las que se encuentran en un cristal. No obstante, las fluctuaciones son suficientes como para destruir cualquier regularidad entre las configuraciones de las moléculas lejanas (puede haber excepciones como por ejemplo en el caso de moléculas largas que generan anisotropía en los líquidos, lo que se conoce como cristales líquidos), característico de la ausencia de orden a largo alcance de los líquidos. Por todo lo dicho, se tratarán las mezclas líquidas como cuasicristales, y se utilizará la terminología aplicable a la red cristalina a pesar de que no existe una red precisa. El modelo de red deja de ser útil cerca del punto crítico pues las distancias entre las moléculas aumentan rápidamente al aproximarse a éste.

b) Cada molécula ocupa una y sólo una celda.

c) Todas las celdas están ocupados. Como consecuencia de ello y de la hipótesis b), el número de nudos de la red es igual al número de moléculas.

d) El problema de los $N$ cuerpos se reduce a un problema de un cuerpo mediante un esquema matemático equivalente al del campo autoconsistente de Hartree-Fock de la Mecánica Cuántica, lo que implica que se suprime la correlación entre las posiciones de las partículas, y por tanto, se postula la independencia de los movimientos de las moléculas confinadas en sus celdas. Por ello, estos modelos no son apropiados cerca de los puntos críticos en donde las correlaciones entre las moléculas son muy importantes.

e) El campo de fuerzas en el que se mueve una molécula, el cual se debe al resto de moléculas y es función del tiempo pues tanto la molécula en cuestión como el resto se mueven dentro de sus celdas, se puede reemplazar por un potencial promedio generado por los vecinos más próximos y correspondiente a la situación en la que todos los primeros vecinos próximos se encuentran en sus posiciones de energía potencial más baja, es decir, en los nudos de la red.

f) Suponiendo que las celdas son esferas, es suficiente suponer que dicho potencial promedio es esféricamente simétrico respecto del centro de la celda. Si por el contrario, las moléculas no son esféricas, los campos de fuerza no son centrales. Para hacerlos tratables, se sustituyen por potenciales efectivos dependientes de la temperatura.

Los llamados modelos de red rígida se basan en el conjunto de premisas anteriores, al cual se añade la hipótesis simplificadora siguiente: 
g) El volumen es constante, es decir, no hay volumen de mezcla. Tal y como Guggenheim desarrolló el modelo de red, se supuso que a presiones similares o inferiores a la atmosférica, todos los términos $P \cdot V$ ó $P \cdot \mathrm{d} V$ que aparecen en las relaciones termodinámicas son despreciables. Esto conduce a la indistinguibilidad entre $G$ y $F$ y entre $F$ y $H$, es decir, $G \approx F, U \approx H$. Sin embargo, este razonamiento solo es válido para gases. En el caso de líquidos y sólidos, la presión que aparece en las ecuaciones es la suma de la presión atmosférica y la presión interna, la cual, para el caso de los líquidos, puede ser mil veces mayor que la atmosférica, por lo que nunca debe despreciarse. No obstante, el hecho de que el volumen sea constante es el responsable de que en los modelos de red se verifiquen las igualdades: $G_{\mathrm{m}}^{\mathrm{E}}=F_{\mathrm{m}}^{\mathrm{E}}, U_{\mathrm{m}}^{\mathrm{E}}=H_{\mathrm{m}}^{\mathrm{E}} \mathrm{y}$ $C_{P, \mathrm{~m}}^{\mathrm{E}}=C_{V, \mathrm{~m}}^{\mathrm{E}}$.

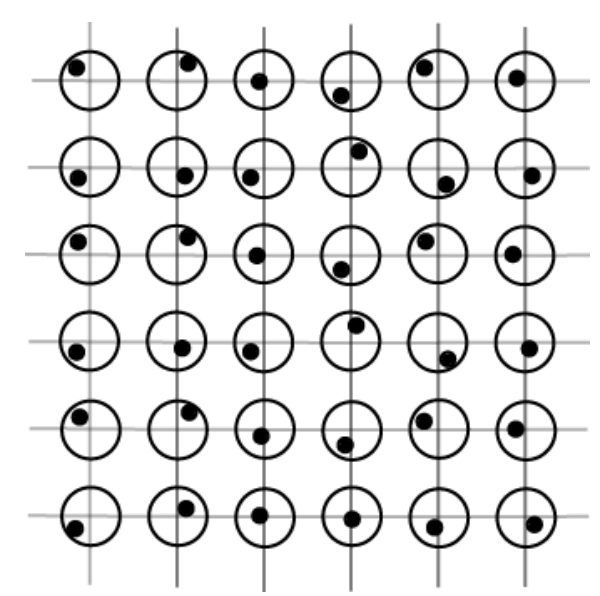

Ilustración 6-4: red bidimensional cuadrada $(z=4)$, con celdas circulares. Las moléculas están confinadas en sus celdas

Por lo tanto, los modelos de red rígida presentan la grave limitación de no ser capaces de explicar los valores del coeficiente de dilatación térmica ni los volúmenes de mezcla. Además, tanto los modelos de red rígida como los de celdas presentan varias limitaciones comunes: en primer lugar, las ecuaciones que se obtienen para las funciones termodinámicas de las mezclas no se reducen a las propiedades de los componentes puros cuando las concentraciones de todos ellos, excepto la de uno, son iguales a cero; en segundo lugar, son incompatibles con un conjunto de hechos experimentales [179] entre los que se pueden destacar la existencia de $\mathrm{P}_{4}$ líquido subenfriado a temperaturas hasta $-71^{\circ} \mathrm{C}$, los valores del coeficiente de temperatura de difusión a volumen constante del $\mathrm{I}_{2}$ en $\mathrm{CCl}_{4}$, los iguales volúmenes molares parciales a dilución infinita del $I_{2}$ en distintos disolventes con volúmenes molares diferentes, etc.; por último, la experiencia demuestra que cada molécula se mueve de forma casi browniana, de modo que la hipótesis de que las moléculas vibran en torno a posiciones fijas de una red o dentro de sus correspondientes celdas carece de fundamentos físicamente aceptables. Sin embargo, si la red se observa como una red de recuento de estados, y no como una red geométrica, parte de estos inconvenientes son superados.

Los modelos de celdas y de red son interesantes puesto que introducen algunos problemas (transición orden-desorden, efectos orientacionales, cáculo del factor combinatorial, etc.) que también aparecen cuando se utilizan modelos más refinados. Además, forman la base teórica de modelos más complicados por lo que es justificable la breve exposición que aquí se da de sus hipótesis y resultados básicos. 
Consideremos primero el caso de sustancias puras. Un primer modelo sencillo de red rígida (modelo de potencial suave) consiste en sustituir $W$ por su valor promedio sobre todas las posibles configuraciones [180] en el interior de la celda, y por infinito en los límites de ésta. Dicho valor promedio es evidentemente proporcional al número de moléculas y negativo (puesto que las fuerzas promedio son repulsivas) luego se puede escribir como $-N \cdot \chi$, donde cada molécula se mueve libremente en un potencial uniforme $-\chi$, que es el potencial promedio debido a todas las posibles configuraciones de los primeros vecinos cercanos de una molécula dada, el cual depende del volumen por molécula $v_{f}=V / N$, llamado volumen libre. En este caso, la función de partición configuracional es:

$$
\Omega(T)=\left[e \cdot v_{f} \cdot e^{\chi(v) / k_{\mathrm{B}} \cdot T}\right]^{N}
$$

Un modelo de red rígida menos simple es el modelo de oscilador armónico de Mie [181], resultante de suponer que cada molécula se mueve en su celda en torno al nudo de la red como un oscilador armónico tridimensional de frecuencia $v$ y es similar al modelo de cristal de Einstein [182], [183]. Si $\chi_{0}$ es el potencial de cada molécula en su posición de equilibrio, es decir, en el nodo de la red y $r$ es la distancia de una molécula cualquiera al punto de su energía mínima, entonces el potencial de una molécula a distancia $r$ de su punto de equilibrio es $\chi_{0}+m \cdot(2 \cdot \pi \cdot v)^{2} \cdot r^{2} / 2, \mathrm{y}$ :

$$
\Omega(T)=\left[e \cdot v_{f} \cdot e^{\chi_{0} / k_{\mathrm{B}} \cdot T}\right]^{N}
$$

donde,

$$
v_{f}=\left(\frac{k_{B} \cdot T}{2 \cdot \pi \cdot m \cdot v^{2}}\right)^{3 / 2}
$$

es el volumen libre efectivo del modelo de oscilador armónico.

La principal desventaja del modelo de Mie radica en el hecho de que solamente proporciona una distinción cuantitativa entre las fases líquida y sólida, expresada por la diferencia entre los valores de los parámetros $v$ y $\chi_{0}$, y no hace ninguna aclaración sobre la dependencia de estos parámetros con las variables de estado.

El potencial del modelo de Mie básicamente se obtiene como una hipótesis ad hoc. Lennard-Jones y Devonshire [184]-[185] fueron los primeros en obtener con éxito una función para el potencial de interacción. Partiendo de las premisas generales propias de los modelos de celdas y añadiendo las llamadas aproximación delta y de suavizado (las cuales no se detallan aquí pero se pueden consultar en $[92,16.2 \alpha-\beta]$ ) se obtiene el llamado modelo de Lennard-Jones y Devonshire (LDD), que se expone a continuación.

Sea $a$ la distancia entre nudos de la red que son vecinos próximos. Supongamos que una molécula se encuentra fija en un nudo y que otra se mueve dentro de su celda, una esfera de radio $r$ cuyo centro se encuentra a la distancia $a$ de la otra molécula. El promedio de la energía potencial de interacción mutua entre ambas partículas es:

$$
\langle\varepsilon(r)\rangle=\frac{1}{2} \cdot \int_{0}^{\pi} \varepsilon\left[\left(r^{2}+a^{2}-2 \cdot a \cdot r \cdot \cos \theta\right)^{1 / 2}\right] \cdot \operatorname{sen} \theta \cdot \mathrm{d} \theta
$$

donde $\varepsilon(r)$ es la energía potencial de interacción mutua entre dos moléculas. Si $z$ es el número de coordinación, la energía potencial promedio $W(r)$ de $z$ vecinos próximos 
dentro de una celda de radio $r<a$ es:

$$
W(r)=z \cdot\langle\varepsilon(r)\rangle
$$

Para poder continuar, debe asumirse una forma para $\varepsilon(r)$. Lenard-Jones y Devonshire adoptaron la siguiente:

$$
\varepsilon(r)=-2 \cdot\left|\varepsilon^{*}\right| \cdot\left(\frac{r^{*}}{r}\right)^{6}+\left|\varepsilon^{*}\right| \cdot\left(\frac{r^{*}}{r}\right)^{12}
$$

donde $\varepsilon^{*}$ es el valor mínimo de $\varepsilon$, el cual ocurre a la distancia $r^{*}$. Sustituyendo (6.120) y (6.122) en (6.121) se tiene que:

$$
W(r)-W(0)=z \cdot\left|\varepsilon^{*}\right| \cdot\left[\left(\frac{r^{*}}{r}\right)^{12} \cdot l\left(\frac{r^{2}}{a^{2}}\right)-2 \cdot\left(\frac{r^{*}}{r}\right)^{6} \cdot m\left(\frac{r^{2}}{a^{2}}\right)\right]
$$

donde $W(0)=\lim _{r \rightarrow 0} W(r)$ y $l(y), g(y)$ son las funciones siguientes:

$$
\begin{gathered}
l(y)=\left(1+12 \cdot y+50 \cdot y^{2}+12 \cdot y^{3}+y^{4}\right) \cdot(1-y)^{-10}-1 \\
m(y)=(1+y) \cdot(1-y)^{-4}-1
\end{gathered}
$$

Es útil definir las cantidades siguientes:

$$
\begin{gathered}
\Lambda^{*}=-z \cdot \varepsilon^{*} \\
\gamma=\frac{a^{3}}{v} \\
V^{*}=\frac{\left(r^{*}\right)^{3}}{\gamma}=\frac{v}{a^{3}} \cdot\left(r^{*}\right)^{3}
\end{gathered}
$$

donde $v$ es el volumen por molécula $V / N$. Sea $-N \cdot \chi_{0}$ la energía total del sistema cuando cada molécula se encuentra en su posición de equilibrio, es decir, en los centros de su celda, referida a un origen de potenciales a distancia infinita de las moléculas. En estas condiciones se puede demostrar que la función de partición del sistema es:

$$
Z=\frac{\left(2 \cdot \pi \cdot m \cdot k_{B} \cdot T\right)^{3 / 2}}{h^{3}} \cdot e^{\chi_{0} / k_{B} \cdot T} \cdot 2 \cdot \pi \cdot N \cdot a^{3} \int_{0}^{0.25} y^{1 / 2} \cdot e^{p(y)} \cdot \mathrm{d} y
$$

donde,

$$
p(y)=\frac{\Lambda^{*}}{k_{B} \cdot T} \cdot\left[-\left(\frac{V^{*}}{V}\right)^{4} \cdot l(y)+2 \cdot\left(\frac{V^{*}}{V}\right)^{2} \cdot m(y)\right]
$$

la función de partición configuracional es:

$$
\Omega(T)=e^{N} \cdot e^{N \cdot \chi_{0} / k_{B} \cdot T} \cdot\left(2 \cdot \pi \cdot a^{3}\right)^{N} \cdot\left[\int_{0}^{0.25} y^{1 / 2} \cdot e^{p(y)} \cdot \mathrm{d} y\right]^{N}
$$

Si ahora se define la cantidad: 


$$
v_{f}=2 \cdot \pi \cdot a^{3} \cdot \int_{0}^{0.25} y^{1 / 2} \cdot e^{p(y)} \cdot \mathrm{d} y=2 \cdot \pi \cdot \gamma \cdot v \cdot g
$$

donde

$$
g=\int_{0}^{0.25} y^{1 / 2} \cdot e^{p(y)} \cdot \mathrm{d} y
$$

Así, la función de partición configuracional tiene la forma dada por la expresión (6.1 18):

$$
\Omega(T)=\left[e \cdot v_{f} \cdot e^{\chi_{0} / k_{\mathrm{B}} \cdot T}\right]^{N}
$$

Este es un resultado general de todos los modelos de celdas. En todos ellos se encuentra que la función de partición configuracional depende de $v_{f}$, por lo que a los modelos de celdas también se les llama de volumen libre. En cada modelo particular el volumen libre viene dado por la ecuación siguiente:

$$
v_{f}=\int_{0}^{\infty} r^{2} \cdot \mathrm{d} r \int_{0}^{\pi} \operatorname{sen} \theta \cdot \mathrm{d} \theta \int_{0}^{2 \cdot \pi} \mathrm{d} \varphi \cdot \mathrm{e}^{-\left[\frac{W(r)-W(0)}{k_{\mathrm{B}} \cdot T}\right]}
$$

donde el origen de coordenadas está situado en el centro de la celda, supuesta esférica, y $W(r)$ es el potencial de interacción de cada molécula, el cual sólo depende de la distancia al centro de la celda pues el movimiento de cada molécula se supone independiente del resto (hipótesis d de los modelos de celdas).

El modelo de Lennard-Jones puede mejorarse si se tienen en cuenta las interacciones entre moléculas que no son vecinos próximos [187]. En este caso, la función de partición configuracional viene dada por (6.118) en donde $v_{f}$ está dado por (6.132) y $\chi_{0}$ debe sustituirse por:

$$
\chi_{0}=\Lambda^{*} \cdot\left[-1.2 \cdot\left(\frac{V^{*}}{V}\right)^{2}+0.5 \cdot\left(\frac{V^{*}}{V}\right)^{4}\right]
$$

Como resultado de la teoría de LJD, las propiedades de equilibrio de cada sustancia están perfectamente determinadas por los valores de los parámetros $\varepsilon^{*}, r^{*}, z$ y $\gamma$. Como la teoría es solamente aplicable de manera estricta a moléculas con simetría esférica, para las cuales la forma más estable de la red es la cúbica centrada en las caras $(z=12$, $\gamma=\sqrt{2}$ ), las propiedades de equilibrio de cada sustancia dependen solamente de $\varepsilon^{*}$ y $r^{*}$, o alternativamente, de $\Lambda^{*}$ y $V^{*}$. Por análisis dimensional se puede deducir que la ecuación de estado tiene la forma funcional siguiente:

$$
\frac{P \cdot V}{k_{\mathrm{B}} \cdot T}=\phi\left(\frac{k_{\mathrm{B}} \cdot T}{\Lambda^{*}}, \frac{V}{V^{*}}\right)
$$

Debido a la complicada forma de $W(r)$ no existe solución analítica para $\phi$, aunque existen tablas de la función de partición y sus derivadas respecto el volumen y la temperatura que permiten comparar la teoría con los datos experimentales [184]-[190]. A pesar de no tener solución analítica, la ecuación (6.136) sigue siendo válida pues correlaciona las relaciones $P V T$ de diferentes sistemas. De hecho, es una expresión exacta general de la ley de los estados correspondientes. 
Aunque el modelo de LJD es solamente aplicable a moléculas con simetría esférica, Prigogine et al [186] lo intentaron generalizar al caso de moléculas sin simetría esférica en términos parecidos al tratamiento de polímeros en los modelos de red rígida.

El modelo LJD se ha extendido para su aplicación en mezclas, en gran parte por Prigogine y sus colaboradores. El modelo resultante más sencillo se basa en suponer que las moléculas tienen simetría esférica, las moléculas de las diferentes especies son de igual tamaño y que la mezcla es al azar [189], [191], [192]. Así, el tratamiento resultante es parecido al de las disoluciones estrictamente regulares. No obstante, el modelo se ha extendido a moléculas que no tienen simetría esférica [193], a mezclas de moléculas con diferente tamaño [194]-[196], e incluso se ha eliminado la hipótesis de mezcla al azar [195], [197] demostrando que los efectos orden-desorden de las mezclas consideradas son generalmente pequeños.

El principio de los estados correspondientes aplicado a las mezclas líquidas y formulado de forma general parte de la hipótesis de que los potenciales intermoleculares de los diferentes componentes tienen las misma forma funcional cuando se expresa en función de la distancia entre los centros de las moléculas (o entre los centros de los segmentos en la adaptación a $r$-méros). Estrictamente hablando, el potencial intermolecular debe ser paramétrico en la distancia y la energía. Si se conocen las propiedades termodinámicas en función de la temperatura y la densidad de un líquido de referencia, o sustancia equivalente, las de cualquier otro de la familia de líquidos correspondientes a éste se determinan mediante dos factores de escala, uno para la distancia de separación entre los centros de las moléculas y otro para la magnitud del potencial intermolecular. Así, las propiedades de un líquido dado pueden determinarse por medio de estos dos parámetros, que pueden ser representados mediante una temperatura característica $T^{*}$ y una presión característica $p^{*}$. En el caso de $r$-méros se necesita un tercer factor adicional que puede representarse mediante el número de grados de libertad de traslación equivalentes.

La principal ventaja del modelo de celdas de Prigogine sobre los modelos de mezclas de red rígida como el de Guggenheim es que predice valores no nulos de los volúmenes de mezcla.

Las hipótesis b) y d) generan las principales fuentes de error de los modelos de celdas, pues la hipótesis b) hace ignorar todas aquellas configuraciones en las que puede haber celdas ocupadas por más de una molécula y la hipótesis d) elimina la correlación de los movimientos de las moléculas debido a la reducción del problema global al de un único cuerpo. Si se elimina la hipótesis b), es decir, si en lugar de suponer que cada celda está ocupada solamente por una molécula, se supone que existen fluctuaciones en el número de ocupación de las celdas, las cuales pueden considerarse como fluctuaciones de densidad a escala molecular, en las ecuaciones de la entropía aparece un término adicional Ilamado entropía comunal [187], [198]-[200]. De esta manera también se tienen en cuenta, al menos en parte, los efectos debidos a la correlación de las moléculas dentro de una misma celda.

El modelo LJD, como el resto de los modelos de celda, no es satisfactorio sobre todo debido a la excesiva estructura molecular que introduce. En dicho modelo se supone que el promedio del número de coordinación es fijo y que depende solamente del tipo de red, lo cual está en contra de la información experimental de que se dispone. Por ejemplo, los experimentos de difracción de rayos $X$ del argón [201] indican que el 
número de coordinación disminuye desde $\approx 12$ en la fase sólida hasta $\approx 6$ en la fase líquida a temperatura cercana a la crítica. Así, se hace necesario un refinamiento de la teoría LJD. En primer, lugar, se introduce la posiblidad de que las celdas tengan números de ocupación múltiple, como se ha explicado en el párrafo anterior, lo que elimina la restricción de que las fluctuaciones locales de densidad sean nulas. En segundo lugar, se introduce la posibilidad de que existan celdas vacías, lo que da lugar al modelo de agujeros [92, 16.3ß], [202]-[206], el cual proporciona mejoras respecto del modelo LJD para gases moderadamente densos, mientras que en el rango de densidades líquidas, mejoras en algunos aspectos se combinan con el empeoramiento en otros. El modelo de agujeros evolucionó hacia el modelo de estructuras significativas [207]-[209], en el que un líquido se considera como una mezcla de grados de libertad de tipo sólido y gaseoso. Este modelo surge como un intento de explicar el cambio de volumen de una sustancia cuando funde, que puede ser del orden de un $12 \%$. El análisis de rayos $X$ dictamina que las distancias intermoleculares medias en líquidos son prácticamente iguales que en los sólidos. ¿Cómo justificar el aumento de volumen de fusión? Una interpretación plausible consiste en suponer que en el líquido existen, a escala microscópica zonas que conservan una estructura cristalina, junto con otras de baja densidad en donde existen espacios intermoleculares vacíos, equivalentes a la estructura gaseosa. Estos espacios vacíos explicarían el aumento de volumen conservando las distancias intermoleculares. Cualquier molécula de la zona cristalina que adquiera la energía térmica suficiente para vencer las fuerzas que la ligan a sus vecinas puede saltar a la zona de baja densidad, adquiriendo propiedades gaseosas. Por el contrario, cualquier molécula gaseosa puede ser atrapada en la zona cristalina, pasando a formar parte de éstas. Así, en un líquido se distinguen tres tipos de estructura: cristalina, gaseosa y una representada por moléculas en tránsito entre una y otra. El objetivo del modelo de estructuras significativas es predecir las propiedades de los líquidos en base a las propiedades conocidas de los sólidos y gases correspondientes, sin hacer ningún uso explícito de las fuerzas intermoleculares. Aunque no está libre de objeciones, los resultados predichos por este modelo para las propiedades de los líquidos puros son mucho más satisfactorios que los obtenidos por otros modelos de celdas. Su aplicación a mezclas [210], [211] proporciona una buena concordancia con los resultados experimentales de las propiedades de exceso excepto para $C_{P, \mathrm{~m}}^{\mathrm{E}}$.

La introducción de huecos y de celdas con ocupación múltiple no termina de tener en cuenta la correlación entre las moléculas de manera completa. Para hacerlo deben considerarse todas o alguna de las correlaciones siguientes:

a) En la distribución de moléculas entre celdas que poseen números de ocupación múltiples, incluyendo huecos.

b) En el movimiento de varias moléculas de la misma celda.

c) En el movimiento de moléculas de celdas distintas.

El caso general, que permite los tres tipos de correlaciones, admite varias formulaciones, todas ellas equivalentes al modelo de clusters de celdas [212], [213]. El concepto básico de este modelo consiste en la sustitución de la celda única del modelo LJD por un enjambre de celdas, o conjunto de $l$ celdas adyacentes. Una descripción detallada de este modelo puede consultarse en la bibliografía [92, 16.4ß], [212]-[214]. El modelo de

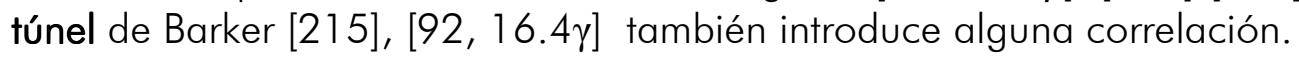




\subsubsection{Modelo de Guggenheim-Barker-Flory-Huggins}

El modelo de red para mezclas es esencialmente una extensión del modelo de red rígida a sistemas multicomponentes. Por simplificación, supondremos mezclas binarias de moléculas de los tipos A y B. La función de partición configuracional de cada componente puro está dada por la ecuación (6.118), es decir:

$$
\Omega_{\mathrm{i}}^{0}(T)=\left[e \cdot v_{f, \mathrm{i}} \cdot e^{\chi_{\mathrm{i}} / k_{\mathrm{B}} \cdot T}\right]^{N_{\mathrm{i}}}
$$

donde $\mathrm{i}=\mathrm{A}$ ó B, $N_{\mathrm{i}}$ es el número de moléculas del componente i, $\chi_{\mathrm{i}}$ es la energía de cada molécula del tipo i en su posición de equilibrio, es decir, en su posición de energía mínima y $-N_{\mathrm{i}} \cdot \chi_{\mathrm{i}}$ es la energía del componente puro i cuando todas sus moléculas se encuentran en sus posiciones de equilibrio.

Para extender el modelo de red rígida a mezclas, a las hipótesis a)-g) propias del mismo para el caso de líquidos puros, se añaden las siguientes:

h) El volumen libre de cada componente tiene el mismo valor en la mezcla que en estado puro.

i) Las moléculas de las diferentes especies tienen tamaño y forma similares y simetría esférica, lo que permite intercambiar sus posiciones en la red. Además, su empaquetamiento en la red es máximo e igual tanto en estado puro como en la mezcla, es decir $z_{\mathrm{A}}=z_{\mathrm{B}}=z=12$.

i) La energía de interacción molecular $W(r)$ es igual a la suma de las energías de interacción de los vecinos próximos, que se considera como un parámetro ajustable independiente de la temperatura. Estrictamente hablando no se requiere que las energías de interacción solo se deban a los vecinos próximos, sino que la diferencia de interacciones entre moléculas idénticas y distintas necesita ser considerada para vecinos próximos, lo cual está perfectamente justificado en el caso de mezclas líquidas de no electrolitos.

Como en todo modelo de red rígida los volúmenes molares y los volúmenes libres no se alteran, es decir, no hay volumen de exceso. Así, el volumen en cualquier configuración es constante e igual a $N_{\mathrm{A}} \cdot V_{\mathrm{A}}+N_{\mathrm{B}} \cdot V_{\mathrm{B}}$.

Las hipótesis enunciadas dan lugar al conocido modelo de disoluciones estrictamente regulares [77, cap 8], [152] de Guggenheim, el cual es completamente equivalente al modelo de Ising de sistemas magnéticos [88, pp 306], en el que se tratan dichos sistemas como un conjunto de spines en una red.

Es fácil ver que $-2 \cdot \chi_{i} z^{-1}$ es la energía de interacción promedio de una par de moléculas iguales del tipo i. Como en la mezcla la red contiene moléculas de ambos tipos, existirán contribuciones a $W(r)$ de pares de moléculas $\mathrm{AA}, \mathrm{BB}$ y $\mathrm{AB}$. Para tener en cuenta la contribución a $W(r)$ de los pares $A B$, se define la cantidad $w$ de modo que

$$
\frac{-2 \cdot \chi_{A}-2 \cdot \chi_{B}+2 \cdot w}{2 \cdot z}=\frac{-\chi_{A}-\chi_{B}+w}{z}
$$

es la energía de interacción promedio de un par AB. $w$ se llama energía de intercambio [216, pp 144], y es tal que si partiendo de ambos cristales puros e intercambiando una molécula $A$ por una molécula $B$, dejando el resto de ellas intactas, el aumento total de energía en el sistema es $2 \cdot w$. En este proceso se rompen $z$ pares AA, $z$ pares BB y se 
crean $2 \cdot z$ pares $A B$. El desconocimiento acerca de las energías de interacción intermoleculares obliga a considerar $w$ como un parámetro ajustable.

Si $x$ es la fracción molar del componente B, y $1-x$ la de A, entonces $N_{\mathrm{A}}=N \cdot(1-x)$ y $N_{\mathrm{B}}=N \cdot x$, donde $N$ es igual al número de nudos de la red, supuesto igual al número de moléculas. Se define $X$ de modo que $z \cdot X$ es el número total de pares de vecinos próximos del tipo $\mathrm{AB}$ en una configuración dada. Así, el número de vecinos de las moléculas del tipo i que no son del tipo j es $z \cdot\left(N_{\mathrm{i}}-X\right)$, el número de pares ii es $z \cdot\left(N_{\mathrm{i}}-X\right) / 2$ y $z \cdot N / 2$ es el número total de pares de vecinos próximos. Así, la energía de interacción total es:

$$
\begin{gathered}
W=\frac{1}{2} \cdot z \cdot\left(N_{\mathrm{A}}-X\right) \cdot\left(-2 \cdot \frac{\chi_{\mathrm{A}}}{z}\right)+\frac{1}{2} \cdot z \cdot\left(N_{\mathrm{B}}-X\right) \cdot\left(-2 \cdot \frac{\chi_{\mathrm{B}}}{z}\right)+z \cdot X \cdot \frac{\chi_{\mathrm{A}}-\chi_{\mathrm{B}}+w}{z}= \\
=-\chi_{\mathrm{A}} \cdot N_{\mathrm{A}}-\chi_{\mathrm{B}} \cdot N_{\mathrm{B}}+X \cdot w
\end{gathered}
$$

Sustituyendo $W$ en la función de partición configuracional (6.17) se tiene:

$$
\Omega(T)=e^{\left(\chi_{\mathrm{A}} \cdot N_{\mathrm{A}}+\chi_{\mathrm{B}} \cdot N_{\mathrm{B}}\right) / k_{\mathrm{B}} \cdot T} \cdot \frac{1}{N_{\mathrm{A}} !} \cdot \frac{1}{N_{\mathrm{B}} !} \cdot \int \ldots \int e^{-X \cdot w / k_{\mathrm{B}} T} d \vec{r}_{1_{\mathrm{A}}} \cdot \ldots d \vec{r}_{N_{\mathrm{A}}} \cdot d \vec{r}_{1_{\mathrm{B}}} \cdot \ldots d \vec{r}_{N_{\mathrm{B}}}
$$

El modelo de disoluciones estrictamente regulares se reduce al modelo de mezcla ideal sin más que suponer que $w=0$, siendo inmediata la solución al problema. En caso contrario, el cálculo de la gran función de partición es equivalente al cálculo de la misma en el problema de lsing tridimensional para sistemas magnéticos. Aunque hoy en día ello se puede hacer de manera casi exacta con la técnica del grupo de renormalización, existen varios métodos clásicos que resuelven el problema de manera aproximada. En primer lugar, (6.140) puede evaluarse, al menos en principio, con cualquier grado de exactitud como una expansión en serie de potencias de $w / z \cdot k_{\mathrm{B}} \cdot T$ [217]-[219]. Desafortunadamente la serie converge muy despacio, sobre todo cerca de la región crítica. En segundo lugar, un tratamiento que se ha utilizado en muchas ocasiones [220]-[223] es la llamada aproximación de orden cero o de mezcla al azar, en la que la entidad elemental a considerar es la molécula, la cual está situada en un campo de fuerzas debido al promedio del resto de moléculas. Este tratamiento es equivalente a la aproximación de Bragg-Williams [224], [225] para resolver el problema de Ising y para el estudio de aleaciones metálicas y superredes. La esencia de esta aproximación es asumir una distribución completamente aleatoria de los dos tipos de molécula, lo que conduce a un valor promedio de $X$ igual a:

$$
\langle X\rangle=\frac{N_{\mathrm{A}} \cdot N_{\mathrm{B}}}{N_{\mathrm{A}}+N_{\mathrm{B}}}=N \cdot x_{1} \cdot x_{2}
$$

Es decir,

$$
\langle X\rangle^{2}=\left(N_{\mathrm{A}}-\langle X\rangle\right) \cdot\left(N_{\mathrm{B}}-\langle X\rangle\right)
$$

Es conveniente definir la cantidad $\tilde{X}$ tal que:

$$
\langle X\rangle=\tilde{X}-T \cdot \frac{\mathrm{d} \tilde{X}}{\mathrm{~d} T}
$$

es decir, 


$$
\tilde{X}=T \cdot \int_{0}^{1 / T}\langle X\rangle \cdot \mathrm{d} \frac{1}{T}
$$

En la aproximación de orden cero (6.141), $\tilde{X}$ es igual a $\langle X\rangle$. La sustitución de $\langle X\rangle$ en la ecuación (6.141) por $X$ en (6.140) permite obtener de manera sencilla ecuaciones para las funciones de mezcla y exceso, coeficientes de actividad, separación de fases, mezcla crítica, etc. Por ejemplo:

$$
\begin{aligned}
F_{\mathrm{m}, V}^{\mathrm{E}}(\mathrm{DISP})= & G_{\mathrm{m}, V}^{\mathrm{E}}(\mathrm{DISP})=x_{1} \cdot x_{2} \cdot N \cdot w \\
U_{\mathrm{m}, V}^{\mathrm{E}}(\mathrm{DISP})= & H_{\mathrm{m}, V}^{\mathrm{E}}(\mathrm{DISP})=x_{1} \cdot x_{2} \cdot N \cdot w \\
& S_{\mathrm{m}, V}^{\mathrm{E}}(\mathrm{DISP})=0 \\
& V_{\mathrm{m}, V}^{\mathrm{E}}(\mathrm{DISP})=0
\end{aligned}
$$

El paréntesis (DISP) indica que las ecuaciones son válidas en la aproximación de orden cero, es decir, bajo la hipótesis de mezcla al azar (cuando sólo hay fuerzas de tipo DISPersivo). El subíndice $V$ se debe a que en los modelos de red rígida no hay cambio de volumen, por lo que los resultados teóricos sólo pueden compararse con los valores experimentales a $V=$ cte.

El estudio de las capacidades caloríficas de exceso es importante no solo desde el punto de vista práctico sino también teórico, pues sirve como test de los diferentes modelos. Ello se debe a que el orden incremental de las derivadas de los potenciales termodinámicas magnifica las deficiencias del modelo en consideración, y precisamente las capacidades caloríficas se calculan a partir de las derivadas segundas de los potenciales termodinámicos respecto de $T$ [177]. En la aproximación de orden cero del modelo de disoluciones estrictamente regulares, se tiene:

$$
C_{\mathrm{m}, V}^{\mathrm{E}}(\mathrm{DISP})=C_{\mathrm{m}, P}^{\mathrm{E}}(\mathrm{DISP})=\left(\frac{\partial U_{\mathrm{m}}^{\mathrm{E}}(\mathrm{DISP})}{\partial T}\right)_{V}=0
$$

Las ecuaciones (6.147) y (6.148) vienen a decir que la entropía de mezcla a volumen constante de una disolución estrictamente regular en la aproximación de orden cero es igual a la entropía de mezcla de las disolución ideal, es decir, que la entropía de exceso a volumen constante es igual a cero, lo que encaja con la definición de Hildebrand de disolución regular. Y es que este modelo de disoluciones estrictamente regulares en la aproximación de orden cero lleva a las mismas consecuencias que la teoría de mezclas regulares desarrollada por Hildebrand [226] y Scatchard [227] independientemente, quiénes liberaron a la teoría de van Laar de las limitaciones asociadas a la utilización de la ecuación de van der Waals o de cualquier otra ecuación de estado. Es útil definir los llamados parámetros de solubilidad $\delta_{i}$ de modo que el logaritmo de los coeficientes de actividad de cada componente se puede escribir en función del volumen molar del mismo $V_{\mathrm{i}}$, de su fracción de volumen en la mezcla $\Phi_{\mathrm{i}}$ y de parámetros de solubilidad $\delta_{\mathrm{i}}$. En mezclas binarias:

$$
\ln f_{\mathrm{i}}=\frac{1}{R \cdot T} \cdot V_{\mathrm{i}} \cdot \Phi_{\mathrm{j}}^{2} \cdot\left(\delta_{1}-\delta_{2}\right)^{2}
$$


La teoría de disoluciones regulares predice solamente desviaciones positivas respecto de la ley de Raoult, lo que es consecuencia de que la adopción de la hipótesis de mezcla al azar lleva implícita la consideración de únicamente fuerzas de tipo dispersivo. Por esta razón funciona bastante bien sólo en mezclas de moléculas apolares [228], excepto en disoluciones de compuestos fluorocarbonados.

A temperaturas altas las moléculas tienden a distribuirse de manera aleatoria, de modo que la hipótesis de mezcla al azar es tanto mejor cuanto mayor sea la temperatura. Sin embargo, a temperaturas menores las diferencias en las interacciones entre unos tipos de moléculas y otros favorecen las distribuciones con menor energía de red, es decir, existen interacciones preferenciales y orientacionales, lo que puede originar inmiscibilidad 0 transiciones orden-desorden. Estos efectos pueden discutirse comparando los resultados obtenidos en la aproximación de orden cero con los obtenidos en la aproximación de orden uno o cuasiquímica [229], que es equivalente a la aproximación de Bethe [230] para el estudio del problema de Ising, de aleaciones metálicas y de superredes, y aplicado por Rushbrooke [231] al estudio de mezclas estrictamente regulares. Esta aproximación es una mejora a la de Bragg-Williams. La esencia de la misma es asumir que los diferentes pares de moléculas no interfieren unos con otros (es decir, la entidad elemental es un dímero que interacciona con el campo promedio debido al resto de dímeros) y proponer una forma combinatorial aproximada para el número de configuraciones del conjunto $g\left(N_{\mathrm{A}}, N_{\mathrm{B}}, X\right)$ con $X, N_{\mathrm{A}}$ y $N_{\mathrm{B}}$ dados:

$$
g\left(N_{\mathrm{A}}, N_{\mathrm{B}}, X\right)=\frac{\left(N_{\mathrm{A}}+N_{\mathrm{B}}\right) !}{N_{\mathrm{A}} ! \cdot N_{\mathrm{B}} !} \cdot \frac{\left[\frac{z}{2} \cdot\left(N_{\mathrm{A}}-X^{*}\right)\right] ! \cdot\left[\frac{z}{2} \cdot\left(N_{\mathrm{B}}-X^{*}\right)\right] ! \cdot\left[\left(\frac{z}{2} \cdot X^{*}\right) !\right]^{2}}{\left[\frac{z}{2} \cdot\left(N_{\mathrm{A}}-X\right)\right] ! \cdot\left[\frac{z}{2} \cdot\left(N_{\mathrm{B}}-X\right)\right] ! \cdot\left[\left(\frac{z}{2} \cdot X\right) !\right]^{2}}
$$

donde $X^{*}$ es el valor de $X$ correspondiente con la distribución aleatoria:

$$
X^{*}=\frac{N_{\mathrm{A}} \cdot N_{\mathrm{B}}}{N_{\mathrm{A}}+N_{\mathrm{B}}}
$$

La función de partición configuracional es, teniendo en cuenta (6.139), igual a:

$$
\Omega(T)=\sum g\left(X, N_{\mathrm{A}}, N_{\mathrm{B}}\right) \cdot e^{\left(\chi_{\mathrm{A}} \cdot N_{\mathrm{A}}+\chi_{\mathrm{B}} \cdot N-X \cdot w\right) / k_{B} \cdot T}
$$

Lo que conduce a los mismos resultados que si se supone la siguiente forma de $\langle X\rangle$ :

$$
\langle X\rangle^{2}=\left(N_{\mathrm{A}}-\langle X\rangle\right) \cdot\left(N_{\mathrm{B}}-\langle X\rangle\right) \cdot e^{-2 \cdot w / z \cdot k_{B} \cdot T}
$$

Puesto que $2 \cdot w / z$ es la energía requerida para destruir un par AA y un par BB, y crear dos pares $\mathrm{AB}$ y como $\langle X\rangle,\left(N_{\mathrm{A}^{-}}\langle X\rangle\right) / 2$ y $\left(N_{\mathrm{B}^{-}}\langle X\rangle\right) / 2$ son proporcionales al número de pares $A B, A A$ y $B B$ respectivamente, entonces (6.154) lleva a una ecuación de forma equivalente a la ley de acción de masas entre especies químicas gaseosas en equilibrio, sustituyendo las especies químicas por los diferentes pares. Por este motivo al presente tratamiento se le llama cuasiquímico.

La aproximación cuasiquímica permite hacer una primera discusión acerca de las inmiscibilidad (mezclas endotérmicas) y transiciones orden-desorden (mezclas exotérmicas). En efecto, de la comparación entre (6.142) y (6.154) se observa que si la mezcla es endotérmica, es decir $w>0, X$ es menor que en la aproximación cero, 
predominando los pares de moléculas iguales hasta el punto de existir una temperatura (temperatura de disolución crítica) por debajo de la cual se produce un cambio de fase, dejando de ser ambos componentes miscibles. Si la mezcla es exotérmica, es decir $w<0$, la mezcla puede realizarse a cualquier composición y temperatura, y $X$ es mayor que en la aproximación de orden cero, predominando la formación de pares de moléculas diferentes, siendo mayor el número de éstos cuanto menor sea la temperatura. Así, mientras que a temperaturas altas las moléculas están distribuidas al azar en la red de manera casi completa (desorden), a medida que la temperatura disminuye las moléculas tienden a colocarse en la red de manera alternativa (orden), llegando a formarse complejos químicos $A B$ a temperaturas lo suficientemente bajas, en casos límite. Es lo que se llama transición orden-desorden. Aunque el tratamiento cuasiquímico sólo da una visión aproximada del problema, la solución exacta de Onsaguer en redes bidimensionales [26], [232] demuestra que excepto en las proximidades de los puntos críticos, la concordancia entre los valores de las funciones de exceso obtenidos mediante la formulación exacta de Onsaguer (que da cuenta perfectamente de los efectos orden-desorden), la aproximación cuasiquímica y la aproximación de orden cero es lo suficientemente buena como para despreciar los efectos orden-desorden, siendo la aproximación de orden cero buena en la mayoría de los casos. De hecho, Kirkwood demostró rigurosamente [233] que los efectos de orden local son relativamente pequeños (del orden del $7 \%$ en comparación con la teoría de disoluciones estrictamente regulares, para una mezcla equimolar, a la temperatura crítica de la mezcla, si $z=8$ y con $w$ independiente de la temperatura).

La sustitución de $\langle X\rangle$ en la ecuación (6.154) por $X$ en (6.140) permite obtener de manera sencilla ecuaciones para las funciones de mezcla y exceso, coeficientes de actividad, separación de fases, mezcla crítica, etc. Por ejemplo, algunas propiedades de exceso son:

$$
\begin{gathered}
F_{\mathrm{m}, V}^{\mathrm{E}}(\mathrm{QUAC})=G_{\mathrm{m}, V}^{\mathrm{E}}(\mathrm{QUAC})=\frac{1}{2} \cdot R \cdot T \cdot z \cdot \sum_{\mathrm{i}} x_{\mathrm{i}} \cdot \ln \frac{\beta+1-2 \cdot\left(1-x_{\mathrm{i}}\right)}{x_{\mathrm{i}} \cdot(\beta+1)} \\
U_{\mathrm{m}, V}^{\mathrm{E}}(\mathrm{QUAC})=H_{\mathrm{m}, V}^{\mathrm{E}}(\mathrm{QUAC})=\frac{2}{\beta+1} \cdot x_{1} \cdot x_{2} \cdot N \cdot w \\
S_{\mathrm{m}, V}^{\mathrm{E}}(\mathrm{QUAC})=\frac{U_{\mathrm{m}, V}^{\mathrm{E}}(\mathrm{QUAC})-F_{\mathrm{m}, V}^{\mathrm{E}}(\mathrm{QUAC})}{T} \\
V_{\mathrm{m}, V}^{\mathrm{E}}(\mathrm{QUAC})=0 \\
C_{\mathrm{m}, V}^{\mathrm{E}}(\mathrm{QUAC})=C_{\mathrm{m}, P}^{\mathrm{E}}(\mathrm{QUAC})=\frac{2 \cdot \eta^{2}}{z \cdot \beta} \cdot\left(\frac{U_{\mathrm{m}, V}^{\mathrm{E}}}{R \cdot T}\right)^{2}
\end{gathered}
$$

donde,

$$
\begin{gathered}
\eta=e^{w / z \cdot k_{\mathrm{B}} \cdot T} \\
\beta^{2}=1+4 \cdot x_{1} \cdot x_{2} \cdot\left(\eta^{2}-1\right)=\left(1-2 \cdot x_{1}\right)^{2}+4 \cdot x_{1} \cdot x_{2} \cdot \eta^{2}
\end{gathered}
$$

El paréntesis (QUAC) quiere decir que las ecuaciones escritas se han resuelto en la aproximación cuasiquímica (QUAsi-Chemical). 
El modelo de disoluciones estrictamente regulares en la aproximación cuasiquímica consta de varios defectos que residen en sus hipótesis de partida. A parte de las limitaciones debidas a las hipótesis a)-g) inherentes a todo modelo de celdas discutidas en la sección 6.4.1, la revisión de las restricciones que son resultado de las hipótesis h)-i) propias de este modelo y de la aproximación cuasiquímica, muestra los defectos siguientes:

a) En primer lugar, la no interferencia entre dímeros distintos, consecuencia de la aproximación cuasiquímica, es más falsa cuanto mayor sea el empaquetamiento de la red. Ello se puede corregir mediante la consideración de tripletes, cuartetes, etc. de moléculas lo cual, sin embargo, no lleva a una corrección significativa de los resultados obtenidos [234].

b) En segundo lugar, no se han tenido en cuenta las interacciones con vecinos que no son los más próximos. Sin embargo, se ha demostrado que el efecto sobre los valores calculados de las diferentes cantidades cuando se tienen en cuenta estas interacciones es despreciable en casi todas las situaciones prácticas [235].

Los defectos a) y b) no son despreciables en las proximidades de los puntos críticos, donde las fluctuaciones y la correlación entre todas las moléculas son muy importantes y no se puede considerar que los pares de moléculas (y mucho menos las moléculas) sean independientes. Los efectos cooperativos a distancia deben ser tenidos en cuenta, es decir, las interacciones entre moléculas que no son vecinos próximos. Por esta razón, ni la aproximación de orden cero ni la cuasiquímica predicen valores correctos de los exponentes críticos. El problema debe abordarse en términos de la formulación de Onsaguer en redes 2-d o de la teoría del grupo de renormalización en el caso 3-d. Por ejemplo, se ha utilizado el método del grupo de renormalización de Migdal-Kadanoff (MKRG) [236], [237] modificado por Walter y Vause [238], [239], el cual permite resolver el problema de manera casi exacta, salvo por las limitaciones inherentes al modo de construcción de la red normalizada. Ello se ha hecho en el conjunto estadístico $T, V, N_{\mathrm{A}}+N_{\mathrm{B}} \mathrm{y} \mu_{\mathrm{A}}-\mu_{\mathrm{B}}$ constantes [240], cuya función de partición es:

$$
\psi^{\prime}=e^{\frac{N \cdot z \cdot g}{2}} \cdot \sum_{\text {conf }} e^{-z \cdot X \cdot \omega+\left(N_{\mathrm{A}}-N_{\mathrm{B}}\right) \cdot \Delta \tilde{\mu}}=e^{\frac{N \cdot z \cdot g}{2}} \cdot L
$$

donde la suma se extiende a todas las posibles configuraciones de la red, $L$ es la función de partición configuracional en el conjunto de Gibbs dado y se han utilizado las cantidades $\Delta \tilde{\mu}, \omega$ y $g$, definidas mediante las ecuaciones siguientes:

$$
\begin{gathered}
\Delta \tilde{\mu}=\frac{2 \cdot\left(\mu_{\mathrm{A}}-\mu_{\mathrm{B}}\right)-2 \cdot\left(\chi_{\mathrm{A}}-\chi_{\mathrm{B}}\right)}{4 \cdot k_{\mathrm{B}} \cdot T} \\
\omega=\frac{w}{k_{B} \cdot T} \\
g=-\frac{\chi_{\mathrm{A}}+\chi_{\mathrm{B}}}{2 \cdot k_{\mathrm{B}} \cdot T}
\end{gathered}
$$

El método permite obtener las ecuaciones recursivas siguientes: 


$$
\begin{gathered}
\omega^{\prime \prime}=\ln \left\{\frac{\left[\cosh \left(\lambda \cdot \omega^{\prime}+8 \cdot \Delta \tilde{\mu}^{\prime} / z\right) \cdot \cosh \left(\lambda \cdot \omega^{\prime}-8 \cdot \Delta \tilde{\mu}^{\prime} / z\right)\right]^{1 / 2}}{\cosh \left(8 \cdot \Delta \tilde{\mu}^{\prime} / z\right)}\right\} \\
g^{\prime \prime}=-4 \cdot \omega+\frac{1}{2} \cdot \ln \left[4 \cdot \operatorname { c o s h } ( \lambda \cdot \omega ^ { \prime \prime } + 8 \cdot \Delta \tilde { \mu } ^ { \prime } / z ) \cdot \operatorname { c o s h } \left(\lambda \cdot \omega^{\prime}-8 \cdot \Delta \tilde{\mu}+\frac{z}{4} \cdot \ln \left\{\frac{\cosh \left(\lambda \cdot \omega^{\prime}+8 \cdot \Delta \tilde{\mu}^{\prime} / z\right)}{\cosh \left(\lambda \cdot \omega^{\prime}-8 \cdot \Delta \tilde{\mu}^{\prime} / z\right)}\right\}\right.\right. \\
N^{\prime \prime}=N^{\prime} / 8 \\
L^{\prime \prime}=\sum_{\operatorname{conf}} e^{-z \cdot X^{\prime \prime} \cdot \omega^{\prime \prime}+\left(N_{\mathrm{A}}{ }^{\prime \prime}-N_{\mathrm{B}}{ }^{\prime \prime}\right) \cdot \Delta \tilde{\mu}^{\prime \prime}}
\end{gathered}
$$

donde $\lambda$ es un parámetro ajustable (igual a 4 en el MKRG) y la función de partición configuracional $L$ ' de la red renormalizada está dada por:

$$
L^{\prime}=2^{N^{\prime} / 2} \cdot e^{\frac{N^{\prime \prime} \cdot z \cdot g^{\prime \prime}}{2}} \cdot L^{\prime \prime}
$$

c) En tercer lugar, la independencia con la temperatura del parámetro ajustable $w$ sólo es razonable en el caso de mezclas de moléculas cuyos campos de fuerza son simétricamente esféricos. En el resto de casos, esta hipótesis no está justificada [241], [242]. En ellos, el carácter no central de las fuerzas de interacción molecular dificulta su tratamiento pues la dependencia con la orientación es difícil de manejar, lo que conduce a la utilización de parámetros de interacción promedio que dependen de la temperatura, siendo dichos parámetros no energías sino energías libres. En el caso que nos ocupa, la cantidad $2 \cdot w$, asociada a la destrucción de un par AA y de otro BB para la formación de dos pares $A B$, sin alterar el resto, es la llamada energía libre cooperativa, y la introducción de una dependencia de $w$ con la temperatura permite dar una descripción de los sistemas en los que hay presentes fuerzas orientacionales. Las ecuaciones resultantes para las propiedades de equilibrio del sistema permanecen idénticas al caso de independencia con $T$, salvo en aquellas que provengan de la diferenciación respecto de la temperatura de la energía libre de Helmholtz. Por ejemplo, las funciones de exceso en la aproximación cuasiquímica son [243]:

$$
\begin{gathered}
F_{\mathrm{m}, V}^{\mathrm{E}}(T, \mathrm{QUAC})=G_{\mathrm{m}, V}^{\mathrm{E}}(T, \mathrm{QUAC})=\frac{1}{2} \cdot R \cdot T \cdot z \cdot \sum_{\mathrm{i}} x_{\mathrm{i}} \cdot \ln \frac{\beta+1-2 \cdot\left(1-x_{\mathrm{i}}\right)}{x_{\mathrm{i}} \cdot(\beta+1)} \\
U_{\mathrm{m}, V}^{\mathrm{E}}(T, \mathrm{QUAC})=H_{\mathrm{m}, V}^{\mathrm{E}}(T, \mathrm{QUAC})=\frac{2}{\beta+1} \cdot x_{1} \cdot x_{2} \cdot N \cdot u \\
S_{\mathrm{m}, V}^{\mathrm{E}}(T, \mathrm{QUAC})=\frac{U_{\mathrm{m}, V}^{\mathrm{E}}(T, \mathrm{QUAC})-F_{\mathrm{m}, V}^{\mathrm{E}}(T, \mathrm{QUAC})}{T}= \\
=\frac{1}{T} \cdot\left[\frac{2}{\beta+1} \cdot x_{1} \cdot x_{2} \cdot N \cdot u-F_{\mathrm{m}}^{\mathrm{E}}(T, \mathrm{QUAC})\right] \\
V_{\mathrm{m}, V}^{\mathrm{E}}(T, \mathrm{QUAC})=0
\end{gathered}
$$




$$
\begin{gathered}
C_{\mathrm{m}, V}^{\mathrm{E}}(T, \mathrm{QUAC})=C_{\mathrm{m}, P}^{\mathrm{E}}(T, \mathrm{QUAC})=N \cdot R \cdot x_{1} \cdot x_{2} \cdot \frac{2}{\beta+1} \cdot\left[\frac{4 \cdot x_{1} \cdot x_{2} \cdot \eta^{2}}{z \cdot \beta \cdot(\beta+1)} \cdot\left(\frac{u}{k_{\mathrm{B}} \cdot T}\right)^{2}+\frac{v}{k_{\mathrm{B}}}\right]= \\
=\frac{2 \cdot \eta^{2}}{z \cdot \beta} \cdot\left(\frac{U_{\mathrm{m}, V}^{\mathrm{E}}}{R \cdot T}\right)^{2}+x_{1} \cdot x_{2} \cdot \frac{2}{\beta+1} \cdot\left(\frac{v}{k_{B}}\right)
\end{gathered}
$$

donde se han utilizado los parámetros $u$ y $v$ definidos como:

$$
\begin{gathered}
u=\frac{\mathrm{d}(w / T)}{\mathrm{d}(1 / T)}=w-T \cdot \frac{\mathrm{d} w}{\mathrm{~d} T} \\
v=\frac{\mathrm{d} u}{\mathrm{~d} T}=\frac{\mathrm{d}}{\mathrm{d} T}\left(w-T \cdot \frac{\mathrm{d} w}{\mathrm{~d} T}\right)=-T \cdot \frac{\mathrm{d}^{2} w}{\mathrm{~d} T^{2}}
\end{gathered}
$$

El paréntesis ( $T$, QUAC) quiere decir que las ecuaciones se han obtenido en la aproximación cuasiquímica, suponiendo dependencia con la temperatura de las energías libres cooperativas, es decir, que las fuerzas no son centrales.

Es bien sabido que la aproximación de orden cero se obtiene a partir de la cuasiquímica en el límite $z \rightarrow \infty$. Si se aplica dicha condición a las ecuaciones (6.172)-(6.176) se obtienen los resultados de la aproximación de orden cero cuando $w$ depende de la temperatura. Por ejemplo, la entropía de exceso es:

$$
S_{\mathrm{m}, V}^{\mathrm{E}}(T, \mathrm{DISP})=-N \cdot x_{1} \cdot x_{2} \cdot \frac{\mathrm{d} w}{\mathrm{~d} T}
$$

que contrasta con el valor nulo de la teoría de disoluciones estrictamente regulares en la aproximación cero con $w$ independiente de la temperatura. Por tanto, (6.179) puede verse como la contribución a la entropía de exceso en una mezcla al azar debida a la dependencia $w=w(T)$, es decir, a que las fuerzas no son centrales. Así mismo, la diferencia de este valor con (6.174) es la contribución a la entropía de exceso dado que la mezcla no es al azar (NR, non-random):

$$
S_{\mathrm{m}, V}^{\mathrm{E}}(T, \mathrm{NR})=S_{\mathrm{m}, V}^{\mathrm{E}}(T, \mathrm{QUAC})-S_{\mathrm{m}, V}^{\mathrm{E}}(T, \mathrm{DISP})
$$

Si este valor es muy pequeño, la hipótesis de mezcla al azar puede considerarse válida.

La dependencia más sencilla que puede proponerse es de tipo lineal $w=\mathrm{U}^{0}-T \cdot \mathrm{S}^{0}$ [152], [244]. Sin embargo, este tipo de dependencia en $w$ no implica dependencia de $U_{m}^{\mathrm{E}}$ (ni de $H_{m}^{\mathrm{E}}$ ), por lo que la derivada respecto de la temperatura de $U_{m}^{\mathrm{E}}$ a $V$ constante, $C_{\mathrm{m}, V}^{\mathrm{E}}$, es cero, tanto en la aproximación cuasiquímica como en la de orden cero (límite $z \rightarrow \infty$ ), lo cual está en contra de lo que se espera para el comportamiento de esta propiedad termodinámica en mezclas de moléculas polar + apolar del mismo tamaño para las cuales McGlashan estableció [245] y Cobos demostró [243] que $C_{\mathrm{m}, V}^{\mathrm{E}}<0$.

Otra posibilidad [82], [124], [243], [246] es introducir una energía libre cooperativa del tipo $w=\mathrm{a}+\mathrm{b} / T$, donde a y $\mathrm{b}$ son constantes. En este caso, $(\mathrm{d} w / \mathrm{d} T)=-\mathrm{b} / T^{2}$, de forma que $u=\mathrm{a}+2 \cdot \mathrm{b} / T=w+\mathrm{b} / T$ y $v=-T^{2}\left(\mathrm{~d}^{2} w=\mathrm{d} T^{2}\right)=-2 \cdot \mathrm{b} / T^{2}$, siendo $(\mathrm{d} v / \mathrm{d} T)=4 \cdot \mathrm{b} / T^{3}$. De esta forma $C_{V}^{\mathrm{E}}(z \rightarrow \infty)=-2 \cdot(6.179)$ que es negativa, como debe ser. A pesar de poseer un fundamento teórico más sólido, pues la dependencia propuesta procede del valor medio de la polarización que proporciona la Física Estadística clásica cuando describe un 
dieléctrico homogéneo formado por moléculas polares situadas en un campo eléctrico uniforme (ecuaciones de Debye-Langevin y Clausius-Mosotti), no es capaz de justificar correctamente todas las propiedades termodinámicas de las mezclas liquidas y aleaciones. Es necesaria una forma funcional más complicada, por ejemplo $w=\mathrm{a}+\mathrm{b} \cdot T+\mathrm{c} \cdot T^{2}, w=\mathrm{a}+\mathrm{b} \cdot T+\mathrm{c} \cdot \ln T$, etc.

d) En cuarto lugar, es común encontrarse con mezclas de moléculas cuyo tamaño y forma no sean similares, luego la hipótesis i) no es cierta. Para tratar tales casos la hipótesis b) de los modelos de celda debe eliminarse, y se supone que cada molécula ocupa un conjunto de sitios adyacentes de la red. El estudio de este tipo de mezcla se ha desarrollado sobre todo en el contexto del estudio del comportamiento de las disoluciones de polímeros, el cual es importante tanto en la manufactura de los mismos como en algunas de sus aplicaciones, como por ejemplo en la eliminación de disolventes e impurezas en películas poliméricas utilizadas para envolver alimentos o medicamentos, o en el hinchamiento de polímeros para impregnarlos de aditivos químicos como los colorantes, etc [161].

Es conveniente realizar las siguientes definiciones:

- Se llama $r$-méro a una sustancia cuyas moléculas están constituidas por $r$ elementos o segmentos, cada uno de los cuales ocupa un nudo de la red.

- Cuando dos segmentos de moléculas diferentes son vecinos próximos, se dice que dichos segmentos están en contacto, y ello permite definir los contactos. Se asume que la energía potencial de interacción total es igual a la suma de las energías de interacción de los diferentes contactos.

- Se dice que un $r$-méro es heterogéneo si las energías de interacción de los contactos de sus segmentos con los de otras moléculas dependen del segmento en cuestión, lo que equivale a decir que hay presentes fuerzas muy localizadas dentro de las moléculas. Si por el contrario, todos los segmentos que constituyen las moléculas son energéticamente iguales, se dice que el polímero es homogéneo.

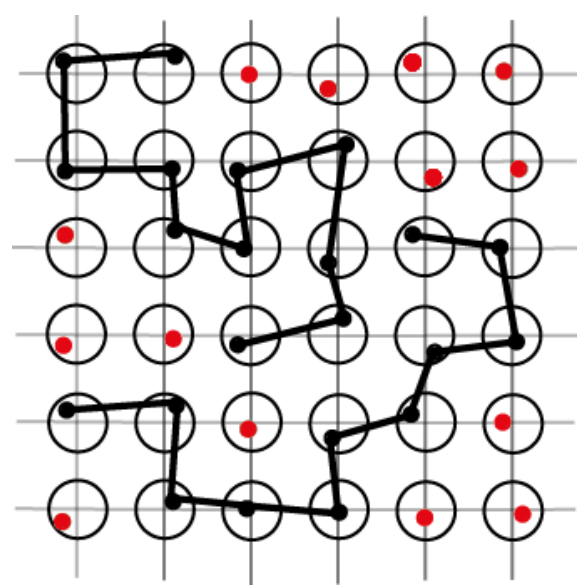

Ilustración 6-5: modelo de red para polímeros. Se muestra un 11-méro en disolución con un monómero

Sea una mezcla de varios $r_{\mathrm{i}}$-méros. Es inmediato ver que el número total de nudos de la red es $N_{\mathrm{S}}=\sum r_{\mathrm{i}} \cdot N_{\mathrm{i}}$. Se define $q_{\mathrm{i}}$ tal que cada molécula del $r_{\mathrm{i}}$-méro tiene $q_{\mathrm{i}} z$ nudos de la red que son vecinos próximos, donde es posible encontrar la relación geométrica 
siguiente, suponiendo que las moléculas no pueden cruzarse ni formar anillos y cuya demostración se puede consultar en el libro de Miller [247, pp 18]:

$$
q_{\mathrm{i}} \cdot z=r_{\mathrm{i}} \cdot z-2 \cdot r_{\mathrm{i}}+2
$$

Una molécula interacciona en $q_{\mathrm{i}} \cdot z$ nudos próximos. Así, se puede suponer que la superficie de una molécula está dividida en $q_{\mathrm{i}} \cdot z$ puntos de contacto, cada uno de los cuales tiene una energía de interacción distinta si el polímero es heterogéneo o igual si es homogéneo. Por simplificación, supondremos que los polímeros son homogéneos. No obstante, el desarrollo del modelo para polímeros heterogéneos fue realizado por Tompa [248] y Barker [249]-[252]. Barker mejoró el modelo suponiendo que los puntos de contacto pueden moverse o vibrar y que, si hay cambios en la extensión del movimiento posible o en las frecuencias de los grados de libertad vibracional, debe haber efectos entrópicos que pueden ser tenidos en cuenta si en lugar de considerar parámetros de interacción puramente energéticos se considera que son energías libres cooperativas dependientes de la temperatura [251], de modo similar a como se hizo en el apartado c) anterior. El modelo resultante, da lugar a ecuaciones muy complicadas que pueden consultarse en las referencias citadas y ha sido aplicado a mezclas de alcoholes con alcanos.

Conviene señalar el hecho de que el modelo puede ampliarse para el caso de moléculas que forman anillos o pueden cruzarse sin más que ignorar la relación geométrica (6.181), como hizo Staverman [253]. Aunque se encuentran diferencias entre las cantidades termodinámicas de la mezcla calculadas con el modelo resultante respecto del modelo para moléculas que no pueden cruzarse, dichas diferencias se eliminan al calcular las propiedades de exceso. Por lo tanto, las funciones de exceso que se presentan en los siguientes párrafos son igualmente válidas tanto para moléculas que se cruzan o forman anillos como para moléculas que no [254].

Se define la fracción de sitios ocupados por el polímero i, que coincide con la fracción de segmentos de dicho polímero, a la cantidad:

$$
\varphi_{\mathrm{i}}=\frac{r_{\mathrm{i}} \cdot N_{\mathrm{i}}}{N_{\mathrm{S}}}
$$

y se define la fracción de contactos:

$$
\theta_{\mathrm{i}}=\frac{q_{\mathrm{i}} \cdot N_{\mathrm{i}}}{N_{\mathrm{q}}}
$$

donde $N_{\mathrm{q}}=\sum_{\mathrm{i}} q_{\mathrm{i}} \cdot N_{\mathrm{i}}$ es el número total de contactos en la mezcla. Se verifican las relaciones siguientes:

$$
\sum_{\mathrm{i}} \varphi_{\mathrm{i}}=1 \quad \sum_{\mathrm{i}} \theta_{\mathrm{i}}=1
$$

La principal dificultad del problema reside en el cálculo del factor combinatorial $g\left(N_{\mathrm{i}}\right)$ correspondiente al número de formas de distribuir las moléculas en la red, el cual ha sido llevado a cabo de manera más o menos aproximada por diferentes autores [255][261]. Si la mezcla es atérmica $\left(H_{\mathrm{m}}^{\mathrm{E}}=0, G_{\mathrm{m}}^{\mathrm{E}}=-T \cdot S_{\mathrm{m}}^{\mathrm{E}}\right)$, es decir, si las propiedades de la mezcla como un todo dependen exclusivamente de factores entrópicos (por tanto las energías de intercambio son idénticamente nulas) este factor combinatorial se puede calcular bajo la hipótesis de que la mezcla es al azar, suponiendo que la ocupación de 
un nudo de la red está influenciada por la ocupación de los sitios adyacentes y no de los lejanos. El resultado que se obtiene es el siguiente:

$$
g\left(N_{\mathrm{i}}\right)=\prod_{\mathrm{i}}\left(\frac{\rho_{\mathrm{i}}}{\sigma_{\mathrm{i}}}\right)^{N_{\mathrm{i}}} \cdot \frac{\left(N_{\mathrm{S}}\right) !}{\prod_{i} N_{\mathrm{i}} !} \cdot\left[\frac{\left(N_{\mathrm{q}}\right) !}{\left(N_{\mathrm{S}}\right) !}\right]^{z / 2}
$$

donde $\rho_{\mathrm{i}}$ es el llamado parámetro de flexibilidad de la cadena, que es igual al número de maneras en las que un polímero se puede distribuir en la red. Por ejemplo, para un monómero es uno, para un dímero es igual a $z$, etc.; $\sigma_{i}$ se llama número de simetría de la cadena (por ejemplo, es igual a 2 en cadenas de hidrocarburos). Este factor combinatorial conduce a la siguiente ecuación para la entropía molar de exceso:

$$
S_{\mathrm{m}, V}^{\mathrm{E}}(\mathrm{COMB})=-R \cdot \sum_{\mathrm{i}} x_{\mathrm{i}} \cdot\left(\ln \frac{\varphi_{\mathrm{i}}}{x_{\mathrm{i}}}+\frac{1}{2} \cdot z \cdot q_{\mathrm{i}} \cdot \ln \frac{\theta_{\mathrm{i}}}{\varphi_{\mathrm{i}}}\right)
$$

conocida como ecuación de Guggenheim-Staverman, que da cuenta de los efectos entrópicos puramente combinatoriales asociados con las diferencias de tamaño y forma entre las moléculas (COMB). Los valores de $g$ y de $S_{\mathrm{m}, V}^{\mathrm{E}}$ quedan determinados solamente por los parámetros geométricos característicos de las moléculas, no existiendo dependencia con la temperatura.

Excepto por un pequeño factor $f_{0}$ que da cuenta de la posibilidad de que las moléculas puedan plegarse hacia sí mismas, la ecuación (6.186) es idéntica a la de Huggins [256]. De hecho, si $f_{0}=0$, la ecuación de Huggins se transforma en la (6.186).

Es de destacar que aunque se ha supuesto que la mezcla es al azar (aproximación cero), el índice de coordinación $z$ es finito. Si en las ecuaciones anteriores se introduce la suposición de que $z \rightarrow \infty$, se obtiene la famosa ecuación de Flory-Huggins ( $F-H)$ para la entropía molar de exceso [257]:

$$
S_{\mathrm{m}, V}^{\mathrm{E}}(\mathrm{F}-\mathrm{H})=-R \cdot \sum_{\mathrm{i}} x_{\mathrm{i}} \cdot \ln \frac{\varphi_{\mathrm{i}}}{x_{\mathrm{i}}}
$$

Lógicamente, si $r_{\mathrm{i}}=1 \forall$ i se recupera el modelo de disoluciones estrictamente regulares en la aproximación de orden cero, es decir, el modelo de disolución regular de Hildebrand y Scatchard $\left(S_{\mathrm{m}, V}^{\mathrm{E}}=0\right)$.

Por no jugar los efectos energéticos papel alguno en las mezclas atérmicas, ya que las energías de intercambio son nulas por definición, el tratamiento cuasiquímico para obtener el factor combinatorial $g$ no tiene sentido.

No se han encontrado disoluciones que sean estrictamente atérmicas, salvo las mezclas ideales y las mezclas de poliestireno + tolveno o + etilbenceno y polidimetilsiloxano + hexametildisiloxano. No obstante, la importancia del modelo de mezclas atérmicas es fundamental puesto que proporciona una base importante para el estudio de mezclas no atérmicas. Además, en aquellas mezclas en las que $H_{\mathrm{m}}^{\mathrm{E}}$ sea muy pequeño, a pesar de no ser cero, la ecuación de Flory-Huggins proporciona una buena aproximación para $S_{\mathrm{m}, V}^{\mathrm{E}}$.

En el caso de mezclas no atérmicas $\left(H_{\mathrm{m}}^{\mathrm{E}} \neq 0, G_{\mathrm{m}}^{\mathrm{E}}=H_{\mathrm{m}}^{\mathrm{E}}-T \cdot S_{\mathrm{m}}^{\mathrm{E}}\right)$, los efectos energéticos 
no son despreciables, pues las energías de intercambio ya no son idénticamente nulas. El factor combinatorial puede calcularse, como sabemos, bajo la adopción de dos hipótesis generales diferentes: aproximación cero o de Bragg-Williams, y aproximación cuasiquímica o de Bethe.

En la aproximación cero, se supone que a pesar de que los efectos energéticos ya no son despreciables, no son lo suficientemente importantes como para impedir la mezcla al azar de los diferentes segmentos presentes en la mezcla. De esta manera se asegura que la entropía de mezcla es independiente de la energía de intercambio $w$. Esto permite adoptar para la entropía de exceso la ecuación (6.186), obtenida para las mezclas atérmicas. Por su parte, el resto de propiedades de exceso y mezcla se pueden consultar en la monografía de Guggenheim [152].

En la aproximación cuasiquímica se supone que el número de los diferentes tipos de contactos están dados por las ecuaciones del equilibrio cuasiquímico. Como sabemos, la hipótesis de la no interferencia de pares es la esencia de esta aproximación y es equivalente a introducir un orden de corto alcance en el modelo de Ising tridimensional. Hay que destacar que la no aleatoriedad que introduce el tratamiento cuasiquímico es relativamente pequeña.

En el proceso de obtención de las ecuaciones características del modelo mediante el método cuasiquímico es posible separar los efectos entrópicos combinatoriales de los efectos energéticos. Así, la entropía de exceso se puede escribir como la suma de dos contribuciones (COMB) y (RES):

$$
S_{\mathrm{m}, V}^{\mathrm{E}}=S_{\mathrm{m}, V}^{\mathrm{E}}(\mathrm{COMB})+S_{\mathrm{m}, V}(\mathrm{RES})
$$

Como los efectos entrópicos combinatoriales dependen únicamente del factor combinatorial $g$ obtenido bajo la hipótesis de mezcla al azar (que coincide con el de las mezclas atérmicas), podemos utilizar para la contribución (COMB) la ecuación de Guggenheim-Staverman o de Flory-Huggins. En cuanto a la parte residual (RES), ella es responsable de la entalpía de mezcla no nula. Suponiendo que las fuerzas no son centrales, es decir, que $w$ depende de la temperatura, se tiene para dicha entalpía de mezcla y para la capacidad calorífica de exceso el resultado siguiente [262]:

$$
\begin{gathered}
U_{\mathrm{m}, V}^{\mathrm{E}}(T, \mathrm{RES})=H_{\mathrm{m}, V}^{\mathrm{E}}(T, \mathrm{RES})= \\
=R \cdot T \cdot \frac{x_{1} \cdot x_{2} \cdot q_{1} \cdot q_{2}}{q_{1}+\left(q_{2}-q_{1}\right) \cdot x_{1}} \cdot \frac{2}{\beta+1} \cdot\left[\frac{u}{k_{\mathrm{B}} \cdot T}\right] \\
C_{\mathrm{m}, V}^{\mathrm{E}}(T, \mathrm{RES})=C_{\mathrm{m}, P}^{\mathrm{E}}(T, \mathrm{RES})= \\
=\frac{2 \cdot x_{1} \cdot x_{2} \cdot q_{1} \cdot q_{2}}{(\beta+1) \cdot\left[q_{1}+\left(q_{2}-q_{1}\right) \cdot x_{1}\right]} \cdot\left[\frac{4 \cdot x_{1} \cdot x_{2} \cdot q_{1} \cdot q_{2} \cdot \eta^{2}}{z \cdot \beta \cdot(\beta+1) \cdot\left[q_{1}+\left(q_{2}-q_{1}\right) \cdot x_{1}\right]^{2}} \cdot\left(\frac{u}{k_{\mathrm{B}} \cdot T}\right)^{2}+\frac{v}{k_{\mathrm{B}}}\right]
\end{gathered}
$$

$\eta, u$ y $v$ vienen dada por las ecuaciones $(6.160),(6.177)$ y $(6.178)$ respectivamente, y $\beta$ :

$$
\beta^{2}=1+4 \cdot \frac{N_{1} \cdot q_{1} \cdot N_{2} \cdot q_{2}}{N_{q}^{2}} \cdot\left(\eta^{2}-1\right)
$$

La mayoría de los modelos de disoluciones consideran que la entropía de exceso es la suma de las contribuciones combinatorial y residual, como ocurre en el modelo aquí 
desarrollado. No obstante, mientras que la contribución combinatorial suele ser común, dada por una de las ecuaciones (6.186) y (6.187), la contribución residual es propia de cada modelo. Así se obtienen los modelos UNIQUAC, DISQUAC, etc. Por otra parte, a veces se utiliza un término semiempírico para la contribución residual. Por ejemplo, puede suponerse que $G_{\mathrm{m}, V}(\mathrm{RES})$ es justo igual a la entalpía de exceso dada por la teoría de Scatchard-Hildebrand, $w \cdot \varphi_{1} \cdot \varphi{ }_{2} \cdot\left(r_{\mathrm{A}} \cdot N_{\mathrm{A}}+r_{\mathrm{B}} \cdot N_{\mathrm{B}}\right)$, y obtener la entropía residual $S_{\mathrm{m}, V}(\mathrm{RES}) \operatorname{de}-\left[\partial G_{\mathrm{m}, V}(\mathrm{RES}) / \partial T\right]_{P}=-\left[\partial H_{\mathrm{m}, V}(\mathrm{RES}) / \partial T\right]_{P}$.

Las mezclas no atérmicas en la aproximación cuasiquímica son a las atérmicas lo que las mezclas estrictamente regulares a las mezclas ideales. Formalmente el paso de un caso al otro se puede realizar haciendo que el índice de coordinación medio verifique $z \rightarrow \infty$, pero su deducción puede obtenerse para $z$ finito de manera independiente. Es la condición de mezcla al azar lo característico de las aproximaciones cero, no el que $z$ tieda a infinito. En este contexto, el índice de coordinación medio es una medida del "grado de orientación" de las moléculas en la disolución, interpretándose que $z \rightarrow \infty$ da cuenta de la falta de orientación en las interacciones, es decir, de los efectos de tamaño y forma predominantes (aún con $H_{\mathrm{m}}^{\mathrm{E}} \neq 0$ ), mientras que $z$ finito (y pequeño) permite caracterizar interacciones orientadas que predominan sobre los efectos de forma y tamaño.

El modelo de red con moléculas de diferente tamaño también ha intentado resolverse en términos de la teoría del grupo de renormalización. No obstante, existe un detalle sutil a tener en cuenta, relacionado con el hecho de que el modelo de spines distribuidos en una red en el que cada spin ocupa más de un punto de la misma, no puede resolverse mediante los métodos convencionales de la teoría del grupo de renormalización, pues para tener en cuenta efectos de tamaño se necesita usar algún enunciado especial del principio de los estados correspondientes, como el de Fox [263]. Una descripción detallada de este método puede consultarse en el artículo de McMahon [264].

e) Por último, se puede introducir una corrección debida a que la mezcla no se produce a volumen constante. Es decir, se elimina la hipótesis de red rígida. La contribución a las diferentes propiedades de exceso por la condición $V \neq c t e$. viene dada por las ecuaciones (2.202) y (2.203). En concreto, la entropía de exceso a presión constante es:

$$
S_{\mathrm{m}}^{\mathrm{E}} \approx S_{\mathrm{m}, V}^{\mathrm{E}}+\frac{\alpha}{\kappa_{T}} \cdot V_{\mathrm{m}}^{\mathrm{E}}=S_{\mathrm{m}, V}^{\mathrm{E}}+\frac{1}{T} \cdot\left(P+P_{\mathrm{int}}\right) \cdot V_{\mathrm{m}}^{\mathrm{E}}=S_{\mathrm{m}, V}^{\mathrm{E}}+\Delta S_{\mathrm{m}}(V \neq \mathrm{cte})
$$

En resumen, el modelo de red aplicado a mezclas no atermicas, no al azar de moléculas de distinto tamaño, que no tienen simetría esférica, permitiendo cambios de volumen en la mezcla, conduce a la ecuación siguiente para la entropía de exceso:

$$
\begin{aligned}
& S_{\mathrm{m}}^{\mathrm{E}}(1)=S_{\mathrm{m}, V}^{\mathrm{E}}(\mathrm{COMB})+S_{\mathrm{m}, V}(\mathrm{RES})+\Delta S_{\mathrm{m}}(V \neq \mathrm{cte})= \\
= & -R \cdot \sum_{\mathrm{i}} x_{\mathrm{i}} \cdot\left(\ln \frac{\varphi_{\mathrm{i}}}{x_{\mathrm{i}}}+\frac{1}{2} \cdot z \cdot q_{\mathrm{i}} \cdot \ln \frac{\theta_{\mathrm{i}}}{\varphi_{\mathrm{i}}}\right)+S_{\mathrm{m}, V}(\mathrm{RES})+\frac{\alpha}{\kappa_{T}} \cdot V_{\mathrm{m}}^{\mathrm{E}}
\end{aligned}
$$

En la aproximación $z \rightarrow \infty$, se tiene:

$$
S_{\mathrm{m}}^{\mathrm{E}}(1, z \rightarrow \infty)=S_{\mathrm{m}, V}^{\mathrm{E}}(\mathrm{F}-\mathrm{H})+S_{\mathrm{m}, V}(\mathrm{RES})+\Delta S_{\mathrm{m}}(V \neq \mathrm{cte})=
$$




$$
=-R \cdot \sum_{\mathrm{i}} x_{\mathrm{i}} \cdot \ln \frac{\varphi_{\mathrm{i}}}{x_{\mathrm{i}}}+S_{\mathrm{m}, V}(\mathrm{RES})+\frac{\alpha}{\kappa_{T}} \cdot V_{\mathrm{m}}^{\mathrm{E}}
$$

Las ecuaciones para la mezcla al azar, que son las mismas que para mezclas atérmicas, se obtienen de las ecuaciones (6.193) y (6.194) suponiendo que $S_{\mathrm{m}, V}(\mathrm{RES})=0$ :

$$
\begin{aligned}
& S_{\mathrm{m}}^{\mathrm{E}}(1, \mathrm{DISP})=S_{\mathrm{m}, V}^{\mathrm{E}}(\mathrm{COMB})+\Delta S_{\mathrm{m}}(V \neq \mathrm{cte})= \\
& =-R \cdot \sum_{\mathrm{i}} x_{\mathrm{i}} \cdot\left(\ln \frac{\varphi_{\mathrm{i}}}{x_{\mathrm{i}}}+\frac{1}{2} \cdot z \cdot q_{\mathrm{i}} \cdot \ln \frac{\theta_{\mathrm{i}}}{\varphi_{\mathrm{i}}}\right)+\frac{\alpha}{\kappa_{T}} \cdot V_{\mathrm{m}}^{\mathrm{E}}
\end{aligned}
$$

En mezclas de moléculas con tamaños similares, en la aproximación cuasiquímica:

$$
\begin{gathered}
S_{\mathrm{m}}^{\mathrm{E}}(2)=S_{\mathrm{m}, V}^{\mathrm{E}}(T, \mathrm{QUAC})+\Delta S_{\mathrm{m}}(V \neq \mathrm{cte})= \\
\frac{1}{T} \cdot\left[\frac{2}{\beta+1} \cdot x_{1} \cdot x_{2} \cdot N \cdot u-F_{\mathrm{m}}^{\mathrm{E}}(T, \mathrm{QUAC})\right]+\frac{\alpha}{\kappa_{T}} \cdot V_{\mathrm{m}}^{\mathrm{E}}
\end{gathered}
$$

Si la mezcla es al azar:

$$
\begin{gathered}
S_{\mathrm{m}}^{\mathrm{E}}(2, \mathrm{DISP})=S_{\mathrm{m}, V}^{\mathrm{E}}(T, \mathrm{DISP})+\Delta S_{\mathrm{m}}(V \neq \mathrm{cte})= \\
=-N \cdot x_{1} \cdot x_{2} \cdot \frac{\mathrm{d} w}{\mathrm{~d} T}+\frac{\alpha}{\kappa_{T}} \cdot V_{\mathrm{m}}^{\mathrm{E}}
\end{gathered}
$$

Si los parámetros no dependen de la temperatura:

$$
\begin{gathered}
S_{\mathrm{m}}^{\mathrm{E}}(3)=S_{\mathrm{m}, V}^{\mathrm{E}}(\mathrm{QUAC})+\Delta S_{\mathrm{m}}(V \neq \mathrm{cte})= \\
\frac{1}{T} \cdot\left[\frac{2}{\beta+1} \cdot x_{1} \cdot x_{2} \cdot N \cdot w-F_{\mathrm{m}}^{\mathrm{E}}(\mathrm{QUAC})\right]+\frac{\alpha}{\kappa_{T}} \cdot V_{\mathrm{m}}^{\mathrm{E}}
\end{gathered}
$$

Si además, la mezcla es al azar:

$$
S_{\mathrm{m}}^{\mathrm{E}}(3, \mathrm{DISP})=S_{\mathrm{m}, V}^{\mathrm{E}}(\mathrm{DISP})+\Delta S_{\mathrm{m}}(V \neq \mathrm{cte})=\frac{\alpha}{\kappa_{T}} \cdot V_{\mathrm{m}}^{\mathrm{E}}
$$

Diferentes comparaciones entre las ecuaciones (6.193)-(6.199) permiten discutir los diferentes efectos importantes. Por ejemplo, la resta (6.193)-(6.195) permite escribir la contribución a la entropía de exceso debida a las desviaciones de la composición local respecto de la global, es decir, debida a los efectos de no aleatoriedad (NR) en la distribución de las moléculas, cuando sus tamaños son diferentes (SD):

$$
\Delta S_{\mathrm{m}}(\mathrm{NR}, \mathrm{SD})=S_{\mathrm{m}}^{\mathrm{E}}(1)-S_{\mathrm{m}}^{\mathrm{E}}(1, \mathrm{DISP})=S_{\mathrm{m}, V}(\mathrm{RES})
$$

Lo que permite hacer discutir si la validez de la hipótesis de mezcla al azar. En definitiva, permite evaluar los cambios orden-desorden.

La resta (6.196)-(6.198) permite escribir la contribución a la entropía de exceso de las fuerzas no centrales en mezclas no al azar de moléculas de tamaño y forma similar:

$$
\Delta S_{\mathrm{m}}(\text { no-central, QUAC })=S_{\mathrm{m}}^{\mathrm{E}}(2)-S_{\mathrm{m}}^{\mathrm{E}}(3)=-\frac{2}{\beta+1} \cdot x_{1} \cdot x_{2} \cdot N \cdot \frac{\mathrm{d} w}{\mathrm{~d} T}
$$

Lo que facilita la discusión de los efectos orientacionales en este tipo de mezclas. 
Si la mezcla fuese al azar, la contribución de las fuerzas no centrales estaría dada por la diferencia (6.197)-(6.199):

$$
\Delta S_{\mathrm{m}}(\text { no-central,DISP })=S_{\mathrm{m}}^{\mathrm{E}}(2, \mathrm{DISP})-S_{\mathrm{m}}^{\mathrm{E}}(3, \mathrm{DISP})=-N \cdot x_{1} \cdot x_{2} \cdot \frac{\mathrm{d} w}{\mathrm{~d} T}
$$

La resta (6.196)-(6.197) proporciona la contribución a la entropía de exceso debida a la no aleatoriedad de la distribución de las moléculas en mezclas de moléculas polares de igual tamaño:

$$
\Delta S_{\mathrm{m}}(\mathrm{NR})=S_{\mathrm{m}}^{\mathrm{E}}(2)-S_{\mathrm{m}}^{\mathrm{E}}(2, \mathrm{DISP})
$$

El estudio de las capacidades caloríficas también es importante, y se puede hacer un análisis de las diferentes contribuciones debidas a la no aleatoriedad de la mezcla y a los efectos orientacionales. De hecho, el modelo de red permite explicar la forma en W de $C_{\mathrm{m}, P}^{\mathrm{E}}$ como función de la composición propia de mezclas del tipo componente muy polar + alcano [266]-[283] que están cercanas a la temperatura crítica superior de mezcla (UCST). Mientras que dicho comportamiento se intentó explicar mediante la aparición de efectos conformacionales de manera similar a como se explicaron los efectos Patterson y Wilhelm [168]-[178], lo cierto es que una discusión en términos de las desviaciones de la composición local respecto de la global, es decir, en términos de la no aleatoriedad de la mezcla, puede ser suficiente [82], [243], [265], [283]. En concreto, para mezclas de moléculas polares de igual tamaño, las ecuaciones exactas de Cobos [243] para las capacidades caloríficas permiten escribir de manera exacta las contribuciones Random (DISP) y Non-Random (NR):

$$
\begin{gathered}
C_{\mathrm{m}, P}^{\mathrm{E}}(T, \mathrm{DISP})=R \cdot x_{1} \cdot x_{2} \cdot \frac{v}{k_{\mathrm{B}}} \\
C_{\mathrm{m}, P}^{\mathrm{E}}(T, \mathrm{NR})=R \cdot x_{1} \cdot x_{2} \cdot\left[\frac{8 \cdot x_{1} \cdot x_{2} \cdot \eta^{2}}{z \cdot \beta \cdot(\beta+1)^{2}} \cdot\left(\frac{u}{k_{\mathrm{B}} \cdot T}\right)^{2}+\left(\frac{2}{\beta+1}-1\right) \cdot \frac{v}{k_{\mathrm{B}}}\right]
\end{gathered}
$$

Las ecuaciones exactas anteriores son comparativamente similares a las ecuaciones aproximadas obtenidas por Saint-Victor y Patterson [265]. Naturalmente, dichas ecuaciones no son válidas a la temperatura crítica, pues la aproximación cuasiquímica no es válida.

Se puede demostrar que es condición necesaria y suficiente para que $C_{\mathrm{m}, P}^{\mathrm{E}}$ tenga doble mínimo (forma de W) que:

$$
\frac{v}{k_{\mathrm{B}}} \in\left[-\frac{1}{z}\left(\frac{u}{k_{\mathrm{B}} \cdot T}\right)^{2}, 0\right]
$$

donde $u$ y $v$ vienen dados por (6.177) y (6.178). Por ejemplo, $w=\mathrm{a}+\mathrm{b} / T$. La condición anterior es una restricción muy fuerte sobre la forma funcional $w=w(T)$. Es inmediato ver que independientemente de la estructura de la moléculas, la contribución $C_{\mathrm{m}, P}^{\mathrm{E}}(\mathrm{DISP})$ es siempre negativa y que $C_{\mathrm{m}, P}^{\mathrm{E}}(\mathrm{NR})$ es positiva, cóncava hacia abajo en $x=0.5$ y cóncava hacia arriba en los extremos de composición. Además, a dilución infinita la mezcla es ideal, luego al azar. Es decir, en los extremos de dilución, $C_{\mathrm{m}, P}^{\mathrm{E}} \approx C_{\mathrm{m}, P}^{\mathrm{E}}(\mathrm{DISP})<0$. Por lo 
tanto, si el efecto non-random supera al random, es decir, si la contribución debida a que las fuerzas no centrales o a que existen interacciones preferenciales es importante, se explica la forma en $\mathrm{W}$ de $C_{\mathrm{m}, P}^{\mathrm{E}}$ sin hacer alusión alguna a los efectos de isomería conformacional ya que el modelo de Cobos no tiene en cuenta la estructura de las moléculas. En la bibliografía se pueden consultar otras propiedades matemáticas de $C_{\mathrm{m}, P}^{\mathrm{E}}$ así como una discusión similar para mezclas de moléculas de diferente tamaño [243], [262].

Para concluir con este apartado, se enumeran algunas de las numerosas variaciones del modelo de red que pueden encontrarse en la bibliografía: se ha desarrollado una teoría de campo cristalino para disoluciones de polímeros, que no es más que la teoría de Flory-Huggins resuelta exactamente, es decir, eliminando las simplificaciones introducidas [284]-[287]; se ha desarrollado una teoría de campo cristalino que incluye interacciones específicas como puentes de hidrógeno; se ha modificado el modelo permitiendo la posibilidad de que existan sitios de la red vacíos [288]-[291]; se han introducido correcciones para tener en cuenta las interacciones fuertes (enlaces de hidrógeno) entre los polímeros en forma de ecuación de estado [292]. A pesar de todas las posibles modificaciones, el modelo de red ha sido superado por la versión de superficies de interacción de Bondi [293], empleada más tarde por Kehiaian para formular el modelo DISQUAC, que permite obtener una representación correcta de las funciones de exceso $G_{\mathrm{m}}^{\mathrm{E}} ; H_{\mathrm{m}}^{\mathrm{E}}, C_{\mathrm{P}, \mathrm{m}}^{\mathrm{E}}$ y de los equilibrios entre fases ELV, ELL y ESL, como se expone más adelante.

\subsubsection{Modelos de composición local}

Estos modelos se basan en el concepto de composición local $x_{\mathrm{ij}}$ introducido por Wilson [294]. $x_{\mathrm{ij}}$ es la fracción molar del componente i en la esfera de influencia de una molécula del tipo j. De su definición por la ecuación (6.109) se extraen los dos corolarios siguientes:

$$
\begin{aligned}
& \sum_{\mathrm{i}} x_{\mathrm{ij}}=1 \\
& x_{\mathrm{ij}}=x_{\mathrm{ji}}
\end{aligned}
$$

El concepto de composición local ayuda a discernir si la distribución de las moléculas es o no al azar.

Aunque una comparación de los principales modelos basados en el concepto de composición local puede consultarse en la bibliografía [161, cap 6], [295], a continuación se exponen las principales características de algunos de ellos:

a) Modelo UNIQUAC: Abrams y Prausnitz [296] utilizaron el modelo de disoluciones estrictamente regulares en la aproximación cuasiquímica de Guggenheim para mezclas no atérmicas que contienen moléculas de tamaños diferentes y que pueden cruzarse, adoptando una forma especial de la contribución residual. Por ello, a la ecuación resultante para $G_{\mathrm{m}}^{\mathrm{E}}$ se le llamó UNIQUAC (de su acrónimo en inglés UNIversal QUAsiChemical), la cual fue deducida nuevamente por Maurer y Prausnitz [297] y otros autores [298]-[300] utilizando argumentos fenomenológicos basados en la teoría de dos fluidos, evitando en parte las inconsistencias reveladas por diversos 
autores [301]-[303] que surgen cuando la ecuación UNIQUAC se deduce utilizando modelos de composición local.

La ecuación UNIQUAC es:

$$
G_{\mathrm{m}}^{\mathrm{E}}=G_{\mathrm{m}}^{\mathrm{E}}(\mathrm{COMB})+G_{\mathrm{m}}(\mathrm{RES})=-T \cdot S_{\mathrm{m}}^{\mathrm{E}}(\mathrm{COMB})+G_{\mathrm{m}}(\mathrm{RES})
$$

Donde $S_{\mathrm{m}}^{\mathrm{E}}(\mathrm{COMB})$ es el mismo que en la ecuación (6.186) y:

$$
G_{\mathrm{m}}(\mathrm{RES})=-R \cdot T \cdot \sum_{\mathrm{i}} x_{\mathrm{i}} \cdot q_{\mathrm{i}} \cdot \ln \left(\sum_{j} \theta_{\mathrm{j}} \cdot \tau_{\mathrm{ji}}\right)
$$

con

$$
\tau_{\mathrm{ji}}=e^{-\frac{g_{\mathrm{ji}}-g_{\mathrm{ii}}}{R \cdot T}}
$$

En la ecuación UNIQUAC no aparece la energía de intercambio del modelo de red. En su lugar, aparecen los dos parámetros ajustables, $\tau_{\mathrm{ji}}$ y $\tau_{\mathrm{ji}}$, que caracterizan la energía de interacción entre los sitios i y j. Es aplicable a un gran número de mezclas líquidas de no electrolitos miscibles completa o parcialmente, con moléculas polares o apolares como alcanos, alcoholes, nitrilos, cetonas, aldehídos, ácidos orgánicos, etc., explicando desviaciones positivas o negativas y moderadas o grandes respecto del comportamiento ideal. Además puede generalizarse fácilmente a mezclas de más de dos componentes en base al conocimiento solamente de parámetros de los sistemas binarios y los componentes puros. La principal desventaja es que la aplicabilidad de la ecuación a sistemas parcialmente miscibles depende de la elección de la unidad de tamaño de los segmentos.

b) Ecuación de Wilson: Según Wilson, la distribución de las moléculas en torno a una central está determinada por la ecuación siguiente:

$$
\frac{x_{\mathrm{ij}}}{x_{\mathrm{kj}}}=\frac{x_{\mathrm{i}} \cdot e^{-g_{\mathrm{ij}} / R \cdot T}}{x_{\mathrm{k}} \cdot e^{-g_{\mathrm{kj}} / R \cdot T}}
$$

donde $g_{\mathrm{ij}}=g_{\mathrm{ji}}$ es proporcional a la energía de interacción del par de moléculas i y j dada por (6.138), y es $g_{\text {ii }}$ proporcional a la energía de interacción entre pares de moléculas similares, es decir, proporcional a $-2 \cdot \chi_{i} \cdot z^{-1}$. La ecuación es equivalente a la condición (6.154) dada por Guggenheim de que la mezcla no sea al azar.

Wilson [294] propuso de manera fenomenológica la ecuación siguiente para $G_{\mathrm{m}}^{\mathrm{E}}$ :

$$
G_{\mathrm{m}}^{\mathrm{E}}=R \cdot T \cdot \sum_{\mathrm{i}} x_{\mathrm{i}} \cdot \ln \frac{\xi_{\mathrm{i}}}{x_{\mathrm{i}}}
$$

Donde $\xi_{\mathrm{i}}$ es la fracción de volumen local del componente i torno a una molécula central del mismo tipo:

$$
\xi_{\mathrm{i}}=\frac{x_{\mathrm{i}} \cdot V_{\mathrm{i}}^{0} \cdot e^{-g_{\mathrm{ii}} / R \cdot T}}{\sum_{j} x_{\mathrm{j}} \cdot V_{\mathrm{j}}^{0} \cdot e^{-g_{\mathrm{jj}} / R \cdot T}}
$$

De las ecuaciones anteriores es claro intuir que si $g_{\mathrm{ij}}=g_{\mathrm{ji}}$ es decir, si las energías de interacción entre diferentes pares de moléculas son iguales, las composiciones locales coinciden con las globales, $x_{\mathrm{ij}}=x_{\mathrm{i}}$, lo que significa que la mezcla es al azar. En ese caso, 
la ecuación (6.213) se convierte en la ecuación de Flory-Huggins (6.187), que como se ha visto es la solución correcta al problema de la mezcla de moléculas de tamaño distinto distribuidas en una red en la aproximación de orden cero. No obstante, si las diferencias entre las energías de interacción de pares de moléculas distintas son diferentes, la distribución de las moléculas es no aleatoria.

La ecuación de Wilson es particularmente útil en disoluciones de componentes polares o asociados donde las ecuaciones de Margules con 3 subíndices o las de Van Laar no son adecuadas. La ecuación de Wilson tiene la ventaja de que sólo consta de dos parámetros. No obstante, no es válida en mezclas en las que el logaritmo natural de los coeficientes de actividad presente algún extremo en función de la composición, lo que es poco habitual. Además, solamente puede utilizarse en mezclas de líquidos que son completamente miscibles, ya que no es capaz de predecir la miscibilidad parcial.

c) Modelo NRTL (Non-Random Two Liquids): se basa en el modelo de dos fluidos de Scott [304] y en el concepto de composición local. Su principal ventaja sobre el modelo de Wilson es que es aplicable a sistemas tanto parcial como completamente miscibles. Para Renon y Prausnitz [305], la distribución de las moléculas en torno a una central no está determinada por la ecuación (6.212) sino por la siguiente:

$$
\frac{x_{\mathrm{ij}}}{x_{\mathrm{kj}}}=\frac{x_{\mathrm{i}} \cdot e^{-\alpha \cdot g_{\mathrm{ij}} / R \cdot T}}{x_{\mathrm{k}} \cdot e^{-\alpha \cdot g_{\mathrm{kj}} / R \cdot T}}
$$

Donde $\alpha$ es una constante propia del carácter de la distribución no aleatoria de las moléculas ( $\alpha=0$ en mezclas aleatorias) y suele tomarse igual a 0.3 .

La ecuación para $G_{\mathrm{m}}^{\mathrm{E}}$ que se obtiene es la siguiente:

$$
\frac{G_{\mathrm{m}}^{\mathrm{E}}}{R \cdot T}=x_{1} \cdot x_{2} \cdot\left(\frac{\tau_{21} \cdot e^{-\alpha \cdot \tau_{21}}}{x_{1}+x_{2} \cdot e^{-\alpha \cdot \tau_{21}}}+\frac{\tau_{12} \cdot e^{-\alpha \cdot \tau_{12}}}{x_{2}+x_{1} \cdot e^{-\alpha \cdot \tau_{12}}}\right)
$$

donde $\tau_{\mathrm{ji}}$ están dados por la ecuación (6.211), los cuales deben considerarse dos parámetros ajustables.

El desconocimiento acerca de las energías de interacción intermolecular hace que las ecuaciones propuestas por cada modelo sean más útiles si se emplean como ecuaciones empíricas en las cuales los parámetros que aparecen se obtienen del ajuste a los datos experimentales. Lo mismo ocurre con otras ecuaciones como las de Van der Waals, Margules, Scatchard-Hamer [306], etc., las cuales son simplificaciones de una ecuación general que relaciona las diversas contribuciones a $G_{\mathrm{m}}^{\mathrm{E}}$ a causa de las interacciones de grupos de 2, 3, 4, etc. moléculas de compuestos distintos, como demostró Wohl [307].

\subsubsection{Teoría de disoluciones conformacionales}

Los modelos de celdas no son completamente satisfactorios. Ello se debe en parte a que deben tenerse en cuenta otras propiedades de los componentes puros, además de las que dan cuenta del tamaño molecular y la energía de interacción. Además, por lo general, fluidos diferentes tienen volúmenes libres diferentes, lo que debe tenerse en cuenta especialmente en disoluciones de moléculas de tamaño molecular muy distinto, como ya se ha explicado. Consecuentemente los modelos de celdas tienen la principal debilidad de que las propiedades termodinámicas de los componentes puros no se 
obtienen a partir de las ecuaciones proporcionadas por el modelo al hacer que todas las concentraciones, excepto la del componente en cuestión, sean cero. Esta desventaja es superada en parte si se utiliza la teoría de disoluciones conformacionales [308]-[310]. En esta teoría, las funciones de exceso se obtienen mediante un cálculo perturbativo de primer orden, sin hacer uso de ningún modelo. Por ello, se podría pensar que la teoría es de validez general, algo que no es cierto ya que en su desarrollo es necesario suponer que el principio de los estados correspondientes es aplicable, lo que limita la utilización de la teoría a aquellos sistemas en los que dicho principio es válido (líquidos simples clásicos para los que la energía potencial de interacción es aditiva por pares, siendo la energía de interacción entre pares de moléculas una función universal de dos parámetros propios de cada especie molecular).

La teoría predice que todas las propiedades de exceso deben tener el mismo signo, lo que está en contradicción con los resultados experimentales. Por lo tanto, un cálculo perturbativo de primer orden no conduce a una teoría correcta de las disoluciones. A pesar de ello, la teoría conformacional tiene interés como guía para el desarrollo de teorías perturbativas de mayor orden, las cuales deben utilizar un modelo microscópico.

\subsubsection{Modelos de potencial promedio}

$\mathrm{Ni}$ los modelos de celdas ni la teoría de disoluciones conformacionales conducen a resultados satisfactorios. Ello condujo al desarrollo de un conjunto de métodos que en cierto sentido aúnan ideas de ambas y de otras teorías: inicialmente se introdujeron heurísticamente la dependencia con la concentración del potencial de interacción, propia de los modelos de celdas, así como el principio de los estados correspondientes, contenido en la teoría de disoluciones conformacionales. Posteriormente, se incluyeron cálculos perturbativos y el concepto de fluido equivalente de la teoría de van der Waals. Así, se obtienen los llamados modelos de potencial promedio [92, cap. 17.10], [311][313] que se basan en la idea de que la mezcla puede considerarse como una mezcla de $m$ fluidos hipotéticos cuyos tamaños moleculares y energías potenciales característicos son promedios ponderados con la composición, el tamaño y la energía potencial de los componentes puros de la mezcla.

Las principales limitaciones de los modelos de potencial promedio son las siguientes:

a) Se desprecian las contribuciones a las propiedades termodinámicas de algunas fluctuaciones dependientes de la composición, lo cual es razonable solamente si las diferencias entre las fuerzas intermoleculares son pequeñas y si el número de vecinos próximos es grande.

b) Se presupone que la forma del potencial de interacción es conocida, y que el potencial promedio utilizado guarda la forma de los líquidos puros sólo si dicha forma es sencilla, como por ejemplo del tipo Lennard-Jones (ver ecuación (6.122)). En el caso de potenciales más complejos, las funciones de exceso no pueden expresarse como función de las propiedades de los líquidos puros y las fuerzas intermoleculares solamente, sino que se debe utilizar un modelo estadístico concreto.

c) El modelo sólo es útil en sustancias en las que el principio de los estados correspondientes es aplicable.

El extenso número de modelos resultantes fue esquematizado por Scott [31 1], siendo los más importantes los siguientes: 
a) Modelo de un fluido [314]-[317]: la mezcla está representada por un líquido hipotético cuyo potencial de interacción intermolecular es el promedio de todas las interacciones en la mezcla. Las ecuaciones se pueden reducir a las de un líquido puro y dan el valor exacto para los términos de primer orden de la teoría conformacional. Los términos de segundo orden son similares a los dados por el modelo de celdas. Al contrario que en el resto de modelos de potencial promedio, no utiliza ningún modelo estadístico. No obstante, tiene el inconveniente de que los efectos de orden molecular son despreciados, es decir, se supone que la mezcla es aleatoria.

b) Modelo de dos fluidos [304], [314]: la disolución está representada por dos líquidos puros hipotéticos e independientes, en el sentido de que la función de Gibbs de exceso de la mezcla es la suma de las funciones de Gibbs de cada líquido. El potencial de interacción de los dos líquidos se obtiene promediando por separado. El modelo de dos fluidos en cierto sentido corrige el de un fluido pues tiene en cuenta el orden molecular.

c) Modelo de tres fluidos: la mezcla está representada por tres líquidos independientes, cuyos potenciales de interacción corresponde a las interacciones A-A, B$B$ y A-B que ocurren en la mezcla. El principal fallo de este modelo es que sobreestima el orden molecular.

\subsubsection{Teorías tipo van der Waals}

En términos modernos [319], la teoría de van der Waals [162] no es más que un modelo de esferas rígidas tratado mediante técnicas perturbativas.

Se basa en las ideas siguientes:

a) La estructura de un fluido está caracterizada principalmente por fuerzas repulsivas de corto alcance.

b) Las fuerzas atractivas (dispersivas, dipolares, puentes de hidrógeno, etc.) son tenidas en cuenta suponiendo que las moléculas están inmersas en un campo promedio efectivo dependiente de la temperatura.

La teoría de van der Waals es una corrección al modelo de gas ideal, en la que se tiene en cuenta que las moléculas tienen tamaño no nulo y que interaccionan. Lo primero se traduce en el hecho de que el volumen disponible para que estas se muevan dentro del recipiente no es $V$ sino $v_{f}$, el volumen libre. Lo segundo, se tiene en cuenta suponiendo que existe un potencial de interacción, que se supone aditivo por pares:

$$
W\left(\vec{R}_{i}\right)=\frac{1}{2} \cdot \sum_{1 \leq \mathrm{j} \leq N} u\left(\vec{X}_{\mathrm{i}}, \vec{X}_{\mathrm{j}}\right)=\frac{1}{2} \cdot \sum_{1 \leq \mathrm{j} \leq N} u\left(R_{\mathrm{ij}}\right)=\frac{1}{2} \cdot \sum_{1 \leq \mathrm{j} \leq N} u(R)
$$

Luego la energía de interacción promedio es,

$$
\langle W\rangle=\frac{1}{2} \cdot \frac{N}{V} \cdot \int g(R) \cdot u(R) \cdot 4 \cdot \pi \cdot R^{2} \cdot \mathrm{d} R
$$

Como conclusión, mientras que la función de partición de un gas ideal confinado en un recipiente de volumen $V$ es:

$$
Z=\frac{Z_{1}^{N}}{N !}=\frac{Z_{\mathrm{t}, 1}^{N} \cdot Z_{\text {int, } 1}{ }^{N}}{N !}=\frac{1}{N !} \cdot\left(\frac{V}{\Lambda^{3}}\right)^{N} \cdot Z_{\text {int, } 1}{ }^{N}=\left(\frac{e}{N}\right)^{N} \cdot\left(\frac{V}{\Lambda^{3}}\right)^{N} \cdot Z_{\text {int }, 1}{ }^{N}
$$


donde $Z_{\mathrm{t}, 1}$ es la función de partición de las traslaciones de los centros de masas de las moléculas en ausencia de interacción y $Z_{\text {int, } 1}$ es la función de partición de los grados de libertad internos (aunque no estrictamente internos pues aquí están incluidas las rotaciones de la molécula como un todo), la función de partición de van der Waals generalizada es [320]:

$$
\begin{gathered}
Z=\left(\frac{e}{N} \cdot \frac{v_{f}}{\Lambda^{3}}\right)^{N} \cdot Z_{\mathrm{int}, 1}{ }^{N} \cdot\left(e^{-\beta \cdot\langle W\rangle}\right)^{N}=\frac{1}{\Lambda^{3 \cdot N}} \cdot Z_{\mathrm{int}, 1}{ }^{N} \cdot\left(\frac{e}{N} \cdot v_{f} \cdot e^{-\beta \cdot\langle W\rangle}\right)^{N}= \\
=\frac{1}{\Lambda^{3 \cdot N}} \cdot Z_{\mathrm{int}, 1}{ }^{N} \cdot \Omega
\end{gathered}
$$

Si se considera que el fluido está estructurado como una red para calcular $v_{f} y\langle W\rangle$, se obtendría para $\Omega$ la misma función que en el modelo de celdas.

La ecuación de estado es:

$$
\begin{gathered}
p=k_{\mathrm{B}} \cdot T \cdot\left(\frac{\partial \ln Z}{\partial V}\right)_{T, N}=N \cdot k_{\mathrm{B}} \cdot T \cdot\left(\frac{\partial \ln v_{f}}{\partial V}\right)_{T, N}-N \cdot\left(\frac{\partial\langle W\rangle}{\partial V}\right)_{T, N}+N \cdot k_{\mathrm{B}} \cdot T \cdot\left(\frac{\partial \ln Z_{\text {int }, 1}}{\partial V}\right)_{T, N}= \\
=p_{1}+p_{2}+p_{3}
\end{gathered}
$$

Para el volumen libre van der Waals utilizó la cantidad siguiente:

$$
v_{f}=V-\frac{N}{N_{A}} \cdot b=V-N \cdot \frac{4 \cdot \pi \cdot \sigma^{3}}{6}
$$

donde se ha supuesto que cada molécula tiene un núcleo duro de radio $\sigma / 2$, es decir, que cuando dos moléculas se acercan, la distancia más pequeña posible entre sus centros es $\sigma$.

Suponiendo que la función de distibución es independiente de la temperatura y del volumen la integral de la ecuación (6.218) es constante y se iguala a la cantidad $-2 \cdot a / N_{A}{ }^{2}$, donde $a$ es una constante positiva. Luego:

$$
\langle W\rangle=-\frac{a \cdot N}{V \cdot N_{A}^{2}}<0
$$

Con todo esto, la función de partición de van der Waals es:

$$
Z=\frac{1}{N !} \cdot \frac{1}{\Lambda^{3 \cdot N}}\left(V-\frac{N \cdot b}{N_{A}}\right)^{N} \cdot\left(e^{\beta \cdot \frac{a \cdot N}{V \cdot N_{A}^{2}}}\right)^{N} \cdot Z_{\text {int, } 1}{ }^{N}
$$

La teoría de van der Waals supone que las moléculas son esferas rígidas. Por tanto, los grados de libertad internos y las libraciones de las moléculas son independientes del ambiente molecular, es decir, de la densidad, por lo tanto, $\left(\partial Z_{\text {int }, 1} / \partial V\right)_{T, N}=0$, pudiendo sin embargo ser función de la temperatura. Esta aproximación es razonable en el caso de moléculas monoatómicas poliatómicas pequeñas y apolares (líquidos normales de acuerdo a la clasificación de líquidos en normales y asociados) y se traduce en que la contribución $p_{3}$ a la ecuación (6.221) es cero. En conclusión, la ecuación térmica de estado es: 


$$
p=k_{\mathrm{B}} \cdot T \cdot\left(\frac{\partial \ln Z}{\partial V}\right)_{T, N}=\frac{R \cdot T}{v-b}-\frac{a}{v^{2}}
$$

donde $v$ es un volumen molar:

$$
v=V \cdot \frac{N_{A}}{N}
$$

Sin embargo, en el caso de los líquidos asociados de acuerdo con la clasificación de líquidos en normales y asociados, los grados de libertad internos de las moléculas (rotaciones, vibraciones, rotaciones conformacionales, etc.) dependen además de la temperatura, de la densidad. Así, mientras que una molécula pequeña puede rotar y vibrar casi sin verse afectada por su entorno, una molécula mayor puede rotar y vibrar sólo cuando sus moléculas vecinas están lejos (baja densidad). A densidades altas, los vecinos interfieren en los grados de libertad internos. Por lo tanto no es correcto suponer que $Z_{\text {int, } 1}$ sea sólo dependiente de la temperatura, sino que también lo será de la densidad. Ahora bien, pueden existir modos (como los modos de vibración de alta frecuencia) que no se vean afectados por el ambiente molecular. Por lo tanto, es una aproximación aceptablemente buena suponer que a densidades altas, se puede realizar la factorización siguiente:

$$
Z_{\text {int, } 1}(T, \rho)=Z_{\text {int, } 1}^{1}(T) \cdot Z_{\text {int, }}^{2}(T, \rho)
$$

Donde $Z_{\text {int,1 }}^{1}$ representa la contribución a la función de partición de las rotaciones,

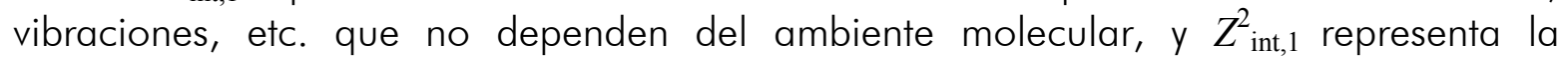
contribución a la función de partición de las rotaciones, vibraciones, etc. que sí dependen del ambiente molecular.

Se pueden proponer diferentes expresiones para $Z_{\text {int,1. }}^{2}$ Prigogine [214] sugirió que los grados de libertad internos que dependen del ambiente molecular pueden considerarse a efectos prácticos como si fuesen grados de libertad de traslación de los centros de masas de las moléculas. Esta aproximación no es válida a bajas densidades [321]. Así, mientras que $3 \cdot N$ es el número de grados de libertad de traslación de $N$ moléculas de un líquido normal, $3 \cdot N \cdot(c-1)$ es el número de grados de libertad internos que dependen del ambiente molecular y $3 \cdot N \cdot c$ es el número de grados de libertad traslacionales equivalentes, es decir, que dependen del ambiente molecular, (luego de la densidad o el volumen). Así:

$$
Z_{\text {int, } 1}^{2}(T, \rho)=\left(\frac{v_{f}}{\Lambda^{3}}\right)^{c-1}
$$

Por lo tanto, la función de partición (6.220) se puede escribir como sigue:

$$
Z=\left(\frac{e}{N}\right)^{N} \cdot\left(\frac{v_{f}^{1 / 3}}{\Lambda}\right)^{3 \cdot N \cdot c} \cdot\left[Z_{i n t, 1}^{1}(T)\right]^{N} \cdot\left(e^{-\beta \cdot\langle W\rangle}\right)^{N}
$$

Así, la ecuación térmica e estado es:

$$
p=3 \cdot N \cdot k_{\mathrm{B}} \cdot T \cdot c \cdot\left(\frac{\partial \ln v_{f}^{1 / 3}}{\partial V}\right)_{T, N}-N \cdot\left(\frac{\partial\langle W\rangle}{\partial V}\right)_{T, N}
$$


Para generalizar el modelo a mezclas es necesario definir reglas de mezcla además de realizar una aproximación del tipo teoría de $n$ fluidos para calcular las propiedades de la mezcla como el promedio de $n$ fluidos [161].

La ecuación de estado puede mejorarse si además de permitir la dependencia de $Z_{\text {int, } 1}$ con el volumen y la temperatura, se acepta que el potencial de interacción es función de la densidad y de la temperatura, y si se busca una mejor expresión para $v_{f}$. Por ejemplo, puede tomarse como expresión del volumen libre la de Carnahan-Starling [322]:

$$
\frac{v_{f}}{v}=e^{-\frac{4 \cdot \eta-3 \cdot \eta^{2}}{(1-\eta)^{2}}}
$$

donde $\eta=\pi \cdot \rho \cdot \sigma^{3} / 6$, $\sigma$ es el diámetro de la molécula, supuesta esférica, y $\rho=N / V$.

Dependiendo de las expresiones escogidas para $v_{f}$, el potencial de interacción $\varphi$ y para $Z_{\text {int, } 1}^{2}(T, \rho)$, se pueden encontrar diferentes ecuaciones de estado [323]-[330], por ejemplo la ecuación de estado de Flory, que se expone en la siguiente sección.

\subsubsection{Modelo de Flory}

La teoría de Flory [331]-[337] nació como un intento de superar los modelos de celdas y los diversos modelos que se obtienen por la aplicación del principio de los estados correspondientes. Flory criticó el excesivo orden que los modelos de celda introducen en la estructura de los líquidos y que el cumplimiento del principio de los estados correspondientes está ligado estrechamente con las reglas de mezcla que se introducen ad hoc en los modelos.

Mientras que los modelos de celdas suponen que el volumen libre de los diferentes componentes puros es idéntico y sólo tienen en cuenta las posibles configuraciones de las moléculas en la red, lo que da lugar a la contribución combinatorial de las propiedades termodinámicas, el modelo de Flory tiene en cuenta las diferencias entre las propiedades de los distintos componentes puros, que son las responsables de la contribución residual que afecta a los valores de las propiedades termodinámicas de la mezcla, explica el volumen de exceso y contribuyen a la ecuación de estado.

Otro logro del modelo de Flory es que es capaz de explicar la miscibilidad parcial a bajas y altas temperaturas [338], [339], mientras que el modelo de red sólo es capaz de hacerlo a bajas temperaturas [340], [341].

\subsubsection{Hipótesis. Modelo de Flory para líquidos puros}

Para aplicar el modelo de Flory debe suponerse que el líquido está confinado en el volumen $V$, que está formado por $N$ moléculas, y que éstas están constituidas por $r$ segmentos, dotados los últimos de $s$ puntos de contacto, o simplemente contactos. Los contactos interaccionan por parejas. La suma de todas estas interacciones da como resultado la energía interna del líquido. Además, se asume que los grados de libertad internos pueden separarse en los que dependen del ambiente molecular y los que no, del igual manera a como hizo Prigogine y se ha explicado en la sección anterior. Los que no dependen del ambiente molecular no contribuyen a las funciones de exceso, pues se anulan al restar las funciones de los componentes puros. Además, al no ser función del ambiente molecular, es decir, del volumen, tampoco contribuyen a la ecuación de estado. Los grados de libertad que sí que dependen del entorno molecular son tratados equivalentemente como traslaciones de los centros de masas, de modo que 
el número de grados de libertad de traslación equivalentes de cada segmento no es 3 sino $3 \cdot c$, con $c<1$ para dar cuenta de los posibles ligaduras entre segmentos. Como se ha reiterado en la sección anterior, esta aproximación sólo es razonable en densidades altas. Por lo tanto, debe tenerse en cuenta que este modelo sólo es aplicable a fluidos en altas presiones, es decir, a fluidos con densidades de líquidos, luego lejos del punto crítico. Una consecuencia es que la ecuación de estado que se obtiene del modelo no se reduce a la del gas ideal en el límite de presión nula.

En definitiva:

a) $\quad s$ es el número de contactos por segmento. Coincide con $q \cdot z / r$ del modelo de red.

b) $\quad r$ es el número de segmentos por molécula. La definición de segmento es algo arbitraria, aunque suele ser apropiado tomarlo como una porción isométrica de la molécula. En cualquier caso, no hay razón alguna para pensar que coincida con el mismo tipo de segmento escogido en el modelo de red.

c) $\quad N$ es el número de moléculas en el líquido.

d) $\quad V$ es el volumen que ocupa el líquido.

e) $3 \cdot N \cdot r \cdot c$ es el número total de grados de libertad de traslación equivalentes.

En lo que sigue será necesario utilizar las cantidades siguientes:

a) $\quad v_{\mathrm{s}}^{*}$ es el volumen compacto por segmento.

b) $\quad v_{\mathrm{m}}^{*}$ es el volumen compacto por molécula. Es claro que los volúmenes compactos por molécula y por segmento están relacionados:

$$
v_{\mathrm{m}}^{*}=r \cdot v_{\mathrm{s}}^{*}
$$

c) $\quad v_{\mathrm{m}}$ es el volumen disponible por molécula. $v_{\mathrm{s}}$ es el volumen disponible por segmento:

$$
\begin{gathered}
v_{\mathrm{m}}=V / N \\
v_{\mathrm{s}}=V / r \cdot N=v_{\mathrm{m}} / r
\end{gathered}
$$

Se supone que la energía de interacción entre las moléculas es igual a la suma de las interacciones entre los pares de contactos. Así, si la energía de interacción entre dos contactos es:

$$
\bar{\varepsilon}=-\frac{\eta}{v_{s}}
$$

entonces, la energía de interacción total será la suma de las interacciones entre todos los pares de contactos presentes en el líquido $(N \cdot r \cdot s / 2)$ :

$$
E_{0}=-\frac{N \cdot r \cdot s}{2} \cdot \frac{\eta}{v_{s}}
$$

que coincide con la energía interna $U$ del líquido. $\eta$ es una constante que caracteriza la intensidad de la interacción entre un par de contactos en el líquido. La energía es de

\footnotetext{
* En este caso se habla del número de grados de libertad equivalentes para cada segmento, no para cada molécula.
} 
tipo van der Waals, ecuación (6.223). Aicart et al. [342] propusieron utilizar un potencial tipo Lenard-Jones, sin obtener mejoras significativas.

En lo que respecta a la función de partición, ésta puede obtenerse a partir de la ecuación de van der Waals generalizada (6.229), suponiendo que $N \cdot\langle W\rangle=E_{0}$ y $v_{f}=2 \cdot r \cdot v_{\mathrm{s}}^{*} \cdot\left(\bar{v}^{1 / 3}-1\right)^{3}$. Sin embargo, originalmente Flory procedió de manera diferente. Generalizó la función de partición de Tonks para un sistema tridimensional de $r \cdot N$ segmentos, cada uno de volumen $v_{\mathrm{s}}^{*}$, que no pueden superponerse. La función de partición canónica es:

$$
Z=Z(\mathrm{COMB}) \cdot\left[\gamma \cdot\left(v_{\mathrm{s}}^{1 / 3}-v_{\mathrm{s}}^{* 1 / 3}\right)^{3} \cdot e^{3}\right]^{c \cdot r \cdot N} \cdot e^{-\beta \cdot E_{0}}=Z(\mathrm{COMB}) \cdot Z(\mathrm{RES})
$$

$\gamma$ es un factor geométrico que permite pasar de un sistema monodimensional a uno tridimensional. $Z(C O M B)$ es el factor combinatorio que surge de considerar las diferentes permutaciones en que se pueden disponer los segmentos en el líquido. Para este factor puede utilizarse por ejemplo el del modelo de red en la aproximación $z \rightarrow \infty$ para mezclas atérmicas de moléculas de diferente tamaño, es decir, el que conduce a la ecuación de Flory-Huggins (6.187). El factor combinatorial junto con los factores $\mathrm{e}^{3 \cdot c \cdot r \cdot N}$ y $\gamma^{N}$ desaparecen al derivar la función de partición respecto el volumen, es decir, no contribuyen a la ecuación de estado. Además, la contribución de $Z(C O M B)$ a la entalpía de mezcla es cero pues la ecuación de Flory-Huggins es válida sólo para mezclas atérmicas.

Derivando $k_{\mathrm{B}} \cdot T \cdot \ln Z$ respecto de $V$ a $T$ y $N$ constantes se obtiene la ecuación de estado de Flory, que es similar a la de Eyring \& Hirschfelder [326]. En forma reducida es la siguiente:

$$
\frac{\bar{p} \cdot \bar{v}}{\bar{T}}=\frac{\bar{v}^{1 / 3}}{\bar{v}^{1 / 3}-1}-\frac{1}{\bar{v} \cdot \bar{T}}
$$

donde se han utilizado las variables reducidas, definidas como sigue:

$$
\begin{gathered}
\bar{v}=v_{\mathrm{s}} / v_{\mathrm{s}}^{*}=v_{\mathrm{m}} / v_{\mathrm{m}}^{*} \\
\bar{T}=T / T^{*} \\
\bar{p}=P / p^{*}
\end{gathered}
$$

donde los parámetros característicos de líquido $T^{*}$ y $p^{*}$ se definen como sigue:

$$
\begin{gathered}
T^{*}=\frac{s \cdot \eta}{2 \cdot v_{\mathrm{s}}^{*} \cdot c \cdot k_{\mathrm{B}}} \\
p^{*}=\frac{s \cdot \eta}{2 \cdot\left(v_{\mathrm{s}}^{*}\right)^{2}}
\end{gathered}
$$

Es útil observar que los parámetros característicos verifican las relaciones siguientes:

$$
\begin{gathered}
p^{*} \cdot v_{\mathrm{s}}^{*}=c \cdot k_{\mathrm{B}} \cdot T^{*} \\
p^{*} \cdot v_{\mathrm{m}}^{*}=r \cdot c \cdot k_{\mathrm{B}} \cdot T^{*} \\
\frac{E_{0}}{k_{\mathrm{B}} \cdot T}=-\frac{c \cdot r \cdot N}{\bar{v} \cdot \bar{T}}
\end{gathered}
$$


Puede utilizarse el modelo de Flory en la aproximación de presión reducida $\bar{p} \approx 0$, la cual es aceptable a presión ambiente. De este modo, la ecuación de estado se simplifica a la siguiente:

$$
\bar{T}=\frac{\bar{v}^{1 / 3}-1}{\bar{v}^{4 / 3}}
$$

Las siguientes expresiones permiten determinar los parámetros característicos de un líquido puro a partir de su volumen por molécula y sus coeficientes térmicos. Se derivan de la ecuación de estado de Flory (6.238), y de las definiciones termodinámicas de los coeficientes térmicos:

$$
\begin{gathered}
v_{\mathrm{m}}^{*}=v_{\mathrm{m}} \cdot\left[\frac{3 \cdot T \cdot \alpha_{P}+3 \cdot\left(1-2 \cdot P \cdot \kappa_{T}\right)}{4 \cdot T \cdot \alpha_{P}+3 \cdot\left(1-2 \cdot P \cdot \kappa_{T}\right)}\right]^{3} \\
p^{*}=(T \cdot \gamma-P) \cdot \bar{v}^{2} \\
T^{*}=T \cdot\left(\frac{\bar{v}^{4 / 3}}{\bar{v}^{1 / 3}-1}\right) \cdot\left(\frac{1}{1+\bar{p} \cdot \bar{v}^{2}}\right)
\end{gathered}
$$

donde:

$$
\begin{aligned}
& \alpha_{P}=\frac{1}{V} \cdot\left(\frac{\partial V}{\partial T}\right)_{P} \\
& \gamma=\left(\frac{\partial P}{\partial T}\right)_{V}=\frac{\alpha_{P}}{\kappa_{T}}
\end{aligned}
$$

Si bien las ecuaciones anteriores permiten determinar los parámetros característicos de forma exacta, el procedimiento propuesto por Flory hace uso de la aproximación $\bar{p} \approx 0$, de modo que las expresiones anteriores se reducen a las siguientes:

$$
\begin{gathered}
v_{\mathrm{m}}^{*}=v_{\mathrm{m}} \cdot\left[\frac{3 \cdot T \cdot \alpha_{P \approx 0}+3}{4 \cdot T \cdot \alpha_{P \approx 0}+3}\right]^{3} \\
p^{*}=T \cdot \gamma_{P \approx 0} \cdot \bar{v}^{2} \\
T^{*}=T \cdot\left(\frac{\bar{v}_{P \approx 0}^{4 / 3}}{\bar{v}_{P \approx 0}^{1 / 3}-1}\right)
\end{gathered}
$$

En la referencia [343] se comparan los resultados obtenidos de las expresiones con y in la aproximación $\bar{p} \approx 0$ para una serie de 14 líquidos orgánicos. En todos ellos se encontró que el error cometido por esta aproximación para $v_{\mathrm{m}}^{*}$ es menor de $0.01 \%$, para $p^{*}$ es menor de $0.03 \%$ y para $T^{*}$ menor de $0.06 \%$. Ello permite establecer un criterio sobre la precisión con la que deben publicarse los parámetros característicos: las incertidumbres con la que deben darse $v_{\mathrm{m}}^{*}, p^{*}$ y $T^{*}$ no deben ser menores de 0.01 $\mathrm{cm}^{3} \cdot \mathrm{mol}^{-1}, 2 \cdot 10^{5} \mathrm{~Pa}$ y $3 \mathrm{~K}$ respectivamente.

Otro método que permite determinar los parámetros característicos es ajustar directamente los datos PVT a la ecuación de estado (6.238) en un amplio intervalo de presión y temperatura. 
En algunas referencias que utilizan el modelo de Flory, es habitual publicar el volumen característico $v_{\mathrm{m}}^{*}$, la presión característica $p^{*}$ y la temperatura característica $T^{*}$ de cada componente en una mezcla. Analizando la ecuaciones (6.248)-(6.250) y (6.253)-(6.255) se puede comprobar que basta con publicar solamente dos y no tres magnitudes características, ya que conocidas dos es posible determinar la tercera.

Supóngase el siguiente caso. Se determinan las magnitudes características $v_{\mathrm{m}, \mathrm{i}}^{*} p_{\mathrm{i}}^{*}$ y $T^{*}{ }_{\mathrm{i}}$ de los componentes de una mezcla según las ecuaciones (6.253)-(6.255) válidas en la aproximación $\bar{p} \approx 0$, truncando los valores de estos parámetros característicos con la precisión arriba indicada. Puesto que $T^{*}{ }_{\mathrm{i}}$ es función de $v_{\mathrm{m}, \mathrm{i}}^{*}$ y $p_{\mathrm{i}}^{*}$, el valor truncado de $T^{*}{ }_{\mathrm{i}}$ deja de ser consistente con los valores truncados de $v_{\mathrm{m}, \mathrm{i}}^{*}$ y $p_{\mathrm{i}}^{*}$. A la hora de calcular las propiedades termodinámicas de la mezcla, por ejemplo la entalpía de exceso (ecuación (6.283), este minúsculo error se amplifica en aquellos términos donde aparecen los factores $x_{\mathrm{i}} \cdot v_{\mathrm{m}, \mathrm{i}}^{*} \cdot p_{\mathrm{i}}^{*}$. Estos factores toman valores del orden de $50000 \mathrm{~J} \cdot \mathrm{mol}^{-1}$, lo que se traduce en un error en la entalpía de exceso del orden de $1 \mathrm{~J} \cdot \mathrm{mol}^{-1}$. Si bien este error es muy pequeño, no es aceptable en determinados casos en los que las entalpías de exceso sean pequeñas en valor absoluto, como por ejemplo en los extremos del rango de concentración. Por todo lo dicho, se recomienda que para el cálculo de la entalpía de exceso únicamente se utilicen los valores del volumen característico $v_{\mathrm{m}, \mathrm{i}}^{*}$ y la presión característica $p_{i}^{*}$, o en su defecto, que se calcule el valor de la temperatura característica $T^{*}$ i para que sea consistente con los valores truncados de $v_{\mathrm{m}, \mathrm{i}}^{*}$ y $p_{\mathrm{i}}^{*}$

\subsubsection{Hipótesis. Modelo de Flory para mezclas}

Una vez establecido el modelo de Flory para líquidos puros, el siguiente paso es su generalización a mezclas. Aunque existe una versión para mezcla multicomponentes [344], aquí se presentará la teoría para mezclas binarias. Se supone que la mezcla de dos líquidos puros es a su vez otro líquido descrito por el modelo de Flory. Los parámetros propios de la mezcla deben definirse de acuerdo con las reglas de mezcla que relacionan dichos parámetros con los de los líquidos puros. Para ello, es necesario definir las cantidades siguientes:

a) Fracción de contactos. Es el cociente entre el número de contactos del líquido i y el número total de contactos en la mezcla:

$$
\theta_{\mathrm{i}}=\frac{s_{\mathrm{i}} \cdot r_{\mathrm{i}} \cdot N_{\mathrm{i}}}{\sum s_{\mathrm{j}} \cdot r_{\mathrm{j}} \cdot N_{\mathrm{j}}}
$$

b) Fracción de segmentos. Es el cociente entre el número de segmentos del líquido i y el número total de segmentos en la mezcla.

$$
\varphi_{\mathrm{i}}=\frac{r_{\mathrm{i}} \cdot N_{\mathrm{i}}}{\sum r_{\mathrm{j}} \cdot N_{\mathrm{j}}}
$$

Las reglas de mezcla se escriben fácilmente en función de las cantidades anteriores:

c) Número de segmentos. Es el número total de segmentos en la mezcla:

$$
r \cdot N=\sum r_{\mathrm{j}} \cdot N_{\mathrm{j}}
$$


De modo que:

$$
r=\frac{\sum r_{\mathrm{j}} \cdot N_{\mathrm{j}}}{N}=\sum r_{\mathrm{j}} \cdot x_{\mathrm{j}}
$$

donde $x_{\mathrm{j}}$ es la fracción molar del componente $\mathrm{j}$.

a) Número de contactos. Es el número total de contactos en la mezcla:

$$
s \cdot r \cdot N=\sum s_{\mathrm{j}} \cdot r_{\mathrm{j}} \cdot N_{\mathrm{j}}
$$

De modo que:

$$
s=\frac{\sum s_{\mathrm{j}} \cdot r_{\mathrm{j}} \cdot N_{\mathrm{j}}}{r \cdot N}=\sum s_{\mathrm{j}} \cdot \varphi_{\mathrm{j}}
$$

b) Número de grados de libertad. El número de grados de libertad de traslación equivalentes en la mezcla es la suma del número de grados de libertad de traslación equivalentes en cada uno de los líquidos puros.

$$
3 \cdot c \cdot r \cdot N=\sum 3 \cdot c_{\mathrm{i}} \cdot r_{\mathrm{i}} \cdot N_{\mathrm{i}}
$$

De modo que:

$$
c=\frac{\sum c_{\mathrm{i}} \cdot r_{\mathrm{i}} \cdot N_{\mathrm{i}}}{r \cdot N}=\sum c_{\mathrm{i}} \cdot \varphi_{\mathrm{i}}
$$

c) Volumen compacto. Se asume que el volumen compacto de los segmentos de la mezcla, así como de los líquidos puros, es el mismo:

$$
v_{\mathrm{s}}^{*}=v_{\mathrm{s}, \mathrm{i}}^{*}
$$

Esto implica que las diferencias de tamaño entre las diferentes moléculas se deben a diferencias en el número de segmentos, $r$.

Heintz [345] amplió el modelo al caso en el que los volúmenes compactos de los segmentos son distintos.

d) Energía interna. Mezcla al azar. Los puntos de contacto de la mezcla interaccionan por parejas, distinguiéndose tres posibilidades en el caso de mezclas binarias $A+B$ :

- los dos puntos de contacto pertenecen a moléculas del líquido A. Designamos la energía de estas interacciones como $-\eta_{\mathrm{AA}} / v_{\mathrm{s}}$. El número de estas interacciones es $A_{\mathrm{AA}}$.

- los dos puntos de contacto pertenecen a moléculas del líquido B. Designamos la energía de estas interacciones como $-\eta_{\mathrm{BB}} / v_{\mathrm{s}}$. El número de estas interacciones es $A_{\mathrm{BB}}$.

- los dos puntos de contacto pertenecen a moléculas diferentes. Designamos la energía de estas interacciones como $-\eta_{\mathrm{AB}} / v_{\mathrm{s}}$. El número de estas interacciones es $A_{\mathrm{AB}}$.

Se admitirá que la energía de las interacciones entre moléculas iguales de la mezcla es la misma que tendría en el líquido puro, luego $\eta_{\mathrm{i}}=\eta_{\mathrm{ii}}$.

De acuerdo con esta notación la energía interna de la mezcla se escribe como:

$$
E_{0}=-\left(A_{\mathrm{AA}} \frac{\eta_{\mathrm{A}}}{v_{s}}+A_{\mathrm{BB}} \frac{\eta_{\mathrm{B}}}{v_{s}}+A_{\mathrm{AB}} \frac{\eta_{\mathrm{AB}}}{v_{s}}\right)
$$


Los valores de $A_{\mathrm{AA}}, A_{\mathrm{BB}}$ y $A_{\mathrm{AB}}$ deben ser compatibles con el número de contactos del primer y del segundo líquido en la mezcla. Esto quiere decir que:

$$
\begin{gathered}
s_{\mathrm{A}} \cdot r_{\mathrm{A}} \cdot N_{\mathrm{A}}=2 \cdot A_{\mathrm{AA}}+A_{\mathrm{AB}} \\
s_{\mathrm{B}} \cdot r_{\mathrm{B}} \cdot N_{\mathrm{B}}=2 \cdot A_{\mathrm{BB}}+A_{\mathrm{AB}}
\end{gathered}
$$

El sistema de ecuaciones permite escribir la energía interna como:

$$
E_{0}=-\frac{N \cdot r \cdot s}{2 \cdot v_{s}} \cdot\left(\theta_{\mathrm{A}} \cdot \eta_{\mathrm{A}}+\theta_{\mathrm{B}} \cdot \eta_{\mathrm{B}}-\frac{A_{\mathrm{AB}}}{N \cdot r \cdot s} \cdot \Delta \eta\right)
$$

donde:

$$
\Delta \eta=\eta_{\mathrm{A}}+\eta_{\mathrm{B}}-2 \cdot \eta_{\mathrm{AB}}
$$

Por lo tanto, la energía interna de la mezcla es función de dos parámetros característicos de la mezcla $A_{\mathrm{AB}}$ y $\Delta \eta$. Para estimar el valor de $A_{\mathrm{AB}}$ se utiliza la hipótesis de mezcla al azar, según la cual, dado un contacto, el resto de los contactos de la mezcla tienen la misma probabilidad de formar con este una interacción. Se puede encontrar [346] que la hipótesis de mezcla al azar conduce al valor siguiente para $A_{\mathrm{AB}}$ :

$$
A_{\mathrm{AB}}=s \cdot r \cdot N \cdot \theta_{\mathrm{A}} \cdot \theta_{\mathrm{B}}
$$

Como conclusión de la adopción de la hipótesis mezcla al azar, es de esperar que el modelo proporcione resultados aceptables en sistemas caracterizados por interacciones dispersivas o débilmente polares y en disoluciones cuyos componentes sean de la misma naturaleza química. Así, el modelo describe razonablemente las propiedades termodinámicas de mezclas de un compuesto no polar (benceno) [347] o débilmente polar ( $N, N, N$-trialquilamina [348], monoéter ramificado o lineal [349], etc.) y un alcano, de mezclas de componentes de naturaleza similiar (1-alcohol + 1-alcohol [350], 2metoxietanol + hidroxiéter [351]).

Como consecuencia de (6.270), la energía interna en la hipótesis de mezcla al azar es:

$$
E_{0}=-\frac{N \cdot r \cdot s}{2 \cdot v_{s}} \cdot\left(\theta_{\mathrm{A}} \cdot \eta_{\mathrm{A}}+\theta_{\mathrm{B}} \cdot \eta_{\mathrm{B}}-\theta_{\mathrm{A}} \cdot \theta_{\mathrm{B}} \cdot \Delta \eta\right)
$$

El parámetro $\Delta \eta$ puede escribirse en función del parámetro $\chi_{A B}$ llamado parámetro energético de la mezcla, que se define de manera análoga a $p^{*}$ en la ecuación (6.243):

$$
\chi_{\mathrm{AB}}=\frac{s_{\mathrm{A}} \cdot \Delta \eta}{2 \cdot\left(v_{s}^{*}\right)^{2}} \neq \chi_{\mathrm{BA}}=\frac{s_{\mathrm{B}} \cdot \Delta \eta}{2 \cdot\left(v_{s}^{*}\right)^{2}}
$$

El papel de $\chi_{A B}$ es análogo al de $w$ del modelo de red rígida, aunque posee unidades de presión. Además, no es una energía libre, por lo que no puede depender de la temperatura. Sin embargo, Heintz y Lichtentahler [352] intentaron introducir en $\chi_{\mathrm{AB}}$ una dependencia con la temperatura, además de cierto orden a corto alcance mediante un mecanismo equivalente al utilizado para explicar el paramagnetismo por medio del acoplamiento entre los momentos magnéticos de spin y el campo magnético externo aplicado, lo que no es sino un intento de extender la teoría al caso análogo de la aproximación cuasiquímica de la red rígida, es decir, una aproximación de orden uno.

A partir de las reglas de mezcla anteriores es posible derivar las siguientes expresiones para las magnitudes características de la mezcla: 


$$
\begin{gathered}
v_{\mathrm{m}}^{*}=r \cdot v_{\mathrm{s}}^{*}=v_{\mathrm{m}, \mathrm{A}}^{*} \cdot x_{\mathrm{A}}+v_{\mathrm{m}, \mathrm{B}}^{*} \cdot x_{\mathrm{B}} \\
T^{*}=\frac{s \cdot \eta}{2 \cdot v_{\mathrm{S}}^{*} \cdot c \cdot k_{\mathrm{B}}}=\frac{p^{*}}{\frac{\varphi_{\mathrm{A}} \cdot p_{\mathrm{A}}^{*}}{T_{\mathrm{A}}^{*}}+\frac{\varphi_{\mathrm{B}} \cdot p_{\mathrm{B}}^{*}}{T_{\mathrm{B}}^{*}}} \\
p^{*}=\frac{s \cdot \eta}{2 \cdot\left(v_{\mathrm{s}}^{*}\right)^{2}}=\varphi_{\mathrm{A}} \cdot p_{\mathrm{A}}^{*}+\varphi_{\mathrm{B}} \cdot p_{\mathrm{B}}^{*}-\varphi_{\mathrm{A}} \cdot \theta_{\mathrm{B}} \cdot \chi_{\mathrm{AB}}
\end{gathered}
$$

La fracción de segmentos $\varphi_{i}$ es una cantidad necesaria para realizar muchos cálculos, por ejemplo, los cálculos de la entalpía y el volumen de exceso. Entre las reglas de mezcla adoptadas, la (6.264), que establece la igualdad de los volúmenes compactos de los segmentos de la mezcla y de los líquidos puros, permite manipular la definición de fracción de segmentos, ecuación (6.257) hasta la forma siguiente:

$$
\varphi_{\mathrm{i}}=\frac{v_{\mathrm{m}, \mathrm{i}}^{*} \cdot x_{\mathrm{i}}}{v_{\mathrm{m}, \mathrm{A}}^{*} \cdot x_{\mathrm{A}}+v_{\mathrm{m}, \mathrm{B}}^{*} \cdot x_{\mathrm{B}}}
$$

La fracción de puntos de contacto $\theta_{\mathrm{i}}$ también es una cantidad importante. Para su determinación, es útil definir el llamado parámetro geométrico de la mezcla $S_{\mathrm{AB}}$ :

$$
S_{\mathrm{AB}}=\frac{S_{\mathrm{A}}}{S_{\mathrm{B}}}
$$

La fracción de puntos de contacto escrita en función del parámetro geométrico $S_{\mathrm{AB}}$ :

$$
\theta_{\mathrm{B}}=\frac{\varphi_{\mathrm{B}}}{\varphi_{\mathrm{B}}+S_{\mathrm{AB}} \cdot \varphi_{\mathrm{A}}}
$$

Para estimar $S_{\mathrm{AB}}$ se supone que las moléculas en los líquidos son esferas de volumen compacto $v_{\mathrm{m}, \mathrm{i}}^{*}$ y que el número de contactos de estas moléculas esféricas $r_{\mathrm{i}} \cdot s_{\mathrm{i}}$ es proporcional a su superficie, es decir:

$$
\frac{r_{\mathrm{A}} \cdot s_{\mathrm{A}}}{r_{\mathrm{B}} \cdot s_{\mathrm{B}}}=\left(\frac{v_{\mathrm{m}, \mathrm{A}}^{*}}{v_{\mathrm{m}, \mathrm{B}}^{*}}\right)^{2 / 3}
$$

Por otro lado, de la hipótesis de la igualdad de los volúmenes compactos de los segmentos en la mezcla y en los líquidos puros, ecuación (6.264), se deriva la ecuación siguiente:

$$
\frac{r_{\mathrm{A}}}{r_{\mathrm{B}}}=\frac{v_{\mathrm{m}, \mathrm{A}}^{*}}{v_{\mathrm{m}, \mathrm{B}}^{*}}
$$

De (6.279) y (6.280) se tiene una relación para $S_{\mathrm{AB}}$ :

$$
S_{\mathrm{AB}}=\frac{s_{\mathrm{A}}}{S_{\mathrm{B}}}=\left(\frac{v_{\mathrm{m}, \mathrm{A}}^{*}}{v_{\mathrm{m}, \mathrm{B}}^{*}}\right)^{-1 / 3}
$$

que puede determinarse a partir de datos estructurales tabulados [353].

La entalpía molar de exceso es toda debida a $Z(R E S)$ y es igual a:

$$
H_{\mathrm{m}}^{\mathrm{E}}(\mathrm{RES})=H_{\mathrm{m}}^{\mathrm{E}}=U_{\mathrm{m}}^{\mathrm{E}}+P \cdot V_{\mathrm{m}}^{\mathrm{E}}=
$$




$$
=N_{A} \cdot \frac{E_{0}(\text { mezcla })-E_{0}(\text { A puro })-E_{0}(\text { B puro })}{N}+P \cdot V_{\mathrm{m}}^{\mathrm{E}}
$$

En la aproximación $\bar{p} \approx 0$, la entalpía y la energía interna coinciden. En ese caso, sustituyendo en la ecuación anterior las expresiones que han sido obtenidas para el modelo se llega a la ecuación siguiente:

$$
H_{\mathrm{m}}^{\mathrm{E}}=U_{\mathrm{m}}^{\mathrm{E}}=N_{A} \cdot\left[v_{\mathrm{m}, \mathrm{A}}^{*} \cdot x_{\mathrm{A}} \cdot p_{\mathrm{A}}^{*} \cdot\left(\frac{1}{\bar{v}_{\mathrm{A}}}-\frac{1}{\bar{v}}\right)+v_{\mathrm{m}, \mathrm{B}}^{*} \cdot x_{\mathrm{B}} \cdot p_{\mathrm{B}}^{*} \cdot\left(\frac{1}{\bar{v}_{\mathrm{B}}}-\frac{1}{\bar{v}}\right)+\frac{v_{\mathrm{m}, \mathrm{A}}^{*} \cdot x_{\mathrm{A}} \cdot \theta_{\mathrm{B}} \cdot \chi_{\mathrm{AB}}}{\bar{v}}\right]
$$

El término que depende de $\chi_{A B}$ se llama término interaccional o de contacto [354], y representa la contribución a la entalpía de exceso atribuible a la diferencia entre los pares A-B y la media entre los pares A-A y B-B. El resto son términos de la contribución de la ecuación de estado. Puede ser muy grande, a veces dominando al término de contacto.

La expresión anterior se puede manipular para llegar a la siguiente:

$$
\begin{gathered}
\frac{H_{\mathrm{m}}^{\mathrm{E}}}{N_{A}}=\frac{U_{\mathrm{m}}^{\mathrm{E}}}{N_{A}}=\frac{v_{\mathrm{m}, \mathrm{A}}^{*} \cdot x_{\mathrm{A}} \cdot p_{\mathrm{A}}^{*}}{\bar{v}_{\mathrm{A}}}+\frac{v_{\mathrm{m}, \mathrm{B}}^{*} \cdot x_{\mathrm{B}} \cdot p_{\mathrm{B}}^{*}}{\bar{v}_{\mathrm{B}}}- \\
-\frac{1}{\bar{v}} \cdot\left(v_{\mathrm{m}, \mathrm{A}}^{*} \cdot x_{\mathrm{A}} \cdot p_{\mathrm{A}}^{*}+v_{\mathrm{m}, \mathrm{B}}^{*} \cdot x_{\mathrm{B}} \cdot p_{\mathrm{B}}^{*}-v_{\mathrm{m}, \mathrm{A}}^{*} \cdot x_{\mathrm{A}} \cdot \theta_{\mathrm{B}} \cdot \chi_{\mathrm{AB}}\right)
\end{gathered}
$$

Igualmente, se puede manipular la expresión de la temperatura característica $T^{*}$ de la mezcla, ecuación (6.274), para incluir la expresión de la fracción de segmentos en función de los volúmenes característicos, ecuación (6.276).

$$
T^{*}=\frac{v_{\mathrm{m}, \mathrm{A}}^{*} \cdot x_{\mathrm{A}} \cdot p_{\mathrm{A}}^{*}+v_{\mathrm{m}, \mathrm{B}}^{*} \cdot x_{\mathrm{B}} \cdot p_{\mathrm{B}}^{*}-v_{\mathrm{m}, \mathrm{A}}^{*} \cdot x_{\mathrm{A}} \cdot \theta_{\mathrm{B}} \cdot \chi_{\mathrm{AB}}}{\frac{v_{\mathrm{m}, \mathrm{A}}^{*} \cdot x_{\mathrm{A}} \cdot p_{\mathrm{A}}^{*}}{T_{\mathrm{A}}^{*}}+\frac{v_{\mathrm{m}, \mathrm{B}}^{*} \cdot x_{\mathrm{B}} \cdot p_{\mathrm{B}}^{*}}{T_{\mathrm{B}}^{*}}}
$$

Combinando ambas expresiones, se deriva una nueva relación para la entalpía de exceso:

$$
\frac{H_{\mathrm{m}}^{\mathrm{E}}}{N_{A}}=\frac{U_{\mathrm{m}}^{\mathrm{E}}}{N_{A}}=\frac{v_{\mathrm{m}, \mathrm{A}}^{*} \cdot x_{\mathrm{A}} \cdot p_{\mathrm{A}}^{*}}{\bar{v}_{\mathrm{A}}}+\frac{v_{\mathrm{m}, \mathrm{B}}^{*} \cdot x_{\mathrm{B}} \cdot p_{\mathrm{B}}^{*}}{\bar{v}_{\mathrm{B}}}-\frac{1}{\bar{v} \cdot \bar{T}} \cdot\left(v_{\mathrm{m}, \mathrm{A}}^{*} \cdot x_{\mathrm{A}} \cdot p_{\mathrm{A}}^{*} \cdot \bar{T}_{\mathrm{A}}+v_{\mathrm{m}, \mathrm{B}}^{*} \cdot x_{\mathrm{B}} \cdot p_{\mathrm{B}}^{*} \cdot \bar{T}_{\mathrm{B}}\right)
$$

En lo que respecta al volumen de exceso, éste puede determinarse a partir de la ecuación de estado de Flory aplicada a la mezcla y a los componentes puros, pues dicha ecuación permite calcular los volúmenes:

$$
v_{\mathrm{m}}^{\mathrm{E}}=v_{\mathrm{m}}-\left(v_{\mathrm{m}, \mathrm{A}} \cdot x_{\mathrm{A}}+v_{\mathrm{m}, \mathrm{B}} \cdot x_{\mathrm{B}}\right)=\left(v_{\mathrm{m}, \mathrm{A}}^{*} \cdot x_{\mathrm{A}}+v_{\mathrm{m}, \mathrm{B}}^{*} \cdot x_{\mathrm{B}}\right) \cdot\left(\bar{v}-\varphi_{\mathrm{A}} \cdot \bar{v}_{\mathrm{A}}-\varphi_{\mathrm{B}} \cdot \bar{v}_{\mathrm{B}}\right)
$$

Donde el volumen molar de exceso es el producto del volumen de exceso por molécula y el número de Avogadro:

$$
V_{\mathrm{m}}^{\mathrm{E}}=v_{\mathrm{m}}^{\mathrm{E}} \cdot N_{A}
$$

A veces es útil el volumen de exceso reducido:

$$
\bar{v}^{\mathrm{E}}=\bar{v}-\bar{v}^{0}=\frac{v_{\mathrm{m}}}{v_{\mathrm{m}, \mathrm{A}}^{*} \cdot x_{\mathrm{A}}+v_{\mathrm{m}, \mathrm{B}}^{*} \cdot x_{\mathrm{B}}}-\left(\bar{v}_{\mathrm{A}} \cdot \varphi_{\mathrm{A}}+\bar{v}_{\mathrm{B}} \cdot \varphi_{\mathrm{B}}\right)=
$$




$$
=\frac{v_{\mathrm{m}}-\left(v_{\mathrm{m}, \mathrm{A}} \cdot x_{\mathrm{A}}+v_{\mathrm{m}, \mathrm{B}} \cdot x_{\mathrm{B}}\right)}{v_{\mathrm{m}, \mathrm{A}}^{*} \cdot x_{\mathrm{A}}+v_{\mathrm{m}, \mathrm{B}}^{*} \cdot x_{\mathrm{B}}}=\frac{v_{\mathrm{m}}^{\mathrm{E}}}{v_{\mathrm{m}, \mathrm{A}}^{*} \cdot x_{\mathrm{A}}+v_{\mathrm{m}, \mathrm{B}}^{*} \cdot x_{\mathrm{B}}}=\frac{v_{\mathrm{m}}^{\mathrm{E}}}{v_{\mathrm{m}}^{*}}
$$

donde se ha utilizado el volumen reducido ideal:

$$
\bar{v}^{0}=\bar{v}_{\mathrm{A}} \cdot \varphi_{\mathrm{A}}+\bar{v}_{\mathrm{B}} \cdot \varphi_{\mathrm{B}}
$$

Si el volumen de la mezcla fuese aditivo, el volumen reducido de la mezcla coincidiría con $\bar{v}^{0}$. La temperatura reducida ideal asociada a $\bar{v}^{0}$ en la aproximación $\bar{p} \approx 0$ es:

$$
\bar{T}^{0}=\frac{\left(\bar{v}^{0}\right)^{1 / 3}-1}{\left(\bar{v}^{0}\right)^{4 / 3}}
$$

El volumen de exceso puede escribirse como la suma de las contribuciones por la ecuación de estado y por el parámetro energético $\chi_{A B}$ igual que la entalpía de exceso, ecuación (6.283). No obstante, el primer término puede desdoblarse en otros dos, de modo que, el volumen de exceso es la suma de tres contribuciones [355], [356]:

$$
v_{\mathrm{m}}^{\mathrm{E}}=v_{\mathrm{m}}^{\mathrm{E}}\left(\chi_{\mathrm{AB}}\right)+v_{\mathrm{m}}^{\mathrm{E}}(\bar{v})+v_{\mathrm{m}}^{\mathrm{E}}\left(p^{*}\right)
$$

donde,

$$
\begin{gathered}
\frac{v_{\mathrm{m}}^{\mathrm{E}}\left(\chi_{\mathrm{AB}}\right)}{x_{\mathrm{A}} \cdot v_{\mathrm{m}, \mathrm{A}}^{*}+x_{\mathrm{B}} \cdot v_{\mathrm{m}, \mathrm{B}}^{*}}=\frac{\left(\bar{v}^{1 / 3}-1\right) \cdot \bar{v}^{2 / 3} \cdot \Psi_{\mathrm{A}} \cdot \theta_{\mathrm{B}} \cdot\left(\chi_{\mathrm{AB}} / p_{\mathrm{A}}^{*}\right)}{\frac{4}{3} \cdot \bar{v}^{-1 / 3}-1} \\
\frac{v_{\mathrm{m}}^{\mathrm{E}}(\bar{v})}{x_{\mathrm{A}} \cdot v_{\mathrm{m}, \mathrm{A}}^{*}+x_{\mathrm{B}} \cdot v_{\mathrm{m}, \mathrm{B}}^{*}}=-\frac{\left(\bar{v}_{\mathrm{A}}-\bar{v}_{\mathrm{B}}\right)^{2} \cdot\left(\frac{14}{9} \cdot \bar{v}^{-1 / 3}-1\right) \cdot \Psi_{\mathrm{A}} \cdot \Psi_{\mathrm{B}}}{\left(\frac{4}{3} \bar{v}^{-1 / 3}-1\right) \cdot \bar{v}} \\
\frac{v_{\mathrm{m}}^{\mathrm{E}}\left(p^{*}\right)}{x_{\mathrm{A}} \cdot v_{\mathrm{m}, \mathrm{A}}^{*}+x_{\mathrm{B}} \cdot v_{\mathrm{m}, \mathrm{B}}^{*}}=\frac{\left(\bar{v}_{\mathrm{A}}-\bar{v}_{\mathrm{B}}\right) \cdot\left(p_{\mathrm{A}}^{*}-p_{\mathrm{B}}^{*}\right) \cdot \Psi_{\mathrm{A}} \cdot \Psi_{\mathrm{B}}}{p_{\mathrm{B}}^{*} \cdot \Psi_{\mathrm{A}}+p_{\mathrm{A}}^{*} \cdot \Psi_{\mathrm{B}}}
\end{gathered}
$$

En estas ecuaciones se ha utilizado la fracción de energía de contacto $\Psi_{\mathrm{i}}$ :

$$
\Psi_{\mathrm{i}}=\frac{\varphi_{\mathrm{i}} \cdot p_{\mathrm{i}}^{*}}{\varphi_{\mathrm{A}} \cdot p_{\mathrm{A}}^{*}+\varphi_{\mathrm{B}} \cdot p_{\mathrm{B}}^{*}}
$$

El primer término de la ecuación (6.292) se llama término interaccional; el segundo se llama término de curvatura de $\bar{v}$, que siempre es negativo; el tercero se llama término en $p^{*}$, que puede ser positivo o negativo.

Si los volúmenes de exceso son pequeños, como suele ocurrir en la mayoría de los sistemas estudiados, el volumen reducido de exceso puede determinarse mediante la ecuación aproximada siguiente:

$$
\bar{v}^{\mathrm{E}}=\left(\frac{\partial \bar{v}}{\partial T}\right) \cdot\left(\bar{T}-\bar{T}_{0}\right)=\frac{\left(\bar{v}^{0}\right)^{7 / 3}}{\frac{4}{3}-\left(\bar{v}^{0}\right)^{1 / 3}} \cdot\left(\bar{T}-\bar{T}_{0}\right)
$$


$\chi_{\mathrm{AB}}$ está incluido en $\bar{T}$ (véase la ecuación (6.285). Así, en la ecuación del volumen reducido de la mezcla:

$$
\bar{v}=\bar{v}^{\mathrm{E}}+\bar{v}^{0}
$$

aparece explícitamente $\chi_{A B}$, lo que hace que esta aproximación sea muy útil para realizar los ajustes de la teoría de Flory a los datos de volumen de exceso, evitando el tratamiento implícito del parámetro $\chi_{\mathrm{AB}} \mathrm{O}$ de complicadas ecuaciones para $\bar{v}$.

En la aproximación $\bar{p} \approx 0$, la función de Gibbs y la función de Helmholtz coinciden. Su exceso es:

$$
\begin{gathered}
G_{\mathrm{m}}^{\mathrm{E}}=F_{\mathrm{m}}^{\mathrm{E}}=H_{\mathrm{m}}^{\mathrm{E}}-T \cdot S_{\mathrm{m}}^{\mathrm{E}}=H_{\mathrm{m}}^{\mathrm{E}}(\mathrm{RES})-T \cdot\left[S_{\mathrm{m}}^{\mathrm{E}}(\mathrm{COMB})+S_{\mathrm{m}}(\mathrm{RES})\right]= \\
=G_{\mathrm{m}}^{\mathrm{E}}(\mathrm{COMB})+G_{\mathrm{m}}(\mathrm{RES})
\end{gathered}
$$

donde, como se ha dicho unos párrafos más arriba, puede tomarse $S_{\mathrm{m}}^{\mathrm{E}}(\mathrm{COMB})$ como la dada por la ecuación de Flory-Huggins, y $S_{\mathrm{m}}(\mathrm{RES})$ es:

$$
\begin{gathered}
S_{\mathrm{m}}(\mathrm{RES})=-\left(\frac{\partial F(\mathrm{RES})}{\partial V}\right)_{T, N}=k_{\mathrm{B}} \cdot T \cdot\left(\frac{\partial \ln Z(\mathrm{RES})}{\partial V}\right)_{T, N}= \\
=-3 \cdot N_{A} \cdot \sum x_{\mathrm{i}} \cdot\left\{v_{\mathrm{m}, \mathrm{i}}^{*} \cdot p_{\mathrm{i}}^{*} \cdot \frac{1}{T_{i}^{*}} \cdot \ln \left(\frac{\bar{v}_{\mathrm{i}}^{1 / 3}-1}{\bar{v}^{1 / 3}-1}\right)\right\}
\end{gathered}
$$

Si las moléculas de ambos componentes tienen tamaño y forma similar, la entropía de exceso combinatorial viene descrita apropiadamente por la de la mezcla ideal, es decir, cero. En ese caso, $S_{\mathrm{m}}^{\mathrm{E}}=S_{\mathrm{m}}(\mathrm{RES})$ e igual a:

$$
S_{\mathrm{m}}^{\mathrm{E}}=S_{\mathrm{m}}(\mathrm{RES})=-3 \cdot N_{A} \cdot \sum\left[\frac{x_{\mathrm{i}} \cdot v_{\mathrm{m}, \mathrm{i}}^{*} \cdot p_{\mathrm{i}}^{*} \cdot \bar{T}_{\mathrm{i}}}{T} \cdot \ln \left(\frac{\bar{v}_{\mathrm{i}}^{1 / 3}-1}{\bar{v}^{1 / 3}-1}\right)\right]
$$

Esta ecuación marca una clara diferencia con la teoría de disoluciones regulares, en la que la entropía de exceso a volumen constante es igual a cero.

En lo que respecta a la función de Gibbs de exceso, en las anteriores condiciones se tiene que $G_{\mathrm{m}}^{\mathrm{E}}=G_{\mathrm{m}}($ RES) e igual a:

$$
\frac{G_{\mathrm{m}}^{\mathrm{E}}}{N_{A}}=\frac{F_{\mathrm{m}}^{\mathrm{E}}}{N_{A}}=\sum x_{\mathrm{i}} \cdot\left\{v_{\mathrm{m}, \mathrm{i}}^{*} \cdot p_{\mathrm{i}}^{*} \cdot\left[\left(\frac{1}{\overline{v_{\mathrm{i}}}}-\frac{1}{\bar{v}}\right)+3 \cdot \bar{T}_{\mathrm{i}} \cdot \ln \left(\frac{\bar{v}_{\mathrm{i}}^{1 / 3}-1}{\bar{v}^{1 / 3}-1}\right)\right]\right\}+\frac{v_{\mathrm{m}, \mathrm{A}}^{*} \cdot x_{\mathrm{A}} \cdot \theta_{\mathrm{B}} \cdot \chi_{\mathrm{AB}}}{\bar{v}}
$$

Recuérdese que estas ecuaciones son resultados de aceptar que la mezcla sea al azar. Por lo tanto, en aquellas mezclas en las que los efectos de interacción preferencial y/u orientacional sean importantes, o las interacciones son importantes, los resultados no serán correctos. Para sobrepasar esta limitación, Flory introdujo un parámetro energético adicional $Q_{12}$ de manera que la expresión resultante para $G_{\mathrm{m}}^{\mathrm{E}}$ es:

$$
\frac{G_{\mathrm{m}}^{\mathrm{E}}}{N_{A}}=\frac{F_{\mathrm{m}}^{\mathrm{E}}}{N_{A}}=\sum x_{\mathrm{i}} \cdot\left\{v_{\mathrm{m}, \mathrm{i}}^{*} \cdot p_{\mathrm{i}}^{*} \cdot\left[\left(\frac{1}{\bar{v}_{\mathrm{i}}}-\frac{1}{\bar{v}}\right)+3 \cdot \bar{T}_{\mathrm{i}} \cdot \ln \left(\frac{\bar{v}_{\mathrm{i}}^{1 / 3}-1}{\bar{v}^{1 / 3}-1}\right)\right]\right\}+
$$




$$
+\frac{v_{\mathrm{m}, \mathrm{A}}^{*} \cdot x_{\mathrm{A}} \cdot \theta_{\mathrm{B}} \cdot \chi_{\mathrm{AB}}}{\bar{v}}-v_{\mathrm{m}, \mathrm{A}}^{*} \cdot x_{\mathrm{A}} \cdot \theta_{\mathrm{B}} \cdot T \cdot Q_{\mathrm{AB}}
$$

La introducción de este parámetro es puramente empírica y no mejora las prestaciones.

El coeficiente de dilatación térmica se calcula simplemente a partir de su definición y de la ecuación de estado de Flory:

$$
\alpha_{P}=\frac{1}{T^{*}} \cdot \frac{\bar{v}^{4 / 3}}{\bar{p} \cdot \bar{v} \cdot\left(\bar{v}^{1 / 3}-\frac{2}{3}\right)+\frac{4}{3}-\bar{v}^{1 / 3}}
$$

en la aproximación $\bar{p} \approx 0$,

$$
\alpha_{P \approx 0}=\frac{1}{T^{*}} \cdot \frac{\bar{v}^{4 / 3}}{\frac{4}{3}-\bar{v}^{1 / 3}}
$$

Las compresibilidades también se pueden calcular. De su definición y la ecuación de estado se tiene:

$$
\kappa_{T}=\frac{1}{p^{*} \cdot \bar{T}} \cdot\left[\frac{\bar{v}^{-2 / 3}}{3 \cdot\left(\bar{v}^{1 / 3}-1\right)} \cdot \frac{1}{\bar{T}} \cdot\left(\bar{p}-\frac{1}{\bar{v}^{2}}\right)\right]^{-1}
$$

en la aproximación $\bar{p} \approx 0$,

$$
\kappa_{T, P \approx 0}=\frac{3 \cdot \bar{v}^{2} \cdot\left(\bar{v}^{1 / 3}-1\right)}{p^{*} \cdot\left(4-3 \bar{v}^{1 / 3}\right)}
$$

Haciendo uso de las expresiones habituales se puede relacionar esta cantidad con la compresibilidad isoentrópica y con la velocidad del sonido.

Los potenciales químicos y otras propiedades molares parciales pueden determinarse. Las ecuaciones pueden consultarse, por ejemplo, en la referencia [343].

\subsubsection{Estudio de la hipótesis de mezcla al azar. Determinación de $\chi_{A B}$}

A continuación se introduce una interpretación del parámetro energético $\chi_{A B}$ que permite evaluar si una mezcla verifica la hipótesis de mezcla al azar. El parámetro $\chi_{A B}$ en el modelo de Flory es una magnitud característica de la mezcla que es independiente de la composición de mezcla si se verifica la hipótesis de mezcla al azar. En caso contrario, este parámetro no es constante.

De la ecuación (6.268), que expresa la energía interna de una mezcla, y de la definición del parámetro energético $\chi_{A B}$, ecuación (6.272), se obtiene la siguiente relación:

$$
E_{0}=-\frac{N \cdot r \cdot s}{2 \cdot v_{s}} \cdot\left(\theta_{\mathrm{A}} \cdot \eta_{\mathrm{A}}+\theta_{\mathrm{B}} \cdot \eta_{\mathrm{B}}\right)-A_{\mathrm{AB}} \cdot \frac{\chi_{\mathrm{AB}} \cdot\left(v_{s}^{*}\right)^{2} / s_{\mathrm{A}}}{v_{s}}
$$

La ecuación anterior se deriva de aplicar todas las hipótesis del modelo de Flory salvo la hipótesis de mezcla al azar. Cuando se aplica dicha hipótesis, ecuación (6.270), la ecuación anterior se reduce a: 


$$
E_{0}=-\frac{N \cdot r \cdot s}{2 \cdot v_{s}} \cdot\left(\theta_{\mathrm{A}} \cdot \eta_{\mathrm{A}}+\theta_{\mathrm{B}} \cdot \eta_{\mathrm{B}}\right)-N \cdot r \cdot s \cdot \theta_{\mathrm{A}} \cdot \theta_{\mathrm{B}} \cdot \frac{\chi_{\mathrm{AB}}(\mathrm{R}) \cdot\left(v_{s}^{*}\right)^{2} / s_{\mathrm{A}}}{v_{s}}
$$

Se ha seguido la notación de la sección6.4.1.1 en cuanto a que el paréntesis (R) implica que la mezcla es al azar (Random). Igualando ambas expresiones se obtiene la relación:

$$
A_{\mathrm{AB}} \cdot \chi_{\mathrm{AB}}=N \cdot r \cdot s \cdot \theta_{\mathrm{A}} \cdot \theta_{\mathrm{B}} \cdot \chi_{\mathrm{AB}}(\mathrm{R})
$$

Considérense las mezclas de dos líquidos y las medidas de su entalpía de exceso. El parámetro energético de la mezcla puede determinarse por correlación de todas estas medidas, valor que se denota como $\chi_{A B}=\chi_{A B}(R)$, o bien, calculado para cada medida, valor que se designa como $\chi_{A B}(x)$. Si todas las hipótesis del modelo de Flory, incluida la de mezcla al azar, fuesen válidas, los valores del parámetro energético $\chi_{\mathrm{AB}}$ y $\chi_{\mathrm{AB}}(x)$ serían iguales, es decir, dicho parámetro debe de ser independiente de la concentración. [357]-[360]. Sin embargo, si la hipótesis de mezcla al azar no se verifica, $\chi_{A B}$ y $\chi_{A B}(x)$ no coinciden, pues cuando la mezcla no es al azar el parámetro $\chi_{A B}(x)$ depende de la composición. En esta situación existen dos casos posibles:

a) $\quad \chi_{\mathrm{AB}}(x)>\chi_{\mathrm{AB}}$, lo que implica $A_{\mathrm{AB}}<s \cdot r \cdot N \cdot \theta_{\mathrm{A}} \cdot \theta_{\mathrm{B}}$. Es decir el número de interacciones entre contactos de moléculas diferentes es menor que el predicho bajo la hipótesis de mezcla al azar.

b) $\quad \chi_{\mathrm{AB}}(x)<\chi_{\mathrm{AB}}$, lo que implica $A_{\mathrm{AB}}>s \cdot r \cdot N \cdot \theta_{\mathrm{A}} \cdot \theta_{\mathrm{B}}$. Es decir el número de interacciones entre contactos de moléculas diferentes es mayor que el predicho bajo la hipótesis de mezcla al azar.

En el razonamiento anterior se ha admitido implícitamente que el valor correlacionado por el modelo de Flory del parámetro energético $\chi_{\mathrm{AB}}$ es el valor real para la mezcla, mientras que las variaciones en los valores calculados para cada composición $\chi_{A B}(x)$ son resultado de que la hipótesis de mezcla al azar no se verifica.

En conclusión, el análisis de la dependencia del parámetro de interacción $\chi_{A B}$ con la concentración el parámetro energético es importante, pues permite para estudiar los procesos de creación/destrucción de orden. Por ejemplo, se ha utilizado para dicho fin en mezclas del tipo $B+C_{n}$, donde $B$ es un compuesto no polar, o débilmente polar con forma esférica o laminar [361]-[363]. A continuación se expone el procedimiento que permite determinar de forma analítica el parámetro energético a partir de una única medida de la entalpía de exceso, basado en el expuesto por Howell [364] para la determinación de la entalpía de exceso a composición equimolar.

El procedimiento para evaluar $\chi_{A B}$ consta de los siguientes pasos:

a) Determinación de $\bar{v} \cdot \bar{T}$. Despejando de la ecuación (6.286) se obtiene la siguiente relación:

$$
\bar{v} \cdot \bar{T}=\frac{v_{\mathrm{m}, \mathrm{A}}^{*} \cdot x_{\mathrm{A}} \cdot p_{\mathrm{A}}^{*} \cdot \bar{T}_{\mathrm{A}}+v_{\mathrm{m}, \mathrm{B}}^{*} \cdot x_{\mathrm{B}} \cdot p_{\mathrm{B}}^{*} \cdot \bar{T}_{\mathrm{B}}}{\frac{v_{\mathrm{m}, \mathrm{A}}^{*} \cdot x_{\mathrm{A}} \cdot p_{\mathrm{A}}^{*}}{\bar{v}_{\mathrm{A}}}+\frac{v_{\mathrm{m}, \mathrm{B}}^{*} \cdot x_{\mathrm{B}} \cdot p_{\mathrm{B}}^{*}}{\bar{v}_{\mathrm{B}}}-\frac{H_{\mathrm{m}}^{\mathrm{E}}}{N_{A}}}
$$

b) Determinación de $\bar{v}$. Despejando de la ecuación de estado de Flory en la aproximación $\bar{p} \approx 0$, ecuación (6.247) se obtiene: 


$$
\bar{v}=\left(\frac{1}{1-\bar{v} \cdot \bar{T}}\right)^{3}
$$

c) Determinación de $\bar{T}$. Dividiendo (6.311) por (6.312) $\bar{T}$ :

$$
\bar{T}=\frac{\bar{v} \cdot \bar{T}}{\bar{v}}=\frac{\left(\frac{v_{\mathrm{m}, \mathrm{A}}^{*} \cdot x_{\mathrm{A}} \cdot p_{\mathrm{A}}^{*} \cdot \bar{T}_{\mathrm{A}}+v_{\mathrm{m}, \mathrm{B}}^{*} \cdot x_{\mathrm{B}} \cdot p_{\mathrm{B}}^{*} \cdot \bar{T}_{\mathrm{B}}}{\bar{v}_{\mathrm{A}}^{*} \cdot p_{\mathrm{A}}^{*}}+\frac{v_{\mathrm{m}, \mathrm{B}}^{*} \cdot x_{\mathrm{B}} \cdot p_{\mathrm{B}}^{*}}{\bar{v}_{\mathrm{B}}}-\frac{H_{\mathrm{m}}^{\mathrm{E}}}{N_{A}}\right.}{\left(\frac{1}{1-\bar{v} \cdot \bar{T}}\right)^{3}}
$$

d) Determinación de $\chi_{A B}$. Despejando $\chi_{A B}$ de la ecuación (6.285), que da la temperatura característica $T^{*}$ se obtiene:

$$
\chi_{\mathrm{AB}}=\frac{v_{\mathrm{m}, \mathrm{A}}^{*} \cdot x_{\mathrm{A}} \cdot p_{\mathrm{A}}^{*} \cdot\left(1-\frac{\bar{T}_{\mathrm{A}}}{\bar{T}}\right)+v_{\mathrm{m}, \mathrm{B}}^{*} \cdot x_{\mathrm{B}} \cdot p_{\mathrm{B}}^{*} \cdot\left(1-\frac{\bar{T}_{\mathrm{B}}}{\bar{T}}\right)}{v_{\mathrm{m}, \mathrm{A}}^{*} \cdot x_{\mathrm{A}} \cdot \theta_{\mathrm{B}}}
$$

Un procedimiento similar permite determinar de forma analítica la entalpía de exceso sin la aproximación $\bar{p} \approx 0$.

Existe otro procedimiento para determinar de forma analítica el parámetro energético $\chi_{A B}$ de una mezcla a partir de una única medida del volumen de exceso $V_{\mathrm{m}}^{\mathrm{E}}$, mucho más sencillo:

a) Determinación de $\bar{v}$. Directamente de la definición de volumen reducido se obtiene:

$$
\bar{v}=\frac{v_{\mathrm{m}, \mathrm{A}} \cdot x_{\mathrm{A}}+v_{\mathrm{m}, \mathrm{B}} \cdot x_{\mathrm{B}}+v_{\mathrm{m}}^{\mathrm{E}}}{v_{\mathrm{m}, \mathrm{A}}^{*} \cdot x_{\mathrm{A}}+v_{\mathrm{m}, \mathrm{B}}^{*} \cdot x_{\mathrm{B}}}
$$

b) Determinación de $\bar{T}$. Simplemente, aplicar la ecuación de estado de Flory en la aproximación $\bar{p} \approx 0$, ecuación (6.247):

$$
\bar{T}=\frac{\bar{v}^{1 / 3}-1}{\bar{v}^{4 / 3}}
$$

c) Determinación de $\chi_{A B}$. Despejando $\chi_{A B}$ de la ecuación (6.285), que da la temperatura característica $T^{*}$, se obtiene:

$$
\chi_{\mathrm{AB}}=\frac{v_{\mathrm{m}, \mathrm{A}}^{*} \cdot x_{\mathrm{A}} \cdot p_{\mathrm{A}}^{*} \cdot\left(1-\frac{\bar{T}_{\mathrm{A}}}{\bar{T}}\right)+v_{\mathrm{m}, \mathrm{B}}^{*} \cdot x_{\mathrm{B}} \cdot p_{\mathrm{B}}^{*} \cdot\left(1-\frac{\bar{T}_{\mathrm{B}}}{\bar{T}}\right)}{v_{\mathrm{m}, \mathrm{A}}^{*} \cdot x_{\mathrm{A}} \cdot \theta_{\mathrm{B}}}
$$

Aunque estas ecuaciones no tienen complejidad excesiva, la verdad es que en su implementación en un programa de computador se debe tener especial cuidado. Mientras que aparentemente los fallos que puedan ocurrir no se deben a la precisión de las operaciones intermedias, la opción de optimización de la velocidad de cálculo de algunos compiladores resta calidad a los resultados obtenidos. Además, los valores de los parámetros característicos deben ser consistentes entre si, pues en caso contrario, 
cuando las entalpías de exceso son pequeñas (por ejemplo, en los extremos del rango de concentración) se pueden cometer grandes errores, como se ha indicado al final de la sección 6.4.6.1. Más información acerca de esto se puede encontrar en la referencia [343].

\subsubsection{Teorías de disoluciones asociadas}

Hasta ahora solamente se han tratado los líquidos y las disoluciones cuya función de partición puede factorizarse de acuerdo con (6.22), es decir, los líquidos simples en los que solamente actúan fuerzas de tipo dispersivo y entre dipolos inducidos $\mathrm{y} / \mathrm{o}$ permanentes (llamadas todas ellas fuerzas físicas o no específicas). El tratamiento mecánico-estadístico de los líquidos y las mezclas que forman complejos moleculares por la acción de las llamadas fuerzas específicas o "químicas" (ya sea por enlaces de hidrógeno o por complejos de transferencia de carga) es más complicado, como se dijo en la sección 6.1. Ello solamente es posible en el caso de que el número de enlaces efectivos sea menor que tres, lo que permite definir una entidad independiente supramolecular (dímero, trímero, etc.) consistente con los datos espectroscópicos, a fin de poder tratar a este tipo de líquidos como si fuesen simples. Por lo tanto, para cada complejo molecular puede construirse una función de partición [365].

Es evidente que cuando los tiempos de vida de las unidades supramoleculares complejas son lo suficientemente largos, la formación de las mismas pueda tratarse exactamente mediante los mismos métodos que la formación de moléculas ordinarias e introducir la idea de equilibrio químico entre monómeros y complejos. Precisamente las llamadas teorías de disoluciones asociadas o teorías químicas de disoluciones postulan la existencia de especies químicas diferentes en disolución que se supone que están en equilibrio químico. Por lo tanto, dichas teorías parten de la premisa de que las moléculas en una disolución líquida interaccionan unas con otras para formar una nueva especie química.

Se pueden distinguir dos tipos de reacción: asociación y solvatación. La primera se refiere a la formación de unidades supramoleculares a partir de moléculas idénticas:

$$
\mathrm{i} \cdot \mathrm{A} \Leftrightarrow \mathrm{A}_{\mathrm{i}} \quad \mathrm{i}=1,2, \ldots
$$

La segunda se refiere a la formación de complejos entre moléculas que no son iguales:

$$
i \cdot A+j \cdot B \Leftrightarrow A_{i} B_{j} i, j=1,2, \ldots
$$

Desde un punto de vista macroscópico (termodinámico), la teoría química de disoluciones fue propuesta por primera vez por Dolezalek [366] al mismo tiempo que la teoría de van Laar. Los diferentes puntos de vista sostenidos por ambos investigadores causaron una profunda enemistad entre ellos. La teoría de Dolezalek supone que todas las sustancias químicas distintas forman una disolución ideal y por tanto, la no idealidad de la disolución es aparente, consecuencia de reacciones químicas pues se basa en una composición de la mezcla que es aparente, más que real. Por esta razón la teoría de Dolezalek se llama modelo ideal de disoluciones asociadas.

En toda teoría química de disoluciones se hace la distinción entre los componentes nominales y los componentes reales (o verdaderas). Por ejemplo, en una mezcla binaria $\mathrm{A}+\mathrm{B}, \mathrm{A}$ y $\mathrm{B}$ son los componentes nominales mientras que los monómeros, dímeros, trímeros, etc. de la misma molécula y los complejos de diferentes moléculas son los 
diferentes componentes reales. De esta manera, si $n_{1}$ y $n_{2}$ es el número de moles de las especies nominales $\mathrm{A}$ y $\mathrm{B}$ respectivamente, y $n_{\mathrm{A}}, n_{\mathrm{B}}$ y $\xi_{\mathrm{ij}}=n_{\mathrm{AiBj}}$ es el número de moles de los monómeros de $A$ y $B$ y de los diferentes complejos $A B$, se tiene:

$$
\begin{aligned}
& n_{1}=n_{\mathrm{A}}+\sum_{\mathrm{i}, \mathrm{j}} \mathrm{i} \cdot n_{\mathrm{A}_{\mathrm{i}} \mathrm{B}_{\mathrm{j}}}=n_{\mathrm{A}}+\sum_{\mathrm{i}, \mathrm{j}} \mathrm{i} \cdot \xi_{\mathrm{ij}} \\
& n_{2}=n_{\mathrm{B}}+\sum_{\mathrm{i}, \mathrm{j}}^{\mathrm{j}} \cdot n_{\mathrm{A}_{\mathrm{i}} \mathrm{B}_{\mathrm{j}}}=n_{\mathrm{B}}+\sum_{\mathrm{i}, \mathrm{j}}^{\mathrm{j}} \cdot \xi_{\mathrm{ij}}
\end{aligned}
$$

Independientemente de la ocurrencia de las reacciones (6.318)-(6.319), se asigna a la mezcla de los componentes verdaderos funciones termodinámicas definidas en todo el rango de concentración, a las que se llama funciones fundamentales. Así, a $P$ y $T$ constantes, se tiene que la función de Gibbs fundamental es función de las variables $P$, $T, n_{\mathrm{A}}, n_{\mathrm{B}}$ y $\xi_{\mathrm{ij}}=n_{\mathrm{AiBj}} \forall \mathrm{i}, \mathrm{j}$ :

$$
G=G\left(P, T, n_{\mathrm{A}}, n_{\mathrm{B}}, \vec{n}_{\mathrm{A}_{\mathrm{B}} \mathrm{B}_{\mathrm{j}}}\right)=G\left(P, T, n_{\mathrm{A}}, n_{\mathrm{B}}, \vec{\xi}_{\mathrm{ij}}\right)
$$

Los coeficientes de actividad $f$ y las actividades relativas a están relacionados con los potenciales químicos mediante la relación siguiente (véase el Capítulo 2):

$$
\begin{gathered}
\mu_{\mathrm{A}}=\mu_{\mathrm{A}}^{0}+R \cdot T \cdot \ln f_{\mathrm{A}} \cdot x_{\mathrm{A}}=\mu_{\mathrm{A}}^{0}+R \cdot T \cdot \ln a_{\mathrm{A}} \\
\mu_{\mathrm{B}}=\mu_{\mathrm{B}}^{0}+R \cdot T \cdot \ln f_{\mathrm{B}} \cdot x_{\mathrm{B}}=\mu_{\mathrm{B}}^{0}+R \cdot T \cdot \ln a_{\mathrm{B}} \\
\mu_{\mathrm{A}_{\mathrm{i}} \mathrm{B}_{\mathrm{j}}}=\mu_{\mathrm{A}_{\mathrm{i}} \mathrm{B}_{\mathrm{j}}}^{0}+R \cdot T \cdot \ln f_{\mathrm{A}_{\mathrm{i}} \mathrm{B}_{\mathrm{j}}} \cdot x_{\mathrm{A}_{\mathrm{i}} \mathrm{B}_{\mathrm{j}}}=\mu_{\mathrm{A}_{\mathrm{i}} \mathrm{B}_{\mathrm{j}}}^{0}+R \cdot T \cdot \ln a_{\mathrm{A}_{\mathrm{i}} \mathrm{B}_{\mathrm{j}}}
\end{gathered}
$$

donde $x_{\mathrm{A}}, x_{\mathrm{B}}$ y $x_{\mathrm{A}_{\mathrm{B}} \mathrm{Bj}}$ son las fracciones molares, $\mu_{\mathrm{A}}^{0}, \mu_{\mathrm{B}}^{0}$ y $\mu_{\mathrm{A}_{\mathrm{i}} \mathrm{B}_{\mathrm{j}}}^{0}$ son los potenciales químicos de los componentes reales puros a la misma $P$ y $T$ que la mezcla. Con esto, la función de Gibbs molar de exceso de la mezcla es:

$$
\frac{G_{\mathrm{m}}^{\mathrm{E}}}{R \cdot T}=\frac{\mu^{\mathrm{E}}}{R \cdot T}=\sum_{\mathrm{i}} x_{\mathrm{i}} \cdot \ln f_{\mathrm{i}}=x_{\mathrm{A}} \cdot \ln f_{\mathrm{A}}+x_{\mathrm{B}} \cdot \ln f_{\mathrm{B}}+\sum_{\mathrm{i}, \mathrm{j}} x_{\mathrm{A}_{\mathrm{i}} \mathrm{B}} \cdot \ln f_{\mathrm{A}_{\mathrm{i}} \mathrm{B}_{\mathrm{j}}}
$$

el sumatorio se extiende a todos los complejos presentes efectivamente en el sistema. Se define el potencial de Gibbs fundamental estándar de asociación como:

$$
\Delta \tilde{G}_{\mathrm{ij}}^{0}(P, T)=\mu_{\mathrm{A}_{\mathrm{i}} \mathrm{j}}^{0}-\mathrm{i} \cdot \mu_{\mathrm{A}}^{0}-\mathrm{j} \cdot \mu_{\mathrm{B}}^{0}=\Delta \tilde{H}_{\mathrm{ij}}^{0}-T \cdot \Delta \tilde{S}_{\mathrm{ij}}^{0}
$$

Las variables $\xi_{\mathrm{ij}}$ pueden considerarse como los grados de avance de la reacción (véase el Capítulo 2), de forma que las derivadas parciales de $G$ con respecto a $\xi_{\mathrm{ij}}$ son las afinidades $A_{\mathrm{ij}}$ con signo contrario de las diferentes reacciones (6.318)-(6.319):

$$
A_{\mathrm{ij}}=-\frac{\partial G}{\partial \xi_{\mathrm{ij}}}=\mathrm{i} \cdot \mu_{\mathrm{A}}+\mathrm{j} \cdot \mu_{\mathrm{B}}-\mu_{\mathrm{A}_{\mathrm{i}} \mathrm{B}_{\mathrm{j}}}
$$

Verificándose también:

$$
\begin{aligned}
& \mu_{1}=-\frac{\partial G}{\partial n_{1}}=\mu_{\mathrm{A}} \\
& \mu_{2}=-\frac{\partial G}{\partial n_{2}}=\mu_{\mathrm{B}}
\end{aligned}
$$


Por lo tanto, los potenciales químicos de los monómeros son iguales que los potenciales químicos de los componentes nominales. Este resultado es independiente del modo de asociación y sólo depende de la hipótesis de que los complejos estén en equilibrio químico unos con otros.

La condición de equilibrio químico que caracteriza al conjunto de reacciones (6.318)(6.319) se puede escribir de la forma [78], [367], [368]:

$$
A_{\mathrm{ij}}\left(P, T, n_{\mathrm{A}}, n_{\mathrm{B}}, \vec{\xi}_{\mathrm{ij}}\right)=0 \mathrm{i}, \mathrm{j}=0,1,2, \ldots, \mathrm{i}+\mathrm{j} \geq 2
$$

Se puede demostrar que el sistema de ecuaciones (6.331) posee siempre una solución estable $\xi_{\mathrm{ij}}^{\mathrm{e}}$ correspondiente a un mínimo de la función $G$, es decir, existe:

$$
\xi_{\mathrm{ij}}^{\mathrm{e}}=\xi_{\mathrm{ij}}^{\mathrm{e}}\left(P, T, n_{\mathrm{A}}, n_{\mathrm{B}}\right)
$$

que permite conocer la composición real de equilibrio de la mezcla:

$$
\begin{gathered}
n_{\mathrm{A}_{\mathrm{B}} \mathrm{B}_{\mathrm{j}}}^{\mathrm{e}}=\xi_{\mathrm{ij}}^{\mathrm{e}} \\
n_{\mathrm{A}}^{\mathrm{e}}=n_{1}-\sum_{\mathrm{i}, \mathrm{j}}^{\mathrm{i}} \cdot \xi_{\mathrm{ij}}^{\mathrm{e}} \\
n_{\mathrm{B}}^{\mathrm{e}}=n_{2}-\sum_{\mathrm{i}, \mathrm{j}} \mathrm{j} \cdot \xi_{\mathrm{ij}}^{\mathrm{e}}
\end{gathered}
$$

y por tanto, conocer $G$ mediante (6.322).

En lugar de (6.331) puede encontrarse un sistema equivalente de ecuaciones (Ley de acción de masas), que permite obtener las constantes de equilibrio químico $K_{\mathrm{ij}}$ :

$$
K_{\mathrm{ij}}=\frac{a_{\mathrm{A}_{\mathrm{i}} \mathrm{B}_{\mathrm{j}}}}{a_{\mathrm{A}}^{\mathrm{i}} \cdot a_{\mathrm{A}}^{\mathrm{i}}}=\frac{f_{\mathrm{A}_{\mathrm{i}} \mathrm{B}_{\mathrm{j}}} \cdot x_{\mathrm{A}_{\mathrm{i}} \mathrm{B}_{\mathrm{j}}}}{\left(f_{\mathrm{A}}^{\mathrm{i}} \cdot x_{\mathrm{A}}^{\mathrm{i}}\right) \cdot\left(f_{\mathrm{B}}^{\mathrm{i}} \cdot x_{\mathrm{B}}^{\mathrm{i}}\right)}=e^{-\Delta \tilde{G}_{\mathrm{ij}}^{0} / R \cdot T}
$$

En el equilibrio, los coeficientes de actividad de los componentes nominales vienen dados por:

$$
\begin{aligned}
& f_{1}=\frac{x_{\mathrm{A}}^{\mathrm{e}} \cdot f_{\mathrm{A}}^{\mathrm{e}}}{x_{1} \cdot x_{\mathrm{A}}^{0, \mathrm{e}} \cdot f_{\mathrm{A}}^{0, \mathrm{e}}} \\
& f_{2}=\frac{x_{\mathrm{B}}^{\mathrm{e}} \cdot f_{\mathrm{B}}^{\mathrm{e}}}{x_{2} \cdot x_{\mathrm{B}}^{0, \mathrm{e}} \cdot f_{\mathrm{B}}^{0, \mathrm{e}}}
\end{aligned}
$$

donde $x_{\mathrm{A}}^{0, \mathrm{e}}$ y $f_{\mathrm{A}}^{0, \mathrm{e}}$ son los valores de $x_{\mathrm{A}}^{\mathrm{e}}$ y $f_{\mathrm{A}}^{\mathrm{e}}$ en el componente puro A, y análogamente en el caso de B.

Para establecer las constantes de equilibrio $K_{\mathrm{ij}}$, es necesario conocer el tipo de complejos realmente presentes en la disolución. Aunque los datos espectroscópicos y otros datos de carácter no termodinámico pueden dar idea de ello, la elección de los mismos es un tanto arbitraria, pudiéndose seleccionar tantos como uno quiera. Esta flexibilidad es a la vez una ventaja y un inconveniente de la teoría química de disoluciones. Es una ventaja porque permite explicar el comportamiento de la disolución de cualquier tipo de interacción química y por tanto, en potencia, tiene un gran rango de aplicabilidad. Es una desventaja por dos razones, principalmente: primero, su ambigüedad puede llevar a conclusiones tan absurdas como la que obtuvo el propio Dolezalek al explicar las desviaciones respecto la idealidad en la mezcla argón + 
nitrógeno postulando la existencia de dímeros de argón; segundo, a no ser que haya otro tipo de información, la estequiometría del complejo es otro parámetro ajustable además de la constante de equilibrio. Por tanto, si una determinada estequiometría no se ajusta a los datos experimentales, se puede probar otra y así sucesivamente, hasta al final obtener un ajuste. Sin embargo, este ajuste no tiene significado físico a no ser que haya una evidencia independiente a partir de la estructura molecular de los componentes que verifique la estequiometría supuesta.

Existen tantas teorías de asociación como formas funcionales para (6.326). Sin embargo, sólo se han desarrollado las más simples. La más sencilla de ellas es la teoría de Dolezalek, que como se ha dicho, supone que todas las sustancias químicas distintas forman una disolución ideal. Por tanto,

$$
f_{\mathrm{A}}^{\mathrm{e}}=f_{\mathrm{B}}^{\mathrm{e}}=f_{\mathrm{A}}^{0, \mathrm{e}}=f_{\mathrm{B}}^{0, \mathrm{e}}=1
$$

de forma que la no idealidad es sólo aparente y se debe a la utilización de una composición para la mezcla que no refleja lo que realmente existe en la misma. Dependiendo del tipo de complejos considerados (dímeros, trímeros, etc.) aparecen diferentes modelos, los cuales pueden consultarse en la bibliografía [78, Cap. XXVI], [161, Cap. 7], [369]-[372].

Aunque las teorías de disoluciones asociadas son útiles en muchos casos, son incapaces de predecir lagunas de miscibilidad y no proporcionan una visión satisfactoria de las propiedades de las mezclas líquidas. Además, hemos de preguntarnos si, dado que la explicación de la mayoría de las propiedades de las mezclas de líquidos no asociados se basa en la consideración de interacciones no específicas (o "físicas") y de las diferencias en los tamaños de los componentes ¿̇puede esperarse que las mismas desaparezcan en el caso de que la mezcla esté formada por complejos moleculares en lugar de por monómeros? La respuesta es obvia: NO.

Los dos tipos de teorías, física y química, son situaciones extremas de lo que se piensa hoy en día que es la solución correcta. En algunas situaciones límite, uno de los dos tipos puede dar resultados satisfactorios: cuando las fuerzas entre moléculas son débiles, no formandose especies químicas estables, se pueden aplicar teorías físicas. Por otra parte, cuando las fuerzas entre moléculas son fuertes, pudiéndose formar enlaces químicos, las teorías químicas proporcionan una descripción razonable del sistema. No obstante, en el caso general deben tenerse en cuenta tanto las fuerzas físicas como las "químicas".

De acuerdo con esto, al modelo de disoluciones ideales asociadas se le suelen añadir dos correcciones: una entálpica, que da cuenta de la contribución de las fuerzas no específicas y una entrópica, que tiene en cuenta las diferencias de tamaño entre las diferentes especies verdaderas. De esta manera, todas las funciones termodinámicas estudiadas en secciones previas pueden añadirse a las obtenidas por la teoría de disoluciones asociadas. Así, aparecen de forma natural los modelos de red de disoluciones asociadas [369]-[373], que en determinados casos originan los llamados modelos de disoluciones asociadas regulares, que tienen en cuenta una corrección entálpica, los modelos de disoluciones asociadas atérmicas, que solamente tienen en cuenta la contribución entrópica debida a las diferencias de tamaño entre las especies moleculares verdaderas, etc. Por ejemplo, en cuanto al modelo de disoluciones asociadas atérmicas, puede formularse en la aproximación $z \rightarrow \infty$ de Flory-Huggins o de 
Guggenheim-Staverman. En el primer caso se toma la ecuación (6.187) para la entropía fundamental de exceso, siendo $\varphi_{i}$ las fracciones de volumen las correspondientes a los componentes reales. Esta teoría se ha aplicado a mezclas de alcoholes alifáticos con hidrocarburos parafínicos [374]-[378]. El caso general de las mezclas asociadas no atérmicas sólo se ha desarrollado en casos simples. Así, los modelos de red no atérmicos de disoluciones asociadas sólo se han resuelto en la aproximación de orden cero, en donde los efectos energéticos no impiden la mezcla al azar [379], [380]. También se ha formulado la teoría de Flory de volumen libre en términos de disoluciones asociadas (modelo ERAS).

En cualquier caso, independientemente del modelo utilizado y con el objetivo de evitar un álgebra engorrosa y un gran número de parámetros ajustables, los modelos de disoluciones asociadas con interacciones físicas deben restringirse a mezclas al azar en las que la estequiometría de los complejos sea simple. En este caso, puede demostrarse que las funciones termodinámicas de exceso pueden desdoblarse formalmente en la suma de dos términos independientes, uno asociado a la parte puramente "química" (CHEM), es decir, a las interacciones específicas (puentes de hidrógeno o fuerzas presentes en los complejos por transferencia de carga) y otro con la parte "física" (PHYS), es decir, con las fuerzas no específicas (fuerzas dispersivas y entre dipolos inducidos y/o permanentes). De esta manera, se tiene [354]:

$$
X^{\mathrm{E}}=X^{\mathrm{E}}(\mathrm{CHEM})+X^{\mathrm{E}}(\mathrm{PHYS})
$$

Entre los diferentes modelos de disoluciones que hacen uso de esta propiedad (6.340), se encuentran DISQUAC, ERAS, LFAS, etc. [161].

\subsubsection{Modelo ERAS}

El modelo ERAS (Extended Real Associated Solution) [381] combina un modelo de asociación real [371], [375], [378], [382] con la ecuación de estado de Flory.

Previamente a este modelo, la mayoría de las aproximaciones aplicadas para determinar las propiedades de exceso de las mezclas asociado (1) + no asociado (2), habían sido desarrolladas para predecir las funciones $G_{\mathrm{m}}^{\mathrm{E}}$ y $H_{\mathrm{m}}^{\mathrm{E}}$ usando teorías de red rígida que tienen en cuenta la autoasociación del componente (1), pero que son incapaces de predecir el $V_{\mathrm{m}}^{\mathrm{E}}$, puesto que presentan la restricción de no permitir cambios de volumen durante el proceso de mezcla. Precisamente, las ecuaciones de estado, lo que predicen son esos cambios de volumen, por tanto la combinación de estas ecuaciones, junto con un modelo de asociación ofrece una solución al problema de los modelos de red rígida.

Originalmente, el modelo ERAS fue desarrollado para predecir las propiedades de exceso de las mezclas binarias alcohol + alcano [381], [383]. Posteriormente, un modelo más generalizado permitió su aplicación a otros sistemas formados por dos componentes asociados [384]-[395]. Por lo tanto, hoy en día el campo de aplicación del modelo ERAS se dirige a las mezclas donde se espera que existan efectos de autoasociación (1-alcohol [381], [383]; 2-alcoxietanol [396], amina secundaria + alcano [397]), o de solvatación entre un componente auto-asociado y otro polar (1-alcohol + monoéter [398], [399]), o entre dos componentes auto-asociados (1-alcohol + amina [391], [389]). En estos casos, la descripción de las propiedades termodinámicas de los sistemas considerados es excelente. Para las mezclas en las que existen grandes 
diferencias entre los valores experimentales de $H_{\mathrm{m}}^{\mathrm{E}}$ y los resultados teóricos, dichas discrepancias deben de considerarse como una valiosa fuente de información, ya que indican que los posibles efectos de asociación y solvatación son de menor importancia que los efectos puramente físicos. Así, en estos casos los malos resultados obtenidos a partir del modelo ERAS son coherentes con los encontrados cuando se aplica la teoría de Flory, pues el parámetro de Flory $\chi_{A B}$ debe depender débilmente de la concentración, como debe ser en una mezcla al azar.

Las hipótesis en las que se basa el modelo ERAS son las siguientes:

a) Cualquier función termodinámica puede desglosarse de acuerdo con la ecuación (6.340).

b) Se supone que la asociación se produce únicamente de forma lineal y consecutiva. Esta asociación queda caracterizada por la constante de equilibrio $K_{\mathrm{A}}$, que se supone independiente de la longitud de la cadena de las especies asociadas:

$$
A_{i}+A \Leftrightarrow A_{i+1}
$$

i varía entre 1 a $\infty$. Naturalmente, puede existir asociación entre los dos componentes de la mezcla. En el caso más simple de una solución con un componente auto-asociado A, y otro componente polar que no muestra ese comportamiento B, la solvatación entre ellos se expresa por la ecuación

$$
A_{i}+B \Leftrightarrow A_{i} B
$$

Aquí también se supone que las correspondientes constantes de equilibrio, $K_{\mathrm{AB}}$, son independientes de la longitud de la especie polimérica. Su dependencia con la temperatura viene dada por la relación de Van't Hoff:

$$
K_{\mathrm{A}}=K_{\mathrm{A} 0} \cdot e^{-\frac{\Delta h_{\mathrm{A}}^{*}}{R}\left(\frac{1}{T}-\frac{1}{T_{0}}\right)}
$$

donde $\Delta h_{\mathrm{A}}^{*}$ es la variación de entalpía asociada a la reacción (6.341), que es igual a la energía del enlace puente de hidrógeno, y toma valores muy cercanos a $-H_{\mathrm{m}, \mathrm{A}}^{\mathrm{E}, \infty}(\mathrm{A}+n$-alcano $) . K_{\mathrm{A} 0}$ es la constante de asociación a temperatura $T_{0}$. Estos valores son necesarios para poder determinar la "contribución química" de las propiedades de exceso, pero es preciso añadir otro parámetro, $\Delta v_{\mathrm{A}}^{*}$, el cambio de volumen producido cuando se forman las cadenas lineales, es decir, cuando se forma un puente de hidrógeno de acuerdo con la reacción (6.341). $\Delta v_{\mathrm{A}}^{*}$ es negativo, indicando, que la interacción por puentes de hidrógeno del componente asociado, hace que se produzca una contracción en el volumen de "core" de las moléculas.

c) Las contribuciones físicas a las funciones de exceso $X^{\mathrm{E}}$ (PHYS) se deben a interacciones no polares del tipo van der Waals e incluye efectos de volumen libre, es decir, se derivan del modelo de Flory. Naturalmente, la ecuación de estado (6.238) se escribe en función de variables reducidas. Sin embargo, el procedimiento para obtener las variables reducidas difiere del original aplicado en el modelo de Flory. En este caso, $p_{\mathrm{i}}^{*}, v_{\mathrm{i}}^{*}$ y $T_{\mathrm{i}}^{*}$ son calculados a partir de datos $P V T\left(\rho, \alpha_{P}\right.$ y $\left.\kappa_{T}\right)$ y además, presentan una 
dependencia con los parámetros $K_{\mathrm{i}}, \Delta h_{\mathrm{i}}^{*}$ y $\Delta v_{\mathrm{i}}^{*}$ tal y como se muestra en las ecuaciones siguientes [389].

$$
\begin{gathered}
v_{\mathrm{i}}^{*}=v_{\mathrm{i}} \cdot\left(\frac{1+\left(\alpha_{i}-\alpha_{i}^{*}\right) \cdot T}{1+4 / 3 \cdot\left(\alpha_{i}-\alpha_{i}^{*}\right) \cdot T}\right) \\
\alpha_{i}^{*}=\Delta v_{\mathrm{i}}^{*} \cdot \Delta h_{\mathrm{i}}^{*} \cdot \frac{\left(4 \cdot K_{\mathrm{i}}+1\right)^{1 / 2}-2 \cdot K_{\mathrm{i}} \cdot\left(4 \cdot K_{\mathrm{i}}+1\right)^{-1 / 2}-1}{2 \cdot K_{i} \cdot v_{\mathrm{i}}^{*} \cdot R \cdot T^{2}} \\
p_{i}^{*}=\left(\alpha_{i}-\alpha_{i}^{*}\right) \cdot T \cdot \bar{v}_{\mathrm{i}}^{2} \cdot\left(\kappa_{T \mathrm{i}}-\alpha_{\mathrm{i}}^{*} \cdot T \cdot \frac{\Delta v_{\mathrm{i}}^{*}}{\Delta h_{\mathrm{i}}^{*}}\right)^{-1}
\end{gathered}
$$

de manera que para un componente inerte $(\mathrm{i}=\mathrm{B})$, es sabido que $\Delta v_{\mathrm{B}}^{*}, \Delta h_{\mathrm{B}}^{*}, K_{\mathrm{B}}$ y $\alpha_{\mathrm{B}}^{*}$, son nulos.

Combinando las ecuaciones (6.238) y (6.344)-(6.346) se tiene:

$$
T_{\mathrm{i}}^{*}=\frac{\bar{v}_{\mathrm{i}}^{4 / 3}}{\bar{v}_{\mathrm{i}}^{4 / 3}-1} \cdot T
$$

Las contribuciones "químicas" a la entalpía y al volumen de exceso, para mezclas formadas por un componente asociado A y uno inerte $B$, vienen dadas por las expresiones:

$$
\begin{gathered}
H^{\mathrm{E}}(\mathrm{CHEM})=x_{\mathrm{A}} \cdot K_{\mathrm{A}} \cdot \Delta h_{\mathrm{A}}^{*} \cdot\left(\varphi_{1 \mathrm{~A}}-\varphi_{1 \mathrm{~A}}^{0}\right)-\left[\frac{p^{*} \cdot x_{\mathrm{A}} \cdot \Delta v_{\mathrm{A}}^{*} \cdot K_{\mathrm{A}} \cdot\left(\varphi_{1 \mathrm{~A}}-\varphi_{1 \mathrm{~A}}^{0}\right)}{\bar{v}}\right] \\
V^{\mathrm{E}}(\mathrm{CHEM})=x_{\mathrm{A}} \cdot \bar{v} \cdot K_{\mathrm{A}} \cdot \Delta v_{\mathrm{A}} \cdot\left(\varphi_{1 \mathrm{~A}}-\varphi_{1 \mathrm{~A}}^{0}\right)
\end{gathered}
$$

donde $x_{\mathrm{A}}$ es la fracción molar del componente asociado, $\varphi_{1 \mathrm{~A}}$ y $\varphi_{1 \mathrm{~A}}^{0}$ son las fracciones de volumen de los monómeros en la mezcla y en el componente puro, respectivamente. Estas fracciones de volumen están relacionadas con la constante de asociación $K_{\mathrm{A}}$ :

$$
\begin{gathered}
\varphi_{1 \mathrm{~A}}=\frac{2 \cdot K_{\mathrm{A}} \cdot \Phi_{\mathrm{A}}+1-\sqrt{4 \cdot K_{\mathrm{A}} \cdot \Phi_{\mathrm{A}}+1}}{2 \cdot K_{\mathrm{A}}^{2} \cdot \Phi_{\mathrm{A}}} \\
\varphi_{1 \mathrm{~A}}^{0}=\lim _{\Phi_{\mathrm{A}} \rightarrow 1}\left(\varphi_{1 \mathrm{~A}}\right)=\frac{2 \cdot K_{\mathrm{A}}+1-\sqrt{4 \cdot K_{\mathrm{A}}+1}}{2 \cdot K_{\mathrm{A}}^{2}}
\end{gathered}
$$

con:

$$
\Phi_{\mathrm{A}}=\frac{x_{\mathrm{A}} \cdot v_{\mathrm{A}}^{*}}{x_{\mathrm{A}} \cdot v_{\mathrm{A}}^{*}+x_{\mathrm{B}} \cdot v_{\mathrm{B}}^{*}}=1-\Phi_{\mathrm{B}}
$$

$\Phi_{\mathrm{A}}$ y $\Phi_{\mathrm{B}}$ son las fracciones de volumen de "core", que difieren de las fracciones de volumen del modelo de Flory $\varphi_{\mathrm{i}}$ pues $v_{\mathrm{A}}^{*}$ y $v_{\mathrm{B}}^{*}$ se calculan de diferente modo. $v_{\mathrm{A}}^{*}$ y $v_{\mathrm{B}}^{*}$ son los volúmenes reducidos del componente asociado y del alcano, respectivamente. Se refieren al volumen de "core", de manera, que para el componente asociado, este 
valor es más pequeño que el correspondiente al de sus monómeros, ya que aparece una contracción en el volumen, cuantificada con el término $\Delta v_{\mathrm{A}}^{*}$.

Las variables reducidas de mezcla, $p^{*}$ y $T^{*}$ se determinan mediante las reglas de mezcla siguientes, análogas a las de Flory (6.273)-(6.275):

$$
\begin{gathered}
v^{*}=x_{\mathrm{A}} \cdot v_{\mathrm{A}}^{*}+x_{\mathrm{B}} \cdot v_{\mathrm{B}}^{*} \\
T^{*}=\frac{p^{*}}{\left(\Phi_{\mathrm{A}} \cdot p_{\mathrm{A}}^{*} / T_{\mathrm{A}}^{*}\right)+\left(\Phi_{\mathrm{B}} \cdot p_{\mathrm{B}}^{*} / T_{\mathrm{B}}^{*}\right)} \\
p^{*}=p_{\mathrm{A}}^{*} \cdot \Phi_{\mathrm{A}}+p_{\mathrm{B}}^{*} \cdot \Phi_{\mathrm{B}}-\Phi_{\mathrm{A}} \cdot \Theta_{\mathrm{B}} \cdot X_{\mathrm{AB}}
\end{gathered}
$$

Donde $\Theta_{B}$ es la fracción de superficie del componente B en la mezcla, definida a partir de la siguiente relación:

$$
\Theta_{\mathrm{B}}=1-\Theta_{\mathrm{A}}=\frac{\Phi_{\mathrm{B}}}{\Phi_{\mathrm{B}}+S_{\mathrm{AB}} \cdot \Phi_{\mathrm{A}}}
$$

donde el parámetro geométrico de la mezcla es:

$$
S_{\mathrm{AB}}=\frac{s_{\mathrm{A}}}{S_{\mathrm{B}}}=\left(\frac{v_{\mathrm{A}}^{*}}{v_{\mathrm{B}}^{*}}\right)^{-1 / 3}
$$

que difiere del del modelo de Flory porque así lo hacen $v_{\mathrm{i}}^{*}$.

El parámetro de interacción $X_{\mathrm{AB}}$ de la ecuación (6.355) describe las interacciones no dipolares de Van der Waals entre moléculas de diferente tipo. Es el único parámetro ajustable de la contribución física.

Para poder aplicar las ecuaciones (6.348) y (6.349), $\bar{v}$ se determina usando las ecuaciones (6.238), (6.355) y (6.354).

Las contribuciones "físicas" a la entalpía y al volumen de exceso vienen dadas por:

$$
\begin{gathered}
H^{\mathrm{E}}(\mathrm{PHYS})=v^{*} \cdot\left(\frac{\Phi_{\mathrm{A}} \cdot p_{\mathrm{A}}^{*}}{\bar{v}_{\mathrm{A}}}+\frac{\Phi_{\mathrm{B}} \cdot p_{\mathrm{B}}^{*}}{\bar{v}_{\mathrm{B}}}-\frac{p^{*}}{\bar{v}}\right) \\
V^{\mathrm{E}}(\mathrm{PHYS})=v^{*} \cdot\left(\bar{v}-\Phi_{\mathrm{A}} \cdot \bar{v}_{\mathrm{A}}-\Phi_{\mathrm{B}} \cdot \bar{v}_{\mathrm{B}}\right)
\end{gathered}
$$

Las ecuaciones anteriores pueden transformarse en las siguientes, equivalentes a las del modelo de Flory (6.283) y (6.287):

$$
\begin{gathered}
H^{\mathrm{E}}(\mathrm{PHYS})=\left(x_{\mathrm{A}} \cdot v_{\mathrm{A}}^{*}+x_{\mathrm{B}} \cdot v_{\mathrm{B}}^{*}\right) \cdot\left(\frac{\Phi_{\mathrm{A}} \cdot p_{\mathrm{A}}^{*}}{\bar{v}_{\mathrm{A}}}+\frac{\Phi_{\mathrm{B}} \cdot p_{\mathrm{B}}^{*}}{\bar{v}_{\mathrm{B}}}-\frac{p^{*}}{\bar{v}}\right)= \\
=x_{\mathrm{A}} \cdot v_{\mathrm{A}}^{*} \cdot p_{\mathrm{A}}^{*} \cdot\left(\frac{1}{\bar{v}_{\mathrm{A}}}-\frac{1}{\bar{v}}\right)+x_{\mathrm{B}} \cdot v_{\mathrm{B}}^{*} \cdot p_{\mathrm{B}}^{*} \cdot\left(\frac{1}{\bar{v}_{\mathrm{B}}}-\frac{1}{\bar{v}}\right)+\frac{x_{\mathrm{A}} \cdot v_{\mathrm{A}}^{*} \cdot \Theta_{\mathrm{B}} \cdot X_{\mathrm{AB}}}{\bar{v}} \\
V^{\mathrm{E}}(\mathrm{PHYS})=\left(x_{\mathrm{A}} \cdot v_{\mathrm{A}}^{*}+x_{\mathrm{B}} \cdot v_{\mathrm{B}}^{*}\right) \cdot\left(\bar{v}-\Phi_{\mathrm{A}} \cdot \bar{v}_{\mathrm{A}}-\Phi_{\mathrm{B}} \cdot \bar{v}_{\mathrm{B}}\right)
\end{gathered}
$$

Las contribuciones química y física a $C_{\mathrm{p}}^{\mathrm{E}}$ [396] son: 


$$
\begin{gathered}
C_{P}^{\mathrm{E}}(\mathrm{CHEM})=\frac{x_{\mathrm{A}} \cdot K_{\mathrm{A}} \cdot \Delta h_{\mathrm{A}}^{*}}{R \cdot T^{2}} \cdot\left(\Delta h_{\mathrm{A}}^{*}-\frac{p^{*} \cdot \Delta v_{\mathrm{A}}^{*}}{\bar{v}}\right) \cdot\left[\frac{\varphi_{1 \mathrm{~A}} \cdot\left(1-K_{\mathrm{A}} \cdot \varphi_{1 \mathrm{~A}}\right)}{1+K_{\mathrm{A}} \cdot \varphi_{1 \mathrm{~A}}}-\frac{\varphi_{1 \mathrm{~A}}^{0} \cdot\left(1-K_{\mathrm{A}} \cdot \varphi_{1 \mathrm{~A}}^{0}\right)}{1+K_{\mathrm{A}} \cdot \varphi_{1 \mathrm{~A}}^{0}}\right]+ \\
+x_{\mathrm{A}} \cdot K_{\mathrm{A}} \cdot\left(\varphi_{1 \mathrm{~A}}-\varphi_{1 \mathrm{~A}}^{0}\right) \cdot\left(\frac{\Phi_{\mathrm{A}} \cdot \Theta_{\mathrm{B}}\left(\mathrm{d} X_{\mathrm{AB}} / \mathrm{d} T\right)+p^{*} \cdot \bar{\alpha}}{\bar{v}}\right) \\
C_{P}^{\mathrm{E}}(\mathrm{PHYS})=v^{*} \cdot\left(-\frac{\Phi_{\mathrm{A}} \cdot p_{\mathrm{A}}^{*} \cdot \bar{\alpha}_{\mathrm{A}}}{\bar{v}_{\mathrm{A}}}-\frac{\Phi_{\mathrm{B}} \cdot p_{\mathrm{B}}^{*} \cdot \bar{\alpha}_{\mathrm{B}}}{\bar{v}_{\mathrm{B}}}+\right. \\
\left.+\frac{p^{*} \cdot \bar{\alpha}+\Phi_{\mathrm{A}} \cdot \Theta_{\mathrm{B}} \cdot\left(\mathrm{d} X_{\mathrm{AB}} / \mathrm{d} T\right)}{\bar{v}}\right)
\end{gathered}
$$

La expresión (6.362) puede reducirse a la expresión (6.364) cuando $\Delta h_{A}^{*}>\Delta v_{A}^{*} \cdot p^{*} / \bar{v}$, como es en el caso de los alcoholes, y se considera despreciable el término $\left(\varphi_{1 A}-\varphi_{1 A}^{\cdot}\right)$ :

$$
C_{P}^{\mathrm{E}}(\mathrm{CHEM})=\frac{x_{\mathrm{A}} \cdot K_{\mathrm{A}} \cdot\left(\Delta h_{\mathrm{A}}^{*}\right)^{2}}{R \cdot T^{2}} \cdot\left[\frac{\varphi_{1 \mathrm{~A}} \cdot\left(1-K_{\mathrm{A}} \cdot \varphi_{1 \mathrm{~A}}\right)}{1+K_{\mathrm{A}} \cdot \varphi_{1 \mathrm{~A}}}-\frac{\varphi_{1 \mathrm{~A}}^{0} \cdot\left(1-K_{\mathrm{A}} \cdot \varphi_{1 \mathrm{~A}}^{0}\right)}{1+K_{\mathrm{A}} \cdot \varphi_{1 \mathrm{~A}}^{0}}\right]
$$

En las ecuaciones (6.362) y (6.363), $\bar{\alpha}_{A}, \bar{\alpha}_{B}$ y $\bar{\alpha}$ se calculan a partir de la expresión:

$$
\bar{\alpha}_{i}=\frac{3 \cdot\left(\bar{v}_{\mathrm{i}}^{1 / 3}-1\right)}{T \cdot\left(4-3 \cdot \bar{v}_{\mathrm{i}}^{1 / 3}\right)}
$$

En cuanto a los coeficientes de actividad, las contribuciones químicas y físicas de cada componente son de la forma:

$$
\begin{gathered}
\ln f_{\mathrm{A}}(\mathrm{CHEM})=\ln \left(\frac{\varphi_{1 \mathrm{~A}}}{x_{\mathrm{A}} \cdot \varphi_{1 \mathrm{~A}}^{0}}\right)-\frac{\varphi_{1 \mathrm{~A}}}{1-K_{\mathrm{A}} \cdot \varphi_{1 \mathrm{~A}}}-\frac{v_{\mathrm{A}}^{*} \cdot \Phi_{\mathrm{B}}}{v_{\mathrm{B}}^{*}}+\frac{\varphi_{1 \mathrm{~A}}^{0}}{1-K_{\mathrm{A}} \cdot \varphi_{1 \mathrm{~A}}^{0}} \\
\ln f_{\mathrm{B}}(\mathrm{CHEM})=\ln \left(\frac{\Phi_{\mathrm{B}}}{x_{\mathrm{B}}}\right)+\Phi_{\mathrm{A}}-\frac{v_{\mathrm{B}}^{*} \cdot \varphi_{1 \mathrm{~A}}}{v_{\mathrm{A}}^{*} \cdot\left(1-K_{\mathrm{A}} \cdot \varphi_{1 \mathrm{~A}}\right)} \\
\ln f_{\mathrm{A}}(\mathrm{PHYS})=\frac{p_{\mathrm{A}}^{*} \cdot v_{\mathrm{A}}^{*} \cdot 3 \cdot \bar{T}_{\mathrm{A}} \cdot\left[\ln \left(\frac{\bar{v}_{\mathrm{A}}^{1 / 3}-1}{\bar{v}^{1 / 3}-1}\right)+\frac{1}{\bar{v}_{\mathrm{A}}}-\frac{1}{\bar{v}}\right]+v_{\mathrm{A}}^{*} \cdot \Theta_{\mathrm{B}}^{2} \cdot \frac{X_{\mathrm{AB}} / \bar{v}-T \cdot Q_{\mathrm{AB}}}{R \cdot T}}{\ln f_{\mathrm{B}}(\mathrm{PHYS})=}=\frac{p_{\mathrm{B}}^{*} \cdot v_{\mathrm{B}}^{*} \cdot 3 \cdot \bar{T}_{\mathrm{B}} \cdot\left[\ln \left(\frac{\bar{v}_{\mathrm{B}}^{1 / 3}-1}{\bar{v}^{1 / 3}-1}\right)+\frac{1}{\bar{v}_{\mathrm{B}}}-\frac{1}{\bar{v}}\right]+}{+v_{\mathrm{B}}^{*} \cdot\left(1-\Theta_{\mathrm{B}}\right)^{2} \cdot S_{\mathrm{BA}} \cdot \frac{X_{\mathrm{AB}} / \bar{v}-T \cdot Q_{A B}}{R \cdot T}}
\end{gathered}
$$

donde $Q_{\mathrm{AB}}$ caracteriza la contribución entrópica debido a la diferencia de interacciones intermoleculares.

Conocidos los coeficientes de actividad, es fácil calcular la función de Gibbs de exceso:

$$
G^{\mathrm{E}}(\mathrm{CHEM})=R \cdot T \cdot\left[x_{\mathrm{A}} \cdot \ln \frac{\varphi_{\mathrm{A} 1}}{x_{\mathrm{A}} \cdot \varphi_{1 \mathrm{~A}}^{0}}+x_{\mathrm{B}} \cdot \ln \frac{\Phi_{\mathrm{B}}}{x_{\mathrm{B}}}+K_{\mathrm{A}} \cdot x_{\mathrm{A}} \cdot\left(\varphi_{1 \mathrm{~A}}-\varphi_{1 \mathrm{~A}}^{0}\right)\right]
$$




$$
\begin{gathered}
G^{\mathrm{E}}(\mathrm{PHYS})=H^{\mathrm{E}}(\mathrm{PHYS})-T \cdot v^{*} \cdot\left[\Theta_{\mathrm{B}} \cdot \Phi_{\mathrm{A}} \cdot Q_{\mathrm{AB}}-3 \cdot \Phi_{\mathrm{A}} \cdot \frac{p_{\mathrm{A}}^{*}}{T_{\mathrm{A}}^{*}} \cdot \ln \left(\frac{\bar{v}_{\mathrm{A}}^{1 / 3}-1}{\bar{v}^{1 / 3}-1}\right)-\right. \\
\left.-3 \cdot \Phi_{\mathrm{B}} \cdot \frac{P_{\mathrm{B}}^{*}}{T_{\mathrm{B}}^{*}} \cdot \ln \left(\frac{\bar{v}_{\mathrm{B}}^{1 / 3}-1}{\bar{v}^{1 / 3}-1}\right)\right]
\end{gathered}
$$

El modelo ERAS permite evaluar la entalpía de vaporización de los compuestos puros:

$$
\Delta H_{v}=\frac{p_{\mathrm{i}}^{*} \cdot v_{\mathrm{i}}^{*}}{\bar{v}_{\mathrm{i}}}-\frac{\Delta h_{\mathrm{i}}^{*}}{2 \cdot K_{\mathrm{i}}} \cdot\left(2 \cdot K_{\mathrm{i}}+1-\sqrt{4 \cdot K_{\mathrm{i}}+1}\right)+R \cdot T
$$

El primer término proviene de las interacciones de Van der Waals y los efectos de volumen libre, mientras que el segundo se debe a la asociación intermolecular consecuencia de la existencia de puentes de hidrógeno.

El modelo ERAS puede extenderse a mezclas formadas por dos componentes asociados, por ejemplo, los sistemas binarios: 1-alcohol + amina primaria o secundaria. Las contribuciones químicas a la entalpía, volumen y función de Gibbs de exceso vienen dadas por las ecuaciones siguientes:

$$
\begin{gathered}
H^{\mathrm{E}}(\mathrm{CHEM})=x_{\mathrm{A}} \cdot \Delta h_{\mathrm{A}}^{*} \cdot K_{\mathrm{A}} \cdot\left(\varphi_{1 \mathrm{~A}}-\varphi_{1 \mathrm{~A}}^{0}\right)+x_{\mathrm{B}} \cdot \Delta h_{\mathrm{B}}^{*} \cdot K_{\mathrm{B}} \cdot\left(\varphi_{1 \mathrm{~B}}-\varphi_{1 \mathrm{~B}}^{0}\right)+ \\
+\frac{x_{\mathrm{A}} \cdot \Delta h_{\mathrm{AB}}^{*} \cdot K_{\mathrm{AB}} \cdot \varphi_{1 \mathrm{~B}} \cdot\left(1-K_{\mathrm{A}} \cdot \varphi_{1 \mathrm{~A}}\right)}{\left[\left(V_{\mathrm{B}}^{\mathrm{mol}} / V_{\mathrm{A}}^{\mathrm{mol}}\right) \cdot\left(1-K_{\mathrm{B}} \cdot \varphi_{1 \mathrm{~B}}\right)+K_{\mathrm{AB}} \cdot \varphi_{1 \mathrm{~B}}\right]}-\frac{p^{*} \cdot V^{\mathrm{E}}(\mathrm{CHEM})}{\bar{v}^{2}} \\
V^{\mathrm{E}}(\mathrm{CHEM})=\bar{v} \cdot\left[x_{\mathrm{A}} \cdot \Delta v_{\mathrm{A}}^{*} \cdot K_{\mathrm{A}} \cdot\left(\varphi_{1 \mathrm{~A}}-\varphi_{1 \mathrm{~A}}^{0}\right)+x_{\mathrm{B}} \cdot \Delta v_{\mathrm{B}}^{*} \cdot K_{\mathrm{B}} \cdot\left(\varphi_{1 \mathrm{~B}}-\varphi_{1 \mathrm{~B}}^{0}\right)+\right. \\
\left.+\frac{x_{\mathrm{A}} \cdot \Delta v_{\mathrm{AB}}^{*} \cdot K_{\mathrm{AB}} \cdot \varphi_{\mathrm{B}} \cdot\left(1-K_{\mathrm{A}} \cdot \varphi_{1 \mathrm{~A}}\right)}{\left(V_{\mathrm{B}}^{\mathrm{mol}} / V_{\mathrm{A}}^{\mathrm{mol}}\right) \cdot\left(1-K_{\mathrm{B}} \cdot \varphi_{1 \mathrm{~B}}\right)+K_{\mathrm{AB}} \cdot \varphi_{1 \mathrm{~B}}}\right] \\
G^{\mathrm{E}}(\mathrm{CHEM})=R \cdot T \cdot\left[x_{\mathrm{A}} \cdot \ln \left(\frac{\varphi_{1 \mathrm{~A}}}{x_{\mathrm{A}} \cdot \varphi_{1 \mathrm{~A}}^{0}}\right)+x_{\mathrm{B}} \cdot \ln \left(\frac{\varphi_{1 \mathrm{~B}}}{x_{\mathrm{B}} \cdot \varphi_{1 \mathrm{~B}}^{0}}\right)-\frac{V_{\mathrm{M}}}{V}+\frac{x_{\mathrm{A}} \cdot V_{\mathrm{A}}^{\mathrm{mol}}}{V_{\mathrm{A}}^{0}}+\frac{x_{\mathrm{B}} \cdot V_{\mathrm{B}}^{\mathrm{mol}}}{V_{\mathrm{B}}^{0}}\right]
\end{gathered}
$$

donde $\varphi_{1 \mathrm{~A}}$ у $\varphi_{1 \mathrm{~B}}$ son las soluciones de las ecuaciones siguientes:

$$
\begin{gathered}
\Phi_{\mathrm{A}}=\frac{\varphi_{1 \mathrm{~A}}}{\left(1-K_{\mathrm{A}} \cdot \varphi_{1 \mathrm{~A}}\right)^{2}} \cdot\left[1+\frac{\left(V_{\mathrm{A}}^{\mathrm{mol}} \cdot K_{\mathrm{AB}} \cdot \varphi_{1 \mathrm{~B}}\right)}{V_{\mathrm{B}}^{\mathrm{mol}} \cdot\left(1-K_{\mathrm{B}} \cdot \varphi_{1 \mathrm{~B}}\right)}\right] \\
\Phi_{B}=\frac{\varphi_{1 \mathrm{~B}}}{\left(1-K_{\mathrm{B}} \cdot \varphi_{1 \mathrm{~B}}\right)^{2}} \cdot\left[1+\frac{\left(K_{\mathrm{AB}} \cdot \varphi_{1 \mathrm{~A}}\right)}{\left(1-K_{\mathrm{AB}} \cdot \varphi_{1 \mathrm{~A}}\right)}\right]
\end{gathered}
$$

y $\Phi_{\mathrm{i}}(\mathrm{i}=\mathrm{A}, \mathrm{B})$ son las fracciones de volumen de "core" dadas por la ecuación (6.352) y $\varphi_{1 \mathrm{i}}^{0}=\lim _{\Phi_{\mathrm{i}} \rightarrow 1}\left(\varphi_{1 \mathrm{i}}\right)$ de (6.351). En la ecuación (6.375):

$$
\frac{1}{V}=\frac{\varphi_{1 \mathrm{~A}}}{V_{\mathrm{A}}^{\mathrm{mol}} \cdot\left(1-K_{\mathrm{A}} \cdot \varphi_{1 \mathrm{~A}}\right)}+\frac{\varphi_{1 \mathrm{~B}}}{V_{\mathrm{B}}^{\mathrm{mol}} \cdot\left(1-K_{\mathrm{B}} \cdot \varphi_{1 \mathrm{~B}}\right)}+
$$




$$
+\frac{K_{\mathrm{AB}} \cdot \varphi_{1 \mathrm{~A}} \cdot \varphi_{1 \mathrm{~B}}}{V_{\mathrm{B}}^{m o l} \cdot\left(1-K_{\mathrm{A}} \cdot \varphi_{1 \mathrm{~A}}\right) \cdot\left(1-K_{\mathrm{B}} \cdot \varphi_{1 \mathrm{~B}}\right)}
$$

donde,

$$
\begin{aligned}
& \frac{1}{V_{\mathrm{A}}^{0}}=\lim _{\Phi_{\mathrm{A}} \rightarrow 1} \frac{1}{V} \\
& \frac{1}{V_{\mathrm{B}}^{0}}=\lim _{\Phi_{\mathrm{A}} \rightarrow 0} \frac{1}{V}
\end{aligned}
$$

Las contribuciones físicas de $H_{\mathrm{m}}^{\mathrm{E}}, V_{\mathrm{m}}^{\mathrm{E}}$ y $G_{\mathrm{m}}^{\mathrm{E}}$, para sistemas formados por dos componentes asociados, vienen dadas por las ecuaciones (6.358), (6.359) y (6.371), es decir, coinciden con aquellas expresiones encontradas para las mezclas compuestas por un componente asociado y uno inerte.

\subsubsection{Métodos de contribución de grupos funcionales}

La utilización de los MMCCGGFF (acrónimo en español de Métodos de Contribución de Grupos Funcionales) fue sugerida por primera vez por Langmuir en 1925 [400] aunque no fue hasta 1959 que comenzaron a emplearse de manera sistemática para el estudio de las propiedades de exceso de disoluciones de no electrolitos [401]- [404]. Todos estos métodos tienen en cuenta que la estructura de cada molécula está formada por un conjunto de grupos funcionales diferentes. Las hipótesis básicas [405], [406] de ellos son:

a) Dada una definición conveniente de grupo funcional, la energía potencial de interacción intermolecular, $W$, está dada por la suma de las energías potenciales de interacción entre los diferentes grupos, lo que define el modelo microscópico de interacción molecular utilizado por estos métodos.

b) Cada grupo funcional se comporta independientemente de la molécula a la que pertenece.

c) Diferentes grupos funcionales tienen diferentes tamaños. Por eso se asigna a cada grupo funcional un área superficial propia. Para estimar tanto la superficie como el volumen de estos grupos funcionales, puede adoptarse por ejemplo el método de Bondi [353].

En consecuencia, a la hora de interaccionar con otros grupos se considera que cada interacción (o contacto) contribuye siempre en la misma cantidad a la energía de interacción entre las moléculas, y que el número (frecuencia) de dichos contactos depende de su área superficial (superficie de contacto). Por eso las propiedades de exceso de las mezclas líquidas se obtienen a partir de las energías interfaciales de interacción entre grupos (cada una característica del par de grupos distintos en contacto), sopesadas estadísticamente de acuerdo con la fracción de superficie de los respectivos tipos de superficies en las moléculas respectivas, y de acuerdo con la fracción de superficie total de los diversos componentes en la mezcla. Es lo que Langmuir denominó "Principio de acción de superficies independientes".

Pese a su carácter aproximado, los MMCCGGFF son muy importantes ya que sus dos características básicas, independencia del comportamiento de cada grupo funcional, e independencia de la superficie de contacto de cada grupo, ambos respecto de la 
molécula a la que el grupo pertenece, transfieren al método un carácter predictivo, puesto que una vez obtenida la información de las interacciones grupol-grupo2 a partir de la correlación de datos experimentales de sistemas binarios, se pueden calcular las interacciones molécula-molécula de cualquier par de moléculas que contengan los mismos grupos, aunque no haya disponibles datos experimentales. Además, resulta ventajoso que el número de grupos funcionales distintos sea mucho menor que el número de moléculas diferentes.

Mientras que los modelos de mezclas líquidas no basados en los métodos de contribución de grupos expresan las propiedades termodinámicas en función de uno o varios parámetros característicos de la mezcla, los cuales, en general, se desconoce como estimarlos, y deben ser ajustados, el método de contribución de grupos funcionales permite expresar el comportamiento termodinámico de las mezclas como resultado de las interacciones entre grupos funcionales, lo que supone un ahorro significativo del número de parámetros ajustables.

No tiene sentido aplicar métodos de contribución de grupos a compuestos con un solo grupo, que nunca han sido investigados en mezclas con moléculas polisegmentadas. Esto conforma el límite inferior de aplicabilidad de los métodos de contribución de grupos funcionales. Por el contrario, cuando se define un grupo que es muy grande con respecto el promedio de las distancias intermoleculares, su potencial de interacción puede ser tan complejo que ninguna teoría pueda describirlo convenientemente, alcanzando el límite superior de aplicabilidad del método. Afortunadamente, entre ambos límites hay un margen suficiente como para aplicar los MMCCGGFF a una gran cantidad de mezclas de compuestos orgánicos.

Por las razones expuestas, la aplicación de un método de contribución de grupos requiere, en primer lugar, una definición clara del conjunto de grupos funcionales considerados, puesto que la calidad de las predicciones depende de la identificación realizada de cada grupo funcional. El factor más importante que condiciona la definición de cada grupo funcional está dado por los efectos intramoleculares. En efecto, la hipótesis de que cada grupo funcional se comporta independientemente de la molécula a la que pertenece es solamente cierta si el grupo funcional se encuentra en un ambiente intramolecular dado, siendo el más común el debido a la presencia de segmentos de cadenas de alcanos, es decir, de los grupos $\mathrm{CH}_{2}$ y $\mathrm{CH}_{3}$. La proximidad de dos o más grupos iguales o distintos dentro de la molécula cambia drásticamente los parámetros de la energía de interacción. En estos casos, se debe redefinir el grupo funcional: el nuevo grupo funcional será uno mayor, que contendrá varios grupos funcionales simples, teniendo cuidado de no alcanzar el límite superior de aplicabilidad (Véase la llustración 1.1).

Muchos modelos de contribución de grupos comienzan expresando $G_{\mathrm{m}}^{\mathrm{E}}$ como la suma de las contribuciones combinatorial (diferencias de tamaño, forma, cruce de moléculas, etc.) y residual (debido a que las mezclas son no atérmicas, es decir, a las interacciones entre las moléculas). Los logaritmos de los coeficientes de actividad se pueden escribir pues como:

$$
\ln f_{\mathrm{i}}=\ln f_{\mathrm{i}}(\mathrm{COMB})+\ln f_{\mathrm{i}}(\mathrm{RES})
$$

donde, 


$$
\ln f_{\mathrm{i}}(\mathrm{RES})=\sum_{s} v_{\mathrm{si}} \cdot\left(\ln r_{\mathrm{s}}-\ln r_{\mathrm{s}}^{(\mathrm{i})}\right)
$$

$v_{\mathrm{si}}$ es el número de grupos del tipo s en la molécula del tipo $\mathrm{i} ; r_{\mathrm{s}}^{(\mathrm{i})}$ es el coeficiente de actividad del grupo s cuando el componente i se encuentra en estado puro; $r_{\mathrm{s}}$ es el coeficiente de actividad para el grupo s en la mezcla, definido a partir de la ecuación siguiente:

$$
G_{\mathrm{s}}=\frac{\partial G(\mathrm{RES})}{\partial n_{\mathrm{s}}}=G_{\mathrm{s}}^{\cdot}+R \cdot T \cdot \ln r_{\mathrm{s}}
$$

$G_{\mathrm{s}}^{*}$ es el potencial químico del grupo s puro; $G(\mathrm{RES})$ es la función de Gibbs residual, una función extensiva con respecto al número $n_{\mathrm{s}}$ de grupos del tipo s presentes en la mezcla: $n_{\mathrm{s}}=\sum_{i} v_{\mathrm{si}} \cdot n_{\mathrm{i}}$.

La hipótesis básica a) concierne solamente al modelo de interacción molecular, no al modelo estadístico utilizado para describir el sistema. Por ello, se puede formular un modelo estadístico de contribución de grupos en base a cualquiera de los modelos estadísticos ya descritos, por ejemplo, la versión en términos del método de contribución de grupos funcionales del modelo de disoluciones estrictamente regulares de Guggenheim en las aproximaciones de orden cero y de orden uno [405]-[408], [379], [380], lo que da lugar al modelo DISQUAC que se expondrá detalladamente más adelante.

Wilson y Deal [404], [369] formularon por primera vez de manera coherente un método de contribución de grupos funcionales, utilizando para $\ln f_{\mathrm{i}}(\mathrm{COMB})$ la ecuación de Flory-Huggins, generalizado más tarde para obtener entalpías de exceso [409], y modificado nuevamente [410] para la utilización del principio de congruencia de Brönsted y Koefed [4 1 1] en la obtención de una expresión para ln $f_{\mathrm{i}}(\mathrm{COMB})$.

Utilizando la ecuación de Flory-Huggins para la contribución combinatorial y como forma funcional de los coeficientes de actividad de los grupos la ecuación de Wilson se obtiene el método ASOG (Analytical SOlution Of Groups) [413]-[415], que permite estimar $G_{\mathrm{m}}^{\mathrm{E}}$. Una extensión del método para estimar las $H_{\mathrm{m}}^{\mathrm{E}}$ da lugar al modelo AGSM [416]. Sus principales características son la elección arbitraria de los $v_{\mathrm{si}}$ con el objetivo de dar cuenta del mayor número posible de propiedades, así como en que para los parámetros energéticos de la ecuación de Wilson, los cuales dependen de la temperatura, se han adaptado diferentes formas funcionales que muestran esta dependencia. Además, las limitaciones de la ecuación de Wilson impiden la aplicabilidad general del método.

El método UNIFAC es un MCGGFF basado en la ecuación UNIQUAC. Se ha aplicado con éxito al estudio de ELV, ELL de sistemas binarios y multicomponentes. Sin embargo, se obtienen resultados poco satisfactorios para los coeficientes de actividad a dilución infinita, calores de mezcla y para el estudio de sistemas asimétricos [418]. Por esta razón se desarrollaron diferentes variantes del método. Así, Nagata y Ohta [419] aplican el modelo UNIFAC a las $H_{\mathrm{m}}^{\mathrm{E}}$ utilizando la relación de Gibbs-Helmholtz, logrando ampliar así su campo de trabajo a la predicción de las entalpías de mezcla. Este modelo se utilizó [420], [421] para obtener un conjunto de valores de parámetros de interacción a 
partir de los cuales se pueda estimar las $H_{\mathrm{m}}^{\mathrm{E}}$. Stathis y Tassios [422] propusieron el modelo UNIFAC/ ASSOCIATION, incluyendo un término de contribución química al modelo elaborado anteriormente, para considerar los efectos de asociación de las moléculas. Otra modificación el modelo UNIFAC [423] permite predecir el equilibrio líquido-vapor, el equilibrio líquido-líquido, las entalpías y las capacidades térmicas de exceso, con el mismo conjunto de parámetros. Weidlich y Gmehling [424] modificaron UNIFAC introduciendo una parte combinatorial empírica, parámetros de interacción dependientes de la temperatura y definiendo nuevos grupos funcionales. El método resultante es el conocido UNIFAC-versión Dortmund. La principal ventaja de este modelo es la gran matriz de parámetros de interacción que posee, lo que permite su aplicación a una gran variedad de mezclas [425]-[428].

Muchos otros métodos de contribución de grupos han sido propuestos, entre los que destacan los siguientes: GTASQUAC (Group contribution TAylor Series aproximation for QUAsi-Chemical equilibria), que es un desarrollo en serie de Taylor de la aproximación cuasiquímica en términos de las interacciones entre grupos funcionales [429]. La naturaleza de la expansión en serie solo permite obtener aproximaciones de las ecuaciones cuasiquímicas; COSMO-RS (Conductor-like Screening MOdel for Real Solutions), es un modelo que requiere información cuántica a priori (potencial de interacción), por lo que tiene carácter predictivo [430]; GECUAC (Group-surface Explicit QUAsi-Chemical theory), el cual se ha aplicado a mezclas de líquidos asociados y no asociados [431]-[434]; COSMOSPACE (COSMO Surface-Pair Activity Coefficiennt Equation), el cual proporciona una solución exacta al modelo de red, supuesta la aditividad por pares de las energías de interacción entre las superficies de las moléculas, seguramente mediante algún mecanismo de renormalización [435]. Se puede ver como la solución analítica al modelo de superficies de interacción de los modelos COSMO-RS, UNIFAC y la teoría cuasiquímica de Guggenheim; Modelo de Nitta-Chao [436]; MOQUAC (Molecular Orientation based QUAsi-Chemical theory), que es un modelo que tiene en cuenta la estructura molecular tridimensional y no necesita el uso de ninguna hipótesis que conduzca a la pérdida de información acerca de la geometría molecular [437], [438], etc.

\subsubsection{Modelo de interacción de grupos funcionales de Kehiaian-Grolier-Benson}

Se trata del modelo de red de Guggenheim-Barker, formulado en términos de superficies de interacción mediante el método de contribución de grupos [379], [380], [405]-[408], [439]-[450]. Su base microscópica está mucho mejor establecida que la de otros modelos equivalentes, tanto en lo que respecta al modelo de interacción molecular adoptado, como al modelo estadístico (red de Guggenheim-Barker). En vez de distribuir las moléculas en los nodos de la red, se distribuyen grupos funcionales, los cuales interaccionan por medio de sus superficies.

\subsubsection{Definiciones e hipótesis de partida. Modelo de interacción molecular}

El modelo es un método de contribución de grupos funcionales, luego está basado en las hipótesis discutidas en la sección 6.4.8.

Se considera que en la mezcla líquida hay $\mathrm{n}$ tipos diferentes de molécula (es decir, $\mathrm{n}$ componentes), que cada molécula está integrada por varios grupos funcionales y que hay presentes un total de $\sigma$ grupos funcionales en la mezcla. Sea $N_{\mathrm{i}}$ el número total de 
moléculas del tipo i $(\mathrm{i}=1,2, \ldots, \mathrm{n})$. Por lo tanto, el número total de moléculas en la mezcla, $N$, y la fracción molar del componente i en la mezcla, $x_{\mathrm{i}}$, son:

$$
\begin{gathered}
N=\sum_{\mathrm{i}=1}^{\mathrm{n}} N_{\mathrm{i}} \\
x_{\mathrm{i}}=\frac{N_{\mathrm{i}}}{N}
\end{gathered}
$$

Sea $q_{\mathrm{i}}$ la superficie de contacto de una molécula del tipo i. Se define la superficie total de contacto de todas las moléculas del tipo i en la mezcla, $A_{\mathrm{i}}$, la superficie total de contacto en la mezcla, $A$, y la fracción de superficie de contacto del componente i en la mezcla, $\xi_{\mathrm{i}}$, a partir de las relaciones siguientes:

$$
\begin{gathered}
A_{\mathrm{i}}=N_{\mathrm{i}} \cdot q_{\mathrm{i}} \\
A=\sum_{\mathrm{i}=1}^{\mathrm{n}} A_{\mathrm{i}} \\
\xi_{\mathrm{i}}=\frac{A_{\mathrm{i}}}{A}
\end{gathered}
$$

Sea $r_{\mathrm{i}}$ el volumen de una molécula del tipo i. Como el modelo está basado en el modelo de red de Guggenheim, no hay cambios de volumen. En consecuencia, se define el volumen de la mezcla, $V$, el volumen total de las moléculas del tipo i, $V_{\mathrm{i}}$, y la fracción de volumen del componente i en la mezcla, $\Phi_{\mathrm{i}}$, a partir de las relaciones siguientes:

$$
\begin{gathered}
V_{\mathrm{i}}=N_{\mathrm{i}} \cdot r_{\mathrm{i}} \\
V=\sum_{\mathrm{i}=1}^{\mathrm{n}} V_{\mathrm{i}} \\
\Phi_{\mathrm{i}}=\frac{V_{\mathrm{i}}}{V}
\end{gathered}
$$

Sea $q_{\mathrm{si}}$ la superficie de contacto del grupo funcional de tipo s en una molécula del tipo i. Se define la superficie total de contacto de los grupos tipo s en la mezcla, $A_{\mathrm{s}}$ la fracción de superficie de contacto del grupo tipo s en una molécula i, $\alpha_{\text {si, }}$ y la fracción de superficie total de contacto del grupo tipo s en la mezcla, $\alpha_{s}$ a las cantidades siguientes:

$$
\begin{gathered}
A_{\mathrm{s}}=\sum_{\mathrm{i}=1}^{\mathrm{n}} N_{\mathrm{i}} \cdot q_{\mathrm{si}} \\
\alpha_{\mathrm{si}}=\frac{q_{\mathrm{si}}}{q_{\mathrm{i}}} \\
\alpha_{\mathrm{s}}=\frac{A_{\mathrm{s}}}{A}
\end{gathered}
$$

De acuerdo con estas definiciones se verifican las igualdades siguientes:

$$
\sum_{s} \alpha_{\mathrm{si}}=1
$$




$$
\sum_{s} \alpha_{\mathrm{s}}=1
$$

Cada una de las diferentes maneras de ordenar los grupos funcionales sobre la estructura cuasicristalina definen una configuración. Dada una configuración c, se define $A_{\mathrm{ss}}^{\mathrm{c}}$ como el área de la superficie total de contacto entre dos grupos funcionales del tipo s, y $A_{\mathrm{st}}^{\mathrm{c}}$ como el área de la superficie de contacto entre un grupo funcional del tipo $s$ y otro grupo funcional del tipo $t$, supuestos $s$ y $t$ distintos.

Asumiendo que la superficie de un grupo funcional del tipo s se encuentra en contacto, o bien con otra superficie del mismo tipo s, o bien con una superficie de un tipo diferente $\mathrm{t}$, debe cumplirse la ecuación de conservación de la superficie:

$$
A_{\mathrm{s}}=2 \cdot A_{\mathrm{ss}}^{\mathrm{c}}+\sum_{\substack{\mathrm{t}=1 \\ \mathrm{t} \neq \mathrm{s}}}^{\sigma} A_{\mathrm{st}}^{\mathrm{c}}
$$

De acuerdo con el planteamiento que el método de contribución de grupos conlleva, la energía configuracional total $U^{\mathfrak{C}}(\mathrm{CONF})$ del sistema en una configuración c del sistema, la cual coincide con el promedio de la energía de interacción (véase la ecuación (6.60)), es igual a la suma de las energías de interacción de todos los contactos. Sean $\varepsilon_{\mathrm{ss}}$ y $\varepsilon_{\mathrm{st}}$ las energías de interacción por unidad de superficie de contacto entre dos superficies (ss) ó (st). Entonces:

$$
U^{\mathrm{c}}(\mathrm{CONF})=\sum_{\mathrm{s}=1}^{\sigma} A_{\mathrm{ss}}^{\mathrm{c}} \cdot \varepsilon_{\mathrm{ss}}+\sum_{\mathrm{s}=1}^{\sigma} \sum_{\mathrm{t}>\mathrm{s}}^{\sigma} A_{\mathrm{st}}^{\mathrm{c}} \cdot \varepsilon_{\mathrm{st}}
$$

Con el fin de simplificar la expresión anterior se define $A_{\mathrm{st}}^{\mathrm{c}}=A_{\mathrm{ts}}^{\mathrm{c}}$, de modo que:

$$
U^{\mathrm{c}}(\mathrm{CONF})=\sum_{\mathrm{s}=1}^{\sigma} A_{\mathrm{ss}}^{\mathrm{c}} \cdot \varepsilon_{\mathrm{ss}}+\frac{1}{2} \cdot \sum_{\mathrm{s}=1}^{\sigma} \sum_{\mathrm{t} \neq \mathrm{s}}^{\sigma} A_{\mathrm{st}}^{\mathrm{c}} \cdot \varepsilon_{\mathrm{st}}
$$

También es conveniente definir la energía de intercambio de los grupos $\mathrm{s}$ y $\mathrm{t}, \Delta \varepsilon_{\mathrm{st}}$, equivalente a la energía de intercambio del modelo de red, $w$ (véase la ecuación (6.138)):

$$
\Delta \varepsilon_{\mathrm{st}}=\varepsilon_{\mathrm{st}}-\frac{\varepsilon_{\mathrm{ss}}+\varepsilon_{\mathrm{tt}}}{2}
$$

Utilizando (6.397) y (6.400), la ecuación (6.399) se transforma en la siguiente:

$$
U^{\mathrm{c}}(\mathrm{CONF})=\frac{1}{2} \cdot \sum_{\mathrm{s}=1}^{\sigma} A_{\mathrm{s}} \cdot \varepsilon_{\mathrm{ss}}+\frac{1}{2} \cdot \sum_{\mathrm{s}=1}^{\sigma} \sum_{\mathrm{t} \neq \mathrm{s}}^{\sigma} A_{\mathrm{st}}^{\mathrm{c}} \cdot \Delta \varepsilon_{\mathrm{st}}
$$

Concretamente, en mezclas de moléculas homogéneas, formadas por un solo tipo de superficies $\circ$ grupos, donde $\Delta \varepsilon_{\mathrm{st}}=0$, la energía configuracional no depende de la configuración del sistema, de modo que para una composición dada $N_{\mathrm{i}}$ posee un valor constante e igual a:

$$
U^{*}(\mathrm{CONF})=\frac{1}{2} \cdot \sum_{\mathrm{s}=1}^{\sigma} A_{\mathrm{s}} \cdot \varepsilon_{\mathrm{ss}}
$$




\subsubsection{Modelo estadístico. Función de partición configuracional}

El modelo KGB adopta como modelo estadístico para describir el sistema el modelo de red de Guggenheim. Así pues, igual que en el modelo de red, la función de partición configuracional viene dada por:

$$
\Omega=\sum_{\mathrm{c}} e^{-U^{\mathrm{c}} / k_{\mathrm{B}} \cdot T}
$$

donde el sumatorio se extiende a todas las configuraciones posibles del sistema, cada una de ellas caracterizada por su energía configuracional $U^{\mathfrak{c}}$ (ecuación (6.401)). Sea $g_{\mathrm{c}}$ el factor combinatorio del sistema, es decir, el número de configuraciones correspondientes a un valor dado de $U^{\mathfrak{c}}$. Entonces, la ecuación anterior se reescribe:

$$
\begin{aligned}
& \Omega=\sum_{\mathrm{c}} g_{\mathrm{c}}\left(z, A, A_{\mathrm{ss}}^{\mathrm{c}}, A_{\mathrm{st}}^{\mathrm{c}}\right) \cdot e^{-U^{\mathrm{c}} / k_{\mathrm{B}} \cdot T} \approx\left[g_{\mathrm{c}}\left(z, A, A_{\mathrm{ss}}^{\mathrm{c}}, A_{\mathrm{st}}^{\mathrm{c}}\right) \cdot e^{-U^{\mathrm{c}} / k_{\mathrm{B}} \cdot T}\right]_{\mathrm{máx}}= \\
= & g\left(z, A, A_{\mathrm{ss}}, A_{\mathrm{st}}\right) \cdot e^{-U / k_{\mathrm{B}} \cdot T}=g\left(z, A, A_{\mathrm{ss}}, A_{\mathrm{st}}\right) \cdot e^{-\left(\frac{1}{2} \cdot \sum_{\mathrm{s}=1}^{\sigma} A_{\mathrm{s}} \cdot \varepsilon_{\mathrm{ss}}+\frac{1}{2} \sum_{\mathrm{s}=1}^{\sigma} \sum_{\mathrm{t} \neq \mathrm{s}}^{\sigma} A_{\mathrm{st}} \cdot \Delta \varepsilon_{\mathrm{st}}\right) / k_{\mathrm{B}} \cdot T}
\end{aligned}
$$

La ecuación anterior es aproximada pues se ha utilizado el método del término máximo. Sin embargo, existe un procedimiento que permite expresar la función de partición de forma similar pero sin perder la exactitud. Este procedimiento consiste en suponer que $\varepsilon_{\mathrm{ss}}$ y $\Delta \varepsilon_{\text {st }}$ son funciones desconocidas de la temperatura, $\omega_{\mathrm{ss}}$ y $\Delta \omega_{\mathrm{st}}$ respectivamente:

$$
\Omega=g\left(z, A, A_{\mathrm{ss}}, A_{\mathrm{st}}\right) \cdot e^{\left.-\left(\frac{1}{2} \cdot \sum_{\mathrm{s}=1}^{\sigma} A_{\mathrm{s}} \cdot \omega_{\mathrm{ss}}(T)+\frac{1}{2} \cdot \sum_{\mathrm{s}=1}^{\sigma} \sum_{\mathrm{t} \neq \mathrm{s}}^{\sigma} A_{\mathrm{st}} \cdot \Delta \omega_{\mathrm{st}} T\right)\right) / k_{\mathrm{B}} \cdot T}
$$

Al igual que en el modelo de red (véase la sección 6.4.1.1), la dependencia con la temperatura de estos parámetros energéticos no introduce cambios en la forma de las propiedades de exceso salvo en aquellas que se obtienen por diferenciación respecto de la temperatura del potencial de Helmholtz $F$.

$U$ y $g$ se llaman energía configuracional y factor combinatorial de equilibrio, respectivamente, y son tales que el término $g \cdot e^{-U / k_{\mathrm{B}} \cdot T}$ es mucho mayor que el resto en el sumatorio y se obtienen calculando el máximo de la función $g_{\mathrm{c}} \cdot e^{-U \mathrm{c} / k_{\mathrm{B}} \cdot T}$. Para ello basta con calcular las derivadas parciales de $\ln \Omega$ respecto las variables $A_{\mathrm{st}}^{\mathrm{c}}$ y resolver el sistema de ecuaciones obtenido al igualar a cero todas estas derivadas. Pero antes de ello es necesario escribir $g$ como función de $A_{\mathrm{st}}^{\mathrm{c}}$. Para ello se sigue el mismo razonamiento utilizado por Guggenheim en la aproximación cuasiquímica del modelo de red. Por tanto, se admite que existe un cierto número de diferentes maneras de realizarse un contacto entre dos elementos dados de superficie, el cual coincide con el número de coordinación $z$ y representa el número de posibles orientaciones que pueden existir entre dos superficies de contacto. Por tanto, $z \cdot A / 2$ es el número total de orientaciones. $z \cdot A_{\mathrm{ss}}$ es el número de orientaciones para los contactos entre grupos funcionales del tipo s, y $z \cdot A_{\mathrm{st}}$ es el número de orientaciones para los contactos entre un grupo funcional del tipo s y otro del tipo t.

El número de configuraciones posibles del sistema para una energía $U^{\mathfrak{c}}$ no puede resolverse, como sabemos, de manera exacta. En lugar de ello, existen dos aproximaciones: la aproximación de orden cero o de Bragg-Williams, y la aproximación de orden uno, cuasiquímica o de Bethe. En la aproximación de orden cero se supone 
que a pesar de que los efectos energéticos no son despreciables, no son lo suficientemente importantes como para impedir la mezcla al azar de los diferentes grupos funcionales presentes en la mezcla. Por el contrario, la aproximación cuasiquímica tiene en cuenta la no aleatoriedad del proceso de mezcla, considerando que cada grupo es capaz de interaccionar con sus vecinos más próximos, dando lugar a orientaciones privilegiadas de interacción. El número de dichas orientaciones está caracterizado por el índice de coordinación de la red, pues $z$ determina el número de grupos que se encuentran en torno al grupo de interés y que son capaces de interaccionar con él. La esencia de la aproximación cuasiquímica es suponer que durante la mezcla los pares de contactos entre grupos distintos que se forman no interfieren los unos con los otros, lo cual no es realmente cierto pero es un truco matemático que proporciona resultados aceptables en determinadas condiciones, sobre todo lejos de los puntos críticos.

a) En la aproximación cuasiquímica, el factor combinatorio tiene una expresión similar a la (6.151) del modelo de red de Guggenheim en la aproximación cuasiquímica:

$$
g_{\mathrm{c}}\left(z, A, A_{\mathrm{ss}}^{\mathrm{c}}, A_{\mathrm{st}}^{\mathrm{c}}\right)=h\left(N_{\mathrm{A}}, N_{\mathrm{B}}\right) \cdot \frac{\left(\frac{z}{2} \cdot A\right) !}{\prod_{\mathrm{s}}\left(z \cdot A_{\mathrm{ss}}^{\mathrm{c}}\right) ! \cdot \prod_{\mathrm{s}} \prod_{\mathrm{s} \neq \mathrm{t}}\left(\frac{z}{2} \cdot A_{\mathrm{st}}^{\mathrm{c}}\right) !}
$$

$h\left(N_{\mathrm{A}}, N_{\mathrm{B}}\right)$ es un factor que trata de corregir el hecho de cuando se suma $g_{\mathrm{c}}$ a todas las configuraciones posibles no proporciona el número total de configuraciones correcto. Hay que destacar que en la mayoría de los trabajos en donde aparece explicado el modelo, se indica incorrectamente que dicho factor tiene en cuenta la interferencia de los contactos de los diferentes grupos. Si así fuese, estaría resuelto el caso general y no se estaría hablando de una aproximación, como realmente y por desgracia es el caso.

Para hacer uso de la ecuación (6.405) es necesario determinar que valores de $A_{\text {ss }}$ y $A_{\text {st }}$ maximizan la expresión:

$$
g_{\mathrm{c}}\left(z, A, A_{\mathrm{ss}}, A_{\mathrm{st}}\right) \cdot e^{-\left(\frac{1}{2} \cdot \sum_{\mathrm{s}=1}^{\sigma} A_{\mathrm{s}} \cdot \omega_{\mathrm{ss}}(T)+\frac{1}{2} \cdot \sum_{\mathrm{s}=1}^{\sigma} \sum_{\mathrm{t} \neq \mathrm{s}}^{\sigma} A_{\mathrm{st}}^{\mathrm{c}} \Delta \omega_{\mathrm{st}}(T)\right) / k_{\mathrm{B}} \cdot T}
$$

El procedimiento seguido es el siguiente, el cual utiliza la aproximación de Sterling $\ln N ! \approx N \cdot \ln N-N$ :

$$
\begin{gathered}
\frac{\partial \ln \Omega}{\partial A_{\mathrm{st}}}=0 \\
\frac{\partial}{\partial A_{\mathrm{st}}}\left[\ln g_{\mathrm{c}}-\frac{\frac{1}{2} \cdot \sum_{\mathrm{s}=1}^{\sigma} A_{\mathrm{s}} \cdot \omega_{\mathrm{ss}}(T)+\frac{1}{2} \cdot \sum_{\mathrm{s}=1}^{\sigma} \sum_{\mathrm{t} \neq \mathrm{s}}^{\sigma} A_{\mathrm{st}}^{\mathrm{c}} \cdot \Delta \omega_{\mathrm{st}}(T)}{k_{\mathrm{B}} \cdot T}\right]=0 \\
-\frac{z}{2} \ln \left(\frac{A_{\mathrm{st}}^{2}}{4 \cdot A_{\mathrm{ss}} \cdot A_{\mathrm{tt}}}\right)-\frac{\Delta \omega_{\mathrm{st}}(T)}{k_{\mathrm{B}} \cdot T}=0 \\
A_{\mathrm{st}}^{2}=4 \cdot A_{\mathrm{ss}} \cdot A_{\mathrm{tt}} \cdot\left(\eta_{\mathrm{st}}\right)^{2}
\end{gathered}
$$




$$
\eta_{\mathrm{st}}=e^{-\frac{\Delta \omega_{\mathrm{st}}(T)}{z \cdot k_{\mathrm{B}} \cdot T}}
$$

La energía configuracional de equilibrio de la mezcla en la aproximación cuasiquímica se puede escribir en función del parámetro $\eta_{\text {st }}$ como sigue:

$$
U(\mathrm{CONF}, \mathrm{QUAC})=\frac{1}{2} \cdot\left(\sum_{\mathrm{i}=1} N_{\mathrm{i}} \cdot q_{\mathrm{i}}\right) \cdot\left(\sum_{\mathrm{s}=1}^{\sigma} \alpha_{\mathrm{s}} \cdot \omega_{\mathrm{ss}}+\sum_{\mathrm{s}=1}^{\sigma} \sum_{\mathrm{t} \neq \mathrm{s}}^{\sigma} X_{\mathrm{s}} \cdot X_{\mathrm{t}} \cdot \eta_{\mathrm{st}} \cdot \Delta \omega_{\mathrm{st}}\right)
$$

Mientras que para $N_{\mathrm{i}}$ moléculas del componente puro i, resulta ser:

$$
U_{\mathrm{i}}(\mathrm{CONF}, \mathrm{QUAC})=\frac{1}{2} \cdot N_{\mathrm{i}} \cdot q_{\mathrm{i}} \cdot\left(\sum_{\mathrm{s}=1}^{\sigma} \alpha_{\mathrm{si}} \cdot \omega_{\mathrm{ss}}+\sum_{\mathrm{s}=1}^{\sigma} \sum_{\mathrm{t} \neq \mathrm{s}}^{\sigma} X_{\mathrm{si}} \cdot X_{\mathrm{ti}} \cdot \eta_{\mathrm{st}} \cdot \Delta \omega_{\mathrm{st}}\right)
$$

El problema de maximización se reduce a resolver el sistema de ecuaciones (6.397) y (6.411):

$$
\begin{gathered}
A_{\mathrm{s}}=2 \cdot A_{\mathrm{ss}}+\sum_{\substack{\mathrm{t}=1 \\
\mathrm{t} \neq \mathrm{s}}}^{\sigma} A_{\mathrm{st}} \\
A_{\mathrm{st}}^{2}=4 \cdot A_{\mathrm{ss}} \cdot A_{\mathrm{tt}} \cdot\left(\eta_{\mathrm{st}}\right)^{2}
\end{gathered}
$$

Para ello, es útil realizar el cambio de variables siguiente:

$$
\begin{gathered}
A_{\mathrm{ss}}=\frac{1}{2} \cdot A \cdot X_{\mathrm{s}}^{2} \\
A_{\mathrm{st}}=A \cdot X_{\mathrm{s}} \cdot X_{\mathrm{t}} \cdot \eta_{\mathrm{st}}
\end{gathered}
$$

con el cual se consigue reducir el sistema de ecuaciones (6.397) y (6.411) al siguiente:

$$
X_{\mathrm{s}} \cdot\left(X_{\mathrm{s}}+\sum_{\mathrm{t} \neq \mathrm{s}} X_{\mathrm{t}} \cdot \eta_{\mathrm{st}}\right)=\alpha_{\mathrm{s}} \mathrm{s}, \mathrm{t}=1,2, \ldots, \sigma
$$

Este sistema de ecuaciones no lineal debe resolverse numéricamente. Sus soluciones permiten calcular $g\left(z, A, A_{\mathrm{ss}}, A_{\mathrm{st}}\right), \Omega$, las propiedades termodinámicas configuracionales, de exceso, etc. Por ejemplo:

$$
\begin{gathered}
g\left(z, A, A_{\mathrm{ss}}, A_{\mathrm{st}}\right)=h\left(N_{\mathrm{A}}, N_{\mathrm{B}}\right) \cdot \frac{\left(\frac{z}{2} \cdot A\right) !}{\prod_{\mathrm{s}}\left(z \cdot A_{\mathrm{ss}}\right) ! \cdot \prod_{\mathrm{s}} \prod_{\mathrm{s} \neq \mathrm{t}}\left(\frac{z}{2} \cdot A_{\mathrm{st}}\right) !} \\
\Omega=g\left(z, A, A_{\mathrm{ss}}, A_{\mathrm{st}}\right) \cdot e^{\left.-\left(\frac{1}{2} \cdot \sum_{\mathrm{s}=1}^{\sigma} A_{\mathrm{s}} \cdot \omega_{\mathrm{ss}}(T)+\frac{1}{2} \cdot \sum_{\mathrm{s}=1}^{\sigma} \sum_{\mathrm{t} \neq \mathrm{s}}^{\sigma} A_{\mathrm{st}} \cdot \Delta \omega_{\mathrm{st}} T\right)\right) / k_{\mathrm{B}} \cdot T} \\
F(\mathrm{CONF}, \mathrm{QUAC})=k_{B} \cdot T \cdot \ln \Omega \\
U(\mathrm{CONF}, \mathrm{QUAC})=-T^{2} \cdot\left(\frac{\partial F(\mathrm{CONF}, \mathrm{QUAC}) / T}{\partial T}\right)_{V, N}=k_{B} \cdot T^{2} \cdot\left(\frac{\partial \ln \Omega}{\partial T}\right)_{V, N}
\end{gathered}
$$

La función de Helmholtz molar de exceso se puede calcular a partir de la ecuación (6.19) y (6.419), lo que permite calcular el potencial químico de exceso del componente 
i en la mezcla como sigue, realizando el conjunto de transformaciones necesarias [354] y teniendo en cuenta que en los métodos de contribución de grupos se considera el desdoblamiento de las magnitudes termodinámicas en parte combinatorial y parte residual, ecuación (6.381):

$$
\begin{aligned}
\frac{\mu_{\mathrm{i}}^{\mathrm{E}}}{R \cdot T} & =\frac{\partial\left(F_{\mathrm{m}}^{\mathrm{E}} / k_{\mathrm{B}} \cdot T\right)}{\partial N_{\mathrm{i}}}=\frac{\mu_{\mathrm{i}}^{\mathrm{E}}(\mathrm{COMB})}{R \cdot T}+\frac{\mu_{\mathrm{i}}(\mathrm{RES})}{R \cdot T}= \\
& =\ln \frac{\Phi_{\mathrm{i}}}{x_{\mathrm{i}}}+z \cdot q_{\mathrm{i}} \cdot \sum_{\mathrm{s}} \alpha_{\mathrm{si}} \cdot \ln \frac{X_{\mathrm{s}} \cdot \alpha_{\mathrm{si}}}{X_{\mathrm{si}} \cdot \alpha_{\mathrm{s}}}
\end{aligned}
$$

donde $\mu_{\mathrm{i}}^{\mathrm{E}}(\mathrm{COMB})$ es la contribución combinatorial al potencial químico de exceso, la cual se ha supuesto representada por la ecuación de Flory-Huggins (6.187), aunque se podía haber adoptado la ecuación análoga sin la aproximación $z \rightarrow \infty$, ecuación (6.186). $\mu_{\mathrm{i}}(\mathrm{RES})=\mu_{\mathrm{i}}(\mathrm{QUAC})$ es la contribución residual al potencial químico de exceso en la aproximación cuasiquímica del modelo adoptado.

Conocido el potencial químico de exceso, la función de Helmholtz molar de exceso y el potencial de Gibbs molar de exceso pueden calcularse a partir de las ecuaciones (2.196) y (2.197), teniendo en cuenta que en el modelo de red rígida $V_{\mathrm{m}}^{\mathrm{E}}=0$, lo que permite escribir $G_{\mathrm{m}}^{\mathrm{E}}=F_{\mathrm{m}}^{\mathrm{E}}$. Dicha ecuación puede modificarse y escribirse:

$$
F_{\mathrm{m}}^{\mathrm{E}}=G_{\mathrm{m}}^{\mathrm{E}}=\sum x_{\mathrm{i}} \cdot \mu_{\mathrm{i}}^{\mathrm{E}}
$$

Que de acuerdo con (6.422) y (6.187) se escribe:

$$
\begin{aligned}
& \frac{F_{\mathrm{m}}^{\mathrm{E}}}{R \cdot T}=\frac{G_{\mathrm{m}}^{\mathrm{E}}}{R \cdot T}=\frac{G_{\mathrm{m}}^{\mathrm{E}}(\mathrm{COMB})+G_{\mathrm{m}}(\mathrm{RES})}{R \cdot T}= \\
& =\sum x_{\mathrm{i}} \cdot\left[\ln \frac{\Phi_{\mathrm{i}}}{x_{\mathrm{i}}}+z \cdot q_{\mathrm{i}} \cdot \sum_{\mathrm{s}} \alpha_{\mathrm{si}} \cdot \ln \frac{X_{\mathrm{s}} \cdot \alpha_{\mathrm{si}}}{X_{\mathrm{si}} \cdot \alpha_{\mathrm{s}}}\right]
\end{aligned}
$$

donde $G_{\mathrm{m}}($ RES $)=G_{\mathrm{m}}(\mathrm{QUAC})$, es la parte residual de $G_{\mathrm{m}}$.

La energía interna molar de exceso, que es igual a la energía molar de mezcla, se obtiene fácilmente a partir de las ecuaciones (6.413) y (6.414):

$$
\begin{array}{r}
U_{\mathrm{m}}^{\mathrm{E}}(\mathrm{RES}, \mathrm{QUAC})=U_{\mathrm{m}}^{\mathrm{E}}(\mathrm{QUAC})=U_{\mathrm{m}}(\mathrm{CONF}, \mathrm{QUAC})-\sum_{\mathrm{i}} x_{\mathrm{i}} \cdot U_{\mathrm{i}}^{0}(\mathrm{CONF}, \mathrm{QUAC})= \\
=\frac{1}{2} \cdot\left(\sum_{\mathrm{i}=1} x_{\mathrm{i}} \cdot q_{\mathrm{i}}\right) \cdot\left[\sum_{\mathrm{s}=1}^{\sigma} \sum_{\mathrm{t} \neq \mathrm{s}}^{\sigma}\left(X_{\mathrm{s}} \cdot X_{\mathrm{t}}-\sum_{i} \xi_{\mathrm{i}} \cdot X_{\mathrm{si}} \cdot X_{\mathrm{ti}}\right) \cdot \eta_{\mathrm{st}} \cdot \Delta \omega_{\mathrm{st}}\right]
\end{array}
$$

Como es bien sabido, la energía interna molar de exceso no tiene parte combinatorial. Además, coincide con la entalpía molar de exceso $H_{\mathrm{m}}^{\mathrm{E}}$ pues en el modelo de red rígida $V_{\mathrm{m}}^{\mathrm{E}}=0$.

Es de todos conocido que el término $P \cdot V_{\mathrm{m}}^{\mathrm{E}}$ es despreciable frente a $F_{\mathrm{m}}^{\mathrm{E}}$ y $U_{\mathrm{m}}^{\mathrm{E}}$ en las mezclas líquidas a presiones moderadas, de modo que las identificaciones $G_{\mathrm{m}}^{\mathrm{E}} \approx F_{\mathrm{m}}^{\mathrm{E}}$ y $U_{\mathrm{m}}^{\mathrm{E}} \approx H_{\mathrm{m}}^{\mathrm{E}}$ son aceptables con buen grado de exactitud. Podría pensarse también ahora 
que la aparición de energías de intercambio dependientes de la temperatura es consecuencia de que el modelo no tiene en cuenta efectos de volumen libre. Sin embargo, es de destacar que en el modelo de Flory, expuesto en este Capítulo, ocurre exactamente lo mismo, siendo necesario introducir un parámetro adicional al que justifica $F_{\mathrm{m}}^{\mathrm{E}}$ y $U_{\mathrm{m}}^{\mathrm{E}}$ a fin de ajustar $G_{\mathrm{m}}^{\mathrm{E}}$. Ello prueba que la dependencia con la temperatura de las energías de intercambio no es un efecto de volumen libre, o al menos en el sentido que de éste proporciona la teoría de Flory. Por esto y para realizar de una manera coherente la comparación del modelo con los resultados experimentales, se introducen los parámetros de interacción $g_{\text {st }}$ y $h_{\text {st, }}$ que son una generalización empírica de $\Delta \omega_{\mathrm{st}}$ y $\Delta \varepsilon_{\mathrm{st}}$ y por lo tanto contienen información adicional que el modelo original no contempla, de modo que las ecuaciones obtenidas puedan asociarse a los valores de $G_{\mathrm{m}}^{\mathrm{E}}$ y $H_{\mathrm{m}}^{\mathrm{E}}$. Dichos parámetros se definen según las ecuaciones siguientes:

$$
\begin{gathered}
g_{\mathrm{st}}=N_{A} \cdot \Delta \omega_{\mathrm{st}}(T) \\
g_{\mathrm{ss}}=N_{A} \cdot \omega_{\mathrm{ss}}(T) \\
\frac{h_{\mathrm{st}}}{k_{\mathrm{B}} \cdot T}=N_{A} \cdot\left[-T \cdot \frac{\partial \Delta \omega_{\mathrm{st}} / k_{\mathrm{B}} \cdot T}{\partial T}\right] \\
\frac{h_{\mathrm{ss}}}{k_{\mathrm{B}} \cdot T}=N_{A} \cdot\left[-T \cdot \frac{\partial \omega_{\mathrm{ss}} / k_{\mathrm{B}} \cdot T}{\partial T}\right]
\end{gathered}
$$

De manera que:

$$
\eta_{\mathrm{st}}=e^{-\frac{g_{\mathrm{st}}}{z \cdot R \cdot T}}
$$

Luego, la entalpía molar de exceso es igual a:

$$
\begin{gathered}
H_{\mathrm{m}}^{\mathrm{E}}(\mathrm{QUAC})=U_{\mathrm{m}}^{\mathrm{E}}(\mathrm{QUAC})= \\
=\frac{1}{2} \cdot\left(\sum_{\mathrm{i}=1} x_{\mathrm{i}} \cdot q_{\mathrm{i}}\right) \cdot\left[\sum_{\mathrm{s}=1}^{\sigma} \sum_{\mathrm{t} \neq \mathrm{s}}^{\sigma}\left(X_{\mathrm{s}} \cdot X_{\mathrm{t}}-\sum_{i} \xi_{\mathrm{i}} \cdot X_{\mathrm{si}} \cdot X_{\mathrm{ti}}\right) \cdot \eta_{\mathrm{st}} \cdot h_{\mathrm{st}}\right]
\end{gathered}
$$

Donde $\eta_{\text {st }}, X_{\mathrm{s}}$ y $X_{\mathrm{si}}$ vienen dados por:

$$
\begin{aligned}
& \alpha_{\mathrm{s}}=X_{\mathrm{s}} \cdot\left(X_{\mathrm{s}}+\sum_{\mathrm{t} \neq \mathrm{s}} X_{\mathrm{t}} \cdot \eta_{\mathrm{st}}\right) \\
& \alpha_{\mathrm{si}}=X_{\mathrm{si}} \cdot\left(X_{\mathrm{si}}+\sum_{\mathrm{t} \neq \mathrm{s}} X_{\mathrm{ti}} \cdot \eta_{\mathrm{st}}\right)
\end{aligned}
$$

b) Establecidas las ecuaciones generales del modelo en la aproximación cuasiquímica, sus equivalentes en la aproximación de orden cero se obtienen formalmente cuando se realiza el paso al límite $z \rightarrow \infty\left(\eta_{\mathrm{st}} \rightarrow 1\right)$ en las mismas, lo que conduce a la hipótesis de mezcla al azar. En este caso, el procedimiento de maximización proporciona el sistema de ecuaciones siguiente, similar al (6.397) y (6.411): 


$$
\begin{aligned}
& A_{\mathrm{s}}^{*}=2 \cdot A_{\mathrm{ss}}^{*}+\sum_{\substack{\mathrm{t}=1 \\
\mathrm{t} \neq \mathrm{s}}}^{\sigma} A_{\mathrm{st}}^{*} \\
& \left(A_{\mathrm{st}}^{*}\right)^{2}=4 \cdot A_{\mathrm{ss}}^{*} \cdot A_{\mathrm{tt}}^{*}
\end{aligned}
$$

Se define el cambio de variables siguiente:

$$
\begin{aligned}
& A_{\mathrm{ss}}^{*}=\frac{1}{2} \cdot A \cdot\left(X_{\mathrm{s}}^{*}\right)^{2} \\
& A_{\mathrm{st}}^{*}=A \cdot X_{\mathrm{s}}^{*} \cdot X_{\mathrm{t}}^{*}
\end{aligned}
$$

Con este cambio de variables, la solución al sistema de ecuaciones (6.434)-(6.435) proporciona los valores de las superficies de equilibrio siguientes:

$$
\begin{aligned}
& X_{\mathrm{s}}^{*}=\alpha_{\mathrm{s}} \\
& X_{\mathrm{si}}^{*}=\alpha_{\mathrm{si}}
\end{aligned}
$$

Deshaciendo el cambio de variables con esta solución, reobtiene un relación similar a la (6.141) que exprsa la hipótesis de mezcla al azar en la teoría de disoluciones estrictamente regulares.

$$
\begin{gathered}
A_{\mathrm{ss}}^{*}=\frac{1}{2} \cdot A \cdot\left(\alpha_{\mathrm{s}}\right)^{2} \\
A_{\mathrm{st}}^{*}=A \cdot \alpha_{\mathrm{s}} \cdot \alpha_{\mathrm{t}}
\end{gathered}
$$

Por tanto, queda comprobado que el límite $z \rightarrow \infty$ conduce a la hipótesis de mezcla al azar. Las siguientes expresiones determinan $g^{*}\left(z, A, A_{\text {ss }}^{*} A_{\text {st }}^{*}\right), \Omega^{*}$, la función de Helmholtz $F^{*}$ y la energía interna $U^{*}$ para este límite:

$$
\begin{gathered}
g^{*}\left(z, A, A_{\mathrm{ss}}^{*}, A_{\mathrm{st}}^{*}\right)=h\left(N_{\mathrm{A}}, N_{\mathrm{B}}\right) \cdot \frac{\left(\frac{z}{2} \cdot A\right) !}{\prod_{\mathrm{s}}\left(z \cdot A_{\mathrm{ss}}^{*}\right) ! \cdot \prod_{\mathrm{s}} \prod_{\mathrm{s} \neq \mathrm{t}}\left(\frac{z}{2} \cdot A_{\mathrm{st}}^{*}\right) !} \\
\Omega^{*}=g^{*}\left(z, A, A_{\mathrm{ss}}^{*}, A_{\mathrm{st}}^{*}\right) \cdot e^{-\left(\frac{1}{2} \sum_{\mathrm{s}=1}^{\sigma} A_{\mathrm{s}} \cdot \omega_{\mathrm{ss}}(T)+\frac{1}{2} \cdot \sum_{\mathrm{s}=1}^{\sigma} \sum_{t \neq \mathrm{s}}^{\sigma} A_{\mathrm{st}}^{*} \cdot \Delta \omega_{\mathrm{st}}(T)\right) k_{\mathrm{B}} \cdot T} \\
F^{*}(\mathrm{CONF}, \mathrm{DISP})=k_{B} \cdot T \cdot \ln \Omega^{*} \\
U^{*}(\mathrm{CONF}, \mathrm{DISP})=-T^{2} \cdot\left(\frac{\partial F^{*}(\mathrm{CONF}, \mathrm{DISP}) / T}{\partial T}\right)_{V, N}=k_{B} \cdot T^{2} \cdot\left(\frac{\partial \ln \Omega^{*}}{\partial T}\right)_{V, N}
\end{gathered}
$$

La energía interna molar de exceso, que coincide con la entalpía molar de exceso, se calcula de modo similar al caso de la aproximación uno mediante $U^{*}(C O N F, D I S P)$ $U_{\mathrm{i}}(\mathrm{CONF}, \mathrm{DISP})$, luego:

$$
H_{\mathrm{m}}^{\mathrm{E}}(\mathrm{DISP})=U_{\mathrm{m}}^{\mathrm{E}}(\mathrm{DISP})=H_{\mathrm{m}}^{\mathrm{E}}(z \rightarrow \infty)=U_{\mathrm{m}}^{\mathrm{E}}(z \rightarrow \infty)=
$$




$$
=\frac{1}{2} \cdot\left(\sum_{\mathrm{i}=1} x_{\mathrm{i}} \cdot q_{\mathrm{i}}\right) \cdot\left[\sum_{\mathrm{i}} \sum_{\mathrm{j}} \xi_{\mathrm{i}} \cdot \xi_{\mathrm{j}} \cdot h_{\mathrm{ij}}^{*}\right]
$$

donde:

$$
h_{\mathrm{ij}}^{*}=-\frac{1}{2} \cdot\left[\sum_{\mathrm{s}=1}^{\sigma} \sum_{\mathrm{t} \neq \mathrm{s}}^{\sigma}\left(\alpha_{\mathrm{si}}-\alpha_{\mathrm{sj}}\right) \cdot\left(\alpha_{\mathrm{ti}}-\alpha_{\mathrm{tj}}\right) \cdot h_{\mathrm{st}}^{*}\right]
$$

La ecuación (6.446) puede reescribirse como sigue:

$$
H_{\mathrm{m}}^{\mathrm{E}}(\mathrm{DISP})=U_{\mathrm{m}}^{\mathrm{E}}(\mathrm{DISP})=\frac{1}{2} \cdot\left(\sum_{\mathrm{i}=1} x_{\mathrm{i}} \cdot q_{\mathrm{i}}\right) \cdot\left[\sum_{\mathrm{s}} \sum_{\mathrm{t} \neq \mathrm{s}}\left(\alpha_{\mathrm{s}} \cdot \alpha_{\mathrm{t}}-\sum_{i} \xi_{\mathrm{i}} \cdot \alpha_{\mathrm{si}} \cdot \alpha_{\mathrm{ti}}\right) \cdot h_{\mathrm{st}}^{*}\right]
$$

Obsérvese que en la expresión de la entalpía de exceso en la aproximación cuasiquímica, ecuación (6.431), se utilizan tanto los parámetros $h_{\mathrm{st}}$ como los parámetros $g_{\text {st }}$. Estos últimos aparecen en la definición de $\eta_{\text {st }}$ ecuación (6.430). Es costumbre correlacionar simultáneamente $h_{\text {st }}$ y $g_{\text {st }}$ a un conjunto de medidas de entalpías y energías libres de Gibbs de exceso. El caso de la contribución dipersiva es diferente, ya que al verificarse que $\eta_{\mathrm{st}} \rightarrow 1$, la expresión de la entalpía de exceso sólo depende de los parámetros $h_{\text {st }}^{*}$.

Por su parte, la función de Gibbs de exceso es:

$$
\begin{aligned}
F_{\mathrm{m}}^{\mathrm{E}}(\mathrm{DISP}) & =G_{\mathrm{m}}^{\mathrm{E}}(\mathrm{DISP})=F_{\mathrm{m}}^{\mathrm{E}}(z \rightarrow \infty)=G_{\mathrm{m}}^{\mathrm{E}}(z \rightarrow \infty)= \\
& =\lim _{z \rightarrow \infty}\left[G_{\mathrm{m}}^{\mathrm{E}}(\mathrm{COMB})+G_{\mathrm{m}}(\mathrm{RES})\right]
\end{aligned}
$$

Donde, la parte combinatorial es la misma que la dada en la ecuación (6.424):

$$
\lim _{z \rightarrow \infty}\left[G_{\mathrm{m}}^{\mathrm{E}}(\mathrm{COMB})\right]=G_{\mathrm{m}}^{\mathrm{E}}(\mathrm{COMB})=R \cdot T \cdot \sum x_{\mathrm{i}} \cdot \ln \frac{\Phi_{\mathrm{i}}}{x_{\mathrm{i}}}
$$

y la parte residual es:

$$
\lim _{z \rightarrow \infty}\left[G_{\mathrm{m}}(\mathrm{RES})\right]=G_{\mathrm{m}}(\mathrm{DISP})=\frac{1}{2} \cdot\left(\sum_{\mathrm{i}} x_{\mathrm{i}} \cdot q_{\mathrm{i}}\right) \cdot\left(\sum_{\mathrm{i}} \sum_{\mathrm{j}} \xi_{\mathrm{i}} \cdot \xi_{\mathrm{j}} \cdot g_{\mathrm{ij}}^{*}\right)
$$

donde:

$$
g_{\mathrm{ij}}^{*}=-\frac{1}{2} \cdot \sum_{\mathrm{s}=1}^{\sigma} \sum_{\mathrm{t} \neq \mathrm{s}}^{\sigma}\left(\alpha_{\mathrm{si}}-\alpha_{\mathrm{sj}}\right) \cdot\left(\alpha_{\mathrm{ti}}-\alpha_{\mathrm{tj}}\right) \cdot g_{\mathrm{st}}^{*}
$$

\subsubsection{DISQUAC (DISpersive-QUAsi Chemical)}

Como se ha puesto de manifiesto en la descripción del modelo de red, y por extensión se verifica ahora, el índice de coordinación $z$ es una medida del grado de orientación de los grupos que constituyen las moléculas que forma la mezcla. De este modo, el límite $z \rightarrow \infty$ da cuenta de la falta de orientación en las interacciones, siendo los efectos de tamaño y forma asociados a las moléculas los que determinan completamente las propiedades de la mezcla. La hipótesis de mezcla al azar se verifica entonces con un alto grado de verosimilitud, de manera que las ecuaciones obtenidas para esta aproximación cero permiten justificar plenamente las mezclas de sustancias apolares o de un componente poco polar con otro inerte, donde las fuerzas dispersivas representan 
la contribución ás importante. El conocido desplazamiento hacia el componente más pequeño en la simetría de las curvas $H_{\mathrm{m}}^{\mathrm{E}}=H_{\mathrm{m}}^{\mathrm{E}}(x), G_{\mathrm{m}}^{\mathrm{E}}=G_{\mathrm{m}}^{\mathrm{E}}(x)$ se justifica así plenamente, siendo los parámetros geométricos $\left(q_{\mathrm{i}}, r_{\mathrm{i}}\right)$ los que caracterizan por entero dichas funciones de exceso.

En mezclas de moléculas con polaridad considerable, en donde las interacciones entre los grupos dependen claramente de la orientación, la forma de las funciones de exceso se distorsina con respecto la que sería de esperar por efecto de los parámetros geométricos (mezcla al azar). Así, en las mezclas de un componente polar con uno apolar, el máximo de la curva $H_{\mathrm{m}}^{\mathrm{E}}=H_{\mathrm{m}}^{\mathrm{E}}(x)$ se desplaza hacia concentraciones bajas en el componente polar, independientemente de los tamaños relativos de ambos. La aproximación cuasiquímica es capaz de justificar este comportamiento para el caso en donde los efectos de orientación son débiles, distorsionando la forma de las funciones de exceso en la dirección adecuada. El índice de coordinación $z$ da cuenta en este caso de la no aleatoriedad en la distribución de las moléculas de la mezcla, consecuencia de los efectos de orientación, de manera que cuanto más pequeño sea $z$ más se desplazan las funciones de exceso en la forma indicada. Por tanto, puede decirse que aunque no se da una relación explícita entre $z$ y la anisotropía del potencial de interacción entre grupos, $z$ representa un verdadero factor de no aleatoriedad, al menos de una manera un tanto primitiva.

El método hasta aquí desarrollado ha sido aplicado para la caracterización de tipos muy diversos de mezclas líquidas de no electrolitos, tanto en la aproximación cero como en la cuasiquímica. Ha permitido, por ejemplo, dar una justificación rigurosa de los efectos conformacionales Patterson y Wilhelm [451], incluso en lo que respecta a $C_{P, \mathrm{~m}}^{\mathrm{E}}$, representando una mejora sustancial respecto los modelos de red más antiguos. Sin embargo, su aplicación a determinados tipos de sistemas ha demostrado ciertas limitaciones que le restan validez general [452], [453]. Esta falta de generalidad del modelo se debe sobre todo a dos razones:

a) De acuerdo con la interpretación de $z$, diferentes clases de mezclas requieren diferentes valores de $z$, ya que el grado de orientación varía de unas a otras. Ahora bien, en mezclas que contengan tres o más grupos de variada polaridad, donde para cada contacto se necesitaría un valor diferente de $z$, nos encontramos que el modelo no es capaz de dar cuenta de ello, pues sólo reconoce un único valor de $z$. Por lo tanto, la hipótesis simplificadora de que $z$ es el mismo para todos los contactos es un lastre.

b) La segunda dificultad es consecuencia de que para valores finitos de $z$ la parte residual de $G_{\mathrm{m}}^{\mathrm{E}}$ tiene un límite superior. Así, para sistemas con $G_{\mathrm{m}}^{\mathrm{E}}$ grande y positiva y $H_{\mathrm{m}}^{\mathrm{E}}$ muy asimétrica, no puede encontrarse un valor de $g_{\mathrm{st}}$ adecuado que sea compatible con ambas funciones de exceso. Si bien la aproximación cero carece de límite alguno que impida su aplicación general, la dependencia específica y única con respecto a los parámetros geométricos que ella conlleva no permite dar cuenta de los desplazamientos típicos asociados a las orientaciones.

Visto todo lo anterior, parece claro que una aproximación físicamente más realista que permita formular un modelo falto de las anteriores dificultades debe de tener en cuenta una contribución dispersiva (al azar) para cada contacto, suplementada eventualmente por una contribución electrostática no aleatoria, en la que cada contacto posea su 
propio z. A la espera de la formulación general de un modelo que posea estas características, Kehiaian et al [405], [406], [439], [441], [452], [453] formularon una ampliación sencilla del modelo expuesto en las secciones anteriores, que supera, en primera aproximación, las dificultades mencionadas, conocido como modelo DISQUAC (DISpersive QUAsi-Chemical). Dicha formulación es análoga a la teoría de mezclas asociadas no atérmicas expuesta en la sección 6.4.7. En dicha teoría, las funciones de exceso pueden desglosarse como la suma de dos contribuciones (ecuación (6.340)):

$$
X^{\mathrm{E}}=X^{\mathrm{E}}(\mathrm{CHEM})+X^{\mathrm{E}}(\mathrm{PHYS})
$$

La contribución "química" está suplementada por una contribución "física" del tipo mezcla al azar. En DISQUAC, la misma contribución de mezcla al azar suplementa a las expresiones de la aproximación cuasiquímica. La relación inmediata entre los enlaces químicos secundrios característicos de las mezclas asociadas y el grado de orientación entre grupos característicos del modelo de red para $z$ finito y pequeño es, así, evidente y justifica plenamente la equivalencia de ambas formulaciones. De esta manera, cada contacto polar o no polar viene caracterizado por un parámetro de intercambio dispersivo, mientras que para el caso de contactos polares aparecen dos parámetros adicionales: el parámetro de intercambio cuasiquímico, y el índice de coordinación. Con ello, la presencia de un término dispersivo evita la acotación de las funciones de exceso, eliminando la segunda de las dificultades indicadas unas líneas arriba. Además, los pesos relativos de las partes dispersiva $(z \rightarrow \infty)$ y cuasiquímica ( $z$ finito y pequeño) permiten graduar de forma simple el grado de orientación asociado a los diferentes contactos, lo que elimina la primera dificultad.

En el modelo así construido, los términos dispersivo y cuasiquímico se calculan independientemente y simplemente se suman. Por ello, las superficies de contacto de equilibrio no se definen de manera única: hay un conjunto para la contribución dispersiva y otro diferente para la cuasiquímica.

Por todo lo visto, se pueden escribir las funciones molares de exceso correspondientes a una mezcla binaria de la siguiente forma:

$$
\begin{gathered}
G_{\mathrm{m}}^{\mathrm{E}}=G_{\mathrm{m}}^{\mathrm{E}}(\mathrm{COMB})+G_{\mathrm{m}}(\mathrm{RES})=G_{\mathrm{m}}^{\mathrm{E}}(\mathrm{COMB})+G_{\mathrm{m}}(\mathrm{DISP})+G_{\mathrm{m}}(\mathrm{QUAC}) \\
H_{\mathrm{m}}^{\mathrm{E}}=H_{\mathrm{m}}^{\mathrm{E}}(\mathrm{RES})=H_{\mathrm{m}}^{\mathrm{E}}(\mathrm{DISP})+H_{\mathrm{m}}^{E}(\mathrm{QUAC})
\end{gathered}
$$

La parte combinatorial puede venir dada por ejemplo por la ecuación de Flory-Huggins:

$$
G_{\mathrm{m}}^{\mathrm{E}}(\mathrm{COMB})=R \cdot T \cdot \sum x_{\mathrm{i}} \cdot \ln \frac{\Phi_{\mathrm{i}}}{x_{\mathrm{i}}}
$$

La parte dispersiva del potencial de Gibbs de exceso es la dada en la ecuación (6.451):

$$
G_{\mathrm{m}}(\mathrm{DISP})=\frac{1}{2} \cdot\left(\sum_{\mathrm{i}} x_{\mathrm{i}} \cdot q_{\mathrm{i}}\right) \cdot\left(\sum_{\mathrm{i}} \sum_{\mathrm{j}} \xi_{\mathrm{i}} \cdot \xi_{\mathrm{j}} \cdot g_{\mathrm{ij}}^{*}\right)
$$

Mientras que la parte cuasiquímica procede de la ecuación (6.424):

$$
G_{\mathrm{m}}(\mathrm{QUAC})=R \cdot T \cdot \sum_{i} x_{\mathrm{i}} \cdot z \cdot q_{\mathrm{i}} \cdot\left(\sum_{\mathrm{s}} \alpha_{\mathrm{si}} \cdot \ln \frac{X_{\mathrm{s}} \cdot \alpha_{\mathrm{si}}}{X_{\mathrm{si}} \cdot \alpha_{\mathrm{s}}}\right)
$$


En cuanto a la entalpía de exceso, la contribución dispersiva está dada por la ecuación (6.446):

$$
H_{\mathrm{m}}^{\mathrm{E}}(\mathrm{DISP})=\frac{1}{2} \cdot\left(\sum_{\mathrm{i}=1} x_{\mathrm{i}} \cdot q_{\mathrm{i}}\right) \cdot\left[\sum_{\mathrm{i}} \sum_{\mathrm{j}} \xi_{\mathrm{i}} \cdot \xi_{\mathrm{j}} \cdot h_{\mathrm{ij}}^{*}\right]
$$

y la cuasiquímica está dada por la ecuación (6.431):

$$
H_{\mathrm{m}}^{\mathrm{E}}(\mathrm{QUAC})=\frac{1}{2} \cdot\left(\sum_{\mathrm{i}=1} x_{\mathrm{i}} \cdot q_{\mathrm{i}}\right) \cdot\left[\sum_{\mathrm{s}=1}^{\sigma} \sum_{\mathrm{t} \neq \mathrm{s}}^{\sigma}\left(X_{\mathrm{s}} \cdot X_{\mathrm{t}}-\sum_{i} \xi_{\mathrm{i}} \cdot X_{\mathrm{si}} \cdot X_{\mathrm{ti}}\right) \cdot \eta_{\mathrm{st}} \cdot h_{\mathrm{st}}\right]
$$

donde $h_{\text {st }}, g_{\text {st }} h_{\text {st }}{ }^{*} g^{*}$ st son los parámetros de interacción cuasiquímicos y dispersivos asociados al contacto $(s, t)$ respectivamente, que cumplen (6.426)-(6.429).

Las superficies de contacto en el quilibrio $X_{\mathrm{s}}$ y $X_{\mathrm{t}}$ en la aproximación cuasiquímica se obtienen al resolver el sistema de $\sigma$ ecuaciones:

$$
\alpha_{\mathrm{s}}=X_{\mathrm{s}} \cdot\left(X_{\mathrm{s}}+\sum_{\mathrm{t} \neq \mathrm{s}} X_{\mathrm{t}} \cdot \eta_{\mathrm{st}}\right)
$$

Mientras que $X_{\mathrm{si}}$ y $X_{\mathrm{ti}}$ son las soluciones de para $x_{\mathrm{i}}=1$ (componente puro i-ésimo).

Para concluir, hay que darse cuenta de lo siguiente: en la comparación de las ecuaciones generales del modelo con los resultados experimentales se utilizan valores de $H_{\mathrm{m}}^{\mathrm{E}}$ y $G_{\mathrm{m}}^{\mathrm{E}}$ obtenidos a diferentes temperaturas, con lo cual se obtienen valores de $h_{\mathrm{st}}, g_{\mathrm{st}}$, $h^{*}{ }_{\text {st }} g_{\text {st }}^{*}$ que, verificando (6.426)-(6.429), dependen de la temperatura. Con el objetivo de reducir todos los valores a una temperatura de referencia $T_{0}$ (que se toma igual a 298.15 K), se supone que dicha dependencia viene dada por una ecuación del tipo:

$$
\frac{g_{\mathrm{st}}^{\prime *}(T)}{R \cdot T}=C_{\mathrm{st}, 1}^{\mathrm{DIS} / \mathrm{QUAC}}+C_{\mathrm{st}, 2}^{\mathrm{DIS} / \mathrm{QUAC}} \cdot\left(\frac{T_{0}}{T}-1\right)+C_{\mathrm{st}, 3}^{\mathrm{DIS} / \mathrm{QUAC}} \cdot\left[\ln \left(\frac{T_{0}}{T}\right)-\frac{T_{0}}{T}+1\right]
$$

Como consecuencia de las ecuaciones (6.426)-(6.429), se tiene:

$$
\frac{h_{\mathrm{st}}^{\prime *}(T)}{R \cdot T}=C_{\mathrm{st}, 2}^{\mathrm{DIS} / \mathrm{QUAC}} \cdot\left(\frac{T_{0}}{T}\right)-C_{\mathrm{st}, 3}^{\mathrm{DIS} / \mathrm{QUAC}} \cdot\left(\frac{T_{0}}{T}-1\right)
$$

Las constantes $C_{\mathrm{st}, \mathrm{i}}^{\mathrm{DIS} / \mathrm{QUAC}} C_{\mathrm{st}, \mathrm{i}}(\mathrm{i}=1,2,3)$ son números independientes de la temperatura y de las unidades energéticas escogidas, llamados coeficientes de intercambio, cuyo significado es el siguiente:

$$
\begin{aligned}
C_{\mathrm{st}, 1}^{\mathrm{DIS} / \mathrm{QUAC}} & =\frac{g_{\mathrm{st}}^{/^{*}}\left(T_{0}\right)}{R \cdot T_{0}} \\
C_{\mathrm{st}, 2}^{\mathrm{DIS} / \mathrm{QUAC}} & =\frac{h_{\mathrm{st}}^{\prime^{*}}\left(T_{0}\right)}{R \cdot T_{0}} \\
C_{\mathrm{st}, 3}^{\mathrm{DIS} / \mathrm{QUAC}} & =\frac{c_{\mathrm{p}, \mathrm{st}}^{/ *}\left(T_{0}\right)}{R}
\end{aligned}
$$

Donde se ha definido $c_{\mathrm{p}, \mathrm{st}}^{\prime *}$ como: 


$$
c_{\mathrm{p}, \mathrm{st}}^{\nu^{*}}(T)=\frac{\mathrm{d} h_{\mathrm{p}, \mathrm{st}}^{/ *}(T)}{\mathrm{d} T}
$$

Cuya misión consiste en dar cuenta del comportamiento de la capacidad calorífica de exceso a presión constante:

$$
C_{P}^{\mathrm{E}}(T)=\frac{\mathrm{d} H^{\mathrm{E}}(T)}{\mathrm{d} T}
$$

En resumen, se puede decir que la justificación de las propiedades de las mezclas líquidas mediante el modelo DISQUAC se reduce a la obtención de los coeficientes de intercambio (dispersivos y cuasiquímicos) para los diferentes contactos $(\mathrm{s}, \mathrm{t})$ que están presentes en la mezcla. Estos se ajustan mediante la comparación de las ecuaciones generales con los datos experimentales de $H_{\mathrm{m}}^{\mathrm{E}}$ y $G_{\mathrm{m}}^{\mathrm{E}}$ (y $C_{P, \mathrm{~m}}^{\mathrm{E}}$ si fuese necesario). 
CAPÍTULO 6 


\section{BIBLIOGRAFÍA}

[1]: Wilson, K.G.; Kogut, J.; Phys. Reps., 12 (2) (1974), 76.

[2]: Solé, R. V.; Manrubia, S. C.; "Orden y Caos en Sistemas Complejos", Ediciones UPC, POLITEXT (2001).

[3]: Haken, H.; "Synergetics, an Introduction. Non-Equilibrium Phase Transitions and Self-Organization in Physics, Chemistry, and Biology", Springer Verlag Berlin Heidelberg New York (1977).

[4]: Anderson, P.W.; Science, 177 (4047) (1972), 393

[5]: Laughlin, R.B.; Pines, D.; PNAS, 97 (1) (2000), 28.

[6]: Wagensberg, J.; Mundo Científico, 196 (1998).

[7]: Kolmogorov, A.; Theor. Comp. Sci., 207 (2) (1998), 387.

[8]: Haken, H.; "Information and Self-Organization. A Macroscopic Approach to Complex Systems", $3^{\text {th }}$ edition, Springer Berlin Heidelberg (2006).

[9]: Boltzmann, L.; Wiener Berichte, 66 (1872), 275.

[10]: Gibbs, J.W.; "The Collected Works of J. Willard Gibbs", Two Volumes, Longmans, Green and Co. New York (1931).

[1 1]: Bertalanffy, L. v.; "General Systems Theory", George Braziller, Inc. New York (1968).

[12]: Baxter, R. J.; "Exactly Solved Models in Statistical Mechanics", $3^{\text {th }}$ imp., Academic Press, London (1989).

[13]: van Hove, L.; Physica, 16 (1950), 137.

[14]: Lieb, E. H. and Mattis, D. C.; "Mathematical Physics in One Dimension", Academic Press, New York and London (1966).

[15]: Lenard, A.; J. Math. Phys., 2 (1961), 682.

[16]: Baxter, R. J.; Proc. Camb. Phil. Soc. 59 (1963), 779.

[17]: Baxter, R. J.; Phys. Fluids, 7 (1964), 38.

[18]: Baxter, R. J.; Phys. Fluids, 8 (1965), 687.

[19]: Felderhof, B. U.; Physica, 65 (1973), 421. 
[20]: Felderhof, B. U.; Physica, 66 (1973), 279.

[21]: Felderhof, B. U.; Physica, 66 (1973), 509

[22]: Felderhof, B. U.; Phys. Letters., 44A (1973), 437.

[23]: Montroll, E. W; Nuovo Cimento, Suppl., 6 (1949), 265.

[24]: Berlin, T. H.;Kac, M.; Phys. Rev., 86 (1952), 821.

[25]: Stanley, H. E.; Phys. Rev., 176 (1968), 718.

[26]: Onsaguer, L.; Phys. Rev., 65 (1944), 117.

[27]: Birgeneau, R. J.; Guggenheim, H. J.; Shirane, G.; Phys. Rev., B8 (1973), 304.

[28]: Als-Nielsen, J.; Birgeneau, R. J.; Guggenheim, H. J.; Shirane, G; Phys. Rev., B12 (1975), 4963.

[29]: Ruelle, D.; "Azar y caos", Alianza Editorial S.A., Madrid (1993).

[30]: H. Poincaré, "Sur le probléme des trois corps et les equations de la dynamique", withdrawn version 1889 and Acta Mathematica 13 (1890), 1-270.

[31]: Petrosky, T.; Prigogine, I.; Chaos, Solitons and Fractals, 11 (2000), 373.

[32]: Petrosky, T.; Prigogine, I.; Chaos, Solitons and Fractals, 7 (1996), 441.

[33]: Petrosky, T.; Prigogine, I.; Adv. Chem. Phys., 99 (1997), 1.

[34]: Petrosky, T.; Prigogine, I.; "Extension of classical dynamics. The case of anharmonic lattices", en: P. Pronin, G. Sardanashvily (Eds.), "Gravity, Particles and Space-time", World Scientific, Singapore, (1996).

[35]: Callen, H.B.; "Thermodynamics, And an Introduction to Thermostatistics", $2^{\text {nd }}$ Ed., John Wiley \& Sons, Inc (1985).

[36]: Hawking, S.; "Gödel and the End of Physics", a Lecture in MIT (2003).

[37]: Yang, C.N.; Lee, T.D.; Phys. Rev., 87 (1952), 404.

[38]: Yang, C.N.; Lee, T.D.; Phys. Rev., 87 (1952), 410.

[39]: Dorfman, J.R.; "An Introduction to Chaos in Nonequilibrium Statistical Mechanics", Cambridge University Press (1999).

[40]: Prigogine, I.; "Non-Equilibrium Statistical Mechanics", Interscience Pub, John Wiley \&Sons, New York-London (1962).

[41]: Balescu, R.; "Equilibrium and Nonequilibrium Statistical Mechanics", Wiley Interscience Pub, John Wiley\&Sons, New York-London-Sidney-Toronto (1975).

[42]: Shannon, C.E.; Weaver, W.; "The Mathematical Theory of Communication", University of Illinois Press, Urbana (1949).

[43]: Jaynes, E.T.; Phys. Rev., 106 (1957), 620.

[44]: Jaynes, E.T.; Phys. Rev., 108 (1957), 171.

[45]: Jaynes, E. T.; "Information Theory and Statistical Mechanics", en Statistical Physics,

K. Ford (ed.), Benjamin, New York (1963), 181. 
[46]: Jaynes, E. T.; "Foundations of Probability Theory and Statistical Mechanics", en Delaware Seminar in the Foundations of Physics, M. Bunge (ed.), Springer-Verlag, Berlin, (1967), 77.

[47]: Jaynes, E. T.; "Where do we Stand on Maximum Entropy?" en The Maximum Entropy Formalism, R. D. Levine and M. Tribus (eds.), M. I. T. Press, Cambridge, MA (1979), 15.

[48]: Jaynes, E.T.; "Probability Teory. The Logic of Science", Cambridge University Press (2003).

[49]: Mayer, J.; Khairy, K.; Howard, J.; Amer. J. Phys., 78(6) (2010), 648.

[50]: Fisher, M.E.; Review of Modern Physics, 46 (4) (1974), 597.

[51]: Maris, H. J.; Kadanoff, L. P.; Amer. J. Phys., 46 (1978), 652.

[52]: Fisher, M.E.; Reviews of Modern Physics, 70 (2) (1998), 653.

[53]: Kadanoff, L. P.; "Scaling universality and operator algebras", en "Phase Transitions and Critical Phenomena", Ed. C. Domb and M. S. Green, Vol. 5a. Academic Press, New York, (1976).

[54]: Wilson, K. G.; Scient. Am., (1979), 158.

[55]: Solé, R. V.; "Phase Transitions", Princeton University Press (2011).

[56]: Istrail, S; Proceedings of the 32nd ACM Symposium STOC00, ACM Press, Portland OR. (2000), 87.

[57]: Nicolis, G., and Prigogine, I.; "Self-Organization in Nonequilibrium Systems". New-York, Wiley (1977).

[58]: Nicolis, G., and Prigogine, I.; "Exploring Complexity", New York, Freeman (1990).

[59]: Kauffman, S. A.; "The Origins of Order", Oxford UK, Oxford University Press (1993).

[60]: Gell-Mann, M.; "The Quark and the Jaguar", New York, Freeman (1994).

[61]: Solé, R. V.; Goodwin, B. C.; "Signs of Life: How Complexity Pervades Biology". New York, Basic Books (2001).

[62]: Morowitz, H. J. "The Emergence of Everything. How the World Became Complex". Oxford, UK, Oxford University Press (2002).

[63]: Mitchell, M.; "Complexity: A Guided Tour". New York, Oxford University Press (2009).

[64]: Sornette, D.; "Why Stock Markets Crash". Princeton, Princeton University Press (2003).

[65]: Mikhailov A.; "Foundations of Synergetics, Volume 1: Distributed Active Systems". Berlin, Springer (1994).

[66]: Mikhailov A.; Calenbuhr V.; "From Cells to Societies". Berlin, Springer (2002).

[67]: Landau, L. D.; Lifshitz, E. M.; "Mecanica Cuantica No Relativista", Volumen 3 Del Curso De Física Teórica; Editorial Reverté, S. A., Barcelona (1983).

[68]: Born, M., Oppenheimer, J.R.; Ann. Phys., 84; 457, 1927 
[69]: Pauling, L.; Wilson, E.B.; "Introduction to Quantum Mechanics: With Applications to Chemistry"; Dover Books on Physics Inc., (1985).

[70]: Wilson, E.B.; "Molecular Vibrations: The Theory of Infrared and Raman Vibrational Spectra", Dover Books on Chemistry Inc., (2003).

[71]: Flory, P.J.; Pure and Appl. Chem., 50 (1978), 255.

[72]: Flory, P.J.; Pure and Appl. Chem., 52 (3) (1980), 241.

[73]: Flory, P.J.; Pure and Appl. Chem., 56 (3) (1984), 305.

[74]: Münster, A.; "Statistical Thermodynamics", Vol 1, Springer-Verlag, Berlin (1969).

[75]: Goldstein, H.; "Mecánica Clásica”, Editorial Reverté, S.A., Barcelona (2002).

[76]: Landsberg, P.T.; "Thermodynamics and Statistical Mechanics", Oxford University Press, (1978).

[77]: Fowler, R.; Guggenheim, E.A.; "Statistical Thermodynamics", Cambridge University Press, (1939).

[78]: Prigogine, I; Defay, R.; "Chemical Thermodynamics", Longmans, $3^{\text {rd }}$ edition (1965).

[79]: Badger, R.M.; Baver, S.H.; J. Chem. Phys., 5 (1937), 605.

[80]: Arunan, E.; Desiraju, G.R.; Klein, R.A.; Sadlej, J.; Scheiner, S.; Alkorta, I.; Clary, D.C.; Crabtree, R.H.; Dannenberg, J.J.; Hobza, P.; Kjaergaard, H.G.; Legon, A.C.; Mennucci, B.; Nesbitt, D.J. ;Pure Appl. Chem. 83 (8) (2011), 1619.

[81]: Pimentel, G.C.; Mcclellan, A.L.; "The Hydrogen Bond"; W. H. Freeman And Company, San Francisco And London (1960).

[82]: Wilhelm, E.; Pure and Appl. Chem., 77 (2005), 1317.

[83]: Smith, J.M.; Van Ness, H.C.; Abbott, M.M.; "Introducción a la Termodinámica en Ingeniería Química", 5a ed., McGraw-Hill, Inc. (1997).

[84]: Mulliken, R.S.; Person, W.B.; "Molecular Complexa: A Lecture and Reprint Volume", Wiley-Interscience, Nueva York (1969).

[85]: Mayer, J.E.; Mayer, M.G.; "Statistical Mechanics", John Wiley \& Sons, Inc., New York (1940).

[86]: Ono, S.; J. Chem. Phys., 19 (1951), 504.

Filpatrick, J.E.; J. Chem. Phys., 21 (1953), 274.

[87]: Pathria, R.K., "Statistical Mechanics", $2^{\text {nd }}$ ed., Butterworth-Heinemann, Jordan Hill, Oxford (1996).

[88]: Hill, T.L.; "Statistical Mechanics. Principles and Selected Applications", Dover Pubplications, Inc, Mineola, New York (1987).

[89]: Born, M.; Fuchs, K.; Proc. Roy. Soc. (London), A166 (1938), 391.

[90]: Kahn, B.; Uhlenbeck, G.E.; Physica, 5 (1938), 399.

[91]: Barker, J.A.; Henderson, D.; Reviews of Modern Physics, 48 (4) (1976), 587.

[92]: Münster, A.; "Statistical Thermodynamics", Vol. 2, Springer-Verlag, Berlin (1974). 
[93]: Ben-Naim, A.; "Molecular Theory of Solutions", Oxford University Press Inc., New York (2006).

[94]: Gray, C.G.; Gubbins, K.E.; "Theory of Molecular Fluids", Vol. 1, Oxford University Press, Inc., New York (1984).

[95]: Boublik, T.; Nezbeda, I.; Hlavaty, K.; "Statistical Thermodynamics of Simple Liquids and Their Mixtures", Elsevier, Amsterdam (1979).

[96]: Hill, T.; "An Introduction to Statistical Mechanics", Addison Wesley Publishing Company, Inc., Massachusetts (1960).

[97]: Steele, W.A.; Chem. Soc. Faraday Discuss., 66 (1978), 138.

[98]: Freasier, B.C.; Jolly, D.; Bearman, R.J.; Molec. Phys., 31 (1976), 255.

[99]: Nezbeda, I.; Molec. Phys., 33 (1977), 1287.

[100]: Yarnell, J.L.; Katz, M.J.; Wenzel, R.G.; Koenig, S.H.; Phys. Rev., A7 (1973), 2130.

[101]: Copley, J.R.D.; Lovesey, S.W.; Rep. Prog. Phys., 38 (1975), 461.

[102]: Chen, Y.-D.; Steele, W.A.; J. Chem. Phys., 54 (1971), 103.

[103]: Kirkwood, J.G.; J. Chem. Phys., 3 (1935), 300.

[104]: Yvon, J.; Actualités Scientifíques et Industrielles, 203 (1935).

[105]: Bogoliubov, N. N.; J. Phys. (Moscow) 10 (1946), 256.

[106]: Born, M.; H. S. Green; Proc. R. Soc. Lond. A188 (1946), 10.

[107]: de Boer, J.; van Leeuwen, J. M. J. ; Groeneveld, J.; Physica, 30 (1964), 2265.

[108]: Rushbrooke, G. S.; Physica, 26(1960), 259.

[109]: Verlet, L.; Levesque, D.; Physica, 28 (1962), 1124.

[1 10]: Percus, J. K.; Yevick, G.J.; Phys. Rev., 110 (1958), 1.

[1 11 1]: Chandler, D.; A. Rev. Phys. Chem., 29 (1978), 441.

[112]: Lorentz, H.A. ; Ann. Phys., 12 (1881), 127.

[1 13]: Berthelot, D. ; C. R. Hebd. Séanc. Acad. Sci., Paris 126 (1898), 1703, 1857.

[1 14]: Kohler, F.; Fischer, J.; J. Mol. Structure, 85 (1982), 245.

[1 15]: Kohler, F.; Ber. Bunsenges. Phys. Chem., 81 (1) (1977), 1037.

[1 16]: Kohler, F.; Pfenning, A.; Pure and Appl. Chem., 61 (6) (1989), 1041.

[1 17]: Lebowitz, J.L.; Phys. Rev., 133 (1964), A895.

[1 18]: Henderson, D.; Barker, J.A.; J. Chem. Phys., 49 (1968), 3377.

[1 19]: Leonard, P.J.; Henderson, D.; Barker, J.A.; Trans. Faraday Soc., 66 (1970), 2439.

[120]: Chambers, M.V.; McDonald, I.R.; Molec. Phys., 29 (1975), 1053.

[121]: Chapman, W.G.; Gubbins, K.E.; Jackson, G.; Radosz, M.; Fluid Phase Equilib., 52 (1989), 31.

[122]: Chapman, W.G.; Gubbins, K.E.; Jackson, G.; Radosz, M., Ind. Eng. Chem. Res., 29 (1990), 1709. 
[123]: Song, Y.; Lambert, S.M.; Prausnitz, J.M.; Macromolecules, 27 (1994), 441.

[124]: Rowlinson, J.S., Swinton, F.L.; "Liquids and Liquids Mixtures", Butterworths Monographs in Chemistry (1982).

[125]: Boublik, T.; Fluid Phase Equilib., 1 (1977), 37.

[126]: Solana, J.R.; "Perturbation Theories for the Thermodynamic Properties of Fluids and Solids", CRC Press Taylor \& Francis Group (2013).

[127]: McQuarrie, D.A.; "Statistical Mechanics", Harper \& Row Pub., New York (1976).

[128]: Kirkwood, J.G.; Buff, F.P.; J. Chem. Phys., 19 (4) (1951), 774.

[129]: Ben-Naim, A.; J. Chem. Phys. 67 (11) (1977), 4884.

[130]: Ben-Naim, A., "Water Solutions, An Introduction to Molecular Theory", Plenum, New York (1974).

[131]: Donkersloot, M.C.A.; J. Sol. Chem., 8 (1979), 293.

[132]: Matteoli, E.; Lepori, L.; J. Chem. Soc., Faraday Trans., 91 (1995), 431.

[133]: Matteoli, E. J.; Phys. Chem. B, 101 (1997), 9800.

[134]: Shulgin, I.L.; Ruckenstein, Eli.; J. Phys. Chem. B, 103 (1999), 2496.

[135]: Ruckenstein, Eli.; Shulgin, I.L.; "Thermodynamics of Solutions. From Gases to Pharmaceutics to Proteins", Springer, (2009).

[136]: Shulgin, I.L.; Ruckenstein, Eli.; Phys. Chem. Chem. Phys., 10 (2008), 1097.

[137]: Shulgin, I.L.; Ruckenstein, Eli.; J. Phys. Chem. B, 110 (2006), 12707.

[138]: Ben-Naim, A.; J. Phys. Chem. B, 111 (2007), 2896.

[139]: Matteoli, E.; Lepori, L.; J. Phys. Chem. B, 111 (2007), 3069.

[140]: Ben-Naim, A.; J. Phys. Chem. B, 112 (2008), 5874.

[141]: Shulgin, I.L.; Ruckenstein, Eli.; J. Phys. Chem. B, 112 (2008), 5876.

[142]: Matteoli, E.; Lepori, L. ; J. Phys. Chem. B, 112 (2008), 5878.

[143]: McMillan Jr., W.G.; Mayer, J.E.; J. Chem. Phys., 13 (1945), 276.

[144]: Ornstein, L.S.; Zernike, F.; Proc. Acad. Sci. Amsterdam, 17 (1914), 793.

[145]: Kato, T.; J. Chem. Phys., 88 (1984), 1248.

[146]: Zaitsev, A.L., Petrenko, V.E.; Kessler, Y.M.; J. Sol. Chem., 18 (1989), 115.

[147]: Pandey, J.D.; Verma, R.; Chem. Phys., 270 (2001), 429.

[148]: Rajand, D.; Verma, R.; Nautiyal, T.; Soni, N.K.; Pandey, J.D.; Phys. And Chem. Liqu., 47 (3) (2009), 287.

[149]: González, J.A.; Alonso, I.; Alonso Tristán, C.; García de la Fuente, I.; Cobos, J.C.; Fluid Phase Equilib., 337 (2013), 125.

[150]: Matteoli, E. Lepori, L.; J. Chem. Phys., 80 (6) (1984), 2856.

[151]: Aicart, E.; Tardajos, G.; Díaz Peña, M.; J. Chem. Thermodyn., 12 (1980), 1085.

[152]: Guggenheim, E. A.; "Mixtures", Clarendon Press, Oxford (1952). 
[153]: Zielkiewicz, J.; J. Phys. Chem., 99 (1995), 4787.

[154]: Sandler, S.I.; Fluid Phase Equilib., 19 (1985), 233.

[155]: Mansoori, G.A.; Ely, J.F.; "Density expansion (DEX) mixing rules. The 9th international CODATA Conference, Jerusalem, Israel (1984).

[156]: Rubio, R. G.; Prolongo, U.; Cabrerizo, M.; Díaz Peña, M.; Renuncio, J. A. R.; Fluid Phase Equilib., 26 (1986), 1.

[157]: Zielkiewicz, J.; Phys. Chem. Chem. Phys., 5 (2003), 1619.

[158]: Rowlinson, J.S.; Ind. Eng. Chem., 59 (1967), 28.

[159]: Kohler, F.; Ber. Bunsenges Phys. Chem., 81 (1977), 1037.

[160]: Shing, K.S; Gubbins, K.E.; ACS Advances in Chem. Series, 204. Haile, J.M.; Mansoori, G.A., Eds.; Amer. Chem. Soc. (1983), 73.

[161]: Prausnitz, J. M., Lichtenthaler, R.N., Gomes de Azevedo, E., "Termodinámica Molecular de los Equilibrios Entre Fases", $3^{a}$ edición, Prentice Hall (2000).

[162]: Van der Waals, J.D.; "Die Kontinuität des gasförmigen und flüssigen Zustandes", Leipzig, 1873.

[163]: Van Laar, J.J.; Z. Phys. Chem., 72 (1910), 723.

[164]: Wilhelm, E.; Thermochim. Acta, 94 (1985), 47.

[165]: Goodstein, D. L., "States of Matter", Dover Publications, Inc, Mineola, New York (2002).

[166]: Nicolas, M.; Malineau, M.; Reich, R.; Phys. Chem. Liq., 10 (1980), 11.

[167]: Mozo Ruiz, I.; "Estudio experimental y teórico de mezclas binarias de n-butan-1-ol, di-n-butiléter y celosolvas". Tesis Doctoral. Departamento de Física Aplicada. Facultad de Ciencias. Universidad de Valladolid (2010).

[168]: Costas, M.; Patterson, D.; Thermochim. Acta, 120 (1987), 161.

[169]: Grolier, J.-P.E. ; Faradizadeh, A.; Kehiaian, H.V.; Thermochim. Acta, 53 (1982), 157.

[170]: Grolier, J.-P.E.; Inglese, A.; Roux, A.H.; Wilhelm, E.; in S.A. Newman (Ed.), Chemical Engineering Thermodynamics, Ann Arbor Science Publishers, Ann Arbor, MI, (1982), 483.

[171]: Heintz, A.; Lichtenthaler, R.N.; Angew. Chem. Int. Ed. Engl., 21 (1982), 184.

[172]: Kehiaian, H.V.; Pure and Appl. Chem., 57 (1985), 15.

[173]: Patterson, D.; Pure and Appl. Chem., 47 (1976), 305.

[174]: Patterson, D.; J. Sol. Chem., 23 (1994), 105.

[175]: Roux, A.H.; Grolier, J.-P.E.; Inglese, A.; Wilhelm, E.; Ber. Bunsenges. Phys. Chem., 88 (1984), 986.

[176]: Wilhelm, E.; Thermochim. Acta, 28 (1979), 59.

[177]: Wilhelm, E.; Thermochim. Acta, 69 (1983), 1.

[178]: Wilhelm, E.; Thermochim. Acta, 94 (1985), 47. 
[179]: Hildebrand, J.H.; Scott, R.L.; "Regular Solutions", Prentice-Hall, Inc., Englewood Cliffs, New Jersey (1962).

[180]: Guggenheim, E.A.; Proc. Roy. Soc. A, 135 (1932), 181.

[181]: Mie, G.; Ann. Phys. 11 (1903), 657.

[182]: Einstein, A.; Ann. Der. Phys., 22 (180) (1907), 800.

[183]: Einstein, A.; Ann. Der. Phys., 34 (170) (1911), 590.

[184]: Lennard-Jones, J.E.; Devonshire, A.F.; Proc. Roy. Soc. A, 163, (1937), 53.

[185]: Lennard-Jones, J.E.; Devonshire, A.F.; Proc. Roy. Soc. A, 165, (1938), 1.

[186]: Prigogine, I.; Trappeniers, N.; Mathot, V.; J. Chem. Phys., 21 (1953), 559.

[187]: Hirschfelder, J.O.; Curtiss, C.F.; Bird, R.B.; "The Molecular Theory of Gases and Liquids", Wiley, New York (1954).

[188]: Hill, T.H.; J. Phys. Chem., 51 (1947), 1219.

[189]: Prigogine, I.; Garikian, G.; J. Chem. Phys., 45 (1948), 273.

[190]: Wentorf, R.H.; Buehler Jr, R.J.; Hirschfelder, JO.; Curtiss, C.F.; J. Chem. Phys., 18 (1950), 1484.

[191]: Prigogine, I.; Mathot, V.; J. Chem. Phys., 20 (1) (1952), 49.

[192]: Mathot, V.; Desmyter, A.; J. Chem. Phys., 21 (5) (1953), 782.

[193]: Prigogine, I.; Trappeniers, N.; Mathot, V.; J. Chem. Phys., 21 (1953), 560.

[194]: Prigogine, I.; Bellemans, A.; J. Chem. Phy., 21 (1953), 561.

[195]: Salsburg, Z.W.; Kirkwood, J.G.; J. Chem. Phys., 20 (10) (1952), 1538.

[196]: Salsburg, Z.W.; Kirkwood, J.G.; J. Chem. Phys., 21 (12) (1952), 2169.

[197]: Saroléa, L.; J. Chem. Phys., 21 (1953), 182.

[198]: Prigogine, I.; Jeener, J.; Physica, 20 (1954), 514.

[199]: Pople, J.A.; Phil. Mag., 41 (1951), 459.

[200]: Englert-Chowles, A.; J. Chem. Phys., 20 (1952), 925.

[201]: Einsenstein, A.; Gingrich, N.S.; Phys. Rev., 42 (1942), 261.

[202]: Eyring, H.; J. Chem. Phys., 4 (1936), 283.

[203]: Cernuschi, F. Eyring, H.; J. Chem. Phys., 7 (1939), 547.

[204]: Ono, S.; Mem. Fac. Eng. Kyushu Univ. 10 (1947), 190.

[205]: Peek, H.M.; Hill, T.L.; J. Chem. Phys., 18 (1950), 1252.

[206]: Rowlinson, J.S.; Curtiss, C.F.; J. Chem. Phys., 19 (1951), 1519.

[207]: Eyring, H.; Ree, T.; Hirai, N.; Proc. Nat. Acad. Sci. U. S., 44 (1958), 683.

[208]: Eyring, H.; Ree, T.; Proc. Nat. Acad. Sci. U. S., 47 (1961), 526.

[209]: Eyring, H.; Jhon, M.S.; "Significant Liquid Structures", Wiley, New York (1969).

[210]: Liang, K.; Eyring, H.; MARCHI, R.P.; Proc. Nat. Acad. Soc., 52 (1964), 1107. 
[2 1 1]: Miner, B.A.; Eyring, H.; Proc. Nat. Acad. Soc., 53 (1965), 1227.

[212]: De Boer, J.; Physica, 20 (1954), 655.

[213]: Cohen, E.G.D.; De Boer, J.; Salsburg, Z.W.; Physica, 21 (1955), 137.

[214]: Prigogine, I. "The Molecular Theory of Solutions", Interscience Publishers, Inc., New York (1957).

[215]: Barker, J.A.; Aust. J. Chem., 13 (1960), 187.

[216]: Hildebrand, J.H.; Scott, R.L.; "Solubility of Non Electrolytes", Reinhold (1950).

[217]: Kirkwood, J.G.; J. Chem. Phys., 6 (1938), 70.

[218]: Rushbrooke, G.S.; Scoins, H.J.; Wakefield, A.J.; Discuss. Faraday Soc., 15 (1953), 57.

[219]: Ter Haar, D.; "Elements of Statistical Mechanics", Reinehart, New York (1954).

[220]: Porter, A.W.; Trans. Faraday Soc., 16 (1920), 336.

[221]: Van Laar, J.J.; Lorenz, R.; Z. anorg. Chem., 145 (1925), 239.

[222]: Heitler, W.; Ann. Phys., 80 (1926), 629.

[223]: Hildebrand, J.H.; J. Amer. Chem. Soc., 51 (1929), 69.

[224]: Bragg, W.;Williams, E.; Proc. Roy. Soc. A, 145 (1935), 699.

[225]: Bragg, W.;Williams, E.; Proc. Roy. Soc. A, 151 (1935), 540.

[226]: Hildebrand, J.H.; J. Amer. Chem. Soc., 51 (1929), 66.

[227]: Scatchard, G.; Chem. Rev., 8 (2) (1931), 321

[228]: Scott, R.L.; Annu. Rev. Phys. Chem., 7 (1956), 43.

[229]: Guggenheim, E.A.; Proc. Roy. Soc. A, 148 (1935), 304.

[230]: Bethe, H.A.; Proc. Roy. Soc. A, 150 (1935), 552.

[231]: Rushbrooke, G.S.; Proc. Roy. Soc. A, 166 (1938), 296.

[232]: Prigogine, I.; Saroléa, L.; Van Hove, L.; Trans. Faraday Soc., 48 (1952), 485.

[233]: Kirkwood, J.G. ; J. Phys. Chem., 43 (1939), 97.

[234]: Guggenheim, E.A.; McGlashan, M.L.; Proc. Roy. Soc. A, 206 (1951), 335.

[235]: Guggenheim, E.A.; McGlashan, M.L.; Trans. Faraday Soc., 47 (1951), 929.

[236]: Migdal, A.A.; Soviet. Phys. JETP, 42 (1976), 743.

[237]: Kadanoff, L.P.; Ann. Phys., 100 (1976), 359.

[238]: Walker, J.S.; Vause, C.A.; Phys. Letters. 79 A (1980), 421.

[239]: Walker, J.S.; Vause, C.A.; J. Chem. Phys., 79 (1983), 2660.

[240]: McMahon, P.D.; Glandt, E.D.; Walker, J.S.; Chem. Eng. Science, 43 (10) (1988), 2561.

[241]: Guggenheim, E.A.; Trans. Faraday Soc., 44 (1948), 1007.

[242]: Guggenheim, E.A.; Supp. Nuovo Cimento, 6 (1949), 181. 
[243]: Cobos, J.C.; Fluid Phase Equilib., 133 (1997), 105.

[244]: Fuchs, R.; Krenzer, L.; Gaube, J.; Ber. Bunsenges. Phys. Chem., 88 (1984), 642.

[245]: McGlashan, M.L.; Pure and Appl. Chem., 8 (1964), 157.

[246]: Kalali, H.; Kohler, F.; Svedia, P.; Fluid Phase Equilib., 20 (1985), 75.

[247]: Miller, A.R. ; "Theory of High Polymer Solutions ", Oxford University Press, New York (1948).

[248]: Tompa, H.; J. Chem. Phys., 21 (1955), 250.

[249]: Barker, J.A.; J. Chem. Phys., 20 (1952), 1526.

[250]: Barker, J.A.; Brown, I.; Smith, F.; Discuss. Faraday Soc., 15 (1953), 142.

[251]: Barker, J.A.; Smith, F.; J. Chem. Phys., 22 (1954), 375.

[252]: Barker, J.A.; "Lattice Theories of the Liquid State", Pergamon Press (1963).

[253]: Staverman, A.J.; Recl. Trav. Chim. Pays-Bas, 69 (1950), 163-1 74.

[254]: Bronnenberg, R.A.H. ; "MOQUAC, a New Expression for the Excess Gibbs Free Energy Based on Molecular Orientations", Doctoral Thesis, RWTH Anchen University, Germany (2012).

[255]: Chang, T.S.; Proc. Roy. Soc. A, 169 (1939), 512.

[256]: Huggins, M.; Ann. N. Y. Acad. Sci., 43 (1942), 9.

[257]: Flory, P.; J. Chem. Phys., 10 (1942), 51.

[258]: Miller, A.R.; Proc. Cambridge Phil. Soc., 39 (1943), 54.

[259]: Guggenheim, E.A.; Proc. Roy. Soc. A, 183 (1944), 203.

[260]: Mathot, V.; Thése, Brussels (1949).

[261]: Guggenheim, E.A.; McGlashan, M.L.; Proc. Roy. Soc. A, 203 (1950), 435.

[262]: Fernández Martínez, J.M.; "Estudio de la hipótesis de la mezcla al azar", Tesis Doctoral. . Departamento de Termodinámica y Física Aplicada. Facultad de Ciencias. Universidad de Valladolid (1996).

[263]: Fox, J.R.; Fluid Phase Equilib., 37 (1987), 123.

[264]: McMahon, P.D.; Fluid Phase Equilib., 58 (1990), 51.

[265]: Saint-Victor, M.E.; Patterson, D.; Fluid Phase Equilib., 35 (1987), 237.

[266]: Andreoli-Ball, L.; Costas, M.; Patterson, D.; Rubio, R.G.; Masegosa, R.M.; Cáceres, M.; Ber. Bunsenges. Phys. Chem., 93 (1989), 882.

[267]: Andreoli-Ball, L.; Patterson, D.; Can. J. Chem., 68 (1990), 1077.

[268]: Andreoli-Ball, L.; Sun, S.J.; Trejo, L.M.; Costas, M.; Patterson, D.; Pure and Appl. Chem., 62 (1990), 2097.

[269]: Benson, G.C.; Kumaran, M.K.; Treszczanowicz, T.; D'Arcy, P.J.; Halpin, C.J.; Thermochim. Acta, 95 (1985), 59.

[270]: Grolier, J.-P.E.; Inglese, A.; Wilhelm, E.; J. Chem. Thermodyn., 16 (1984), 67. 
[271]: Grolier, J.-P.E.; Benson, G.C.; Can. J. Chem., 62 (1984), 949.

[272]: Inglese, A.; Grolier, J.-P.E.; Wilhelm, E.; Fluid Phase Equilib., 15 (1984), 287.

[273]: Kalali, H.; Kohler, F.; Svejda, P.; Fluid Phase Equilib., 20 (1985), 75.

[274]: Kalali, H.; Kohler, F.; Svejda, P.; Monatsh. Chem., 118 (1987), 1.

[275]: Kimura, F.; D'Arcy, P.J.; Sugamori, M.E.; Benson, G.C.; Thermochim. Acta 64 (1983), 149.

[276]: Lainez, A.; Rodrigo, M.M.; Wilhelm, E.; Grolier, J.-P.E.; J. Sol. Chem., 21 (1992), 45.

[277]: Lainez, A.; Wilhelm, E.; Grolier, J.-P.E.; Monatsh. Chem., 125 (1994), 877.

[278]: Pintos, M.; Bravo, R.; Baluja, M.C.; Paz Andrade, M.I.; Roux-Desgranges, G.; Grolier, J.-P.E.; Can. J. Chem., 66 (1988), 1179.

[279]: Rubio, R.G.; Caceres, M.; Masegosa, R.M.; Andreoli-Ball, L.; Costas, M.; Patterson, D.; Ber. Bunsenges. Phys. Chem., 93 (1989), 48.

[280]: Smith, J.; Andreoli-Ball, L.; Patterson, D.; J. Chem. Soc. Faraday Trans., 88 (1992), 2875.

[281]: Trejo, L.M.; Costas, M.; Patterson, D.; J. Chem. Soc. Faraday Trans., 87 (1991), 3001.

[282]: Wilhelm, E.; Lainez, A.; Grolier, J.-P.E.; Fluid Phase Equilib., 49 (1989), 233.

[283]: Wilhelm, E.; Thermochim. Acta, 162 (1990), 43.

[284]: Freed, K.F.; J. Phys. A: Math. Gen., 18 (1985), 871.

[285]: Bawendi, M.G.; Freed, K.F.; Mohanty, U.; J. Chem. Phys., 87 (1987), 5534.

[286]: Madden, W.G.; Pesci, A.I.; Freed, K.F.; Macromolecules, 23 (1990), 1181.

[287]: Madden, W.G.; Dudowicz, G.J.; Freed, K.F.; Macromolecules, 23 (1990), 4803.

[288]: Sánchez, I.C.; Lacombe, R.H.; J. Phys. Chem., 80 (1976), 2352.

[289]: Sánchez, I.C.; Lacombe, R.H.; J. Phys. Chem., 80 (1976), 2568.

[290]: Costas, M. ; Sanctury, B.C.; J. Phys. Chem., 85 (1981), 3153.

[291]: Panayiotou, C.; Vera, J.H.; Polym. J., 14 (1981), 681.

[292]: Panayiotou, C.; Sánchez, I.C.; Macromolecules, 24 (1991), 6231.

[293]: Bondi, A.; J. Phys. Chem., 68 (1964), 441.

[294]: Wilson, G.M.; J. Amer. Chem. Soc., 86 (1964) 127.

[295]: Wu, O.-Y.; Cui, Y.; Donohue, M.D.; Ind. Eng. Chem. Res. 37, (1998), 2936.

[296]: Abrams, D.S.; Prausnitz, J.M.; A. I. Ch. E. J., 21 (1975) 116.

[297]: Maurer, G.; Prausnitz, J.M.; Fluid Phase Equilib., 2 (1978), 91.

[298]: Vera, J.H.; Fluid Phase Equilib., 8 (1982), 315.

[299]: Panayiotou, C.; Vera, J.H.; Fluid Phase Equilib., 5 (1980), 55.

[300]: Kemeny, S., Rasmussen, P.; Fluid Phase Equilib., 7 (1981), 197. 
[301]: Flemr, V. Coll. Czech. Chem. Commun., 41 (1976), 3347.

[302]: McDermott, C.; Ashton, N.; Fluid Phase Equilib., 1 (1977), 33.

[303]: Fischer, J.; Fluid Phase Equilib., 10 (1983), 1.

[304]: Scott, R.L.; J. Royal Netherlands Chem. Soc., 75 (1956) 787.

[305]: Renon, H.; Prausnitz, J.M.; A. I. Ch. E. J., 14 (1968) 135.

[306]: Scatchard, G.; Hamer, W.J.; J. Amer. Chem. Soc., 57 (1935), 1805.

[307]: Wohl, K.; Trans. A. I. Ch. E. J., 42 (1946), 215.

[308]: Longuet-Higgings, H.C.; Proc. Roy. Soc., A205 (1951), 247.

[309]: Brown, W.B.; Longuet-Higgings, H.C.; Proc. Roy. Soc., A209 (1951), 416.

[310]: Cook, D.; Longuet-Higgings, H.C.; Proc. Roy. Soc., A209 (1951), 28.

[31 1]: Scott, R.L.; J. Chem. Phys., 25 (1956), 193.

[312]: Hicks, C.P.; J.Chem. Soc. Faraday Trans. II, 72 (1972), 423.

[313]: McGlashan, M.L.; Trans. Faraday Soc. 66 (1970), 18.

[314]: Prigogine, I.; Bellemans, A.; Englert-Chwoles, A.; J. Chem. Phys., 24 (3) (1956), 518.

[315]: Salsburg, Z.W.; Wojtowizc, P.J.; Kirkwood, J.G.; J. Chem. Phys. 26 (1957), 1533.

[316]: Salsburg, Z.W.; Wojłowizc, P.J.; Kirkwood, J.G.; J. Chem. Phys. 27 (1957), 505.

[317]: Brown, W.B.; Phil. Trans. A, 250 (175) (1957), 221.

[318]: Nosanow, L.H.; J. Chem. Phys., 30 (1959), 1596.

[319]: Zwanzig, R. W.; J. Chem. Phys., 22 (1954), 1420.

[320]: Vera, J.H.; Prausnitz, J.M.; Chem. Eng. J., 3 (1972), 1.

[321]: Scott, R.L.; Van Konynenburg, P.H.; Discuss. Faraday Soc., 49 (1970), 87.

[322]: Carnahan, N.F.; Starling, K.E.; J. Chem. Phys., 51 (1969), 635.

[323]: Wilson, G. M.; Advan. Cryog. Eng., 9 (1964), 168.

[324]: Wilson, G. M.; Advan. Cryog. Eng., 11 (1966) 392.

[325]: Egelstaff, P. A.; "An Introduction to the Liquid State", Academic Press, London (1967).

[326]: Eyring, H.; J. 0. Hirschfelder, J. Phys. Chem., 41 (1937), 249.

[327]: Pryde, J. A., "The Liquid State", Hutchinson, London (1966).

[328]: Rice, S. A.; Gray, P.; "The Statistical Mechanics of Simple Liquids", Wiley, New York (1965).

[329]: Rowlinson, J. S.; Contemp. Phys., 5 (1964), 359.

[330]: Rowlinson, J. S.; Repts. Progr. Physics, 28 (1965), 169.

[331]: Flory, P.J.; Orwoll, R.A.; Vrii, A.; J. Amer. Chem. Soc., 86 (1964), 3507.

[332]: Flory, P.J.; Orwoll, R.A.; Vrii, A.; J. Amer. Chem. Soc., 86 (1964), 3515. 
[333]: Flory, P.J.; J. Amer. Chem. Soc., 87 (1965), 1833.

[334]: Abe, A.; Flory. P.J.; J. Amer. Chem. Soc., 87 (1965), 1838.

[335]: Orwoll, R.A.; Flory, P.J.; J. Amer. Chem. Soc., 89 (1967), 6814.

[336]: Orwoll, R.A.; Flory, P.J.; J. Amer. Chem. Soc., 89 (1967), 6822.

[337]: Flory, P.J.; Discuss. Faraday Soc., 49 (1970), 7.

[338]: Patterson, D.; Macromolecules, 2 (1969), 672.

[339]: Patterson, D.; Delmas, G.; Discuss. Faraday Soc., 49 (1970), 98.

[340]: Siow, K.G.; Delmas, G.; Patterson, D.; Macromolecules, 5 (1972), 29.

[341]: Zeman, L.; Patterson, D.; J. Phys. Chem., 73 (1972), 1214.

[342]: Aicart, E.; Tardajos, G.; Diaz Peña, M.; J. Sol. Chem., 12 (1) (1983), 41.

[343]: Riesco, N.; "Aplicación del Modelo de Flory al Estudio de las Propiedades de las Mezclas Entre Hidroxiéteres", Trabajo de Licenciatura; Universidad de Valladolid, Departamento de Termodinámica y Física Aplicada (1999).

[344]: Pouchly, J. Patterson, D.; Macromolecules, 9 (1976), 574.

[345]: Heintz, A.; Ber. Bunsenges. Phys. Chem., 83 (1979), 155.

[346]: Riesco, N.; "Contribución Experimental y Teórica al Estudio de las Propiedades Termodinámicas de las Mezclas Binarias entre Compuestos Orgánicos", Tesis Doctoral; Universidad de Valladolid, Departamento de Termodinámica y Física Aplicada (2003).

[347]: Aicart, E.; Menduiña, C.; Arenosa, R.L.; Tardajos, G.; J. Sol. Chem., 12 (1983), 703.

[348]: Riesco, N.; González, J.A.; Villa, S.; García de la Fuente, I.; Cobos, J.C.; Phys. Chem. Liq., 41 (2003), 309.

[349]: Wang, L.; Benson, G.C.; Lu, B.C.-Yu; Thermochim. Acta, 213 (1993), 83.

[350]: Benson, G.C.; Pflug, H.D.; J. Chem. Eng. Data, 15 (1970), 382.

[351]: Riesco, N.; Villa, S.; González, J.A.; García de la Fuente, I.; Cobos, J.C.; Thermochim. Acta, 362 (2000), 89.

[352]: Heintz, A.; Lichtentahler, R.N.; Ber. Bunsenges. Phys. Chem., 84 (1980), 890.

[353]: Bondi, A.; "Physical Properties of Molecular Crystals, Liquids and Glasses", John Wiley \& Sons, New York (1968).

[354]: Cobos Hernández, J.C.; "Estudio termodinámico de mezclas líquidas de alcoxietanoles con disolventes orgánicos". Tesis Doctoral. Departamento de Física Aplicada II. Facultad de Ciencias. Universidad de Valladolid, 1987.

[355]: Tra Van, H.; Patterson, D.; J. Sol. Chem., 11 (1 1) (1982), 793.

[356]: Patterson, D. ; Delmas, G. ; Discuss. Faraday Soc., 49 (1970), 98.

[357]: González, J.A.; Riesco, N.; Mozo, I.; García de la Fuente, I.; Cobos, J.C.; Ind. Eng. Chem. Res., 46 (2007), 1350.

[358]: Heintz, A.; Ber. Bunsenges. Phys. Chem., 89 (1985), 172. 
[359]: González, J.A.; Riesco, N.; Mozo, I.; García de la Fuente, I.; Cobos, J.C.; Ind. Eng. Chem. Res., 48 (2009), 7417.

[360]: González, J.A.; García de la Fuente, I.; Cobos, J.C.; Mozo, I.; Alonso, I.; Thermochim. Acta, 514 (2011), 1.

[361]: Battacharyya, S.N.; Patterson, D.; J. Phys. Chem., 83 (1979), 2979.

[362]: Tardajos, G.; Aicart, E.; Costas, M.; Patterson, D.; J. Chem. Soc., Faraday Trans. 1, 82 (1986), 2977.

[363]: Battacharyya, S.N.; Costas, M.; Patterson, D.; Tra H.-V.; Fluid Phase Equilib., 20 (1985), 27.

[364]: Howell, P.J.; Skillerne de Bristowe, B.J.; Stubley, D.; J. Chem. Soc. (A), (1971), 397.

[365]: Marsh, K.; Kohler, F.; J. Mol. Liquids, 30 (1985), 13.

[366]: Dolezalek, F.; Z. Phys. Chem., 64 (1908), 727.

[367]: Tejerina, F.; "Termodinámica", Tomo II (Aplicaciones), Ed. Paraninfo, Madrid (1977).

[368]: Münster, A.; "Classical Thermodynamics", Wiley (1970).

[369]: Acree, W.E.; "Thermodynamic Properties of Non-Electrolytes Solutions", Jr. Academic Press (1984).

[370]: Kehiaian, H.; Treszczanowicz, A.; Bull. Soc. Chim. France, 5 (1969), 1561.

[371]: Kehiaian, H.; Bull. Acad. Polon. Sci., Ser. Sci. Chim., 16 (1968), 165.

[372]: Treszczanowicz, A.; Kehiaian, H.; Bull. Acad. Polon. Sci., Ser. Sci. Chim., 18 (1970), 729.

[373]: Kehiaian, H.; Treszczanowicz, A.; Bull. Acad. Polon. Sci., Ser. Sci. Chim., 14 (1966), 891.

[374]: Scatchard, G.; Chem. Rev., 44 (1949), 7.

[375]: Kretschmer, C.B.; Wiebe, R.; J. Chem. Phys., 22, (1954), 1697.

[376]: Smith, F.; Brown, I.; Aust. J. Chem., 26 (1973), 691.

[377]: Smith, F.; Brown, I.; Aust. J. Chem., 26 (1973), 705

[378]: Renon, H.; Prausnitz, J.M.; Chem. Eng. Sci., 22 (1967), 299; Errata 1891.

[379]: Kehiaian, H.V.; en "Thermochemistry and Thermodynamics", MTP Intern. Review of Science, Physical Chemistry (series one, Volume 10), H.A. Skinner, Ed. Butterworths, London (1972).

[380]: Kehiaian, H.V.; en "Chemical Thermodynami Data on Fluids and Fluid Mixtures", Conf. Proc., NPL, Teddington, Middlesex, U.K., Published by IPC Sci. and Technol. Press (1978), 121.

[381]: Heintz, A.; Ber. Bunsenges Phys. Chem., 89 (1985), 172.

[382]: Kehiaian, H.; Bull. Acad. Polon. Sci., Ser. Sci. Chim., 16 (1968), 171.

[383]: Bender, M.; Heintz, A.; Fluid Phase Equilib., 89 (1993), 197. 
[384]: Funke, H.; Wetzel, M.; Heintz, A.; Pure and Appl. Chem., 61 (1989), 1429.

[385]: Reimann, R.; Heintz, A.; J. Sol. Chem., 20 (1991), 29.

[386]: Letcher, T.M.; Bricknell, B.C.; J. Chem. Eng. Data, 41 (1996), 639.

[387]: Mohren, S.; Heintz, A.;, Fluid Phase Equilib., 133 (1997), 247.

[388]: Heintz, A.; Naicker, P.K.; Verevkin, S.P.; Pfestorf, R.; Ber. Bunsenges. Phys. Chem., 102 (1998), 953.

[389]: Heintz, A.; Papaioannou, D.; Termochim. Acta, 310 (1998), 69.

[390]: González, J.A.; García de la Fuente, I.; Cobos, J.C.; Can. J. Chem., 78 (2000), 1272.

[391]: González, J.A.; García de la Fuente, I.; Cobos, J.C.; Fluid Phase Equilib. 168 (2000), 31.

[392]: Villa, S.; Riesco, N.; García de la Fuente, I.; González, J.A.; Cobos, J.C.; Fluid Phase Equilib., 190 (2001), 113.

[393]: Villa, S.; Riesco, N.; García de la Fuente, I.; González, J.A.; Cobos, J.C.; Fluid Phase Equilib., 198 (2002), 313.

[394]: Villa, S.; Riesco, N.; García de la Fuente, I.; González, J.A.; Cobos, J.C.; J. Sol. Chem., 32, (2003) 179.

[395]: Bender, M.; Hauser, J.; Heintz, A.; Ber. Bunsenges. Phys. Chem., 95, (1991), 801.

[396]: González, J.A.; Villa, S.; Riesco, N.; García de la Fuente, I.; Cobos, J.C.; Can. J. Chem., 81 (2003), 1.

[397]: Villa, S.; González, J.A.; García de la Fuente, I.; Riesco, N.; Cobos, J.C.; J. Sol. Chem., 31 (2002), 1019.

[398]: Garriga, R.; Pérez, P.; Gracia, M.; Ber. Bunsenges. Phys. Chem., 101 (1997), 1466.

[399]: Kammerer, K.; Lichtenthaler, R.N.; Thermochim. Acta., 310 (1998), 61.

[400]: Langmuir, I.; Colloid Symp. Monogr., 3 (1925) 48.

[401]: Pierotti, G.J.; Deal, C.H.; Derr, E.L.; Ind. Eng. Chem., 51 (1959), 95.

[402]: Redlich, O.; Derr, E.L.; Pierotti, G.J.; J. Amer. Chem. Soc., 81 (1959), 2283.

[403]: Papadopoulos, M.N.; Derr, E.L.; J. Amer. Chem. Soc., 81 (1959, 2285.

[404]: Wilson, G.M. ; Deal, C.H. ; Ind. Eng. Chem. Fund., 1 (1962), 20.

[405]: Kehiaian, H.V.; Fluid Phase Equilib., 13 (1983), 243.

[406]: Kehiaian, H.V.; Pure and Appl. Chem., 57 (1) (1985), 15.

[407]: Kehiaian, H.V.; Grolier, J.-P.E.; Benson, G.C.; J. Chem. Phys., 75 (1978), 1031.

[408]: Kehiaian, H.V.; Sosnkowska-Kehiaian, K.; Hryniewicz, R.; J. Chem. Phys., 68 (1971), 922.

[409]: Scheller, W.A.; Ind. Eng. Chem. Fundam., 4 (1965), 459.

[410]: Ratcliff, G.A.; Chao, K.C.; Can. J. Chem. Eng., 47 (1969), 148. 
[4 1 1 1]: Bronsted J.N., Koefoed J., Det. Kgl. Danske Videnske Selsk., Mat. Fys. Medd.,22 (1946), 1 .

[412]: Kuo, C.-M.; Robinson, R.L.; Chao, K.-C.; Ind. Eng. Chem. Fundam., 9 (1970), 564.

[413]: Derr, E.L.; Deal, C.H.; “Distillation 1969”, Brigton, England, Int. Conf. Distillation (1969).

[414]: Deal, C.H.; Derr, E.L.; Ind. Eng. Chem., 60 (1968), 28.

[415]: Deal, C.H.; Derr, E.L.; I. Chem. Eng. Symposium Series, 32 (1969), 3.

[416]: Nguyen, T.H.; Ratcliff, G.A.; Can. J. Chem. Eng., 52 (1974), 641.

[417]: Fredenslund, A.; Jones, R.L.; Prausnitz, J.M.; A. I. Ch. E. J., 21 (1975), 1086.

[418]: Gmehling, J.; Pure and Appl. Chem., 75 (7) (2003), 875.

[419]: Nagata, I.; Ohta, T.; Chem. Eng. Sci., 33 (1978), 177.

[420]: Rupp, W.; Hetzel, S.; Ojuni, I. ; Tassios, D. ; Ind. \& Eng. Chem Proc. Des. Develop., 23 (1984), 391.

[421]: Dang, D.; Tassios, D.P.; Ind. \& Eng. Chem. Proc. Des. Develop., 25 (1986), 22.

[422]: Sathis, P.J.; Tassios, D.P.; Ind. \& Eng. Chem. Proc. Des. Develop., 24 (1985), 701.

[423]: Larsen, B.L.; Rasmussen, P.; Fredenslund, A.; Ind. Eng. Chem. Res., 26 (1987), 2274.

[424]: Weidlich, U.; Gmehling, J.; Ind. Eng. Chem. Res., 26 (1987), 1372.

[425]: Gmehling, J.; Li, J.; Schiller, M.; Ind. Eng. Chem. Res., 32 (1993), 178.

[426]: Gmehling, J.; Lohmann, J.; Jakob, A.; Li, J.; Joh, R.; Ind. Eng. Chem. Res., 37 (1998), 4876.

[427]: Gmehling, J.; Wittig, R.; Lohmann, J.; Joh, R.; Ind. Eng. Chem. Res., 41 (2002), 1678.

[428]: Jakob, A.; Grensemann, H.; Lohmann, J.; Gmehling, J.; Ind. Eng. Chem. Res., 45, (2006).

[429]: Lacmann, B.; Schäfer, B.; Brennecke, D.; Li, J.; Pfennig, A.; Phys. Chem. Chem. Phys. 1 (1999), 115.

[430]: Klamt, A.; "COSMO-RS: From Quantum Chemistry to Fluid Phase Thermodynamics and Drug Design", Elsevier (2005).

[431]: Larsen, B. I.; Rasmussen, P.; Fluid Phase Equilib., 28 (1986), 1.

[432]: Egner, K.; Gaube, J.; Pfennig, A.; Ber. Bunsenges. Phys. Chem., 101 (1997), 209.

[433]: Egner, K.; "Ein neues Modell zur Berechnung der Exzessgrößen stark nichtidealer Mischungen und Messungen ternärer Exzessenthalpien", Ph.D. thesis, RWTH University AVT -Thermal Process Engineering (1998).

[434]: Egner, K.; Gaube, J.; Pfennig, A.; Fluid Phase Equilib. 158-160 (1999), 381.

[435]: Klamt, A.; Krooshof, G.J.P.; Taylor, R.; A. I. Ch. E. J., 48 (10) (2002), 2332. 
[436]: Nitta, T.; Turek, E.A.; Greenkorn, R.A.; Chao, K.C.; A. I. Ch. E. J., 23 (1977), 144.

[437]: Bronneberg, R.; Pfennig, A.; Fluid Phase Equilib., 338 (2013), 63.

[438]: Bronneberg, R.; "MOQUAC, a New Expression for the Excess Gibbs Free Energy Based on Molecular Orientations", Ph.D. thesis, RWTH Aachen University - AVT-Thermal Process Engineering (2012).

[439]: Villamañán, M.A.; Tesis Doctoral, Universidad de Valladolid (1979).

[440]: Ait-Kaci, A.; Thése de troisieme cycle. Lyon, France (1979).

[441]: Sarmiento Escalona, F.; Tesis Doctoral, Universidad de Santiago de Compostela (1982).

[442]: Grolier, J.-P.E.; Sosnkowska-Kehiaian, K.; Hryniewicz, R.; J. Chim. Phys., 68 (1971), 922.

[443]: Grolier, J.P.E.; Kehiaian, H.V.; J. Chim. Phys., 70 (1973), 807.

[444]: Teszczanowicz, T.; Bull. Acad. Polon. Si., Ser. Sci. Chim., 23 (1975), 161.

[445]: Karbali Ghassemi, M.H.; Thése de Doctorat, Université de Provence, Marseille, France (1977).

[446]: Wilhem, E. ; Inglese, A. ; Grolier, J.-P.E.; Kehiaian, H.V.; Monatsh Chem., 109 (1978), 235.

[447]: Kehiaian, H.V.; Grolier, J.-P.E.; Kechavarz, M.-R.; Benson, G.C.; Fluid Phase Equilib., 5 (1980), 159.

[448]: Kehiaian, H.V.; Grolier, J.-P.E.; Kechavarz, M.-R.; Benson, G.C.; Kiyohara, O.; Handa, Y.P.; Fluid, Phase Equilib., 7 (1981), 95.

[449]: Ferino, I.; Marongiu, B.; Solinas, V.; Torrazza, S.; Kehiaian, H.V.; Fluid Phase Equilib., 9 (1982), 49.

[450]: Kehiaian, H.V.; Sandler, S.I.; Fluid Phase Equilib., 17 (1984), 139.

[451]: Roux, A.H.; Grolier, J.-P.E. ; Inglese, A. ; Wilhelm, E. ; Ber. Bunsenges. Phys. Chem., 88 (1984), 986.

[452]: Kehiaian, H.V.; Marongiu, B.; Fluid Phase Equilib., 21 (1985), 197.

[453]: Pintos Barral, M.; Tesis Doctoral, Universidad de Santiago de Compostela (1980). 
CAPÍTULO 6 
CAPITULO 7

\section{Aplicación de los modelos teóricos a los datos experimentales}

"Truth in science can be defined as the working hypothesis best suited to open the way to the next better one."

Konrad Lorenz

— n el presente capítulo se presentan los valores calculados de los diferentes parámetros que aparecen en las ecuaciones de los modelos teóricos utilizados para el análisis de las mezclas consideradas en esta memoria, así como las diferentes propiedades termodinámicas calculadas mediante dichos modelos. También se discuten tanto la validez de los mismos como el comportamiento de los parámetros hallados.

\subsection{MODELO DISQUAC}

En la presente memoria el modelo se ha aplicado a las mezclas siguientes:

a) anilina +2 -propanona, 2-butanona, 2-pentanona y 2-heptanona.

b) $\quad \mathrm{N}$-metilanilina +2 -propanona, 2-butanona, 2-pentanona y 2-heptanona.

c) di-n-propilamina +2 -propanona, 2-butanona, 2-pentanona y 2-heptanona.

d) di- $n$-butilamina +2 -propanona, 2-butanona, 2-pentanona y 2 -heptanona.

e) $N, N, N$-trietilamina +2 -propanona, 2-butanona, 2-pentanona y 2 -heptanona.

f) $\quad N, N$-dimetiletilamina ó $N, N$-dietilmetilamina +2 -propanona.

g) piridina +2 -propanona

h) $N$-metilanilina + heptano, octano, decano, ciclohexano, tolveno y benceno.

i) $\quad N, N$-dimetilanilina + ciclohexano.

i) metanol ó 1-butanol $+\mathrm{N}$-metilanilina. 
k) metanol ó 1 -butanol $+N, N$-dimetilanilina.

l) 1-dimetilamino-2-propanona + heptano y hexadecano.

m) 5-dimetilamino-2-pentanona + heptano y hexadecano.

n) 1-dietilamino-2-propanona + hexano, heptano, dodecano y hexadecano.

o) 1-propanol $+N, N, N$-trietilamina +2 -butanona.

Como puede observarse en la lista anterior, no sólo se ha aplicado DISQUAC a las mezclas estudiados experimentalmente en este trabajo, sino que también se ha aplicado a otros sistemas en los que aparecen los grupos amino y cetona y de los que se han encontrado datos experimentales en la bibliografía. La razón de ello es analizar el valor predictivo de los métodos de contribución de grupos para estas mezclas, consecuencia de sus dos características básicas: independencia del comportamiento de cada grupo funcional e independencia de la superficie de contacto de cada grupo, todo ello respecto de la molécula a la que el grupo pertenece. Como ya se ha explicado en el Capítulo 6, una vez conocida la información de las interacciones grupol-grupo2, a partir de la correlación de datos experimentales de sistemas clave se pueden calcular las interacciones molécula-molécula de cualquier par de moléculas que contengan los mismos grupos, aunque no haya disponibles datos experimentales.

Antes de aplicar DISQUAC es necesario saber que en el contexto de dicho modelo, en las mezclas arriba listadas aparecen los siguientes tipos de grupos funcionales:

a) Tipo a, alifático. En concreto, $\mathrm{CH}_{3}$ y $\mathrm{CH}_{2}$ presentes en las moléculas de $N$-metilanilina, $N, N$-dimetilanilina, aminas lineales secundarias (di- $n$-etilamina, di- $n$ propilamina y di-n-butilamina), tri-n-alquilaminas $(N, N, N$-trietilamina, $N, N$ dietilmetilamina, $N, N$-dimetiletilamina), 2-alcanonas (2-propanona, 2-butanona, 2pentanona y 2-heptanona), amino-cetonas (1-dimetilamino-2-propanona, 1-dietilamino2-propanona y 5-dimetilamino-2-pentanona), alcanos (hexano, heptano, decano, dodecano y hexadecano), tolveno y alcoholes (metanol, 1-propanol y 1-butanol).

b) Tipo b, aromático. En concreto $\mathrm{C}_{6} \mathrm{H}_{6}$ presente en la molécula de benceno y $\mathrm{C}_{6} \mathrm{H}_{5}$ presente en las moléculas de aminas aromáticas (anilina, $N$-metilanilina, piridina y $N, N$-dimetilanilina).

c) Tipo c, alquilo cíclico, $\mathrm{c}-\mathrm{CH}_{2}$ presente en la molécula de ciclohexano.

d) Tipo $\mathrm{h}$, hidroxilo $(\mathrm{OH})$ presente en las moléculas de los 1-alcoholes (metanol, 1propanol y 1-butanol).

e) Tipo k, carbonilo (CO). Presente en las moléculas de las cetonas (2-propanona, 2-butanona, 2-pentanona y 2-heptanona) y de las amino-cetonas (1-dimetilamino-2propanona, 1-dietilamino-2-propanona y 5-dimetilamino-2-pentanona).

f) Tipo $\mathrm{n}$, amino. En concreto, $\mathrm{NH}_{2}$ presente en moléculas de aminas primarias (anilina); $\mathrm{NH}$ presente en moléculas de aminas secundarias ( $N$-metilanilina, di- $n$-etilamina, di- $n$-propilamina y di- $n$-butilamina); $\mathrm{N}$ presente en moléculas de aminas terciarias (piridina, $N, N$-dietilmetilamina, $N, N$-dimetiletilamina, $N, N, N$-trietilamina y $N, N$-dimetilanilina) y amino-cetonas (1-dimetilamino-2-propanona, 1-dietilamino-2propanona y 5-dimetilamino-2-pentanona).

En el modelo DISQUAC aparecen dos tipos de parámetros característicos diferentes: geométricos y de intercambio o de interacción. Como sabemos, los parámetros 
geométricos dan cuenta del modelo de interacción molecular adoptado, mientras que los coeficientes de intercambio están asociados con el modelo estadístico de cuasired que constituye el sustrato microscópico sobre el que se asienta dicho modelo.

Respecto a los parámetros geométricos, ya se ha visto en el capítulo anterior que cada molécula del tipo i está caracterizada por los parámetros siguientes: superficie de contacto, $q_{\mathrm{i}}$; volumen, $r_{\mathrm{i}}$; fracción de superficie del grupo tipo s, $\alpha_{\mathrm{si}}$.

De acuerdo con el planteamiento que los MMCCGGFF conllevan, y que es introducido por el modelo molecular adoptado, las propiedades estructurales de cada molécula se obtienen aditivamente a partir de las de los grupos funcionales de los que se considera que está formada. Para la caracterización de los grupos funcionales se ha adoptado el método de Bondi [1], [2] que considera cada átomo como una esfera y donde el volumen, $V_{\mathrm{g}}$ y la superficie, $A_{\mathrm{g}}$, de cada grupo se calculan basándose en la geometría de los átomos que lo forman, unidos por enlaces con superficies interpenetrantes. La fracción de superficie (y por tanto de volumen) disponible para un posible contacto se calcula a partir de los radios covalentes y de van der Waals. Los parámetros geométricos, es decir, los incrementos relativos de volumen y superficie de cada grupo g utilizados en el presente estudio, se denotan $r_{\mathrm{g}}$ y $q_{\mathrm{g}}$ respectivamente, y aparecen en la Tabla 7-1. Como es habitual en cálculos de este tipo, $r_{\mathrm{g}}$ y $q_{\mathrm{g}}$ son incrementos relativos de grupo referidos al volumen y la superficie de la molécula de metano $\left(17.12 \cdot 10^{-6}\right.$ $\mathrm{m}^{3} \cdot \mathrm{mol}^{-1}$ y $2.96 \cdot 10^{-5} \mathrm{~m}^{2} \cdot \mathrm{mol}^{-1}$ respectivamente), que arbitrariamente se toman como unidades de superficie y de volumen:

$$
\begin{gathered}
r_{\mathrm{g}}=\frac{V_{\mathrm{g}}}{V_{\mathrm{CH}_{4}}} \\
q_{\mathrm{g}}=\frac{A_{\mathrm{g}}}{A_{\mathrm{CH}_{4}}}
\end{gathered}
$$

Tabla 7-1: incrementos relativos de grupo $r_{\mathrm{g}}$ y $q_{\mathrm{g}} \cdot{ }^{\mathrm{a}}[3] ;{ }^{\mathrm{b}}[4] ;{ }^{\mathrm{c}}[5] ;{ }^{\mathrm{d}}[6] ;{ }^{\mathrm{e}}[7] ;{ }^{\mathrm{f}}[8] ; \mathrm{g}[9]$

\begin{tabular}{ccc}
\hline grupo & $r_{\mathrm{g}}$ & $q_{\mathrm{g}}$ \\
\hline $\mathrm{CH}_{3}$ & $0.79848^{\mathrm{a}}$ & $0.73103^{\mathrm{a}}$ \\
$\mathrm{CH}_{2}$ & $0.59755^{\mathrm{a}}$ & $0.46552^{\mathrm{a}}$ \\
$\mathrm{C}_{6} \mathrm{H}_{5}$ & $2.67757^{\mathrm{a}}$ & $1.837933^{\mathrm{a}}$ \\
$\mathrm{C}_{6} \mathrm{H}_{6}$ & $2.8248^{\mathrm{b}}$ & $2.0724^{\mathrm{b}}$ \\
$\mathrm{OH}$ & $0.46963^{\mathrm{c}}$ & $0.50345^{\mathrm{c}}$ \\
$\mathrm{CO}$ & $0.68341^{\mathrm{d}}$ & $0.55172^{\mathrm{d}}$ \\
$\mathrm{NH}$ & $0.61565^{\mathrm{e}}$ & $0.6000^{\mathrm{e}}$ \\
$\mathrm{NH}$ & $0.47196^{\mathrm{f}}$ & $0.34138^{\mathrm{f}}$ \\
$\mathrm{N}$ & $0.25290^{\mathrm{g}}$ & $0.07930^{\mathrm{g}}$ \\
\hline
\end{tabular}

El volumen total de la molécula $i, r_{i}$, su superficie total, $q_{i}$, y la superficie total del grupo s en la molécula del tipo i, $q_{\mathrm{si}},(\mathrm{i}=1,2, \ldots, \mathrm{n} ; \mathrm{s}=1,2, \ldots, \sigma)$ se calculan de forma aditiva utilizando los correspondientes incrementos de grupo. Las fracciones de superficie de contacto del grupo tipo s en una molécula i, $\alpha_{\text {si }}$, se calculan según su definición:

$$
\alpha_{\mathrm{si}}=\frac{q_{\mathrm{si}}}{q_{\mathrm{i}}}
$$


Los valores de $r_{\mathrm{i}}$ y $q_{\mathrm{i}}$ de todas las moléculas a las que se ha aplicado el modelo DISQUAC en este trabajo están dados en la Tabla siguiente:

Tabla 7-2: volúmenes totales $\boldsymbol{r}_{\mathbf{i}}$ y superficies totales $\boldsymbol{q}_{\mathrm{i}}$ de cada molécula calculados por la suma simple de los incrementos relativos de grupo de la Tabla $7-1 ;{ }^{\circ}[3]$

\begin{tabular}{|c|c|c|}
\hline Molécula & $r_{\mathrm{i}}$ & $q_{\mathrm{i}}$ \\
\hline hexano & 3.98716 & 3.32414 \\
\hline heptano & 4.58471 & 3.78966 \\
\hline octano & 5.18226 & 4.25518 \\
\hline decano & 6.37736 & 5.18622 \\
\hline dodecano & 7.57246 & 6.11726 \\
\hline hexadecano & 9.96266 & 7.97934 \\
\hline ciclohexano & $3.5187^{a}$ & $2.5966^{a}$ \\
\hline tolveno & 3.47605 & 2.56896 \\
\hline benceno & 2.82480 & 2.07240 \\
\hline metanol & 1.26811 & 1.23448 \\
\hline 1-propanol & 2.46321 & 2.16552 \\
\hline 1-butanol & 3.06076 & 2.63104 \\
\hline 2-propanona & 2.28037 & 2.01378 \\
\hline 2-butanona & 2.87792 & 2.4793 \\
\hline 2-pentanona & 3.47547 & 2.94482 \\
\hline 2-heptanona & 4.67057 & 3.87586 \\
\hline anilina & 3.29322 & 2.43793 \\
\hline$N$-metilanilina & 3.94801 & 2.91034 \\
\hline piridina & 2.93047 & 1.91723 \\
\hline$N, N$-dimetilanilina & 4.52743 & 3.37929 \\
\hline di-n-etilamina & 3.26402 & 2.73448 \\
\hline di-n-propilamina & 4.45912 & 3.66552 \\
\hline di-n-butilamina & 5.65422 & 4.59656 \\
\hline$N, N$-dimetiletilamina & 3.24589 & 2.73791 \\
\hline$N, N$-dietilmetilamina & 3.84344 & 3.20343 \\
\hline$N, N, N$-trietilamina & 4.44099 & 3.66895 \\
\hline 1-dimetilamino-2-propanona & 3.9293 & 3.28963 \\
\hline 1-dietilamino-2-propanona & 5.1244 & 4.22067 \\
\hline 5-dimetilamino-2-pentanona & 5.1244 & 4.22067 \\
\hline
\end{tabular}

En lo que respecta a los coeficientes de intercambio, que caracterizan el modelo estadístico utilizado, hay que decir que cada par de grupos en contacto $(\mathrm{s}, \mathrm{t})$ está descrito por el conjunto de parámetros dispersivos $C_{\mathrm{st}, l}^{\mathrm{DIS}}$ y cuasiquímicos $C_{\mathrm{st}, l}^{\mathrm{QUAC}}\left(l=1, G_{\mathrm{m}}^{\mathrm{E}}\right.$; $l=2, H_{\mathrm{m}}^{\mathrm{E}} ; l=3, C_{P, \mathrm{~m}}^{\mathrm{E}}$ ), junto con el número de coordinación $z$ (relacionado con la parte cuasiquímica), que da cuenta de la no aleatoriedad en las distribución de las moléculas en la mezcla, como sabemos. Se utiliza el valor $z=4$ para todos los contactos polares, lo que constituye una de las mayores desventajas del modelo, aunque se elimina parcialmente suponiendo que los parámetros de interacción dependen de la estructura. La estimación de los parámetros de intercambio debe realizarse mediante la comparación de las ecuaciones generales del modelo con los mejores datos experimentales disponibles de $G_{\mathrm{m}}^{\mathrm{E}}$ y $H_{\mathrm{m}}^{\mathrm{E}}$ (y $C_{P, \mathrm{~m}}^{\mathrm{E}}$ si fuese necesario), cuya dependencia con la concentración se intenta representar de la manera más precisa posible, partiendo de aquellas clases de mezclas (llamadas sistemas clave) en donde sólo un 
tipo de contacto es desconocido. En particular, los datos experimentales de $G_{\mathrm{m}}^{\mathrm{E}}$ permiten encontrar los valores de las superficies de contacto en equilibrio $X_{\mathrm{s}}$ y $X_{\mathrm{t}}$ para la aproximación cuasiquímica (ecuación 6.416) a través de $\eta_{\text {st }}$ (ecuación 6.429) que es además imprescindible para poder ajustar $H_{\mathrm{m}}^{\mathrm{E}}$ (ecuación 6.430). Así es evidente la necesidad de disponer de datos de $G_{\mathrm{m}}^{\mathrm{E}}$ lo más fiables posibles. En la presente memoria, como no se han realizado mediciones experimentales que permitan obtener valores de $G_{\mathrm{m}}^{\mathrm{E}}$, éstos han sido tomados de la bibliografía.

El ajuste se realiza mediante un programa [10] que utiliza un algoritmo de ajuste para funciones no lineales de tipo Marquardt [1 1] que minimiza la función siguiente:

$$
F\left(C_{\mathrm{st}, 1}^{\mathrm{DIS} / \mathrm{QUAC}}, C_{\mathrm{st}, 2}^{\mathrm{DIS} / \mathrm{QUAC}}, C_{\mathrm{st}, 3}^{\mathrm{DIS} / \mathrm{QUAC}}\right)=\sum_{1}^{N_{G}} \frac{\left(G_{\mathrm{m}, \mathrm{exp}}^{\mathrm{E}}-G_{\mathrm{m}, \mathrm{cal}}^{\mathrm{E}}\right)^{2}}{N_{G}}+\sum_{1}^{N_{H}} \frac{\left(H_{\mathrm{m}, \text { exp }}^{\mathrm{E}}-H_{\mathrm{m}, \mathrm{cal}}^{\mathrm{E}}\right)^{2}}{N_{H}}
$$

donde $N_{G}$ y $N_{H}$ es el número de datos experimentales disponibles para $G_{\mathrm{m}}^{\mathrm{E}}$ y $H_{\mathrm{m}}^{\mathrm{E}}$ respectivamente.

En ausencia de datos experimentales, los correspondientes coeficientes de intercambio se estiman por interpolación o extrapolación de aquellos que han sido previamente hallados, teniendo en cuenta la variación relativa de dichos coeficientes de intercambio con la estructura molecular.

En la Tabla 7-3 se muestran los valores de los coeficientes de intercambio utilizados. En su obtención se han tenido en cuenta las siguientes observaciones:

a) Mezclas amina lineal secundaria + cetona: estas mezclas están caracterizadas por los tres tipos de contacto siguientes: $(\mathrm{a}, \mathrm{k}),(\mathrm{a}, \mathrm{n})$ y $(\mathrm{k}, \mathrm{n})$. Los parámetros de intercambio para los contactos $(\mathrm{a}, \mathrm{n})$ y $(\mathrm{a}, \mathrm{k})$ son conocidos de estudios anteriores de mezclas de amina lineal secundaria con alcanos [8] y cetonas con alcanos [6]. En consecuencia, solamente se han tenido que ajustar a los datos de $G_{\mathrm{m}}^{\mathrm{E}}$ [12] y $H_{\mathrm{m}}^{\mathrm{E}}$ los parámetros de intercambio dispersivos $C_{\mathrm{st}, l}^{\mathrm{DIS}}$ y cuasiquímicos $C_{\mathrm{st}, l}^{\mathrm{QUAC}}$ de los contactos $(\mathrm{k}, \mathrm{n})$.

b) Mezclas amina aromática + cetona: estas mezclas están caracterizadas por los seis tipos de contacto siguientes: $(\mathrm{a}, \mathrm{b}),(\mathrm{a}, \mathrm{k}),(\mathrm{a}, \mathrm{n}),(\mathrm{b}, \mathrm{k}),(\mathrm{b}, \mathrm{n})$ y $(\mathrm{k}, \mathrm{n})$. Los contactos $(\mathrm{a}, \mathrm{b})$ están representados solamente por parámetros dispersivos, y han sido determinados a partir de las propiedades termodinámicas de las mezclas alquilbenceno + alcano [13]. Los parámetros de intercambio para los contactos $(\mathrm{b}, \mathrm{k})$ se han obtenido a partir de los resultados experimentales de las mezclas cetona + compuesto aromático [6], [14]. Análogamente, los parámetros de intercambio para los contactos $(a, n)$ y $(b, n)$ se han obtenido a partir de los datos de las mezclas anilina, $N$-metilanilina o piridina + n-alcano o compuesto aromático [este trabajo], [15], [16]. Los parámetros de intercambio para los contactos $(\mathrm{a}, \mathrm{k})$ son conocidos de estudios anteriores de mezclas de cetonas con alcanos [6]. Por tanto, sólo se han ajustado a los datos de $G_{\mathrm{m}}^{\mathrm{E}}$ [17] y $H_{\mathrm{m}}^{\mathrm{E}}$ los parámetros de intercambio $C_{\mathrm{st}, l}^{\mathrm{DIS}}$ y $C_{\mathrm{st}, l}^{\mathrm{QUAC}}$ de los contactos $(\mathrm{k}, \mathrm{n})$.

c) Mezclas tri- $n$-alquilamina + cetona: estas mezclas están caracterizadas por los tres tipos de contacto siguientes: $(\mathrm{a}, \mathrm{k}),(\mathrm{a}, \mathrm{n})$ y $(\mathrm{k}, \mathrm{n})$. Los parámetros de intercambio para los contactos $(\mathrm{a}, \mathrm{n})$ y $(\mathrm{a}, \mathrm{k})$ son conocidos de estudios anteriores de mezclas de amina 
terciarias con alcanos [9], [18]-[20] y cetonas con alcanos [21], [6]. Los parámetros de interacción de los contactos $(\mathrm{a}, \mathrm{n})$ son solamente dispersivos y se ha demostrado que dependen de los grupos alquílicos unidos al átomo de nitrógeno [9], [18]-[20], y en el caso de las mezclas con $N, N, N$-trietilamina, también de la longitud del alcano considerado [20], consecuencia del efecto Patterson. Por tanto, sólo se han ajustado a los datos de $G_{\mathrm{m}}^{\mathrm{E}}$ [22]-[24] y $H_{\mathrm{m}}^{\mathrm{E}}$ [25] los parámetros de intercambio $C_{\mathrm{st}, l}^{\mathrm{DIS}}$ y $C_{\mathrm{st}, l}^{\mathrm{QUAC}}$ de los contactos $(\mathrm{k}, \mathrm{n})$.

d) Mezclas $N$-metilanilina $+n$-alcano, benceno o tolueno: estas mezclas están caracterizadas por los tres tipos de contactos siguientes: $(\mathrm{a}, \mathrm{b}),(\mathrm{a}, \mathrm{n})$ y $(\mathrm{b}, \mathrm{n})$. Los contactos $(\mathrm{a}, \mathrm{b})$ han sido determinados a partir de las propiedades termodinámicas de las mezclas alquilbenceno + alcano [13], como ya se ha dicho. Los parámetros de intercambio asociados a los contactos $(\mathrm{a}, \mathrm{n})$ y $(\mathrm{b}, \mathrm{n})$ han sido calculados ajustando simultáneamente los datos experimentales de $G_{\mathrm{m}}^{\mathrm{E}}$ y $H_{\mathrm{m}}^{\mathrm{E}}$ disponibles [26].

e) Mezclas $N$-metilanilina + ciclohexano: estas mezclas están caracterizadas por los seis tipos de contacto siguientes: $(\mathrm{a}, \mathrm{b}),(\mathrm{a}, \mathrm{c}),(\mathrm{a}, \mathrm{n}),(\mathrm{c}, \mathrm{n}),(\mathrm{b}, \mathrm{c})$ y $(\mathrm{b}, \mathrm{n})$. Los contactos $(\mathrm{a}, \mathrm{c})$ y $(\mathrm{b}, \mathrm{c})$ están descritos solamente por parámetros dispersivos, y han sido obtenidos a partir de las propiedades termodinámicas de las mezclas ciclohexano $+n$-alcano [29] o alquilbenceno [13], respectivamente. Como los parámetros de interacción para los contactos $(\mathrm{a}, \mathrm{n})$ y $(\mathrm{b}, \mathrm{n})$ ya son conocidos, solamente deben considerarse los contactos $(\mathrm{c}, \mathrm{n})$. Esto se ha hecho asumiendo que $C_{\mathrm{an}, l}^{\mathrm{QUAC}}=C_{\mathrm{cn}, l}^{\mathrm{QUAC}}(l=1,2,3)$. Esta regla es general, y es válida para muchos tipos de mezclas como cetona lineal, carbonato orgánico lineal, ácido anhídrido alcanoico, cloroalcano, alcoholes amida, anilina, $\ldots+n$-alcano o ciclohexano (ver las referencias citadas en [15] y [26]).

f) Mezclas $N$-metilanilina + alcoholes: estas mezclas están caracterizadas por los seis tipos de contactos siguientes: $(\mathrm{a}, \mathrm{b}),(\mathrm{a}, \mathrm{h}),(\mathrm{a}, \mathrm{n}),(\mathrm{b}, \mathrm{h}),(\mathrm{b}, \mathrm{n})$ y $(\mathrm{h}, \mathrm{n})$. Los parámetros de interacción para los contactos $(\mathrm{a}, \mathrm{h})$ y $(\mathrm{b}, \mathrm{h})$ son conocidos del estudio de las mezclas alcohol + alcano [5] o + alquilbenceno [27], [28]. Como los parámetros de interacción para los contactos $(\mathrm{a}, \mathrm{b}),(\mathrm{a}, \mathrm{n})$ y $(\mathrm{b}, \mathrm{n})$ son conocidos, solamente deben determinarse los de los contactos (h,n).

g) Mezclas amino-cetona + $n$-alcano: estos sistemas están caracterizadas por los tres tipos de contacto siguientes: $(\mathrm{a}, \mathrm{k}),(\mathrm{a}, \mathrm{n})$ y $(\mathrm{k}, \mathrm{n})$. Como ya se ha mencionado, los parámetros de intercambio para los contactos $(\mathrm{a}, \mathrm{n})$ y $(\mathrm{a}, \mathrm{k})$ son conocidos de estudios anteriores de sistemas amina o cetona + alcano. Por tanto sólo se han ajustado a los datos experimentales [30] los parámetros de intercambio dispersivos $C_{\mathrm{st}, l}^{\mathrm{DIS}}$ y cuasiquímicos $C_{\mathrm{st}, l}^{\mathrm{QUAC}}$ de los contactos $(\mathrm{k}, \mathrm{n})$.

h) Mezcla 1-propanol $+N, N, N$-trietilamina +2 -butanona: se ha supuesto que en estas mezclas no existen interacciones ternarias, es decir, que están caracterizadas por los seis tipos de contactos siguientes: $(\mathrm{a}, \mathrm{h}),(\mathrm{a}, \mathrm{k}),(\mathrm{a}, \mathrm{n}),(\mathrm{h}, \mathrm{n}),(\mathrm{k}, \mathrm{n})$ y $(\mathrm{h}, \mathrm{k})$. Todos ellos han sido calculados con anterioridad en el estudio de los sistemas alcohol + alcano, cetona + alcano, amina terciaria + alcano, alcohol + amina terciaria, cetona + amina terciaria y alcohol + cetona. En consecuencia, ningún parámetro de intercambio se ha ajustado a los datos experimentales, y en este caso el modelo adquiere carácter totalmente predictivo. No obstante, como se explica más adelante, los resultados obtenidos no son muy precisos, debido a que las interacciones entre tríos de grupos son 
importantes, al menos en este caso particular.

Tabla 7-3: Coeficientes de intercambio dispersivos $C_{\mathrm{st}, l}^{\mathrm{DIS}}$ y cuasiquímicos $C_{\mathrm{st}, l}^{\mathrm{QUAC}}(l=1$, energía de

Gibbs de exceso; $l=2$, entalpía de exceso; $l=3$, capacidad calorífica de exceso) para los contactos (s,t) en las mezclas de interés. ${ }^{a}$ valor estimado; ${ }^{b}$ valor conjeturado

\begin{tabular}{|c|c|c|c|c|c|c|}
\hline Sistema & $C_{\mathrm{st}, 1}^{\mathrm{DIS}}$ & $C_{\mathrm{st}, 2}^{\mathrm{DIS}}$ & $C_{\mathrm{st}, 3}^{\mathrm{DIS}}$ & $C_{\mathrm{st}, 1}^{\mathrm{QUAC}}$ & $C_{\mathrm{st}, 2}^{\mathrm{QUAC}}$ & $C_{\mathrm{st}, 3}^{\mathrm{QUAC}}$ \\
\hline \multicolumn{7}{|c|}{ anilina } \\
\hline 2-propanona & 1.65 & -11.3 & -22 & -1.5 & 3.5 & -2 \\
\hline 2-butanona & 1.65 & 5.45 & -22 & -1.5 & -2.2 & -2 \\
\hline 2-pentanona & 1.65 & 6.8 & -22 & -1.5 & -2.2 & -2 \\
\hline 2-heptanona & 1.65 & 7.4 & & -1.5 & -2.2 & -2 \\
\hline \multicolumn{7}{|c|}{$N$-metilanilina } \\
\hline$n$-alcano & 2.03 & 11 & 12 & 6 & 11.5 & 20 \\
\hline ciclohexano & $2^{a}$ & 12.1 & 18 & 6 & 11.5 & 20 \\
\hline $\begin{array}{l}\text { benceno o } \\
\text { tolueno }\end{array}$ & 3 & 11 & 28 & 1.25 & 5 & 4 \\
\hline metanol & $-5^{a}$ & 7 & -25 & 0.5 & 2.85 & 5 \\
\hline etanol & $-5^{b}$ & $16^{\mathrm{b}}$ & $-25^{b}$ & 1.5 & 2.85 & 5 \\
\hline 1-propanol & $-2^{b}$ & $19^{b}$ & $-25^{b}$ & 3 & -3.5 & 5 \\
\hline$\geq 1$-butanol & $-0.5^{b}$ & 21.5 & $-25^{b}$ & 3 & -3.5 & 5 \\
\hline 2-propanona & 1.65 & 36.8 & -22 & -1.5 & -10.65 & -2 \\
\hline$\geq 2$-butanona & 1.65 & 36.8 & -22 & -1.5 & -10 & -2 \\
\hline \multicolumn{7}{|c|}{ piridina } \\
\hline 2-propanona & 4.12 & 22.8 & -22 & -1.5 & -10.65 & -2 \\
\hline \multicolumn{7}{|c|}{$\mathrm{N}, \mathrm{N}$-dimetilanilina } \\
\hline$n$-alcano & 38 & 112 & $12^{b}$ & 6 & 11.5 & 20 \\
\hline ciclohexano & 40 & 116 & $18^{b}$ & 6 & 11.5 & 20 \\
\hline $\begin{array}{c}\text { benceno o } \\
\text { tolveno }\end{array}$ & 45 & 104 & $28^{b}$ & 1.25 & 0.2 & 4 \\
\hline metanol & $20^{b}$ & 118 & $-25^{b}$ & 0.5 & -2.4 & 5 \\
\hline 1-butanol & $120^{\mathrm{b}}$ & 163 & $-25^{b}$ & 3 & -10 & 5 \\
\hline \multicolumn{7}{|c|}{ di-n-etilamina } \\
\hline 2-propanona & -0.5 & 15 & -22 & 0.9 & -4 & -2 \\
\hline \multicolumn{7}{|c|}{ di- $n$-propilamina } \\
\hline 2-propanona & -0.5 & 7.2 & -22 & 0.9 & -2 & -2 \\
\hline 2-butanona & -0.5 & 1.95 & -22 & 0.9 & -0.4 & -2 \\
\hline$\geq 2$-pentanona & -0.5 & -3.3 & -22 & 0.9 & 1.4 & -2 \\
\hline \multicolumn{7}{|c|}{ di-n-butilamina } \\
\hline 2-propanona & -0.5 & 9.3 & -22 & 0.9 & -2 & -2 \\
\hline 2-butanona & -0.5 & 3.4 & -22 & 0.9 & -0.4 & -2 \\
\hline$\geq 2$-pentanona & -0.5 & -2.7 & -22 & 0.9 & 1.4 & -2 \\
\hline \multicolumn{7}{|c|}{$N, N, N$-trietilamina } \\
\hline 2-propanona & 19 & 49.2 & & -4 & -4.4 & \\
\hline$\geq 2$-butanona & 19 & 27.4 & & -4 & -0.2 & \\
\hline 3-pentanona & 19 & 32 & & -4 & -0.2 & \\
\hline \multicolumn{7}{|c|}{$N, N$-dimetiletilamina ó $N, N$-dietilmetilamina } \\
\hline 2-propanona & 30 & 110 & & -4 & 0.5 & \\
\hline \multicolumn{7}{|c|}{ 1-dimetilamino-2-propanona } \\
\hline$n$-alcano & $51^{\mathrm{b}}$ & 196 & & -4 & -4.4 & \\
\hline \multicolumn{7}{|c|}{ 5-dimetilamino-2-pentanona } \\
\hline$n$-alcano & $51^{\mathrm{b}}$ & 213 & & -4 & -0.2 & \\
\hline \multicolumn{7}{|c|}{ 1-dietilamino-2-propanona } \\
\hline$n$-alcano & $51^{\mathrm{b}}$ & 100 & & -4 & -0.2 & \\
\hline
\end{tabular}


En las llustraciones 7-1 a 7-12 se representan los resultados experimentales y calculados mediante el modelo DISQUAC de $H_{\mathrm{m}}^{\mathrm{E}}$ para las mezclas de interés. Además, en las llustraciones 7-13 a 7-18 se representan tanto los resultados experimentales como los calculados con DISQUAC de los equilibrios líquido-líquido, sólido-líquido y líquido-vapor de algunas mezclas con aminas. Como se observa en la Tabla 7-4, las coordenadas de los puntos críticos, $\left(T_{\mathrm{c}}, x_{1 \mathrm{c}}\right)$, pueden obtenerse con DISQUAC con buen grado de exactitud. Los cálculos se han realizado bajo la hipótesis de que las funciones de exceso son analíticas cerca de los puntos críticos, mientras que, en realidad, las propiedades termodinámicas no son analíticas, aunque pueden expresarse en términos de las leyes de escala con exponentes críticos universales y funciones de escala universales [33], [34].

Tabla 7-4: valores experimentales y calculados con DISQUAC coordenadas de los puntos críticos, $\left(T_{\mathrm{c}}, x_{1 \mathrm{c}}\right)$, de mezclas $\mathrm{N}$-metilanilina + alcano

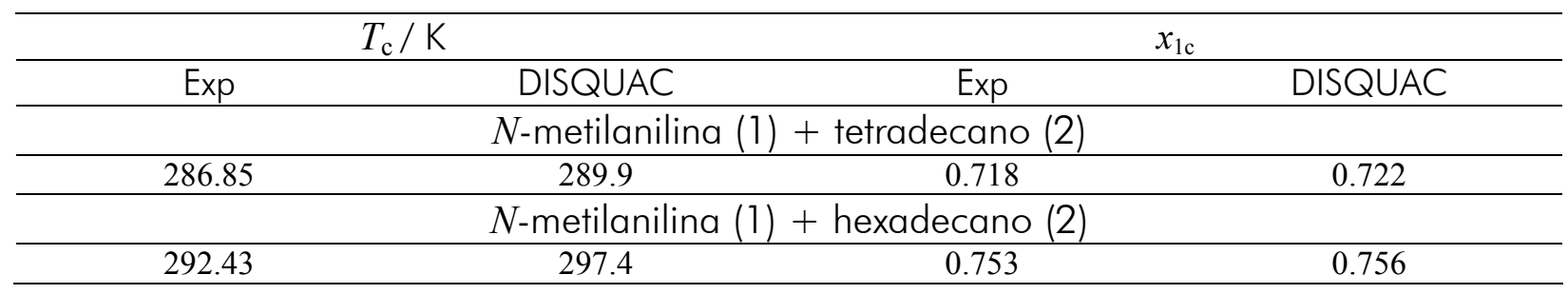

En las tablas $7-5$ y $7-6$ se comparan los valores calculados y experimentales de $G_{\mathrm{m}}^{\mathrm{E}}$ y $H_{\mathrm{m}}^{\mathrm{E}}$, respectivamente, a $x_{1}=0.5$ de los sistemas de interés para los que hay datos disponibles en la bibliografía, o bien se han determinado en este trabajo. Con el objetivo de proporcionar información más detallada, en dichas tablas también se comparan las desviaciones estándar del ajuste Redlich-Kister a los datos experimentales con las desviaciones relativas de la presión, $\sigma_{r}(P)$, y de la entalpía de exceso, $\operatorname{des}\left(H_{\mathrm{m}}^{\mathrm{E}}\right)$, calculadas de acuerdo con las expresiones:

$$
\begin{gathered}
\sigma_{r}(P)=\sqrt{\frac{1}{N} \cdot \sum_{1}^{N}\left(\frac{P_{\exp }-P_{\text {cal }}}{P_{\text {exp }}}\right)^{2}} \\
\operatorname{des}\left(H_{\mathrm{m}}^{\mathrm{E}}\right)=\sqrt{\frac{1}{N} \cdot \sum_{1}^{N}\left(\frac{H_{\mathrm{m}, \mathrm{exp}}^{\mathrm{E}}-H_{\mathrm{m}, \mathrm{cal}}^{\mathrm{E}}}{H_{\mathrm{m}, \text { exp }}^{\mathrm{E}}\left(x_{1}=0.5\right)}\right)^{2}}
\end{gathered}
$$

donde $N$ es el número de datos experimentales disponible para cada sistema estudiado y $P_{\text {cal }}$ y $H_{\mathrm{m}, \mathrm{Eal}}^{\mathrm{E}}$ son los valores de $P$ y $H_{\mathrm{m}}^{\mathrm{E}}$ calculados mediante DISQUAC.

En vista de los resultados obtenidos, se puede afirmar que el modelo representa adecuadamente las propiedades termodinámicas de los sistemas analizados. 
Tabla 7-5: valores experimentales y calculados con DISQUAC de las energías de Gibbs de exceso, $G_{\mathrm{m}}^{\mathrm{E}}$, a composición $x_{1}=0.5$ y temperatura $T$ de mezclas amina (1) + cetona (2). ${ }^{a}[12] ;{ }^{b}[14] ;{ }^{c}[22] ;{ }^{d}[23] ;{ }^{e}[24] ;{ }^{f}[35]$

\begin{tabular}{|c|c|c|c|c|c|c|}
\hline & \multirow[t]{2}{*}{$T / \mathrm{K}$} & \multirow[t]{2}{*}{$N$} & \multicolumn{2}{|c|}{$G_{\mathrm{m}}^{\mathrm{E}} / \mathrm{J} \cdot \mathrm{mol}^{-1}$} & \multicolumn{2}{|c|}{$\sigma_{r}(P)$} \\
\hline & & & Exp & DISQUAC & Exp & DISQUAC \\
\hline \multicolumn{7}{|c|}{ di-n-etilamina } \\
\hline \multirow[t]{3}{*}{ 2-propanona } & 298.03 & 13 & $393^{a}$ & 395 & $0.0007^{a}$ & 0.001 \\
\hline & 347.97 & 13 & $369^{a}$ & 365 & $0.0005^{a}$ & 0.002 \\
\hline & 398.10 & 13 & $351^{a}$ & 330 & $0.004^{a}$ & 0.006 \\
\hline \multicolumn{7}{|c|}{ anilina } \\
\hline \multirow[t]{3}{*}{ 2-propanona } & 277.35 & 13 & $-278^{b}$ & -340 & $0.013^{b}$ & 0.053 \\
\hline & 313.15 & 15 & $-240^{b}$ & -231 & $0.004^{b}$ & 0.014 \\
\hline & 386.67 & 15 & $117^{b}$ & 113 & $0.007^{\mathrm{b}}$ & 0.029 \\
\hline \multicolumn{7}{|c|}{$N, N, N$-trietilamina } \\
\hline 2-propanona & 318.15 & 23 & $778^{c}$ & 762 & $0.010^{c}$ & 0.029 \\
\hline \multirow[t]{6}{*}{ 2-butanona } & 293.15 & 12 & $623^{d}$ & 602 & $0.011^{d}$ & 0.016 \\
\hline & 303.15 & 9 & $560^{\mathrm{e}}$ & 597 & $0.001^{\mathrm{e}}$ & 0.020 \\
\hline & 320.15 & 12 & $594^{d}$ & 588 & $0.004^{d}$ & 0.009 \\
\hline & 323.15 & 20 & $531^{\mathrm{e}}$ & 586 & $0.002^{\mathrm{e}}$ & 0.024 \\
\hline & 346.15 & 12 & $544^{d}$ & 573 & $0.004^{d}$ & 0.015 \\
\hline & 373.15 & 12 & $543^{d}$ & 556 & $0.002^{d}$ & 0.010 \\
\hline \multicolumn{7}{|c|}{ piridina } \\
\hline 2-propanona & 303.15 & 23 & $155^{f}$ & 158 & $0.004^{f}$ & 0.006 \\
\hline
\end{tabular}

Tabla 7-6: valores experimentales y calculados con DISQUAC de las entalpías de exceso, $H_{\mathrm{m}}^{\mathrm{E}}$, a composición $x_{1}=0.5$ y temperatura $T$ de mezclas amina (1) + cetona (2), amina (1) + alcano (2), alcohol (1) + amina (2), amino-cetona (1) + alcano (2) y 1 -propanol (1) $+N, N, N$-trietilamina (2) +2 -butanona (3). ${ }^{a}[36]$; ${ }^{b}[37] ;$; [38]; d[39]; ${ }^{\mathrm{e}}[25] ;{ }^{\mathrm{f}}[30] ;$

\begin{tabular}{|c|c|c|c|c|c|c|}
\hline & \multirow[t]{2}{*}{$T / \mathrm{K}$} & \multirow[t]{2}{*}{$N$} & \multicolumn{2}{|c|}{$H_{\mathrm{m}}^{\mathrm{E}} / \mathrm{J} \cdot \mathrm{mol}^{-1}$} & \multicolumn{2}{|c|}{$\operatorname{des}\left(H_{\mathrm{m}}^{\mathrm{E}}\right)$} \\
\hline & & & Exp & DISQUAC & Exp & DISQUAC \\
\hline \multicolumn{7}{|c|}{ anilina } \\
\hline 2-propanona & 298.15 & 10 & -1236 & -1222 & 0.007 & 0.013 \\
\hline 2-butanona & 298.15 & 10 & -1165 & -1148 & 0.009 & 0.017 \\
\hline 2-pentanona & 298.15 & 11 & -1029 & -1001 & 0.010 & 0.021 \\
\hline 2-heptanona & 298.15 & 14 & -692 & -672 & 0.009 & 0.025 \\
\hline \multicolumn{7}{|c|}{$N$-metilanilina } \\
\hline heptano & 298.15 & 9 & 1675 & 1681 & 0.009 & 0.030 \\
\hline octano & 298.15 & 9 & 1767 & 1790 & 0.007 & 0.025 \\
\hline decano & 298.15 & 9 & 1913 & 1980 & 0.004 & 0.032 \\
\hline \multirow[t]{2}{*}{ ciclohexano } & 298.15 & 9 & $1617^{a}$ & 1635 & 0.009 & 0.017 \\
\hline & 323.15 & 9 & $1780^{a}$ & 1725 & & 0.013 \\
\hline \multirow[t]{2}{*}{ benceno } & 298.15 & 17 & $457^{b}$ & 451 & 0.009 & 0.026 \\
\hline & 323.15 & 10 & $503^{b}$ & 493 & 0.006 & 0.022 \\
\hline tolveno & 298.15 & 9 & 497 & 485 & 0.008 & 0.044 \\
\hline metanol & 298.15 & 16 & $426^{c}$ & 431 & 0.016 & 0.020 \\
\hline 1-butanol & 313.15 & 14 & $1372^{d}$ & 1355 & 0.009 & 0.016 \\
\hline 2-propanona & 298.15 & 9 & -668 & -675 & 0.012 & 0.013 \\
\hline
\end{tabular}




\begin{tabular}{|c|c|c|c|c|c|c|}
\hline \multicolumn{7}{|c|}{ Tabla 7-6 (continuación) } \\
\hline 2-butanona & 298.15 & 9 & -812 & -798 & 0.007 & 0.014 \\
\hline 2-pentanona & 298.15 & 9 & -835 & -859 & 0.004 & 0.035 \\
\hline 2-heptanona & 298.15 & 9 & -741 & -718 & 0.004 & 0.024 \\
\hline \multicolumn{7}{|c|}{ di-n-propilamina } \\
\hline 2-propanona & 298.15 & 9 & 648 & 643 & 0.009 & 0.016 \\
\hline 2-butanona & 298.15 & 10 & 398 & 396 & 0.010 & 0.010 \\
\hline 2-pentanona & 298.15 & 9 & 281 & 272 & 0.011 & 0.025 \\
\hline 2-heptanona & 298.15 & 9 & 161 & 163 & 0.011 & 0.019 \\
\hline \multicolumn{7}{|c|}{ di- $n$-butilamina } \\
\hline 2-propanona & 298.15 & 9 & 933 & 927 & 0.004 & 0.015 \\
\hline 2-butanona & 298.15 & 9 & 620 & 618 & 0.006 & 0.014 \\
\hline 2-pentanona & 298.15 & 9 & 454 & 444 & 0.009 & 0.013 \\
\hline 2-heptanona & 298.15 & 9 & 277 & 275 & 0.011 & 0.015 \\
\hline \multicolumn{7}{|c|}{$N, N, N$-trietilamina } \\
\hline \multirow[t]{3}{*}{ 2-propanona } & 298.15 & 19 & 1062 & 1066 & & 0.006 \\
\hline & 303.15 & 19 & $1071^{\mathrm{e}}$ & 1085 & & 0.023 \\
\hline & 313.15 & 19 & $1073^{\mathrm{e}}$ & 1121 & & 0.034 \\
\hline \multirow[t]{3}{*}{ 2-butanona } & 298.15 & 19 & 745 & 746 & & 0.016 \\
\hline & 303.15 & 19 & $747^{\mathrm{e}}$ & 752 & & 0.015 \\
\hline & 313.15 & 19 & $764^{\mathrm{e}}$ & 762 & & 0.009 \\
\hline \multirow[t]{3}{*}{ 2-pentanona } & 298.15 & 19 & 584 & 599 & & 0.079 \\
\hline & 303.15 & 19 & $600^{\mathrm{e}}$ & 603 & & 0.023 \\
\hline & 313.15 & 19 & $612^{e}$ & 608 & & 0.018 \\
\hline 2-heptanona & 298.15 & 19 & 415 & 395 & & 0.043 \\
\hline \multirow[t]{2}{*}{3 -pentanona } & 303.15 & 19 & $520^{\mathrm{e}}$ & 535 & & 0.14 \\
\hline & 313.15 & 19 & $549^{e}$ & 537 & & 0.078 \\
\hline \multicolumn{7}{|c|}{$N, N$-dimetiletilamina } \\
\hline 2-propanona & 298.15 & 19 & $690^{f}$ & 695 & & 0.044 \\
\hline \multicolumn{7}{|c|}{$N, N$-dietilmetilamina } \\
\hline 2-propanona & 298.15 & 19 & $930^{f}$ & 921 & & 0.035 \\
\hline \multicolumn{7}{|c|}{ 1-dimetilamino-2-propanona } \\
\hline hexano & 298.15 & 19 & $1066^{f}$ & 1061 & & 0.028 \\
\hline heptano & 298.15 & 19 & $1159^{f}$ & 1147 & & 0.036 \\
\hline dodecano & 298.15 & 19 & $1501^{f}$ & 1485 & & 0.069 \\
\hline hexadecano & 298.15 & 19 & $1674^{f}$ & 1680 & & 0.120 \\
\hline \multicolumn{7}{|c|}{ 5-dimetilamino-2-pentanona } \\
\hline hexano & 298.15 & 19 & $721^{f}$ & 712 & & 0.068 \\
\hline heptano & 298.15 & 19 & $782^{f}$ & 777 & & 0.021 \\
\hline dodecano & 298.15 & 19 & $1040^{f}$ & 1042 & & 0.041 \\
\hline hexadecano & 298.15 & 19 & $1206^{f}$ & 1201 & & 0.019 \\
\hline \multicolumn{7}{|c|}{ 1-dietilamino-2-propanona } \\
\hline hexano & 298.15 & 19 & $752^{f}$ & 829 & & 0.067 \\
\hline heptano & 298.15 & 19 & $829^{f}$ & 760 & & 0.058 \\
\hline dodecano & 298.15 & 19 & $1085^{f}$ & 1106 & & 0.085 \\
\hline hexadecano & 298.15 & 19 & $1243^{f}$ & 1273 & & 0.110 \\
\hline \multicolumn{7}{|c|}{ 1-propanol+ $N, N, N$-trietilamina +2 -butanona } \\
\hline & 298.15 & 54 & -927 & 521 & 0.015 & 0.280 \\
\hline
\end{tabular}




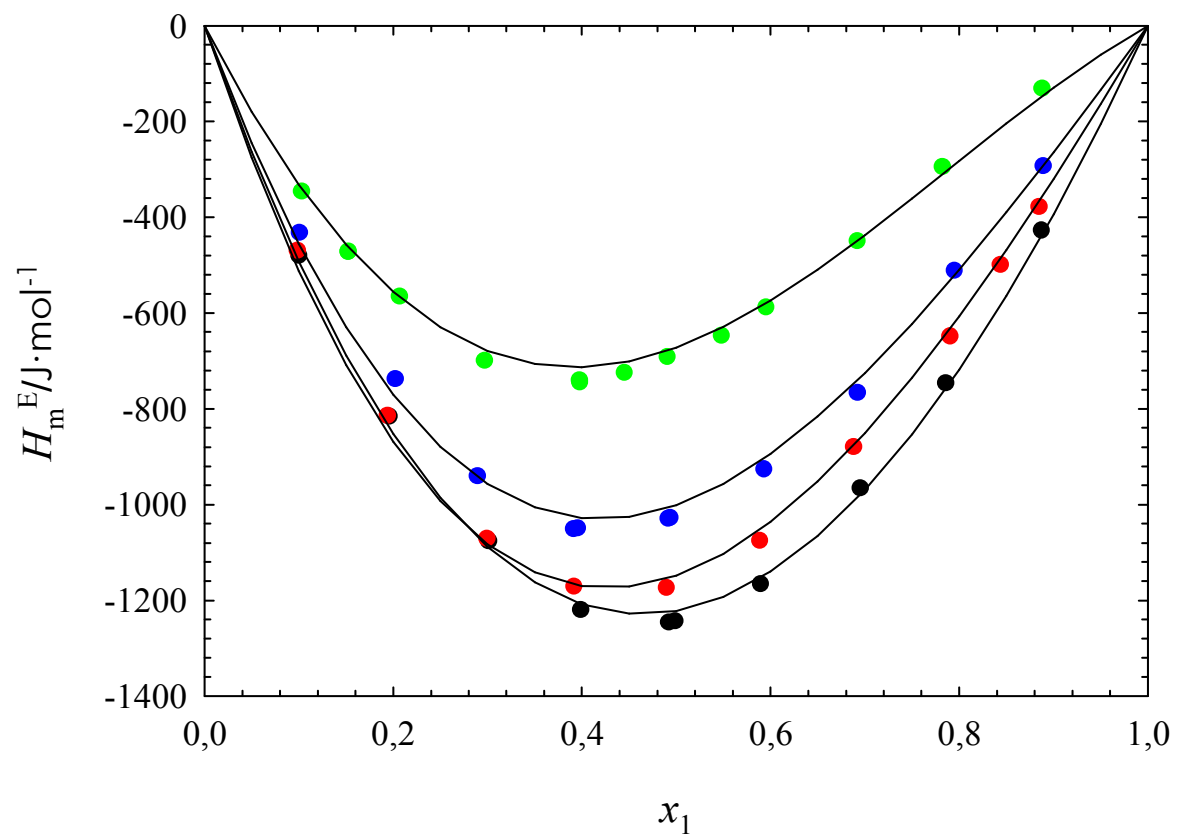

Ilustración 7-1: $H_{\mathrm{m}}^{\mathrm{E}}$ de mezclas anilina (1) + cetona (2) a $T=298.15 \mathrm{~K}$. Puntos, resultados experimentales: -2-propanona; $\cdot 2$-butanona; $\bullet 2$-pentanona; $\cdot 2$-heptanona. Línea continua, resultados del modelo DISQUAC con los coeficientes de intercambio de la Tabla 7-3.

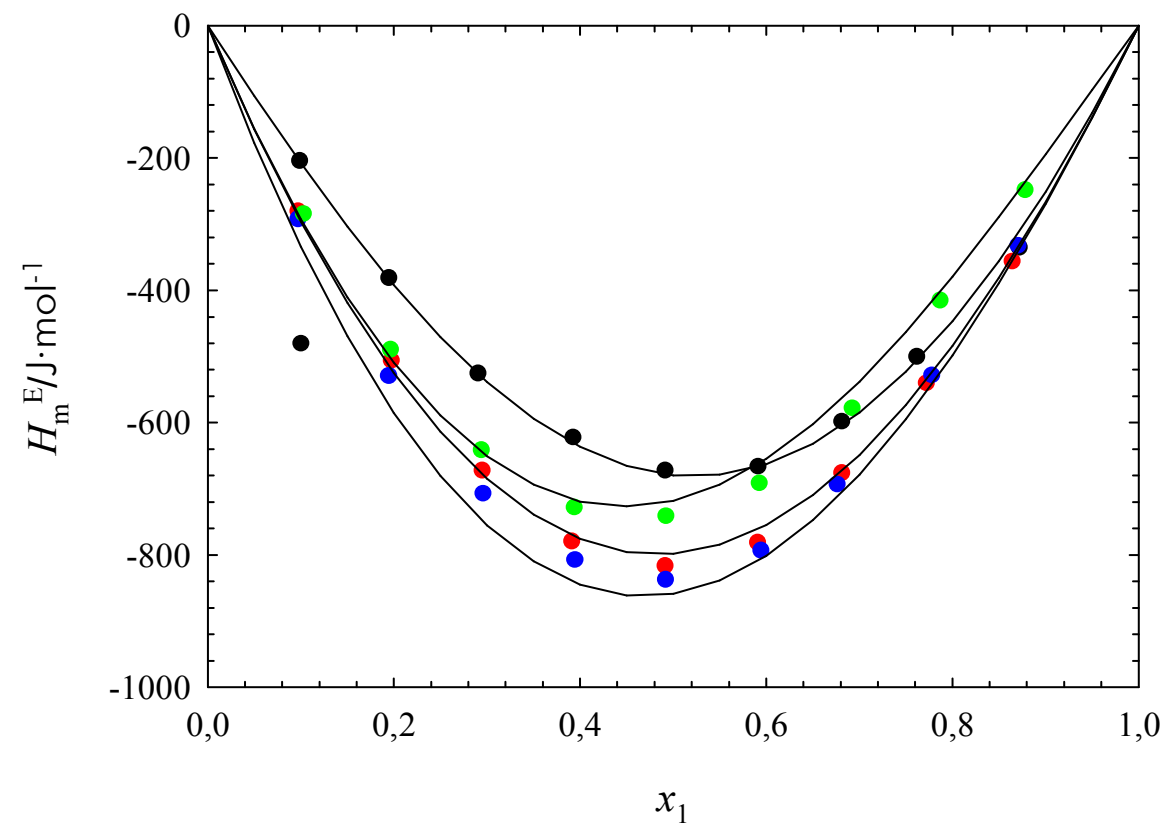

Ilustración 7-2: $H_{\mathrm{m}}^{\mathrm{E}}$ de mezclas $N$-metilanilina (1) + cetona (2) a $T=298.15 \mathrm{~K}$. Puntos, resultados experimentales: $\bullet 2$-propanona; $\bullet 2$-butanona; $\bullet 2$-pentanona; $\bullet 2$-heptanona. Línea continua, resultados del modelo DISQUAC con los coeficientes de intercambio de la Tabla 7-3. 


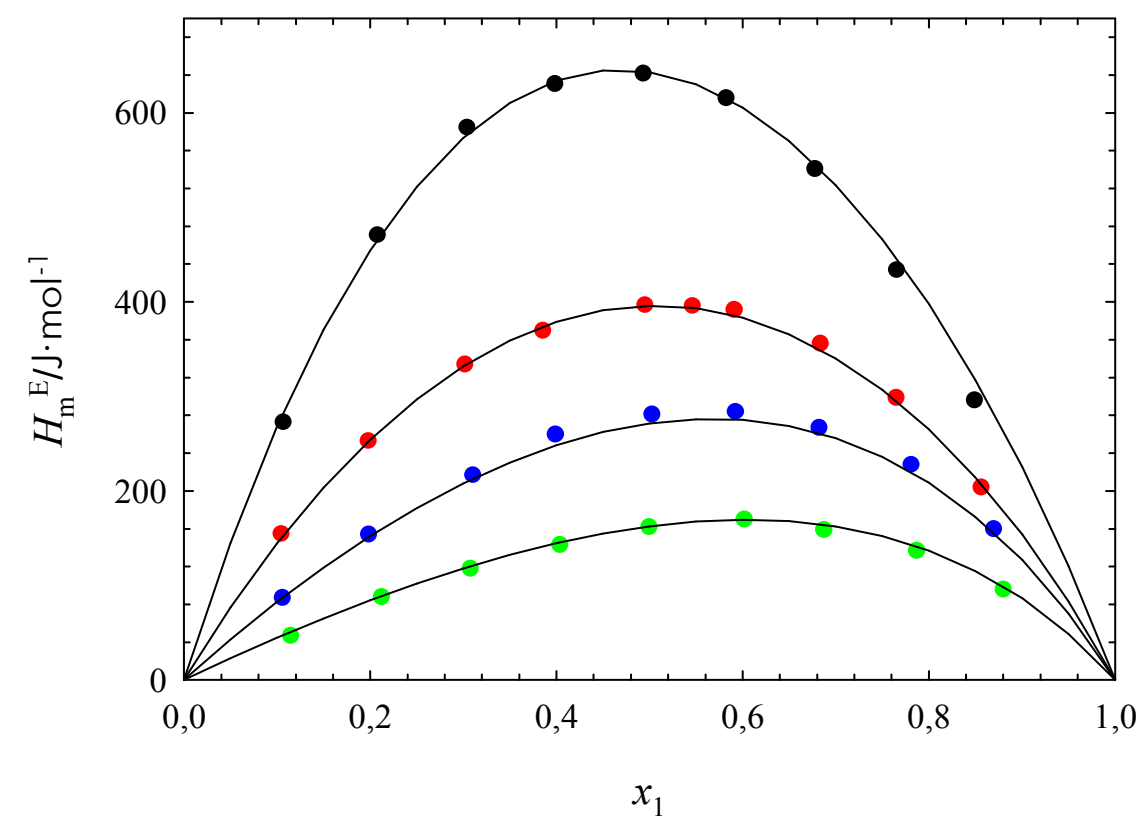

Ilustración 7-3: $H_{\mathrm{m}}^{\mathrm{E}}$ de mezclas di-n-propilamina (1) + cetona (2) a $T=298.15 \mathrm{~K}$.. Puntos, resultados experimentales: $\bullet 2$-propanona; $\bullet 2$-butanona; $\bullet 2$-pentanona; 2 -heptanona. Línea continua, resultados del modelo DISQUAC con los coeficientes de intercambio de la Tabla 7-3.

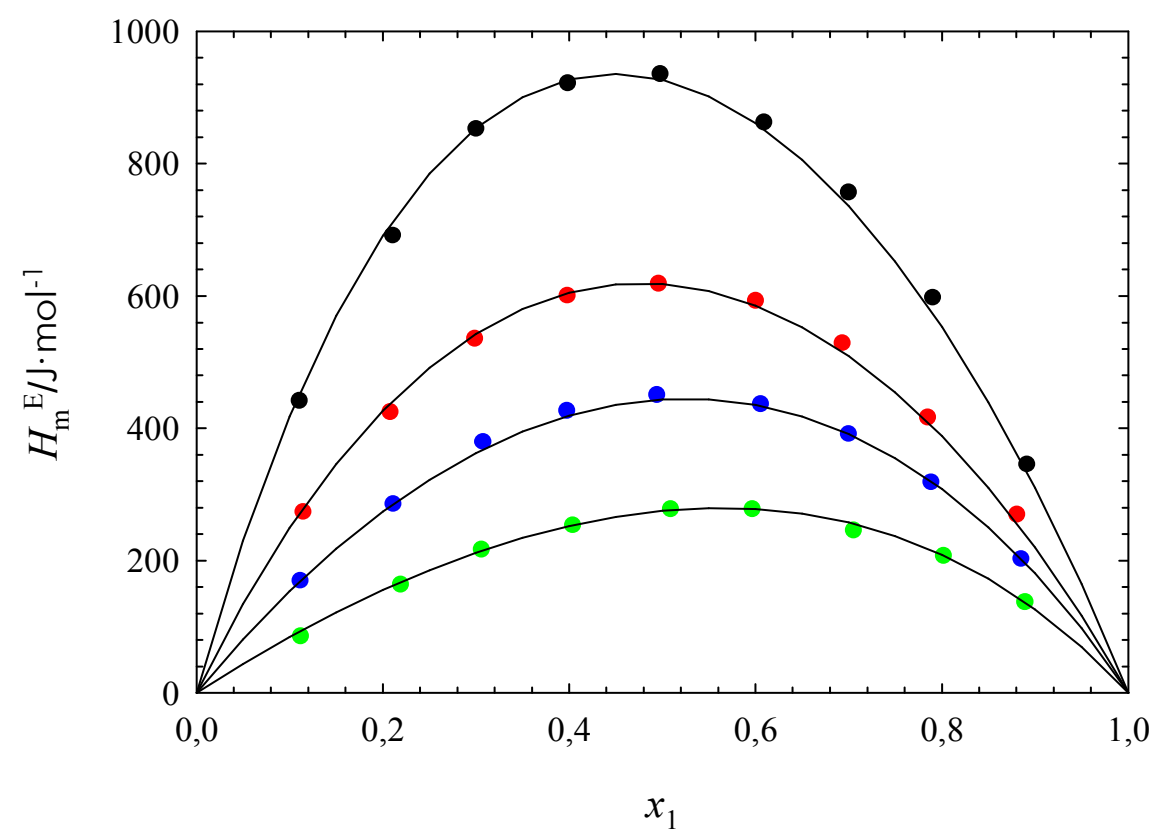

Ilustración 7-4: $H_{\mathrm{m}}^{\mathrm{E}}$ de mezclas di- $n$-butilamina (1) + cetona (2) a $T=298.15 \mathrm{~K}$.. Puntos, resultados experimentales: $\bullet 2$-propanona; $\cdot 2$-butanona; $\cdot 2$-pentanona; 2 -heptanona. Línea continua, resultados del modelo DISQUAC con los coeficientes de intercambio de la Tabla 7-3. 


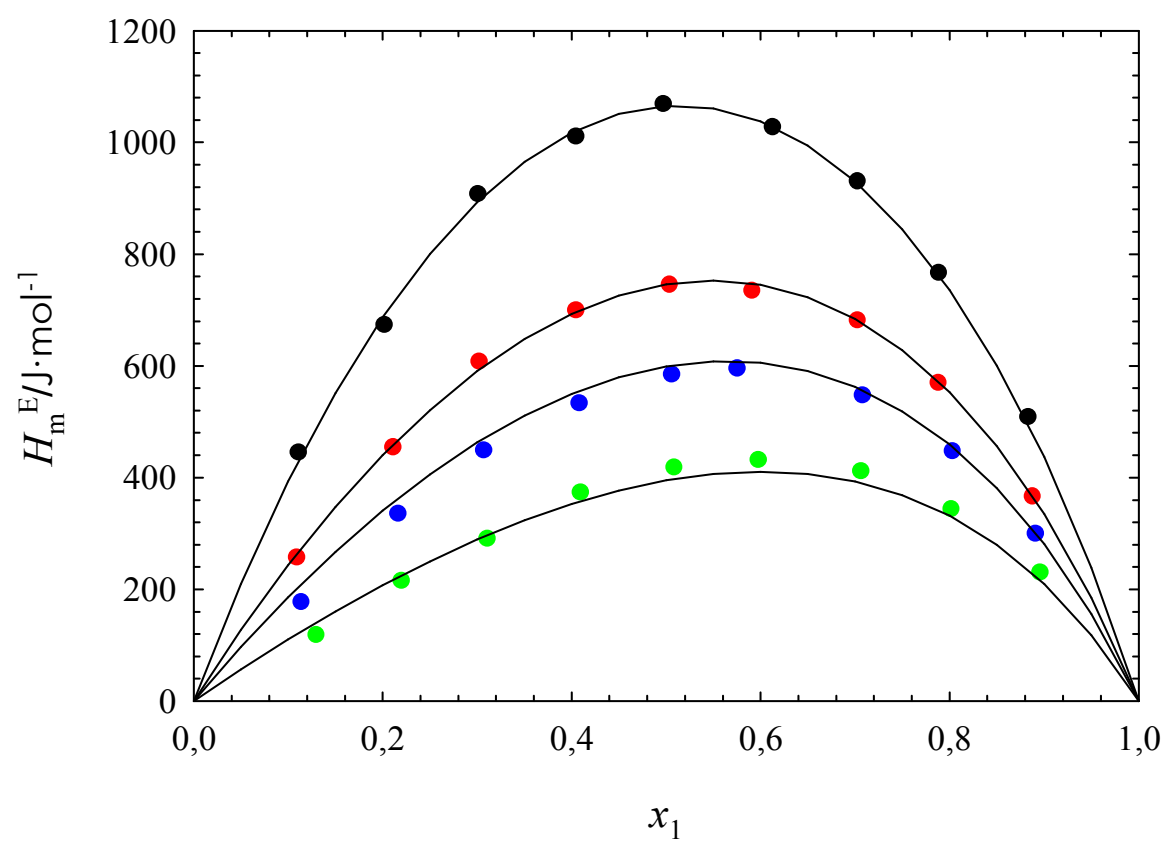

Ilustración 7-5: $H_{\mathrm{m}}^{\mathrm{E}}$ de mezclas $N, N, N$-trietilamina (1) + cetona (2) a $T=298.15 \mathrm{~K}$.. Puntos, resultados experimentales: $\bullet 2$-propanona; $\bullet 2$-butanona; $\bullet 2$-pentanona; $\bullet 2$-heptanona. Línea continua, resultados del modelo DISQUAC con los coeficientes de intercambio de la Tabla 7-3.

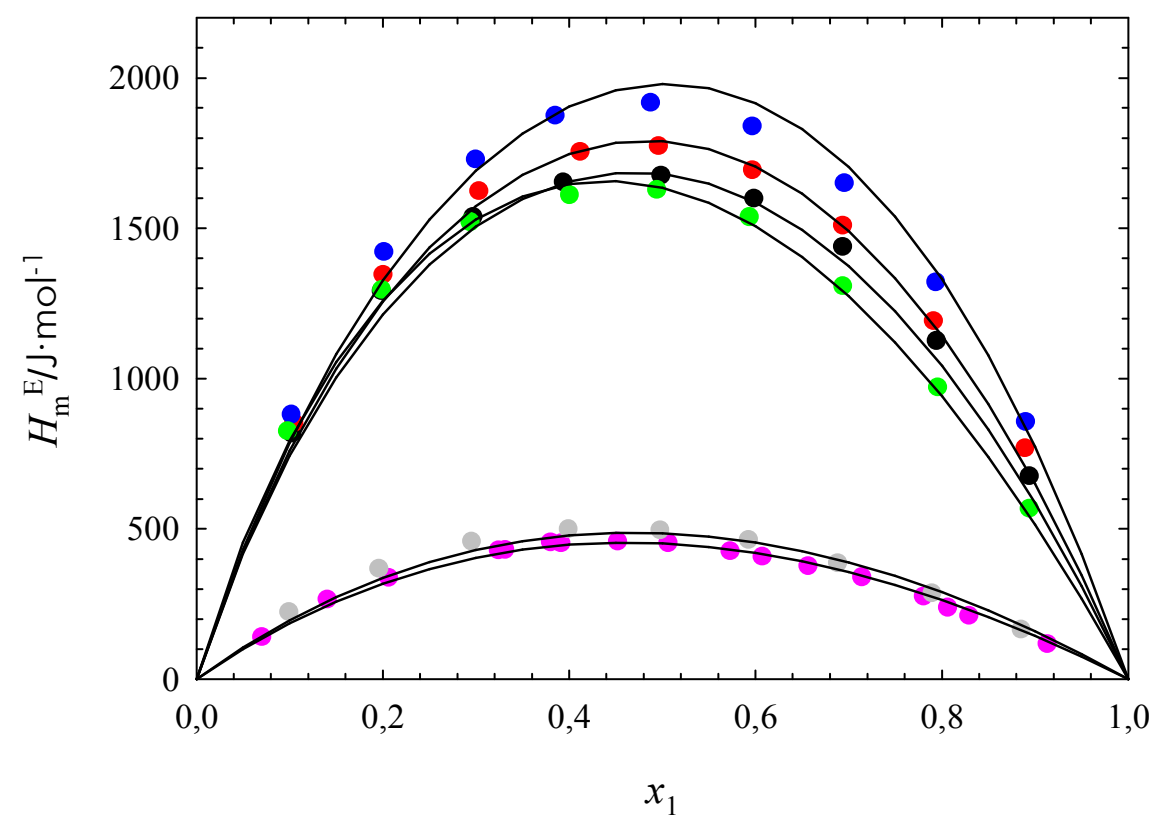

Ilustración 7-6: $H_{\mathrm{m}}^{\mathrm{E}}$ de mezclas $N$-metilanilina (1) + hidrocarburo (2) a $T=298.15 \mathrm{~K}$.. Puntos, resultados experimentales: •heptano; $\bullet$ octano; $\bullet$ decano; ${ }^{\bullet}$ ciclohexano; • benceno; •tolveno. Línea continua, resultados del modelo DISQUAC con los coeficientes de intercambio de la Tabla 7-3. 


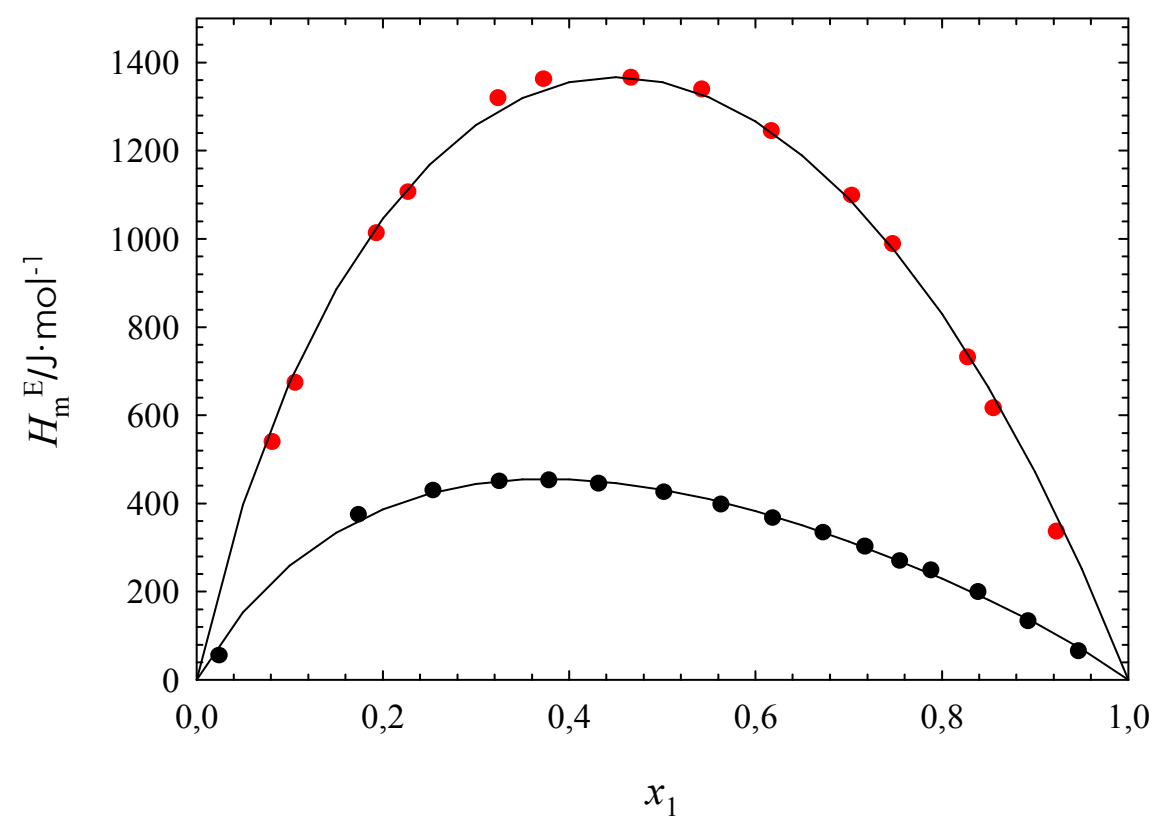

Ilustración 7-7: $H_{\mathrm{m}}^{\mathrm{E}}$ de mezclas alcohol (1) $+N$-metilanilina (2). Puntos, resultados experimentales: - metanol a $T=298.15 \mathrm{~K}$; $\bullet 1$-butanol a $T=313.15 \mathrm{~K}$. Línea continua, resultados del modelo DISQUAC con los coeficientes de intercambio de la Tabla 7-3.

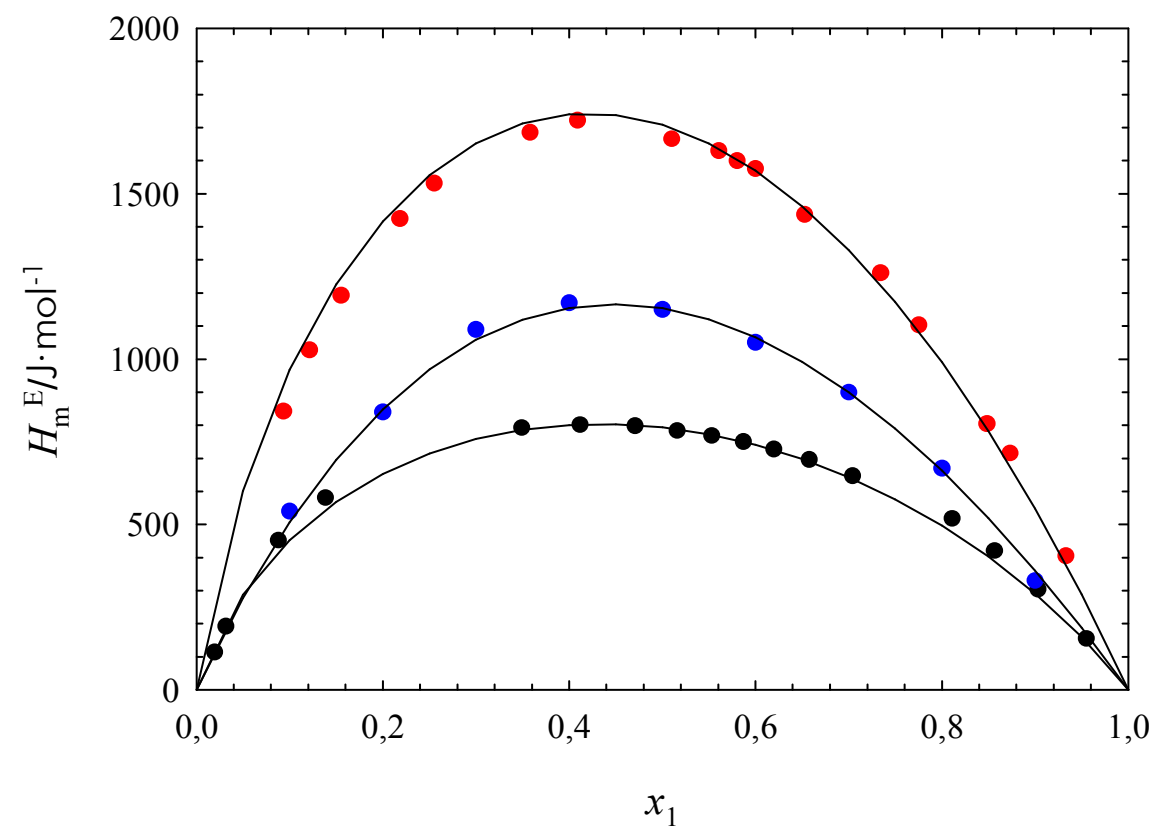

Ilustración 7-8: $H_{\mathrm{m}}^{\mathrm{E}}$ de mezclas alcohol (1) $+N, N$-dimetilanilina (2) y $N, N$-dimetilanilina (1) + ciclohexano (2) a $T=298.15 \mathrm{~K}$.. Puntos, resultados experimentales: • metanol [38]; • 1-butanol [39]; - ciclohexano [36]. Línea continua, resultados del modelo DISQUAC con los coeficientes de intercambio de la Tabla 7-3. 


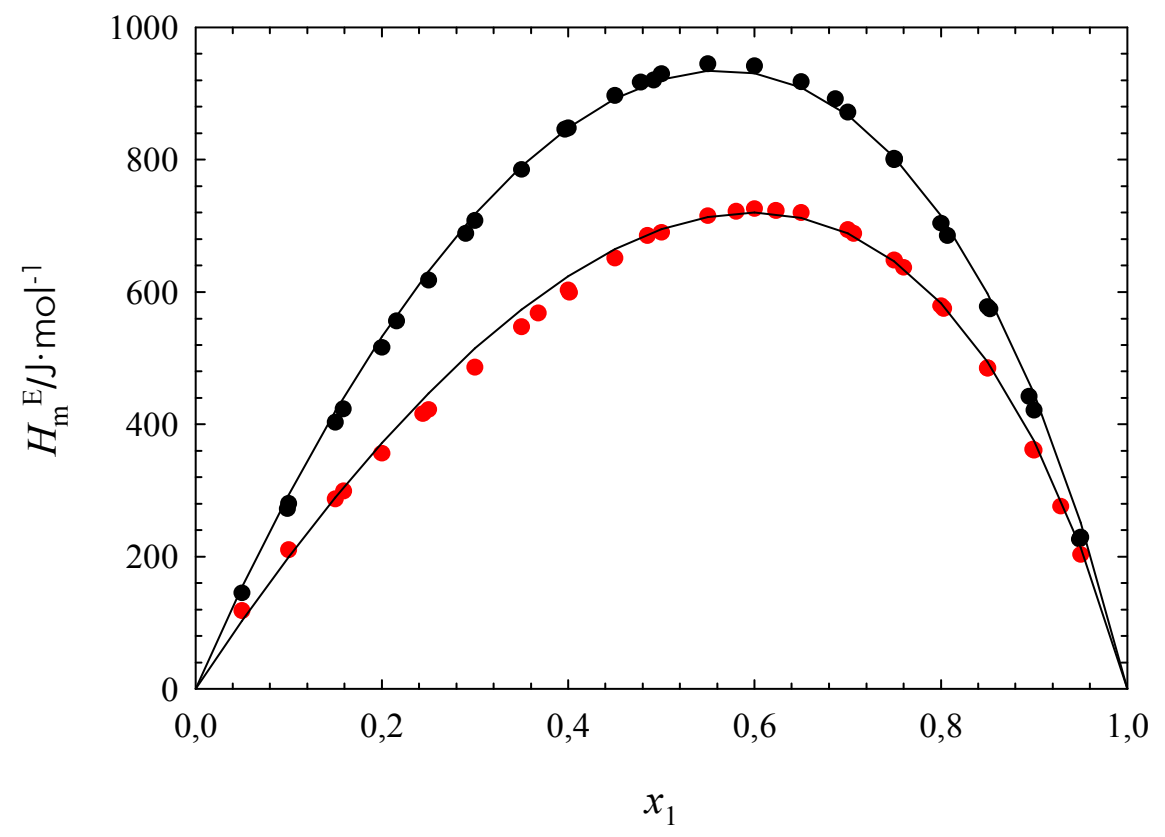

Ilustración 7-9: $H_{\mathrm{m}}^{\mathrm{E}}$ de mezclas amina (1) + 2-propanona (2) a $T=298.15 \mathrm{~K}$.. Puntos, resultados experimentales: $\bullet N, N$-dietilmetilamina; $-N, N$-dimetiletilamina. Línea continua, resultados del modelo DISQUAC con los coeficientes de intercambio de la Tabla 7-3.

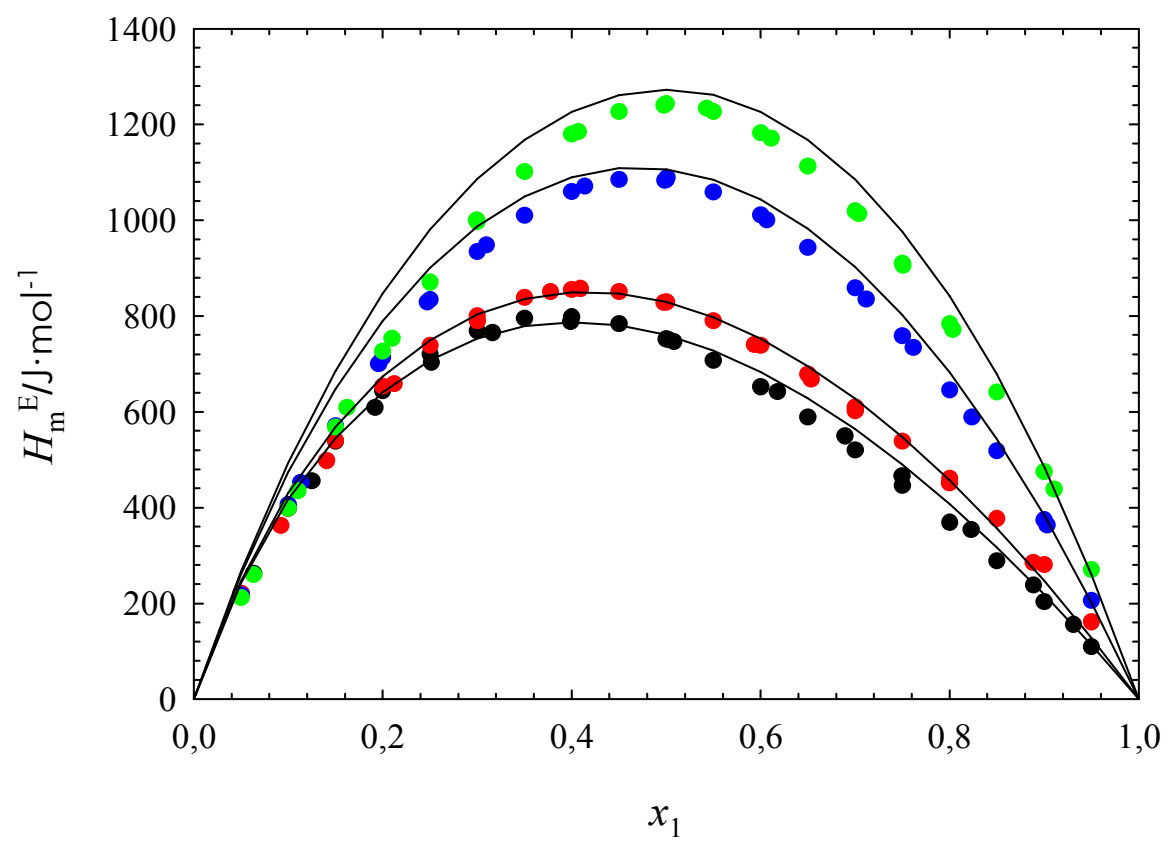

Ilustración 7-10: $H_{\mathrm{m}}^{\mathrm{E}}$ de mezclas 1-dietilamino-2-propanona (1) $+n$-alcano (2) a $T=298.15$ K.. Puntos, resultados experimentales: • hexano; •heptano; • dodecano; • hexadecano. Línea continua, resultados del modelo DISQUAC con los coeficientes de intercambio de la Tabla 7-3. 


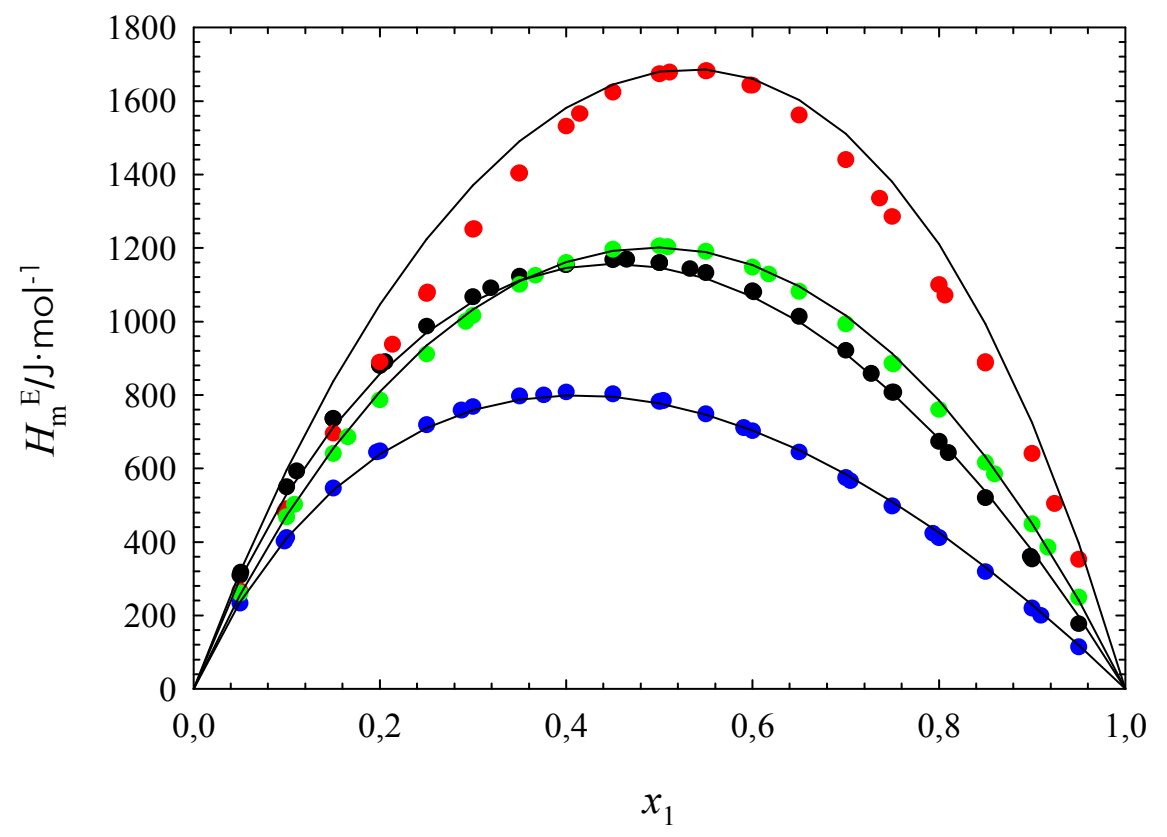

Ilustración 7-11: $H_{\mathrm{m}}^{\mathrm{E}}$ de mezclas amino-cetona (1) $+n$-alcano (2) a $T=298.15 \mathrm{~K}$.. Puntos, resultados experimentales: $\bullet 1$-dimetilamino-2-propanona $(1)+$ heptano $(2) ; \cdot 1$-dimetilamino-2-propanona $(1)+$ hexadecano(2); $\bullet$-dimetilamino-2-pentanona (1) + heptano $(2) ; \cdot 5$-dimetilamino-2-pentanona (1) +hexadecano (2). Línea continua, resultados del modelo DISQUAC con los coeficientes de intercambio de la Tabla 7-3.

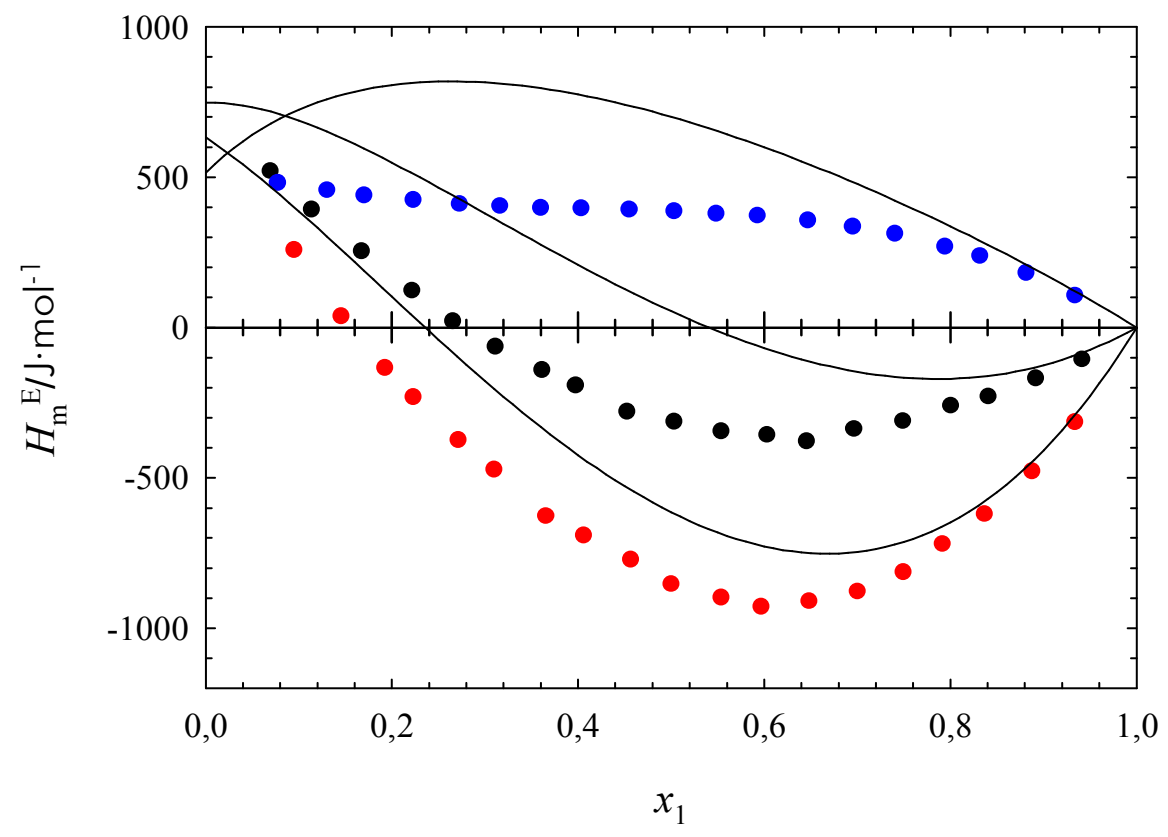

Ilustración 7-12: $H_{\mathrm{m}}^{\mathrm{E}} 1$-propanol (1) $+N, N, N$-trietilamina (2) +2 -butanona (3) en función de la composición del 1-propanol $x_{1}$ a $298.15 \mathrm{~K}$ para los cocientes: $\bullet x_{2} / x_{3}=2.9529 ; \bullet x_{2} / x_{3}=1.0249 ; \bullet x_{2} / x_{3}$ $=0.3264$. Línea continua, resultados del modelo DISQUAC con los coeficientes de intercambio de la Tabla 7-3. 


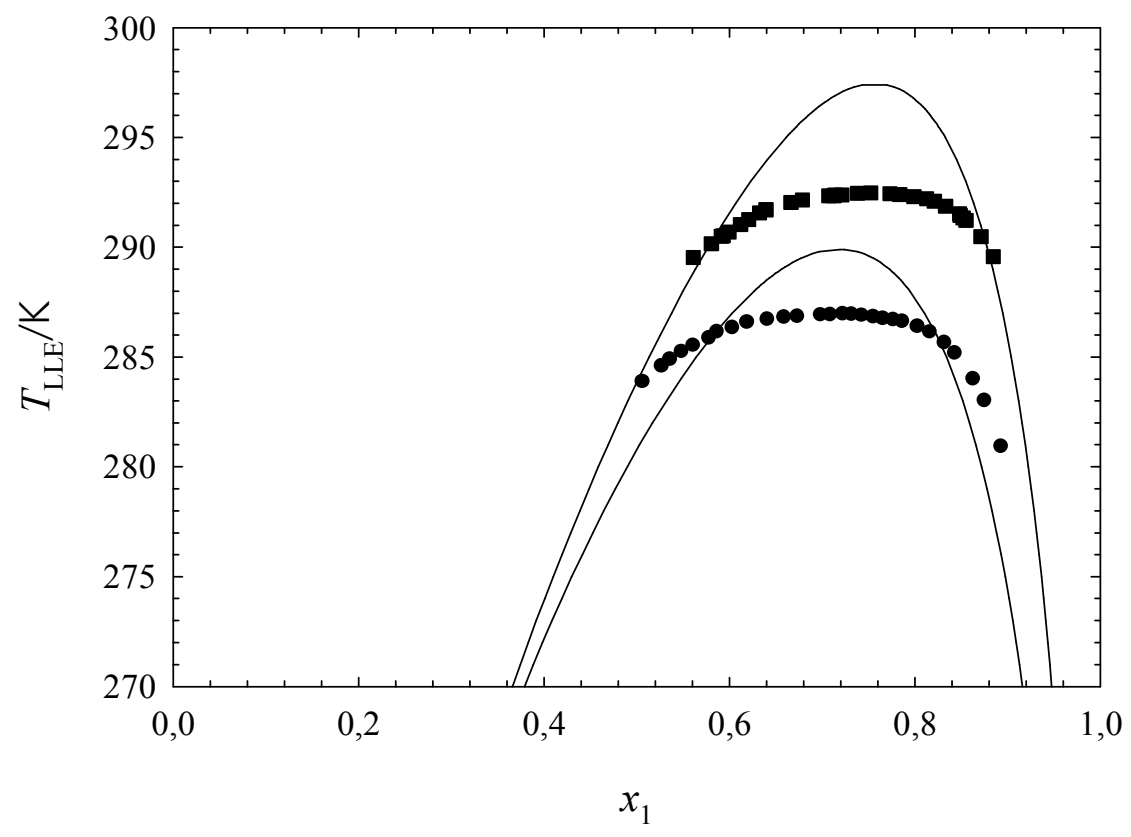

Ilustración 7-13: equilibrio líquido-líquido de mezclas $N$-metilanilina (1) + alcano (2). Puntos, resultados experimentales: - tetradecano; - hexadecano. Línea continua, resultados del modelo DISQUAC para la representación $T_{\mathrm{LLE}} \mathrm{vs} x_{1}$

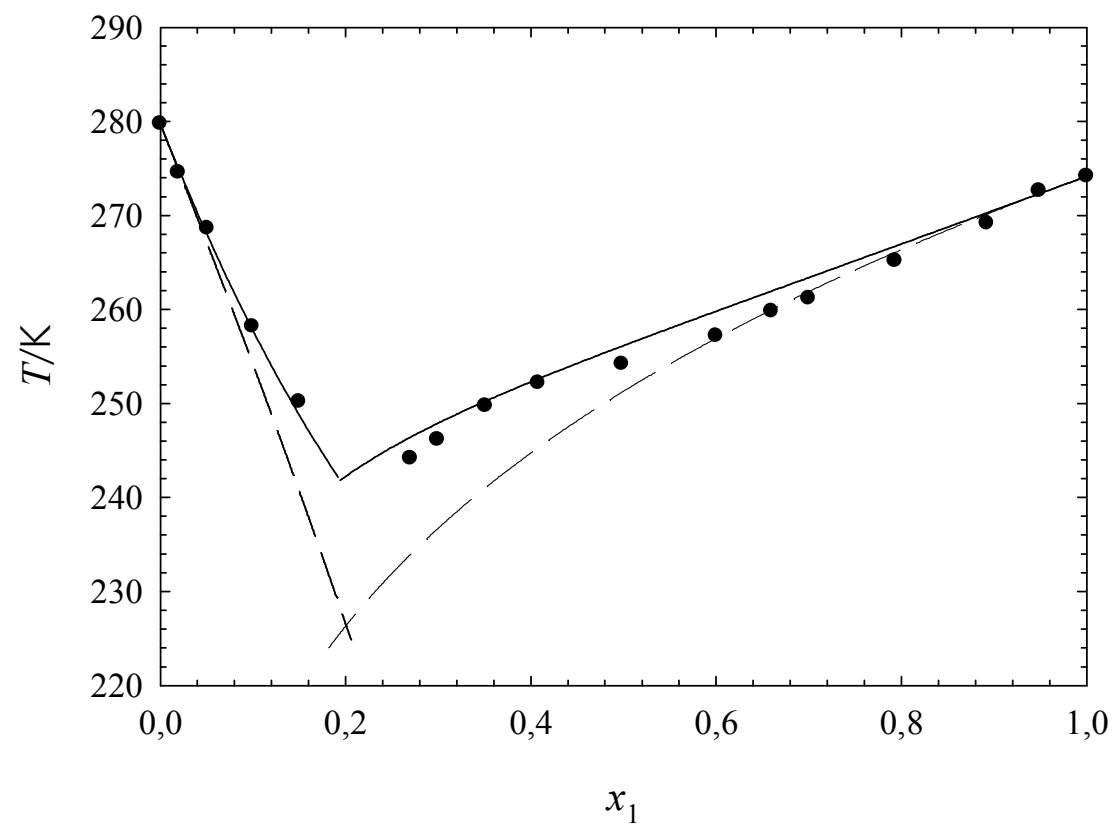

Ilustración 7-14: equilibrio sólido-líquido de la mezcla $N, N$-dimetilanilina (1) + ciclohexano (2). Puntos, resultados experimentales [40]. Línea continua, resultados del modelo DISQUAC. Línea discontinua, curva de solubilidad ideal. 


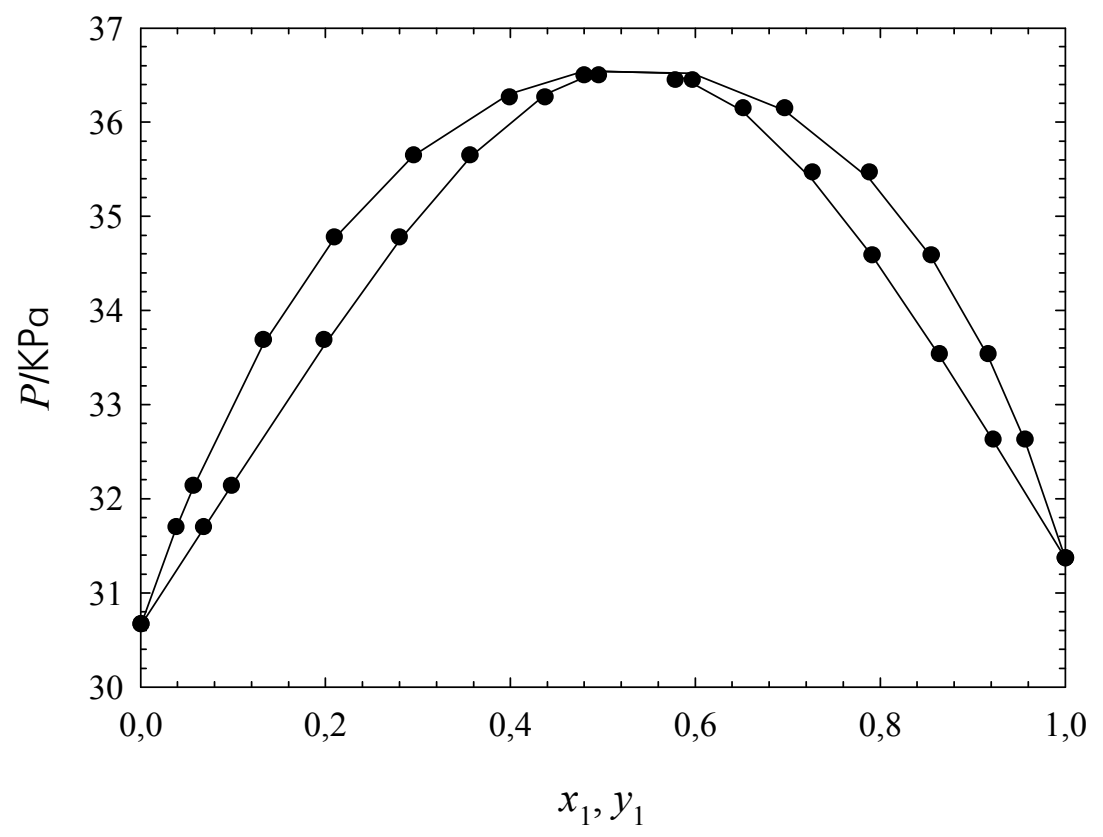

Ilustración 7-15: equilibrio líquido-vapor de la mezcla di-n-metilamina (1) + 2-propanona (2) a 298.15K. Puntos, resultados experimentales [12]. Línea continua, resultados del modelo DISQUAC con los coeficientes de intercambio de la Tabla 7-3.

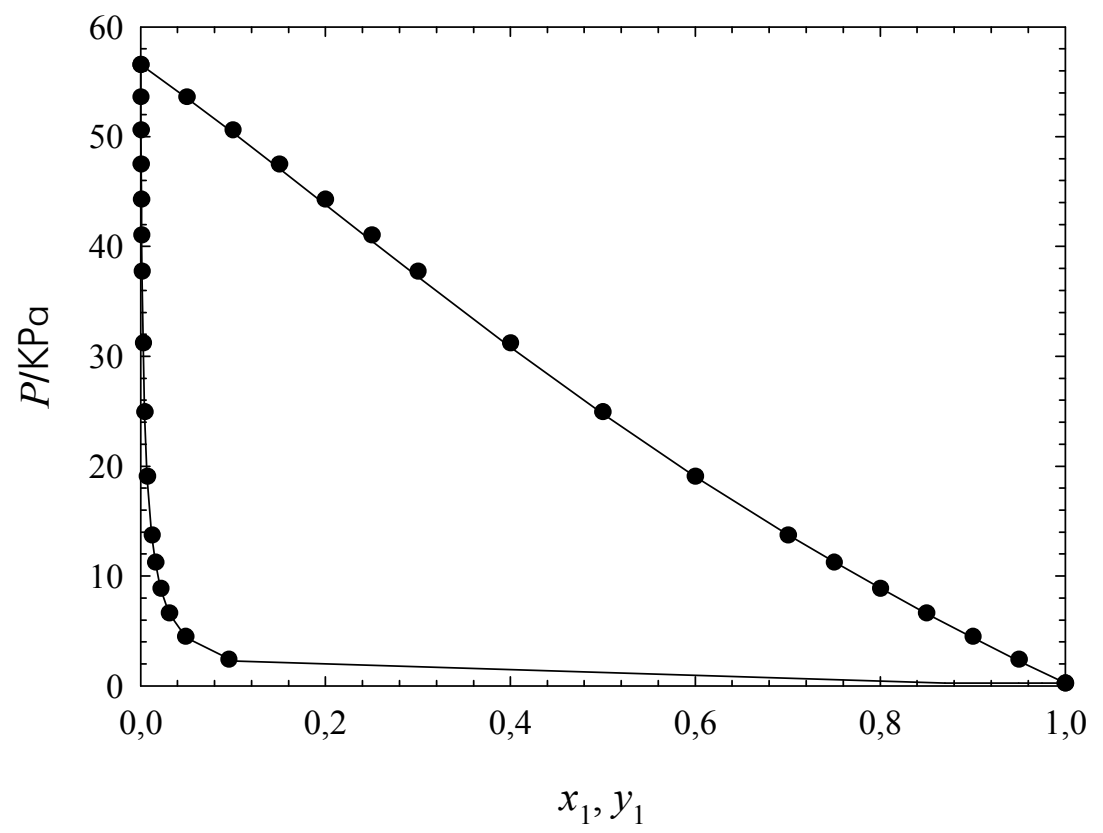

Ilustración 7-16: equilibrio líquido-vapor de la mezcla anilina (1) + 2-propanona (2) a 313.15 K. Puntos, resultados experimentales [17]. Línea continua, resultados del modelo DISQUAC con los coeficientes de intercambio de la Tabla 7-3. 


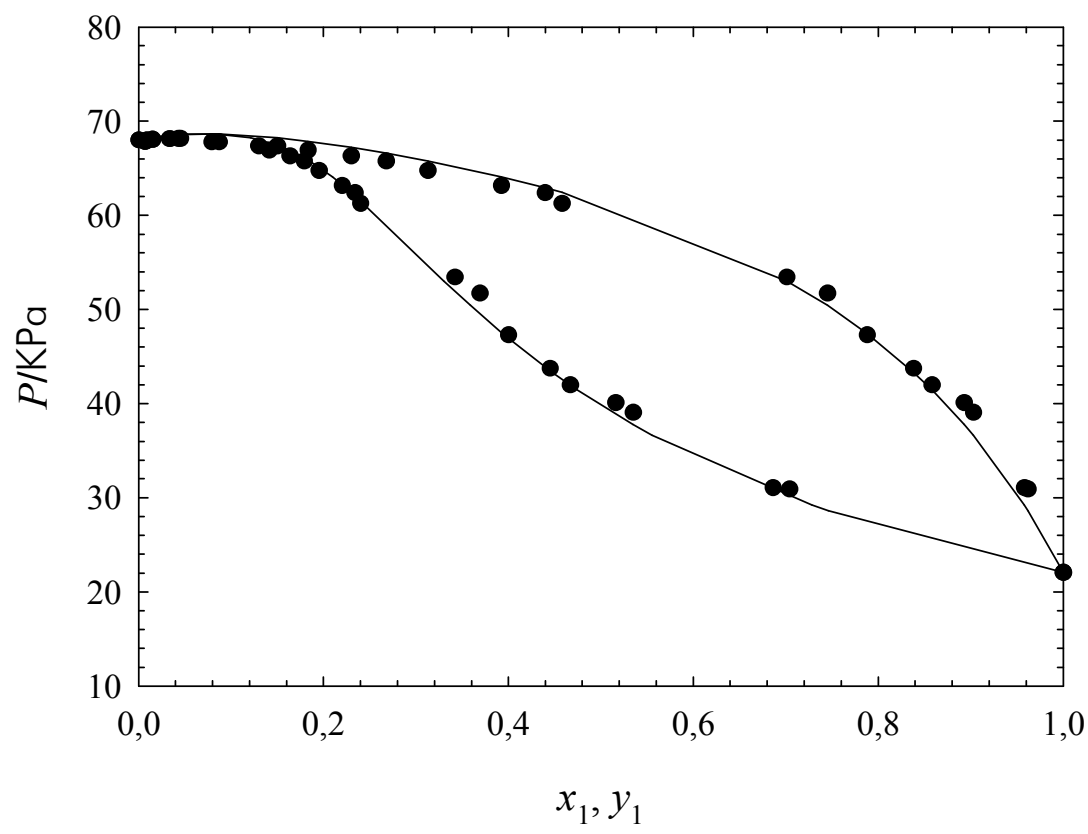

Ilustración 7-17: equilibrio líquido-vapor de la mezcla $N, N, N$-trietilamina (1) + 2-propanona (2) a 318.15 K. Puntos, resultados experimentales [22]. Línea continua, resultados del modelo DISQUAC con los coeficientes de intercambio de la Tabla 7-3.

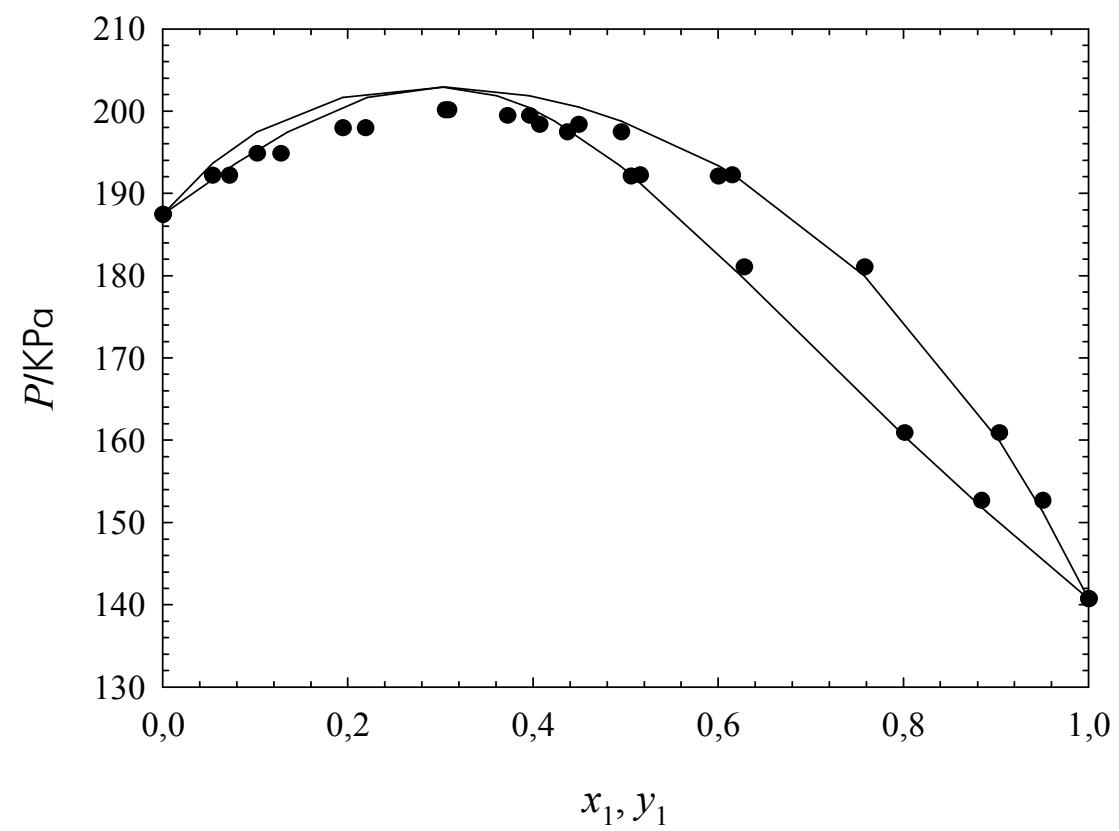

Ilustración 7-18: equilibrio líquido-vapor de la mezcla $N, N, N$-trietilamina (1) + 2-butanona (2) a $373.15 \mathrm{~K}$. Puntos, resultados experimentales [23]. Línea continua, resultados del modelo DISQUAC con los coeficientes de intercambio de la Tabla 7-3. 


\subsection{MODELO ERAS}

Como es bien sabido, el modelo ERAS únicamente puede aplicarse a aquellos sistemas en los que existen efectos de asociación y/o solvatación. Sin embargo, proporciona información no sólo acerca de $H_{\mathrm{m}}^{\mathrm{E}}$, sino también de $V_{\mathrm{m}}^{\mathrm{E}}$, así como de sus magnitudes derivadas. En este trabajo, el modelo ERAS ha sido aplicado a los sistemas siguientes:

a) anilina +2 -propanona, 2-butanona, 2-pentanona y 2-heptanona.

b) $\quad N$-metilanilina +2 -propanona, 2-butanona, 2-pentanona y 2 -heptanona.

c) di-n-propilamina +2 -propanona, 2-butanona, 2-pentanona y 2-heptanona.

d) di- $n$-butilamina +2 -propanona, 2-butanona, 2-pentanona y 2-heptanona.

e) $N$-metilanilina + heptano, octano, decano, ciclohexano, tolveno y benceno.

f) metanol y 1 -butanol $+N$-metilanilina.

Los parámetros reducidos de los componentes puros $\left(p_{\mathrm{i}}^{*}, v_{\mathrm{i}}^{*}\right.$ y $\left.T_{\mathrm{i}}^{*}\right)$ a $T=298.15 \mathrm{~K}$ se calculan a partir de datos experimentales de $\rho_{0}, \alpha_{P 0}$ y $\kappa_{T 0}$ (véase la Tabla 7-7).

Para hacer aplicable el modelo a temperaturas distintas de $298.15 \mathrm{~K}$, se deben utilizar las ecuaciones siguientes para $\rho, \alpha_{P}$ y $\gamma=\alpha_{P} / \kappa_{T}[41]$ :

$$
\begin{gathered}
\rho=\rho_{0} \cdot e^{-\alpha_{0} \cdot \Delta T} \\
\alpha=\alpha_{0}+\alpha_{0}^{2} \cdot\left(7+4 \cdot \alpha_{0} \cdot T\right) \cdot \Delta T / 3 \\
\gamma=\gamma_{0}-\gamma_{0} \cdot\left(1+2 \cdot \alpha_{0} \cdot T\right) \cdot \Delta T / T
\end{gathered}
$$

Los parámetros ajustables a las propiedades de exceso $H_{\mathrm{m}}^{\mathrm{E}}$ y $V_{\mathrm{m}}^{\mathrm{E}}$ son $K_{\mathrm{A}}, K_{\mathrm{B}}, K_{\mathrm{AB}}, \Delta h_{\mathrm{A}}^{*}$, $\Delta h_{\mathrm{B}}^{*}, \Delta h_{\mathrm{AB}}^{*}, \Delta v_{\mathrm{A}}^{*}, \Delta v_{\mathrm{B}}^{*}, \Delta v_{\mathrm{AB}}^{*}$ y $X_{\mathrm{AB}}$, mientras que $Q_{\mathrm{AB}}$ se obtiene del ajuste a los datos de $G_{\mathrm{m}}^{\mathrm{E}}$. El parámetro $X_{\mathrm{AB}}$ es un parámetro energético de interacción que representa la diferencia de las interacciones intermoleculares dispersivas entre las moléculas de A y $B$ en la mezcla y en los líquidos puros, siendo el único parámetro ajustable de la contribución física a las funciones de exceso $H_{\mathrm{m}}^{\mathrm{E}}$ y $V_{\mathrm{m}}^{\mathrm{E}}$ [42], [43]. Por su parte, $Q_{\mathrm{AB}}$ sólo aparece en la expresión de $G_{\mathrm{m}}^{\mathrm{E}}$ y caracteriza la contribución entrópica a la diferencia de interacciones intermoleculares.

Dependiendo de la naturaleza de los componentes a mezclar, el modelo ERAS se aplica de uno u otro modo. Así, dados dos componentes A y B tales que ninguno forma complejos ni en estado puro ni en la mezcla, los parámetros $K_{\mathrm{i}}, \Delta h_{\mathrm{i}}^{*}$ y $\Delta v_{\mathrm{i}}^{*}$ son todos iguales a cero y el modelo ERAS se transforma en el modelo de Flory, con $X_{\mathrm{AB}}=\chi_{\mathrm{AB}}$. $\mathrm{Si}$ solamente uno de ellos forma complejos en estado puro (A) y no existe solvatación cruzada con el segundo componente, sólo los parámetros correspondientes al componente $\mathrm{A}, K_{\mathrm{A}}, \Delta h_{\mathrm{A}}^{*}$ y $\Delta v_{\mathrm{A}}^{*}$, y el energético de interacción $X_{\mathrm{AB}}$ son no nulos. Si existiese solvatación cruzada, también habría que considerar los parámetros $K_{\mathrm{AB}}, \Delta h_{\mathrm{AB}}^{*}$ y $\Delta v_{\mathrm{AB}}^{*}$, además de $X_{\mathrm{AB}}$. El caso más complicado se corresponde con aquellos sistemas en los que tanto $A$, como $B$ están asociados, y en los que existe solvatación cruzada. 
En las mezclas de interés aquí, lo anterior se traduce en las tres situaciones diferentes siguientes ( $\mathrm{A} \equiv$ amina, $\mathrm{B} \equiv$ cetona, $\mathrm{C} \equiv$ alcano, $\mathrm{D} \equiv$ alcohol):

a) Mezclas $N$-metilanilina (1) + alcano (2). $K_{\mathrm{C}}, \Delta h_{\mathrm{C}}^{*}$ y $\Delta v_{\mathrm{C}}^{*}$ son nulos por ser los alcanos compuestos no asociados, al contrario que $K_{\mathrm{A}}, \Delta h_{\mathrm{A}}^{*}$ y $\Delta v_{\mathrm{A}}^{*}$. No existe solvatación cruzada entre los componentes, luego los parámetros a ajustar en estas mezclas son $K_{\mathrm{A}}, \Delta h_{\mathrm{A}}^{*}, \Delta v_{\mathrm{A}}^{*}$ y $X_{\mathrm{AC}}$.

b) Mezclas amina (1) + cetona (2). $K_{\mathrm{B}}, \Delta h_{\mathrm{B}}^{*}$ y $\Delta v_{\mathrm{B}}^{*}$ son nulos por ser las cetonas compuestos no asociados al contrario que $K_{\mathrm{A}}, \Delta h_{\mathrm{A}}^{*}$ y $\Delta v_{\mathrm{A}}^{*}$, los que se determinan siguiendo una estrategia similar a la utilizada en la aplicación de los métodos de contribución de grupos, es decir, a partir de los datos disponibles de $H_{\mathrm{m}}^{\mathrm{E}}$ y $V_{\mathrm{m}}^{\mathrm{E}}$ para las mezclas amina + alcano [15], [16], [44]. $K_{\mathrm{AB}}, \Delta h_{\mathrm{AB}}^{*}, \Delta v_{\mathrm{AB}}^{*}$ y $X_{\mathrm{AB}}$ son los parámetros que deben ajustarse a los valores experimentales de $H_{\mathrm{m}}^{\mathrm{E}}$ y $V_{\mathrm{m}}^{\mathrm{E}}$ de las mezclas amina (1) + cetona (2).

c) Mezclas alcohol (1) + amina (2). $K_{\mathrm{A}}, \Delta h_{\mathrm{A}}^{*}, \Delta v_{\mathrm{A}}^{*}, K_{\mathrm{D}}, \Delta h_{\mathrm{D}}^{*}$ y $\Delta v_{\mathrm{D}}^{*}$ se determinan a partir de los datos disponibles en la literatura de $H_{\mathrm{m}}^{\mathrm{E}}$ y $V_{\mathrm{m}}^{\mathrm{E}}$ para las mezclas amina + alcano y alcohol + alcano, respectivamente. $K_{\mathrm{DA}}, \Delta h_{\mathrm{DA}}^{*}, \Delta v_{\mathrm{DA}}^{*}$ y $X_{\mathrm{DA}}$ son los parámetros a ajustar con los valores experimentales de $H_{\mathrm{m}}^{\mathrm{E}}$ y $V_{\mathrm{m}}^{\mathrm{E}}$ de las mezclas alcohol (1) + amina (2).

Todos los parámetros se dan en las Tabla $7-7$ y Tabla 7-8, junto con $V_{\mathrm{i}}$ (volumen molar) $\alpha_{P \mathrm{i}}, \kappa_{T 1}, p_{\mathrm{i}}^{*}$ y $v_{\mathrm{i}}^{*}$, necesarios para los cálculos.

Tabla 7-7: parámetros del modelo ERAS para los compuestos puros a $T=298.15 \mathrm{~K} .{ }^{a}[16]$; b [42], [43], $[45] ;{ }^{\mathrm{c}}[47] ;{ }^{\mathrm{d}}[46] ;{ }^{\mathrm{e}}[15] ;{ }^{\mathrm{f}}[44]$

\begin{tabular}{|c|c|c|c|c|c|c|c|c|}
\hline & $\begin{array}{c}V_{\mathrm{i}} / \\
\mathrm{cm}^{3} \cdot \mathrm{mol}^{-1}\end{array}$ & $\alpha_{P \mathrm{i}} / \mathrm{K}^{-1}$ & $\begin{array}{c}\kappa_{T_{1}} / \\
\mathrm{TPa}^{-1}\end{array}$ & $K_{\mathrm{i}}$ & $\begin{array}{c}\Delta h_{\mathrm{i}}^{*} / \\
\mathrm{kJ} \cdot \mathrm{mol}^{-1}\end{array}$ & $\begin{array}{c}\Delta v_{\mathrm{i}}^{*} / \\
\mathrm{cm}^{3} \cdot \mathrm{mol}^{-1}\end{array}$ & $\begin{array}{c}V_{\mathrm{i}}^{*} / \\
\mathrm{cm}^{3} \cdot \mathrm{mol}^{-1}\end{array}$ & $\begin{array}{l}P_{\mathrm{i}}^{*} / \\
\mathrm{MPa}\end{array}$ \\
\hline heptano & $147.47^{a}$ & $1.256^{\mathrm{b}}$ & $1460.6^{b}$ & 0 & 0 & 0 & $113.88^{a}$ & $430.5^{a}$ \\
\hline octano & $163.51^{a}$ & $1.164^{b}$ & $1302.4^{b}$ & 0 & 0 & 0 & $127.70^{a}$ & $444.5^{a}$ \\
\hline decano & $195.89^{a}$ & $1.051^{\mathrm{b}}$ & $1109.6^{b}$ & 0 & 0 & 0 & $155.71^{a}$ & $453.3^{a}$ \\
\hline ciclohexano & $108.75^{a}$ & $1.220^{c}$ & $1140^{c}$ & 0 & 0 & 0 & $84.23^{a}$ & $531.9^{a}$ \\
\hline benceno & $89.42^{a}$ & & & 0 & 0 & 0 & $69.33^{a}$ & $622.8^{a}$ \\
\hline tolveno & $106.87^{a}$ & & & 0 & 0 & 0 & $84.73^{a}$ & $555.2^{a}$ \\
\hline metanol & $40.75^{a}$ & $1.196^{c}$ & $1248^{c}$ & $986^{d}$ & $-25.1^{d}$ & $-5.6^{d}$ & $32.12^{a}$ & $426.9^{a}$ \\
\hline 1-propanol & $75.15^{a}$ & $1.004^{c}$ & $1026^{c}$ & $197^{d}$ & $-25.1^{d}$ & $-5.6^{d}$ & $61.24^{a}$ & $396.9^{a}$ \\
\hline 1-butanol & $91.99^{a}$ & $0.9493^{c}$ & $949.2^{c}$ & $175^{d}$ & $-25.1^{d}$ & $-5.6^{d}$ & $75.51^{c}$ & $406.9^{c}$ \\
\hline anilina & 91.53 & 0.85 & 467.9 & $14.8^{\mathrm{e}}$ & $-15^{\mathrm{e}}$ & $-12^{e}$ & 79.82 & 541.6 \\
\hline$N$-metilanilina & 109.11 & 0.82 & 522.1 & 6 & -12.5 & -5.2 & 92.37 & 569.4 \\
\hline di-n-propilamina & 137.92 & 1.24 & 1216.4 & $0.55^{f}$ & $-7.5^{f}$ & $-2.8^{f}$ & 107.17 & 488.4 \\
\hline di-n-butilamina & 171.08 & 1.07 & 1054 & $0.16^{f}$ & $-4.5^{f}$ & $-2.8^{f}$ & 135.41 & 485 \\
\hline 2-propanona & 73.96 & 1.45 & 1317.5 & 0 & 0 & 0 & 54.69 & 619 \\
\hline 2-butanona & 90.15 & 1.31 & 1175.9 & 0 & 0 & 0 & 68.92 & 571.1 \\
\hline 2-pentanona & 107.46 & 1.198 & 1098.77 & 0 & 0 & 0 & 83.50 & 538.3 \\
\hline 2-heptanona & 140.77 & 1.06 & 968.9 & 0 & 0 & 0 & 111.73 & 517.7 \\
\hline
\end{tabular}


Tabla 7-8: parámetros del modelo ERAS para las mezclas amina (1) + cetona o alcano (2) y alcohol (1) + amina (2) a $T=298.15 \mathrm{~K}$.

\begin{tabular}{|c|c|c|c|c|}
\hline & $K_{\mathrm{AB}}$ & $\Delta h_{\mathrm{AB}}^{*} / \mathrm{kJ} \cdot \mathrm{mol}^{-1}$ & $\Delta v_{\mathrm{AB}}^{*} / \mathrm{cm}^{3} \cdot \mathrm{mol}^{-1}$ & $X_{\mathrm{AB}} / \mathrm{MPa}$ \\
\hline \multicolumn{5}{|c|}{ anilina } \\
\hline metanol & 60 & -40.58 & & 2 \\
\hline 2-propanona & 2 & -28.7 & -16 & 1.9 \\
\hline 2-butanona & 2 & -28.7 & -16.5 & 1.9 \\
\hline 2-pentanona & 2 & -28.7 & -16.5 & 2.2 \\
\hline 2-heptanona & 2 & -26.5 & -16.5 & 2.2 \\
\hline \multicolumn{5}{|c|}{$N$-metilanilina } \\
\hline heptano & 0 & 0 & 0 & 40.5 \\
\hline octano & 0 & 0 & 0 & 40.5 \\
\hline decano & 0 & 0 & 0 & 39 \\
\hline ciclohexano & 0 & 0 & 0 & 52.4 \\
\hline benceno & 2.8 & -8 & -4.6 & 16 \\
\hline tolveno & 2.8 & -8 & -4.6 & 12 \\
\hline metanol & 8 & -25 & 25 & 1 \\
\hline 1-propanol & 5 & -15 & 20 & 5 \\
\hline 1-butanol & 3 & -15 & 20 & 18.5 \\
\hline 2-propanona & 1.05 & -22 & -8 & 8 \\
\hline 2-butanona & 1.4 & -22 & -7.2 & 8 \\
\hline 2-pentanona & 1.65 & -22 & -7.1 & 8 \\
\hline 2-heptanona & 1.85 & -22 & -6.8 & 8 \\
\hline \multicolumn{5}{|c|}{ di-n-propilamina } \\
\hline 2-propanona & 0.40 & -15 & -12.1 & 55.1 \\
\hline 2-butanona & 0.40 & -15 & -9.5 & 38.5 \\
\hline 2 -pentanona & 0.40 & -15 & -8.7 & 29 \\
\hline 2-heptanona & 0.40 & -15 & -8 & 18.2 \\
\hline \multicolumn{5}{|c|}{ di- $n$-butilamina } \\
\hline 2-propanona & 0.15 & -15 & -18.4 & 56 \\
\hline 2-butanona & 0.15 & -15 & -14 & 37.2 \\
\hline 2-pentanona & 0.15 & -15 & -12.8 & 26.8 \\
\hline 2-heptanona & 0.15 & -15 & -11.5 & 16.9 \\
\hline
\end{tabular}

En las llustraciones 7-19 a 7-27 se representan los resultados experimentales de $H_{\mathrm{m}}^{\mathrm{E}}$ y de $V_{\mathrm{m}}^{\mathrm{E}}$, junto con los calculados mediante el modelo ERAS de algunas de las mezclas de interés. En ellas se representan las contribuciones física y química a $H_{\mathrm{m}}^{\mathrm{E}}$ y $V_{\mathrm{m}}^{\mathrm{E}}$, lo que permite realizar una interpretación más profunda de los resultados obtenidos.

En las tablas $7-9$ y $7-10$ se comparan los valores experimentales con los calculados mediante el modelo ERAS de $H_{\mathrm{m}}^{\mathrm{E}}$ y $V_{\mathrm{m}}^{\mathrm{E}}$ a $x_{1}=0.5$. Con el objetivo de proporcionar información más detallada, en dichas tablas también se incluye la desviación relativa de la entalpía de exceso, $\operatorname{des}\left(H_{\mathrm{m}}^{\mathrm{E}}\right)$, calculada de acuerdo con la expresión (7.5), siendo en este caso $H_{\mathrm{m}, \mathrm{E} a l}^{\mathrm{E}}$ los valores de calculados mediante ERAS.

En vista de los resultados obtenidos, se puede afirmar que el modelo representa adecuadamente tanto $H_{\mathrm{m}}^{\mathrm{E}}$ como $V_{\mathrm{m}}^{\mathrm{E}}$ en todos los sistemas analizados, excepto del sistema 1-propanol $+N$-metilanilina (del cual sólo se conocen datos de $V_{\mathrm{m}}^{\mathrm{E}}$ ), lo que se 
debe casi con total seguridad a que estos datos no son muy fiables.

Tabla 7-9: valores experimentales y calculados con ERAS de las entalpías de exceso, $H_{\mathrm{m}}^{\mathrm{E}}$, a composición $x_{1}=0.5$ y temperatura $T$ de mezclas amina (1) + cetona (2), amina (1) + alcano (2) y alcohol (1) + amina (2). ${ }^{\mathrm{a}}[36] ;{ }^{\mathrm{b}}[37] ;{ }^{\mathrm{c}}[38] ;{ }^{\mathrm{d}}[39]$

\begin{tabular}{|c|c|c|c|c|c|c|}
\hline & \multirow[t]{2}{*}{$T / \mathrm{K}$} & \multirow[t]{2}{*}{$N$} & \multicolumn{2}{|c|}{$H_{\mathrm{m}}^{\mathrm{E}} / \mathrm{J} \cdot \mathrm{mol}^{-1}$} & \multicolumn{2}{|c|}{$\operatorname{des}\left(H_{\mathrm{m}}^{\mathrm{E}}\right)$} \\
\hline & & & Exp & ERAS & Exp & ERAS \\
\hline \multicolumn{7}{|c|}{ anilina } \\
\hline 2-propanona & 298.15 & 10 & -1236 & -1236 & 0.007 & 0.052 \\
\hline 2-butanona & 298.15 & 10 & -1165 & -1112 & 0.009 & 0.040 \\
\hline 2-pentanona & 298.15 & 11 & -1029 & -1012 & 0.010 & 0.058 \\
\hline 2-heptanona & 298.15 & 14 & -692 & -679 & 0.009 & 0.145 \\
\hline \multicolumn{7}{|c|}{$N$-metilanilina } \\
\hline heptano & 298.15 & 9 & 1675 & 1667 & 0.009 & 0.047 \\
\hline octano & 298.15 & 9 & 1767 & 1782 & 0.007 & 0.037 \\
\hline decano & 298.15 & 9 & 1913 & 1878 & 0.004 & 0.040 \\
\hline ciclohexano & 298.15 & 9 & $1617^{a}$ & 1607 & 0.009 & 0.037 \\
\hline benceno & 298.15 & 17 & $457^{b}$ & 460 & 0.009 & 0.158 \\
\hline & 323.15 & 10 & $503^{b}$ & 508 & 0.006 & 0.090 \\
\hline tolveno & 298.15 & 9 & 497 & 491 & 0.008 & 0.092 \\
\hline metanol & 298.15 & 16 & $426^{c}$ & 425 & 0.016 & 0.115 \\
\hline 1-butanol & 313.15 & 14 & $1372^{d}$ & 1373 & 0.009 & 0.064 \\
\hline 2-propanona & 298.15 & 9 & -668 & -690 & 0.012 & 0.130 \\
\hline 2-butanona & 298.15 & 9 & -812 & -801 & 0.007 & 0.059 \\
\hline 2-pentanona & 298.15 & 9 & -835 & -827 & 0.004 & 0.041 \\
\hline 2-heptanona & 298.15 & 9 & -741 & -749 & 0.004 & 0.053 \\
\hline \multicolumn{7}{|c|}{ di-n-propilamina } \\
\hline 2-propanona & 298.15 & 9 & 648 & 645 & 0.009 & 0.063 \\
\hline 2-butanona & 298.15 & 10 & 398 & 403 & 0.010 & 0.066 \\
\hline 2-pentanona & 298.15 & 9 & 281 & 288 & 0.011 & 0.092 \\
\hline 2-heptanona & 298.15 & 9 & 161 & 161 & 0.011 & 0.062 \\
\hline \multicolumn{7}{|c|}{ di-n-butilamina } \\
\hline 2-propanona & 298.15 & 9 & 933 & 946 & 0.004 & 0.095 \\
\hline 2-butanona & 298.15 & 9 & 620 & 628 & 0.006 & 0.089 \\
\hline 2-pentanona & 298.15 & 9 & 454 & 455 & 0.009 & 0.090 \\
\hline 2-heptanona & 298.15 & 9 & 277 & 282 & 0.011 & 0.093 \\
\hline
\end{tabular}


Tabla 7-10: valores experimentales y calculados con ERAS de los volúmenes de exceso, $V_{\mathrm{m}}^{\mathrm{E}}$, a composición $x_{1}=0.5$ y temperatura $T=298.15 \mathrm{~K}$ de mezclas amina (1) + cetona (2), amina (1) + alcano (2) y alcohol (1) + amina (2). ${ }^{a}[48]$

\begin{tabular}{|c|c|c|}
\hline & \multicolumn{2}{|c|}{$V_{\mathrm{m}}^{\mathrm{E}} / \mathrm{J} \cdot \mathrm{mol}^{-1}$} \\
\hline & Exp & ERAS \\
\hline \multicolumn{3}{|c|}{ anilina } \\
\hline 2-propanona & -1.183 & -1.152 \\
\hline 2-butanona & -1.246 & -1.111 \\
\hline 2-pentanona & -1.096 & -1.075 \\
\hline 2-heptanona & -0.861 & -0.691 \\
\hline \multicolumn{3}{|c|}{$N$-metilanilina } \\
\hline heptano & -0.227 & -0.176 \\
\hline ciclohexano & 0.462 & 0.452 \\
\hline tolveno & -0.214 & -0.212 \\
\hline 1-propanol $(T=303.15 \mathrm{~K})$ & $-0.274^{a}$ & -0.344 \\
\hline 2-propanona & -0.727 & -0.728 \\
\hline 2-butanona & -0.669 & -0.666 \\
\hline 2-pentanona & -0.687 & -0.689 \\
\hline 2-heptanona & -0.509 & -0.504 \\
\hline \multicolumn{3}{|c|}{ di- $n$-propilamina } \\
\hline 2-propanona & 0.243 & 0.241 \\
\hline 2-butanona & 0.144 & 0.147 \\
\hline 2-heptanona & -0.089 & -0.084 \\
\hline \multicolumn{3}{|c|}{ di-n-butilamina } \\
\hline 2-propanona & 0.417 & 0.410 \\
\hline 2-butanona & 0.264 & 0.279 \\
\hline 2-heptanona & 0.009 & 0.010 \\
\hline
\end{tabular}




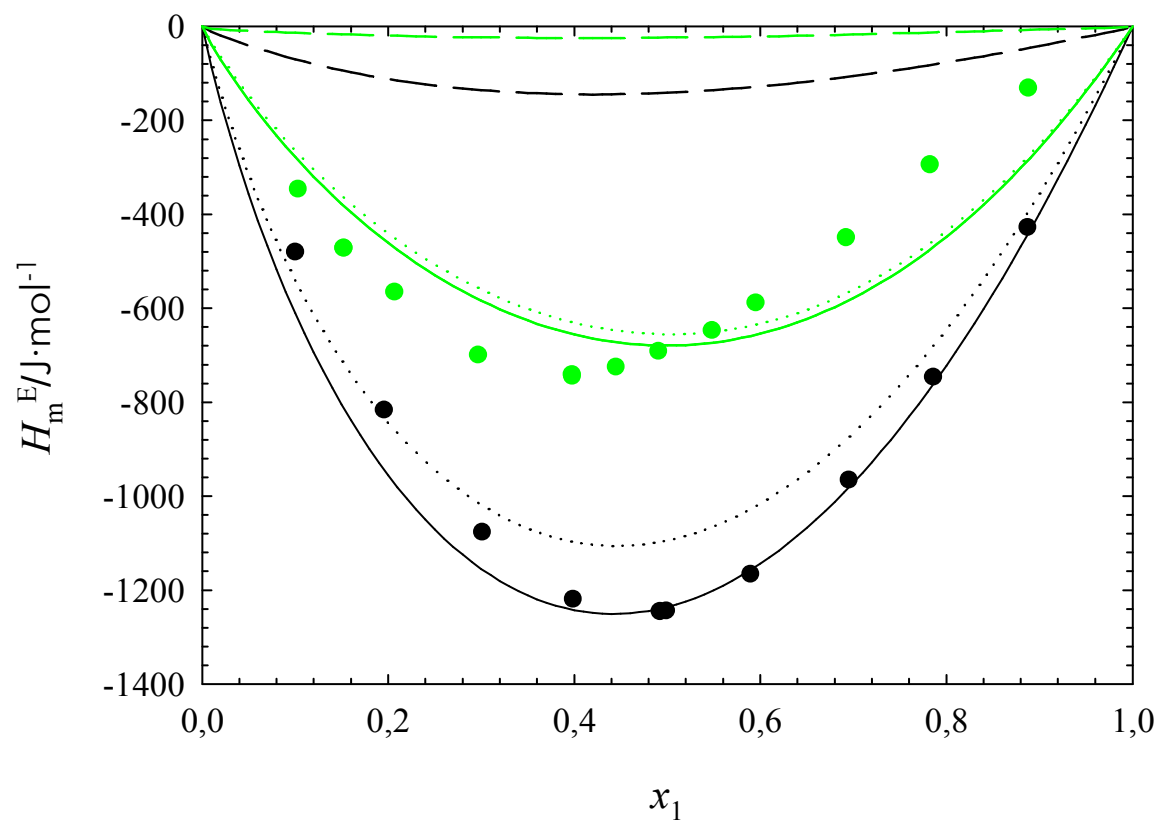

Ilustración 7-19: $H_{\mathrm{m}}^{\mathrm{E}}$ de mezclas anilina (1) + cetona (2) a $T=298.15 \mathrm{~K}$. Puntos, resultados experimentales: - 2-propanona; 2 -heptanona. Línea continua, resultados del modelo ERAS con los parámetros de la Tabla 7-8. - - contribución física. ….. contribución química

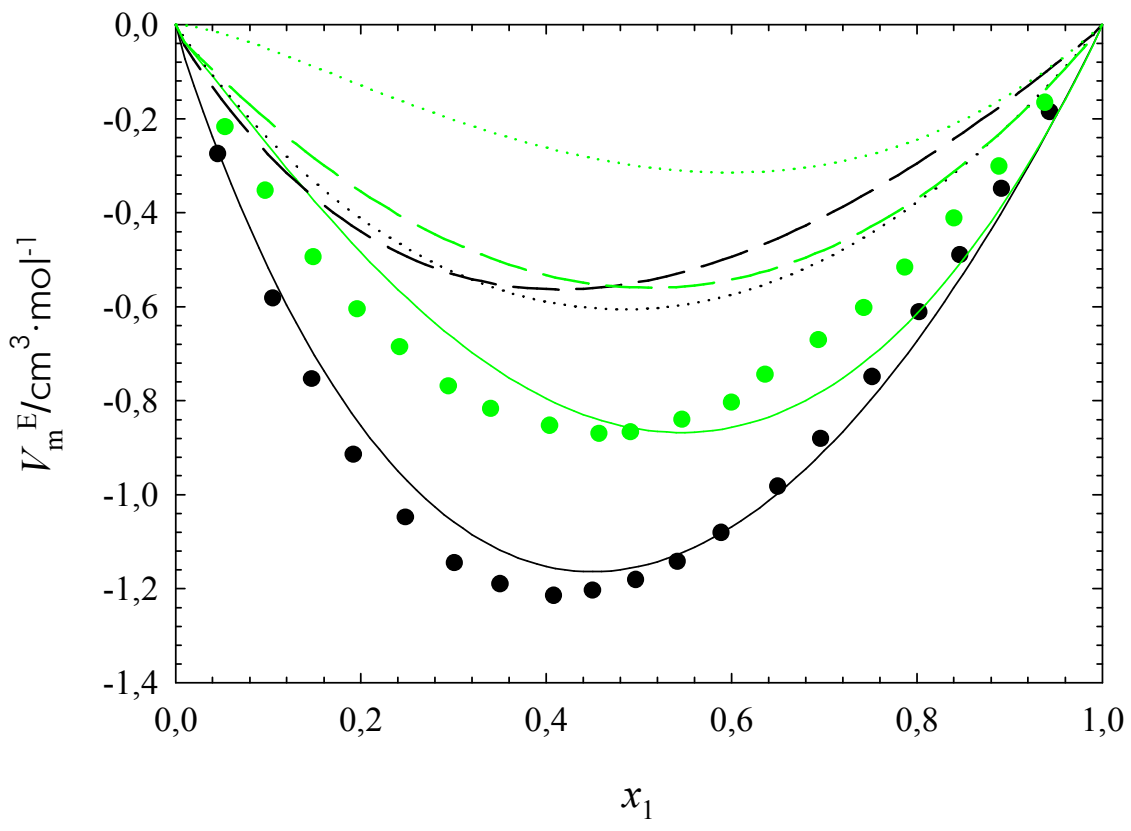

Ilustración 7-20: $V_{\mathrm{m}}^{\mathrm{E}}$ de mezclas anilina (1) + cetona (2) a $T=298.15 \mathrm{~K}$. Puntos, resultados experimentales:

- 2-propanona; • 2-heptanona. Línea continua, resultados del modelo ERAS con los parámetros de la Tabla 7-8. - - contribución física. …… contribución química 


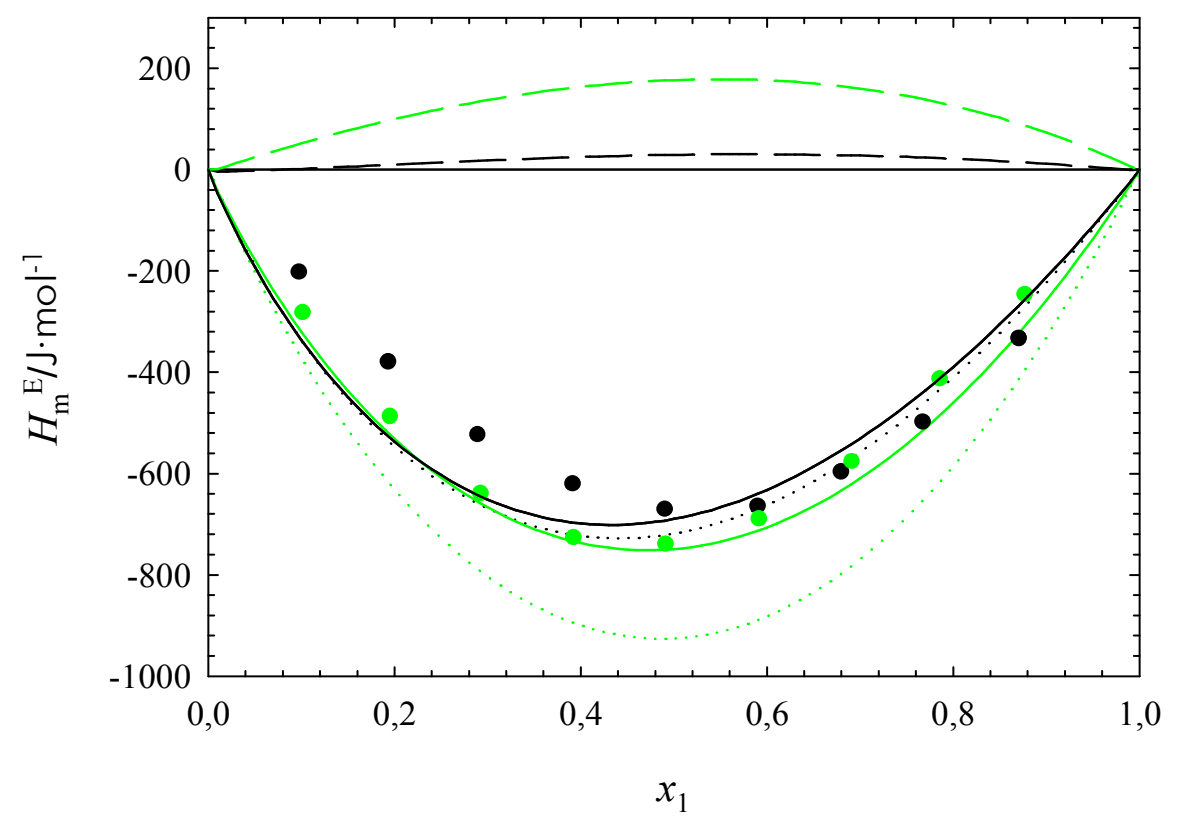

Ilustración 7-21: $H_{\mathrm{m}}^{\mathrm{E}}$ de mezclas $N$-metilanilina (1) + cetona (2) a $T=298.15 \mathrm{~K}$. Puntos, resultados experimentales: $\bullet 2$-propanona; 2 -heptanona. Línea continua, resultados del modelo ERAS con los parámetros de la Tabla 7-8. — — contribución física. …… contribución química

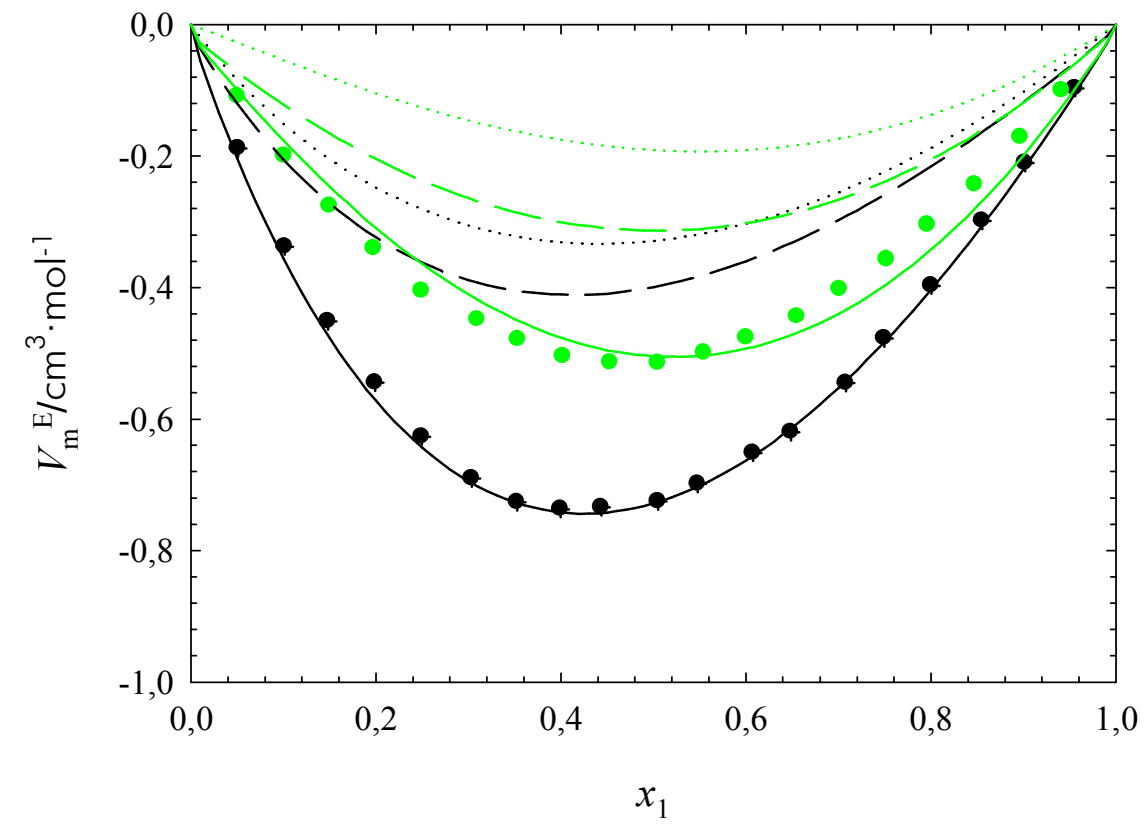

Ilustración 7-22: $V_{\mathrm{m}}^{\mathrm{E}}$ de mezclas $N$-metilanilina (1) + cetona (2) a $T=298.15 \mathrm{~K}$. Puntos, resultados experimentales: -2-propanona; 2 -heptanona. Línea continua, resultados del modelo ERAS con los parámetros de la Tabla 7-8. — - contribución física. … contribución química 


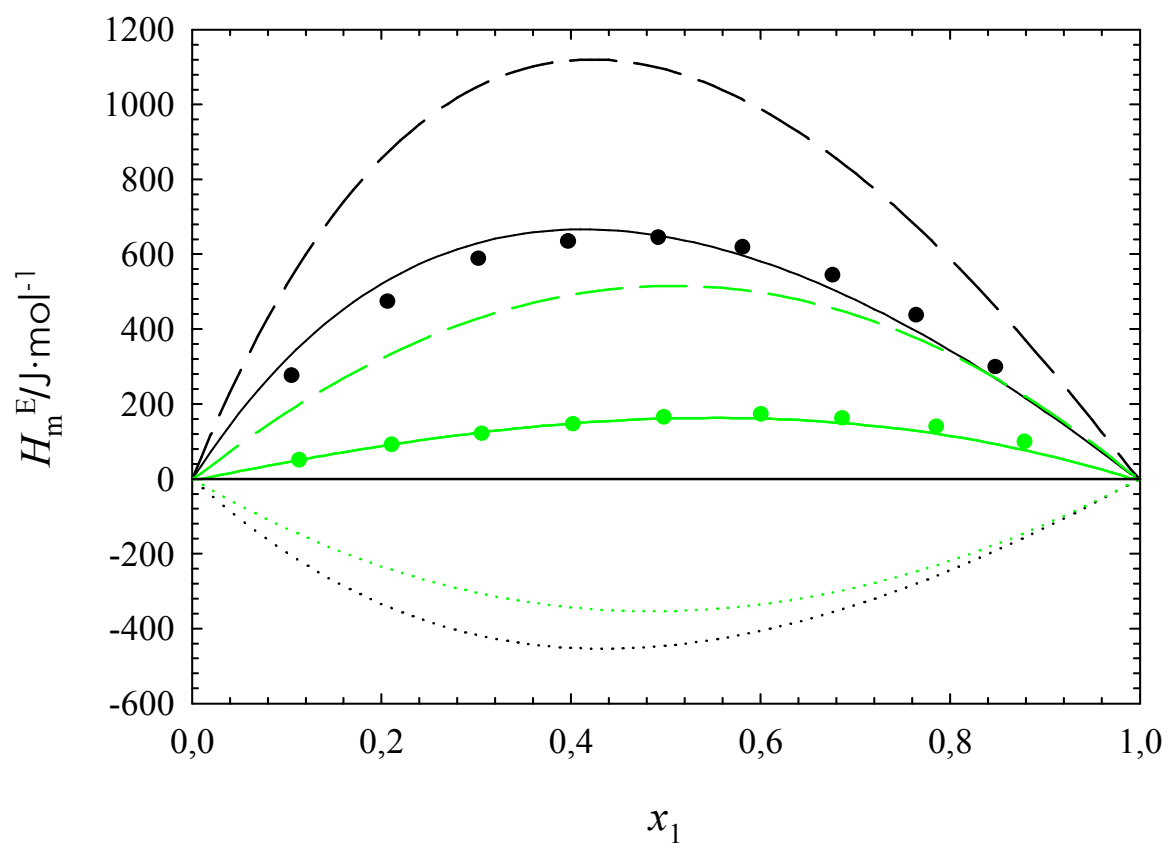

Ilustración 7-23: $H_{\mathrm{m}}^{\mathrm{E}}$ de mezclas di- $n$-propilamina (1) + cetona (2) a $T=298.15 \mathrm{~K}$. Puntos, resultados experimentales: - 2-propanona; 2 -heptanona. Línea continua, resultados del modelo ERAS con los parámetros de la Tabla 7-8. - - contribución física. ….. contribución química

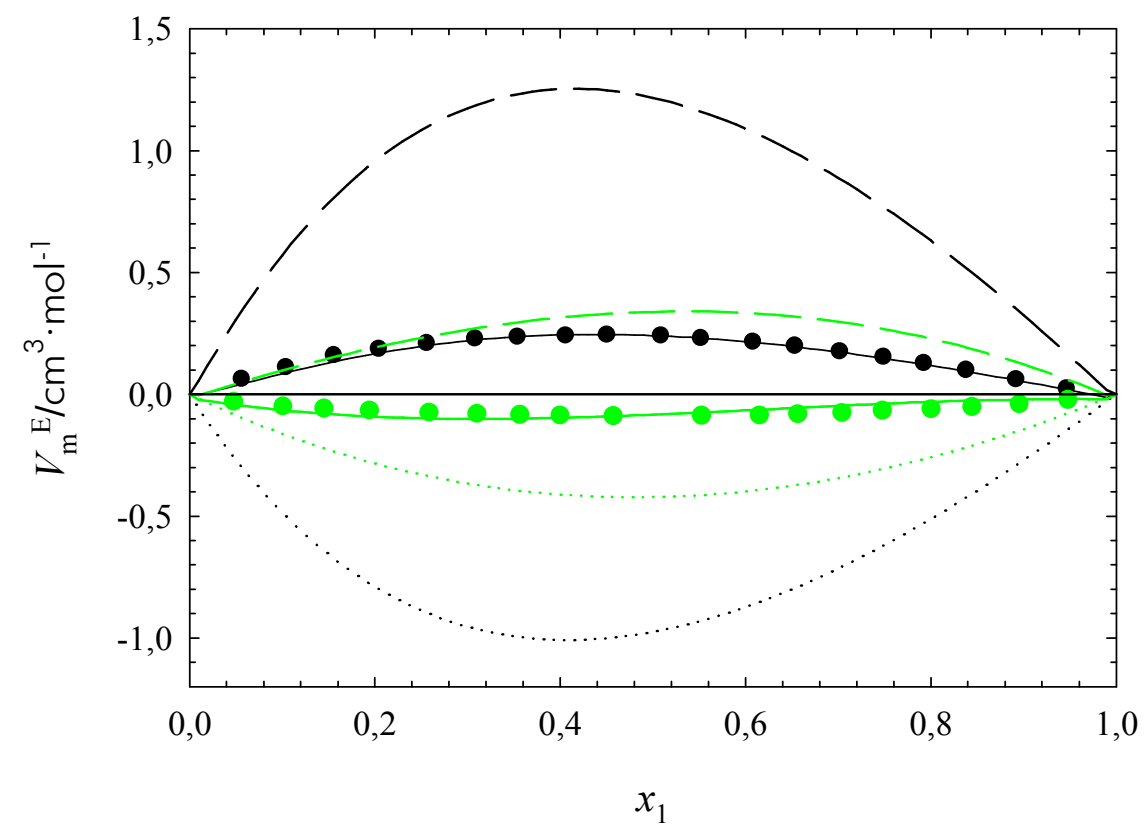

Ilustración 7-24: $V_{\mathrm{m}}^{\mathrm{E}}$ de mezclas di- $n$-propilamina (1) + cetona (2) a $T=298.15 \mathrm{~K}$. Puntos, resultados experimentales: - 2-propanona; 2 2-heptanona. Línea continua, resultados del modelo ERAS con los parámetros de la Tabla 7-8. - - contribución física. ….. contribución química 


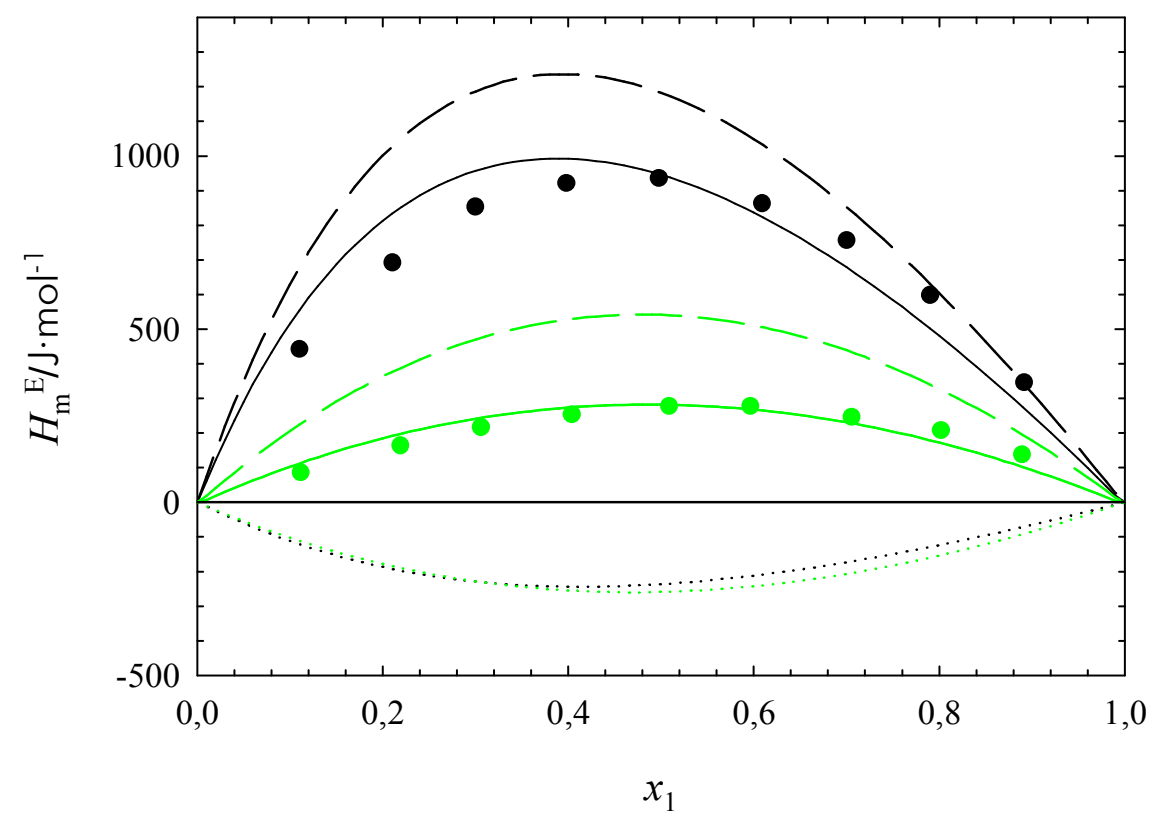

Ilustración 7-25: $H_{\mathrm{m}}^{\mathrm{E}}$ de mezclas di-n-butilamina (1) + cetona (2) a $T=298.15 \mathrm{~K}$. Puntos, resultados experimentales: - 2-propanona; - 2-heptanona. Línea continua, resultados del modelo ERAS con los parámetros de la Tabla 7-8. — — contribución física. …… contribución química

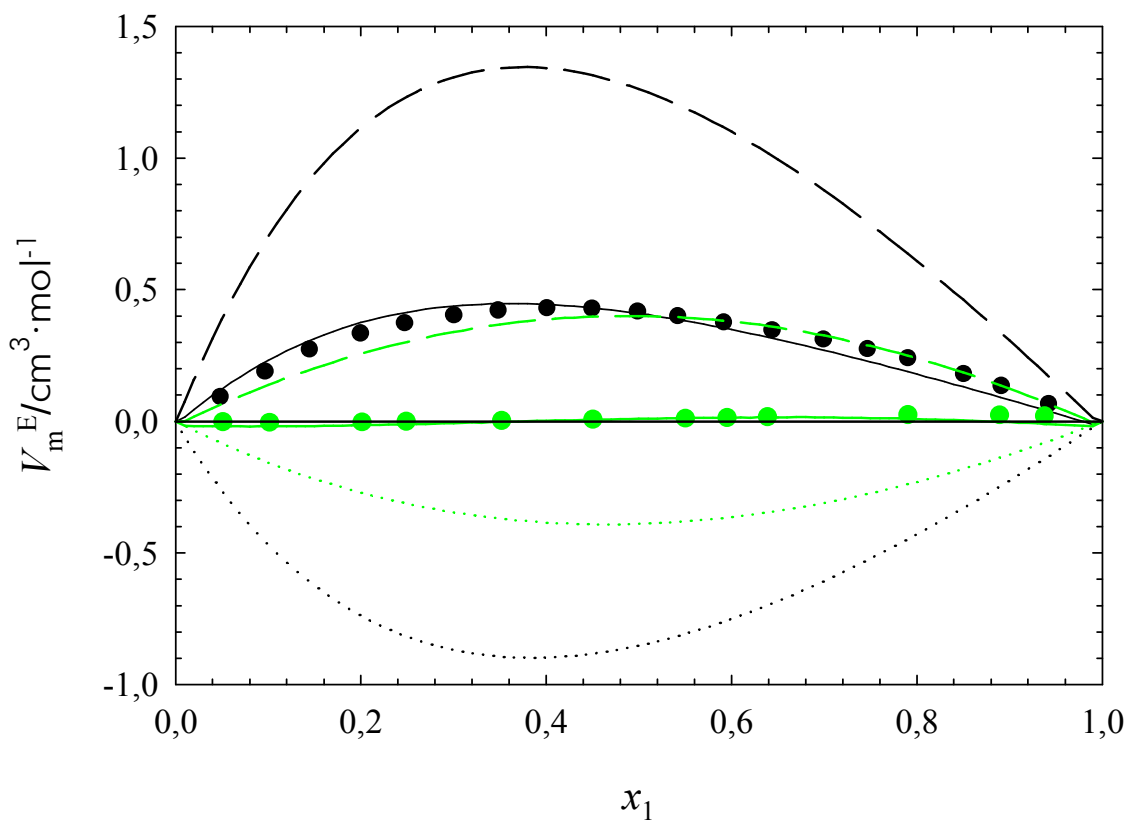

Ilustración 7-26: $V_{\mathrm{m}}^{\mathrm{E}}$ de mezclas di-n-butilamina (1) + cetona (2) a $T=298.15 \mathrm{~K}$. Puntos, resultados experimentales: - 2-propanona; - 2-heptanona. Línea continua, resultados del modelo ERAS con los parámetros de la Tabla 7-8. — - contribución física. …. contribución química 


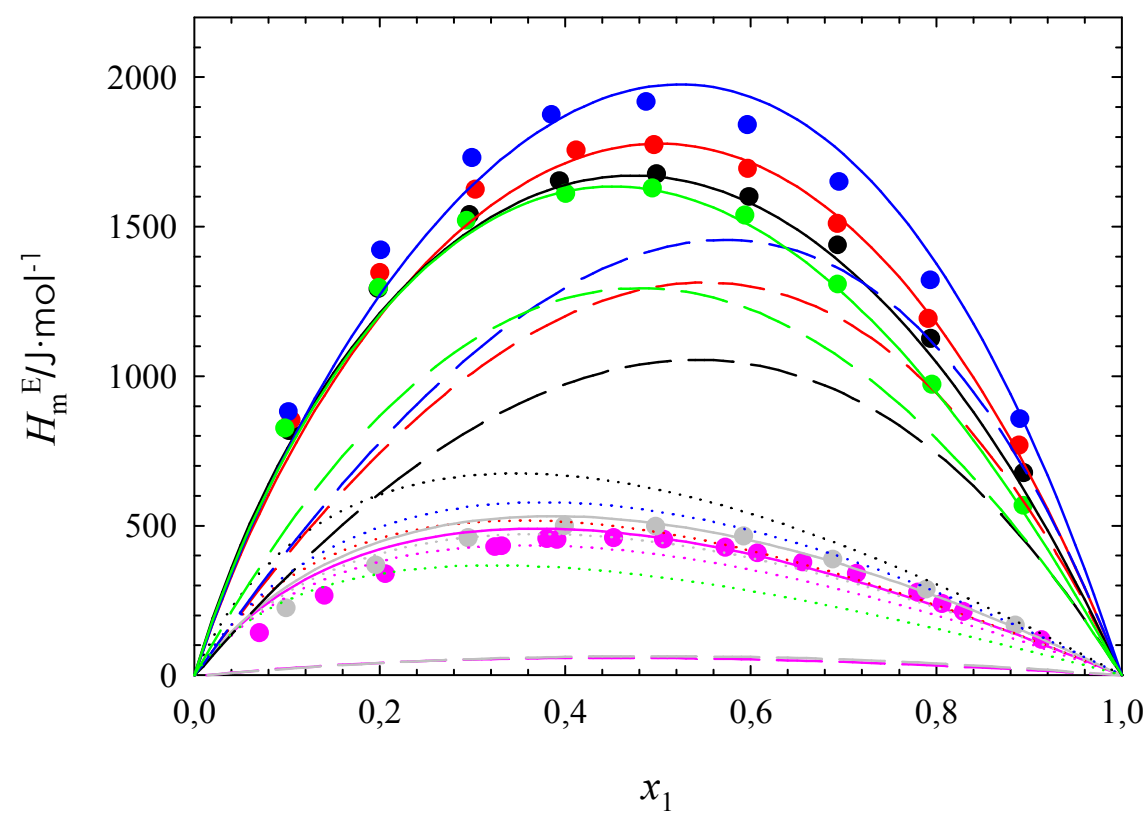

llustración 7-27: $H_{\mathrm{m}}^{\mathrm{E}}$ de mezclas $N$-metilanilina (1) + hidrocarburo (2) a $T=298.15 \mathrm{~K}$. Puntos, resultados experimentales: - heptano; •octano; • decano; 'ciclohexano; • benceno; •tolveno. Línea continua, resultados del modelo ERAS con los parámetros de la Tabla 7-8. - - contribución física. contribución química

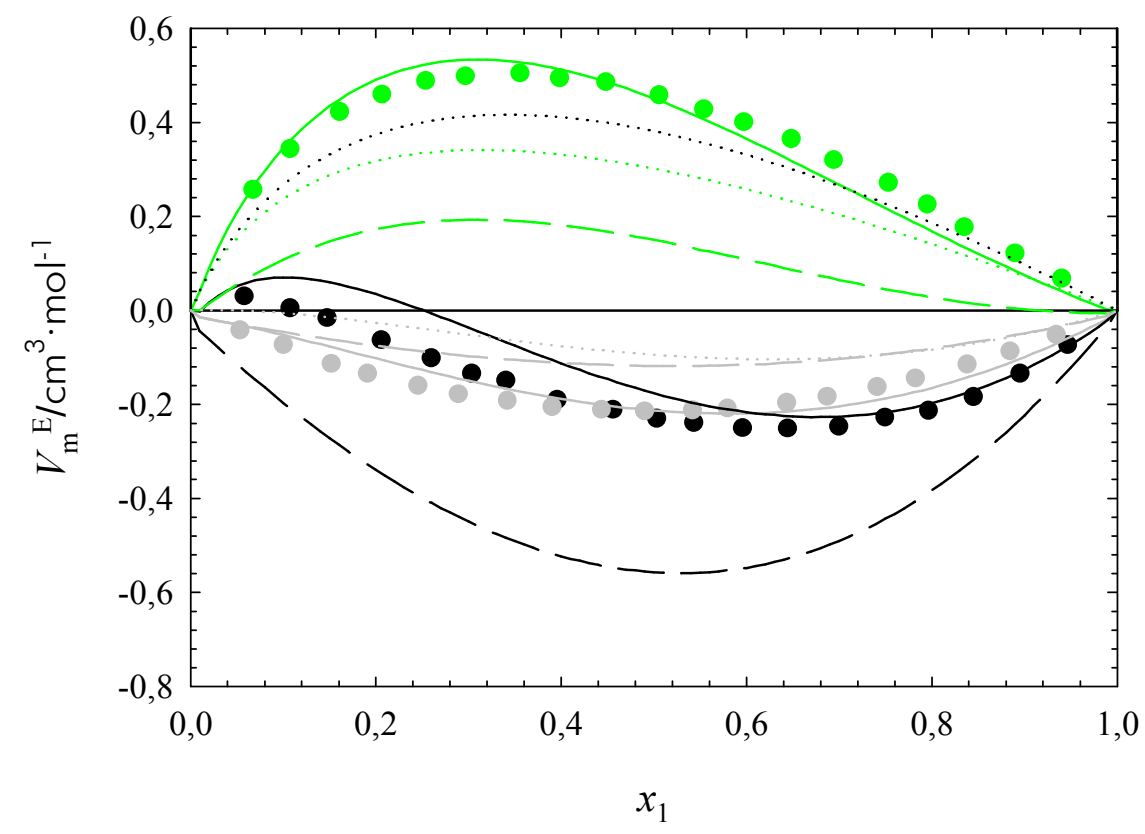

Ilustración 7-28: $V_{\mathrm{m}}^{\mathrm{E}}$ de mezclas $N$-metilanilina (1) + hidrocarburo (2) a $T=298.15 \mathrm{~K}$. Puntos, resultados

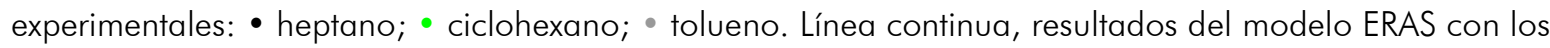
parámetros de la Tabla 7-8. — - contribución física. ….. contribución química 


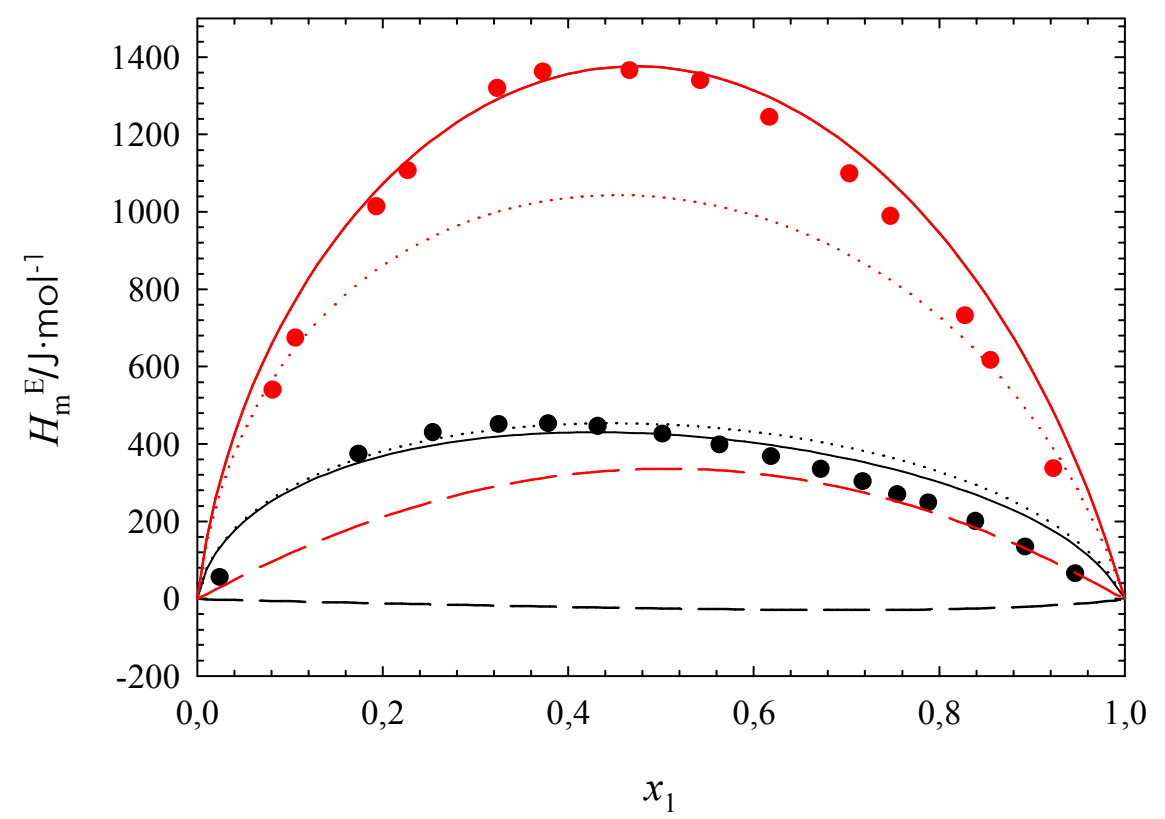

Ilustración 7-29: $H_{\mathrm{m}}^{\mathrm{E}}$ de mezclas alcohol (1) $+N$-metilanilina (2). Puntos, resultados experimentales:

- metanol a $T=298.15 \mathrm{~K}$; -1 -butanol a $T=313.15 \mathrm{~K}$. Línea continua, resultados del modelo ERAS con los parámetros de la Tabla 7-8. — - contribución física. … contribución química

\subsection{DISCUSIÓN DE LOS RESULTADOS}

\subsubsection{Modelo DISQUAC}

DISQUAC proporciona resultados muy buenos tanto de los equilibrios entre fases como de $H_{\mathrm{m}}^{\mathrm{E}}$ para las mezclas consideradas, como puede verse en las llustraciones 7-1 a 7-18 y en las Tablas 7-5 y 7-6. Es importante resaltar el hecho de que DISQUAC representa la asimetría de las curvas de las entalpías de exceso de los sistemas dados. Sin embargo, no es capaz de estimar los valores de $V_{\mathrm{m}}^{\mathrm{E}}$, por ser un modelo de red rígida.

La dependencia de los parámetros de interacción con la estructura molecular merece ser examinada. Del análisis de la Tabla 7-3, se pueden obtener las conclusiones siguientes, agrupadas de acuerdo con la clase de sistema considerada:

\subsubsection{Mezclas amina + alcano}

Los mismos coeficientes $C_{\mathrm{sn}, l}^{\text {QUAC }}(l=1,3 ; \mathrm{s}=\mathrm{a}, \mathrm{b}, \mathrm{c}, \mathrm{h})$ se pueden utilizar en sistemas con anilina, $N$-metilanilina, o incluso $N, N$-dimetilanilina, lo que remarca la importancia de los efectos relacionados con el anillo aromático en estas mezclas

\subsubsection{Mezclas amina aromática o amina lineal secundaria + cetona}

Un resultado importante es que, fijada la amina, los coeficientes $C_{\mathrm{kn}, l}^{\mathrm{DIS} / \mathrm{QUAC}}(l=1,3)$ son constantes en una serie homóloga, es decir, son independientes de la longitud de la cetona (Tabla 7-3). Un comportamiento similar se ha hallado al investigar muchas otras 
mezclas en términos del modelo DISQUAC [50], [51], por lo que parece ser una regla del modelo. Además, dichos coeficientes $C_{\mathrm{kn}, l}^{\mathrm{DIS} / \mathrm{QUAC}}(l=1,3)$ son los mismos para mezclas con anilina, $N$-metilanilina, o incluso $N, N$-dimetilanilina y piridina. Lo mismo ocurre en las mezclas con di- $n$-propilamina o di- $n$-butilamina.

En cuanto a los coeficientes $C_{\mathrm{kn}, 2}^{\mathrm{QUAC}}$ de las mezclas con amina aromática, disminuyen según la secuencia: $N, N$-dimetilanilina $>$ piridina $\equiv N$-metilanilina $>$ anilina. Este comportamiento puede deberse a la mayor superficie aromática de la amina primaria respecto de la secundaria y de la terciaria. En lo que respecta a las mezclas con di- $n$ propilamina o di- $n$-butilamina, los coeficientes $C_{\mathrm{kn}, 2}^{\mathrm{QUAC}}$ son idénticos, lo que sugiere que en dichas mezclas los contactos $(\mathrm{k}, \mathrm{n})$ se diferencian principalmente en intracciones dispersivas.

Los coeficientes $C_{\mathrm{kn}, l}^{\mathrm{DIS} / \mathrm{QUAC}}(l=1,2)$ para los sistemas anilina $\mathrm{O} N$-metilanilina son muy diferentes a aquellos de las mezclas con di-n-propilamina o di-n-butilamina, lo que ensalza la importancia del anillo aromático en las interacciones entre las moléculas.

El modelo ha sido aplicado con éxito a la mezcla piridina (1) + 2-propanona (2) (véase la Tabla 7-5), de la que solamente se han encontrado en la bibliografía datos experimentales sobre el equilibrio líquido-vapor [35], [49], suponiendo que los parámetros de intercambio cuasiquímicos son los mismos que para las mezclas con $\mathrm{N}$ metilanilina.

\subsubsection{Mezclas amina lineal terciaria + cetona o amino-cetona + alcano}

En ambos tipos de sistema, los parámetros dispersivos son mucho mayores que los cuasiquímicos (ver la Tabla 7-3). En consecuencia, la contribución cuasiquímica a $H_{\mathrm{m}}^{\mathrm{E}}$ está esencialmente determinada por la ruptura de las interacciones cetona-cetona, y el contacto $(\mathrm{k}, \mathrm{n})$ puede considerarse principalmente de tipo dispersivo.

La disminución que se observa en $C_{\mathrm{kn}, 2}^{\mathrm{DIs}}$ al sustituir la $N, N$-dimetiletilamina $\circ N, N$ dietilmetilamina por $N, N, N$-trietilamina puede deberse a efectos estéricos. En mezclas con las dos primeras, estos efectos se tienen en cuenta utilizando valores diferentes para los coeficientes $C_{\mathrm{an}, 2}^{\mathrm{DIS}}$. La misma tendencia se ha encontrado en las mezclas amina terciaria + alcano [9] y en las mezclas con 1-dietilamino-2-propanona.

La utilización de los mismos parámetros de intercambio en las mezclas amino-cetona + alcano que en las mezclas $N, N, N$-trietilamina + cetona conduce a resultados muy pobres, lo que confirma que en este tipo de sistemas, los efectos intramoleculares (efectos de proximidad) son importantes, aumentando a medida que la distancia entre los grupos carbonilo y amino disminuye, siendo el caso límite las amidas, en las que carece de sentido hablar separadamente de tales grupos y debe definirse el grupo amida.

\subsubsection{Mezclas ternarias}

DISQUAC también se ha aplicado en la predicción de $H_{\mathrm{m}}^{\mathrm{E}}$ de la mezcla ternaria 1-propanol (1) $+N, N, N$-trietilamina (2) +2 -butanona (3), únicamente a partir de la información disponible para los correspondientes sistemas binarios, e ignorando las posibles interacciones ternarias $(\mathrm{s}, \mathrm{t}, \mathrm{m})$, es decir, entre tríos de grupos. Mientras que este 
método ha resultado de gran éxito en un gran número de sistemas estudiados [52]-[55] (por ejemplo, en las mezclas 1-propanol (1) $+N, N, N$-trietilamina (2) + heptano (3) ó 1 propanol (1) + benceno (2) + 2-butanona (3)), en este caso en concreto se han encontrado grandes diferencias entre los valores experimentales y los determinados utilizando DISQUAC (ver Tabla 7-6 e llustración 7-12), lo que revela la importancia de las interacciones ternarias. El hecho de que DISQUAC represente adecuadamente $H_{\mathrm{m}}^{\mathrm{E}}$ de los sistemas binarios relacionados, refuerza esta conclusión (véase la Tabla 7-11). Además, debe señalarse que la forma "anómala" de $H_{\mathrm{m}}^{\mathrm{E}}$ para el cociente $x_{2} / x_{3}=0.3264$ es muy difícil de representar para cualquier modelo teórico.

Tabla 7-11: valores experimentales y calculados con DISQUAC de entalpías de exceso, $H_{\mathrm{m}}^{\mathrm{E}}$, a composición $x_{1}=0.5$ y temperatura $T=298.15 \mathrm{~K}$ de las mezclas binarias relacionadas con la mezcla 1 -propanol $(1)+$ $N, N, N$-trietilamina (2) +2 -butanona (3). ${ }^{a}[56] ;{ }^{b}[57]$

\begin{tabular}{cccccc}
\hline & $N$ & \multicolumn{2}{c}{$H_{\mathrm{m}}^{\mathrm{E}} / \mathrm{J} \cdot \mathrm{mol}^{-1}$} & \multicolumn{2}{c}{$\operatorname{des}\left(H_{\mathrm{m}}^{\mathrm{E}}\right)$} \\
\hline & & $\operatorname{Exp}$ & DISQUAC & $\operatorname{Exp}$ & DISQUAC \\
\hline $\begin{array}{c}\text { 1-propanol (1) + } \\
N, N, N \text {-trietilamina (2) } \\
\text { 1-propanol (1) }+ \\
\begin{array}{c}\text {-butanona (2) } \\
N, N, N \text {-trietilamina (1) }\end{array}\end{array}$ & 13 & $-1424^{\mathrm{a}}$ & -1413 & $0.005^{\mathrm{a}}$ & 0.037 \\
2-butanona (2) & 14 & $1252^{\mathrm{b}}$ & 1251 & $0.001^{\mathrm{b}}$ & 0.012 \\
\hline
\end{tabular}

\subsubsection{Modelo ERAS}

Los resultados obtenidos con el modelo ERAS son de peor calidad que los proporcionados por DISQUAC, como puede inducirse de la comparación entre los valores de $\operatorname{des}\left(H_{\mathrm{m}}^{\mathrm{E}}\right)$ calculados mediante ambos métodos (Tablas 7-6 y 7-9). Ello sugiere que los efectos relacionados con la asociación y/o solvatación son menos importantes que los efectos procedentes de las interacciones físicas. La aplicación del modelo ERAS reafirma esta conclusión para aquellos sistemas con valores altos de $X_{\mathrm{AB}}$ (relacionados con valores pequeños de $K_{\mathrm{i}},\left|\Delta h_{\mathrm{i}}^{*}\right|, K_{\mathrm{AB}}$ y $\left|\Delta h_{\mathrm{AB}}^{*}\right|$ ) puesto que en aquellos sistemas en los que la autoasociación y / O la solvatación cruzada son importantes, los valores de $X_{\mathrm{AB}}$ no son mayores de $20 \mathrm{MPa}$.

Como en el caso de DISQUAC, la dependencia de los parámetros del modelo con la estructura molecular merece ser examinada. Del análisis de las Tablas 7-7 y 7-8 se pueden obtener las conclusiones siguientes, agrupadas de acuerdo con la clase de sistema considerada:

\subsubsection{Mezclas amina + alcano y alcohol + amina}

Los parámetros ERAS de la $N$-metilanilina $\left(K_{\mathrm{A}}=6 ; \Delta h_{\mathrm{A}}^{*}=-12.5 \mathrm{~kJ} \cdot \mathrm{mol}^{-1}\right)$ son, en valor absoluto, menores que los de la anilina $\left(K_{\mathrm{A}}=14.8 ; \Delta h_{\mathrm{A}}^{*}=-15 \mathrm{~kJ} \cdot \mathrm{mol}^{-1}\right)$. Esto indica que la autoasociación de la $N$-metilanilina es menor que en la anilina, lo que está de acuerdo con las conclusiones obtenidas en el Capítulo 5. Por otra parte, los valores de $\Delta h_{\mathrm{A}}^{*}$ para estas aminas son consistentes con los valores de $H_{\mathrm{m}, 1}^{\mathrm{E}, \infty}$ (véase la Tabla 5-2). Además, ERAS proporciona buenas estimaciones de las entalpías de vaporización, $\Delta H_{v}$. 
Por ejemplo, para la $N$-metilanilina predice el valor $55.3 \mathrm{~kJ} \cdot \mathrm{mol}^{-1}$, mientras que el valor experimental es $53.1 \mathrm{~kJ} \cdot \mathrm{mol}^{-1}$ [58].

Los elevados valores del parámetro $X_{\mathrm{AB}}$ de las mezclas $N$-metilanilina $+n$-alcano (Tabla 7-8) revelan que las interacciones físicas en estas mezclas son importantes, lo que está en consonancia con el hecho de que la contribución física a $H_{\mathrm{m}}^{\mathrm{E}}$ en estos sistemas sea tan importante (ver la llustración 7-27). Por el contrario, el parámetro $X_{\mathrm{AB}}$ es menor en las mezclas de $N$-metilanilina con hidrocarburos aromáticos (Tabla 7-8), lo que sugiere la existencia de solvatación entre las moléculas diferentes. Por ello, en estos sistemas la contribución química a $H_{\mathrm{m}}^{\mathrm{E}}$ es mayor que la física (llustración 7-27). Un comportamiento similar se encuentra en las mezclas alcohol + anilina, en las que el parámetro $X_{\mathrm{AB}}$ es pequeño (Tabla 7-8), y la contribución química a $H_{\mathrm{m}}^{\mathrm{E}}$ es mayor que la física (Ilustración 7-29). Los valores absolutos de $K_{\mathrm{AB}}$ y $\Delta h_{\mathrm{AB}}^{*}$ de las mezclas alcohol + anilina son mucho mayores que en las mezclas con $N$-metilanilina, lo que sugiere que la solvatación en las mezclas con anilina es mayor (véanse los valores de la Tabla 7-8 para las mezclas metanol + amina).

Es notorio el hecho de que el modelo ERAS no represente con buena exactitud las entalpías de exceso de las mezclas con metanol (véase $\operatorname{des}\left(H_{\mathrm{m}}^{\mathrm{E}}\right)$ de la Tabla 7-9) lo que puede deberse a los pequeños valores de $X_{\mathrm{AB}}$ utilizados en dichas mezclas, lo que hace sobreestimar la contribución química a $H_{\mathrm{m}}^{\mathrm{E}}$ de estas mezclas. Las mismas consideraciones son válidas en las mezclas $N$-metilanilina + benceno o tolveno. Es interesante ver que el modelo de Flory mejora la representación de $H_{\mathrm{m}}^{\mathrm{E}}$ para estas mezclas $\left(\operatorname{des}\left(H_{\mathrm{m}}^{\mathrm{E}}(N\right.\right.$-metilanilina $(1)+$ benceno $\left.(2))\right)=0.044 ; \operatorname{des}\left(H_{\mathrm{m}}^{\mathrm{E}}(N\right.$-metilanilina $(1)+$ benceno (2)) $)=0.068$. Valores obtenidos utilizando $\chi_{\mathrm{AB}}=22.8 \mathrm{MPa}$ y $22.6 \mathrm{MPa}$, respectivamente), lo que significa que la hipótesis de mezcla al azar es válida en gran medida para estas disoluciones, y ello explica las discrepancias observadas entre los valores experimentales y calculados con ERAS de $H_{\mathrm{m}}^{\mathrm{E}}$.

Mencionar, finalmente, que ERAS proporciona resultados aceptables para los volúmenes de exceso, como puede verse en la llustración 7-28.

\subsubsection{Mezclas amina + cetona}

En las mezclas anilina o $N$-metilanilina + cetona, los valores de $\Delta h_{\mathrm{AB}}^{*}$ (Tabla 7-8) son consistentes con los valores de $\Delta H_{\mathrm{N}-\mathrm{co}}$ (véase la Tabla 5-2). Por el contrario, en las mezclas con di- $n$-propilamina o di- $n$-butilamina, existen diferencias considerables entre los valores de $\Delta h_{\mathrm{AB}}^{*}$ y $\Delta H_{\mathrm{N}-\mathrm{CO}}$, lo que se debe a que el modelo ERAS sobreestima la contribución química a $H_{\mathrm{m}}^{\mathrm{E}}$ de estas mezclas. A pesar de ello, la variación relativa de $\left|\Delta h_{\mathrm{AB}}^{*}\right|$ y $\left|\Delta H_{\mathrm{N}-\mathrm{CO}}\right|$ es similar en mezclas que contienen una cetona fija, pues disminuyen siguiendo la secuencia siguiente: anilina $>N$-metilanilina $>$ di- $n$ propilamina $>$ di-n-butilamina (llustración 7-30). Para tales mezclas, $K_{\mathrm{AB}}$ también disminuye en el mismo orden (llustración 7-32). Esto concuerda con el hecho de que $H_{\mathrm{m}}^{\mathrm{E}}$ sea más negativo en las mezclas con aminas aromáticas y significa que las interacciones entre las moléculas distintas son mayores en dichas mezclas, como ya se discutió en el Capítulo 5. 
Los pequeños valores del parámetro $X_{\mathrm{AB}}$ y grandes de $K_{\mathrm{AB}}$ y de $\left|\Delta h_{\mathrm{AB}}^{*}\right|$ en las mezclas anilina $\circ \mathrm{N}$-metilanilina + cetona revelan que la solvatación entre moléculas diferentes es importante, y que existen contribuciones químicas muy importantes a $H_{\mathrm{m}}^{\mathrm{E}}$, siendo mayores cuanto más pequeños sean los valores de $X_{\mathrm{AB}}$. Además, $X_{\mathrm{AB}}$ aumenta con el tamaño de la cetona (Ilustración 7-33). Esta variación puede deberse al gran número de interacciones entre las moléculas de la amina aromática que se rompen en el proceso de mezcla, cuando la superficie alifática aumenta.

Por otra parte, las mezclas con di- $n$-propilamina o di- $n$-butilamina se comportan de manera distinta. Los mayores valores de $X_{\mathrm{AB}}$ y menores de $K_{\mathrm{AB}}$ así como de $\left|\Delta h_{\mathrm{AB}}^{*}\right|$, respecto de las mezclas con mezclas anilina $\circ \mathrm{N}$-metilanilina, revelan la existencia de una contribución física grande y positiva a $H_{\mathrm{m}}^{\mathrm{E}}$ que puede deberse a las rupturas de las interacciones cetona-cetona (véanse las llustraciones 7-23 y 7-25). Además, $X_{\mathrm{AB}}$ disminuye con la longitud de la cetona (Ilustración 7-33), lo que está en consonancia con el hecho de que, como se explica en el Capítulo 5, dichas interacciones son menores cuanto mayor es la longitud de la cetona, debido al apantallamiento estérico del grupo carbonilo en las cetonas más largas.

ERAS también proporciona resultados aceptables para los volúmenes de exceso, como puede verse en las llustraciones 7-20, 7-22, 7-24 y 7-26. Los grandes y negativos valores de $\Delta v_{\mathrm{AB}}^{*}$ constituyen otra prueba más de la existencia de fuertes interacciones entre las moléculas distintas y de efectos estructurales.

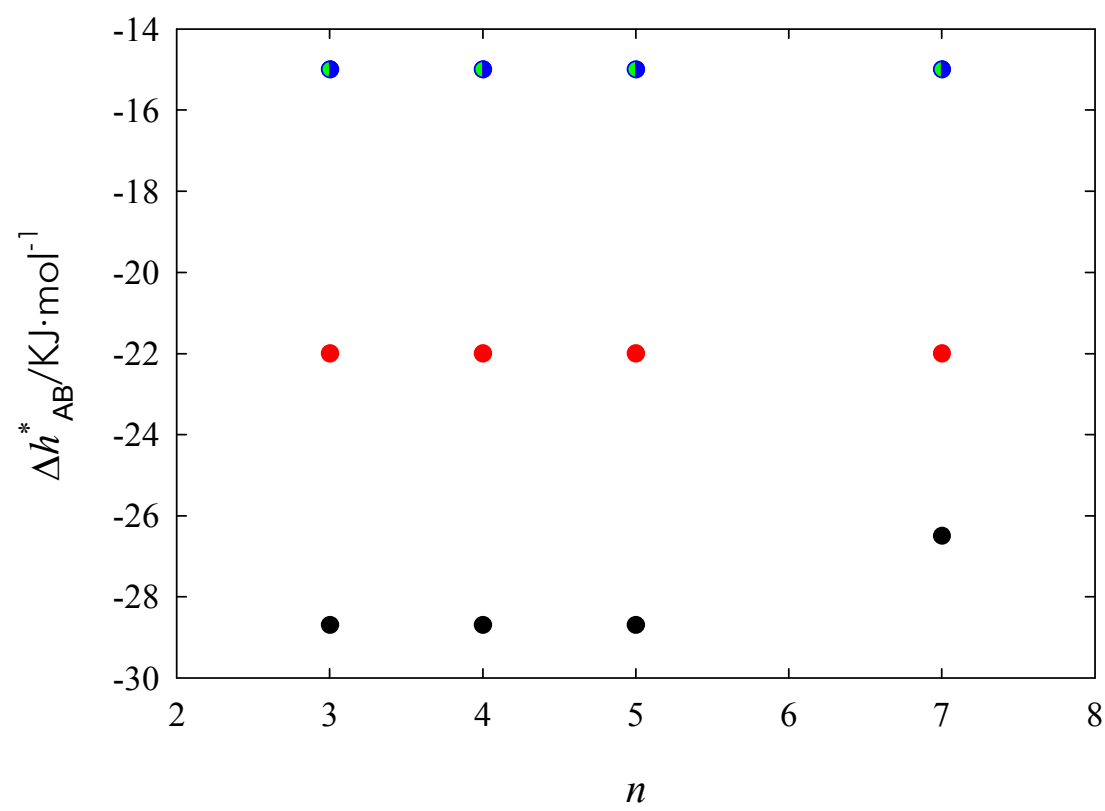

llustración 7-30: $\Delta h_{\mathrm{AB}}^{*}$ en función del número de carbonos de la cetona, $n$, para las mezclas amina (1) + cetona (2): • anilina; $・ N$-metilanilina; $・$ di- $n$-propilamina; $・$ di- $n$-butilamina 


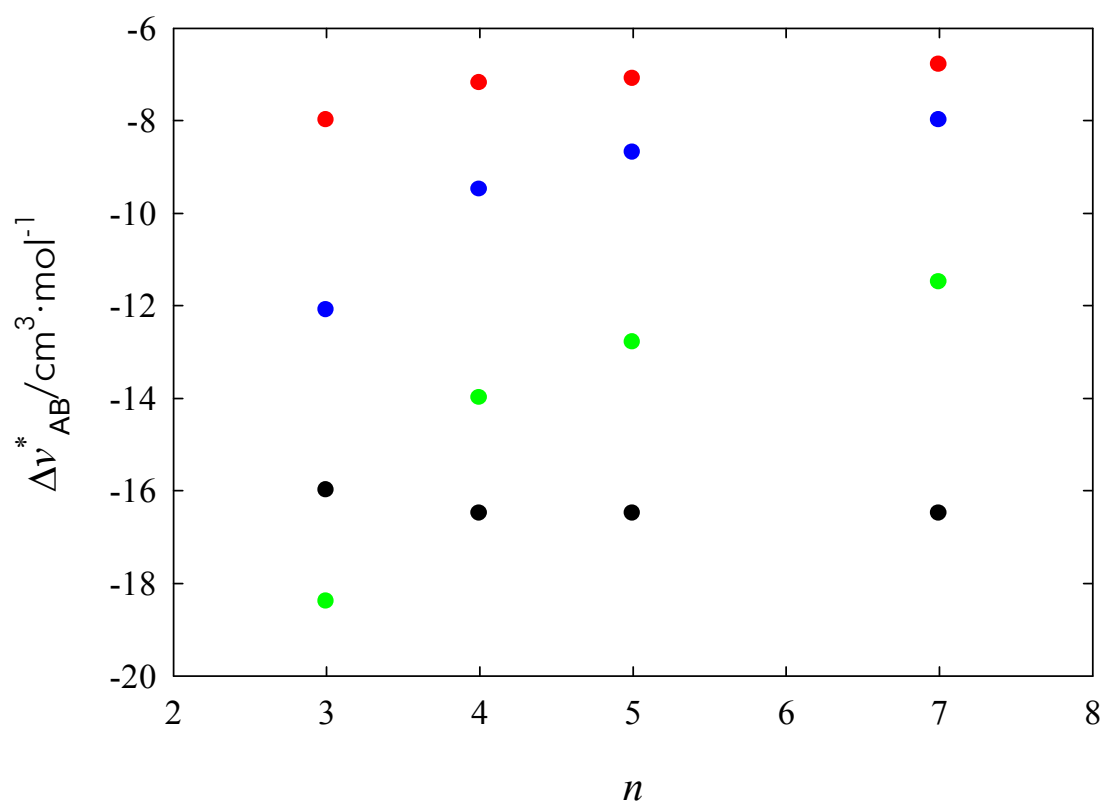

Ilustración 7-31: $\Delta v_{\mathrm{AB}}^{*}$ en función del número de carbonos de la cetona, $n$, para las mezclas amina (1) + cetona (2): • anilina; $・ N$-metilanilina; $・$ di- $n$-propilamina; $・$ di- $n$-butilamina

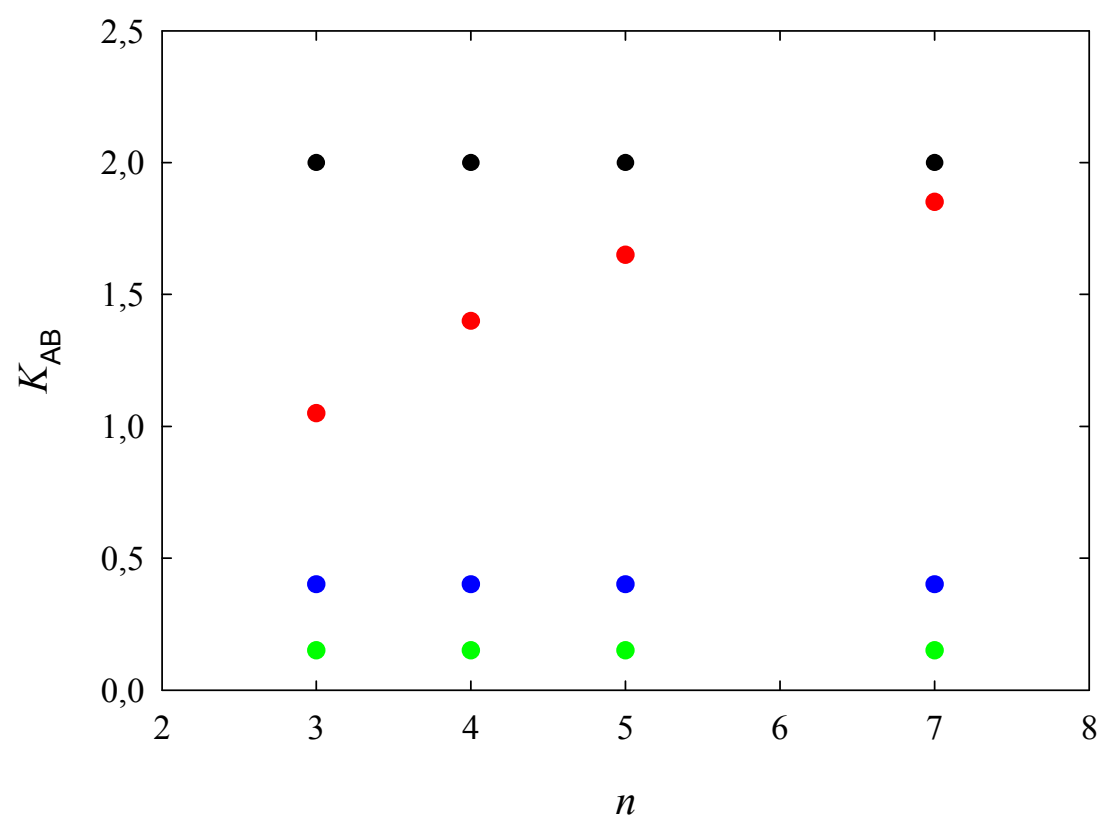

llustración 7-32: $K_{\mathrm{AB}}$ en función del número de carbonos de la cetona, $n$, para las mezclas amina (1) + cetona (2): • anilina; $・ N$-metilanilina; $・$ di- $n$-propilamina; $・$ di- $n$-butilamina 


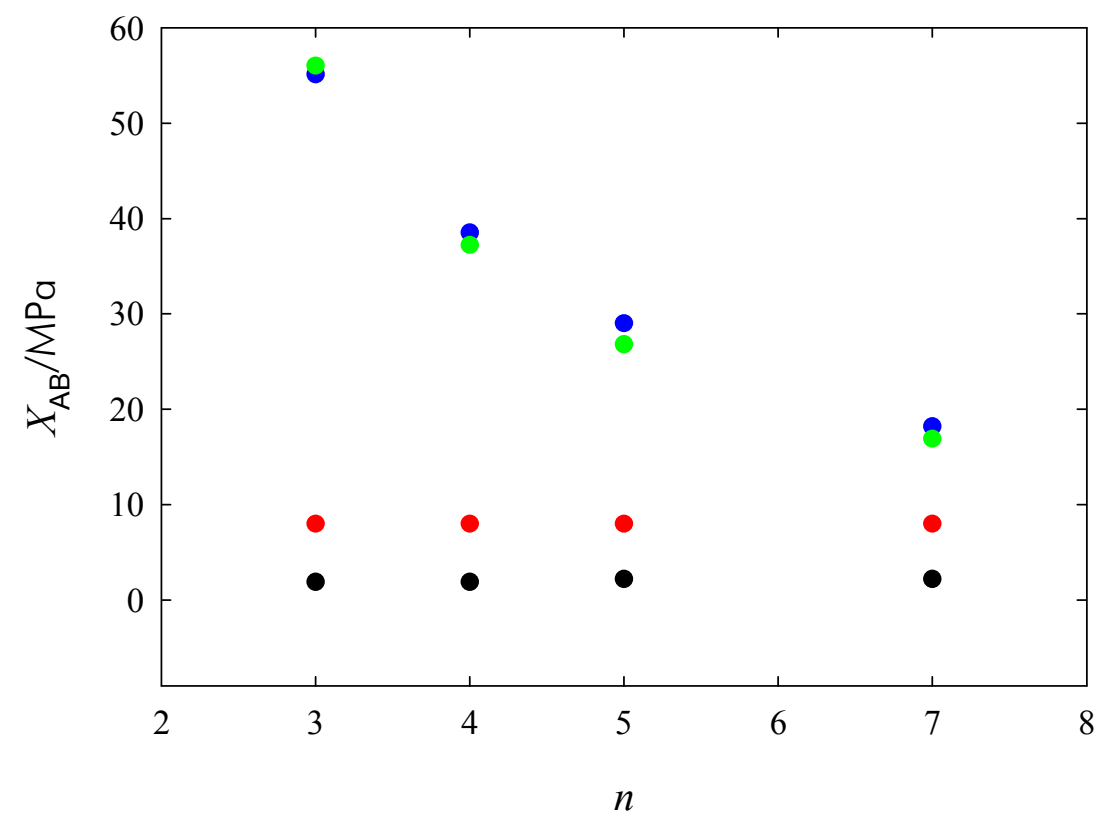

Ilustración 7-33: $X_{\mathrm{AB}}$ en función del número de carbonos de la cetona, $n$, para las mezclas amina (1) + cetona (2): • anilina; $N$-metilanilina; $・$ di- $n$-propilamina; $・$ di- $n$-butilamina 


\section{BIBLIOGRAFÍA}

[1]: Bondi, A.; J. Phys. Chem., 68 (1964), 441.

[2]: Bondi, A.; "Physical Properties of Molecular Crystals, Liquids and Glasses", John Wiley \& Sons, New York (1968).

[3]: Kehiaian, H.V.; Grolier, J.-P.E.; Benson, G.C.; J. Chem. Phys., 75 (1978), 1031.

[4]: González, J.A.; García de la Fuente, I.; Cobos, J.C.; Ber. Bunsenges. Phys. Chem., 100 (1996), 1746.

[5]: González, J.A.; García de la Fuente, I.; Cobos, J.C.; Casanova, C.; Ber. Bunsenges. Phys. Chem., 95 (1991), 1658.

[6]: Delitala, C.; Marongiu, B.; Porcedda, S.; Fluid Phase Equilib., 142 (1998), 1.

[7]: Velasco, I.; Fernández, J.; Otín, S.; Kehiaian, H.V.; Fluid Phase Equilib., 69 (1991), 15.

[8]: Tiné, M.R.; Kehiaian, H.V.; Fluid Phase Equilib., 32 (1987), 211.

[9]: Ferino, I.; Marongiu, B.; Solinas, V.; Torrazza, S.; Fluid Phase Equilib., 9 (1982), 49.

[10]: González López, J.A.; "Estudio termodinámico de las mezclas líquidas de cetonas con alcanos mediante el modelo DISQUAC. Comparación con las predicciones del modelo UNIFAC". Tesis Doctoral. Departamento de Física Aplicada II. Facultad de Ciencias. Universidad de Valladolid, 1987.

[1 1]: Bevington, P.R.; Robinson, D.K.; "Data Reduction and Error Analysis for the Physical Sciences", Thrid edition, Mc Graw Hill, New York (2003).

[12]: Srivastava, R.; Smith, B.D.; J. Chem. Eng. Data, 30 (1985), 308.

[13]: Cannas, A.; Marongiu, B.; Porcedda, S.; Thermochim. Acta, 311 (1998), 1.

[14]: González, J.A.; Alonso, I.; Alonso-Tristán, C.; García de la Fuente, I.; Cobos, J.C.; Fluid Phase Equilib., 33 (2013), 125.

[15]: González, J.A.; Mozo, I.; García de la Fuente, I.; Cobos, J.C.; Can. J. Chem., 83 (2005), 1812.

[16]: González, J.A.; Alonso, I.; Alonso-Tristán, C.; García de la Fuente, I.; Cobos, J.C.; J. Chem. Thermodyn., 56 (2013), 89.

[17]: Maher, P.J.; Smith, B.D.; J. Chem. Eng. Data, 25 (1980), 61. 
[18]: Marongiu, B.; Porcedda, S.; Thermochim. Acta, 158 (1990), 61.

[19]: González, J.A.; García de la Fuente, I.; Cobos, J.C.; Can. J. Chem., 78 (2000), 1272.

[20]: Riesco, N.; González, J.A.; Villa, S.; García de la Fuente, I.; Cobos, J.C.; Phys. Chem. Liq., 41 (2003), 309.

[21]: Kehiaian, H.V.; Porcedda, S.; Marongiu, B.; Lepori, L.; Matteoli, E.; Fluid Phase Equilib., 63 (1991), 231.

[22]: Kietz, E.; Bittrich, H.J.; Hauthal, W.H.; Z. Phys. Chem., 266 (1985), 281.

[23]: Owens, J.L.; Brady, C.J.; Freeman, J.R.; Aiche Symposium Ser. No 256, 83 (1987), 18.

[24]: Kalali Emghani, S.H.; PhD Thesis (1982).

[25]: Kokkonen, P.; J. Chem. Thermodyn., 18 (1986), 761.

[26]: González, J.A.; Cobos, J.C.; García de la Fuente, I.; Fluid Phase Equilib., 224 (2004), 169.

[27]: González, J.A.; García de la Fuente, I.; Cobos, J.C.; Casanova, C.; Fluid Phase Equilib., 93 (1994), 1.

[28]: González, J.A.; Mozo, I.; Fernández, M.; García de la Fuente, I.; Cobos, J.C.; J. Mol. Liq., 133 (2007), 77.

[29]: González, J.A.; García de la Fuente, I.; Cobos, J.C.; Casanova, C.; Ait-Kaci, A.; Fluid Phase Equilib., 112 (1995), 63.

[30]: Ferino, I.; Marongiu, B.; Solinas, V.; Torraza, S.; Int. DATA Ser., Sel. Data Mixtures Ser. A, 1 (1985), 52.

[31]: Rowlinson, J.S.; Swinton, F.L.; "Liquids and Liquid Mistures", $3^{\text {rd }}$ ed., Butterworths Monographs in Chemistry, London (1982).

[32]: González, J.A.; García de la Fuente, I.; Cobos, J.C.; Fluid Phase Equilib., 168 (2000), 31.

[33]: Rowlinson, J.S.; Swinton, F.L.; "Liquids and Liquid Mistures", $3^{\text {rd }}$ ed., Butterworths Monographs in Chemistry, London (1982).

[34]: González, J.A.; García de la Fuente, I.; Cobos, J.C.; Fluid Phase Equilib., 168 (2000), 31.

[35]: Byer, S.M.; Gibbs, R.E.; Van Ness, H.C.; A. I. C. E. J., 19 (1973), 245.

[36]: Sosnkowska-Kehiaian, K.; Kehiaian, H.V.; Bull. Acad. Pol. Sci., Ser. Sci. Chim., 14 (1966), 573.

[37]: Pannetier, G.; Abello, L.; Bull. Soc. Chim. Fr., (1965), 2048.

[38]: Nakanishi, K.; Tohuara, H.; J. Chem. Thermodyn., 18 (1986), 657.

[39]: Pikkarainen, L.; J. Sol. Chem., 17 (1988), 1093.

[40]: Allal, F.; Dahmani, A.; Fluid Phase Equilib., 190 (2001), 33. 
[41]: Allen, G.; Chai, Z.; Chong, C.L.; Higgings, J.S.; Tripathi, J.; Polymer, 25, (1984), 239.

[42]: Heintz, A.; Papaioannou, D.; Thermochim. Acta, 310 (1998), 69.

[43]: Heintz, A.; Naicker, P.K.; Verevkin, S.P.; Pfestorf, R.; Ber. der Bunsenges. Phys. Chem., 102 (1998), 953.

[44]: Villa, S.; González, J.A.; García de la Fuente, I.; Riesco, N.; Cobos, .C.; J. Sol. Chem., 31 (2002), 1019.

[45]: González, J.A.; Mozo, I.; García de la Fuente, I.; Cobos, J.C.; Riesco, N.; J. Chem. Thermodyn., 40 (2008), 1495.

[46]: Wang, L.; Benson, G.C.; Lu, B.C.; J. Chem. Thermodyn., 20 (1988), 975.

[47]: Riddick, J. A.; Bunger, W. B., Sakano, T. K. "Organic Solvents, Physical properties and methods of purification", $4^{a}$ ed. Techniques of Chemistry Volume II, a Wiley Interscience Publication.

[48]: Saleh, M.A.; Alauddini, M.; Begum, S.; Phys. Chem. Liquids, 39 (2001), 453.

[49]: Ratkovics, F.; Laszlo-Parragi, M.; Fluid Phase Equilib., 17 (1984), 97.

[50]: González, J.A.; Domanska, A.; Phys. Chem. Chem. Phys., 3 (2001), 1034.

[51]: González, J.A.; García de la Fuente, I.; Cobos, J.C.; Fluid Phase Equilib., 301 (2011), 145.

[52]: González, J.A.; Mozo, I.; García de la Fuente, I.; Cobos, J.C.; Ind, Eng. Chem. Res., 43 (2004), 7622.

[53]: González, J.A.; García de la Fuente, I.; Cobos, J.C.; Casanova, C.; Ber. Bunsenges. Phys. Chem., 98 (1994), 106.

[54]: González, J.A.; Carmona, J.; García de la Fuente, I.; Cobos, J.C.; Thermochim. Acta, 326 (1999), 53.

[55]: González, JA.; Carmona, J.; Riesco, N.; García de la Fuente, I.; Cobos, J.C.; Can. J. Chem., 79 (2001), 1447.

[56]: Tamura, K.; Nagata, I.; J. Chem. Thermodyn., 23 (1991), 359.

[57]: Bender, M.; Hauser, J.; Heintz, A.; Ber. Bunsenges. Phys. Chem., 95 (7) (1991), 801.

[58]: Liessmann, G.; Schmidt, W.; Reiffarth, S.; Data compilation of the Saechsische Olefinwerke Boehlen (1995). 
CAPITULO 7 
En este capítulo se enumeran brevemente todo el trabajo presentado en esta memoria.

a) Se ha realizado una amplia y profunda revisión de los conceptos fundamentales de la Termodinámica de los sistemas mono y multicomponentes, prestando especial atención tanto a las características matemáticas de las propiedades molares parciales, como a las definiciones de mezcla ideal y de las funciones termodinámicas de mezcla y de exceso. También se han presentado las principales funciones empleadas para el ajuste de los datos experimentales de las funciones de exceso.

b) Tras el calibrado del densímetro y analizador del sonido Anton-Paar DSA 5000 a 298.15 K y presión atmosférica, se procedió a la determinación de los volúmenes molares de exceso, $V_{\mathrm{m}}^{\mathrm{E}}$, del sistema test ciclohexano + benceno. Posteriormente se determinaron experimentalmente los volúmenes de exceso a $293.15 \mathrm{~K}, 298.15 \mathrm{~K}$ y $303.15 \mathrm{~K}$ y presión atmosférica y las compresibilidades isoentrópicas de exceso y los coeficientes de expansión térmica de exceso a presión constante 298.15 K y presión atmosférica de los siguientes sistemas binarios:

- $\quad$ piridina + 2-propanona, 2-butanona, 2-pentanona o 2-heptanona.

- $\quad$ anilina + 2-propanona, 2-butanona, 2-pentanona o 2-heptanona.

- $\quad N$-metilanilina + 2-propanona, 2-butanona, 2-pentanona o 2-heptanona.

- di-n-propilamina + 2-propanona, 2-butanona o 2-heptanona.

- di- $n$-butilamina + 2-propanona, 2-butanona o 2-heptanona.

- $\quad N, N, N$-trietilamina +2 -propanona, 2-butanona o 2-heptanona.

- $\quad N$-metilanilina + heptano, ciclohexano o tolveno. 
c) Se ha realizado una sucinta revisión bibliográfica acerca de las técnicas experimentales para la medida de $V_{\mathrm{m}}^{\mathrm{E}}$, comparando ventajosamente la técnica aquí utilizada frente a otras conocidas.

d) Tras el calibrado del microcalorímetro Tian-Calvet, a 298.15 K y presión atmosférica, se procedió a la determinación de las entalpías molares de exceso, $H_{\mathrm{m}}^{\mathrm{E}}$, del sistema test ciclohexano + benceno. Posteriormente se determinaron experimentalmente las entalpías de exceso a $298.15 \mathrm{~K}$ y presión atmosférica de los siguientes sistemas:

- $\quad$ anilina + 2-propanona, 2-butanona, 2-pentanona o 2-heptanona.

- $\quad N$-metilanilina + 2-propanona, 2-butanona, 2-pentanona o 2-heptanona.

- di-n-propilamina + 2-propanona, 2-butanona, 2-pentanona o 2-heptanona.

- di- $n$-butilamina + 2-propanona, 2-butanona, 2-pentanona o 2-heptanona.

- $\quad N, N, N$-trietilamina + 2-propanona, 2-butanona, 2-pentanona o 2-heptanona.

- $\quad N$-metilanilina + heptano, octano, decano, ciclohexano o tolveno.

- $\quad$ propanol $+N, N, N$-trietilamina +2 -butanona

e) En el caso de las mezclas binarias, todas las funciones termodinámicas de exceso determinadas experimentalmente $\left(V_{\mathrm{m}}^{\mathrm{E}}\right.$ y $\left.H_{\mathrm{m}}^{\mathrm{E}}\right)$ han sido ajustadas a un polinomio tipo Redlich-Kister. En el caso del sistema ternario 1-propanol $+N, N, N$-trietilamina +2 butanona, los datos fueron ajustados siguiendo las indicaciones de Tamura y Nagata (ref. 199 del Capítulo 4). Tanto en sistemas binarios como en el ternario, el número de coeficientes significativos se evaluó mediante un test- $F$ al $95 \%$ de confianza.

f) Para poner de relieve las ventajas e inconvenientes de la técnica utilizada para la determinación de $H_{\mathrm{m}}^{\mathrm{E}}$, se ha realizado una profunda, aunque algo breve, revisión bibliográfica sobre las principales técnicas calorimétricas conocidas.

g) Las medidas de las entalpías y los volúmenes de exceso de las mezclas arriba citadas han sido interpretadas en términos de dos contribuciones: una contribución interaccional, que depende de la intensidad y del número de interacciones entre las moléculas de la mezcla, y una contribución estructural, que es resultado del cambio en la distribución geométrica de estas moléculas, consecuencia de distintos efectos como las diferencias de forma y de tamaño entre las diferentes moléculas.

h) Se ha realizado una amplia y profunda revisión de la mayoría de las teorías de líquidos y de disoluciones de no electrolitos formuladas a día de hoy, poniendo especial énfasis en las limitaciones de la Mecánica Estadística Tradicional al intentar explicar las propiedades de aquellos sistemas cuyos componentes tienen la capacidad de formar complejos (asociación y solvatación).

i) Se han estudiado las mezclas mencionadas en el contexto de los modelos teóricos DISQUAC y ERAS. Se han obtenido los parámetros que caracterizan esas mezclas. Los resultados obtenidos se han interpretado comparándolos con los resultados obtenidos para mezclas de series similares.

i) Se ha hecho una breve descripción de las principales propiedades de interés de aminas y cetonas. 
k) Por último, se ha procedido al análisis del espectro de absorción infrarroja de las mezclas siguientes:

- benzilamina + 2-butanona.

- ciclohexilamina +2-butanona.

- hexilamina +2 -butanona.

- $\quad$ anilina +2 -butanona.

- $\quad$ piridina +2 -butanona.

- $\quad N$-metilanilina +2 -butanona.

- di-n-propilamina +2 -butanona.

- di-n-butilamina +2 -butanona.

- $\quad N, N, N$-trietilamina +2 -butanona.

Lo que ha esclarecido el hecho de que en algunas de dichas mezclas se producen reacciones químicas entre sus componentes. 
CAPITULO 8 


\section{Elección del número de coeficientes del ajuste. Calidad del ajuste a los datos}

\section{A. 1 CONSIDERACIONES GENERALES SOBRE EL AJUSTE A UNA FUNCIÓN}

La elección de un número $n$ apropiado de coeficientes para representar un conjunto de $m$ datos experimentales es, hasta cierto punto, subjetiva por parte del experimentador, aunque es cierto que se puede establecer una relación entre el número de coeficientes y las propiedades de la mezcla [1]. Por ejemplo, en lo que respecta al ajuste de las propiedades de exceso a un polinomio tipo Redlich-Kister (R-K), ecuación (2.208), si todos los coeficientes son nulos, la mezcla será ideal. Si uno de los componentes puros de la mezcla está asociado, dará lugar a una contribución en $\ln f_{1}$ y $\ln f_{2}$ aproximadamente del carácter del término que acompaña al coeficiente $\mathrm{A}_{2}$ en las ecuaciones (2.206) y (2.207). En sistemas en los que las moléculas diferentes se asocian fuertemente (solvatación), este término suele disminuir por lo que tales sistemas pueden representarse sólo con tres coeficientes (dos en la ecuaciones (2.206) y (2.207)).

Sin embargo existe un método de estadística matemática que sugiere la elección de un número $u$ otro de coeficientes. El procedimiento es el siguiente: una vez tomados experimentalmente los datos de la función de exceso en cuestión, $F_{\mathrm{i}}^{\mathrm{E}}$ (observado) $=F_{\mathrm{i}}^{\mathrm{E}}$, $\mathrm{i}=1, \ldots, m$, se buscan los coeficientes de los ajustes con $1,2, \ldots, n$ parámetros, por el método de los mínimos cuadrados. A la hora de aplicar el método, hay que darse cuenta de una cosa: para los datos con los que se ha trabajado, en el caso de los volúmenes de exceso por ejemplo, cada valor es el promedio de 20 datos individuales, luego, cada valor promedio $V_{\mathrm{m}, \mathrm{i}}^{\mathrm{E}}$ (observado) tendrá una desviación estándar muestral o varianza muestral, $s_{\mathrm{i}}^{2}$, que en general no será igual para los $m$ datos que se van a ajustar a la ecuación R-K. Como se ha dicho en el Capítulo 2, hay que verificar la homogeneidad o no de las dispersiones estándar generales, $\sigma_{i}^{2}$, mediante el método de Kokren. Una vez verificada esta homogeneidad, se puede aplicar el método de mínimos cuadrados con las ecuaciones correspondientes, sin tener en cuenta los pesos estadísticos, y con un valor de la estimación de la varianza de la media $S^{2}$ igual a: 


$$
S^{2}=\frac{\sum_{\mathrm{i}=1}^{m} s_{\mathrm{i}}^{2}}{m}
$$

Para los datos de la entalpía de exceso, la comprobación de la homogeneidad de las dispersiones estándar generales no tiene sentido tal y como se han obtenido en el presente trabajo puesto que cada dato se obtiene solamente una vez, por lo que no hay error accidental.

El método de los mínimos cuadrados se basa en hacer mínima la cantidad siguiente:

$$
\chi^{2}=\sum_{\mathrm{i}=1}^{m}\left[\frac{F_{\mathrm{i}}^{\mathrm{E}}(\text { observado })-F_{\mathrm{i}}^{\mathrm{E}}(\text { ajustado })}{\sigma_{\mathrm{i}}}\right]^{2}
$$

donde $\sigma_{\mathrm{i}}$ es la desviación cuadrática media del punto i-ésimo. La cantidad $\chi^{2}$ sigue una distribución chi-cuadrado [2]. En el caso en el que la homogeneidad de las dispersiones se cumpla, $\sigma_{\mathrm{i}}=\sigma_{\mathrm{j}}=\sigma \approx s$, y además cada punto experimental se ajuste a una función $F_{\mathrm{i}}^{\mathrm{E}}($ ajustado, $n)$ de tipo Redlich-Kister,

$$
F_{\mathrm{i}}^{\mathrm{E}}(\text { ajustado, } n)=F^{\mathrm{E}}\left(x_{\mathrm{i}}\right)=x_{1, \mathrm{i}} \cdot\left(1-x_{1, \mathrm{i}}\right) \cdot \sum_{\mathrm{j}=1}^{n} A_{\mathrm{j}} \cdot\left(2 \cdot x_{1, \mathrm{i}}-1\right)^{\mathrm{j}}
$$

Se tiene que:

$$
\chi^{2}(n)=\sum_{\mathrm{i}=1}^{m} \frac{1}{s^{2}} \cdot\left[F_{\mathrm{i}}^{\mathrm{E}}-x_{1, \mathrm{i}} \cdot\left(1-x_{1, \mathrm{i}}\right) \cdot \sum_{\mathrm{j}=1}^{n} A_{\mathrm{j}} \cdot\left(2 \cdot x_{1, \mathrm{i}}-1\right)^{\mathrm{j}}\right]^{2}
$$

Cada función de ajuste tiene una desviación estándar propia, representativa del error que se comete al ajustar los puntos experimentales a dicha función. Si $F^{\mathrm{E}}(x)$ es lineal en los $n$ parámetros y para $m$ puntos de datos, toma el valor:

$$
s^{2}(n)=\frac{1}{m-n} \cdot \frac{\sum_{\mathrm{i}=1}^{m}\left\{\left(1 / \sigma_{\mathrm{i}}^{2}\right) \cdot\left[F_{\mathrm{i}}^{\mathrm{E}}-F^{\mathrm{E}}\left(x_{\mathrm{i}}\right)\right]^{2}\right\}}{(1 / m) \cdot \sum_{\mathrm{i}=1}^{m}\left(1 / \sigma_{\mathrm{i}}^{2}\right)}=\frac{1}{v} \cdot \sum_{\mathrm{i}=1}^{m}\left\{w_{\mathrm{i}} \cdot\left[F_{\mathrm{i}}^{\mathrm{E}}-F^{\mathrm{E}}\left(x_{\mathrm{i}}\right)\right]^{2}\right\}
$$

a la cantidad $m-n=v$ se la llama número de grados de libertad y $w_{\mathrm{i}}$ es el factor de ponderación que viene dado por la ecuación siguiente:

$$
w_{\mathrm{i}}=\frac{\frac{1}{\sigma_{\mathrm{i}}^{2}}}{\frac{1}{m} \cdot \sum_{\mathrm{j}=1}^{m} \frac{1}{\sigma_{\mathrm{j}}^{2}}}
$$

que es el inverso de la varianza $\sigma_{\mathrm{i}}^{2}$ que describe las incertidumbres de cada punto, normalizado al promedio de todos los factores de ponderación. Si $\sigma_{\mathrm{i}}=\sigma_{\mathrm{j}}=\sigma \approx s, w_{\mathrm{i}}=1$, y entonces, la varianza del ajuste es:

$$
s^{2}(n)=\frac{\sum_{\mathrm{i}=1}^{m}\left[F_{\mathrm{i}}^{\mathrm{E}}-F_{\mathrm{i}}^{\mathrm{E}}\left(x_{1, \mathrm{i}}\right)\right]^{2}}{m-n}
$$


Un primer criterio en la determinación del número de coeficientes del ajuste es considerar suficiente que $s(n)$ sea menor que el 1\% del valor máximo (o mínimo, según sea el caso) del conjunto de datos experimentales que se están ajustando.

\section{A.2 TEST $\chi^{2}$}

La calidad del ajuste de un conjunto de datos experimentales a una ecuación dada se puede medir en términos de un test $\chi^{2}$.

Una vez definida la cantidad $\chi^{2}$ para cada ajuste, que sigue una ley de distribución $\chi^{2}$, se puede relacionar la varianza del ajuste $s^{2}(n)$ con $\chi^{2}(n)$ en términos de la nueva variable $\chi_{v}^{2}$ :

$$
\chi_{v}^{2}=\frac{\chi^{2}(n)}{v}=\frac{s^{2}(n)}{\left\langle\sigma_{\mathrm{i}}^{2}\right\rangle}
$$

donde $\left\langle\sigma_{\mathrm{i}}^{2}\right\rangle$ es el promedio ponderado de las varianzas individuales:

$$
\left\langle\sigma_{\mathrm{i}}^{2}\right\rangle=\frac{\frac{1}{m} \cdot \sum_{\mathrm{i}=1}^{m}\left[\left(\frac{1}{\sigma_{\mathrm{i}}^{2}}\right) \cdot \sigma_{\mathrm{i}}^{2}\right]}{\frac{1}{m} \cdot \sum_{\mathrm{i}=1}^{m} \frac{1}{\sigma_{\mathrm{i}}^{2}}}=\left(\frac{1}{m} \cdot \sum_{\mathrm{i}=1}^{m} \frac{1}{\sigma_{\mathrm{i}}^{2}}\right)^{-1}
$$

y es igual a $\sigma^{2}$ si todas las incertidumbres son iguales: $\sigma_{\mathrm{i}}=\sigma_{\mathrm{j}}=\sigma \approx S$.

La varianza estimada del ajuste, $s^{2}(n)$, es característica tanto de la dispersión de los datos respecto su valor medio como de la exactitud del ajuste. La definición de $\chi^{2}$ y su clara relación con $s^{2}(n)$ hace de ella una medida conveniente de la bondad del ajuste.

Si la función de ajuste es una buena aproximación de la función principal, entonces la varianza estimada del ajuste $s^{2}(n)$ debería ser casi igual a la varianza general de los datos, $\sigma^{2}$ y el valor de $\chi^{2}$ v debería aproximarse a la unidad. Además, la probabilidad tomará un valor cercano a 0.5 :

$$
P\left(\chi^{2}<x^{2}\right)=P_{\chi}\left(\chi^{2}, v\right)=\int_{x^{2}}^{\infty} p_{\chi}\left(\chi^{2}, v\right) \cdot \mathrm{d} x^{2} \approx 0.5
$$

$p_{\chi}\left(\chi^{2}, v\right)$ es la función densidad de probabilidad, la cual puede consultarse en cualquier libro de estadística matemática, por ejemplo, en el libro de Bevington [2].

Si la función de ajuste no es apropiada para describir los datos, las desviaciones serían mayores y la varianza estimada será demasiado grande, de manera que $\chi^{2} v>1$ y la probabilidad sería mucho menor de 0.5 . Sin embargo, un valor de $\chi^{2} v<1$ no significa necesariamente que el ajuste sea mejor. Simplemente es consecuencia del hecho de que existe una incertidumbre en la determinación de $s^{2}(n)$ y los valores observados de $\chi^{2}$ fluctuarán de experimento a experimento. Un valor muy pequeño de $\chi^{2}$ v puede indicar un error en la asignación de las incertidumbres de las variables medidas. Como se ve, este test puede dar lugar a cierta controversia. Además, no es un test que compare cómo mejora la calidad del ajuste al introducir un parámetro nuevo, sino que simplemente sirve para afirmar si un ajuste es bueno o no. No obstante, para dar con el 
criterio adecuado, es necesario definir la cantidad $\chi^{2}$, puesto que como se verá a continuación, forma parte de la piedra angular del posterior razonamiento.

\section{A.3 TEST $F$. TEST DEL TÉRMINO ADICIONAL}

Sean dos variables aleatorias $\chi_{1}^{2}$ y $\chi_{2}^{2}$ que siguen la distribución chi-cuadrado, con número de grados de libertad $v_{1}$ y $v_{2}$ respectivamente. Entonces, la variable aleatoria:

$$
f=\frac{\chi_{1}^{2} / v_{1}}{\chi_{2}^{2} / v_{2}}=\frac{\chi_{1, v}^{2}}{\chi_{2, v}^{2}}
$$

sigue una distribución $F$, cuya función densidad de probabilidad depende de $v_{1}$ y $v_{2}$. Hágase notar que el resultado del cociente cambia dependiendo, como es lógico, de cuál es el numerador y cual el numerador. Como norma general, en el numerador irá colocada la cantidad mayor.

Si las dispersiones de ambas variables aleatorias son homogéneas e iguales a $\sigma_{1}$ y $\sigma_{2}$ respectivamente, y $n$ y $l$ son el número de parámetros de cada uno de los ajustes, entonces:

$$
f=\frac{s_{1}^{2}(n) / \sigma_{1}^{2}}{s_{2}^{2}(n) / \sigma_{2}^{2}}
$$

Los valores de la función de distribución acumulativa (probabilidad) $P_{\mathrm{F}}\left(F, v_{1}, v_{2}\right)$ están tabulados. Normalmente, se establece una probabilidad del 95\% (nivel de significación $\beta=0.05$ ), y conocidos $v_{1}$ y $v_{2}$, por las tablas se determina el valor de $F$.

La utilidad directa de la distribución $F$ es que se puede emplear como test para verificar una hipótesis estadística concreta: se admitirá como cierta, con nivel de confianza $\beta$, si una vez hallado el valor experimental de $f$, éste es menor que el valor tabulado $F\left(1-\beta, v_{1}, v_{2}\right)$; pero también puede utilizarse para determinar el número de coeficientes significativos de un ajuste, en su variante del test del término adicional. Este se basa en el hecho de que la distribución chi-cuadrado obedece a la ley interna de aditividad. Por ello, podemos formar una nueva variable aleatoria como la diferencia entre dos variables aleatorias que tienen una distribución chi-cuadrado, y seguirá obedeciendo tal distribución. En nuestro caso, si ajustamos un conjunto de $m$ datos experimentales a una función de ajuste con $n$ parámetros, el valor resultante de la cantidad $\chi^{2}(n)$ asociada a los datos y el ajuste tendrá $m-n$ grados de libertad. Si añadimos un nuevo parámetro a la ecuación de ajuste, el valor correspondiente de la cantidad $\chi^{2}(n+1)$ tendrá $m-n-1$ grados de libertad. La diferencia entre ambas cantidades, por lo dicho en este párrafo, obedece a la ley de distribución $\chi^{2}$, con un solo grado de libertad. Si dividimos esta resta por la cantidad $\chi^{2}{ }_{v}(n+1)$, obtendremos una nueva variable aleatoria $F_{\chi}$ que obedece a la ley de distribución $F$ con $v_{1}=1$ y $v_{2}=m-n-1$ :

$$
F_{\chi}=\frac{\chi^{2}(n)-\chi^{2}(n+1)}{\chi^{2}(n+1) /(m-n-1)}=\frac{\Delta \chi^{2}}{\chi_{v}^{2}}
$$

Recuérdese que $n$ es el número de coeficientes del ajuste y $m$ es el número de datos experimentales, y que: 


$$
\chi_{v}^{2}=\frac{\chi^{2}(n)}{v}=\frac{s^{2}(n)}{\left\langle\sigma_{\mathrm{i}}^{2}\right\rangle}
$$

En el caso que nos ocupa, en el que se ha verificado la hipótesis de homogeneidad de las varianzas los datos, $\sigma_{\mathrm{i}}=\sigma$, y $m-n=v$, el cociente anterior es igual a:

$$
\frac{\chi^{2}(n)}{m-n}=\frac{s^{2}(n)}{\sigma^{2}}
$$

luego:

$$
F_{\chi}=\frac{s^{2}(n) \cdot(m-n)-s^{2}(n+1) \cdot(m-n-1)}{s^{2}(n+1)}
$$

Si $F_{\chi}>F(\beta, 1, m-n-1)$, podemos tener la certeza de que el nuevo coeficiente no debe eliminarse, es decir, el término añadido es estadísticamente significativo. En caso contrario, el nuevo coeficiente debe considerarse nulo y podemos cortar el desarrollo en serie de potencias de $x_{1}$ en el término $n$-ésimo con una certeza del $100 \cdot(1-\beta) \%$. 


\section{Propiedades físico-químicas y características de interés biológico, fisiológico e industrial de las moléculas estudiadas}

Como se expone en el Capítulo 1, la elección de aminas y cetonas como sustancias a estudiar no ha sido al azar, sino que está en consonancia con el progreso del subproyecto NCO del proyecto T.O.M. Además de ser importantes para el avance de dicho proyecto, aminas y cetonas gozan de gran interés biológico, biotecnológico, industrial, fisiológico, etc., lo que justifica las primeras secciones de este anexo. Además, se da una breve exposición de las principales propiedades fisicoquímicas de ambos tipos de compuestos, que sirven para la correcta interpretación teórica de los datos termodinámicos obtenidos, así como de sus propiedades espectroscópicas fundamentales, lo que ha servido para interpretar los espectros de absorción infrarroja que se presentan en el Anexo C.

\section{B.1 FORMACIÓN BIOLÓGICA Y CICLO DEL NITRÓGENO}

El nitrógeno es el componente mayoritario de la atmósfera $(\approx 78 \%)$, y es tan esencial para muchos procesos biológicos, que sin él no existiría la vida en la Tierra. De hecho, es el componente básico de todos los aminoácidos, proteínas y ácidos nucleicos como el ADN y el ARN, y es fundamental para realizar el proceso de fotosíntesis en las células clorofiladas de las plantas.

A pesar de todo, el nitrógeno molecular $\mathrm{N}_{2}$, que es como se encuentra en la atmósfera, es inerte y la mayoría de los seres vivos no pueden aprovecharlo directamente. Solamente unos pocos organismos vivos unicelulares son capaces de hacerlo. La mayoría de las plantas no pueden aprovechar el nitrógeno gaseoso de manera directa, y necesitan amoniaco o algunos nitratos como fuente de nitrógeno. Los animales, más complejos en cuanto a estructura celular, funcionalidad y especialización, necesitan compuestos nitrogenados orgánicos, los cuales suelen contener al grupo amino. Por lo tanto, se necesitan ciertos procesos químicos, de origen biológico o no (por ejemplo, fijación, mineralización, nitrificación o desnitrificación) para convertir el nitrógeno gaseoso en su forma más adecuada, llamado nitrógeno fijo o reactivo, que sea aprovechable por los organismos vivos. Nótese que aunque lo llamemos nitrógeno 
reactivo, en realidad estaremos hablando, como se ha dicho, de amoniaco, nitratos, aminas, aminoácidos,... Al conjunto total de estos procesos se le llama ciclo del nitrógeno, y básicamente se basa en el hecho de que plantas, animales y ciertos microorganismos disfrutan entre sí de relaciones simbióticas de manera que se sostiene la balanza entre el nitrógeno atmosférico y el fijado. Entre estos microorganismos nos encontramos con: bacterias fijadoras de nitrógeno, de vida libre o en simbiosis con algunas especies vegetales (por ejemplo, la bacteria Rhizobium, en simbiosis con legumbres como la alubia o el guisante), que gracias a la enzima nitrogenasa son capaces de combinar nitrógeno molecular con oxígeno o hidrógeno, para producir óxidos de nitrógeno o amoniaco; bacterias nitrificantes, que pueden convertir el amoniaco $u$ otros compuestos nitrogenados procedentes de plantas $O$ animales en nitratos, los cuales pueden ser asimilados nuevamente por las plantas y éstas sintetizar nuevos compuestos nitrogenados. Entre estos últimos, se incluyen algunos de los aminoácidos los cuales se encuentran necesariamente en la dieta de los animales herbívoros mientras que los carnívoros obtienen los mismos aminoácidos a través del consumo de herbívoros. Cuando animales y/o plantas mueren, los compuestos nitrogenados procedentes de ellos se vuelven a convertir en nitratos, mediante la función de las bacterias nitrificantes, y así el ciclo de conservación del nitrógeno reactivo se completa. Alternativamente, el nitrógeno de los nitratos puede volver a la atmósfera mediante la actuación de las llamadas bacterias desnitrificantes.

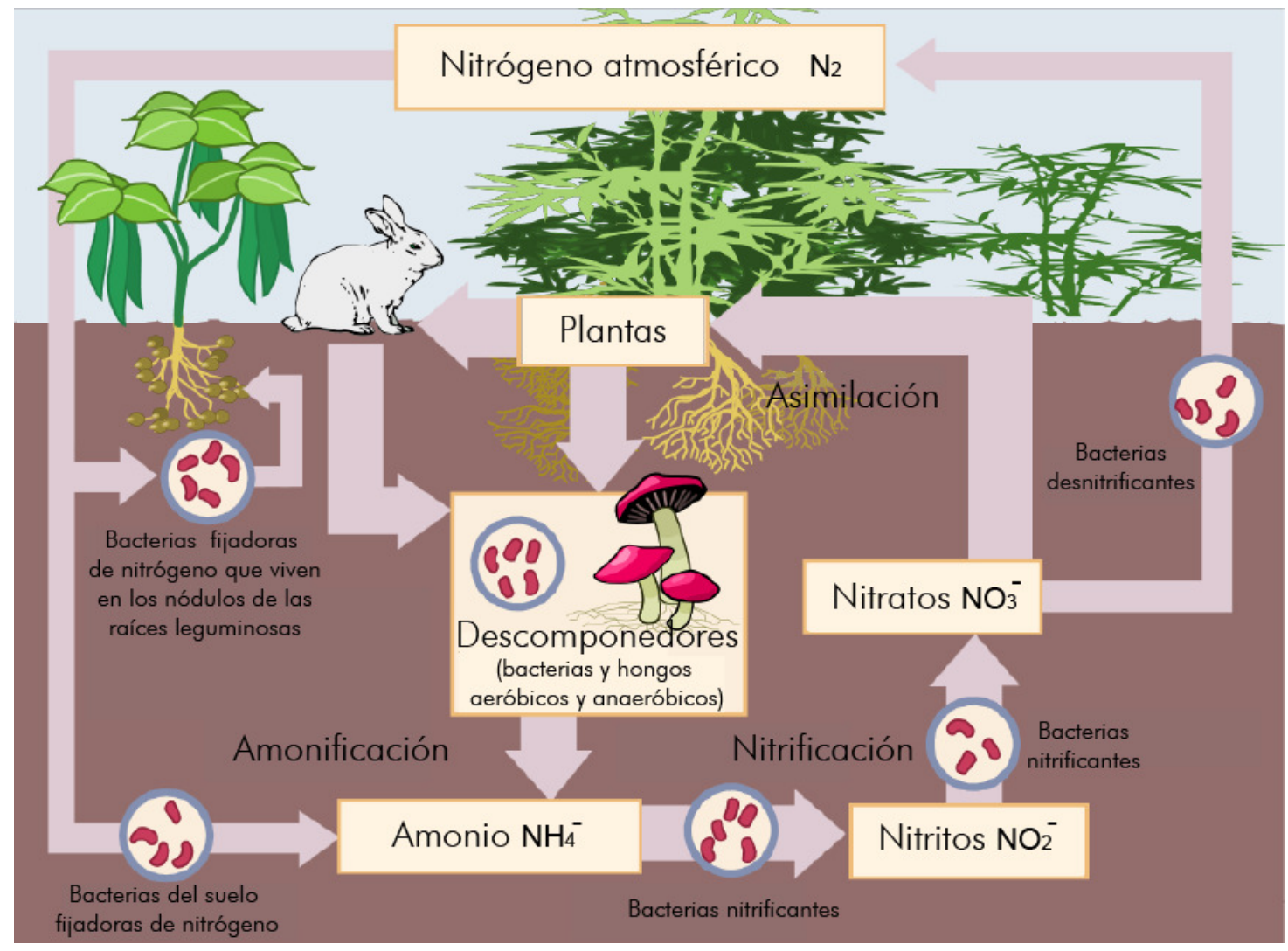

Ilustración B-1: ciclo del nitrógeno

No todos los animales obtienen los aminoácidos esenciales a partir de la ingesta de plantas. Algunos, como los rumiantes, pueden asimilar directamente los aminoácidos producidos por la bacteria simbiótica que se encuentra en su intestino. Esta bacteria rompe la celulosa, estructura principal de los carbohidratos de las plantas, y sintetiza todos los aminoácidos requeridos para el crecimiento y desarrollo del animal, a partir 
de una fuente fija de nitrógeno simple. Por ello, las cabras, por ejemplo, pueden sobrevivir a partir de una dieta de amoniaco y heno.

La secuencia de reacciones por las que el nitrógeno libre o combinado se convierte en amino-compuestos aún se desconoce, pero lo que sí está claro es que los pasos en cada secuencia están todos catalizados enzimáticamente. La habilidad de las diferentes formas de vida para utilizar nitrógeno libre o combinado está gobernada por la presencia de enzimas particulares. Puesto que todas las enzimas son proteínas, y por lo tanto, derivados de los amino-compuestos más importantes en la naturaleza, los $\alpha$-L-aminoácidos, cualquier argumento relacionado con la formación puramente biológica de amino-compuestos es un razonamiento circular. Esto implica que en alguna edad temprana, antes de la aparición de la vida en la Tierra, los aminoácidos debieron formarse directamente a partir de los elementos de la atmósfera, mediante procesos no biológicos o abióticos. De hecho se ha demostrado que el paso de una descarga eléctrica a través de mezclas de metano, amoniaco y vapor de agua (posibles constituyentes de la atmósfera terrestre primigenia) produce algunas formas de $\alpha$-aminoácidos, aunque éstos siempre ocurren en mezclas racémicas de las formas $L y$ D. En sistemas biológicos, las proteínas funcionales y estructurales están constituidas casi exclusivamente de L-aminoácidos. Este razonamiento induce a pensar que los aminoácidos están entre los amino-compuestos formados más pronto en la historia de la evolución de las especies, puesto que, como se ha dicho, son los precursores de los catalizadores naturales de los procesos metabólicos.

Además, parecen ser los primeros productos sintetizados por plantas y microorganismos tras la ingestión de compuestos nitrogenados.

Estas especulaciones sobre el origen de los $\alpha$-L-aminoácidos nos muestran la importancia de éstos, y por ende, de los amino-compuestos, en los sistemas biológicos.

\section{B.2 EL GRUPO CARBONILO EN BIOLOGÍA Y FISIOLOGÍA}

Los compuestos con el grupo funcional carbonilo se encuentran entre las sustancias orgánicas de interés bioquímico. Los compuestos carbonílicos aparecen en las reacciones metabólicas de todas las formas de vida animal, vegetal y microbiana, como productos finales o intermediarios de las reacciones, estando todas estas catalizadas enzimáticamente.

De entre estas reacciones metabólicas, son señalables las que tienen lugar en el cuerpo humano participando los hidratos de carbono, ácidos grasos, aminoácidos, alcoholes,... procedentes de los alimentos y de las bebidas alcohólicas ingeridos. Los hidratos de carbono, formados por los grupos carbonilo e hidroxilo, desempeñan funciones importantes en el organismo: la glucosa y el almidón constituyen sus principales fuentes de energía; la fructosa, que es un azúcar encontrado en los vegetales, las frutas y la miel y que combinada con la glucosa forma la sacarosa o azúcar común, también actúa como fuente de energía; la celulosa, que a pesar de no ser digerible a no ser que se disponga de la enzima celulasa necesaria para romper los enlaces $\beta$-1-4-glucosídicos, como ocurre en las termitas y los rumiantes, es necesario incluirla en la dieta humana puesto que al mezclarse con las heces facilita la digestión y la defecación; etc. El metabolismo de todos estos compuestos tiene como objetivo 
principal la producción de energía. En estos procesos se forman nuevas especies químicas, algunas de las cuales puede ser nocivas para la salud, sobre todo si se generan en grandes proporciones. Este es el caso de la oxidación del etanol en acetaldehído o etanal. El consumo de grandes cantidades de etanol provoca un serio aumento de acetaldehído en la sangre, lo que puede producir nauseas, disminución de la tensión arterial o sudoraciones. Otro ejemplo es la descomposición de ácidos grasos, por la eliminación del grupo amino de los aminoácidos por cetogénesis en las mitocondrias del hígado y del riñón, en los llamados cuerpos cetónicos (ácido acetoacético, y ácido $\beta$-hidroxibutírico). Debido a que la acetilcoenzima (acetil-CoA), formada en la oxidación de los ácidos grasos, sólo entra en el ciclo de Krebs (ciclo del ácido cítrico) si la degradación de las grasas y los carbohidratos está adecuadamente equilibrada, en caso contrario el exceso de acetil-CoA se desvía para formar los cuerpos cetónicos. Esto suele ocurrir cuando hay presentes pocos carbohidratos en la sangre. En estas condiciones, el cuerpo comienza a utilizar como fuente de energía los ácidos grasos procedentes de las reservas de grasa en lugar de la glucosa, dando como resultado los cuerpos cetónicos. Debido a que muchas veces los ácidos grasos de cadena larga no son aprovechables por un gran número de tejidos, los cuerpos cetónicos pueden actuar como fuentes energéticas importantes, especialmente durante el ayuno y la inanición (condiciones ambas propicias para la falta de glucosa y otros hidratos de carbono). En particular, en estas condiciones, el cerebro depende en gran medida de los cuerpos cetónicos para la síntesis de lípidos y obtención de energía.

Una parte del ácido acetoacético sufre descarboxilación no enzimática originado la aparición de acetona (una cantidad insignificante en condiciones normales), que es expulsada del cuerpo al orinar y respirar. La exhalación de acetona es la responsable de un olor afrutado característico en el aliento.

Los cuerpos cetónicos siempre están presentes en la sangre, aumentando su concentración durante el ejercicio intenso y prolongado. También están presentes en abundancia en las mujeres embarazadas y los recién nacidos. No obstante, en condiciones normales de funcionamiento del organismo, en la sangre hay homeóstasis ácido-base entre los cuerpos cetónicos, de carácter ácido, y sus bases conjugadas, la cual se lleva a cabo mediante los mecanismos de la respiración, absorción de iones de hidrógeno en los tejidos de las proteínas y los huesos, procesos renales, etc. Cuando el organismo es incapaz de mantener eficazmente la homeóstasis ácido-base, éste entra en un estado metabólico llamado cetoacidosis, asociado con la elevada concentración de los cuerpos cetónicos. La cetoacidosis puede producir deshidratación o estrés. En casos severos, la acumulación de los ácidos acetoacético, y $\beta$-hidroxibutírico provoca la disminución del $\mathrm{pH}$ de la sangre $(<7.3)$, lo que puede ser fatal.

La diabetes es la causa patológica más común de cetoacidosis: grandes cantidades de cuerpos cetónicos se producen en respuesta al bajo nivel de insulina y altos niveles de hormonas contrareguladoras. También puede producirse en casos agudos de intoxicación alcohólica, sobre todo tras una intoxicación de metanol o etilenglicol, más que tras la ingesta de etanol. La falta grave de hidratos de carbono por inanición severa es otra causante de la cetoacidosis.

Los cuerpos cetónicos son relevantes para las enfermedades neurológicas como el Alzheimer y la enfermedad de Parkinson [3]. La investigación también ha demostrado que los cuerpos cetónicos desempeñan un papel fundamental en la reducción de las 
crisis epilépticas con la llamada dieta cetogénica alta en grasas y con casi cero carbohidratos [4], lo cual causa cierta controversia puesto que la principal desventaja de las dietas basadas en la exclusión de hidratos de carbono, como, las Atkins o Dunkan, es precisamente la producción de grandes cantidades de cuerpos cetónicos, lo que provoca a largo plazo la cetoacidosis.

Otros compuestos carbonílicos que desempeñan importantes roles biológicos son los esteroides, muchos de las cuales como la testosterona, la progesterona y la cortisona son cetonas. En los mamíferos, cumplen importantes funciones:

a) Reguladoras de los niveles de sal y la secrección de bilis.

b) Estructurales: el colesterol es un esteroide que forma parte de la estructura de las membranas de las células junto con los fosfolípidos, y además, a partir del colesterol se sintetizan el resto de esteroides.

c) Hormonales: hormonas sexuales masculinas y femeninas, corticoides, etc.

d) Los esteroides anabólicos pueden provocar efectos adversos profundos sobre el hígado.

Los aldehídos son compuestos carbonílicos que también son importantes. Por ejemplo, el retinal, es un aldehído que en combinación con la proteína opsina en la retina del ojo forma la rodopsina, y es el principal compuesto involucrado en el proceso de la visión; los aldehídos alifáticos de cadena de 7 a 18 carbonos están implicados en el fenómeno de la bioluminiscencia de algunas bacterias [5]. En este mecanismo, se produce luz cuando el flavin-mononucleótido (FMN) y un aldehído se incuban en presencia de oxígeno molecular, gracias a la enzima luciferasa. El aldehído se combina aparentemente con el oxígeno formando un peróxido intermediario, que al descomponerse en el ácido correspondiente libera suficiente cantidad de energía como para excitar algún producto de la reacción de manera que al volver a su estado fundamental, se emite luz.

La reducción de los ácidos carboxílicos, ésteres y anhídridos que aparece en la síntesis de cetonas y aldehídos es muy importante ya que es un mecanismo que aparece en el metabolismo de las plantas.

Con lo expuesto en estas dos primeras secciones, queda más que resuelta la pregunta, si ésta existía, de por qué hacer un estudio de compuestos con los grupos amino y carbonilo, al margen del proyecto T.O.M. No obstante, por si quedase alguna duda al respecto, en la sección siguiente se exponen algunas aplicaciones industriales y efectos biológicos en los que se ven involucrados este tipo de sustancias.

\section{B.3 AMINAS Y CETONAS DE USO COTIDIANO}

Las aminas pueden encontrarse en multitud de situaciones cotidianas. De hecho, en la atmósfera, se han llegado a detectar entre 150 aminas y 30 aminoácidos [6]. Puede encontrarse histamina y tiramina en alimentos que se están pudriendo o fermentando. La etilamina, la dimetilamina y la isopropilamina se utilizan ampliamente en la producción de herbicidas (sales de mono isopropilamina de la molécula de glifosato) de nueva generación, caracterizados por su baja toxicidad ya que no contaminan el suelo ni el agua. La dimetilamina se utiliza también en la industria farmacéutica para la 
obtención de anestésicos, y en la industria textil. Se utilizan, así mismo, como surfactantes, lubricantes, inhibidores de la corrosión, aditivos en la fabricación de asfaltos y aditivos de las gasolinas. Las aminas aromáticas, como la anilina y sus derivados, tienen aplicaciones semejantes. La anilina se utiliza ampliamente como intermediario en la síntesis de colorantes, en la manufactura de caucho, antioxidantes, productos farmacéuticos, tintas de marcar, agentes blanqueantes en óptica, reveladores fotográficos, resinas, barnices, perfumes... También se utiliza en la fabricación de electrodos en celdas de combustible debido a la nube de electrones deslocalizados del anillo bencénico, que junto con los que rodean al $\mathrm{N}$ hacen de este compuesto un buen conductor. Por la misma razón, otras aminas aromáticas juegan un papel importante en el desarrollo de polímeros conductores, como el polipirrol. El 1-H-imidazol es una amina que contiene los grupos $-\mathrm{NH}$ y $>\mathrm{N}-$ en un anillo aromático de 5 eslabones. Resulta ser de gran importancia porque es la molécula a partir de la cual se pueden obtener líquidos iónicos, excelentes disolventes para un gran número compuestos orgánicos. Su baja presión de vapor hace que sean de gran aplicación ya que pueden sustituir a otros compuestos orgánicos mucho más volátiles, con las consiguientes ventajas medioambientales. La piridina, se encuentra en un sinfín de compuestos relacionados con la vida (pero casi nunca en su forma pura), como en la enzima NAD, responsable del intercambio de electrones e hidrogeniones en la producción de la energía de todas las células, la nicotina, la vitamina B3, la vitamina B6, etc. Se encuentra en disolventes, reactivos para la síntesis de fármacos, insecticidas, herbicidas, sazonadores, colorantes, adhesivos, pinturas, explosivos, desinfectantes, y químicos para el caucho. Se pueden encontrar trazas de piridina como componentes volátiles de compuestos orgánicos que se generan en los procesos de tostación y enlatado, como es el caso del pollo frito, sukiyaki, panceta frita, queso Beaufort, aroma del café, té negro y miel de girasol. El humo del tabaco y de la marihuana contienen piridina.

En la industria farmacéutica el grupo amino en un anillo bencénico aparece con frecuencia, dado que las aminas aparecen en muchos de los compuestos biológicos más importantes que se conocen. Ellas funcionan en los organismos vivos como biorreguladores, neurotransmisores, en mecanismos de defensa y en muchas otras funciones más. Así, la estructura tipo quinolina es de importancia entre los compuestos heterocíclicos que presentan propiedades antimicrobiales. Derivados de la quinolina, como la quinina, cloroquina o mefloquina se utilizan en el tratamiento de la malaria. Muchos alcaloides ${ }^{*}$, como la morfina o la nicotina, también contienen el grupo amino en anillos aromáticos. Y otras, como la adrenalina, o la mescalina son moléculas biológicamente importantes.

No obstante, no todos los efectos de las aminas son beneficiosos, y de hecho hay una gran variedad de estudios que demuestran que determinadas aminas aromáticas, y heterocíclicas, son las principales causantes de la aparición de cáncer de vesícula y de pulmón, por ejemplo, el 4-aminodifenilo (presente en el humo del tabaco) y la bencidina. Los animales carnívoros están sujetos a la aparición de cáncer de mama y de colon, debido a la aparición de aminas heterocíclicas en la superficie de la carne y el pescado, una vez cocinados. Estas aminas también son causantes de cáncer de páncreas y de próstata. La aparición de aminas heterocíclicas durante la cocción puede

\footnotetext{
- Se llaman alcaloides a aquellos metabolitos secundarios de las plantas sintetizados, generalmente, a partir de aminoácidos. Los metabolitos secundarios cumplen funciones no esenciales en ellas, de forma que su ausencia no es fatal, ya que no intervienen en el metabolismo primario de las plantas.
} 
reducirse con la utilización de antioxidantes, de prolina, o eliminando creatina mediante una breve irradiación de microondas, antes de freír o hervir el alimento en cuestión. Por otra parte, el efecto de las aminas heterocíclicas disminuirse mediante el consumo de alimentos que contengan antioxidantes como los vegetales, la soja o el té [7].

La absorción de anilina, ya sea por inhalación del vapor o mediante el contacto del líquido con la piel, produce anoxia debido a la formación de metahemoglobina. La exposición prolongada puede producir la cianosis, asociada a la cefalea, somnolencia, disnea, debilidad y pérdida de conocimiento. Si no se actúa inmediatamente, puede producir la muerte.

La piridina pura es nociva si se inhala, ingiere, o si se absorbe por vía cutánea. Reduce la fertilidad masculina y femenina y es carcinógeno. Los síntomas de exposición más comunes son: cefalea, tos, dificultad respiratoria, laringitis, náuseas y vómitos.

La $N$-metilanilina es nociva por inhalación y contacto con la piel. Produce anoxia. Los síntomas de exposición son irritación de nariz y garganta, tos, dificultad respiratoria, cefalea, fatiga, y color azulado de la piel y los labios. A niveles altos de exposición puede producir colapso nervioso y muerte. La exposición repetida puede producir daños en el hígado, los riñones y anemia.<smiles>CNC</smiles><smiles>CC(C)N</smiles><smiles>CN(C)C</smiles><smiles>Nc1ccccc1</smiles><smiles>c1ccncc1</smiles>

$N, N$-dimetilamina isopropilamina $N, N, N$-trimetilamina

anilina

piridina<smiles>CCCCNCCCC</smiles>

di-n-butulamina<smiles>CCCNCCC</smiles>

di-n-propilamina<smiles>c1cc[nH]c1</smiles>

pirrol<smiles>c1c[nH]cn1</smiles>

1-H-imidazol<smiles>c1ccc2ncccc2c1</smiles>

quinolina<smiles>CNc1ccccc1</smiles>

$N$-metilanilina<smiles>CN1CCCC1c1cccnc1</smiles>

nicotina<smiles>CNCC(O)c1ccc(O)c(O)c1</smiles>

adrenalina 
<smiles>COc1cc(CCN)cc(OC)c1OC</smiles>

mescalina

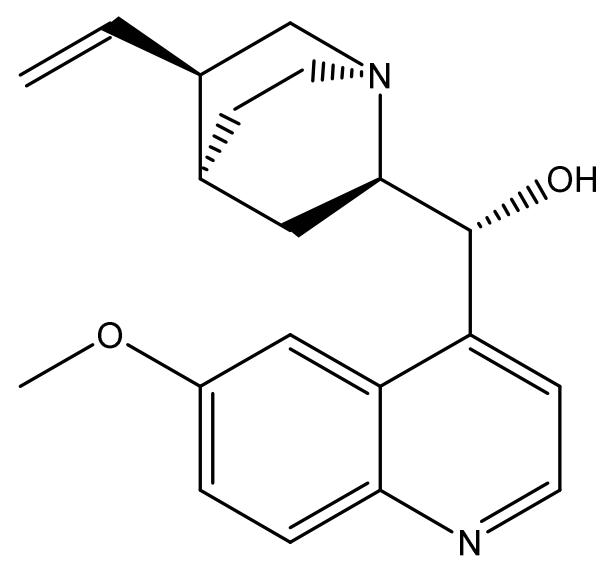

quinina

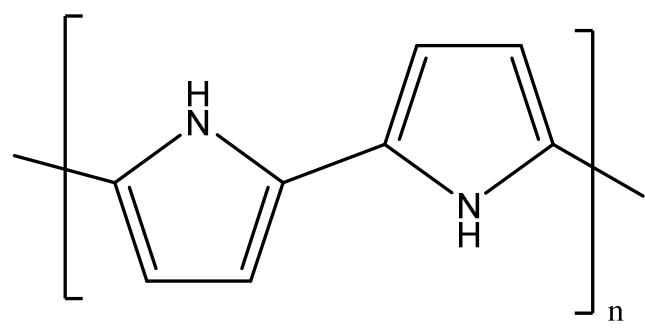

polipirrol

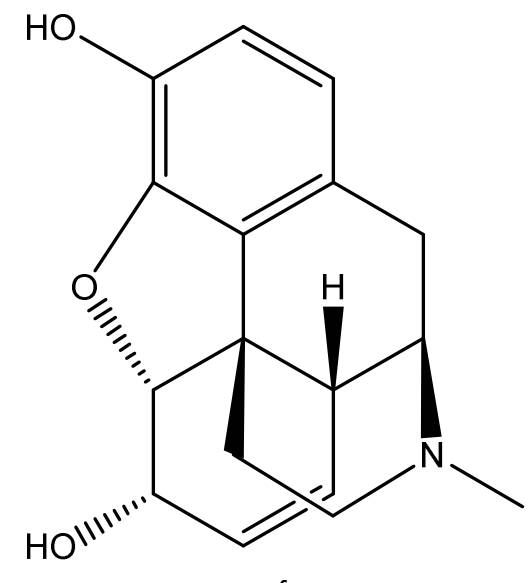

morfina

Ilustración B-2: algunas aminas de interés biológico, científico y tecnológico

Respecto a las cetonas, se puede decir que son producidas a gran escala en la industria como disolventes, precursores de polímeros, perfumes, sazonadores y productos farmacéuticos. La combustión de hidrocarburos es un proceso de oxidación incontrolada que dan cetonas, entre otros compuestos. En términos de producción mundial, las cetonas más importantes son la 2-propanona (acetona), la 2-butanona y la ciclohexanona. La acetona es uno de los pocos compuestos orgánicos que es soluble infinitamente en agua y que además disuelve la mayoría de compuestos orgánicos. Por esta razón y por su baja temperatura de ebullición $\left(56^{\circ} \mathrm{C}\right)$, que permite poder eliminar sus restos por evaporación, es uno de los disolventes más ampliamente utilizados en la industria, y forma parte de las pinturas, barnices, resinas, pulimentos y recubrimientos. La 2-butanona también se utiliza como disolvente. La dihidroxiacetona es un ingrediente activo de los bronceadores que reacciona con las células muertas de la capa más externa de la piel proporcionando una coloración más oscura. La 2-heptanona es la responsable del olor de muchas frutas y del queso azul y también puede encontrarse en el aceite de clavo. La acetofenona se utiliza en perfumería, como disolvente orgánico, así como en la síntesis de productos farmaceuticos. La acroleína, producida por la ruptura de los triglicéridos de la carne cuando se cocina, es la responsable del olor de las barbacoas, y del sabor del caramelo. El olor de las palomitas se debe a la metil 2piridilcetona...

En cuanto a los aldehídos, el más utilizado comúnmente es el formaldehído (metanal). El formaldehído, un gas en condiciones normales, disuelto en agua puede utilizarse para broncear, embalsamar cadáveres, preservar especimenes biológicos, esterilizar instrumentos, como desinfectante, fungicida e insecticida para las plantas y vegetales, 
en la composición de algunos cosméticos y productos de higiene personal como champúes, cremas para baño, etc., aunque su mayor aplicación está en la síntesis de ciertos materiales poliméricos, que se utilizan no sólo como plásticos sino también como adhesivos y recubrimientos. Por ejemplo, la baquelita es un producto de la reacción entre el formaldehído y el fenol. Otros polímeros similares se producen a partir de reacciones entre el metanal y la urea o la melanina, como la Formica y el Melmac. Recientemente los informes del IARC (el acrónimo en inglés de International Agency for Research on Cancer) lo han clasificado como carcinógeno para el ser humano. Otros aldehídos aparecen como condimentadores del sabor de manera natural, como el benzaldehído, responsable del sabor de las almendras frescas, cerezas albaricoques y melocotones, y el cinamaldehído, que aparece en el aceite de la canela...<smiles>C=CC(=O)OCC(C)=O</smiles><smiles>CCCC(C)=O</smiles>

2-pentanona<smiles>CCCCCC(C)=O</smiles>

2-heptanona<smiles>O=C1CCCCC1</smiles>

ciclohexanona<smiles>O=Cc1ccccc1</smiles>

benzaldehído<smiles>CC(=O)c1ccccc1</smiles>

acetofenona<smiles>CC(=O)c1ccccn1</smiles>

metil 2-piridil cetona<smiles>C=CC=O</smiles>

acroleína<smiles>C[C@]12CC[C@]3(C)[C@@]4(C)CCC(=O)C=C4CC[C@]3(C)[C@@H]1CC[C@@H]2O</smiles><smiles>CC(=O)[C@H]1CC[C@]2(C)[C@]1(C)CC[C@]1(C)[C@@]3(C)CCC(=O)C=C3CC[C@]12O</smiles> 
<smiles>CC(=O)CC(=O)O</smiles>

ácido acetoacètico<smiles>CC(O)CC(=O)O</smiles>

ácido beta-Hidroxibutírico<smiles>CC(=O)C(O)O</smiles>

dihidroxiacetona<smiles>O=C[C@H](O)[C@H](O)[C@H](O)[C@H](O)CO</smiles><smiles>O=C(CO)[C@@H](O)[C@H](O)[C@H](O)CO</smiles>

llustración B-3: algunos compuestos de interés biológico, científico y tecnológico (continuación)

\section{B.4 PROPIEDADES FÍSICOQUÍMICAS DE LAS AMINAS}

La forma general de una amina es:<smiles>[R]N([R])[R]</smiles>

donde $\mathrm{R}, \mathrm{R}^{\prime}$ y $\mathrm{R}^{\prime \prime}$ son átomos de hidrógeno, o radicales (o grupos) alquílicos. El número de radicales orgánicos unidos al átomo de nitrógeno determina que la molécula sea clasificada como amina primaria (un grupo orgánico), secundaria (dos grupos) o terciaria (tres grupos). Si al menos uno de los radicales sustituyentes es un grupo arilo, entonces la amina, a parte de ser primaria, secundaria o terciaria, se dice que es aromática. Por ejemplo, la anilina. Si no hay grupos arilo, la amina será alifática.

El amoniaco $\mathrm{NH}_{3}$ es la amina alifática más simple, estando el resto derivadas de él:

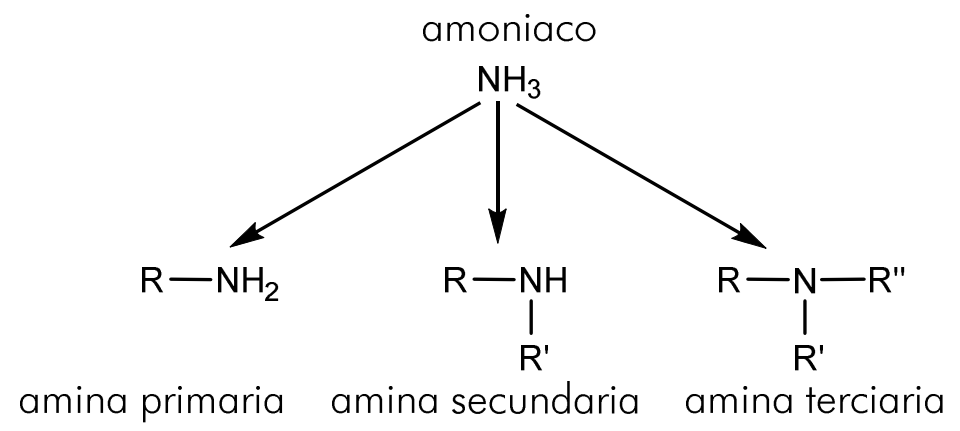

Ilustración B-4: esquema de la estructura de las aminas alifáticas a partir del amoniaco

De hecho, la mayoría de las aminas alifáticas tienen propiedades físicas y químicas similares a las del amoniaco. Todas las aminas alifáticas tienen un par de electrones 
libres en el átomo de nitrógeno y tres pares de electrones enlazantes. Así, todas las aminas alifáticas tienen cuatro pares de electrones en torno al átomo de nitrógeno, por lo que sería de esperar que los ángulos $\mathrm{R}-\mathrm{N}-\mathrm{R}$ sean cercanos a $109^{\circ}$. La molécula de amoniaco es piramidal, considerando que el par aislado de electrones no enlazantes ocupa uno de los vértices del tetraedro, siendo el ángulo del enlace $\mathrm{H}-\mathrm{N}-\mathrm{H}$ igual a $107^{\circ}$; tanto la forma de la molécula como el valor de dicho ángulo se pueden explicar admitiendo que los orbitales del átomo de nitrógeno tienen hibridación $\mathrm{sp}^{3}$. El par de electrones libres provoca una compresión del ángulo que forman entre sí los orbitales híbridos, reduciéndolo de $109^{\circ}$ a $107^{\circ}$. En las aminas, como la trimetilamina, $\left(\mathrm{CH}_{3}\right)_{3} \mathrm{~N}$, el ángulo del enlace $\mathrm{C}-\mathrm{N}-\mathrm{C}$ es mayor que el formado en la molécula de amoniaco porque los grupos alquilo, al ser más voluminosos que los átomos de hidrógeno, abren ligeramente el ángulo (efecto estérico), como se puede observar en la siguiente figura:

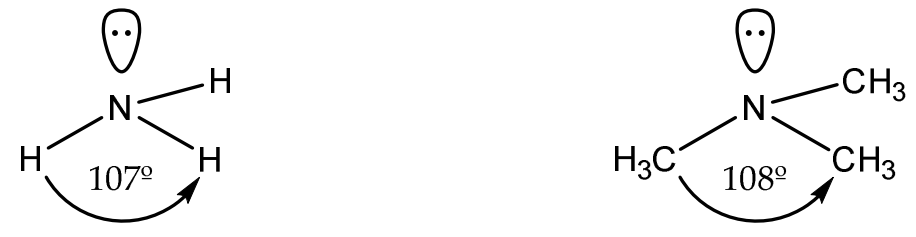

Ilustración B-5: efecto estérico en la molécula de trimetilamina

En las aminas alifáticas, al presentar una estructura piramidal, orbitales híbridos $\mathrm{sp}^{3}$, ángulos de enlace de $108^{\circ}$, un par de electrones sin compartir en uno de los orbitales $\mathrm{sp}^{3}$ y pudiendo ser los tres grupos alquílicos diferentes, nos encontramos con una configuración en la que el átomo de nitrógeno se convierte en un centro de simetría quiral. Las dos configuraciones asimétricas están en equilibrio a temperatura ambiente con una pequeña barrera energética $\left(6 \mathrm{Kcal} \cdot \mathrm{mol}^{-1}\right.$ ó $25.14 \mathrm{KJ} \cdot \mathrm{mol}^{-1}$ ) por lo que su interconversión es muy rápida y no permite el aislamiento de los enantiómeros correspondientes.

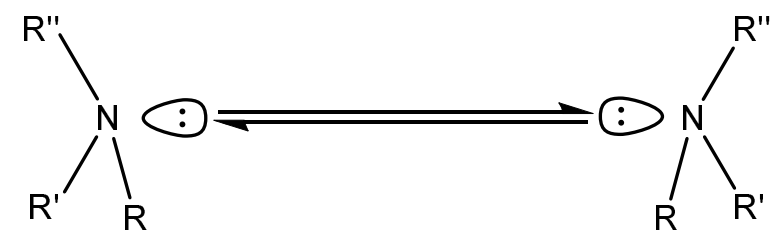

Ilustración B-6: enantiómeros de una amina terciaria

La estructura electrónica del enlace $\mathrm{N}-\mathrm{H}$ en el amoniaco fue investigada de manera cuidadosa por Tovard [8] desde el punto de vista teórico. Como la estructura electrónica de este enlace en las aminas alifáticas probablemente sea similar, los resultados principales obtenidos para el amoniaco se mantienen para las aminas alifáticas. Tovard calculó la densidad electrónica del $\mathrm{NH}_{3}$ de acuerdo con la función de onda de Moccia [9]. Se puede observar que las nubes de densidad electrónica toman valores negativos en torno al átomo de nitrógeno y positivos en torno al de hidrógeno [10, pp 16]. Es como si hubiese transferencia de electrones desde el hidrógeno hasta el nitrógeno, lo que concuerda con el hecho de que el $\mathrm{N}$ sea más electronegativo que el $\mathrm{H}$. Algunos experimentos de difracción de electrones han confirmado este efecto sobre la distribución de la densidad de electrones en el amoniaco.

El efecto del primer potencial de ionización es la cesión de uno de los electrones que existen en la región donde está localizado el par de electrones libres. Por ello es de esperar que el primer potencial de ionización sea de similar magnitud para todo este 
conjunto de moléculas, independientemente de que sean aminas primarias, secundarias o ternarias; por ejemplo, en el amoniaco, $I_{1}=10.5 \mathrm{e}^{-\mathrm{V}}$, y en la trimetilamina, $I_{1}=9 \mathrm{e} \mathrm{V}$.

En cuanto al momento dipolar eléctrico de las aminas alifáticas, a parte de la contribución al mismo del par de electrones libres, contribuyen los momentos dipolares de los otros enlaces, por lo que el valor resultante varía de manera significativa de unas molécula a otras: por ejemplo, para el amoniaco, $\mu=1.46 \mathrm{D}$, y para la trimetilamina, hemos encontrado en la bibliografía valores desde $0.61 \mathrm{D}$ [1 1] hasta $0.86 \mathrm{D}$ [12].

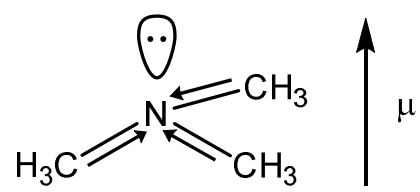

Ilustración B-7: contribuciones al momento dipolar de la trimetilamina

En cuanto a las aminas aromáticas, muy poco puede decirse sobre su geometría. Ni siquiera en el caso de la más simple, la anilina, se dispone de un modelo suficientemente fiable. La mayor parte de esta molécula es plana con casi total seguridad, estando el nitrógeno en el plano del anillo aromático. La organización geométrica es muy parecida a la del benceno, aunque la disposición de los dos átomos de hidrógeno del grupo $\mathrm{NH}_{2}$ se desconoce. Algunos estudios sugieren que si el grupo $\mathrm{NH}_{2}$ estuviera en el mismo plano que el anillo, la molécula de p-diaminobenceno no sería polar, lo cual está en contra de los resultados experimentales que facilitan el valor $1.5 \mathrm{D}$ para el momento dipolar. Por otra parte, el estudio mediante difracción de rayos $X$ de la estructura geométrica del 2-bromo-4-dimetilamino-2-cianoestilbeno indica que el grupo dimetilamino está en el mismo plano que los anillos aromáticos vecinos. Esta discrepancia se puede solucionar suponiendo que el grupo amino y el anillo aromático no son coplanares en las fases líquida o gaseosa, pero sí en la fase sólida, como ocurre en el cristal utilizado para observar el patrón de difracción de rayos $X$.

En la bibliografía no se ha en contrado ningún estudio preciso sobre la distribución electrónica en las aminas aromáticas. Tal información solamente se puede obtener a partir de la teoría. Consideremos el caso de la anilina. Como es una molécula similar a la de benceno, tendremos en cuenta los datos disponibles para esta molécula. Se sabe que el benceno es un hidrocarburo de fórmula molecular $\mathrm{C}_{6} \mathrm{H}_{6}$ que se puede presentar mediante las dos estructuras resonantes siguientes, propuestas por Kekulé:
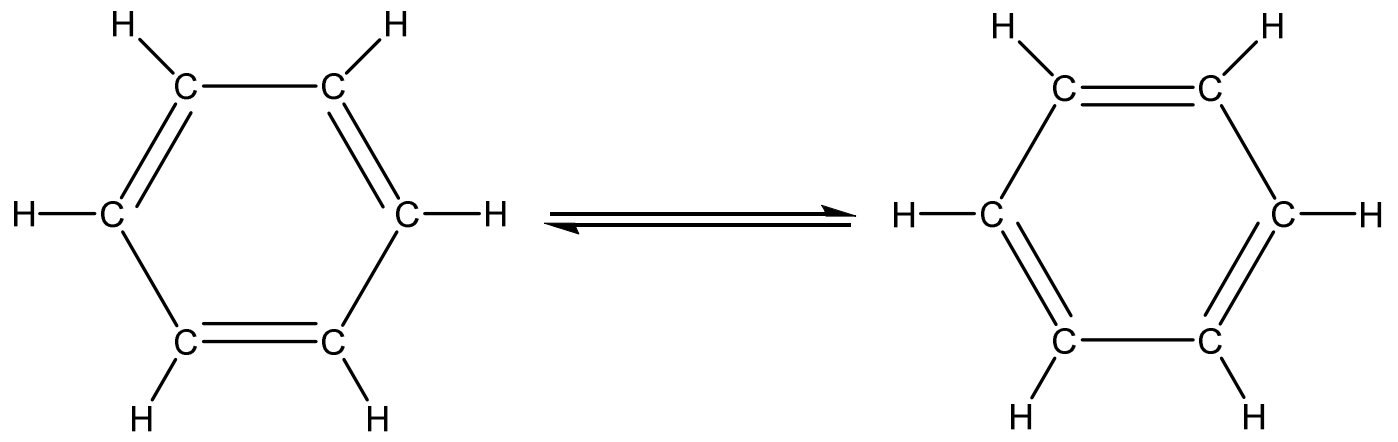

Ilustración B-8: estructuras resonantes del benceno

El que exista esta resonancia se explica con el hecho de que la molécula de benceno es más estable que si estuviese representada por una sola estructura de Kekulé, lo cual 
puede verse en términos de la energía de resonancia [13]. Esta estabilidad explica el hecho de que el benceno y otras moléculas aromáticas sean muy poco reactivos.

Se sabe que todos los enlaces C-C tienen la misma longitud, $1.39 \AA$, y que el anillo bencénico es plano. Además, la longitud del enlace $\mathrm{C}-\mathrm{C}$ en el benceno tiene un valor intermedio entre la longitud del enlace simple C-C $(1.48 \AA)$ y la del enlace doble $(1.34 \AA)$. De acuerdo con lo anterior, en realidad el benceno es un híbrido en resonancia cuyos enlaces $\pi$ están deslocalizados, con un orden de enlace de aproximadamente $1^{1 / 2}$ entre los átomos de carbono adyacentes. Por tanto, el benceno se puede describir como un anillo formado por seis átomos de de carbono con hibridación $\mathrm{sp}^{2}$, enlazados entre sí mediante enlaces $\sigma \mathrm{Csp}^{2}$-Csp ${ }^{2}$. Cada uno de los átomos de carbono se enlaza además a un átomo de hidrógeno mediante enlace $\sigma \mathrm{Csp}^{2}-\mathrm{H} 1 \mathrm{~s}$. Todos los ángulos de enlace C$\mathrm{C}$ son $120^{\circ}$. Como los átomos de carbono presentan hibridación $\mathrm{sp}^{2}$, cada átomo de carbono tiene un orbital $p$ perpendicular al plano del anillo que se solapa con los orbitales $\mathrm{p}$ de los carbonos contiguos para formar dos círculos de densidad electrónica $\pi$, uno por encima y otro por debajo del plano molecular, encontrándose en estas nubes 6 electrones deslocalizados.

En el caso de la anilina, deben tenerse en cuenta los orbitales atómicos de los átomos del grupo $\mathrm{NH}_{2}$, esto es, hay que introducir los orbitales $\mathrm{N} 1 \mathrm{~s}, \mathrm{~N} 2 \mathrm{~s}, \mathrm{~N} 2 \mathrm{p}_{\mathrm{x}}, \mathrm{N} 2 \mathrm{p}_{\mathrm{y}}, \mathrm{N} 2 \mathrm{p}_{\mathrm{z}}$ del nitrógeno y los orbitales $\mathrm{s}$ de los dos nuevos átomos de hidrógeno. Sean $\mathrm{H}_{7} 1 \mathrm{~s}$ y $\mathrm{H}_{8} 1 \mathrm{~s}$ estos dos últimos orbitales. Asumamos que, como como el benceno, la anilina es una molécula plana. El orbital N1s estará asociado con la primera capa del nitrógeno. Los orbitales $\mathrm{N} 2 \mathrm{~s}, \mathrm{~N} 2 \mathrm{p}_{\mathrm{x}}, \mathrm{N} 2 \mathrm{p}_{\mathrm{y}}$ estarán combinados de tal manera que producirán tres orbitales trigonales híbridos $\mathrm{sp}^{2}$ que denominaremos $\mathrm{Nt}_{r}, \mathrm{Nt}^{\prime}{ }_{\mathrm{r}}$ y $\mathrm{Nt}^{\prime \prime}{ }_{\mathrm{r}}$ los cuales se utilizan para formar los enlaces de dos electrones localizados siguientes: $\mathrm{C}-\mathrm{N}, \mathrm{N}-\mathrm{H}_{7}, \mathrm{~N}-\mathrm{H}_{8}$, como se ve en la llustración B-11. El enlace deslocalizado de seis electrones puede extenderse sobre el átomo de nitrógeno cuando no se utiliza el orbital $\mathrm{N} 2 \mathrm{p}_{\mathrm{z}}$. Además, el átomo de nitrógeno introduce sus dos electrones libres dentro del enlace deslocalizado del anillo aromático.

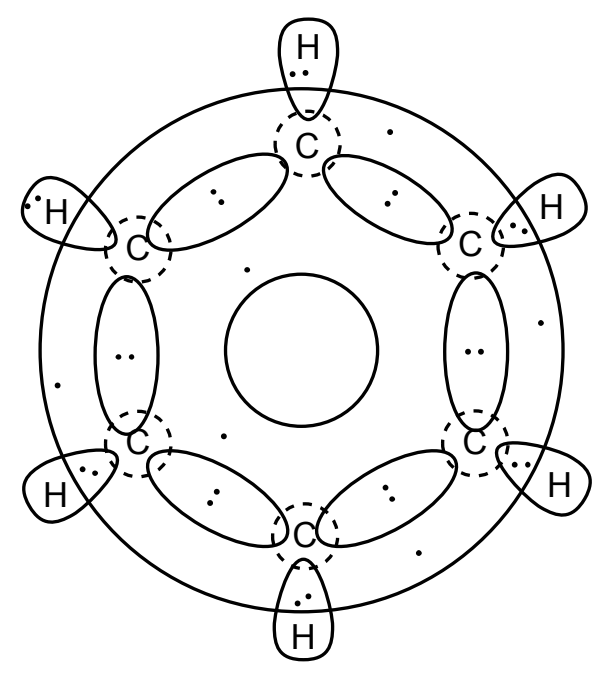

Ilustración B-9: vista frontal de la descomposición aproximada en orbitales del benceno 


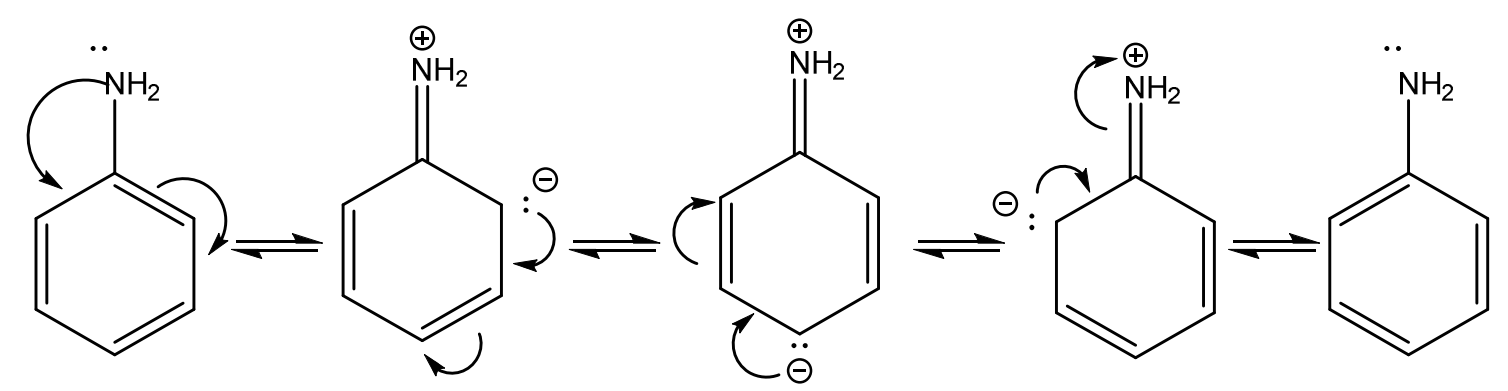

Ilustración B-10: estabilización de la anilina: Las estructuras 1 y 5 son generales para cualquier derivado del benceno. Las estructuras 2, 3, y 4, sin embargo, deslocalizan el par de electrones sin compartir del nitrógeno en las posiciones orto y para del anillo. Esta deslocalización del par de electrones lo hace menos asequible al protón, y lo que es más importante, estabiliza a la amina aromática.

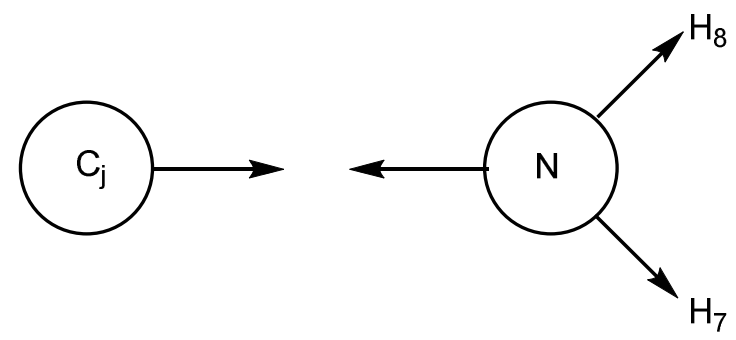

Ilustración B-11: orbitales híbridos del nitrógeno en la anilina

Una de las propiedades más importantes de las aminas es su basicidad. En las aminas, la basicidad radica en la disponibilidad del par de electrones del nitrógeno. Mientras mayor sea la disponibilidad, más básica es la amina. Si estos electrones se deslocalizan en la molécula, están menos disponibles y disminuye su basicidad. La anilina y otras aminas aromáticas son bases más débiles que las aminas alifáticas, debido a que la base libre está estabilizada por resonancia (ver la llustración B-10). El par de electrones del nitrógeno que se encuentran en un orbital $\mathrm{sp}^{3}$ en la anilina está interactuando con la nube $\pi$ del anillo bencénico por lo que estabiliza la base por resonancia.

En el caso de aminas aromáticas como la piridina, la quinolina, la isoquinolina y la acridina, entre otras, se ha observado experimentalmente la existencia de una protonación (adición de un protón a un átomo, molécula o ión; lo que conlleva el aumento de la concentración $\left[\mathrm{BH}^{+}\right]$y disminución de [B]) en el(los) nitrógeno(s) que pertenece(n) a el(a los) anillo(s) aromático(s). Por tanto, el valor del $p K$ no está relacionado con la protonación del grupo amino, sino del nitrógeno en el anillo. Este estudio es extremadamente interesante porque permite determinar las perturbaciones que el grupo amino lleva al heteroátomo. En la llustración B-1 1 se puede observar que el grupo amino es capaz de aumentar significativamente la carga electrónica de ciertos átomos del anillo aromático. Por tanto, es de esperar que este grupo sea capaz de aumentar la facilidad de protonación del heteroátomo y entonces aumentar el valor de $p K_{a}$. A $20^{\circ} \mathrm{C}$, el $p K_{a}$ de la piridina es 5.23 y el de la p-aminopiridina 9.17. La constante de disociación del equilibrio base/ácido conjugado se reduce en un factor de 10000 como resultado de la presencia del grupo amino, es decir, el compuesto con el grupo amino es mucho más básico que la piridina por sí sola.

Los efectos de hibridación también juegan un papel importante en la basicidad de las aminas. Cuanto mayor es el carácter s de un orbital mayor es la atracción que ejerce el núcleo sobre los electrones, haciendo más difícil la posibilidad de ceder sus pares de electrones libres, teniendo por este motivo un carácter menos básico. Por ejemplo, la 
piridina es una base más débil que las aminas alifáticas, como la piperidina. En la piridina, el par de electrones no enlazantes ocupan un orbital con hibridación $\mathrm{sp}^{2}$ (33\% de carácter s) y en la piperidina ocupan un orbital con hibridación $\mathrm{sp}^{3}(25 \%$ de carácter s). En la piridina, el par electrónico solitario está en un orbital con más carácter s que el orbital que ocupa el par de electrones solitarios de la piperidina y por tanto los electrones están menos disponibles para unirse a un protón.
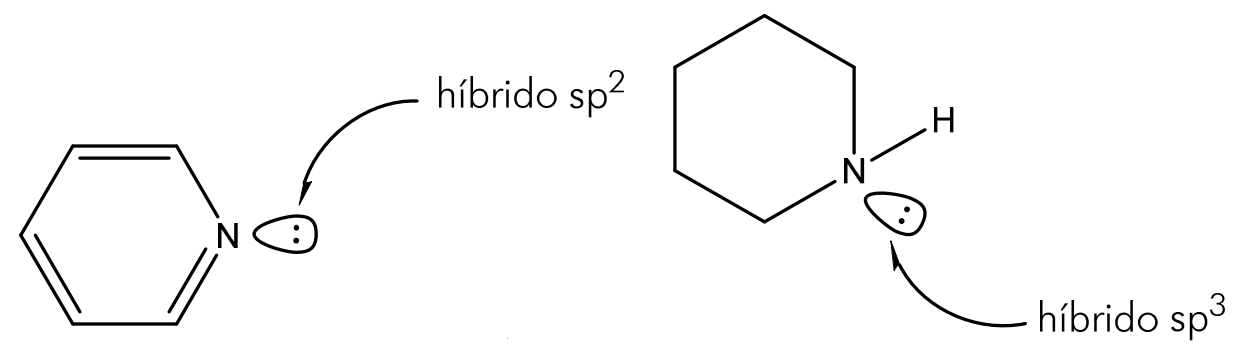

Ilustración B-12: hibridación de la piridina (izq.) y la piperidina (drcha.)

La principal característica de las aminas es que son capaces de asociarse consigo mismo o con otras moléculas distintas mediante dos tipos de enlaces puente de hidrógeno, representados de la siguiente forma:

$$
-\mathrm{N}-\mathrm{H} \cdots \mathrm{X} \quad-\mathrm{N} \cdots \mathrm{H}-\mathrm{X}
$$

En el primer tipo de enlace, la amina actúa como un ácido protónico débil, luego cede un hidrógeno en el enlace puente de hidrógeno. Por esta razón, este tipo de enlace sólo se da en aminas primarias o secundarias. En el segundo tipo de enlace, la amina actúa, como una base débil, compartiendo su par de electrones libres, por lo que puede ser una amina primaria, secundaria o terciaria. Ambos tipos de enlace son relativamente débiles en comparación con el enlace covalente, por lo que se pueden investigar mediante espectroscopia infrarroja. Las frecuencias de los modos de elongación de los enlaces $\mathrm{H} \cdots \mathrm{X}$ y $\mathrm{N} \cdots \mathrm{H}$, la forma e intensidad de las bandas de absorción, y el efecto de la temperatura y la concentración deben ser observadas con atención.

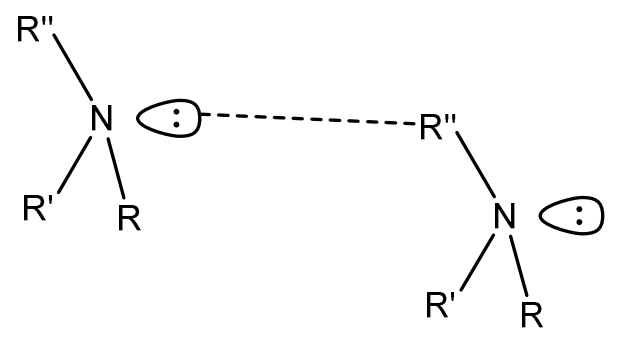

Ilustración B-13: autoasociación por puentes de hidrógeno entre aminas $1^{\text {as }} y / o ́$ as

El enlace por puente de hidrógeno formado entre las aminas influye sobre gran cantidad de propiedades físicas y químicas, como se aprecia en la siguiente tabla: 
Tabla B-1: Influencia del enlace por puente de hidrógeno en las propiedades de un sistema [14]

\begin{tabular}{|c|c|c|}
\hline Propiedad & $\begin{array}{l}\text { Puente de hidrógeno } \\
\text { intramolecular }\end{array}$ & Puente de hidrógeno intermolecular \\
\hline Estructura del compuesto & $\begin{array}{l}\text { Se puede encontrar en las } \\
\text { posiciones orto, cis, para, } \\
\text { diecuatorial y ecuatorial-axial }\end{array}$ & $\begin{array}{l}\text { Se puede encontrar en las } \\
\text { posiciones meta, para y trans }\end{array}$ \\
\hline Geometría del enlace & Casi siempre arqueado & Casi siempre recto \\
\hline Peso molecular & Normal & Aumenta \\
\hline Densidad & & Aumenta \\
\hline Volumen molecular & Disminuye & Disminuye \\
\hline Viscosidad & Disminuye & Aumenta \\
\hline Tensión superficial & Disminuye & Aumenta \\
\hline $\begin{array}{l}2^{\circ} \text { coeficiente del virial } \\
\text { (ec. De Berthelot) }\end{array}$ & Normal & Aumenta en valor absoluto \\
\hline Presión de vapor & Normal & Disminuye \\
\hline Punto de ebullición & Normal & Aumenta \\
\hline Punto de fusión & Normal & Aumenta \\
\hline Conductividad térmica & Normal & Aumenta \\
\hline Poder disolvente & Normal & $\begin{array}{l}\text { Aumenta si el soluto forma puentes } \\
\text { de hidrógeno con el disolvente }\end{array}$ \\
\hline Conductividad acústica & Normal & Aumenta \\
\hline Conductividad eléctrica & Normal & $\begin{array}{l}\text { Aumenta si se forma una red } \\
\text { de puentes de hidrógeno }\end{array}$ \\
\hline Constante dieléctrica & $\begin{array}{l}\text { Normal para sólidos, y } \\
\text { variable conforme la forma de la } \\
\text { molécula para los líquidos }\end{array}$ & $\begin{array}{l}\text { Aumenta para sólidos y } \\
\text { variable de acuerdo con la forma } \\
\text { molecular para líquidos }\end{array}$ \\
\hline Momento dipolar & $\begin{array}{c}\text { Independiente de la } \\
\text { concentración y más bajo que } \\
\text { el calculado a partir de la } \\
\text { estructura }\end{array}$ & $\begin{array}{c}\text { Aumenta con la concentración y } \\
\text { mayor que el calculado a partir de } \\
\text { la estructura }\end{array}$ \\
\hline Bandas de absorción electrónica & Desplazada & Desplazada \\
\hline \multirow{2}{*}{$\begin{array}{c}\text { Frecuencia del modo de } \\
\text { elongación } \\
\text { Intensidad de la banda del modo } \\
\text { de elongación }\end{array}$} & $\begin{array}{l}\text { Desplazada a longitudes de onda } \\
\text { menores }\end{array}$ & $\begin{array}{l}\text { Desplazada a longitudes de onda } \\
\text { menores }\end{array}$ \\
\hline & Aumenta un poco & Aumenta mucho \\
\hline
\end{tabular}

Una consecuencia importante de la formación del enlace por puente de hidrógeno $\mathrm{N}-\mathrm{H} \cdots \mathrm{X}$ es el acortamiento de la distancia interatómica $\mathrm{N}-\mathrm{X}$ (la cual puede determinarse mediante cálculos realizados a partir del ancho de banda del espectro de resonancia magnética nuclear, por ejemplo) cuando se compara con la suma de los radios de Van der Waals de $\mathrm{N}$ y $\mathrm{X}$. Este efecto debe considerarse cuando se hace un estudio estereoquímico de aminas y/o de compuestos nitrogenados, especialmente en la fase sólida, ya que pueden aparecer efectos estéricos y distorsiones muy significativas. Hágase notar que mientras que el enlace $\mathrm{N}-\mathrm{X}$ se acorta, el enlace $\mathrm{N}-\mathrm{H}$ se alarga. En la Tabla B-2 se resumen varios enlaces de puente de hidrógeno en los que está implicado un átomo de nitrógeno, y sus longitudes, comparadas con las distancias dadas por la suma de los radios de Van der Waals. 
Tabla B-2. Longitudes del enlace de hidrógeno y distancias de Van der Waals para algunos compuestos cristalinos [10, pp 142]

\begin{tabular}{|c|c|c|c|}
\hline$A-H \cdots B$ & Tipo de enlace $\mathrm{A}-\mathrm{H}$ & $\begin{array}{l}\text { Distancia } \\
\mathrm{A}-\mathrm{B} / \AA\end{array}$ & $\begin{array}{c}\text { Distancia de Van der } \\
\text { Waals / } \AA\end{array}$ \\
\hline $\mathrm{O}-\mathrm{H} \cdots \mathrm{N}$ & Todos los enlaces $\mathrm{O}-\mathrm{H}$ & $2.80 \pm 0.09$ & 3.05 \\
\hline $\mathrm{N}^{+}-\mathrm{H} \cdots \mathrm{O}$ & Amonio & $2.88 \pm 0.13$ & 3.05 \\
\hline $\mathrm{N}-\mathrm{H} \cdots \mathrm{O}$ & Amida & $2.93 \pm 0.10$ & 3.05 \\
\hline $\mathrm{N}-\mathrm{H} \cdots \mathrm{O}$ & Amida & $3.04 \pm 0.13$ & 3.05 \\
\hline $\mathrm{N}-\mathrm{H} \cdots \mathrm{N}$ & Todos los enlaces $\mathrm{N}-\mathrm{H}$ & $3.10 \pm 0.13$ & 3.30 \\
\hline $\mathrm{N}^{+}-\mathrm{H}^{\cdots} \cdot \mathrm{F}^{-}$ & $\mathrm{NH}_{4} \mathrm{~F}$ & 2.69 & 3.00 \\
\hline $\mathrm{N}-\mathrm{H} \cdots \mathrm{F}$ & $\mathrm{NH}_{3} \cdot \mathrm{BF}_{3}$ & $3.01 \pm 0.3$ & 3.00 \\
\hline $\mathrm{N}^{+}-\mathrm{H}^{\prime} \cdots \mathrm{Cl}^{-}$ & $\mathrm{NH}_{2}\left(\mathrm{CH}_{2}\right)_{6} \mathrm{NH} .2 \mathrm{HCl}$ & $3.01,3.07$ & 3.45 \\
\hline $\mathrm{N}-\mathrm{H}^{\cdots} \mathrm{Cl}^{-}$ & Adenina. $\mathrm{HCl} . \frac{1}{2} \mathrm{H}_{2} \mathrm{O}$ & $3.11,3.21$ & 3.45 \\
\hline $\mathrm{N}-\mathrm{H} \cdots \mathrm{Cl}$ & 4,5-Diamino-2-cloropirimidina & $3.51,3.52$ & 3.45 \\
\hline $\mathrm{N}^{+}-\mathrm{H}^{\cdots} \cdot \mathrm{Br}^{-}$ & $\mathrm{NH}_{2}\left(\mathrm{CH}_{2}\right)_{10} \mathrm{CO}_{2} \mathrm{H} . \mathrm{HBr} . \frac{1}{2} \mathrm{H}_{2} \mathrm{O}$ & $3.30,3.44$ & 3.60 \\
\hline
\end{tabular}

Como el nitrógeno es menos electronegativo que el oxígeno, el enlace $\mathrm{N}-\mathrm{H}$ está menos polarizado que el enlace O-H. Por lo tanto, las aminas forman puentes de hidrógeno más débiles que los alcoholes de masas molares semejantes y por tanto tienen puntos de ebullición menores que los de los alcoholes análogos. Las aminas terciarias, que no pueden formar puentes de hidrógeno entre sí, tienen temperaturas de ebullición más bajas que las aminas primarias o secundarias de masas molares semejantes. No obstante las aminas poseen temperaturas de ebullición mayores que las de los alcanos homomorfos de masa molar semejante ya que estos últimos son apolares y las fuerzas de interacción intermoleculares son muy débiles. En la siguiente tabla se comparan los puntos de ebullición de aminas, alcoholes y éteres de masas molares semejantes.

Tabla B-3: punto de ebullición y masa molar para diferentes compuestos de interés

\begin{tabular}{cccc}
\hline Compuesto & Tipo & $M_{\mathrm{m}} / \mathrm{g} \cdot \mathrm{mol}^{-1}$ & $T_{\text {eb }} / \mathrm{K}$ \\
\hline$\left(\mathrm{CH}_{3}\right)_{3} \mathrm{~N}$ & amina terciaria & 59 & 276.15 \\
$\mathrm{CH}_{3}-\mathrm{O}_{-}-\mathrm{CH}_{2} \mathrm{CH}_{3}$ & éter & 60 & 281.15 \\
$\mathrm{CH}_{3}-\mathrm{NH}_{-}-\mathrm{CH}_{2} \mathrm{CH}_{3}$ & amina secundaria & 59 & 310.15 \\
$\mathrm{CH}_{3} \mathrm{CH}_{2} \mathrm{CH}_{2}-\mathrm{NH}_{2}$ & amina primaria & 59 & 321.15 \\
$\mathrm{CH}_{3} \mathrm{CH}_{2} \mathrm{CH}_{2}-\mathrm{OH}$ & alcohol & 60 & 370.15 \\
\hline
\end{tabular}

Todas las aminas, incluso las terciarias, forman puentes de hidrógeno con el agua y los alcoholes. Por esta razón, las aminas de baja masa molar (hasta 6 átomos de carbono) son relativamente solubles en agua y en alcoholes.
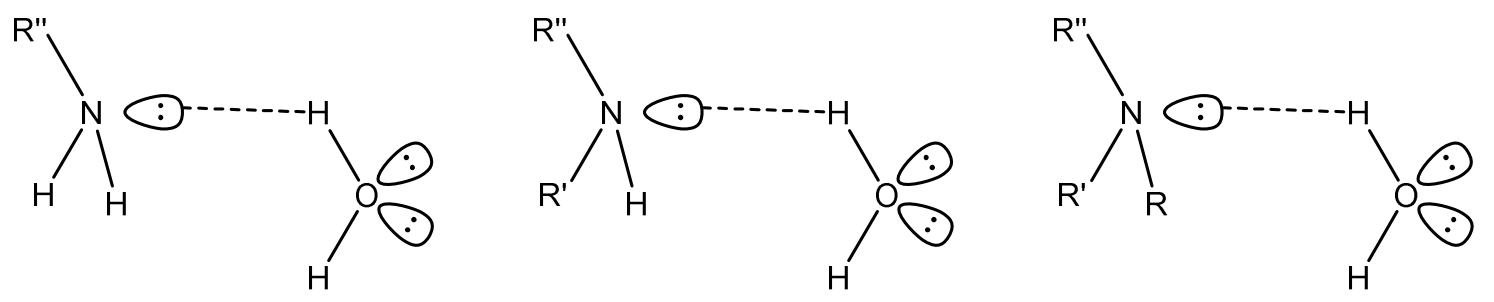

Ilustración B-14: solubilidad de las aminas con el agua: de izq. a drcha.: primaria, secundaria y terciaria 
Se han llevado a cabo diferentes trabajos cuya finalidad es alcanzar una mejor comprensión de la naturaleza de las aminas, así como de sus propiedades tanto en estado puro como en mezclas. La localización del átomo de hidrógeno que participa en el enlace se ha llevado a cabo mediante varios métodos, por ejemplo, difracción de rayos $X$ que, aunque bastante inefectivo para este propósito, debido a las pequeñas amplitudes de dispersión de los átomos de hidrógeno, en algunos casos como en la 4,5-diamino-2-cloropirimidina, ofrecen resultados de confianza. La difracción de electrones se ha empleado con el mismo propósito. La autoasociación de las aminas primarias y secundarias ha sido estudiada mediante scattering de neutrones o de rayos $X$ [15], a través de medidas de las presiones de vapor de las aminas en estado puro o en solución [16]- [19] o mediante métodos de simulación [20].

\section{B.5 PROPIEDADES FISICOQUÍMICAS DE LAS CETONAS}

Entre el átomo de carbono y el de oxígeno del grupo carbonilo se forman enlaces $\sigma$ y $\pi$, estando el último ligeramente deslocalizado. El carbono hibrida en forma $\mathrm{sp}^{2}$, enlazándose uno de los orbitales híbridos con un orbital $2 \mathrm{p}_{\mathrm{x}}$ del oxígeno dando el enlace $\sigma$, mientras que los orbitales $2 p_{z}$ del oxígeno y el carbono se recubren lateralmente formando el enlace $\pi$. Por tanto, en el átomo de oxígeno existen dos pares de electrones libres; un par es principalmente de carácter $2 \mathrm{~s}$, y el otro de $2 \mathrm{p}_{y^{\prime}}$ con el eje de su orbital perpendicular al orbital que forma el enlace $\pi$. Las principales propiedades físico-químicas de los compuestos con el grupo carbonilo están controladas por esta estructura electrónica y también por la posición del grupo carbonilo dentro de la molécula. Así, en un aldehído, el grupo carbonilo se encuentra necesariamente en uno de los extremos de la molécula, mientras que en una cetona se encuentra en cualquier posición intermedia. Como el eje del orbital del par de electrones libres $2 p$ es perpendicular a aquel que forma el enlace $\pi$, este par de electrones libres no puede estar conjugado con el sistema $\pi$, motivo por el cual las propiedades de este par de electrones libres será constante. Las propiedades de los electrones que participan en el enlace $\pi$ del grupo carbonilo también son bastante constantes. Por ambas razones, la explicación de las propiedades fisico-químicas de estos compuestos es relativamente simple.

Las cetonas suelen ser menos reactivas que los aldehídos dado que los grupos alquílicos actúan como donadores de electrones por efecto inductivo.

La principal característica de las sustancias carbonílicas es que son muy polares debido a la diferencia de electronegatividad entre el carbono y el oxígeno, dando una gran contribución la forma de resonancia dipolar en la que al oxígeno se le asocia carga negativa y al carbono, positiva. El dipolo crea fuerzas de atracción débiles entre las moléculas.

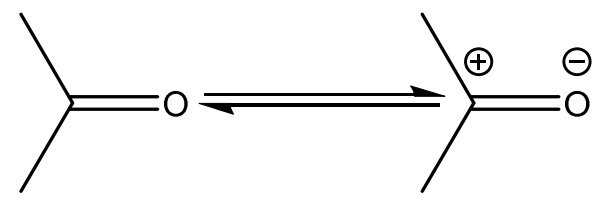

Ilustración B-15: resonancia dipolar del grupo carbonilo 
El momento dipolar puede determinarse mediante el estudio de las constantes dieléctricas de disoluciones o del efecto Stark en transiciones dentro del rango de microondas. Los momentos dipolares de algunos compuestos carbonílicos pueden consultarse en la Tabla B-4. La influencia del efecto inductivo del grupo metilo es clara si se compara el momento dipolar de la acetona (2.9 D) con el del acetaldehído (2.68 D) y el formaldehído (2.339 D). Los grupos metilo adyacentes al carbonilo aumentan la polaridad de éste. En contraste, los átomos de cloro disminuyen la polaridad, como se ve al comparar el formaldehído con el fosgeno (dicloruro de carbonilo).

Tabla B-4: momento dipolar de algunos compuestos con el grupo carbonilo [21, pp 7]

\begin{tabular}{|c|c|c|c|}
\hline Compuesto & & Momento dipolar / D & Método de obtención ${ }^{*}$ \\
\hline $\mathrm{CO}$ & & 0.112 & m.w. \\
\hline $\cos$ & & 0.7124 & m.w. \\
\hline $\mathrm{COCl}_{2}$ & & 1.109 & d. \\
\hline HCOF & & 2.02 & m.w. \\
\hline $\mathrm{HCHO}$ & & 2.339 & m.w. \\
\hline $\mathrm{HCOOH}$ & & 1.415 & m.w. \\
\hline $\mathrm{CH}_{3} \mathrm{OH}$ & & 1.69 & m.w. \\
\hline $\mathrm{ClCOCOCl}$ & & 0.92 & d. \\
\hline $\mathrm{H}_{2} \mathrm{C}=\mathrm{C}=\mathrm{O}$ & & 1.387 & m.w. \\
\hline $\mathrm{CH}_{3} \mathrm{CHO}$ & & 2.68 & $\mathrm{~m}$. \\
\hline $\mathrm{CH}_{3} \mathrm{COBr}$ & & 2.45 & d. \\
\hline $\mathrm{CH}_{3} \mathrm{COCl}$ & & 2.47 & $\mathrm{~d}$ \\
\hline $\mathrm{CH}_{2} \mathrm{ClCHO}$ & & 1.99 & d. \\
\hline $\mathrm{CH}_{3} \mathrm{COCH}_{3}$ & & 2.90 & m.w. \\
\hline Ciclohexanona & & 3.08 & comp. \\
\hline $\mathrm{HC} \equiv \mathrm{CCHO}$ & & 2.46 & m.w. \\
\hline \multirow[t]{2}{*}{$\mathrm{H}_{2} \mathrm{C}=\mathrm{CHCHO}$} & $\mathrm{s}$-cis & 2.6 & m.w. \\
\hline & s-trans & 3.11 & m.w. \\
\hline $\mathrm{C}_{6} \mathrm{H}_{5} \mathrm{CHO}$ & & 2.724 & r.t. \\
\hline $\mathrm{C}_{6} \mathrm{H}_{5} \mathrm{COCH}_{3}$ & & 2.97 & $\mathrm{~d}$. \\
\hline
\end{tabular}

Cabría esperar que en primera aproximación el momento dipolar de un compuesto aromático y del correspondiente alifático fuese similar. Sin embargo, debido a la gran polarizabilidad del grupo fenilo en las proximidades de un grupo polar, el momento dipolar efectivo del compuesto aromático debe ser ligeramente mayor, aunque esto sólo es cierto si los grupos están en las posiciones meta pero no en las para u orto. En cualquier caso, la influencia del efecto de resonancia o mesomérico es muy pequeña. Esto explica el pequeña diferencia entre el momento dipolar del benzaldehído (2.72 D) y el correspondiente compuesto alifático, el n-heptaldehído (2.61 D).

A pesar de la ausencia de hidrógeno en el grupo carbonilo, por lo que ni las cetonas ni los aldehídos pueden formar puentes de hidrógeno entre sí, el elevado momento dipolar es el responsable de las interacciones de tipo dipolo-dipolo que se dan en estas sustancias. Ello explica que estas sustancias tengan temperaturas de ebullición mayores que los alcanos y menores que los alcoholes de igual peso molecular, y que sean

* m.w. = Efecto Stark; comp. = cálculo computacional a partir de valores experimentales obtenidos mediante diferentes métodos; $d$. = método de la constante dieléctrica; r.t. = método del tiempo de relajación. 
menos volátiles que los alcanos y más volátiles que los alcoholes y los ácidos carboxílicos semejantes. Las interacciones moleculares son responsables también de que el aldehído más simple, el metanal, tenga temperatura de ebullición a presión atmosférica igual a $-21^{\circ} \mathrm{C}$, lo que hace de él un gas en condiciones normales, mientras que el siguiente mayor, el etanal, tiene temperatura de ebullición de $+21^{\circ} \mathrm{C}$, próxima a la temperatura ambiente. El resto de aldehídos y cetonas estarán, en condiciones estándar de presión y temperatura $(T=298.15 \mathrm{~K}$ y $P=1 \mathrm{~atm}$.) en fase líquida. No hay grandes diferencias entre los puntos de ebullición de aldehídos y cetonas de igual peso molecular (véase la Tabla B-5).

Tabla B-5: temperaturas de ebullición y solubilidad en agua a presión atmosférica de varios compuestos de masa molecular semejante

\begin{tabular}{cccc}
\hline Compuesto & Masa molecular $/ \mathrm{g} \cdot \mathrm{mol}^{-1}$ & $\begin{array}{c}\text { Temperatura de } \\
\text { ebullición } / \mathrm{K}\end{array}$ & Solubilidad en agua \\
\hline Butano & 58 & 273.15 & No \\
Propanal & 58 & 322.15 & Sí \\
Acetona & 58 & 329.15 & Sí \\
1-propanol & 60 & 370.15 & Sí \\
\hline
\end{tabular}

Las fuerzas dispersivas tipo London entre dipolo instantáneos son las responsables de que la temperatura de ebullición aumente cuando lo hace la longitud de la cadena, ya que cuanto mayor sea la longitud de la cadena, mayores son las fluctuaciones en las posiciones de los electrones dentro de la molécula responsables de los dipolos inducidos, y en consecuencia, mayor será la fuerza intermolecular.

Por otra parte, el oxígeno del grupo carbonilo actúa como nucleófilo débil ante el hidrógeno, por lo que puede formar puentes de hidrógeno con las moléculas de, por ejemplo, agua. Por esta razón, los aldehídos y cetonas son solubles en agua, disminuyendo la solubilidad a medida que aumenta el tamaño de la molécula (véase la Ilustración B-16), debido al mayor carácter alquílico que va adquiriendo la molécula.

El carácter de las propiedades de solubilidad en agua, temperatura de ebullición y volatilidad de las cetonas está relacionado con su capacidad de penetración, lo que es muy relevante en la industria de la perfumería y para su utilización como disolventes.

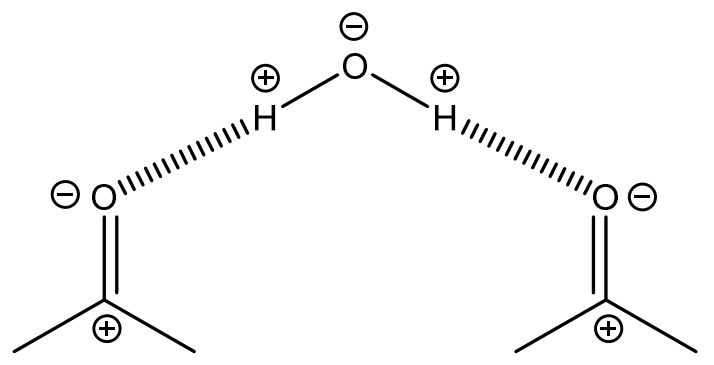

Ilustración B-16: solubilidad de cetonas/aldehídos en agua

Una magnitud relacionada con la polaridad del enlace carbonilo es su longitud de enlace. Ésta disminuye cuando su polaridad aumenta [22], debido al aumento de las fuerzas de atracción culombiana entre los centros de carga positiva y negativa, lo cual explica, por ejemplo, el hecho de que la distancia entre el $\mathrm{C}$ y el $\mathrm{O}$ en el acetaldehído sea mayor que en el formaldehído. 
La longitud del enlace $\mathrm{C}=\mathrm{O}$ en aldehídos, cetonas, ácidos carboxílicos y ésteres está en torno a $1.20 \AA$ (véase la Tabla B-6), de acuerdo con su determinación mediante difracción de electrones y espectroscopia de microondas. En los compuestos inorgánicos con el enlace $\mathrm{C}=\mathrm{O}$, como el monóxido de carbono, el dióxido de carbono, o el ión carbonato, la longitud de éste difiere bastante de $1.20 \AA$.

Tabla B-6: longitud del enlace $\mathrm{C}=\mathrm{O}[21, \mathrm{pp} 4]$

\begin{tabular}{|c|c|c|}
\hline Compuesto & Longitud / $\AA$ & Método de determinación ${ }^{*}$ \\
\hline \multirow[t]{2}{*}{$\mathrm{CO}$} & 1.1282 & Espec. \\
\hline & 1.1308 & M.o. \\
\hline $\mathrm{CO}_{2}$ & 1.1632 & Espec. \\
\hline $\mathrm{C}_{3} \mathrm{O}_{2}$ & 1.16 & D.e. \\
\hline $\cos$ & 1.1637 & M.o. \\
\hline $\mathrm{COCl}_{2}$ & 1.166 & M.o. \\
\hline $\mathrm{COF}_{2}$ & 1.174 & M.o. \\
\hline $\mathrm{CH}_{3} \mathrm{COF}$ & 1.181 & M.o. \\
\hline $\mathrm{CH}_{3} \mathrm{COCl}$ & 1.192 & M.o. \\
\hline $\mathrm{HCOOCH}_{3}$ & 1.200 & M.o. \\
\hline $\mathrm{HCOOH}$ & 1.202 & M.o. \\
\hline $\mathrm{HCHO}$ & 1.2078 & M.o. \\
\hline \multirow[t]{2}{*}{$\mathrm{CH}_{3} \mathrm{CHO}$} & 1.216 & M.o. \\
\hline & 1.208 & D.e. \\
\hline $\mathrm{CH} \equiv \mathrm{CCHO}$ & 1.215 & M.o. \\
\hline $\mathrm{CH}_{3} \mathrm{COCH}_{3}$ & 1.22 & D.e. \\
\hline $\mathrm{CH}_{2}=\mathrm{CHCHO}$ & 1.22 & M.o. \\
\hline $\mathrm{CHOCHO}$ & 1.20 & Espec. \\
\hline p-Benzoquinona & 1.222 & R.x. \\
\hline $\mathrm{CH}_{3} \mathrm{COCN}$ & 1.226 & M.o. \\
\hline \multirow{2}{*}{$\operatorname{co} \frac{2}{3}^{-}$} & 1.264 & R.x. \\
\hline & 1.346 & R.x. \\
\hline $\mathrm{HCOOCH}_{3}(\mathrm{CO})$ & 1.334 & M.o. \\
\hline $\mathrm{CH}_{3} \mathrm{OH}$ & 1.427 & M.o. \\
\hline
\end{tabular}

Cuando se consideran las direcciones del enlace $\mathrm{C}=\mathrm{O}$, la hibridación no es $\mathrm{sp}^{2}$ simple. Por ejemplo, en el estado fundamental de la molécula plana $\mathrm{HCHO}$, el ángulo $\mathrm{H}-\mathrm{C}-\mathrm{H}$ es de $116^{\circ} 31^{\prime}$ [23] y en el acetaldehído el ángulo $\mathrm{C}-\mathrm{C}-\mathrm{O}$ es $123^{\circ} 55^{\prime}$ [24] en lugar de $120^{\circ}$ como sugiere una hibridación $\mathrm{sp}^{2}$ simple.

Es posible relacionar la longitud de enlace del grupo carbonilo en una molécula con su primer potencial de ionización: el primero aumenta cuando disminuye el segundo. Los potenciales de ionización se pueden determinar tanto por la extrapolación de la serie de Rydberg en el espectro ultravioleta en vacío como a partir de los potenciales aparentes de los iones en un espectrómetro de masas (método del impacto de electrones), o bien mediante el estudio de las curvas de eficiencia de fotoionización [25, pp 339]. El primero de estos métodos proporciona la diferencia de energía entre los estados fundamentales del ión y la molécula, ambos estados estando en el nivel vibracional

\footnotetext{
Espec. $=$ espectroscopia; M.o. $=$ espectroscopia de microondas; D.e. $=$ difracción de electrones; R.x.=determinación estructural por Rayos $X$
} 
cero. El valor que se obtiene así del potencial de ionización se llama adiabático. En el segundo método, la transición es considerada tan rápida que los núcleos no cambian sus posiciones. El ión estará en un nivel vibracional por encima del nivel cero, y el valor del potencial de ionización obtenido por este segundo método, llamado vertical, será igual o mayor el del obtenido por el primer método.

Los valores del primer potencial de ionización de algunas moléculas que contienen al grupo carbonilo se pueden observar en la tabla siguiente:

Tabla B-7: Potencial de ionización de algunos compuestos con el grupo carbonilo [21, pp9]

\begin{tabular}{|c|c|c|}
\hline Molécula & Potencial de ionización / e-V & Método de obtención \\
\hline \multirow[t]{3}{*}{$\mathrm{CO}$} & 14.013 & Espec. \\
\hline & 14.01 & Impacto \\
\hline & 14.11 & Impacto \\
\hline \multirow[t]{2}{*}{$\mathrm{CO}_{2}$} & 13.88 & Impacto \\
\hline & 13.788 & Espec. \\
\hline $\cos$ & 11.23 & Espec. \\
\hline $\mathrm{C}_{3} \mathrm{O}_{2}$ & 10.8 & Impacto \\
\hline \multirow[t]{3}{*}{$\mathrm{HCHO}$} & 10.87 & Fotoionización \\
\hline & 10.87 & Impacto \\
\hline & 10.88 & Espec. \\
\hline $\mathrm{CH}_{3} \mathrm{CH}_{2} \mathrm{CHO}$ & 10.14 & Impacto \\
\hline \multirow[t]{2}{*}{$\mathrm{CH}_{3} \mathrm{CHO}$} & 10.25 & Impacto \\
\hline & 10.25 & Fotoionización \\
\hline \multirow{2}{*}{$\mathrm{CH}_{2}=\mathrm{CHCHO}$} & 10.10 & Fotoionización \\
\hline & 10.14 & Impacto \\
\hline $\mathrm{CH}_{3} \mathrm{CH}=\mathrm{CHCHO}$ & 9.81 & Impacto \\
\hline $\mathrm{CH}_{3} \mathrm{CH}_{2} \mathrm{CH}_{2} \mathrm{CHO}$ & 10.14 & Impacto \\
\hline $\mathrm{HCO}$ & 9.88 & Impacto \\
\hline \multirow[t]{2}{*}{$\mathrm{CH}_{3} \mathrm{CO}$. } & 7.08 & Fotoionización \\
\hline & 8.08 & Impacto \\
\hline \multirow[t]{2}{*}{$\mathrm{CH}_{2}=\mathrm{C}=\mathrm{O}$} & 9.607 & Espec. \\
\hline & 9.60 & Fotoionización \\
\hline \multirow{4}{*}{$\mathrm{CH}_{3} \mathrm{COCH}_{3}$} & 9.69 & Fotoionización \\
\hline & 9.68 & Fotoionización \\
\hline & 9.89 & Impacto \\
\hline & 9.92 & Impacto \\
\hline $\mathrm{CH}_{3} \mathrm{COCH}_{3}$, forma enol & 8.2 & Fotoionización \\
\hline $\mathrm{CH}_{3} \mathrm{COCH}_{2} \mathrm{CH}_{3}$ & 9.58 & Impacto \\
\hline $\mathrm{CH}_{3} \mathrm{COCH}_{2} \mathrm{CH}_{2} \mathrm{CH}_{3}$ & $I_{\mathrm{V}}=9.51 ; I_{\mathrm{N}}=9.48$ & Fotoionización \\
\hline \multirow{2}{*}{$\mathrm{CH}_{3} \mathrm{OH}$} & $I_{\mathrm{V}}=9.41 ; I_{\mathrm{N}}=9.37$ & Fotoionización \\
\hline & 10.85 & Fotoionización \\
\hline $\mathrm{HCOOH}$ & 10.9 & Impacto \\
\hline $\mathrm{CH}_{3} \mathrm{COCl}$ & 11.05 & Fotoionización \\
\hline \multirow[t]{2}{*}{$\mathrm{COCl}_{2}$} & 11.02 & Espec. \\
\hline & 11.78 & Fotoionización \\
\hline \multirow[t]{2}{*}{$\mathrm{HCOOCH}_{3}$} & 11.77 & Impacto \\
\hline & 11.14 & Impacto \\
\hline \multirow[t]{3}{*}{$\mathrm{CH}_{3} \mathrm{OCH}_{3}$} & 10.92 & Impacto \\
\hline & 10.00 & Fotoionización \\
\hline & 10.13 & Impacto \\
\hline
\end{tabular}


Respecto a la energía de enlace carbono-oxígeno, en la Tabla B-8 pueden consultarse para muchas moléculas de interés. Si se compara con la Tabla B-6, se observa que la energía del enlace es inversa a la longitud del enlace. Se observa también que en el caso del monóxido de carbono la energía de enlace es elevadísima, lo que sugiere que en esa molécula el carbono y el oxígeno podrían formar un enlace triple.

Tabla B-8: Energías del enlace carbonilo en diferentes moléculas

\begin{tabular}{ccc}
\hline Compuesto & \multicolumn{2}{c}{ Energía de enlace/ Kcal $\cdot \mathrm{mol}^{-1}$} \\
\hline $\mathrm{CO}$ & & 257.3 \\
$\mathrm{CO}_{2}$ & & 192.1 \\
$\mathrm{CH}_{2}=\mathrm{C}=\mathrm{O}$ & $\mathrm{C}=\mathrm{O}$ & 184.8 \\
$\mathrm{HCHO}$ & $\mathrm{O} \cdots \mathrm{H}$ & 160.0 \\
& $\mathrm{C}=\mathrm{O}$ & 2.0 \\
$\mathrm{CH}_{3} \mathrm{COCH}_{3}$ & $\mathrm{O} \cdots \mathrm{H}$ & 160.0 \\
& $\mathrm{C}=\mathrm{O}$ & 3.1 \\
$\mathrm{HCOOH}$ & $\mathrm{C}-\mathrm{O}$ & 160.0 \\
& $\mathrm{C}=\mathrm{O}$ & 106.0 \\
$\mathrm{HCOOCH}_{3}$ & $\mathrm{C}-\mathrm{O}$ & 160.0 \\
& $\mathrm{C}-\mathrm{O}$ & 106.0 \\
$\mathrm{CH}_{3} \mathrm{OH}$ & & 81.2 \\
\hline
\end{tabular}

El extraño comportamiento del formaldehído puede explicarse asumiendo que hay un enlace intramolecular de tipo puente de hidrógeno [26], con energía estimada de 2 $\mathrm{Kcal} \cdot \mathrm{mol}^{-1}$, entre los protones y el átomo de oxígeno del grupo carbonilo.

\section{B.6 PROPIEDADES ESPECTROSCÓPICAS}

Con el objetivo de interpretar correctamente los espectros de absorción infrarroja que aparecen en el apéndice, en esta sección se da una breve exposición de las propiedades espectroscópicas de aminas y cetonas. Las frecuencias de las vibraciones de los núcleos están en el rango del infrarrojo, luego las discusiones acerca del espectro infrarrojo de una sustancia se refieren a dichas vibraciones. Aunque estas vibraciones no son armónicas, la aproximación a un potencial armónico da buenos resultados. En tal caso, la frecuencia de la vibración $v$ estará relacionada con la constante de fuerza $K$ del oscilador mediante la ecuación siguiente:

$$
v=\frac{1}{2 \cdot \pi} \cdot\left(\frac{K}{\mu}\right)^{1 / 2}
$$

Las vibraciones pueden verse como cambios en los enlaces que unen dos núcleos (o átomos), por lo que el estudio de estas vibraciones es fundamental para describir los enlaces entre átomos dentro de la molécula, y en consecuencia, los grupos funcionales (pues un grupo funcional está caracterizado por el enlace que une ambos núcleos).

Se sabe que una molécula de 1 núcleos tiene $3 \cdot 1-6$ (ó $3 \cdot 1-5$ si la molécula es lineal) modos normales de vibración. Cuando todos los núcleos se mueven en el mismo modo normal, todos ellos se desplazan respecto de sus posiciones de equilibrio según un movimiento armónico simple de igual frecuencia y en fase, aunque con amplitudes diferentes. Por lo tanto, todos los núcleos alcanzan las posiciones de equilibrio y de 
máximo desplazamiento a la vez. En la llustración B-17 se observan los tres modos de vibración de la molécula de agua, y los seis del grupo $\mathrm{CH}_{2}$. Sin embargo, el movimiento más general es la superposición de los diferentes modos, lo que da lugar a las vibraciones esqueletales. Por lo tanto, las vibraciones esqueletales más que dar información acerca de las vibraciones del enlace de un grupo funcional dan información acerca de vibraciones de la molécula como un todo. En algunas situaciones muy favorables en las que las existen relaciones muy concretas entre las amplitudes, las frecuencias y las fases iniciales de los diferentes modos, cada núcleo se moverá respecto su posición de equilibrio dibujando en el espacio elipsoides de revolución, superficies de Lisaijous, etc.

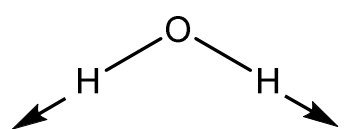

Tensión simétrica

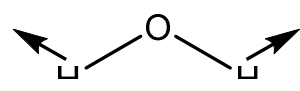

Flexión simétrica<smiles>[CH]O[CH]C</smiles>

Tensión asimétrica

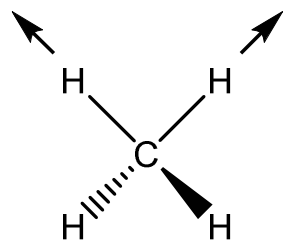

Tensión simétrica

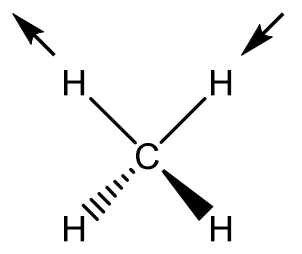

Tensión asimétrica Symmetrical stretching $\left(2925 \mathrm{~cm}^{-1}\right)$ Asymmetrical stretching $\left(2850 \mathrm{~cm}^{-1}\right)$<smiles>CC</smiles>

Flexión asimétrica en el plano Scissoring $\left(1465 \mathrm{~cm}^{-1}\right)$<smiles>[CH2+][CH2+]</smiles>

Flexión simétrica fuera del plano Wagging $\left(1350-1150 \mathrm{~cm}^{-1}\right)$

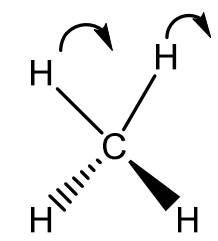

Tensión asimétrica en el plano Rocking $\left(720 \mathrm{~cm}^{-1}\right)$<smiles>[CH2+]</smiles>

Flexión asimétrica fuera del plano Twisting (1350-1150 $\mathrm{cm}^{-1}$ )

Ilustración B-17: modos de vibración de la molécula de agua y del grupo $\mathrm{CH}_{2}$ en la molécula de metano. En el último caso se ha mantenido la denominación en inglés para cada modo normal.

Para explicar los espectros de absorción, es conveniente diferenciar los dos casos siguientes: 
a) Moléculas polares: En este tipo de moléculas, cualquier desplazamiento vibracional de los núcleos respecto de las posiciones de equilibrio provoca un cambio en el momento dipolar eléctrico, el cual puede relacionarse con el índice de absorción de la sustancia, gracias a la ecuación de Clausius-Mossotti. Éste tiene máximos en cada una de las frecuencias naturales del sistema, siendo mayor la absorción cuanto mayor sea el cambio en el momento dipolar.

b) Moléculas apolares: estas moléculas no tienen momento dipolar permanente. Debido a su geometría, existen modos de vibración para los que el momento dipolar inducido sigue siendo cero: son los modos simétricos, como por ejemplo la tensión simétrica. En estos casos el índice de absorción es cero y se dice que la molécula es inactiva a la absorción infrarroja para el modo de tensión simétrica. Es lo que ocurre, por ejemplo, en la molécula $\mathrm{CO}_{2}$. Por el contrario, en los modos asimétricos si existe momento dipolar inducido, de manera que, como en el caso a), se pueda relacionar con el índice de absorción, siendo éste máximo en cada una de las frecuencias naturales de los diferentes modos. En las moléculas diatómicas homonucleares $\left(H_{2}, N_{2}\right.$, $\mathrm{Cl}_{2}$, etc) el momento dipolar inducido siempre es nulo, puesto que los dos átomos que forman la molécula tienen la misma electronegatividad, por lo que no se forman nubes de carga, luego siempre son inactivas a la absorción infrarroja.

En definitiva, independientemente de que la molécula tenga o no dipolo permanente, el espectro de absorción tiene máximos en cada una de las frecuencias naturales de cada uno de los modos, siempre que haya un cambio en el momento dipolar eléctrico de la molécula, lo cual es consecuencia de la relación entre la polarizabilidad y el momento dipolar eléctrico, y la relación de Clasius Massotti. Si no hay momento dipolar inducido, no habrá absorción. Además, la absorción es mayor cuanto mayor sea el cambio del momento dipolar. Por lo tanto, es condición necesaria para que se produzca absorción en el infrarrojo que haya un dipolo inducido. No es condición suficiente, puesto que junto con el cambio del momento inducido se deben cumplir ciertas reglas de selección que se regulan la posibilidad de transición entre los niveles de energía cuánticos.

\section{B.6.1 Aminas}

B.6.1.1 Par de electrones libres

El hecho de que en las aminas el átomo de nitrógeno tenga dos electrones libres fácilmente disponibles para reaccionar es la base de un gran número de métodos de detección y determinación. Además, en muchos casos en los que los productos son aparentemente el resultado de una reacción con los grupos $\mathrm{N}-\mathrm{H}$, la presencia del par de electrones libres es esencial para que la reacción tenga lugar. Por otra parte, este par de electrones libres es el responsable de los enlaces por puente de hidrógeno que se forman en las aminas.

En el espectro de infrarrojo de algunas aminas, las bandas en torno a la región de 2800 $\mathrm{cm}^{-1}$ están asociadas con los electrones libres. Algunas estructuras en las que estas bandas aparecen son: $N$-metil, $N, N$-dimetil (no confundir con $\mathrm{N}$-etil), con bandas de intensidad media-alta; compuestos cíclicos cuando dos o más grupos $\mathrm{C}-\mathrm{H}$ adyacentes están en la posición trans respecto al par de electrones libres (por ejemplo, 10-metiltrans-quinozilidina), con bandas de pequeña intensidad; compuestos como di y $N, N, N$ trietilamina, piperidina, $N$-etilpiperidina,.. , con bandas de pequeña intensidad también. 
Las bandas de los compuestos $N$-metil, pueden ser de ayuda en la caracterización más profunda de la molécula, cuando aparecen en $2810-2820 \mathrm{~cm}^{-1}$ en aminas aromáticas y a $2780-2805 \mathrm{~cm}^{-1}$ en aminas alifáticas.

\section{B.6.1.2 Enlace N-H}

El grupo N-H de las aminas primarias y secundarias tiene las bandas de absorción características que aparecen resumidas en la Tabla B-9:

Tabla B-9: bandas de absorción de los enlaces N-H en aminas [27]-[29]

\begin{tabular}{cc}
\hline Región $/ \mathrm{cm}^{-1}$ & Origen de la banda \\
\hline 1600 & Modos de flexión \\
3400 & $\begin{array}{c}\text { Stretching (fundamental) } \\
\text { Combinación de los modos de } \\
5000\end{array}$ \\
6700 & deformación y stretching \\
10000 & Stretching ( ${ }^{\text {er }}$ armónico) \\
\hline
\end{tabular}

En las aminas primarias, aparece casi siempre una banda intensa entre 1590 y 1650 $\mathrm{cm}^{-1}$, asociada al modo de deformación; en aminas aromáticas, se puede confundir con una banda debida a la vibración esqueletal del anillo aromático, en torno a 1600 $\mathrm{cm}^{-1}$. Sin embargo, la región del espectro más interesante es la asociada a los modos de tensión (también llamados stretching o vibración longitudinal).

El modo de tensión del enlace N-H se corresponde con una banda entre 3500 y 3400 $\mathrm{cm}^{-1}$, en el espectro de las aminas alifáticas secundarias tomadas en disolución diluida con $\mathrm{CCl}_{4}$ para evitar la asociación molecular. Bajo estas condiciones, las aminas primarias muestran dos bandas entre 3500 y $3400 \mathrm{~cm}^{-1}$ correspondientes a los modos de tensión simétrico y antisimétrico. También encontramos dos frecuencias asociadas a los modos de tensión simétrico y antisimétrico en el espectro del amoniaco en aproximadamente la misma región (3506 y $3577 \mathrm{~cm}^{-1}$ ). Por tanto, en la aproximación al potencial de oscilador armónico, se puede decir que la constante de fuerza $K$ asociada a dicho potencial es del mismo orden de magnitud para el amoniaco y para las aminas primarias y secundarias, lo que confirma el hecho de que la estructura electrónica de los enlaces $\mathrm{N}-\mathrm{H}$ no será demasiado diferente en todas estas moléculas.

Como se acaba de decir, aparecen dos bandas en torno a 3400 y $3500 \mathrm{~cm}^{-1}$ en el espectro de las aminas primarias. La banda de menor frecuencia está asociada con el modo simétrico, y la de mayor frecuencia, con el modo antisimétrico, siendo ambas de intensidad media. Este par de bandas está relacionado por la ecuación siguiente:

$$
\lambda_{\text {sim }}^{-1}=0.876 \cdot \lambda_{\text {asim }}^{-1}+345.5 \mathrm{~cm}^{-1}
$$

La intensidad de las bandas asociadas a los modos de tensión son mucho mayores para las aminas aromáticas y algunas aminas heterocíclicas que para aminas alifáticas. En el espectro de las aminas primarias se encuentran bandas de absorción en la región de $5000 \mathrm{~cm}^{-1}$, probablemente debidas a la combinación de modos de tensión y flexión/deformación.

También se observan en el espectro bandas asociadas a los armónicos de los diferentes modos. Por ejemplo, en el caso de aminas aromáticas primarias, se encuentran los primeros armónicos de los modos de vibración longitudinal en torno a $6700 \mathrm{~cm}^{-1}$ 
(simétrico) y $6900 \mathrm{~cm}^{-1}$ (antisimétrico), siendo el primero unas 6 veces más intenso que el segundo. En el caso de aminas aromáticas secundarias sólo se encuentra una banda en esta región, mucho menos intensa. Las aminas alifáticas primarias y secundarias absorben en torno a $6500 \mathrm{~cm}^{-1}$.

La señal del segundo armónico es mucho menos intensa, y solo se puede observar en aminas aromáticas en torno a los $9800 \mathrm{~cm}^{-1}$.

\section{B.6.1.3 Enlace N-C}

La Tabla B-10 resume las bandas de absorción infrarroja asignadas al enlace N-C. En las aminas alifáticas, la banda es difícil de encontrar debido a su baja intensidad y al hecho de que aparece en una región en la que lo hacen otras bandas asociadas a las vibraciones de otros grupos funcionales. Las bandas correspondientes a las aminas aromáticas se pueden utilizar para la confirmación de las estructuras propuestas, a pesar de que la posibilidad de una confusión con bandas pertenecientes a otros grupos también debe mantenerse en mente.

Tabla B-10: bandas de absorción en los modos de tensión de los enlaces N-C [30], [31]

\begin{tabular}{ccc}
\hline Tipo de compuesto & Región $/ \mathrm{cm}^{-1}$ & Intensidad \\
\hline & Aminas alifáticas & \\
& $1020-1220$ & Débil a media \\
\hline Primaria & Aminas aromáticas & \\
Secundaria & $1250-1340$ & Fuerte \\
Terciaria & $1280-1350$ & Fuerte \\
\hline
\end{tabular}

\section{B.6.2 Cetonas}

Aunque como ya hemos visto, son varios los modos de vibración de una molécula, de ahora en adelante sólo hablaremos de los modos de vibración longitudinal (stretching o de tensión).

Todos los compuestos con el grupo carbonilo muestran una banda muy intensa en la región 1650-1850 $\mathrm{cm}^{-1}$ (véase la Tabla B-11). Es fácil identificar un grupo carbonilo mediante una vibración localizada en esta región, pues además de que el conjunto de moléculas con grupos funcionales cuyas frecuencias de vibración son similares a las del grupo carbonilo es muy pequeño $(\mathrm{C}=\mathrm{C},>\mathrm{NH}$ y $-\mathrm{OH})$, son bandas mucho menos intensas que la del carbonilo, salvo en el caso de $-\mathrm{C}-\mathrm{NH}$.

La región en la que aparece este modo de vibración en compuestos similares es muy estrecha. Por ejemplo, en la mayoría de los aldehídos aromáticos se encuentra una banda correspondiente a este modo de vibración en la región 1670-1750 cm-1.

El análisis de las frecuencias del modo de elongación del grupo carbonilo en diferentes tipos de moléculas sugiere que la posición observada de las bandas resulta ser la suma de varios factores entre los cuales están: estado físico del compuesto; efectos inductivos; efectos másicos y electrónicos en las proximidades de los constituyentes; enlace por puente de hidrógeno; enolización; efectos de los disolventes. Cabe señalar que el primer factor es muy determinante, de hecho, para la mayoría de compuestos la frecuencia es mayor en estado gaseoso que para la fase líquida o sólida. Por ejemplo, 
en la ciclohexanona gaseosa, la frecuencia de absorción es $1742 \mathrm{~cm}^{-1}$, y en la fase líquida $1717 \mathrm{~cm}^{-1}$.

Tabla B-1 1: frecuencia del modo de elongación de compuestos con el grupo carbonilo [21, pp 16]

\begin{tabular}{|c|c|c|}
\hline \multirow{2}{*}{ Molécula } & \multicolumn{2}{|c|}{ Frecuencia $v_{\mathrm{C}=\mathrm{O}}$ del modo de elongación $/ \mathrm{cm}$} \\
\hline & Fase vapor & Disolución \\
\hline $\mathrm{CH}_{3} \mathrm{COF}$ & 1869 & \\
\hline $\mathrm{CH}_{3} \mathrm{COCl}$ & 1822 & \\
\hline $\mathrm{CH}_{3} \mathrm{COBr}$ & 1821 & \\
\hline $\mathrm{CH}_{3} \mathrm{CHO}$ & 1743 & \\
\hline$\left(\mathrm{CH}_{3}\right)_{2} \mathrm{CO}$ & 1738,1736 & \\
\hline $\mathrm{CH}_{3} \mathrm{COCN}$ & 1740 & \\
\hline $\mathrm{HCHO}$ & 1744 & \\
\hline $\mathrm{C}_{6} \mathrm{H}_{5} \mathrm{CHO}$ & & 1710 \\
\hline Ciclohexanona & 1742 & 1717 \\
\hline Ciclopentanona & & 1746,1728 \\
\hline Ciclobutanona & 1816 & \\
\hline $\mathrm{HCOOC}_{2} \mathrm{H}_{5}$ & & 1733 \\
\hline $\mathrm{CH}_{3} \mathrm{COOCH}_{3}$ & 1770 & \\
\hline $\mathrm{CH}_{3} \mathrm{COOC}_{2} \mathrm{H}_{5}$ & & 1745 \\
\hline $\mathrm{ClCOOC}_{2} \mathrm{H}_{5}$ & & 1782 \\
\hline $\mathrm{C}_{6} \mathrm{H}_{5} \mathrm{COOC}_{2} \mathrm{H}_{5}$ & & 1725 \\
\hline $\mathrm{CH}_{3} \mathrm{CH}_{2} \mathrm{COOC}_{2} \mathrm{H}_{5}$ & & 1741 \\
\hline $\mathrm{CH}_{3} \mathrm{CH}_{2} \mathrm{CH}_{2} \mathrm{COOC}_{2} \mathrm{H}_{5}$ & & 1739 \\
\hline $\mathrm{C}_{6} \mathrm{H}_{5} \mathrm{COCH}_{3}$ & 1709 & 1689 \\
\hline $\mathrm{C}_{6} \mathrm{H}_{5} \mathrm{COCH}_{2} \mathrm{CH}_{3}$ & & 1694 \\
\hline $\mathrm{COBrF}$ & & 1874 \\
\hline COCIF & & 1868 \\
\hline $\mathrm{COF}_{2}$ & & 1928 \\
\hline $\mathrm{COCl}_{2}$ & & 1827 \\
\hline $\mathrm{HCOCHO}$ & 1730 & \\
\hline $\mathrm{CH}_{2}=\mathrm{CHCHO}$ & 1724 & \\
\hline $\mathrm{CH} \equiv \mathrm{CCHO}$ & 1697 & \\
\hline p-Benzoquinona & $1689,1683,1667$ & \\
\hline p-Aminoacetofenona & & 1677 \\
\hline p-Metilacetofenona & & 1687 \\
\hline p-Fluoroacetofenona & & 1692 \\
\hline p-Nitroacetofenona & & 1700 \\
\hline
\end{tabular}

En la aproximación del potencial armónico, la constante de fuerza $K$ puede cambiar con la distribución de electrones en la molécula. Estas variaciones normalmente se interpretan como resultado de efectos mesoméricos e inductivos. En los grupos funcionales con fuerte efecto inductivo de atracción de electrones, como ocurre en la acetona, la carga negativa del oxígeno disminuye por lo que la frecuencia de vibración aumenta en comparación con la de las cetonas normales. En el caso de efecto inductivo repulsivo, que es mayor, por ejemplo, en el grupo etilo que en el metilo, se genera un aumento de carga negativa en el oxígeno, siendo este efecto el responsable del corrimiento de la frecuencia de vibración desde $1689 \mathrm{~cm}^{-1}$ (acetona) hasta $1694 \mathrm{~cm}^{-1}$ (etilfenilcetona).

El efecto mesomérico o efecto de resonancia ocurre cuando el doble enlace $\mathrm{C}=\mathrm{O}$ está conjugado por otro doble enlace o con un par de electrones libres. En este caso el enlace entre el carbono y el oxígeno es más polar, la constante de fuerza es menor, y 
las bandas correspondientes al modo de elongación están desplazadas hacia frecuencias menores.

Los efectos sobre el desplazamiento de la banda del espectro debido a los otros efectos mencionados pueden consultarse en la bibliografía [21, pp 13]. 


\section{Análisis espectroscópico de algunas mezclas amina+cetona.} Iminas

En la etapa experimental de este trabajo, surgieron varias dificultades. En primer lugar se observaron fluctuaciones en la densidad cuando se intentó medir en mezclas amina primaria +cetona (en concreto, ciclohexilamina, benzilamina, hexilamina ó butilamina + 2-butanona). En segundo lugar, al intentar medir las entalpías de exceso de dichos sistemas, se observó un comportamiento anómalo. Estos resultados sugieren la ocurrencia de una reacción química. En concreto, se estudió la evolución temporal de la densidad de la mezcla hexilamina+2-butanona $\left(x_{1} \approx 0.5\right)$ durante 48 horas y se observó que ésta no cesaba de cambiar. Además, se intentó medir su entalpía de exceso. El voltaje de salida del microcalorimetro Calvet se muestra en la llustración C-1:

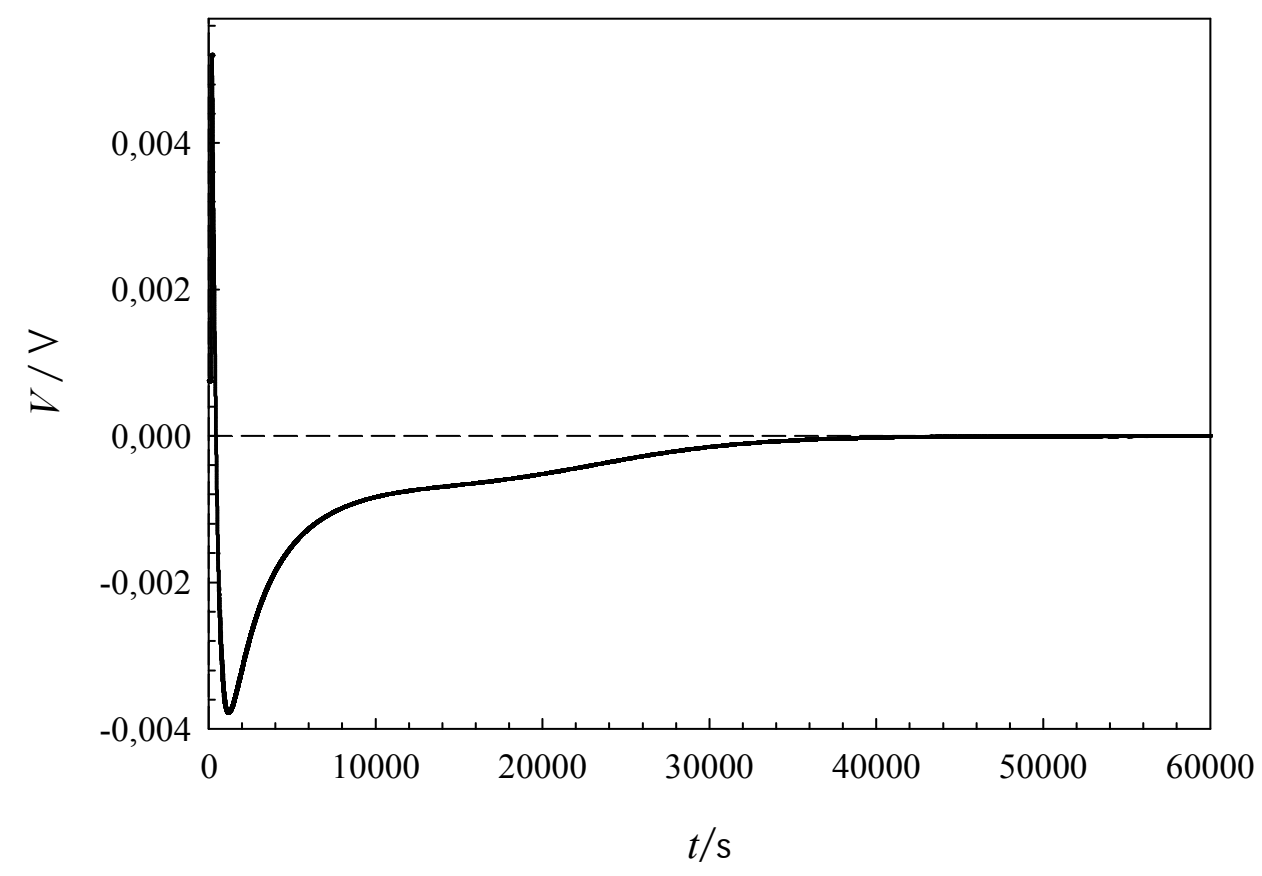

Ilustración C-1: voltaje de salida del microcalorimetro Calvet para la mezcla hexilamina +2-butanona, $x_{1}=0.5025$ 
El calor medido es mucho mayor que el de las mezclas típicas. Además, la mezcla se desarrolla durante un tiempo muy largo $\left(4 \cdot 10^{4} \mathrm{~s}\right)$. Existen dos etapas bien diferenciadas: $1^{a}$, endotérmica; $2^{a}$, exotérmica. Esto se observa mejor en la llustración C-2:

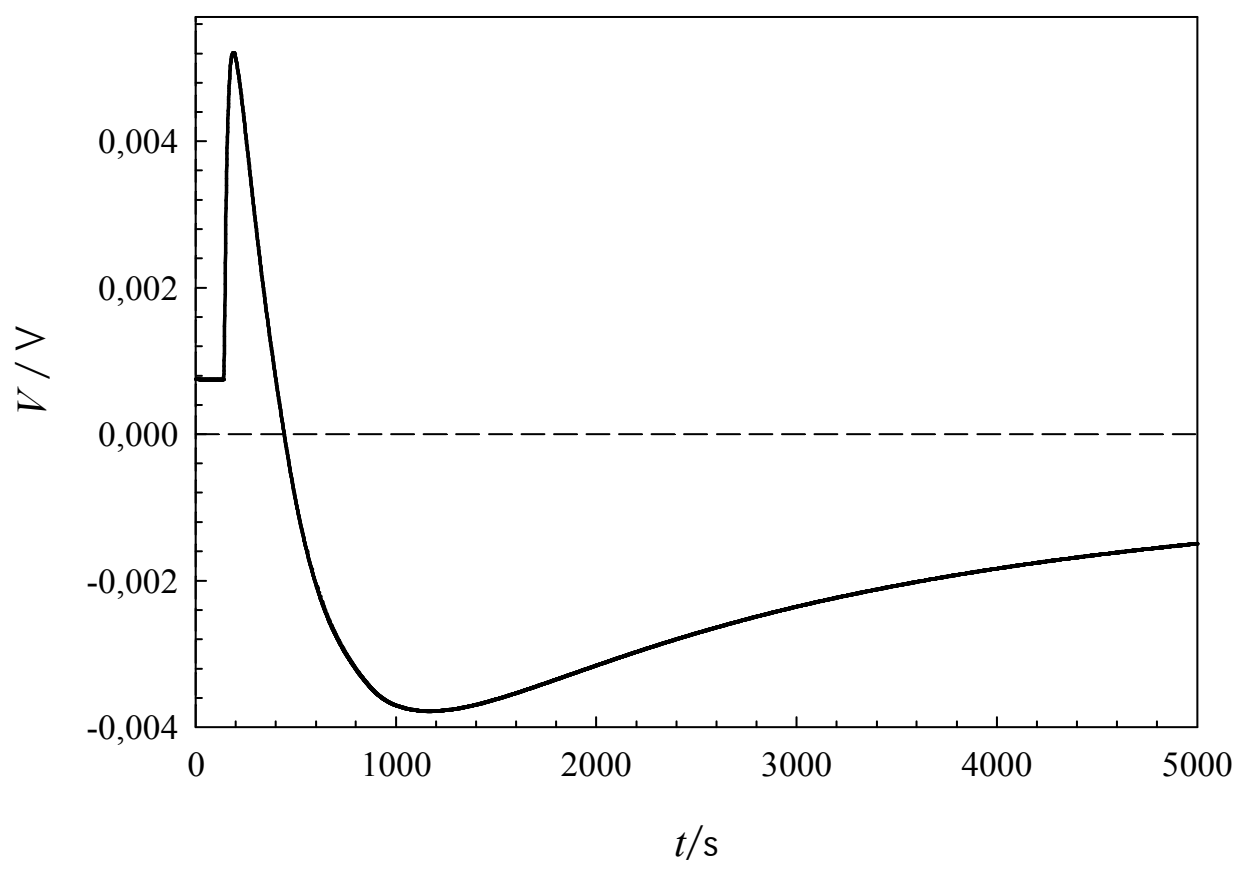

Ilustración C-2: ampliación de la región 0-5000 s del la llustración C-1

En una mezcla típica, como la anilina (1)+2-butanona (2), $x_{1}=0.4894$, la señal de salida tiene una forma completamente diferente (véase la llustración C-3).

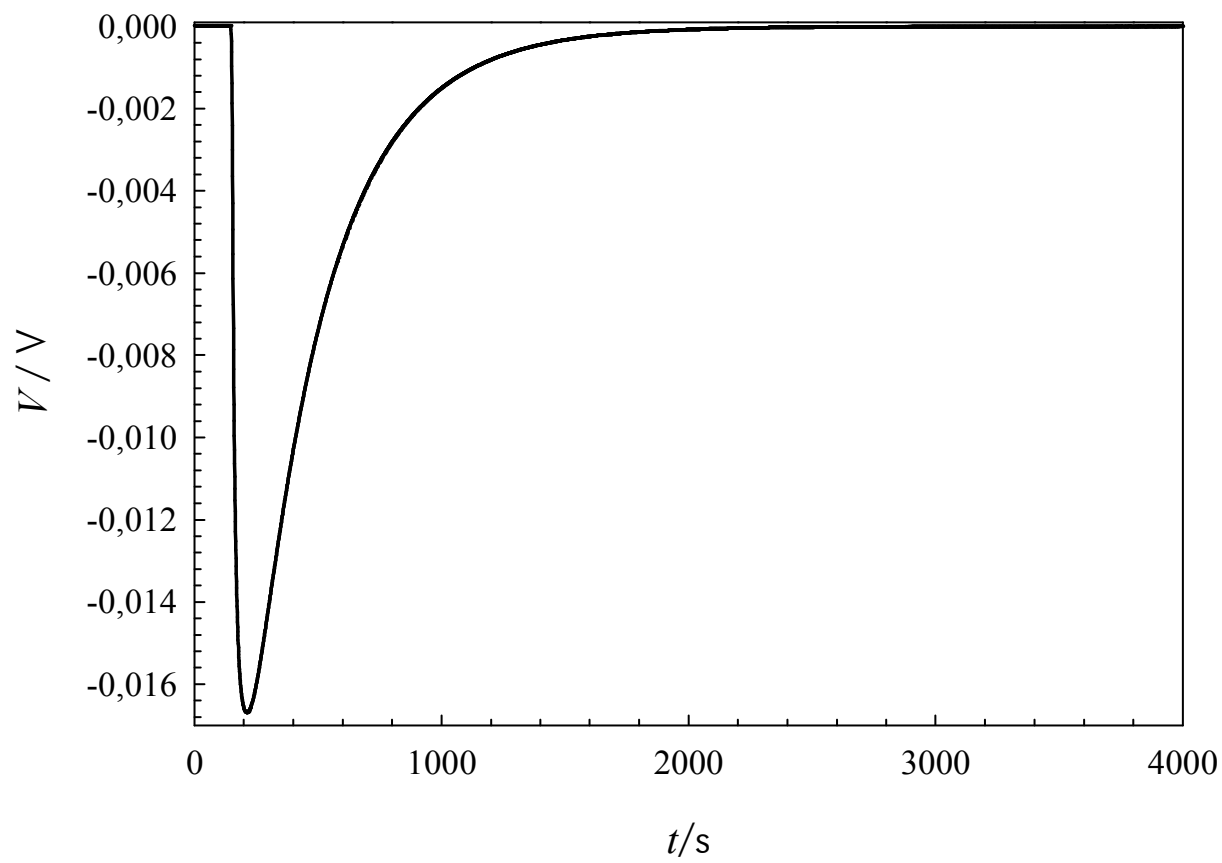

Ilustración C-3: voltaje de salida del microcalorimetro Calvet para la mezcla anilina(1) + 2-butanona, $\mathrm{x}_{1}=0.4894$ 
Estos resultados no eran de esperar, pues las únicas reacciones conocidas entre aminas y cetonas son aquellas que dan lugar a la formación de las llamadas bases de Schiff, iminas, y enaminas las cuales ocurren en condiciones muy particulares, que se enumeran unas líneas más abajo. Las iminas y las bases de Schiff son importantes en numerosos mecanismos bioquímicos, puesto que se pueden formar a partir de aminoácidos, y proteínas.

La condensación de aldehídos y cetonas con aminas primarias la descubrió Schiff en 1864, y por eso las bases resultantes llevan su nombre. En este tipo de reacciones, se produce un enlace doble $\mathrm{R}-\mathrm{N}=\mathrm{C}$ y agua. Las propiedades del enlace $\mathrm{C}=\mathrm{N}$ son similares a las del enlace $\mathrm{C}=\mathrm{O}$ razón por lo cual las iminas tienen propiedades similares a las de los compuestos carbonílicos. Si R es un hidrógeno, el compuesto será una imina primaria, y en caso contrario será en general una base de Schiff, pudiendo ser una imina secundaria si $\mathrm{R}$ es una cadena alquílica, una oxima si $\mathrm{R}$ es el grupo hidroxilo, $\mathrm{OH}, \mathrm{O}$ una hidrazona si $\mathrm{R}$ es $\mathrm{NH}_{2}$. La condensación de aldehídos y cetonas enolizables con aminas secundarias pueden originar enaminas, pero en estas ya no está presente el doble enlace $\mathrm{C}=\mathrm{N}$.

En estas reacciones de condensación el equilibrio está desplazado hacia el compuesto carbonílico y la amina, por lo que si se desea favorecer la formación de la imina se necesita realizar destilación azeotrópica o utilizar agentes deshidratantes como por ejemplo tamiz molecular o sulfato de magnesio.

Las cetonas son, en general, menos reactivas que los aldehídos en lo que a formación de iminas se refiere. Las cetonas con impedimento estérico son en particular poco reactivas. La reactividad de las aminas es paralela a su basicidad, o más correctamente, a su carácter como nucleófilo. Además, la formación de bases de Schiff se ve favorecida por la presencia de radiación ultravioleta.

El mecanismo de formación de iminas comienza por la adición nucleófila de la amina sobre el carbonilo electrófilo formándose un hemianal como intermedio, el cual a continuación pierde una molécula de agua para generar la imina [32]:<smiles>[R]C=[OH+]</smiles><smiles>[R]NC</smiles><smiles></smiles><smiles>[R]C=[NH+]</smiles><smiles>O</smiles>

La reacción requiere catálisis ácida, altas temperaturas y elevados tiempos de reacción. Además, como el agua es un producto de reacción y en la reacción está involucrado el equilibrio, las condiciones más favorables para que se produzca la reacción completamente se tienen en un medio no acuoso, razón por la que se requiere el uso de agentes deshidratantes o de producir la reacción de forma azeótropa.

Es posible la aparición de pares de iminas tautómeras en la condensación de aminas alifáticas con hidrógenos $\alpha$ :
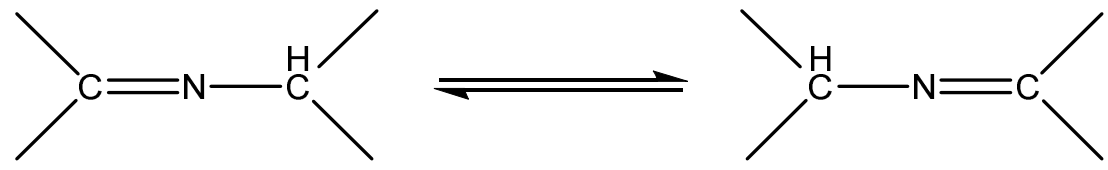
Las condiciones descritas para la síntesis de iminas no eran las presentes en el laboratorio, razón por la cual no era de esperar su formación. No obstante, como se ha dicho, los resultados obtenidos para la densidad y la entalpía de exceso sugerían lo contrario. Por ello se decidió a proceder con el análisis en el tiempo del espectro de absorción infrarroja (IR) de las mezclas en cuestión, pues si entre la amina y la cetona se forman enlaces $\mathrm{C}=\mathrm{N}$, este tendrá modos de vibración característicos, no presentes en la amina y la cetona puras, cuyas frecuencias están en el rango de los infrarrojos. Para ello se utilizó el espectrómetro IR del Departamento de Química Orgánica de la Facultad de Ciencias de la Universidad de Valladolid en colaboración con la profesora M. P. Cuadrado. El método de trabajo utilizado fue el siguiente:

El lunes 10 de octubre de 2011 a primera hora de la mañana se prepararon unos 10 $\mathrm{ml}$ de las siguientes mezclas*:

$$
\begin{aligned}
& \text { benzilamina (1)+2-butanona (2), } x_{1}=0,6528 . \\
& \text { ciclohexilamina (1) }+2 \text {-butanona (2), } x_{1}=0,5043 . \\
& \text { hexilamina (1) }+2 \text {-butanona (2), } x_{1}=0,3203 . \\
& \text { anilina (1) }+2 \text {-butanona (2), } x_{1}=0,4959 . \\
& \text { piridina (1) }+2 \text {-butanona (2), } x_{1}=0,5000 . \\
& N \text {-metilanilina (1) }+2 \text {-butanona (2), } x_{1}=0,4937 . \\
& \text { di-n-propilamina (1) }+2 \text {-butanona (2), } x_{1}=0,4905 . \\
& \text { di-n-butilamina (1) }+2 \text {-butanona (2), } x_{1}=0,4983 . \\
& N, N, N \text {-trietilamina (1) }+2 \text {-butanona (2), } x_{1}=0.4550 .
\end{aligned}
$$

Seguidamente se procedió al análisis espectroscópico de cada una de las mezclas, todos los días de la semana (excepto el miércoles por ser festivo y estar cerrada la Facultad) a la misma hora del día, procurando que el intervalo de tiempo entre espectros fuese de unas 24 horas (excepto de martes a jueves, lógicamente). Se almacenaron las mezclas todo el fin de semana para volver a realizar el espectro por última vez, el lunes 17 de octubre. De esta manera, se puede hacer un estudio de la evolución temporal del espectro de IR de cada sistema en consideración.

En el Anexo B se han tabulado las principales propiedades espectroscópicas de las aminas y las cetonas, por lo que la interpretación de los espectros que se presentan en las siguientes páginas es más sencilla. En ellos se muestra la transmitancia en tanto por ciento en función del inverso de la longitud de onda, en la región desde 4000 hasta 600 $\mathrm{cm}^{-1}$, en donde el código de colores empleado es el siguiente: -, lunes 10 de octubre del 2011; - martes 11 de octubre del 2011; - jueves 13 de octubre del 2011; viernes 14 de octubre del 2011 ; - lunes 17 de octubre del 2011.

Además, se muestra ampliada la región $1760-1580 \mathrm{~cm}^{-1}$, en la que aparecen los picos debidos a los modos de stretching (vibración longitudinal) del doble enlace $\mathrm{C}=\mathrm{O}$ del compuesto carbonílico y del doble enlace $\mathrm{C}=\mathrm{N}$ de la imina, que son los de interés para el objetivo aquí propuesto (se verá que mientras la intensidad del enlace $\mathrm{C}=\mathrm{O}$ disminuye con el paso del tiempo, la intensidad del enlace $\mathrm{C}=\mathrm{N}$ aumenta). Como la intensidad de

\footnotetext{
* Nótese que también se procedió al análisis del espectro de infrarrojos de algunas mezclas que no dieron ningún problema (aminas no primarias) para comparar los diferentes resultados obtenidos.
} 
las bandas depende de la cantidad del líquido que se utilizó en la celda (que es de $\mathrm{NaCl}$ cristalizado) para obtener el espectro, y en la práctica es imposible añadir en cada experiencia la misma cantidad de mezcla, la comparación directa de los espectros no tienen sentido y se debe hacer relativa a algún valor. Para ello, en la Tabla C-1 se muestra la evolución temporal del cociente de la absortancia ${ }^{*} \tau$ del enlace $\mathrm{C}=\mathrm{N}$ dividida por la del enlace $\mathrm{C}=\mathrm{O}$. En ella se observa que el valor de dicho cociente crece en el tiempo hasta llegar a un valor constante, lo que indica que la reacción ha llegado al equilibrio.

A continuación se interpretan los espectros leídos de izquierda a derecha, es decir, en orden decreciente del número de ondas $1 / \lambda$ :

En mezclas con aminas primarias, aparecen dos bandas cercanas a $3400 \mathrm{~cm}^{-1}$ y a 3500 $\mathrm{cm}^{-1}$, debidas a los modos de tensión simétrico y antisimétrico del enlace $\mathrm{H}-\mathrm{N}-\mathrm{H}$, como se observa en las mezclas de 2-butanona + bencilamina, ciclohexilamina, hexilamina o anilina. En aminas secundarias y sus mezclas ( $N$-metilanilina, di- $n$-propilamina y di- $n$ butilamina) solamente aparece un pico en dicha zona del espectro, debido a que sólo hay un enlace $\mathrm{N}-\mathrm{H}$ y por ello sólo hay un modo de vibración de tensión. Si se formasen puentes de hidrógeno, aparecería otra banda en esta región, por eso los espectros de la di-n-propilamina y la di-n-butilamina presentan dos picos en esta zona (uno debido al puente y otro debido al enlace $\mathrm{N}-\mathrm{H}$ ), el espectro de la anilina presenta un tercer pico en esta región, y en los espectros de la bencilamina, la ciclohexilamina y la hexilamina los dos picos debidos a los modos simétrico y antisimétrico del enlace $\mathrm{H}-\mathrm{N}$ se confunden por el ensanchamiento debido al puente de hidrógeno. La $\mathrm{N}$-metilanilina no presenta esta banda debido a la ausencia de puente de hidrógeno. Las aminas terciarias no tienen bandas en esta región por no presentar enlace $\mathrm{N}-\mathrm{H}$ (piridina y $N, N, N$-trietilamina) ni elaces de hidrógeno. Hay que mencionar que a $3500 \mathrm{~cm}^{-1}$ hay una pequeña contribución debida a un armónico del enlace $\mathrm{C}=\mathrm{O}$, por eso el espectro de la mezcla 2butanona + piridina se observa una banda en esta región.

Más a la derecha del espectro, se observan bandas de intensidad moderada (mezclas 2-butanona +amina aromática) a intensa (2-butanona + amina alifática). Esto se debe a que los compuestos aromáticos como la bencilamina, anilina, $N$-metilanilina y la piridina presentan bandas debidas al modo de elongación del grupo arilo en la zona $3080-3030 \mathrm{~cm}^{-1}$ y los compuestos alifáticos presentan picos intensos en la región en torno a 2800-2900 $\mathrm{cm}^{-1}$, debido su carácter alquílico, como la ciclohexilamina, hexilamina, di- $n$-propilamina, di- $n$-butilamina y $N, N, N$-trietilamina y la 2 -butanona. En la región 2780-2820 $\mathrm{cm}^{-1}$ a veces aparecen picos debidos al par de electrones libres de la amina, ya sea ésta alifática o aromática, como se observa en los casos de la ciclohexilamina, $\mathrm{N}$-metilanilina, di- $n$-propilamina, di- $n$-butilamina y $N, N, N$-trietilamina.

La banda en torno a $1720 \mathrm{~cm}^{-1}$ se debe al modo de elongación del doble enlace $\mathrm{C}=\mathrm{O}$ de la 2-butanona.

El pico en la región $1660 \mathrm{~cm}^{-1}$ se debe al doble enlace $\mathrm{C}=\mathrm{N}$, característico de la imina, el cual sólo aparece en las mezclas de 2-butanona + amina primaria, excepto en la

* Cuando radiación electromagnética incide en un medio absorbente, parte de la intensidad $I_{0}$ se absorbe $I_{A}$ y parte atraviesa el medio $I$ : $I_{0}=I+I_{A}$. Se verifica la ley de Beer-Lambert: $I=I_{0} \cdot e^{-A}$, donde $A$ es la absorbancia del medio y $T$ es su transmitancia: $T=I / I_{0}=e^{-A}$. Fácilmente se obtiene que $1=T+\tau$, siendo $\tau=I_{A} / I_{0}$ la absortancia del medio. 
anilina. En estas mezclas se forma imina, y como se ve en la Tabla C-1, la intensidad de la banda asociada al enlace $\mathrm{C}=\mathrm{N}$ relativa a la del enlace $\mathrm{C}=\mathrm{O}$ aumenta hasta llegar a un valor máximo, que es cuando la reacción ha llegado al equilibrio. La anilina no forma imina puesto que en ésta el par de electrones libres del nitrógeno está deslocalizado en el anillo aromático y por ello el átomo de nitrógeno en esta situación no es nucleófilo. En aminas secundarias y terciarias no se forman iminas probablemente por impedimento estérico.

En las aminas primarias (bencilamina, ciclohexilamina y anilina) se observa una banda en torno a $1600 \mathrm{~cm}^{-1}$ debida al modo de deformación del enlace $\mathrm{N}-\mathrm{H}$. En las aminas aromáticas, aparece un pico en estas frecuencias debido a la vibración esqueletal del anillo aromático, y suele aparecer otro en $1500 \mathrm{~cm}^{-1}$ también debido a vibraciones esqueletales. En la di-n-propilamina, y di-n-butilamina se observan estos picos en frecuencias menores a $1580 \mathrm{~cm}^{-1}$ y mayores de 1490 . En la región $1450-1380 \mathrm{~cm}^{-1}$ aparecen bandas debidas a modos de deformación asimétrica de los enlaces C-H de las cadenas alquílicas de la 2-butanona y de las aminas alifáticas. También aparece un pico en 1400-1440 $\mathrm{cm}^{-1}$ debido al modo de deformación asimétrica los grupos $\mathrm{CH}_{2}$ activados contiguos a $\mathrm{C}=\mathrm{O}$ y $\mathrm{C}=\mathrm{C}$, debidos a la cetona y el anillo aromático. En aminas aromáticas y terciarias (anilina, $N$-metilanilina, $N, N, N$-trietilamina y sus mezclas) suelen aparecer dos bandas sin valor práctico, debidas al modo longitudinal del enlace C-N en la región 1360-1000 $\mathrm{cm}^{-1}$.

El pico en torno a $1200 \mathrm{~cm}^{-1}$ no tiene valor práctico y se debe al enlace $\mathrm{C}=\mathrm{O}$ de la cetona. A veces este pico también se debe a vibraciones esqueletales del compuesto alifático (di- $n$-propilamina, di-n-butilamina, $N, N, N$-trietilamina y sus mezclas), aunque a veces puede confundirse con bandas debidas al modo de deformación en el plano del anillo aromático, en caso de ser un compuesto bencénico (caso de la anilina y la $\mathrm{N}$ metilanilina y sus mezclas).

La banda ancha que aparece en aminas no terciarias (bencilamina, ciclohexilamina, hexilamina, di-n-propilamina, di- $n$-butilamina y sus mezclas) por debajo de $1000 \mathrm{~cm}^{-1} \mathrm{se}$ debe a los modos de deformación fuera del plano del enlace $\mathrm{N}-\mathrm{H}$ y carece de valor práctico. En compuestos aromáticos (bencilamina, anilina, piridina, $N$-metilanilina y sus mezclas) puede haber de una a tres bandas en dicha región y se debe a deformaciones fuera del plano del anillo bencénico.

A continuación se muestran todos los espectros obtenidos. En el eje de las abscisas se representa el inverso de la longitud de onda, de mayor a menor (o sea, en orden creciente de la longitud de onda) y en el eje de las ordenadas se representa el tanto por ciento de la transmitancia. Nótese que dependiendo del día, los picos asociados a los mismos modos de vibración pueden ser más o menos intensos, lo cual es función de la cantidad de muestra presente en la celda de medida (salvo en el caso de que haya reacción química), como se ha discutido previamente. 


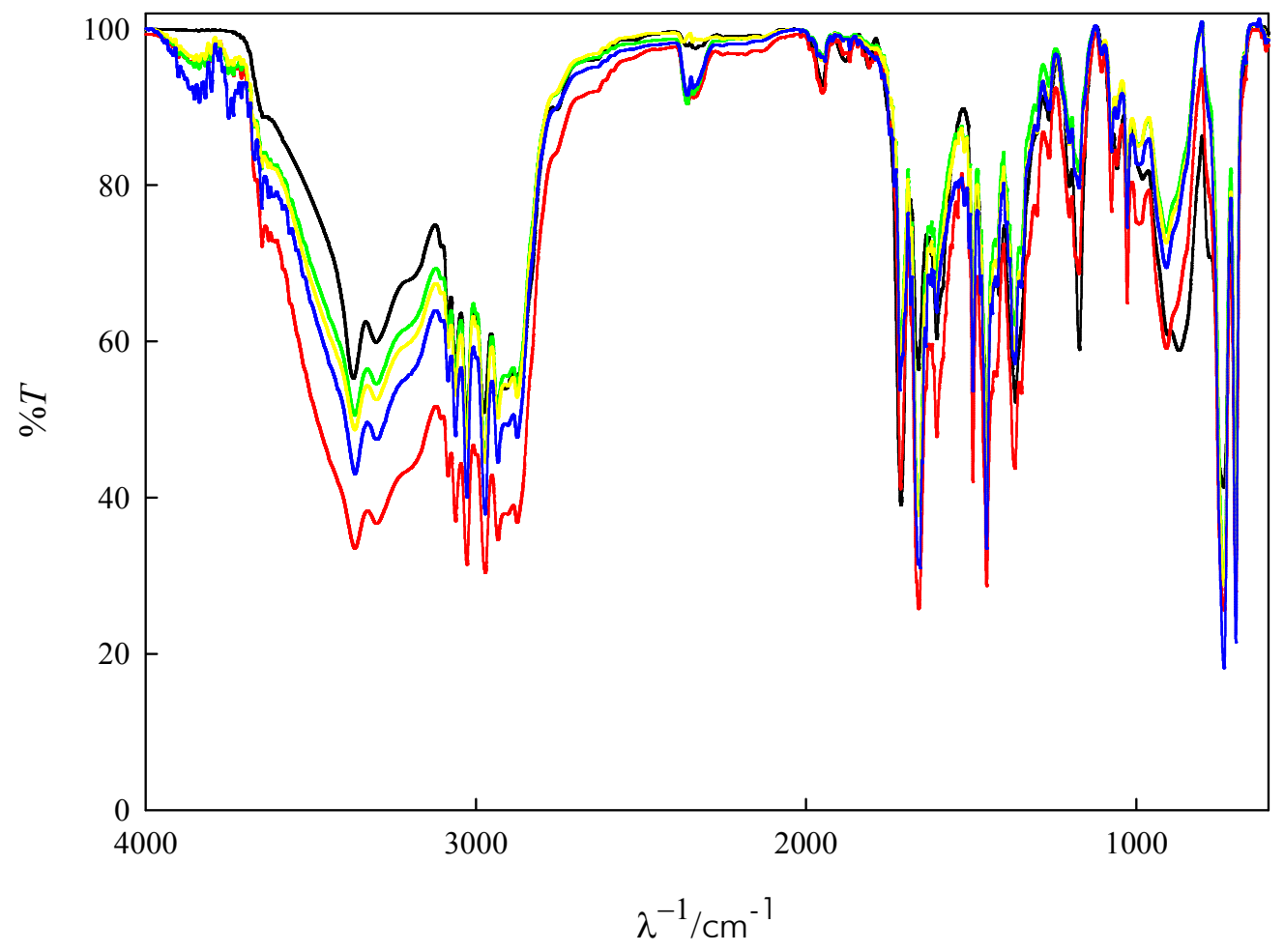

Ilustración C-4: espectro infrarrojo de la mezcla benzilamina (1) +2-butanona (2), $x_{1}=0,6528$

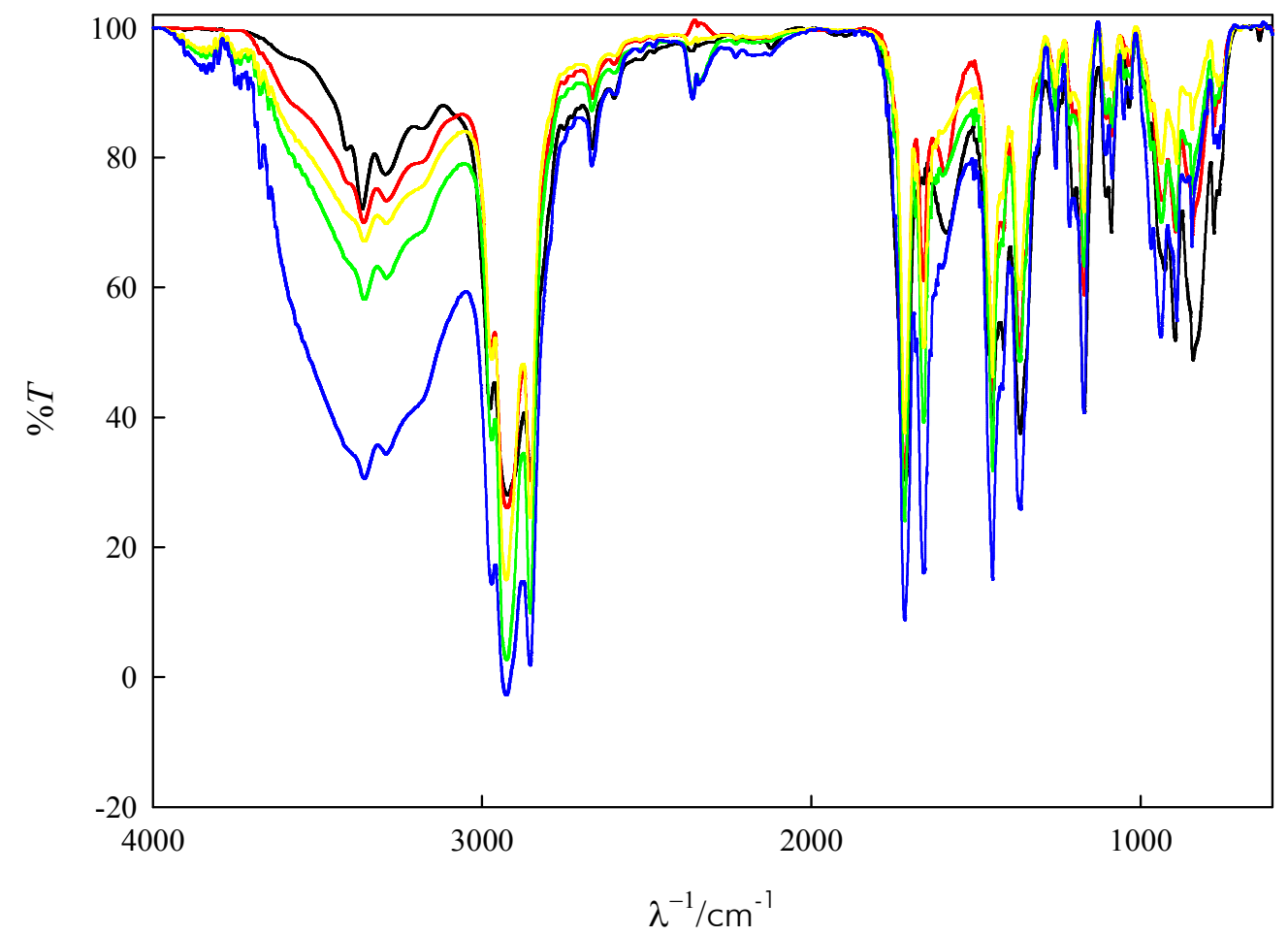

Ilustración C-5: espectro infrarrojo de la mezcla ciclohexilamina (1)+2-butanona (2), $x_{1}=0,5043$ 


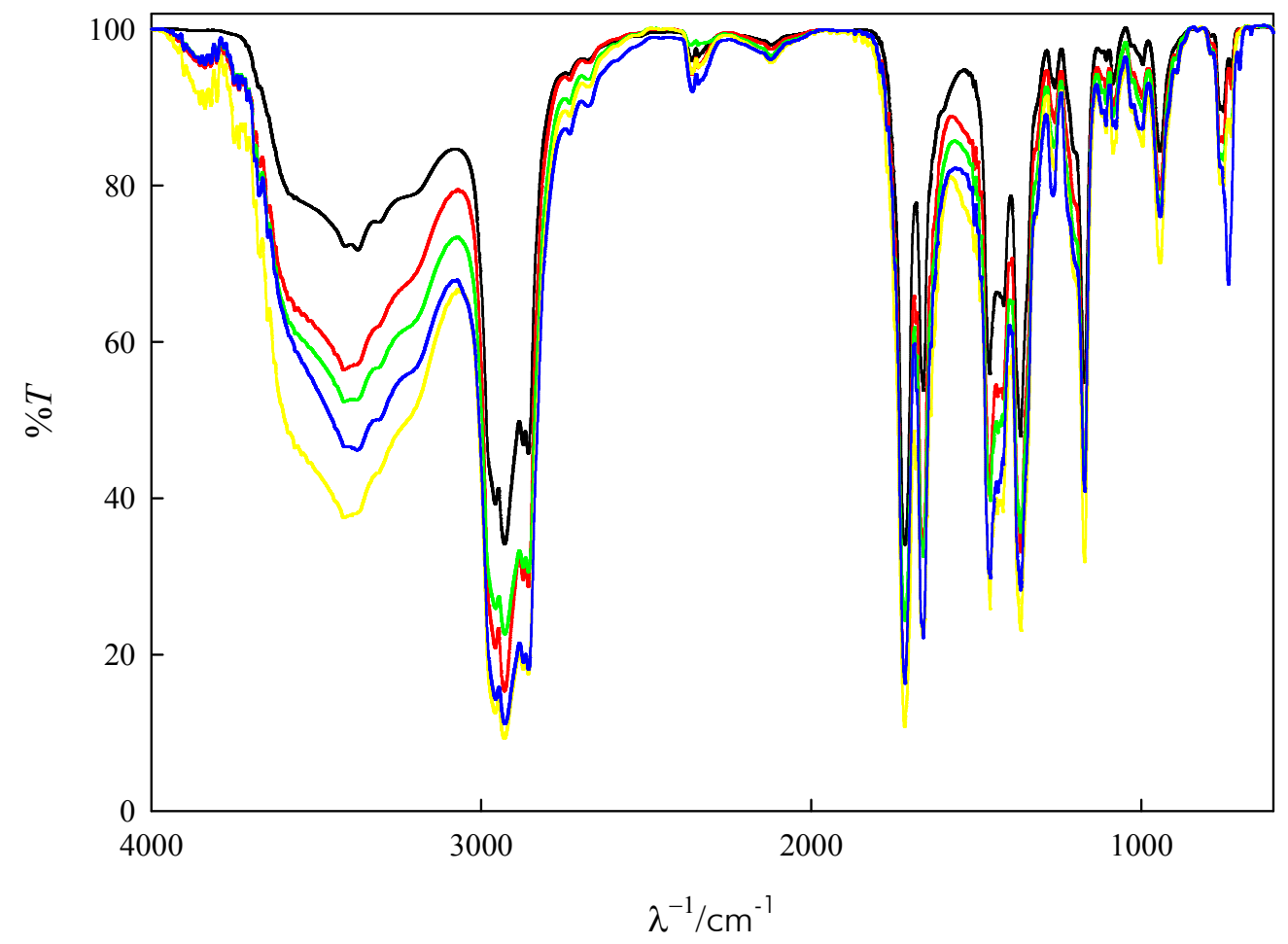

Ilustración C-6: espectro infrarrojo de la mezcla hexilamina (1) +2 -butanona (2), $x_{1}=0,3203$

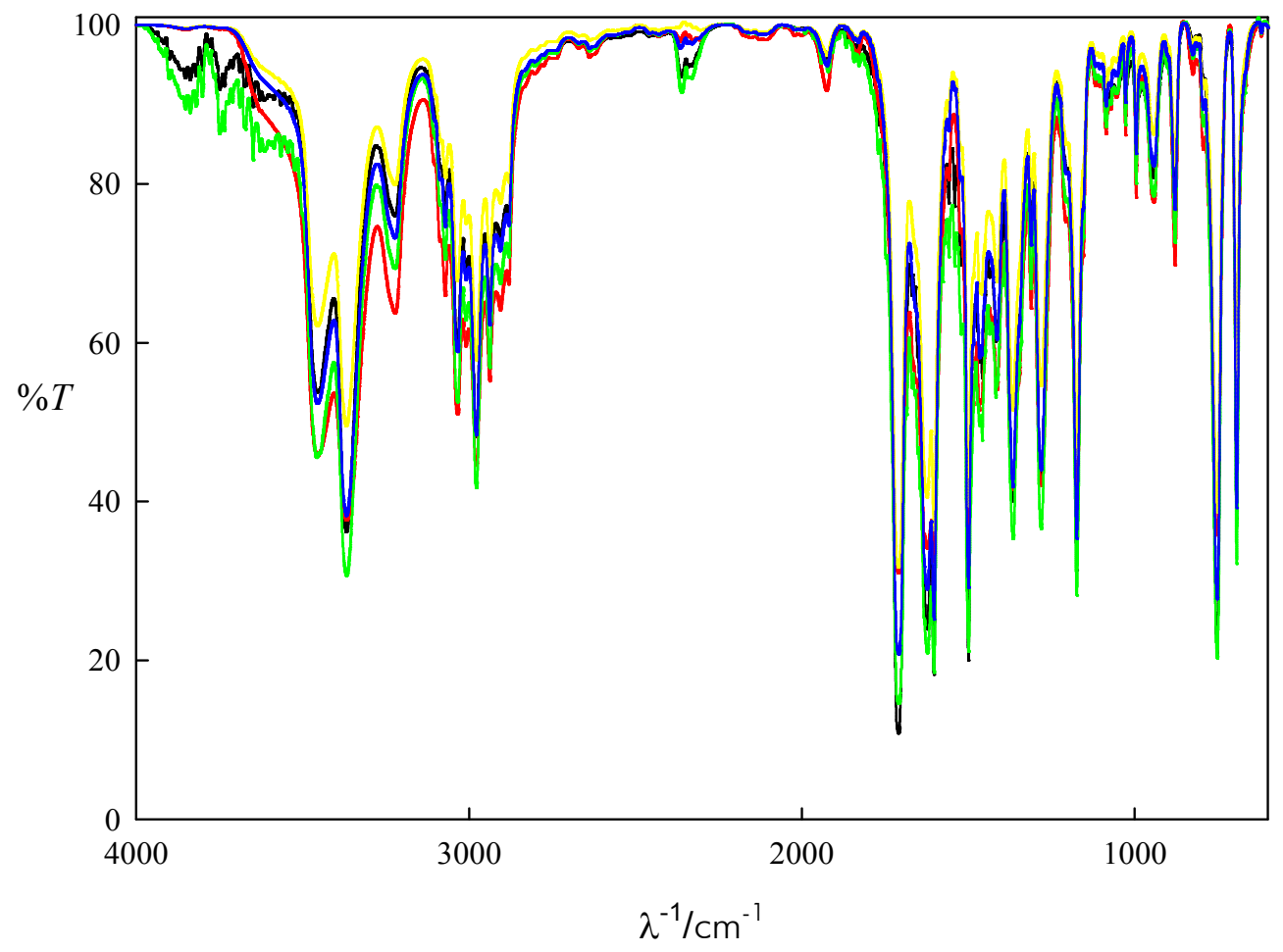

Ilustración C-7: espectro infrarrojo de la mezcla anilina (1) +2-butanona (2), $x_{1}=0,4959$ 


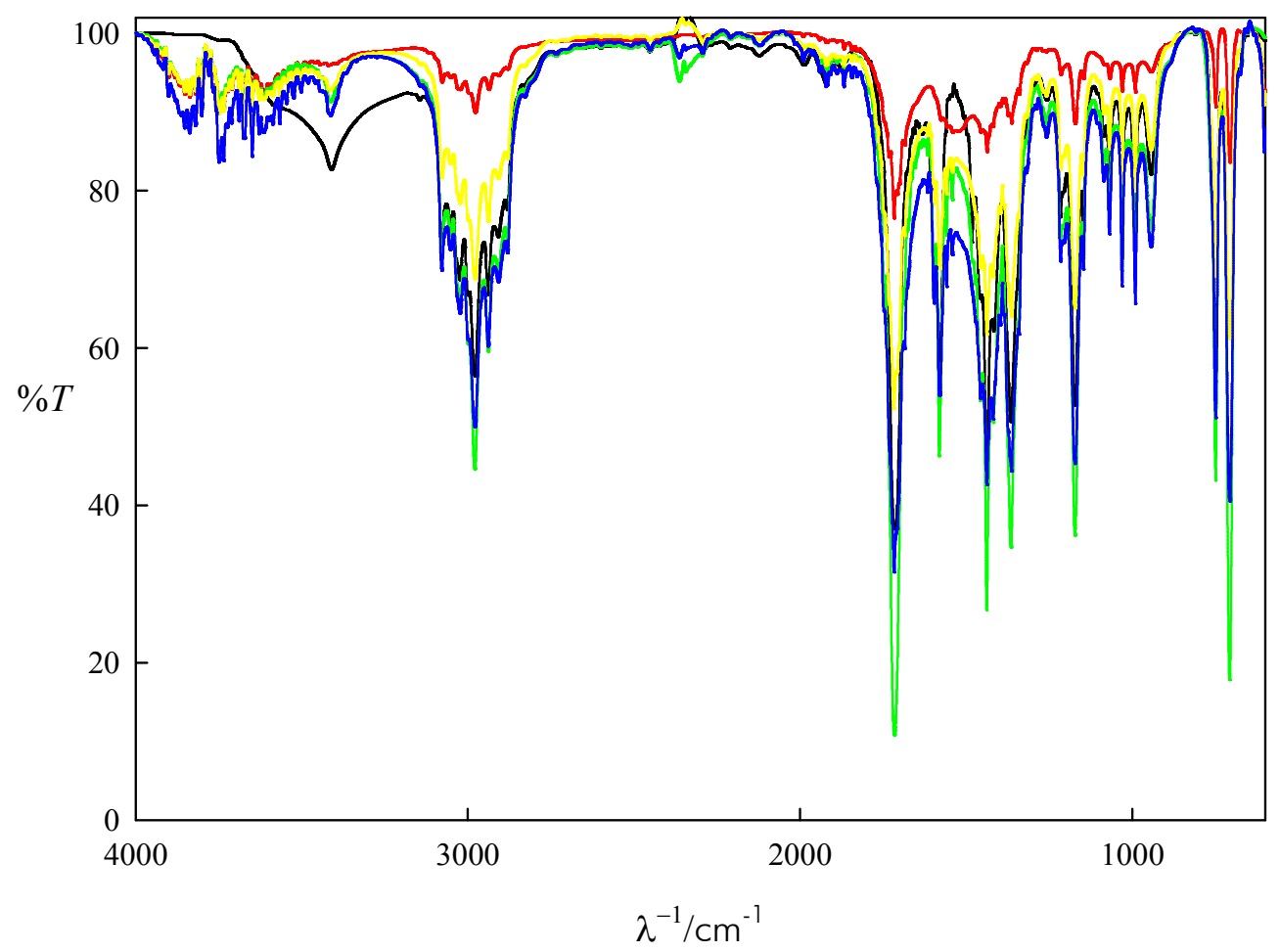

Ilustración C-8: espectro infrarrojo de la mezcla piridina (1) +2-butanona (2), $x_{1}=0,5000$

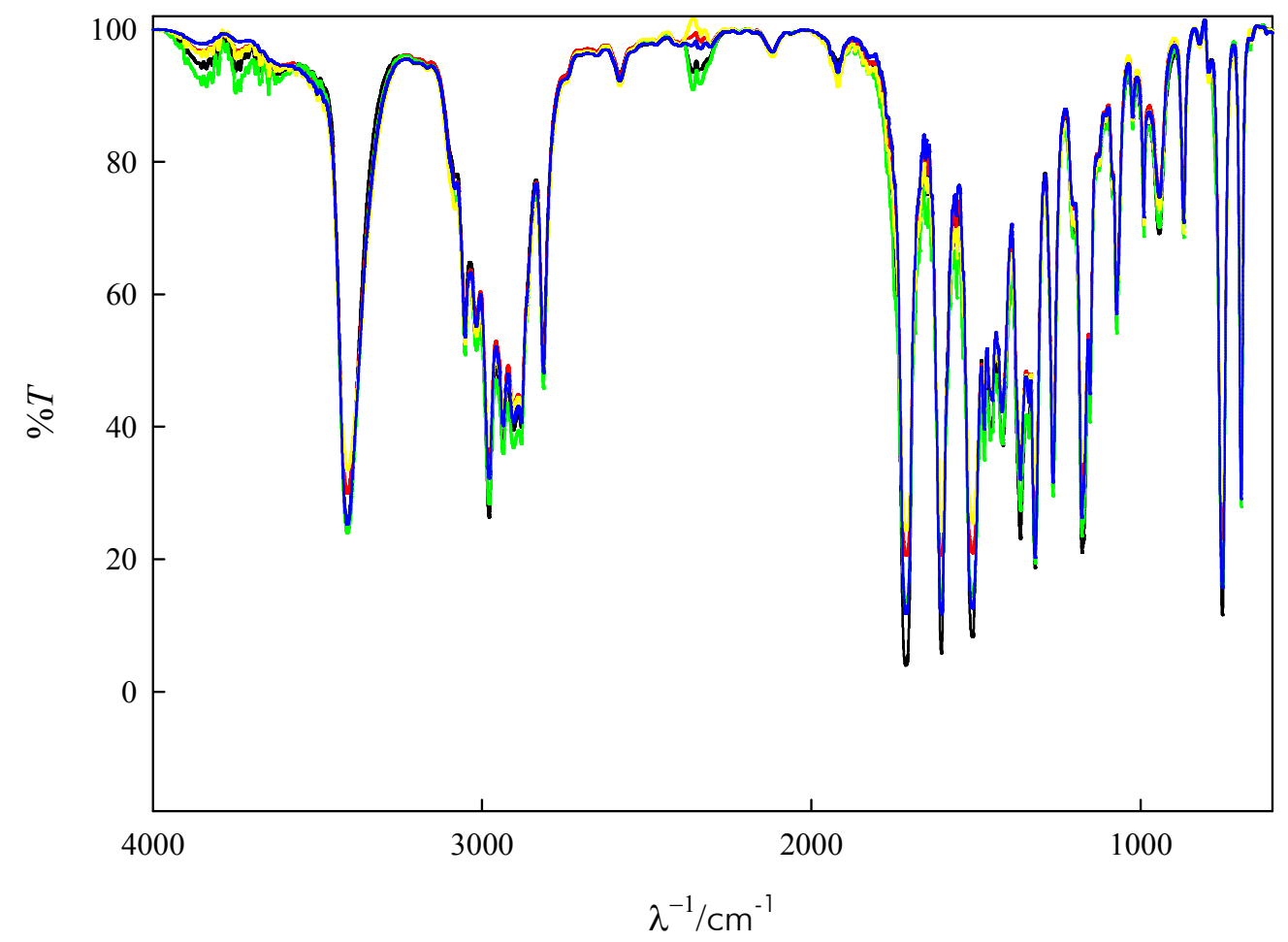

Ilustración C-9: espectro infrarrojo de la mezcla $N$-metilanilina (1) +2-butanona (2), $x_{1}=0,4937$ 


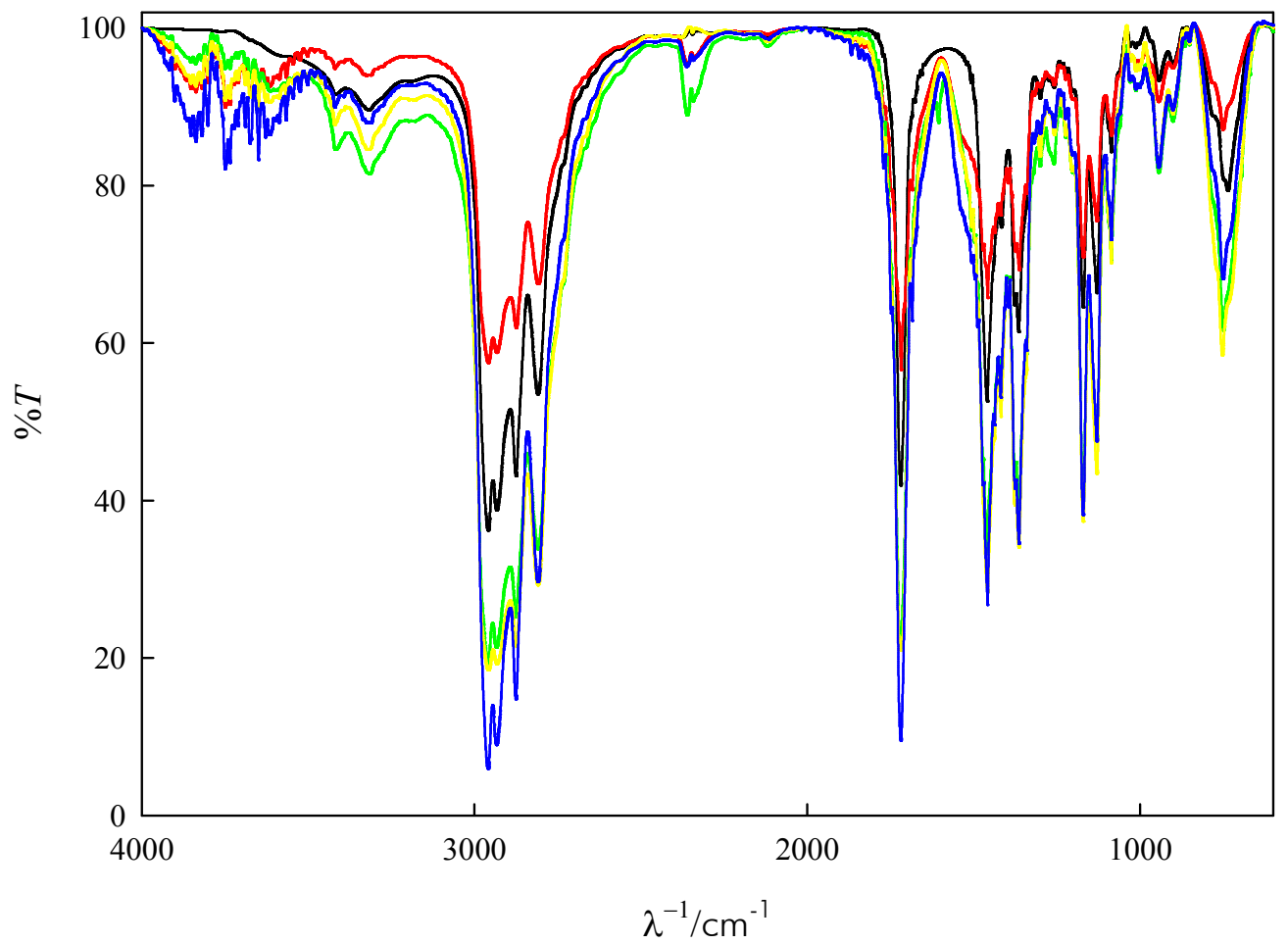

Ilustración C-10: espectro infrarrojo de la mezcla di- $n$-propilamina (1) +2-butanona (2), $x_{1}=0,4905$

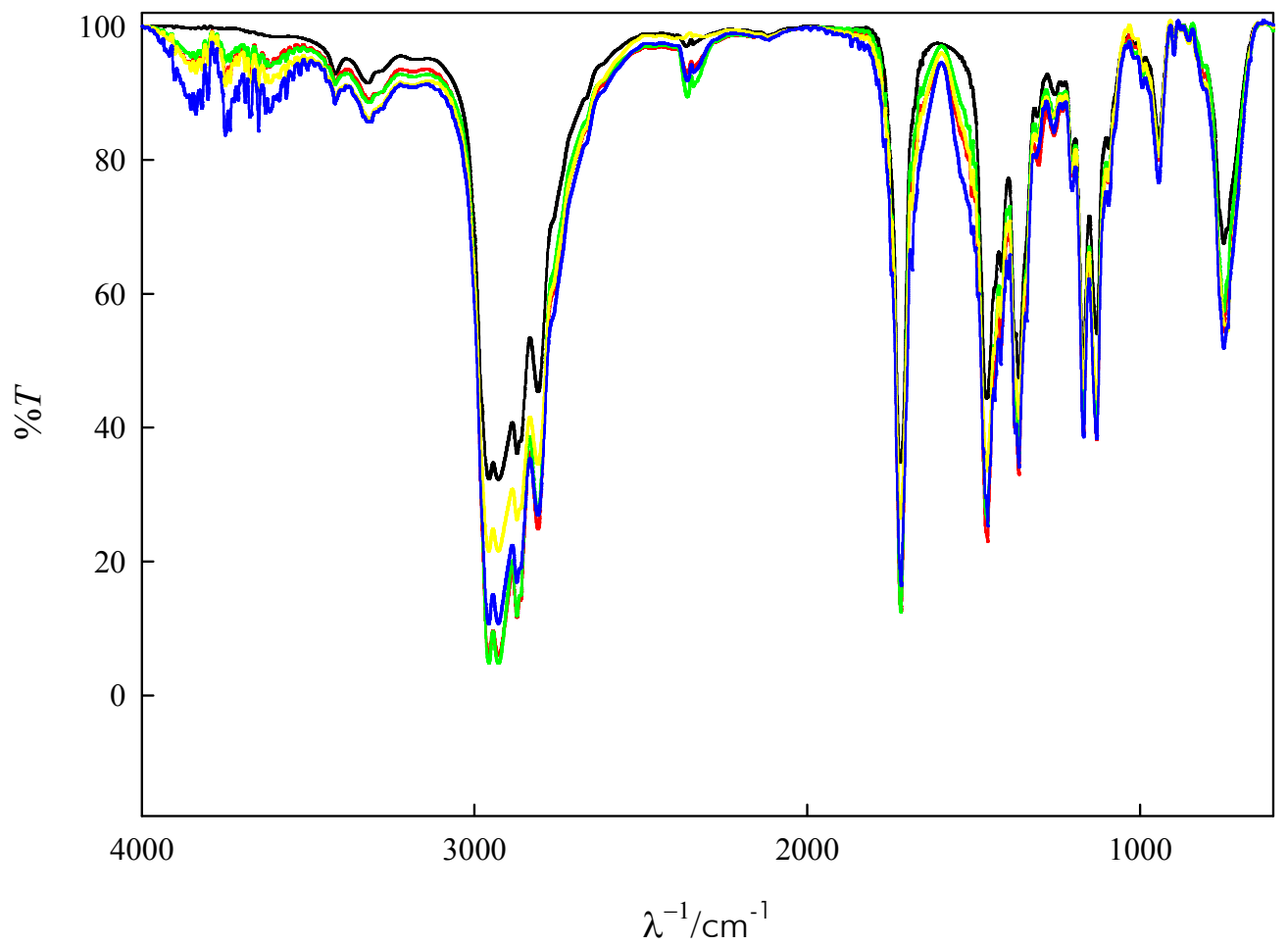

Ilustración C-1 1: espectro infrarrojo de la mezcla di- $n$-butilamina (1), +2-butanona (2), $x_{1}=0,4983$ 


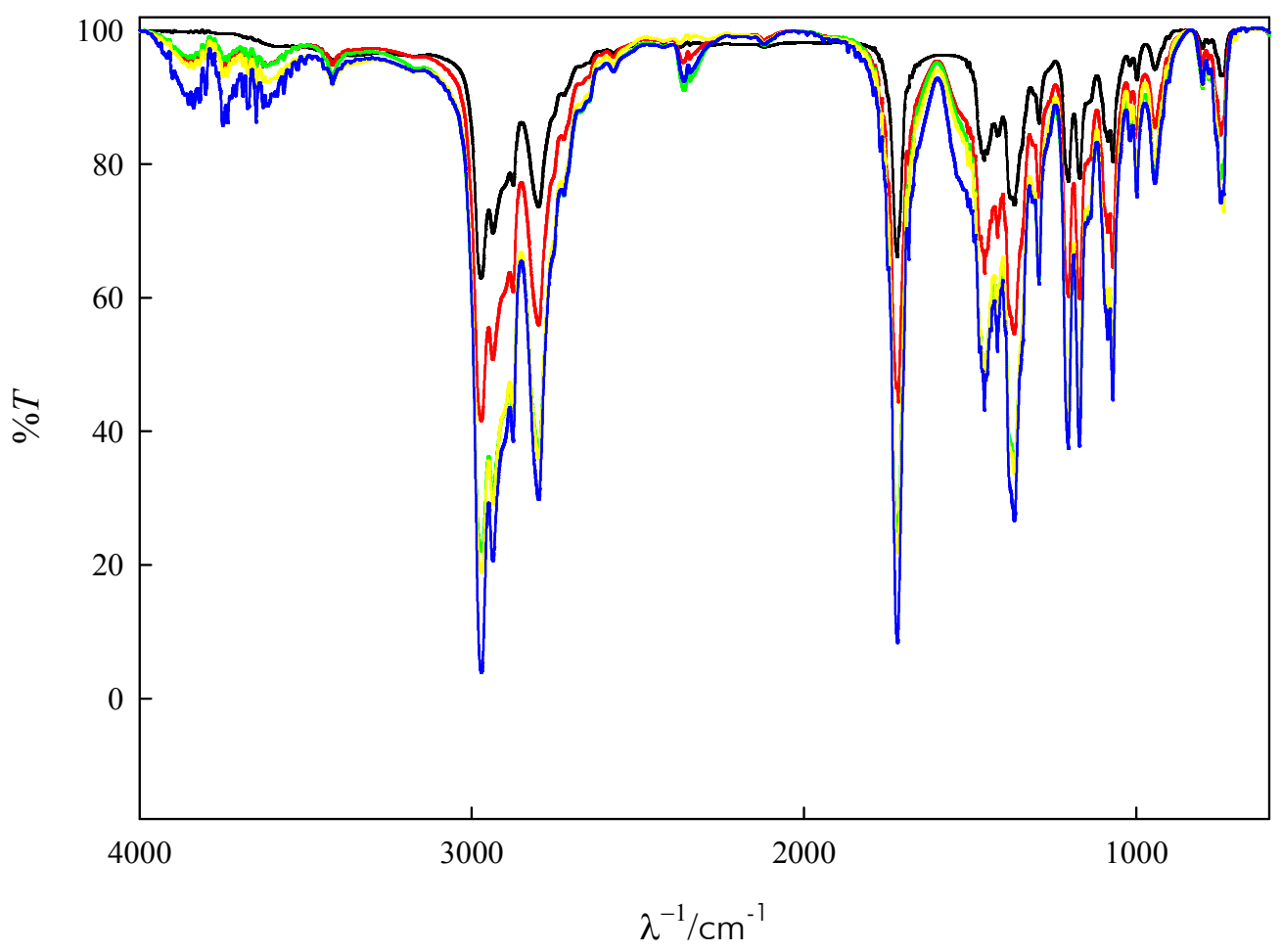

Ilustración C-12: espectro infrarrojo de la mezcla $N, N, N$-trietilamina (1) +2-butanona (2), $x_{1}=0.4550$

En la tabla siguiente se muestran, para aquellas mezclas en las que se detectaron iminas, el número de ondas $1 / \lambda$ de las bandas asociadas a los enlaces $\mathrm{C}=\mathrm{O}$ y $\mathrm{C}=\mathrm{N}$ y el cociente entre las absortancias de dichos enlaces. Se observa que la intensidad relativa del pico del enlace $\mathrm{C}=\mathrm{N}$ crece en el transcurso del tiempo hasta alcanzar un valor casi constante, lo que indica que la reacción química ha llegado al equilibrio.

Tabla C-1: cocientes entre absortancias y frecuencias de los picos de los modos de tensión de los enlaces C=O y $\mathrm{C}=\mathrm{N}$

\begin{tabular}{|c|c|c|c|}
\hline$t$ /horas & $1 / \lambda_{\mathrm{C}=0} / \mathrm{cm}^{-1}$ & $1 / \lambda_{\mathrm{C}=\mathrm{N}} / \mathrm{cm}^{-1}$ & $\tau_{\mathrm{C}=\mathrm{N}} / \tau_{\mathrm{C}=\mathrm{O}}$ \\
\hline \multicolumn{4}{|c|}{ 2-butanona + benzilamina } \\
\hline 0 & 1714 & 1660 & 0.7080 \\
\hline 24 & 1714 & 1660 & 1.2610 \\
\hline 72 & 1716 & 1660 & 1.4946 \\
\hline 96 & 1714 & 1660 & 1.4749 \\
\hline 168 & 1716 & 1654 & 1.4831 \\
\hline \multicolumn{4}{|c|}{ 2-butanona+ciclohexilamina } \\
\hline 0 & 1716 & No hay & 0 \\
\hline 24 & 1716 & 1660 & 0.5730 \\
\hline 72 & 1716 & 1660 & 0.8033 \\
\hline 96 & 1716 & 1660 & 0.7918 \\
\hline 168 & 1716 & 1660 & 0.9220 \\
\hline \multicolumn{4}{|c|}{ 2-butanona+hexilamina } \\
\hline 0 & 1718 & 1660 & 0.7001 \\
\hline 24 & 1716 & 1662 & 0.8005 \\
\hline 72 & 1716 & 1660 & 0.8908 \\
\hline 96 & 1718 & 1662 & 0.8723 \\
\hline 168 & 1718 & 1662 & 0.9295 \\
\hline
\end{tabular}


ANEXO C 


\section{BIBLIOGRAFÍA}

[1]: Redlich, O; Kister, A.T.; Ind. Eng. Chem. 40 (2) (1948), 345.

[2]: Bevington, P.R.; Robinson, D.K.; "Data Reduction and Error Analysis for the Physical Sciences", Thrid edition, Mc Graw Hill, New York (2003).

[3]: Kashiwaya, Y.; Takeshima, T.; Mori, N.; Nakashima, K.; Clarke, K.; Veech, R.L.; PNAS 97, 10, (2000), 5440-5444.

[4]: International Union of Pure and Applied Chemistry. "ketones". Compendium of Chemical Terminology Internet edition.

[5]: McElroy, W.D.; Seliger, H.H.; Advan. Enzymol., 25, (1963), 119.

[6]: Ge, X.; Wexler, A. S.; Clegg, S.L.; Atmospheric Env., 45, (2011), 524.

[7]: Weisburger, J.H.; Mutation Res., 9-20, (2002), 506.

[8]: Tavard,C. ; Thèse Sciences, Sorbonne, Paris, (1966).

[9]: Moccia, R.; J. Chem. Phys., 40, (1964), 2164,2176 y 2186.

[10]: Patai, S.; "The Chemistry of the Amino Group", Interscience Publishers, Great Britain, (1968).

[1 1]: Sänger, R.; Steiger, O.; Gächterk, J.; Helv. Phys. Acta, 5, (1932), 200.

[12]: Lefevre, R.J.W.; Russell, P.; Tans. Faraday. Soc., 43, (1947), 574.

[13]: Pauling, L. General Chemistry, Dover Publications, Inc., New York, (1969), 234.

[14]: Pimentel, G.C.; McClellan, A.L.; The Hydrogen Bond, W. H. Freeman and Company, San Francisco (1960).

[15]: Bertagnolli, H. ; Engelhardt, T. ; Chieux, P. ; Ber. Bunsenges. Phys. Chem., 90, (1986), 512.

[16]: Wolff, H.; Gamer, G.; J. Phys. Chem., 76, (1972), 871.

[17]: Landeck, H.; Wolff, H.; Götz, R.; J. Phys. Chem., 81, (1977), 718.

[18]: Megiel, E.; Kasprzycka-Guttman, T.; Jagielska, A.; Wroblewska, L.; J. Molecular Struct., 569, (2001), 111.

[19]: Wolff, H.; Schiller, O.; Fluid Phase Equilib., 22, (1985), 185.

[20]: Sagarik, K.; Spohr, E.; Chem. Phys., 199, (1995), 73. 
[21]: Patai, S.; "The Chemistry of the Carbonyl Group", Interscience Publishers, Great Britain, (1966).

[22]: Walsh, A.D.; Trans. Faraday Soc., 42, (1946), 56.

[23]: Takagi, K., Oka, T.; J. Phys. Soc. Japan, 18, (1963), 1174.

[24]: Kilb, R.W., Lin, C.C., Wilson, E. B. Jr.; J. Chem. Phys., 26, (1957), 1695.

[25]: Turner, D.W.; Determination of Organic Structures by Physical Methods, Vol2; Academic Press, New York, (1962), 339-400.

[26]: Glockler, G.; J. Phys. Chem., 62, (1958), 1049.

[27]: Nakanishi, K.; Goto, T.; OACl, M.; Bull. Chem. Soc. Japan, 30, (1957), 403.

[28]: Siggia, S.; Hanna, J.G.; Serencha, N.M.; Anal. Chem, 35, (1963), 575.

[29]: Goddu, R.F.; en la referencia 11 de The Chemistry of alkanes, Patai, S., 347.

[30]: Nakanishi, K.; Infrared Absorption Spectroscopy-Practical, Holden Day, San Francisco, and Nankodo, Tokio (1962).

[31]: Bellamy, L. J.; The Infra-red Spectra of Complex Molecules, 2nd ed., Methuen, London, (1958).

[32]: S. Siggia; E. Segal; Anal. Chem., 25, 830 (1953) 

